

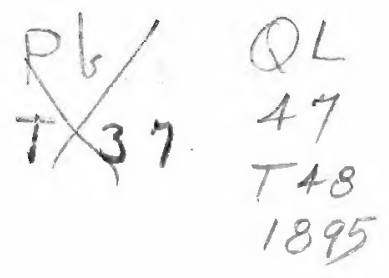

THE

Haskell q. Flamer library THIS BOOK IS THE GIFT OF

Dr. Pierre Augustine Fish Professor of Comparative Physiology, Pharmacology, and Therafenties. Cect.14. 1903.

2767 
Cornell University Library

QL 47.T48 1895

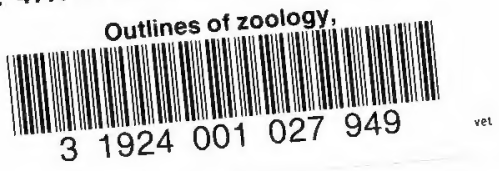


. 
Pa fiak.

Jume 27, 1896 

Pentland's Students' Manuals.

OUTLINES OF ZOOLOGY. 


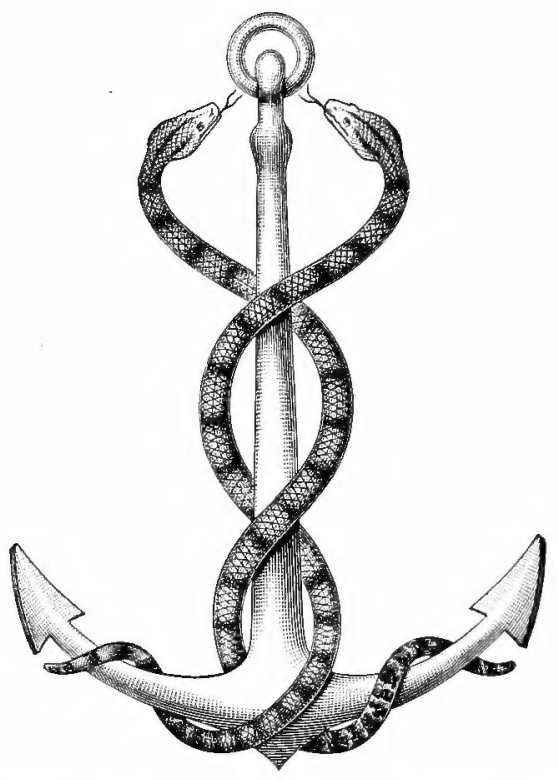

NUNQUAM AIIUD NATURA, ALIUD SAPIENTIA DICIT. 


\section{OUTLINES OF}

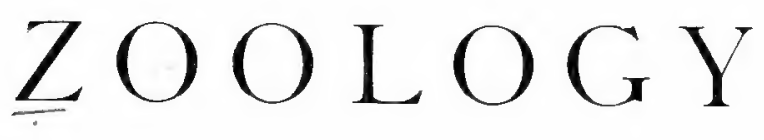

BY

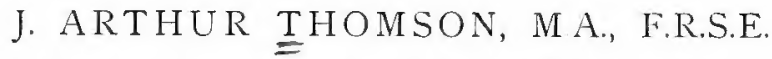

LECTURER ON ZOOLOGY AND BIOLOGY IN THE SCHOOL OF MEDICINE, EDINBURGH; JOINT-AUTHOR OF "THE EVOLUTION OF SEX"; AUTHOR OF "THE STUDY OF ANIMAL LIFE."

SECOND EDITION, REVISED AND ENLARGED, WITH 266 ILLUSTRATIONS.

NEW YORK:

D. A PPLETON \& COY.

I 895 . 


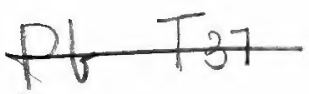

No -168

PRINTED FOR YOUNG J. PENTLAND: EDINBURGH, II TEVIOT PLACE. LONDON, $3^{8}$ WEST SMITHFIELD, E.C.

Q $L$

47

748

1895 


\section{PREFACE TO THE SECOND EDITION.}

THE favourable reception granted to the first edition of this book has already led to a demand for a second. In preparing this, I have endeavoured to take advantage of the suggestions of kindly critics. The book is intended to serve as a manual which students of Zoology may use in the lecture-room, museum, and laboratory, and as an accompaniment to several well-known works, cited in the Appendix, most of which follow other modes of treatment.

To numerous authorities I acknowledge an obvious indebtedness, a detailed recognition of which would be out of place in a book of this kind. But I must thank Dr. Ramsay Traquair for helping me with the chapter on Fishes, Dr. Beard for revising the pages on the general structure of Vertebrates, and Dr. Gilchrist for suggestions as to the chapter on Molluscs. For the imperfections in these chapters I am, of course, alone responsible.

In preparing this revised edition I have been assisted throughout by Miss Marion Newbigin, B.Sc. She has 
also written the chapter on Comparative Physiology. To my assistant, Mr. R. A. Staig, I am indebted for the index.

I wish to express my thanks to my artist friend, Mr. William Smith, for the carefulness with which he has executed many of the illustrations; and I am indebted to Dr. Traquair for allowing me to figure some of the specimens in the Edinburgh Museum of Science and Art. In regard to these illustrations, I may say that in almost every case they have either been derived from original memoirs and works of reference, or drawn from specimens. Of course, no one who has worked with such excellent practical books as that by Marshall and Hurst or Parker's Zootomy, can help being assisted by them in preparing analogous diagrams; but I have refrained from incurring any but an absolutely necessary debt to such books, except in the case of Figure 2I5, which Messrs. Macmillan have kindly permitted me to make use of.

SCHOOL OF MEDICINE,

$$
\text { J. A. T. }
$$

EdINBURGH, March I 895 . 


\section{CONTENTS.}

GENERAL.

CHAPTER I.

General Survey of the Ayimal Kingdom, . . . I

CHAPTER II.

PHYSIOLOGY,

CHAPTER III.

Morphology, .

CHAPTER IV.

EMBRYOLOGY, .

CHAPTER V.

PALFONTOLOGY, 
INVERTEBRATES.

CHAPTER VII.

PAGE

PróTOZOA,

CHAPTER VIII.

SPONGES,

CHAPTER IX.

Ceelentera,

CHAPTER X.

UNSEGMENTED WORMS,

CHAPTER XI.

Segmented Worms or Annelids, . . . . $\quad$ I86

CHAPTER XII.

ECHINODERMS,

\section{CHAPTER XIT.}

227

CHAPTER XIII.

Crustaceans, .

CHAPTER XIV.

Peripatus, Myriopons, and Insects, 285

CHAPTER XV.

Arachnoidea and Palfostraca,

CHAPTER XVI. 


\section{VERTEBRATES.}

CHAPTER XVII

PAGF,

HEMICHORDATA,

CHAPTER XVIII.

UROCHORDATA

CHAPTER XIX.

Cephalochordata,

\section{CHAPTER XIX.}

CIAAPTER XX.
Structure and DeVelopment of Vertebrates,

410

CHAPTER XXI.

Cyclostomata,

CHAPTER XXII.

FISHES,

475

CHAPTER XXIII.

Aмphibia,

CHAPTER XXIV.

Repriles,

560

CHAPTER XXV.

BIRDS, .

595

CHAPTER XXVI. 


\section{GENERAL.}

PAGE

CHAPTER XXVII.

Comparative Physiology, . . . . . 731

CHAPTER XXVIII.

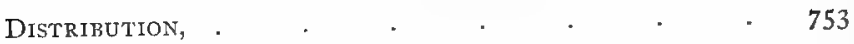

CHAPTER XXIX.

ETIOLOGY,

APPENDIX ON BOOKS, . $\quad . \quad$. $\quad . \quad 773$

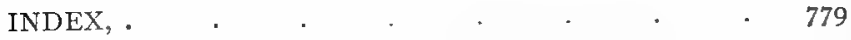




\section{LIST OF ILLUSTRATIONS.}

FIG.

PAGE

I. Diagrammatic expression of classification in a genealogical tree, I I

2. Structure of the cell. (CARNOY), . . . . 44

3. Fertilised ovum of Ascaris. (Boveri), . . . 44

4. Diagram of cell division. (BOVFrI), . . . 45

5. Karyokinesis. (Flemming), . . . . 47

6. Diagrammatic expression of alternation of generations, $\quad 55$

7. Diagram of ovum, showing diffuse yolk granules, . $\quad 57$

8. Forms of spermatozoa, . . . . . . 58

9. Diagram of maturation and fertilisation. (GEDDFs and

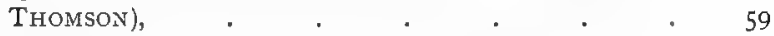

10. Spermatogenesis and polar bodies. (HERTWig and WEISMANN), . . . . . $6 \mathrm{I}$

I 1. Fertilisation in Ascaris megalocephala. (BoverI), , , 62

I2. Modes of segmentation, . . . . . 64

13. Life history of a coral, Monoxenia Darwiniz. (HACkEL), 66

14. Embryos (I) of bird; (2) of man. (His). . . . 69

15. Gradual transitions between Paludiua Neumayri and Ialudina Harnesi. (NEUMAYR), . . . . . 77

16. Life history of $A$ mazba, . . . . . . 87

17. End-to-end union of Gregarines. (FRENZEL), . . 88

18. Life history of Gregarina. (BüтschLI), . . . 89

I9. Life history of Monocystis. (Bütschli), . . . 90

20. Paramacium. (BÜTScHLI), . . . $9 \mathrm{I}$

21. Conjugation of Paranocium aurelia-four stages.

(Maupas), . . . . . . . 92

22. Diagrammatic expression of process of conjugation in Paramacium aurelia. (MAUPAS), . . . . 93

23. Vorticella. (BütschLI), . . . . . . 95

24. Volvox globator. (CоHN), . . . . . . 96

25. Diagram of Protomyxa aurantiaca. (HÆCKEL), . $\quad 99$

26. Formation of shell in a simple Foraminifer. (DrErER), - IOO 
FIG.

PAGE

27. Polystomella. (SCHuLTZE),. - Ior 28. A pelagic Foraminifer-Hastigerina (Glabigerina) Murrayi. (BRADY), . . . . . . . . 102

29. Optical section of a Radiolarian (Actinomma). (HECKEL), IO3 30. A colonial flagellate infusorian-Proterospongia Hackelii. (SAVILIE KENT), . . . . . . 104

31. Simple sponge-Ascetta primordialis. (HACKEL), II7

32. Section of a sponge. (F. E. SchulzE), . , II8

33. Diagram showing types of canal system of sponge. (Korschelt and Heider), . . . . II9

34 Development of Sycandra raphanus. (F. E. ScHuLzF), - I23

35. Diagrammatic representation of development of Oscarella lobularis. (HEIDER), . . . .

36. A. Young Dicyema. B. Female Orthonectid (Rhopalura Giardii). (Whitman and JuLIN), , . . I 27

37. Salinella. (FRENZEL),

38. Diagram of Coelenterate structure, . . . . . I32

39. Hydra, hanging from water-weed. (GREENE), . . I34

40. Minute structure of Hydra. (PARKER and JICKELI), . I37

4I. Development of Hydra. (BRAUER), . . . 139

42. Surface view of Aurelia. (ROMANEs), . . . I44

43. Vertical section of Auralia. (Clads), . . . I45

44. Diagram of life history of Aurelia. (HAEKEL), . . I46

45. Luternaria. (KorotnefF), . . . . I48

46. Structure of sea anemone. (ANDRES), . . . I50

47. Section through sea anemone. (ANDRES), . . I 5 I

48. Diagrammatic sections of Zoantharian and Alcyonarian. (Chun), . . . . . . I53

49. Diagram of a Gymnoblastic Hydromedusa. (ALLMAN), . I56

50. Diagrammatic figure of a simple Turbellarian, . . . I63

5r. Diagrammatic expression of part of the structure of a simple Turbellarian,

52. Structure of liver fluke. (SOMMER), . . . I65

53. Reproductive organs of liver fluke. (SOMMER), , . I66

54. Life-history of liver fluke. (THOMAS), . . . I68

55. Diagram of reproductive organs in Cestode joint. (LEUCKART), . . . . . . . I72

56. Life history of Tania solium. (LEUCKART), . I74

57. Transverse section of the Nemertean Drepanophorus latus.

(BURGER), . . . . . . . I77

58. Transverse section of a Nemertean (Carinella). (BURGER), I78

59. Diagram of structure of a Nematode (Oxymris). (GALEB), I 82 
FIG.

60. Anterior region of earthworm. (HERING),

1'AGE

6I. Transverse section of earthworm. (ClaparÈde), .

I 90

62. Reproductive organs of earthworm. (HERING), .

193

63. Stages in the development of earthworm. (WILsON),

195

64. Arenicola piscatorum. (CUNNINGHAM and RAMAGE),

198

65. Anterior part of nervous system in Arenicola. (VoGT and YUNG), ,

66. Dissection of anterior region of Arenicola. (Cosmovici), .

67. Cross section of Arenicola. (Cosmovici), .

202

68. Development of Polygordizes. (FRAIPONT),

69. Parapodium of a marine Polychæte, Heteronereis. (QUATREFAGES), . . . . . . 210

70. Transverse section of leech. (A. G. BOURNE), . . 217

7 I. Dissection of leech, . . . . . . 219

72. Development of Sagitta. (O. Hertwig), . . . 222

73. Interior of Brachiopod shell, showing calcareous support for the "arms." (DAViDSON), . . . . 226

74. Pluteus larva with rudiment of adult. (Johannes Múller), 228

75. Alimentary system of starfish. (MúlleER and Troschel), 23 I

76. Diagrammatic cross section of starfish arm. (LUDWIG), . 233

77. Ventral half of sea urchin. (TiEDEMANN), . $\quad 238$

78. Dissection of Holothurian. (HUNTER), . . . 242

79. Diagrammatic vertical section through disc and base of one of the arms of Antedon rosaceus. (Milnes Marshall),

80. Stages in development of Echinoderms. (SELENKA), . 247

8I. Forms of Echinoderm larva. (MÜLLER). . . . 249

82. Appendages of Norway lobster, . . . . 258

83. A single eye element or ommatidium of the lobster. (PARKer), . . . . 260

84. Longitudinal section of lobster, showing some of the organs, 262

85. Female reproductive organs of crayfish. (Suckow), - 265

86. Section through the egg of Astacus after the completion of segmentation. (REICHENBACH), . . 266

87. Longitudinal section of later embryo of Astacus. (REICHENBACH), . '. . .

88. Embryo of crayfish, flattened out, with removal of yolk.

(REICHENBACH), . . . . . . 268

89. Acorn shell (Balanus tintinnabulum). (DARWIN), , 273

90. Development of Sacculina. (DELAGE), . . . 274

9I. Zoæa of common shore crab (Carcinus manas). (FAXON), $28 \mathrm{r}$

92. External form of Peripatus. (BALFOUR), . . . 286

93. Dissection of Peripatus capensis. (Balrour), . . 288 
FIG.

PAGE

94. Embryo of Peripatus capensis. (KORSCHeIT and HeIDER), 289 95. Mouth appendages of cockroach. (DUFOUR), . . 294 96. Transverse section of insect. (PACKARD), . . 296 97. Head and mouth parts of bee. (CHeshire), . . 300 98. Nervous system of bee. (CHESHIRE), . . . 302 99. Food canal of bee. (Cheshire), . . . . 303 I00. Joints of cockroach's leg, . . . . . 308 IOI. Young May fly or Ephemerid. (EAton), . . 309 I02. Diagrammatic cross section of an Invertebrate, with a primary body cavity. (ZiegleR), . . . 316

I03. Diagrammatic cross section of an Invertebrate, with a secondary and a primary body cavity. (ZIEGLER), . 316

I04. Diagram of insect embryo. (KoRsCHELT and HElDER), . 320 105. Scorpion, . . . . . . . 328 ro6. Section of lung book. (MACLEOD), . . . 333 I07. Limulus or king crab, . . . . . . 338 108. Young Limulus. (WalcotT), . • . . 340 109. Vertical cross section of a Trilobite, Calymene. (WAI cotT), 34 I I IO. I'eal mollusc. (LANkesteR), . . . . 344 I I I. Stages in Molluscan development, . . . . 344 I12. Chiton. (PRÉTRE), · . . . . 345

I I3. Dorsal view of nervous system of Chiton. (PELSENEER), 346 I14. Proneonenia. Nervous system. (Hubrecht), . . 346 II 5. Dissection of Helix pomatia. (LeUCKART), . . 355 II6. Diagram of larva of Paludina. (ERLANGER), . . 356 II7. Stages in Molluscan development, . . . . 360 II8. Nervous system of Molluses, . . . . . 366 I I9. Structure of Anodonta. (RANkin), . . . . 368 120. Development of Anodonta. (GøTтE), . . . 37 I 12I. Diagram of the structure of Sepia. (PElseneer), - 380 I22. Diagram of circulatory and excretory systems in a Decapod like Sepia. (PELSENEER), . . . . $38 I$ I23. Section of shell of Nautilus. (LENDENFELD), . . $38_{4}$ 124. Male of Balanoglossus kowalewski. (BATESON), . . 392 I25. Transverse section through gill slit region of Ptychodera minuta. (SPENGEL), . . . . 394

I26. Development of Balanoglossus. (BATESON), · . 396 I27. Tornaria larva, from the side. (SPENGEL), • . 397 128. Dissection of ascidian. (HERDMAN), . . . 403 I29. Dissection of ascidian. (HERDMAN), . . . 405 130. Young embryo of ascidian (Clavelina). (VAN BENEDEN and JUI.IN), 
FIG.

PACE

I3I. Section of newly fixed larva of Clavellina. (SEELIGER), .

132. Lateral view of Amphioxus. (RAY LANKESTER),

4 II

I33. Transverse section through pharyngeal region of $A m p h i$ oxus. (RAY LANKESTHR),

4 I 2

I34. Cross section of Amphioxus through the gill-slit region. (BOVERI and HA'scheK),

I35. Early stages in the development of Amphioxus. (IIATSCHEK),

136. Three larval stages of Amphioxus. (RAy LANKESTEl and WILLEY),

I37. Transverse section through an Elasmobranch embryo (diagrammatic). (ZIEGLER), . "

138. Longitudinal section of brain of young dogfish (diagrammatic). (GASKELL),

I39. Origin of pineal body. (BEARD), .

I40. Diagram of the parts of the brain in Vertebrates. (GASKEI.L),

141. Diagrammatic section of spinal cord,

142. Diagram of the eye,

143. Development of the eye. (BALFour and HERTwIG),

I44. Origin of lungs, liver, and pancreas in the chick. (GCETE),

145. Transverse section through a Teleostean embryo (diagrammatic). (ZIEGLER),

146. Diagram of circulation. (Leunis),

147. Transverse section through a Vertebrate embryo. (SEMON), .

I48. Urinogenital system. (BALFOUR), .

149. Mammalian ovum. (HERTwiG), .

150. Median longitudinal section of anterior end of Myxine.

15 I. Respiratory system of hag, from ventral surface,

152. Longitudinal vertical section of anterior end of larval

$$
\text { lamprey. (BALFOUR), . . . . . }
$$

I53. Restored skeleton of Palcospondylus. (TRAQUAIR), . 474

I54. Under surface of skull and arches of skate. (W. K.

I55. Side view of skate's skull. (W. K. PARKER), · . 480 I56. Skeleton of skate, . . . . . . 48

157. Dissection of nerves of skate, . . . . $\quad 483$

158. Side view of chief cranial nerves of Elasmobranchs. 
FIG.

159. Spiral valve of skate.

(T. T. PARKER),

I60. Upper part of the dorsal aorta in the skate. (Monro), .

I6I. Heart and adjacent vessels of skate. (MONRo), .

I62. Urinogenital organs of male skate,

I63. Urinogenital organs of female skate. (MONRO). .

I64. Elasmobranch development. (BALFour),

165. External characters of a Teleostean-a carp. (ILENIS), .

166. Caudal vertebra of haddock,

I67. I)isarticulated skull of cod,

168. Pectoral girdle and fin of cod,

I69. Diagram of Teleostean circulation. (NuHr),

501

I7o. Young skate. (BEARD), .

I7 I. Outline of Acanthodes sulcatus. (TRAQUAIR), . . 507

I72. Pterichthys milleri. (TRAQUAIR), . . . 509

I73. Skeleton of Ceratodus fin. (GegenbauR), . . 5 II

I74. Head region of Protopterus. (W. N. PARKER), . . 514

I75. Vertebral column and pelvic girdle of bull frog, . . 532

I76. Skull of frog-upper and lower surface. (IV. K. PARkeR), 533

177. Pectoral girdle of frog. (EckER), . . . 535

I78. Side view of frog's pelvis. (EСKER), . . . 536

I79. Brain of frog. (ECKER), . . . . . 537

I80. Nervous system of frog- (ECKER), . . $\quad 53^{8}$

18I. Arterial system of frog. (ECKER), . . . 542

182. Venous system of frog. (ECKER), . . . . 544

183. Urinogenital system of male frog. (ECKER), . . 548

184. Urinogenital system of female frog. (EckER). . . 548

185. Division of frog's ovum. (ECKER), . . 550

186. Gastrula stage of newt. (HeRTwiG), . - $55 \mathrm{I}$

187. Dissection of tadpole. (Milnies Marshall and Bles), - 552

I88. Life history of frog. (BRFHM), . . . $\quad 554$

I89. Cæecilian (Ichthyophis) with eggs. (SARASIN), • . 557

190. External appearance of tortoise, . . 563

19I. Carapace of tortoise, . . . 564

192. Internal view of skeleton of turtle, . . . 565

193. Dissection of Chelonian heart. (HuxLEY), . . 566

194. Heart and associated vessels of tortoise. (NUHN). 567

195. Hatteria or Sphenodon. (HAYEK), . . 568

196. Side view of skull of Lacerta. (IV. K. PARkER), $\quad 572$

I97. Heart and associated vessels of lizard. (NUHN), . 574

198. Lung of Chameleo vulgaris, showing air sacs. (WIEDERSHEIM),

199. Snake's head. (NuHN), 
FIG.

200. Skull of grass snake. (W. K. PARKeR), .

PAGE

20I. Lower surface of skull of a young crocodile,

$5^{8} \mathrm{I}$

. $\quad 584$

202. Crocodile's skull from dorsal surface, . . . . 585

203. Half of the pelvic girdle of a young crocodile, . . 586

204. Origin of amnion and allantois. (BALFOUR), . 590

205. Comparison of pelvic girdles of cassowary, Iguanodon, and crocodile, .

206. Position of organs in a bird. (SelenkA), . 597

207. Diagrammatic section of young bird. (GADOw), . 598

208. Disarticulation of bird's skull. (GADOw), . 602

209. Under surface of gull's skull, . . . . 603

2 Io. Wing of dove, . . . . . $\quad . \quad .604$

2I I. Side view of pelvis of cassowary, . . . . . 605

212. Bones of hind leg of eagle, . . . . . 606

213. Brain of pigeon. (BRONN), . . . . 607

214. Diagrammatic section of cloaca of male bird. (GADow), . 608

215. Circulation of pigeon. (PARKER), . . . 609

216. Urinogenital organs of pigeon, . . . . 612

217. Diagrammatic section of egg. (Allen Thomson), . 6 I 3

218. Stages in development of chick. (MARshalL), . . 615

219. Diagrammatic section of embryo within egg. (KENNEL), 6I6

220. Pectoral girdle and sternum of swan, . . . 622

22I. Position of wings in pigeon at maximum elevation. (MAREY), . . . . . . . 623

222. Wings coming down. (MAREY), . . . . 624

223. Wings completely depressed. (MAREY), . . . 624

224. Segmentation of rabbit's ovum. (VAN Beneden), . 648

225. Development of hedgehog. Three early stages. (HuBrecht), 649

226. Two stages in segmented ovum of hedgehog. (HuBrECHT), 650

227. Development of foetal membranes. (HERTWIG), . . 652

228. Diagram of fotal membranes. (TURNER), . . 653

229. View of embryo of rabbit, with its foetal membranes.

(KENNEL), . . . . . . 657

230. Side view of rabbit's skull, . . . . . . 662

23I. Dorsal view of rabbit's skull, . . . . . . 663

232. Under surface of rabbit's skull, . . . . 664

233. Rabbit's fore leg, . . . . . . . . . 665

234. Rabbit's hind leg, . . . . . . . $\quad$. 665

235. Dorsal view of rabbit's brain. (KRAUSE), . . 667

236. Under surface of rabbit's brain. (KRAUSE), . . . 667

237. Diagram of cæcum in rabbit, . . . . 670

238. Duodenum of rabbit. (Claude Bernard), . . 67 I 
xviii

FIG.

PAGE

239. Circulatory system of rabbil. (PARKER and KRAUSE), . 672

240. Vertical section through rabbit's head, . $\because .675$

24I. Urinogenital organs of male rabbit, . . . 677

242. Urinogenital organs of female rabbit, . . . 677

243. Pectoral girdle of Echidna, . . . . 681

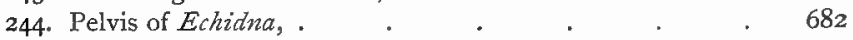

245. Urinogenital organs of male duckmole. (OWEN), . 683

246. Urinogenital organs of female duckmole. (Owen), . 683

247. Lower jaw of kangaroo, . . . . . $\quad .684$

248. Foot of young kangaroo, . . . . . . . $\quad$. 688

249. Foot of ox, . . . . . . . 694

250. Fore leg of pig, . . . . . . . 694

251. Side view of sheep's skull, with roots of back teeth exposed, 695

252. Stomach of sheep. (LEUNIS), . . . . 696

253. Side view of calf's fore leg, . . . . . $\quad 697$

254. Side view of lower part of pony's fore leg, . . 699

255. Side view of ankle and foot of horse, . . . 699

256. Side view of horse's skull, roots of back teeth exposed, . 700

257. Feet of horse and its progenitors. (NEUMAYR), . . 70 I

258. Fore limb of Balcenoptera, . . . . 705

259. Fore limb of whale (Megaptera longimana). (STRUTHERS), 706

260. Pelvis and hind limb of Greenland whale. (Struthers), 707

261. Vertebra, rib, and sternum of Balanoptera, . . 708

262. Lower surface of dog's skull, . . . . 7 I 2

263. Skeleton of fox bat, . . . . . 720

264. Skeleton of male gorilla, . . . . . . $\quad 723$

265. Skull of orang-utan, . . . . . . 726

266. Skull of gorilla, . . . . . . . . $\quad$. 727 
OUTLINES OF ZOOLOGY. 


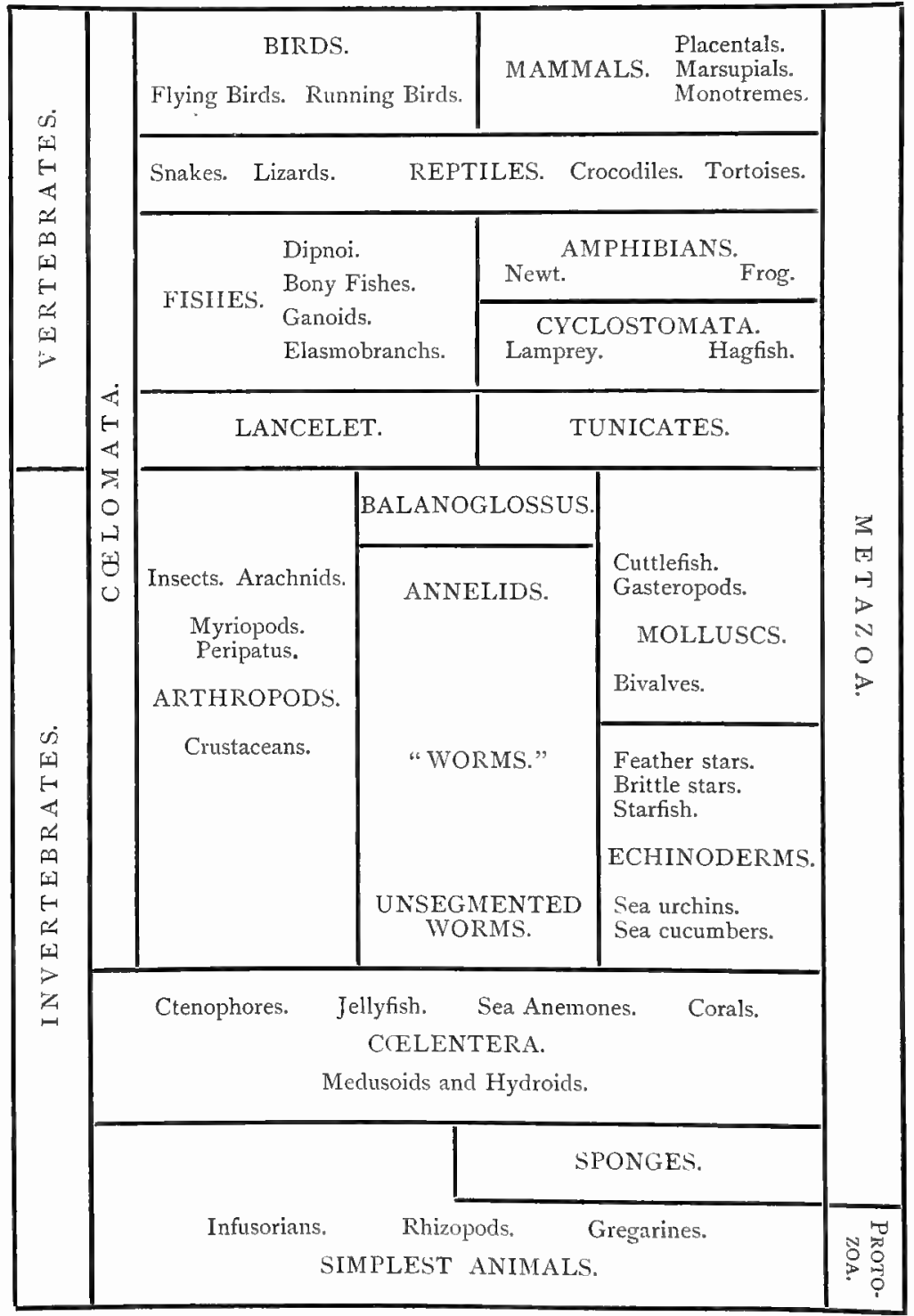




\section{OUTLINES OF ZOOLOGY.}

\section{CHAPTER I.}

GENERAL SURVEY OF THE ANIMAL KINGDOM.

At the outset of our study of Zoology it is useful to take a general survey of the "animal kingdom." Without some such bird's eye view-necessarily very superficial-one is apt not to see the wood for the trees.

\section{Mammals.}

We naturally begin a survey with the animals which are most like man-the monkeys. But neither we nor the monkeys are separated by any structural gulf from the other four limbed, hair bearing animals, to which Lamarck gave the name of Mammals. For although there are many different types of Mammals-such as monkeys and men; horses, cattle, and other hoofed quadrupeds; cats, dogs, and bears; rats, mice, and other rodents; hedgehogs, shrews, and moles, and so on-the common possession of certain characters unites them all in one class, readily distinguishable from Birds or Reptiles.

Among these characters we rank the milk giving of the mother mammals, the growth of hair on the skin, the general presence of convolutions on the front part of the 1 
brain, the occurrence of a muscular partition or diaphragm between the chest and the abdomen, and so on, as we shall afterwards notice in detail. Most mammals are suited for life on land, but diverse types such as seals, whales, and sea cows have taken to the water, while the bats are as markedly suited for aerial life.

Among the mammalian characteristics of great importance are those which relate to the bearing of young, and even a brief consideration of these enables us to see that some mammals are distinguished from others by differences deeper than those which separate whales from carnivores, or rodents from bats. These deep differences may be stated briefly as follows:-(a) Before birth most young mammals are very closely united (by a complex structure called the placenta) to the mothers who bear them. (b) But this close connection between mother and unborn young is only hinted at in the kangaroos and other pouched animals or Marsupials, which bring forth their young in a peculiarly helpless condition, as it were prematurely, and in most cases place them in an external pouch, within which they are sheltered and nourished. (c) In the Australian duckmole and its two relatives, the placental connection is quite absent, for these animals lay eggs as birds and most reptiles do. Besides these differences in the bearing of young, there are others relating to structure which are of great importance, and which seem to warrant the division of Mammals into three sub-classes :-

I. Prototheria, Ornithodelphia, or Monotremes-the egg laying duckmole (Omithorhynchus), Echidna and Proechidna.

2. Metatheria, Didelphia, or Marsupials - the prematurely bearing, usually pouch possessing kangaroos and their relatives.

3. Eutheria, Monodelphia, or Placentals-those in which there is a close (placental) union between the unborn embryo and its mother, e.g., Ungulates, Carnivores, Monkeys.

\section{Birds.}

There can be no hesitation as to the class which we should rank next to Mammals. For Birds are in most respects as highly developed as Mammals, though in a divergent direction. They are characterised by their feathers and wings, and many other adaptations for flight, by their high temperature, by the frequent sponginess and hollow- 
ness of their bones, by the tendency to fusion in many parts of the skeleton, e.g., backbone and ankle, by the absence of teeth in modern forms, by the fixedness of the lungs and their association with numerous air sacs, and so on.

But here again different grades must be distinguished(I) there is the vast majority-the flying birds, who have a breast-bone keel or carina, to which the muscles used in flight are in part attached (Carinatæ); (2) there is the small minority of running birds (ostriches, emu, cassowary, and kiwi), whose wings are incapable of flight, and who have no keel (Ratitæ); and (3) there is an extinct type, Archaopteryx, with markedly reptilian affinities.

\section{Reptiles.}

There are no close relationships between Birds and Mammals, but the old-fashioned Monotremes have some markedly reptilian features, and so have some aberrant living birds, such as the Hoatzin and the Tinamou. Moreover, when we consider the extinct Mammals and Birds, we perceive other resemblances linking the two highest classes of animals to Reptiles.

Reptiles do not form a compact class, but rather an assemblage of classes. In other words, the types of Reptile differ much more widely from one another than do the types of Bird or Mammal. Nowadays, there are five distinct types:- the crocodilians, the unique New Zealand "lizard" (Hatteria), the lizards proper, the snakes, and the tortoises. But the number of types is greatly increased when we take account of the entirely extinct saurians who had their golden age in the inconceivably distant past.

The Reptiles which we know nowadays are scaly-skinned animals, they resemble Birds and Mammals in having a practically or really four chambered heart, in never having gills, and in having during embryonic life two important "fotal membranes," known as the amnion and the allantois.

\section{Amphibians.}

The Amphibians, such as frogs and newts, were once regarded-e.g., by Cuvier--as naked Reptiles, but a more accurate classification has linked them rather to the Fishes. 
Thus Professor Huxley grouped Birds and Reptiles together as Sauropsida; Amphibians and Fishes together as Ichthyopsida-for reasons which shall be afterwards stated. Amphibians mark the transition from aquatic life, habitual among Fishes, to terrestrial life, habitual among Reptiles, for while almost all Amphibians have gills-in their youth at least-all the adults have lungs, and some retain the gills as well. In having limbs which are fingered and toed, and thus very different from fins, they resemble Reptiles. But the two fotal membranes characteristic of the embryonic life of higher Vertebrates are not present in Amphibian embryos, and the general absence of an exoskeleton in modern forms is noteworthy.

\section{Fishes.}

The members of this class are as markedly adapted to life in the water as birds to life in the air. The tail usually forms the locomotor organ, and the limbs are fins. There are also unpaired median fins supported by fin rays. All have permanent gills borne by bony or gristly arches. There is an exoskeleton of scales, and the skin also bears numerous glandular cells and sensory structures.

In many ways Fishes are allied to Amphibians, especially if we include among Fishes three peculiar forms, known as Dipnoi, which show hints of a three chambered heart, and have a lung as well as gills. Other Fishes have a two chambered heart, containing only impure blood, which is driven to the gills, whence, purified, it passes directly to the body.

Apart from the divergent Dipnoi, there are three orders of Fishes:-the cartilaginous Elasmobranchs, such as shark and skate; the Ganoids, such as sturgeon and bony pike; and the Teleosteans or bony fishes, such as cod, herring, salmon, eel, and sole.

\section{Primitive (?) Vertebrates.}

Under this title we include (I) the class of Roundmouths or Cyclostomata; (2) the class of which the lancelet or Amphioxus is the only adequately known type; (3) the class of Tunicates, some of which are called sea-squirts; and (4), with much hesitation, several strange forms, 
especially Balanoglossus, which exhibit structures suggestive of affinity with Vertebrates.

The Cyclostomata, represented by the lamprey (Petromyzon) and the hag (Myxine), and some other forms, probably including an interesting fossil known as Palaospondylus, are sometimes ranked with Fishes under the title Marsipobranchii. But they have no definitely developed jaws, no paired fins, no scales, and are in other ways more primitive.

The lancelet (Amphioxus), for which the class Cephalochorda has been erected, is even simpler in its general structure. Thus there is an absence of limbs, skull, jaws, well-defined brain, heart, and some other structures. The vertebral column is represented by an unsegmented (or unvertebrated) rod, called the notochord, which in higher animals (except Cyclostomata and some fishes) is a transitory organ replaced by a backbone.

The Tunicata or Urochorda form a class of remarkable forms, the majority of which degenerate after larval life. In the larvæ of all, and in the few adults which are neither peculiarly specialised nor degenerate, we recognise some of the fundamental characters of Vertebrates. Thus there is a dorsal supporting axis (a notochord) in the tail region, a dorsal nervous system, gill clefts opening from the pharynx to the exterior, a simple ventral heart, and so on.

Of Balanoglossus and its allies, for which the class Hemichorda or Enteropneusta has been established, it is still difficult to speak with confidence. The possession of gill clefts, the dorsal position of an important part of the nervous system, the occurrence of a short supporting structure on the anterior dorsal surface and other features, have led some to place them at the base of the Vertebrate series.

At this stage, having reached the base of the Vertebrate series, we may seek to define a Vertebrate animal, and to contrast it with Invertebrate forms.

The distinction is a very old one, for even Aristotle clistinguished mammals, birds, reptiles, amphibians, and fishes as "blood bolding," from cuttlefish, shell bearing animals, crustaceans, insects, \&c., which he regarded as "bloodless." He was, indeed, mistaken about the bloodlessness, but the distinctiveness of the higher animals first mentioned has been recognised by all subsequent naturalists, though it was first precisely expressed in 1797 by Lamarck. 
Yet it is no longer possible to draw a boundary line between Vertebrates and Invertebrates with that firmness of hand which characterised the early or, indeed, the pre-Darwinian classifications. For we now know-(I) that Fishes and Cyclostomata do not form the base of the Vertebrate series, for the lancelet and the Tunicates must also be included in the Vertebrate alliance; (2) that Balanoglossus and Cephalodiscus have several Vertebrate-like characteristics; (3) that some of the Invertebrates, especially Chretopods and Nemerteans, show some hints of affinities with Vertebrates. The limits of the Vertebrate alliance have been widened, and though the recognition of their characteristics has become more definite, not less so, the apartness of the sub-kingdom has disappeared.

It does not matter much whether we retain the familiar title Vertebrata, or adopt that of Chordata, provicled that we recognise-(I) that it is among Fishes first that separate vertebral bodies appear in the supporting dorsal axis of the body; (2) that, as a characteristic, the backbone is less important than the notochord, which precedes it in the history alike of the race and of the individual. Nor need we object to the popular title backboned, provided we recognise that the adjective "bony" is first applicable among Fishes, and not even to all of them.

The essential characters of Vertebrates may be summed up in the following table, where they are contrasted, somewhat negatively, with what is true of Invertebrates :-

"Backboneless," Invertebrate
or Non-Chordate.

The greater part of the nervous system is - on the ventral surface.

No corresponding structure is known.

No corresponding structures are known with any certainty.

The eye is usually derived directly from the skin.

The heart, if present, is dorsal.

\section{"BACKBONED," Vertebrate OR CHORDATE.}

The central nerrous systen-brain and spinal cord-is dorsal, and tubular.

There is a dorsal supporting axis or notochord, which is in most cases replaced by a backbone.

Gill-slits or visceral clefts open from the sides of the pharynx to the exterior. In fishes, and at least young amphibians, they are associated with gills and are useful in respiration; in bigher forms they are transitory and functionless, except when modified into other structures.

The essential parts of the eye are formed by an outgrowth from the brain.

The heart is ventral.

INVERTEIBRATES.

Molluscs.

This series of forms includes Bivalves, such as cockle and mussel, oyster and clam; Gasteropods, such as snail and slug, periwinkle and buckie; Cephalopods, such as octopus 
and pearly nautilus. They may be placed highest among Invertebrates since many of them exhibit a concentration of the nervous system greater than occurs elsewhere.

Unlike Vertebrates, and such Invertebrates as Insects and Crustaceans, Molluscs are without segments and without appendages. A muscular protrusion of the ventral surface, known as the "foot," serves in the majority as an organ of locomotion. In most cases, a single or double fold of skin, called the "mantle," makes a protective shell. The nervous system has three chief pairs of nerve centres or ganglia. In many cases, the larval stages are very characteristic.

\section{Arthropods.}

This large series includes Crustaceans, Myriopods, Insects, Spiders, and other forms, which have segmented bilaterally symmetrical bodies and jointed appendages. The skin produces an external cuticle, the organic part of which consists of a substance called chitin, associated in Crustaceans with carbonate of lime. 'The nervous system consists of a dorsal brain, connected, by a nerve ring around the gullet, with a ventral chain of ganglia.

\section{Echinoderms.}

This is a well-defined series, including starfishes, brittle stars, sea urchins, sea cucumbers, and feather stars. The symmetry of the adult is usually radial, though that of the larva is bilateral. A peculiar system, known as the watervascular system, is characteristic, and is turned to various uses, as in locomotion and respiration. There is a marked tendency to deposition of lime in the tissues. The development is strangely circuitous or "indirect."

\section{Segmented "Worms."}

It is hopeless at present to arrange with any definiteness those heterogeneous forms to which the title "worm" is given. For this title is little more than a name for a shape, assumed by animals of varied nature who began to move head foremost and to acquire sides. There is no class of "worms," but an assemblage - a mob-not yet reduced to order. It seems useful, however, to separate those which 
are ringed or segmented, from those which are unsegmented. The former are often called Annelids, and include :Chætopoda, or Bristle-footed worms, e.g., earthworm and lobworm ; and

Hirudinea, or Leeches; and some smaller classes.

\section{Unsegmented "Worms."}

These differ from the higher "worms" in the absence of true segments and appendages, and resemble them in their bilateral symmetry. The series includes Turbellarians or Planarians; the parasitic Trematodes or Flukes; the parasitic Cestodes or Tapeworms; the Nemerteans or Ribbonworms; the frequently parasitic Nematodes or 'Threadworms; and several smaller classes.

As to certain other forms, such as the sea mats (Polyzoa or Bryozoa), the lamp shells (Brachiopoda), and the wormlike Sipunculids, it seems best, at this stage, to confess that they are incertic sedis.

But the general fact is not without interest that in the midst of the well-defined classes of Invertebrates there lies, as it were, a pool from which many streams of life flow, for among the heterogeneous "worms "we detect affinities with Arthropods, Molluscs, Echinoderms, and even Vertebrates.

At this stage we may notice that in all the above forms the typical symmetry is bilateral (in Echinoderms, the radial symmetry belongs only to the adults); that in most types a body cavity or collome is developed ; that the embryo consists of three germinal layers (external ectoderm or epiblast, internal endoderm or hypoblast lining the gut, and a median mesoderm or mesoblast lining the body cavity). In the next two classes (Coelentera and Sponges) the conclitions are different, as may be expressed in the following table, though it is open to question whether the contrast is quite so great as it seems:-

Sponges and Coelentera.

There is no body cavity. There is but one cavity, that of the food canal.

There is no definite middle layer of cells (mesoderm), but rather a middle jelly (mesogloca).

The radial symmetry of the gastrula embryo is retained in the adult, and the Jongitudinal (oral-aboral) axis of the adult corresponds to the long axis of the gastrula.
Higher Animals (Cglomata).

There is a body cavity or cuclome between the food canal and the walls of the body. But this is often incipient, or degenerate.

There is a distinct middle layer of cells (mesoderm) between the external ectoderm and the gut lining endoderm.

The longitudinal axis of the adult does not correspond to the long axis of the gastrula einbryo. 


\section{Calentera.}

This series includes jelly fish, sea anemones, corals, zoophytes, and the like, most of which are equipped with stinging cells, by means of which they paralyse their prey. All but four or five are marine. The body may be a tubular polype, or a more or less bell-like "medusoid," and in some cases the two forms are included in one life cycle. Budding is very common, and many of the sedentary forms "corals"-have shells of lime.

\section{Porifera.}

Sponges, or Porifera, are the simplest many celled animals. In the simplest forms, the body is a tubular, two layered sac, with numerous inhalent pores perforating the walls, with a central cavity lined by cells bearing lashes or flagella, and with an exhalent aperture. But budding, folding, and other complications arise, and there is almost always a skeleton, calcareous, siliceous, or "horny," or both siliceous and horny at once. Water passes in by the small inhalent pores and out by the exhalent aperture. With few exceptions they are marine.

All the animals hitherto mentioned have bodies built up of many cells or unit masses of living matter, but there are other animals, each of which consists of a single cell. These simplest animals are called Protozoa.

Every animal hitherto mentioned, from mammal or bird to sponge, develops, when reproduction takes its usual course, from a fertilisecl egg cell. This egg cell or ovum divides and redivides, and the daughter cells are arranged in various ways to form a "body." But the Protozoa form no "body," they remain single cells, and when they divide, the daughter cells almost invariably go apart as independent organisms.

Here, then, is the greatest gulf which we have hitherto noticed - that between multicellular organisms (Metazoa) and unicellular organisms (Protozoa). But the gulf was bridged, and traces of the bridge remain. For (a) there are a few Protozoa which form loose colonies of cells, and $(b)$ there are multicellular organisms of great simplicity.

\section{Protozoa.}

The Protozoa remain single cells, with few exceptions. Thus they form no "body;" and necessarily therefore they 
have no organs, nor sexual reproduction in the ordinary sense of the phrase. The series includes-

(a) Infusorians, with actively moving lashes of living matter;

(b) Rhizopods, with outflowing threads or processes of living matter;

(c) Gregarines, parasitic forms, without either lashes or outflowing processes.

\section{Note on Classification.}

We naturally group together in the mind those impressions which are like one another. In this lies the beginning of all classification, whether that of the child, the savage, or the zoologist. For there are many possible classifications, varying according to their purpose, according to the points of similarity which have been selected as important. Thus we may classify animals according to their habitats or their diet without taking any thought of their structure.

But a strictly zoological classification is one which seeks to show the natural relationships of animals, to group together those which resemble one another in their real nature or structure. It must therefore be based on the results of comparative anatomy, technically speaking, on "homologies," or real resemblances of structure. Whales must not be ranked with fishes, nor bats with birds.

To a classification based on structural resemblances, two corroborations are necessary, from embryology and from palseontology. On the one hand, the development of the forms in question must be studied; timus no one dreamed that a Tunicate was a Vertebrate until its lifehistory was worked out; on the other hand, the past history must be inquired into, thus the affinity between Birds and Reptiles is confirmed by a knowledge of the extinct forms.

In classification it is convenient to recognise certain grades or degrees of resemblance, which are spoken of as species, genera, families, orders, classes, and so on.

To give an illustration, all the tigers are said to form the species Felis tigris, of the genus Felis, in the family Felidae, in the order Carnivora, within the class Mammalia. The resemblances of all tigers are exceedingly close; well-marked, but not so close, are the resemblances between tigers, lions, jaguars, pumas, cats, etc, which form the genus Felis; broader still are the resemblances between all members of the cat family Felide; still wider those between cats, dogs, bears, and seals, which form the order Carnivora; and lastly, there are the general resemblances of structure which bind Mammals together in contrast to Birds or Reptiles.

It must be understood that the real things are the individual animals, and that a species is a subjective conception within which we include all those individuals who resemble one another so closely that we feel we nced a specific name applicable to them all. And as resemblances which seem important to one naturalist may seem trivial to others, there 
are often wide differences of opinion as to the number of species which a genus contains. In a handful of small shells the "splitters" may recognise 20 species where the "slumpers" see only 3. Thus Hæckel says of calcareous sponges that, as the naturalist likes to look at the problem, there are 3 species, or 21 , or 289 , or 59 I !

But while no rigid definition can be given of a species, seeing that

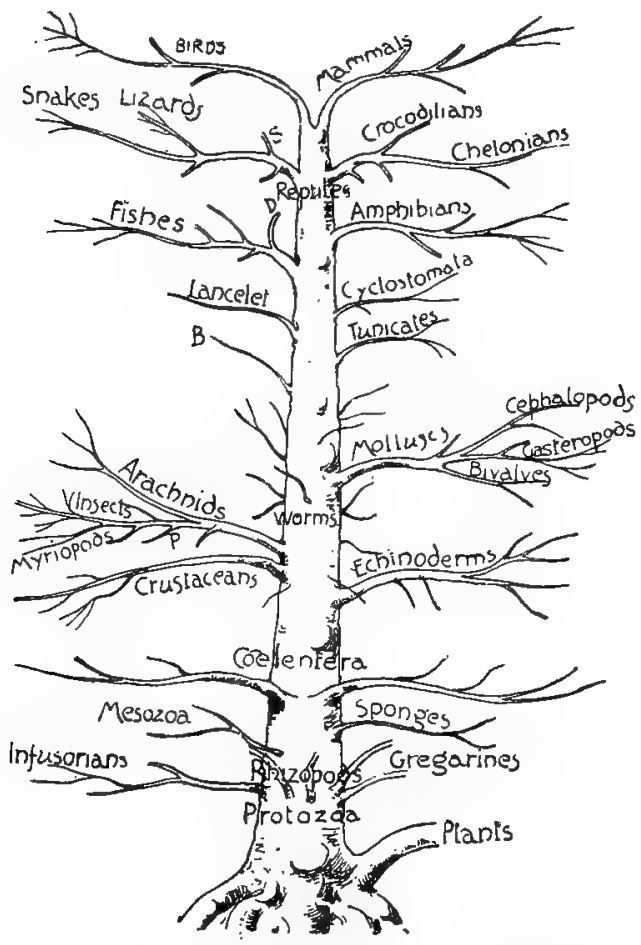

FIG. I. -Diagrammatic expression of classification in a genealogical tree. B indicates possible position of Balanoglossus, D of Dipnoi, S of Sphenodon or Hatteria.

the conception is one of practical convenience and purely relative, there are certain common-sense considerations to be borne in mind-

I. No naturalist now believes, as Linnæus did, in the fixity of 
species; we believe, on the contrary, that one form has given rise to another. At the same time, the common characteristic on the strength of which we deem it warrantable to give a name to a group of individuals must not be markedly fluctuating. The specific character should exhibit a certain degree of constancy from one generation to another.

2. Sometimes a minute character, such as the shape of a tooth or the marking of a scale, is so constantly characteristic of a group of inclividuals that it may be safely used as the index of more important characters. On the other hand, the distinction between one species and another should always be greater than any difference between the members of a family (using the word family here to mean the progeny of a pair). For no one would divide mankind into species according to the colour of eyes or hair, as this would lead to the absurd conclusion that two brothers belonged to different species. Thus it is often doubly unsatisfactory when a species is established on the strength of a single specimen, (a) because the constancy of the specific character is undetermined; (b) because the variations within the limits of the family have not been observer. Indeed, it has happened that one species has been made out of a male and another out of its mate. But the characters of a single specimen are sometimes so distinctive that the zoologist is safe in making it the type of a new species, or even of a new genus.

3. While cases are known where members of different species have paired and brought forth fertile hybrids, this is not common. The members of a species are fertile inter se, but not zisually with members of other species. In fact, the distinctness of species has largely depended on a restriction of the range of fertility.

To sum up, a species is but a relative conception, convenient when we wish to include under one title all the members of a group of individuals who resemble one another in certain characters. There is no absolute constancy in these specific characters, and one species often melts into another with which it is connected by intermediate varieties. At the same time, the characters, on account of which the naturalist gives a specific name to a group of individuals, should be greater than those which distinguish the members of any one family, should show a relative constancy from generation to generation, and should be associated with reprorluctive peculiarities which tend to restrict the range of mutual fertility to the members of the proposed species.

It will be enough now simply to state some of the more important grades of classification :-

Individuals.

Varieties among these individuals.

Species, e.s., Fielis tigris.

Genus, Fetis.

Family, Felida.

Order, Carnivora.

Class, Mammalia.

Phylum or Series, Vertebrata. 
Tabular Survey of Classes.-(For Future Reference.)

METAZOA CHORDATA.

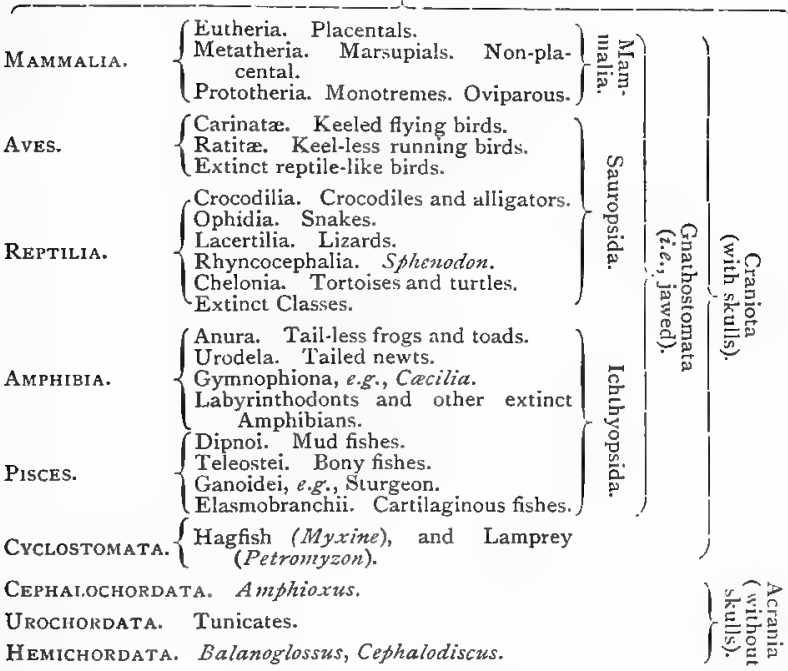

METAZOA NON-CHORDAT'A.

Molzusca. $\left\{\begin{array}{l}\text { Cephalopoda. Cuttle fishes. } \\ \text { Gasteropoda. Snails. } \\ \text { Lamellibranchiata. Hivalves. }\end{array}\right.$

Arthropoda. $\quad \begin{aligned} & \text { Arachroidea. Spiders, scorpions, mites. } \\ & \text { Insecta. } \\ & \text { Myriopoda. Centipedes and millipedes. }\end{aligned}$

Protracheata. Ptripatus.
Crustacea.

(Crinoidea. Feather stars. (Cystoids and Blastoids, extinct.)

Eichinoderma. $\left\{\begin{array}{l}\text { Ophiuroidea. Brittle stars. } \\ \text { Asteroidea. Star fish }\end{array}\right.$

$\left\{\begin{array}{l}\text { Asteroidea. Star fish. } \\ \text { F,chinoidea. Sea urchins. }\end{array}\right.$

Holothuroidea. Sea cucumbers.

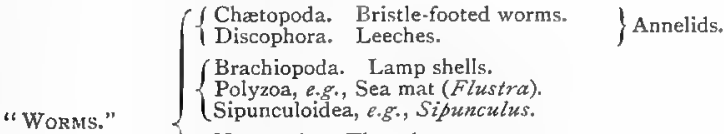

Nematoda. Thread-worms.

Nemertea. Ribbon-worm:

$\left\{\begin{array}{l}\text { Cestoda. Tape-worms. } \\ \text { Trematoda. } \\ \text { Turbellaria. } \\ \text { Planes. }\end{array}\right\}$ Plathelminthes.

Cumentera. $\quad\left\{\begin{array}{l}\text { Ctenophora, e.g. Beroe. } \\ \text { Scyphozoa. Jellyfish and sea anemones. } \\ \text { Hydrozoa. Zoophytes and medusoids. }\end{array}\right.$

Porifera. Sponges.

PROTOZOA.

InFUSORIA. RHIZOPODA. GREGARINIDA.

Simplest forms of animal life. 


\section{CHAPTER II.}

\section{THE FUNCTIONS OF ANIMALS.}

(Physiotogy.)

Most animals live a conscious and active life, busied with the search for food, the wooing of mates, the building of homes, and the tending of young. These and other forms of activity depend upon internal changes within the body. For the movements of all but the very simplest animals are due to the activity of contractile parts known as muscles, which are controlled by nervous centres and by impulseconducting fibres.

But as the work done means expenditure of energy, and is followed by muscular and nervous exhaustion, the necessity for fresh supplies of energy is obvious. This recuperation is obtained from food, but before this can restore the exhausted parts to their normal state, or keep them from becoming, in any marked degree, exhausted, it must be rendered soluble, diffused throughout the body, and so chemically altered that it is readily incorporated into the animal's substance. In other words, it has to be digested.

We may say then that there are two master activities in the animal body, those of muscular and those of nervous parts, to which the other internal activities are subsidiary conditions, turning food into blood and thus repairing the waste of matter and energy, keeping up the supply of oxygen and the warmth of the body, sifting out and removing waste products.

Besides the more or less constantly recurrent activities or functions, which are summed up under the general term "metabolism," there are the processes of growth and repro- 
duction. When income exceeds expenditure in a young animal, growth goes on, and the inherited qualities of the organism are more and more perfectly developed. At the limit of growth, when the animal has reached "maturity," it normally reproduces, that is to say, liberates parts of itself which give rise to new individuals. It is this power of growing and reproducing which most distinguishes an organism from an inanimate thing.

\section{Division of Labour.}

All the ordinary functions of life are exhibited by the simple unicellular animals or Protozoa. Take the Amoeba for example. It moves by contracting its living substance, it draws back sensitively from hurtful influences, it engulfs and digests food, it gets rid of waste, and it absorbs oxygen, without which its living matter cannot continue active or indeed alive. For activity implies, in part, an oxidation, a combustion of material, and respiration in plants and animals alike consists in absorbing oxygen, and in liberating the carbonic acid gas which is one of the waste products both of life and burning.

But all these activities occur in the Amoba within the compass of a unit mass of living matter, - a single cell, physiologically complete in itself. There is no division of labour, there are as yet no parts.

In all other animals, from Sponges onwards, there is a "body" consisting of hundreds of unit masses or cells. It is impossible for these to remain the same, for some are internal and others external, nor would it be well for the organism that all its units should retain the primitive and many sided qualities of Amcebæ. Division of labour, consequent on diversity of conditions, is thus established in the organism. In some cells one kind of activity predominates, in others a second, in others a third. And this division of labour is followed by that complication of structure which we call differentiation.

Thus, in the fresh water Hydra, which is one of the simplest many celled animals, the units are arranged in two layers, and form a tubular body. Those of the outer layer are protective, nervous, and muscular; those of 
the inner layer absorb and digest the food, and are also muscular.

In worms and higher organisms, there is a middle layer in addition to the other two, and this middle layer becomes, for instance, predominantly muscular. Moreover, the units or cells are not only arranged in strands or tissues, each with a predominant function, but become compacted into well-defined parts or organs. None the less should we remember that each cell remains a living unit, and that, in addition to its principal activity, it usually retains others of a subsidiary character.

\section{History.}

Physiologists, or those who study the activities of organisms and of their parts, were at first content to speak of these as the result of " animal and vital spirits," of moods and temperaments.

Stimulated, however, by the anatomists' disclosure of organs, the physiologists soon began to explain the organism as a complex engine of many parts. The muscles were recognised as the mechanism which produced movement, the heart pumped the blood through the body, the brain was the seat of thought, and so on. This was an exceedingly necessary and natural step in analysis. Nor has it yet been thoroughly taken in every case, for there are many organs, especially in backboneless animals, about whose predominant use we are uncertain. But the physiologists of this school sometimes finished their work too quickly. That the liver was an organ for secreting bile was deemed a completely satisfactory statement, until it began to be seen that this organ is the seat of many other activities. Moreover, some thought that it was possible to deduce the function of an organ from its visible structure, as one might infer the use of a piston from its shape. To a certain extent this is true, as when we show how an image is formed on the retina of the eye. But we cannot, in terms of visible structure, explain another function of the eye-that of distinguishing the "colours" of things. In fact, it must be clearly understood that each organ is far more than a I piece of mechanism in a living engine, - that it is a complicated factory of living units, each with subtle and manifold powers,

In I8OI, Bichât analysed the animal body into its component tissues - muscular, nervous, glandular, \&c., and being a physiologist as well as an anatomist, sought to explain the activities of the organism in terms of the contractile, irritable, secretory, or other properties of its tissues. This was a further step in the analysis, and one of great importance.

About forty years later, however, it began to be recognised that the hody was a great city of cells, each with a life of its own. The functions were not merely the activities of organs of various construction, or of tissues with various properties, they were the results of the life of the component units or cells.

Finally, in those last days, the physiologists have touched the bottom 
in their analysis, for they are endeavouring to discover the physical and chemical changes associated with the living stuff or protoplasm itself. These are obviously at the founclation of the whole matter.

\section{Plants and Animals.}

Before we give a sketch of the chief functions in a higher animal, let us briefly consider the resemblances and differences between plants and animals.

(a.) Resemblance in Function. - The life of plants is essentially like that of animals, as has been recognised since Claude Bernard wrote his famous book, Phénomènes de la vie communs aux animaux et aux vépétaux. The beech tree feeds and growes, digests and breathes, as really as does the squirrel on its branches. In regard to none of the main functions is there any essential difference. Many simple plants swim about actively; young shoots and roots also move; and there are many cases in which even the fullgrown parts of plants exhibit movements. Moreover, the tendrils of climbers, the leaves of the sensitive plant, the tentacles of the sun-dew, the stamens of the rock rose, the stigma of the musk, are but a few instances of the numerous plant structures which exhibit marked sensitiveness.

(b.) Resemblance in Structure.-The simplest plants (Protophyta) like the simplest animals (Protozoa) are single cells; the higher plants (Metaphyta) and higher animals (Metazoa) are built up of cells and of various modifications of cells. In short, all organisms have a cellular structure. This general conclusion is known as the Cell Theory or Cell Doctrine (see p. 4I).

(c.) Resemblance in Development.-When we trace the beech tree back to the beginning of its life, we find that it arises from a unit element or egg cell, which is fertilised by intimate union with a male element derived from the pollengrain. When we trace the squirrel back to the beginning of its life, we find that it also arises from a unit element or egg cell, which is fertilised by intimate union with a male cell or spermatozoon. Thus all the many celled plants and animals begin as fertilised egg cells, except in cases of virgin birth (parthenogenesis) or of asexual reproduction. From the egg cell, which divides and redivides after fertilisation, the body of the plant or animal is built up by con- 
tinued division, arrangement, and modification of cells. Thus, plants and animals resemble one another in their essential functions, in their cellular structure, and in their development.

But while there is no absolute distinction between plants and animals, they represent divergent branches of a V-shaped tree of life. It is easy to distinguish extremes, like bird and daisy, less easy to contrast sponge and mushroom, well nigh impossible to decide whether some very simple forms, which Hæckel called "protists," have a bias towards plants or towards animals. But the food which most plants absorb is cruder or chemically simpler than that which animals are able to utilise. Thus plants derive the carbon they require from the carbonic acid gas of the air, whereas only a few (green) animals have this power. Almost all animals depend for their carbon supplies on the sugar, starch, and fat already made by other animals, or by plants. As regards nitrogen, most plants derive this from nitrates and the like, absorbed along with water by the roots; whereas animals obtain their nitrogenous supplies from the complex proteids formed within other organisms. Most plants, therefore, feed at a lower chemical level than do animals, and it is characteristic of them that, in the reduction of carbonic acid, and in the manufacture of starch and proteids, the kinetic energy of sunlight is transformed by the living matter into the potential chemical energy of complex food stuffs. Animals, on the other hand, get their food ready-made; they take the pounds which plants have, as it were, accumulated in pence, and they spend them. For it is characteristic of animals that they convert the potential chemical energy of food stuffs into the kinetic energy of locomotion and other activities. In short, the great distinction-an average one at best-is that most animals are more active than most plants. Let us condense in tabular summary the timehonoured "distinctions between plants and animals." 


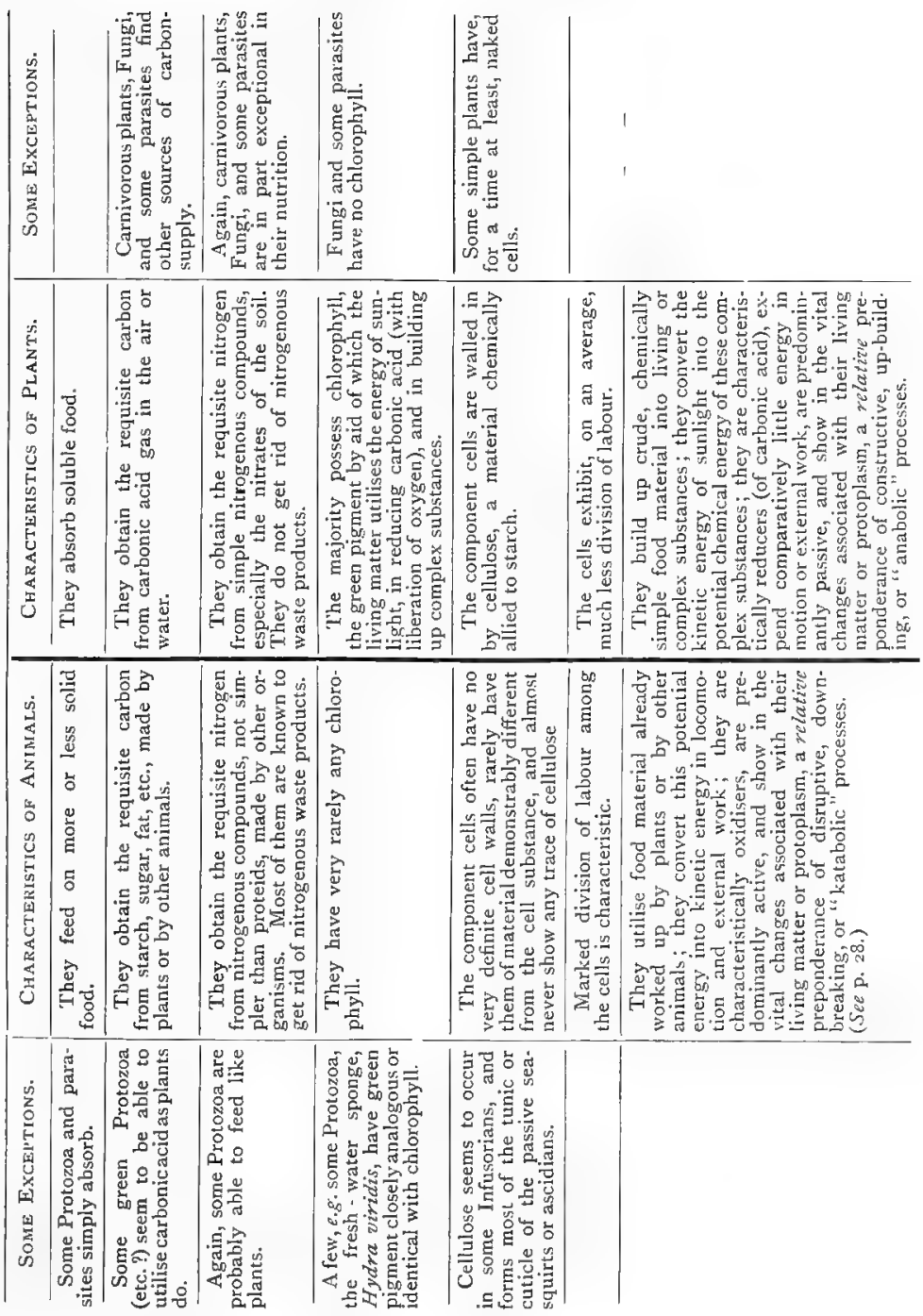




\section{CHIEF FUNCTIONS OF THE ANIMAL BODY.}

We have seen that there are two master activities in animals, those of muscular and of nervous structures, and that the other functions, always excepting reproduction, are subservient to these. Let us now consider the various functions, as they occur in some higher organism, such as man, reserving comparative treatment for a subsequent chapter.

\section{Nervous Activities.}

Life has been described as consisting of action and reaction between the organism and its environment, and it is evident that an animal must in some way feel, or become aware of surrounding influences. In a higher animal we find parts which are specially excitable. 'These are the sensory end-organs: the retina of the eye for light, certain parts of the ear for sound, papillæ on the tongue for taste, part of the lining of the nasal chamber for smell, tactile corpuscles of the skin for pressure and temperature.

All these end-organs are associated with nerves which are stimulated by the excitation of the end-organ, and conduct the stimulus inwards to what are called centres or ganglia.

In Vertebrate animals the brain and spinal cord contain a series of such centres, some of which serve for the perception of the changes produced in the end-organs by the stimulus, while others preside over the activities of the muscles. As we ascend in the scale we find that in addition the brain possesses, to an increasing extent, the power of correlating present and past experiences, and originating or inhibiting action in accordance with the judgment formed.

Thus, nervous activities involve (a) end-organs or sense organs; $(b)$ centres or ganglia ; and $(c)$ the conducting nerves, some of which are afferent (or sensory) passing from endorgans to ganglia, while others are efferent (or motor) passing from centres to muscles. And in whatever part there is activity there is necessarily waste of complex substances and some degree of exhaustion.

It is interesting to notice, as a trimmph of histological technique, that Hodge, Gustav Mann, and others have succeeded in demonstrat- 
ing in nerve cells the structural results (cellular collapse, \&c.) of fatigue, and that in such diverse types as bee, frog, bird, and dog.

\section{Muscular Activity.}

The movements of a unicellular animal are due to the contractility of the living matter, or of special parts of the cell such as cilia (see p. 106). In sponges, there are often specially contractile cells; in most higher animals such cells are aggregated to form the muscles on whose activity all movement depends.

In many of the lower animals, e.g., sea-anemones and seasquirts, the contractile strands consist of long spindle-shaped cells which appear almost homogeneous; these are called smooth muscle fibres. They occur in certain parts of the body in higher Vertebrates, e.g., on the wall of the urinary bladder. A more specialised kind of muscle, prevailing in active animals, consists of fibres which show alternate light and dark cross bands; these are called striped muscle fibres. The two kinds, unstriped and striped, may be seen to pass into one another in the same animal, and in a general way one may think of the former as slowly contracting, the latter as rapidly contracting.

A piece of living muscle consists of fine transparent tubes or fibres, each invested by a sheath or sarcolemma, and the whole muscle is surrounded by connective tissue. It usually runs from one part of the skeleton to another and is fastened to the skeleton by tendons or sinews. It is stimulated by motor nerves, and is richly supplied with blood.

When a muscle contracts, usually under a stimulus propagated along a motor nerve, there is of course a change of shape-it becomes shorter and broader. The source of the energy expended in work done is the "chemical explosion"which occurs in the fibres, for the oxygen stored up (intramolecularly) in the muscle enters into rapid union with a carbohydrate. Heat, $\mathrm{CO}_{2}$, and water are produced as the result of this combustion, and lactic acid is also formed as a bye-product. Besides the chemical change and the change of shape, there are also changes of electrical potential associated with each contraction. 


\section{Digestion.}

The energy expended in doing work or in growth is balanced by the potential energy of the food stuffs taken into the body. These consist of proteids, carbohydrates, fats, water, and salts in varying proportions according to the diet of the animal. Oxygen may also be regarded as forming part of the food.

In some of the lower animals, such as sponges, the food particles are directly engulfed by some of the cells with which they come in contact. Within these cells they are dissolved ; this is known as intracellular digestion. In most cases, however, the food is rendered soluble and diffusible within the food-canal by the action of certain ferments made by the cells which line the gut or form the associated glands. The great peculiarity of these fermenting substances is that a small quantity can act upon a large mass of material without itself undergoing any apparent change. But however digestion be effected, it means making the food soluble and diffusible. In a higher vertebrate, there are many steps in the process.

(a) The first ferment to affect the food, masticated by the teeth and moistened by the saliva, is the ptyalin of the salivary juice, which changes starch into sugar. The juice is formed or secreted by various salivary glands around the mouth.

(b) The food is swallowed, and passes down the gullet to the stomach, where it is mixed with the gastric juice secreted by glands situated in the walls. These walls are also muscular, and their contractions churn the food and mix it with the juice. In the juice there is some free hydrochloric acid and a ferment called pepsin; these act together in turning proteicls into peptones. The juice has also a slight solvent effect on fat, and the acid on the carbohydrates.

(c) The semi-digested food, as it passes from the stomach into the small intestines, is called chyme, and on this other juices act. Of these the most important is the secretion of the pancreas, which contains various ferments, es. trypsin, and affects all the different kinds of organic fool. It continues the work of the stomach, changing proteids into peptones; it continues the work of the salivary juice, changing starch into sugar; it also emulsifies the fat, dividing the globules into extremely small drops, which it tends to split into fatty acids and glycerine.

(d) Into the beginning of the small intestine, the bile from the liver also flows, but this is not of great digestive importance, being rather of the nature of a waste protuct. It scens to have a slight solvent, emulsifying, and saponifying action on the fats; in some animals it has a slight power of converting starch into sugar; by its alkalinity it helps the action of the trypsin of the pancreas (which, unlike pepsin, acts in a 
neutral fluid); it affects cell membranes, so that they allow the passage of small drops of fat and oil; and it is said to have various other qualities.

(e) In addition to the liver and the pancreas, there are on the walls of the small intestine a great number of small glands, which secrete a juice which probably seconds the pancreatic juice. The digested material is in part alssorbed into the blood, and the mass of food, still being digested, is passed along the small intestine by means of the muscular contraction of the walls, known as peristaltic action. It reaches the large intestine and its reaction is now distinctly acid by reason of the acid fermentation of the contents. The walls of the targe intestine contain glands similar to those of the small intestine, and the digestive processes are completed, while absorption also goes on ; so that by the time the mass has reached the rectum, it is semi-solid, and is known as freces. These contain all the indigestible and uniligested remnants of the food and the useless products of the chemical digestive processes.

\section{Absorption.}

But the food must not only be rendered soluble and diffusible, it must be carried to the different parts of the body, and there incorporated into the hungry cells. It is carried by the blood-stream, and in part also by what are called lymph vessels, which contain a clear fluid resembling blood minus red blood corpuscles.

Absorption begins in the stomach by direct osmosis into the capillaries or fine branches of blood vessels in its walls, and a similar absorption, especially of water, takes place along the whole of the digestive tract. But lining the intestines there are special hair-like projections called villi; they contain capillaries belonging to the portal system (blood vessels going to the liver), and small vessels known as lacteals connected with lymph spaces in the wall of the intestine. The lacteals lead into a longitudinal lymph vessel or thoracic duct, which opens into the junction of the left jugular and left subclavian veins at the root of the neck. The contents of the duct in a fasting animal are clear; after a meal they become milky; the change is due to the matters discharged into it by the lacteals. It is probable that nearly all the fat of a meal is absorbed from the intestines by the lacteals, but it is not certain in what measure, if at all, this is true of the other dissolved food stuffs; the greater part certainly passes into the capillaries of the portal system, which are contained in each villus. The peptone or digested proteid, as it passes through the cells of the villi, is changed into other proteids nearly related to those of the blood, for no peptone is found in the portal vein.

\section{Function of the Liver.}

We now know the fate of the fats, and of the proteids of the food, and the manner in which they pass into the blood; but we must follow the starchy material, or carbo- 
hydrates, a little further. The starch, we know, is converted into sugar, and this, with the sugar of the food, passes into the capillaries of the villi, and is carried to the liver. During digestion there is an increase of sugar in the blood vessel going to the liver from the intestine, that is, in the portal vein, but no increase in the hepatic veins, the vessels leaving the liver. The increase must, therefore, be retained in that organ, and we recognise as one of the functions of the liver, the regulation of the amount of sugar in the blood. There is no special organ for the regulation of the amount of fat; the drops pass through the capillary walls, and are retained in the connective tissue.

We must remember that all the products of digestion, except the fat, pass through the liver, which receives everything before it is allowed to pass into the general circulation. Thus, many poisons, especially metals, are arrested by the liver, and many substances which result from digestive processes and would be harmful, are there altered into harmless compounds. The excess of sugar, we have already noted, is stored in the liver. It is converted there into a substance called glycogen, which can be readily retransformed into sugar according to the needs of the system. Glycogen is stored in the muscles also, and is the material chiefly useful as the fuel for the supply of muscular energy and of the warmth of the body. Thus, if an animal be subjected to a low temperature, the glycogen of the liver disappears just as it does during the performance of muscular work.

Another of the many functions of the liver is that in it nitrogenous waste products begin to be prepared for their final elimination by the kidneys.

\section{Respiration.}

There is another most important food stuff to be noticed, namely, the oxygen which is absorbed from the air by the lungs. We may picture a lung as an elastic sponge-work of air chambers, with innumerable blood capillaries in the walls, enclosed in an air-tight box, the chest, the size of which constantly and rhythmically varies. When we take in a breath the size of the chest is increased, the air pressure within is lowered, and the air from without rushes down 
the windpipe until the pressure is equalised. The oxygen of this air combines with a substance called hæmoglobin, contained in the red corpuscles of the blood, and is thus carried to all parts of the body. The protoplasm of the tissues having a stronger affinity for oxygen than has the hæmoglobin, takes as much as it requires. The carbonic acid gas formed as a waste product is absorbed by the serum of the blood, and so in time reaches the lungs. But as the partial pressure of the carbonic acid in the air is lower than it is in the serum, the gas escapes from the latter into the air chambers of the lungs. When the size of the chest is decreased, the pressure is increased, and the gas escapes by the mouth until the pressure is equalised. By the constant repetition of the breathing movements, oxygen is constantly being taken in, and carried to the tissues which are in a marvellous way "hungry" for it, while the waste carbonic acid gas is as constantly being removed.

\section{Excretion.}

We have seen that the blood carries the digested food to the various parts of the body, and that it is also the carrier of oxygen and of the waste carbonic acid gas.

But there is much waste resulting from tissue changes, which is not gaseous. It is cast into the blood stream by the tissues, and has to be got rid of in some way. This is effected by the kidneys, which are really filters introduced into the blood stream. But they are the most marvellous filters imaginable, and give us a good example of the intricacy of life processes. For the kidneys not only cast out of the blood all the waste products that result from the metabolism of proteids, and contain nitrogen; but they maintain the composition of the blood at its normal, rejecting any stuffs that vary from that normal, either qualitatively or quantitatively, doing this work according to laws quite different from the simple ones of diffusion or solubility; thus, sugar and urea are about equally soluble, and yet the sugar is kept in the body, while the urea is cast out. Even substances as insoluble as resins are removed from the blood by the living cells of the kidneys.

A considerable quantity of water, and traces of salts, fats, \&c., leave the body by the skin, but its chief use is to pro- 
tect and to regulate the temperature by variations in the size of its blood vessels.

This completes our sketch $(a)$ of the process by which the food becomes available for the organism as fuel for the maintenance of its life energies, and $(b)$ of the removal of the waste products which are formed as the ashes of life.

There are indeed some organs which we have not mentioned, such as the spleen, which seems to be an area for the multiplication of blood corpuscles, and the thyroid gland, which seems to have to do with keeping the blood at a certain standard of efficiency, but what we have said is perhaps enough to convey a general idea of the processes of life in a higher animal.

In conclusion, it is perhaps useful to remark that when in the course of further studies the student meets with organs which are called by the same name as those found in man or in Mammals, as, for example, the "liver" of the Molluses, he must be careful not to suppose that the function of such a "liver" is the same as in Mammals, for comparatively little investigation into the physiology of the lower types of animal life has as yet been made. At the same time, he must clearly recognise that the great internal activities are in a general way the same in all animals; thus, respiration, whether accomplished by skin, or gills, or air tubes, or lungs, ly help of the red pigment (heemoglobin) of the blood, or of some pigment which is not red, or occurring without the presence of any blood at all, always means that oxygen is absorbed almost like a kind of food by the tissues, and that the carbonic acid gas which results from the oxidation of part of the materinl of the tissues is removed.

\section{Modern Conception of Protoplasm.}

'The activities of animals are ultimately due to physical and chemical changes associated with the living matter or protoplasm. This is a mere truism. We do not know the nature of this living matter; in fact, our most certain knowledge of it is that in our brains its activity is expressed as thought.

When more is known in regard to the chemistry and physics of living matter, it may be possible to bring vital phenomena more into a line with the changes which are observed in inorganic things. At present, however, it is idle to deny that vital phenomena are things apart. Not even the simplest of them can be explained in terms of chemistry and physics. Even the passage of digested food from the gut to the blood vessels is more than ordinary 
physical osmosis; it is modified by the fact that the cells are living.

From the point of view of a student of physics Dr. J. Joly draws the following contrast between an animate and an inanimate body:- "While the transfer of energy into any inanimate material system is attended by effects retardative of the transfer and conducive to dissipation, the transfer of energy into any animate material system is attended by effects conducive to the transfer and retardative of dissipation."

But though we cannot analyse living matter, nor thoroughly explain the changes by which the material of the body breaks down or is built up, we can trace, by chemical analysis, how food passes through various transformations till it becomes a useable part of the living body, and we can also catch some of the waste products formed when muscles or other parts are active.

Apart from any theory, it is certain that waste products are formed when work is done, and that living animals have a marvellous power of rapid repair, of ceaselessly changing, and yet remaining more or less the same. Theory begins when we attempt to make the general idea of waste and repair more precise. In the study of "protoplasm," both morphologist and physiologist have reached their strict limits. Further analysis becomes physical and chemical, and ends in the confession that protoplasm is a marvellous form of matter in motion or a subtle kind of motion of which we can form only a very vague conception.

What is known in regard to the structure of protoplasm cloes not help the physiologist very much. As we shall afterwards see, the micro. scopists discover an intricate network which pervades each unit of living matter, but no physiologist dreams of explaining the life of a cell in terms of its microscopically visible structure. Yet, as Burdon Sanderson says, "We still hold to the fundamental principle that living matter acts by virtue of its structure, provided the term structure be used in a sense which carries it beyond the limits of anatomical investigation, i.e., beyond the knowledge which can be attained either by the scalpel or the microscope." But, in the end, this means that living matter acts in virtue of its peculiar qualities, its characteristic motion, of which we can form only a hypothetical conception, and can give no scientific explanation.

One general idea, however, the study of structure has suggested, which the conclusions of physiologists corroborate. This idea is-that 
a cell consists of a relatively stable living framework, and of a changeful content enclosed by it.

Now, many physiologists regard the framework as the genuine living protoplasm, and the contents as the material upon which it acts. "The framework is the acting part, which lives, and is stable; the content is the acted-on part, which has never lived, and is labile, that is,--in a state of metabolism or chemical transformation." This view naturally learls those who adopt it to regard protoplasm as a sort of ferment acting on less complex material which is brought to it, and which forms the really changeful part of each cell. You will remember that the strange characteristic of a ferment is that it can act on other sulsstances without being itself affected by the changes which it produces, and that it can go on cloing so continuously with a power which has no direct relation to its amount. In these respects, therefore, living matter resembles a ferment.

Somewhat different, however, is another idea, -that the protoplasm is itself the seat of constant change; that it is constantly being unmade and remade. On the one hand, more or less crude food passes into life by an ascending series of assimilative or constructive chemical changes with each of which the material becomes molecularly more complex and more unstable. On the other hand, the protoplasm, as it becomes active or a source of energy, breaks down in a descending series of disruptive or clestructive chemical changes ending in waste products.

The former view, which considers protoplasm as a sort of ferment, restricts the metabolism to the material on which the protoplasm acts. The second view regards protoplasm as the climax or central term of the constructive and disruptive metabolism.

\section{Anabolism and Katabolism.}

All physiologists are agreed that in life there is a twofold process of waste and repair, of discharge and restitution, of activity and recuperative rest. But there is no certainty as to the precise nature of this two-fold process.

In your future physiological studies you will have to consider the power that our eyes have of appreciating those different kinds of light which give rise to sensations of colour. It was in studying these that Professor Hering was led to an interesting theory of living matter. $\mathrm{He}$ supposes that there exist in or about the retina three different "visual substances," which we may call A, B, C. He supposes that each of these is continually undergoing one of two kinds of metabolism. It is either being built up lyy assinilation, or it is being broken down in dis. assimilation. IIe supposes that each of these sulbstances is affected by two kinds of light, and that these two kinds of light have opposite influences on the metabolism of the substance. When we have a sensation of white, or of rell, or of yellow, it is supposed that in one of the three kinds of visual substance disassimilation is preponderant. When we have a sensation of black, or of green, or of blue, it is supposed that in one of the three kinds of visual substances assimilation is preponderant. Excess of disassimilation in A gives us the sensation of white; excess 
of assimilation of $\mathrm{A}$ gives us the sensation of black ; and similarly with red and green for $B$, with yellow and blue for $C$.

But generalising from his studies on colour sensation, Hering was led to regard all life as an alternation of two kinds of activity, both induced by stimulus, the one tending to storage, construction, assimilation of material, the other tending to explosion, disruption, disassimilation. Both processes are, according to Hering, activities; both are dependent upon stimulus; they differ, however, in direction and results.

In your future physiological studies you will also learn of the paths or channels by which the brain sends its mysterious commands to the various parts of the body. You will learn that some of these bear impulses to activity, while others convey commands which send the affected part to rest.

It was in studying and greatly elucidating these interesting facts, that Professor Gaskell was led to a theory of vital action sumewhat different from that of Hering.

Gaskell believes that life means an alternation of two processes, one of them a running down or disruption (katabolism), the other a winding up or construction (anabolism). The disruptive or liatabolic process in which energy is discharged, takes place occasionally and in obedience to stimulus; the constructive or anabolic process of restitution goes on constantly and of itself, i.e., without the necessity of stimulus. Thus Gaskell's theory suggests an alternation of activity and rest, of stimulated disruption and self-regulative construction, while Hering's theory suggests an alternation of two antagonistic kinds of activity, assimilation and disassimilation, both requiring stimulus. The student will find the theories, which I have briefly noticed, discussed in Professor M. Foster's article Physiology, in the Encyclopadia Britannica, and in an address by Professor Burdon Sanderson (British Association Reports, I889, and also published in Natzure, September I889). 


\section{CHAPTER III.}

\section{THE ELEMENTS OF STRUCTURE.}

(MORPHOLOGY.)

Animals may be studied alive or dead, in regard to their activities or in regard to their parts. We may ask how they live, or what they are made of ; we may investigate their functions or their structure. The study of life, activity, function, is physiology; the study of parts, architecture. structure, is morphology.

The first task of the morphologist is to describe structure (descriptive anatomy); the second is to compare the parts of one animal with those of another (comparative anatomy); the third is to generalise, to formulate the "principles of morphology," or the laws of vital architecture.

But just as the physiologist investigates life or activity at different levels, passing from his study of the animal as a unity with certain habits, to consider it as an engine of organs, a web of tissues, a city of cells, and a whirlpool of living matter; so the morphologist has to investigate the form of the whole animal, then in succession its organs, their component tissues, their component cells, and finally, the structure of protoplasm itself. The tasks of morphology and of physiology are parallel.

Morphology thus includes not only the description of external form, not only the anatomy of organs, but also that minute anatomy of tissues and cells and protoplasm which we call histology. Moreover, there is no real difference between studying fossil animals which died and were buried countless years ago, and dissecting a modern frog. The anatomical palæontologist is also a student of morphology. Finally, as the greater part of embryology consists in study- 
ing the anatomy and histology of an organism at various stages of its development, the work of the embryologist is also in the main morphological, though he has also to inform us, if he can, about the physiology of development.

Morphology has been defined by Geddes as "the study of all the statical aspects of organisms," in contrast to physiology, which is concerned with their vital dynamics. In this chapter we shall follow the historical development of morphology, and work from the outside inwards in deeper and deeper analysis.

\section{External Form.}

The form of an animal is due to the interaction of two variables-the protoplasmic material which composes the organism, and the environment which plays upon it. In fact, an animal takes definite form just as a crystal does; in both the shape is determined by the nature of the stuff and by the surrounding influences. Activity, or function, also affects form; but function is merely action and reaction between the animal and its surroundings. Such statements, however, are platitudes; we are far from being able to explain the conditions of growth which lead to this shape or that.

As regards symmetry, animals may be distinguished as:-

(a) Radially Symmetrical ;

(b) Bilaterally Symmetrical ; or

(c) Asymmetrical.

In a radially symmetrical animal, such as a jelly-fish, the body can be halved by a number of vertical planes-it is symmetrical around a median vertical axis. That is, it is the same all round, and has no right or left side. In a bilaterally symmetrical body, such as our own, there is but one plane through which the body can be halved. In an asymmetrical animal, such as a snail, accurate halving is impossible.

Radial symmetry is illustrated by simple Sponges, most Coelentera, and by many adult Echinoderms. As it is the rule in the two lowest classes of Metazoa, and as it is characteristic of the very common embryonic stage known as the gastrula (an oval or thimble shaped sac consisting of two layers of cells), it is probably more primitive than the bilateral symmetry characteristic of most animals above Colentera. Radial symmetry seems best suited for sedentary life, or for aimless floating and driftng. Bilateral symmetry probably arose as it became advantageous for animals to move energetically and in definite directions, to pursue their prey and avoid their enemies. Among many celled animals, some worm type probably cleserves the credit of beginning the profitable habit of moving head-foremost; had some one not taken this step, we should never have known our right hand from our left. 


\section{ORgans.}

We give this name to any well-defined part of an animal, such as heart or brain. The word suggests a piece of mechanism; but the animal is more than a complex engine, and many organs have several different activities to which their visible structure gives little clue.

\section{Differentiation and Integration.}

When we review the animal series, or study the development of an individual, we see that organs appear gradually. 'The gastrula cavity - the future stomach - is the first acquisition, but some would make out that it was primitively a brood chamber. To begin with, it is a simple sac, but it soon becomes complicated by digestive and other outgrowths. The progress of the individual, and of the race, is from simplicity to complexity. When we think over the animal series we also notice that before definite nervous organs appear there is diffuse irritability, before definite muscular organs appear there is diffuse contractility, and so on. In other words, functions come before organs. The attainment of organs implies specialisation of parts, or concentration of functions in particular areas of the body.

Contrast a frog with Hydra, and one of the great facts about the evolution of organs is illustrated. Among the living units which make up a frog, there is much more division of labour than there is among those of Hydra. An excised representative sample of $H y d r a$ will reproduce the whole, but you cannot perform this experiment with the frog. Now, the structural result of this physiological division of labour is differentiation. The animal, or part of it, becomes more complex, more heterogeneous.

Contrast a bird and a sponge, and another great fact about the evolution of organs is illustrated. The bird is more of a unity than a sponge; its parts are more closely knit together and more adequately subordinated to the life of the whole. We call this kind of progress, integration. Differentiation involves the acquisition of new parts and powers, these are consolidated and harmonised as the animal becomes more integrated. 


\section{Correlation of Organs.}

It is of the very nature of an organism that its parts should be mutually dependent. The organs are all partners in the business of life, and, if one member suffer, others also are affected. This is especially true of certain organs which have developed and evolved together, and are knit by close physiological bonds. Thus, the circulatory and the respiratory systems, the muscular and the skeletal systems, the brain and the sense organs, are very closely united, and we say that they are correlated. A variation, for better or worse, in one system often brings about a correlated variation in another, but sometimes we cannot trace the connection.

\section{Homologous Organs.}

Organs which arise from the same primitive layer of the embryo (see Chapter IV.), have something in common. But when a number of organs arise in the same way, from the same embryonic material, and are at first fashioned on the same plan, they have still more in common. Nor will this fundamental sameness be affected though the final shape and use of the various organs be very different. We call organs which are thus structurally and developmentally similar, homologous. Thus, the nineteen pairs of appendages on a crayfish are all homologous ; the three pairs of "jaws" in an insect are homologous with the insect's legs; and it is also true that the fore-leg of a frog, the wing of a bird, the flipper of a whale, the arm of a man, are all homologous. On the other hand, the wing of a bird and the wing of an insect, which resemble one another in being organs of flight, are not the least alike in structure; this is expressed by saying that they are only analogous. Yet two organs may be both homologous and analogous, e.g., the wing of a bird and the wing of a bat, for both are fore-limbs, and both are organs of flight. Sometimes two organs or two organisms-deeply different in structure-have a marked superficial resemblance, simply because both have arisen in relation to similar conditions of life. Thus a burrowing amphibian, a burrowing lizard, and a burrowing snake, resemble one another in being limbless, but this "convergence" of form does not indicate any relationship between them.

To describe such cases the term homoplastic is used. 


\section{Change of Function.}

Division of labour involves restriction of functions in the several parts of an animal, and no higher Metazoa could have arisen if all the cells had remained with the many-sided qualities of Amœbæ. Yet we must avoid thinking about organs as if they were necessarily active in one way only. For many organs, e.g., the liver, have several very distinct functions, and we know how wondrously diverse are the activities in our brains. In addition to the main function of an organ there are often secondary functions; thus, the wings of an insect may be respiratory as well as locomotor, and part of the food canal of Tunicates and Amphioxus is almost wholly subservient to respiration. Moreover, in organs which are not very highly specialised, it seems as if the component elements retained a considerable degree of individuality, so that in course of time what was a secondary function may become the primary one. Thus Dohrn, who has especially emphasised this idea of function change, says : "Every function is the resultant of several components, of which one is the chief or primary function, while the others are subsidiary or secondary. The diminution of the chief function and the accession of a secondary function changes the total function; the secondary function becomes gradually the chief one ; the result is the modification of the organ." Notice, in illustration, how the structure known as the allantois is an unimportant bladder in the frog, while in Birds and Reptiles it forms a foetal membrane (chiefly respiratory) around the embryo, and in most Mammals forms part of the placenta which effects nutritive connection between offspring and mother.

\section{"Substitution of Organs."}

The idea of several changes of function in the evolution of an organ, suggests another of not less importance which has been emphasised by Kleinenberg. An illustration will explain it. In the early stages of all vertebrate embryos, the supporting axial skeleton is the notochord, $-\mathrm{a}$ rod developed along the dorsal wall of the gut. From Fishes onwards, this embryonic axis is gradually replaced in development by the vertebral column or backbone; the notochord does not become the backbone, but is replaced 
by it. It is a temporary structure, around which the vertebral column is constructed, as a tall chimney may be built around an internal scaffolding of wood. Yet, it remains as the sole axial skeleton in Amphioxus, likewise in great part in hag and lamprey, but becomes less and less persistent in Fishes and higher Vertebrates, as its substitute, the backbone, develops more perfectly. Now, what is the relation between the notochord and its substitute the backbone, seeing that the former does not become the latter? Kleinenberg's suggestion is that the notochord supplies the stimulus, the necessary condition, for the formation of the backbone. Of course, we require to know more about the way in which an old-fashioned structure may stimulate the growth of its future substitute, but the general idea of one organ leading on to another is suggestive. It is consistent with our general conception of development - that each stage supplies the necessary stimulus for the next step; it also helps us to understand more clearly how new structures, too incipient to be of use, may persist.

\section{Rudimentary Oroans.}

In many animals there are structures which attain no complete development, which are rudimentary in comparison with those of related forms, and seem retrogressive when compared with their promise in embryonic life. But it is necessary to distinguish various kinds of rudimentary structures. (a) As a pathological variation, probably due to some germinal defect, or to the insufficient nutrition of the embryo, the heart of a mammal is sometimes incompletely formed. Other organs may be similarly spoilt in the making. They illustrate arrested development. Some animals lose, in the course of their life, some of the promiseful characteristics of their larval life; thus parasitic crustaceans at first free-living, and sessile sea squirts at first free-swimming, always undergo degeneration. The retrogression can be seen in each lifetime. But the little Kiwi of New Zealand, with mere apologies for wings, and many cave fishes and cave crustaceans with slight hints of eyes, illustrate degeneration which has taken such a hold of the animals that the young stages also are degenerate. The retrogression cannot be seen in each lifetime, evident as it 
is when we compare these degenerate forms with their ancestral ideal. (c) But among "rudimentary organs" we also include structures somewhat different, e.g., the gill clefts which persist in embryonic reptiles, birds, and mammals, though they serve no obvious purpose, or the embryonic teeth of whalebone whales. These are "vestigial structures," traces of ancestral history, and intelligible on no other theory. The gill clefts are used for respiration in all vertebrates below reptiles; the ancestors of whalebone whales doubtless had functional teeth. In regard to these persistent vestigial structures, it must also be recognised that we are not warranted in calling them useless. Though they themselves are not functional, they may sometimes be, as Kleinenberg suggests, necessary for the growth of other structures which are useful.

Classification of Organs. - We may arrange the various parts of the body physiologically, according to their share in the life. Thus, some parts have most to do with the external relations of the aninals; such are locomotor, prehensile, food-receiving, protective, aggressive, and copulatory organs. Of internal parts, the skeletal structures are passive; the nervous, muscular, and glandular parts are active. The reproductive organs are distinct from all the rest. They are often called "gonads," and should never be called glands. For by a gland we mean an organ which secretes, an organ whose cells produce and liberate some definite chemical substance, such as a digestive ferment. Whereas the gonads are organs in which certain celis, kept apart from the specialisation characteristic of most of the "body cells" or "somatic" cells, are multiplied, and eventually liberated.

Another classification of organs is embryological, i.e., according to the embryonic layer from which the various parts arise. Thus, the outer layer of the embryo (the ectoderm or epiblast) forms in the adult (I) the outer skin or epidermis; (2) the nervous system: (3) much at least of the sense organs: the inner layer of the embryo (the endodern or hypoblast) forms at least an important part (the "mid gut") of the food canal, and the lusis of outgrowths (lungs, liver, pancreas, \&c.) which may arise therefron, and also the notochord of Vertebrates: the mirddle layer of the embryo (the mesoderm or mesoblast) forms skeleton, connective swathings, muscle, \&c.

It is important to atopt some order of description. It is obviously prejuclicial to the success of your work and to the heal th of your brains, to describe an animal in any order that occurs to you, to skip from foodcanal to kidney, or from heart to reproductive organs. Therefore, in my descriptions I shall follow, almost consistently, this order of treatment:Mode of life, form, external appentages, skin, skeleton, muscle, nervous system, sense organs, food canal, hody cavity, vascular system, respiratory system, excretory system, reproductive system, development. 


\section{Tissues.}

Zoological anatomists, of whom Cuvier may be taken as a type, analyse animals into their component organs, and discover the homologies between one animal and another. But as early as x 80r, Bichât had published his Anatomie Générale, in which he carried the analysis further, showing that the organs were composed of tissues, contractile, nervous, glandular, \&c. In 1838-9, Schwann and Schleiden formulated the "cell theory," in which was stated the result of yet deeper analysis - that all organisms have a cellular structure and origin. The simplest animals (Protozoa) are typically single cells or unit masses of living matter; as such all animals begin; but all, except the simplest, consist of hundreds of these cells united into more or less homogeneous companies (tissues) which may be compacted, as we have seen, into organs. If we think of the organism as a great city of cells, the tissues represent streets (like some of those in Leipzig) in each of which some one kind of function or industry predominates.

Since Leydig gave a strong foundation to comparative histology in his remarkable Lehrbuch der Histologie des Menschen und der Thiere (Frankfurt, I 857), the study has been prosecuted with great energy, and has been constantly stimulated by improvements in microscopic apparatus and technique.

The student should read the introductory chapters in one of the numerous works on histology, so as to gain a general idea of the characters of the different tissues.

There are four great kinds,-epithelial, connective, muscular, and nervous.

\section{(a) Epithelial Tissue}

is illustrated by the external layer of the skin (epiclermis), the internal (endothelial) lining of the food canal and its outgrowths, the lining of the body cavity, \&c. ; by the early arrangements of cells in all embryos; and by the simplest Metazoa, such as Hydra, whose tulbular body is lined by two layers of epithelium. Embryologically and historically, epithelium is the most primitive kind of tissue. It may be single layered or stratified; its cells may be columnar, scale-like, or otherwise. The cells may be close together, or separated by intercellular spaces, and they are often connected by bridges of living matter. Nor are the functions of epithelium less diverse than its forms, for it may be ciliated 
(effecting locomotion, food-wafting, \&c.), or sensitive (and as such forming sense organs), or glandular (liberating certain products or even the whole contents of its cells), or pigmented (and thus associated with respiration, excretion, and protection), or covered externally with a sweated-off cuticle, susceptible of many modifications (especially of protective value).

\section{(b) Connective Tissue.}

This term is somewhat like the title "worms." It includes too many different kinds of things to mean much. It represents a sort of histological lumber room.

The embryologists help us a little, for they have shown that almost all forms of connective tissue are derived from the mesoderm or middle layer of the embryo. As this mesoderm usually arises in the form of outgrowths from the gut, or from ("mesenchyme") cells liberated at an early stage from either (?) of the two other layers of the embryo (ectoderm or endoderm), we may say that connective tissue is primarily derived from epithelium.

The general function of "connective tissue" is to enswathe, to bind, and to support, but the forms assumed are very various.

(a) The cells may be close together, without any intercellular "mortar" or matrix. They may contain large vacuoles, and thus produce the appearance of a network, or they may be laden with fat or with pigment.

(b) In other cases the cells of the connective tissue lie in a matrix, which they exude, or into which they in part die away. Such cells are very often irregular in outline, and give off in most cases fine processes, which traverse the matrix as a network. The fibrous tissue of tendons ancl the different kinds of gristle or cartilage illustrate connective tissue with much matrix. Cartilage is sometimes hardened by the deposition of lime salts in its substance, and then has a slight resemblance to another kind of "connective tissue" - bone. But bone, which is restricted to Vertebrate animals, is quite different from the cartilage which it often succeeds and replaces. It is made by strands or layers of special bone-forming cells (osteoblasts), which may rest on a cartilage foundation, or may be quite independent. These osteoblasts form the bone matrix, and some of them are involved in it, and become the permanent bone cells. These have numerous radiating branches, and are arranged in layers, usually around a cavity or a blood ressel. (There are no blood vessels in cartilage.) The matrix becomes rery rich in lime salts (especially phosphate); and the cartilage foundation, if there was one, is quite destroyed by the new formation. Here we may also note two important fluid tissues, the floating curpuscles or cells of the blood, and those of the borly cavity or "perivisceral "fluid, which is often abundant ancl important in backboneless animals.

\section{(c) Muscular Tissue.}

Origin. - The single celled Amuba moves by flowing out on one side and drawing in its substance on another. It is diffusely contractile, and it has also sensitive, digestive, and other functions.

In Hydra and some other Coelentera the bases of some of the epithelial 
cells which form the outer and inner layers are prolonged into contractile roots. Here then we have cells of which a special part discharges a contractile or muscular function, while the other parts retain other powers.

In other Colentera the muscular cells are still directly connected with the epithelium, but become more and more exclusively contractile. In all other animals the muscular tissue is derived from the mesoderm, which, as we have already mentioned, is not distinctly present in Coelentera. In the majority, the muscle cells arise on the walls of the body cavity, and their origin may often at least be described as epithelial. But in other cases the muscles arise from those wandering "mesenchyme" cells to which we have already referred.

Structure.-A distinction is usually drawn between striped and unstriped muscle fibres, but the distinction seems to be one of degree.

Smooth or unstriped muscle fibres are elongated contractile cells, externally homogeneous in appearance. They are especially abundant in sluggish animals, e.g., Molluscs, and occur in the walls of the gut, bladder, and blood vessels of Vertebrates. They are less perfectly differentiated than striped muscle fibres, and usually contract more slowly.

A striped muscle fibre is a cell, the greater part of which is modified into a set of parallel longitudinal fibrils, with alternating "clear and dark" transverse stripes. Each fibril has certainly a complex internal structure, but according to Haycraft the "stripes" are the optical effects of the ampullated or beaded form of the fibrils, and can be seen even on a collodion film, upon which the muscle fibres are pressed. A residue of unmodified cell substance, with a nucleus or with many, is often to be observed on the side of the fibre, and a slight sheath or sarcolemma forms the "cell wall." Many muscle fibres closely combined, and wrapped in a sheath of connective tissue, form a muscle, which, as every one knows, can contract with extreme rapidity when stimulated by a nervous impulse.

\section{(d) Nervous Tissue.}

Origin.-Beginning again with the Amaba, we recognise that it is diffusely sensitive, and that a stimulus can pass from one part of the cell to another.

In some Colentera some of the external cells seem to combine contractile and nervous functions. Therefore they are sometimes called " neuro-muscular."

But in Hydra there are special nervous cells, whose basal prolongations are connected with the contractile roots already described. This is a neuro-muscular apparatus of the simplest kind. The nerve cells probably receive impressions from without, and transmit them as stimuli to the contractile elements.

In sea anemones and some other Ccelentera, there is an interesting complication, withal very simple. There are superficial sensory cells, connected with subjacent nerve or ganglion cells, from which fibres pass to the contractile elements.

In higher animals the sensory cells are integrated into sense organs, the ganglionic cells into ganglia, while the delicate fibres which form 
the connections between sensory cells and ganglionic cells, and between the latter and muscles, are represented by well-developed nerves.

So far as we know, nervous tissue always arises from the outer or ectodermic layer of the embryo, as we would expect from the fact that this is the layer which, in the course of history, has been most directly subjected to external stimulus.

Structure.-Let us consicler first the ganglionic cells which receive stimuli and shunt them, which regulate the whole life of the organism, and are the physical conditions of "spontaneous" activity and intelligence. The simplest are prolonged at one pole into an outgrowth which branches into an afferent and efferent nerve fibre. Most, however, give off outgrowths from two poles or on all sides. Internally they consist in great part of a network or coil of fine fibrils, amid which lies the usual cell kernel or nucleus. Ganglionic cells, aggregated to form ganglia, generally lie embedded in a fibrous cellular substance called neuroglia, usually regarded as an ensheathing and supporting material.

In all but a few of the simplest Metazoa, the nerve fibres are surrounded by a sheath called the neurilemma, said to be formed by adjacent connective tissue. Several nerve fibres may combine to form a nerve, but each still remains ensheathed in its neurilemma. In Vertebrate animals each nerve filse usually consists of an internal "axis cylinder," the important part, and an external unessential medullary sheath. But even in the higher Vertebrates, "non-medullated" or simply contoured nerve fibres are found in the sympathetic and olfactory nerves, and this simpler type alone occurs in hag, lamprey, and lancelet, as well as in all the Invertel)rates with distinct nerves. Furthermore, nerves are usually surrounded by an enveloping nucleated layer called Schwann's sheath, or else lyy neuroglia.

A nerve fibre consists of numerous fibrils like those seen within a ganglion cell. These are regarded by some as the essential elements in conducting stimuli, while others maintain that the essential part is the less compact, sometimes well-nigh fluid stuff between the fibrils, or that the fibrils are but the walls of tubes within which the essentially nervous stuff lies.

According to some authorities, the nerve fibres are extensive prolongations of the ganglion cells; according to others the neuroglia or other ensheathing elements contribute to the extension of the nerve fibres, or rather special neuroblast cells malie both sheath and fibre.

\section{Cells.}

In discussing tissues, it was necessary to refer to the component cells. Let us now consider the chief characteristics of these elements.

A cell is a unit mass of living matter. Most of the simplest animals and plants (Protozoa and Protophyta) are single cells; eggs and male elements are single cells; in multicellular organisms the cells are combined into tissues and organs. 
Most cells are too small to be distinguished except through lenses; many Protozoa, e.g., large Amoebæ, are just visible to our unaided eyes; the chalk forming Foraminifera are single cells, whose shells are often as large as pin-heads, and some of the extinct kinds were as big as half-crowns; the bast cells of plants may extend for several inches; the largest animal cells are eggs distended with yolk.

History.-The word "cell" was first used in histological description by Hooke ( 1665 ), and Grew (167I-5), but not in a very accurate or definite way. Malpighi (I675) also described minute "utricles," some of which we should call cells.

Leeuwenhoek (Phil. Trans. I674) seems to have been the first to describe single-celled organisms. In the eighteenth century the analysis continued; thus Rösel von Rosenhof described the "Proteus animalcule" or Amaba in 1755, and Fontana, in 1784, discovered the kernel or nucleus of the cell.

But the fact that Bichât, in his Anatomie Gínérale (180r), spealis of tissues only, shows that the import of cells was not realised at the beginning of this century.

In I 835 , Robert Brown showed that a nucleus was normally present in all vegetable cells, and in the same year Johannes Muiller definitely compared the cells of plants with those of the notochord in animals.

The cellular structure and origin of organisms began to be vaguely recognised by many. At length, in $1838-9$, Schwann and Schleiden showed that all but the simplest plants and animals are built up of cells, and develop from cells, thus establishing the famous "cell theory," or, rather cell doctrine :- "There is one universal principle of development for the elementary part of organisms however different, and this principle is the formation of cells." 2

This doctrine was corroborated in many ways. Numerous investigators, Prévost and Dumas (I824), Martin Barry (I838-4I), Reichert I840), Henle (I84I), Kölliker (I843-6), and Remak (I84I-52), showed how the cells of the embryo arise from the division of the fertilised egg cell.

Moreover, Goodsir in $\mathbf{1} 845$, Virchow in 1858 , proved that in all cases, pathological as well as normal, cells arise from pre-existing cells, that onnis cellula e cellula is a general fact of histology.

There was a strong tendency, however, to attach too much importance to the cell wall, and too little to the contained cell substance. The all important protoplasm was not adequately appreciated.

In 1835 , Dujardin describer the "sarcode" of Protozoa, and other animal cells; in 1839 , Purkinje compared the substance of the animal

1 Those interested in history should read the scholarly history of cell lore by Sir William Turner, "The Cell Theory, Past and Present," Inaug. Address to Scottish Microscopical Society (Edin. I89o, also in Vature, 1890). See also Professor M'Kendrick On the Modern Cell Theory (Proc. Phil. Soc., Glasgow, 1888), also his text-book of Physiology. The articles Morphorogy and Protoplasm in the Encyc. Brit., and the article CELL in the new edition of Chambirs's Encyc., should be consulted. 
embryo with the "cambium" of plant cells; in 1846 Von Mohl emphasised the importance of the "protoplasm" in vegetable cells; Ecker (I849) compared the contractile substance of muscles with the living matter of amoebre; Donders also referred the contractility from the wall to the contents; Cohn suspected that the "sarcode" of animals and the "protoplasm" of plants must be "in the highest degree analogous sulstances;" and finally, Max Schultze (186r), accepted the growing belief that plants and animals were made of very similar living matter, and defined the cell as a unit mass of nucleated protoplasm.

Forms of Cells.--The typical and primitive form of cell is a sphere,-a shape naturally assumed by a complex coherent substance situated in a medium different from itself. Most egg cells and many Protozoa retain this primitive form, but the internal and external conditions of life (such as nutrition and pressures) often evolve other shapes,-oval, rectangular, flattened, thread-like, stellate, and so on.

Structure of Cells. - In a cell we may distinguish :-

(a) The general cell substance or cytoplasm, which consists partly of genuinely living stuff or protoplasm, and partly of complex materials not really living;

(b) A specialised kernel or nucleus, with a complex structure, and important, but hardly, as yet, definable functions ;

(c) One or more specialised bodies called central corpuscles or centrosomata which seem to be centres of activity during cell division;

(d) A cell wall, which occurs in very varied form, or may be entirely absent.

(a) The Cell Substance.-When a simple cell is examined in its living state, it often appears approximately homogeneous. Its substance is usually slightly fluid, but it may be firm and compact in passive cells. It is usually translucent, but there are often obscuring granules of different kinds.

In thinking of the cell substance or cytoplasm, we must distinguish the genuinely living protoplasm, of whose nature we know almost nothing, from associated substances, such as proteids, carbohydrates, fats, pigments, \&c., whose chemical composition can be ascertained. The associated substances which often crowd the protoplasm, are due to the chemical ascent of food material towards protoplasm, and to the chemical disruption which protoplasm undergoes or produces as it lives. 
(b) The Nucleus.--Almost every cell contains a nucleus or several. It used to be said that some very simple animals which Hæckel called Monera had no nuclei, but in several they have been recently discovered. In other cases, e.g., some Infusorians, the nuclear material seems to be diffused in the cell substance. The red blood cells of Mammals seem to be distinctly nucleated in their early stages, but there is no trace of a nucleus in those which are full grown. We may safely say that cells without nuclei are very rare, though in some cells the nuclei are less differentiated than in others.

The nucleus is a very important part of the cell, but it is not yet possible to define precisely what its importance is. In fertilisation an essential process is the union of the nucleus of the spermatozoon or male cell with the nucleus of the ovum or female cell (Fig. 3). In cell division, the nucleus certainly plays an essential part. Cells bereft of their nuclei die, or live for a while a crippled life. According to some, the nucleus is important in connection with the nutrition of the cell, and it is generally believed that there are complex actions and reactions between the living matter of the nucleus and that of the cytoplasm.

The nucleus often lies within a little nest in the midst of the cell substance, but it may shift its position from one part of the cell to another. It has a definite margin, but this may be lost, e.g., before cell division begins. Internally, it is anything but homogeneous; at any rate, homogeneous nuclei are rare. Usually there is a network of fine strongly stainable (chromatin) strands, with less stainable (achromatin) substance in the meshes. But in other cells, or at another time in the same cell, the nucleus is seen to contain a coiled (chromatin) thread, or a number of chromatin loops (Fig. 2). Weismann and others believe that these chromatin elements or chromosomes are the bearers of hypothetical bodies whose properties are supposed to determine the nature of an organism and its life. Many nuclei also contain one or more little round bodies or nucleoli, apparently of less importance. The term is applied somewhat vaguely to little aggregations of chromatin, and more properly to vacuole-like bodies, in which some believe that the waste products of the nucleus are collected. 
Within the nucleolus an "endo-nucleolus" has been discovered. Though the nuclei of different cells differ in details, there is a fundamental sameness, both of structure and activity, throughout the world of cells.

(c) The Centrosomes.--When a cell divides into two, the chromatin elements or chromosomes of the nucleus are also divided and separate to form the two daughter nuclei. In this separation extremely fine "archoplasmic" threads have been seen passing to the chromosomes from beside two minute bodies in the cytoplasm. These two bodies are called central corpuscles or centrosomata (Figs. 3 and 4 ); they seem to act like two centres of force. They also occur, in most cases singly, in resting cells, and it seems likely that they are constant parts of the cell, and that they arise from within the nucleus.

(d) The Cell Wall.-To the earlier histologists, who often spoke of cells

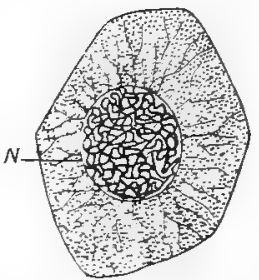

Fit: 2. - Structure of the cell. (After CARNOY.)

n. Nucleus with chromatic coil, note protoplasmic reticulum. as little bags or boxes, the wall seemed of much moment. It is, however, the least important part of the cell. In

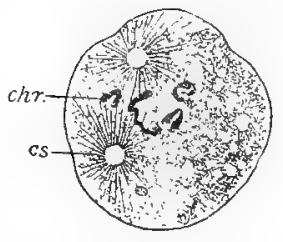

FIG. 3.- Fertilised ovum of Asiaris. (After BOVER1.)

chr. Chromatin elements, two from ovum nucleus and two from sperm nucleus; $c s_{0}$. centrosoma from which "archoplasmic" threads radiate, partly to the chroniosomes.

which are not very plant cells there is usually a very distinct wall, consisting of cellulose. This is a product, not a part, of the protoplasm, though some protoplasm may be intimately associated with it as long as its growth continues. In animal cells there is rarely a very distinct wall chemically distinguishable from the living matter itself. But the margin is often different from the interior, and a slight wall may be formed by a superficial compacting of the threads of the cell network, or by a physical alteration of the cell substance, comparable to the formation of a skin on cooling porridge. In other cases, especially in cells active, such as ova and encysted 
Protozoa, a more definite sheath is formed around the

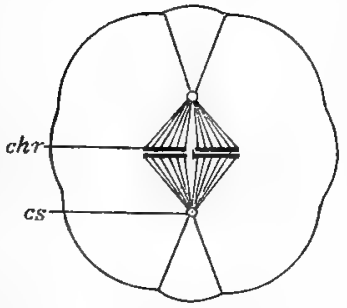

FIG. 4. - Diagram of cell division. (After Boverri.)

chr. Chromosomes forming an equatorial plate; cs. centrosoma. cell substance. Again, animal cells may die off superficially into a "cuticle," sometimes of known chemical composition, as, for instance, the chitin formed by the ectoderm cells in Insects, Crustaceans, and other Arthropods.

In animals, as well as in plants, adjacent cells are often linked by intercellular bridges of living matter.

Cell Division.-Though the division of cells, by which all growth is affected, is a subject with which the physiologist is as much concerned as the morphologist, it will be convenient to discuss it here. The following facts are most important.

(I) We know that there is a striking unity in all cases, and that the nucleus plays an essential part in the process. In most cases the dividing nucleus passes through a series of complex changes known as karyokinesis or mitosis, and these are much the same everywhere, though different kinds of cells have their specific peculiarities. Occasionally, however, both in Protozoa and Metazoa the nucleus divides by simple constriction (direct or amitotic division).

(2) The eventful changes of karyokinesis are as follows:-

(a) The resting stage of the nucleus shows a network or complete coil of filaments (chromatin elements) (Fig. 2).

(b) First Stage. -As division begins, the membrane separating the nucleus from the cell substance disappears, and the chromatin elements are seen as a tangled or broken coil (Fig. 5, I).

(c) Astroid-stage. - The chromatin elements bend into looped pieces, which are disposed in a star, the free ends of the U-shaped loops being directed outwards. Meanwhile, a centrosome has appeared and divided into two separating halves, between which a spindle of fine achromatin threads is formed. This seems to form (at least part of) what is called the nuclear spindle. The centrosomes separate until one lies at each pole of the cell, surrounded by radiating "archoplasmic" threads, which become attached to the chromosomes (Fig. 5, 2). 
(d) Diviston and separation of the loops.-Each of the loops which nake up the star divides longitudizally into two, and each half separates from its neighbour. They lie at first near the equator of the cell, but they are apparently drawn, or driven, to the opposite poles (Fig. 5, 2-3).

(e) Diastroid.-The single star thus forms two daughter stars, which separate further and further from one another towards the opposite poles of the cell, remaining connected, however, by delicate threads (Fig. 5, 3-5).

(f) Each daughter star is reconstituted into a coil or network for each daughter cell, for the cell substance has been constricted meanwhile at right angles to the transverse axis of the spindle. The halves separate in the case of Protozoa, but in most other cases, e.g., growing embryos, they remain adjacent, with a slight wall between them (Fig. 5, 6).

(g) Each daughter nucleus then passes into the normal resting phase. The spindle and usually the centrosomes also disappear.

Flemming gives the following summary of karyokinesis :-

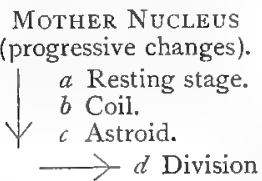

DAUGHTER NuCleUS (regressive changes).

Resting stage.

Coil.

Diastroid.

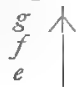

\section{(metakinesis).}

(3) We are far from being able to give even an approximate account of the "mechanism" of cell division. Rapidly progressire research has disclosed many mysteries, but it does not explain them. The nucleus is resolved into a chromatin framework and an achromatin matrix, but we know the nature of neither. The longitudinal division of each loop shows how thorough is the partition of substance and implied qualities. The "central corpuscles," recently discovered, act like centres of force, and the indescribably fine threads, which pass from around these to the chromatin loops, have been credited with motive powers. Similarly the threads of the nuclear spindle are believed by some to draw or drive the chromosomes. But we do not know. The whole process is vital, and therefore inexplicable in terms of matter and motion, so long at least as we do not know the secret of protoplasm.

(4) On the other hand, Leuckart and Spencer have given a general rationale of cell division. Why do not cells grow much larger, why do they almost always divide at a definite limit of growth? Their answer is as follows:- Suppose a young cell has doubled its original mass, that means that there is twice as much living matter to be kept alive. But the living matter is fed, aerated, purified through its surface, which, in growing spherical cells for instance, only increases 
as the square of the radius, while the mass increases as the cube. The surface growth always lags behind the increase of mass. Therefore, when the cell has, let us say, quadrupled its original mass, but by no means quadrupled its surface, difficulties set in, waste begins to gain on repair, anabolism loses some of its ascendancy over katabolism. At the limit of growth, then, the cell divides, halving its mass and gaining new surface. Of course surface may be increased by outflowing processes, just as that of leaves by many lobes; and
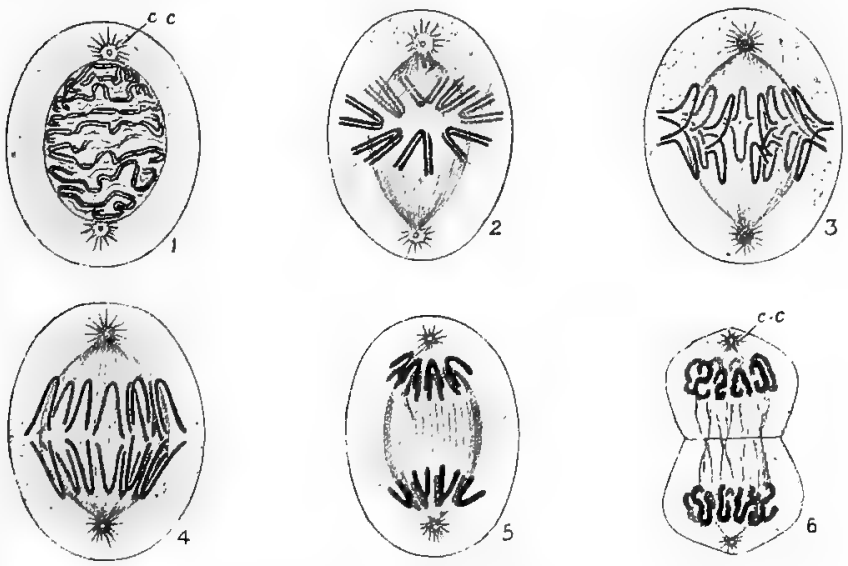

FIG. 5.-Karyokinesis. (After Flemming.)

1. Coil stage of nucleus; $c c_{3}$ central corpuscle.

2. Division of chromatin elements into U-shaped loops, and longitudinal splitting of these (astroid stage).

3.4. Recession of chromatin elements from the equator of the cell (diastroid).

5. Nuclear spindle, with chromatin elements at each pole, and achromatin threads between.

6. Division of the cell completed.

division may occur before the limit of growth is reached, but as a general rationale, applicable to organs and bodies as well as to cells, the suggestion of Leuckart and Spencer is very helpful.

(5) Protoplasm. - Morphological as well as physiological analysis passes from the organism as a whole to its organs, thence to the tissues, thence to the cells, and finally to the protoplasm itself. But although 
we may define protoplasm as genuinely living matter - as "the physical basis of life "-we cannot definitely say how much or what part of an Amoba, or an ovum, or any other cell is really protoplasm. We are able to make negative statements, e.g., the yolk of an egg is not protoplasm. but we cannot make positive statements, or say, This is protoplasm and nought else. Thus, what is spoken of as the structure of protoplasm is really the structure of the cytoplasm.

In regard to this structure, we know that it is very complex, but we are not sure of much more. For different experts see different appearances, even in the same cells.

Thus some, e.g., Frommann, see an intricate network or reticulum with less stable material in the meshes; others, e.g., Flemming, see what looks like a manifold coil of fibrils : and others, e.g., Bütschli, see a foam-like or vacuolar structure. It seems likely that the structure is different at different times, or in different cells.

Professor Biitschli's belief that the cytoplasm has a vacuolar structure is corroborated by his interesting experiments on microscopic foams. Finely powdered potassium carbonate is mixed with olive oil which has been previously heated to a temperature of $50^{\circ}-60^{\circ} \mathrm{C}$., an acid from the oil splits up the potassium carbonate, liberates carbon dioxide, and forms an extremely fine emulsion. Drops of this show a structure like that of cytoplasm, exhibit movements and streamings not unlike those of Amolse, and are, in short, mimic cells. Just as a working model may help us to understand the circulation, so these oil emulsions may help us to understand the living cell, - by bringing the strictly vital phenomena into greater prominence. 


\section{CH A P TER IV.}

\section{THE REPRODUCTION AND LIFE HISTORY OF ANIMALS.}

\section{REPRODUCTION.}

IN the higher animals the beginnings of individual life are hidden, within the womb in mammals, within the egg shell in birds. It is natural, therefore, that early preoccupation with those higher forms should have hindered the recognition of what seems to us so evident, that almost every animal arises from an egg cell or ovum which has been fertilised by a male cell or spermatozoon. The exceptions to this fact are those organisms which multiply by buds or detached overgrowths, and those which arise from an egg cell which requires no fertilisation. Thus Hydra may form a separable bud, much as a rose bush sends out a sucker; thus drone bees "have a mother but no father," for they arise from parthenogenetic eggs which are not fertilised. Apart from these and similar cases, the "ovum theory," which Agassiz called "the greatest discovery in the natural sciences in modern times," is true, - that each organism begins from the division of a fertilised egg cell.

History. - We can realise this discovery better if we consider its history. For a long time, on into the present century, what was called the doctrine of preformation prevailed. According to this theory, development was merely an unfolding ("evolution") of a preformed miniature which lay within the germ. The "ovists" found this miniature model of the future organism in the egg; the "animalculists" found and even figured it within the spermatozoon. "There is no becoming," said Haller, "no part of the body is made from another, all are created at once." But this was not all. The germ was more than a marvellous bud-like miniature of the adult, it included the next 4 
generation, and the next, and the next, and all future generations. Germ lay within germ, preformed in transparency, and in successively smaller miniature, after the fashion of an infinite juggler's box. We laugh at this, but we need not laugh too much, for the preformationists, though wrong and crude in their facts, were right in two of their ideas, - that the germ contains the potentiality of a future organism, and that it has relations, not only to the animal into which it develops, but also to generations following. (See p. 7I.)

In the middle of the seventeenth century, however, Harvey had reached conclusions which might have saved much blundering. Study. ing the development of the chick, - as Greek naturalists had tried to do wellnigh two thousand years before, as we are doing still in our embryological laboratories, - Harvey maintained that every animal was produced from an ovum (oon esse primordium commune onnibus animalibus), and that organs arose by new formation (epigenesis), not by the expansion or "evolution" of some invisible preformation.

But the great champion of epigenesis was Caspar Friedrich Wolff, who, in his doctorial dissertation of 1759 , traced the chick back to a layer of organised particles (the familiar cells of to-day), in which there was no likeness of the future embryo, far less of the adult.

Wolff was long in finding successors, but in I824 Prévost and Dumas described the division of the ovum; in 1827 Von Baer discovered the mammalian ovum; while Wagner, Von Siebold, and others elucidated the real nature of the spermatozoon.

A great step was made in 1838-9, when Schwann and Schleiden formulated the "cell theory," according to which every organism is made up of cells, and starts from a cell. From this date modern em. bryology began.

\section{Sexual Reproduction.}

There is apt to be a lack of clearness in regard to sexual reproduction, because the process which we describe by that phrase is a complex result of evolution. It involves two distinct facts:- $(a)$ the liberation of special germ cells from which new individuals arise; (b) the occurrence of two different kinds of germ cells-ova and spermatozoa, which come to nothing unless they unite (fertilisation). Furthermore, these dimorphic reproductive cells are produced by two different kinds of individuals (females and males), or from different organs of one individual or at different times within the same organ (hermaphroditism).

It is conceivable that organisms might have gone on multiplying asexually, by detaching overgrown portions of themselves which had sufficient vitality to develop into complete forms. But a more economical method is the liberation of special germ cells, in which the qualities of the 
organism are inherent. This is the primary characteristic of sexual, as opposed to asexual multiplication.

It is also conceivable that organisms might have remained approximately like one another in constitution, and at all times very nearly the same, and that they might have liberated similar germ cells capable of immediate development. Such a race would have illustrated the one characteristic of sexual reproduction, the liberation of special germ cells, but it would have been without that other characteristic of sexual reproduction,-the existence of dimorphic germ cells, of different kinds of sexual organs, or of male and female individuals.

\section{The Liberation of Special Germ Cells.}

One must think of this as an economical improvement on the method of starting a new life by asexual overgrowth or by the liberation of buds. Asexual reproduction, as Spencer and Hrckel point out, is a mode of growth in which the bud, or whatever it is, becomes discontinuous from the parent. The buds of a sponge, of a coral, of a sea mat (Polyzoon), or of many Tunicates, remain attached to the parent. If there be a keen struggle for subsistence, this may be disadvantageous; but in some cases, doubtless, the colonial life which results is a source of strength. In the case of Hydra, however, the buds are set adrift; the same is true of not a few worms. This liberation of buds takes us nearer the sexual process of liberating special germ cells. But unless the organism is in very favourable nutritive conditions, in which overgrowth is natural, the liberation of buds is evidently an expensive way of continuing the life of a species. Not only so, but we can hardly thirk of budding even as a possibility in very complex organisms, like snails or birds, in which there is much division of labour. Moreover, the peculiarity of a true germ cell is, that it is unspecialised, continuous in quality with the original germ cell from which the parent arose, and not very liable to be tainted by the mishaps which may befall the "body" which bears it. And, finally, in the mixture of two units of living matter which have had different histories, the possibility of permutations and combinations, in other words, of variation 
is evidently supplied (see p. 63). Thus it is not surprising to find that the asexual method of liberating buds has been replaced in most animals by the liberation of special germ cells, by the more economical and advantageous process of sexual reproduction.

\section{Summary of Modes of Reproduction.}

\section{A. In single celled Animals (Protozoa).}

(I) The almost mechanical rupture of an amoeboid cell, which has become too large for physiological equilibrium (e.g., Schizogenes).

(2) The discharge of numerous superficial buds at once $(e . g$. , Arcella and Pelomyxa).

(3) The formation of one bud at a time (very common).

(4) The ordinary division into two daughter cells at the limit of growth.

(5) Repeated divisions within limited time and within limited space (a cyst). This results in what is called spore formation, "free cell formation," "endogenous multiplication" (e.g., in Gregarines).

\section{B. In many celled Animals (Metazoa).}

\section{(Asexual.)}

(a) The separation of a clump of body cells, e.g., from the surface of some Sponges. (A crude form of budding.)

(b) The formation of definite buds which may or nuay not be liberated; and other forms of asexual multiplication.

\section{(Sexual.)}

(a) The liberation of cells from a simple Metazoon in which there is so little division of labour that the distinction between body cells and reproductive cells is not marked. (Hypothetical.)

(b) The liberation of special reproductive or germ cells, which have not taken part in the formation of the body, and which retain, more or less unaltered, the inherent qualities of the original germ cell from which the parent arose. These special reproductive cells-the ova and spermatozon-are nomally united in fertilisation, but some animals have (parthenogenetic) ova which develop without being fertilised.

\section{The Evolution of Sex.}

A further problem is to account for the two facts $(a)$ that most animals are either males or females, the former liberating actively motile male elements or spermatozoa, the latter 
forming and usually liberating more passive egg cells or ova; and $(b)$ that these two different kinds of reproductive cells usually come to nothing unless they combine.

The problem is partly solved by a clear statement of the facts. Begin with those interesting organisms which are on the border line between Protozoa and Metazoa, the colonial Infusorians of which Volvox is a type (see p. 95). The adults are balls of cells, and the component units are connected by protoplasmic bridges. From such a ball of cells reproductive units are sometimes set adrift, and these divide to form other individuals without more ado. In other conditions, however, when nutrition is checked, a less direct mode of reproduction occurs. Some of the cells become large well fed elements, or ova; others, less successful, divide into many minute units or spermatozoa. The large cells are fertilised by the small. Here we see the formation of dimorphic reproductive cells in different parts of the same organism. But we may also find Volvox balls in which only ova are being made, and others with only spermatozoa. The former seem to be more vegetative and nutritive than the latter; we call them female and male organisms respectively; we are at the foundation of the differences between the two sexes.

All through the animal series, from active Infusorians and passive Gregarines, to feverish Birds and more sluggish Reptiles, we read antitheses between activity and passivity, between lavish expenditure of energy and a habit of storing. The ratio between disruptive (katabolic) processes and constructive (anabolic) processes in the protoplasmic metabolism varies from type to type. We believe that the contrast between the sexes is another expression of this fundamental alternative of variation.

This theory may be confirmed in many ways, e.g., by contrasting the characteristic products of female life,passive ova, with the characteristic products of male life,active spermatozoa; or by comparing the complex conditions (such as abundant food, favourable temperature) which tend to produce female offspring, with the opposite conditions which tend to produce males; or by contrasting the secondary sexual characters of males (e.g., bright colours and smaller size), with the opposite characteristics of females. 


\section{Stages in the History of Fertilisation.}

While it is not difficult to see the advantage of fertilisation as a process which helps to sustain the standard or arerage of a species and as a source of new variations, we can at present do little more than indicate various forms in which the process occurs.

(a) Formation of Plasmodia, the flowing together of numerous feeble cells, as seen in the life history of those very simple Protozoa called Proteomyxa, e.g., Protomyxa, and Mycetozoa, e.g., flowers of $\tan$ (Ethatium septicum).

(b) Multiple Conjugation, in which more than two cells unite and fuse together, as in some Gregarines and in the sun animalcule (Actinospharium).

(c) Ordinary Conjugation, in which two similar cells fuse together, observed in Gregarines and Rhizopods. In ciliated Infusorians, the conjugation may be merely a temporary union, during which nuclear elements are interchanged.

(d) Dimorplic Conjugation, in which two cells different from one another fuse into one, a process well illustrated in Vorticella and related Infusorians, where a small, active, free swimming (we may say, male) cell unites with a fixed individual of normal size, which may fairly be called female (see Fig. 23, p. 94).

(e) Fertitisation, in which a spermatozoon liberated from a Metazoon unites intimately with an ovum liberated from another individual normally of the same species.

\section{Divergent Modes of Sexual Reproduction.}

(a) Hermaphroditism is the combination of male and female sexual functions in varying degrees within one organism. It may be demonstrable in early life only, and disappear as maleness or femaleness predominates in the adult. It may occur as a casualty or as a reversion; or it may be normal in the adult, e.g., in some Sponges and Coelentera, in many "worms," e.g., earthworm and leech, in barnacles and acorn shells, in one species of oyster, in the snail, and in many other Bivalves and Gastropods, in Tunicates and in the hagfish. In most cases, though these animals are bisexual, they produce ova at one period and spermatozoa at another (dichogany). It rarely occurs (e.g., in some parasitic worms) that the ova of a hermaphrodite are fertilised by the spermatozoa of the same animal. Certain facts, such as the occurrence of hermaphrodite organs as a transitory stage in the development of the embryos of many unisexual animals (e.g., frog and bird), make it likely that hermaphroditism is the primitive condition, and that the 
unisexual condition of permanent maleness or femaleness is a secondary differentiation. The cases which we have cited above may be interpreted as due to persistence of the primitive condition, or as reversions to it.

(b) Parthenogenesis, as we know it, is a degenerate form of sexual reproduction, in which ova produced by female organisms develop without being fertilised by male elements. It is well illustrated by Rotifers, in which fertilisation is not known to occur, while in some genera males have never been found; by many small Crustaceans whose males are absent for a season; by aphides, from among which males may be absent for the summer (or in artificial conditions for several years) without affecting the rapid succession of female
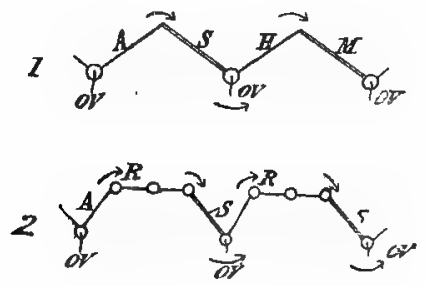

FIG. 6.-Diagrammatic expression of alternation of generations.

I. Hydromedusæ.

ov. Fertilised ovum gives rise to asexual form $A$, which, by budding, produces sexual form or forms $S$; in case of Hydromedusa $A$ is represented by hydroid $(H)$ and, $S$ by medusoid $(M)$.

II. Liver Fluke.

ov. Fertilised ovum gives rise to asexual stages $(A)$ which, from special spore-like cells $(R)$, produce eventually the sexual fluke $(S)$.

generations; by the production of drones in the bee hive,for the eggs which give rise to drones are unfertilised (see p. 60).

(c) Altemation of Generations.-A fixed asexual hydroid or zoophyte (campanularian or tubularian) often buds off and liberates sexual medusoids or swimming bells, whose fertilised ova develop into embryos which become fixed and grow into hydroids (Fig. 49, p. I 56). This is the simplest illustration of alternation of generations, which may be 
defined as the alternate occurrence in one life cycle of two (or more) different forms differently produced.

The liver fluke (Distoma hepaticum) of the sheep produces eggs which when fertilised grow into embryos. Within the latter, certain cells (which can hardly be called eggs) grow into numerous other larvæ of a different form. Within these the same process is repeated, and finally the larvæ thus produced grow (in certain conditions) into sexual flukes (Fig. 54, p. 168). In this case, reproduction by special cells like undifferentiated precocious ova, alternates with reproduction by ordinary fertilised egg cells. So, too, the vegetative sexless "fern plant" gives rise to special spore cells, which develop into an inconspicuous bisexual "prothallus," from the fertilised egg cell of which a "fern plant" springs.

Various kinds of alternation are seen in the life cycle of the fresh water sponge, in the stages of the jelly fish Aurelia, in the history of some "worms" and Tunicates. They illustrate a rhythm between asexual and sexual multiplication, between parthenogenetic and normally sexual reproduction, between vegetative and animal life, between a relatively " anabolic" and a relatively "katabolic" preponderance.

\section{EMBRYOLOGY.}

The Egg Cell or Ovum.-Apart from cases of asexual reproduction and parthenogenesis every multicellular organism begins life as an egg cell with which a male cell or spermatozoon has entered into intimate union.

The most important characteristic of the reproductive cells, whether male or female, is that they retain the essential qualities of the fertilised ovum from which the parent animal was developed.

'The ovum has the usual characters of a cell; its substance is traversed by a fine protoplasmic network; its nucleus or germinal vesicle contains the usual chromatin elements; it has often a distinct sheath representing a cell wall.

In Sponges, the ova are well nourished cells in the middle stratum of the body; in Cœlentera they seem to arise in connection with either outer or inner layer (ectoderm or endoderm); in all other animals, they arise in connection 
with the middle layer or mesoderm, usually on an area of the epithelium lining the body cavity. In lower animals they often arise somewhat diffusely ; in higher animals their formation is restricted to distinct regions, and usually to definite organs-the ovaries.

The young ovum is often amceboid, and that of Hydra retains this character for some time (Fig. 4I, p. I39). The ovum grows at the expense of adjacent cells, or by absorbing material which is contributed by special yolk glands or supplied by the vascular fluid of the body.

The yolk or nutritive capital may be small in amount,

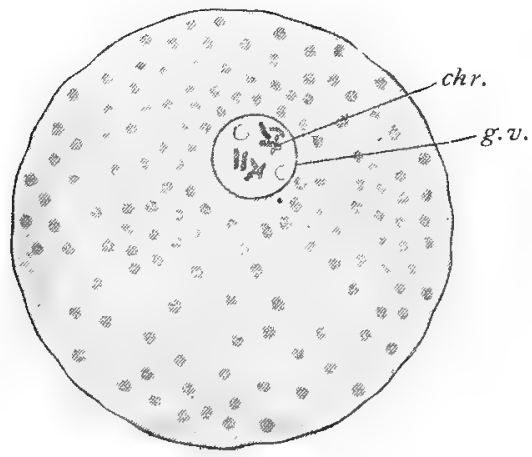

FIG. 7.-Diagram of ovum, showing diffuse yolk granules.

g.v. Germinal vesicle or nucleus; chr. chromatin elements.

and distributed uniformly in the cell as in the ova of Mammals, earthworm, starfish, and sponge; or it may be more abundant, sinking towards one pole as in the egg of the frog, or accumulated in the centre as in the eggs of Insects and Crustaceans; or it may be very copious, dwarfing the formative protoplasm, as in the eggs of Birds, Reptiles, and most Fishes.

Round the egg there are often sheaths or envelopes of various kinds, $(a)$ made by the ovum itself, and then very delicate (e.g., the vitelline membrane); (b) formed by adjacent cells (e.g., the follicular envelope); or $(c)$ formed by 
special glands or glandular cells in the walls of the oviducts (e.g., the "shells" of many eggs). The envelope is often firm, as in the chitinous coat around the eggs of many Insects, and in these cases there is often a little aperture (micropyle) through which alone the spermatozoon can enter. The hard calcareous shells round the eggs of Birds and Tortoises, or the mermaid's purse enclosing the egg of a skate are of course formed after fertilisation. Egg shells must be distinguished from egg capsules or cocoons, e.g., of the earthworm, in which several eggs are wrapped up together.

The Male Cell or Spermatozoon is a much smaller and usually a much more active cell than the ovum. In its
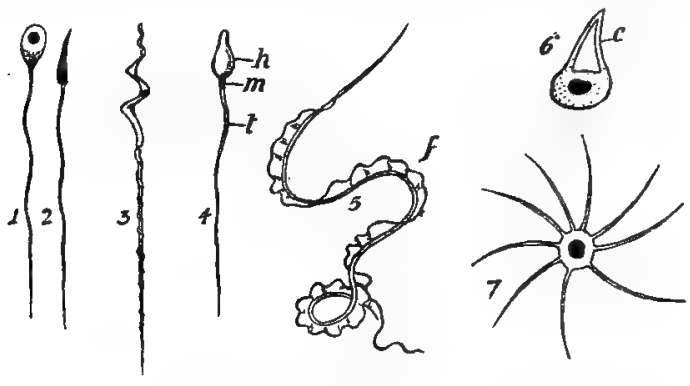

FIG. 8. -Forms of Spermatozoa (not drawn to scale).

I and 2. Immature and mature spermatozoa of snail ; 3. of bird; 4. of man ( $h$, head; $m$, middle portion; $t$, tail); 5 - of salamander, with vibratile fringe $(f) ; 6$. of Ascaris, slightly amoboid with cap (c); 7. of crayfish.

minute size, locomotor energy, and persistent vitality, it resembles a flagellate monad, while the ovum is comparable to an amoba or to one of the more encysted Protozoa.

A spermatozoon has usually three distinct parts: the essential "head," consisting mainly of nucleus, and the mobile "tail" which is often fibrillated, and a small middle portion between head and tail, which is regarded by some as the centrosome. The spermatozon of Threadworms and Crustaceans are sluggish, and inclined to be amoboid (Fig. $8(6,7))$.

Both ova and spermatozoa are true cells, and they are 
complementary, but the spermatozoon has a longer history behind it. The homologue of the ovum is the mother sperm cell or spermatogonium. This segments much as the ovum does, but the cells into which it divides have little coherence. They go apart, and become spermatozoa. There is a striking resemblance between the different ways in which a mother sperm cell divides and the various kinds of segmentation in ova. In most cases the spermatogonium divides into spermatocytes, which usually divide again into spermatides or young spermatozoa.

Maturation of the Ovum.-When the egg cell attains its definite size or limit of growth, it bursts from the ovary or

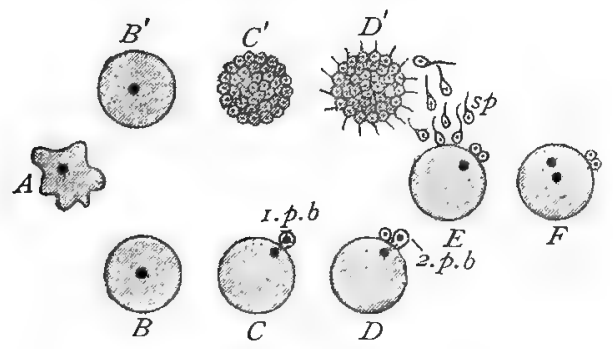

FIG. 9.-Diagram of maturation and fertilisation. (From "Evolution of Sex.")

4. Primitive sex cell, supposed to be amoboid.

$B$. Ovum ; $C$. formation of first polar body $(x . \not B . b) ;$.$D . formation$ of second polar body $(2, p b$.$) .$

$B$ x. Mother sperm cell; $C$ x. the same divided (sperm-morala or polyplast, or spermatogonium).

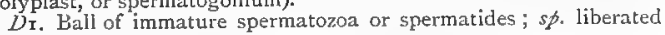
spermatozoa.

$E$. Process of fertilisation; $F$. approach of male and female nuclei within the ovum.

from its place of formation, and in favourable conditions meets either within or outside the body with a spermatozoon from another animal. Before this union between ovum and spermatozoon is effected, generally indeed before it has begun, the nucleus or germinal vesicle of the ovum moves to the periphery and divides twice. This division results in the formation and extrusion of two minute cells or polar 
bodies, the first containing half, the second necessarily a quarter of the nuclear material which composed the germinal vesicle. The nucleus is thus reduced to a quarter of its original chromatin content. It is noteworthy that the second division follows close on the first without the intervention of the "resting stage," which usually succeeds a nuclear division. Moreover, there is this important difference between the formation of polar bodies and ordinary cell division, that the number of nuclear rods or chromosomes suffers reduction, whereas in ordinary karyokinesis the daughter nuclei have as many nuclear rods as the original cell. The extruded polar bodies come to nothing, though they may linger for a time in the precincts of the ovum, and may even divide. The extrusion of polar globules from mature ova seems to be almost universal; but observations are lacking in regard to Birds and Reptiles. Moreover, Weismann and Ischikawa have shown that in all parthenogenetic ova which they have examined, only one polar body is formed. It is said, however, that in the parthenogenetic eggs which become drones (Blochmann), and in those of a moth called Liparis (Platner), two polar bodies are formed. But in neither of these two exceptional cases is the parthenogenesis habitual; thus many of the eggs which the queen bee lays are fertilised, and give rise to queens and workers.

One of the most important results of recent investigations as to polar hodies is due to O. Hertwig and others. It may be briefly stated, with particular reference to the ova of Ascaris megalocephala-the threadworm of the horse. In one variety of this worm (var. bivalens) the germinal vesicle of the ovum contains four nuclear rods, chromosomes, or idants. By doubling these increase to eight (Fig. IO, B); the first polar body goes off with four (Fig. IO, C), and the second with two (Fig. IO, D); leaving two. Two "reducing divisions" have thus occurred. Similarly, the homologue of the ovum, the sperm mother cell contains four chromosomes in its nucleus (Fig. 10, $\mathrm{A}^{1}$ ). By doubling these increase to eight (Fig. IO, B ), and by division the cell forms four spermatozoa, each with two. When fertilisation takes place, the nucleus of the spermatozoon, with two chromosomes, unites with the reduced nucleus of the ovum, also with two chromosomes; and the number is thus raised to four, which is the nomial number in the cells of this variety of Ascaris megalocephala. There is thus a striking parallelism in the history of the two nuclei which unite in fertilisation; both have been subjected to reducing divisions. If this did not occur, each fertilisation would involve a doubling of the number of chromosomes. Weismann interprets the whole process as an arrangement by which the com- 
binations and permutations of nuclear rods and their vital qualities are increased so as to give rise to new variations.

There are, indeed, other interpretations, and the facts are difficult to understand on any theory. Thus Minot, Balfour, Tan Beneden, and others have suggested that the polar bodies are extrusions of male substance from the ovum. Bütschli, Giard, and others interpret the premature division of the ovum as the survival of an ancient habit, and regard the polar bodies as rudimentary or abortive ova.

It may be possible to combine various interpretations: (I) the ovum divides, like any other cell, like the Protozoon ancestors, at its limit of growth; (2) the extrusion does in some way differentiate the ovum and renders fertilisation possible or more profitable; (3) the peculiar reduction involved in the process makes the origin of new variations more certain.

Fertilisation. - In the seventeenth and eighteenth centuries, some naturalists, nicknamed "ovists," believed that

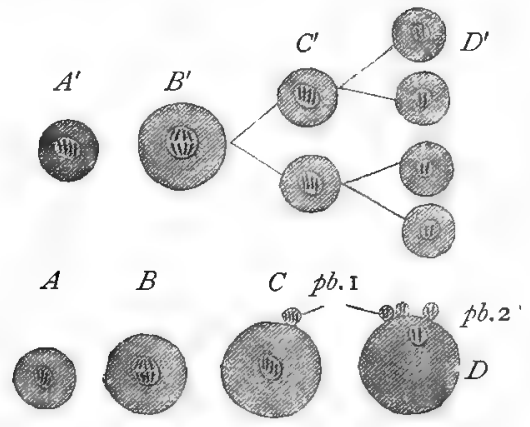

FIG. IO.-Spermatogenesis and Polar bodies. (After Hertwig and WeismanN.)

Ar. Primitive germ cell of Ascaris megaloceshala war. bivalens (4 chromosonies).

BI. Sperm nother cell (8 chromosomes).

Cr. Two spermatocytes formed, each with 4 chromosomes (first reducing division).

Dr. Four spermatozoa formed, each with a chromosomes (second reducing division).

A. Primitive germ cell (4 chromosones).

B. Fully developed ovum ( 8 chromosomes).

C. Formation of first polar body ( $p b . \mathrm{l}$ ) (first reducing division).

D. Formation of second polar body $(p b, z)$ (second reducing division). First polar body may divide into two.

the ovum was all-important, only needing the sperm's awakening touch to begin unfolding the miniature model which it contained. Others, nicknamed "animalculists," were equally confident that the sperm was essential, though it required to be fed by the ovum. Even after it was 
recognised that both kinds of reproductive elements were essential, many thought that their actual contact was unnecessary, that fertilisation might be affected by an aura seminalis. Though spermatozoa were distinctly seen by Hamm and I.eeuwenhoek in 1677 , their actual union with ova was not observed till 1843 , when Martin Barry detected it in the rabbit.

Of the many facts which we now know about fertilisation, the following are the most important :-

(I.) Apart from the occurrence of parthenogenesis in a
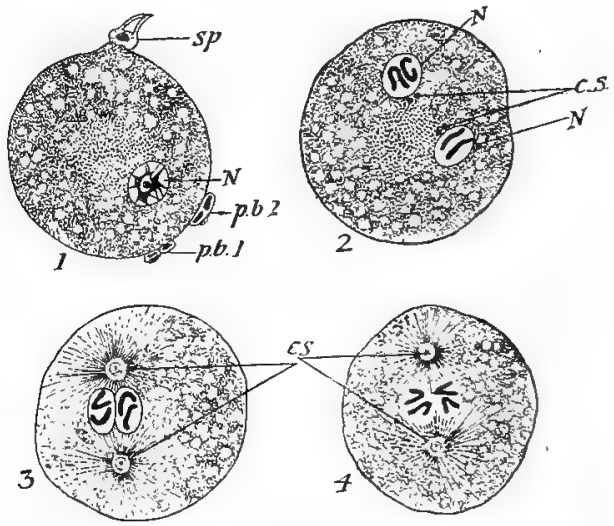

FIG. I I.-Fertilisation in Ascaris megalocephala. (After BOVERI.)

x. Spermatozoon ( $s p$. ) entering ovum, which contains reduced nucleus $(N)$, having given off two polar bodies ( $p, b, x$ and 2 ).

2. Sperm nucleus $(n)$, and ovum nucleus $(N)$, each with two chromatin elements or idants, with centrosomes (c.s.).

3. Centrosomes (C.s.) with "archoplasmic" threads radiating outwards, in part to the chromosomes of the two approximated nuclei.

4. Segmentation spindle before first cleavage.

few of the lower animals, an ovum begins to divide only after a spermatozoon has united with it. After one spermatozoon has entered the ovum, the latter ceases to be receptive, and other spermatozoa are excluded. If, as rarely happens, several spermatozoa effect an entrance into the ovum, the result is usually pathological. It is said, however, 
that the entrance of numerous spermatozoa (polyspermy) is frequent in insects and Elasmobranch fishes.

(2.) The union of spermatozoon and ovum is very intimate; the nucleus of the spermatozoon and the reduced nucleus of the ovum approach one another, combining to form a single nucleus.

(3.) When this combined or segmentation nucleus begins the process of development by dividing, each of the two daughter nuclei which result consists partly of material derived from the sperm nucleus, partly of material derived from the ovum nucleus. In other words, the union is orderly as well as intimate, and the subsequent division is so exact, that the qualities so marvellously inherent in the sperm nucleus (those of the male parent), and in the ovum nucleus (those of the mother animal), are diffused throughout the body of the offspring, and persist in its reproductive cells.

As to the interpretation of these facts, Weismann maintains the importance of the quantitative addition which the sperm nucleus makes to the diminished nucleus of the ovum. At the same time, he finds an important source of transmissible variations in the mingling of the two nuclear substances (amphimixis). Others believe that the mingling diminishes the risk of unfavourable idiosyncrasies being transmitted from parents to offspring. Others emphasise the idea that the sperm supplies a vital stimulus to the ovum, and this seems to be corroborated by the fact well known to breeders that impregnation by a male with certain marked characteristics influences the constitution of the female, and may have an effect on the progeny of sulssequent years and by different males ("telegony").

Segmentation.--The different modes of division exhibited by fertilised egg cells depend in great measure on the quantity and disposition of the passive and nutritive yolk material, which is often called deutoplasm in contrast to the active and formative protoplasm. The pole of the ovum at which the formative protoplasm lies, and at which the spermatozoon enters, is often called the animal pole; the other, towards which the heavier yolk tends to sink, is called the vegetative pole.

In contrasting the chief modes of segmentation, it should be recognised that they are all connected by gradations. 

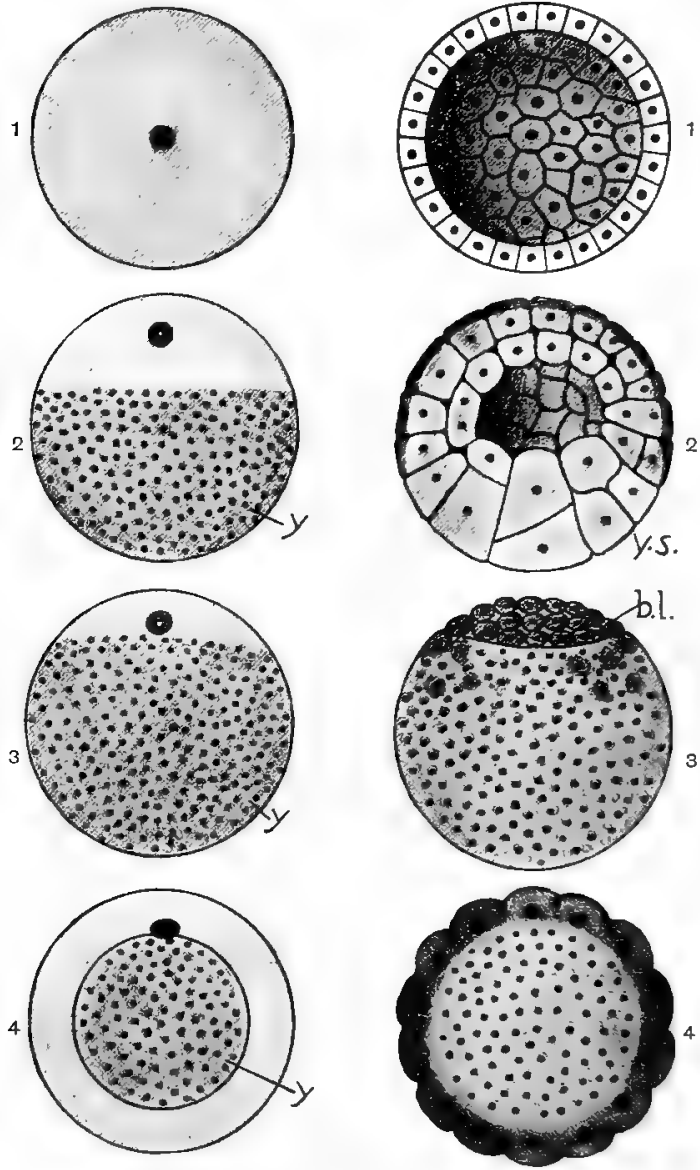

FIG. 12.-Modes of segmentation.

r. Ovum with little yolk segments totally and equally into a blastosphere, e.g., Hydra.

2. Ovum with considerable yolk $(y)$ at lower pole segments wholly hut unequally, e.g., frog ; $\left(y_{\text {s. }}\right.$ ) larger yolk, laden cells.

3. Ovum with much yolk segments partially and discordally, forming blastoderm (bl.), $c \cdot g_{\text {. }}$, bird.

4. Ovum with central yolk $(y)$ segments partially and peripherally, e.g., crayfish. 
A. Complete Division-IIoloblastic Segmentation.

I. Eggs with little and diffuse yolk material divide completcly into approximately equal cells, [or, Ova which are alecithal (i.e., without yolk) undergo approximately equal holoblastic segnentation].

This is illustrated in most Sponges, most Ccelentera, some "worms," most Echinoderms, some Molluscs, all Tunicates, Amphioxus, and most Mammals.

II. Eggs with a little yolk material accumulated towards one pole, divide completely, but into unequal cells,

[or, Ova without very abundant deutoplasm, but with what they have lying towards one pole (tololecithal), undergo unequal holohlastic segmentation].

This is illustrated in some Sponges, some Cnelentera (e.g., Ctenophora), some "worms," many Molluscs, the lamprey, Ganoid Fishes, Ceratoines, Amphibians.

R. Partial Division-Meroblastic Segmentation.

III. Eggs with a large quantity of yolk, on which the formative protoplasm lies as a small clise at one pole, divide partially, and in cliscoidal fashion,

[or, Ova which are telolecithal, and have a large quantity of deutoplasm, undergo meroblastic and discoidal segmentation].

This is illustrated in all Cuttle fishes, all Elasmobranch and Teleostean fishes, all Reptiles and Birds, and also in the Monotremes or lowest Mammals.

IV. Eggs with a considerable quantity of yolk, accumulated in a central core, and surrounded by the formative protoplasm, divicle partially, and superficially or peripherally,

[or, Ova which are centrolecithal undergo meroblastic and superficial segmentation].

This is illustrated by almost all Arthropods, and by them alone.

Summarising the alove, we have :-
A. Complete Division.
I. Equal.
B. Partial Division.
II. Unequal.
$\left\{\begin{array}{l}\text { III. Discoidal. } \\ \text { IV. Peripheral. }\end{array}\right.$

Blastosphere and Morula.-The result of the division is usually a ball of celis. But when the yolk is very abundant (III.) a disc of cells - a discoidal blastoderm-is formed at one pole of the mass of nutritive material which it gradually surrounds.

As the cells divide and redivide, they often leave a large 
central cavity-the segmentation cavity - and a hollow ball of cells-a blastosphere or blastula-results.

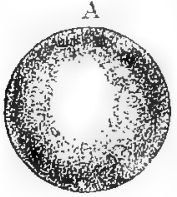

1)
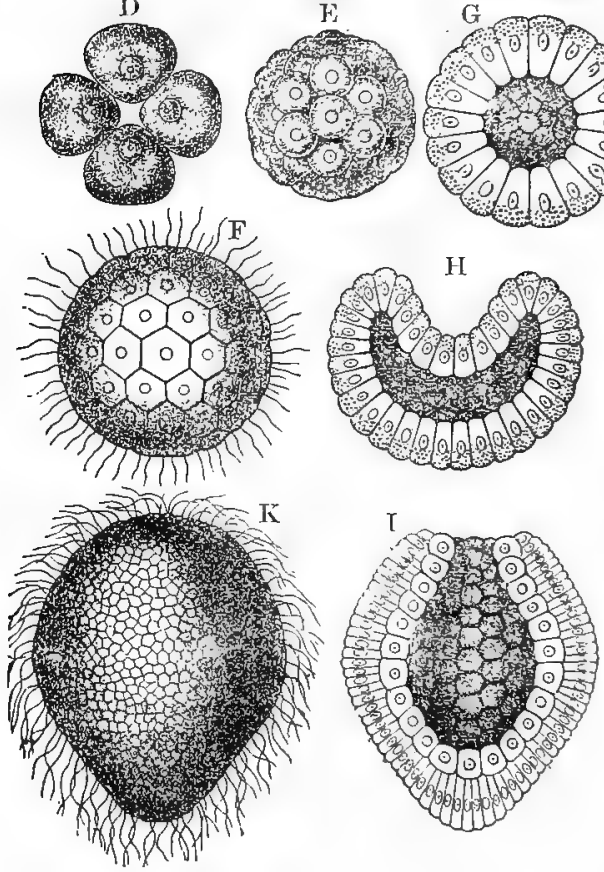

FIG. 13.-Life history of a coral, Monoxenia Darvinii. (From HAEKEL.)

A, B, Ovum. C, Division into two. D, Four cell stage. E, Blastula, F, Free swimming blastula with cilia. G, Section of blastula. H, Beginning of invagination. 1, Section of completed gastrula showing ectoderm, endoderm, and archenteron. $\mathrm{K}$, Free swimming ciliated gastrula. 
But if the so-called "segmentation cavity" be very small or absent, a solid ball of cells or morula, like the fruit of bramble or mulberry, results.

Gastrula.-The next great step in development is the establishment of the two primary germinal layers, the outer ectoderm and the inner endoderm, or the epiblast and the hypoblast.

One hemisphere of the hollow ball of cells may be apparently dimpled into the other, as we might dimple as indiarubber ball which had a hole in it. Thus, out of a hollow ball of cells, a two layered sac is formed --a gastrula formed by invagination or embolé. The mouth of the gastrula is called the blastopore, its cavity the archenteron.

But where the ball of cells is practically a solid morula, the apparent in-dimpling cannot occur in the fashion described above. Yet in these cases the two layered gastrula is still formed. The smaller, less yolk laden cells, towards the animal pole, gradually grow round the larger yolk containing cells, and a gastrula is formed by overgrowth or epibolé.

In the course of our studies, we shall have opportunity to discuss various forms of gastrulation, and some other processes by which two layers are established, such as that called delamination.

Mesoderm.-We are not yet able to make general statements of much value in regard to the origin of the middle germinal layer-the mesoderm or mesoblast. In Sponges and Coelentera it is less distinct than in higher forms, and is usually represented by a gelatinous material (mestgloea) which appears between ectoderm and endoderm, and into which cells wander from these two layers. In the other Metazoa, the middle layer may arise from a few primary mesoblasts or cells which appear at an early stage between the ectoderm and endoderm (e.g., in the earthworm's development); or from numerous "mesenchyme" immigrant cells, which are separated from the walls of the blastula or gastrula (e.g., in the development of Echinoderms); or as calome pouches-outgrowths from the endodermic lining of the gastrula cavity (e.g., in Sagitta, Balanoglossus, Amphioxus); or by combinations of these and other modes of origin. The mesoderm lies or comes to lie be- 
tween ectoderm and endoderm, and it lines the body cavity, one layer of mesoderm (parjetal or somatic) clinging to the ectodermic external wall, the other (visceral or splanchnic) cleaving to the endodermic gut and its outgrowths.

Origin of Organs.-From the outer ectoderm and inner endoderm, those organs arise which are consonant with the position of these two layers, thus nervous system from the ectoderm, digestive gut from the endoderm. 'The middle layer, which begins to be developed in "worms," assumes some of the functions, e.g., contractility, which in Sponges and Coelentera are possessed by ectoderm and endoderm, the only two layers distinctly represented in these classes.

In a backboned animal the embryological origin of the organs is as follows:-

(a) From the Ectodern or Epiblast arise the epidermis and epidermic outgrowths, the nervous system, the most essential parts of the sense organs, infoldings at either end of the gut (fore gut or stomatodreum and hind gut or proctodrum), and perhaps the segmental or primary excretory duct.

(b) From the Endoderm or Hypoblast arise the mid gut (mesenteron) and the foundations of its outgrowths (e.g., the lungs, liver, allantois, $\mathbb{E} c$, of higher Vertebrates), also the axial rod or notochord. According to some authorities, the blood and the vascular system of Vertebrates is in the main endodermic in origin.

(c) From the Mesodern or Alesoblast arise all other structures, e.g., dermis, muscles, connective tissue, bony skeleton, the lining of the body cavity, and perhaps the vascular system. This layer aids in the formation of organs originated by the other two. With it the reproductive organs are associated.

Physiologial Enhyology.-Of the phy siolonical conditions of development, we know relatively little. To investigate them, is one of the tasks of the future. Why toes an egg cell form polar bodies, how is the sperm attracterl to the ovum, why does the fertilised egg cell divide, how does the yolk affect segmentation, what are the conditions of the infolling which forms the endoderm, and of the outfolding which makes the colome pouches, and what do the numerous larval stages mean? 
Generalisations-(I) The Ovum Thenry or Cell Theory.All many celled animals, produced by sexual reproduction, begin at the beginning again. "The Metazoa begin where the Protozoa leave off "- as single cells. Fertilisation does not make the egg cell double; there is only a more complex and more vital nucleus than before. All development takes place by the division of this fertilised egg cell and its descendant cells.

(2) The Gastraa Theory. - As a two layered gastrula stage occurs, though sometimes disguised by the presence of much yolk, in the development of the majority of animals, Hackel concluded that it represents the individual's recapitulation of an ancestral stage. He believes that the simplest stable,
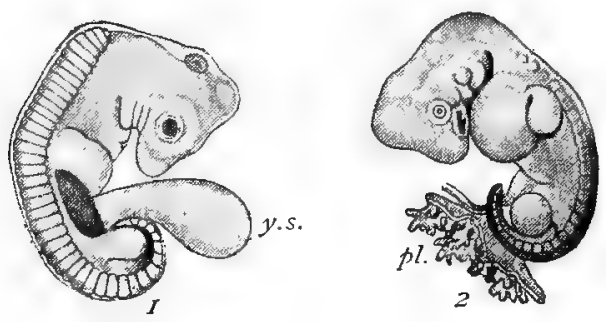

Fig. I4.-Embryos ( I) of bird ; (2) of man. (After IIIs.)

The latter about twenty-seven days old.

$$
y \text {.s. Yolk sac; } p l \text {. placenta. }
$$

many celled animal, was like a gastrula, and this hypothetical ancestor of all Metazoa he calls a gastroc. The gastrula is, on this view, the individual animal's recapitulation of the ancestral gastræa. Rival suggestions have been made: perhaps the original Metazon were balls of cells like Volvox, with a central cavity in which reproductive cells lay; perhaps they were like the planula larva of some Coelenterates-two layered, externally ciliated, oval forms without a mouth.

(3) The Fact of Recapitulation. - It is a matter of experience that we recapitulate in some measure the history of 
our ancestors. Embryologists have made this fact most vivid, by showing that the individual animal develops along a path the stations of which correspond to some extent with the steps of ancestral history.

(I) The simplest animals are single cells (Protozoa).

(2) The next simplest are balls of cells (e.g., Volvox).

(3) The next simplest are twolayered sacs of cells (e.g., $H y^{\prime}(r x)$.
(I) The first stage of development is a single cell (fertilised ovum).

(2) The next is a ball of cells (blastula or morula).

(3) The next is a two layered sac of cells (gastrula).

Von Baer, one of the pioneer embryologists, acknowledged that with several very young embryos of higher Vertebrates before him, he could not tell one from the other. Progress in development, he said, was from a general to a special type. In its earliest stage, every organism has a great number of characters in common with other organisms in their earliest stages; at each successive stage the series of embryos which it resembles is narrowed. "The rabbit begins like a Protozoon as a single cell, after a while it may be compared to the young stage of a very simple vertebrate, afterwards to the young stage of a reptile, afterwards to the young stage of almost any mammal, afterwards to the young stage of almost any rodent, eventually it becomes unmistakably a young rabbit.

Herbert Spencer expressed the same idea, by saying that the progress of development was from homogeneous to heterogeneous, through steps in which the individual history was parallel to that of the race. But Hrekel has illustrated the idea more vividly, and summed it up more tersely than any other naturalist. His "fundanental biogenetic law" reads, "Ontogeny, or the development of the individual, is a shortened recapitulation of phylogeny, or the evolution of the race."

It is hardly necessary to say that the young mammal is never like a worm, or a fish, or a reptile. It is at most like the embryonic stages of these, and it may also be noticed that as our knowledge is becoming more intimate, the individual peculiarities of different embryos are becoming more evident. Thus Professor Sedgwick has recently said that a blind man could distinguish the early stages of 
Elasmobranch and Bird embryos. But this need not lead us to deny the general resemblance.

Moreover, the individual life history is much shortened compared with that of the race. Not merely does the one take place in days, while the other has progressed through ages, but stages are often skipped, and short cuts are discovered. And again, many young animals, especially those "larvæ" which are very unlike their parents, often exhibit characters which are secondary adaptations to modes of life of which their ancestors had probably no experience. In short, the individual's recapitulation of racial history is general, but not precise.

But we do not understand how the recapitulation is sustained. Has the protoplasm of the embryo some unconscious memory of the past? Have the protoplasmic molecules, as Hrckel puts it, learned long since some rhythmic dance which they cannot forget? And, to what extent must there be similarity of external conditions if the recapitulation, "the perigenesis of the plastidules," is to be sustained? For a careful statement of the problem, the student would do well to read the late Professor Milnes Marshall's British Association address on RecaprTulaTTON, now published in his collected papers.

(4) Organic Continuity between Generations.-Heredity. - Every one knows that like tends to beget like, that offspring resemble their parents, and sometimes their ancestors (atavism). Not only are the general characteristics transmitted, but minute features, idiosyncrasies, pathological conditions, innate or congenital in the parents, may be transmitted to the offspring.

Many attempts have been made to explain this, but the first suggestion with any scientific pretensions was that the reproductive cells, which may become offspring, consist of samples accumulated from the different parts of the body.

This was a very old idea, but Herbert Spencer and Charles Darwin gave it new life. According to Darwin's "provisional hypothesis of pangenesis," the reproductive cells accumulate gemmules liberated from all parts of the body. In development these gemmules help to give rise to parts like those from which they originated. This hypothesis has been repeatedly modified, but, except in the general sense that the body may influence its reproductive cells, "pangenesis" is discredited by most biologists.

The idea which is now accepted with general favour is, 
that the reproductive cells, which give rise to the offspring, are more or less directly continuous with those which gave rise to the parent. This idea, suggested by Owen, Hæckel, Rauber, Galton, Jäger, Brooks, Nussbaum, and especially emphasised by Weismann, is fundamentally important.

At an early stage in the development of the embryo the future reproductive cells of the organism are distinguishable from those which are forming the body. 'These, the somatic cells, develop in manifold variety, and, as division of labour is established, they lose their likeness to the fertilised ovum of which they are the descendants. The future reproductive cells, on the other hand, are not implicated in the formation of the "body," but remaining virtually unchanged, continue the protoplasmic tradition unaltered, and are thus able to start an offspring which will resemble the parent, because it is made of the same protoplasmic material, and develops under similar conditions.

A fertilised egg cell with certain characters $(a, b, c)$, develops into an organism in which these characters are variously expressed; but if, at an early stage, certain cells are set apart, retaining the characters, $a, b, c$, in all their entirety, then each of these cells will be on the same footing as the original fertilised egg cell, able to give rise to an organism, almost necessarily to a similar organism.

An early insulation of reproductive cells, directly continuous and therefore presumably identical with the original ovum, has been observed in the development of some "worm types"-(Sagitta, Threadworms, Leeches, Polyzoa), and of some Arthropods (e.g., Moinu among Crustaceans, Chironomus among Insects, Phalangidae among Spiders), in Micrometrus agsregatus among Teleostean fishes, and with less distinctness in some other animals.

In many cases, however, the reproductive cells are not recognisable until a relatively late stage in development, after differentiation has made considerable progress. WVeismann gets over this difficulty by supposing that the continuity is sustained by a specific nuclear substance-the germplasm-which remains unaltered in spite of the differentiation in the body. But it is perhaps enough to say that as all the cells are descendants of the fertilised orum, the reproductive cells are those which retain intact the qualities 
of that fertilised ovum, and that this is the reason why they are able to develop into offspring like the parent.

Finally, it may be noticed in connection with heredity, that there is great doubt to what extent the "body" can definitely influence its own reproductive cells. Animals acquire individual bodily peculiarities in the course of their life, as the result of what they do or refrain from doing, or as dints from external forces. The "body" is thus changed, but there is much doubt whether the reproductive cells within the "body" are affected by such changes. Weismann denies the transmissibility of any characters except those inherent or congenital in the fertilised egg cell, and therefore denies that the influences of function and environment are, or have been, of any importance in the evolution of many celled animals. Such influences affect the body, but do not reach its reproductive cells, and are therefore non-transmissible. Many of the most authoritative biologists are at present of this opinion. On the other hand, many still maintain that profound changes due to function or environment may saturate through the organism, and affect the reproductive cells, and thus the race. The whole question remains under discussion. 


\section{CHAPTER V.}

\section{PAST HISTORY OF ANIMALS.}

Palmontology.

In the two preceding chapters we have noticed two of the great records of the history of animal life,- that preserved in observable structures, and the modified recapitulation discernible in individual development; in this we turn to the third-the geological record. From Morphology many conclusions as to the course of evolution have been drawn; the study of form must indeed, by itself, in time have led to the doctrine of evolution,- that the present is the child of the past. In the early days of the evolution theory the modern science of Embryology was still in its infancy, and could furnish few arguments, and it was the opponents of the new theory rather than its supporters who appealed to Palæontology. They asserted that the palæontological facts refused to lend the support which the theory demanded. To their attacks the evolutionists then chiefly sought to reply by pointing out that the geological record was very incomplete. The numerous investigations which have since been carried on on all sides, now show conclusively that it was imperfection rather of knowledge than of the record which produced the negative results. We must, however, still acknowledge that, except in a relatively few cases, little is known of the ancestors of living animals, and seek for reasons to explain this.

Reasons for the "Imperfection of the Genlogial Record."

If we remember the rule of modern Geology that the past is to be interpreted by the aid of the present, there can be no difficulty in realising that the chances against the pre- 
servation of any given animal are very great. Many are destroyed by other living creatures, or obliterated by chemical agencies. Except in rare instances, only hard parts, such as bones, teeth, and shells, are likely to be preserved, and this at once greatly limits the evidential value of fossils. The primitive forms of life would almost certainly be without hard parts, and have left no trace behind them. A number of extremely interesting forms, such as many worms and the Ascidians, are, for the same reason, almost unrepresented in the rocks. Finally, we cannot suppose that such an external structure as a shell can always be an exact index of the animal within. Some shells, such as Nautilus and some of the Brachiopods, occur as fossils from remote Palæozoic ages onwards, but it is impossible to believe that the animal within has never varied during this period, though we cannot now learn either the nature or the amount of the variation.

After fossilisation has taken place, the rock with its contents may be entirely destroyed by subsequent denudation, or so altered by metamorphic changes that all trace of organic life disappears. Of these fossils which have been preserved only a small percentage are available, for vast areas of fossiliferous rocks are covered over by later deposits, or now lie below the sea or in lands which have not yet been explored.

With all these causes operating against the likelihood of preservation, and of finding those forms that may have been preserved, it is little wonder if the geological record is incomplete; but such as it is, it is in general agreement with what the other evidence, theoretical and actual, leads us to expect as to the relative age of the great types of animal life. Further, those specially favourable cases which have been completely worked out have yielded results which strongly support the general theory.

\section{Probabilities of "fossils" in the various classes of animals.}

But it will be useful to note the probabilities of a good representation of extinct forms in the various classes of animals. Thus, among the Protozoa, the Infusoria have no very hard parts, and have therefore almost no chance of preservation, and the same may be said of forms like Amobæe ; while the Foraminifera and the Radiolaria, having hard structures of lime or silica, have been well preserved. The sponges are well represented by their spicules and skeletons. Of the Coelenterates, except an extinct order known as Graptolites, only the various forms of coral had any parts readily capable of preservation, and remains of these 
are very abundant in the rocks of many anciert seas. But, strange as it may seem, some leautiful remains of jellyfish have been discovered.

Of the great series of "worms," only the tube makers bave left actual remains, the others are known only by their tracks, while of any that may have liver on the land there is no evidence.

The Echinoderms, because of their hard parts, are well represented in all their orders except the Holothurians, where the calcareous structures characteristic of the class are at a minimum.

The Crustacea, being mostly aquatic, and in virtue of their hard skin, are fossilised in great numbers.

The Arachnicla and the Insects, owing to their air breathing habit, are chiefly represented by chance individuals that have been drowned, or enclosed within tree stumps and amber.

The Molluses and Brachiopods are perhaps better preserved than any other animals, since nearly all of them are possessed of a shell specially suitable for preservation.

Among the Vertelrates, some of the lowest are without scales, teeth, or bony skeleton; such forms have therefore left almost no traces.

Fishes, which are usually furnished with a firm outer covering, or with a bony internal skeleton, or with both, are well represented.

The primitive Amphibians were furnished with an exoskeleton of bony plates, and are fairly numerous as fossils. The bones and teeth of the others have been fossilised, though more rarely. Those living in fresh water have left footprints as traces.

The traces of Reptilia depend upon the habits of the various orders, those living in water being oftenest preserved, but the strange flying Reptiles have also left many skeletons behind them.

Of the Birls, the wingless ones are best represented, and then those that lived near seas, estuaries, or lakes.

The history of Mammals is very imperfect, for most of them were terrestrial. But the discoveries of Marsh, Cope, and others show how much may be found by careful search. The aquatic Mammals are fairly well preserved.

\section{"Palcontologianl Series."}

In spite of the imperfection of the "geological record," in spite of the conditions unfavourable to the preservation of many kinds of animals, it is sometimes possible to trace a whole series of extinct forms through progressive changes. Thus a series of fossilised fresh water snails (Planorthis) has been worked out; the extremes are very different, but the intermediate forms link them indissolubly by a marvellously gradual series of transitions. The same fact is well illustrated by another series of fresh water snails (Paludina), and not less strikingly among those extinct Cuttlefishes which are known as Ammonites, and have perfectly preserved shells. Similarly, though less perfectly, the modern crocodiles are linked by many intermediate forms to their extinct 
ancestors, for it is impossible not to call them by that name, and the modern horse to its entirely different progenitors. In short, as knowledge increases, the evidence from Palæontology becomes more and more complete.

In a general way, it is true that the simpler animals precede the more complex in history as they do in structural rank, but the fact that all the great Invertebrate groups are represented in the oldest distinctly stratified and fossiliferous rocks-the Cambrian system-shows that this correspondence is only roughly true. To account for this we must remember that the whole mass of the oldest rocks, known
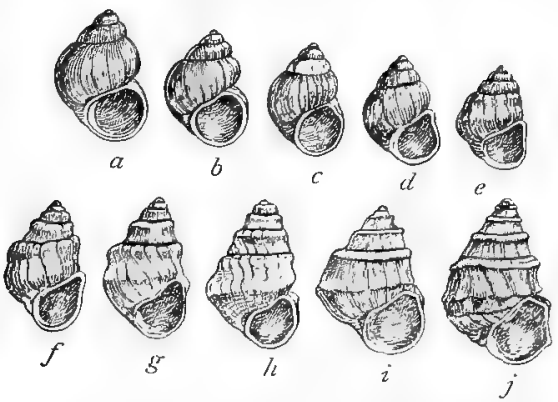

FIG. 15.-Gradual transitions between Paludina Neumayri (a) and Paludina Hamesi (j). (From Neumayr.)

as Archæan or Pre-Cambrian, have been so profoundly altered that, as a rule, only masses of marble and carbonaceous material are left to indicate that forms of life existed when these rocks were laid down. What these early forms of life were, it seems impossible for us to find out, although recent discoveries, for instance, of "annelid tracks" in rocks of possible Pre-Cambrian age in N.W. Scotland, suggest that patient investigation may yet do much towards the solving of the problem.

\section{Extinction of Animals.}

Some animals, such as some of the lamp shells or Brachiopods, have persisted from almost the oldest ages till now, and most fossilised animals have modern representatives which we believe to be their actual 
descendants. That a species should disappear need not surprise us, if we believe in the "transformation" of one species into another. The disappearance is more apparent than real, the species lives on in its modified descendants, "different species" though they be.

But, on the other hand, there are not a few fossil animals which have become wholly extinct, having apparently left no direct descendants. Such are the ancient Trilobites (perhaps remotely connected with our king crab), their allies the Eurypterids, two classes of Echinoderms (Cystoids and Blastoids), many giant Reptiles, and some Mammals.

It is almost certain that there has been no sudden extinction of any animal type. There is no evidence of universal cataclysm, though local floods, earthquakes, and volcanic eruptions occurred in the past, as they do still, with disastrous results to fauna and flora. In many cases, the waning away of an order, or even of a class of animals, may be associated with the appearance of some formidable new competitors; thus Cuttlefish would tend to exterminate Trilobites, just as man is rapidly and often inexcusably annihilating many kinds of beasts and birds. Apart from the struggle with competitors, it is conceivable that some stereotyped animals were unable to accommodate themselves to changes in their surroundings, and also that some fell victims to their own constitutions, becoming too large, too sluggish, too calcareous, in short-too extreme.

\section{Illustrations of the Appearance of Animals in Time.}

Such tables as those given here are apt to be misleading, in that they convey the impression that the great types of structure have appeared suddenly. It must be noted that any apparent abruptness is merely due to incompleteness of knowledge or inaccuracy of expression. The table is a mere list of a few important historical events, lut one must fully realise that they are not isolated facts, that the present lay hidden in the past and has gradually grown out of it. Of the relative length of the periods represented here we know almost nothing, and we are also ignorant of the earliest ages in which life began. But the general result is clear. We find that in the Cambrian rocks, before Fishes appeared, the great Invertebrate classes were represented, though as yet but feel,ly. As we pass upwards they increase in number and in differentiation. Again, Fishes precede Amphibians, Amphibians are historically older than Reptiles, and many types of Reptiles are much older than Birds. In short, in the course of the ages life has been slowly creeping upwards. 


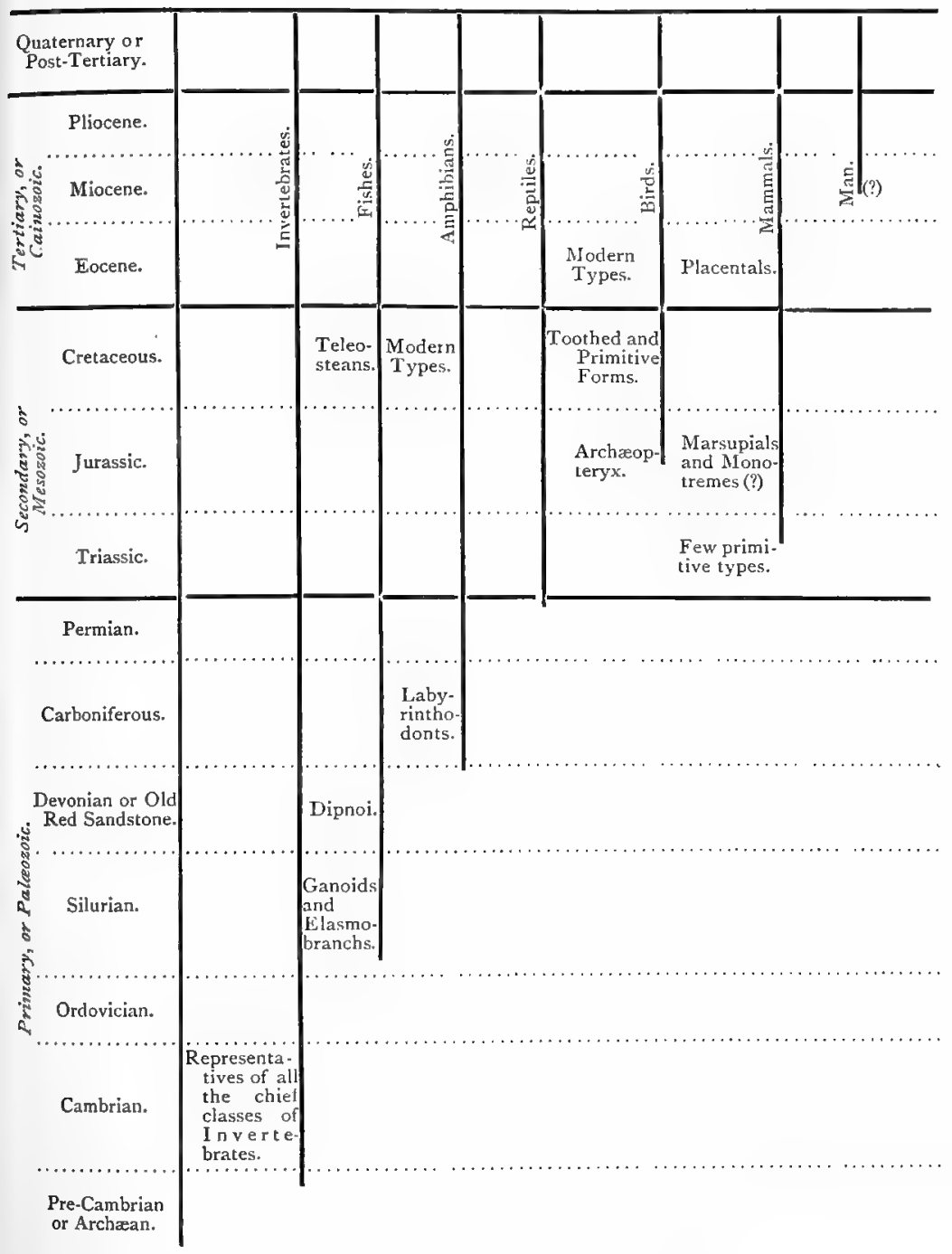




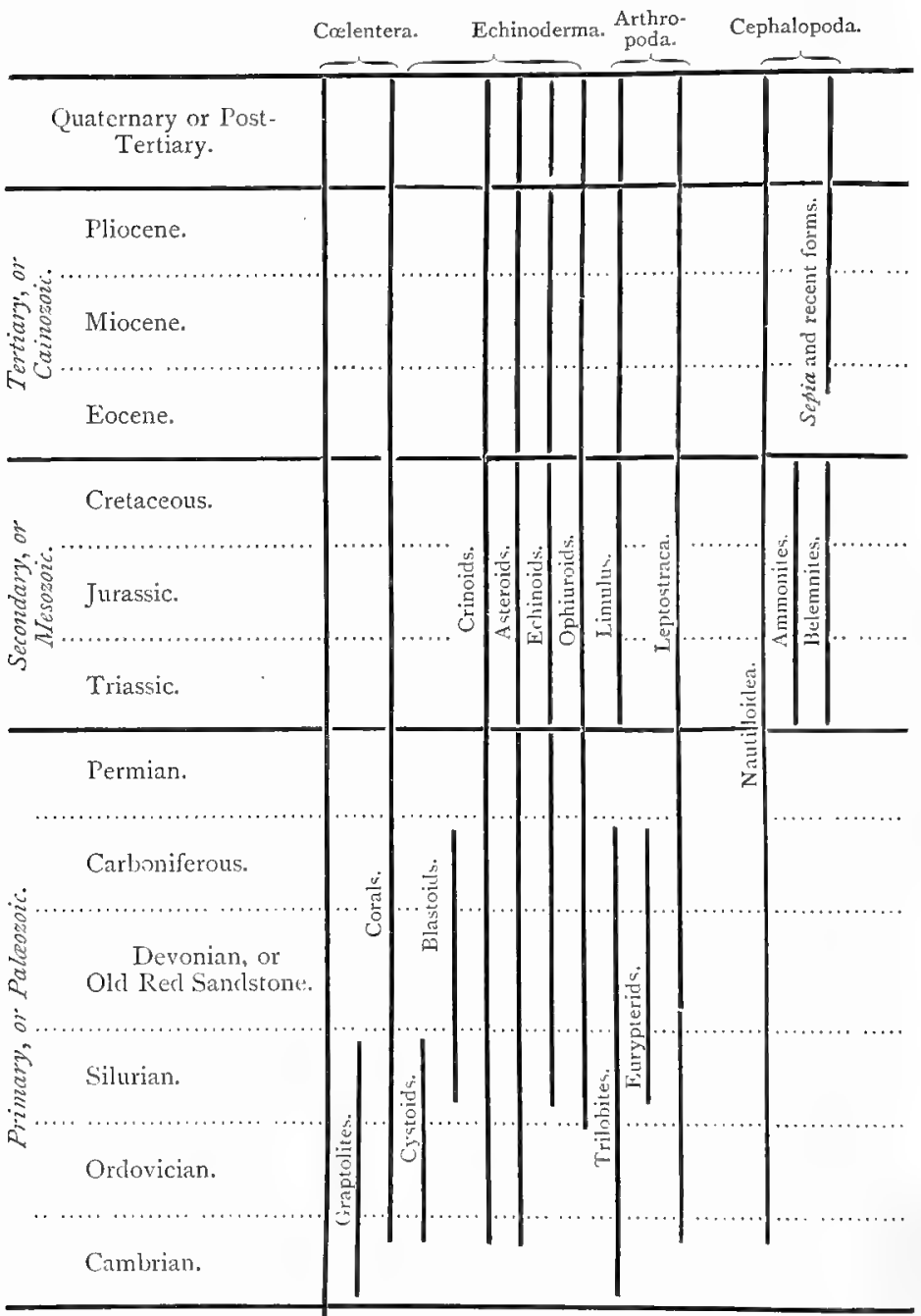

Pre-Cambrian, or Archrean. 


\section{CHAPTER VI.}

\section{TIIE DOCTRINE OF DESCENT.}

WHEN we ask, as we are bound to ask, how the living plants and animals that we know have come to be what they arevery numerous, very diverse, very beautiful, marvellous in their adaptations, harmonious in their parts and qualities, and approximately stable from generation to generation,we may possibly receive three answers. According to one, the plants and animals that we know have always been as they are; but this is at once contradicted by the record in the rocks, which contain the remains of successive sets of plants and animals very different from those which now live upon the earth. According to another, each successive fauna and flora was destroyed by mundane cataclysms, to be replaced in due season by new creations, by new forms of life which arose after a fashion of which the human mind can form no conception. Of such cataclysms there is no evidence, and if it be enough to postulate one creation, we need not assume a dozen. The third answer is, that the present is the child of the past in all things, that the plants and animals now existing arose by a natural evolution from simpler pre-existing forms of life, these from still simpler, and so on back to a simplicity of life such as that now represented by the very lowest organisms.

This third theory is really an old one; it is merely man's application of his idea of human history to the world around him. It was maintained with much concreteness and power by Buffon (I 749), by Erasmus Darwin (I794), and by Lamarck (I80I). Yet in spite of the labours of these thoughtful naturalists and of many others, the general idea of the natural descent of organisms from simpler ancestors, 
was not received with favour until Darwin, in his "Origin of Species" (1859), made it current intellectual coin. By his work and by that of Spencer, Wallace, Hæckel, and many others, the doctrine of descent, the general fact of evolution, has been established, and is now all but universally recognised.

The chief arguments which Darwin and others have elaborated in support of the doctrine of descent, according to which organisms have been naturally erolved from simpler forms of life, may be ranked under three beads- $(a)$ structural, (b) physiological. (c) historical.

\section{EVIDENCES OF EVOLUT-ON.}

(a) Structural.-There are said to be pver a milition living animals of different species. 'Thesespecies are linked together by varieties, which make strict severance often impossible (Fig. I5); they can be rationally arranged in genera, orders, families, and classes, between which there are not a few remarkable connecting links; there is a gradual increase of complexity from the Protozoa upwards along various lines of organisation; it is possible to rank them all on a hypothetical genealogical tree (Fig. I). A little practical experience makes one feel that the facts of classification favour the idea of common descent.

Throughout vast series of animals, we find in different guise essentially the same parts, twisted into most diverse forms for different uses, but yet referable to the same fundamental type. It is difficult to understand this "adherence to type," this "homology" of organs, except on the theory of natural relationship.

There are many rudimentary organs in animals, especially in the higher animals, which remain very slightly developed, and which often disappear without having served any apparent purpose. Such are the "gill slits" or "visceral clefts" in Reptiles, Birds, and Mammals, the teeth of young whalebone whales, the pineal body (a rudimentary eye) in Vertebrates. Only on the theory that they are vestiges of structures which were of use in ancestors are these rudiments 
intelligible. They are relics of past history, comparable, as Darwin said, to the unpronounced letters in many words.

(b) Physiological.-Observation shows that animals are to some extent plastic. In natural conditions they vary in the course of several generations or even in a lifetime. This is especially the case if one section of a species be in any way isolated from the rest, or if the animals be subjected in the course of their wanderings to novel conditions of life. Even apart from markedly changed circumstances, moreover, animals exhibit variations from generation to generation.

The evidence from domesticated animals is very convincing. By careful interbreeding of varieties which pleased his fancy or suited his purposes, man has produced numerous breeds of horses, cattle, sheep, and dogs, which are often distinguished from one another by structural differences more profound than those which separate two natural species. In great measure, however, domestic breeds are fertile with one another, while different species rarely are. The numerous and very diverse breeds of domestic pigeons, which are all derived from the rock dove (Columba livia), vividly illustrate the plasticity or variability of organisms.

It sometimes happens that the offspring of an animal resemble not so much the parent as some other form believed or known to be ancestral. Thus a blue pigeon like the ancestral Columba livia may be hatched in the dove cot, a foal may appear with zebra-like stripes, and in times of famine children may be born who are in some ways ape-like. Such atavisms or reversions are not readily intelligible except on the theory of descent.

(c) Historical. - Among the extinct animals disentombed from the rocks, many form series by which those now existing can be linked back to simpler ancestors. Thus the ancient history of horses, crocodiles, and cuttlefish is known with a degree of completeness which makes it almost certain that the simpler extinct forms were in reality the ancestors of those which now live. Moreover, that many connecting links have been discovered in the rocks, and that the higher animals appear gradually in successive periods of the earth's history, are strong corroborations of the theory.

It is less easy to state in a few words how the facts of 
geographical distribution, or the history of the diffusion of animals from centres where the presumed ancestral forms are or were most at home, favour the doctrine of descent.

The individual life history of an animal-often strangely circuitous or indirect-is interpretable as a modified recapitulation of the probable history of the race. The embryo mammal is at one stage somewhat like an embryonic fish, at another like an embryonic reptile; even in details, the recapitulation, if such we may term it, is sometimes faithful.

Such, in merest outline, is the nature of the evidence which leads us to conclude that the various forms of life have descended or have been evolved from simpler ancestors, and these from still simpler, and so on, back to the mist of life's beginnings.

In accepting this conclusion naturalists are practically unanimous; but in regard to the manner in which the modification of species or the general ascent of life has been brought about, there is much difference of opinion. The fact of evolution is admitted; debate goes on with regard to the factors (see Chap. XXX.). 


\section{CHAPTER VII.}

\section{PROTOZOA-THE SIMPLEST ANIMALS.}

Chief classes:-(1) Rhizopods; (2) Gregarinida, or Sporozoa; (3) Infusoria.

The study of Protozoa is a study of beginnings. For while we know nothing directly about the beginnings of animal life, the Protozoa give us hints of the original relative simplicity. They have remained, almost all of them, unit masses of living matter. And, in virtue of their simplicity, they are in some measure exempt from natural death, which is " the price paid for a body." Moreover, in their variety they exhibit, as it were, a natural analysis of the higher animals, which are built up of many diverse cells.

General Characters. - The Protozoa, the simplest and most primitive animals, are usually very small unit masses of living matter or single cells, and differ from plants in their way of feeding. Most of them feed on small plants or other Protozoa, or on debris, and not a feze are parasitic. Most of them live in water, but many can endure dryness for some time. In one set (Rhizopods) the living matter is zeithout any rind, and flow's out in more or less changeful threads and lobes, by the movements of which the animals engulf their food and glide along. The others have a definite rind, wihich in a large number (Infusorians) bears motile cilia or flagella, but in a minority (Gregarines) is without any obvious locomotor structures. But these three states may occur in the life of one form; in fact, each of the three great classes is marked by the predominant, and not by the exclusive occurrence of the Rhizopod-like, or the Infusorianlike, or the Gregarine-like phase of cell life. Manyhave a skeletal 
framework of lime, fint, or other material, while within the cell there is a special kernel or nucleus, or there may be several. There are also other less constant structures. A Protozoon multiplies by dividing into two drughter units, or into a large number; and two individuals often unite temporarily or permanently, in conjugation, which is analogous to the union of ovum and spermatozoon in higher animals. A few types, instead of remaining single cells, form by division or budding loose colonies, taking a step, as it were, towards the Metazoa.

\section{TYPES OF PROTOZOA.}

\section{First Type-AMeBA.}

Amceba, a type of Rhizopods, especially of those in which the outflowing processes of living matter are blunt and fingerlike (Lobosa).

Description.-Amaba proteus and some other species are found on the muddy bottoms of ponds; $A$. terricola occurs in damp earth. Some are just large enough to be seen with the unaided eye. The diameter is often about one-hundredth of an inch. Each is like a little sac of jelly, and glides over the surface of stone and plant by protruding and retracting blunt processes or pseudopodia. As they move the shape constantly changes, whence the old (I 755) popular name of "Proteus animalcule." Round the margin, which sometimes shows an apparent radial striation, the cell substance is firmer and clearer than it is in the interior, which is granular and more fluid. According to Professor Ray Lankester, the formation of pseudopodia is due to the outflowing of the central fluid substance at places where the firm outer pellicle has been temporarily ruptured. In the centre of the cell lies a single nucleus, and Amaba princeps has numerous nuclei. The food consists of minute Algæ, such as diatoms, or of vegetable débris. It is surrounded by the finger-like processes, and engulfed along with drops of water, which form food vacuoles in the cell substance. After the digestible parts of the food have bcen absorbed, the undigested residue is got rid of at any point of the protoplasm. One or more contractile vacuoles are visible in the cell substance. They 
have an excretory function, and serve to get rid of the finer waste products.

Life History.-In favourable nutritive conditions the Amoeba grows. At the limit of growth it reproduces by dividing into two. In disadvantageous conditions, such as drought, it may become globular, and secreting a cell wall or cyst, lie dormant for a time. With the return of favourable conditions it revives, and, bursting from the cyst with renewed energy, begins anew the cell cycle. The conjugation of two Amcebæ has been observed, and it is said that
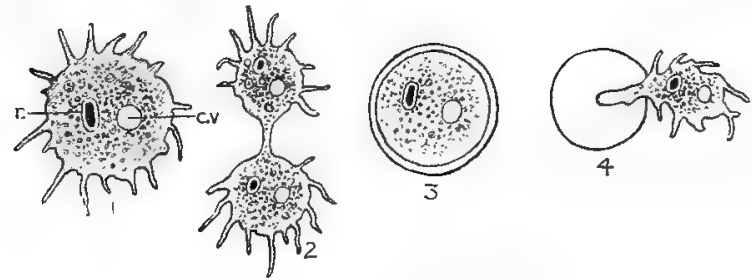

Fig. 16.-Life history of Amoba.

т. $n$. Nucleus, c.v. contractile vacuole.

2. Division into two

3. Encystation.

4. Escape of amcba from its cyst.

spore formation occasionally occurs; of these processes, however, little is certainly known.

Second Type-Gregarina.

Gregitrina, a type of those Gregarinida or Sporozoa in which the cell is divided into two regions by a partition.

Description.-Various species occur in the intestine of the lobster, cockroach, and other Arthropods. When young they are intracellular parasites, but later they become free in the gut. They feed by absorbing diffusible food stuffs, such as peptones and carbohydrates, from their hosts, and store up glycogen within themselves. The maximum size is about one-tenth of an inch. There is a firm cuticle of "protoelastin," which grows inwards so as to divide the cell into a larger nucleated posterior region and a smaller anterior region, and also, in the young stage, forms a small anterior cap. The 
cell substance is divided into a firmer cortical layer and a more fluid central substance. The protoplasm often presents a delicate fibrillar appearance, suggesting that of striated muscle. The nucleus is very distinct, but there are no vacuoles. We may associate the absence of locomotor processes, "mouth," and contractile vacuoles, as well as the thickness of the cuticle and the general passivity, with the parasitic habit of the Gregarines. It is not clearly understood how these and other intestinal parasites have become habituated to resist the action of digestive juices.

Life History. - The young Gregarine is parasitic in one of the lining cells of the gut; it grows, and leaving the cell, remains for a time still attached to it by the cap (Fig. I 8,a. yg.) ; later this is cast off, and the individual becomes free in the gut, while still increasing in size. Two individuals often attach themselves together end to end, but the meaning of this is obscure. Encystation occurs, involving a single unit or two together, and from the division of the encysted cell or cells spores are formed. All the protoplasm is not always used up in forming the spores, but a residue may remain, which forms a network of threads supporting the spores. The cyst is sometimes (as in G. blattarum) complex, with "ducts" serving for the exit of the spores, each of which is surrounded by a firm case. Eventually the cyst bursts, the spore cases are liberated, and from within each of these the single spore emerges to become a cellular parasite. The spore of $G$. gigantea is at first non-nucleated; it gives off two processes, one of which becomes detached, vibratile, and nucleated, while the other seems to come to nothing (Fig. I $8, s p^{2}$ ). The adult of this species is sometimes three-quarters of an inch in length -enormous for a Protozoon.

\section{Third Type-Monocystis.}

Monocystis. - A type of those Gregarinida, or Sporozoa, in which the cell is not divided into two parts by a partition. 
Description.-Two species ( $M$. agilis and M. magna) infest the male reproductive organs of the earthworm so constantly that we are almost always sure of finding them. The full grown adults are visible to the naked eye. They are usually cylindrical, and frequently much elongated, cells, but the shape alters "considerably during the sluggish movements. There is a definite contractile rind, which is sometimes fibrillated, and a central more fluid
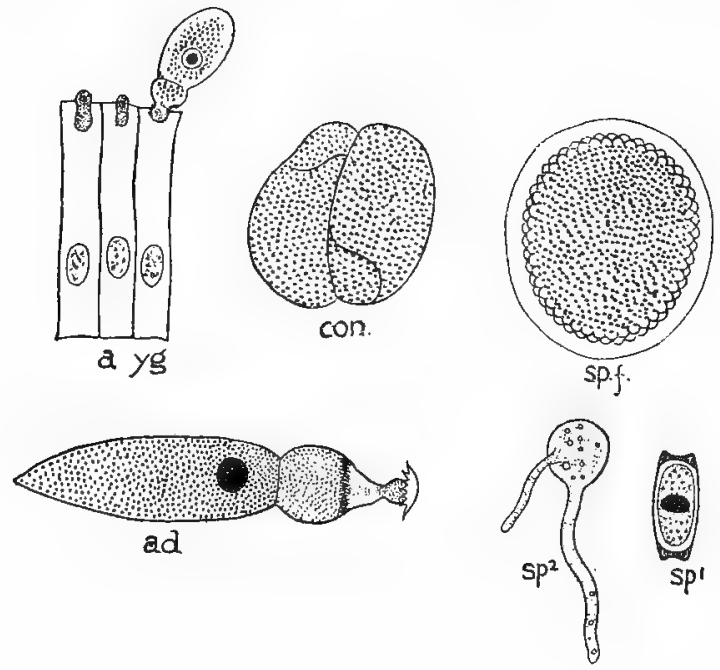

Fig. I8. - Life history of Gregarina. (After Bütschli.)

a.yg. Young forms emerging from intestinal cells.

ad. Adult with deciduous head cap and a cuticular partition dividing cell into two.

con. Two forms conjugating ( $G$. blatiarun).

$s p . f$. Spore formation.

spi. Ripe spore of $G$. blattarum.

spa. Spore of $G$. gigantea, with long vibratile part which breaks off and develops into an adult.

substance, in which the large nucleus floats. In one species there is an anterior projection which resembles the cap of Gregarina, otherwise unrepresented in Monocystis. As in Gregarina, and many parasitic forms, a contractile 
vacuole is absent, but the significance of this is not quite obvious.

Life Fistory. - The young form is parasitic within one of the reproductive cells of the earthworm. It grows, and becomes free from the cell. In the free stage, two individuals may unite in the curious end-to-end manner observed also in Gregarina. Encystation occurs, involving either a single individual or two together. Within the rounded cyst, orderly nuclear division results in the formation of spore forming masses. These form elliptical spore cases, or "pseudonavicellæ," enclosed in a firm sheath, and each spore case seems to contain several, usually eight, spores, lying around a residual core. The spores are considerably larger than those of Gregarina. Eventually the cyst bursts, the spore cases are extruded, the spores emerge from their firm chitinoid cases. The young spore is more

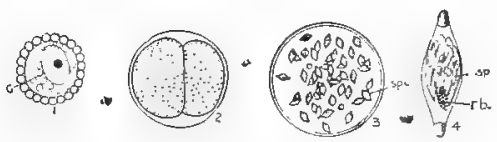

FIG. 19.-Life history of Monocystis. (After BüTSCHLI.)

I. Gregarine lies within a sperm-mother-cell of earthworm.

2. Conjugation of two Gregarines within a cyst.

3. Numerous spore-cases (pseudonavicella) within a cyst.

4. A spore-case with eight spores $(s p$.) and a residual core ( $r b$.$) .$

active than the adult; indeed, in some Gregarines, it is for a brief period flagellate, then amœboid, then like the sluggish adult. Intracellular parasitism and copious food naturally act as checks to activity.

The species of Monocystis occur chiefly in "Worms" and Tunicates; none are known in Arthropods, Molluscs, or Vertebrates.

Fourth Type-Paramocium.

Paramacium.-A type of Infusorians, especially of those which are uniformly covered with short cilia (Holotricha).

Description.-Specimens of Paramacium may be readily and abundantly obtained, by leaving fragments of hay to 
soak for a few days in a glass of water. A few Infusorians have been lying dormant about the plant; they revive and multiply with extraordinary rapidity. They are abundant in most stagnant pools, and are just visible when a test tube containing them is held between the eye and the light. Their food consists of small vegetable particles.

The form is a long oval, the outer portion of the cell substance is differentiated to form a definite rind. With this we may associate the fact that there is now a definite opening, the so-called mouth, which serves for the ingestion
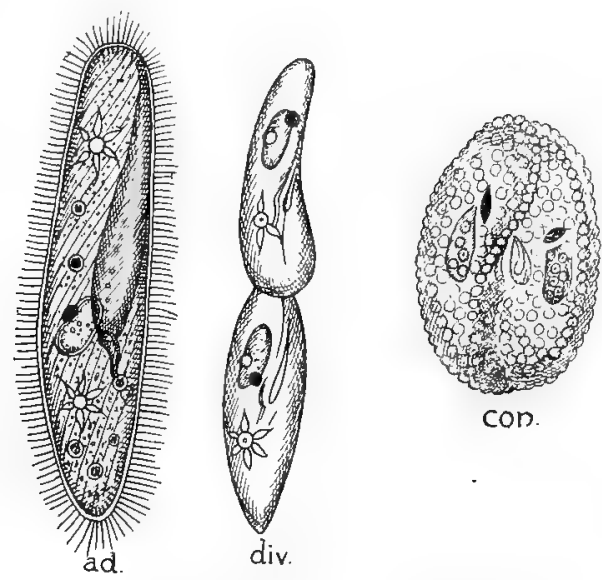

FI(;. 20.-Paramacizm. (After Bútschli).

ad. Adult form, showing cilia, "mouth," contractile vacuoles, e:c.

dit. Transverse division.

con. Conjugation.

of food particles. The surface is uniformly covered with cilia, arranged in regular longitudinal rows; these serve both for locomotion and for driving food particles towards the mouth. Among the cilia on the cortex there are small cavities, in which lie fine protrusible threads ("trichocysts"). These, though parts of a cell, suggest the thread cells of Coelentera, and are probably of the nature of weapons. In the substance of the cell lie two nuclei, the smaller 
"micronucleus" lying by the side of the larger "macronucleus." Food vacuoles occur as in the Amoba. There are two contractile vacuoles, from which fine canals radiate
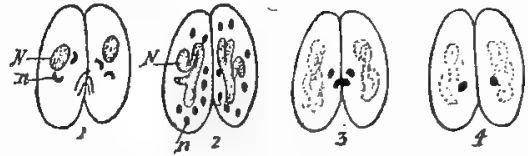

FIG. 2x.-Conjugation of Paramacium aurelia-four stages. (After MAUPAS.)

x. Shows macronucleus $(N)$, and two micronuclei $(n)$ in each of the two conjugates.

2. Shows breaking up of macronucleus, and multiplication of micronuclei to eight.

3. Shows the fertilisation in progress; the macronucleus is vanishing.

4. Shows a single (fertilised) micronucleus in each conjugate.

into the surrounding protoplasm; these discharge into the vacuole, which then bursts to the exterior.

Life History. - Growth is followed by obliquely transverse division into two (Fig 20, div.). One-half includes the "mouth," the other has to make one. As well as this simple fission, a process of transient conjugation also occurs.

FIG. 22.-Diagrammatic expression of process of conjugation in Paramacium aurelia. (After Maupas.)

A. The two microntuclei enlarge.

B. Each divides into two.

C. Eight micronuclei resnlt.

D. Seven disappear ; one (darkened) divides into two.

E. An interchange and fusion occurs, and the conjugates separate.

F. The fertilised micronucleus divides in to two.

G. Each conjugate begins to divide, the micronucleus of each half dividing into two, one of which becomes macronucleus, while the others form the two normal micronuclei. The top line represents four individuals, each with a macronucleus and two micronuclei.

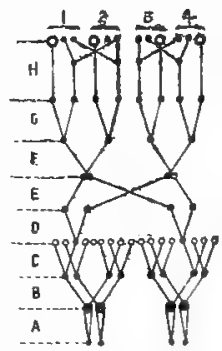

Two individuals approach one another closely, the two nuclei of each break up, an exchange of pieces of the micronucleus takes place; the two then separate, each to reconstruct its two nuclei. This process is necessary for the continued health of the species. 
The details of the conjugating process have been worked out with great care by Maupas and others. They differ slightly in different species; what occurs in $P$. aurelia is summarised diagrammatically in Fig. 22.

The micronuclear elements are represented by two minute bodies. As conjugation begins, these separate themselves from the macronucleus. The macronucleus degenerates, and each micronucleus increases in size (A). Each divides into two (B); another division raises their number to eight (C) ; seven of these seem to be absorbed and disappear, the remaining eighth divides again into what may be called the male and female elements (D); for mutual fertilisation now occurs (E). After this exchange has been accomplished, the Infusorians separate, and nuclear reconstruction begins. The fertilised micronucleus divides into two $(F)$, and each half divides again $(G)$, so that there are four in each cell. Two of these form the macronuclei of the two daughter cells into which the Infusorian proceeds to divide $(\mathbf{H})$; the other two form the micronuclei, but before another division occurs each has again divided. Thus each daughter-cell contains a macronucleus and two micronuclei.

\section{Fifth Type-VORTICELLA.}

Vorticella, or the bell animalcule, is a type of those ciliated Infusorians in which the cilia are restricted to a region round the mouth (Peritricha).

Groups of Vorticella, or of the compound form Carchesium, grow on the stems of fresh water plants, and sometimes are readily visible to the unaided eye as white fringes. In Vorticella each individual suggests an inverted bell with a long flexible handle. The base of the stalk is moored to the water weed, the bell swings in the water, now jerking out to the full length of its tether, and again cowering down with the stalk contracted into a close and delicate spiral. In Carchesium the stalk is branched, and each branch terminates in a bell. Up the stalk there runs, in a slightly wavy curve, a contractile filament, which, in shortening, gives the non-contractile sheath a spiral form. This contractile filament, under a high power, may exhibit a fine striation. (A similar striated structure is seen in some Amœbæ, Gregarines, spermatozoa, \&c., and above all, in striped muscle fibres. It seems to be some structural adaptation to contractility.) The bell has a thickened margin, and within this lies a disc-like lid; in a depression on the left side, between the margin and the disc, there is an opening, the mouth, which leads by a distinct passage into the cell. 
On the side of this passage there is a weak spot, the potential anus, by which useless debris is passed out. The cilia are so arranged as to waft food particles into the mouth and down the passage. There is a large and horseshoe shaped macronucleus, and a small micronucleus. Food vacuoles and contractile vacuoles are present as usual.

Sometimes a Vorticella bell jerks itself off its stalk and

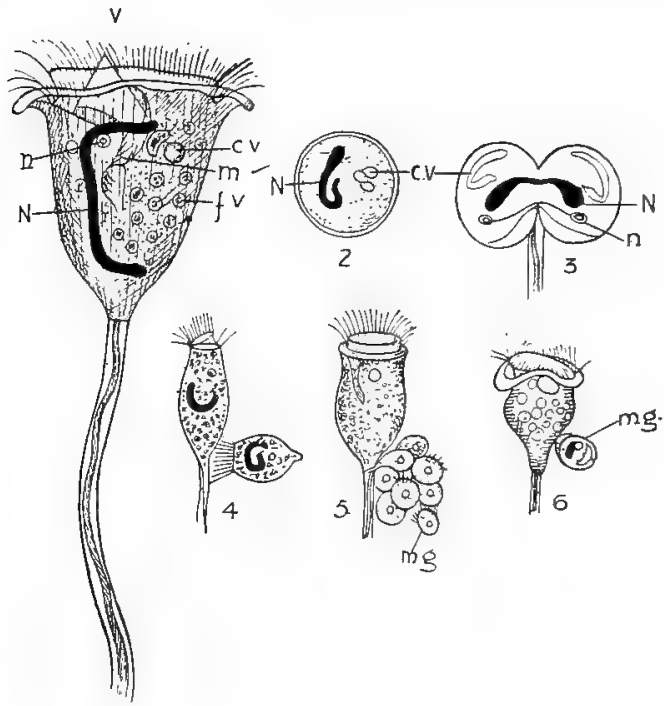

1

FIG. 23.-Vorticella. (After Bütrschli.)

x. Structure. N, Macronucleus: $n$, micronucleus ; $\mathrm{C} \mathrm{V}$, contractile vacuole; $m$, mouth; $f z$, food vacuole ; $r$, vestibule.

2. Encysted individual. 3. Division.

4. Separation of a free swimming unit-the result of a division.

5. Formation of 8 minute units $(m g)$.

6 . Conjugation of microzooid $(m g)$ with one of normal size.

swims about; in other conditions it may form a temporary cyst; normally, the cilia are very active, and the movements of the stalk frequent and rapid. Multiplication may take 
place by longitudinal fission-a bell divides into similar halves, one of these acquires a basal circlet of cilia and goes free, ultimately becoming fixed. Or the division may be unequal, and one, or as many as eight, microzooids may be set free. These swim away by means of the posterior girdle of cilia, and each may conjugate with an individual of normal size. In this case a small active cell (like a spermatozoon) fuses intimately with a larger passive cell, which may be compared to an ovum. The details of the process of fertilisation are analogous to those described in Paramcecinm. It is said that in some cases an encysted Vorticella breaks up into a number of minute spores, but this is doubtful.

\section{Sixth Type-Volvox.}

Volvox is a type of flagellate Infusorians, especially of those with flagella of equal size.

Volvox is found, not very commonly, in fresh water pools, and is usually classed by botanists as a green Alga. It consists of numerous biflagellate individuals, connected by fine protoplasmic bridges, and embedded in a gelatinous matrix, from which their flagella project, the whole forming a hollow, spherical, actively motile colony. In $V$. globator the average number of individuals is about $x 0,000$; in $V$. aureus or minor, 500-rooo. The individual cells are stellate or amoboid in $V$. globator, more spherical in $V$. aureus; each contains a nucleus and a contractile vacuole. At the anterior hyaline end, where the flagella are inserted, there is a pigment spot; the rest of the cell is green, owing to the presence of chlorophyll corpuscles. In consequence of the presence of these, Volvox is holophytic, i.e., it feeds as a plant does.

In its method of reproduction Volvox is of much biological interest and importance. As Klein, one of its best describers, says, it is an epitome of the evolution of sex. Some of the colonies are asexual. In these a limited number of cells possess the power of dividing up to form little clusters of cells, these clusters escape from the envelope of the parent colony, and form new free swimming colonies. In other colonies there are special reproductive cells, which may be called ova and spermatozoa. 
In $V$ globator the two kinds of reproductive cells are usually formed in the same colony, the spermatozoa generally first. Technically, the colony is usually a protandrous hermaphrodite.

In $V$. aztreas the colony is oftenest unisexual or dicecious, i.e., either male or female. But it may be monoecious or hermaphrodite, and then generally protogynous, i.e., producing eggs first.

Whether in a hermaphrodite or in a unisexual colony, the sex cells appear among the ordinary vegetative units; the ova are distinguishable

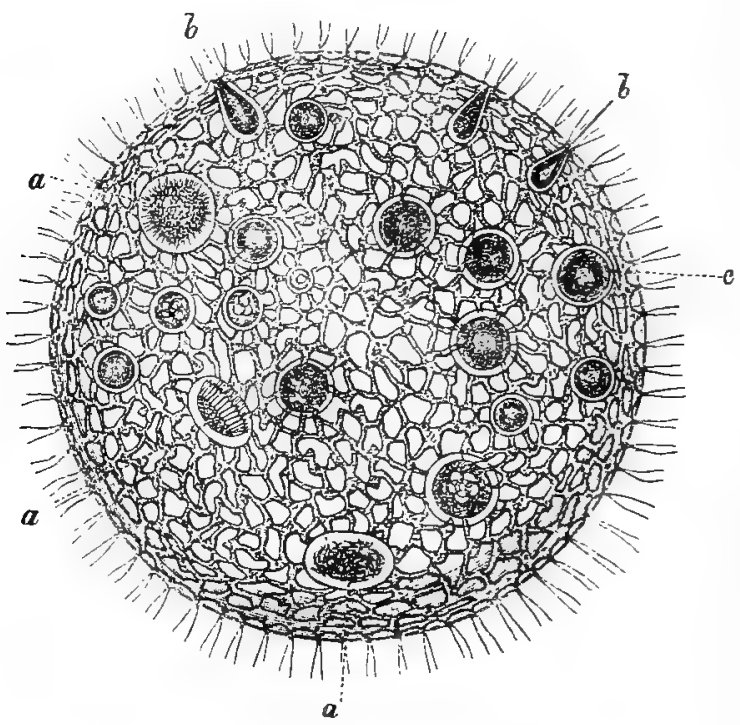

FIG. 24--Volvox globator. (After CoHN.)

$a$. Balls of sperms; $b$. immature ova; $c$. ripe ova.

by their larger size, the "sperm mother cells" divide rapidly and form numerous (32-100 or more) slender spermatozon, each with two cilia. In $V$. globator their bundles may break up within the parent colony; or, as always in $V$. aureus, they may escape intact, and swim about in the water. In any case, the ovum is fertilised by a spermatozoon, and, after a period of encystation and rest, segments to form a new colony. Occasionally, however, this organism, so remarkable a condensation of reproductive possibilities, exhibits a parthenogenetic development of ova.

Here then we have an organism, on the border line between plant 
and animal life, just across the line which separates the unicellular from the multicellular, illustrating the beginning of that important distinction between somatic or body cells and reproductive cells, and occurring in asexual, hermaphrodite, and unisexual phases. Klein records no less than 24 different forms of $V$. aureus from the purely vegetative and asexual to the parthenogenetic, for there may be almost entirely male colonies, almost entirely female colonies, and other interesting transitional stages. Klein has also succeeded to some extent in showing that the occurrence of the various reproductive types depends on outside influences.

\section{General Classification of Protozoa.}

Since the Protozoa are unicellular organisms (except the few which form loose colonies), their classification should be harmonious with that of the cells in a higher animal. This is so. Thus (a) the Rhizopods, in which the living matter flows out in changeful threads or "pseudopodia," as in the common Amoba, are comparable with the white blood corpuscles or leucocytes, many young ova, and other "amaboid" cells of higher animals; (b) the Infusorians which have a definite rind and bear motile lashes (cilia or flagella), e.g., the common Paramocium, may be likened to the cells of ciliated epithelium, or to the active spermatozoa of higher animals; (c) the parasitic Gregarines which have a rind and no motile processes or outflowings, may be compared to degenerate muscle cells, or to mature ova, or to "encysted" passive cells in higher animals.

This comparison has been worked out by Professor Geddes, who also points out that the classification represents the three physiological possibilities-(a) the amœboid units, neither very active nor very passive, form a median compromise; $(b)$ the ciliated Infusorians, which are usually smaller, show the result of a relative predominance of expenditure; $(c)$ the encysted Gregarines represent an extreme of sluggish passivity.

But, as Geddes and others have shown, the cells of a higher animal often pass from one phase to another, - the young amceboid ovum accumulating yolk becomes encysted, the ciliated cells of the windpipe may, to our discomfort, sink into amceboid forms. The same is true of the Protozoa; thus in various conditions the ciliated or flagellate unit may become encysted or amoeboid, while in some of the simplest forms, such as Protomyxa, there is a "cell cycle" in which all the phases occur in one life history.

It is also important to notice Professor Ray Lankester's division of the Protozoa into naked and corticate forms (Gymnomyxa and Corticata). The Gymnomyxa include the primitive forms and the Rhizopods; the Corticata include the two extremes-Gregarines and Infusorians. 


\title{
CLASSIFICATION OF PROTOZOA.
}

\author{
(CoRTICATA.) \\ (GYMNOMYXA.)
}

(Corticata.)

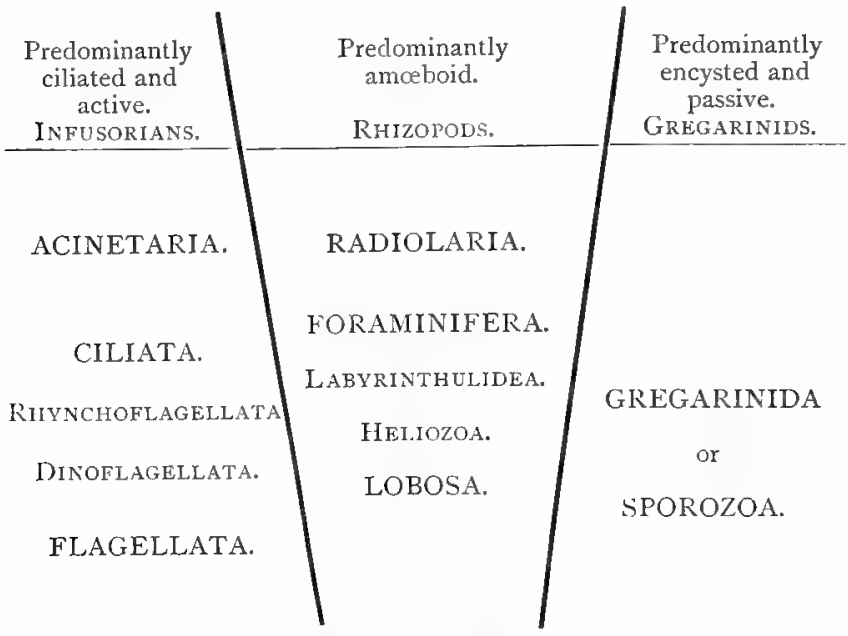

Proteomyxa and MycetozoA.

Primitive ForMS.

\section{SYSTEMATIC SURVEY.}

\section{A.-Primitive Forms.}

1. Proteonyxa.-A class established by Professor Ray Lankester, and described by him as "a lumber room in which obscure, lowly developed, and insufficiently known forms may le kept until they can be otherwise dealt with." They are simple in structure, often parasitic in habit, and protean in their phases. In some no nucleus has yet been detected. They occur in fresh water, in the sea, and parasitically.

Examples.-Protomy'xa, in four phases:- $(a)$ encysted and breaking up into spores, which (b) are briefly flagellate, $(c)$ sink into amoeboid forms, and $(d)$ flow together into a composite "plasmodium." Vam. pyrella, parasitic on fresh-water Algie; Ariherina, with chlorophyll, on Diatoms. Protogenes, the simplest "amneba." Protobathybius, dredged up in masses from the depths. Sichizogenes, multiplying by mere breakage. Monobia, dividing into beautiful colonies.

2. MycetozoA. - Protozoa which live on land and have a fungus-like habit of feeding on decaying vegetable matter. The plasmodial stage in the cycle is predominant. The coated spores are ustally produced in 
little capsules which arise from the surface of the plasmodium, and are often elaborate in structure. The spores may have a brief flagellate activity. and then sink down into anoboid forms, or may be at once little amœbre the amœbre grow and consequently multiply and, after a while, collect into the characteristic fused masses or plasmodia, which sometimes spread over several square inches.
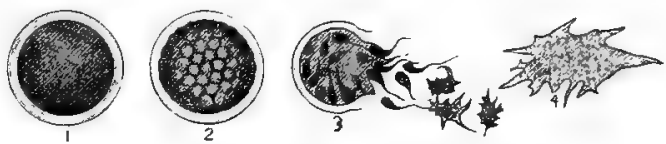

FIG. 25.-Diagram of Protomyxa aurantiaca. (After HÆCkel.)

r. Encysted; 2. Dividing into spores; 3. Escape of spores, at first flagellate, then amneboid ; 4. Plasmodium, formed from fusion of small amœba.

Example.-Fuligo or Ethalium septicum, "flowers of tan"-a large spreading mass found in summer on the bark of the tan yard. This and the other forms are sometimes ranked as plant organisms allied to Fungi, and it is natural that some of these primitive forms should appear to hesitate between the two paths. Krukenberg's discovery of a peptic ferment and an acid in these forms is an interesting illustration of the general similarity of digestive processes in all organisms.

\section{B.-Predominantly Amaboid Protozoa-Rhizopoda.}

3. Loвosa, in which the living matter flows out and in as protean, usually blunt, never interlaced processes. A physical difference between outer and inner portions, one nucleus or more, bubbles of water engulfed along with the fond, special contractile vacuoles, and granules, may generally be observed. They multiply in most cases l,y dividing into two, but in some cases liberate numerous buds (Arcella), or may rarely form spores (Pelomyxa). They sweat off a protecting cyst in unfavourable conditions. Two individuals may unite in conjugation. Most of them occur in fresh water, some in the sea, a few are parasitic.

Examples. - (a) Naked forms:-Amaba, and the giant amoba Pelomyxa; (b) Shelled forms:-Arella, with a firm (chitinoid) shell; secreting gas bubbles which float it ; and Diffugia, shut in except at one end by a membrane, with foreign bodies such as sand grains glued over it. Magosphara (Catallacta), a unique form described by Hreckel-(a) in an encysted phase; (b) as a free swimming colony of ciliated cells (like the embryo of some sponges); (c) as ciliated units produced from the breaking up of $(b) ;(d)$ as amoboid forms resulting from modifications of the active units.

4. LABYRINTHULIDEA, compound forms consisting of a mass of protoplasm spreading out into a network, and of numerous spindle shaped units which travel continually up and down the threarls of the living net. 
Examples,-Labyrinthula (on Algx), Chlamydomyxa (on bog-moss).

5. HeLrozoA, with stiff processes radiating from a spherical body. The outer protoplasm has usually larger vacuoles than the internal portion; there may be numerous nuclei, and one or more contractile vacuoles. Skeletal structures may be entirely alssent (Actinospharium); they may be represented by a jelly-like envelope (Heterophrys); or by loose flinty needles (Raphidiophrys); or, more rarely, by a connected framework (Clathralina). Multiplication by division or by spores. Conjugation occurs. Encystation and spore making, and in some young forms flagellate phases are known; the stiff processes become more

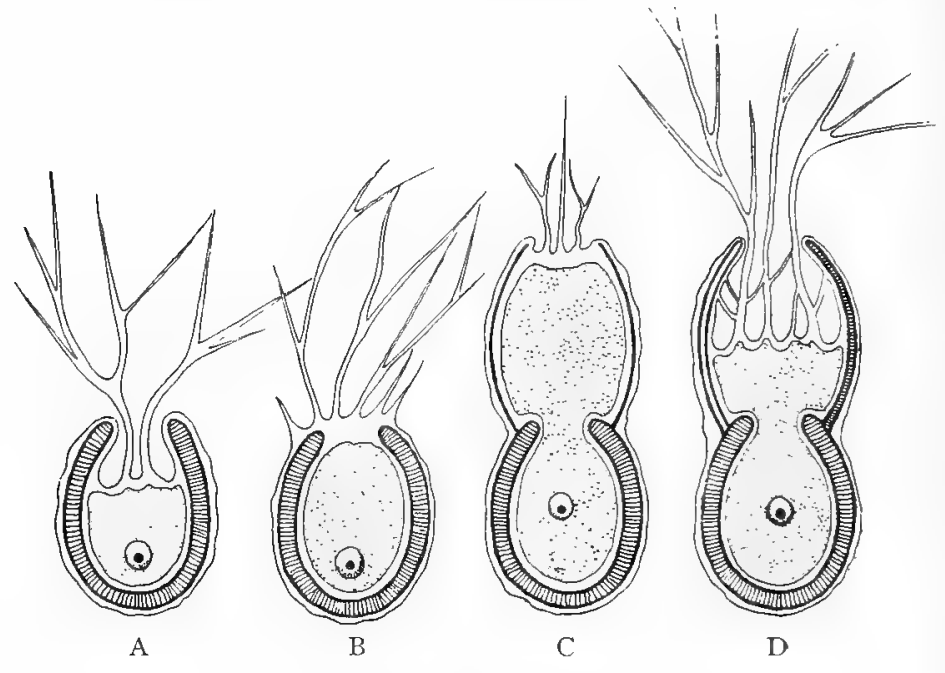

FIG. 26.-Formation of shell in a simple Foraminifer. (After DREYER.)

In $A$ the shell has one chamber; $B, C$, and $D$ show the formation of a second. Note outflowing pseudopodia and the enclosure of the shell by a thin layer of protoplasin; note also the nucleus in the central protoplasm.

amorboid in food catching. Compared with Lobosa, the Heliozoa are passive. The majority occur in fresh water.

Examples. - Actinospherium, Actinophrys sol (sun animalcules); Raphidiophrys, forming colonies; Clathrulina, stalked.

6. Foraminifera. - Predominantly amoboid forms, with fine branching and interlacing processes issuing from the main mass, which is always within a shell, calcareous in the majority, arenaceous or chiti- 
nous in others. A nucleus is present, and often multiplies, apparently in association with reproduction. Vacuoles, contractile or otherwise, seem to be very rare. Conjugation has not been certainly observed. Multiplication may take place by division, but usually by the repeated division of the nucleus and the formation of internal bud spores. The great majority are marine, occurring at all depths. Those from great depths have usually shells of glued sand; the limy forms are found at their best in the shallow water of warm seas, but some occur in the open sea, and sinking down as they die form ooze. They are common as fossils from Silurian strata onwards.

Examples.-Gromia, in both fresh and salt water, with one or two openings to its shell, which is, however, virtually enclosed in the over-

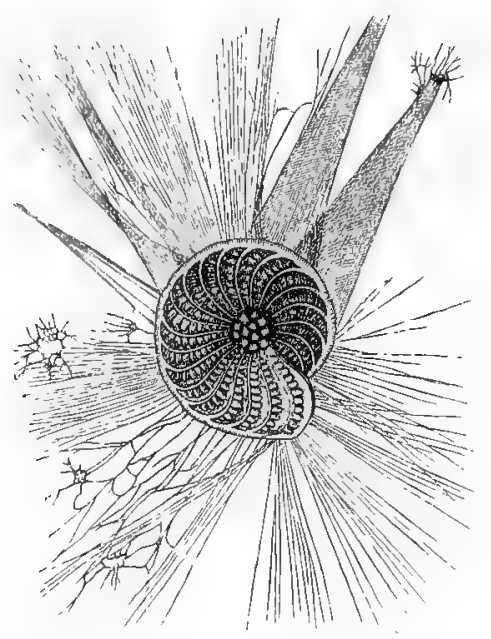

FIG. 27.-Polystomella. (After Schultze.)

flowing protoplasm; Microsromia socialis, in fresh water, forming colonies ; Shepheardella, with an opening at each end of a long membranous case ; Miliolina, with a chambered cell simply coiled, and a single aperture. Such forms are often called Imperforate, in contrast to those whose shells have many pores. Lagena, with a simple flaskshaped cell, with diffuse holes for the processes; Globigerina, a pelagic limy form, with many chambers covered with pores, contributes very largely to the ooze; Hastigerina, a pelagic form, with bubbly protoplasm abundantly overflowing round the shell ; which comes to be internal like a Radiolarian "central capsule" (q v.); Ammodiscus, from the depths, 
with a flinty agglutinate shell; Haliphysena, a form utilising sponge spicules to cover itself, once mistaken for a minute sponge, or for a very simple many celled animal.

Most kinds of chalk consist mainly of the shells of Foraminifera, accumulated on the floor of ancient seas; Nummulites and related fossil forms were as large as shillings or half-crowns.

7. RADIOlaria,-Marine Rhizopods, divided by a nembrane into an inner central capsule (with one or more nuclei), and an outer portion, giving off radiating thread-like pseudopodia. The protoplasm of the

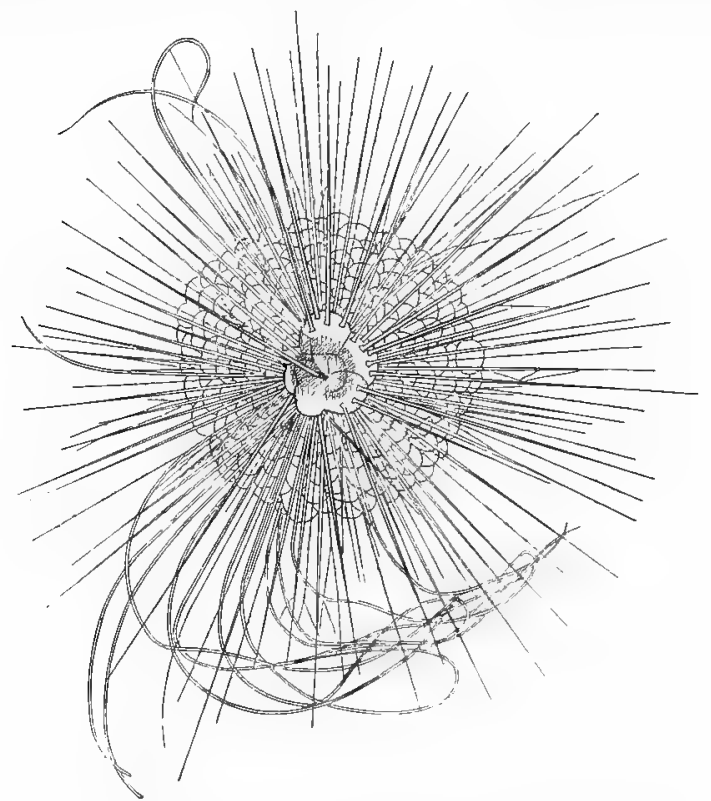

FIG. 28.-A pelagic Foraminifer-Hastigcrina (Globigerina) Marrayi. (After BRADY.)

Note central shell, projecting calcareous spines with a protoplasmic axis; also fine curved pseudopodia and vacuolated protoplasm.

two regions is connecterl by openings in the capsule membrane, and contains many vacuoles. No contractile vacuoles have been seen.

There is usually a skeleton, in most cases siliceous and of complex architecture, in some cases of a horn-like substance, called acanthin. The skeleton may be quite outside the central capsule, or may invade it 
also. Most lead an isolated existence (Monocyttaria); a few form colonies by fusion (Polycyttaria).

Most Radiolarians include unicellular Alga (yellow cells), with which they live in intimate mutual partnership (symbiosis). Division is probably the commonest mode of multiplication, but flagellate sporessometimes of two sizes, small and large, as if male and female-may be formed in the central capsule. Conjugation is still unknown. Professor Lankester notes that the central capsule of a Radiolarian may be compared with the enclosed shell of Hastigerina, and that the character of the protoplasm, which in contrast with that of Foraminifera is abundantly vacuolated, may be associated with the pelagic life, which is rare in the former class. Radiolarians form much of the ooze of the great depths, and occur abundantly as fossils from Palzoozoic times.

Examples. - Thalassicola (no skeleton); Acanthometra (acanthin); Artinomma (flinty skeleton, central capsule with pores all over);

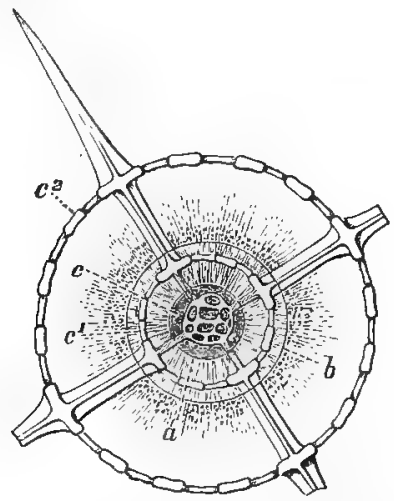

FIG. 29.-Optical section of a Radiolarian (Actinomma). (After HACKEL.)

a. Nucleus ; b. Wall of central capsule ; $c$. Siliceous shell within nucleus ; $c 1$. Middle shell within central capsule ; $c^{2}$. Outer shell in extra-capsular substance. Four radial spicules hold the three spherical shells together.

Eucyrtidium (flinty skeleton, with one perforate area in cone shaped central capsule); Aulosphera (flinty skeleton, central capsule with more than one perforate area); Collozoum and Spharozoum, multicellular colonial forms.

\section{C.-Predominantly Encysted Protosou-Sporozoa.}

8. Gregarinida (or better, perhaps, SporozoA).-- Protozoa of parasitic habit, very passive in adult life, clothed by a definite rind. almost never with any locomotor processes. Found in almost all kinds 
of animals; often, especially when young, within the cells of their host ; deriving their food by absorbing diffusible juices. A single large nuclens; no contractile vacuole. Reproduction by division in early life, but typically by spore formation. An encysted phase precedes the division into encased spores. The young forms escaping from a spore case may be flagellate or amœboid ; but, except in a very few case;, passivity prevails, and the adults are much restricted in their contractile movements. Conjugation, followed by fusion, often precerles encystation; and two forms often occur joined together but not fused.

Examples.-Monocystis, in earthworm; Gregarina, with a cross partition, in food canal of Arthropods ; Eimeria, remaining, except in young stages, within a cell of the host ; Drepanidium, and other forms, in blood corpuscles; Myxidium, with amoboid adult; Sarcocystis, in muscle fibres of Mammals and some other Vertebrates; Coccidium oviforme, a

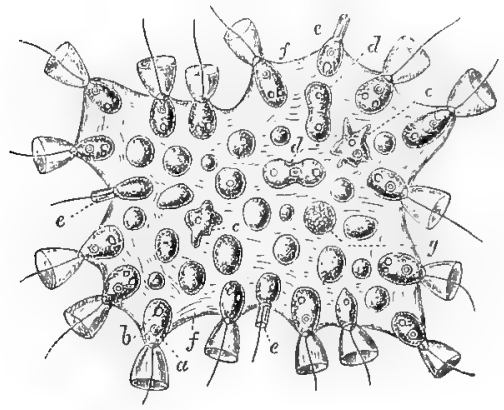

FIG. 30.-A Colonial Flagellate Infusorian-Proterospongia Hrackelii. (After SAVILLF KENT.)

There are about 4 oflagellate individuals. $a$, nucleus; $b$, contractile vacuole; $c$, amoeboid unit in gelatinous matrix; $d$, division of an amceboid unit ; $\varepsilon$, flagellate units with collars contracted ; $f$, byalıne outer membranes; $g$, unit forming spores.

permanent cell parasite, in many Vertebrates, common in the liver of rabbits, \&c.

\section{D.-Predominantly Active Forms (ciliate and flatscllate), generally called Infusorians.}

(Occurring in fresh and sea water, abundant in infusions.)

9. FiagellatA, units with a definite rind, with $\mathbf{I}-3$ actively undulating flagella, often with a distinct apurture for the entrance of food. Reproduction by division into two, or by multiple division within a cyst. Conjugation and encystation are common. Some forms are colonial, and suggest the transition of Metazoa. 
Exanples. - Mastiganceba, possessing a flagellum and amoboid processes ; Euglena, very common in wayside pools, with green or variable colouring matter, probably feeding for the most part like a plant; the colonial Volvox; Codosiga, with stalked colonies, each individual with a collar round the base of the flagellum; Proterospongia, colonial, like a detached piec 3 of sponge. Many, e.g., Monads, live parasitically or in putrid liquids.

IO. Dinoflagrlata, very successful Protozoa, which combine activity and passivity, having two flagella and generally a cellulose coat. The one flagellum projects from a longitudinal groove, the other lies in a transverse groove. Mostly marine.

Examples.-Peridininm and Ceratium.

II. Rirynchoflagellata, large forms, with firm rind and very spongy protoplasm, with two flagella, the larger one striated like a muscle, springing from a deep groove, the smaller one near the aperture for the food.

Examples.-The phosphorescent Noctiluca; Leptodiscus medusoides, disc-like in form, swimming like a miniature medusoid.

I2. Ciliata, provided with numerous cilia, which bend and straighten rapidly, driving the animals along or wafting food particles into the "mouth." There is a definite rind. Beside the large macronucleus there is in most a micronucleus or "paranucleus." There are usually two contractile vacuoles. Multiplication by rapidly succeeding divisions; in rare cases spores seem to be formed. Conjugation has in some cases at least been shown to be associated with intimate interchange of micronuclear material. Parasitic forms, some mouthless, are not uncommon.

Examples. - (a) Peritricha, with a circle of cilia at one end or at both, e.g.. Vorticella; Trichodina, common on Hydra; (b) Heterotricha, with long and short cilia, e.g., the large Stentor, about $\frac{1}{2} \sigma_{\text {th }}$ inch in length; Batantidium coli, in colon of man. (c) Holotricha, uniformly ciliated. e.g., Paranzcizm; Opalina, in intestine of frog, with numerous nuclei, and no contractile vacuoles. (d) Hypotricha, locomotor cilia confined to under surface, e.g., Stylonichia.

I3. ACINETARIA, ciliated when young, and probably derived from the Ciliata, but more passive when adult. They are fixed in adult life, generally stalked, and bear tentacle-like processes often suctorial. The nucleus is sometimes branched. They have one or more contractile vacuoles. They multiply by division, or by the formation of buds which usually remain for a time partly enclosed by the parent. Their food consists of other Protozoa. They represent " an extreme modification of the Protozoon series, in which the differentiation of parts in a unicellular animal reaches its highest point" (Lankester).

Examples. - Acineta, suctorial; Dendrosoma, forming branched colonies, suctorial ; Ophryodendron, non-suctorial. While most Acinetæ seize other Infusorians by means of their suckers, there are others of minute size, e.g., Spharophrya paramaciorum, which penetrate into their prey and become parasites.

Fistory.-Of animals so small and relicate as Protozoa, we do not expect to find distinct relics in the much battered ancient rocks. But there are hints of Foraminifer shells even in the Cambrian; more than hints in the Silurian and Devonian ; and an abundant representation in 
rocks of the Carboniferous and several subsequent epochs. The famous Eosoon canadeuse of Cambrian rocks is regarded by most as a purely mineral formation.

There seem at least to be sufficient relics to warrant Neumayr's generalisation in regard to Foraminifera, that the earliest had shells of irregularly agglutinated particles (Astrorhizidx), that these were succeeded by forms with regularly agglutinated shells, exhibiting types of architecture which were subsequently expressed in lime.

Relics of siliceous Radiolarian shells are also known from Silurian strata onwards, with, perhaps, the exception of the Devonian. Best known are those which form the later Tertiary deposits of Barbados carth, from which Ehrenberg described no fewer than 278 species.

\section{GENERAL NOTES ON THE PJOTOZOA.}

Ordinary Functions-Movement.-The most obvious function of a Protozoon is movement, of which the simplest mode is that termed amcoboid. This is well illustrated by an Ameba. In ordinary conditions it is continually changing its shape, putting forth blunt lobes and drawing others in. With this is usually associated a streaming movement of the granules, while within the cell itself a somewhat similar streaming is often seen, as in many plant cells. Besides the local changes of form seen in the Amorba, a defined contraction, like that of a muscle cell, is illustrated in the contractile filament of the stalk of Vorticella and similar Infusorians; and not less definite are the movements of cilia and flagella, by means of which most Infusorians travel swiftly through the water. Cilia in movement are "bent and straightened alternately," while flagella, which are usually single mobile threads, "exhibit lashing movements to and fro, and are thrown into serpentine waves during these movements."

Considered generally, the movements are of two kinds; either ( $\mathbf{r}$ ) reflex, i.e., responses to external stimulus, as when the I'rotozoon moves towards a nutritive substance, or (2) automatic, i.e., such movements as appear to originate from within, without our being able to point to the immediate stimulus, e.g., the rhythmical pulsations of contractile vacuoles.

While all vital activity or life must remain inexplicable in lower terms until we know the chemical nature of protoplasm, it is useful to compare the movements of Amobre with the movements of drops of fine emulsion, as Professor Buitschli has done in great detail. For in this 
way the strictly vital may be distinguished from what rlepends on known physical conditions.

Dr. Verworn has speculatively suggested that the substance of the amoboid cell is drawn out towards oxygen in the medium, that the chemically satisfied particles make way for their unsatisfied neighbour particles, that external stimulus provokes a molecular disruption, and that the exhausted particles have then to retreat to the nucleus which he regards as a trophic centre.

Sensitiveness. - The Amnba is sensitive to external influences. It shrinks from strong light and obnoxious materials, it moves towards eatable substances. This sensitiveness is, so far as we know, diffuse, - a property of the whole of the cell substance, but the pigment spots of some forms are specialised regions.

Many Protozoa well illustrate a strange sensitiveness to (the physical and chemical stimuli of) objects or substances with which they are not in contact. Thus the simple amoboid Vampyrella will, from a considerable distance, creep directly towards the nutritive substance of an Alga, and the plasmodium of a Myxomycete will move towards a decoction of dead leaves, and away from a solution of salt. The same sensitiveness, technically termed Chemotaxis, is seen when microorganisms move towards nutritive media or away from others, when the spermatozoon (of plant or animal) seeks the ovum, or when the phagocytes (wandering amceboid cells) of a Metazoon crowd towards an intruding parasite or some irritant particle.

Nutrition.-The energy which the Amaba expends in movement, it makes up for by eating and digesting food particles. Most of the free Protozoa live in this manner upon solid food particles, whether plant or animal; a few such as Volvox, in virtue of their chlorophyll, live entirely as do plants; the parasitic forms usually absorb soluble and diffusible substances from their hosts.

Respiration.-Like all living creatures the Amocba respires, that is, its complex substance is continually undergoing a process of oxidation, carbon dioxide being produced as a waste product. Without oxygen none of the activities can be efficiently performed, and if it is long withheld death ensues. In all Protozoa oxygen is simply taken up by the general protoplasm from the surrounding medium, into which the waste carbonic acid is again passed. The bubbles which enter with the food particles assist in respiration. In parasitic forms the method of respiration must be the same as that of the tissue cells of the host. 
Excretion.-Of the details of this process little is certainly known, but the contractile vacuoles are, without doubt, primitive excretory appliances. In the more specialised forms they appear to drain the cell substance by means of fine radiating canals, and then to burst to the exterior. Uric acid and urates are said to be demonstrable as waste products.

Grozeth and Reproduction.-In favourable conditions, when income exceeds expenditure, the Amoba or other Protozoon grows; in reverse conditions, or at the limit of growth, it reproduces. The phenomena of reproduction we will consider in greater detail later on.

Colour.-Pigments are not infrequently present in the Protozoa; we have already noticed the presence of chlorophyll in some forms. With Radiolarians, the so-called "yellow cells" are found almost constantly associated. Each of these cells consists of protoplasm, surrounded by a cell wall, and containing a nucleus. The protoplasm, is impregnated with chlorophyll, the green colour of which is obscured by a yellow pigment. Starch is also present. The cells multiply by fission and continue to live after isolation from the protoplasm of the Radiolarian. All these facts point to the conclusion that the cells are symbiotic Alge, so-called Zoochlorelle. According to some, the "chlorophyll corpuscles" seen in the primitive Archerina, in some flagellate forms, as Euglena, and in many Ciliata, as Stentor, Stylonichia, one species of Paramaccium, Volvox and the allied forms, are also symbiotic Algre, which have lost the power of independent existence. The evidence for this is, however, insufficient, and this explanation will not apply to cases like that of Vorticella viridis, where the green colouring matter is uniformly distributed through the protoplasm. In many çases there is, besides the chlorophyll, a brown pigment, identical with the diatomin of Diatoms. In many of the Flagellata there are one or more bright pigment spots at the anterior end of the cell; these may be specially sensitive areas. In some of the simpler Gregarines the medullary protoplasm is coloured with pigment which is apparently a derivative of the hremoglobin of the host.

Psychical Life- - As to the psychical life of the Protozoa, we find that they often behave in a way which suggests conscious effort and intelligence, but as cut-off fragments also act with apparent reasonableness, and as the nucleus cannot be regarded as a brain, there seems no reason to credit them with more than that diffuse consciousuess which is possibly co-extensive with life. Verworn has decided, after much labour, that the Protozoa do not exhibit what even the most sanguine could call intelligence, but this is 
no reason why he or any other evolutionist should doubt that they have in them the indefinable rudiments of thought.

Structure.-The Protozoa are sometimes called "structureless," but they are only so relatively. For though they have not stomachs, hearts, and kidneys, as Ehrenberg supposed, they are not like drops of white of egg. Our eyes, when aided by the microscope, can distinguish structure in these simplest animals. They are simple as an egg is simple when compared with a bird.

The cell substance consists of a living network or foam, in the meshes or vacuoles of which there is looser material. Included with the latter are granules, some of which are food fragments in process of digestion, or waste products in process of excretion.

The cell substance includes one or more nuclei, specialised areas which are essential to the life and multiplication of the unit. In the Protozoa there are several conditions under which the nucleus may exist.

(I) In some adult forms, and in many spores or young forms, no nucleus has yet been discovered. It is, however, unnecessary to preserve the term "Monera" for such simple forms, as it is probable that nuclear material does exist in some form even in these cases.

(2) In some of the Ciliata the nucleus is diffuse, that is, it exists in the form of a powder scattered through the medullary protoplasm, and is only discernible after cleath by means of careful staining. In Opalinopsis the fine powder sometimes coalesces into a single nucleus.

(3) In the majority of cases, notably in the Gregarines. the nucleus is single, often large, and placed centrally; from a consideration of the cells of Metazoa we may call this the typical case.

(4) In many of the Ciliata, e.g., Paramacium, the nucleus is double. There is a large oblong nucleus and beside it a smaller spherical one.

(5) In Opalina, from the intestine of the frog, and a few other forms, there are very numerous nuclei, arranged in a symmetrical manner in the cell sulstance. In some cases these isolated nuclei have been observed to unite to form one large nucleus just before binary fission takes place. Of these various cases the cliffise condition is apparently very primitive.

The nucleus, when stained and examined uncler high powers, is observed to be complex in structure. It consists of a nuclear network, or a coil of chromatin threads. In the division of many Protozoa, as in the cells of higher animals it plays an important part. During division it passes from the resting to the active condition. The nuclear threads or "chromatin filaments" loosen themselves from their coiled state, and arrange themselves in a star at the equator of the cell, whence they 
divide into two groups, which retreat from one another, and become the daughter nuclei of two daughter cells. In short, karyokinesis has been observed here as elsewhere (see p. 45).

While we cannot at present define the physiological import of the nucleus, we must recognise its importance. Thus, Bruno Hofer has shown that when an Amaba is cut in two, the part with the nucleus lives and grows normally, while the part without any nucleus sooner or later dies ; and Balbiani has observed that in the case of Infusorians cut into pieces, those parts which have nuclei survive, while if no nucleus is present in the fragment, the wound may remain unhealed and death ensues. There seems no reason why one may numbine the view of Weismann that the nucleus bears the essen editary substances with the view that it is a trophic, or, at any rate, a vitul centre in the cell.

In naked Protozoa, the outer part of the colli substance (" ectoplasm") is often clearer and less grantular than the inner part ("endoplasm"), but this difference "is a physical one of little importance. In corticate Protozoa there is a more definite rind or thickened margin of cell substance. Outside this there may be a "cuticle" distinct from the living matter, sometimes consisting of chitin, or gelatin, or rarely of cellulose. The cuticle may form a cyst, which is either a protection during drought, or a sheath within which the unit proceeds to divide into numerous spores. Moreover, the cuticle may become the basis of a shell formed from foreign particles, or made by the animal itself of lime, flint, or " horny " material.

In the cell substance there may be bubbles of water taken in with food particles (food vacuoles), contractile vacuoles, fibres which seem to be specially contractile (in Gregarines), spicules of flint or threads of horn-like material which may build up a connected framework, and the pigments already mentioned.

Reproduction of Protosoa. - Growth and reproduction are on a different plane from the other functions. Growth occurs when income exceeds expenditure, and when constructive or anabolic processes are in the ascendant. Reproduction occurs at the limit of growth, or sometimes in disadvantageous conditions when disruptive or katabolic processes gain some relative predominance.

As it is by cell division that all embryos are formed from the egg, and all growth is effected, the beginnings of this process are of much interest. (a) Some very simple 
Protozoa seem to reproduce by what looks like the rupture of outlying parts of the cell substance. (b) The production of a small bud from a parent cell is not uncommon, and some Rhizopods (e.g., Arcella, Pelomyxa) give off many buds at once. (c) Commoner, however, is the definite and orderly process by which a unit divides into two-ordinary cell division. (d) Finally, if many divisions occur in rapid succession or contemporaneously, and usually within a cyst enclosing the parent cell, i.e., in narrowly limited time and space, the result is the formation of a considerable number of small units or spores. In the great majority of cases, each result of division is seen to include part of the parent nucleus.

A many celled animal multiplies in most cases by liberating reproductive cells-ova and spermatozoa-different from the somatic cells which make up the "body." A Protozoon multiplies by dividing wholly into daughter cells. This difference between Metazoa and Protozoa in their modes of multiplication is a consequence of the difference between multicellular and unicellular life. Each part of a divided Protozoon is able to live on, and will itself divide after a time, whereas the liberated spermatozoa and ova of a higher animal die unless they unite.

By sexual reproduction, we mean $(a)$ the liberation of special reproductive cells from a "body" and (b) the fertilisation of ova by spermatozoa. It is obvious that unicellular Protozoa can show nothing corresponding to sexual reproduction in the first sense. Moreover, Protozoa can live on, dividing and multiplying, for prolonged periods without the occurrence of anything like fertilisation.

So it is often stated as a characteristic of Protozoa that "they have no sexual reproduction." But if this mean that the unicellular Protozoa have no special reproductive cells, then it is a truism. If, however, the statement mean that the Protozoa are without anything corresponding to fertilisation, then it is not true. For in many of the Protozoa, there occurs at intervals a process of "conjugation " in which two individuals unite either permanently or temporarily. This is an incipiently sexual process; it is the analogue of the fertilisation of an ovum by a spermatozoon. 
It is one of the recurrent phases in the life history of some of the simplest Protozoa (Proteomyxa and Mycetozoa) (see p. 98), that a number of amoeboid units flow together into a composite mass, which has been called a "plasmodium."

It is known that more than two individual Gregarines and other forms occasionally unite. To this the term "multiple conjugation" has been applied.

Commonest, however, is the union of two apparently similar individuals, either permanently so that the two fuse into one, or temporarily so that an exchange of material is effected. Permanent conjugation has been observed in several Rhizopods, Infusorians, and Gregarines. Temporary conjugation is well known in not a few ciliated Infusorians, and it is possible that a curious end-to-end union of certain Gregarines is of the same nature, or it may be of the nature of a "plasmodium" formation.

Fourthly, there are some cases where one of the conjugating individuals is larger and less active than the other. Thus in Vorticella, a small free swimming form unites and fuses completely with a stalked individual of normal size. To call this "dimorphic conjugation" is hardly necessary, since it is evidently equivalent to the fertilisation of a passive ovum by an active spermatozoon, one of the well-known characteristics of reproduction in the Metazoa.

In Volvox this is even more obvious, for the small and active cells, both in shape and method of formation, recall the spermatozoa of higher forms. The conjugation of ciliated Infusorians, such as Paramecizm, has been studied with great care by Gruber, Maupas, R. Hertwig, and others, and though their results are not quite harmonious, the main facts are secure. In many ciliated Infusorians there are two nuclear bodies, one large, the other small. The smaller or micronucleus lies by the side of the larger or macronucleus. The micronucleus divides into parts, while the macronucleus degenerates. Two individual Infusorians ( $A$ and $B$ ) lie side by side in close contact, a portion of the micronucleus of $\mathrm{A}$ passes into $\mathrm{B}$, and fuses with a portion of the micronucleus of $\mathrm{B}$, similarly a portion of the micronucleus of $\mathrm{B}$ passes into $A$, and fuses with a portion of the micronucleus of $A$. In short, mutual fertilisation occurs, the conjugating individuals separate, a new micronucleus and a new macronucleus are established in each.

The precise interpretation of the process is to some extent a matter of mere opinion. We may regard it as a mutual rejuvenescence, each unit supplying some substances or qualities which the other lacks; or we may regard it rather as a process by which the average character of the species is sustained, peculiarities or pathological variations of one individual being counteracted by other characters in the neighbour (apparently no near relation) with which it conjugates; or we may see in it a source of variation as the result of new combinations among the essential hereditary substances. The researches of M. Maupas have thrown much light on the facts, and some of his results deserve summary.

It has been often alleged that the subsequent dividing is accelerated by conjugation; but Maupas finds that this is by no means the case. The reverse in fact is true. While a pair of Infusorians (Onyihodromes 
grandis) were engaged in conjugation, a single individual had, by ordinary asexual division, given rise to a family of from forty thousand to fifty thousand individuals. Moreover, the intense internal changes preparatory to fertilisation, and the general inertia during subsequent reconstruction, not only involve loss of time, but expose the Infusorians to great risk. Conjugation seems to involve danger and death rather than to conduce to multiplication and birth.

The riddle was, in part at least, solved by a long series of careful observations. In November I 885 , M. Maupas isolated an Infusorian (Stylonichia pustulata) and observed its generations till March 1886 . By that time there had been two hundred and fifteen generations produced by ordinary division, and since these lowly organisms do not conjugate with near relatives, there had been no conjugation.

What was the result? At the date referred to, the family was observed to have exhausted itself. The members were being born old and debilitated. The asexual division came to a standstill, and the powers of nutrition were lost.

Meanwhile, before the generations had exhausted themselves, several of the individuals had been restored to their natural conditions, where they conjugated with unrelated forms of the same species. One of these was again isolated, and watched for five months. In this case up till the one hundred and thirtieth generation, it was found that on removal to fresh conclitions the organisms were capable of conjugating with unrelated forms. Later this power was lost, and at the one hundred and eightieth generation the indivicluals of the same family were observed making a vain attempt to conjugate with each other.

We thus see that without normal conjugation the whole family becomes senile, degenerates both morphologically and physiologically. Morphologically, the individuals decrease in size, until they measure only a quarter of their original proportions, the micronucleus atrophies completely or partially, the chromatin of the macronucleus gradually disappears, other internal structures also degenerate. Physiologically, the powers of nutrition, division, and conjugation come to a standstill, and this senile decay of the isolated individuals or family inevitably ends in death.

The general conclusion is evident. Sexual union in those Infusorians, dangerous, perhaps, for the individual life, and a loss of time so far as immediate multiplication is concerned, is absolutely necessary for the species. The life runs in strictly limited cycles of asexual division. Conjugation with allied fornis must occur, else the whole life ebbs. Without it, the Protozoa, which some have called "immortal," die a natural death. Conjugation is the necessary condition of their eternal youth.

Bionomics.-Many Protozoa raise organic debris once more into the circle of life, and many form part of the food of higher animals. Thus, those pelagic Foraminifera and Radiolarians, which dying sink to the great oceanic depths, form along with more substantial debris the fundamental food supply in that plantless world. Fundamental, since it 
is plain that the deep sea animals cannot all be living on one another.

Almost every kind of nutritive relation occurs among the Protozoa, Predatory life is well illustrated by most Infusorians, and thorough going parasitism by the Gregarines; Opalina in the rectum of the frog may serve as a type of those which feed on decaying debris, and Volvox of those which are holophytic. Radiolarians, with their partner Algæ, exhibit the mutual benefits of symbiosis, the plants utilising the carbon dioxide of their transparent bearers, the animals being ærated by the oxygen which the plants give off in sunlight, and probably nourished by the carbohydrates which they build up. Some of the parasitic forms, especially among the Sporozoa, are of serious importance to higher animals.

Though Protozoa may be seriously infected by Bacteria, Acineta parasites, some fungi, like Chytridium, \&c., fatal infection is rare, because of the power of intracellular digestion which most Protozoa possess. "The parasite," Metchnikoff says, "makes its onslaught by secreting toxic or solvent substances, and defends itself by paralysing the digestive and expulsive activity of its host; while the latter exercises a deleterious influence on the aggressor by digesting it and turning it out of the body, and defends itself by the secretions with which it surrounds itself." With this struggle should be compared that between phagocytes and Bacteria in most multicellular animals.

Few Protozoa come into direct touch with human life, but man has several Protozoon parasites, e.g., Amoba coli, associated with inflammation of the intestinal mucous membrane, Coccidium oviforme (Sporozoa), affecting the liver, and various Infusorians. On the other hand, the shells of Protozoa deposited as ooze in ancient days, have formed important deposits, such as chalk and Barbadoes Earth.

General Zoological Interest.-..The Protozoa illustrate, in free and single life, forms and functions like those of the cells which compose the many celled animals. Typically, they show great structural or morphological simplicity, but great physiological complexity. Within its single cell, the Protozoon discharges all the usual functions, while in a higher animal distinct sets of cells have been specialised for various 
activities, and each cell has usually one function dominant over the others. The Metazoan cells, in acquiring an increased power of doing one thing, have lost the Protozoan power of doing many things.

'The Protozoa remain at the level represented by the reproductive cells of higher forms, and are comparable to reproductive cells which have not formed bodies. In the sexual colonies of Volvox, however, we see the beginning of that difference between reproductive cells and body cells which has become so characteristic of Metazoa. The Protozoa are self-recuperative, and in normal conditions they are not so liable to "natural death" as are many celled animals. Weismann and others maintain that they are physically immortal.

They illustrate $(a)$ the beginnings of reproduction, from mere breakage to definite division, either into two as in fission, or in limited time and space into many units, as in the formation of spores within a cyst; $(b)$ the beginnings of fertilisation, from "the flowing together of exhausted cells" and multiple conjugation to the specialised sexual union of some Infusorians, where two individuals become closely united; $(c)$ the beginnings of sex, in the difference of size and of constitution sometimes observed between two conjugating units; $(d)$ the beginnings of many celled animals in the associated groups or colonies which occur in several of the Protozoan classes. These colonies show a gradation in complexity. Raphidiophrys and other Heliozoa form loose colonies, which arise by the want of separation of the products of fission. Among the Radiolarians, there are several colonial forms, in these the individuals are united by their extra-capsular protoplasm, but are all equivalent. In Proteropongia the cells show considerable morphological distinctiveness, some are flagellate, some amoboid, some encysted and spore forming. Again, in Volvox, as we noticed above, the cells of the colonies show a distinction into nutritive and reproductive units.

Lastly, in their antithesis of passivity and activity, constructive and destructive preponderance, anabolism and katabolism, the Protozoa illustrate the phases of the cell cycle, and so furnish a key to the variation of higher animals. 


\section{CHAPTER VIII.}

\section{PORIFERA-SPONGES.}
A. Calcarea (Calcispongia).
B. Non-Calcarea.

Hexactinellida.

Demospongize. $\left\{\begin{array}{l}\text { Monaxonida. } \\ \text { Tetractinellida. }\end{array}\right.$

SPONGES seem to have been the first animals to attain marked success in the formation of a "body." For though their details are often complex, their general structure is simpler than the average of any other class of Metazoa, and some of the simplest forms do not rise high above the level of the gastrula embryo. A "body" has been gained, but it shows relatively little division of labour or unified life; it is a community of cells imperfectly integrated. There are no definite organs, and the tissues are, as it were, in the making. Sponges are passive, vegetative animals, and do not seem to have led on to anything higher; but they are successful in the struggle for existence, and are strong in numbers alike of species and of individuals.

\section{General Characters.}

Sponges are diploblastic (two layered) Metasoa, the middle stratum of cells - the mesoglaia-not attaining to the definiteness of a proper mesoderm. There is no calome or body cavity. The longitudinal axis of the body corresponds to that of the embryo; in other zerords, the general symmetry of the gastmila is retained. In these three characters the Sponges agree with the Calentera and differ from higher (triploblastic and calomate) Metazoa.

The body varies sreatly in shape, even within the same 
species. It is traversed by canals, through which currents of water bear food inwards and waste outwards. Numerous minute pores on the surface open into afferent canals, leading into a cavity or cavities lined by endoderm cells, many or all of which are flagellate. To the activity of the flugella the allimportant cuater currents are due. The endodermic or gastric cavity may be a simple tube, or it may have radially outgrozeing chambers, or it may be represented by branched spaces, from which efferent canals lead to the exterior. Where there is a distinct central cavity there is usually

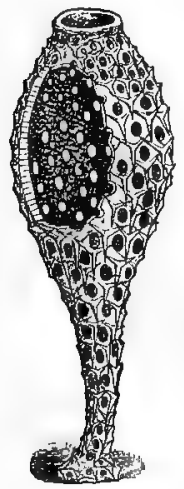

FIr. 3I,--Simple Sponge (Ascetta primordialis).

(After HACKEL.)

Note the vase-like form, the apical osculum, the inhalent pores in the walls. but one large exhalent aperture (osculum), but in other cases there are many exhalent apertures.

The ectoderm is the least important layer; it covers the body, and is perhaps continued into the afferent canals; the endoderm lines most of the internal cavities, and is typically flagellate; the intervening mesogloea contains a skeleton of lime, flint, or spongin; amoeboid cells or phagocytes, important in digestion and excretion; reproductive cells, and other elements.

Budding is very common, and in a few cases buds are set adrift. Both hermaphrodite and unisexual forms occur. The sexually produced embryo is almost always developed within the mesoglaxa, and leaves the sponge as a ciliated larva. With the exception of one family, all are marine.

\section{Description of a Simple Sponge.}

A very simple sponge, such as $A$ scetta, is a hollow vase, moored at one end to rock or seaweed, with a large exhalent aperture at the opposite pole, and with numerous minute inhalent pores through the walls. These walls consist of (r) a flat ectoderm; (2) a mesogloea containing triradiate calcareous spicules, phagocytes, and reproductive elements; and (3) an endoderm lining the central cavity, and composed of collared flagellate cells, almost exactly like some of the monad Infusorians. This simple sponge is not much above the gastrula level; it 
agrees generally with a simple Coelenterate, such as Hydra, but differs from it in the absence of tentacles and stinging cells, and in the greater development of the mesogloca.

\section{More Complicated Forms.}

But a description of a simple sponge like Ascetta conveys little idea of the structure of a complex form such as the bath sponge (Euspongia). Let us consider the origin of complications:-

(a) Sponges-long regarded as plants -are plant-like in being sedentary and passive. They seem also to feed easily and well. Like plants, they form buds, the outcome of surplus nourishment. These buds, like the suckers of a rose bush, often acquire some apparent independence, and the sponge looks like many vases, "not like one. Moreover, as they grow these buds may fuse, like the branches of a tree tied closely together. Thus the structure becomes more intricate.

(b) In the simple sponge the gastric cavity of the vase is completely lined by the collared endoderm cells (Ascon type). But the endoderm may grow out into radial chambers, and the walls of these may also be folded into side aisles (Sycon type). The outgrowing of

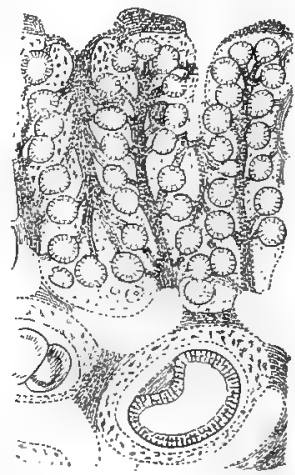

FIG. 32.-Section of a Sponge. (After F. E. Schulze.)

Showing inhalent canals, flagellate chambers, a gastrula forming in the mesogloea, \&c. the endoderm into the mesogloea may be continued even further, and the cells may become pavement-like except in minute flagellate chambers, where the characteristic collared type is retained (Lencon type). (See Fig. 33.)

[Speculatively it may be suggested that the characteristic folding or outgrowth of the endoderm is necessitated by the fact that the endoderm cells are better nourished and multiply more rapidly than those of the ectoderm, which thus fails to keep pace with the inner layer.]

(c) By infoldings of the skin-ectoderm and a subjacent 
sheath of mesoglcea-subdermal spaces may be formed; an outer cortex may be
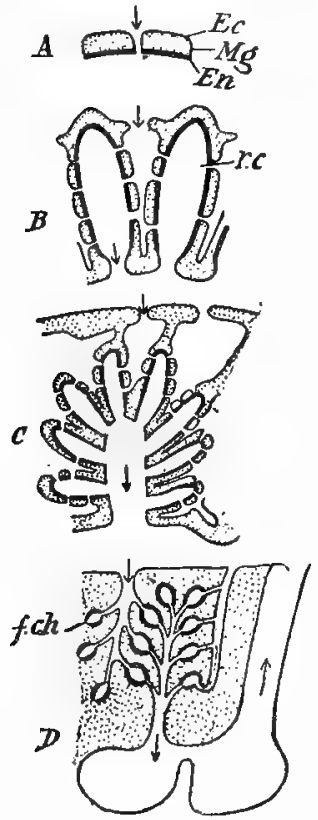

FIG. 33.-Diagram showing types of Canal system. (After KORSCHELT and Heiner.) The flagellate regions are dark throughout, the mesoglcea is dotted, the arrows show the direction of the currents. All the figures represent cross sections through the wall.

A. Simple Ascon type, Ec. ectoderm, En. endoderm, $M g$. mesogloea.

B. Sycon type, with flagellate radial chambers (r.c.).

C. Leucon type, with flagellate side aisles on the main radial chambers.

D. Still more complex type, with small flagellate chanbers, $f$. $c h$. distinctly differentiated from the internal region in which the flagellate chambers occur; the pores may collect into sieve-like areas which open into dome-like cavities; these and many other complications are common.

(d) The ectoderm is usually described as a covering layer of flat epithelium, but flask shaped cells have also been observed (Bidder). It may be folded inwards, as we have noticed, and, according to some, it also lines the incurrent or afferent canals in whole or in part. In a few cases, e.g., Oscarella lobularis, it is ciliated, and its cells may also exhibit contractility, as around the osculum of Ascetta clathrus, though the contractile elements usually belong to the mesogloea.

The endoderm consists typically of collared flagellate cells, but in the more complex sponges these are replaced, except in the flagellate 
The mesogloca contains very varied elements, and illustrates the beginnings of different kinds of tissue. Thus there are migrant amoboid cells (phagocytes); irregular connective tissue cells embedded in a little jelly; spindle shaped connective tissue cells, united into fibrous strands; contractile cells, e.g., those forming a sphincter around the oscula of some forms, such as Pachymatisma; skeleton making cells; pigment containing cells; supposed nerve cells, projecting on the surface, and believed to be connected internally with multipolar (ganglion?) cells; and lastly, the reproductive cells, which are connected by transitional forms with the ordinary phagocytes.

(e) The skeleton consists of calcareous or siliceous spicules, or of spongin fibres, or of combinations of the two last. A calcareous spicule is formed of calcite, with a slight sheath and core of organic matter; a siliceous spicule is formed of colloid silica or opal; the spongin is chemically somewhat like silk. Uniradiate, biradiate, triradiate, quadriradiate, sexradiate, and multiradiate spicules occur, and in a general way it may be said that they are arranged so that they give most architectural stability. Each is formed within a single cell, and may be speculatively regarded as an organised excretion. "During its growth," Prof. Sollas says, "the spicule slowly passes from the interior to the exterior of the sponge, and is finally (in at least some sponges-Geodia, Stelletta), cast out as an effete product." The fibres of spongin are formed as the secretions of mesoglcea cells known as spongioblasts.

\section{Ordinary Functions.}

Excepting the fresh water Spongillida, all Sponges are marine, occurring from between tide marks to great depths. After embryonic life is past, they live moored to rocks, shells, seaweeds, and the like. Their motor activity is almost completely restricted to the lashing movements of the flagella, the migrations of the phagocytes, and the contraction of muscular mesoglocal cells, especially around the exhalent apertures. In the closure of the inhalent pores, sponges show sensitiveness to injurious influences, but how far this is localised in specialised cells is uncertain. 
The most important fact in the life of a Sponge is that which Robert Grant first observed, - - that currents of water pass gently in by the inhalent pores, and more forcibly out by the exhalent aperture or apertures. This may be demonstrated by adding powdered carmine to the water. The instreaming currents of water bear dissolved air and supplies of food, such as Infusorians, Diatoms, and particles of organic debris. The outflowing current carries away waste. When a sponge is fed with readily recognisable substances, such as carmine or milk, and afterwards sectioned, the grains or globules may be found $(a)$ in the collared endoderm cells; (b) in the adjacent phagocytes of the mesogloea; $(c)$ in the phagocytes surrounding the subdermal spaces, if these exist. It is uncertain whether the epithelium of the subdermal spaces or the collared endoderm is the more important area of absorption, but it is certain that the phagocytes play an important part in engulfing and transporting particles, in digesting those which are useful, and in getting rid of the useless. In an extract of several sponges, Krukenberg found a (tryptic) digestive ferment, probably formed within the phagocytes.

Many sponges contain much pigment, thus the lipochrome pigment (see Chap. XXIX.) zoonerythrin is common, and like some others, such as floridine, is regarded as helping in respiration. The green pigment of the fresh water sponge is closely analogous, if not identical, with chlorophyll, and probably renders some measure of holophytic nutrition possible.

\section{Reproduction.}

Sponge growers often cut a sponge into pieces, and bed these out in suitable places. The parts regenerate the whole-a fact which illustrates the relatively undifferentiated state of the sponge body. It is possible that fission may also occur naturally.

The frequent budding is merely a kind of continuous growth, but when buds are set adrift, as sometimes happens, we have discontinuous growth or asexual reproduction.

In the fresh water Spongillidxe there is a peculiar mode of reproduction by statoblasts or gemmules. A number of mesogloeal cells occur 
in a clump, some forming an internal mass, others a complex protective capsule with capstan-like spicules, known as amphidiscs. According to W. Marshall, the life history is as follows: In autumn the sponge suffers from the cold and the scarcity of food, and dies away. But throughout the moribund parent genmules are formed. These survive the winter, and in April or May they float away from the dead parent, and develop into new sponges. Some become short lived males, others more stable females. The ova produced by the latter and fertilised by spermatozoa from the former, develop into a summer generation of sponges, which in turn die away in autumn and give rise to gemmules. The life history thus illustrates what is called alternation of generations (see p. 55). Interpreted from a utilitarian point of view, the formation of gemmules is a life saving expedient. As Prof. Sollas says, "the gemmules serve primarily a protective purpose, ensuring the persistence of the race, while, as a secondary function, they serve for dispersal."

All Sponges produce sex cells, which seem to arise from amcboid mesoglcea cells retaining an embryonic character. In the case of the ovum, the amcboid cell increases in size, and passes into a resting stage; in the case of the male elements, the amceboid cell divides into a spherical cluster of numerous minute spermatozoa. 'The similar origin of the ova and spermatozoa is of interest. Most sponges are unisexual, but many are hermaphrodite. In the latter case, however, either the production of ova or the production of spermatozoa usually preponderates, probably in dependence on nutritive conditions.

\section{Development.}

It is not surprising to find that there is great variety of development in the lowest class of Metazoa; it seems almost as if numerous experiments had been made, none attended with progressive success.

The minute ovum, without any protective membrane, usually lies near one of the canals, and is fertilised by a spermatozoon borne to it by the water. It exhibits a certain power of migration as in some Hydroids. Previous to fertilisation, the usual extrusion of polar bodies has been observed in a few cases, and is doubtless general. Segmentation is total and usually equal, and results in a spherical or oval embryo more or less flagellate. This leaves the parcnt sponge, swims about for a time, then settles down, and undergoes a larval metamorphosis often difficult to understand. It is peculiarly difficult to bring the 
history of the germinal layers in Sponges into line with that in other Metazoa.

(a) In the small calcareous sponge Sycandra raphanus, as described by F. E. Schulze, the segmentation results in a hollow ball of cells-the blastula. A few cells at the lower pole remain large, and are filled with nutritive granules; the other cells divide rapidly and become small, clear, columnar, and flagellate. The large granular cells become temporarily invaginated, forming what is called a "psezedo-
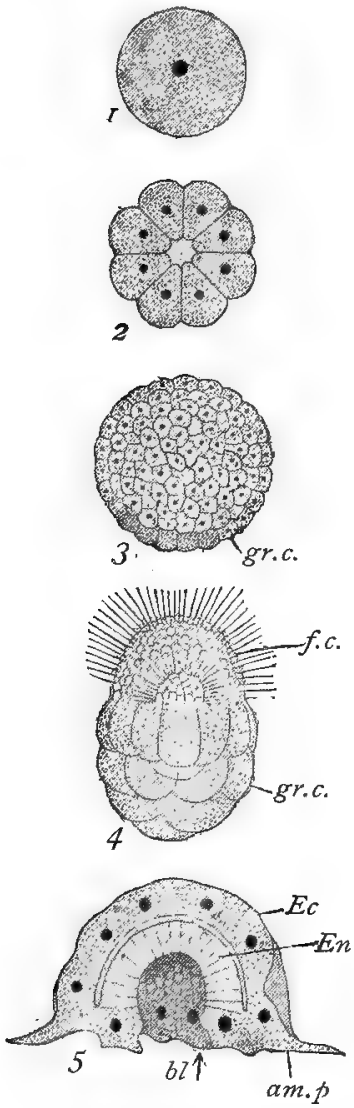
gastrula." This leaves the parent and the granular cells right themselves, forming the posterior hemisphere of the embryo, now called an amphiblastula. It swims for a time actively, but the flagellate cells of the anterior hemisphere are invaginated into or overgrown by the large granular cells, and thus what is generally called the gastrula stage results. This soon settles down, on rock or seaweed, with the blastopore or gastrula mouth downwards, and is moored by amoboid processes from the granular cells, which likewise obliterate the blastopore. The granular cells lose their granules, for the larva is not yet feeding; the now internal flagella disappcar in the absence of the stimulating water; a mesogloa with spicules begins to be formed between the inner

Fig. 34.-Development of Sycandra raphanus. (After F. E. SCHULZE.)

I. Ovum.

2. Section of 16 cell stage.

3. Blastula with 8 granular cells $(g r . c$.$) at lower pole.$

4. Free swimming amphiblastula, with upper hemisphere of flagellate cells (f.c.), and lower hemisphere of granular cells.

5. Gastrula stage settled down. Ec., outer layer (ectoderm?); En., inner layer(endoderm?); bl., closing blastopore; $a m$. p., mooring amœboid processes. 
and outer layer, probably by migrants from the latter. But this disadvantageous state of affairs cannot last. Pores open through the walls, the entrance of water enables the inner cells to recover their flagella, and an exhalent aperture is ruptured at the upper pole. The young sponge is now in an Ascon stage, from which, by the outgrowth (?) of the inner layer into radial chambers, it passes into the permanent Sycon form, grows into a cylinder, and becomes differentiated in detail (Fig. 34).

(b) In Oscarella (Halisarca) lobularis, a sponge without any skeleton, the ovum segments equally into a blastula, which is flagellate all over. This free swimming stage may be invaginated from either pole to form a hemispherical gastrula, which settles mouth downwards. Pores, an osculum, and the mesoglcea are formed as before, and the inner layer becomes folded into flagellate chambers. It may be suggested that this folding is due to the higher nutrition, and consequent disproportionate growth, of the inner layer, for a rapidly growing sac within one of less rapid growth must become folcled on itself (HEIDER).

(c) Another type, seen for instance in a horny sponge, Spongelia, results in a flagellate larva, whose cavity is filled up with what may be called gelatinous connective tissue, from which mesoglcea and endoderm are subsequently differentiated. Such- a larva is called a parenchynula.

As these are not all the types of development which occur among sponges, the general fact is impressive that in this lowest class of Metazoa there has been considerable plasticity in development.

\section{Classification.}

A. Porifera Caliarea, with skeleton of calcareous spicules:-
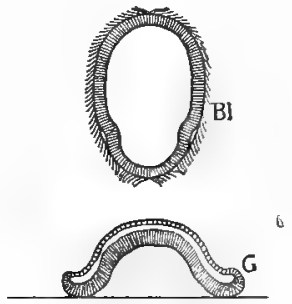

6
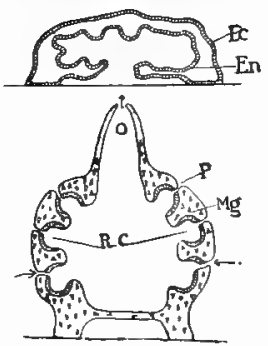

FIG. 35. - Diagrammatic representation of development of Oscarella lobularis. (After HEIDER.)

$B l$. Free swimming blastula with flagella; $G$. gastrula settled down.

Next figure shows folding of Endoderm ( $E n)$.

Lowest figure shows radial chambers $(R . C$.$) . Mesogloca \left(1 I_{g}\right)$; inhalent pore $(P$.$) ; exhalent oscu-$ $\operatorname{lum}(O$.$) .$

Order I. - Homocoela.--Endoderm wholly composed of collared flagellate cells, e.g., Ascetta, Leucosolenia.

Order II.-Heterocola.-Endoderm consists of collared flagellate cells in radial tubes or chambers, and of flat epithelium elsewhere, e.g., Grantia, Sycon. 
$B$. Porifera non-Calcarea, skeleton of silica or of spongin, or of both.

(I) Hexactinellida, with sexradiate siliceous spicules, canal system usually simple, with Sycon chambers. The members live chiefly in deep water, e.g., Venus' Flower Basket (Euplectella) and the Glass Rope Sponge (Hyalonema).

(2) Monaxonida, with siliceous spicules (which are not quadri- or sex-radiate), or with "horny" skeleton, or with both.

Order I,-Monaxona, with spicules only, e.g., Mermaid's Gloves (Chalina oculata), Crumb of Bread Sponge (Halichondria or Amorphina panicea), Fresh Water Sponge (Spongilla).

Order II.-Ceratosa, "horny" sponges with or without spicules, e.g., the Bath Sponge (Euspongia).

(3) Tetractinellida, mostly with quadriradiate spicules, or with trimes, in which a main shaft bears at one end three branches diverging at equal angles, e.g., Tetilla, Geodia, Pachymatisma, Plakina.

There are also a few sponges (Myxospongix) without any skeleton, perhaps survivals of primitive types (Oscarella, Halisarca) or degraded forms (Chondrosia).

History. -Sponges, as one would expect, date back almost to the beginning of the geological record. Thus the siliceous Protospongria occurs in Cambrian rocks, and in the next series-the Silurian-the main groups are already represented. From that time till now they have continued to abound and vary.

Bionomics.-Sponges are living thickets in which many small animals play hide-and-seck. Many of the associations are practically constant and harmless, but some burrowing worms do the sponges much damage. 'The spicules and a frequently strong taste or odour doubtless save sponges from being more molested than they are; the numerous phagocytes wage successful war with intruding microorganisms. Some sponges, such as Clione on oyster shells, are borers, and others smother forms of life as passive as themselves. Several crabs, such as Dromia, are masked by growths of sponge on their shells, and the free transport is doubtless advantageous to the sponge till the crab casts its shell. A compact orange coloured sponge (Suberites domuncula) of peculiar odour often grows round a whelk shell tenanted by a hermit crab, and gradually eats into the shell substance. Within several sponges, minute Algæ live, like the "yellow cells" of Radiolarians, in mutual partner- 
ship or symbiosis. Finally, sponges deserve mention as factors in human civilisation.

General Zoological Interest and Position.-Sponges have this great interest, that they form the first successful class of Metazoa. They illustrate the beginnings of a "body"the beginnings of tissues. Along with the Coelentera, from which it is the almost unanimous opinion that they must be held distinct, they differ markedly from the triploblastic, Colomate Metazoa, which do not retain the radial symmetry of the gastrula.

Their origin is wrapped in obscurity, though there is much to be said for the view that they are the non-progressive descendants of primitive gastrula-like ancestors of sluggish constitution. It does not seem likely that they have led on to anything higher, they rather represent a by-road in Metazoan evolution. 


\section{APPENDIX TO SPONGES. MESOZOA.}

The title Mesozoa was applied by Van Beneden to some very simple organisms which appear to occupy a very humble position in the Metazoan series. The name suggests a grade between the Protozoa and the Metazoa. Hæckel called some of them Gastræadæ, regarding them

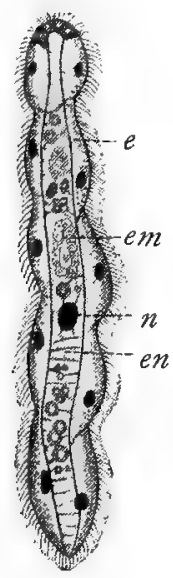

A

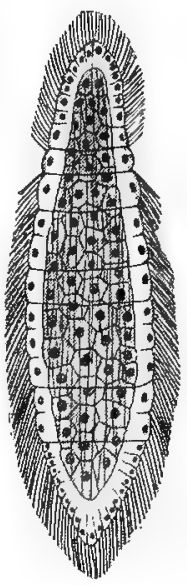

B

F1G. 36.-A. Young Dicyema. (After Whi'man.)

B. Female Orthonectid (Rhopalura Giardii). (After JULiN.)

$e$. Ectoderm; $e n$, inner endoderm cell with nucleus $(n)$; and embryo (ent).

as slight modifications of the hypothetical gastrula-like ancestors of the Metazoa, while Hatschek, comparing them to precociously reproductive planulæ speaks, of them as Planuloidæ. It is also possible that some of them may be 
parasitic degencrations of Turbellarian-like worms. It will be enough here merely to notice four types:-

(I.) Dicyemidæ (type Dicyema) occur as parasites in Cephalopods; the body consists of a ciliated outer layer, enclosing a single multinucleate inner cell, within which egg-like germs develop, apparently without fertilisation, into dimorphic embryos (see Fig. 36, A).

(2.) Orthonectidxe (type Rhopalura) occur as parasites in Turbellarians, Brittle stars, and Nemerteans; the body is slightly ringed, and consists of a ciliated outer layer, a subjacent sheath of contractile fibres, and an internal mass of cells, among which ova and spermatozoa appear. The sexes are separate and dimorphic (see Fig. 36 B.).

(3.) Professor F. E. Schulze has discovered a small marine organism-Trichoplax adharens -in the form of a thin, three layered, externally ciliated plate; and Monticelli records a similar form under the title Treptoplax adharens.

(4.) Professor J. Frenzel has discovered in brine solutions a minute Turbellarianlike organism-Salinella salve-whose body consists of one layer of cells. There is an anterior mouth, a ciliated food canal, and a posterior anus. The ventral surface is finely ciliated, the rest of the cells bears short bristles. The animal reproduces by transverse fission, but conjugation and encystation also occur. The larva is unicellular.

These forms are obviously of much interest to those who ponder over the possible nature of the earliest multicellular animals. 


\title{
CHAPTER IX.
}

\author{
CELLENTERA.
}

Class t. Hydrozoa $\left\{\begin{array}{l}\text { Hydromedusæ, } \\ \text { Siphonophorz, }\end{array}\right.$ Class I. HydrozoA $\left\{\begin{array}{l}\text { Hydromedusæ. } \\ \text { Scyphomeduse, }\end{array}\right.$
Class 2. Scyphozoa $\left\{\begin{array}{l}\text { Acraspeda, } \\ \text { Acrinozoa. }\end{array}\right.$
Class 3. Ctenophora.

THE Cœlentera - including zoophytes, jelly fish, sea anemones, corals, and the like-form a very large series of Accelomate Metazoa, i.e., of multicellular animals without a body cavity. Their simplest forms are not much above the level of the simplest sponges, but the series has been more progressive. Thus many illustrate the beginnings of definite organs. In their variety they seem almost to exhaust the possibilities of radial symmetry, and some types may be regarded as pioneers of the yet more progressive bilateral "worms." Many are very vegetative, deserving the old name of zoophytes (which should rather be read backwards - Phytozoa), and in their budded colonies afford most interesting illustrations of organic co-operation and division of labour.

\section{General Characters.}

The Colentera are simple, usually marine, forms in which the primary long axis of the gastrula becomes the long axis of the adult, zehich is almost always radially symmetrical about this axis. There is no body cavity or calome, distinct from the primitive digestive cavity (enteron) and its outgrowths. In the lower nembers of the series, the primary opening of this cavity becomes the mouth of the adult, but in the more specialised types there is an (ectodernic) oral invagination, which forms a gullet tube. Between the ectoderm and endoderm of the body wall, there is a supporting layer, or 
mesoglaa, of jelly-like consistency. In the simplest cases this is quite devoid of cells, but secondarily, these may migrate into it from the endoderm. Stinging cells of zarying complexity are almost invariably present, but in most members of the class Ctenophora they are modified into adhesive cells.

The Colentera exhibit two divergent types of structure, which recur constantly, in modified forms, throughout the group, and may even be both present in the course of one life history, when they illustrate the phenomenon of alternation of generations or metagenesis. Of the two, the more primitive type is the sessile tubular hydroid, which may be compared to a gastrula, fixed by one end, and furnished with a crown of tentacles placed round the central aperture of the other pole. The other derived form, which has become specialised in various directions, is the active medusoid or jelly fish type. In several divisions the formation of a calcareons "skeleton" by the hydroid type may result in the production of "corals." Multiplication by budding is common, and often results in the formation of colonies, some of which shou considerable division of labour.

The preservation of the primary axis, the absence of true mesoderm and of a calome, are often said to distinguish Calentera and Sponges from other Metasoa, or Calomala, but the results of recent researches on the nature of the mesoderm seen to rob this distinction of part of its pricision.

\section{General Survey.}

The Coelentera, or "Nettle animals" of the Germans, include a large number of familiar and beautiful forms of life. The graceful zoophytes which fringe shells and stones, and the tiny transparent bells which float in the pools; the sea anemones which cluster in the nooks of the rocks, and the active jelly fish which swim on the waves, are but different expressions of the antithesis so characteristic of the series, and illustrate, the former in the class Hydrozoa, the latter in the Scyphozoa, the two physiological tendencies of the Colentera. The delicate irridescent globes, which represent the third class, the Ctenophora, illustrate the climax of activity, for among them there is no sessile hydroid type.

In our survey of the series, however, we must pass over 
these familiar types, and begin with the little fresh water Hydra, which is often to be found attached to the stems and leaves of water plants. The structure here is extremely simple, but the simplicity is probably due to degeneration. 'This Hydra was first described in I 703 by Leeuwenhoek, and was studied by some of the older naturalists, such as Rösel von Rosenhof and the Abbé Trembley, with much eagerness. In favourable conditions it may be frequently observed giving off daughter buds, which remain for a time attached to the parent, and then separate as independent Hydre. The bud itself, before leaving the parent, may also bud, so that three generations are present. If we suppose this process of gemmation, combined with imperfect separation of the units, to continue indefinitely, we can understand the formation of hydroid colonies, such as the zoophytes, where the colony is supported by an organic axis of varying complexity.

The members of such a colony would, however, with an exception which we will consider later, be all similar and equivalent, and this is by no means true of all hydroid colonies. In Hydractinia, for example, which is common on shells at the shore, the colony consists of polypes of varied structure and function. It may be that these differences are caused by differences in nutrition, the fact at any rate is that some of the polypes are nutritive "persons," like Hydra in appearance; some are mouthless reproductive "persons," which produce sperms and eggs, and so eventually start a new colony; others also mouthless, are long, slender, sensitive, and abundantly furnished with stinging cells; while the little protecting spines at the base of the colony may perhaps be abortive "persons." All these polypes are united by connecting canals at the base, and all are fed at the expense of the nutritive "persons." Hydractinia thus exhibits division of labour among the members of the colony, and a tendency towards this is common in the Colentera.

If we now return to the simpler zoophyte colony, we find that this tendency can be recognised even here. Like Hydractinia, the colony at intervals exhibits reproductive "persons," different from the ordinary polypes. These, as in Hydractinia, may be sessile and mouthless, or they may 
after a time become detached and float away as delicate, pulsating, swimming bells. These swimming bells are male and female, they give rise to male and female elements, and so to embryos, which, after a time, settle down and form new zoophyte colonies. This is an instance of the phenomenon of alternation of generations (see p. 55).

Again, just as the predominance of passivity is exhibited in Hydractinia and some zoophytes, where the active
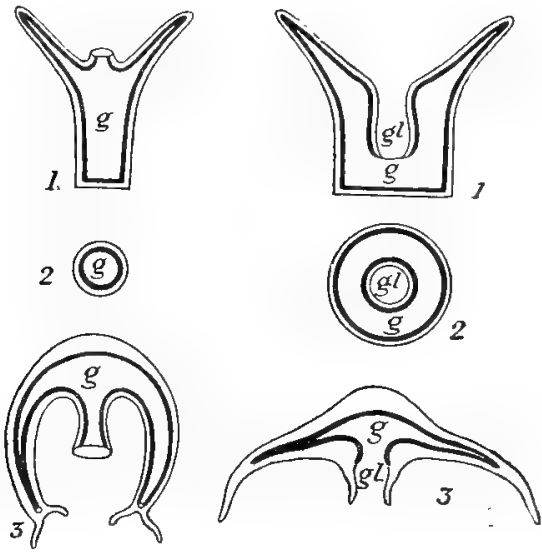

FIG. 38.-Diagram of Celenterate structure, endoderm darker throughout.

r. To left, shows longitudinal section of Hydra; to right, of Sea anemone. $g$, gut; $g$., incipient gullet.

2. To left, shows crosy section of $\mathrm{H}_{\mathrm{y}} \mathrm{dra}$; to right, of Sea anemone.

3. To left, shows vertical section of Craspedote Medusoid (with velum); to right, of Acraspedote Medusa, without velum. $g$, gut; gl., guilet.

Note anatomical correspondence of the polype and medusoid forms.

swimming bell is left out of the life history, so the predominance of activity is exhibited in the permanent medusoids, e.g., Geryonia, where the colonial hydroid stage is omitted, and the enbryo becomes at once a swimming bell. Finally, the medusoids themselves may become colonial, and we have active floating colonies, like those of the 
Portugese man-of-war, which show, on a different plane, as much division of labour as Hydractinia.

All these types belong to the class Hydrozoa, but the same general conclusions apply to the next class, that of the sea anemone and jelly fish. The jelly fish present a strong general resemblance to the medusoids, but are separated from them by their usually greater size, as well as by greater complexity and distinct anatomical differences. It is in accordance with this increased complexity that the alternation of active and passive forms, though as real, is less obvious. Yet even here we find one type (Pelagia) always locomotor, another (Aurelia) whose early life is sedentary, and others (Iucernarians) which in their adult life are predominantly passive, and attach themselves by a stalk.

The sea anemones and their numerous allies may be regarded as bearing a relation to the jelly fish, somewhat similar to that which the hydroid polypes bear to the swimming bells (Fig. 38). They are, however, much more complicated in structure than the hydroids. Simple forms are much commoner than in the Hydrozoa, but the colonial type is nevertheless very frequent. The colonies may be supported by an organic framework only, but very commonly there is a tendency to accumulate lime in the tissues, which results in the formation of corals.

It may be well to note here explicitly that various polypoid types may form corals. In fact the formation of a framework of carbonate of lime may be regarded as an expression of the sedentary constitution. The most important reef building corals are included in the Scyphozoa, but among the Hydrozoa the Millepores form very considerable lime sheathed colonies.

The corals present many problems of great interest. How do they get their carbonate of lime? Is that salt particularly abundant about the reefs, or is there, as Irvine and Murray suggest, an interaction between the waste products of the polypes and the sulphate of lime abundant in sea water? On what do they feed? Do their bright pigments, as Hickson suggests, enable them to utilise carbonic acid after the manner of plants? We may think also of the struggle for standingroom among the coral polypes, and of the struggle for existence among the many brightly coloured animals which browse and hide on the reef.

Finally, as the corals are predominantly passive, so there is a climax of activity in the Ctenophores, which move by 
cilia united into combs, and often shine with that "phosphorescence" which is an expression of intensity of life in many active animals. The Ctenophores have probably arisen from a modified Hydrozoon medusoid.

As to diet, the active Ctenophores are carnivorous, attaching themselves by adhesive cells to one another, or to other small animals; many of the larger forms, e.g., sea anemones and jelly fish are able to engulf booty of considerable size; the majority, however, feed on small organisms, in seizing and killing which the tentacles and stinging cells are actively used, but what the corals eat no one seems to know.

Types of C(Elentera.

lirst Type-Hydra, illustrative of the Class Hrdrozos.

General Life. - The genus Hydra is represented by several species, e.g., the green Hydra qiridis and the brownish Hydra fusca, both widely distributed in fresh water. It is one of the simplest of Coelentera, for the body is but a two-layered tube, with a crown of (6-1o) hollow tentacles around the mouth, and with no organs except those concerned in reproduction. The body is usually fixed by its base to some aquatic plant, often to the underside of a duckweed leaf. It may measure $\frac{1}{4} \frac{1}{2}$ inch in length, but it is as thin as a needle, and contracts into a minute knob.

The animal sways its body and tentacles in the water, and it can also loosen its base, lift itself by its tentacles, stand on its head, or creep by looping movements. Usually, how-

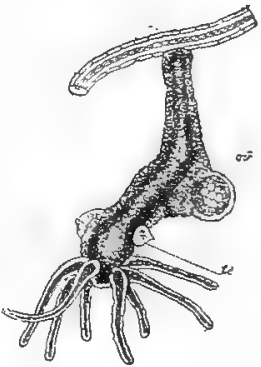

FIG. $\quad$ 39. - $H_{y^{\prime}} d r \alpha_{2}$ hanging from water weerl.

(After GReEne.)

$0 \%$, Ovary. $t$, Testes. ever, it prefers a quiet life. It feeds on small organisms, which are paralysed or killed by stinging cells on the tentacles, and are swept into the tubular cavity of the body by the action of flagella on the internal cells. Sometimes animals as large as water fleas (e.g., Dafhnia) are caught, and in part digested. Infusorians (Euplotes, etc.) are of ten 
seen wandering to and fro on the surface of the Hydra, but these wonted visitors do not seem to provoke the stinging cells to action.

So simple is Hydra that a cut of tentacle, or a fragment, containing samples of the various kinds of cells in the body, and not too minute, may grow into an entire animal. Thus the Hydra may be multiplied by being cut in pieces. If the animal be turned inside out (a delicate operation), the status quo is soon restored. The Abbé Trembley who first made this experiment thought that the out-turned inner layer or endoderm assumed the characters of the outer layer or ectoderm, and that the inturned ectoderm assumed the characters of endoderm. But this is not the case. Either the animal rapidly rights itself by turning outside in, or, if this be prevented, the inturned ectoderm disappears internally, and by growing over the out-turned endoderm, from the lips downwards, restores the normal state.

In favourable nutritive conditions, the $H y d r a$ forms buds, and on these a second generation of buds may be developed. A check to nutrition or some other influence causes the buds to be set adrift. Besides this asexual mode of multiplication, the usual sexual reproduction occurs.

General Structure. - The tubular body consists of two layers of cells, i.e., the animal is diploblastic. The cavity is the gut, and it is continuous with the hollow tentacles. These, when fully extended, may be longer than the body. The mouth is slightly raised on a disc or hypostome. Of the two layers of cells, the outer or ectoderm is transparent, the inner or endoderm usually contains abundant pigment. On the tentacles especially, even with low power, one can see numerous clumps of clear stinging cells. The male organs appear as ectodermic protuberances a short distance below the bases of the tentacles; the ovary, with a single ovum, is a larger bulging further down. Both male and female organs may occur on the same animal, either at one time or at different times, but often they occur on different individuals. The buds have the same structure as the parent body, but in origin they appear to be wholly ectodermic.

Minute Structure. - The outer layer or ectoderm includes the following different kinds of cells :-

(I) Large covering or epithelial cells, within or between some of 
which lie the stinging cells. The epithelial cells are somewhat conical, broader externally than internally, and in the interspaces lie interstitial cells. By certain methods, a thin shred can be peeled off the external surface of the ectoderm cells. This is a cuticle, i.e., a pellicle no longer living, produced by the underlying cells.

(Ia.) Many of these large cells have contractile basal processes, or roots, running parallel to the long axis of the body, and lying on a middle lamina which separates ectoderm from endoderm (Fig. 40, E). The cells themselves are contractile, but there is special contractility in the roots. Like the muscle cells of higher animals, they contract under certain stimuli, and are often called "neuro-muscular." But the discovery of special nerve cells (Jickeli) shows that even in Hydra there is a differentiation of the two functions of contractility and irritability.

(2.) Small stinging cells or cnidoblasts occur abundantly on the upper parts of the body, especially on the tentacles. Each contains a protrusible structure called a nematocyst. This consists of a sack, the neck of which is doubled in as a pouch, usually learing internal barbs, and prolonged into a long, hollow, spirally coiled filament or lasso. This lasso is bathed in a fluid, presumably poisonous, for it is able to paralyse small animals. On its free surface the stinging cell usually bears a delicate trigger hair or cnidocil. Uncler stimulus, whether directly from the outside or from a nerve cell, the cnidoblast contracts, and the pressure of the fluid causes the forcible evagination of the barbed pouch and the long lasso. Besides the ordinary stinging cells, there are others of small size which do not seem to explode.

(3.) Scattered about there are minute nerve cells, with fine connections, especially with the muscular and the stinging cells (Fig. 40, B).

(4.) Small interstitial or indifferent units fill up chinks in the ectoderm, and seem to grow into reproductive, stinging, and other cells.

(5.) Granular glandular cells on the basal disc or "foot " probably secrete a glutinous substance. They are also said to put out pseudopodia and so move the animal slowly.

The inner layer or endoderm is less varied in structure, as is to be expected from the fact that it is not, like the ectoderm, exposed to the varying action of the environment. Its cells are pigmented, often vacuolated, and most of them are either flagellate or amoboid. The pigment bodies in $H$. viridis seem comparable to the chlorophyll corpuscles of plants ; in $H$. fusia they are brownish and without chlorophyll. The active lashing of the flagella causes currents which waft food in and waste out. If some small animal, stung by the tentacles, is thus wafted in, it may be directly engulfed by the amceboid processes of some of the cells, and it has been noticed that the same cell may be at one time flagellate and at another time amoboid ( $f$. the cell cycle, p. 97). After this direct absorption the foorl is digested within the cells, and while some of the clark granules seen in these cells may be decomposed pigment bodies, others seem to be particles of indigestible dibris. Thus Hydra illustrates what is called intracellular digestion (T. I. Parker), such as occurs in Sponges, some other Coelentera, and some simple "worms." But, accorrling to Miss Creenwood, the lood is digested in the gut cavity, and sulssequently alsorbed. It seems likely that both intracellular and extracellular digestion occur. 
Some of the endoderm cells have muscular roots like those of the ectoderm. They lie on the inner side of the middle lamina, in a transverse or circular direction. A few cells near the mouth and base are described as glandular, and the presence of a few stinging cells has been recorded, though some suggest that the last are discharged ectodermic nematocysts which have been swallowed.

The middle lamina, representing the mesogloea, is a thin homogeneous plate, on each side of which lie the muscular roots of ectodermic and endodermic cells (Fig. 40, D).

It is bistorically interesting to notice the important step which was
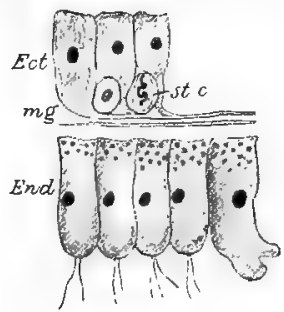

A

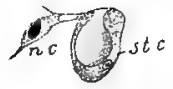

B

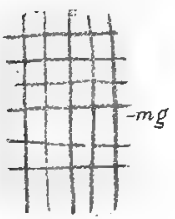

D

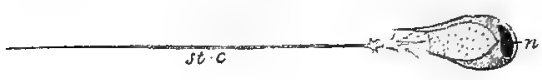

C

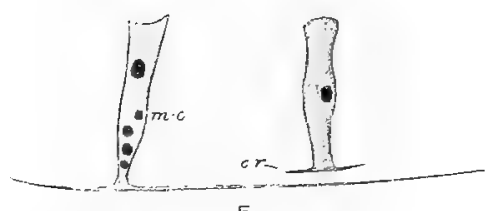

E

FIG. 40.-Minute structure of Hydra. (After T. J. PARIER and JICKELI.)

A. Ect. ectoderm; mg. mesogloeal plate; st. c. stinging cell ; Emd. endoderm with flagella and amoeboid processes.

B. nc. nerve cell, and st.c. stinging cell together.

C. Stinging cell with ejected thread; $n$. nucleus.

D. Mesogloal plate with contractile roots resting on it.

E. m.c. muscular cell with contractile roots, c.r.

made when, in 1849 . Huxley definitely compared the outer and inner layers of the Coelentera with the epiblast and hypoblast which embryologists were beginning to demonstrate in the clevelopment of higher animals. Not long afterwards Allman applied to the two layers of Hydroids, the terms ectoderm and endoderm. 
The division of labour among the cells of Hydra is not very strict, but already the essential characteristics of ectoderm and endoderm are evident. We may summarise these as follows, comparing them with the characteristics of epiblast and hypoblast in higher animals :-

\begin{tabular}{l|l|}
\hline Outer Laver. & Median I.AYER. \\
\hline $\begin{array}{l}\text { The ectoderm forms- } \\
\text { Covering cells, } \\
\text { Stinging cells, } \\
\text { Nerve cells, } \\
\text { Muscle cells, \&c. }\end{array}$ & \\
\hline $\begin{array}{l}\text { The embryonic epiblast } \\
\text { of higher animals grows } \\
\text { into epidermis, nervous } \\
\text { system, and essential parts } \\
\text { of sense organs. }\end{array}$ & $\begin{array}{c}\text { The mesoblast of higher } \\
\text { animals becomes muscular, } \\
\text { connective, skeletal tissue. }\end{array}$
\end{tabular}

INNER LAYER.

The endoderm formsDigestive cells lining the food canal, and also Muscle cells, \&c.

The embronic hypoblast of higher animals always lines the digestive part of the food canal.

The Reproductive Organs.-(a) From nests of repeatedly dividing interstitial cells, several ( $\mathrm{I}-2 \mathrm{O})$ simple male organs or testes are formed. Each consists merely of a clump of male elements or spermatozoa, bounded by the distended ectoderm. Through this the spermatozoa are extruded at intervals, and one may fertilise the ovum of the Hydra. In other words, self-fertilisation, which is very rare among animals, may occur. The spermatozoon is a motile cell, with a minute cylindrical "head" consisting of nucleus, a minuter middle piece, and a long thread-like vibratile tail (Fig. 4I, I).

(b) Usually there is but one female organ or ovary, but in $H$. fusca as many as eight have sometimes been observed. The ovary arises like the testes from a nest of interstitial cells, one of which becomes the ovum. In rare cases there are two ova. The ovum is at first amceboid and transparent, but like many other ova it feeds on its neighbours, loses its amoeboid form, and becomes rich in nutritive material. It also becomes pigmented (Fig. 4I, 2).

Development. ${ }^{1}$ - The ovmo of Hydra is, as we have seen, the successful central cell in a nest of interstitial elements which form the rudimentary ovary. It is at first amoboid, and becomes more and more rich at the expense of its neighbours. Their remains (perhaps nuclei) accumulate within the ovum as "yolk spherules" or "pseudo-cells." With increase of size the ovum changes its form from amceboid to calielike, and from that to spherical. Around the spherical ovum a gelatinous sheath is formed. When the limit of growth is reached the nucleus or germinal vesicle divides twice in the usual way, and two polar bolies are extruded at the distal pole. Thereafter, the ectoderm of the parent Hydra yields to the increasing strain put upon it and ruptures, allowing the ovum to protrule. By a broad hase it still remains, however, attached to the parent, and in this state it is fertilised, the spermatozoon entering by the distal pole (Fig. 4I, 4).

1 As the accounts given by various investigators do not agree, it may be noted that we have here followed that of Brauer ( 1891 ). 
The segmentation which follows is total and equal, and results in the formation of a blastosphere (F'ig. 4I, 5). By inwandering, or by division of the cells of the blastosphere, an intermal endoderm is formed, and this formation takes place on all sides. In a word, it is multipolar. The segmentation cavity of the blastosphere is thus filled up, and the two layers become rifferentiated from one another.

The outer or ectodermic layer forms $(a)$ an external "chitinoid" shell of several layers; $(b)$ an internal membrane, homogeneous, thin, and elastic; and $(c)$ the future ectoderm of the adult. In Hydra fiesca the cgg is separated from the parent before the shell is formed, and is fastened by its gelatinous sheath to aquatic plants; in $H$. viridis and $H$. srisea the egg falls off after the outer shell has been formed. In all species the separation from the parent appears to be followed by a period of quiescence lasting from one to two months.

Within the shell, differentiation at length recommences, but it proceeds
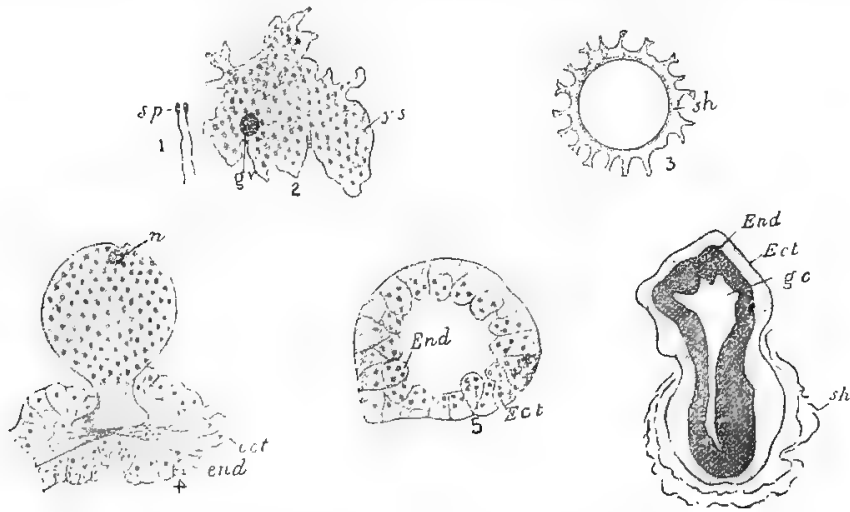

FIG. 4I.-Development of Hydra. (After BRAUER.)

I. $s p$, spermatozoa. 2. Amœboid ovum; g.7., germinal vesicle or nucleus; y.s., yolk spherules.

3. Ovum with lobed envelope $(s / h)$ around it.

4. Ovum protruding; $x_{\text {. }}$, the nucleus ; $c c t$, the ruptured ectoderm; cnd., the endoderm.

5. Section of blastosphere-Ect., ectoderm; End., endodermbeing formed.

6. Section of larva. Ect., ectoderm; End., endoderm ; g.c., gutcavity; sh., ruptured envelopes.

slowly. Interstitial cells arise in the ectoderm; a micldle lamella is formed; a gastric cavity begins to appear in the midst of the endoderm. Thereafter the shell bursts, and development proceeds more rapidly. The embryo elongates, acquires a mouth by rupture at the distal (sometimes called vegetative) pole. The inner sheath is also lost, and the young Hydra fixes itself and begins to live as its parent or parents dirl.

The multipolar formation of the encloderm is perhaps a prinitive 
mode; it occurs only in Colentera, and in those which have no free swimming blastosphere stage. Perhaps, as Brauer suggests, it was originally characteristic of free swimming spherical blastospheres, which rotated in any direction. But when the blastosphere is oval, and swims with one pole always forwards, the food particles are swept towards the posterior pole. It is therefore advantageous that the formation of the endoderm should occur there, either by immigration of cells, or by gastrular invagination (Korschelt and Heider).

\section{Forms like Hydra.}

Even simpler than Hydra is Protohydra, a form without tentacles, occurring both in the sea and in fresh water. A similar fresh water form has been described under the name of Microhydra, and a strange simple polype-Polypodium -whose history is incompletely known, has been found as a parasite on the eggs of sturgeons. But further details in regard to all these forms are much wanted.

Second Type of Celentera-A Medusoid. Class HydrozOA. Sub-Class Hydromedusæ or Craspedota.

Hydra is too simple to be thoroughly typical of the Hydrozoa. The class includes the hydroid colonies or zoophytes, which may be compared to Hydra with many buds, and also free medusoid forms, which may be $(a)$ liberated nembers of a hydroid colony, or $(b)$ independent organisms. Besides these there are complex colonies of medusoid forms (Siphonophoræ).

'The hydroid type, except in minor details, usually resembles Hydra. In some cases the tentacles are solid, instead of hollow as in Hydra, and they may be arranged in two circles,-an outer and an inner (Tubularia). In some of the hydroid colonies, notably the Millepores and Hydractinia, the polypes are very dissimilar to one another, and have become specialised for the performance of different functions.

The medusoid type is like an inflated hydroid adapted for swimming. It is bell shaped, and down the middle of the bell hangs a prolongation - the manubrium - which terminates in the mouth. Around the margin of the bell there is a little shelf, the velum or craspedon, which projects inwards, and is furnished with muscle cells. The margin of the bell also bears tentacles, usually hollow, and abundantly furnished with stinging cells (Fig. 38,3 ). 
On the convex surface of the bell the ectoderm forms simply an epithelial layer; on the concave surface it is differentiated into muscle cells on the velum, the manubrium, and the tentacles, nerve cells at the base of the velum, and stinging cells on the tentacles. The endoderm is ciliated; it lines the food space, and extends also into the tentacles. The mesogloea forms a thickened jelly, present more especially on the convex (ex-umbrellar) surface.

The mouth opens into the canal of the manubrium, which leads to the central cavity of the dome. With this a varying number of unbranched radial canals communicate; these also open into a marginal circular vessel, which again communicates with the cavities of the tentacles. Digestion is intracellular, and probably goes on throughout the whole of this "gastro-vascular" system.

The movements of the bell are caused by the contractions of the muscle cells mentioned above.

The nervous system consists of a double ring of nerve fibres around the margin of the bell. With these are associated ganglionic cells, which apparently control the muscular contractions.

Sense organs may be present, in the form of "eyes," at the base of the tentacles (Ocellatre), or there may be "auditory" vesicles developed as pits in the velum (Vesticulatæ).

The reproductive organs develop either in the manubrium or in the course of the radial canals. The products always (?) ripen in the ectoderm, and often seem to arise there; but Weismann and others have shown that the reproductive cells of a medusoid derived from a hydroid, or of the reduced and fixed reproductive persons of many hydroids, have considerable powers of migration, and may originate (sometimes in the endoderm) in the hydroid colony at some distance from the place where they are matured within the medusoid bud. The sexes are usually separate. The commonest kind of free swimming larva is the planula, which is oval, ciliated, and diploblastic, devoid of an opening, and usually of a central cavity. In those medusoids which arise as the liberated sexual persons of a fixed asexual hydroid colony, the planula settles down and develops into a new hydroid. 
In many Hydrozoa, as has been already noticed, the sexual persons are not set free, but remain attached as buds to the parent hydroid. "These fixed "gonophores" show many stages of degeneration; some, notably in the floating colonies of Siphonophoræ, differ little structurally from true medusoids, while others, as in Hydractinia, are simply small closed sacs enclosing the genital products. (Fig. 49.)

Third Type of Celentera. The common Jelly fish-Aureliu aurita. Class ScyphozoA. Sub-Class Scyphomedusæ or Acraspeda.

This medusa is almost cosmopolitan, and in the summer months occurs abundantly around the British coasts. We often see hundreds gently swimming in shoals, and many are washed shorewards and stranded on flat beaches. The glassy disc usually measures about four inches in diameter, but may be twice as large. The jelly fish feeds on small animals, such as crustaceans, which are entangled and stung to death by the long lips.

\section{External Appearance.}

The animal consists of a gelatinous disc, slightly convex on its upper (ex-umbrellar) surface, and bearing on the centre of the other (sub-umbrellar) surface a four cornered mouth, with four long much-frilled lips. The circumference of the disc is fringed by numerous short hollow tentacles, by little lappets, and by a continuation of the sub-umbrella forming a delicate muscular flap or velarium. Conspicuously bright are the four reproductive organs which lie towards the under surface. Nor is it difficult to see the numerous canals which radiate from the central stomach across the disc, the eight marginal sense organs, and the muscle strands on the lower surface.

\section{The Three Layers.}

The ectoderm which covers the external surface bears stinging cells, especially on the tentacles, and to this layer belong sensory and nervous cells aggregated at eight centres, also a plexus of ganglion cells beneath the skin on the under surface, and, finally, the muscle cells. According to some, the ectoderm lines part of the mouth tube or manubrium. The endoderm lines the digestive cavity, is continued out 
into its radiating canals, and is ciliated throughout. The mesoglcea is a gelatinous coagulation containing wandering amoboid cells from the endoderm. The whole animal is very watery, indeed the solid parts amount to not more than ten per cent. of the total weight.

\section{Neroous System.}

The nervous system consists $(a)$ of a special area of nervous epithelium, associated with each of the eight sense organs, and (b) of numerous much elongated bipolar ganglion cells lying beneath the epithelium on the under surface of the disc. This condition should be contrasted with that in Craspedote medusoids, but too much must not be made of the contrast, for a nerve ring is described in Cubomedusæ, one of the orders of Acraspedote jelly fish. In Aurelia, the sense organs are less differentiated than in many other jelly fish. Each of the eight organs, protected in a marginal niche, consists of a pigmented spot, a clubshaped projection with numerous calcareous "otoliths" in its cells, and a couple of apparently sensitive pits or grooves. We are not warranted in calling these organs "optic," "auditory," and "olfactory," in Aurelia at any rate. The sense organs arise as modifications of tentacles, and are often called "tentaculocysts" or "rhopalia." Their cavities are in free communication with branches of the radial canals.

\section{Muscular System.}

Between the plexus of nerve cells and the sub-umbrellar mesogloea, there are cross-striped muscle fibres, each of which has a large portion of non-contractile cell substance attached to it. They lie in ring-like bundles, and by their contractions the medusa moves. Unstriped muscle fibres are found about the tentacles and lips.

\section{Alimentary System.}

The four corners of the mouth are extended as four much frilled "arms," each with a ciliated groove and stinging cells, and with an axis of mesoglcea. They exhibit considerable mobility. Their crumpled and mobile bases surround and almost conceal the mouth. A short tube, the "manubrium" or gullet, connects the mouth witl the 
central digestive cavity which occupies the centre of the disc. From this central chamber, sixteen gastro-vascular canals of approximately equal calibre radiate to the circumference, where they open into a circular canal, with which the hollow tentacles are connected. Eight of the radial canals are straight, but the other eight are branched, and thus in an adult Aurelia the total number of canals is large. These canals are really due to a partial obliteration of the gastric cavity, to a fusion of its ex-umbrellar and subumbrellar walls along definite lines. They are all lined by ciliated endoderm.

Where the manubrium or tube from the mouth passes into the central digestive cavity, there are four strong pillars of thickened sub-umbrellar material. Outside each of these

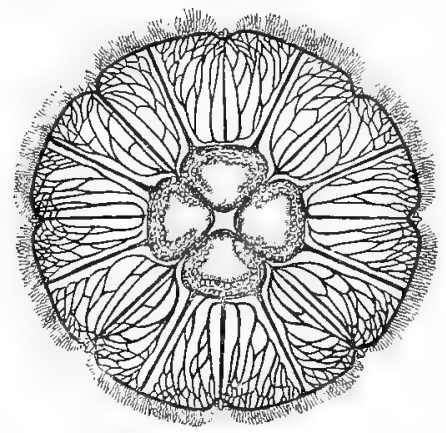

FIG. 42.- Surface view of Aurelia. (From Romanes.)

Showing four genital pockets in centre, much branched radial canals, eight peripheral niches for sense organs, and peripheral tentacles.

pillars, and still near the base of the manubrium, there are four patches where the sub-umbrellar surface remains thin. These are the gastro-genital membranes, lined internally by germinal epithelium (Fig. 43, R).

To the inside of these genital organs, within the digestive cavity, are four groups of mobile gastric filaments $(g . f$. , Fig. 43), which are very characteristic of jelly fish. In appearance these are very similar to the small tentacles of the margin, and, like them, are hollow. They are covered with endoderm--with ciliated, glandular, muscular, and stinging cells. 
The body is mapped out into regions by the following convention. The first tentacles to appear in the larva are four in number, and correspond to the four angles of the mouth; the radii on which they appear are called "perradial." Halfway between these, four "interradials" are then developed. Then eight "adradials" may follow, between perradii and interradii.

\section{Reproductive System.}

The sexes are separate. The reproductive organs -ovaries or testes - consist of plaited ridges of germinal epithelium, situated on the four patches already mentioned, within sacs which are derived from and communicate with the floor of the gastric cavity. They are of a reddish violet colour, and at first of a horse-shoe shape, with the closed part of the curve directed outwards. Afterwards the ridges become circular and extend all round the walls of the sacs in which they lie.

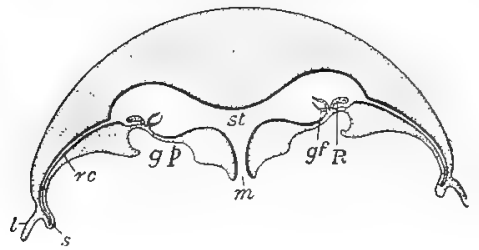

FIG. 43--Vertical section of Aurelia. (After Claus.) m., Mouth; st., stomach ; r.c., radial canal ; $R$., reproductive
organs; $g f$. gastric filaments ; g.p., genital pocket : t., marginal
tentacle; s., sense organ; the shaded part is mesogløa.

But the sub-umbrellar surface is modified beneath each genital sac in such a way that the sac comes to lie in a subgenital cavity communicating with the exterior (s.p., Fig. 43). The contractions of the umbrella produce a rhythmic movement of the water which enters the sub-genital sacs, and this constant renewal of the water suggests some respiratory significance for the sacs. It must be understood that the genital sacs containing the plaited ridges of germinal epithelium communicate with the gastric cavity only, while the sub-genital cavities containing water and enveloping the genital sacs communicate with the exterior only.

The ova and spermatozoa pass from the frills of germinal epithelium into the sacs, and thence into the gastric cavity. They find exit by the mouth, but young embryos may be 
found swimming in the gastro-vascular canals, and also within the shelter of the long lips.

Life History of Aurelia.-According to the most recent investigation, the fertilised ovum divides completely, but not quite equally, to form a blastosphere with a very narrow slit-like cavity. From the larger celled hemisphere, single cells migrate into the cavity, and fill this up entirely with a solid mass of endoderm. The archenteron arises as a central cleft in this cell mass, and opens to the exterior temporarily by the primitive mouth. During these processes the embryo elongates, the

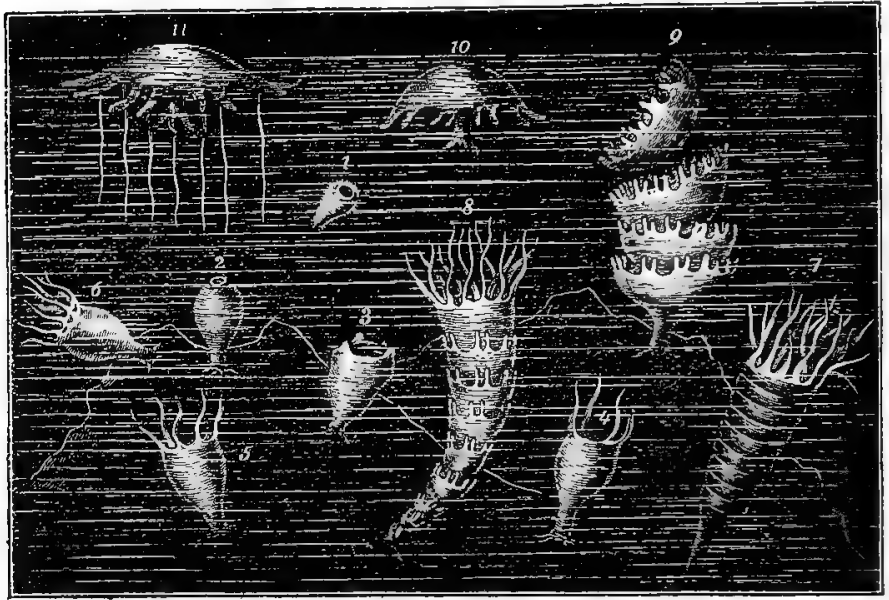

FIG. 44,-Diagram of life history of Aurelia. (After H.ACKEL.)

I, Free swimming embryo; 2-6, various stages of Hydra-tuba; 7-8, Strobila stage; 9, liberation of Ephyræ; IO-II, growth of Ephyre into Meduse.

outer cells become ciliated, the mouth closes, and the embryo swims freely as an oval planula.

After a short period of free life, this planula settles down on a stone or seaweed, attaching itsclf by the pole where the mouth formerly opened. At a very early stage the mesoglcea appears between the two layers. At the free pole an ectodermic invagination next occurs, an opening breaks through at its lower end, and thus a gullet lined with ectoderm ${ }^{1}$ is formed, which hangs freely in the general cavity. During

1 The statement as to the ectodermic gullet is due to Götte (1887); its existence is denied by Claus, who is followed by Chun. 
this process there are formed first two and then four diverticula of the general cavity, which are arranged round the gullet above, and open freely into the digestive cavity below. In the gullet region these are separated by broad septa, which are continued into the lower region of the body as four interradial ridges or teniolie. Although the development is different, these may perhaps be compared to the mesenteries of Anthozoa. The tentacles bud out from the region of the mouth, the first four corresponding in position to the four pouches. Interradially above the four septa, four narrow funnel shaped invaginations arise, these are produced by the ingrowth of ectoderm, which then forms the muscle fibres which run down the treniole (contrast the endodermic nuscles of Anthozoa).

The larva now forms a "Hydra-tuba" or "Scyphistoma," it is about an eighth of an inch in height. By lateral budding, or by the formation of creeping stolons, it may give rise to larva like itself. The gradual widening of the central cavity renders the gullet tube less obvious, and results in an increasing resemblance to the medusa type.

In late autumn, however, a more fundamental change occurs. IVe will begin with the simplest case. (a) Occasionally, as has been observed by Hæckel, the Scyphistoma becomes detached and converted into a free swimming Ephyra, which in turn becomes a jelly fish. (b) In Aurelia, in unfavourable conditions, a furrow appears round the upper region of the Scyphistoma, the upper portion is converted into an Ephyra, and floats away, while the lower portion reforms its oral region by regeneration, and produces another Ephyra. (c) In ordinary conditions the Scyphistoma elongates, and displays a succession of annular constrictions. This stage, often compared to a pile of saucers, is technically called a Strobila. Each disc is separated off in its turn as a free swimming Ephyra, which becomes a jelly fish. The still undivided basal portion may rest for a time, and then undergo further constriction. This is probably an abbreviation of the primitive mode of development.

In the conversion of the Scyphistoma into the Ephyre, the diverticula coalesce into a general cavity, the entrances to the septal invaginations probably persist as the sub-genital pits, the gastric filaments sprout out from the remains of the septa, and so mark the place where the ectodermal gullet passed into the endodermal cavity.

The first Ephyra differs from those which come after it in bearing the original tentacles of the Hydra-tuba. From its margin eight bifid lobes grow out, each embracing the base of a perradial or interradial tentacle. The bases of these eight tentacles become the sense organs or rhopalia. The other eight adradial tentacles atrophy. On the Ephyree which follow there are at first no tentacles, only the eight bificl marginal lobes which bear the sense organs in their niches.

This development illustrates alternation of generations. From the fertilised ovum a fixed asexual Scyphistoma results. This grows into a Strobila, from which transverse buds or Ephyræ are liberated. Each of these grow into a sexual jelly fish, producing ova or spermatozoa. The first two cases mentioned ( $a$ and $b$ ) show how readily this alternation might pass into a "direct" development.

Relatives of Aurelia.-The Medusie, or true jelly fish, include forms which agree with the Anthozoa, in relative complexity of structure as 
compared with IIydrozoa, in the possession of an ectodermal gullet (see footnote on page 146), but differ in possessing ectodermal septal muscles and in some histological features. Among those closely allied to Aurelia, some, e.g., Pelagia, have a direct development without the intervention of Scyphistoma or Strobila stages, but this may occur exceptionally in Aurelia. Cyanea is often very large, "it may measure $7 \frac{1}{2}$ feet across the bell, with tentacles Izo feet long." Chrysaora is hermaphrodite, and has diffuse sperm sacs even upon the arms. In the Rhizostome-e.g., Cassiopeia and Pilema, the mouth is obliterated, and replaced by numerous small

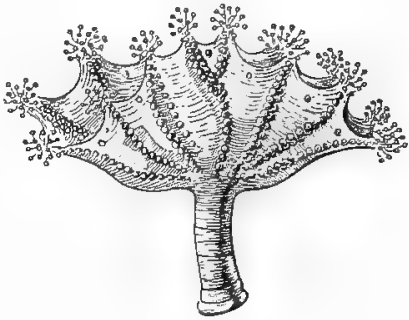

FIG. 45-- Lucernaria. (After pores on the four double arms. Luernaria and its allies are interesting sessile forms which have been compared to sexual Scyphistomas-that is, are regarded as persistently larral forms.

\section{Contrast between Hydrozoon and Scyphozoon medusoids.}

The majority are small "swimming

A flap or velum (craspedon) projects inwards from the margin of the bell.

No taniolæ, nor gastric filaments.

A double nerve ring around the margin.

Naked sense organs either optic or auditory. They are usually derived from the skin, but the auditory sacs may be modified tentacles.

Reproductive organs on the radial canals or by the side of the manubrium. The reproductive cells are usually derived from the ectoderm.

With the exception of the Trachymedusa, all arise as the liberated reproductive persons of Hydroid colonies.

True Hydrozoa.

\section{Scyphozoon. (ACRASpeda.)}

Many are large "jelly fish."

No velum. (The zelariun of Aurelia is a mere fringe, very inconspicuous in the adult, and not inturned.)

In the Scypbistoma there are four tæniolæ, from part of which the gastric filaments of the adult grow.

Eight separate nervous centres beside the sense organs, and a sub-umbrellar nervous plexus.

Sense organs are modified tentacles, and probably have almost always a triple function. They are usually protected by a hood.

Reproductive organs in special pockets on the floor of the gastric cavity. The reproductive cells arise in the endoderm.

Have no connection with hydroids, but may have a small sedentary polype stage (or Scyphistoma) in the course of their life history.

Probably more nearly related to Anthozoa than to Hy'drozoa.

We may note here that Chun, while agreeing provisionally to the separation of the Acraspeda from the Hydrozoa, strongly opposes their association with the Anthozoa, basing his opposition especially on the existence of Scyphistomas of great simplicity (e.s., Spongitiola). 
Fourth Type of Ceelentera. A Sea Anemone, such as Tealia crassicornis.

Class, ScyphozoA. Sub-Class, Anthozon or ActinozoA.

Most sea anemones live fixed to the rocks about lowwater mark. Some, e.g., Tealia crassicornis, are often half buried in sand; a few are unattached. The sedentary forms are able to shift their positions by short stages. They feed on small animals, - molluscs, crustaceans, worms, which are caught and stung by the tentacles, but many must depend largely on minute organisms, while others may be seen trying to engulf molluscs decidedly too large for them. A few anemones, without pigment or with little, have symbiotic Algæ in their endoderm cells; the bright pigments of many others seem to help in respiration. Besides the normal sexual reproduction (in which the young are sometimes developed within the parent), some sea anemones exhibit a power of asexual multiplication by detaching portions from near the base, and fission occurs in a few forms.

\section{External Appearance.}

The cylindrical body is fixed by a broad base; it bears circles of hollow tentacles around the oral disc; the mouth is usually a longitudinal slit. The tentacles are contracted when the animal is irritated, and the whole body can be much reduced in size. Just below the margin of the oral disc there is a powerful sphincter muscle, this contracts, and pulls together the body wall over the mouth and retracted tentacles. Water may pass out gently or otherwise by a pore at the tip of each tentacle, and long white threads, richly covered with stinging cells, are often ejected through the walls of the body. In certain states, especially if dying, the sea anemone protrudes its gullet, and turns itself partially inside out.

\section{General Structure of the Body.}

The Anthozoon polype differs markedly from the Hydroid -not only because an invagination from the oral disc inwards has formed a gullet tube, which hangs down freely 
into the general cavity ; but also because a number of partitions or mesenteries extend from the body wall towards this gullet. Some of the partitions are "complete," i.e., they reach the gullet; others are "incomplete," i.e., do not extend so far inwards. The complete mesenteries are attached to the oral disc above, to the side of the gullet, and to the base, and all the mesenteries are ingrowths of the body wall. The cavity of the anemone is thus divided into a number (some

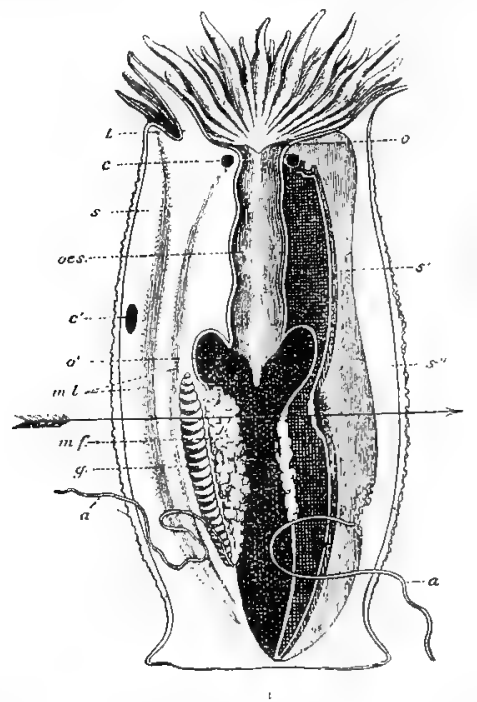

FIG. 46.--Structure of Sea anemone. (After ANDREs.)

$t$, Tentacles; 0 , mouth ; as, cesophagus ; $c, c^{\prime}$, apertures through a mesentery ; $a, a^{\prime}$, acontia; $g$, genital organs on mesentery; $m . f$, mesenteric filaments; $m . l$, longitudinal muscles; s, primary septum or mesentery ; $s^{\prime}$, secondary septum; $s^{\prime \prime}$, tertiary septum; $z^{\prime}$, base of gut cavity.

multiple of six) of radial chambers. These are in communication at the base, so that food particles from the gullet may pass into any of the chambers between the partitions. Moreover, each partition is perforated, not far from the mouth, by a pore, besides which there is often amother nearer 
the body wall. The tentacles are continuous with the cavities between the mesenteries, and thus all the parts of the body are in communication. The mouth is usually a longitudinal slit, and its two corners are often richly ciliated. The gullet is marked with longitudinal grooves, two of which, the "siphonoglyphes," correspond to the angles of the mouth, and are especially broad and deep. Along these two grooves, and by these two corners, food particles ustually pass in ; but in some, one side is an incurrent, the other an excurrent channel. Occasionally, only one corner of the mouth and side of the gullet is thus modified. The gullet often extends far down into the cavity of the anemone. It admits of a certain amount of extrusion. The mesenteries bear $(a)$

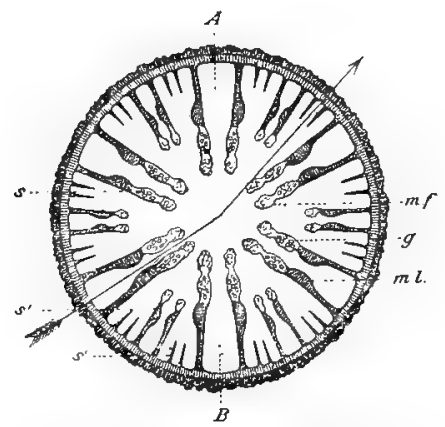

FIG. 47.- Section through Sea anemone (across arrow in Figure 46). (After ANDRES.)

A. B., directive septa; m.f., mesenteric filaments; $g$, genital organs; $m . l$, longitudinal muscles ; $s$, primary septum ; $s^{\prime}$, secondary septa; $s^{\prime \prime}$, tertiary septum. The arrow enters between two primary septa (an intra-septal cavity) and passes out between two Lertiary septa.

mesenteric filaments; (b) retractor muscles; (c) ridges of reproductive cells, almost always either ova or spermatozoa, rarely both; and $(d)$ in some cases offensive threads (acontia), rich in stinging cells, and extrusible through the body wall. The mesenteric filaments seem to be closely applied to the food and perhaps secrete digestive juice. Intracellular digestion also occurs. Sea anemones have no sense organs; the sapphire beads, which are so well seen at 
the bases of the outermost tentacles of the common Actinia mesembryanthemum, are batteries of stinging cells. The nervous system is uncentralised, and consists of superficial sensory cells connected with a plexus of sub-epithelial ganglion cells.

The Layers of the Body. -The ectoderm which clothes the exterior is continued down the inside of the gullet. The endoderm lines the whole of the internal cavity, including mesenteries and tentacles. The mesogloea is a supporting plate between these two layers, and forms a basis for their cells.

The ectoderm consists of ciliated, sensory, stinging, and glandular cells, and also of sub-epithelial muscle and ganglion cells based on the mesogloea, but mainly restricted to the circumoral region.

The endoderm consists mainly of flagellate cells, with muscle fibres at their roots. These form the main muscle bands of the wall, the mesenteries, and the gullet. Nor are glandular and even sensory cells wanting from the endoderm.

The Mesenteries, - In sea anemones and nearly related Anthozoa twelve primary mesenteries are first formed. These are grouped in pairs, and the cavity between the members of a pair is called intra-septal, in contrast to the inter-septal cavities between adjacent pairs. In these inter-septal chambers other mesenteries afterwards appear in pairs. Two pairs of mesenteries, however, differ from all the rest, those, namely, which are attached to each corner of the mouth and to the corresponding grooves of the gullet. These two pairs of mesenteries are called "directive," and they divide the animal into bilaterally symmetrical halves. Anatomically, a pair of directive mesenteries differs from the other paired mesenteries, because the retractor muscles which extend in a vertical ridge along them, are turned away from one another, and run on the inter-septal surfaces, whereas in the other mesenteries the retractor muscles run on the intra-septal surface, those of a pair facing one another. The arrangement of these muscles is of great importance in classifying Anthozoa. It is possible that the mesenteries are homologous with the treniole of jelly fish, and the mesenteric with the gastric filaments.

From the above description, it will be noticed that the fundamental radial symmetry of the Coelentera has here become profoundly modified.

Development.-Comparatively little is known in regard to the early stages of development in sea anemones. From the fertilised ovum, a blastosphere may result which by invagination becomes a gastrula. Or the two layers may be established by a process known as delamination, in which a single layer of cells is divided into an inner endodernic and an outer ectodermic layer.

Related Forms. - The sea anemones are classified in the sub-class Anthozoa or Actinozoa, and along with many corals are distinguished as Zoantharia or IIexacoralla from the Alcyonaria or Octocoralla, like Alcyonizen and related corals. This contrast is not perfectly satisfactory, but it rests on such distinctions as the following :- 
ANTHOZOA OR ACTINOZOA.

Zoantharia, Hexacoralla, e.g., Sea Anemone,

Many are simple, many colonial.

Tentacles usually simple, usually some multiple of six, often dissimilar.

Mesenteries usually some multiple of six, complete and incomplete.

Retractor muscles never as in Alcyonaria.

Two gullet grooves or siphonoglyphes, or only one.

Dimorphism only in some Antipatharia, and in one Madrepore coral.

Calcareous skeleton if present is derived from the basal ectoderm.

Types.

Actiniaria. Sea anemones.

Madreporaria. Reef building corals.

Antipatharia Black corals.
Alcyonaria, Octocoralla, e.g.

Dead Men's Fingers

All colonial, except a small family includ. ing Monoxenia and Haimea.

Tentacles eight, feathered, uniform.

Mesenteries eight, complete.

Retractor muscles always on one (ventral) side of each mesentery.

One (ventral) gullet groove or siphonoglyphe, or none.

Occasional dimorphism among members of a colony.

There are usually calcareous spicules (of ectodermic origin) in the mesogloea.

$$
\text { Examples. }
$$

Alcyonizur (Dead men's fingers), with diffuse spicules of lime.

Tubipora (Organ pipe coral), with spicules fused into tubes and transverse platforms.

Corallium mbrum (Red coral), with an axis of fused spicules.

$I$ sis, with an axis of alternately limy and "horny" joints.

Penratula (Sea pen), a free phosphorescent colony, with a "horny" axis possibly endodermic.

Heliopora, blue coral.

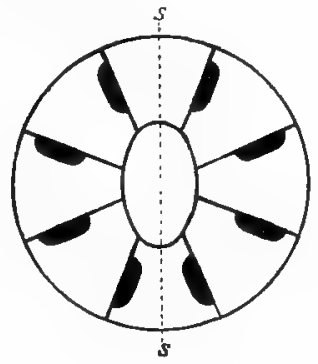

FIG. 48.- $Z$, Diagrammatic section of Zoantharian; $A$, of Alcyonarian. (After CHUN.)

The line $S-S$ in $Z$ is through the siphonoglyphes $(a)$. The retractor muscles are represented by dark thickenings on the mesen. teries-all on one (the ventral) side in Alcyonaria.

Coral Making. - We have alrearly noticed that there are "corals" among the Hydrozoa, viz., the Millepores. Leaving these out of account, we have to recognise that both divisions of Anthozoa include many corals. 
With the doubtful exception of the Sea pens and their allies, in which the axial skeleton is believed by some to be endodermic, the "coral " is due to ectoderm cells, which either remain in the ectoderm or wander into the mesogloea.

Taking as a basis the hard parts only, corals may be classified in various ways:-

According to Composition-

(1.) Discontinuous calcareous spicules-Alcyonium, \&c.; these may also occur along with some forms of (2).

(2.) Continuous skeleton.

(a) Organic and "horny," e.g., axis of many Gorgonids, axis of Pennatulids.

(b) "Horny" and calcareous, e.g., axis of $J$ sis.

(c) Wholly caleareous, in the great majority.

According to extent of the hard parts-

(I.) Diffuse spicules, e.g., Alcyonizm.

(2.) Fused in an external tube, e.g., Tubipora (Organ pipe coral).

(3.) Fused in an axis, e.g., Corallizum nubrum (Red coral).

(4.) Invading the outer wall (theca), the base, and forming calcareous septa between the mesenteries, and often, also, a central pillar (columella), e.g., massive reef building corals.

The terms Sclerodermic and Sclerobasic were formerly much used in the description of corals. The former denoted corals in which the hard parts are laid down by the individual polypes themselves, and support their soft tissues, as in Tubipora, Fungia, and numerous others; the latter was used in describing cases, like the Red Coral, the Sea Pens, \&c. where there is a calcareous skeleton in the connecting substance of the colony.

According to position of the hard parts-

(I.) "Exoskeletal," more or less directly continuous with the ectoderm, e.g., in Madrepore corals (reef builders), like Astrac, Fungia, Madrepora; in Gorgonids, Gorgonia and Isis.

(2.) "Mesoskeletal," i.e., in the mesogloea,

e.g., spicules of Alcyonizm, fused spicules of Tubipora, axis of Corallizm.

\section{Systematic Classification of the Celentera.}

The Colentera are often classified as follows :-
A. Hydrozoa,
$\left\{\begin{array}{l}\text { Craspedota. } \\ \text { Acraspeda. }\end{array}\right.$
$\left\{\begin{array}{l}\text { Hydromeduse. } \\ \text { Siphonophore }\end{array}\right.$
B. Actinozon,
Alcyonaria.
C. Ctenophora.
Zoantharia, 
The complex structure of the Acraspeda, or true jelly fishes, together with the special points already noticed, seems, however, to justify their association with the sea anemones rather than with the simpler Craspedote forms. The classes are then arranged thus:-
A. Class Hydrozoa, $\left\{\begin{array}{l}\text { Order I. Hydromeduse, } \\ \text { Order 2. Siphonophoræ. }\end{array}\right.$
B. Class Scyphozoa.

C. Class Ctenophora.

\section{A. Class Hydrozoa.}

There are two types, polypoid and medusoid, which may be combined in one life history. The mouth leads directly into the gastric cavity. The mesogloea is simple, and without migrant cells. The reproductive cells seem to be usually ectodermic.

I. Order Hydromedusæ.-Simple or colonial forms in which the sexually reproductive persons are either liberated as free swimming medusoids, or are sessile gonophores.

(a) IIydrophora.-Two types are included here. The first includes the Tubularians, Hydractinia, and other forms in which the polypes are not enclosed in the protective sheath which often surrounds the colony (gymnoblastic), and in which the free medusoid forms, when present, have their genital organs placed in the wall of the manubrium (Anthomedus $x$ ), and are furnished with ocelli placed at the base of the tentacles. Hydra and its allies may be included here.

Examples :-

Syncorynze sarsiz, the free medusoid of which is called Sarsia tubulosa.

Bougainvillea ramosa liberates the medusoid Margelis ramosa. Cordylophora lacustris and Tubularia larynx have sessile gonophores.

The second type includes Campanularians, Sertularians, Plumularians, and others, in which the protective sheath surrounding the colony is continued into little cups enclosing the polypes (calyptoblastic). The free medusoids have their gonads placed in the course of the radial canals (Leptomedusa), and are either "ocellate" or "vesiculate." 
Examples:-

Plumularia and Sertularia have sessile gonophores.

Campanularia geniculata liberates the medusoid Obelia geniculata.

(b) Hydrocorallinæe-Colonial forms which suggest the Hydractiniae in their polymorphism and division of labour, but are distinguished by their power of taking up lime, and so forming "corals." The colonies are complex and divergent, the medusoid persons are probably sessile gonophores, but a simple male medusoid has been described. Millepora, Stylaster.

(c) Trachymedusa.-These exist only in the medusoid form, and are divided into two groups, Trachomedusa and Narcomedusæ, according to the position of the gonads.

Geryonia, Carmanina, Cunina, Aeginopsis.

2. Order Siphonophore.-Free swimming colonies of modified medusoid persons (medusomes), with much division of labour. Physalia (Portugese Man-of-War), Diphyes, Velella, Porpita.

\section{B. Class ScyphozoA.}

There are two types-polypoic and medusoid-very rarely occurring in one life history. The gastric cavity has partitions with gastric or mesenteric filaments, and there is an ectodermic gullet. The mesogloea generally contains migrant cells. The reproductive cells are endodermic.

I. Sub-class Scyphomeduse, or Acraspeda-

Jelly fish with gastric filaments, sub-genital pits, and no velum-

(I.) Lucernarize, - Sessile forms. Lucemaria.

(2.) Discomeduse.-Active forms, often with complicated life history, Aurelia, $P \varepsilon$ lagia, Cyanea, Khisostoma.

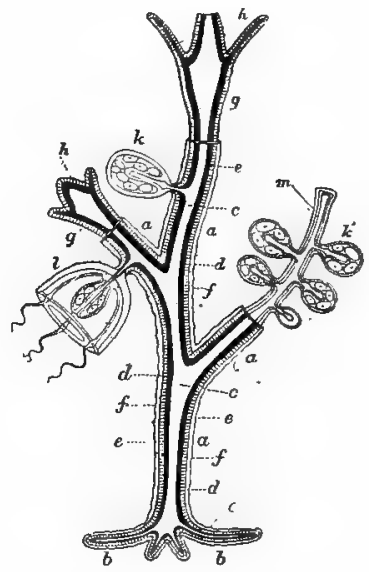

FIG. 49.-Diagram of a gymnoblastic Hydromedusæ. (After AllmaN.)

$a$, Stem ; $b$, root; $c$, gut cavity; $d$, endoderm (dark); $c_{2}$ ectoderm; $f$, horny perisare; $g$, hydra like "person" (hydranth); $\mathrm{g}^{1}$, the same, contracted; $h$, hypostome bearing mouth; $k$, sac like reproductive bud (sporosac); $m$, a modified hydranth (blastostyle) bearing sporosacs; $l_{3}$ medusoid "person."

(3.) Conomeduse.-Forms with broad pseudu-velum, and other peculiar features. Chaybulea.

(4.) Peromedusa. - Forms with four tentaculocysts only. Pericolpa. 
II. Sub-class Anthozoa, or Actinozoa-

Polypoid forms with well developed gullet and septa, and circumoral tentacles.

(I.) Zoantharia or Hexacoralla.

(a) Actiniaria. Sea anemones. Actinia, Anemonia, Tealia, Cerianthus.

(b) Madreporaria. Stone or reef corals. Astrea, Madrepora, Funoia, Mreandrina.

(c) Antipatharia. "Horny" black corals, with an axial skeleton, and occasional dimorphism between nutritive and reproductive "persons," Antipathes.

(2.) Alcyonaria, or Octocoralla.

Alcyonizm (Dead men's fingers), Tubipora (Organ pipe coral), Corallium (Red coral), Gorgonia, Pennatula (Sea pen), Monoxemia (non-colonial).

The Rugosa, or Tetracoralla, include extinct, or almost entirely extinct, forms, with numerous septa in some multiple of four.

\section{Class Ctenophora.}

Delicate free swimming organisms, generally globular in form, moving by means of eight meridional rows of ciliated plates, or comb-like combinations of cilia. The stinging cells are usually modified into "adhesive cells." The mouth is at one pole, and leads into an ectodermic gullet. The gastric cavity is usually much branched. The mesenchyme is very well developed, and includes muscular and connective cells. At the aboral pole there is a sensory organ, including an "otolith," which seems of use in steering. Here, also, there are two excretory apertures. Except in Beroe and its near relatives, there are two retractile tentacles. All are hermaphrodite. The development is direct. They are pelagic, very active in habit, carnivorous in diet, and often phosphorescent. According to Lang, they have affinities with Planarian "worms," but this is very uncertain.

Examples:-

(a) With tentacles, Cydippe and the ribbon shaped Venus' Girdle (Cestum V'eneris).

(b) Without tentacles, Beroe.

History.-Of corals, as we would expect, the rocks preserve a faithful record, and we know, for instance, that in the older (Palrozoic) strata, they were represented by a distinct series (Rugosa or Tetracoralla), of which we have at most two or three survivors. We often talk of the imperfection of the geological record, and rightly, for much of the library has been burned, nany of the volumes are torn, whole chapters are wanting, and many pages are blurred. But this imperfect record sometimes surprises us, as in the quite distinct remains of ancient jelly fish, which animals, as we know them now, are apparently little more than animated sea water. IVe should also grasp the conception, with which Lyell first impressed the world, of the uniformity of natural processes throughout the long history of the earth. Thus in connection with Colentera we learn that there were great coral reefs in the incalcul- 
ably distant past, just as there are coral reefs still. So in the Cambrian rocks, which are next to the oldest, there are on sandy slabs markings exactly like those which are now left for a few hours, when a large jelly fish stranded on the flat beach slowly melts away. On the other hand, some forms of life which lived long ago, seem to have been very clifferent from any that now remain, witness, for example, the very abundant Graptolite fossils, which, though probably Coelentera, do not fit well into any of our modern classes.

Pedigree.-As to the pedigree of the Coelentera, the facts of individual life history, and the scientific imagination of naturalists, help us to construct a genealogical tree-a hypothetical statement of the case. Thus it seems very likely that the ancestral many celled animals-ancestral to Sponges, Ccelentera, and all the rest-were small two layered tubular or oval forms. The many celled animals must have begun as clusters of cells; the question is, what sort of clusters-spheres of one layer of cells, or mouthless ovals, or little discs of cells, or two layered thimble-like sacs? Possibly there were many forms, but Hæckel and other naturalists were led to fix their attention especially on the two layered sac or gastrula, because this form keeps continually cropping up as an embryonic stage in the life history of animals, whether sponge or coral, earthworm or starfish, mollusc or even vertebrate, and also because this is virtually the form which is exhibited by the simplest.sponges (Ascones), the simplest Coelentera (Hydra), and even by the simplest "worms" (Turbellarians).

If we begin in our survey with such a gastrula-like ancestor, the probabilities are certainly in favour of the supposition that it was a free swimming organism. A gradual perfecting of the locomotor characteristics might yield the two medusoid types of which we have already spoken. But we know that the common jelly fish Aurelia has a prolonged larval stage which is sedentary, vegetative, and prone to bud. If we suppose with W. K. Brooks that many forms, less constitutionally active than others, relapsed into this sedentary state, with postponed sexuality, and with a preponderant tendency to bud, we can understand how polypes arose, and these of two types, one nearer the jelly fish and Lucernarians and leading on to sea anemones and corals, the other nearer the swimming bell type and leading on to a terminus in Hydra. It is certainly suggestive that 
we have jelly fish wholly free (Pelagia), jelly fish with a sedentary larval life (Aurelia), jelly fish predominantly passive (Lucernaria), and related polypes (Sea anemones, \&c.), which only occasionally rise into free activity; while in the other series we have medusoid types always free (Trachymedusæ), others which are liberated from (Campanularian and Tubularian) sedentary hydroids, other (Sertularian and Plumularian) zoophytes whose buds though often medusoidlike are not set free, and finally, Hydra, which, though it may creep on its side, or walk on its head, is predominantly a sedentary animal, without any youthful free swimming stage. It must be noticed that the most frequent larval form is the planula, so that if we regard the gastrula as the ancestral type, the life history is not here a recapitulation of the race history.

GENERAL SCHEME OF CCELNTERA.

\begin{tabular}{|c|c|c|}
\hline \multirow{2}{*}{\multicolumn{2}{|c|}{ Predominaitly Passive. }} & PredominantLy ACTIVE. \\
\hline & & $\begin{array}{c}\text { C. CTENOPHORA, e.g., Leroe, } \\
\text { Venus' Girdle. } \\
\text { (Active climax.) }\end{array}$ \\
\hline \multirow{3}{*}{$\begin{array}{l}\text { B. } \\
\text { SCYPHO- } \\
\text { 2OA. }\end{array}$} & $\begin{array}{l}\text { II. Anthozoa or Actinozoa. } \\
\text { (Zoantharia) Sea anemones } \\
\text { and related corals. } \\
\text { (Alcyonaria) Dead Men's } \\
\text { Fingers and related } \\
\text { corals. }\end{array}$ & $\begin{array}{l}\text { The embryos are free swimmers, and a } \\
\text { few adults also are locomotor. }\end{array}$ \\
\hline & $\begin{array}{l}\text { I. }\left\{\begin{array}{l}\text { Scyphomedusa or } \\
\text { Acraspeda. }\end{array}\right. \\
\text { c. Adult Lucernarians } \\
\text { usually attached. } \\
\text { b. Sedentary larval stage. } \\
\text { a. No fixed stage. }\end{array}$ & $\begin{array}{l}\text { c. Free embryos. } \\
\text { b. Aurelia type of jelly fish. } \\
a . \text { Pelagia type of jelly fish. }\end{array}$ \\
\hline & ANCESTRAL & GASTR止A. \\
\hline $\begin{array}{l}Y \\
\text { A. } \\
\text { HYDO- } \\
\text { ZOA. }\end{array}$ & $\begin{array}{l}\text { I. No fixed stage. } \\
\text { 2. No fixed stage. } \\
\text { 3. Many Hydroid colonies. } \\
\text { (Campanularians and } \\
\text { Tubularians.) } \\
\text { 4. Many Hydroid colonies, } \\
\text { whose reproductive per- } \\
\text { sons are not liberated. } \\
\text { 5. Coralline Millepores. }\end{array}$ & $\begin{array}{l}\text { 1. Trachymedusa (always locomotor). } \\
\text { 2. Siphonophora (locomotor colonies of } \\
\text { modified medusoids). " } \\
\text { 3. Liberated reproductive "persons" of } \\
\text { these colonies. } \\
\text { 4. No free stage, except as embryos. } \\
\text { 5. No known free stage. } \\
\text { ocomotor stage. }\end{array}$ \\
\hline
\end{tabular}


Bionomics.-The Ccelentera are almost all marine. In fresh water we find the common Hydra, the minute Microhydra without tentacles, the strange Polypodium, which in early life is parasitic on sturgeons' eggs, the compound Cordylophora, occurring in canals and in brackish water, and the fresh water Medusoid (Limnocodium) found in a tank in the Regent's Park Botanic Gardens, and another similar form recently discovered in Africa. Most of the active swimmers are pelagic, but there are also a few active forms in deep water. Many polypes anchor upon the shells of other animals which they sometimes mask, and there are most interesting constant partnerships between hermit crabs and sea anemones, e.g., Bernhardus prideauxii and Adamsia palliata.

The hermit crab is masked by the sea anemone, and may be protected by its stinging powers; the sea anemone is carried about by the hermit crab and may get crumbs from its abundantly supplied table. This illustrates a mutually beneficial partnership or commensalism, which, however, in some other animals, may degenerate into parasitism. 


\section{CHAPTER X.}

\section{UNSEGMENTED "WORMS."}

\section{Chief Classes.}

I. Turbellaria |Plathelminthes

2. Trematoda

3. Cestoda or

4. Nematoda.

5. Nemertea.

THE title "worms" is hardly justifiable except as a convenient name for a shape. For there is no class of worms, the animals to which the name is applied forming a heterogeneous mob, a collection of classes whose relationships are imperfectly discerned.

But the zoological interest of the diverse types, sometimes called "worms," is great. For amid the diversity we discern affinities with Cœlentera, Echinoderms, Arthropods, Molluscs, and Vertebrates.

Moreover, it is likely, as has been already noted, that certain "worms" were the first definitely to abandon the more primitive radial symmetry, to begin moving with one part of the body always in front, to acquire head and sides. And if one end of the body constantly experienced the first impressions of external objects, it seems plausible that sensitive and nervous cells would be most developed in that much stimulated, over-educated, region. But a brain arises from the insinking of ectodermic cells, and its beginning in the cerebral ganglion of the simplest "worms" is thus in part explained.

Again it may be noted that with worm types begins the series of triploblastic cœlomate animals, i.e., of those which 
have a well-defined mesoderm, and a mesoderm lined internal cavity distinct from the gut. But the appearance of a well-developed coelome is very gradual.

It is not at present possible to have much confidence in preferring one arrangement of the many classes of "worms" to another, but it seems useful to separate the segmented Annelids from the unsegmented types.

\section{Class Turbellaria. Planarians, \&c.}

Turbellarians are unsegmented "worms," living in fresh, brackish, or salt water. They represent the beginning of definite bilateral symmetry.

The ectoderm is ciliated, and contains peculiar rod-like bodies (rhabdites), and occasionally stinging cells. A pair of ganglia in the head region give off lateral nerve cords, and there are usually simple sense organs. The food canal has a muscular pharynx, is often branched, and is always blind. In diet the Turbellarians are carnivorous. There are no special respiratory or circulatory organs; the body cavity is represented at most by small spaces; the excretory system usually consists of two longitudinal canals whose branches end internally in ciliated (flane) cells. Excepting two genera, the Turbellarians are hermaphrodite, and the reproductive organs usually show some division of labour, e.g., in the development of a yolk gland, which seems to have arisen as an over-nourished (hypertrophied) part of the ovary.

\section{Classification.}

A. Rhabdoccelida. - Small fresh water and marine forms. The body tends to be cylindrical. The food canal is very slightly branched or quite straight or absent.

(I) Acœla. Degenerate forms without intestine, e.g., Conzoluta, which contains green cells, regarded by some as symbiotic Algre.

(2) Rhabdocoela." With straight intestine, e.g., Vortex; Microstoma, a unisexual fresh water genus, with stinging cells, forming temporarily united asexual chains, sometimes of sixteen individuals, suggesting the origin of a segmented type; Graffilla and Anoplodinm, parasitic (cf. next class).

(3) Alloioccela. With Iobed or irregular gut. All marine except one from Swiss lakes (Plagiostoma Lemani). 
B. Dendrocclida. Larger, flatter forms with branched intestine.

(I) Tricladicla. Elongated flat "Planarians"; the mouth and tubular pharynx lie behind the middle of the body; intestine with three main branches, themselves branched; two ovaries, numerous yolk glands and testes, a common genital aperture, e.g., Planaria and Dendrocalum (in fresh water), the former sometimes divides transversely; Gunda segmentata (marine) showing hints of internal segmentation; Geodesmus and Bipalizm (in damp earth).

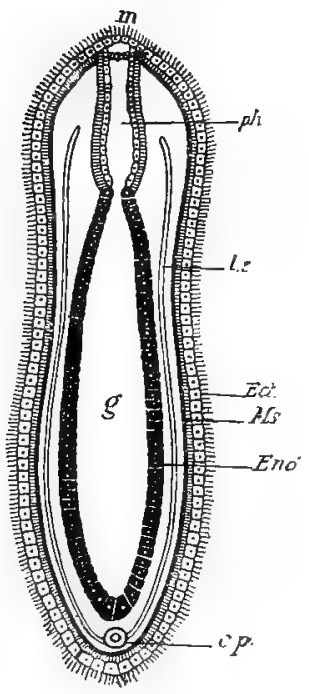

FIG. 50.--Diagrammatic figure of a simple Turbellarian.

$m$, Mouth; phe, pharynx; $g$, digestive part of gut; l.e, longitudinal excretory vessels; $e . p$, excretory pore; $E c t$, ciliated Ectoderm: $M S$, mesoderm; End, endoderm.

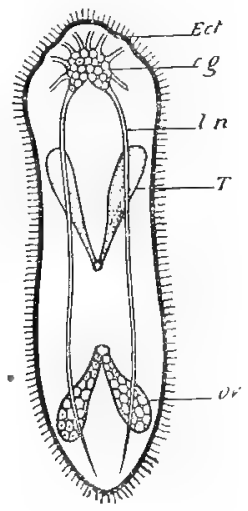

FIG. 5I.-Diagrammatic expression of part of the structure of a simple Turbellarian.

Ect, Ciliated ectoderm; c.g, cerebra ganglion; $l . n$, lateral nerve; $T$, tester; ow, ovary.

(2) Polycladida. Large leaf-like marine "Planarians," with numerous intestinal branches diverging from a central stomach; with numerous ovaries and testes, without yolk glands, mostly with two genital apertures.

e.g., Cycloporus (showing beginning of anus). Leptoplana, Thysanozoon. 
Relationships. -Two remarkable forms Caloplana (Kowalewsky) and Ctenoplana (Korotneff) seem in some ways intermerliate between Turbellarians and Ctenophora. Thus they have an aboral sense organ, and retractile branched tentacles; the branching of the food canal is slightly suggestive of that in Ctenophora; and Ctenoplana has eight dorsal bands of ciliated combs. The resemblance has been made much of by Lang and others, but, apart from direct affinity, there are likely to be resemblances of "convergence" (see p. 33) between forms not far removed from a common stock-that of the primitive Metazoa.

The occasional presence of a retractile proboscis and of a ciliated groove on each side of the brain is suggestive of two characteristics of Nemerteans.

The Turbellaria are also related to the next class-the Trematodes.

\section{Class Trematoda. Flukes, \&c.}

The Trematodes are leaf-like or roundish external or internal parasites. With their mode of life a'e may associute the absence of cilia on the surface of the adults, the avellformed and apparently cellular "cuticle," the presence of attaching suckers (occasionally with hooks), and the rarity of sense organs. It is likely that they have arisen from free Turbellarian-like ancestors, and they resemble the Turbellarians in being unsegmented, in having anterior nerve centres from which nerves pass backward and forward, in the nudimentary nature of the body cavity, in the ramifying system of fine excretory canals, in the hermaphrodite and usually conplex reproductive system. The alimentary canal is usually forked, often much branched, and alreays ends blindly. In many cases the animals are self-impregnating, but cross fertilisation also occurs. The development of the external parasites is usually direct, of the internal parasites usually indirect, involving alternation of generations. They occur in or on all sorts of Vertebrates, but those which have an indirect development, and require two hosts to complete their life cycle, often pass part of their life in some Invertebrate.

\section{Type, The Liver Fhtke (Fusciola (Distoma) hepatica).}

The adult fluke lives in large numbers in the liver and bile duct of the sheep. It sometimes occurs in cattle, horses, and other domestic animals, and rarely in man. 
In the sheep it causes the serious disease called liver rot. The animal is flat, oval, and leaf-like, measures about an

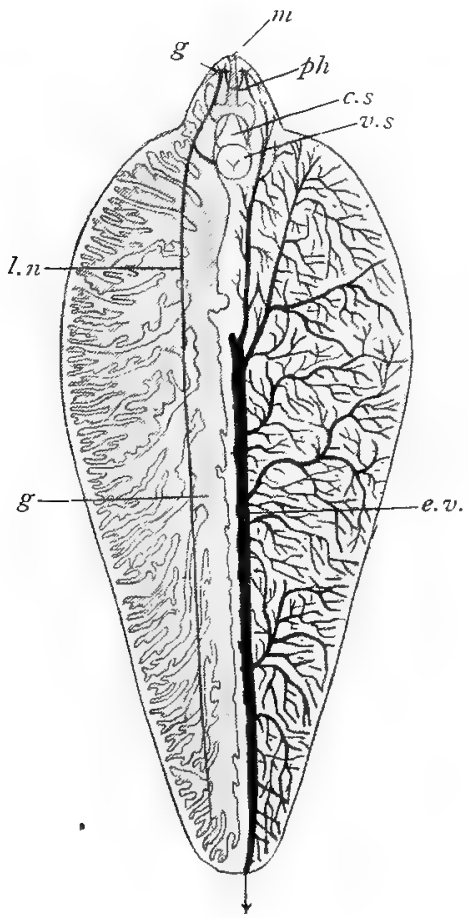

FIG. 52.-Structure of Liver Fluke. (After Sommer.) From ventral surface. The branched gut $\left(g^{n} n\right.$.) and the lateral nerve (l.n.) are shown to the left, the branches of the excretory vessel $(e . v$. $)$ to the right.

$m$, Mouth; ph, pharynx; $g$, lateral head ganglion; $v, s$, ventral sucker; c.s, position of cirrus sac; an arrow indicates the excretory aperture.

inch in length by half an inch across the broadest part, varies from reddish brown to grayish yellow in colour. As the word Distoma suggests, there are two suckers, -an 
anterior, perforated by the mouth; a second, imperforate a little further back on the mid ventral line.

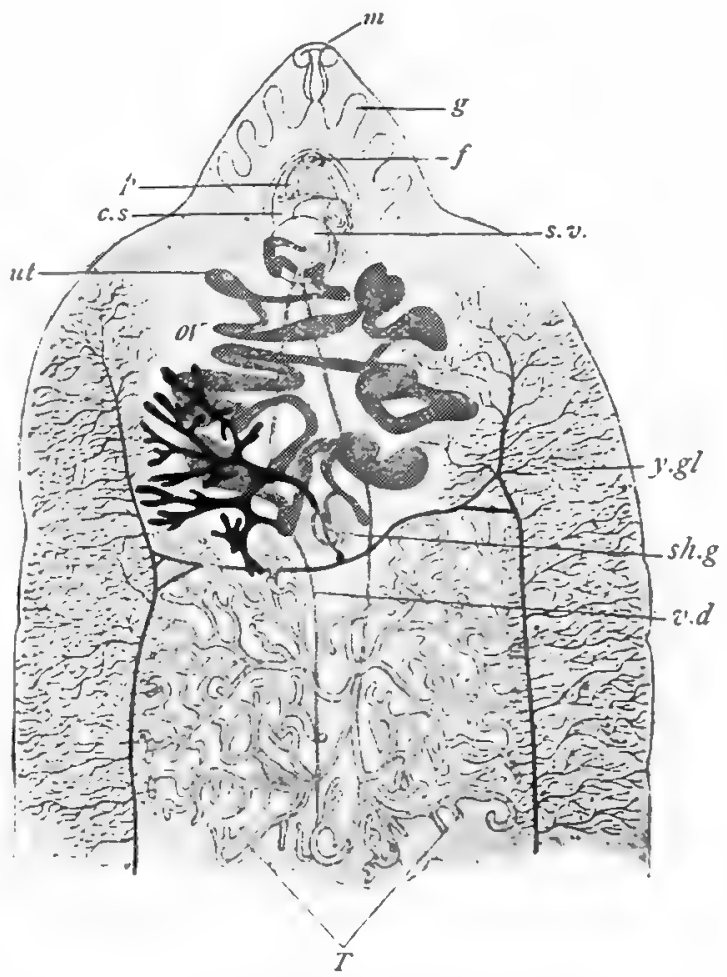

Fit: 53.-Reproductive Organs of Liver Fluke. (After SOMMER).

f. Female aperture.

s.v. Seninal vesicle.

y. $\%$ l. Diffuse yolk glands.

shig. Shell gland.

ir.d. Vas deferens.

T. Testes (anterior). ov. Ovary (dark).

ut. Uterus.

c.s. Cirrus sac.

p. Penis.

m. Mouth.

g. Anterior lobes of gut. 
which sends branches throughout the body. The nervous system consists of a ganglionated collar round the pharynx, from which nerves go forward and backward; of these, the two which run laterally are most important. Although the larva has eye spots to start with, there are no sense organs in the adult. 'The body cavity is represented only by a few small spaces. Into these there open the ciliated ends of much branched excretory tubes, which unite posteriorly, and communicate with the exterior by a terminal pore. The reproductive system is hermaphrodite and complex. From much branched testes, spermatozon pass by a pair of ducts (vasa deferentia) into a seminal vesicle lying in front of the ventral sucker. Thence they are expelled by an ejaculatory duct, which passes through a muscular protrusible penis. The retracted penis and the seminal vesicle lie in a space or "cirrus sac" between the ventral sucker and the external male genital aperture. The ovary is also branched, but less so than the testes. From its tubes ova are collected into an ovarian duct. Nutritive cells are gathered from very diffuse yolk glands, collected in a reservoir, and pass by a duct into the end of the aforesaid ovarian duct. At the junction of the yolk duct and the ovarian duct there is a shell gland, which secretes the "horny" shells of the eggs, and from near the junction, a fine canal (the Laurer-Stieda canal) seems to pass direct to the exterior, opening on the dorsal surface. The meaning of this is still somewhat uncertain. In some cases it is said to be a copulatory duct; in others it is regarded as a safety valve for overflowing products. From the junction of the ovarian duct and the duct from the yolk reservoir, the eggs (now furnished with yolk cells, accompanied by spermatozoa, and encased in shells) pass into a wide convoluted median tube, the oviduct or uterus, which opens to the exterior at the base of the penis. Self fertilisation is probably normal, but in some related forms cross fertilisation has been observed.

Life History.-The fertilised and segmented eggs pass in large numbers from the bile duct of the sheep to the intestine, and thence to the exterior. A single fluke may produce towards half a million embryos, which illustrates the prolific reproduction often associated with the luxurious 

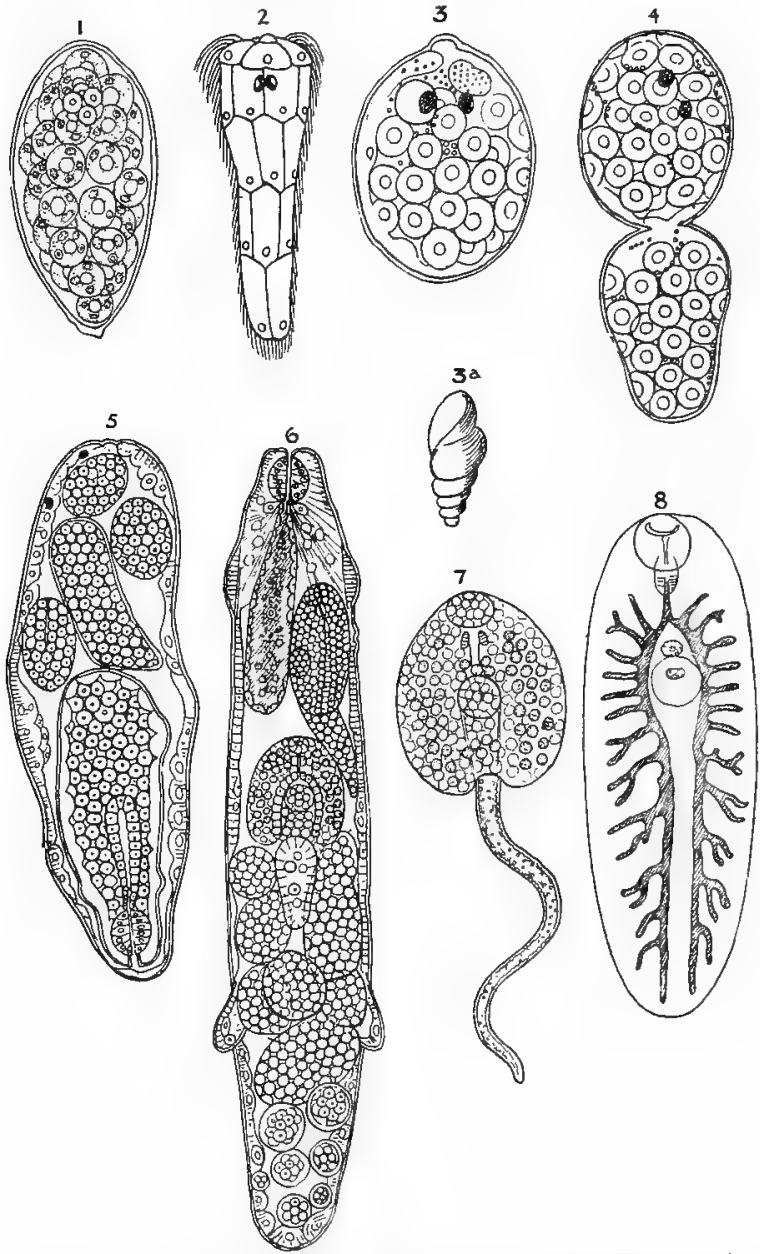

FIG. 54--Life history of Liver Fluke. (After Thomas.)

I. Developing embryo in egg case; 2. free swimming ciliated embryo; 3. sporocyst; $3 a$. Lymncea truncatula; 4 . division of sporocyst ; 5. sporocyst with redize forming within it ; 6 . redia with more redie forming within it ; 7 . tailed cercaria ; 8 . young fluke. 
conditions of parasitism, and almost essential to the continuance of species whose life cycles are full of risks. Outside of the host, but still within the egg case, the embryo develops for two or three weeks, and eventually escapes at one end of the shell. Those which are not deposited in or beside pools of water must die. The free embryo is conical in form, covered with cilia, provided with two eye spots, and actively locomotor. By means of its cilia it swims actively in the water for some hours, but its sole chance of life depends on its meeting a small amphibious water-snail (Lymncea truncatula), into which it bores its way. In an epidemic among horses and cattle in the Hawaiian Islands, the host was $L$. cahuensis (Lutz). Within the snail, e.g., in the pulmonary chamber, the embyro becomes passive, loses its cilia, increases in size, and becomes a sporocyst. Sometimes this sporocyst divides transversely (Fig. 54, 4).

Within the sporocyst certain cells behave like parthenogenetic ova. Each segments into a ball of cells or morula, which is invaginated into a gastrula, and grows into another form of larva-the redia. "These redize burst out of the sporocyst, and migrate into the liver or some other organ, killing the snail if they are very numerous. Indeed the death of the snail is probably necessary for the escape of the final larve. Each redia is a cylindrical organism with a short alimentary canal (Fig. 54, 6).

Like the sporocysts, the rediæ give rise internally to more embryos, of which some are simply rediæe over again, while the last set are quite different,-long tailed cercaria, with two suckers and a forked food canal. These emerge from the rediæ, wriggle out of the snail, pass into the water, and moor themselves to stems of damp grass. There they lose their tails and become encysted. If the encysted cercaria on the grass stem be eaten by a sheep, it grows, in about six weeks, into the adult sexual fluke.

To recapitulate, the developing embryo becomes a free swimming form, which bores into a snail, and changes into a sporocyst.

From certain cells of the sporocyst rediæ are developed, and these may similarly give rise to other redia.

Eventually, within the redize the tailed cercaria are formed, 


\section{and these in favouring circumstances grow into the adult flukes.}

The above history has been indepenclently worked out by Leuckart and Thomas.

It will be noted that the sporocyst is the modified emuryo, but that it has the power of giving rise asexually to redir. These develop, however, from special cells of the sporocyst which we may compare to precociously developed parthenogenetic ova. Though the reproduction is asexual, it is not comparable to budding or division. The same power is possessed by the rediæe, and there are thus several (at least two) asexual generations between the embryo and the adult. Finally, it must be clearly understood that the cercaria is the young fluke.

The disease of liver rot in sheep is common and disastrous. It has been known to destroy a million sheep in one year in Britain alone, and in the winter, $1879-80$, the mortality attributed to fluke disease was estimated at three millions. It is especially common after wet seasons, and in damp districts. The preventives suggested are drainage of pastures and dressings of lime and salt; destruction of the eggs, the snails, infected manure, and diseased sheep. It is usual to give the infected sheep some salt and a little dry food.

\section{Classification.}

Trematodes with direct development-Monogenetic.

e.g., Polystonum integerrimum. This form with many suckers is often found in the blarlder of the frog. It attaches itself in its youth to the gills of tadpoles, passes thence through the food canal to the bladder, where it develops slowly for years.

Gyrodactylus, found on the gills and fins of fresh-water fishes. It is viviparous, but the embryo, before it is extruded, itself contains an embryo, and this in turn another, so that three generations of embryos are represented simultaneously.

Diplozoon paradoxum, consists of two individuals united. The single embryo (Diporpa) is at first free swimming, but becomes a parasite on the gills of a minnow, and there two individuals unite very closely and permanently.

Tristomum, with three suckers, is not uncommon on the skin of some marine fishes.

Trematodes with indirect development-Digenetic.

e.g., Fasciola or Distoma.

Bilharzia, or Gynacophorus hamatobizs, a dangerous parasite of man, widely distributed in Africa. It infests the urinary and visceral blood vessels. The sexes are separate, and the male carries the female inserted in a groove.

Mlonostomum, a form with one sucker.

The relationships of the class are on the one hand with the free living Turbellarians, on the other hand with the parasitic Cestorles. 


\section{Class Ceston.1. 'Tapeworms.}

The Cestodes are internal parasites, zehose life history includes a bladderworm (proscolex) and a tapezworm (strobila) stage, the former in a Vertebrate or Invertebrate host, the latter (with one exception) in the gut of a Vertebrate. In a few cases the body is unsegmented, e.g., Archigetes and Caryophyllæus, with one set of gonad's; in a ferv cases, e.g., Ligula, there is a serial repetition of gonads without distinct segmentation of the body; in most cases, e.g., Tænia and Bothriocephalus, the body of the tapezvorm forms a chain of numerous joints or proglottides, each with a set of gonads. Thus the class includes transitions from unsegmented to segmented forms, but the latter are imperfectly integrated. The general form of the body is tape-like and bilaterally svmmetrical, arith hooks, grooves, or suckers ensuring attuchment to the gut of the host. The nervous system consists of longitudinal nerve strands and anterior ganglionated commissures; there are no special sense organs. There is no alimentary sy'stem; the parasite floating in the digested food of its host absorbs soluble material by its general surface. There is no vascular nor respiratory system, and the body cavity is represented merely by irregular spaces. Into some of these spaces there open ciliated funnets, the ends of the fine branches of longitudinal excretory tubes, which are connected transversely at each joint and open terminally by one or more pores. All tapeworms are hermathrodite, and most, if not all, are probably self-fertilising. The male reproductive organs include diffuse testes, a vas deferens, and a protrusible terminal cirrus. The female organs include a pair of ozaries, yolk glands, a shell gland, a vagina by which spermatozoa enter, a receptacle for storing spermatosou, and a uterus in which the ova develop. The embry'o develops within another host into a proscolex or bladderworm stage, which forms a "head" or scolex. When the host of the bladderworm is eaten by the final host, the scolex develops into an adult sexual tapererorm. With the conditions of endoparasitic life, we may' associate the occurrence of fixing organs, the absence of sense organs, the low though somewhat complex character of the nervous system, the entive absence of a food canal, and the prolific reproduction.

Life History of Tania solium.-This is one of the most 
frequent of the tapeworms infesting man. In its adult state it is often many feet in length, and is attached by its "head" to the wall of the intestine. The head bears four suckers and a crown of hooks, and buds off a long chain of joints, which develop complex reproductive organs as they get

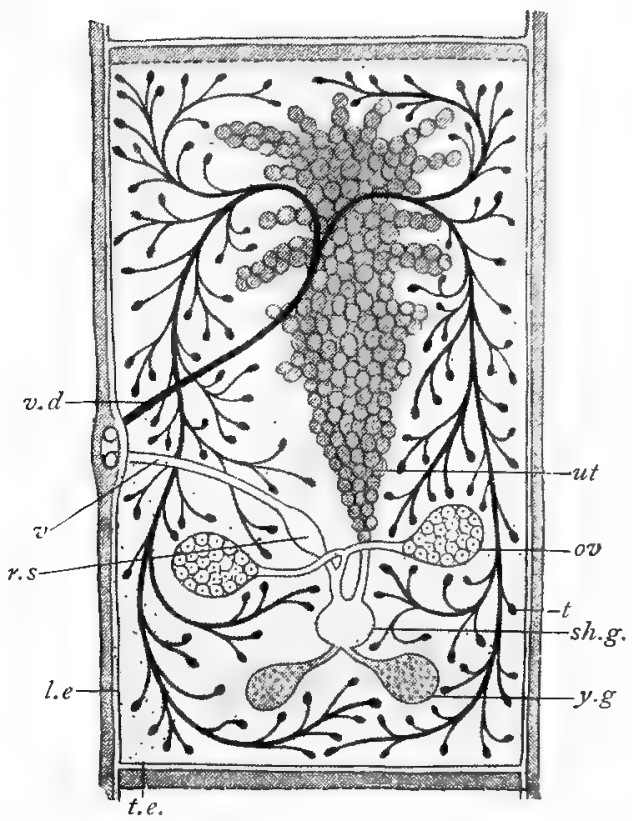

FIG. 55.-Diagram of reproductive organs in Cestode joint. (Constructed from LEUCKART.)

\footnotetext{
ov., Ovary, with short oviduct; $a t$., uterus; $t$., diffuse testes ; sh.g., shell gland; $y . g$., yolk gland; $z \cdot . t$., vas deferens; $\%$. vagina; $r . s .$, receptaculum seninis; l.e., longitudinal excretory duct $5 ; t . e .$, transverse bridges connecting these.

The dotted lines above and below represent the anterior and posterior borders of the proglottis.
}

shunted further and further from the head. The last of the joints or proglottides, is liberated (singly or along with 
others) and passes down the intestine of its host to the exterior. It has some power of muscular contraction, and is distended with little embryos within firm egg shells. When the proglottis ruptures, these egg cases are set free.

In certain circumstances, the embryos, within their firmly resistent egg shells, may. be swallowed by the omnivorous pig. Within the alimentary canal of this animal the egg shells are dissolved, and the embryos bearing six anterior hooks are liberated. They bore their way from the intestine into the muscles or other structures, and there encyst. They increase in size and become passive, vegetative, asexuall "bladderworms." A bud from the wall of the bladder or proscolex grows into the cavity of the same, and forms the future "head" or scolex. It is afterwards everted, and then the bladderworm consists of a small head attached by a short neck to a relatively large bladder. But this remains quiescent, and without power of further development, unless the pig be eaten by some other Vertebrate.

When man unwittingly eats "measly" pork, that is pork infested with bladderworms, an opportunity for further development is afforded. The bladder is lost, and is of no importance, but the "head" or scolex fixes itself to the wall of the intestine. There it is copiously and richly nourished, and buds off asexually a chain of joints.

As these joints are pushed by younger interpolated buds further and further from the head, they become sexually mature, developing complex hermaphrodite reproductive organs. The ova produced in these are fertilised, apparently by spermatozoa from the same joints; the proglottis becomes distended with ripe eggs and developing embryos. These ripe joints are liberated, and the vicious circle may recommence. Happily, however, the chances are thirtyfive millions to one against the embryo becoming an adult.

The above history is true mutatis mutandis for many other tapeworms. It will be observed that the embryo grows into a proscolex or bladder, which burls off a scolex or head, which, in another host. buxds off the chain of proglottides, but as it is virtually the same animal throughout, the life history does not include an " alternation of generations." It is doubtful, however, what term should be applied to those cases in which the bladderworm (Co'nutrus and Echinococtus) forms not one head 
only but many, each of which is capable of becoming an adult tapeworm. The only known exception to the fact that sexual tapeworms are parasites

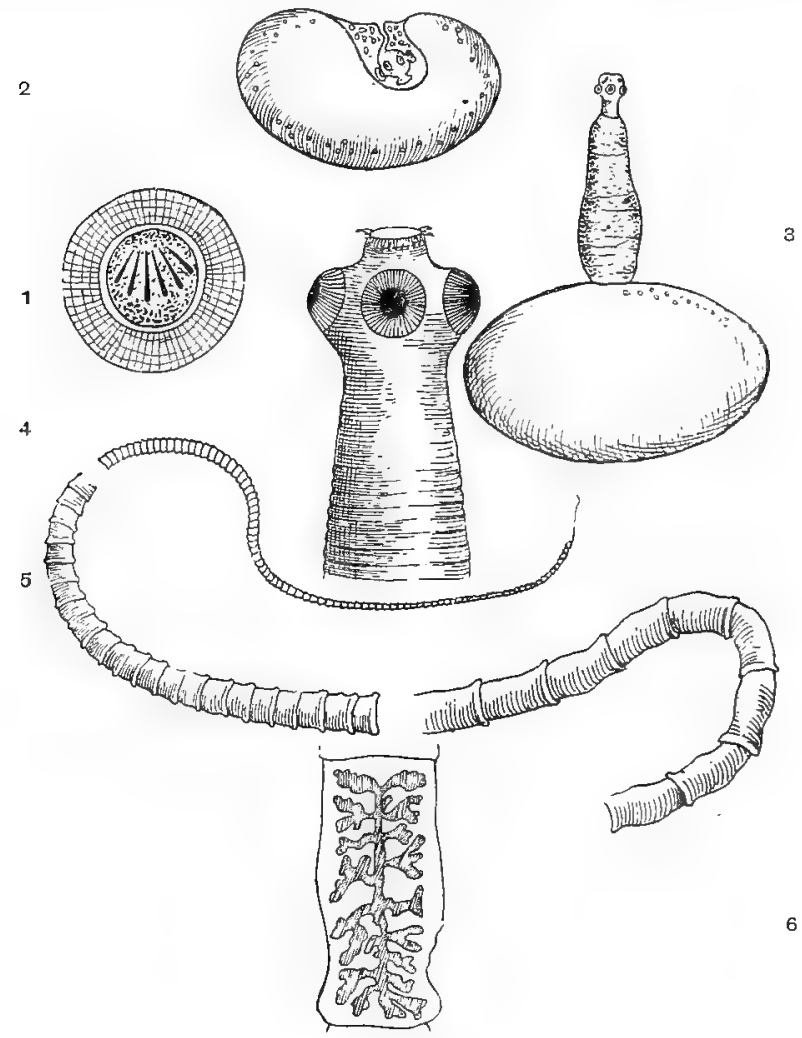

FIG. 56.-Life history of Tamia solium. (After LeUCkART.)

I. Six-hooked embryo in egg case; 2. proscolex or bladderworm stage with invaginate head; 3. bladderworm with evaginated head ; 4. enlarged head of adult, showing suckers and hooks; 5 . general view of the tapeworm from small head, and thin neck to the ripe joints ; 6. a ripe joint or proglottis with uterus.

of Vertebrates, is Arhigetes sicboldii, a simple cestode which is sexual within the small fresh water worm Tubifex rivulorum. 


\section{Life Histories.}

Adult, Sexual, or Tapeworm STAGE.

I. Tania solizm, in man, with four suckers and many hooks.

2. Tenia saginata or nediocanellata, in man, with four suckers, but no hooks.

3. Bothriocephalus latus, in man, with two lateral suckers, but with no hooks, with less distinct separation of the proglottides than in Tania.

4. Tenia echinococcus, in dog.

5. Tania conurus, in dog.

6. Taria serrata, in dog.

7. Tania cucumerina, in cat.

8. Tenia elliptica, in dog.
Non-Sexual, Proscolex, or BladdekWORM STAGE.

I. Cysticercus cellulosa, in muscles of the pig.

2. Bladderworm in cattle.

3. The ciliated, free swimming embryo becomes a parasite in the pike or burbot, but without a distinct bladder-like stage.

4. Echinococcus veterinorum, in domestic animals, and sometimes in man, producing brood capsules, which give rise to many "heads."

5. Comurus cirebralis, causing sturdie in sheep, producing numerous "heads."

6. Cysticercus pisiformis, in rabbit.

7. Cysticercus fasciolaris, in mouse.

8. Cysticercus in dog louse or perhaps in flea.

The Cestodes are closely connected with Trematodes by such forms as Amphilina, Caryophylleus, Archigetes. Zoologically, they are interesting, on account of their life histories, the degeneration associated with their parasitism, the prevalence of self-impregnation, and the complexity of the reproductive organs. Practically, they are of importance as parasites of man and domestic animals. The medical student should consult Leuckart's great work, The Parasites of MIan, part of which has been translated by W. E. Hoyle (Edin. I886).

\section{Class Nimertea. Nemertines.}

The Nemertines are zorm-like animals, unsegmented, and generally elongate in form. Almost all are marine; most, if not all, are carnivorous. Among their characteristics, the following are most noteworthy :- The skin is ciliated: there is a remarkable retractile proboscis; the head bears a pair of ciliated pits; the nervous system consists of a brain, a commissure around the proboscis, and two lateral nerve cords; there is a colonic vascular system, a pair of anterior nephridia, and a simple reproductive system. The sexes are usually separate. In some the development includes a peculiar pelagic larval stage; in others there is no metamorphosis.

\section{External Appearance.}

Some are ribbon-like, others thread-like, and the cross section is generally a flattened cylinder. They vary greatly 
in size, from a Lineus, $r 2$ feet long, to the small pelagic Pelagonemertes, which is under an inch. There are no appendages. 'The colours are often bright.

\section{Skin.}

The ectoderm is covered with numerous short cilia, and many of its cells are also glandular, secreting mucus which often forms a tube around the animal, or is exuded in movement. Some of the glandular cells extend into the subjacent cutis, which consists in part of connective tissue.

\section{Muscular System.}

The Nemertines are remarkably contractile, and in some cases the spasms result in the breakage of the body. The muscles are circular and longitudinal, but their arrangement is variable even in individuals.

\section{Body Cavity.}

In the adult there is no distinct coelome, the space between the gut and the body wall being filled up with connective tissue. In the larvæ, however, a body cavity may be seen, either as an archicoele, i.e., persistent segmentation cavity (Lineus obscurus), or as a scbizoccele, i.e., a space formed by the cleavage of the mesoderm into two layers (Pilidium-larvæ). In the adult, however, only the blood spaces and the cavity of the proboscis sheath are colomic.

\section{Nervous System.}

In the head there is a brain, generally four lobed, with a commissural ring surrounding the proboscis and its sheath ; from the lower brain lobes two longitudinal nerve stems run along the sides, and are sometimes united posteriorly above the anus (Fig. 57, ln).

Hubrecht suggests that the nerve stems and the brain may "be looked upon as local accumulations of nervous tissue in what was in more primitive ancestors a less highly differentiated nervous plexus, situated in the body wall," as in many Celentera. In some cases (Schizonemertea) this nerve plexus persists, and then the longitudinal stems do not give off regular peripheral branches as is the case in another sub-class (Hoplonemertea) where there is no definite plexus.

It is interesting to find that in Drepanophorus the lateral nerve stems are approximated ventrally, and in Langia, dorsally; for these two 
approximations tend towards the two positions most characteristic of the nervous systems of Annelids and Arthropods on the one hand, and of Vertebrates on the other.

\section{Lateral Organs.}

On each side of the head there is a ciliated pit communicating with the exterior through an open slit or groove, and communicating internally either with the brain itself, or with adjacent and associated nervous tissue. In those cases in which the development has been studied these so-called

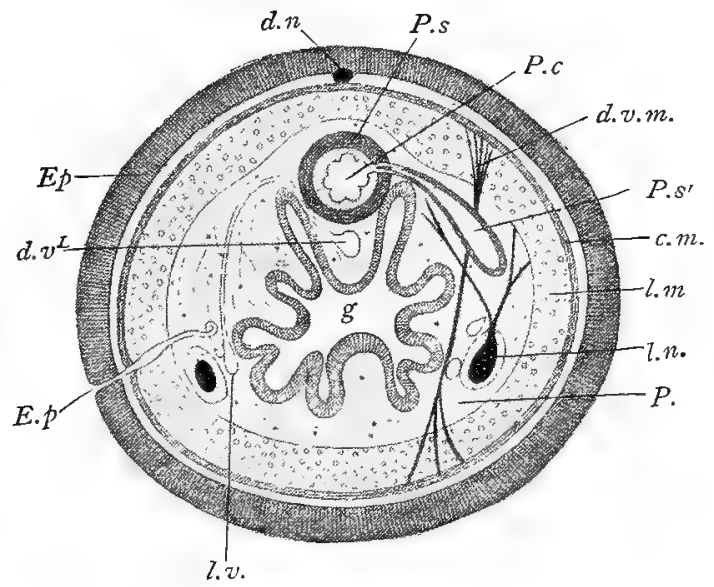

F1G. 57.-Transverse section of the Nemertean Drepanophorus latus. (After Bürger.)

$d^{\prime} \cdot n$., Dorsal nerve; $P . s .$, proboscis sheath ; $P . c .$, proboscis cavity ; P.s., sac of proboscis cavity; $d . z^{\prime} . m$., dorso-ventral muscles; c.mi., circular muscles; $l . m$. ., longitudinal muscles; $l . n$., lateral nerve with

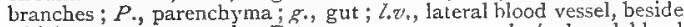
which excretory vessel; $E . p_{n}$, excretory pore; $d_{. \nu} ;$, dorsal blood vessel ; $E$ p., epidermis.

lateral organs arise from epiblastic insinkings and osophageal outgrowths. In the most primitive genus, Carinella, they are absent, except in one species. It has been suggested that they conduce to the respiration of the brain, which is rich in hæmoglobin, and they have even been compared with gill slits. In some forms the groove through which they 
open to the exterior is rhythmically contractile. It has also been suggested that they are sensory.

\section{Sense Organs.}

Nemertines are very sensitive, and in many this is to be associated with the superficial nerve plexus already mentioned. Tactile papillæ and hairs are also present in some. Eyes and eye spots are of general occurrence, and in some cases otocyst sacs have been observed.

\section{Alimentary System.}

A ventral mouth leads into a plaited cesophagus, which is followed by an intestine with regularly arranged lateral cæca.

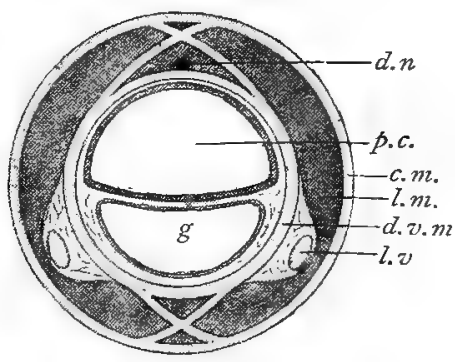

Fig. 58. -Transverse section of a Nemertean-Carinella. (After BüRGER.)

d.n., Dorsal nerve; p.c., proboscis cavity; $g_{*}$, gut; c.m., circular muscles ; l.m., longitudinal muscles; $d . v . m$. , dorso-ventral muscles; i.t., lateral vessel.

Between the cæca run transverse muscle partitions. The anus is terminal. In the adults of the primitive genus, Carinella, the cæca are absent, but they are present in the larva.

\section{The Proboscis.}

In a cavity along the dorsal median line there lies a remarkable organ, known as the proboscis. It is protruded and retracted through an opening above, or, in a few cases, within the mouth, but it has no connection with the ali- 
mentary system. The proboscis is a hollow muscular structure, very richly innervated, and is sometimes protruded with such force that it separates from the body, and then "often retains its vitality for a long time, apparently crawling about as if it were itself a worm" (Hubrecht). It has been compared in its retracted state to a glove finger drawn in by a thread attached to its tip, the thread being the retractor muscle. But in front of the attachment of the retractile muscle there is a non-eversible glandular region which secretes an irritant fluid. In many cases there is a stylet at the tip of the eversible portion, and if this be absent, there are stinging cells or adhesive papilla. There is a hint of a similar structure in some Turbellarians, and the organ is usually interpreted as one which was originally tactile, but which has become secondarily aggressive. It is protruded by the muscular contraction of the walls of the proboscis sheath, which forms a closed cavity containing fluid and surrounding the proboscis. (Fig. 57, P.s.)

\section{Vascular System.}

In the majority there are three longitudinal blood vessels or blood spaces, a median and two laterals, which unite anteriorly and posteriorly, and also communicate by numerous transverse vessels. The vessels or spaces are remnants of a cœlome. The blood is a colourless fluid, sometimes at least with corpuscles in which hæmoglobin may be present.

\section{Excretory System.}

In most, if not all, there are two coiled nephridia, one on each side of the cesophagus-opening anteriorly. (Fig. 57, E.p.)

\section{Reproductive System.}

The sexes are usually separate, and the reproductive organs are always simple. They consist of simple sacs, arranged in a series on each side between the intestinal creca, and communicating with the exterior by fine pores. The ova are often laid in gelatinous tubes, and are probably fertilised shortly before or at the time of extrusion. In three or four forms known to be viviparous the fertilisation must, of course, be internal. 


\section{Development.}

(x.) In Cerebratulus, etc., the larva is adapted for pelagic life, and is known as the Pitidium. "In exterual shape it resembles a helmet with spike and ear lobes, the spike being a strong and long flagellum or a tuft of long cilia, the ear lobes, lateral ciliated appendages" (Hubrecht). (2.) In Lineus there is a sedentary larva, which has been interpreted as a reduced Pilidium, and is known as the "larva of Desor." (3.) In Hoplonemertea, the development is direct without metamorphosis.

\section{Habits.}

Most Nemertines are marine, creeping about in the mud, under stones, among seaweed, and the like; many are able to swim; Pelagonemertes is pelagic; a few live in fresh water; Malacobdella lives in the mantle cavity of marine bivalves, and two others occur on crabs. Most seem to be carnivorous, eating other "worms." Many break readily into pieces when stimulated, and the Schizonemertea are able to regenerate what they lose in this way.

Classification (after Hubrecht):-

I. Palieonemertea: No deep head fissure; nostylet; mouth behind brain.

e.g., Carinella, Cephalothrix, Carinoma, Polia.

2. Schizonennertea: A deep head fissure with a ciliated duct to the brain; lateral nerves between the longitudinal and inner circular muscles; mouth behind brain. e.g., Lineus, Cerebratulus, Langia.

3. Hoplonemertea: No deep head fissures; Iateral nerves inside the muscles; stylet present ; mouth generally in front of brain.

e.g., Amphiporas, Nemertes, Drepanophorus, Malacobdella, The last has no head fissures nor spines on the prohoscis, but bears a posterior sucker.

Relationships. - Some of the characteristics of the Nemerteans are hinted at among the Turbellarians. Professor Hubrecht has maintained that Nemerteans exhibit affinities with Vertebrates. (See Chapter XX.)

Class Nematoda. Threadworms, Hairworms, \&c.

The Nematodes are unsegmented, more or less thread-like "norms," some of which are free living and others parasitic. The body is covered by a cuticle, often thick. Ciliated epithe- 
lium is altogether absent. From a nerve ring around the pharynx six nerves run forwards and six backwards. An alimentary canal, consisting of fore, mid, and hind gut, is usually developed. There is no vascular nor respiratory system, but there is usually a body cavity, and there are two excretory tubes opening ly an anterior ventral pore. The sexes are usually separate and the reproductive organs simple. The life history is often intricate.

\section{Form.}

The body is usually cylindrical in cross section and tapering at each end. The male is usually smaller than the female, and his tail, concerned in copulation, bears sensory papillæ, and usually some spines and a "bursa."

\section{Body Wall.}

(a.) Most externally there is a chitinoid, often wrinkled, cuticle, thick in the larger forms, and perhaps of service in enabling the animals to resist drought and digestive juices. With its presence may be associated the almost entire absence of cutaneous glands, and the entire absence of cilia. (b.) Beneath this is the sub-cuticula or hypodermis, usually thickened in four longitudinal lines-median dorsal, ventral, and lateral. (c.) Beneath the hypodermis is a layer of longitudinal muscles, which sometimes lie in groups defined by the above mentioned lines. Many of the Nematodes are very agile.

\section{Nervous System.}

Around the pharynx there is a nerve ring from which six nerves run forwards and six backwards. One of the latter runs along the median dorsal line - a unique position in an Invertebrate. Here and there on the ring and on the nerves there are ganglionic cells, but any aggregation of these into ganglia is rare. Some of the free living forms have eye spots; and probably all Nematodes have sensory papillie on various parts of the body.

\section{Alimentary System.}

The mouth is terminal or almost terminal; the anus is ventral and posterior, and occasionally terminal. As 
the food consists chiefly of juices either from a living host or from putrefying organic matter, it is not surprising to find that the alimentary canal has usually but a narrow cavity. In some forms, e.g., Spharularia from the bee, it degenerates altogether. Normally it consists of three parts, a fore gut or oesophagus, lined by the inturned cuticle, a mid gut or mesenteron of endodermic origin, and a usually short hind gut or rectum, lined by the cuticle. When the external cuticle is shed, so is that of the fore gut and hind gut (cf. Crayfish).

\section{Body Cavity.}

A colome is developed and contains a clear fluid, which probably discharges some of the functions of the absent blood. There are no amoboid phagocytes.

\section{Excretory System.}

Imbedded in each lateral line there is a long tube containing clear fluid, probably drained from the surrounding tissues. The two longitudinal tubes unite anteriorly, and open in a ventral excretory pore near the head.

\section{Reproductive System.}

The sexes are separate, except in Angiostomum which is hermaphrodite and selffertilising. In the male, the testis is usually unpaired, - a coiled tube gradually differentiating into vas deferens, seminal vesicles, and ejaculatory duct. The genital aperture is close to the anus, and beside it there are sensory papillæ, and often spicules, and peculiar membranous folds of varied form which constitute what is called the copulatory "bursa." The spermatozoa have not the typical form, and are sluggish.

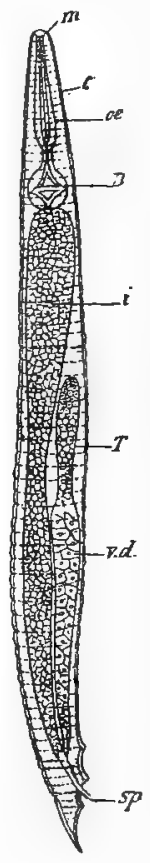

FIG. 59.-Illustrating the structure of a Nematode (Oxyuris). (After (GALEB.)

m., Mouth; c., cuticular ring; $\alpha$, , cesophagus; $B$., bulb containing teeth; $i$, intestine; $T$., testis; ". $d$., vas deferens; $s p$. penial spine at anus.

In the female, the ovary is a single or paired tube which passes grad- 
ually into an oviduct, a uterus, and a short vagina. The genital aperture is ventral, usually about the middle of the body, but it is occasionally far forward or far back.

\section{Development.}

The ova meet the spermatozoa at the junction of uterus and oviduct. Segmentation is total and may take place before or after laying. Indeed the embyro may be hatched within the uterus. Before the embryo exhibits adult characteristics, several, e.g., three, moultings of the cuticle usually occur.

\section{LifE Histories.}

I. The embryo grows directly into the adult, and both live in fresh or salt water, damp earth, and rotting plants-Enoplidre, e.g., Enoplus.

2. The larva are free in the earth, the sexual adults are parasitic in plants, or in Vertebrate animals, e.g., Tylenchus scandens, a common parasite on cereals; Strongylus and Dochmius in man.

3. The sexual adults are free, the larve are parasitic in insects, e.g., Alermis. The fertilised females of Spherularia bombi pass from the earth into the body cavity of humble-bee and wasp, whence their larve bore into the intestine and eventually emerge.

4. The larva are parasitic in one animal, the sexual aclults in another which feeds on the first. Thus Ollulanus passes from mouse to cat, Cucullanus from Cyclops to perch.

There are other life histories, and many degrees of parasitism. The most remarkable form is Angiostomum (or Ascaris, or Leptodera) nigrovenoszm. In damp earth males and females occur, the progeny of which pass into the lungs of frogs and toads. There they mature into hermaphrodite animals (the only example among Nematodes), which produce first spermatozoa and then ova. They are self-impregnating, and the young pass out into the earth as males or females. Ifere there is alternation of generations, and a somewhat similar story might be told of Rhabdonema strongrloides from the intestine of man and Leptodera appendiculata from the snail.

There are several quaint reproductive abnomalities, thus - the female Spherularia bombi, which gets into the body cavity of the humblebee, has a prolapsed uterus, larger than itself; the male of Trichodes crassicarda passes into the uterus of the female. 


\section{Parasitic in Man.}

\begin{tabular}{|c|c|c|c|}
\hline NAME. & Position. & HISTORY. & $\begin{array}{c}\text { RESULT ON } \\
\text { HOST. }\end{array}$ \\
\hline $\begin{array}{l}\text { Ascaris lunbri- } \\
\text { coides (common). }\end{array}$ & Small intestine. & $\begin{array}{l}\text { Probably enter } \\
\text { the body as larvæ, } \\
\text { along with vege- } \\
\text { table food or impure } \\
\text { water. Julus gut- } \\
\text { tulatus perbaps an } \\
\text { intermediate host. }\end{array}$ & $\begin{array}{l}\text { Rarely danger- } \\
\text { ous, but may per- } \\
\text { forate intestine, and } \\
\text { cause abscesses. }\end{array}$ \\
\hline $\begin{array}{l}\text { Oxyuris vermi- } \\
\text { cularis (common). }\end{array}$ & $\begin{array}{l}\text { From stomach to } \\
\text { rectum, mostly in } \\
\text { crecum. }\end{array}$ & $\begin{array}{l}\text { From food or } \\
\text { water. }\end{array}$ & $\begin{array}{l}\text { Rarely more than } \\
\text { discomfort. }\end{array}$ \\
\hline $\begin{array}{l}\text { Trichocephalus dis- } \\
\text { par (common). }\end{array}$ & Cæcum and colon. & $"$, & " \\
\hline $\begin{array}{l}\text { Dochmius (Anchy- } \\
\text { lostona) duadcnalis } \\
\text { (Europe, Egypt } \\
\text { Brazil). } \\
\text { Rhabdone na a } \\
\text { strongyloides. }\end{array}$ & $\begin{array}{l}\text { Small intestine. } \\
\text { Associated with } \\
\text { Dochmius. }\end{array}$ & $\begin{array}{l}\text { The larva seem } \\
\text { to live freely in the } \\
\text { earth. }\end{array}$ & $\begin{array}{l}\text { Dangerous } \\
\text { æmia. }\end{array}$ \\
\hline $\begin{array}{l}\text { Filaria sanguinis } \\
\text { hominis (Australia, } \\
\text { China, India, Egypt, } \\
\text { and Brazil). }\end{array}$ & $\begin{array}{l}\text { Mature female in } \\
\text { lymphatic glands, } \\
\text { embryos in blood. }\end{array}$ & $\begin{array}{l}\text { Larva in a Mos- } \\
\text { quito. }\end{array}$ & $\begin{array}{l}\text { Elephantiasis, } \\
\text { and hamaturia. }\end{array}$ \\
\hline $\begin{array}{l}\text { Dracunculus (Fit- } \\
\text { aria) medinensis } \\
\text { (Guineaworm) in } \\
\text { Arabia, Egypt, } \\
\text { Abyssinia, etc. }\end{array}$ & $\begin{array}{l}\text { The fenale is I-6 } \\
\text { feet long, encysts } \\
\text { beneath skin. The } \\
\text { male is not known, } \\
\text { though his tail is } \\
\text { said to have been } \\
\text { seen. }\end{array}$ & $\begin{array}{l}\text { Larva in a } C y \text { - } \\
\text { clops. }\end{array}$ & Skin abscesses. \\
\hline Trichina spiralis. & $\begin{array}{l}\text { Becomes sexually } \\
\text { mature in the intes- } \\
\text { tine; embryos, pro- } \\
\text { duced rapidly and } \\
\text { viviparously, bore } \\
\text { their way to } \\
\text { muscles, and be- } \\
\text { come encysted. }\end{array}$ & $\begin{array}{l}\text { From "trichi- } \\
\text { nosed" pig's muscle } \\
\text { to man. }\end{array}$ & $\begin{array}{l}\text { Inflammatory pro- } \\
\text { cesses, often fatal, } \\
\text { are brought about } \\
\text { by the migration } \\
\text { of the young worms } \\
\text { fron intestine to } \\
\text { muscles. }\end{array}$ \\
\hline
\end{tabular}

Trichina. - The formidable Trichina deserves fuller notice. It is best known as a parasite in man, pig, and rat, but occurs also in hedgehog, fox, marten, dog, cat, rabbit, ox, and horse. The sexual forms live in the intestine, the female about 3 millimetres in length, the male about half as long. After impregnation, the female brings forth numerous embryos viviparously, 60 to 80 at a time, and altogether about I500. These bore through the wall of the intestine into the body cavity or blood vessels, and work their way, especially through connective tissue, to the muscle fibres. There they grow, coil themselves spirally, and become encysted within a sheath, at first membranous and afterwards calcareous. In these cysts, which may be sometimes counted in millions, the young Trichinre remain passive, unless the flesh of their host be eaten 
by another, pig eating rat, man eating pig. In the alimentary canal of the new host the capsule is dissolved, the embryos are set free, and become rapidly reproductive.

Among the numerous other parasitic Nematodes the following may be noted:-The giant palisade worm (Eustrongylurs gigas) occurs in the renal region of domestic animals, \&c. ; the female may be 3 feet long. The armed palisade worm (Strongylus armatus) occurs in the intestine and intestinal arteries of horse, causing aneurisms, colic, \&c. The young forms are swallowed from stagnant water, bore from grut into arteries, become adult, return to gut, copulate and multiply. Various other species of Strongylus occur in sheep, cattle, \&c. The large Ascaris megalocephala and the much smaller Oxyuris curvula are not uncommon in horses. Syngamus trachealis occurs in the trachea of birds, causing "gapes." Various species of Tylenchus, especially $T$. devastatrix and $T$. scandens (or $T$. tritici), destroy cereal and other crops. Various species of Heterodera (especially $H$. schachti $i$ and $H$. radicicola) infest the roots of many cultivated plants-e.g., turnip, radish, cabbage.

\section{Classification.}

At present the Nematodes are usually classified in families-Ascaridie, Anguillulidre, \&c. With these we need not concern ourselves here, but it is important to notice that the Gordiidxe, (e.g., Gordizes aquaticus-the horse hair worm) are very different from all the others. In the aduit the mouth is shut and the food canal is partly degenerate. The aduits live freely in fresh water; there are two larval forms, the first in aquatic molluscs, young insects, \&c., the second in adult insects, fish, frog, \&c.

\section{Class Acanthocephala.}

For a single genus Echinor/hynchus, whose larve live in Arthropods, and the acluits in Vertebrates, a special class, ACANTHOCEPiAla, has been established. We may provisionally place this genus, which has about a hundred species, beside Nematodes, but the relationship does not seem to be very close. Mouth and gut are absent. The anterior end bears a protrusible hooked proboscis.

Eihinorlynnchus proteus of Pike, larva in the Amphipod Gammarus pulex.

3 angustatus of Perch, larva in the Isopod Asellus aquaticus.

9)

gigas of Pig, larva in young Cockchafers. 


\section{CHAPTER XI.}

SEGMENTED WORMS OR ANNELIDA.

Chief classes-Chetopoda, Discophora.

THE Annelida do not form a well defined phylum, but in. clude segmented worms, in which the segmentation of the body is usually visible externally. There is usually a well developed body cavity, which communicates with the exterior by paired nephridia or segmental organs. The nervous system consists typically of dorsal cerebral ganglia, a commissural ring round the gullet, and a ventral ganglionated chain. Not infrequently the nephridia function also as genital ducts. The development is either indirect, when it includes a larval Trochosphere stage, or direct.

In habit, form, and structure the Annelids exhibit much diversity of type. The Chrtopods, represented on the one hand by the familiar earthworm, and on the other by the marine worms, best exhibit the structure upon which the Annelid type is founded. It seems, however, that with these we may also include the aberrant Echiuridx-e.g., Echiurus and Bonellia. A few forms of primitive type (the Archi-Annelida), and the Myzostomata, which are degenerate parasites found on Crinoids, may also be appended to the class Chrtopoda. The divergent leeches (Discophora) are probably Annelids which have become modified in consequence of a peculiar habit. Finally, some zoologists provisionally include Sagitta (Chætognatha) in this series as an Annelid with three segments, and also the Rotifers (Rotatoria), since their adult form somewhat resembles the Trochosphere larvæ of many Annelids.

According to Lang, the Chretopods are derived from a 
leech-like type, this from a Polyclade Turbellarian, and this from a Ctenophore. According to Sedgwick, the Annelids are derived from an Actinozoon-like ancestor. But we cannot here discuss these possibilities, nor the difficult questions concerned with the meaning of segmentation or metamerism.

\section{Class ChæтороDA. Worms with Bristles.}

Segmented animals with setce developed in little skin sacs, either on a uniform body wall or on special loconotor protrusions known as parapodia. The segments, indicated externally by rings, are often marked internally by partitions running across the body cavity, which is usually well developed. The nervous system generally consists of a double ventral chain of ganglia, connected with a pair of dorsal or cerebral centres, by means of a ring round the beginning of the gat. Two excretory tubes or nephridia are typically present in each segment, and they or their modifications may also function as reproductive ducts. The reproductive elements are formed on the lining membrane of the body cavity, and the development is either direct or with a metamorphosis.

The two prominent divisions of this class may be contrasted as follows :-

Oligochat, e.g., Earthworm.

With no parapodia, and with few sete.

Other external appendages are also wanting, except that Branchiura has gills.

Hermaphrodite.

Development direct.

Living in fresh water or in the soil.
PoLycheta, e.g., Nireis.

With parapodia and with numerous seta: With antennæ, gills, and cirri.

Sexes usually separate.

A metamorphosis in development. Marine.

Type of Oligocheta. The Earthworm (Lumbricus).

Earthworms eat their way through the ground, and form definite burrows, which they often make more comfortable by a lining of leaves. The earth swallowed by the burrowers is reduced to powder in the gut, and, robbed of some of its decaying vegetable matter, is discharged on the surface as the familiar "worm castings." By the burrowing the earth is loosened, and ways are opened for plant roots and rain drops; the internal bruising reduces mineral matter to more useful form; while, in burying the surface with earth 
brought up from beneath, the earthworms have been ploughers before the plough. Darwin calculated that there were on an average over 53,000 earthworms in an acre of arable ground, that ten tons of soil per acre pass annually through their bodies, and that they cover the surface with earth at the rate of three inches in fifteen years. $\mathrm{He}$ was therefore led to the conclusion that earthworms have been the great soil makers, or more precisely, that the formation of vegetable mould was mainly to be placed to their credit. According to Gilbert White (I777), "the earth without worms would soon become cold, hard bound, void of fermentation, and consequently sterile;" while Darwin (I88I) said that " it may be doubted whether there are many other animals which have played so important a part in the history of the world as have these lowly organised creatures."

Though without eyes, earthworms are sensitive to light and persistently avoid it, remaining underground during the day unless rain floods their burrows, and reserving their public life for the night. Then, prompted by "love" and hunger, they roam about on the surface, leaving on the moist roadway the trails which we see in the morning. More cautiously, however, they often remain with their tails fixed in their holes, while with the rest of their body they move slowly round and round. 'The nocturnal peregrinations, the labour of eating and burrowing, the transport of leaves to their holes, the collection of little stones to protect the entrance to the burrows, include most of the activities of earthworms, except as regards pairing and egg laying, of which something will afterwards be said. When an earthworm is halved with the spade it does not necessarily die, for the head portion may grow a new tail, while a decapitated worm has even been known to grow a new head and brain. The earthworm is much persecuted by numerous enemies, e.g., centipedes, moles, and birds. The male reproductive organs are always infested by unicellular parasites Gregarines of the genus Monocystis, and little threadworms seem almost always to occur in the excretory tubes.

\section{Form and External Characters.}

The earthworm is often alout six inches long, with a pointed head end, and a cylindrical body rather flattened posteriorly. The successive 
rings seen on the surface mark true segments. The mouth is orerarched by the most anterior (pre-oral) segment, while the food canal terminates at the blunt posterior end. The skin is covered by a thin transparent cuticle, traversed by two sets of fine lines which break up the light and produce a slight irridescence. On a region extending from the 3 Ist to the 38 th ring, the skin of mature worms is swollen and glandular, forming the clitellum or saddle, which helps the worms as they unite in pairs, and perhaps forms the slimy stuff which hardens into cocoons. The middle line of the back is marked by a special redness of the skin. On the sides and ventral surface, we feel and see four rows of tiny bristles or setxe, which project from little sacs, are worked by muscles, and assist in locomotion. These bristles are fixed like pins into the ground, at times so firmly that even a bird finds it difficult to pull the worm from its hole. As each of the four longitudinal rows is

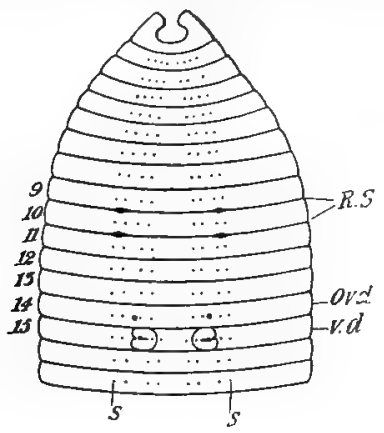

FIG. 6o.-Anterior region of Earthworm. (After HERING.)

Note the eight setæ $(s)$ on each segment.

R.S., Spots between 9-10, IO-II, indicate openings of receptacula seminis; Ovd., openings of oviducts on segment $34 \quad$ v.d., openings of vasa deferentia on segment 15 .

double, there are obviously eight bristles to each ring. On the skin of the ventral surface, there are not a few special apertures, which should be looked for on a full grown worm, but careful examination of several specimens is usually necessary. Almost always plain on the $15^{\text {th }}$ ring are the two swollen lips of the male ducts, less distinct on the 14 th are the apertures of the oviducts through which the eggs pass, while on each side, between segments 9 and IO, IO and II, are the openings of two receptacula seminis or spermathece into which male elements from another earthworm pass, and from which they again pass out to fertilise the eggs of the earthworm when these are laid. Each segment contains a pair of excretory tubes, which have minute ventral-lateral apertures, while on the middle line of the back, between every two adjacent rings, there are minute pores, through which fluid from the body cavity may exude. 
Skin and Bristles.

Outermost lies the thin cuticle, on which intersecting lines produce interference of light and irridescence. Like any other cuticle, it is produced by the cells which lie beneath, and it is perforated by the apertures previously mentioned. The epidermis clothing the worm is a single layer of cells, of which most are simply supporting or covering elements, while many are slightly modified, as glandular or mucous cells, and as nervous cells. As the latter are connected with afferent fibres which enter the nerve cord, the skin is diffusely sensitive. In a few species the skin is slightly phosphorescent. The bristles, which are longest on the genital segments, are much curved, and lie in small sacs of the skin, in which they can be replaced after breakage.

\section{Muscular Sy'stem.}

The earthworm moves by the contraction of muscle cells, which are arranged in hoops underneath the skin, and in longitudinal bands more internally. The special muscles about the mouth and pharynx have considerable powers of grasping, while less obvious muscular elements occur in the wall of the gut, in the partitions which run internally between the segments, and on the outermost portions of the excretory tubes.

\section{The Body Cavity.}

Unlike the leech, the earthworm has a very distinct body cavity, through the middle of which the gut extends, and across which run the partitions or septa incompletely separating successive segments. In this cavity there is some fluid with cellular elements, of which the most numerous are yellow cells detached from the walls of the gut. Possible communications with the exterior are by the dorsal pores, and also by the excretory tubes which open internally into the cavities of the segments.

\section{The Nervous System.}

Along the middle ventral line lies a chain of nerve centres or ganglia, really double from first to last, but compactly united into what to unaided eyes seems a single 
cord. As the segments are very short, the limits of the successive pairs of ganglia are not very evident, especially in the anterior region, but they are plain enough on a small portion of the cord examined with the microscope, when it may also be seen that each of the pairs of ganglia gives off nerves to the walls of the body. Anteriorly, just behind the mouth, the halves of the cord diverge and ascend, forming a ring around the pharynx. They unite above in two dorsal or cerebral ganglia. These form the earthworm's "brain," and give off nerves to the adjacent pre-oral segment or prostomium, on which are numerous sensitive cells. These, coming in contact with many things, doubtless receive impressions, which are transmitted by the associated nerves to the "brain." As Mr. Darwin observed that earthworms seized hold of leaves in the most expeditious fashion, taking the sharp twin leaves of the Scotch fir by their united base, we may credit the earthworms with some power of profiting by experience; moreover, as they deal deftly with leaves of which they have no previous experience, we may even charitably grant them a modicum of intelligence. From the nerve collar uniting the dorsal ganglia with the first pair on the ventral cord, nerves are given off to the pharynx or gut, forming what is called a "visceral system." The earthworm has no special sense organs, but we have just mentioned sensitive cells, which are particularly abundant on the head end of the worm. By them the animal is made aware of the differences between light and darkness, and of the approaching tread of human feet, not to speak of the hostile advances of a hungry blackbird. The sense of smell is also developed. The afferent or sensory nerve fibres from the nervous cells of the skin enter the nerve cord and bifurcate into longitudinal branches, which end freely in the nearest ganglia. In this the earthworm's nervous system suggests that of Vertebrates.

Two facts in regard to minute structure deserve attention. The nerve cells, instead of being confined to special centres or ganglia, as they are in Arthropods, occur diffusely along with the nerve fibres throughout the course of the cord. Along the dorsal surface of the ventral nerve cord there run three peculiar tubular fibres, with firm walls and clear contents. These "giant fibres," which do not seem to be nervous, but are rather supporting elements, have been dignified by the name of neurochord. 


\section{Alimentary System.}

Earthworms eat the soil for the sake of the plant debris which it may contain, and also, indeed, because they must swallow as they tunnel. In eating they are greatly helped by the muscular nature of the pharynx, whence the soil passes down the gullet or oesophagus, first into a swollen crop, then into a strong walled grinding gizzard, and finally through a long digestive and absorptive stomach intestine. On the gullet are three pairs of cesophageal or calciferous glands-the products of which are limy and able to affect the food chemically, probably counteracting the acidity of the decaying vegetable matter. The long intestine has its internal surface increased by a dorsal fold, which projects inwards along the whole length. In this "typhlosole," and over the outer surface of the gut, the yellow cells are crowded. There is no warrant for calling these hepatic or digestive. Structurally they are pigmented cells of the peritoneal epithelium, which here, as in most other animals, lines the body cavity and the outside of the gut. As to their function we know that they absorb particles from the intestine, and go free into the body cavity, whence, as they break up, their debris may pass out by the excretory tubes. When a worm has been made to eat powdered carmine, the passage of these useless particles from gut to yellow cells, from yellow cells to body cavity, and thence out by the excretory tubes, has been traced. Various ferments have been detected in the gut, a diastatic ferment turning the starchy food into sugars, and otherspeptic and tryptic-not less important. The wall of the stomach intestine from without inwards, as may be traced in sections, is made up of pigmented peritoneum, muscles, capillaries, and an internal ciliated epithelium. In the other parts of the gut the innermost lining is not ciliated, but covered with a cuticle.

\section{Vascular System.}

'The fluid of the blood is coloured red with hæmoglobin, and contains small corpuscles. Along the median dorsal line of the gut a prominent blood vessel extends, another (supra-neural) runs along the upper surface 
of the nerve cord, another (infra-neural) along the under surface, while two small lateral-neurals pass along each side of this same cord. All these longitudinal vessels, of which the first three are most important, are parallel with one another ; the first three meet in an anterior network on the pharynx ; the dorsal and the supra-neural are linked together in the region of the gullet by five or six pairs of contractile vessels or "hearts." The precise path of the blood is not

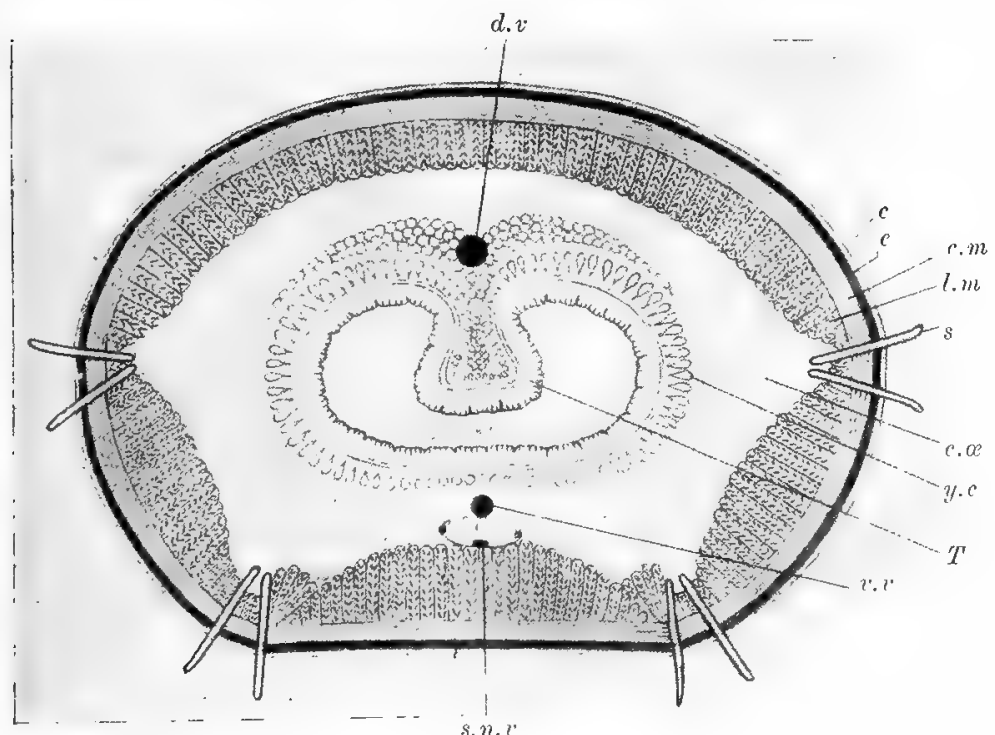

FrG. 6I.-Transverse section of Earthworm.

(After Clapartide.)

c., Cuticle; e., epidermis: c.m., circular muscles; l.mt., longitudinal muscles; s., a seta; c.c., coelome; $y_{. c}$, yellow cells; 17 ., typhlosole; $v_{*} \sigma_{*}$, ventral blood vessel; s.n.\%., sub-neural vessel below nerve cord; $d_{.2 \%}$, dorsal vessel.

known, but the distribution of vessels to skin, nephridia, and alimentary canal is readily seen.

Respiration is effected by the distribution of blood on the general surface of the skin. 


\section{Excretory System.}

As we have mentioned, small particles may pass from the gut to the body cavity, and thence to the exterior by the excretory tubes. There is a pair of these little kidneys, nephridia or segmental organs, in each segment except the first four. Each opens internally into the segment in front of that on which its other end opens to the exterior. They remove little particles from the body cavity, but probably get finer waste products from the associated blood vessels. Nephridia occur in many animals, in most young Vertebrates as well as among Invertebrates, but they are never seen more clearly than in the earthworm. When a nephridium is carefully removed, along with a part of the segment septum through which it passes, and examined under the microscope, the following three parts are to be seen : $(a)$ an internal ciliated funnel, $(b)$ a trebly coiled ciliated tube, at first transparent then glandular and granular, and (c) a muscular duct opening to the exterior. Minute particles swept into the ciliated funnel pass down the ciliated coils of the tube, and out by the muscular part which opens just outside of the ventral bristles. The coiled tube consists in part at least of a series of intracellular cavities, that is to say, it runs through the middle of the cells which compose it; the external muscular portion arises from an invagination of skin.

\section{Reproductive System}

The earthworm is hermaphrodite, and its reproductive organs are somewhat difficult to demonstrate with completeness. To see them it will be necessary to dissect several earthworms with special attention to individual parts.

(a) The Male Organs consist of two pairs of testes, three pairs of seminal vesicles, and a paired vas deferens.

(I) The testes lie near the nerve cord on the septa between segments IO and II; each is "a white translucent body of irregular quadrangular form, rarely more than onetenth of an inch in diameter." (Fig. 62,T.)

(2) Mother sperm cells, which give rise by division to young spermatozoa, pass from the testes to the much lobed seminal vesicles, where the spermatozoa are matured. 
These vesicles (Fig. 62, s.v.) are very prominent, and seem to be outgrowths of the septa between segments nine to twelve. Among the spermatozoa there are parasitic Gregarines (Monocystis) in various stages of development.

(3) The spermatozoa pass from the seminal vesicles into two vasa deferentia or male ducts. These open to the exterior on the I5th segment. Each vas deferens bears two ciliated funnels, which collect spermatozoa in segments IO and II, and soon unite in one duct.

(b) The Female Organs consist of two ovaries, and two oviducts each of which has a side receptacle for the eggs.

(I) The two ovaries are small bodies situated near the

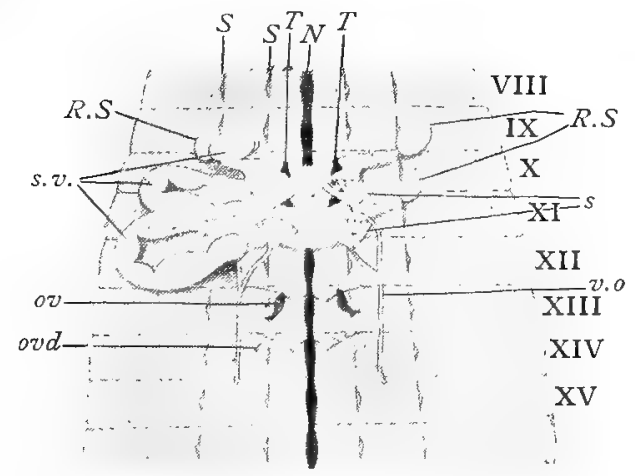

FIG. 62,-Reproductive organs of Earthworm. - (After HeRING.)

\footnotetext{
$N$, Nerve cord; $T$, anterior testes ; $S$., sacs of setæ; R.S., receptacula seminis; s., seminal furnels; v.o., vas deferens; ord., oviduct; ov., ovary; s.z , seminal vesicles; $V I I 1 .-X V$., segments.
}

nerve cord on the septum between segments 12-13. Each is pear shaped, the stalk of the pear being a string of ripe ova. They are more likely to be seen than the testes.

(2) The two oviducts open internally on the anterior face of the septum between I3-I4, and externally on the ventral surface of segment I4. Into the wide ciliated internal mouths, which lie opposite the ovaries, the ripe eggs pass. 
(3) The egg sac or receptaculum ovorum, near the internal mouth of each oviduct, is a posterior diverticulum of the septum between segments I3-I4. Within it a few mature ova are stored.

(c) Two pairs of receptacula seminis or spermathecæ receive spermatozoa from another earthworm, and liberate them to fertilise the eggs of this one. They are white globular sacs, opening in the grooves between segments 9-10 and ro-II. According to some, these spermathecæ not only receive and store spermatozoa, but make them into packets or spermatophores. Others say that the glands of the clitellum make these packets. At any rate minute thread-like packets of spermatozoa are formed, and a pair of them may often be seen adhering to the skin of the earthworm about the saddle region.

When two worms unite sexually they lie apposed in opposite directions, the head of the one towards the tail of the other. What happens is that spermatozoa of the one pass into the receptacula of the other.

When the eggs of an earthworm are liberated they are surrounded by a sheath of gelatinous stuff said by some to be secreted by the saddle. As this is peeled off towards the head a spermatophore is also enclosed.

\section{Development of the Earthrorm.}

Many cocoons are made about the same time, and each contains numerous ova, and also packets of sperms, so that fertilisation takes place outside the body. These cocoons are buried in the earth a few inches below the surface. They measure about a quarter of an inch in length.

The favourite time for egg laying is during the spring and summer, though it may be continued throughout the whole year. The earthworm of the dung heap ( $L$. foxtidus) makes this a habit, induced probably by the warmth of its habitat.

Of the many ova of the earthworm $L$. terrestris, only one comes to maturity, while of L. fotidus a few, and of $L$. communis two may do so. But in the last species the two embryos are often twins formed from one ovum, separation taking place at the gastrula stage. 
The whole process of growth, until leaving the egg, lasts from two to three weeks, the time varying however with the temperature.

The ovum is surrounded by a vitelline membrane, and is laden with yolk granules. It seems that several polar cells are formed, probably by division of the two primary ones separated from the ovum. Segmentation is slightly unequal, and exhibits considerable variation even within the limits of a species.

In about twenty-four hours, a nearly spherical, one layered blastosphere or blastula is formed. It consists of only about thirteen cells. During the next twenty-four hours the cells increase in number rapidly, but the blastula remains one layered. Two cells lying together do not take part in this division; they are rather larger than the rest, and their inner ends project into the cavity and are soon cut off. Gradually these large cells begin to sink in, giving rise to more daughter cells, and at last are quite included in the cavity. Thus there arise two parallel rows of cells within the blastula, and these define the longitudinal axis of the embryo. This is the beginning of the mesoblast which will form all the muscles of the trunk, and which thus takes origin from two primary mesoblasts.

After five to six pairs of secondary mesoblasts have been formed, the blastula begins to flatten, and to elongate, becoming an oval disc. The cells of the lower surface become clearer, and the hypoblast is thus defined. The cells of the upper surface are smaller, and become very much flattened; they compose the epiblast. The mesoblasts lie side by side near one end, forming two rows extending forwards and downwards, but divergent, because of the flattening of the blastula. The hypoblast now becomes concave, and thus the blastopore arises, occupying the whole of the lower surface. The sides close in and the blastopore becomes a slit, which further closes from behind forwards leaving only a small opening,-the future mouth. During these processes the cells at the anterior tip of the blastopore, which will give rise to the præoral lobe, undergo no change, but the mesoblast has been active.

As gastrulation proceeds, the mesoblast rows grow forwards and upwards until they come near each other above the 
anterior tip of the blastopore, while their middle portions are carried downwards until they lie on the ventral surface. Over them the epiblast is thickened in two bands. Two longitudinal rows of epiblast cells near the anterior end, and ending behind in large cells, sink in just as the primary mesoblasts did. The thickening now extends ventrally until the two bands meet, and passing into the blastopore forms the stomatodæum. Even before this the embryo has begun to swallow the albumen in which it floats.

There are now two lateral bands of cells called the germ bands, composed of three layers: outside is the thickened epiblast, next, the rows of cells which sank in, and inmost the mesoblast rows. "The mesoblast rows have met in the middle line by dividing and widening out into a pair of flattened plates, but they still end behind in the two primary mesoblasts. Coelomic cavities develop in the plates, and the anterior ends meet above the mouth. The epiblastic rows which sank in (there were eight of them, four on each side of the median line and each ending in a large mother cell) go on growing. The mother cells are apparently carried backwards as the embryo lengthens, leaving a trail of daughter cells behind them. The cells so formed also divide, the embryo rapidly lengthening and finally becoming 
vermiform. The two inner rows (neuroblasts) give rise to the nervous system, the next two rows on either side (nephridioblasts) form parts of the nephridia, while of the fourth row nothing definite is known. Each row, ending behind in a single cell, widens out and deepens as it is traced forwards, the neuroblasts are much further forwards than the mesoblasts, with the nephridioblasts just behind them. The neural and mesoblastic rows can be traced round the mouth and help to form the prostomium, the others fade away at the sides of the stomatodreum. The mesoblast rows grow to meet one another on the median dorsal line.

Let us sum up this complex history :-

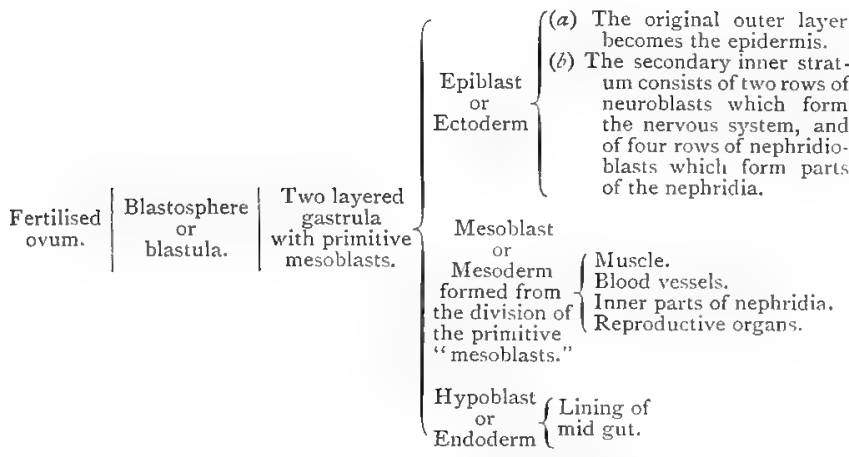

General Development of the Organs.-Though it will involve a slight repetition, we shall now describe the origin of the various organs.

The skin arises from the original outer wall of the gastrula. The "setigerous glands," within which the sete develop, and from which they push their way to the exterior, arise partly from the rows of cells started by the nephridioblasts, and partly in all probability from the outermost of the four cell rows previously mentioned. The double ventral nere cord arises from the neuroblasts. The two cerelral ganglia originate, according to Kleinenberg, independently of the ventral cord from a median unpaired apical plate of ectoderm, while according to Wilson they arise along with the ventral cord, and have their foundations in the thickened anterior end of each of the two neural rows.

The history of the excretory system is complex. (a) At the anterior end of young embryos, a group of ectoderm cells, dorsal in position, forms a larval excretory organ, which wholly disappears in later stages. (b) Next appear two ciliated canals in the anterior region, closed inter- 
nally, opening on the head. These are known as "provisional nephridia" or "head kidneys." They degenerate as the permanent excretory organs develop. (c) The numerous permanent nephridia are for the most part ectodermic, arising from the rows of nephridial cells already described. Two parts of each nephridium, however, have a mesoblastic origin, viz., the innermost part or the ciliated funnel, and the peritoneal investment which ensheaths the whole organ.

By the invagination of the blastosphere, a globular gastrula cavity is formed. This forms the archenteron, - the future mid gut,-and elongates with the growth of the embryo. To the completion of the entire alimentary canal, however, two other processes are necessary, an intucking of ectoderm from in front-the stomatodaum or "fore gut"which pushes the archenteron backwards and forms the future pharynx, and a similar in-tucking of ectoderm from behind-the proctodaum or "hind gut"-which meets and fuses with the archenteron, and forms the anus and a small portion of the posterior gut.

The mesoderm begins with the two primary mesoblasts already described. These multiply and form mesoderm bands, which, insinuating themselves between ectoderm and endoderm, proceed to surround the gut. At the same time, some of the mesoderm cells become migratory, wander on to the head, and also surround the gut, before the final trunk musculature is completed. The migratory, mesoblasts of the trunk appear to form a special larval musculature precociously developed, in order to enable the embryo to manage the enormous mass of albumen (absorbed from the capsule) with which its body is distended. The mesoderm bands grow in strength, and form a complete ring encircling the archenteron.

Origin of the body cavity. - The mesoderm bands, growing in strength, become two layered. These two layers separate, the inner (splanchnic) cleaving to the gut, the outer (somatic) clinging to the body wall. The space between them is the body cavity or calome. But as the separation of somatic and splanchnic layers takes place, partitions are also formed transversely, to become the septa which partition off the body cavity into a series of segments. The cavity of the pre-oral segment or prostomium differs somewhat from that of the others, heing from the first unpaired, instead of including two lateral cavities one on each side of the gut.

As to the blood vessels, the ventral or sub-intestinal appears first, as a space between the wall of the archenteron and the underlying mesoderm; the dorsal vessel has a double origin, arising from the fusion of two lateral vessels which develop like the ventral. The important point is, that the blood vessels are at first long lacunar spaces, which gradually acquire definite walls. By and by the "hearts" and other complications in the vascular system appear.

The reproductive organs, though probably arising from cells which have kept to some extent apart from the formation of the embryo, certainly appear in association with the mesoderm.

The above is the account of the development of Lambrizus given by Wilson; another investigator, Bergh, differs from Wilson in several important points. First, with regard to the nomenclature of the constituents of the germ bands. 
WJLSON,

Two inner primitive, Neuroblasts. cells.

Next three cells on either side.

Posterior cell of each side.
BERGH.

Neuroblasts.

fOuter or anterior $\left\{\begin{array}{c}\text { Two nephridioblasts, } \\ \text { one lateral cell, }\end{array}\right.$

Primitive mesoblast.
Inner or posterior myoblast.

According to Bergh the germ band consists originally of three cells on each side, the neuroblast, the primary inner myoblast, the outer myoblast. The primary inner myoblast later gives origin to the three inner myoblasts, nephriclioblasts, and lateral cell of Wilson.

Further, Bergh states that at an early stage a "nerve plexus" arises on the mid-ventral line, probably from the ectoderm, and that this unites with the neuroblastic rows to produce the nervous system of the aduit.

\section{Type of Polycheta. The Lob Worm (Arenicola piscatorum).}

\section{Habits.}

On the flat sandy beach uncovered at low tide, the "castings" of the lob worm are very numerous. There the fishermen seek the worms for bait, and have to dig deep, for the burrowers rapidly retreat far into the sand. The burrows of the lob worm are cylindrical tubes, lined by a yellowish green secretion, and the surrounding sand is often discoloured by some change in which the organic juices reduce the iron constituents to lower oxides. The tubes are at first vertical, and afterwards oblique or horizontal.

The lob worm burrows like the earthworm-eating the sand for the sake of the organic particles or small organisms which it contains. The sandy castings, which pass from the end of the food canal, and are got rid of at the mouth of the tube, fall into spiral coils. When getting rid of the castings, the worm lies with its tail upwards and its head downwards, or with its body bent like a bow; when the 
tide comes in, the mouth may protrude. The animal is able to turn in its burrow.

The young are pelagic, and it is said by some that the adults are active and swim about at certain seasons.

\section{External Appearance.}

The lob worm varies in length from eight inches to a foot, and at its thickest part is about half an inch in diameter. There are three regions in the body:-(a) the anterior seven segments, of which all but the first have bristles; $(b)$ the gill bearing region of thirteen segments; (c) the thinner posterior part of variable length, without either

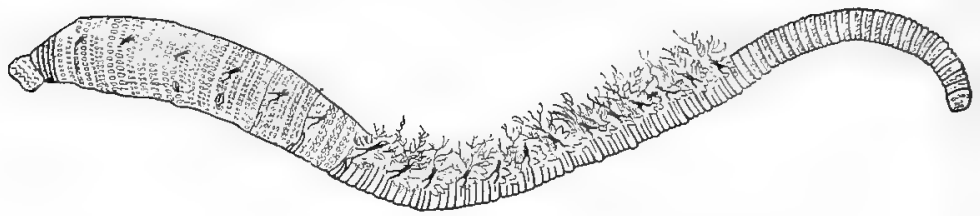

Fig. 64.-Arenicola piscatorum. (After Cunnivghum and RAMAgE.)

Note anterior protrusion of mouth; setæe on anterior region; setze and gills on median region; thinner tail region often longer than shown.

bristles or gills. The head lobe is very small; there are no tentacles or eyes. Anteriorly a soft proboscis is protruded.

Skin and Muscles.

Each segment is marked by several rings; there are numerous warts on the posterior region. Most externally lies the cuticle, then the pigmented epidermis, then the circular and the longitudinal muscle fibres.

\section{Appendages.}

Unlike many of the marine Annelids which have on each segment well-developed outgrowths or parapodia, divided into a dorsal notopodium and a ventral neuropodium, Arenicola has very rudimentary appendages. This reduction of appendages must be associated with the animal's mode of life; the same is true of many tube inhabiting worms. The first segment has no trace of appendages, 
the next nineteen have rudiments. The dorsal part consists of a tuft of bristles, whose bases are enclosed in a sac; - the ventral part, separated by a short interval, bears several hooks.

\section{Nervous System.}

The nervous system is in its general features like that of the earthworm, but ganglia are not developed. In the

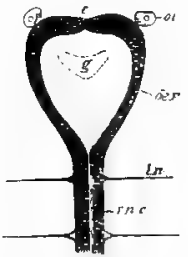

FIG. 65.-Anterior part of nervous system in Arenicola. (After VOGT and YUNG.)

c., Cerebral part on dorsal surface; a. $r$., csophageal ring; $g$, gullet; v.n.c., ventral nerve cord ; $2 . n$., lateral nerves; ot., otocyst. ventral nerve cord, the ring round the gullet, and the slight cerebral enlargement which represents a brain, nerve cells occur diffusely scattered among the nerve fibres. Along the dorsal surface of the nerve cord run two "giant fibres" like those in the earthworm.

In some species at least, the head lobe is distinctly sensory and there are two ciliated "neck organs." Otherwise sense organs are represented only by a pair of otocyst sacs, one on each side of the oesophageal nerve ring. These sacs, like those which occur in many other Invertebrates, seem to have to do rather with the direction of the animal's morements than with hearing. Prof. Ehlers notes an interesting series:-In $A$. Claparedii, there are simply two open grooves; in $A$. marina, the sacs have open necks and contain foreign particles; in $A$. Grubbii and $A$. antillensis, the sacs are closed and contain intrinsic otoliths of lime.

\section{Food Canal.}

The mouth is at the end of a protrusible cup-like proboscis; the gullet has smooth walls, and bears an anterior and a larger posterior pair of glands which secrete a yellowish fluid perhaps digestive; the succeeding part of the gut is covered with yellow cells and many blood vessels, and is divided into rings; the terminal portion is full of sand from which the nutritive matter has been absorbed; the anus is at the very end.

\section{The Body Cavity.}

The body cavity is spacious, except in the tail region, and contains a fluid. Anteriorly there are three transverse, 
partly muscular, partitions or mesenteries which moor the gullet; in the tail region there are many such; the median part of the gut swings freely. Posteriorly there are also oblique partitions which divide the segments into a median and two lateral chambers.

\title{
The Vascular System.
}

The blood has a bright red colour. It flows forward in a dorsal vessel, running along the mid-dorsal line of the

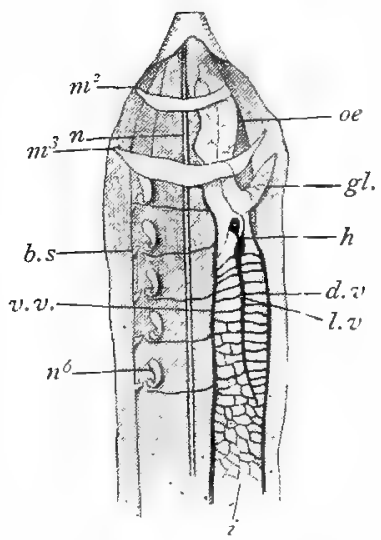

FIg. 66.-Dissection of anterior region of Arenicola.

(After Cosmovici.)

\begin{abstract}
m.2., m.3. Second and third mesenteric septa; $n$. , ventral nerve-

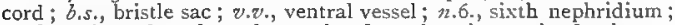
$i$, intestine; $l . q$, lateral vessel; $d . v$, dorsal vessel; $h$., heart; $g l .$, one of the two larger vesophageal glands; $\alpha_{n}$, cesoplagus.
\end{abstract}

gut, backward in a ventral vessel below the gut. Two sub-intestinal vessels lie between the ventral vessel and the gut, and receive tributaries from the anterior gills. (Some deny that the sub-intestinal vessel is double.) On each side of the digestive part of the gut there is a lateral vessel.

Just behind the posterior pair of cesophageal glands lies a very contractile heart. It consists of two lateral chambers 
or ventricles, each of which receives blood from the dorsal vessel, from a sub-intestinal vessel, and from a lateral vessel, and drives blood into the ventral vessel. Each of the lateral vessels before entering the heart expands into a kind of auricle.

The longitudinal vessels are all connected by transverse branches. From the ventral vessel arise afferent branchial vessels. From the seven posterior gills efferent branches enter the dorsal vessel; while those from the six anterior gills join the sub-intestinals. Each efferent vessel gives off

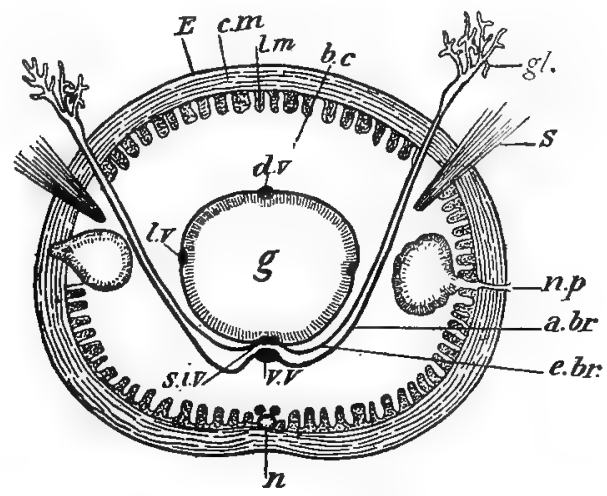

Fig. 67.-Cross section of Arenicola, (After Cosmovici.)

E. Epidermis ; c.m., circular muscles; l.m., longitudinal muscles; b.c., body cavity; gl., gill ; s., setæ; $n_{.} p_{*}$, nephridial pore; $\boldsymbol{a}_{\text {.br., }}$ afferent branchial; e.t.r., efferent branchial; $n_{\text {. }}$, ventral nerve-cord with blood vessels above; $d . v$., dorsal vessel; $z . v$., lateral vessel;

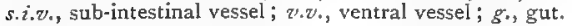

a branch to the skin, while the dorsal and sub-intestinal vessels give off numerous branches to the walls of the gut. It seems that the flow of the blood is not always quite the same.

\section{Respiratory System.}

There are thirteen pairs of gills. Each is a tuft of thread-like branches, through the thin walls of which the 
red blood shines. As the papillæ on the proboscis are hollow and contain vessels, they are doubtless of respiratory significance. Indeed, the gills may be regarded as exaggerated papillæ.

\section{Excretory System.}

In the anterior region, from the fifth to the tenth segments, there are six pairs of nephridia. Each consists of three parts - a funnel opening into the body cavity, a glandular portion, and a bladder communicating with the exterior.

\section{Reproductive System.}

The sexes are separate and similar. The reproductive organs are very simple, and lie on the peritoneal membrane of the body cavity. They are developed in close association with the nephridia. The reproductive cells are liberated into the body cavity, and there matured. In August and September they pass out by the nephridia. Little is known in regard to the development, beyond the fact that the young are for a time free swimming pelagic forms.

\section{Development of Polychata.}

As an example of the development of the marine Chrotopods, we may take Eupomatus, which has been investigated by Hatschek. Here segmentation is complete but somewhat unequal, and results in the formation of a blastula, with its upper hemisphere composed of small (ectodermic) cells, and the lower of large (endodermic) cells. Among these latter are two spherical cells - the primitive mesoblasts. Invagination takes place in the usual way to form a gastrula, the primitive mesoblasts divide and form mesoblastic bands. During these processes the external form has altered consiclerably. The apical (aboral) region of the gastrula becomes tilted forward, an ectodermic invagination arises posteriorly, and uniting with the archenteron produces hind-gut and anus, while a similar insinking anteriorly, in the region of the blastopore, forms fore-gut and mouth. The larral gut so formed has a clistinct ventral curve. Cilia appear on the surface at an early stage, and now form a distinct pre-oral ring, and also a less constant post-oral ring. At the apex of the pre-oral region an ectodermic thickening takes place, this gives rise to an apical ganglion with which sensory structures are often associated. The mesodermic bands give rise to muscle cells used in swimming, and also to the "head kidneys"-a pair of larval excretory tules. The larva so formed is a typical Trochosphere, such as occurs in the great majority of Polycheta, in a more or less modified 

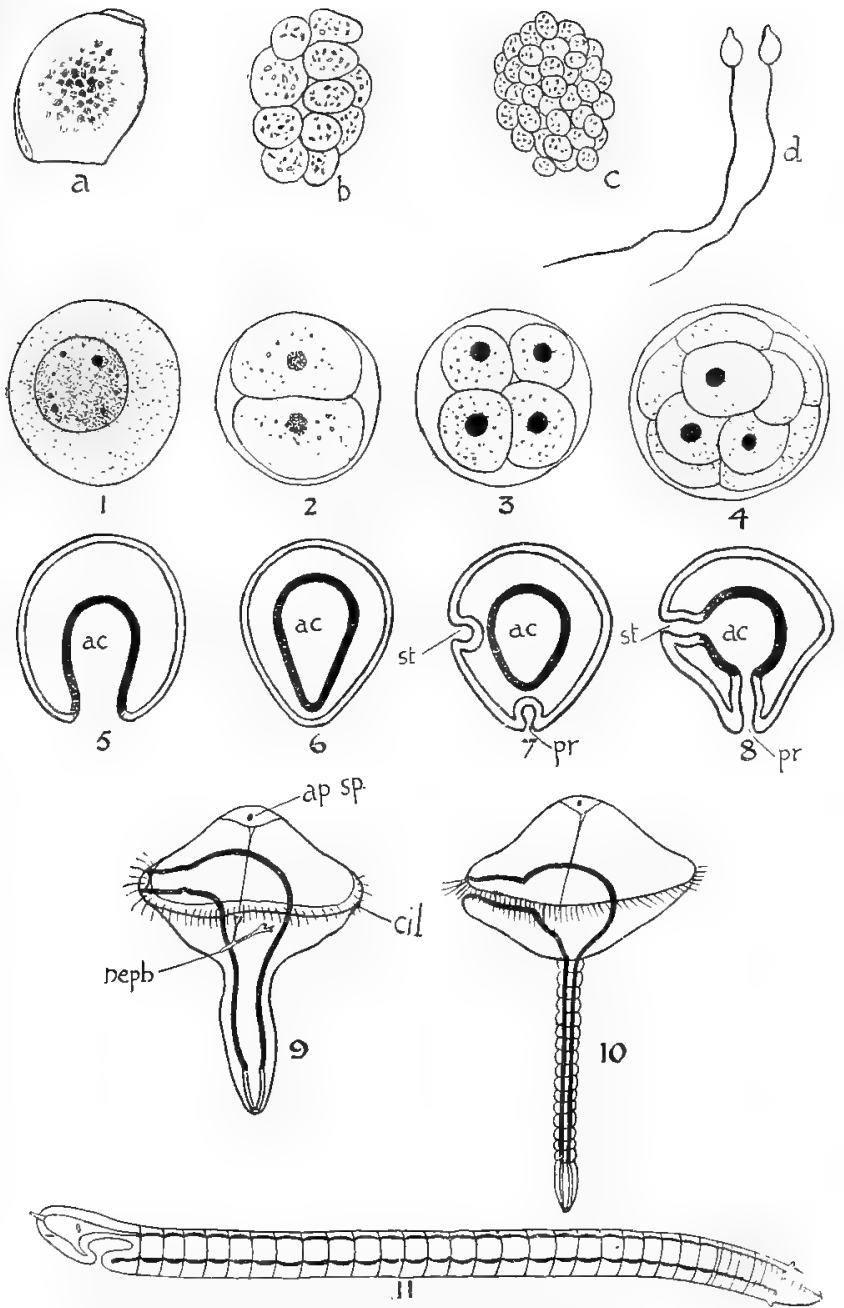

FIG. 68.-Development of Folygordius, (After FRAIPONT.)

$a .$, Mother sperm cell ; $b_{\text {. }}, c_{\text {. }}$, sperm morule ; $d$., spermatozoa.

I. Ovum with large nucleus; 2. 2ncell stage; 30 4-cell stage; 4. blastosphere; 5. Gastrula; $a c_{1}$, archenteron; 6 . closure of gastrula mouth or blastopore; 7. formation of stomatodæum (st.), and proctodæum ( $f r$.$) , invaginated to meet archenteron$ $(a c) ;$.8 . complete gut formed; $g$. elongation of larva; $a p$. sp., apical spot; cil., ciliated ring; neph., primitive nephridia; to. formation of posterior cegments; rr. Form of adult Polygordius. 
guise in many other worm-types, and also in Molluscs. We may summarise the chief characters of the Trochosphere thus:-

(I) There is a prominent pre-oral region with an apical ganglion and a ring of cilia.

(2) The gut has a distinct ventral curve and a threefold origin.

(3) The larval body cavity is simply the persistent segmentation cavity, and in it posteriorly lie the primitive mesoblasts.

The Trochosphere is a free swimming pelagic larva which, among worms, corresponds largely to the future head region of the adult. Its metamorphosis into the adult probably takes place in the most primitive fashion in the little worm Polygordizts. We shall, therefore, follow it there (Fig. 68).

In the larva, which is a typical Trochosphere, the first sign of segmentation appears in the bands of mesoblast. These tecome divided into successive segments, while at the same time the posterior region of the larva elongates greatly, carrying the larval gut backwards with it. Meanwhile, a cavity appears in each of the mesoblastic segments. These cavities, taken together, form the adult body cavity; the outer and inner walls form the somatic and splanchnic layers; the posterior and anterior walls of adjacent segments fuse to form the septa of the adult worm; the inner (splanchnic) walls of the primitive segments on each side fuse above and below the gut to form the dorsal and ventral supporting mesenteries of the gut. The head region is at first disproportionately large, but later by an independent process of growth becomes reduced. The larva abandons its pelagic life and becomes adult.

Comparing the development of Polychreta with this, we find that the Trochosphere is often modified, and that segmentation tends constantly to appear at an earlier stage. As a further step in the same direction, we may note that in some Polychrta the Trochosphere stage is no longer recognisable as such.

\section{$A$ general contrast of the modes of Development in different Annelids.}

A.
"Larval" Types
as in
marine Chretopods,
Polygordius, \&c.

Development indirect.

A free swimming Trochosphere stage, with trunk almost or wholly suppressed, with head region greatly developed, with ardaptations to free marine life.
"F."

as in

Earthworm, Leech, \&c.

Development direct, within egg capsule ; Trochosphere stage almost or wholly suppressed.

Lumbricus type Clepsine type
with little nutri- with much nutri-
tive material in tive material in
ovun, with gas- ovum, with gas-
trula formed by trula therefore
invagination (em- formed by over-
bolic).




\section{Oligochicta.}

\section{General Survey of Chatopoda.}

Of Lumbricus there are many species, e.g., the common earthworm L. terrestris, the dunghill worm L. fatidues, and L. communis or trapezoides, whose ova usually form twins. We may conveniently include under the title "carthworms" a great array of animals more or less like Lumbricus, and usually described as terriculous Oligochreta. These are arranged by Beddard in four main groups -LUMBRICINI, GEOSCOLECINI, ACANTHODRILINI, and EUDRILINI, with a divergent group Moniligastres. It is enough for us to notice here that the modern classification is chiefly based on the modifications of the excretory system. The largest "earthworm" is a Tasmanian speciesMegascolides gippslandicus - measuring about six feet in length, said to make a gurgling noise as it retreats underground.

To these must then be added a number of families, Tubificidu, Enchytroida, \&c., which live in mud and water, and are often called limicolous Oligochreta. Of these a very common representative is the little river worm Tubifex rivulortm, often found in the mud of brooks, and well suited in its transparency and small size for microscopic examination. Also notable is the fresh water Nais, with remarkable powers of asexual budding. Another interesting ally of Tubifex is Branchiura, which has paired contractile gills on each of the posterior segments of its body, thus resembling a Polychrete.

The leech-like Branchiobdella, which is parasitic on the crayfish, is apparently an abnormal Oligochrete.

\section{Polyihata.}

Living in surroundings usually very different from those of the more or less subterranean earth-and mud-worms, the marine Polychicta have a richer development of external structures, and a more complex life history. From the sides of the body rings distinct outgrowths form the first genuine legs. These, known as parapodia, bear bundles of firm bristles, and are typically divisible into a ventral neuropodium and a dorsal notopodium. Each of these is usually furnished with a probably tactile process, the two being known respectively as the notopodial cirrus and the neuropodial cirrus. With the notopodium the first true gills, which contain prolongations of the body cavity, are often associated, but the respiratory plates which occur in the sea mouse, dxc., are probably metamorphosed dorsal cirri. Parapodia are absent from the anterior region, but this is frequently well furnished with tactile cirri, as well as with eyes, "ear sacs," and other sensitive structures. The eyes show an interesting series of gradations from simple pigment spots to very complicated structures (e.g., Alciope), exhibiting cornea, crystalline lens, retina, \&c. In many cases in these marine worms the blood is red as it is in most Oligochzetes, but it may be colourless (Aphrodite), green (Sabella), or yellow. The pigment is usually dissolved in the plasma, and its variations in character and amount among nearly allied forms are of great interest to the comparative physiologist. The gut is frequently branched and of large calibre. In some cases (Capitellida) it possesses an accessory communicating tube (Nebendarm) which is of interest, 
having been compared to the notochord of Vertebrates. The sexes are usually separate, the reproductive organs simple and devoid of accessory structures. The nephridia function as genital ducts. There is a metamorphosis in development.

(a) Some of these marine Polychretes lead a free and more or less active life, crawling between tidemarks or on the sea bottom, burrowing in the sand, or swimming in the open water. These Errantia have wellcleveloped appendages, and a large pre-oral scgment, ancl are generally furnished with eyes and well-developed antenne. Gills are usually associated with the dorsal parts of the paraporia. Most of them feed on other animals, and have sharp "horny jaws," while the anterior part of the gut is protrusible as a proboscis.

Nereis and Nephthys are two common genera, species of which may be unearthed by digging in the sand close to rocks, though at times these or other species are seen swimming freely. The sea mouse, Apluroizte, has irridescent bristles, a feltwork of matted hair covering large gill plates which lie along its back, a very large muscular pharynx, and a gut with numerous irregular branches extending throughout the body. A very common shore form, a little like a small Aphrodite, is Polynoe. As an actively errant worm, with well developed eyes, Alciope may be noted, and the family of Syllidee is remarkable for the unusually prolific asevual budding, which sometimes results in a chain or even an irregular

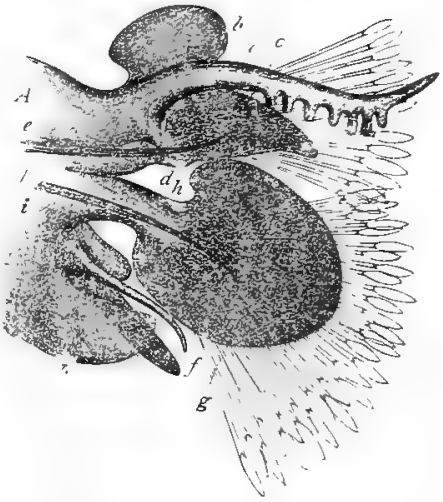

FIG. 69.-Parapoclium of a Marine Polychiete, Heleronereis. (From QUATREFAGES.)

$A$, Notopodium; $B$, neuropodium; $\pi$, notopodial cirrus; $f$, neurupulial cirrus; $b, c, g$, gill plates; $c, i$, tufts of bristles.

branched aggregate of indlividuals. As the cuticle is often irridescent, and as the red blood may shine through the skin, these marine worms are frequently beautiful. The list of nymb hand goddesses has been the source of such titles as Nereis, Aphrodite, Eunice, and Hermione, and one can almost believe the legend, according to which a specialist on Errantia christened his daughters after his seven favourites.

(b) Other marine Polychata, however, lead a more sluggish life within various kinds of tules, limy, sandy, papery, or gelatinous. As one would expect, their paraporlia are minute, apt to degenerate, and often used solely for clambering within the tube. The pre-oral region is small, but the anterior rings usually bear gills, cirri, and tentacles, often in rich profusion. These Sedentaria rarcly have a protrusible pharynx, and 
never " jaws." Most of them feed on minute Algæ swept in by the cilia on the tentacles and other structures about the mouth.

The fisherman's lob-worm (Arenicola) burrows on the sandy shore like Lumbricus in the frelds. Common also on the shore within a tube of ghed sand particles is Terebella or Lanice conchileya, where the excretory tubes are partly united by a longitudinal tube in a manner suggestive of the segmental duct which connects the nephridia of a young Vertebrate. The twisted limy tubes of Serpula are common outside shells and all sorts of marine objects, and the animal bears a stopper or operculum, with which it closes the mouth of its tube, but through which it probably at the same time breathes. In deep water, within a yellow parchment-like tube, Chatopterus may be dredged, perhaps the strangest form of all.

\section{Echiuride.}

In holes in the rocks on some of the warmer European coasts lives a curious "worm"-Bonellia viridis, of a beautiful green colour, with a globular body and a long, grooved, anteriorly forked, pre-oral protrusion. Such, at least, is the female, but the male is microscopic in size, hopelessly degenerate, living parasitically in or on his mate. The male resembles in some ways a Turbellarian, is mouthless and gutless, and little else than a migratory spermatophore. By means of cilia, it moves from one part of the female to another, and fertilises the eggs in a modified excretory tube, which serves the female Bonellia as a uterus. Here illustrated in extreme, we see the usual inequality (in size) between the sexes.

Less abnormal than Bonellia are the genera Echizurus and Thalassena.

In this small sub-order the aduits have, at most, indistinct traces of the segments which the young forms exhibit. Nor are there parapodia, cirri, or gills, but setae are always represented (except in the male Bonellia) by two anterior bristles, and in Echizurus by posterior spines as well. The nerve cord is unsegmented, and there is but a slight anterior ring without a brain. The anterior part of the body forms a muscular, well-innervated, ciliated proboscis, with the mouth deeply situated at its base; the gut is much coiled, bears a curious adjacent tube known as the "collateral intestine," and a pair of excretory "anal glands" opening into the body cavity by ciliated funnels. There is a terminal anus. There are dorsal and ventral blood vessels, and two or three pairs of nephridia, one or more of which function as reproductive ducts. The sexes are separate, and the reproductive elements are formed on the walls of the body cavity, into which they are liberated. There is a metamorphosis in development, the larva differing from the adults in many ways, e.g., in being segmented.

\section{Appendix (I) to Chatopoda.}

Primitive Chatopods and Annelios (Archi-Chrotopoda and Archi-Annelida).

An aberrant Chretopod type is represented by Saccocirms, a small marine "worm" with many primitive characteristics. The body is 
segmented, and very uniform throughout; the pre-oral region is small, but the mouth segment is large; there are bundles of setre on the rings; the nervous system remains embedkled in the epidermis.

More prinitive, however, are the Archi-Annelida represented by Polygordius, Protodritus, and Histriodrilus-all marine. The small body is segmented and uniform; there are no setre, parapodia, cirri, or gills, but the head bears a few tentacles; as in Saccocirrus, the pre-oral region is small, and the segment around the mouth is large; the very simple ncrvous system is retained in the epidermis.

Polygordizes is a thin worm, an inch or more in length, living at slight depths in sand or fine gravel, often along with the lancelet. It has a few external cilia about the mouth in a pair of head-pits, and sometimes on the body; it moves like a worm, but has no bristles. It feeds like an earthworm, or sometimes nnore discriminatingly on unicellular organisms. The females are usually larger than the males, and in some species break up at sexual maturity. The development includes a metamorphosis, and the larvae seem to throw some light on the nature of the ancestral Annelids. They are ciliated, free swimming, light loving, surface animals, feeding on minute pelagic organisms, seeking the clepths as age advances. According to some, the larva represents a primitive unsegmented ancestral Annelid with medusoid affinities; according to others, the larval characteristics are adaptive to the mode of life, and without historic importance.

Protodrilus is even smaller than Polygordius, with more cilia, mobile tentacles, and two fixing lobes on the posterior extremity; the movements are Turbellarian-like, the reproductive organs hermaphrodite, the development direct. Histriodrilzes is parasitic on the eggs of the lobster.

\section{Appendix (2) to Chatopoda.}

\section{Parasitic and Degenerate Chatopods. Myzostomata.}

The remarkable forms (Myzostoma) included in this small class, live parasitically on feather stars, on which they form galls. They are regarded as divergent offshoots from primitive Annelids, the larval form showing some distinctly Chretopod characters. The minute dise-like body is unsegmented, and bears five pairs of parapodia, each with a grappling hook, with which five pairs of suckers usually alternate. There are also abundant cirri. The skin is thick, the body muscular, the nervous system is concentrated in a ganglionic mass; which encircles the gullet, and gives off abunclant branches. There is a protrusible proboscis and a branched gut; the mouth and anus are ventral. The ova arise in the reduced body cavity, and pass by three meandering oviducts to the anal aperture. The testes are paired, branched, and ventral, with associated ducts, which open anteriorly on the side of the body. The sexual relations are interesting, for one species is hermaphrodite and another unisexual, between which there is an intermediate species with ovaries and rudimentary testes. The hermaphrodite form may bear on its body dwarfish males, analogous to the complemental pigmies on some hermaphrodite barnacles. 


\section{Class Discophora or Hrrudinea. Leeches.}

This class inchudes forms in which the body is elongated or flattened, devoid of appendages or bristles, and marked externally by rings, which are much more numerous than the segments as displayed in the internal structure. The body cavity is much reduced and communicates werth the welldeveloped vascular system. The nephridia are numerous and segmentally arranged. There is always a posterior sucker, and the mouth is frequently suctorial also. Almost all are hermaphrodite, the male organs are numerous and usually segmentally arranged. The nephridia do not function as ducts for the reproductive organs.

Leeches show several very distinct Annelid characters, but on the other hand differ from ringed worms and agree with flat worms in having suckers, in the absence of bristles, in the frequently flattened form and other features. It is impossible to say how far these resemblances with flat worms are due to the adoption of a peculiar mode of nutrition, but the evidence on the whole seems to be in favour of Annelid affinities.

Most leeches are worm-like aquatic animals, with blood sucking propensities, but some live in moist soil, and others keep to the open surface, while the parasitic "vampire" habit, familiarly illustrated by the apothecary's ancient panacea, is in many cases replaced by carnivorous habits and predatory life. The medicinal leech (Hirudo) is typical of the majority, for it lives in ponds and marshes, and sucks the blood of snails, fishes, frogs, or of larger available victims. The giant leech (Macrobdella valdiviana), said to measure $2 \frac{1}{2}$ feet in length, is subterranean and carnivorous, while the wiry land leeches (Hamadipsa, \&c.), of Ceylon and other parts of the East move in rapid somersaults along the ground, fasten on to the legs of man or beast, and gorge themselves with blood. By attaching the head end by the mouth and loosening the tail sucker, then fixing the tail and extending the anterior region, many leeches move very quickly and deftly, while at other times, or in other forms, the mode of locomotion is by graceful serpentlike swimming, or by gentle gliding after the manner of snails. The hungry horse leeches, "whose daughters cry 
Give, Give," are species of Hamopis, greedily suctorial though their teeth are too small to be useful in bloodletting; but the popular name is also applied to species of the common genus Aulastoma, whose members are carnivorous. Other common leeches are species of Nephelis, predacious forms with indiscriminating appetites, and the little Clepsine, also common in our ponds, notable for its habit of carrying its young about on its belly. Numerous marine forms prey upon fishes and other animals, e.g., the "skate sucker" Pontobdella, with a leathery skin rough with knobs, and Branchellion on the Torpedo, remarkable for numerous leaf-like respiratory plates on the sides of its body. Perhaps the strangest habitat is that of Lophobdella, which lives on the lips and jaws of the crocodile.

\section{Type. The Medicinal Leech (Hirudo medicinalis).}

This is the commonest and most familiar of leeches, once so constantly used in the practice of medicine that leech became synonymous with medical practitioner. It lives in ponds and sluggish streams, and though not common in Britain, is very abundant in many regions of the Continent, where leech farms, formerly of great importance, are still to be seen. Leeches feed on the blood of fishes, frogs, and the like, and are still caught in the old fashion on the bare legs of the callous collector. As animals are naturally averse to bloodletting and hard to catch, leeches make the most of their opportunity, and feed very greedily. They gorge themselves with blood and keep on slowly digesting it for many months, it may be indeed for a year. Watched in a glass jar, the leech will be seen to move by alternately fixing and loosening its oral and posterior suckers, while some slight provocation, such as some drops of chloroform or alcohol, will induce the animal to swim about both actively and gracefully. At times it may also be seen to cast off from its skin thin transparent shreds of cuticle,--a process which, in natural conditions, usually occurs after a heavy meal, when the animal as if in indigestion spasmodically contracts its body, or rubs itself on the stems of water plants. Numerous eggs are laid together in cocoons in the damp earth near the edge of the pool. Thence after a 
direct development, young leeches emerge and make for the water.

External Features.-The leech usually measures from two to six inches in length, and appears cylindrical or strap-like, according to its state of contraction. The slimy body shows over a hundred skin rings, its torsal surface is beautifully marked with longituclinal pigmented bands, while the ventral surface is mottled irregularly; the suctorial mouth is reaclily distinguished from the unperforated hind sucker, above which on the dorsal surface the alimentary canal may be seen to end.

It is, however, necessary to consider the external characters in greater detail. As already noted, the rings of the body are merely superficial wrinkles; it is therefore not difficult to realise that there may be doubt as to their exact limits, and that the apparent number may differ according as they are counted from the dorsal or ventral surface. According to Whitman's precise investigations, $102 \mathrm{skin}$ rings in all are represented, and these correspond to 26 somites or true segments. These segments may be recognised externally by consp)icuous pigment spots ("segmental papille"), which in the micldle region of the body occur on every fifth ring. In type, therefore, five rings correspond to a segment, but at either end of the body the number of rings is abbreviated. In the head region a pair of "eyes" occurs on each of the 1 st, 2 nd, 3 rel, 5 th, and 8 th rings; these are homologous with "segmental papille," and therefore in this region eight rings correspond to five segments.

Careful examination of the surface of the body will show further, the swollen protrusion of the male organs on the mikdle ventral line between rings 30 and $3 I$, the aperture of the female organs five rings further back, and also on the ventral surface seventeen pairs of small lateral apertures, through which a whitish fluid may be squeezed -the apertures of the excretory organs. The skin of segments 9-II is especially glandular, and forms the so-called clitellum or saddle, the secretion of which forms the cocoon for the eggs.

\section{The Skin.}

The skin is so closely connected with the connective and muscular tissue lying beneath that little can be seen of its structure except in sections. Most extemally lies the cuticle - a product of the epidermis - periodically shed as we have already noticed. In this shedding some of the genuine epidermis cells are also thrown off. These are somewhit hammer-like units with the heads turned outwards, while the spaces between the thick handles contain pigment and the fine branches of blood vessels. As the latter come very near the surface a respiratory absorption of oxygen and outward passage of carbonic acid is readily effected. Opening between the epidermal elements, but really situated much deeper, are numerous long necked, flask shaped glandular 
cells, the coutents of which form the mucus so abundant on the skin. Underneath the epidermis there is much connective tissue, and not a little pigment, yellow and green, brown and black in colour.

\section{The Muscular System.}

The muscular system consists of spindle shaped cells arranged externally in circular bands like the hoops of a barrel, internally in longitudinal strands like staves. Besides these there are numerous muscle bundles running diagonally through the body, or from dorsal to ventral surface, and there are other muscles associated with the lips, tooth plates, and pharynx.

\section{The Body Cavity.}

The body cavity is almost quite obliterated in the adult leech, where the predominant connective tissue has filled up nearly every chink and crevice. It is to be seen in the embryo, and its remnants may be detected here and there in the adult. The virtual absence of the body cavity, and the spongy compactness of the whole animal, make the leech a tedious subject to dissect.

\section{Nervous System.}

The nervous system mainly consists of a pair of dorsal ganglia lying above the pharynx, and of a double nerve cord with twenty-three ganglia lying along the middle ventral line. 'The dorsal (or supra oesophageal) ganglia are connected with the most anterior (or sub-oesophageal) pair on the ventral chain, by a narrow nerve ring surrounding the beginning of the gut. From the dorsal centres nerves proceed to the "eyes" and anterior sense spots, from the ventral centres the general body is innervated, and from the beginning of the ventral chain special nerves supply the alimentary canal, forming what is called a visceral system.

\section{The Sense Organs.}

The sense organs of the leech are ten so-called "eyes," besides numerous sense spots usually occurring on every fifth skin ring. The eyes are arranged round the edge of the mouth, and look like little black spots. Microscopic examination shows them to be definite cups, surrounded by connertive tissue with black pigment, and containing clear strongly refracting cells, each in connection with a fibre of the optic nerve. 
It has been shown (Whitman) that the eyes of leeches are serially homologous with the segmental sense organs. At the one extreme there are purely tactile organs, at the other extreme there are purely visual organs, and between these there are compound sense organs, in part tactile and in part visual, - a series which is full of suggestiveness in regard to the evolution of sense organs (cf. of the series sensitive setæ in the crayfish. "The visual organs of the leech are not able to form

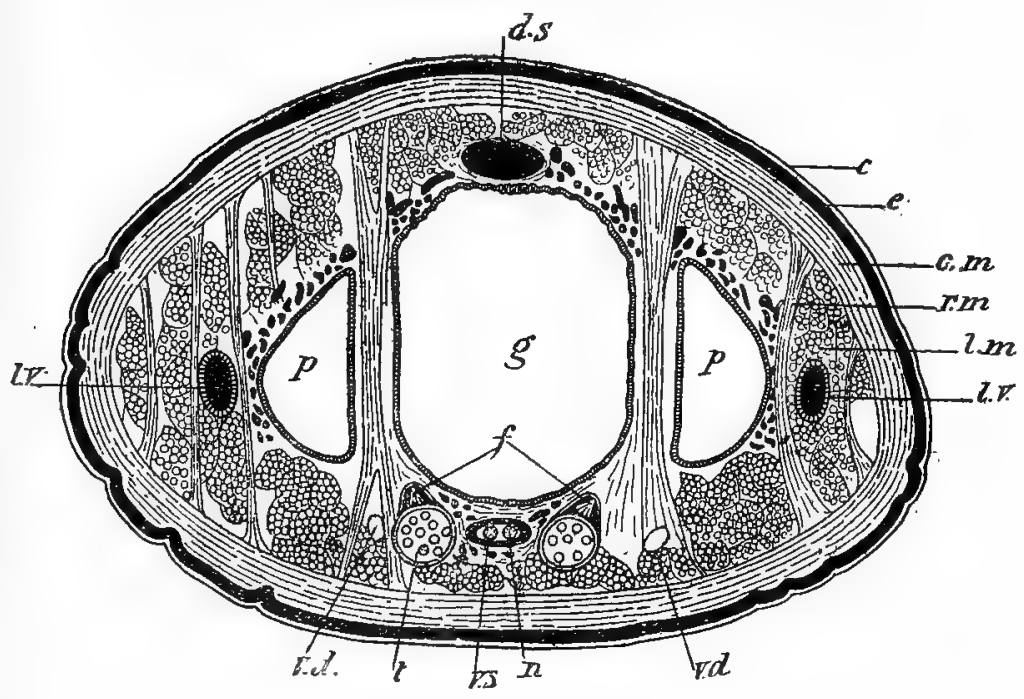

F1G. 70.-Transverse section of Leech. (Simplified from A. G. BOURNE.)

$c$, Cuticle; $\varepsilon_{\text {. }}$ epidermis; $c . m$., dermis and outer muscles (circular and oblique); l.m., longitudinal muscles (the peculiar connective tissue is hardly indicated); $\eta_{m} m$, radial muscles; $l . v$, lateral blood vessel ; d.s., dorsal blood sinus ; v.s., ventral sinus enclosing nerve cord $(n) ; g_{1}$, median part of crop, with lateral pockets $(p)$; $t$., testes ; $f$., nephridial funnel; $v$.d., vas deferens.

images of external objects, but the animals are exquisitely sensitive to alterations of light.

\section{The Alimentary System.}

When the leech has firmly fastened itself to its prey by the hind sucker, it brings its muscular mouth into action, pressing the lips tightly on the skin, and protruding three chitinous tooth plates which lie within. Each of these 
tooth plates is worked by muscles, and is like a semicircular saw, for the edge bears from 60 to roo small teeth. Rapidly these saws cut a triangular wound, whence the flowing blood is sucked into the mouth by the muscular pharynx. The process may be observed and felt by allowing a hungry leech to fasten on your arm. As the blood passes down the pharynx, it is influenced by the secretion of salivary cells which lie among the muscles, and exude a ferment which prevents the usual clotting. The blood greedily sucked in gradually fills the next region of the gut - the crop-which bears on each side eleven storing pockets. These become wider and more capacious towards the hind end, the largest terminal pair forming two great sacs on each side of the comparatively narrow posterior part of the gut. As all the pockets point more or less backwards, it is evident why a leech to be emptied of the blood which it has sucked must be pressed from behind forwards. The pockets filled, the leech drops off its victim, seeks to retire into more private life, and digests at leisure. The digestion does not take place in the pockets, but in a small area just above the beginning of the terminal part or rectum. "This rectum, running between the two last pockets, is separable from the true stomach just mentioned by a closing or sphincter muscle. It ends in a dorsal anus above the hind sucker.

\section{The Vascular System.}

The vascular system consists of four main vessels running longitudinally, one above the gut, one round about and obscuring the nerve cord, and one on each side of the body. These are all connected with one another by looping vessels, and all give off numerous branches which riddle the spongy body. The main side vessels are most distinct, are contractile throughout, and give off to the skin, gut, and excretory organs, a rich supply of branches. The dorsal and ventral vessels, though quite distinct, are less delinite, being rather blood spaces than well-formed vessels. 'That the lateral vessels do most of the work of circulation is certain, but the precise course of the blood is not satisfactorily known. 'The blood itself is a red fluid with floating colourless cells diverse in form. 


\section{The Excretory System.}

The excretory system includes seventeen pairs of excretory

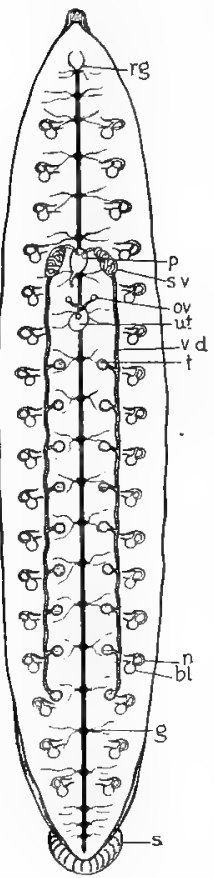

FIG.7 I.-Dissection of Leech.

rg., Nerve ring around cesophagus, here incomplete; p., penis; s. $\%$.; seminal vesicle; $\sigma \tau^{\prime}$., ovary ; ut., uterus ; v.d., vas deferens; $t$., one of the testes; $n$., nephridium with bladder (bl.); g., a cord ; s., posterior sucker. ganglion on ventral nerve

tubules or nephridia, opening laterally on the ventral surface, ending blindly within the body, but extracting waste products from the blood vessels which cover their walls. Each consists of two parts, a twisted horse shoe shaped glandular region where the actual excretory function is discharged, and a spherical, internally ciliated bladder opening to the exterior. Within the latter there is a whitish fluid in which microscopic examination shows numerous waste crystals. The nephridia secrete a clear fluid which helps to keep the skin moist, and thus makes respiratory diffusion easier.

The Reproductive System.

The leech, like many other Invertebrates, is hermaphrodite, containing both male and female reproductive organs. The essential male organs or testes are diffuse, being represented by nine pairs, lying on each side of the nerve cord in the middle region of the body. Each is a firm globular body, within which mother sperm cells divide into balls of sperms. 'The spermatozoa pass from each testis by a short canal leading into a wavy longitudinal vas deferens. This duct followed towards the head forms a coil (so-called seminal vesicle) as it approaches the ejaculatory organ or penis. From the coil on each side the sperms pass into a swollen sac at the base of the penis, where by the viscid secretion of special ("prostate") glands, they are glued together into packets or spermatophores. These pass up 
the narrow canal of the muscular penis, pass out on the middle ventral line between rings thirty and thirty-one, and are transferred in copulation to the female duct of another leech.

The female organs are more compact. The two small tubular and coiled ovaries are enclosed in a spherical vesicle, the walls of which are continued as two oviducts which unite together in a convoluted common duct. This is surrounded by a mass of glandular cells, which exude a glairy fluid into the duct. Finally, the duct opens into a relatively large muscular sac-the "uterus," which opens through a sphincter muscle on the middle ventral line between rings thirty-five and thirty-six.

The favourite breeding time is in spring Two leeches fertilise one another, uniting in reverse positions so that the penis of each enters the uterus of the other. Spermatophores are passed from one to the other, and the contained sperms may remain for a long time within the uterus, or, liberated from their packets, may work their way up the female duct, meeting the eggs at some point, or reaching them even in the ovaries.

The development has been most carefully worked out for the little leech Clepsine, and we shall follow it there.

\section{Development of Clepsine.}

The eggs are laid in water, and surrounded by a cocoon ; they are large, and contain much food yolk.

Cleavage is complete but unequal. At the four cell stage, there are three sub-equal smaller cells and one larger posterior cell, which marks the future hind end of the body. From each of these cells a small cell is cut off, and in this way four macromeres and four micromeres are produced. The number of micromeres is continually increased by the splitting off of cells from the macromeres, so that a disc of small cells is formed. Except for this continued splitting off of small cells, three of the four macromeres remain passive for a considerable period; they contain most of the food yolk, and serve as reservoirs of nutriment. The other, or posterior, macromere divides into two cells of unequal size. the larger speedily again divides into two primitive mesoblasts, the smaller clivides into eight symmetrically arranged cells, the neuro-nephroblasts. At this time free nuclei appear in the other three macromeres (entohlasts) without any corresponding process of cell division, these surround themselves with protoplasm, and form the encloderm cells lining the gut. The clisc of small cells is now spreading over the surface of the entoblasts, over the neuro-nephroblasts, and over the primitive mesoblasts, which 
have sunk slightly inwards. The small cells are ectodermic, they contribute to the formation of the epiclermis, and apparently form also the ectoderm of the head region. The ectodernic structures of the body, on the other hand, are formed by the eight neuro-nephroblasts. These, together with the two mesoblasts underlying them, undergo continuous division in a forward direction, and so produce long rows of cells-the germ bands. The two germ bands are widely separated posteriorly, but commence to unite anteriorly, the union travelling backward. As the neuro-nephroblasts must be regarded as ectodermic in origin, we see that the spreading of the micromeres over the surface of the egg, and the union of the germ bands, constitute together the delayed epibolic gastrulation.

Each germ band consists of three layers, first a thin epidermic stratum, then the layer of the neuro-nephroblasts, and finally the mesodermic layer. Of the neuro-nephroblasts, the inner two form the ventral nerve chain, the next two on either side the nephridia, while the fate of the outer on each side is unknown. The mesoblast rows give rise to the mesoderm, the gut is formed by the entoblasts, and an anterior ectodermic invagination forms the pharynx. At this stage the embryos leave the cocoon and attach themselves to the ventral surface of the mother. A little later the form of the body becomes approximated to that of the adult, and an anus is formed by the fusion of ectoderm and endoclerm.

The most interesting point about this development is that, although the method of gastrulation differs widely from that of Lumbricus, the history of the germ bands shows very marked resemblances in the two forms. It can hardly be that these resemblances are due to adaptation, so that we must consider that development confirms the view which is otherwise probable, that the leeches are true Annelida. In Clepsine the eight neuro-nephroblasts are not, as in Lambricus, obviously ectodermic in origin, but are early covered over by the ectodermic micromeres. Both analogy and the future course of development, however, prove that they do, nevertheless, belong to the outer layer, and that their position is due to a hastening of events.

\section{Classification.}

I. Rhychobdellidre, in which the fore part of the pharynx can be protruded as a proboscis. There is an anterior as well as a posterior sucker. The blood plasma is colourless. The ova are large and rich in yolk; the embryos are hatched at an advanced stage, and soon leave the cocoon, which contains no albuminous fluid. e.g., Clepsine, Pontobdella, Branchellion.

2. Gnathobdellide, in which there is no proboscis, but the pharynx usually bears three tooth plates. The mouth is suctorial. The blood plasma is red. The ova are small and without much yolk; the enbryos are hatched at an early stage, and swim about in the nutritive albuminous fluid of the cocoon.

$$
\text { e.g., Hirudo, Hiemopis, Homadipsa, Aulastoma, Nepletis. }
$$




\section{Appendix (I) to Annelid Series.}

\section{Class Chetognatha. Arrow Worms.}

There are two little marine "worms," Sagitta and Spadella, which are so different from all others, that they have been placed in a class by themselves. It is possible to regard them as Annelids with three segments.

The translucent body, which is about an inch long, has three distinct regions, - a head bearing a ventral mouth with spines and bristles (whence the name Chrtognatha), a median region with lateral fins, and a trowel-like tail.

The nervous system consists of a supra-œsophageal ganglion in the head, a sub-cesophageal about the middle of the body, long commissures between them, and numerous nerves from both. There are two eyes and various patches of sensitive cells.

The food canal is complete and simple; it lies in a spacious ciliated body cavity, which arises in the embryo as two pockets (coelome pouches) from the primitive gut cavity or archenteron. Corresponding to the external divisions, the cavities of head, body and tail are distinct.
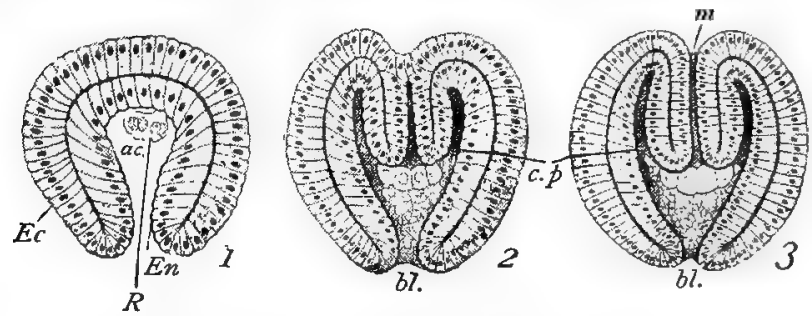

FIG. 72.-Development of Sagitta (after O. HeRTwIG), illustrating formation of a body cavity by pockets from the archenteron, and early separation of reproductive cells $(R$.$) .$

$E_{c .,}$ Ectoderm ; En., endoderm ; ac., archenteron; $R$., reproductive cells; $b l$., blastopore; $c p$. , caelome pouches; $m_{\text {. }}$, mouth; x. section of gastrula; 2 and 3 . origin of coelome pouches.

There is no vascular system, nor are there any certain nephridia. It is possible that the latter may be represented by the genital ducts.

The animals are hermaphrodite, and the simple reproductive organs lie near one another posteriorly. The two ovaries project into the body cavity, and their ducts open laterally where body and tail meet. The two testes project into the cavity of the tail ; and their ducts have internal ciliated funnels, and open on the tail. It is interesting to know that two reproductive cells are set apart at a very early stage, and that each divides into the rudiment of an ovary and of a testis.

The development is very regular. The eggs undergo complete segmentation; a gastrula is formed by the invagination of a hollow ball of cells; the body cavity arises in the form of two pockets from the gastrula cavity or archenteron. 


\section{Appendix (2) to Annelid Series. \\ Class Rotatoria. Rotifers.}

Rotifers are beautiful minute animals, abundant in fresh water, also found in damp moss, and in the sea. They owe their name and the old-fashioned title of wheel animalcules to the fact that the rapict movements of cilia on their anterior end produce the appearance of a rotating wheel. The food seems to consist of small organisms and particles caught in the whirlpool marle by the lashing cilia. The little animals are tenacious of life, and can survive prolonged drought. If they are left dry for long, however, they die, though the ova may survive and sulsequently develop.

The body is usually microscopic, and is sometimes (e.g., in Melicerta and Floscularia) sheltered within an external tube. There is no internal segmentation, but there are sometimes external rings, and a ventral outgrowth or "foot" is sometimes segmented. The anterior end bears, on a retractile ridge, the ciliated ring or "trochal apparatus."

The nervous system is a single dorsal ganglion with a few nerves. An unpaired eye and some tufts of sensory hairs are usually present.

The food canal extends along the body in a well-developed colome, and the fore gut contains a mill in which two complex hammers beat upon an anvil. The canal ends posteriorly on the dorsal surface between the body and the foot, and as the terminal portion also receives the excrefory canals and the oviduct, it is called a cloaca.

There is no vascular system, but a nephridial tube of a primitive type lies on each side of the body, and opens posteriorly into the cloaca.

The sexes are separate; the reproductive organs are simple. Except in the marine parasite Seison and two other forms, the males are dwarfed and degenerate, destitute even of a true food canal. In many cases at least, sexual union (effected by a penis) seems to be ineffective, and there is no doubt that many, if not most, Rotifers are parthenogenetic. The females lay three different kinds of eggs, according to their conditions and constitution - either small ora, which become males, or thin shelled "summer ova," or thick shelled "resting or winter ova," the two last developing into females. Many species, however, are viviparous. We include the Kotifers besicle the Annelicls proper, because it seems possible to regard them as derived from ancestors somewhat like Annelid larvæ.

Rotifers living in fixed tubes or envelopes,-MTelicerla, Floscularia, Stephanoceros.

Free Rotifers,-Notommata, Hydatina, Brachionus.

Parasitic on the marine Crustacean Nebalich,-Seison.

Pedalion occupies a unique position; it has hints of appendages and a peculiar jumping motion.

Equally incerta sedis, but plausibly regarcled as a specialised Trochosphere, is the genus Dinophilus, with the nature of which advanced students should make themselves acquainted.

At this stage I may also mention that there are several sets of small worm-like animals of which we know very little. It is quite possible 
that some of them may lecome of great interest to the systematic zoologist, but we do not yet understand what places in the system they should occupy. Moreover, as they are small, unfamiliar, and unknown to myself, I shall simply refer the curious to what more complete works say about the Gasterotricha, Echinoderidae, Demoscolecidre, and Chretosomidre.

\section{Aspendix (3) to Annelin Series. \\ Class Sipunculoidea, e.g., Sipunculus.}

Marine worms usually living in the sand. The body is elongated and apparently unsegmented. The oral or anterior region can be invaginated by special muscles. There are no setre. They are sometimes, but perhaps erroneously, placed beside Echiuride as Gephyrea Achæta. The nervous system consists of an cesophageal ring, and a median ventral nerve cord, which shows slight hints of segmentation. There is a spacious body cavity.

SipunCULid.s.
The anus is dorsal and anterior, and
the food canal is usually in a spiral : the
mouth is surrounded by tentacles. There
is a closed vascular system, with branches
to the tentacles.
An anterior pair of nephridia serve also
as genital ducts, removing the repro-
ductive cells from the body cavity. The
sexes are separate.
Examples-Sipunculus.
Phascolosoma.

\section{PRiAPUlide.}

The alimentary canal is straight or slightly looped, and the anus is dorsal and posterior. There are no tentacles.

There is no vascular system.

No anterior nephridia, but a pair of tubes open besile the anus, and are said to be excretory in the young, genital in the adult. The sexes are separate.

Examples-Priapulus. Halicryptus.

\section{Appendix (4) to Annelid Series.}

Under the old term Molluscoidea are sometimes included the three classes--Phoronoidea, Polyzoa or Bryozoa, and Brachiopoda. Prof. Lang includes them along with Sipunculoids in the provisional group Prosopygii.

The Molluscoiclea are characterised by the presence of a true coelome, formed in development by the folding off of pouches fiom the archenteron, and by the shortening of the dorsal region of the body, which results in the close approximation of mouth and anus. The mouth is typically furnished with ciliated tentacles, and is often overhung by an epistome; both tentacles and epistome, when present, contain spaces which are part of the body cavity. Except in Polyzoa, two or four nephridia are present, and serve also as genital ducts. There is always a metamorphosis in levelopment, and the larve are peculiar.

\section{Class Phoronoidea.}

The crown of tentacles is shaped like a horse shoe, each tentacle is supported by an internal skeleton. The nervous system lies in the 
ectoderm, and consists of a ring round the mouth, and of a cord down the left side of the body. There is a closed vascular system with nucleated red cells. The body cavity is well-developed. The sexes are united. The larva, known as an Actinotrocha, is a much modified trochosphere.

Phoronis, the only genus, is a worm-like marine animal, always found enclosed in a fixed leathery tube, and social in habit.

\section{Class PolyzoA.}

As usually defined the class includes two sub-classes, the Ectoprocta and the Entoprocta, but it seems doubtful whether the Entoprocta should not be raised to the dignity of a distinct class.

The Ectoprocta include fresh water and marine forms in which the anus is outside the basis of the tentacles. The nervous system is represented by a ganglion placed between the mouth and anus. There is no vascular system. In Cristatella, at least, there are two nephridia. All are colonial and bud very freely; the marine forms show considerable division of labour among the members of the colony.

(a) Tentacles in a crescent-Fresh water, Cristatella, Lophopus, etc.

(b) Tentacles in a circle-Marine, except Paludicella; Flustra, the common sea-mat ; Membranipora, encrusting seaweed, etc. ; Cellepora, very calcareous; Alcyonidium, gelatinous.

The Fntoprocta include the colonial Pedicellina, with a few allied genera, and Urnatella, also the non-colonial Loxosoma, in which the buds separate as soon as they are formed. All are stalked and minute. The anus is included within the tentacular circle. In the metamorphosis of Pedicellina, there is an elongation of the dorsal region of the body, and a consequent approximation of the mouth and anus on the shortened ventral surface. There is no apparent body cavity in the adult, and the mesoderm arises from two primitive mesoblasts. The nephridia are anterior, minute, and do not serve as genital ducts, but resemble the "head kidneys" of Annelid trochospheres. In all these three respects the Entoprocta cliffer from the Ectoprocta, and from the Molluscoidea generally, but the significance of this is uncertain, more especially as it is possible that the differences may in part arise from defective observation.

\section{Class Brachiopoda.}

The Brachiopods or Lampshells are quaint marine animals, once very numerous, but now decadent. The borly is enveloped dorsally' and ventrally by two folds of skin or mantle, these secrete a shell, usually of lime, but sometimes organic. The development of this shell has apparently modified both the position and the relations of the organs. There is no real resemblance between a Brachiopod shell and that of a bivalve Mollusc, except that both consist of two valves. In Brachiopods these lie dorsally and ventrally, in Lamellibranchs they are lateral : moreover, in Brachiopods the ventral valve is usually the larger. It is hardly necessary to say that the Brachiopod organism is not the least like a Mollusc. 
A considerable part of the space between the valves of the shell is filled up by two long "arms," which are coiled in a spiral, and often supported by a calcareous skeleton. These arise in development from the specialisation of a horse-shoe shaped "lophophore," such as is characteristic of the Polyzoa. The mouth is placed between the arms, and opens into the ciliated food canal. This may end blindly, or may be furnished with an anus placed near the mouth; in Crania the anus is dorsal and posterior. The muscular system is well-developed, the shell is both opened and closed by means of muscles. There is a nervering round the gullet, with a slight brain and an inferior ganglion. Sensory structures in many cases perforate the valves. Above the gut lies the heart, which is connected with blood vessels. Two (or more rarely four) nephridia open near the mouth, and

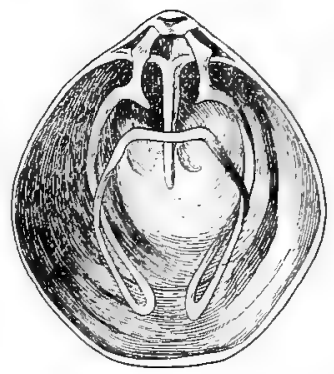

FIG. 73.- Interior of Brachiopod Shell, showing calcareous support for the "arms." (After Davinson.) serve also as genital ducts. The posterior region of the body often forms a stalk by which the shell is moored, but in many this stalk is absent, and the animal is directly attached to the substratum. The sexes are sometimes separate, but perhaps some are hermaphrodite. There is a metamorphosis in the development, and the larve resemble those of Polyzoa. Of the details little is yet known.

\begin{tabular}{l|l}
\multicolumn{1}{c|}{ Testicardines. } & \multicolumn{1}{c}{ Ecardines. } \\
\hline The valves are hinged. & $\begin{array}{l}\text { There is no hinge. } \\
\text { There is no anus. }\end{array}$ \\
Terebratula. Waldheimia. & $\begin{array}{l}\text { Crania. } \\
\text { Lingula, persistent since Palaozoic } \\
\text { ages. }\end{array}$
\end{tabular}




\title{
CHAPTER XII
}

\author{
ECHINODERMA.
}

Class I. Holothuroidea (Scytoderma). Sea Cucumbers.

2. Echinoidea. Sea Urchins | EleutherozoA

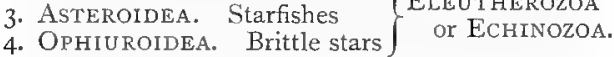

5. CrinoIDEA. Feather stars

6. Blastoldea. Extinct $\}$ Pelmatozoa.

IN contrast to the "worms," the Echinoderms form a well-defined series. They may be described as sluggish marine animals, generally of radiate symmetry, with a tendency to form limy skeletons. The radial symmetry led the older zoologists to place the Echinoderma near Coelentera, but the larval Echinoderm is more specialised than most of the larval "worms," and is bilateral in its symmetry. It seems likely that the adult radial symmetry is an adaptation to sedentary life, and that the Echinoderms represent an offshoot of some "worm" stock. Yet it is interesting to notice that in both Colentera and Echinoderma the nervous system shows a marked absence of centralisation, which may be connected with the absence of a definite head region, and this again with the relatively sedentary habit.

General Characters. - The Echinoderms include forms in which the bilateral symmetry of the larva is replaced in the adult by radial symmetry. In addition to the dominant radial symmetry, the adults showe to a varying. extent a tendency towards a biluteral form, but this is never the same as that of the larva, nor is it equivalent in the different types. Lime is always deposited in the 
mesodermic tissues (mesenchyme), and in consequence there is frequently a very complete skeleton. From the primitive gut of the larva, pouches grow out to form the usually spacious colone and the characteristic water vascular system. The branches of this system, together with the nerves, exhibit in most cases a typical five-rayed arrangement. In development there is a marked distinction between mesoblast derived from gut pouches, and mesenchyme produced by immigrant amoboid cells. There is usually a very striking circuitousness or indirectness in development.

The Echinoderms are all marine. By reason of their

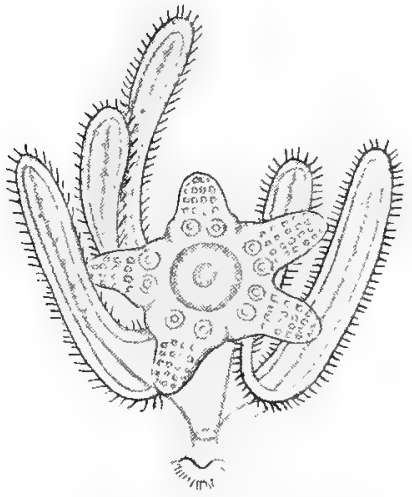

FIG. 74-- Pluteus larva with rudiment of adult.

(After Johannes Múllekr.)

durable skeletons, they are extremely well represented as fossils, yet this does not alter the fact that the group is well-defined, and shozes no close relation to any other, whether in its living or extinct representatives.

The average habit is suggish, and this may be correlated with the constant development of lime in the tissues. This pozer of forming skeletal substance is indeed so dep-seated that lime may appear in almost any of the orinans of the body. The diet is vegetarian (most sea urchins), carnivorous (starfishes), or consists of the organic particles found in sand 
and mud, the Holothurians in particular practising this worm-like mode of nutrition.

Most Echinoderms have to a remarkable extent the pozer of casting off and regenerating portions of their body. This power is frequently reckoned as one of their means of defence, but they often mutilate themselves merely as a consequence of unfavourable conditions of life. The self-mutilation, or autotomy, as it is called, seems to be entirely a reflex action, not voluntary.

The peculiar water vascular system attains great development, and has usually respiratory or locomotor functions. It is possible that in some cases it may also function as an organ of excretion. Well-defined excretory organs are conspicuously rare. Soluble waste products seem generally to diffuse out into the water, while the insoluble are here, as in sea squirts, stored up in the tissues in the form of granular masses.

The Holothurians are in form nearest to the supposed worm-like ancestor, and are perhaps primitive forms, which do not lead up to any of the other classes. From primitive unspecialised Cystoids, the Echinozoa, and Pelmatozoa have perhaps taken origin. Of the Echinozoa the Asteroidea and Ophiuroidea are very closely related, ancl seem to be connected by fossil forms.

In our survey of the group it is more convenient to begin with the familiar starfishes than with the more primitive forms. The general characters of each class may be read from the synoptic table at the end.

\section{Class Asteroidea. Starfish.}

The description applies especially to the common fiverayed starfish (Asterias or Asteracanthion rubens). It is often seen in shore pools exposed at low water, but its haunts are on the floor of the sea at greater depths. There it moves about sluggishly in any direction by means of its tube feet.

Form.-Each of the five arms bears a deep ventral groove in which the tube feet are lodged. The mouth is in the middle of the ventral surface, the food canal ends about the centre of the dorsal disc. With this flat, five-rayed form, the II-I 3 rayed sun star (Solaster), the pincushionlike Goniaster, and the flat pentagonal Palmipes, should be contrasted. 
Integument.-(a) The body is covered by a ciliated ectoclerm. This inclucles supporting, glandular, and sensory cells, and beneath it there is a network of nerve fibrils with ganglionic cells.

(b) The micllle layer of the integument consists of a double stratum of ground substance, the outer part of which contains the chief limy structures except the ambulacral ossicles which are formed more internally. There is also a thin muscular layer. The whole of this middle layer is formed in development from the mesenchyme tissue.

(c) Internally the body wall is lined by a ciliated epithelium, derived in development from the wall of the colomic pouches. (See Development.)

Between two of the arms lies the perforated madreporic plate, the entrance to the water vascular system, thus defining the bivium, while the other three arms constitute the trivizm.

\section{The Calcareous Skeleton.}

In association with the inner mesodermic layer of the integument, there is developed on the ventral surface of each arm a double series of sloping plates. These two series meet dorsally, like rafters, in the middle line of the arm, forming an elongated shed. The rafter-like plates are called ambulacral ossicles; the groove which they bound lodges the nerve cord, the blood vessel, the water vessel, and the tube feet of each arm.

In asscciation with the outer mesodermic layer of the integument, numerous smaller plates are developed, e.g., the adambulacrals, which articulate with the outer lower ends of ambulacrals. The dorsal surface bears a network of little ossicles, and many of these bear spines. Peculiarly modified spines, known as pedicellarice, look like snapping scissor blades mounted on a single soft handle. They have been seen gripping Algæ and the like, and probably keep the surface of the starfish clean.

\section{Muscular System.}

A starfish is not very muscular, but it often bends its arms upwards by means of the muscular layer noted above, and may sometimes be seen tightly embracing an oyster. Other muscles affect the size of the ventral grooves, and muscular elements also occur on the protrusible part of the stomach, and in connection with the water vascular system. 


\section{Nervous System.}

Underneath the ciliated ectoderm lies a network of nerve fibrils, with some ganglionic cells. But besides these diffuse elements there is a pentagon around the mouth, and a nerve along each arm. The system is not separable from the skin.

\section{Sense Organs.}

A red eye spot, sensitive to light, lies on the terminal ossicle at the tip of each arm, and is usually upturned. It

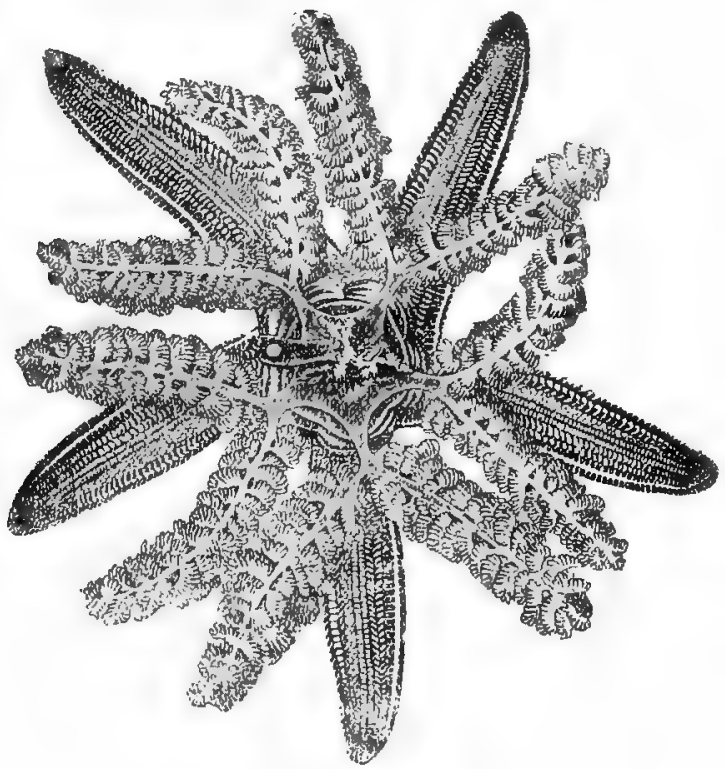

FiG. 75.-Alimentary system of Starfish. (After MüLLEK and TrosCheL.)

The dorsal surface has been removed; the digestive caca, the stomach, \&c., are shown.

is a modified tentacle, bearing numerous little cups, lined by sensitive and pigmented cells, containing clear fluid, and covered by cuticle. The skin is diffusely sensitive. The terminal tube foot of each ray seems to be olfactory. 


\section{Alimentary System.}

The starfish is fond of young oysters and other bivalves, and may be found with part of its stomach extruded over them. This protrusible or cardiac portion of the stomach is glandular and sacculated, and bulges slightly towards the arms; it is followed by an upper or pyloric portion, giving off five branches, each of which divides into two large digestive cæca, a pair in each arm (Fig 75.) These glands contain a yellowish pigment (enterochlorophyll) and secrete tryptic, peptic, and diastatic ferments. From the short tubular intestine between the stomach and the almost central dorsal anus two little outgrowths are given off, perhaps homologous with the "respiratory trees" of Holothuroids. Some parts of the food canal are ciliated.

\section{Body Cavity.}

The colome is distinct, though not much of it is left unoccupied either in the disc or in the arms. It is lined by ciliated epithelium, and contains a fluid with amcboid cells. A few of these have a pigment which probably aids in respiration ; others are phagocytes, which get rid of injurious particles through the "skin gills;" others continue the work of digestion.

\section{Water Vascular System.}

When we watch a starfish crawling up the side of a rock we see that scores of tube feet are protruded from the ventral groove of each arm, that these become long and tense, and that their sucker-like terminal discs are pressed against the hard surface. There they are fixed, and towards them the starfish is gently lifted. The protrusion is effected by the internal injection of fluid into the tube feet, the fixing is due to the subsequent withdrawal of the water producing a vacuum between the ends of the tube feet and the rock.

As to the course of the fluid, it is convenient to begin with the madreporic plate, which lies between the bases of two of the arms (the bivizum). This plate is a complex calcareous sieve, with numerous perforating canals and external pores. It may be compared to the rose of a watering pan, but the holes are much more numerous, and lead into small canals which converge into a main ciliated canal. The latter rums down through the body, and is like a complex calcareous filter. It is called the stone canal. 
The stone canal leads into a water ring round about the mouth. From this circumoral ring are given off nine glandular bodies (Tiedemann's hodies), and five radial tubes, one for each of the arms. Considerations of symmetry suggest that there should be ten glandular bodies, lout the stone canal has taken the place of one. In many starfishes there are five or ten little reservoirs (Polian vesicles) opening into the circumoral ring, but in Asterias rubens these are hardly clistinguishable from the first ampullee of the radial vessels.

Along each arm, then, there runs a radial vessel. It lies in the ambulacral groove beneath the shelter of the rafter-like ossicles. From it branches are given off to the bases of the tube feet, but from each of these bases a canal ascends between each pair of ambulacral ossicles, and expands into an ampulla or reservoir on the dorsal or more internal sicle. The fluid in the system may pass from the radial vessels into the tube feet, and from the tube feet it can flow back, not into the radial vessel,

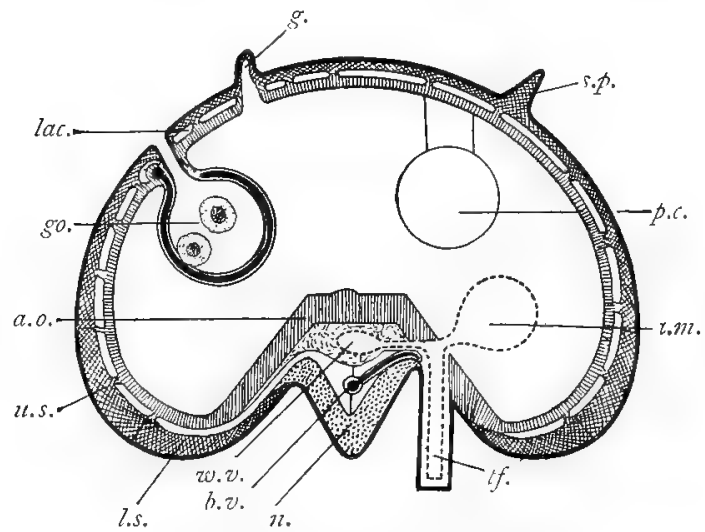

FIG. 76.-Diagrammatic cross section of starfish arm. (After Ludwig.)

n., radial nerve; $b_{.} z^{\prime}$, radial blood vessel according to Ludwig, septum in blood vessel according to others; zu.zr., radial water vessel ; am., ampulla; $t f$. , tube foot; $p_{\text {. }}$, , a pyloric cæcum cut across; s.p., a calcareous spine; $g$., a skin gill; lac., spaces in the skin; go., ova in ovary; a.o., ambulacral ossicle.

but into the ampullie. There are muscles on the walls of the tube feet, ampullee, and vessels. At the end of each arm, there is a long unpaired tube foot, which seems to act as a tactile tentacle, and has also olfactory significance.

To recapitulate, the madreporic plate leads into the stone canal, this passes into the ring round the mouth with its nine resicles, from the ring radial vessels run along the arms, they give off branches to the tube feet, and the base of each tube foot communicates with an ampulla. 
Vascular System.-We have not yet reached certainty in regard to this system. German authorities, e.g.n Ludwig, describe (I) a radial blood vessel above the nerve in each arm; (2) a circumoral vessel around the month; (3) a heart lying beside the stone canal and leading into (4) an aboral ring which gives off vessels to the genital organs.

But others say that the so-called "heart" is a solid glandular organ, that the aboral ring is merely the connecting strand or rhachis of the genital organs, and that the radial and circumoral vessels described are really thickened septa within the true vessels.

French authorities describe $(a)$ a radial perihremal space or blood vessel divided by a median mesentery, and $(b)$ the union of these in a circumoral ring. But the latter encloses (c) another annular vessel with which a sinus $(d)$ surrounding the stone canal communicates. Finally, an aboral pentagon (e) gives off five pairs of genital blood vessels.

\section{Respiratory System.}

From the dorsal surface and sides of a starfish in a pool, numerous transparent processes may be seen hanging out into the water. They are the simplest possible respiratory structures, contractile outgrowths of the skin, with cavities continuous with the coelome, and are called "skin gills." It is likely that pigmented cells of the body cavity fluid act like rudimentary red blood corpuscles; the water vascular system may help in aeration; and the whole body is of course continually washed with water.

\section{Excretory System.}

The "skin gills" are said to have an excretory function; for phagocytes, bearing waste, seem to traverse their walls. It may also be that excretion is somehow concerned in forming the carbonate of lime skeleton, but facts are wanting.

\section{Reproductive System.}

The sexes are separate, and they are like one another, both externally and internally. The organs develop periodically, and lie in pairs in each arm. Each is branched like an elongated bunch of grapes, and is surrounded by a blood sinus. Each has a separate duct, which opens on a porous plate, between the bases of the arms on the dorsal surface. In Asterina gibbosa, however, the eggs are extruded ventrally. The eggs are fertilised in the water, and the free swimming larva, which will be described along with those of of the other classes, is known as a Bipinnaria or as a Brachiolaria. 


\section{Other Starfishes.}

Astropecten and most forms related to it have blind food canals; Brisinga has 9-12 long arms, arising abruptly from a small disc as in Brittle stars, and has no ampulla, eye spots, or skin gills ; Luidia has three-bladed pedicellarice; in most forms the genital ducts end on plates with a single aperture, and so on.

The commonest European forms are species of Asterias or Asteracanthion, Astropecten, Cribrella, Solaster, Goniaster.

The largest are such as Asterias gigantea (from the Pacific coast of N. America), measuring 2 feet in diameter, or Pycnopodia helianthoides, about a yard in diameter, and with over twenty arms.

There are many deep sea forms, such as the ophiuroid-like Brisinga, the widely distributed Hymenaster, and the blue Porcellenaster carnlezs, but the majority occur in water of no great depth.

Parental care is incipient among Asteroids, for a large Asterias has been seen sheltering its young within its arms : there is a definite brood pouch in the form of a sort of tent on the dorsal surface of Pteraster.

Many Asteroids break very readily, or throw off their arms when these are seized. Professor Forbes describes how a fine specimen of Luidia thus escaping him gave a "wink of derision" as it passed over the side of the boat. The lost parts are slowly regenerated, and strange forms are often found in process of regrowth. Thus the "comet form" of starfish occurs when a separated arm proceeds to grow the other four. Asteroidea first occur in Silurian strata.

\section{Class Ophiuroidea. Brittle stars, e.g., the common Ophiopholis bellis.}

The body of a brittle star differs from that of a starfish in the abruptness with which the arms spring from the central disc (cf. Brisinga). These arms are muscular, and useful in wriggling and clambering; they do not contain outgrowths of the gut, nor reproductive organs. Moreover there is no ambulacral groove, and the tube feet which project on the sides are too small to be of locomotor service. The madreporic plate is situated on the ventral surface, usually on one of the plates around the mouth. The food canal ends blindly.

'The reproductive organs lie in pairs between the arms, and open into pockets or bursæ formed from inturnings of the skin, which communicate with the exterior by slits opening at the bases of the arms. Water currents pass in and out of these pockets, which probably have both respiratory and excretory functions. 
The free swimming larva is a Pluteus, very like that of Echinoids.

Ophiuroids are first found in Silurian strata.

I. Euryalida. Skin without plates, arms simple or branched and capable of being rolled up.

Astrophyton. Gorgonocephahus.

2. Ophiurida. Skin with plates, arms simple.

Ophiopholis, Ophiocoma, Ophiothrix, are common genera.

Amphizura squanata is hermaphrodite.

Class EchiNoIDEA. Sea Urchins, e.g., the common Echinus edulis, Strongylocentrotus lividus.

Most sea urchins live off rocky coasts, and not a few shelter themselves sluggishly in holes. They move by means of their tube feet and spines, and seem to feed on seaweeds, and on the organic matter found in mud and other deposits. After the perils of youth are past, the larger forms have few formidable enemies.

\section{Form, Skin, and Skeleton.}

The hard and prickly body is more or less spherical. The food canal begins in the middle of the lower surface; it ends at the opposite pole in the middle of an apical disc formed of a central plate surrounded by five "ocular" and five "genital" plates. The ocular or radial plates bear eye specks ; the genital or basal plates bear the apertures of the genital ducts, but one of the five is modified as the madreporic plate. From pole to pole run ten meridians of calcareous plates which fit one another firmly; five of these (in a line with the ocular plates) are known as ambulacral areas, for through their plates the locomotor tube feet are extruded; the five others (in a line with the genital plates) are called inter-ambulacral areas, and bear spines, not tube feet. Altogether, therefore, there are ten meridians, and each meridian area has a double row of plates. On the dry shell from which the spines bave been scraped, the ambulacral plates are seen to be perforated by small pores, four pairs or so to each plate. Through each pair of pores a tube foot is connected with an internal ampulla. In the starfish the ambulacral areas are wholly ventral, and the 
apical area seen on the dorsal surface of the young forms is not demonstrable in the adult.

The "posterior" ambulacra, those between which the modified basal or madreporic plate lies, are often distinguished as the "bivium," the other three form the "trivium," and the middle one of the three is "anterior."

On the shell there are obviously many spines, most abundant on the inter-ambulacral areas. Their bases fit over ball-like knobs, and are moved upon these by muscles. But besides these, there are two modified forms of spines, (a) the minute pedicellaria, with three snapping blades on a soft stalk, and sometimes with apical glands; and (b) small globular sphæridia, which show some structural resemblances to otocysts. It is said that like true otocysts they are concerned with the perception of direction of motion.

In front of the mouth project the tips of five teeth, which move against one another, grasping and grinding small particles. 'They are fixed in five large sockets, and along with fifteen other pieces form "Aristotle's lantern," a complex masticating apparatus, of whose history we know little. It surrounds the pharynx, and is swayed about and otherwise moved by muscles, many of which are attached to five beams which project inward from the margin of the shell round about the mouth.

As in other Echinoderms, the skeleton of lime is mesodermic. The shell is covered externally by a delicate ciliated ectoderm, beneath which, in a thin layer of connective tissue, there is a network of nerve fibres, and some ganglion cells. Internally, there is another thin layer of connective tissue, and a ciliated epithelium lining the body cavity. The skeleton grows by the formation of new plates around the apical disc, and also by the individual increase of each. In a few forms the shell retains some plasticity.

\section{Nervous System.}

The nervous system consists of a ring around the mouth, of radial branches running up each ambulacral area, and of the superficial network. Tube feet, sphæridia, pedicellariæ, and spines are all under nervous control, and each radial nerve ends in the "eye specks" of the apical "ocular plates." It is probable that all the tube feet are sensory, and 
this is certainly the main function of ten which lie near the mouth.

\section{Alimentary Canal.}

The alimentary canal passes through Aristotle's lantern, and the intestinal portion lies in two and a half coils around the inside of the shell to which it is moored by mesenteries. It contains fine gravel, sand, and some organic debris. It ends near the centre of the apical disc, whence the pedicellariæ have been seen removing the fæeces.

Accompanying the first coil of the gut is a canal or "siphon," which opens into the gut at both ends. According to Cuénot, a current of water traverses this tube, which thus, by reason of its thin walls, carries oxygen to the cor-

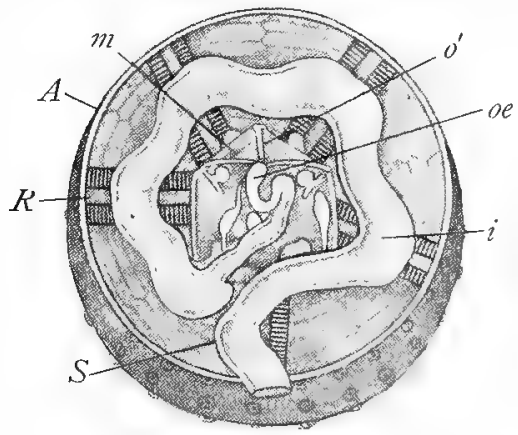

Frg. 77.-Ventral half of Sea Urchin. (From CARUS, after TIEDEMANN.)

$o^{\prime}$, Aristotle's lantern in centre; oe, œsophagus; $i$, intestine; $S$, intestinal blood vessel; $R$, radial water vessel in an ambulacral area ; $A$, an inter-ambulacral area; $n$, muscles of the lantern.

puscles of the body fluid. The spacious body cavity is lined by ciliated epithelium and contains a "perivisceral" fluid, whose corpuscles have a respiratory pigment (echinochrome). When the fluid of a perfectly fresh sea urchin is emptied out, the contained corpuscles unite in plasmodia, forming composite amceboid clots (cf. Proteomyxa, \&c.).

\section{Water Vascular System.}

The madreporic plate communicates with a membranous stone canal, which runs downwards into a circular vessel 
near the upper end of the lantern. This gives off five interradial transparent vesicles, and five radial vessels which run down the sides of the lantern and up each ambulacral area. Each radial vessel gives off numerous lateral branches, which communicate with the internal ampullæ and thence with the external tube feet. When the tube feet are made tense with fluid, they extend beyond the limit of the spines, and are attached to the surface of the rock over which the sea urchin slowly drags itself. The sucker at the tip of each tube foot bears small calcareous plates regularly arranged, indeed there is hardly any part of an Echinoderm in which lime may not be deposited. Before bending upwards from the base of the lantern, each radial vessel gives off a branch to two large tentacle-like tube feet without attaching discs. The five pairs lie near the mouth, and are sensitive.

The Blood Vascular System is not readily traced, and there is uncertainty as to many points. Along the stone canal lies an enigmatical structure, to which such names as "plexiform organ," "ovoill gland," "dorsal organ," and "heart" are given. Its structure is like that of the smaller glandular enlargements found on the vascular system. According to some, it gives origin to some of the amoeboid cells of the body cavity fluid. It is connected superiorly with the five genital organs, inferiorly with a circular vessel surrounding the pharynx at the top of the lantern, within and beneath the water ring. This vascular ring seems to be connected, by branches at least, with the five pockets of the water ring. A distinct vessel arises from the ring and runs along the inner or ventral surface of the intestine, while another on the opposite side seems to originate from capillaries. It is likely enough that there may be radial blood vessels or spaces in the ambulacral areas. The fluid cannot be distinguished from that of the body cavity; it contains corpuscles, some of which have pigment.

\section{Respiratory and Excretory Systems.}

On the area round about the mouth there are ten hollow outgrowths, which resemble the skin gills of starfishes. As already mentioned, the pigmented cells of the body cavity fluid seem able to absorb oxygen. The water vascular system plays here a very important part in respiration. Waste products seem simply to accumulate in the tissues, but Hartog maintains that the water vascular system helps in excretion. 


\section{Reproductive System.}

The sexes are separate, and like one another. Five branched yellow-brown ovaries or rose-white testes lie interradially under the apex of the shell, and open by separate ducts on the five genital or basal plates. In spring the apical disc may be seen covered with orange ova or milky-white spermatozoa.

The eggs are fertilised externally by sperms wafted from adjacent sea urchins, and the free swimming larva, which we shall afterwards describe, is called a Pluteus.

Classification of Echinoidea.

I. Palæo-echinoidea. Extinct forms, apparently with a plastic test, of overlapping and variable plates. They appear in Lower Silurian rocks.

2. Desmosticha. Regular and symmetrical sea urchins like Echinus. e.g., Cidaris, without external gills.

Diadema, a species has been described as covered with compound eyes.

Cyanosona urens, the spines contain a poison apparatus. Echinothuridae have flexible tests.

3. Clypeastroidea. Shield shaped, and often flat. The food canal ends outside the apical disc on the posterior inter-radius.

$$
\text { e.g., Clypeaster. }
$$

4. Petalosticha. Heart shaped. The mouth is ex-centric, the food canal ends away from the apical disc. There are no masticating organs. On the dorsal surface the ambulacral areas dilate from the apex outwards, and contract again towards the margin in the form of "petals." The anterior area is often different from the other four.

e.g., Spatangzes.

Hemiaster and some others carry their young among their spines.

\section{Class Holothuroidea. Sea Cucumbers.}

The Holothurians do not at first sight suggest the other Echinoderms, for they are like plump worms, and the calcareous skeleton is not prominent. But closer examination shows the characteristic pentamerous symmetry, and the occurrence of calcareous plates in the skin. These seem to be absent in the unique pelagic Pelagothuria.

Holothurians occur in most seas, from slight to very great depths. Their food consists of small animals, and of 
organic particles from the sand. Some of them grasp little things in their waving tentacles, and then plunge these into the pharynx. The muscles of a captured Holothurian often over contract and eject the viscera at the ends or through a side rupture; in this way the animal may sometimes escape, and the viscera can be regrown.

The worm-like body is often regular in form, with five equidistant longitudinal bands along which tube feet emerge. But three of these "ambulacral areas" are often approximated on a flattened ventral sole, leaving two on the convex dorsal surface, and there are other modifications of form.

The walls of the body are tough and muscular, and the skeleton is represented by scales, plates, wheels, and anchors of lime scattered in the skin, by plates around the gullet, and on a few other regions.

The nervous system consists of a circumoral ring in which the five radial nerves running in the ambulacral areas unite, and from which nerves to the tentacles arise. Sense organs are represented by the tentacles, which sometimes have "ear sacs" at their bases, and by tactile processes on the dorsal surface of some of the creeping forms.

From the terminal or ventral mouth, surrounded by five, ten, or more tentacles, the food canal coils to the opposite pole. There it expands in a cloacal chamber sometimes contractile, and from this are given off in many forms a pair of much branched "respiratory trees," which extend forward in the body cavity. These are constantly supplied with fresh water by means of the rhythmic contractions of the cloaca, and seem in the adult to be respiratory in function. The body fluid sometimes contains a red pigment, said to be identical with the hæmoglobin of vertebrates. Arising from the base of the left respiratory tree there are remarkable structures, known as Cuvierian organs, consisting of numerous tubes, which are in most cases glandular. The Holothurian can eject these tubes through the cloaca, the wall of which is apparently ruptured in the process. The tubes are very viscid, and seem to grow longer in the water; they will adhere to almost everything but the Holothurian itself.

The water vascular system may consist of a ring around 16 
the mouth communicating $(a)$ with the tentacles, $(b)$ with five radial vessels, one for each ambulacral area, (c) with a "Polian vesicle" or more than one pendent in the body

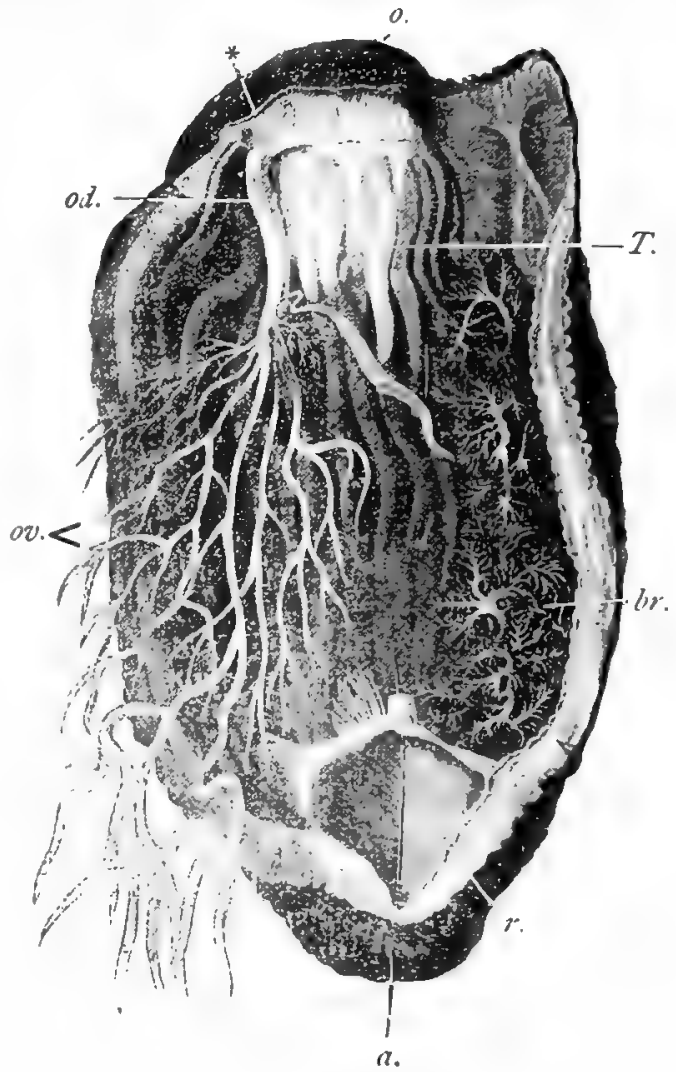

FIG. 78.-Dissection of Holothuria. (After HUNTER.)

The gut has been removed; there is a bristle through anus $(a)$ and cloaca $(r) ; o$, mouth; $T$, the retracted tentacles; $b r, a$ respiratory tree; $o n$, the ovary ; $o d$, the oviduct; *, a bristle at genital aperture.

cavity, $(d)$ with a "stonce canal " which usually hangs freely in the body cavity, and opens into it. The radial ressels may 
have ampullæ and tube feet, as in sea urchins. But there are many divergences, especially in the reduction of the tube feet areas. Instead of tube feet, or along with them, there are often conical processes or papillæ without terminal discs. These are especially common on the dorsal surface. The blood vascular system is not very definite, and seems to consist mainly of spaces in the connective tissue, e.g., around the pharynx and along the intestine.

The sexes are usually separate. The reproductive organs do not exhibit radial symmetry, and are branched tubes which open within or just outside the circle of tentacles. They and other internal organs of Holothurians are often very brightly coloured. The larva is, in most cases, what we shall afterwards describe as an Auricularia. Sometimes, however, the larval stage is skipped, as in Cucumaria crocea and Psolus ephippiger where the eggs and young are attached to the back of the mother. In Cucumaria lavigata there is an invaginated brood pouch; in Synapta vivipara and others the body cavity serves as a brood pouch.

The calcareous plates of Holothurians are found as far back as Carboniferous strata.

\section{Classification.}

I. Elasipoda : primitive deep sea forms, bilaterally symmetrical, with tube feet on the ventral surface only, and with papilla on the back. The stone canal often opens externally by a pore. There are no respiratory trees.

$$
\text { e.g., Kolga, Elpidia. }
$$

2. Pedata : with well-developed tube feet and papillæ. e.g., Holothuria, Cucunaria, Psolus.

3. Apoda : without radial canals, tube feet, or respiratory trees.

e.g., Synapta, a remarkable animal, especially apt to break in pieces; pinnate tentacles; hermaphrodite; with beautiful calcareous anchors and plates in the skin.

Semper has described a strange animal, Rhopalodina lageniformis, from the Congo coast. It is like a globular flask, with mouth and anus close together at the narrow end, with ten ambulacral areas.

Class Crinoidea. Feather Stars.

Commonest Type, Antedon rosaceus.

The feather stars or sea lilies differ from other Echinoderms in being fixed permanently or temporarily by a jointed 
stalk. The modern Comatulids, e.g., the rosy feather star (Comatula or Antedon rosaceus) leave their stalk at a certain stage in life; but the other Crinoids, e.g., Pentacrinus, are permanently stalked like almost all the extinct stone lilies or encrinites once so abundant. Most of them live in deep water, and many in the great abysses. An anchorage is found on rocks and stones, or in the soft mud, and great numbers grow together-a bed of sea lilies. The free Comatulids swim gracefully by bending and straightening their arms, and they have grappling "cirri" on the aboral side, where the relinquished stalk was attached. By these cirri they moor themselves temporarily. Small organismsDiatoms, Protozoa, minute Crustaceans-are wafted down ciliated grooves on the arms to the central mouth, which is of course on the upturned surface. Some members of the class, e.g., Comatula, are infested by minute parasitic "worms" (Myzostomidæ) allied to Chætopods, which form galls on the arms. A lost arm can be replaced, and even the visceral mass may be regenerated completely within a few weeks after it has been lost.

The animal consists of (I) a cup or calyx, (2) an oral disc forming the lid of this cup, (3) the radiating "arms," and (4) the stalk supporting the whole.

The calyx consists of the topmost segment of the stalk, a centro-dorsal plate, and several rows of radial plates, which lead on to the brachial plates of the arms. When Comatulids break off from their larval stalk, they carry with them the centro-dorsal plate, (C.D. in Fig. 79), which becomes the central part of their calyx, and bears the cirri.

The oral disc, turned upwards, is supported by plates. Here the anus also is situater. The arms usually branch in dichotomous fashion, and thus ten, twenty, or more may arise from the original five. But the growing point continues to fork dichotomously, like the leaf of many ferns, and as each alternate fork remains short, a double series of lateral "pinnules" results. The arms are supported by calcareous plates. The stalk usually consists of numerons joints, especially in extinct forms, in some of which it measured over fifty feet in length. Except in Holopus, and in the stalked stage of Antedon, the stalk bears lateral cirri.

The nervous system is remarkable in being double. On the upturned surface of each arm, beneath the food wafting ciliated grooves, there is a subepithelial nervous band, probably in great part sensory (Fig. 79, h). These bands are united in a ring or plexus around the mouth. So far the Crinoid is like a starfish. But on the dorsal surface the main mass lies-an antambulacral motor and sensory nervous system, consisting of a central capsule, with branches to the cirri and to the arms ( $d$ and $a$ in Fig. 79). Cuénot asserts that this system, though to a very slight extent, 
is also represented in starfishes. Apart from the superficial epithelium there are no sensory structures.

The ciliated food canal descends from the mouth into the culp, and curves up again to the anus, which is usually ex-centric in position. The last part of the gut is expanded to form an anal tube, which during life is in constant movement, and has apparently a respiratory function. From the cuip, where the body cavity is in great part filled with connective tissue and organs, two calomic canals extend into each of the arms. They communicate at the apices of the arms and pinnules, and currents pass up one and down the other.

The blood vascular system consists of a circumoral ring, which is connected with a raclial vessel under each ambulacral nerve, and with a circumoesophageal plexus. There is also a "plexiform organ," "lying interradially in the disc anteriorly to the mouth" ( $g$ in Fig. 79). It encloses the barren central part of the reproductive system (the axial genital

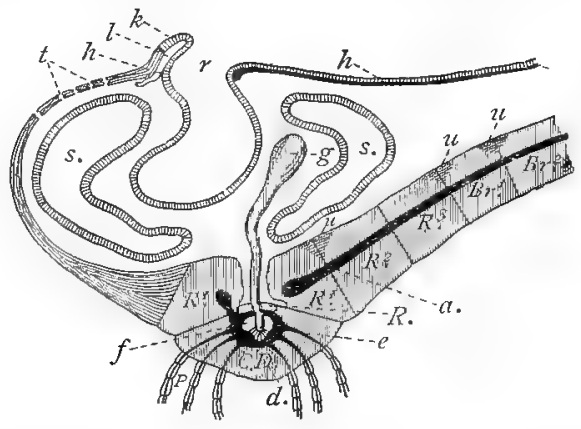

FIG. 79.-Diagrammatic vertical section through disc and base of one of the arms of Antedon Rosaceus. (After Milnes Marshall.)

The section is interradial on the left, radial on the right. $t$, ciliated openings in body wall; $h$, subepithelial ambulacral nerve; $L$, water vascular canal ; $k$., tentacle; $\%$, mouth ; $s$., intestine; $g$., central plexus, with "chambered organ" at its base; $R_{1}-R_{3}$.",

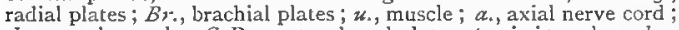
$d$. , central capsule ; C.D., centro-dorsal plate ; $\not$., cirri ; $e_{0}$, branches from central capsule to cirri.

stolon), and has connections with the above mentioned plexus, with the vessels to the organs, and with a strange "chambered organ" which lies within the central aboral nervous system.

The water vascular system consists as usual of a circumoral ring and radial vessels. These lie under the corresponding blood vascular system. But the system is divergent in several ways; $(a)$ water passes into it by several ciliated and branched water tubes which hang from the ring, and from the origins of the radial vessels, into the body cavity; $(b)$ water 
passes from the exterior into the body cavity by numerous (I 500 on the disc of Antedon rosacea) ciliated water pores which pierce the disc, and sometimes the arms also; $(c)$ " the rarlial water vascular vessels give off alternately to the right and left, in groups of three each, delicate tubular branches, respiratory in function, which form the tentacles homologous with tube feet."

The sexes are separate, and a process suggestive of sexual union has been observed in Antedon. The reproductive organs extend as tubular strands from the clisc along the arms, but are rarely functional except in the pinnules, from each of which the elements burst out by one duct in females, by one or two fine canals in males.

There are about 400 living species in twelve genera, but about 1500 species in 200 genera are known from the rocks. The class is obviously decadent. It is represented in the Cambrian, and attained its maximum development in Silurian, Devonian, and Carboniferous times.

The oval ciliated larva of Antedon, the only one known, is less quaint than that of other Echinoderms.

\section{Classification of Crinoidea.}

I. Palro-crinoidea (= Tesselata). Palrozoic forms. The symmetry of the calyx is not always pentamerous.

2. Neo-crinoidea (= Articulata + Holopus and Marsupites).

Mesozoic and recent. The calyx always has pentamerous symmetry. The recent forms include the stalked Pentacrinus, Rhizocrimus, $\mathcal{S}^{*} c_{\text {. }}$, and the free Comatulids, which pass through a stalked Pentacrinus stage, e.g., Antedon.

Holopus is a remarkable deep sea form with direct ancestors in the Upper Silurian. Marsupites is an extinct Crinoid which had no stalk.

\section{Class Blastoidea. Wholly extinct.}

The Blastoids are first found in the Upper Silurian, later than Cystoids and Crinoids; they had their golden age in the Carboniferous and Devonian times, but then disappeared. Their body was ovate, with five ambulacral areas, with each groove of which jointed pinnules were associated.

\section{Class Cystoidea. Wholly extinct.}

The Cystoids are first found in the Lower Silurian rocks, had their golden age in Upper Silurian times, and died out in the Carboniferous ages. Their body was ovate or globular, sessile or shortly stalked, covered with polygonal plates often irregularly arranged. Some (according to Bell, the more primitive) types were "never fixed, and had not fixed ancestors." They seem usually to have borne two to five feeble, unbranched arms.

Both Cystoids and Blastoids seem to have been half smothered in lime, and perhaps this is in part the explanation of their extinction. 


\section{Development of Echinoderms.}

The ovum undergoes total segmentation, and a hollow ball of cells or blastosphere results. Apart from two alleged cases of delamination, the gastrula is always formed by the invagination of this blastosphere. Ectoderm and endoderm, or epiblast and hypoblast, are thus established.

The mesoblast has a twofold origin: (a) from "mesenchyme" cells, which immigrate from the invaginated hypoblast into the segmentation cavity; (b) by the outgrowing of one or more colome pouches from the gastrula cavity or archenteron. It is thus that the body cavity and the rudiments of the water vascular system arise.

According to Hertwig's fundamental thesis this double

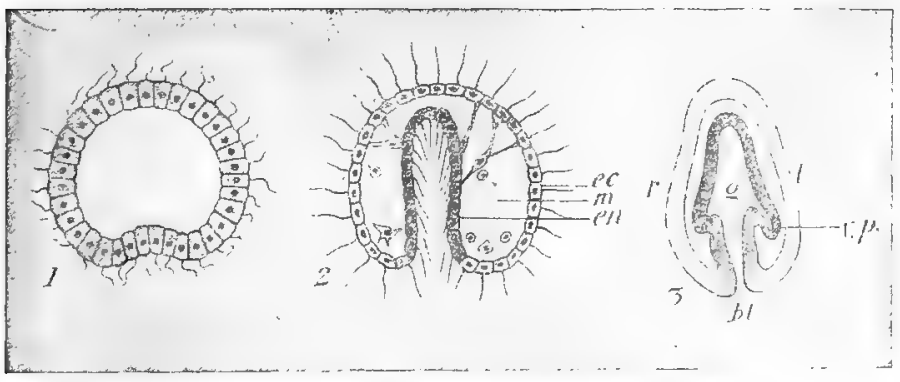

FIG. 80.-Stages in development of Echinoderms. (After Selenka.)

I. Section of blastula of Synapta digitata (Holothuroid) with a hint of gastrulation; 2 . Section of Gastrula of Toxopnenstes brevispinosus (sea urchin); $\epsilon$., ectoderm; $e n$., endoderm; $m$., segmentation cavity with mesenchyme cells in it; 3 . Section of larva of Asterina gibbosa (starfish); bl., blastopore; $G$., mesenteron; $\nu$, $p$., vaso-peritoneal vesicle; $r$. and $l$, right and left sides.

origin is a primitive condition, and the mesenchyme here, as always, is non-epithelial and gives rise to the connective tissues and to the vascular system. On the other hand, it has been asserted that in Echinoderms the mesenchyme is not purely a "packing tissue," but may acquire a distinctly epithelial character. Many of the early mesenchyme cells are calciferous, combining to form the larva skeleton. 
The larva is, first of all, a slightly modified, diffusely ciliated gastrula. It becomes more modified, but preserves a bilateral symmetry. In Holothuroids, Echinoids, Asteroids, and Ophiuroids, the larva becomes quaintly modified by the outgrowth of external processes, and the formation of special ciliated bands. The larva of Crinoids (i.e., of Antedon only) is not so divergent.

The larva does not grow directly into the adult. On the contrary the adult arises, for the most part, from new growth within the larva. The structures peculiar to the larva are absorbed, or in part thrown off. Only in a very few cases is the development direct.

Following the excellent account of Echinoderm development, in the Vergleichende Entreicklungsgeschichte der wirbellosen Thieren (Jena, I890), by Korschelt and Heider, we distinguish four stages :-

I. The formation of the primary serminal layers, of the mesenchyme, and of the mouth and anus.

Ectoderm and endoderm are established by the invagination of the blastosphere. The result is a ciliated gastrula. From the invaginating endoderm, somewhat amœboid cells are liberated into the persisting segmentation cavity, and form the mesenchyme tissue alluded to above. The gastrula cavity or archenteron is the larval mid gut; the blastopore, or mouth of the gastrula, seems usually to become the anus; but an invagination taking place at the other end forms a short fore gut or stomatodæum.

\section{The formation of the enterocal (body cavity) and the hyarocal (wrater vascular systemi).}

There is a close connection between the origin of the body carity and that of the water vascular system. Both are the results of an outgrowth or of outgrowths from the gastrula cavity or archenteron, into the surrounding space between endoderm and ectoderm. As they have a common origin, the outgrowth or outgrow ths which give rise to enterocœel and hydrocœl may be termed vaso-peritoneal.

There is not perfect agreement as to this united origin, but the follow. ing facts are generally recognised.

In Holothuroids there is a single outgrow th which gives rise to both body cavity and water vascular system.

In Echinoids, Asteroids, and Ophiuroids, there are two outgrowths, from the left of which the water vascular system arises.

In Crinoids (Antedon), there are three outgrowths, that which gives rise to the water vascular system being independent of the pair which form the body cavity.

In most cases a dorsal pore bringing the hydrocol into communication with the exterior has been detected. 


\section{The differentiation of the typical larval forms.}

The celebrated comparative anatomist and physiologist, Johannes Muiller, was the first to show that the varions types of Echinoderm larvæ might be derived from one fundamental form.

"This fundamental type is an elongated, oval or pear shaped larva, which is somewhat flattened on its ventral side. It has arisen from a gastrula, whose blastopore has become the anus, while the archenteron is bent towards the ventral surface, where it communicates by the larval mouth with the exterior. Besices these two apertures, the larva has a third, namely, the dorsal pore of the water vascular system. The cilia, with which the larva was at first uniformly covered, partly disappear, and persist only in restricted regions or ciliated bands." "Korschelt and Heider.)

Crinoids.-The simplest Echinoderm larva is that of Antedon, a somewhat modified oval, with five transverse rings of cilia (the most anterior is less distinct), and a posterior terminal tuft.

Holothuroids. The larva of Holothuroids (an Auricularia) is much quainter. Its diffuse cilia are succeeded by a wavy longitudinal band,
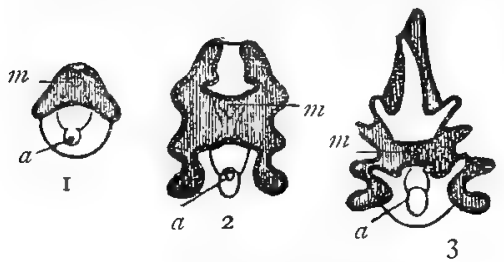

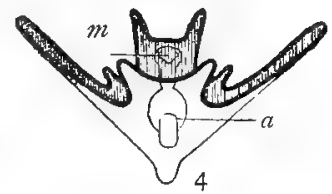

FIG. 8I.-Forms of Echinoderm Larva, (After MúlLer.)

m., Mouth; a., anus. The dark lines indicate the ciliated bands.

I. Supposed primitive type from which the various forms may be derived.

2. Auricularia of Holothurian.

3. Bipinnaria of Asteroid.

4. Pluteus of Ophiuroid.

which in the Pupa stage breaks into transverse rings, usually five in number. The pre-oral region becomes large.

Asteroids. Nearest the Auricularia is the larva of starfishes, which has the same enlarged pre-oral region. There are two ciliated bands, of which the ad-oral is smaller, the ad-anal much larger. They are extended peripherally by the development of soft arms, and such a larva is known as a Bipinnaria. But this may be succeeded by a Brachiolaria stage, in which three warty arms are formed at the anterior dorsal end, independently of the ciliated bands.

Ophiuroids and Eclinoids. In the Pluteus larve characteristic of these classes, the pre-oral region remains small, while the post-anal region becomes large. There is one undulating ciliated band, the course of which is much modified by the growth of six long arms, with 
temporary calcareous supports. This quaint form is often compared to a six-legged easel.

\section{The modification of the larza into the aduit Echinoderm.}

This history is so intricate and so difficult to understand without models, that it may be better simply to state that the development is indirect, that the adult is a new formation within the larva, retaining the water vascular system and mid gut, but absorbing or rejecting the provisional larval structures. As certain parts are broken down, others are built up, chiefly through the agency of the wandering amœboid cells of the mesenchyme. The first steps in the upbuilding of the adult, and especially of its skeleton, are to some extent parallel in the five classes.

One of the most important changes is that from bilateral to radial symmetry. In connection with this, it has been conjectured that the primitive ancestor was bilaterally symmetrical, and that the radiate symmetry was acquired by early sessile or sedentary Echinoderms, such as the Cystoids. As we have already seen, the adults in the different classes tend to acquire an independent and secondary bilateral symmetry.

It is very difficult to compare the Echinoderm larvæ, even in their simplest form, with those of other animals. The nearest type is perhaps the Tomaria of Balanoglossus, but it again is very unique. One naturally tries to compare the Echinoderm larva with the Trochosphere of Annelids, but the differences are very marked.

\section{Pedigree and Relationships of Echinoderms.}

Concerning the exact relationships of the different classes of Echinoderma, there is still considerable doubt. The following account is based upon the views set forth by Professor Jeffrey Bell, but the student will do well to realise that in this, as in most problems of phylogeny, there is little certainty.

The Holothurians have no aboral system of plates, and the radial symmetry does not effect the reproductive organs. These two negative characters, combined with some positive ones, may indicate that the Holothurians are primitive, and, as is certainly suggested by their external appearance, have affinities with the supposed "worm-like" ancestors of Echinoderms.

Again, some members of the heterogenous class of Cystoids are extremely primitive, luut differ from the Holothurians in the possession of an aboral system of plates, alternately radial and interradial. From this primitive Cystoidean stock, two branches diverge. The one leads to the sessile Cystoids, Blastoids, and Crinoids (Pelmatozoa), the other to the free Echinoidea, Asteroidea, and Ophiuroidea. Of these the existing Asteroidea and Ophiuroidea are late divergences from a common stock. 


\begin{tabular}{|c|c|c|c|c|c|c|c|}
\hline 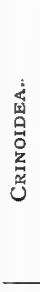 & 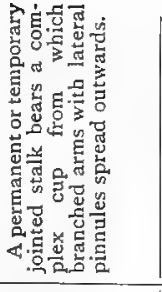 & 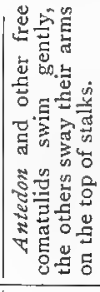 & 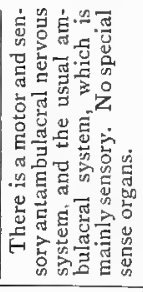 & 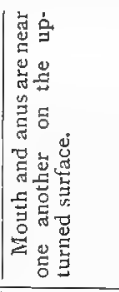 & 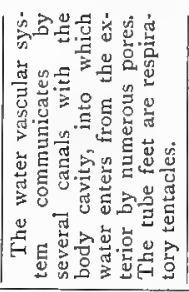 & 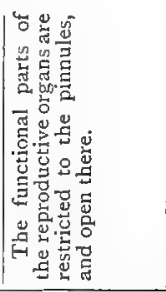 & 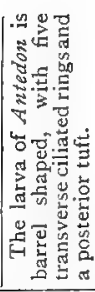 \\
\hline $\begin{array}{l}\dot{4} \\
0 \\
0 \\
0 \\
0 \\
0 \\
0 \\
0 \\
0 \\
0\end{array}$ & 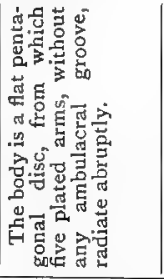 & 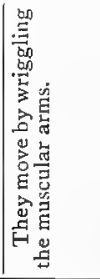 & 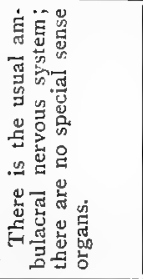 & 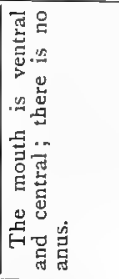 & 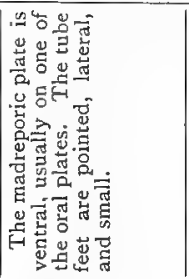 & 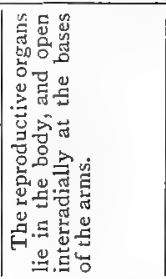 & 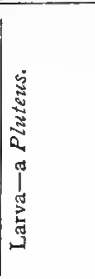 \\
\hline 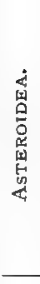 & 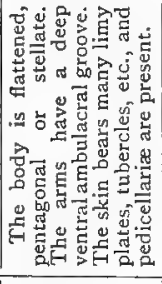 & 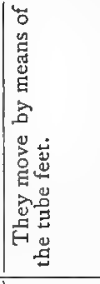 & 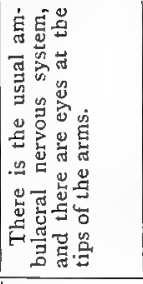 & 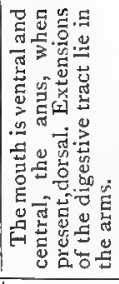 & 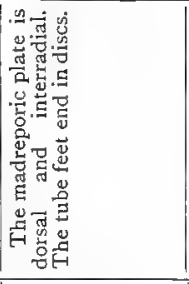 & 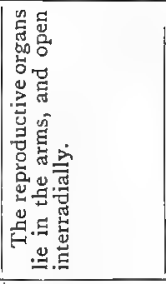 & 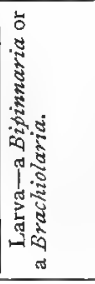 \\
\hline 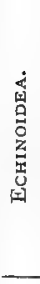 & 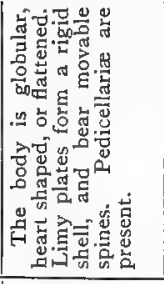 & 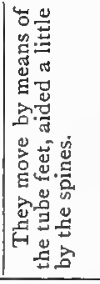 & 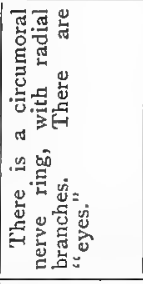 & 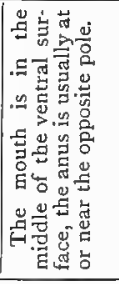 & 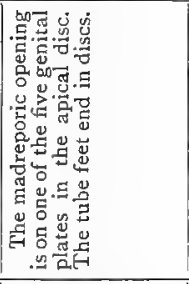 & 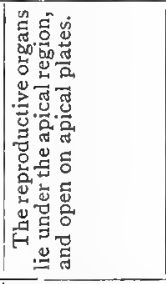 & 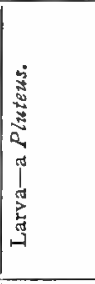 \\
\hline 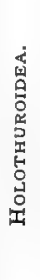 & 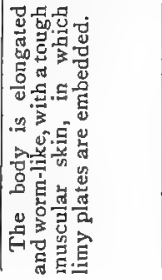 & 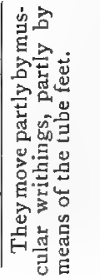 & 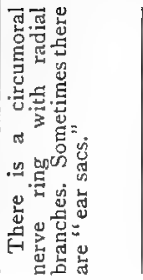 & 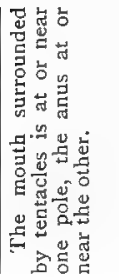 & 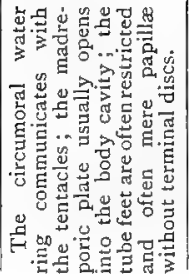 & 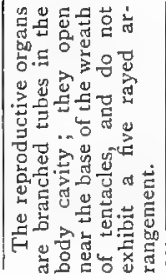 & 热 \\
\hline
\end{tabular}




\title{
CHAPTER XIII.
}

\author{
CRUSTACEA.
}

Series Arthropoda :- Classes Crustacea. Prototracheata. -Peripatzes. Myriopona. - Centipedes and Millipedes. InseCta. Arachnoldea. - Spiders, Mites, Scorpions. Palmostraca. King crab, Eurypterids, and Trilobites.

MORE than half the known species of animals are included in the Arthropod series, for of insects alone there are said to be more species than of all other animals taken together.

The Arthropods are in some ways like Annelids, - in the bilateral symmetry, in the division of the body into successive segments, some or all of which bear appendages, in the plan of the nervous system, and so on. Furthermore, Peripatus, which has air tubes or tracheæ somewhat similar to those of Myriopods and Insects, has nephridia like those of some Annelids; and the biramose appendages of a simple Crustacean like Apus may be compared with the parapodia of an Annelid. But we cannot, as yet, do more than recognise certain possibilities of pedigree.

It is also difficult to discern the relationships of the classes. Crustaceans, most of which are aquatic and breathe by gills, are often opposed to the others (Tracheata), most of which are terrestrial or aerial, and breathe by trachere or air tubes, or possible modifications of these. But the King crab (Limulus) is aquatic, and so were the extinct Eurypterids and Trilobites. In other respects these three types are very divergent, and as they have been much bandied about from Crustaceans to Arachnoids, it seems convenient to keep them in a separate class as Palæostraca. 
General Characteristics of Arthropods (to which primitive, parasitic, and degenerate forms present exceptions).

The body is bilaterally symmetrical, and consists of numerous segments variously grouped. Several or all of the segments bear paired jointed appendages variously modified. The cuticle is chitinous. Ciliated epithelium is absent, except in Peripatus. The dorsal brain is connected by a ring round the gullet with a double chain of ventral ganglia. Above the food canal lies the heart. The true or primitive colome is always small in the adult; the apparent body cavity is of secondary origin, and has in a great part a blood carrying or vascular function. The sexes are almost alneays separate, the reproductive organs and ducts are usually paired. There is often some metamorphosis in the course of development. In habit the Arthropods are predominantly active.

\section{Class Crustacea.}

General Characteristics of Crustaceans (to which primitive, parasitic, and degenerate forms offer exceptions).

With the exception of the land crabs, wood lice, and sand hoppers, the Crustaceans live in water and breathe by gills or through the skin. The head carries two pairs of antenna and other appendages; the thorax or median part of the body, sometimes distinct from, sometimes fused to the head, also bears limbs; the posterior region or abdomen is usually segmented, and often furnished zerith appendages. The typical appendage consists of two branches and a basal portion to which gills may be attached. To the chitin of the cuticle, carbonate of lime is added.

\section{A Type of Crustacea. The fresh water Crayfish (Astacus fuviatilis).}

(Most of the following description will apply also to the Lobsters (Honarus and Palinuints), and to the Norway Lobster (Nephrops norvegicus), often called a crayfish).

$$
\text { Mode of Life. }
$$

The fresh water crayfish lives in streams, and burrows in the banks. It is not found in Scotland, but occurs here 
and there in England and Ireland, and is common on the Continent. It is absent from districts where the water contains little lime. The food is very varied-from roots to water rats; cannibalism also occurs. The animals swim backwards by powerful tail strokes, or creep forwards on their "walking legs." Their life is tolerably secure, but frequent moultings occur which are expensive and hazardous. When hatched the young are like miniature adults; for a time they cling beneath the tail of the mother.

\section{External Appearance.}

The head and thorax are covered by a continuous (cephalothoracic) shield; the abdomen shows obviously distinct segments movable upon one another. As indicated by the appendages, there are three groups of segments or metameres - five in the head, eight in the thorax, six in the abdomen, as well as an unpaired piece or telson on which the food canal ends. (According to some authorities there are twelve segments in the cephalothorax, and seven in the abdomen.) Each of the nineteen segments bears a pair of appendages. Among other external characters may be noticed the stalked movable eyes, the two pairs of feelers, the mouth with six pairs of appendages crowded round it, the gills under the side flaps of the thorax, and the varied post-oral appendages.

The Body Wall consists of:-
(I) The external shell or cuticle, composed of various strata of chitin, coloured with pigments, hardened with lime salts ;

(2) The ectoderm, epidermis, or hypodermis, which makes and remakes the cuticle;

(3) An internal connective tissue layer or dermis, with pigment, blond vessels, and nerves. Internal to this lie the muscles.

Between the rings and at the joints the cuticle contains no lime, and is therefore pliable. As a sacrificed product of epidermic cells, it is dead and cannot expand. Hence, as long as the animal continues to grow periodic moulting is necessary. The old husk becomes thinner, a new one is formed beneath it, a split occurs across the back just behind the shield, the animal withdraws its cephalothorax and then its abdomen, and an empty but complete shell is left behind. 
The moulting is preceded by an accumulation of glycogen in the tissues, and this is probably utilised in the rapid growth which intervenes between the casting of the old and the hardening of the new shell.

How thorough the ecdysis or cuticle casting is, will be appreciated when we notice that the covering of the eyes, the hairs of the ears, the lining of the fore gut and hind gut, the gastric mill, and the tendinous inward prolongations of the cuticle to which some of the muscles are attached are all got rid of and renewed. The moults occur in the warm months, eight times in the first year, five times in the second, thrice in the third, after which the male moults twice, the female once a year, till the uncertain limit of growth is reached. It is not clearly known in what form the animals procure the carbonate of lime which is deposited in the chitinous cuticle, but experiments made by Mr. Irvine at Granton Marine Station proved that a carbonate of lime shell could be formed by crabs even when the slight quantity of carbonate of lime in sea water was replaced by the chloride. Moulting is an expensive and exhausting process, and great mortality is associated with the process itself or with the defenceless state which follows. The process is a disadvantage attendant on the advantage of armature. Inequalities in the legs are usually due to losses sustained in combat, but these are gradually repaired by new growth.

The surface of the body bears hairs or bristles of various kinds. These have their roots in the epidermis, and are made anew at each moult. There are simple glands beneath the gill flaps, and on the abdomen of the female there are cement glands, the viscid secretion of which serves to attach the eggs.

\section{Appendages.}

The limbs of a Crustacean usually exhibit considerable diversity ; in different regions of the body they are adapted for different work; yet all have the same typical structure, and begin to develop in the same way. In other words, they are serially homologous organs, illustrating division of labour. Typically each consists of a two-jointed basal piece (protopodite), and two jointed branches rising from this-an internal endopodite and an external exopodite; but in many the outer branch disappears. The protopodite has usually two joints, a basal or proximal coxopodite, and a distal basipodite; the five joints which the endopodite frequently exhibits are named from below upwards-ischio-; mero-, carpo-, pro-, dactylo-podites-details of some use in the comparison and identification of species. 


\section{THE APPENDAGES OF THE CRAYFISH.}

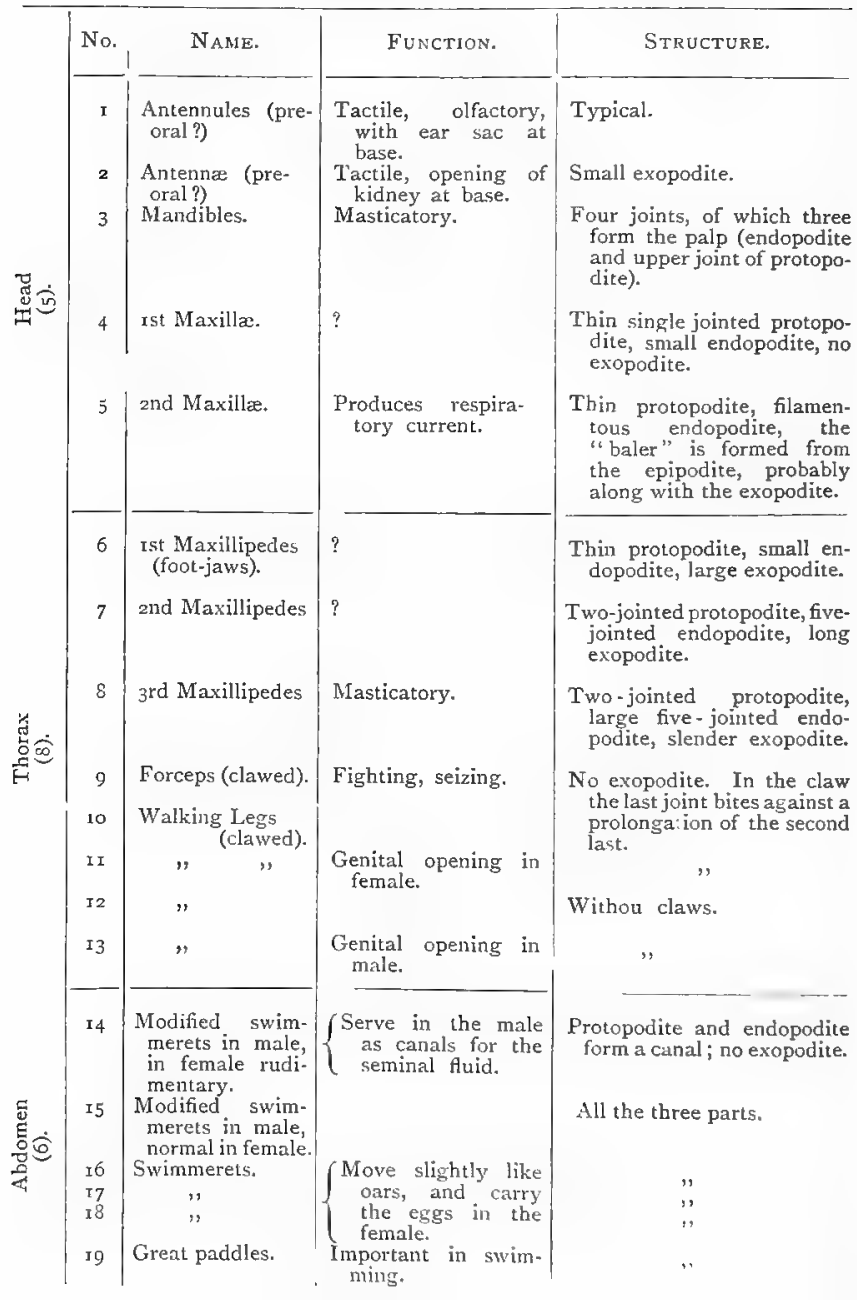


We can fancy how the Crustacean form of limb might arise from the biramose parapodium of a Polychæe. The hard chitinous cuticle of the Arthropod makes joints possible and necessary. In regard to the foregoing list it should be noted that the eye stalks are no longer included in the series since their development is not like that of the limbs, and, moreover, that though the two pairs of antenne lie far in front of the mouth, it is possible that they were originally post-oral. With many of the thoracic appendages, gills, plate-like epipodites, and setre are associated.

It is interesting to connect the structure of the appendages with their functions. Thus it may be seen that the great paddles are fully spread when the crayfish drives itself backwards with a stroke of its tail, while in straightening again the paddles are drawn inwards, and the outer joint of the exopodite bends in such a way that the friction is reduced.

It is likely that some of the crowded mouth parts, e.g. , the first maxillæ, are almost functionless. The hard toothed knob which forms the greater part of the mandible is obviously well adapted to its crushing work.

In connection with the skeleton, the student should also notice the beak (rostrum) projecting between the eyes; the triangular area (epistoma) in front of the mouth, and the slight upper and lower lips; how the gills are protected by lateral flaps of the body wall ; that each posterior segment consists of a dorsal arch (tergum), side flaps (pleura), a ventral bar (sternum), while the little piece between the pleura and the socket of the limb is dignified by the name of epimeron. The hindmost piece (telson) on which the food canal ends ventrally is regarded by some as a distinct segment, by others as an unpaired appendage. The most difficult fact to understand clearly, is that the cuticle of certain mouth parts (e.g., the mandibles), and of the ventral region of the thorax, is folded inwards, forming chitinous "tendons" or insertions for muscles, protecting the ventral nerve cord and venous blood sinus, and above all, constituting the complex, apparently, but not really, internal, "endophragmal " skeleton of the thorax.

\section{Muscular System.}

The muscles are white bundles of fibres. On minute examination these show clearly that transverse striping which is always well-marked in rapidly contracting elements. They are inserted on the inner surface of the cuticle, or on its internal foldings (apodemata). The most important sets are-(I) the dorsal extensors or straighteners 


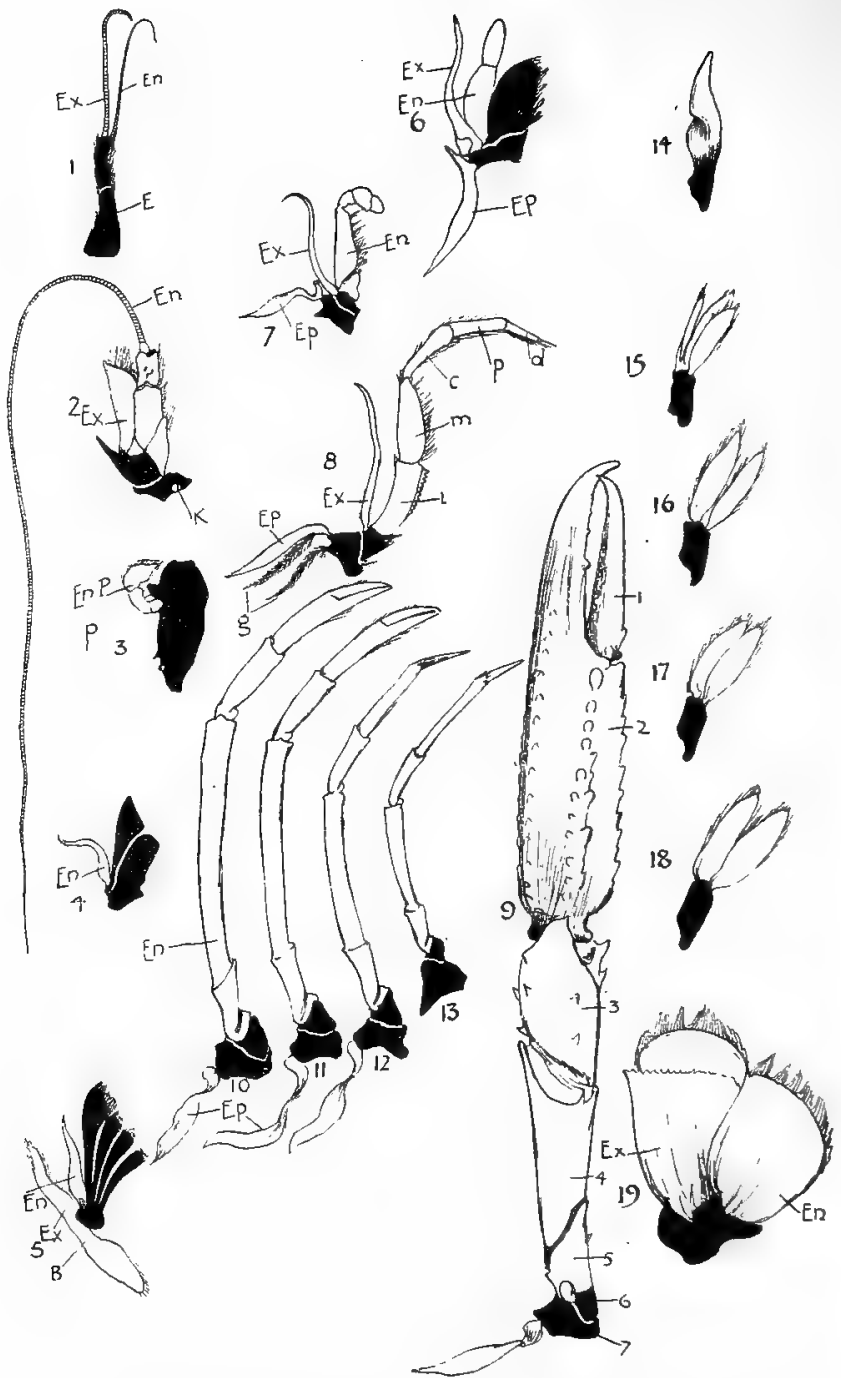

FIG. 82.-Appendages of Norway Lobster.

$E x$, Exopodite; $E_{n}$, endopodite; protopodite dark throughout; $E_{p}$, epipodite. r. Antennule, $E$, position of ear ; 2. antenna, $K$, opening of kidney; 3 . mandible, $P$, palp; 4 . first maxilla ; 5 . second maxilla, $b$, baler ; 6 . first maxillipede ; 7 . second maxillipede ; 8 . third maxillipede-the basal joint of protopodite is called coxopodite, the next basipodite; the five joints of the endopodite are called,-ischiopodite (i); meropodite $(m)$; carpopodite $(c)$; propodite $(p)$; dactylopodite $(d)$; 9 . forceps ; (7) coxopodite, (6) basipoc I5-x. small swimnerets; 
of the tail; (2) the twisted ventral muscles, most of which are flexors or benders of the tail, which have harder work, and are much larger than their opponents; (3) those moving the appendages; (4) the bands which work the gastric mill.

\section{Nervous System.}

The supra-cesophageal nerve centres or ganglia, forming the brain, have been shunted far forward by the growth of the pre-oral region. We thus understand how the nerve ring round the gullet, connecting the brain with the ventral chain of twelve paired ganglia, is so wide.

The dorsal or supra-œsophageal ganglia are three lobed, and give off nerves to eyes, antennules, antennæ, and food canal, besides the commissures to the sub-cesophageal centres.

The sub-œsophageal ganglia, the first and largest of the ventral dozen, innervate the six pairs of appendages about the mouth. There are other five ganglia in the thorax, and six more in the abdomen.

Though the ganglia of each pair are in contact, the ventral chain is double, and at one place, between the $4^{\text {th }}$ and 5 th ganglia, an artery (sternal) passes between the two halves of the cord. From each pair of ganglia nerves are given off to appendages and muscles, and apart from the brain, these minor centres are able to control the individual movements of the limbs. In the thoracic region the cord is well protected by the cuticular archway already referred to.

From the brain, and from the commissure between it and the sub-œsophageal ganglia, nerves are given off to the food canal, forming a complex visceral or stomato-gastric system. Similarly from the last ganglia of the ventral chain, nerves go to the hind gut. If the brain be regarded as the fusion of two pairs of ganglia, as the development suggests, and the sub-œsophageal as composed of six fused pairs, then these, along with the eleven other pairs of the ventral chain, give a total of nineteen nerve centres, - a pair for each pair of appendages.

\section{Sensory System.}

A skin clothed with chitin is not likely to be in itself very sensitive, but some of the setre are. These are 
not mere outgrowths of the cuticle, but are continuous with the living epidermis beneath, and though some are only fringes, both experiments and histological examination show that others are tactile.

On the under surface of the outer fork of the antennules, there are special innervated setæ which have been credited with a smelling function.

Other likewise specialised hairs have sunk into a sac at the base of the antennules, and are spoken of as auditory. The sac opens by a bristleguarded slit on the inner upper corner of the expanded basal joint, and contains a gelatinous fluid and small "otoliths" which seem to be foreign particles. This "ear" is somehow connected with directing the animal's movements. In some other Crustaceans, the auditory hairs are lodged in an open depression; this has become an open sac in the Crayfish, a closed bag in the Crab.

Small hairs on the upper lip of the mouth have been said to have a tasting function, but imagination is apt to help conclusions as to the precise nature of the sensitiveness of such simple structures.

The stalked eyes, which used to be regarded as appendages, arise in development from what are called "procephalic lobes" on the head. They are compound eyes, that is, they consist of a multitude of elements, each of which is structurally complete in itself. On the outside there is a

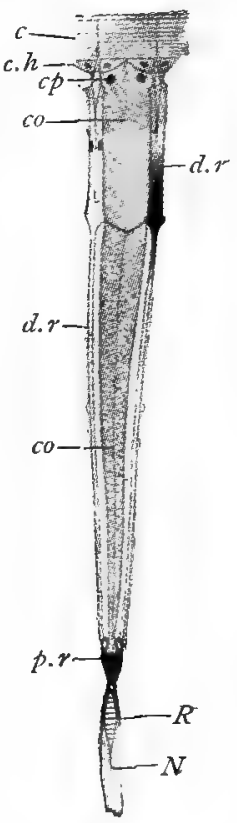

FIG. 83. -A single eye element or ommatidium of the Lobster. (After G. H. PARKER.)

$c$, Cornea; $c . h$, corneal bypodermis ; $c p$, cap of crystalline cone; co, crystalline cone; $d . r$, distal retinula elements; $p, r$, proximal retinula elements; $R$, rhabdome; $N$, nerve fibre. cuticular cornea, divided into square facets, one for each of the optic elements. Then follows a focussing layer, corresponding to the epidermis, consisting of many crystalline 
cones. Each crystalline cone is composed of four crystalline cells, which taper internally. Internal to each crystalline cone lie a number of retinula cells. The innermost of these surround four little red rods, united closely into what is called a rhabdome. At its base, a nerve fibre enters from the adjacent optic ganglion at the end of the optic nerve. Thus each element consists of corneal facet, crystalline cone, and retinula, and the retinula consists of internal rhabdome, and external retinula cells. Between the individual optic elements, lie some pigment cells. Opinions differ as to the visual powers of Crustaceans, but their eyes are able to form images of external objects, and these images are erect, not inverted as in the eyes of Vertebrates.

\section{Alimentary System.}

The food canal consists of three distinct parts, a fore gut or stomatodæum developed by an intucking from the anterior end of the embryo, a hind gut or proctodæum similarly invaginated from the posterior end, and a mid gut or mesenteron which represents the original cavity of the gastrula.

The mouth has been shunted backwards from the anterior end of the body, so that the antennules and antennæ lie far in front of it. The fore gut, which is lined by a chitinous cuticle, includes a short gullet, on the walls of which there are small glands hypothetically called "salivary," and a capacious gizzard, or "stomach," which is distinctly divided into two regions. In the anterior (cardiac) region there is a complex mill ; in the posterior (pyloric) region there is a sieve of numerous hairs. The mill is very complex, but there is no difficulty in dissecting it carefully, nor in seeing at once that there are supporting "ossicles" on the walls with external muscles attached to them, and internally projecting teeth which clash together and grind the food. Three of the teeth are conspicuous; a median dorsal tooth is brought into contact with two large laterals. On each side of the anterior part of the gizzard, there are two limy discs or gastroliths, which are broken up before moulting, and though quite inadequate to supply sufficient carbonate of lime for the new skeleton, seem to have some relation to this process. The occurrence of chitinous cuticle, hairs, 
teeth, and gastroliths in the "stomach," is intelligible when the origin of the fore gut is remembered, and so is the dismantled state of this region when moulting occurs.

The mid gut is very short, but it is the digestive and absorptive region. From it, there grows out on each side a large digestive gland with two ducts. This gland is more than a "liver," more even than a "hepatopancreas." It absorbs peptones and sugar, and makes glycogen like the Vertebrate liver, its digestive juices are comparable to those of the pancreas and the stomach of higher animals. The hind gut is long and straight. It is lined by a chitinous

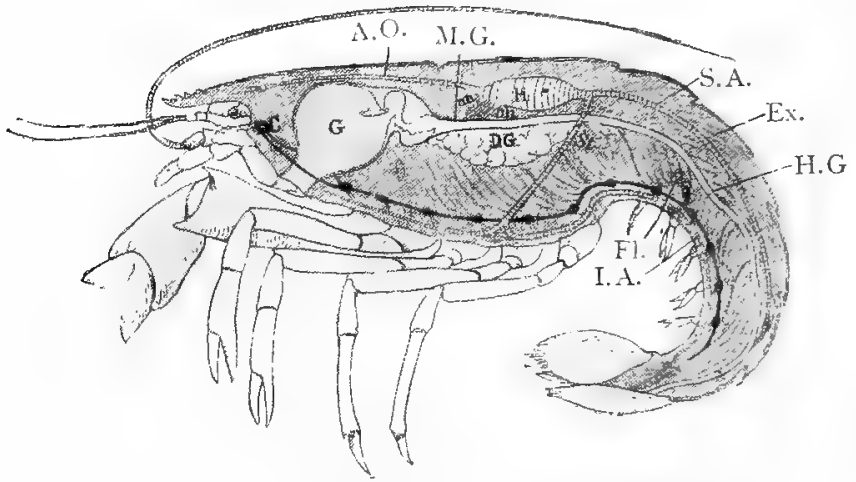

FIG. 84.-Longitudinal Section of Lubster, showing some of the organs.

$H$, Heart ; $A . O$, ophthalmic artery ; $a . a$, antennary artery ; $a . h$, hepatic artery; $S t$, sternal artery; $S . A$, superior abdominal artery ; $M . G$, mid gut: $D . G$, digestive gland ; $H . G$, hind gut ; $E_{\mathcal{X}}$, extensor muscles of the tail; $F l$, flexor muscles of the tail; $I . A$, inferior abdominal artery; $G$. gizzard ; $C$, cerebral ganglia.

cuticle, as its origin suggests. There are a few minute glands on its walls.

\section{Body Carity.}

The space between the gut and the body wall is for the most part filled up by the muscles and the organs, but there are interspaces left which contain a fluid with amoeboid cells. These interspaces seem to represent enlarged blood sinuses (a hæmocoele) rather than a true hody cavity or 
cœlome. One of the spaces forms the pericardium, or chamber in which the heart lies.

\section{Vascular System.}

Within this non-muscular pericardium, and moored to it by thin muscular strands, lies the six-sided heart, which receives pure blood from the gills (vi $\hat{a}$ the pericardium) and drives it to the body.

The arterial system is well developed. Anteriorly, the heart gives off a median artery to the eyes and antennules, a pair of arteries to the antennæ, and a pair to the digestive gland. Posteriorly, there issues a single vessel, which at once divides into a superior abdominal, running along the dorsal surface, and a sternal which goes vertically through the body. This sternal passes between the connectives joining the $4^{\text {th }}$ and $5^{\text {th }}$ ventral ganglia, and then divides into an anterior and posterior abdominal branch. All these arteries are continued into capillaries.

From the tissues the venous blood is gathered up in channels, which are not sufficiently defined to be called veins. It is collected in a ventral venous sinus, and passes into the gills. Thence purified by exposure on the water-washed surfaces, it returns by six vessels on each side to the pericardium. From this it enters the heart by six large and several smaller apertures, which admit of entrance but not of exit.

The blood contains amneboid cells, and the fluid or plasma includes a respiratory pigment, hæmocyanin (bluish when oxidised, colourless when deoxidised), and a lipochrome pigment, called tetronerythrin. Both of these are common in other Crustaceans.

\section{Respiratory System.}

Twenty gills-vascular outgrowths of the body wall - lie on each side of the thorax, sheltered by the flaps of the shield. A current of water from behind forwards is kept up by the activity of the baling portion, or scaphognathite, of the second maxilla. Venous blood enters the gills from the ventral sinus, and purified blood leaves them by the six channels leading to the pericardium.

Observed superficially, the gills look somewhat like feathers with plump barbs, but their structure is much more 
complex. The most important fact is that they present a large surface to the purifying water, while both the stem and the filaments which spring from it contain an outer canal continuous with the venous sinus, and an inner canal communicating with the channels which lead back to the pericardium and heart.

Three sets of gills are distinguishable. To the basal joints of the six appendages from the second maxillipede to the fourth large limb inclusive, the podobranchs are attached. They come off with the appendages when these are pulled carefully away, and each of them bears in addition to the feathery portion a simple lamina or epipodite. The membranes between the basal joints of the appendages and the body, from the second maxillipede to the fourth large limb inclusive, bear a second set, the arthrobranchs, which have no epipodites. In connection with the second maxillipede there is a single arthrobranch, in connection with each of the five following appendages there are two, so that there are eleven arthrobranchs altogether. There remain three pleurobranchs, one on the epimeron of the fifth large limb, and two others quite rudimentary on the two preceding segments. The bases of the podobranchs bear long setæ.

In Nephrops and the common lobster the number and arrangement of the gills is slightly different.

\section{Excretory System.}

A kidney or "green gland" lies behind the base of each antenna, and its opening is marked by a conspicuous knob on the basal joint of that appendage. Each kidney consists of a dorsal sac communicating with the exterior, and of a ventral coiled tube which forms the proper renal organ. The latter is supplied with blood from the antennary and abdominal arteries, and forms as waste products uric acid and greenish guanin. Each kidney may be regarded as homologous with a nephridium.

In Palcemon, the kidney is connected by a glandular duct with a delicate dorsal "nephro-peritoneal sac," possibly ccelomic: more probably an enlargement of the nephridial system.

The crayfish has also, near the gills, small branchial glands which excrete carcinuric acid from the blood, and also help in phagocytosis, that important process in which wandering amœboid cells resist infection and help to repair injuries (cf. possible function of thymus in Fishes).

\section{Reproductive Organs.}

The male crayfish is distinguished from the female by his slightly slimmer build, and by the copulatory modification 
of the first two pairs of abdominal appendages. In both sexes the gonads are three lobed, and communicate with the exterior by paired ducts.

The testes consist of two anterior lobes lying beneath and in front of the heart, and of a median lobe extending backwards. Each lobe consists of many tubules within which the spermatozoa develop. From the junction of each of the anterior lobes with the median lobe, a genital duct or vas deferens is given off. This has a long coiled course, is in part glandular, and ends in a short muscular portion opening on the last thoracic limb. The spermatozoa are at first disclike cells, they give off on all sides long pointed processes like those of a Heliozoon, and remain very sluggish. The

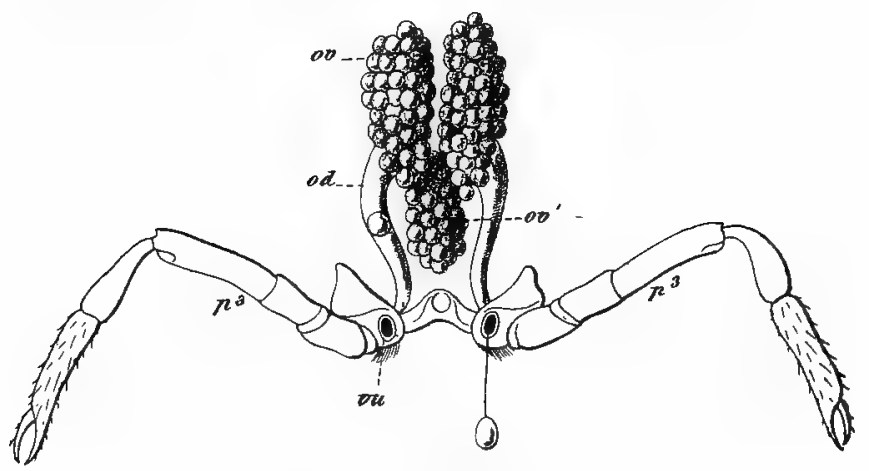

FIG. 85.-Female reproductive organs of Crayfish. (After Suckow.)

ov, Ovaries ; ov', fused posterior part ; od, oviduct: $v u$, female aperture on the second walking leg.

seminal fluid is milky in appearance, and becomes thicker in its passage through the genital ducts. It is possible that the genital ducts represent modified nephridia, and that the cavities of the gonads are coelomic.

The ovaries are like the testes, but more compact. The eggs are liberated into the cavity of the organ, and pass out by short thick oviducts opening on the second pair of walking legs. As they are laid they seem to be coated with the secretion of the cement glands of the abdomen, and the 
mother keeps her tail bent till the eggs are glued to the small swimmerets.

Before this, however, sexual union has occurred. The male seizes the female with his great claws, throws her on her back, and deposits the seminal fluid on the ventral surface of the abdomen. The fluid flows down the canal formed by his first abdominal appendages, and these seem to be kept clear by the movements of the next pair, which

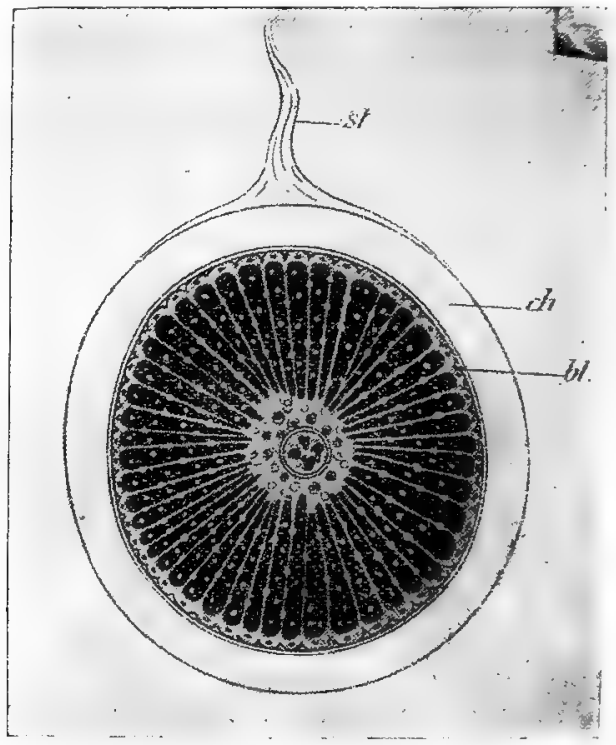

FIG. 86.- Section through the egg of Astacus after the completion of segmentation. (After ReICHENBACH.)

st. Stalk of the egg; $c /$, chorion envelope ; $b l$, peripheral blastoderm, within which are the yolk pyramids (dark).

are also modified. On the abdomen of the female the agglutinated spermatozon doubtless remain until the eggs are laid, when fertilisation in the strict sense is achieved.

The Derelopment has been very fully worked out, and is of interest in being direct, without the metamorphosis so common among the 
Arthropoda. The spherical ovum is surrounded by a cuticular vitelline membrane, and contains a considerable quantity of yolk. After fertilisation the segmentation nucleus divides in the usual way into 2,4 , 8 , and so on, but this nuclear division is not followed by division of the plasma. Eventually the nuclei, each surrounded by a small amount of protoplasm, approach the surface of the egg and arrange themselves regularly round it. The peripheral protoplasm then segments round these nuclei, and thus we have a central core of unsegmented yolk enveloped by a peripheral ring of rapidly dividing cells. In the central yolk free nuclei may be frequently found, these are the so-called yolk

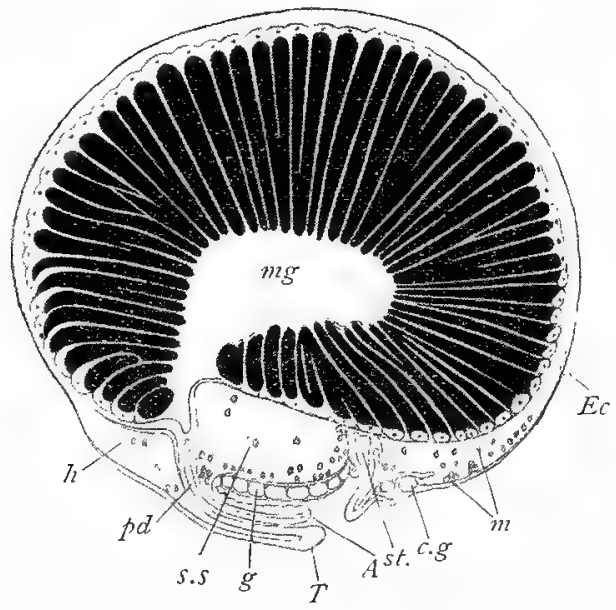

FIG. 87.-Longitudinal section of later embryo of Astacus. (After ReICHenBACH.)

ec, Ectoderm; $m$, mesoderm cells ; cg, cerebral ganglia ; st, stomatodæum; $A$, anus: $T$, telson; $g$, ventral ganglia ; $s s$, sternal sinus; $m d^{\prime}$, proctodzum ; $h$, heart ; mg , mid gut ; yolk pyramids (dark).

nuclei. Such a type of segmentation is called peripheral or centrolecithal, and is very characteristic of Arthropod eggs.

Over a particular region of the segmented egg, known as the "ventral plate," the cells begin to thicken; at this region an invagination occurs, which represents the gastrula. At the anterior lip of the blastopore the mesoderm appears, being many celled from the first. Soon the blastopore closes; the cavity of the gastrula thus becomes a closed sac-the future mid gut. The cells of this archenteron take up the core of yolk 


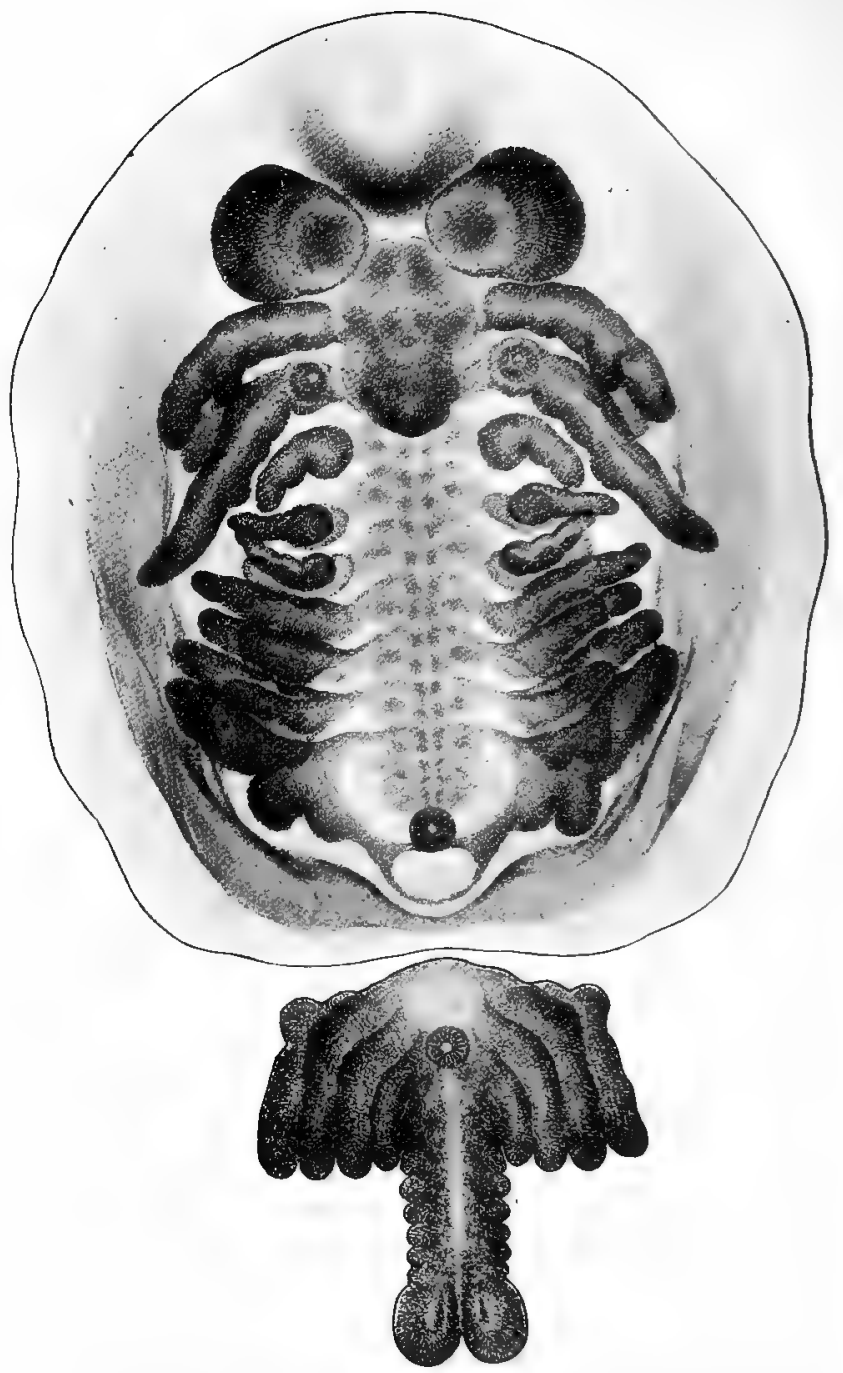

FIG. 88.-Embryo of Crayfish, flattened out, with removal of yolk, magnified about 40 times. (After ReIcHeNBACH.)

Note rudiments of eyes and appendages, and in the middle line the nervous system. 
into themselves in a way which early suggests their future digestive function. On the surface of the egg there have already appeared ectodermic thickenings - the so-called eye folds, - rudiments of the appendages, and of the thoracic and abdominal regions.

In the later stages invaginations of the ectoderm form the fore and hind gut, which grow inward from opposite ends to meet the endodermic mid gut, also the ear sac and the green glands. The gills are formed in great part from ectodermic outgrowths or evaginations. From the mid gut the digestive gland is budded out. The heart, the blood vessels, blood, and muscles are due to the mesoderm.

As usual, the nervous system arises from an ectodermic thickening. The eye arises partly from the optic ganglia of the "brain," partly from the "eye folds," and partly from the epidermis.

When the young crayfish are hatched from the egg shells, they still cling to these, and thereby to the swimmerets of the mother. In most respects they are miniature adults, but the cephalothorax is convex and relatively large, the rostrum is bent down between the eyes, the tips of the claws are incurved and serve for firm attachment, and there are other slight differences. The noteworthy fact is that the development is completed within the egg case, and that it is continuous withuut metamorphosis. The shortened life history of the crayfish is interesting in relation to its fresh water habitat, where the risks of being swept away by currents are obviously great; but it must also be remembered that the tendency to abbreviate development is a general one. There is some maternal care in the crayfish, for the young are said sometimes to return to the mother after a short exploration on their own account.

\section{Systematic Survey of the Class Crustacea.}

(I) Entomostraca, lower forms.

They are usually small and simple.

The number of segments and appendages is very variable.

The larva is generally hatched as a simple unsegmented Nauplius. There is no gastric mill.
(2) Malacostraca, higher forms.

They are usually larger and more complex.

The head consists of 5 , the thorax of 8 , the abdomen of $6(7$ in Leptostraca) segments.

The larva is usually higher than a Nauplius.

There is a gastric mill.
I. Phyllopoda, $\left\{\begin{array}{l}\text { Apus, Branchi- } \\ \text { pus, and Artemia } \\ \text { (brine-shrimps), } \\ \text { Daphnia,Moina, } \\ \text { Polyphemzes. }\end{array}\right.$

2. Ostracoda, Cypris, Cypridina.

3. Copepoda, Cyclops, Argulus, many parasites.

4. Cirripedia, acorn shells and barnacles, e.g., Balanus and Lepas.
Leptostraca, e.g., Nebalia.

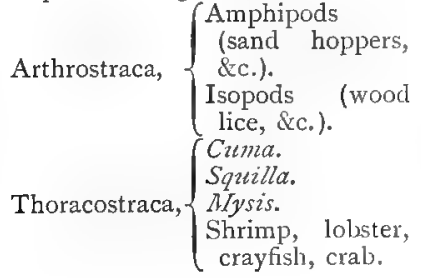


First Sub-Class. Entomostraca.

These are the more primitive Crustaceans, often small and simple, with a variable number of segments and appendages. The newly hatched larva is usually a Nauplius. The adult may retain the unpaired simple frontal eye, which is always found in the Nauplius, and has no gastric mill.
Order I. Phyllopoda.
Order 2. Ostracoda.
Order 3. Copepoda.
Order 4. Cirripedia.

Order I. Phyllopoda. In these at least four pairs of swimming feet bear respiratory plates. The body is generally well segmented, and is protected by a shield-like or bivalve shell. The mandibles are without palps, and the maxillae are rudimentary.

(a) Branchiopoda. The body has numerous segments and (I0-20 or more) appendages with respiratory plates. The shell is rarely absent, usually shield-like or bivalved. The heart is a long dorsal vessel with numerous openings. The eggs are able to survive prolonged desiccation in the mud.

Branchipus, a beautifully coloured fresh water form, with hardly any shell.

Artemia. Brine shrimps. Periodically parthenogenetic. By gradually changing the salinity of the water, Schmankewitsch was able, in the course of several generations, to modify $A$. salina into $A$. mühlenhausii, and vice versa. Artemia fertilis is one of the four animals known to occur in the dense waters of Salt Lake. Limnadia, with bivalve shell. Periodically parthenogenetic. A mollusc-like livalve shell is still more marked in Estheria.

Apus, a fresh water form with a large dorsal shield. Periodically parthenogenetic. One species hermaphrodite.

Of these Apus is certainly the most interesting. It is over an inch in length, and therefore a giant among Entomostraca. It has an aImost world wide distribution. "It possesses peculiarities of organisation which mark it out as an archaic form, probably standing nearer to the extinct ancestors of the Crustacea than almost any other living member of the group." The appendages are very numerous and mostly leaf-like. They may be regarded as representing a primitive type of Crustacean limb. Professor Ray Lankester enumerates them as follows:-

\footnotetext{
Pre-oral. $\quad\left\{\begin{array}{l}\text { I. Antenna. } \\ \text { 2. Second antenna. (This is sometimes absent, and }\end{array}\right.$ apparently always in certain species.)

Oral. 
Thoracic 6. First thoracic foot (leg-like).

(Pre-genital). The 16th in the female carries an egg sac or brood chamber. There are eleven thoracic rings on the body.

Abdominal $\{$ I7-68. Fifty-two abdominal feet, to which there corres(Post-genital). $\{$ pond only seventeen rings on the body.

The large dorsal shield is not attached to the segments behind the one bearing the maxillipedes. Many of the thin limbs doubtless function as gills. The genital apertures are on the 16 th appendages. The anus is on the last segment of the body.

There is a pair of ventral ganglia to each pair of limbs; the ventral nerve cords are widely apart; and the cephalic ganglion is remarkably isolated. Professor Ray Lankester called this cephalic ganglion an "archi-cerebrum," to emphasise its preoral position and its distinctness from the posterior ganglia. Subsequent research has shown, however, that in Apres, as in other Crustaceans, the cephalic ganglion is a "syn-cerebrum, i.e., it is composed of pre-oral ganglia fused with post-oral ganglia which have been shunted forwards.

(b) Cladocera. Small laterally compressed "water fleas," with few and somewhat indistinct segments. The shell is usually bivalved. The head often projects freely. The second pair of antennæ are large, two-branched, swimming appendages, and there are $4-6$ pairs of other swimming organs. The heart is a little sac with one pair of openings. An excretory organ (the shell or maxillary gland) opens in the region of the second maxillae. It is the Entomostracan equivalent of the antennary green gland of Malacostraca. The males are usually smaller and much rarer than the females. The latter have a brood chamber between the shell and the back. Within this many broods are hatched throughout the summer. Periodic parthenogenesis (of the "summer ova") is very common. "Winter eggs," which require fertilisation, are set adrift in a part of the shell modified to form a protective cradle or ephippium.

Daphnia, Moina, Sida, Polyphenus, Leptodora, and many other "water fleas" are extraordinarily abundant in fresh water, and form part of the food of many fishes. A few occur in brackish and salt water.

Order 2. Ostracoda. Small Crustaceans, usually laterally compressed, with an indistinctly segmented or unsegmented body, rudimentary abdomen, and bivalve shell. There are only seven pairs of appendages.

Cypris (fresh water), Cypridina (marine).

Order 3. Copepoda. Elongated Crustaceans, usually with distinct segments. There is no dorsal shell. There are five pairs of biramose thoracic appendages, but the last may be rudimentary or absent. The abdomen is without limbs, and of its five segments the first two are sometimes united. The females carry the eggs in external ovisacs. Many are ecto-parasitic, especially on fishes ("fish lice ") 
and are often very degenerate. The free living Copepods form an important part of the food supply of fishes.

Cyclops, free and exceedingly prolific in fresh water. Cetochilus free and abundant in the sea.

Sapphirina, a broad flat marine form about quarter of an inch long, occasionally parasitic. The male surpasses all animals in the brilliancy of its " phosphorescent" colour.

Chondracanthus. As in many other cases, the parasitic females carry the pigmy males attached to their body.

Caligus, a very common genus of "fish lice."

Lernea, Penella, etc. The adult females are parasitic, and almost worm-like. The males and the young are free. That the males are often free and not degenerate, while their mates are parasitic and retrogressive, may be understood by considering ( $I$ ) the greater vigour and activity associated with maleness; (2) the fact that parasitism affords safety and abundance of nutrition to the females during the reproductive period.

Argulus, a divergent form temporarily parasitic on carp, \&c. It has a shield-like cephalothorax and a small cleft abdomen. A protrusible spine projects in front of the blood sucking mouth; the mandibles and first maxillæ are adapted for piercing; the second maxille or maxillipedes for adhesion. There are four pairs of two-branched swimming appendages. There are two large compound eyes. The female has no ovisacs; the eggs are laid on foreign objects.

Order 4. Cirripedia. Barnacles and acorn shells, and some allied degenerate parasites.

Marine Crustaceans, which in adult life are fixed head downwards. The body is indistinctly segmented, and is enveloped in a fold of skin, usually with calcareous plates. The anterior antennæe are involved in the attachment, the posterior pair are rudimentary. The oral appendages are small, and in part atrophied. In most there are six (or less frequently four) pairs of two-branched thoracic feet, which sweep food particles into the depressed month. The abdomen is rudimentary. There is no heart. The sexes are usually com. bined, but dimorphic unisexual forms also occur. The hermaphrodite individuals occasionally carry pigmy or "complemental" males. The spermatozoa are mobile, which is unusual among Crustacea.

Lepas, the ship barnacle, is as an adult attached to floating logs and ship bottoms. The anterior end by which the animal fixes itself is drawn out into a long flexible stalk, containing a cement gland, the ovaries, \&c., and involving in its formation the first pair of antenne and the front lobe of the head. The second antenne are lost in larval life. The mouth region bears a pair of small mandibles and two pairs of small maxillae, the last pair united into a lower lip. The thorax has six pairs of two-branched appendages, and from the encl of the rudimentary 
abdomen a long penis projects At the base of this lies the anus. Around the body there is a fold of skin, and from this arise five calcareous plates, an unpaired dorsal carina, two scuta right and left anteriorly, two terga at the free posterior end. The nervous system consists of a brain, an osophageal ring, and a ventral chain of five or more ganglia. There is a fused pair of rudimentary eyes. No special circulatory or respiratory organs are known. Two excretory (?) tubes lead from (colomic) cavities to the base of the second maxillie, and are probably comparable with shell glands and with nephridia. There is a complete food canal and a large digestive gland. Beside the latter lie the branched testes, whose vasa deferentia unite in an ejaculatory duct in the penis. From the much branched ovaries in the stalk, the oviducts pass to the first thoracic legs, where they pass into a cement making sac, opening to the exterior. The eggs are found in flat cakes between the external fold of skin and the body.

The life history is most interesting. Nauplius larvæe escape from the

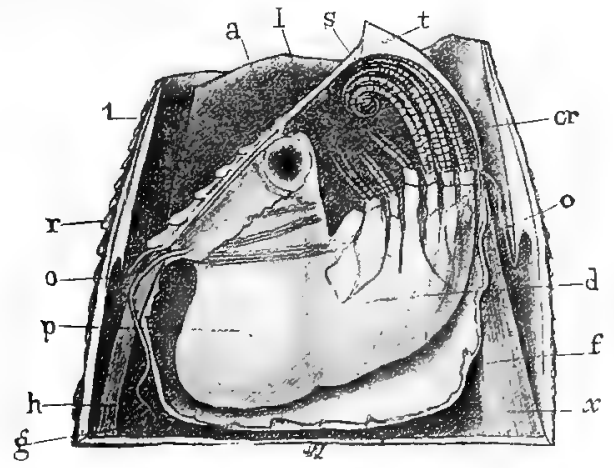

FIG. 89.-Acorn shell (Balanus tintinnabulum). (After DARWIN.)

$t$, Tergum ; $s$, scutum ; $d$, opening of oviduct ; $f$, mantle cavity ; $x$, depressor muscle of tergum; $g$, depressor muscle of scutum; $h$, oviduct ; $r$, ourer shell in section; $a$, adductor muscle of scuta.

egg cases, and after moulting several times become like little Cyprid water fleas. The first pair of appendages become suctorial, and after a period of free swimming, the young barnacle settles down on some floating object, mooring itself by means of the antennary suckers, and becoming firmly glued by the secretion of the cement glands. During the settling and the associated metamorphosis, the young barnacle fasts, living on a store of fat previously accumulated. Many important changes occur, the valved shell is developed, and the adult form is gradually assumed. While the early naturalists, such as Gerard (I597), regarded the barnacle as somehow connected with the barnacle goose, 
and zoologists, before J. Vaughan Thompson's researches (I829), were satisfied with calling Cirripedes divergent Molluses, we now know clearly that they are somewhat degenerate Crustaceans. We do not know, however, by what constitutional vice, by what fatigue after the exertions of adolescence, they are forced to settle down to sedentary life.

The food consists of small animals, which are swept to the mouth by the waving of the curled legs. Growth is sonewhat rapid, but the usual skin casting is much restricted except in one genus. Neither the valves, nor the uniting membranes, nor the envelope of the stalk, are moulted, though disintegrated portions may be removed in flakes and renewed by fresh formations. In the allied genus Scalpellum, some are like Lepas, hermaphrodites, without complementary males (Sc. balanoides); others are hermaphrodite, with complementary males (Sc. villoszm), \&c.; and others are unisexual, but the males are minute and parasitic (Sc. regium).

Balanus, the acorn shell, encrusts the rocks in great numbers between high and low water marks. It may be described, in Huxley's graphic words, as a crustacean fixed by its head, and kicking the food into its mouth with its legs. The body is surrounded, as in Lepas, by a fold of skin, which forms a rampart of six or more calcareous plates, and a fourfold lid, consisting of two scuta and two terga. When covered by the tide, the animal protrudes and retracts between the valves of the shell six pairs of curl-like thoracic legs. The structure of the acorn shell is
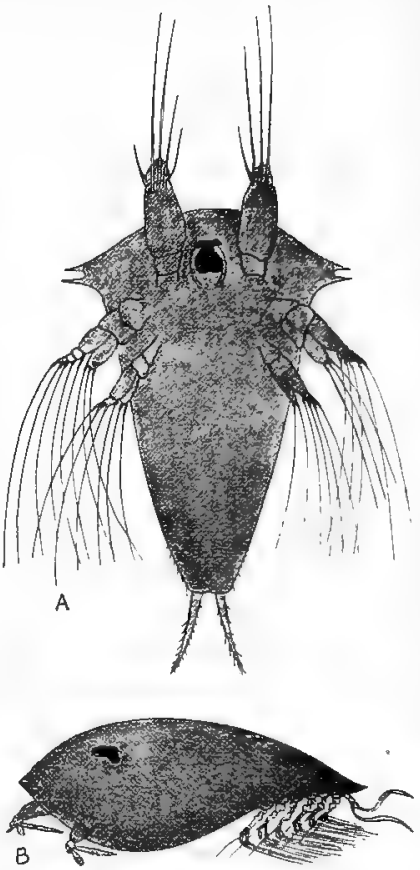
in the main like that of the barnacle, but there is no stalk.

FIG. 90.-Development of Sacculina. (After Del.Age.)

(Not drawn to scale.)

A. Free swimming Nauplius, with three pairs of appendages; B. Pupa stage; C. Adult protruding from the tail of a crab.

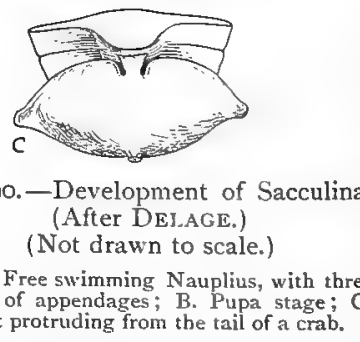


The life history also is similar. A Nauplius is hatched. It has the usual three pairs of legs, an unpaired eye, and a delicate dorsal shield. It moults several times, grows larger, and acquires a firmer shield, a longer spined tail, and stronger legs. Then it passes into a Cypris stage, with two side eyes, six pairs of swimming legs, a bivalve shell, and other organs. As it exerts itself much but does not feed, it is not unnatural that it should sink down as if in fatigue. It fixes itself by its head and antennæe, and is glued by the secretion of the cement gland. Some of the structures, e.g., the bivalve shell, are lost; new structures appear, e.g. the characteristic Cirriped legs and the shell. Throughout this period, which Darwin called the "pupa stage," there is external quiescence, and the young creature continues to fast. The skin of the pupa moults off ; the adult structures and habits are gradually assumed. At frequent periods of continued growth, the lining of the shell and the cuticle of the legs are shed. In spring these glassy cast coats are exceedingly common in the sea. Acorn shells feed on small marine animals. They fix themselves not to rocks only, but also to shells, floating objects, and even to whales and other animals.

Alcippe and Cryptophialus (with only three or four pairs of feet) live in the shells of other Cirripedes or of Molluscs ; Proteolepas is parasitic in the mantle of other Cirripedes, and like a grub.

On the ventral surface of the abdomen of crabs, Sacculina, the most degenerate of all parasites, is often found. Its complete history has been beautifully worked out by Professor Delage. It is in shape an ovoid sac, and is attached about the middle of a segment. On the lower surface of the sac there is a cloacal aperture, opening into a large brood chamber, usually distended with eggs contained in chitinous tubes. The brood chamber surrounds the central "visceral mass," consisting of a nerve ganglion, a cement gland which secretes the egg cases, and the hermaphrodite reproductive organs; of digestive or vascular systems there is no trace. The parasite is attached by a peduncle, dividing up, within the body of the crab, into numerous "roots," which have been compared to the placenta of a mammalian foetus. The "roots" ramify within the body of the crab, and by them the Sacculina obtains nutrition and gets rid of its waste products; it is therefore practically, even at this stage, an endoparasite. The larvæ leave the brood chamber as Nauplii, they moult rapidly and become Cyprid larva. These fix themselves by their antenna to young crabs, at the uncalcified membrane surrounding the base of the large bristles of the back or appendages. The thorax and abdomen are cast off entirely; the structures within the hear region contract ; eyes, tendons, pigment, the remaining yolk, and the carapace are all lost; and a little sac remains, which passes into the interior of the crab. Eventually it reaches the abdomen, and, as it approaches maturity, the integuments of the crab are dissolved beneath it, and the sac-like body protrudes; essentially, however, Sacculina is always endoparasitic. It appears to live for three years, during which time the growth of its host is arrested, and no moult occurs. 


\section{Second Sub-Class. Malacostraca.}

These are higher Crustaceans in which the body consists of three regions with a constant number of segments, five to the head, eight to the thorax, and six to the abdomen (except in forms like Nebalia, which have seven). The terminal piece or telson of the abdomen is regarded by some zoologists as a distinct segment. Apart from this telson, and also the segment next to it in Nebalia, all the segments bear paired appendages. More or less of the thorax is fused to the head region, and the anterior thoracic limbs are usually auxiliary to mastication. Two compound lateral eyes and a gastric mill are always present. There is an antennary excretory gland, probably comparable with the Entomostracan maxillary gland. The female genital apertures are on the third last pair of thoracic legs, the male apertures on the last pair. Very few are hatched in the Nauplius stage, many, however, at the Zora level, while others have no metamorphosis at all.

Legion I. Leptostraca. Nebalia.

Legion 2. Arthrostraca, with three orders, Anisopoda, Isopoda, Amphipoda.

Legion 3. Thoracostraca, with four orders, Cumacea, Stomatopoda, Schizopoda, Decapoda.

Legion I. Leptostraca.

Marine Crustaceans of great systematic interest, because they retain in many ways the simplicity of ancestral forms, and link Malacostraca to Phyllopods. The most important genus is Nebalia.

A bivalve shell covers the whole of the lank body, except the last four abdominal segments; the hearl is free from the thorax; the eight segments of the thorax are free from one another, and the plate-like appendages resemble those of Phyllopods; the abdomen has seven segments and a telson with two forks; the elongated heart extends into the abdomen, and has seven pairs of lateral apertures or ostia. Nebalia and its congeners are probably related to certain ancient fossil forms from Palzozoic strata-Hymenocaris, Ceratiocaris, \& c.

Legion 2. Arthrostraca. (Edriophthalmata, sessile eyed.)

There is no shell fold or shield, except in the order Anisopoda. The first thoracic segment (rarely with the addition of the second) is fused to the head, the corresponding appendages serve as maxillipedes, the other thoracic segments (seven or six) are free. The eyes are sessile. The heart is elongated. 
Order I. Anisopoda. The fusion of the first two thoracic segments to the head, the presence of a cephalothoracic shield, and other divergent features distinguish Tanais, Apsendes, $\hat{\sigma}^{5} c .$, from the Isopoda.

Order 2. Isopoda. The body is flattened from above downwards. The first thoracic segment is fused to the head, while the other six or seven are free, and there is no cephalothoracic shield. The abdomen is usually short, and its appendages, usually overlapped by the first pair, are plate-like, and function in part as respiratory organs.

The "wood lice" (Onisius, Porcellio) are familiar animals which lurk in damp places under stones and bark, and devour vegetable refuse. Some related forms (e.g., Armadillo), which roll themselves up, are called "pill bugs." In the terrestrial forms there is obviously a departure from the ordinarily aquatic habit of Crustaceans, and the exopodites of some of the abdominal appendages have tubular air passages.

Asellus is a very common form, living in both fresh and salt water.

Idotea is not uncommon among the shore rocks.

The "gribble" (Limnoria lignorum) is a destructive marine Isopod which eats into wood.

Among the marine Cymothoidee which are often parasitic on fishes, some, $e_{0}$. . Cynnothoe, are remarkable in their sexual condition, for they are hermaphrodites in which the male organs mature and become functional when the oviducts are still closed, while at a later period in life the male organs are lost and the animals become functionally female.

The Bopyrida infest the gill chambers of other Crustaceans, e.g., prawns. The pigmy males are usually carried about by their mates.

Among the parasitic Cryptoniscida, we again find hermaphrodites with associated pigny males. In not a few cases they seriously affect the reproductive organs of their male hosts.

Many of these Isopods, like not a few other Crustaceans, are extremely interesting to those who care to think about the problem of sex. Thus, to cite one other instance, the males and females in the genus Gnathia are so unlike, that they have been mistakenly referred to different sub-families.

Order 3. Amphipoda. The body is laterally compressed. In most it is only the first thoracic segment which is fused to the head, in the "no-body-crabs" (Caprellide), and "whale lice" (Cyamidce), two segments are involved. The thoracic limbs bear respiratory appendages. Of the six pairs of legs which the abdomen usually bears, the anterior three are usually more strongly developed as swimmers, while the posterior three-directed backwards-are used in jumping. 
Gammarus pulex is very, common in fresh water. Other species occur on the sea-shore. There also the "Beach fleas " (Talit'us and Orchestia) are exceedingly abundant. On solid ground they move on their sides in a strange fashion, but they swim very swiftly.

Hyperia, Phrouima, and many marine Amphipods, have a habit of living as commensals with other animals.

Caprella, a common marine gymnast on Hydroids, \&c., has the trunk of the body reduced to the quaintest possible minimum.

Legion 3. Thoracostraca. (Podophthalmata, with stalked eyes.)

Several or all of the thoracic segments are fused to the head, and there is a cephalothoracic shield overlapping the gills. The two eyes are stalked except in Cumacea.

Order I. Cumacea. The cephalothoracic shield is small, and four or five thoracic segments are left uncovered and free. The eyes are sessile and adjacent or fused. There are two pairs of maxillipedes. The females have no abdominal appendages except on the last segment. The genera are marine, e.g., Cuma or Diastylis.

Order 2. Stomatoporla. The shield is still small and does not cover the three posterior thoracic segments. The body is somewhat flattened, the abdomen is very strong. Five anterior thoracic appendages are directed towards the mouth, and serve to catch food, and to clamber. The five anterior abdominal legs carry feathery gills, the sixth pair forming swimming paddles. The elongated heart extends into the aludomen, which also contains the reproductive organs. The genera are marine, e.g., Squilla.

Order 3. Schizopoda. A delicate shield covers the whole of the thorax, but there is still some freedom as to one or more of the posterior thoracic segments. The eight thoracic appendages are uniformly biramose, but the first two may serve as maxillipedes. The abdominal appendages of the male are strongly developed, those of the female are weak except the last, which in both sexes form parldles. They are marine forms, e.g., D/ysis (withont gills on the thoracic legs), Lophogaster and Euphausia (with gills on the thoracic legs). The last named starts in life as a Nauplius. As an adult it has luminous organs on the eye-stalks, thoracic legs, and abdominal segments.

Order 4. Decapoda. The shield is large and firm, and is fixed to the dorsal surface of all the thoracic segments. Of the thoracic appendages, the first three pairs are maxillipedes, the five other pairs are jointed walking legs (whence the term Decapod).

Sub-order I. Macrura. Abdomen long. Homarus (lobster); Nephrops (Norway lobster, sea crayfish); Astacus (fresh water crayfish); Palimums (rock lobster), whose larva was long known as the glass crab (Phyllosoma); Penaus, a shrimp which passes through Nauplius, Zozea, and Mysis stages; Lacifer and Sergestes are also hatched at a stage antecedent to the Zoxa; Crangon vulgaris (the British 
shrimp); Palcemon, Pandalus, Hippolyte (prawns); Galathea (with the abdomen bent inwards); Pagurus, Eupagurus (hermit crabs) ; Birgus latro (the terrestrial robber or palm crab), in which the upper part of the gill cavity is shut off to form a "lung," the walls having numerous vascular plaits. Opinion seems to incline against recognising a separate suborder (Anomura) for the soft-tailed hermit crabs.

Sub-order 2. Brachyura. Abdomen short, and bent under the thorax. It is narrow in the male, and does not usually bear more than two pairs of appendages; it is broader in the female, and bears four paired appendages. The ventral ganglia have fused into an oval mass. Cancer (edible crab); Carcinus manas (shore crab); Portznus (swimming crab); Maia (spider crab); Lithodes (stone crab); Porcellana; Dromia (often covered by a sponge); Pinnotheres (living inside bivalves); Gelasimus (fiddler crab, a very adept burrower); Telphusa (a fresh water crab); Gecarcinzes (land crabs, only visiting the sea at the breeding season).

History.-Fossil Crustaceans are found in Cambrian strata, but the highest forms (Decapoda) were not firmly established till the Tertiary period. Some of the genera, e.g., the Branchiopod Estheria, living from Devonian ages till now, are remarkably persistent and successful. How the class arose, we do not know: it is probable that types like Nebalia give us trustworthy hints as to the ancestors of the higher Crustaceans; it is likely that the Phyllopods, e.g., Apus, bear a similar relation to the whole series; the Copepocls also retain some primitive characteristics; but it is difficult apart from mere guessing to say anything definite as to the more remote ancestry.

We naturally think of a segmented worm type as a plausible starting-point for Crustaceans, and it is not difficult to understand how a development of cuticular chitin would tend to produce a flexibly jointed limb out of an unjointed parapodium, how the mouth might be shunted a little backwards, and two appendages and ganglia a little forwards, and how division of labour would result in the differentiation of distinct regions.

\section{General Notes on Crustaceans.}

Of a class that includes animals so diverse as crabs, lobsters, shrimps, "beach fleas," "wood lice," barnacles, acorn shells, and "water fleas," it is difficult to state general characteristics, other than those facts of structure which we have already summarised.

Admitting the parasitism of many Crustaceans, and the sedentary life of barnacles and acorn shells, we must still 
allow that great activity characterises the class. With this may be connected the brilliant colouring, the power of colour change, and the phosphorescence of many forms.

Except some primitive and degenerate forms, all are segmented. The typical appendage consists of a basal piece with two jointed branches. The cuticle is always chitinous, and often very much calcified. The abundance of chitin may, to some extent, explain the absence of cilia in Crustaceans and other Arthropods. The rigidity of the cuticle partially explains the necessity of frequent moults. As the muscles contract very rapidly, they illustrate the striated condition with great clearness. In crabs and some others the ventral ganglia are concentrated. Sensory organs are generally well developed; both "eyes" and "ears" may occur away from the head. Much of the alimentary canal, which is almost always simple, consists of fore gut and hind gut. These are anterior and posterior invaginations of skin which meet the mid gut or archenteron-the original gastrula cavity - and are especially large in the higher Crustaceans or Malacostraca. 'The frequent presence of a gastric mill is quite intelligible, for it occurs in the fore gut. The body cavity is never very large, being mainly filled up with muscles and organs, and of a true colome there is little trace. In the blood hæmocyanin is the commonest respiratory pigment. In the body or skin lipochrome pigments, such as those which change from bluish green to red as the lobster is boiled, frequently occur. Of modes of respiration, there are many grades,--by the general surface, by currents of water in and out of the posterior part of the food canal, by thin plates on the legs, by well-formed gills. We miss the numerous excretory nephridia of Annelids; the greenglands of lobsters, \&c., probably represent a pair ; the shellglands of Phyllopods and Copepods and some other structures seem to be in part at least excretory. It is possible that shell making is an organised method of getting rid of some waste products. There are many peculiarities connected with reproduction; thus parthenogenesis for prolonged periods is common among "water fleas"; hermaphroditism occurs in barnacles, acorn shells, ic.; the hermaphrodites are sometimes accompanied by pigmy "complemental" males; the two sexes are often very 
diverse. The spermatozoa are usually exceptional in being very slightly motile. Some appendages are often modified for copulation or for carrying the eggs.

Development.-The ova of most Crustacea show considerable similarity to those of Astacus, and the segmentation is typically of the kind already described. But while this is the most typical case for Crustacean, and, indeed, for Arthropod

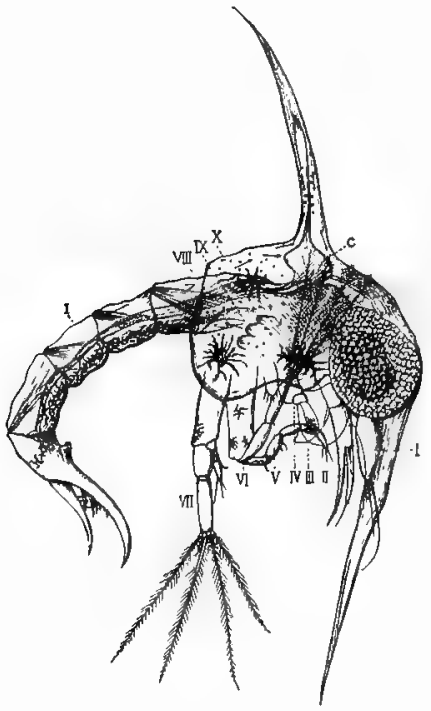

FIG. 91.-Zorea of common Shore Crab (Carcinuts mucras). (After Faxos.) The appendages are numbered. development, it is possible, within the limits of the class Crustacea, to trace out a complete series, in which the first term is a segmentation of the complete and equal type, like that of a worm, and the last the purely peripheral. In the same way, though gastrulation is usually much disguised, we find all cases from an invagination of the simplest embolic type (Lucifer), and through the condition described for. Astacus, to the formation of endoderm by the ingrowth of a solid plug of cells (Arthrostraca, \&c.).

Compared with Astacus, however, the most important point we have to notice is the frequent occurrence of a very striking metamorphosis in the life history. In other words, the larva hatched from the egg is rarely like the parent, and only acquires the adult characters after a series of profound changes. In some cases (Nebalia, Mysis) a metamorphosis takes place within the egg-cases, and in the few forms in which development seems to be direct, slight traces of metamorphosis are found. 
Almost all the lower Crustaceans and the higher forms Euphausia and Penaus are hatched in a Nauplius stage. In the remaining cases the Nauplius stage is indicated within the egg by the moulting of a larval cuticle (so in Astacus). The Nauplius is characterised by a typically rounded body, and by the presence of three pairs of appendages, which are the only obvious indications of segmentation. The first pair of appendages are unbranched and bear larval sense organs, the next two are biramose swimming organs. There is an unpaired median eye, but no heart and frequently no hind gut. The three pairs of appendages become the first and second pairs of antennæ and the mandibles of the adult. The head region of the Nauplius becomes the head region of the adult, the posterior region also persists, the new growth of segments and appendages takes place (with numerous moultings) in the region between these.

The second important form of larva is the Zoæa, which has all the appendages on to the last maxillipedes inclusive, an unsegmented abdomen, and two lateral compound eyes in addition to the unpaired one of the Nauplius stage. Most Decapoda are hatched in the Zoæa stage.

(a) The crayfish (Astacus) is hatched almost as a miniature adult. The development is therefore very direct in this case.

(b) The lobster (Homarzes) is hatched in a Mysis stage, in which the thoracic limbs are two-branched and used for swimming. After some moults it acquires adult characters.

(c) Crabs are hatched in the Zocea form, and pass with moults through a Megalopa stage, in which they resemble certain Hermit Crabs. The abdomen is subsequently tucked in under the thorax.

(d) Pencus (a kind of shrimp) is hatched as a Nauplius, becomes a Zocza, then a Mysis, then an adult. Its relative Lucifer starts as a Meta-Nauplizes with rudiments of three more appendages than the Nauplius. Another related form, Sergestes, is hatched as a Protozocea, with a cephalothoracic shield and an unsegmented abdomen. Thus there are two grades between Nauplius and Zora.

Three facts must be borne in mind in thinking over the life histories of crayfish, lobster, crab, and Pencus:-(I) there is a general tendency to abbreviate development, and this is of more importance when metamorphosis is expensive and full of risks ; (2) there is no doubt that larva exhibit characters which are related to their own life rather than to that of the adult ; (3) it is a general truth, that in its individual development the organism has to recapitulate to some extent the evolution of the race, that ontogeny recapitulates phylogeny. But while there can be no 
doubt that the metamorphoses of these Crustaceans is to some extent interpretable as a recapitulation of the racial history, for there were unsegmented animals before segmented forms arose, and the Zoca stage is antecedent to the Mysis, \&c., yet it does not follow that ancestral Crustaceans were like Nauplii. On the contrary, the Nauplizes must be regarded as a larval reversion to a type much simpler than the ancestral Crustacean. Moreover, this idea of recapitulation offers a philosophical rather than a material explanation of the facts.

\section{Bionomics.}

Most Crustaceans are carnivorous and predatory; others feed on dead creatures and organic debris in the water; a minority depend upon plants.

Parasitism occurs in over 700 species, in various degrees, and of course with varied results. Most of the parasites keep to the outside of the host (e.g., Fish lice), and suck nourishment by their mouths; the Rhizocephala (e.g., Sacculina), send ramifying absorptive roots through the body of the host. Sometimes the parasitism is temporary ( $A r-$ gulus); sometimes only the females are parasitic (e.g., in Lernoa). The parasites tend to lose appendages, segmentation, sense organs, \&c., but the reproductive organs become more fertile. The hosts, e.g., crabs infested by Rhizocephala, are sometimes materially affected, and even rendered incapable of reproducing.

Some Crustaceans live not as parasites but as commensals with other animals, doing them no harm, though sharing their food. Thus there is a constant partnership between some hermit crabs and sea anemones. The hermit crab is concealed and protected by the sea anemone; the latter is carried about by the Crustacean and gets fragments of food.

Masking is also common, especially among crabs. Some will cut the tunic off a sea squirt and throw it over their own shoulders. Many attain a mask more passively, for they are covered with hydroids and sponges, which settle on the shell. There is no doubt, however, that some actively mask themselves, for besides those known to use the Tunicate cloak, others have been seen planting seaweeds on their backs. The protective advantage of masking both in offence and defence is very obvious.

The intelligence of crabs and some of the higher Crustaceans is well developed. Maternal care is frequent. 
Fighting is very common, but the loss of limbs is readily repaired.

Deep sea Crustaceans are very abundant, and often remarkable "for their colossal size, their bizarre forms, and brilliant red colourings;" some are blind, others are brilliantly phosphorescent. Yet more abundant are the pelagic Crustaceans (especially Entomostraca and Schizopods); they are often transparent except the eyes, often brightly coloured or phosphorescent. Many Crustaceans live on the shore, and play a notable part in the struggle for existence which is so keen in that densely crowded region. The lower Crustaceans are abundantly represented in fresh water, in pools, streams, and lakes. A few, such as wood lice and land crabs, are terrestrial, and some blind forms occur in caves. 


\section{CHAPTER XIV.}

\section{PEIRIPATUS, MYRIOPODS, AND INSECTS.}

Series Arthropoda. Sub-division Tracheata Antennata.

Classes Prototracheata.-Peripatus. Myriopoda.-Centipedes and Millipedes. InSECTA,-Insects.

These three classes form a series of which winged insects are the climax. The type Peripatus is archaic, and links the series to the Annelids; the Myriopods lead on to the primitive wingless insects.

We may speak of the series as 'Tracheata Antennata, for all breathe by tracheæ-tubes which carry air to the recesses of the body - and all have antennæ.

First Class of Tracheata Antennata- Prototracheata, including one genus, Peripatus.

General Characters. - The body is worm-like in form, soft skinned, and without external segmentation.

There is a pair of prominent pre-oral antennce.

The true appendages are - a pair of jazes in the mouth, a pair of slime secreting oral papilla, numerous pairs of short, imperfectly jointed legs, each with two claws, and a pair of anal papillce. The legs contain peculiar (coxal) glands.

Respiration is effected by numerous trachece, whose openings are somewhat scattered on the surface of the body. The heart is simply an elongated dorsal vessel with valvular openings. There is a series of excretory tubes or nephridia. The halves of the ventral nerve cord are widely separate.

The single genus Peripatus is represented by numerous 
(twelve) species, widely distributed; in its possession of trachee and nephridia it is an interesting connecting link; in many ways it seems to be an old fashioned survivor of an archaic type.

The species of Peripatus are beautiful animals. Professor Sedgwick says-"'The exquisite sensitiveness and continually changing form of the antennæ, the wellrounded plump body, the eyes set like small diamonds on the side of the head, the delicate feet, and, above all, the rich colouring and velvety texture of the skin, all combine to give these animals an aspect of quite exceptional beauty." As to their habits, Mr. Hatchett Jackson says-" "They live under stones, in rotting wood, \&c., in moist places, are nocturnal in habit, and feed on insects, \&c., which they ensnare by the ejection of slime from the oral papilla." To their shy habits, their persistence is possibly in part due. They are able to move quickly, somewhat after the fashion of Millipedes, especially like Scolopendrella. Young forms roll up when touched, and have been seen to $\operatorname{climb}$ up vertical glass plates.

The species acknowledged by Sedgwick are:-Four from South Africa- $P$. capensis, $P$. balfouri, and $P$. brevis from Table Mountain, and $P$. moseleyi from near Williamstown; two from Australasia- $P$. nowe zealandice from New Zealand, and $P$. leuckarti from Queensland; seven from neotropical regions- $P$. edwards $i i$ from Caracas, $P$. inthumi or demeraranus from Demerara, $P$. trinidadensis and $P$. torquatus from Trinidad, $P$. iuliformis from St. Vincent, $P$. chilensis from Chili, $P$. quitensis from Ecuador, besides which there are some doubtful forms. The list shows how widely this remarkable genus is distributed.

As the different species have similar habits, and live in very similar conclitions, the differences between them perhaps illustrate purely constitutional variations.

\section{A more Detailed Account of Peripatus.}

Form.-The body suggests an Annelid or a caterpillar, but, apart from the appendages, there is no external segmentation. Over the soft skin are numerous minute warts with small bristles. The mouth is ventral and anterior; the anus terminal and posterior. 
Appendages. - The two large, ringed antennee do not seem to be homologous with limbs. The first pair of appendages-clouble sickle. like jaws-lie in the mouth cavity. A little further back are two oral papillx from which slime is exuded. Then there are the I4-42 stumplike legs, each with two terminal chitinous claws. In the young $P$. capensis the leg is said to be five-jointed, but in the adults there is no trace of this. In respect to its legs, therefore, Peripatus is hardly an Arthropod.

Skin. - The chitinous cuticle, ordinarily thick in Arthropods, is delicate. The ectoderm [hypodermis, or epidermis] is a single layer of cells.

The Muscular System is very well developed. (I) Externally there is a layer of circular muscles; (2) within this lies a double layer of diagonal fibres; (3) internally there are strong longitudinal bundles. Finally, in connection with this internal layer, there are fibres which divide the body cavity into a median and two lateral compartments. The median includes heart, gut, slime glands, reproductive organs; the laterals include the nerve cords, the salivary glands; the legs contain nephridia and coxal or crural glands. Striped, rapidly contracting muscles are characteristic of Arthropods, but in Peripatus the muscles are unstriped, excepting those which work the jaws and are perhaps the most active.

The Nervous System consists of a dorsal brain and two widely separate lateral ventral nerve cords. These are connected transversely by numerous commissures, are slightly swollen opposite each pair of legs to which they give off nerves, and are united posteriorly over the anus. There are only hints of ganglia, but there is a continuous layer of ganglionic cells. The brain is very homogeneous, simpler than that of most Insects. From the brain nerves pass to the antenna, \&c., and two viscerals or sympathetics, soon uniting, innervate the anterior part of the gut. Sense organs are represented by two simple eyes on the top of the head. These are most like the eyes of some marine Annelids. Behind each there lies a special optic lobe connected with the brain, but the eye itself arises as a dimple in the skin.

Alimentary Cana!. - Round about the mouth, papillat seem to have fused to form a " mouth cavity," which includes the mandibles, a median pad or tongue, and the opening of the mouth proper. The mouth leads into a muscular pharynx, into which opens the common duct of two large salivary glands, which extend far back along the body. Mouth, pharynx, and short cesophagus are lined by a chitinous cuticle, like that of the exterior. The long digestive region or mid gut extends from the second leg nearly to the end of the body. Its walls are plaited. Finally, there is a short rectum, lined by a chitinous cuticle.

Circulatory System. - The dorsal blood vessel forms a long contractile heart. It lies within a pericardial space, and receives blood by segmentally arranged apertures with valves. The circulation is mostly in ill-defined spaces in the apparent body cavity or "hremocole."

The Respiratory System consists of very long and very fine unbranched trachex, which are widely distributed in the body; a number open together to the exterior in flask-like depressions. These openings or stigmata are diffuse and irregular in Peripatus edwardsiz, but in $P$. capensis 
there is a dorsal and ventral row on each side. In $P$. nove zeaiandice the trachea are said to be branched.

The Excretory System.-A pair of nephridia lie in each segment. Each consists of an internal terminal funnel, a looped canal, and a wide vesicle which opens near the base of each leg. They are not very different from those of many Annelids, but their occurrence in a Tracheate is remarkable. The salivary glands and the genital ducts are probably modified nephridia. It may be noted, too, that the same is perhaps true of the "coxal glands" of Limulus and of the antennary glands of Crustaceans.

Crural or Coxal Glands lie in the legs and open to the exterior. Their meaning is uncertain, their occurrence is variable. Thus in $P$. edwardsiz they occur in the males only, in $P$. capensis they are present in both sexes. In the male of $P$. capensis the last pair are very long (a.g., Fig. 93). The large mucus glands, which pour forth slime from the oral papillæe, are regarded as modified coxal glands.

Reproductive System.-(a) Female (of P. edwardsii).-From the two ovaries, which are surrounded by one connective tissue sheath, the eggs

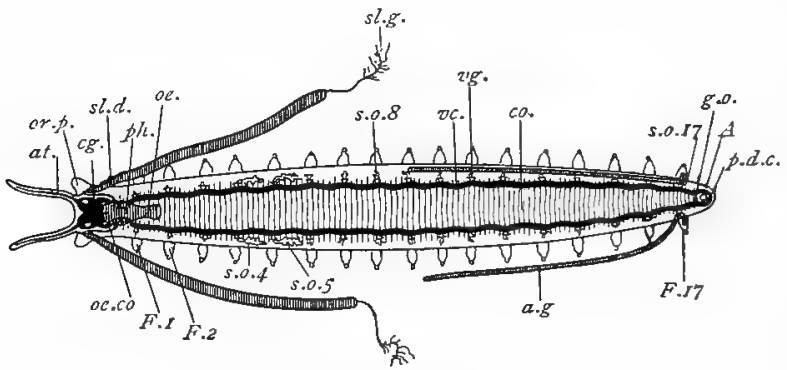

FIG. 93.-Dissection of Peripatus capensis.

(After BALFouR.)

at., Antennz; or.p., oral papillæ; c.g., cerebral ganglia ; sl.d., duct of slime gland $\left(s, g_{1}\right) ; s .0 .8$, segmental organ or nephridium eighth; $\eta_{\text {. }}$., ventral nerve connected by transverse commissures (co.) with its fellow; $v . g$, last crural gland; 5.0 .17 , seventeenth nephridium ; g.o., genital aperture; $A$, anus; $\phi_{\text {. }}$ l.c.u. $_{\text {. }}$ posterior commissure; $F, r 7$, seventeenth appendage: $a, g$., last crural gland; $F . I, F .2$, first and second legs; oe.co., cesophageal nerve commissure.

pass by two long ducts leading to a common terminal vagina opening between the second last legs. These ducts are for the most part uteri, but on what may be called the oviduct portions adjoining the ovaries, there are two pairs of pouches- $(a)$ a pair of receptacula seminis (for storing the spermatozoa received during copulation), and a pair of receptacula ovorum for storing fertilised eggs.

The eggs are hatched in the uteri, and all stages are there to be found in regular order. The young embryos seem to be connected to the wall 
of the uterus by what has been called a "placenta," so suggestive is it of mammalian gestation. The older embryos lose this "placenta," but each lies constricted off from its neighbours. When born the young resemble the parents except in size and colour. In $P$. novce zealandice, the ova pass from the ovary into the uterus in December, and the young are born in July-a long period of gestation.

(b) Male (of $P$. edwardsiz). The male elements are produced in small testes, pass thence into two seminal vesicles, and onwards by two vasa deferentia into a long single ejaculatory duct, which opens in front of the anus. In the ejaculatory duct the spermatozoa are made into a long packet or spermatophore, which is attached to the female during
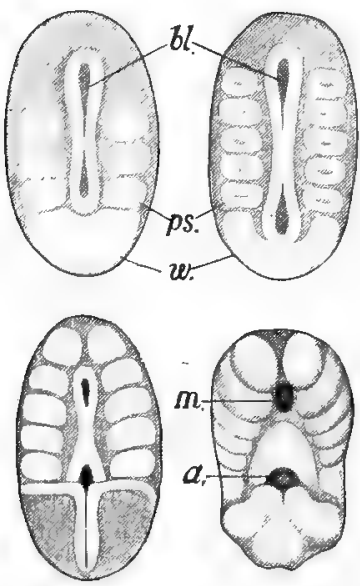

FIG. 94.-Embryos of Peripatus capenssis, showing closure of blastopore and curvature of embryo. (After KORSCHELT and HEIDER.)

$a$, Anus ; $b l$, blastopore ; $m$, mouth ; $p s$, primitive segments; $z \ell$, zone of proliferation. copulation.

[While it is characteristic of Arthropods, in which the development of chitin is so predominant, that ciliated epithelium is absent, it seems that in Peripatus, which is much less chitinous than the others, ciliated cells occur in some parts of the reproductive ducts, and perhaps also at the internal funnels of the nephridia. This is indeed what one would expect.]

Development of Peripatus.There is a strange variety of development in different species of this genus. Thus there is much yolk in the ovum of $P$. nove zealandice, extremely little in that of $P$. capensis. In the former species the yolk has a manifold origin; it is said to arise in the protoplasm or the ovum itself from the breaking up of the germinal vesicle, from surrounding follicle cells, and from yolk present within the ovary. In $P$. capensis and $P$. balfour $i$ spermatozoa reach the ovary, and there probably the ova are fertilised, but in $P$. nove zealandia the spermatozoa are confined to the receptaculum seminis, near which fertilisation seems to occur. In the maturation of the ova of $P$. capensis and $P$, balfour $i$ two polar bodies are extruded as usual, but none have been observed in the case of $P$. nove zealandice.

In $P$. capensis the "segmentation" is remarkable, for true cleavage of cells does not occur. The fully "segmented" ovum does not exhibit the usual cell limits. It is a protoplasmic mass-or syncytium-with many nuclei. Even when the body is formed, the continuity of cells 
persists, nor does the adult lack traces of it. To Professor Sedgwick, this singular fact suggested the theory that the Metazoa may have begun as multinucleate Infusorian-like animals.

The gut appears as a large vacuole within the multinucleated mass, and a gastrula stage is thus established.

In the ova of $P$. nove zealandia, which have much yolk, a superficial multiplication of nuclei forms a sort of blastoderm, which spreads over almost the entire ovum. The segmentation in this case has been called centrolecithal (the type characteristic of Arthropods), but it is again true that for a long time the cells do not exist as well defined units. It has been said, indeed, that "the embryo is formed by a process of crystallising out in situ from a mass of yolk, among which is a protoplasmic reticulum containing nuclei."

From these examples the student will perceive how difficult it is to give a succinct account of the development of Peripatus.

\section{Development of Organs.}

The hypodermis is ectodermic, the cuticle an external product thereof.

The muscles are as usual derived from the mesoderm, which arises from two ventral mesodermic strands. These are subsequently divided into hollow segments. The true body cavity or colome is represented by the original cavities of the mesoderm segments. In the adult this series of truly colomic cavities is hardly represented except by the innermost portions of the nephridia. The apparent body cavity is a secondary cavity, consisting, for the most part, of blood carrying or vascular spaces, subsequently established in the mesoderm. It is divided into five regions, the central space, the two lateral cavities, and the cavities of the legs.

The appendages are outgrowths of the body wall. They, and all the segmentally arranged parts, develop progressively from in front backwards.

The nervous system is derived from ectodermic thickenings which sink inwards. It develops from in front of the mouth backwards.

The food canal consists of the long endodermic mid gut or mesenteron (the gastrula cavity), of an anterior ectodermic invagination forming pharynx and gullet (fore gut or stomatodæum), and of a short posterior ectodermic invagination forming the rectum (hind gut or proctodrum).

The nephridia have a twofold origin. The internal funnel is derived directly from part of a mesodermic segment or vesicle. The rest of the nephridium is invaginated from the ectoderm.

The reproductive organs arise on the epithelium of a persistent portion of the true colome or primitive body cavity.

Zoological Position of Peripatus. - Professor Lang, in his work on Comparative Anatomy, summarises the synthetic characters of Peripatus
as follows :- 
Annelid Characteristics.

Segmentally arranged nephridia as in Chætopods.

Segmentally arranged coxal glands, like similar glands in some Chætopods.

The muscular ensheathing of the body.

Less important are the stumplike legs and the simple eyes.
Tracheate Characteristics.

The presence of tracheæ.

The nature of the heart and the lacunar circulation.

The modification of appendages as mouth organs.

The form of the salivary glands.

The smallness of the genuine body cavity or colome.

The ladder like character of the ventral nervous system (cf. primitive Molluscs, Phyllopod Crustaceans, and Nemerteans) is probably primitive. That salivary glands and genital ducts are homologous with nephridia is a fact of much morphological interest. It is possible that the slime glands are modifications of coxal or crural glands, and that the latter are homologous with the parapodial glands of some Annelids. It is not certain that the antennæ, jaws, and oral papillæ of Peripatus precisely correspond to the antennæ, mandibles, and first maxillæ of Insects.

Our general conclusion is that Peripatus is an archaic type, a survivor of forms which were ancestral to Tracheata and closely related to Annelids.

Second Class of Tracheata Antennata. Mrriopoda. Centipedes and Millipedes.

These animals retain a worm-like shape; the numerous rings of the body and the appendages they bear are very uniform; there is little division of labour. It would be rash to assert that any of the modern Myriopods are stages in the pedigree of Insects, but it is likely that the two classes are branches from one base. Simple wingless insects, known as Collembola and Thysanura, are closely approached by such Myriopods as Scolopendrella.

Both centipedes and millipedes live on land, but two or three of the latter (e.g., a species of Geophilus) occur on the seashore. Most are very shy animals, lurking in dark places and avoiding the light.

The head bears a pair of antennæ, and two pairs of appendages-mandibles and maxillæ. The limbs are sixor seven-jointed, clawed, and very uniform. They have many more legs than insects, but they make less of them. The nervous system, heart, excretory tubules, \&c., are like those of Insects. 
The development in many ways suggests and leads up to that of Insects.

$$
\text { MYRIOPODA. }
$$

\begin{tabular}{l} 
Centipedes. \\
Chilopoda. \\
\hline Carnivorous. \\
Poisonous. \\
Body usually flat.
\end{tabular}

A pair of appendages to each segment.

Many-jointed antenne.

Toothed cutting mandibles.

Each maxilla consists of an external palp, and a bilobed median portion.

The next appendage is leg-like. Then follows a large basilar plate, beside which are the two poison claws. ture.

A single posterior genital aper-

\section{Examples Scolopendra.} Lithobizes.
Millipedes,

Diplopoda (or Chilognatha).

Vegetarian.

Harmless,

Body cylindrical.

By theimperfect separation of the segments all but the most anterior seem to have two pairs of appendages each, and also two paired ganglia, and two pairs of stigmata.

Seven-jointed antennæ.

Broad masticating mandibles.

Maxillee are represented by a four-lobed plate.

No basilar plate.

Genital apertures open on the second or third pair of limbs.

$$
\begin{aligned}
& \text { Examples-Julus. } \\
& \text { Geophilus. }
\end{aligned}
$$

In reference to habitat, it is interesting to note that at least two myriopods-Geophilus submarinus and Linotinia maritima, occur on British coasts.

As distinct from the two chief sub-classes, it is perhaps necessary to recognise other two-Pauropoda, e.g., Pauropzis, and Symphyla, e.g., Scolopendrella. The last-named approaches closely to the most primitive insects (Collembola and Thysanura).

Third Class of Tracheata Antennata. INsEctA.

Insects occupy a position among the backboneless animals like that of birds among the Vertebrates. The typical members of both classes have wings and the power of true flight, richly aërated bodies, and highly developed nervous and sensory organs. Both are very active and brightly 
coloured. They show parallel differences between the sezes, and great wealth of species within a narrow range. One expects to find that insects, like birds, have a high body temperature.

General Characters. - Like other Arthropods, Insects have segmented bodies, jointed legs, chitinous armature, and a ventral chain of ganglia linked to a dorsal brain. Compared with Peripatus and Myriopods, adult insects showe concentration of the body segments, decrease in the number and increase in the quality of the appendages, and wings withal.

Insects are terrestrial and aërial, and rarely aquatic animals; usually zeinged as adults, breathing by means of trachee, and often with a metamorphosis in the course of their groweth.

The body is divided into three distinct regions, - head, thorax, and abdomen. The head bears three pairs of mouth appendages (= legs), and a pair of pre-oral out-growethsthe antennce; the thorax bears a pair of legs on each of its three segments, and, typically, a pair of wings on each of the posterior two; the abdomen has no appendages, unless rudimentary modifications of these be represented by stings, ovipositors, \&c.

\section{First Type of Insects, Periplaneta (or Blatta). The Cock ROACH.}

The cockroaches found in Britain are immigrants, either from the East ( $P$. orientalis), or from America ( $P$. americana); the two species closely resemble one another. They are omnivorous in their diet, and active in their habits, but they hide during the day and feed at night. They are ancient insects, for related forms occurred in Silurian ages; they are average types, for they are neither very simple nor very highly specialised. Their position is among the Orthoptera, in the same order as locusts and grasshoppers. The young are hatched as miniature adults, except that wings are absent ; in other words, there is no metamorphosis in development.

The skin consists of an external chitinous cuticle and a subjacent cellular layer-the epidermis or hypodermisfrom which the cuticle is formed. The newly hatched cockroaches are white, the adults are dark brown. 
External Characters.

The Heav.
It is vertically
elongated and
separated from
the thorax by a
neck.

The Thorax.

It consists of three segments :-

(a) prothorax,

(b) mesothorax,

(c) metathorax,

(Each segment is bounded by a dorsal tergum, and ventral sternum.)

The Abdomen.

It consists of ro (or I I) distinct segments, with terga and sterna as in the thorax.
Appendages of the Head.

I. A pair of stout toothed mand. ibles working sideways.

2. The first maxillæ, each consisting $(\alpha)$ of a basal piece or protopodite with two jointsa basal cardo, a distal stipes;

(b) of a double endopodite borne by the basal piece, and consisting of an inner lacinia and a softer outer galea;

(c) of an exopodite or maxillary palp also borne by the basal piece, and consisting of five joints.

3. The second pair of maxilla, fused together as the "labium," consisting (a) of a fused basal piece or protopodite with two joints-a basal submentum, a smaller distal mentum; on each side this protopodite bears

(b) a double endopodite (ligula) consisting of an inner lacinia, and an outer paraglossa;

(c) an exopodite or labial palp, consisting of three joints.

The Appendages of The Thorax.

(a) First pair of legs.

(b) Second pair of legs.

(c) Third pair of legs. Each leg consists of many joints-a basal "coxa" with a small "trochanter" at its distal end, a "femur," a "tibia," a six-jointed tarsus or foot ending in a pair of claws.

\section{Appendages (?) OF THE ABDOMEN.}

Two cigar-shaped tactile anal cerci, attached under the edges of the last tergum, are possibly relics of the last abdominal appendages.

The ninth sternum of the male bears a pair of styles, possibly relics of appendages.

Both sexes have complex hard structures (gonapophyses) beside the genital apertures. They are possibly relics of appendages.
Other Structures on the HEAD.

The antenna (probably not homologous with appendages), long, slender, many jointed, tactile.

The large black compound eyes.

The "upper lip" or labrum, in front of the mouth.

The white oval patches near the bases of the antenna, possibly sensory.

\section{Other STRUCTURES ON THE} Thorax.

(b) A pair of elytra or wing. covers (modified wings) rudimentary in female of $P$. orientalis.

(c) A pair of membranous wings, sometimes used in flight, folded when not in use, absent in female of $P$. orientalis.

Between the segments of the thorax are two pairs of respiratory apertures or stigmata.

Other Structures on the ABDonen.

A pair of stigmata occur between the edges of the terga and sterna in the first eight abdominal seg. ments. There are therefore twenty stigmata in all. The anus is terminal, beneath the tenth tergum of the abdomen; a pair of "podical plates" the beside it.

The genital aperture is terminal, ventral to the anus.

The opening of the spermatheca - the female's receptacle for sperma. tozoa-lies on the ninth sternum of the abdomen 
Moulting, which involves a casting of the cuticle, of the internal lining of the tracheæ, \&c., occurs some seven times before the cockroach attains in its fifth year to maturity.

The muscles, which move the appendages, and produce abdominal movements essential to respiration, are markedly cross striped.

Nervous System.-A pair of supra-œesophageal or cerebral ganglia lie united in the head. As a brain, they receive

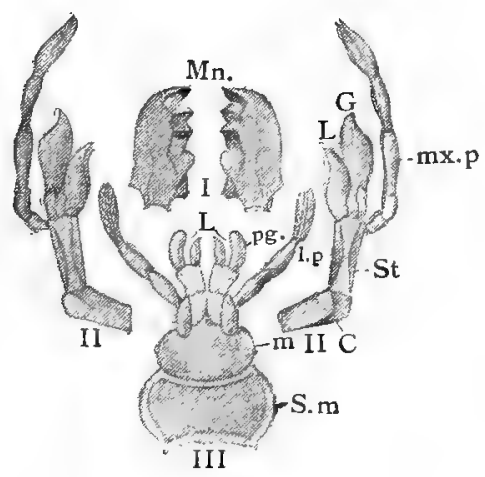

FIG. 95.-Mouth appendages of Cockroach. (After DUFOUR.)

I. $M n$, Mandibles; II. First Maxilla ; $c$, cardo; st, stipes; $L$, lacinia; $G$, galea; mx.p, maxillary palp; III. Second Maxillæ or Labium; s.m, submentum; $m$, mentum ; $L$, laciniz ; $p g$, paraglossa ; l.p, labial palp.

impressions by antennary and optic nerves. By means of a paired commissure surrounding the gullet, they are connected with a double ventral chain of ten ganglia. Of these, the first or sub-œsophageal pair are large, and give off nerves to the mouth parts, \&c. ; from each of the ganglia of the thorax and the abdomen nerves are given off to adjacent parts. There are three pairs of ganglia in the thorax, and six 
in the abdomen, of which the last is the largest. From the cesophageal commissures two visceral nerves are given off, which form in a somewhat complex manner the innervation, of gullet, crop, and gizzard. Besides the large compound eyes, there are other sensory structures-some of the hairs on the skin, the maxillæ (to some extent organs of taste), the antennæ (tactile and olfactory), the anal cerci (tactile), and possibly the oval white patches on the head.

Alimentary System.-(x) The fore gut (stomatodæum) is lined by a chitinous cuticle continuous with that of the outer surface of the body. It includes $(a)$ the buccal or mouth cavity, in which there is a tongue-like ridge, and into which there opens the duct of the salivary glands; $(b)$ the narrow gullet or œsophagus; (c) the swollen crop; $(d)$ the gizzard

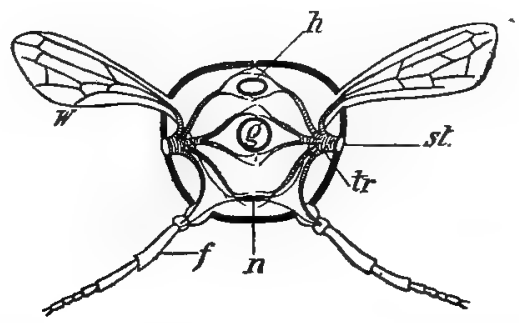

FIG. 96.-Transverse section of Insect. (After PACKARD.)

$h$, Heart ; $g$, gut ; $n$, nerve cord ; st, stigma ; $t r$, trachea ; $w$, wing; $f$, femur of leg.

with muscular walls, six hard cuticular teeth, and some bristly pads.

There is a pair of diffuse salivary glands on each side of the crop, and between each pair of glands a salivary receptacle. The ducts of the two salivary glands on each side unite, the two ducts thus formed combine in a median duct, and this unites with another median duct formed from the union of the ducts of the receptacles. The common duct opens into the mouth.

(2) The mid gut (mesenteron) is lined by endoderm. It is short and narrow, and with its anterior end seven or eight 
club-shaped digestive outgrowths are connected. These seem to have a pancreatic function.

(3) The hind gut (proctodæum) is lined by a chitinous cuticle. It is convoluted and divided into narrow ileum, wider colon, and dilated rectum with six internal ridges. From the beginning of the ileum, the excretory Malpighian tubules are given off.

Respiratory System. - The tracheal tubes, which have ten pairs of lateral apertures or stigmata, ramify throughout the body.

Circulatory System.-The chambered heart lies along the mid dorsal line of abdomen and thorax. It receives blood by lateral valvular apertures from the surrounding pericardial space, and drives it forwards by a slender aorta. The blood circulates, however, within ill-defined spaces in the body.

The Excretory System consists of sixty or so fine (Malpighian) tubules, which rise in six bundles from the beginning of the ileum, and twine through the "fatty body" and in the abdominal cavity.

\section{Reproductive System}

OF THE MALE.
The testes are paired organs, sur-
rounded by the fatty body below
the 5th and 6th abdominal
terga. They atrophy in the
adult.

From the testes, two narrow ducts or vasa deferentia lead to two seminal vesicles.

These seminal vesicles (the "mushroom-shaped gland ") open into the top of the ejaculatory duct.

This duct opens on the Ioth sternum. Beside the aperture there are copulatory structures (gonapophyses). With the ejaculatory duct a gland is associated.
OF THE FEMALE.

The ovaries are paired organs, in the posterior abdominal region, each consisting of eight ovarian tubes. These are bead-like strings of ova at various stages of ripeness.

From the ovarian tubes of each side, eight eggs pass at a time into a short wide oviduct.

The two oviducts unite and open in a median aperture between the 8th and gth abdominal sterna. Beside the aperture are hard structures (gonapophyses) which help in the egg laying. Here also a pair of "colleterial" glands pour out their cementing secretion by two apertures. The spermatheca is a paired sac with a single aperture on the gth abdominal sternum. 
Sixteen ova, one from each ovarian tube, are usually enclosed within each egg capsule. The latter is formed from the secretion of the colleterial glands. Each egg is enclosed in an oval shell, on which there are several little holes (micropyles), through one of which a spermatozoon enters. Spermatozoa, from the store within the spermatheca, are included in the egg capsule. The development is similar to that of other insects, and it has already been mentioned that there is no metamorphosis.

At an early stage in development, some cells associated with the mesoderm are set apart as reproductive cells, and originally these have a segmental arrangement as in Annelids; at a later stage other mesoderm cells join these, some forming ova, others epithelial cells around the latter. The distinction between truly reproductive cells and associated epithelial cells, which is said to be late of appearing in some of the higher insects, is established at a very early stage in the cockroach.

Second Type of Insects. The British Hive BeE (Apis mellifica.)

This is a much more highly specialised type than the cockroach. It belongs to the order Hymenoptera.

The Hive Bee (Apis mellifica) is a native of this country, and is the species most commonly found domesticated. It is the only British representative of the genus Apis, and exhibits, in its most fully developed form, the social life which is foreshadowed among the Humble Bees. As a consequence of this social life, there is much division of labour, which expresses itself alike in habit and in structure. The males (drones) take no part in the work of the colony, and have solely a reproductive function; the females are divided into two groups-the queen bees and the workers. In the workers, which do, in fact, perform all the work of the hive, the vegetative organs attain their highest degree of development, but the reproductive organs are normally abortive and functionless. In the queens, of which there is but one adult to each hive, the enormous development of the reproductive organs seems to act as a check upon the vegetative organs, which are of less advanced type than those of the workers. The workers are further divisible into nurses, which are young and do not leave the hive, being 
occupied with the care of the larva, and the foraging bees, which are older workers, and gather the food to supply the whole colony.

In considering the relation between the life of the Hive Bee and that of many allied forms (Bombus, \&c.), it is important to notice that the habit of laying up stores of food material for the winter, enables the colony, and not merely an individual, to survive, and must thus have greatly assisted in the evolution of sociality.

The body shows the usual division into head, thorax, and abdomen, and varies considerably in the three different types, being smallest in the workers. It is entirely covered with hairs, some of which are sensitive, while others are used in pollen gathering, \&c.

The head bears antennæ, which are composed of a long basal and numerous smaller joints. They are marvellously sensitive, serving to communicate impressions, and also containing organs of special sense. A pair of compound eyes, largest in the drones, and three median ocelli are also present in the head region. Of the true appendages of the head, the mandibles are in the workers very powerful and used for many purposes connected with comb building. In the first maxillæ, the maxillary palps are aborted, but internal lacinia, external galea, and basal stipes and cardo are present as usual. The second pair of maxillæ are much modified to form the labium or so-called lower lip. The united basal joints form the mentum and sub-mentum. From the mentum at either side springs the long labial palp, which represents the outer fork of the typical appendage. The inner fork is divided into two parts at each side, of these the inner (laciniæ) are united and much elongated, the two outer or paraglossæ are free and closely apposed to the lacinix; the whole structure is known as the ligula. When the bee is engaged in sucking honey from a flower, the maxillæ and labial palps are closely apposed to the ligula, and thus an air-tight tube is formed. When not in use, the whole structure is folded back upon itself.

In the queen and in the drone the mouth parts are shorter, and are not used in honey gathering.

The thoracic appendages consist as usual of three pairs of legs, which have the usual parts. On the first leg, at 
the junction of the tibia and the first tarsal joint, there is a complicated mechanism which is employed in cleaning the antennæ; this is present in all three forms, and varies with the size of the antennæ. In the workers the third leg is remarkably modified for pollen gathering purposes. The first tarsal joint bears regular rows of stiff straight hairs on which the pollen grains are collected ; they are borne to the hive in the pollen basket, placed at the back of the tibia, and furnished with numerous hairs. In queen and drone, these special arrangements of hairs are absent.

The second and third thoracic segments bear each a pair of wings. These are largest in the drones and relatively smallest in the queen, who flies but seldom. At the base of each wing there is a respiratory spiracle.

In the adult queen and worker, the abdomen is divided into six segments; in the drone, into seven. There are no abdominal appendages. On the ventral surface in the worker, but not in the queen or drone, there are four pairs of wax pockets or glands, which secrete the wax which, after mastication with saliva, is employed in

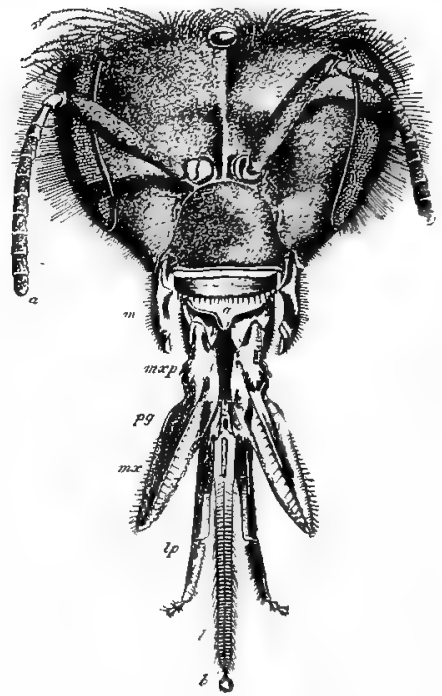

FIG. 97.- Head and mouth parts of Bee. (After Cheshire.)

$a$, Antenna; $n$, mandible; $g$, gum flap or epipharynx; mx.p, maxillary palp; $p g$ and $n x$, galen and lacinia; $2 . p$, labial palp; $l$, ligula ; $b$, bouton at end. building the combs. The abdomen also bears in queen and worker five pairs of spiracles, but in the drone, on account of the additional segment, there are six pairs. The total number of spiracles is thus fourteen for queen and worker, and sixteen for the drone. The posterior region of the abdomen bears 
the complicated sting. In the worker, this consists of a hard incomplete sheath, which envelops two barbed darts. The poison flows down a channel lying between the darts and the sheath. Ramifying through the abdomen are found the two slender coiled tubes which constitute the poison gland. At the posterior end of the body these unite and open into a large poison sac. When a bee uses its sting, the chitinous sheath first pierces the skin, and then the wound is deepened by the barbed and pointed darts, while at the same time poison is steadily pumped down the channel mentioned above, and pours out by minute openings at the bases of the darts. The poison contains formic acid, and is fatal to the bee if directly introduced into its blood. Associated with the sting there are a pair of delicate tactile palps. In the queen, the sting is curved and more powerful, but it is apparently only used in combat with a rival. In the worker, the sting, and with it a portion of the gut, is usually lost after use, and, in consequence, death ensues ; the queen, on the other hand, can withdraw her sting from the wound with considerable ease. There is no trace of sting in the drone, as is natural when we consider that it is merely a modification of an ovipositor.

\section{Nervous System.}

In the adult this exhibits considerable fusion of parts. 'The supra-œsophageal ganglia are very large, and send large lateral extensions to the compound eyes. This "brain" is best developed in the active workers. The sub-oesophageal mass is formed by the fusion of three pairs of ganglia. In the thorax there are two pairs of ganglia, of which the second supplies the wings and the two last pairs of legs. In the worker there are five pairs of abdominal ganglia, but in the queen and drone only four. The sense organs are the simple and compound eyes, and the antennæ, which are furnished with numerous sensitive structures.

\section{Alimentary System.}

The cesophagus is a narrow tube which runs down the thoracic region. In the abdominal region it expands into the crop or honey sac. The crop opens by a complicated 
orifice, with a remarkable stopper arrangement, into the digestive region or chyle stomach, which is separated by a pylorus from the coiled small intestine. The inner wall of the small intestine bears numerous rows of chitinous teeth set in longitudinal ridges, and is perforated by the apertures of the excretory tubules. At the junction of the small with the large intestine, there are six brownish plates, perhaps functioning as valves.

In connection with the anterior region of the gut, there is a very complicated series of glands. First, we have in the workers only, on
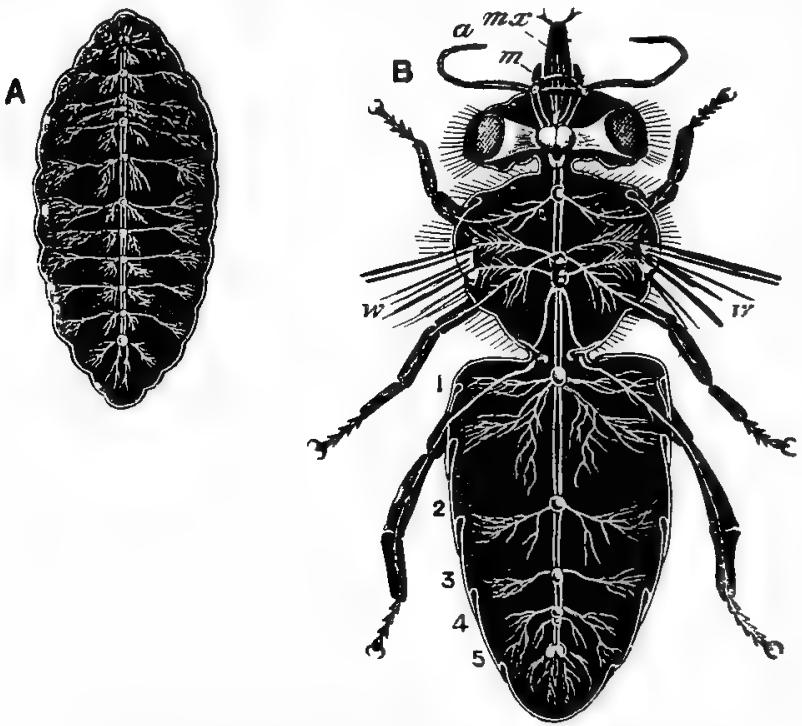

FIG. 98--Nervous system of Bee. (After CHeshiRe.)

A, Of larva; $\mathrm{B}$, of adult ; $a$, antenna ; m $x$, maxilla ; $m$, mandible ; $w$, origin of wing; $\mathrm{x}-5$, abdominal ganglia.

either side of the head, a long coiled gland which is intracellular in type. It is largest in the so-called "nurses" which feed the young, and diminishes in size later. According to Mr. Cheshire, this gland secretes a nitrogenous fluid which is furnished to all the larve in their early stages, but is supplied to the future queen during the whole of the 
feeding period, and also during the period of egg laying; this secretion was formerly termed "royal jelly." In addition to this pair of glands, there are in the worker three other gland systems. Of these, the second and third pairs have a common central outlet on the mentum, and

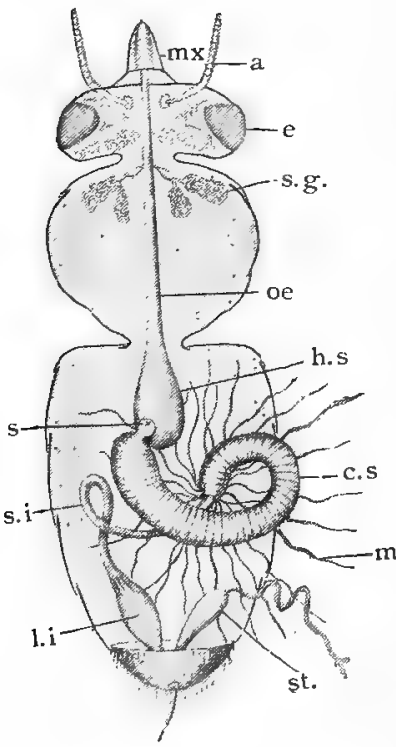

FIG. 99.-Food canal of Bee. (In part after CheshiRe.)

$m x$, Maxilla ; $\boldsymbol{a}$, antenna ; $\ell$, eye ; s. $g$, salivary glands; $\alpha$, oesophagus; $h . s$, honey sac; $s$, stopper; c.s, chylific stomach; $m$ t.t, malpighian tubules : s.i, small intestine; $l . i$, large intestine; st, sting. workers, avail themselves of the general food supply of the colony, and do not themselves collect honey.

\section{Other Systems.}

The respiratory system is represented by the ramifying tracheal tubes. "They open to the exterior by the lateral spiracles, which can be completely closed. In connection 
with the tracheæ there are large air sacs which aid greatly in flight.

The circulatory system is in essentials the same as that of the cockroach. The blood contains a few nucleated amoboid corpuscles.

The excretory system consists of numerous fine Malpighian tubules which open into the small intestine.

\section{Reproductive System.}

In the drone the reproductive organs consist of a pair of testes, each furnished with a narrow vas deferens, expanding at its distal end into a seminal vesicle. The seminal vesicles open into the ejaculatory duct, and at their junction a large paired mucus gland opens. When maturity is reached the testes diminish in size, while the spermatozoa accumulate in the terminal expanded part of the ejaculatory duct, and there become aggregated into a compact spermatophore. With the terminal portion of the male duct copulatory organs are associated.

Mating takes place only once in the life of the queen, and is followed by the death of the drone.

In the queen the large ovaries occupy considerable space in the abdominal region. As usual, each consists of numerous (100-I 50) ovarian tubes containing ova in various stages of development. The ovarian tubes open into the right and left oviducts, which again unite to form the common oviduct. With the anterior portion of the common duct the globular spermatheca is associated. In connection with it there is a gland corresponding to the mucus gland of the male. The oviduct terminates in a copulatory pouch.

Previous to laying, the eggs are fertilised by sperms set free from the spermatheca. In the case of drone eggs this liberation of spermatozoa does not take place, and the eggs in consequence are parthenogenetic. Queens which have never mated, or which have exhausted their stock of male elements, habitually lay drone eggs, but those which are laying abundant fertilised eggs at times also lay unfertilised eggs. This withholding of spermatozoa is said to be "voluntary," and related to the needs of the colony, but the physiological reason is unknown.

The workers possess female organs similar in type to those of the queen, but of an extremely rudimentary nature.

The eggs are laid singly in the cells of the comb, at the rate of about two per minute, for weeks together. They are of the usual insect type. According to the size of the cell in which it is deposited, and the food with which it is furnished, the fertilised ovum develops into a worker or into a queen. The development takes place within the cell, and includes a complete metamorphosis. 
吾覃

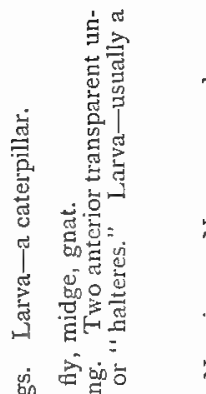

先

点焉

突

हूँ

을

i⿱

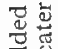

胥.

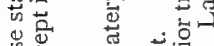

y

i.

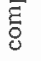

乙

당

$\infty$ 을

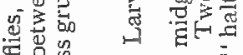

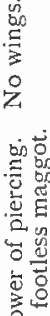

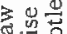

की

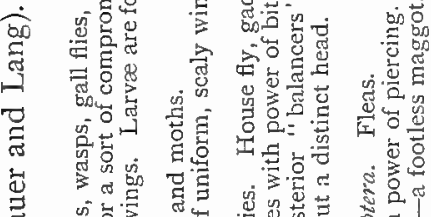

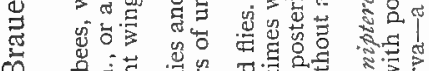

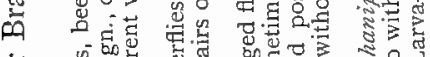

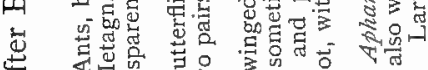

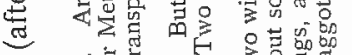

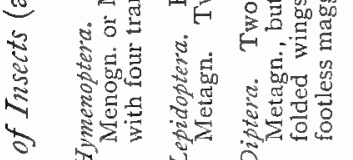

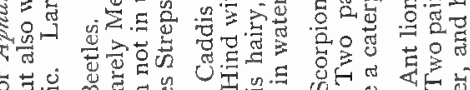

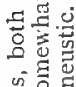

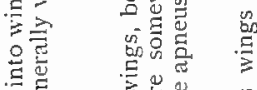

品

$\stackrel{0}{4}$

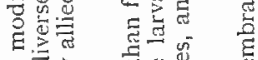

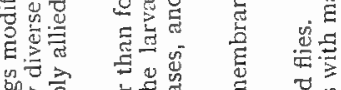

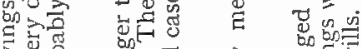

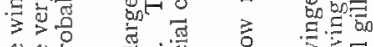

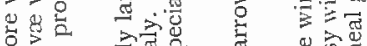

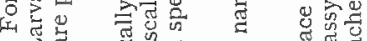

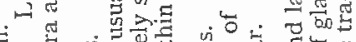

Бें प्य

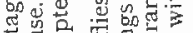

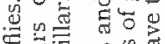

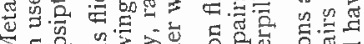

节

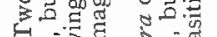

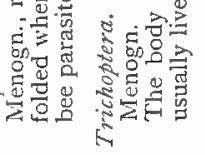

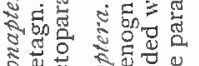

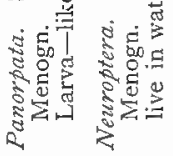

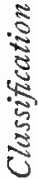

苗

in $\dot{H}$

号

손

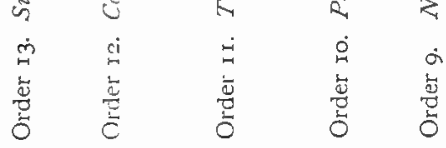

$\begin{array}{ll}0 & 0 \\ 0 & 5 \\ 0 & 0 \\ 0 & 0\end{array}$

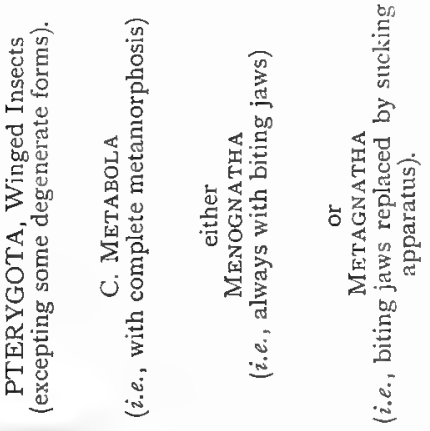




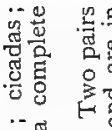

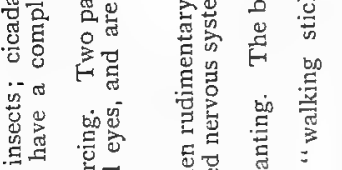

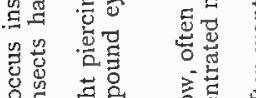

8

क्य

흐용

点

$\geqslant$ - का

范

氖导

量

4

हु

(1)

要过

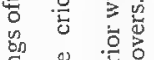

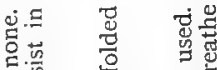

$\underset{\square}{\square}$

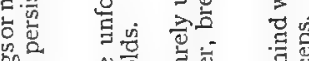

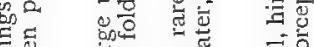

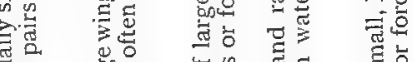

bis

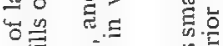

क्षे

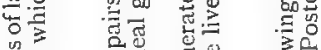

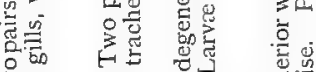

३

焉 它焉

各 ᄒ⿱宀匕

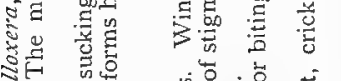

है

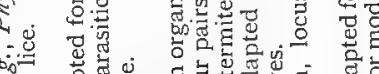

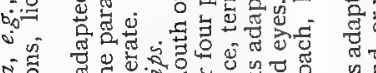

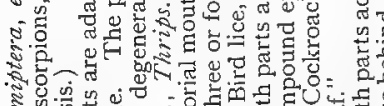

언.

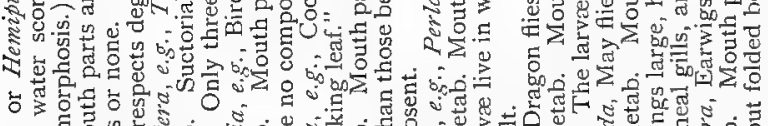

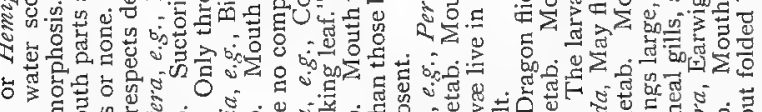

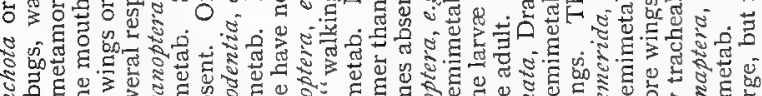
点 से

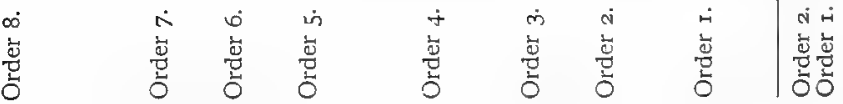

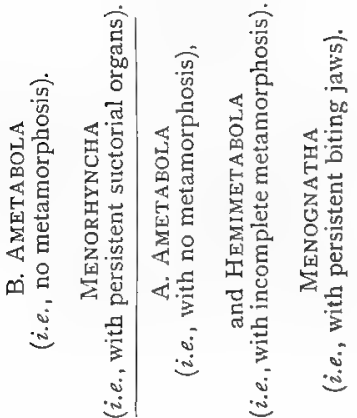




\section{GENERAL NOTES ON INSECTS.}

The main characteristics of insects have already been described in the two types chosen, but we here revise them in general terms.

\section{Form.}

The body of an adult insect may be divided into three distinct regions :-

I. The undivided head, which consists of at least three fused segments, as it bears three pairs of appendages.

2. The median thorax, divided into pro-, meso-, and meta-thoracic segments, each with a pair of legs, the last two often with wings.

3. The abdomen with about eleven rings, usually without trace of limbs.

But this is only the crude anatomy of form. One must think of the long dragon fly with outspread wings, and of the compact cockchafer, of the thin-waisted wasps and longbodied butterflies, of house fly and cricket, of large moths and beetles, and the almost invisible insect parasites.

\section{Appendages.}

Insects "feel their way," test food, and apparently communicate impressions to one another by means of a pair of jointed feelers or antennæ, situated in front of the head. Unlike the organs of a similar name in Crustaceans, the antennæ are not usually ranked among the appendages strictly so-called. They seem to be pre-oral outgrowths.

It was a step of some importance in morphology when Savigny showed that the three pairs of appendages about the mouth were homologous with the other appendages, i.e., were masticatory legs.

(r.) Furthest forward lie two mandibles, the biting and cutting jaws. These are single jointed, and thus differ from the organs of the same name in the crayfish, which bear a three-jointed palp in addition to the hard basal part. In those insects which suck and do not bite, e.g., adult butterflies, the mandibles are reduced.

(2.) Next in order is the first pair of maxille. Each maxilla consists of a basal piece (protopodite), an inner fork (endopodite), and an outer fork (exopodite). I use these names from Crustacean terminology, 
after the example of Marshall and Hurst. The entomologists divide the protopodite into a lower joint the cardo, and an upper the stipes, the endopodite into an internal lacinia, and an external galea, while the exopodite is called the maxillary palp.

(3.) The last pair of oral appendages or second maxilla are partially fused, and form what is called the labium. The lower and upper joints of their fused protopodites are called submentum and mentum; the endopodites on each side are double as in the first maxillæ, and consist of internal lacinia and external paraglossa; the exopodites are called the labial palps.

The three pairs of thoracic legs consist of many joints, are usually clawed and hairy at their tips, and vary greatly according to their uses. Think, for instance, of the hairy feet by aid of which the fly runs up the smooth window pane, of the muscular limbs of grasshoppers, of the lank length of those which characterise "daddy-long-legs," of the pollen

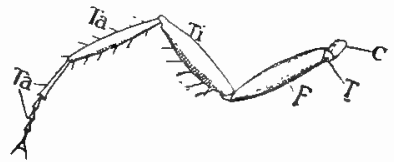

\author{
FIG. 100.-Joints of Cockroach's Leg. \\ $C$, Coxa ; $T$, trochanter ; $F$, femur ; $T$; , tibia ; $T a$, tarsus with \\ terminal claws.
}

baskets on bees, of the oars of water beetles. In identifying insects from a book it is needful to recognise the joints of the legs by the names which entomologists have transferred to them from human osteology, viz., the superior coxa with projecting trochanter, the stout femur, the tibia, and finally numerous tarsal joints.

\title{
Wings.
}

Wings are flattened hollow sacs, which grow out from the two posterior segments of the thorax. They are moved by muscles, and traversed by "veins" or "nervures," which include air-tubes, nerves, and vessel-like continuations of the body cavity. Most insects have two pairs, but many sluggish females and parasites like lice and fleas have lost them. On the other hand, there is no reason to believe 
that the very simplest wingless insects, known as Collembola and Thysanura, ever had wings.

There are many interesting differences in regard to wings in the various order of Insects. Thus, in beetles, the front pair form wing covers or elytra, in the little bee parasites-Strepsiptera-they are twisted rudiments, in flies the posterior pair are small knobbed stalks (halteres

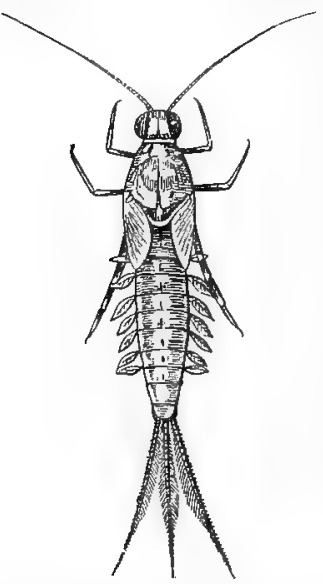

FIG. IOI.-Young May fly or Ephemerid. (After EATON.)

Showing tracheal gills, and wings appearing in front of them. or balancers), in bees the wings on each side are hooked together. When the insect is at rest, the wings are usually folded neatly on the back; but dragon flies and others keep them expanded, butterflies raise them like a single sail on the back, moths keep them flat. Many wings bear small scales or hairs and are often brightly coloured. Professor Eimer maintains that the arrangement of the nervures and the colouring of butterfly wings are certain marks of the progress and relationships of species. It is well known that the colours also vary with sex, climate, and surroundings. Most interesting are those cases in which the colours of an insect harmonise exactly with those of its habitat, or make it a mimetic copy of some more successfully protected neighbour.

As to the origin of wings, this at least should be remembered, that in many cases they are of some use in respiration as well as in locomotion. Seeing that the power of flight is evidently an accomplishment which the original insects did not possess, the theory seems plausible that wings were originally respiratory outgrowths, which by-and-by became useful for aërial locomotion. This view is consistent with an idea, which grows in favour with evolutionists, that new organs develop by the predominance of some new function in organs which had some prior significance. Moreover, we can fancy that an increase in respiratory efficiency brought about by the outgrowths in question would quicken the whole life, and would tend to raise insects into the air, just as terrestrial insects can be made to frisk and jump when placed in a vessel with relatively more oxygen than there is in the atmosphere. Finally, we must note that the aquatic larvæ of some insects, e.g., May flies, have a series of respiratory outgrowths from the sides of the abdomen, the so-called "tracheal gills," which in origin and appearance are like young wings.

Insects excel in locomotion. with the quadrupeds; they fly with the birds; they glide 
with the serpents, and they swim with the fish." They beat the elastic air with their wings, and though there cannot be so much complexity of movement as in birds where the individual feathers move, the insect wing is no rigid plate, and its up and down motions are complex. They can soar rapidly, but their lightness often makes horizontal steering difficult. The wind often helps as well as hinders them; thus the insects which fly in and out of the windows of express trains are probably in part sucked along. Marey calculates the approximate number of wing strokes per second at 330 for the fly, 240 for the humble bee, I 90 for the hive bee, II 0 for the wasp, 28 for the dragon $\mathrm{fly}_{1}, 9$ for a butterfly. It has been found that for short distances a bee can out-fly a pigeon.

\section{Skin.}

As in other Arthropods, the epidermis (or hypodermis) of Insects forms a firm cuticle of chitin, which in the exigencies of growth has sometimes to be moulted. This cuticle is often finely marked, so that the animal seems iridescent, and there are many different kinds of scales, hairs, and spines. Chitin is not favourable to the development of skin glands, but most insects have "salivary glands," opening in or near the mouth, bees have wax-making glands opening on the abdomen, aphides have " honey-dew" tubes, not a few have poison bags, and many larvæ besides silkworms have organs from which are exuded the threads of which a cocoon is made.

\section{Muscular System.}

In very active animals like Insects, we of course find a highly developed set of rapidly contracting striped muscles. These work the wings, the legs, and the jaws. The resulting movements have this further significance that they help in the respiratory interchange of gases, and in the circulation of the blood.

\section{Nervous System.}

It is often remarked as marvellous that ants and bees, with brains smaller than pin heads, should be so clever. The more we know about an ant, "the more the wonder grows, so small a head should carry all it knows," or seems 
to know. But these statements imply forgetfulness of the relative size of brain to body, and tend moreover to exaggerate the importance of mere size. The complexity of a brain is the important fact, not its size, and there is no doubt that the cleverer insects (ants, bees, and wasps), have more complex brains than the others. As in other Arthropods, the nervous system consists $(a)$ of a dorsal brain or supraœsophageal ganglionic mass, and $(b)$ of a double ventral nerve cord with a number of paired ganglia of which the most anterior (the sub-œesophageal) are linked to the brain by a ring commissure around the gullet, and $(c)$ of nerves given off from the various ganglia to the sense organs, the alimentary canal, and the other organs. In many of the higher insects the ganglia of the ventral nerve cord are in some degree concentrated, and the adults are usually more centralised than the larvæ.

\section{Sensory Structures.}

Animals so much alive as Insects, and in surroundings so stimulating as many of them enjoy, have naturally highly developed sense organs.

Two compound eyes are present on the head of all adults except the primitive Collembola, the degenerate lice, the likewise parasitic fleas, and blind insects which live in caves or other dark places. Each eye contains a large number of similar elements, in each of which we can distinguish (I) a cuticular or corneal facet; (2) a glassy lens-like portion; (3) a retinal portion in association with which are fibres from the optic nerve, and there are also pigmented cells between the elements (cf. p. 259).

Simple eyes or ocelli are present in addition to the compound eyes in the adults of many insects, e.g., ants, bees, and wasps ; they occur without the accompaniment of compound eyes in Collembola, lice, and fleas, and they are usually the only eyes possessed by larvæ. They have only one lens (monomeniscous), whereas the compound forms have many lenses (polymeniscous). Their structure varies greatly, and their use is very uncertain.

Auditory (or chordotonal) organs have been found in all orders of Insects (except as yet the Thysanoptera), and occur both in the larve and in the adults. Their essential structure is as follows :-A nerve ends in 
a centre or ganglion near the skin, some of the cells of this ganglion grow out into long sensitive rods enclosed in a tiny sheath, the rods are directly or indirectly connected with the epidermis above them. "They are found in groups of $2-200$ in various parts of the body, antennæe, palps, legs, wings, in the halteres of Diptera, and upon the dorsal aspect of the abdomen." Quite different from these, and occurring in flies alone, on the hind end of the larva, or at the base of the adult's feelers, are little bags with fluid in which clear globules float. We do not know how much or how little Insects hear, but the "song" of male Cicadas and crickets does not fall on deaf ears.

In addition to the "eyes" and "ears" there are innervated hairs (tactile, tasting, olfactory) on the antenne and mouth parts of many insects. Not a few have been shown to possess a diffuse or dermatoptic sense, by which, for instance, they can, when blinded, find their way out of a dark box.

Many Insects produce sounds which often express a variety of emotions. We hear the whirr of rapidly moving wings in flies, the buzz of leaf-like structures near the openings of the air-tubes in many Hymenoptera, the scraping of legs against wing ribs in grasshoppers, the chirping of male crickets which rub one wing against its neighbour, the piping of male Cicadas which have a complex musical instrument, the voice of the death's-head moth which expels air forcibly from its mouth. The death watch taps with his head on wooden objects, as if knocking at the door behind which his mate may be hidden. In some cases the sounds are simply automatic reflexes of activity; in many cases they serve as alluring love calls, and they may also serve as expressions of fear and anger, or as warning alarms.

\section{Alimentary System.}

The diet of Insects is very varied. Some, such as locusts, are vegetarian, and destroy our crops; others are carnivorous (we need not specify the homœopathist's leech) and suck the blood of living victims, or devour the dead; the bees flit in search of nectar from flower to flower, while the ant lion lurks in his pit of sand for any unwary stumbler; the termites gnaw decaying wood; some ants keep aphides as cows ("vaccæ formicarum," Linnæus called them), whose sweet juices they lick; and a great number of larva devour the flesh and vegetables in which they are born.

It is important to have some vivid idea of the diversity of diet, for the many modifications of mouth organs, in beetle and bee, in caterpillar and butterfly, as well as differences in the alimentary canal itself, are associated with the way in which the insect feeds.

For purposes of classification, the following distinctions in regard to the mouth organs are very useful :- 
(a) The mouth parts may be similar in all stages of life, and adapted for biting. In this case the term Menognatha (i.e., permanently jawed) is applied:-

e.g., to earwigs, dragon flies, the cockroach order (Orthoptera), the beetle order (Coleoptera).

(b) The mouth parts may be similar in all stages of life, and adapted for sucking. In this case the term MENORHyncha (i.e., permanently with a sucking proboscis) is applied :-

e.g., to bugs of all sorts (Rhynchota or Hemiptera).

(c) The mouth parts may be adapted for biting in the larva, for suck. ing in the adult. In this case the term MetagnathA (i.e., with changed jaws) is applied :-

e.g., to butterflies and moths.

The alimentary canal consists of fore gut, mid gut, and hind gut, of which the mid gut is endodermic and the result of the original gastrula cavity (archenteron), whereas the other two regions are fore and hind invaginations of the ectoderm, and therefore lined by a chitinous cuticle.

The fore gut conducts food, and includes mouth cavity, pharynx, and oesophagus, the latter being often swollen into a storing crop, or continued into a muscular gizzard with grinding plates of chitin.

The mid gut is digestive and absorptive, often bearing a number of glandular outgrowths or cæca, and varies in length (in beetles at least) in inverse proportion to the nutritive and digestible quality of the food.

The hind gut is said to be partly absorptive, but is chiefly a conducting intestine, often coiled and terminally expanded into a rectum with which glands are frequently associated.

In association with the alimentary canal are various glands :-

(a) The salivary glands, which open in or near the mouth. They are usually paired on each side, and provided with a reservoir. They arise as invaginations of the ectoderm near the mouth. Their secretion is mainly diastatic in function, i.e., it changes starchy material into sugar by means of a ferment. Along with these may be ranked the "spinning glands" of caterpillars, \&c., which also open at the mouth. They secrete material which hardens into the threads used for the cocoon.

(b) From the beginning of the mid gut, blind outgrowths sometimes arise (in some Orthoptera, \&c.), which are apparently digestive. They are sometimes called pyloric cæca. In other cases (some beetles) there may be more numerous and smaller glandular outgrowths on the external wall of the mid gut.

(c) From the hind gut arise numerous fine Malpighian tubes, which are certainly excretory in function. 


\section{Respiratory System.}

The body of an insect is traversed by a system of air tubes (tracheæ), which open laterally by special apertures (stigmata), and by means of numerous branches conduct the air to all the recesses of the tissues. In animals which breathe by gills or lungs the blood is carried to the air ; in insects the air permeates the whole body. But how does the air pass in and out? In part, no doubt, there is a slow diffusion; in part, the movements of the wings and legs will help; but there are also special expiratory muscles. We see their action when we watch a drone fly panting on a flower. Inspiration is passive, as in birds, and depends on the elasticity of the skin and of the tracheal walls; expiration is active, and depends on special muscles. These are chiefly situated in the abdomen, but in some beetles (at least) they are also present in the metathorax.

The tracheæ seem to arise as tubular ingrowths of skin, and, primitively, each segment probably contained a distinct pair. But their number has been reduced, and many are often connected into a system. With the doubtful exception of one of the primitive Collembola, and the certain exception of caterpillars, no insects have any tracheal openings in the head region. There are rarely more than two pairs in the thorax, there are often six to eight pairs in the abdomen, the maximum total is ten pairs. Each trachea is kept tense throughout the greater part of its course by internal chitinous thickenings, which apparently have a spiral course. The branches of the tracheæ penetrate into all the organs of the body, carrying oxygen to every part. The very efficient respiration of insects must be kept in mind in an appreciation of the general activity of their life.

As the conditions of larval life are often different from those of the adult insects, the mode of respiration may also differ in details.

In insects without marked metamorphosis, and even in some beetles in which the metamorphosis is complete, the young insect and the adult both breathe by trachere with open stigmata. Both are said to be " holopneustic."

When the larve live in water, the tracheal system is closed, otherwise the creatures would drown. This closed condition is termed "apneustic." These larvze (of dragon flies, May flies, and some others) 
breathe by "tracheal gills"-little wing-like outgrowths from the sides of the abdomen, rich in trachea-or by tracheal folds within the rectum, in and out of which water flows. In either case, an interchange of gases between the trachex and the water takes place. In adult aërial life, the tracher of the body acquire stigmata, and the insect becomes "holopneustic."

In most insects with complete metamorphosis, the larva (e.g., caterpillar or grub) has closed stigmata on the last two segments of the thorax (those which will bear wings), but there is a pair of open stigmata on the prothorax. In the adult the reverse is true.

There are some other modifications, for instance what obtains in the parasitic larvæ of some flies, e.g., gad flies. In these the stigmata are open only at the end of the body. In all cases, however, the stigmata of the adult are already present as rudiments in the larva, though they may not open till adolescence is over.

\section{Circulatory System.}

As the respiratory system is very efficient, establishing the possibility of gaseous interchange between the inmost recesses of the body and the external medium, it is natural that the blood vascular system should not be highly developed. Within a dorsal part of the body cavity, known as the pericardium, the heart lies, swayed by special muscles. It is a long tube, usually confined to the abdomen, usually of eight chambers, with paired valvular openings on its sides, through which blood enters from the pericardium. The blood is driven forwards, the posterior end of the heart being closed, and there is usually an anterior aorta or main blood vessel. But, for the most part, the blood circulates in spaces within what is commonly called the body cavity. Such a circulation is often described as lacunar. The blood may be colourless, yellow, red, or even greenish, and, in some cases, hæmoglobin, the characteristic blood pigment of Vertebrates, has been detected. The cells of the blood are amceboid.

\section{Body Cavity.}

One is apt to use this term in two senses-for the primitive body cavity or colome, and for the apparent body cavity of the adult. In discussing the development of Peripatus, Sedgwick notes the following characteristics of a true coelome:-It is a cavity which (I) does not communicate with the vascular system; (2) does communicate by nephridial pores with the exterior; (3) has the reproductive elements developed on its lining; (4) develops either as one or more diverticula from the 
primitive enteron (or gut), or as a space or spaces in the unsegmented or segmented mesoderm. Now, in Arthropods the apparent body cavity

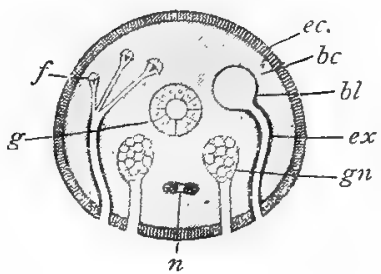

FIG. I02.-Diagrammatic cross section of an Invertebrate, with a primary body cavity $(b c)$ which is shaded. (After ZIEGLER.)

ec., Ectoderm; $b l$., bladder of nephridium (as in Crustaceans); ex., excretory duct; $g n$., genital organ; $n_{\text {., }}$ ventral nerve cord; $g_{*}$, gut: $f_{*}$, funnels of nephridia (as in worms).

of the adult is not a true cœelome, it consists of a set of secondarily derived vascular spaces; it has been called a pseudocœle or a hæmocœle.

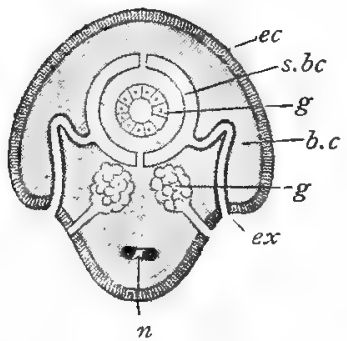

FIG. I03.-Diagrammatic cross section of an Invertebrate, with a secondary and a primary body cavity. (After ZiEGLER.)

ec., Ectoderm; s.bc., secondary body cavity (as in Lamellibranchs); $g$. gut; $b . c_{n}$, primary body cavity (shaded); $c_{x}$., excretory aperture of nephridium; gm., genital organ; $n_{\text {. }}$, ventral nerve ganglia.

The true cœlome of Arthropods is very much restricted in the adult, all the more so that most Arthropods (e.g., Insects) have no distinct nephridia. 
But the apparent body cavity in which the organs lie, and in which the blood circulates, is well developed in Insects. It includes, inter alia, a peculiar fatty tissue, which seems to be a store of reserve material, which is especially large in young insects before metamorphosis, and is also interesting as one of the seats of "phosphorescence" in those insects which glow.

\section{Excretory System.}

Although no structures certainly homologous with nephridia have yet been demonstrated in Insects, the excretory system is well developed. From the hind gut (proctodæum), and therefore of ectodermic origin, arise fine tubes, or in some cases solid threads, which extend into the apparent body cavity. Their number varies from two (in some Lepidoptera for instance) to one hundred and fifty (in the bee). They twine about the organs in the abdominal cavity, and their excretory significance is inferred from the fact that they contain uric acid.

\section{Reproductive System.}

Among Insects the sexes are always separate and often different in appearance. The males are more active, smaller, and more brightly coloured than the females. Darwin referred the greater decorativeness of the males to the sexual selection exercised by the females. The handsomer variations succeeded in courtship better than their rivals. Wallace referred the greater plainness of females to the elimination of the disadvantageously conspicuous in the course of natural selection. There may be truth in both views, but both require to be supplemented by the consideration, in part accepted by Wallace, that the "secondary sexual characters" of both sexes are the natural and necessary expressions of their respectively dominant constitutions. 


\section{Reproductive Organs.}

MALE.

The paired testes usually consist of many small tubes.

Two ducts (vasa deferentia), conducting spermatozoa (perhaps in part comparable to nephridia).

An unpaired terminal and ejaculatory duct, paired and with two apertures in Ephemerids only; sometimes formed by a union of the vasa deferentia, sometimes by an external invagination meeting the vasa deferentia.

From the vasa deferentia or from the ejaculatory duct, a paired or unpaired seminal vesicle for storing spermatozoa.

Various accessory glands, whose secretion sometimes unites the spermatozoa into packets or spermatophores.

Sometimes a copulatory penis.

Often external hard pieces.
Female.

The paired ovaries usually consist of many small tubes (ovarioles).

Two ducts (oviducts), conducting the ova (perhaps in part comparable to nephridia).

An unpaired terminal region or vagina, paired and with two apertures in Ephemerids; usually formed from an external invagination meeting the united ends of the oviducts.

Near or from the vagina, a receptaculum seminis for storing spermatozoa received from a male during copulation.

Various accessory glands, e.g., those which secrete the material surrounding the eggs.

Sometimes a special bursa copulatrix in the vagina.

Often external hard pieces, $e_{-} g_{*}$, ovipositor.

\section{Some Peculiarities in Reproduction.}

Many Insects, such as aphides, silk moth, and queen bee, are exceedingly prolific. The queen termite lays thousands of eggs "at the rate of about sixty per minute" !

The store of spermatozoa received by the female, and kept within the receptaculum seminis, often lasts for a long time,-for two or three years in some queen bees. Sir John Lubbock gives the remarkable instance of an aged queen ant, which laid fertile eggs thirteen years after the last union with a male.

Parthenogenesis, or the development of ova which are unfertilised, occurs normally, for a variable number of generations, in two Lepidoptera and one beetle, in some coccus insects and aphides, and in certain saw flies and gall wasps. It occurs casually in the silk moth and several other Lepidoptera, seasonally in aphides, in larval life in some midges (Miastor, Chironomus), and partially or "voluntarily" when the queen bee lays eggs which become drones. Parthenogenetic ova (in water fleas, Rotifers, \&c.), are believed to form only one polar body; the egg which becomes a drone forms two as usual, but the case of the bee is in several respects exceptional.

A few insects hatch their young within the body, or are "viviparous." This is the case with parthenogenetic summer aphides, a few flies, the little bee parasites Strepsiptera, and a few beetles. 


\section{Development of the Orum.}

The tubes which compose the ovaries and lead into the oviducts begin as thin filaments, the ends of which are usually connected on each side. Those thin filaments consist of indifferent germinal cells, all of them potential ova, and of mesodermic epithelial cells, which form the ovarian tubes, \&c., and are connected anteriorly to the pericardial wall.

But in most cases only a minority of these cells become ova, the others become nutritive cells, which are absorbed by the ova, and follicle cells which line the walls of the ovarian tubes and help to furnish the egg shells.

There may be, indeed, ovarian tubes without nutritive cells (e.g., in Orthoptera), and then each tube is simply a bead-like row of ova, which become larger and larger as they recede from the thin terminal filaments and approach the oviducts. In other cases, the bead-like row consists of ova alternating with clumps of nutritive cells (e.g., in Hymenoptera and Lepidoptera). In other cases, the nutritive cells mostly remain in the terminal region, but their products pass down to the receding ova.

As there are numerous ovarian tubes in each ovary, and as the same process of oogenesis is going on in each, numerous eggs are ready for liberation at the same time, and are simultaneously discharged into the oviduct of each side.

The eggs are large and contain much yolk. In relatively few cases yolk is almost absent, as for example, in the summer eggs of the Aphides, which are hatched within the body, and in some forms where the young are endoparasitic. The ovum is surrounded by a vitelline membrane, and also by a firm chitinous shell, secreted by the follicular cells, which is often sculptured in a characteristic manner. This shell is pierced by one or more minute holes (micropyles). Through a micropyle the spermatozoon finds entrance, sometimes (as in the cockroach) after moving round and round the shell in varying orbits.

\section{Development.}

The ripe egg usually consists of a central yolk-containing mass, surrounded by a thin sheath of protoplasm. As is usual for Arthropods, 
the segmentation is peripheral or centrolecithal. The central nucleus divides up into several nuclei, which, being united by protoplasmic cords, form for a time a central syncytium. Later, these nuclei emigrate into the peripheral protoplasm, which segments around them, thus a peripheral layer of similar epithelial cells is formed. Some of the nuclei may be left behind in the central yolk to form the yolk nuclei, or, what is probably the more primitive condition, these are formed by subsequent immigration from the blastoderm.

The next process is the appearance of differentiation among the similar cells of the blastoderm. Over a special area-the ventral plate-(cf. Astaczes) the cells increase in number and become cylindrical in shape; over the rest of the egg the cells flatten out and become much thinner. In the middle of the ventral plate, a slight groove is formed by rapid
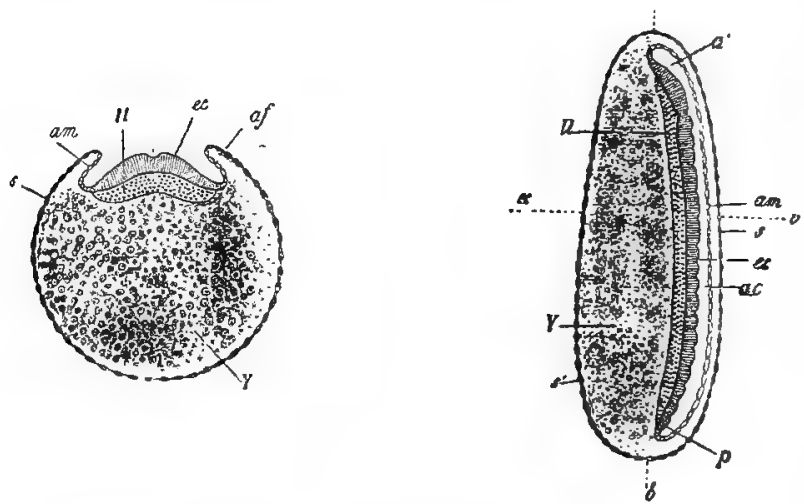

F1G. 104.-Diagram of Insect embryo. (From KORSCHELT and HeIDER.)

A transverse section before union of amnion folds, and a longitudinal median section after union of amnion folds; $a$, anterior pole of ovum ; $a^{\prime}$, anterior end of blastoderm ; am, amnion ; s, serosa ; $\alpha . c$, amniotic cavity; $\not$, posterior pole of ovum ; $e c$, ectoderm; $l$, lower germinal layer; $y$, yolk.

multiplication of the cylindrical cells. This represents the disguised gastrulation, the open roof of the groove being the much elongated blastopore. The surrounding cylindrical cells unite over this open roof, the groove usually flattens out, and thus we have formed a two layered germinal streak which spreads forwards and backwards over the egg, and early exhibits externally transverse division into segments. The upper layer is the ectoderm, the lower includes the rudiments of both mesoderm and endoderm. 
Meanwhile another very important event has taken place. We saw that while the cells of the ventral plate increased in depth, the remaining cells flattened out laterally; at the point where the two kinds of cells unite, on either side of the ventral plate, a double fold arises. The two folds unite over the surface of the ventral plate, forming a membranous arch over it. The internal fold is called "amniotic," the outer "serous," from their resemblance to the similar envelopes in the embryos of higher Vertebrates. The folds take no direct part in the development of the embryo.

We must now return to the germinal streak. The gastrula groove may persist as a tube after closure of the blastopore, but it is usually compressed by the ectoderm, or never exists as a distinct cavity. The greater part of the lower stratum of the germinal streak consists of mesoderm. This becomes divided into successive segments at each side, each containing a primitive coelomic cavity, perhaps continuous with the gastrula cavity. The endoderm arises as paired clusters of cells, found only at the anterior and posterior ends of the primitive streak. These clusters increase rapidiy and form long endodermal streaks which curve downwards so as to enclose the yolk. The streaks meet and fuse, first ventrally and later dorsally, thus constituting the mid gut. The yolk nuclei previously mentioned have meanwhile increased rapidly, forming yolk cells which absorb the yolk. These cells are included in the endodermic mid gut, and there break up. As the endoderm grows round the yolk, it is accompanied by a layer (splanchnic) of the mesoblast. Fore and hind gut are formed by invaginations which fuse with the mid gut.

In the later stages of development the primitive ccelomic pouches lose their cross partitions, become filled with mesenchymatic cells, and practically obliterated. The body cavity of the adult is formed by the appearance of lacunæe in the cells of the mesenchyme.

The tracheæ arise as segmentally repeated invaginations of the ectoderm. The openings of the invaginations form the stigmata. From the hind gut arise the Malpighian tubules, which are therefore ectodermic. The development of the other organs is similar to that of the Crustacea.

In summarising the development of Insecta, one must specially note the peripheral segmentation, the formation of the two-layered germinal streak, the presence of an over arching blastodermic fold, the segmentation of the mesoderm, and the formation of the mid gut by the union of endodermic bands.

\section{Metamorphosis of Insects.}

(I.) In the lowest Insects-namely, in the old-fashioned wingless Thysanura and Collembola, the hatched young are miniature adults. By gradual growth, and after several moultings, they attain adult size. 
Similarly the newly hatched earwigs, young of cockroaches and locusts, of lice, aphides, termites, and bugs, are very like the parents, except that they are sexually immature, and that there are no wings, which indeed are absent from some of the adults.

These insects are called ametabolic, i.e., they exhibit no marked change or metamorphosis.

(2.) In Cicadas there are slight but most instructive differences between larvæ and adults. The adults live among herbage, the young on the ground, and the diversity of habit has associated differences of structure, as in the burrowing fore legs of the larva. Moreover, the larva acquires the characters of an adult after a quiescent period of pupation.

The differences between larva and adult are more striking in May flies, dragon flies, and the related Plecoptera (e.g., Perla), for in these the larvæ are aquatic, with closed respiratory apertures, with tracheal gills or folds, while the adults are winged and aërial, and breathe by open tracheæ.

These insects are called hemimetabolic, i.e., they have a partial or incomplete metamorphosis.

(3.) Very different is the life history of all other sets of Insects-ant lions, caddis flies, flies, fleas, butterflies and moths, beetles, ants, and bees. From the egg there is hatched a larva (maggot, grub, or caterpillar), which lives a life very different from the adult, and is altogether unlike it in form. The larva feeds voraciously, grows, rests, and moults. Having accumulated a rich store of reserve material in its "fatty body," it finally becomes for some time quiescent, as a pupa, nymph, or chrysalis, often within the shelter of a cocoon. During this period there are great transformations; wings bud out, appendages of the adult pattern are formed, reconstruction of other organs is effected. Finally, out of the pupal husk emerges a miniature winged insect of the adult or imago type.

These insects are called holometabolic, i.e., they exhibit a complete metamorphosis.

Two kinds of larvæe occur among insects. (a) In many ametabolic and hemimetabolic forms, the larva is somewhat like one of the lowly Thysanuran insects (Campodea), and is 
therefore called campodeiform. It has the regions of the body well defined, three pairs of locomotor thoracic limbs, and mouth parts adapted for suction. (b) The other type is worm-like or eruciform, e.g., the caterpillars of butterflies and moths, with three pairs of limbs; the more modified grubs of bees, \&c., with distinct head, but without limbs; and the degenerate maggots of flies, \&c., not only limbless, but with an ill-defined head. But the caterpillar has often several pairs of abdominal pro-legs, which may be homologous with legs, and other abdominal appendages are known on the larvæ of other insects, and even in the embryos of some whose larvæ are campodeiform. These facts make it likely that the primitive form had many legs.

The larvæ of Insects vary enormously in habit and in structure, and exhibit numerous adaptations to conditions of life very different from those of the parent. Thus caterpillars, which are usually plump and tense, so that a peck from a bird's bill may cause them to bleed to death, even if no immediate destruction befall them, are protectively adapted in many different ways. Their colours are often changed in harmony with those of their surroundings, some palatable forms are saved by their superficial resemblance to those which are nauseous, a few strike "terrifying attitudes," others are like pieces of plants.

But for our purpose it is perhaps more important to recall the differences between the respiration of some larvæ and that of the adult, between the apneustic larva of the dragon fly and the holopneustic winged tyrant. Likewise of great importance, and supplying a basis for classification, are the changes in connection with the mouth organs. The main facts may be summarised in a terse sentence from the monumental work of Rolleston and Hatchett Jackson "Forms of Animal Life," Oxford, 1888). "The mouth parts may be similar in all stages of life, and then are either adapted for biting (Menognatha, i.e., jaws persistent), or for sucking (Menorhyncha, i.e., proboscis persistent), or else they are adapted in the larva for biting, in the adult for sucking, the change commencing in the pupa, and rarely affecting the larval stage (Metagnatha, i. e., jaws changed)."

\section{Internal Metamorphosis.}

In Insects with no marked metamorphosis, or with an incomplete one merely, the organs of the larva develop gradually into those of the adult. But in Insects with complete metamorphosis, there is a marvellous internal reconstruction during the later larval, and especially during the quiescent pupal stage. Most of the larval organs are disrupted and partially absorbed by amœboid cells, their debris being used in building new structures. Parts of larval 
organs which have not been highly specialised form the foundations of new adult structures. Of special importance are certain ingrowths of the larval skin (the epi- or hypodermis) which form what are called "imaginal discs," from which arise the wings, legs, and epidermis of the imago or perfect insect. The reconstruction is very thorough; most of the musculature, much of the tracheal system, part of the mid gut, \&c., are gradually replaced by the corresponding organs of the adult. Yet there is no abruptness; the absorption and replacement of organs is perfectly gradual.

\section{Bronomics.}

The average insect is active, but between orders (e.g., ants, bees, and wasps versus aphides, coccus insects, and bugs), between nearly related families, between the sexes (e.g., male and female cochineal insect), between caterpillar and pupa, we read the constantly recurrent antithesis between activity and passivity.

The average length of life is short. Queen bees of five years, queen ants aged thirteen, are rare exceptions. In many cases death follows as the rapid nemesis of reproduction. But though the adult life is often very short, the total life may be of considerable length, witness some Ephemerids which in their adult life of winged love-making may be literally the flies of a day, while their aquatic larval stages may have lived for two years or more.

The relation between the annual appearance of certain insects and that of the plants which they visit, the habits of hibernation in the adult or larval state, the occasional "dimorphism" between winter and summer broods of butterflies should be noticed.

The prolific multiplication of many insects may lead to local and periodic increase in their numbers, but great increase is limited by the food supply and the weather, by the warfare between insects of different kinds, by the numerous insects parasitic on others, by the appetite of higher animals,-fishes, frogs, ant-eaters, insectivores, and, above all, birds.

There is a great variety of protective adaptation. 'The young of caddis flies are partially masked by their external 
cases of pebbles and fragments of stem; many caterpillars and adult insects harmonise with the colour of their environment; leaf insects, "walking sticks," moss insects, scale insects, have a precise resemblance to external objects which must often save them; a humming bird moth closely resembles a humming bird; many palatable insects and larvæ have a mimetic resemblance to others which are nauseous or otherwise little likely to be meddled with. Many insects may be saved by their hard chitinous armour, by their disgusting odour or taste, by their deterrent discharges of repulsive formic acid, \&c., by simulation of death, by active resistance with effective weapons.

Many flowers depend for cross fertilisation upon insects which carry the pollen from one to another. Many insects depend for food on the nectar and pollen of flowers. Thus many flowers and insects are mutually dependent. But many insects injure plants, and many plants exhibit structures which tend to save them from attack. On the other hand, there may be "partnerships" between insects and plantswitness the "myrmecophilous" (ant loving) plants which shelter a bodyguard of ants, by whom they are saved from unwelcome visitors. And again, the formation of galls by some insects which lay their eggs in plants, and the insect catching proclivities of some carnivorous plants, should be remembered.

Most insects are terrestrial and aërial; the majority live in warm and temperate countries, but they are represented almost everywhere, even above the snow line, in arctic regions, in caves. Even on the sea the "Challenger" explorers found the pelagic Halobates, a genus of bugs. The distribution of Insects is mainly limited by food supplies and climate, for their powers of flight are often great, and their opportunities of passive dispersal by the wind, floating logs, \&c., are by no means slight.

Many insects are more or less parasitic, either externally as adults, e.g., fleas, lice, bird lice, plant lice, \&c., or internally as larvæ, e.g., the maggots of gad flies on cattle, and a great number of borers within plants.

We need only mention Hessian fly, Phylloxera, Colorado beetle, Weevils, Locusts, to suggest many more which are of much economic importance as injurious insects. On the 
other hand, our indebtedness to hive bee and silk moth, to cochineal and lac insects, to those which destroy injurious insects, and to those which carry pollen from flower to flower, is obvious.

Finally, we must at least mention that in ants, bees, wasps, and termites we find illustration of various grades of social life, and marvellous exhibitions of instinctive skill as well as some intelligence.

\section{Pedigree.}

Insects must have appeared relatively early, for remains of a cockroach-like form have been found even in Silurian strata. The higher forms with complete metamorphosis appear much later (e.g., beetles in the Carboniferous ages), but it seems that the Palæozoic Insects were mostly generalised types, prophetic of, rather than referable to, the modern orders.

As to the pedigree of Insects, the wingless Collembola and Thysanura are doubtless primitive. They lead us back to some of the less specialised Myriopods (e.g., Scolopendrella), back further to Peripatzes, which helps to link the Tracheate to the Annelid series.

But though the primitive wingless insects, the simple types of Myriopods, and Peripatus represent ascending steps in evolution, what the actual path has been we do not know. 


\section{CHAPTER XV.}

\section{ARACHNOIDEA AND PALÆOSTRACA.}

Class Arachnoidea-Spiders, Scorpions, Mites, \&c.

The class Arachnoidea is far from being a coherent unity. Its subdivisions are numerous and diverse, and a statement of general characters is consequently difficult.

The anterior segments, about seven in number, are fused into a cephalothorax, which bears six pairs of appendages. The most anterior of these appendages may be turned in front of the mouth, but there are no pre-oral outgrowths like the antennce of Insects and Myriopods. The first two pairs of appendages (chelicerce and pedipalps) generally have to do with seizing and holding the food; the others are walkinglegs. But although six pairs occur in most, there may be more or less. The abdomen is generally, but not always, without appendages; it may be segmented or unsegmented; it is generally distinct from, but may be fused to, the cephalothorax. A plate-like internal skeleton, called the endosternite, is often present. Respiration may be by tubular trachea, or by lung books (chambered trachece?), or by both, and many would include the Branchiate Palcestraca along with Arachnoidea. In the tracheate forms there are never more than four pairs of stigmata. An elongated dorsal heart usually lies in the abdomen. The position of the genital aperture or apertures is usually on one of the anterior abdominal segments. Except Tardigrada, all have separate sexes.

Order I. SCORPIONIDE

Scorpions are elongated Arachnids, restricted to warm countries, lurking under stones or in holes during the day, 
but active at night. The Scorpio afer of the East Indies attains a length of 6 inches, but most are much smaller. They feed on insects, spiders, and other small animals. The "tail," with the venomous sting at its tip, is usually curved over the anterior part of the body, and can reach forward to kill the prey caught by the anterior appendages, or can be

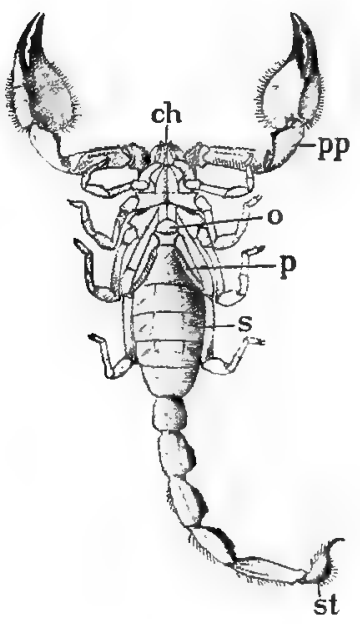

FIG, 105.-Scorpion.

$c h$, Cheliceræ ; $\not p h$, pedipalps ; 0 , genital operculum; $\not$., pectines; $s$, stigma of a lung book on the pra-abdomen; st, sting or post anal piece.

suddenly straightened to strike backwards. When man is stung, the poison seems to act chiefly on the red blood corpuscles, and though never or very rarely fatal, may cause much pain. It has been said that scorpions commit suicide when surrounded by fire or otherwise fatally threatened, but 
it has been answered that they do not sting themselves, that they could not if they would, and that, even if they could, the poison would have no effect !

The body is divided into (I) a cephalothorax or "prosoma " of six segments, whose terga fuse into a carapace, and (2) an abdomen which includes a broad seven-segmented "mesosoma," and a narrow five-segmented "metasoma." At the end of the latter there is a post-anal curved spine or "telson," containing a paired, compressible poison gland opening at the sharp tip. There is a strong cuticle of chitin, and also an interesting internal piece of skeleton (the endosternite), partly chitinoid, but also resembling fibro-cartilage, which lies in the cephalothorax above the nerve cord, and serves for the insertion of muscles.

The appendages are-

(I.) Small three-jointed chelate cheliceræe or falces just above the mouth, used in holding prey.

(2.) Large, six-jointed, chelate pedipalps. These seize the prey; their basal joints help in mastication, and in some cases they produce rasping sounds.

(3-6.) Four pairs of seven-jointed, non-chelate walking legs. The basal joints of the first two pairs help in connection with the mouth.

Apparently equivalent to a first pair of abdominal appendages is a small notched plate or operculum which covers or bears the genital aperture or apertures.

Apparentiy of the nature of appendages are the comb-like, probably tactile, pectines on the second abdominal segment.

Six other pairs of abdominal appendages are present in the embryo, but they abort.

The nervous system consists of a dorsal brain, a ring round the gullet, and a ventral nerve cord. The simple eyes situated on the carapace are innervated from the brain, the first six appendages from the collar and the subœsophageal ganglion. Behind the latter there are seven ventral ganglia in the eleventh to seventeenth segments inclusive.

Scorpions seize small animals with their pedipalps, hold them close to the small mouth by their cheliceræ, sting them if need be, and suck their blood and juices. The pharynx serves as a suction pump, a narrow gullet leads to a slight enlargement, into which a pair of salivary glands open; from the narrow mid gut several large digestive outgrowths arise; 
then follow one or two pairs of Malpighian tubes; the hind gut ends in a ventral anus beneath the base of the sting. The narrowness of the gut may be associated with the fluid nature of the food.

The body cavity is for the most part filled up with organs, muscles, and connective tissue. A pair of coxal glands, perhaps excretory and nephridial, but apparently closed in the adult, lie near the base of the last two walking legs.

The blood contains amoboid corpuscles and the respiratory pigment hæmocyanin. An eight-chambered heart, within a pericardium, lies along the back of the mesosoma. It gives off lateral arteries from the posterior end of each of its chambers, is continued backwards in a posterior aorta, and forwards in an anterior aorta. The latter supplies the head, and divides into two branches encircling the gullet and reuniting in a ventral artery above the nerve cord. From capillaries the blood is gathered into a ventral venous sinus, is purified in the lung books, and thence returns by veins to the pericardium, finding its way by valved lateral openings (ostia) into the anterior end of each heart chamber.

On the ninth to twelfth segments lie slit-like stigmata, the openings of four pairs of lung books. Each lung book is like a little purse with numerous (over a hundred) compartments. Air fills the much-divided cavity, and blood circulates in the lamellæ, which form the partitions. These lung books or pulmonary sacs are believed by some to be chambered or plaited tracheæ, while Professor Ray Lankester regards them as invaginated modifications of gill books such as Limulus possesses.

The testes consist of two pairs of longitudinal tubes, united by cross bridges; the vas deferens, with a terminal copulatory modification, opens under the operculum on the first abdominal segment. The ovary consists of three longitudinal tubes, united by cross ducts, and two oviducts open on the under surface of the genital operculum.

Fertilisation is internal; the ova begin their development in the ovary, and complete it in the oviduct. The segmentation is discoidal, the ova are hatched within the mother. The young, thus born "viviparously," are like miniature adults, and adhere for some time after birth to the body of the mother. 
The race of scorpions is of very ancient origin, for one has been found in Silurian strata, and others nearly resembling those now alive are found in the Carboniferous.

\section{Examples.-Scorpio, Eusiorpius, Buthus, Androctonus.}

Order 2. PSeudoscorpionidre. "Book Scorpions," e.g., Chelifer, Chernes.

Minute animals, most abundant in warm climates, under bark, in books, under the wing covers of insects. \& c. They are like miniature scorpions, but without the long tail and sting. Their food probably consists of the juices of insects ; the chelicerae are minute suckers, the pedipalps like those of scorpions. The abdomen is broad, with IO-I I segments. They breathe by tubular trachex, and have spinning glands.

Order 3. Pedipalpr. "Whip Scorpions," e.g., Thelyphonzes, Phrynus.

Small animals, found in warm countries. The abdomen is depressed, well-defined from the thorax, and has II-I2 segments. The chelicera are simply clawed, but are poisonous; the pedipalps are simply clawed or else truly chelate. The first pair of limbs are like antennæ. Respixation by two pairs of lung sacs. In Thelyphonus there is a long terminal whip.

Order 4. Phalangidee (or Opilionina). " Harvest-men," e.g., Phalangium.

The small spider-like " harvest men" are noted for their extremely long legs, by which they stalk slowly along avoiding the glare of day. The broad six-segmented abdomen is not distinct from the thorax; the chelicere are chelate; the pedipalps are like legs. Respiration by tubular trachex. The harvest men are sometimes called daddy-long legs, but we reserve that name for the crane fly (Tiputa oleracea). Nor are they to be confused with the troublesome "harvest bugs " (Trombidium holosericeum), which are minute red mites. The harvest men do not trouble us, but feed on small insects.

Order 5. Solfugidex or Solifugre, e.g., Galeodes or Solpuga.

Active, pugnacious, venomous, nocturnal little animals, found in the warmer parts of the earth. The head and abdomen are distinct from the thorax. The thorax has three segments, the abdomen nine or ten. The cheliceræe are chelate, the pedipalps like long legs. The respiration is by means of tubular trachere. The segmentation of the thorax is remarkable.

\section{Order 6. Araneide. Spiders.}

Spiders are found almost everywhere upon the earth, and a few are at home in fresh water. Most of them live on the juices of insects, and many form webs in which their victims are snared. They may be divided according to habit into 
the wanderers who spin little, and the sedentary forms who spin much. The body consists of an unsegmented cephalothorax and a soft unsegmented abdomen, separated by a narrow waist. The chitinous cuticle varies in hardness, hairiness, and colouring; it has as usual to be moulted as the spider grows. Thus the young garden spider moults eight times in its first year.

There are six pairs of appendages :-

(I.) The two jointed cheliceræ or falces, whose terminal joint bends down on the other in "sub-chelate" fashion, and is perforated by the duct of a poison gland.

(2.) The leg-like, usually six-jointed, non-chelate pedipalps, whose basal joint helps in mastication, while the terminal joint in the male expands as a reservoir for the spermatozoa and serves as a copulatory organ.

(3-6.) Four pairs of terminally clawed walking legs. The most anterior pair are much used as feelers. In the embryo there are four pairs of abdominal appendages which abort.

The nervous system is of the usual Arthropod type, but shows much centralisation. Thus the ventral ganglia are fused into one large centre in the cephalothorax, a condition comparable to that in crabs. There are two or three rows of simple eyes on the cephalothorax, whose focal distance is very short, spiders trusting most to their exquisite sense of touch by which they discriminate the various vibrations on a web line. The senses of smell, hearing, and taste are also present, but little is known in regard to the organs.

Body cavity, endosternite, and coxal glands generally resemble those of scorpions.

The spider usually sucks the blood and juices of its prey, and behind the gullet lies a powerfully suctorial region, strengthened by chitinous plates, and worked by muscles. From the small mid gut arise five pairs of long cæca, a pair running forwards and a pair passing into the bases of each pair of legs and then back again. These cæca sometimes anastomose. Further back, the mid gut gives off numerous digestive outgrowths which fill a large part of the abdomen. Their secretion digests proteids. Terminally there is a large cloaca, and where the intestine joins this, four excretory Malpighian tubes are given off.

A three-chambered heart, containing colourless blood, lies within a pericardium near the dorsal surface of the 
abdomen. It gives off an anterior and a posterior aorta and lateral vessels; and the circulation corresponds in general to that of the scorpion.

In a few forms, united as Tetrapneumones, respiration is effected by four "lung books"; the large bird-catching Mygale is an example. In the vast majority (Dipneumones) there are two "lung books," and tubular tracheæ in addition. The stigmata of the lung books lie on the anterior ventral surface of the abdomen; the tracheæ open posteriorly near the spinnerets, or just behind the openings of the lung books, or at both places.

The spinnerets (4-6) lie posteriorly, a little in front of the anus. They are movable organs, perforated by numerous (often many hundred)

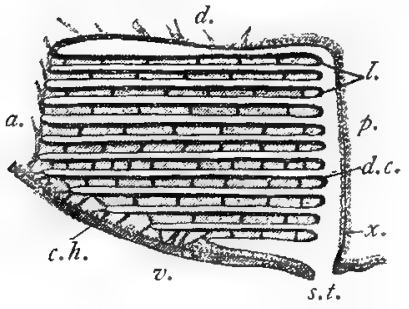

Fig. 106.-Section of Lung book. (After MACLEOD.)

$d$, Dorsal ; $z$, ventral ; $l$, lamella ; $p$, pos. terior; $\alpha$, anterior; $d . c$, dorsal chamber; $x$, posterior wall; st, stigma; $c h$, one of the interlamellar chambers. fine tubes which project as "spinning spools." The tubes are connected with numerous compressible glands secreting liquid silk. There are various kinds of glands, both the amount and nature of the silk secretion being under the spinner's control.

The males are usually smaller and often more brightly coloured than their mates. From the paired testes in the anterior part of the abdomen, two vasa deferentia pass to a common aperture beside the openings of the lung books. From the paired ovary two oviducts likewise arise and open into a uterus, whose external aperture is surrounded in the mature female by a complex genital armature or epigynium. Here also in most females are the openings of two receptacula seminis, in which the sperms received from a male are stored, and from which they pass by a pair of internal ducts to the oviducts, there to fertilise the ova. Allusion has already been made to the fact that the sperms of the male, after emission, may be stored up in the last 
joint of the palps. The ova are usually surrounded by silken cocoons, which are carried about by the mother or carefully hidden in nooks or nests.

Spinning.-Compression of the spinning glands causes a flow of liquid silk through the fine spools of the spinnerets. The extremely thin filaments from each spinneret unite into a thread, and the thread of one spinneret is often combined with that from the others. In this way a compound thread of exquisite fineness, though rivalled by a quartz fibre, is produced, but two or four separate threads are often exuded at the same time. Before beginning to "spin," the spider often presses the spinnerets against the surface to which the thread is to adhere, and draws the filaments out by slowly moving away. Often, however, the filaments ooze out quite apart from any attachment. The legs are also much used in extending and guiding the thread, and some spiders have on the hind legs a special comb of stiff hairs.

One of the most important ways in which the secreted threads are used is in forming a web. The common garden spider (Epeira) makes a web which is a beautiful work of unconscious art, and very effective as a snare for insects. The spider first forms "foundation lines" around the selected area; it then swings across the area with the first "ray" which it fixes firmly; another and another is formed, all intersecting in one centre. Secondly, it starts from the centre, and moves from ray to ray in a long wide spiral gradually outwards, leaving a strong spiral thread as it goes. Thirdly, the spider moves in a closer spiral from the circumference inwards, biting away the former spiral, replacing it by another, which is viscid and adhesive. It is to this that the web chiefly owes its power of catching insects which light there. There is usually a special thread running to the adjacent hole or nest, and the entire fabric is marvellously sensitive, for the spider feels rather than sees when a victim is caught.

The spun threads are used in many other ways. They line the nest, and form cocoons for the eggs. They often trail behind the spiders as they creep; they greatly assist locomotion, and are used in marvellous feats of climbing. Small and young spiders often stand on tip-toe on the top of a fence, secrete a parachute of threads, and allow themselves to be borne by the wind. The fallen threads are known as gossamer.

Courtship.-The males are usually much smaller than the females. It is calculated that the disproportion is sometimes such as would be observed if a man 6 feet high and 150 pounds in weight were to marry a giantess of 75-90 feet high, 200,000 pounds in weight. It may be that the smallness of the males is mainly due to the fact that they are males; others explain it by saying that the smaller the males are, the less likely they are to be caught by their frequently ferocious mates. It is difficult, however, to understand how this characteristic smallness, though perhaps advantageous and likely to be favoured by natural selection, can be entailed on the male offspring only. But this difficulty in regard to inheritance is one which besets many similar interpretations.

The males are often more brilliantly coloured than the females, perhaps, again, because they are males, though what the physiological 
connection between the male constitution and bright colours in this case is we cannot tell till the nature of the pigments is known. Wallace has spoken of the frequent brilliancy of males as due to their greater vitality, and refers the relative plainness common in females to their greater need for protection. Darwin referred the greater decorativeness of males to the fact that those which varied in this direction found favour in the eyes of their mates, were consequently more successful in reproduction, and thus tended to entail brilliancy on their male successors. But we naturally ask how the brilliancy began, and how its enhancement is transmitted to males alone. In the "Evolution of Sex," Professor Geddes and I have recognised that sexual selection may help to establish the brilliancy of males, and that natural selection may help to keep the females plain, but have also sought to associate decorative and other differences between the sexes with the more fundamental qualities of maleness and femaleness.

I have introduced this subject here, because it affords a pleasant interlude in our systematic survey, and because it serves to illustrate some of the problems of evolution.

Two American observers, Mr. and Mrs. Peckham, have made a series of studies on the courtship of spiders more careful than any others of the kind.

They find "no evidence that the male spiders possess greater vital activity; on the contrary, it is the female that is the more active and pugnacious of the two." They find, "no relation, in either sex, between development of colour and activity; the Lycosidæ, which are among the most active of all spiders, having the least colour development, while the sedentary orb-weavers show the most brilliant hues." "In the numerous cases where the male differed from the female by brighter colours and ornamental appendages, these adornments were not only so placed as to be in full view of the female during courtship, but the attitudes and antics of the male spider at that time were actually such as to display them to the fullest extent possible." "The males were much more quarrelsome in the presence of the females, and to a great extent lost their tendency to fight when the mating season was over."

The courtship is prolonged and elaborate, the females are not only coy but often savage. The male's love-making is often cut short by his death at the hands or cheliceræ of his desired mate. Of course we must be careful not to exaggerate the subtlety of the mental processes involved in the courtship of animals ; we must also beware of regarding it too crudely.

"The fact that in Attidæ the males vie with each other in making an elaborate display, not only of their grace and agility, but also of their beauty, before the females; and that the females, after attentively watching the dances and tournaments which have been executed for their gratification, select for their mates the males which they find most pleasing, points strongly to the conclusion that the great differences in colour and in ornament between the males and females of these spiders are the result of sexual selection."

It is still, however, quite possible that the colouring and decorations may have arisen as natural outcrops of the male constitution, the characteristics of which are by no means limited to greater vitality or activity. 


\section{Classification of Spiders.}

I. Tetrapneumones.

Four lung books and no tracher.

Mygale, a large lurking spider which has been known to kill small birds, but usually eats insects. Atypus, Cteniza, and others make neat trap door nests.

2. Dipneumones.

Two lung books and trachere as well.

Here are included the web spinners, e.g., Epeira, wolf spiders, e.g., Lycosa, Tarantula, the latter with poisonous qualities which have been much exaggerated; jumping spiders, the family Attidæ, e.g., Attus salticus. The common house spider is Tegenaria domestica; the commonest garden spider is Epeira diademata. Argyroneta aquatica fills an aquatic silken nest with bubbles caught at the surface.

Order 7. ACARINA, Mites and Ticks, e.g., Cheese mite (Tyroglyphus).

Mites are minute Arachnoids inclined to parasitism. They occur in the earth or in water, salt and fresh, or on animals and plants. They feed on the organisms they infest or upon organic debris.

The abdomen is fused with the cephalothorax, both are unsegmented. According to the mode of life, the mouth parts are adapted for biting or for piercing and sucking. Respiration may be simply through the skin ; in the majority there are trachex with two stigmata. A heart seems usually absent, but it is present in Gamasus. Many of the young have only three pairs of legs when hatched, but soon gain another pair. When some mites are starved or desiccated, and to some extent die, certain cells in the body unite within a cyst, and are able in favourable conditions to regrow the animal.

Examples-

(a) Without trachere. Cheese mite (Tyroglyphus). Itch mite (Sarcoptes scabiei), causing a loathsome disease. S. canis causes "mange" in dogs. Follicle mite (Demodex folliculorum), common in the hair follicles of man and domestic animals. Gall mites (Phytoptus), on plants.

(b) With tracheæ. Harvest mites (Trombidium), minute para. sites often troublesome in summer. What is often called the red spider (Tetrarhyzus telearius), spins webs, and lives socially under leaves. Water mites, e.g., Hydrachna, on water beetles, and Atax, on gills of fresh water mussels. Beetle mites (Gamasus), often found on carrion beetles. Ticks (Ixodes), on dogs, cattle, \&c.

\section{Aberrant Orders or Classes.}

Order 8. Linguatulida. Pentastomum tanioides.

This strange animal is parasitic in the nasal and frontal cavities of the dog and wolf. It is worm-like in form, externally ringed, without any oral appendages, but with two pairs of movable hooks near the 
mouth. There are no sense organs nor trachere. The sexes are separate, the males smaller than the females.

Embryos within egg cases pass from the nostrils of the dog. If they happen to be swallowed by a rabbit or a hare, or it may be some other mammal, the embryos hatch in the gut and penetrate to liver or lung. There they encyst, moult, and undergo metamorphosis. The final larval form is not so unlike an Arachnoid as the adult is. Liberated from its encystment, it moves about within its host, but will not become adult or sexual unless its host be eaten by dog or wolf. There are a few other species occurring in Reptiles, Apes, and even man, but their history is not adequately known.

Order 9. TARDigrada. Water Bears or Sloth animalcules, e.g., Macrobiotus.

Microscopic animals, sometimes found about the damp moss of swamps or even in the roof gutters of houses. The body is somewhat worm-like, with four pairs of clawed limbs like little stumps, with mouth parts resembling those of some mites, and adapted for piercing and sucking. There is no abdomen. There is a food canal, a brain and a ventral chain of four ganglia, sonetimes even a pair of simple eyes, but no respiratory or vascular organs. They are the only hermaphrodite Arachnoids, if they are Arachnoids. The eggs, which are developed in the cast skin of the parent, undergo total segmentation but little is known in regard to their development.

The water bears are said to have great powers of successfully resisting desiccation, but perhaps it is the enclosed eggs which do so, developing rapidly when favourable conditions return.

Some authorities dignify (8) and (9) as classes; for reasons of practical expediency I continue to call them orders.

\section{Class Palæostraca.}

The three following orders, - Xiphosura, Eurypterina, and Trilobita may be united under this title. 'They live or lived in water, and have or had gills in association with the limbs. The recently discovered antennæ of Trilobites, together with the markedly biramose character of some of their limbs, suggests an affinity with Crustacea, but, on the other hand, the affinities of the Xiphosura seem to be distinctly Arachnoid.

Order I. Xiphosura.

There is one living genus, the King Crab or Horse-shoe Crab (Limulus). 
The King Crab lives at slight depths off the muddy or sandy shores of the sheltered bays and estuaries of North America, from Maine to Florida, in the West Indies, and also on the Molucca Islands, \&c., in the far East. The body consists of a vaulted cephalothorax shaped like a horse-shoe, and an almost hexagonal abdomen ending in a long spine. Burrowing in the sand, Limulus arches its body at the joint between cephalothorax and abdomen, and pushes forward with legs and spine. It may also walk about under water, and even rise a little from the bottom. It is a hardy animal, able to survive exposure on the shore or even some freshening of the water. Its food consists chiefly of worms.

The King Crab is interesting in its structure and habits, and also because it is the only living representative of an old race. Since Ray Lankester published in I88I a famous paper entitled "Limulus an Arachnid," it has been generally, though not unanimously recognised, that the King Cral,'s relationships among modern animals are with Arachnoidea, not with Crustacea.

The hard, horse-shoe-shaped chitinous cephalothoracic shield is vaulted, but the internal cavity is much smaller than one would at first sight suppose; the well-defined abdomen shows some hint of being divisible into meso- and metasoma ; the long sharp spine is (like the scorpion's sting) a post-anal telson.

On the concave under surface of the cephalothorax, there are six (or seven) pairs of limbs, as in spiders and scorpions:-

(I) A little pair of 3-jointer cheliceræ in front of and bent towards the mouth. (They are chelate in the female, simply clawed in the male.)

(2-6) Five pairs of 6-jointed walking legs, the bases of which surround the mouth and help in mastication. The last of these ends in two-flat plates, which help in digging. The other appendages are ustally chelate, except the first in the male.

(7) Then follows on the abdomen a double "operculum" overlapping the rest. The genital apertures lie on its posterior surface. Some refer this operculum to the cephalothorax.

(8-I2) Under the operculum lie five pairs of flat plates beaning remarkable respiratory organs ("gill books"). These appendages show hints of the exopodite and endopodite structure characteristic of Crustaceans. At any rate in the young they serve also as swimming organs.

As in the scorpion, there is an internal skeletal structure, or endosternite, lying between the gullet and the nerve ring, serving for the 
attachment of muscles. It should be noted, however, that an analogous structure occurs in Apus and some other Crustaceans.

The Nervous System. - The supra-oesophageal brain gives off nerves to the eyes. United to the brain are two ganglionated and transversely connected commissures forming a long oval cesophageal ring, giving off nerves to the limbs, and continued into a ganglionated abdominal cord. Ensheathing ring, ventral cords, and some of the nerves are numerous blood vessels.

There are two "compound" eyes lying towards the sides of the cephalothoracic shield, and in front of these two more median simple eyes. The compound eyes are covered by a layer of chitin continuous with that of the shield, and the various eye elements are so remarkably

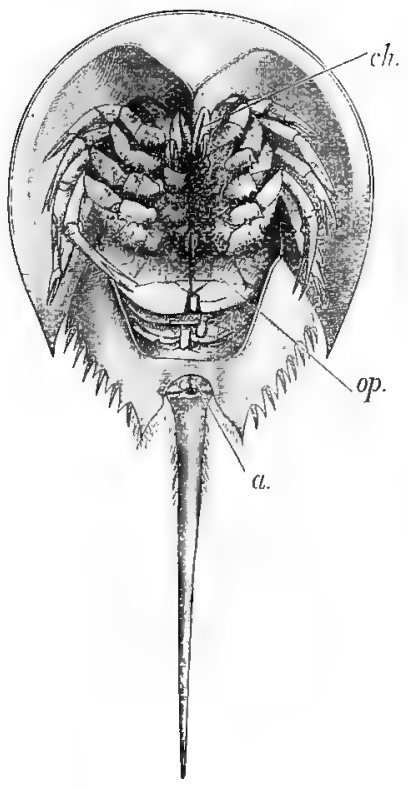

FIG. 107. -Limulus or King Crab. ch., Chelicara ; op., operculum; a., anus. distinct from one another, that the eye might be called a group of simple eyes.

The Food Canal. - Worms and the like seized by some of the pincers, are partly masticated by the bases of the five posterior cephalothoracic legs. The mouth leads into a suctorial pharynx, with chitinous folds; thence the fore gut bends upwards and forwards into a crop. Separated from this by a valve is the mid gut which extends along the cephalothorax and abdomen, and in the former bears two pairs of large yellow hepato-pancreatic outgrowths. The hind gut is short and ends in front of the base of the spine.

Two large reddish glands lie in the cephalothorax, and open in young forms at the bases of the fifth appendages. They also open internally, and may be compared with the coxal glands of spider and scorpion, with the shell gland of Entomostraca, and with nephridia (?).

The Vascular System. - The heart lies within a pericardium and is partially divided into eight chambers, with eight pairs of valved ostia. Hrmocyanin is present as usual as the respiratory pigment of the blood, and there are oval corpuscles. From an anterior aorta, like that of the scorpion, two vessels are given off which bend backward, unite with lateral arteries from each chamber of the heart, and form a collateral vessel on each sicle of the heart. These 
unite in a posterior clorsal artery. From the anterior aorta two other branches unite in a ring around the nerve collar which gives off vessels to the limbs, and is continued backwards around the nerve cord. From capillaries, the blood is gathered into a ventral venous sinus, whence it passes to the respiratory organs, and thence to the pericardium and heart.

The Respiratory Organs or gill books are borne by the last five appendages. Each looks like a much plaited gill, or like a book with over a hundred hollow leaves. Their leaf-like folds are externally washed by the water, within them the blood flows. The leaves of the gill books are compared to the leaves of the lung books of scorpions. If this homology is correct the gill books are evaginations, the lung books invaginations, of the skin.

The Reproductize System.-The males are smaller than the females. The testes are very diffuse, the two vasa deferentia open on the internal surface of the operculum, and the spermatozoa, which are viluratile, are shed into the water. The ovaries form two much branched but connected sacs; the oviducts are separate, and enlarge before they open beneath the operculum.

Spawning occurs in the spring and summer months. The ova and spermatozoa are deposited in hollows near high water mark. Some of the early stages of development, still imperfectly known, present considerable resemblance to corresponding stages in the scorpion. In the larve, both cephalothorax and abdomen show signs of segmentation, but these disappear. The spine is represented only by a very short plate, and the larva presents a striking superficial re. semblance to a Trilobite.

It seems likely that Limulus is linked to the extinct Eurypterids by some fossil forms known as Hemiaspidre, e.g., Hemiaspis, Belinurus.

\section{Order 2. EuRYpterina (= Merostomata), e.g., Eurypteris.}

Gigantic extinct forms found from Ordorician to Carbeniferous strata. The body is divided into hearl, thorax, and abdomen. The head is small and unsegmented. The thorax is composed of six distinct segments, the abdomen of six with a terminal telson, which was sometimes a pointed spine, sometimes paddle shaped. There is, however, some doubt as to the exact nomenclature of the regions. On the head are borne six pairs of appendages of varying shape, two lateral compound eyes, and two median ocelli. On the ventral surface of the thorax, there are five pairs of gills covered by flat plates, of which the 
most anterior pair are very large, and form the so-called operculum (cf., Limulus). The surface of the body was covered with scales. Some of the Eurypterids reached a length of six feet.

This order is sometimes placed near the Crustacea, but the general opinion seems to be that which links them through Limulus to Arachnoids.

Order 3. Trilobita. Trilobites, e.g., Calymene, Phacops, Asaphus.

Extinct forms chiefly found in Cambrian and Oxdovician strata, but extending up to the Carboniferous. The body as found is divisible into three parts, 一the unsegmented head shield, often prolonged backwards at the angles; the flexible thorax of a varying number of segments; the unsegmented abdomen, or pygidium. A median longitudinal ridge, or rachis, divides the body into three longitudinal portions.

Traces of limbs are only rarely preserved. In the head region there are four pairs, apparently simple. Antenna have been recently found in this region. The thorax and abdomen were furnished with biramose

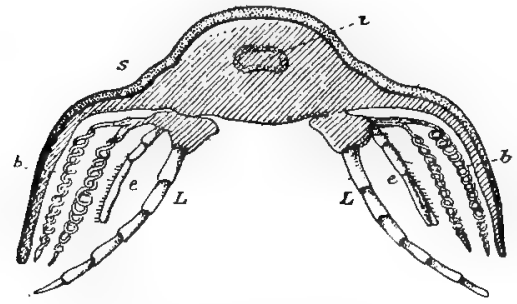

FIG. I09.-Vertical cross section of a Trilobite, Calynene. (After WALCOTT.)

i., Intestine ; s., shield ; $L$., endopodite ; e., exopodite ; 6., epipodial parts.

appendages with long jointed endopodite, short exopodite, and a gill (or epipodite? ) of varying shape. In the abdominal region, the gills were. perhaps rudimentary.

Trilobites are often found rolled up in a way that reminds one of some wood lice. So abundant are they in some rocks that even their development has been studied with some success.

The limbs seem to be more like those of Crustaceans than those of Arachnoids, and the recent discovery of antennæe accentuates the resemblance; but the marked affinities with Limulus, according to the views of most authorities, justify the continued association with Arachnoids. It is, perhaps, most logical to regard Trilobites as an offshoot from a stock ancestral to both Arachnoids and Crustaceans. 
Incertie Sedis.

\section{Pantopoda or Pycnogonide.}

These are marine Arthropods, sometimes called sea spiders. Their affinities are uncertain, but perhaps they may be ranked between Crustaceans and Arachnoids. Many climb about seaweeds and hydroids near the shore, but some live at great depths. The body consists of an anterior proboscis, a cephalothoracic region with three fused and three free segments, and an unsegmented rudimentary abdomen. In most there are seven pairs of appendages, into five of which outgrowths of the mid gut extend. The sexes are separate, and the males usually carry the eggs attached to the third pair of appendages. The larvæ are at first unsegmented, with three pairs of appendages.

Examples:-Pycnogonum, Nymphon, Ammothea. 


\title{
CHAPTER XVI.
}

\author{
MOLLUSCA.
}

Classes I. AmphineurA-A small class of bilaterally symmetrical forms, e.g., Chiton. 2. Gasteropoda, e.g., Snails. 3. SCAPhoPODA-A small class, of which the best known is Dentalizm. 4. Lamellibranchiata-Bivalves. 5. Cephalopoda-Cuttlefishes.

THE series of Molluscs stands in marked contrast to that of Arthropods, for the body of the Mollusc is unsegmented, and there are no appendages. The general habit of life is also very different, for, although there are active Molluscs and sluggish Arthropods, it is true as an average statement that Molluscs are sluggish and Arthropods active. Though the pedigree is unknown, there does not seem to be any possible ancestry for Molluscs less remote than the stock from which Turbellarians and other unsegmented "worms" have sprung.

General Characters.-Molluscs are unsegmented and without appendages. The symmetry is fundamentally bilateral, but this is lost in most Gasteropods. The "foot" - a muscular protrusion of the ventral surface, is very characteristic; it usually serves for locomotion, but is much modified according to habit. Typically, a projecting dorsal fold of the body wall forms a mantle, or pallium (Fig. I Io, c.), which often secretes a single or bilobed shell covering the viscera; but both mantle and shell may be absent. There are three chief pairs of ganglia-cerebrals, pedals, and pleurals, with connecting commissures, and oflen zeith accessory ganglia, especially two viscerals on a loop connecting the pleurals (Figs. I10, I 8). Except in Lamellibranchs, in which the head region is degenerate, there is in the mouth a chitinous 
ribbon or radula, usually bearing numerous small teeth, and moved by special muscles, the whole structure being known as the odontophore. A portion of the true body cavity or calome

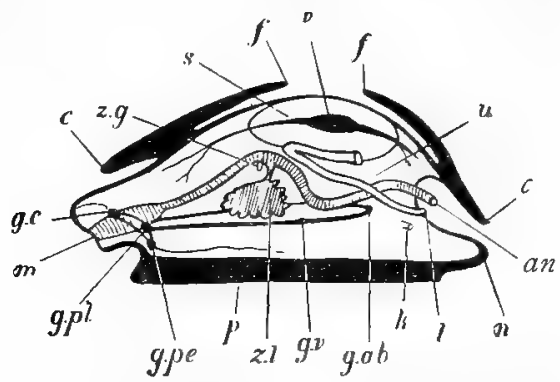

Fig. I IO._Ideal Moilusc. (After Ray Lankester.)

m., Mouth ; g.c., cerebral ganglia ; $c$., edges of mantle skirt; $z . g=$, duct of right digestive gland; $s$, pericardial cavity; $f_{*}$, edges of shell sac; $v_{\text {., }}$ ventricle of heart; $u_{\text {. }}$, nepbridium; an., anus; $n_{\text {. }}$,

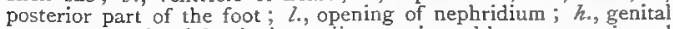
aperture; g.ab., abdominal ganglion on visceral loop; $g . v$, visceral ganglion; $z . l$., left digestive gland; $p$. , foot; $g . p e$. , pedal ganglion ; $g . p l$, pleural ganglion.

usually persists as the pericardium at least (Fig. iro, s.), and communicates with the exterior through the nephridium or nephridia. The vascular system is almost always well
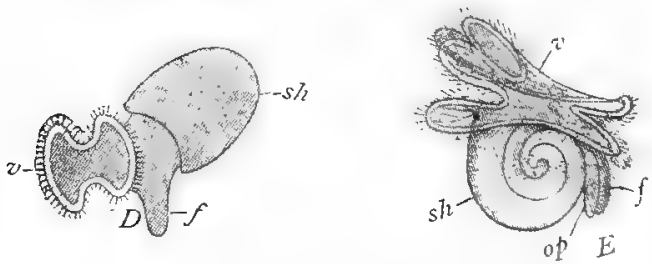

FIG. I I I. - Stages in Molluscan development.

$D$., Larva of Heteropod (after Gegenbaur); $s /$, shell covering visceral hump; $z_{1}$, velum ; $f_{\text {. }}$ foot.

E., Larva of Atlanta (after Gegenbaur); $z$, velum ; sh., shell; f., foot ; op., operculum.

developed, but part of the circulation is in most cases through ill defined spaces or lacunce. Respiratory organs are most typically represented by a pair of vascular processes of the 
body wall (ctenidia or gills), but one or both of these may be absent. At the base of the gills there is generally an olfactory organ or osphradium. Frequently there are two larval stages, the Trochosphere, which resembles the same stage in some Annelids, and the more characteristic Veliger; but the development is often direct.

\section{Class I. Amphineura.}

Syn. Gasteropoda Isopleura, e.g., Chiton.

General Characters.-The Amphineura are marine Molluscs, more or less elongated in form, with bilateral. symmetry. They are often ranked along with Gasteropods. The mouth is anterior, the anal

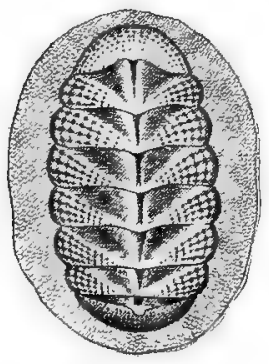

FIG. II 2.-Chiton. (After PRÉTRE.) and nephridial apertures are posterior. The mantle, which bears cuticular spicules, covers at least a great part of the body. The nervous system consists of a cerebral commissure and two paired longitudinal cords, with ganglionic cells, but at most very feeble ganglia, which run the whole length of the body. Of these paired cords the pedals are connected by numerous cross commissures, and the viscerals or pallials are united posteriorly by a commissure above the rectum. The bilateral symmetry is shozen internally, e.g., in the paired nephridia, auricles, and genital ducts. The class is of ancient origin, dating from the Silurian. There are twe orders:-Polyplacophora, e.g., Chiton, and Aplacophora, e.g., Neomenia.

\section{Ist Order of Amphineura, Polyplacophora (Chitonidæ).}

The members of this order, represented on British coasts by several species of Chiton, are sluggish, usually vegetarian, animals, occurring from the shore to great depths. The foot is generally as long as the body; the mantle covers the back and bears eight shell plates (Fig. II2), perforated, in many cases at least, by numerous sensory organs, which may be in part optic; numerons gills lie in a regular row along a groove on each side between the mantle and the foot. 
In most cases the eight shell plates are jointed on one another, and the animal can roll itself up. The uncovered parts of the mantle bear spicules. Ganglia, in the strict sense, are scarcely developed, but there is a supra-cesophageal ganglionic commissure from which the visceral and pedal cords extend backwards along the whole length of the body. There are no special sense organs on the head, which is but slightly

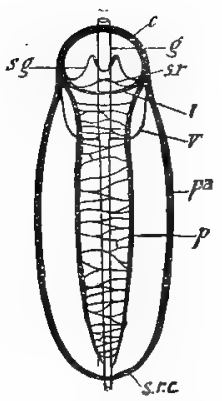

FIG. I 13,-Dorsal view of nervous system of Chiton. (After PELSENEER.)

c., Cerebral commissure; $g$, gut (above all the commissures except cerebral and supra-rectal); pa., pallial or visceral loop with suprarectal commissure (s.r.c.); b., pedal nerves united by numerous transverse branches; s.g., stomatogastric commissure; s. $\%$, subradular commisstire; $l$, , labial commissure; $v$, visceral commissure.

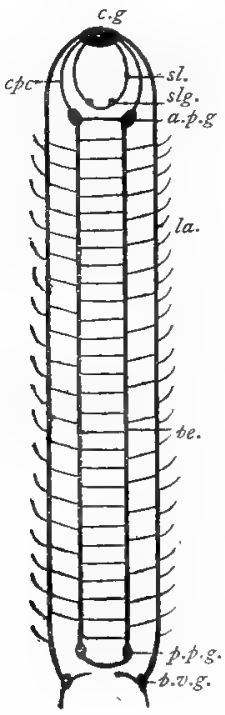

Fig. II4.-Proneomenia. Nervous system (From $\mathrm{H}_{3} \mathrm{uB}$ RECHT.)

c.g., Cerebral ganglia; slg., sublingual; a.p.g., anterior pedal; $p_{.}, g_{.}$. , posterior pedal ; f. $x^{\prime}, g$. posterior viscerals; sl., sublingual connectives; $c p c$, cerebro-pedal connective; pe., longitudinal pedal nerves; la., longitudinal lateral nerves.

differentiated; but the pallial sunse organs are ustally numerous and varied. A twisted gut runs through the body, surrounded by a diffuse digestive gland. There is a raclula in the mouth. The heart is median and posterior, and consists of $n$ ventricle and $2-8$ auricles. Numerous gills lie in a regular row along a groove on each side between the mantle and the foot. There are two symmetrical nephridia opening posteriorly. 
The sexes are separate; a single reproductive organ extends dorsally between gut and intestine almost the whole length of the body; the genital ducts are paired and open posteriorly in front of the excretory apertures. The ova with chitinous spiny shells are usually retained for some time by the female between the mantle and the gills. The segmentation is holoblastic, and a gastrula is formed by invagination.

\section{2nd Order of Ampinevra, Aplacophora, e.g*, Neomenia, Proneomenia, and Chatoderma.}

The members of this order are worm-like animals, in which the mantle envelops the whole body and bears numerous spicules but no shell. There are two families, Neomeniidre and Chretodermidx.

Of Neomeniidæ, six genera are known. They have a longitudinal pedal groove, an intestine without distinct digestive gland, two nephridia with a common aperture, and hermaphrodite reproductive organs. The Chretodermidx, represented by one genus Chatoderma, are cylindrical in form, without a pedal groove, with a radula bearing one tooth, with a distinct digestive gland, and with two nephridia opening separately into a posterior cavity, which also contains two gills. The sexes are separate.

There seem to be more than merely superficial resemblances between these simple Molluscs and such worm types as Turbellarians. It seems justifiable to speak of the Amphineura as primitive Molluscs, but the Aplacophora are perhaps rather degenerate than primitive.

\section{Class II. Gasteropoda, e.g., Snail, Whelk, Limpet.}

General Characters.-Gasteropods are more or less asymmetrical Molluscs. The head region, which is well developed, remains symmetrical, and so does the foot, which is typically a flat creeping organ. But the visceral mass or hump, with its mantle fold, is more or less twisted forwards and to the right. Thus the pallial, anal, nephridial, and genital apertures usually lie on the right side, more or less anteriorly. A further asymmetry is shown by the twisting of the morphologically right gill to the left side, while the original left gill is usually lost. Similarly, one of the nephridia, probably that which is morphologically the left, tends to disappear, and in most cases only one persists - topographically on the left side. The main torsion must be distinguished from the sfiral twisting which the visceral hump often exhibits, and from the frequently associated spiral coiling of the univalve shell. Moreover, a superficial secondary bilateral symmetry tends to 
be acquired by free swimming forms, e.g., Heteropods. The foot usually contains a mucus gland, and tends to be divided into three regions-the pro-, meso-, and meta-podium. There is a single reproductive organ and genital duct.

\section{A type of Gasteropoda-The snail (Helix). \\ Mode of Life.}

The common garden snail ( $H$. aspersa) and its larger neighbour species (H. pematia), rare in England but abundant on the Continent, are so like one another, except in size, that the same description will serve for both. They are thoroughly terrestrial animals, breathing air directly through a pulmonary chamber. They drown (slowly) when immersed in water. Their food consists of leaves and other parts of plants, but they sometimes indulge in strange vagaries of appetite. They are hermaphrodite, but their sexual relations are by no means simple. The breeding time is spring, and the eggs are laid in the ground. In winter snails bury themselves, usually in companies, cement the mouths of their shells with hardened mucus and a little lime, and fall into a state of "latent life" in which the heart beats feebly. In such a state they have been known to survive for years.

\section{General Appearance.}

A snail actively creeping shows a well developed head, with two pairs of retractile horns or tentacles, of which the longer and posterior bear eyes. The foot, by the muscular contraction of which the animal creeps, is very large; it leaves behind it a trail of mucus. The viscera protrude, as if ruptured, in a dorsal hump, which is spirally coiled and protected by the spiral shell. On slight provocation the the animal retracts itself within its shell, a process which drives air from the mantle cavity, and thus promotes respiration. Around the mouth of the shell is a very thick mantle margin or collar, by which the continued growth of the shell is secured. On the right side of the expanded animal, close to the anterior edge of the shell, there is a large aperture through which air passes into and out of the mantle cavity. Within the same aperture is the terminal 
opening of the ureter. The food canal ends slightly below and to the right of the pulmonary aperture. All the three openings are close together. The anterior termination of ureter and food canal is one of the results of the twisting of the visceral mass forwards to the right. But still further forward, at the end of a slight groove which runs along the right side of the neck, indeed quite close to the mouth, is the genital aperture. Lastly, an opening just beneath the mouth leads into the large mucus gland of the foot.

\section{The Shell.}

The shell, a right-handed spiral, is a cuticular product made and periodically enlarged by the mantle margin. Chemically it consists of carbonate of lime and an organic basis (conchiolin). The outermost layer is coloured, without lime, and easily rubbed off : the median layer is thickest, and looks like porcelain; the innermost layer is pearly. The twisted cavity of the shell is continuous, and the viscera extend to the uppermost and oldest part.

As the shell is gradually made, the inner walls of the coils form a central pillar (columella), as on a staircase, and to this the animal is bound by a strong (columellar) muscle. Many Gasteropods bear a horn-like shell lid (operculum) on their foot, but Helix has none; the "epiphragm" with which the shell is sealed in winter, consists of hardened mucus, plus phosphate and a smaller quantity of carbonate of lime. It is formed very quickly from the collar region when cold weather sets in, has no organic connection with the animal, such as binds an operculum to the foot of the whelk, and is loosened off in the mildness of spring.

\section{External Appearance after the Shell is Removed.}

If the shell is removed carefully, so that nothing is broken except the columellar muscle, many structures can be seen without any dissection. The skin of the head and foot should be contrasted $(a)$ with the thick collar of the mantle; $(b)$ with the loose roof of the mantle or pulmonary chamber; $(c)$ with the exceedingly delicate, much stretched, and always protected skin of the visceral hump. It is important to realise that the snail has an "enlargement of the liver" and a great rupture-like hump of viscera on the dorsal surface, that this has been coiled spirally, and that there is the yet deeper torsion forward to the right. 
A great part of the hump consists of the greenish brown digestive gland, in which the bluish intestine coils behind the mantle chamber; on the left lies the triangular and greyish kidney; the whitish reproductive organ lies in the second last and third last coil of the spiral.

\section{The Skin.}

The skin varies greatly in thickness. It consists of a single layered epidermis and a more complex dermis, including connective tissue and muscle fibres. There are numerous cells from which mucus, pigment, and lime are secreted; those forming pigment and lime are especially abundant on the collar, where they contribute to the growth of shell.

\section{Muscular System.}

The most important muscles are- $(a)$ those of the foot; (b) those which retract the animal into its shell, and are in part attached to the columella; (c) those which work the radula in the mouth; $(d)$ the retractors of the horns; and $(e)$ the retractor of the penis. The muscle fibres usually appear unstriated. There is much connective tissue, some of the cells of which contain glycogen, pigment, and lime.

\section{Nervous System.}

This is concentrated in a ring around the gullet. Careful examination shows that this ring consists dorsally of a pair of cerebral ganglia, connected ventrally with a pair of pedals and a pair of pleuro-viscerals, which, according to some authorities, have a median abdominal ganglion lying between them (Fig. I I 8 ).

The cerebrals give off nerves to the head, e.g., to the mouth, tentacles, and otocysts, and also two nerves which run to a pair of small buccal ganglia, lying beneath the junction of gullet and buccal mass. The pedals give off nerves to the foot, the viscerals to the mantle and posterior organs.

\section{Sense Organs.}

An eye, innervated from the brain, is situated on one side of the tip of each of the two long horns. It is a cup invaginated from the epidermis, lined posteriorly by a single 
layer of pigmented and non-pigmented retinal cells, filled with a clear vitreous body perhaps equivalent to a lens, and closed in front by a transparent "cornea," and strengthened all round by a firm "sclerotic." How much a snail sees we do not know, but it detects swift movements. Though the eye is by no means very simple, the snail soon makes another if the original be lost, and this process of regeneration has been known to occur twenty times in succession.

The otocysts appear as two small white spots on the pedal ganglia. Each is a sac of connective tissue, lined by epithelium which is said to be ciliated in one region, containing a fluid and a variable number of oval otoliths of lime, innervated by a delicate nerve from the cerebral ganglia.

Though no osphradium or smelling patch, comparable to that which occurs at the base of the gills in most Molluscs, has been discovered in Helix, the snail is repelled or attracted by odours; it shrinks from turpentine, it smells strawberries from afar. This sense of smell seems to be located in the horns, for a dishorned snail has none. The tips of both pairs of horns bear sensory cells connected with ganglionic tissue and nerve fibres within.

Other sensory cells, probably of use in tasting, lie on the lips ; and there are many others, which may be called tactile, on the sides of the foot, and on various parts of the body. In short, the snail is diffusely sensitive.

\section{Alimentary System.}

'The snail files the leaves of plants by means of the radula or toothed ribbon which lies in the mouth, and it grasps the debris with its lips.

The radula is a long strip of membrane, bearing several longitudinal rows of minute chitinoid teeth. It rests on a cartilaginous pad on the floor of the mouth cavity, and is moved (backwards and forwards, and up and down) in a curve, by protractor and retractor muscles. The whole apparatus, including radula teeth, membrane, and pad, is called the odontophore. The radula wears away anteriorly, but is added to posteriorly within a radula sac which projects from the floor of the buccal cavity. Its action on 
leaves may be compared very roughly to that of a file, but its movements within the mouth also produce a kind of suction which draws food particles inwards. In this suction the muscular lips and the cilia in the mouth cavity assist.

Altogether apart from the radula, lying on the upper surface of the buccal chamber, sometimes visible when the snail opens its mouth, is a hard, crescent shaped jaw plate.

The ducts of two large salivary glands open on the dorsal surface of the buccal cavity, and there are numerous distinct glandular cells close to the entrance of the two ducts. The salivary glands are large lobed structures, and extend far backward on the crop. They consist of hundreds of glandular cells or unicellular glands, which secrete a clear fluid stuff. This travels up the ducts, and is forced, in part at least, by muscular compression, into the buccal cavity. While some say that this fluid converts starch into sugar (after the usual fashion of saliva), other authorities deny that it has any effect upon the food. Similar glands are found in all Gasteropods, while they are entirely absent in Lamellibranchs. In some boring Gasteropods the secretion contains $2-4$ per cent. of free sulphuric acid.

The gullet extends backward from the buccal cavity, and expands into a storing crop; this is followed by a stomach surrounded by the digestive gland; thence the intestine extends, and after coiling in the visceral hump, passes forward to end on the right side anteriorly beside the respiratory aperture. The digestive tract is muscular, and in part ciliated internally.

A large part of the visceral spiral is occupied by the so-called "liver," a digestive gland of many qualities, producing juices which digest all kinds of food, making glycogen, storing phosphate of lime, and containing a greenish pigment called enterochlorophyll. It is possible that the phosphate of lime may be used to form the autumnal epiphragm, but the most important fact is that the gland is more than a "liver," more even than a "hepato-pancreas," it is a complex digestive gland, producing several digestive ferments. 


\section{Vascular System.}

'The blood of the snail contains some colourless amceboid cells, and a respiratory pigment called hæmocyanin, which gives the oxidised blood a blue tint, and is very common among Molluscs.

The heart, consisting of a ventricle and an auricle, lies within a pericardial chamber on the dorsal surface, to the left side, behind the mantle cavity.

From the ventricle pure blood flows by cephalic and visceral arteries to the head, foot, and body, passes into fine ramifications of these arteries, and thence into spaces among the tissues. Authorities differ as to the existence of capillaries, but the distinction between these and narrow channels is of no physiological importance. From spaces among the tissues the blood is collected in larger venous spaces, and eventually in a pulmonary sinus around the mantle cavity, on the roof of which there is a network of vessels. There the blood is purified. Most of it returns directly to the auricle by a large pulmonary vein, but some passes first through the kidney.

\section{Respiratory System.}

Most Gasteropods, e.g., the dog whelk (Purpura), the buckie (Buccinum), the periwinkle (Littorina), breathe by gills covered over by a fold of the mantle. The snail being entirely terrestrial has a pulmonary or lung cavity, formed by the mantle fold. On the roof of this cavity the blood vessels are spread out. Air passes into and out of the pulmonary chamber by the respiratory aperture. When the animal is retracted within its shell, the freshening of the air in the pulmonary chamber takes place by slow diffusion, but when the snail extends itself at full length, the chamber is rapidly filled with air, and it is even more rapidly emptied when the animal withdraws into the shell again.

\section{Excretory System.}

There is a single triangular greyish kidney behind the pulmonary chamber, between the heart and the rectum. It is a sac with plaited walls, and excretes nitrogenous waste products, which pass out by a long ureter running along the 
right side of the pulmonary chamber, and opening close beside the anus. From two sources the kidney is supplied with blood $(a)$ from the pulmonary chamber, and $(b)$ from the heart by a renal artery. As in most other Molluscs, the kidney communicates by a small aperture with that part of the body cavity which forms the pericardial sac. Thus, as in earthworm, lobworm, \&c., the cœlome has a nephridial connection with the exterior.

\section{Reproductive System.}

The snail is hermaphrodite, and its reproductive organs exhibit much division of labour.

(a) The essential reproductive organ (the ovotestis) is a whitish body near the apex of the visceral spire. It consists of numerous cylindrical follicles, in each of which both ova and spermatozoa are formed, but not at the same time. Simultaneous formation of elements so different is probably very rare.

(b) A much convoluted hermaphrodite duct of a white colour conducts the sex cells from the ovotestis, and leads to the base of a large yellowish albumen gland.

(c) This tongue shaped albumen gland varies in size with the age and sexual state of the snail. It forms gelatinous proteid material, which envelops and probably nourishes the ova.

(d) The ova and spermatozoa pass from the hermaphrodite duct towards the head along a common duct, but not at the same time. Moreover their paths are different, for the portion of the duct down which the ova travel is much plaited, while the path which the spermatozoa follow is a less prominent groove, incompletely separated from the other. Both paths are glandular, and the glands on the male side are often called prostatic.

(e) At the base of this common duct, a distinct vas deferens diverges to the left and leads into a muscular penis, which can be protruded at the single genital aperture and retracted by a special muscle. Before the vas deferens enters the penis, a long process or flagellum is given off. It is like the lash of a whip, and is as long as the common duct. Within it a spermatophore is partly formed, but seems to be completed in the penis. This spermatophore is laden with 
a large number of spermatozoa, and is transferred by the penis into the genital aperture of another snail.

$(f)$ Continued from the oviducal side of the common duct, there is a separate ciliated oviduct. This has a short course, and ends in the common genital aperture. Before it reaches this, however, the oviduct is associated with two structures. The first of these is a long process, as long as the common duct, beside which it runs, in appearance

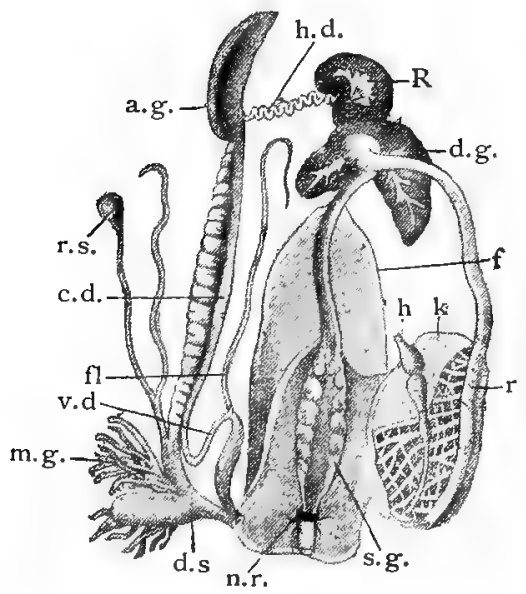

FIG. I 15.-Dissection of Helix pomatia. (Mainly after LEUCKART.)

m.r., Nerve ring; s.g., salivary glands on the crop; $f$. , foot; $d . g$. , digestive gland opening in to mid gut; $h$. heart; $k$. kidney; $\mu$, rectum; $R$. hermaphrodite organ in terminal part of digestive gland; h.d., hermaphrodite duct; a.g., albumen gland; c.d., common duct, with more convoluted oviducal part; $v, d$. , vas deferens entering penis; $f$., flagellum; r.s. receptaculum seminis, with a branch from its duct ; m.g., mucus glands ; d.s., dart sac.

suggesting the flagellum, but expanding at its free end into a globular sac-the receptaculum seminis. It is into this long duct and sac that a spermatophore from another snail passes, and is after some days dissolved, liberating hundreds of spermatozoa. By these spermatozoa the ova of this snail 
are fertilised. The second structure associated with the female duct is a conspicuous mucus gland, formed of two sets of finger-like processes. The mucus secretion of this gland is very abundant during copulation, and as it contains not a little lime, it is possible that it may form the calcareous shells of the eggs.

(g) Finally, between the entrance of oviduct and penis into the terminal aperture there lies a firm cylindrical structure, larger than the penis and with muscular walls. It is the Cupid's Dart Sac, and contains a pointed calcareous arrow (spiculum amoris), which is jerked out previous to copulation. The dart is sometimes found adhering to the skin of a snail, and after copulation the sac is empty, soon, however, to be refilled.

When two snails pair, the genital apertures are dilated,

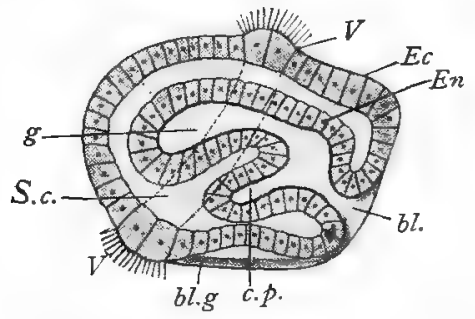

Fig. I I6.-Diagram of larva of Paludina. (After ERLANGer.)

Ec., ectoderm; En., endoderm; $\%$ velum, with cilia; $g$, gut cavity; S.c., segmentation cavity; c.p., coelome pocket from gut; bl.g., blastopore groove closed, except at $b l$., which becomes the anus.

the protruded penis of one is inserted into the aperture of the other, and the transference of a spermatophore is thus effected.

The eggs are laid in the earth in June and July. Each is surrounded by gelatinous material acquired in the oviduct, and by an elastic but calcareous shell.

Segmentation is total but slightly unequal. As the snail is a terrestrial Gasteropod, there is no trochosphere larva nor more than a slight hint of the characteristic Molluscan velum. A miniature adult is hatched in about three weeks. 
The study of development may be more profitably followed in the pond snail Limnaus, where gastrula, trochosphere, and veliger can be readily seen.

\section{Classification of Gasteropoda.}

Sub-Class I, Streptoneura.

Sub-Class II. Euthyneura.

(I) Zygobranchia, e.g., limpet.

(2) Azygobranchia, e.g., whelk.

(1) Opisthobranchia, e.g., Aplysia.

(2) Pulmonata, e.g., Snail.

I. Streptoneura (i.e., with twisted nerves). In this division the torsion of the body has twisted the visceral nerve loop in the form of a figure 8 . The sexes are separate.

(1) Zygobranchs. Both gills may persist, or both may degenerate, their functions being then discharged by folds of the mantle. Both nephridia persist, that on the right side being the larger, and serving also as a genital duct.

Examples-

Limpet (Patella), Ear shell (Haliotis).

(2) Azygobranchs. The morphologically left gill, nephridium, and osphradium disappear, those morphologically right persist topographically on the left. It may also be that the genital duct is a modification of the morphologically left nephridium.

Examples-

Periwinkle (Littorina), Buckie (Buccinum), Dog whelk (Purpura), Cone shells (Conus), Murex, Ianthina; and also the pelagic Heteropods, with foot adapted for swimming, e.g., Atlanta (large sheli), Carinaria (small shell), Pterotrachea (no shell).

II. Euthyneura (i.e., with straight nerves). The torsion of the visceral hump is less complete, and the visceral loop is untwisted. All are hermaphrodite. The shell is often light, and may be absent from the adult. The mantle may also be much reduced. The gill (if present) is behind the heart (Opisthobranch condition), whereas in the Streptoneura it was almost always in front (Prosobranch condition).

(I) Opisthobranchia. Small visceral hump, rarely any shell in the adult; anus behind, and heart in front of gill when this is present.

(a) Tectibranchia. Well developed mantle fold and functional gill, usually with delicate shell. Bulla, Aplysia, Dolabella, Umbrella.

(b) Nudibranchia. Mantle fold rudimentary and shell absent in the adult. The gill is either much modified or absent. 
As Tectibranchia must also be included, the Pteropoda, the Winged Snails or Sea Butterflies, which have become modified for pelagic life. They have a secondarily acquired apparent symmetry, and swim by two large lateral lobes of the foot ("parapodia"). They often swim actively in shoals, and occur in all seas. They afford food for whales, \&c., and the shells of some are abundant in the ooze. They include:-

(a) Thecosomata, with mantle fold and shell, diet of minute animal or vegetable organisms, closely related to Bulla and its allies.

Examples-Hyalea, Cymbulia.

(b) Gymnosomata, without mantle fold or shell in the adult. Closely allied to Aplysia and its allies. Actively carnivorous.

Examples-Clio, Pneumoderma.

(2) Pulmonata. Air breathing, without gill, the edge of the mantle has fused with the body wall, forming a pulmonary chamber with a small aperture, e.g., Helix (snail); Limax (grey slug); Arion (black slug); Limnetus, Planorbis, and Ancylus (common fresh water snails).

\section{General Notes on Gasteropods.}

From a form in essentials similar to Chiton, except for its eight shells, we may consider that the Gasteropods proper have been developed. They are all more or less asymmetrical, but we must notice-(I) that this want of symmetry does not affect the head or the foot, but only the dorsal viscera, which are more or less twisted round to the right side towards the head; (2) the torsion must be distinguished from the frequent spiral twisting of the visceral hump and of the shell ; (3) the torsion occurs in variable degree, and some forms, especially free swimmers, have a superficial symmetry.

The current explanation of the asymmetry, which has been recently elaborated by Lang, is as follows :-

If we begin with a form something like a Chiton, but with a simple shell, we must suppose the head and foot to become increasingly specialised, and at the same time to acquire an increasing freedom of movement; during the process the viscera will tend to become more and more limited to a special region of the body, and a "visceral hump" will thus be formed. The shell becomes limited to this region, but the contractility of head and foot, which enables these to be drawn into the shell, must be correlated with the increasing size and complexity of this structure. As, however, shell and visceral hump become larger, they become too heavy to be carried in the primitive position on the back of the animal, and incline to one side. There is, therefore, a onesided pressure, which results in an increased growth relatively of the opposite side, and so in a deep seated twisting, which brings the originally posterior anus to an anterior position near the mouth, and produces a tendency to the suppression of one of the originally paired 
gills, nephridia, \&c. According to Lang, during the torsion an increased growth of the upper surface of the visceral mass is necessary in order to avoid rupture, and thus the superficial spiral coiling is produced; this is reflected in the coiling of the shell. In one series of the Gasteropods the visceral nerve loop, running from the cerebral and pleural to the visceral ganglia, is "caught in the twist," and twines like a figure 8 (Streptoneural condition), in the others the same visceral loop is short and untwisted (Euthyneural condition). In both groups we find forms with coiled shells, but among the Euthyneura there is a tendency to lose the shell, the visceral hump becoming at the same time inconspicuous, while a superficial appearance of symmetry is produced. The deep seated torsion of the organs is, however, still retained.

It is not very uncommon to find, either as a constant occurrence or as an occasional variation, spirally coiled shells with a reversed or left handed spiral. In some of these cases the superficial coiling of the visceral hump, as well as the deep seated torsion, is also left handed; but in others we find that the internal structure retains the normal arrangement.

Mode of Life.

From the number of diverse types which the class includes, it is evident that few general statements can be made about the life of Gasteropods. We are safe in saying, however, that though the majority are sluggish when compared with Crustaceans, they are active when compared with Lamellibranchs.

The locomotion effected by the contractions of the muscular foot is usually a leisurely creeping, but there are many gradations between the activity of Heteropods in the open sea, the gliding of fresh water snails (Limncus) foot upwards across the surface of the pool, the explorations of the periwinkles on the sand of the shore, and the extreme passivity of limpets (Patella), which move only for short distances at a time from their resting places on the rocks.

Statistics are neither interesting nor reliable, for there is much difference of opinion as to the limits of species and varieties, but we may notice that the number of terrestrial snails and slugs, breathing the air directly by means of a pulmonary chamber, is estimated at over 6000 living species, while the aquatic Gasteropods are reckoned at about Io, ০oo, most of which are marine. Of this myriad, about 9000 are streptoneural, the relatively small minority are euthyneural opisthobranchs and nudibranchs, with light shells or none. The Heteropods and some opisthobranchs live in the open sea ; the great majority of aquatic Gasteropods frequent the 
shore and the sea bottom at relatively slight depths; the deep sea forms are comparatively few.

Gasteropods rarely feed at such a low level as bivalves do, indeed some of them are fond of eating bivalves. Most prosobranchs (streptoneural), with a respiratory siphon and a shell notch in which this lies, are carnivorous, e.g., the buckies (Buccinum) and "dog whelks" (Purpura); on the other hand, those without this siphon, and with an unnotched shell mouth, feed on plants, e.g., the seaweed eating periwinkles (Littorina). The vegetarian habits of most land snails and slugs are known to all. Many Gasteropods, both marine and terrestrial, are very voracious and indiscriminate in their meals; others are as markedly specialists or epicures. Some marine forms, partial to Echinoderms, have got over the difficulty of eating such hard food by secreting dilute sulphuric acid, which is said to change the carbonate of lime in the starfish into the more brittle and readily pulverised sulphate. Only a few Gasteropods are parasitic, e.g., Eulima and Stylifer on Echinoderms, and the extremely degenerate Entoconcha mirabilis,-_-within the Holothurian Synapta.

\section{Life History.}

The eggs of Gasteropods are usually small, without much yolk, but surrounded by a jelly, the surface of which hardens. In the snail and in some others there is an egg shell of lime.

Sexual union occurs between hermaphrodites as well as between separate sexes, and fertilisation is effected inside the genital duct. Development sometimes proceeds within the parent, but in most cases 
the fertilised eggs are laid in gelatinous clumps, or within special capsules. The free swimming Ianthina carries the eggs in capsules attached to a large raft-like float towed by the foot. On the shore one often finds numerous egg capsules of the "buckie" (Buccinum undatum) united in a ball about the size of an orange. Under the ledges of rock are many little yellowish cups, the egg capsules of the dog whelk (Purpura lapillus). In the buckie and whelk, and in some other forms, there is a struggle for existence-an infant cannibalism-in the cradle, for out of the numerous embryos in each capsule only a few reach maturity, those that get the start eating the others as they develop.

The segmentation of the ovum is total, but somewhat unequal; a gastrula is formed by invagination or by overgrowth according as there is less or more yolk; the gastrula becomes a trochosphere with a pre-oral ring of cilia; the trochosphere grows into a veliger with a lobed ciliated cushion or velum, a visceral dome, a dorsal shell gland which soon disappears, and an incipient ventral foot. In terrestrial snails like Helix, the life history is abbreviated. In the water snail Limnaus, Ray Lankester has detected the persistence of the velum in the circumoral lobes of the adult.

Past History. - As the earth has grown older the Gasteropods have increased in numbers. A few have been disinterred from the Cambrian rocks; thence onwards they increase. Most of the Palæozoic genera are now quite extinct, but many modern families trace their genealogy to the Cretaceous period. Those with respiratory siphons were hardly, if at all, represented in Palzozoic ages, and the terrestrial air breathers are comparatively modern. Zoological statisticians estimate the number of Gasteropods at 23,000, of which 7000 are extinct, I6,000 alive. But besides the numerical success which may be inferred from these figures, it is important to notice that not a few types have persisted from early ages.

\section{Bionomics.}

As voracious animals, with irresistible raspers, Gasteropods commit many atrocities in the struggle for existence and decimate many plants. Professor Stahl shows, however, that there are more than a dozen different ways in which plants are saved from snails,-by crystals, acids, ferments, \&c. ; and like an orthodox Darwinian he regards these 
plants as the survivors of a multitude, which did not become sufficiently gritty or poisonous. As food and bait many Gasteropods are very useful; their shells have supplied tools and utensils and objects of delight; the juices of Purpura and Murex furnished the Tyrian purple, more charming than all aniline.

\section{Class III. SCAPHOPODA.}

Very different from Gasteropoda are the Scaphopoda, of which Dentalium (Elephant's tooth shell) is the commonest genus. Their position is uncertain. Some place them nearer bivalves, others nearer Cephalopods. They burrow in the sand at considerable depths off the coasts of many countries. The mantle has originally two folds, which fuse ventrally, and the shell becomes cylindrical, like an elephant's tusk. It is open at both ends. The larger opening (directed downwards in the sand) is anterior, the concave side of the shell is dorsal. The small cylindrical head bears at its extremity a mouth surrounded by numerous "tentacles," while at its base there is a double cluster of ciliated contractile processes possibly representing gills. The foot is long, with three small terminal lobes. It is used in slow creeping, and is protruded at the anterior opening. There are cerebral and pleural ganglia, near one another in the head, pedal ganglia in the foot, and a long untwisted visceral loop with olfactory ganglia near the posterior anus. Sense organs are represented by otocysts beside the pedal ganglia. There is an odontophore with a simple radula. The food consists of minute animals. There is no heart, but colourless blood circulates in the body cavity. There are two nephridial apertures, one on each side of the anus; the nephridial chamber is perforated by the intestine. The sexes are separate; the reproductive organ is simple and clorsal in position; the elements pass out by the right nephridium. The gastrula is succeeded by a free swimming stage, in which there is a hint of a velum and a rudimentary shell gland.

Examples.-Dentalium, Entalium. About forty widely distributed species are known. Dentalizem entale occurs off British coasts. The genus occurs as a fossil from Carboniferous (or perhaps earlier) strata onward.

\section{Class IV. Lamellibranchiata or Bivalves.}

(Synonyms. Acephala, Conchifera, Pelecypoda, Lipocephala, \&c.)

Examples._Cockles, Mussels, Clams, and Oysters.

Lamellibranchs are bilaterally symmetrical Molluscs, in which the body is compressed from side to side and the foot 
more or less ploughshare-like. The head (or prostomium) region remains undeveloped, zeithout tentacles or eyes; the mouth is without radula, horny jawe, or salivary glands, but there is a pair of labial palps on each side. The mantle skirt is divided into two flaps, which secrete the two valves of the shell, now lateral instead of dorsal in position. The valves are united by a dorsal elastic ligament, and closed by two transverse adductor muscles or by one. Internal bilateral symmetry is marked by the paired nature and disposition of the nephridia, auricles, gills, digestive and reproductive organs. The gills (ctenidia) consist of numerous gill filaments which typically growe together into large plates (hence the title Lamellibranch). There are usually three pairs of ganglia: (a) cerebro-pleurals in the head; (b) pedals in the foot; (c) viscerals at the posterior end of the body. The heart consists of a ventricle and two auricles, and is surrounded by a pericardium which is coelomic in origin, and communicates with the exterior by means of the two nephridia. Reproductive organs are always simple, and the sexes are usually separate. The typical development includes trochosphere and veliger stages. Most Lamellibranchs feed on microscopic organisms and particles; the distribution is very wide both in salt and fresh zeater; the average habit is sedentary or sluggish.

\section{Type of Lamellibranchiata: 'The Fresh water Mussel (Anodonta cygnea).}

The fresh water mussel lives in rivers and ponds. It lies with its head end buried in the mud, or ploughs slowly along by means of its wedge-like foot. Its food consists of minute plants and animals, which are wafted in at the posterior end by the currents produced by the ciliated gills.

\section{External Appearance.}

The bivalve is four to six inches long; its valves are equal and united in a dorsal hinge by an elastic ligament, an uncalcified part of the shell; on the ventral surface when the valves gape the foot protrudes; the anterior end is rounded, the posterior end is more pointed, and it is there that the water currents flow in (ventrally) and out (dorsally). In bivalves the ligament is generally posterior 
to the dorsal knob or umbo-the oldest part of the shelland the umbo generally points towards the anterior end. The greenish-brown soft ("horny") layer of the shell is often worn away near the umbo on each side, and then displays the median "prismatic" layer of lime. Internally there is a pearly layer. Lines of growth on the shell mark the position of the margin in former years, the youngest part being obviously at the edge.

The shell is a cuticular structure, i.e., it is made by the epidermis of the mantle. It consists mostly of calcium carbonate plus an organic substance called conchiolin.

\section{Internal Appearance.}

When the right half of the shell is folded back, the anterior and posterior closing muscles being carefully cut close to the gently raised valve, the mantle folds are seen lining the shell and forming posteriorly the ventral inhalent and dorsal exhalent lips. The ventral lips have papillary processes. (In some bivalves, e.g., Mya, the lips of the mantle are prolonged into retractile siphons.) Internal to the mantle two gill plates lie on each side; projecting from between these is the foot, muscular ventrally, softer dorsally; the median dorsal pericardium is just beneath the ligament; the ventricle shines through its walls, and the dark-coloured kidneys are seen through its floor. Below the anterior closing (adductor) muscle, the large mouth will be found, bordered beneath by two lip processes (labial palps) on each side; above the posterior closing muscle the food canal ends. The whole space between the two mantle flaps is called the mantle cavity, and it is divided by a slight partition at the bases of the gills into a large ventral infrabranchial chamber, and a small dorsal supra-branchial chamber which ends at the exhalent orifice.

On the valve of the shell folded back, are seen a number of concentric (pallial) lines, marking the gradual extension of the mantle and the consequent growth of the shell. A few small pearls may also be seen; they are formed by the enclosure of some minute grains of sand in the prismatic layer. The following muscles are inserted on the shell and leave impressions :- 
(a) The anterior adductor.

(b) The posterior adductor.

(c) The anterior retractor of the foot continuous with $(a)$.

(d) The protractor of the foot a little below $(a)$.

(e) The posterior retractor of the foot continuous with $(b)$.

As the shell grows the insertion of the muscles and the attachment of the mantle change, and the traces of this shifting are visible.

Skin.

There is much ciliated epithelium about Anodonta, especially on the internal surface of the mantle, on the gills, and on the labial palps ; and little pieces cut from an animal incompletely dead (e.g., from the oyster which many of us swallow half alive) have by means of their cilia a slight power of motion. The skin of the foot is not ciliated but glandular; on the mantle edge sensitive and glandular cells are abundant, but usually in inverse ratio to one another.

\section{Muscular System.}

The shell is closed and kept closed by the action of the two adductor muscles. When these are relaxed under nervous control, the elasticity of the hinge ligament opens the valves. A book with an elastic binding, stretched when the book is closed by clasps, would in the same way open when unclasped. It is easier for the mussel to open the valves of its shell than to keep them shut. The foot is a muscular protrusion of the ventral surface, under the control of three muscles-a retractor and a protractor anteriorly, and a posterior retractor. Its upper portion contains some coils of gut and the reproductive organs; its lower region is very muscular. The protrusion or extension of this locomotor organ is mainly due to an inflow of blood, which is prevented from returning by the contraction of a sphincter muscle round the veins. In moving, the animal literally ploughs its way along the bottom of the pond or river pool, and leaves a furrow in its track. The muscle fibres are of the slowly contracting non-striped sort. 


\section{Nervous System.}

There are three pairs of nerve centres:-

(a) Cerebro-pleural ganglia, lying above the mouth on each side on the tendon of the anterior retractor of the foot, connected to one another by a commissure, connected to the two other pairs of ganglia $(b)$ and $(c)$, by long paired connectives, and giving off some nerves to mantle, palps, \&c.
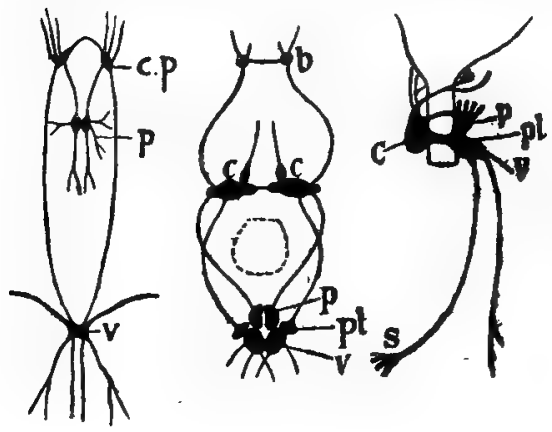

FIG. I I 8. - Nervous system of Molluscs.

Tothe left that of Anodonta; to the right that of Octopus; in the middle that of Helix. In the last two the position of the gullet is shown.

$c_{. p .}$, Cerebro pleural ganglia; p., pedals ; $\eta_{.}$, viscerals; $c_{.}$, cerebrals; $\not l$., pleurals ; $b$., buccals; s., stellate ganglion.

(b) Pedal ganglia, lying close together about the middle of the foot, united by connectives to $(a)$, giving off nerves to the foot, and having beside them two small ear sacs, each with a calcareous otolith, and with a nerve said to be derived from the connective between $(a)$ and $(b)$.

(c) Visceral ganglia (also called parieto-splanchnic or osphradial), lying below the posterior adductor, connected to $(a)$ by two long connectives, and giving off nerves to mantle, muscles, \&c., and to a patch of "smelling cells" at the bases of the gills. 


\section{Sense Organs.}

Unlike not a few bivalves which have hundreds of "eyes" on the mantle margin, Anodonta has no trace of any. The ear sac, originally derived from a skin pit, is sunk deeply within the foot and is of doubtful use. The "smelling patch" or "osphradium" at the base of the gills, has perhaps water testing qualities. There are also "tactile" cells about the mantle, labial palps, \&c.

\section{Alimentary System.}

The mouth lies between the anterior adductor and the foot, and beside it lie the ciliated, vascular, and sensitive labial palps, two on each side. It opens immediately into the gullet, for the pharynx of other Molluscs, with all its associated structures, is absent in Lamellibranchs. The short wide gullet leads into a large stomach surrounded by the paired digestive gland (hepato-pancreas (?)), whose juices are partly analogous with those of the Vertebrate liver and pancreas. Part of the food digested by these juices in the stomach is compacted in autumn into a "crystalline style". a mass of reserve food stuffs, and similar but less solid material is found in the intestine. On this supply the mussel tides over the winter. Some authorities, however, maintain that the style is a glandular secretion, protecting the lining of the gut from injury. Similar structures are found in several Gasteropods. The intestine, which has in part a folded wall like that of the earthworm, coils about in the foot, ascends to the pericardium, passes through the ventricle of the heart, and ends above the posterior adductor at the exhalent orifice.

\section{Vascular System.}

The heart lies in the middle line on the dorsal surface, within a portion of the body cavity called the pericardium, and consists of a muscular ventricle which has grown round the gut and drives blood to the body, and of two transparent auricles-one on each side of the ventricle-which receive blood returning from the gills and mantle. The colourless blood passes from the ventricle by an anterior 
and a posterior artery, flows into ill-defined channels, is collected in a "vena cava" beneath the floor of the pericardium, passes thence through the kidneys, where it loses nitrogenous waste, to the gills, where it loses carbonic acid and gains oxygen, and returns finally by the auricles to the ventricle. The blood from the mantle, however, returns directly to the auricles without passing through kidneys or gills, but probably freed from its waste none the less. The so-called "organ of Keber" consists of "pericardial glands"

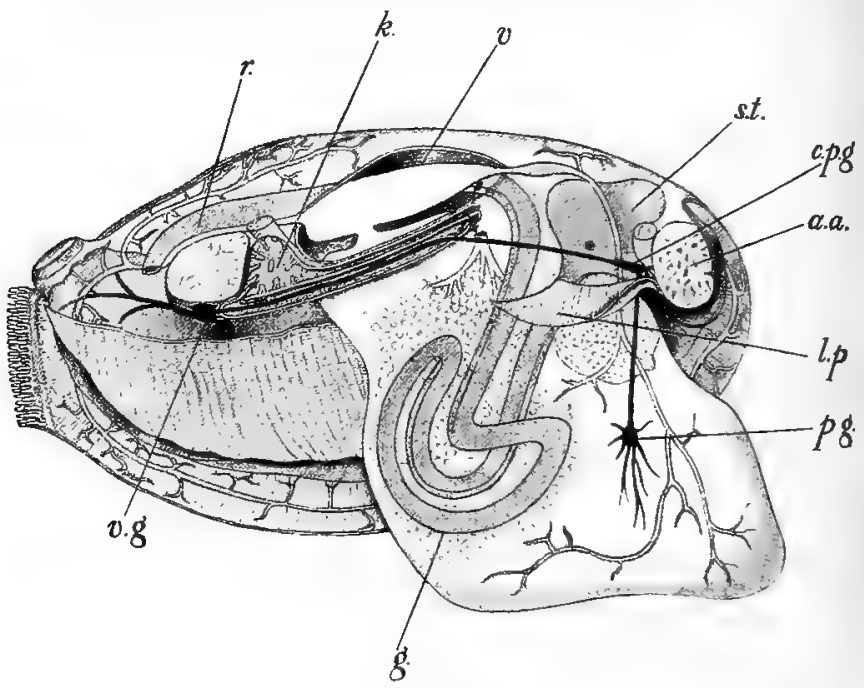

FIG. I I9.-Structure of Anodonta. (After RANkIN.)

aa., Anterior adductor; c.p.g., cerebro-pleural ganglia; st., stomach; $v$, ventricle, with an auricle opening into it; $k$., kidney, above which the posterior retractor of foot ; $r_{\text {. }}$, rectum, ending above posterior adductor; $\tau \cdot g$., visceral ganglia with connectives (in black) from cerebro pleurals ; $g$., gut coiling in foot; $p . g$. , pedal ganglia in foot, where also are seen branches of the anterior aorta and the reproductive organs; l.p., labial palps behind mouth.

on the epithelium of the pericardial cavity. They seem to be connected with excretion. Many of the cells lining the blood channels secrete glycogen, the principal product of the Vertebrate liver. 


\section{Respiratory System.}

Lying between the mantle flaps and the foot there are on each side two large gill plates, whence the title Lamellibranch. They are richly ciliated, their internal structure is like complex trellis work, their cavities communicate with the supra-branchial chamber. "Ctenidia" they are often called, because they are more than gills; not only are they surfaces on which blood is purified by the washing water currents (a respiratory function), but some of their many cilia waft food particles to the mouth (a nutritive function), and in the females the outer gill plate shelters and nourishes the young larvæ (a reproductive function). The water may pass through the gills to the supra-branchial chamber and thence out again, or over the gills to the mouth, and thence into the supra-branchial chamber. It is likely that the mantle has no small share in the respiration.

The precise structure and attachment of the gill plates is complex, but it is important to understand the following facts:- $-(a)$ a cross section of the two gill plates on one side has the form of a W, one half of which is the outer, the other the inner gill plate; $(b)$ each of these gill plates consists of a united series of gill filaments, which descend from the centre of the $\mathrm{W}$ and then bend up again; (c) adjacent filaments are bound together by fusions and bridges both horizontal and vertical, so that each gill plate becomes like a complex piece of basket work; $\left(a^{2}\right)$ both gill plates begin by the downward growth of filaments from a longitudinal "ctenidial axis," the position of which on cross section is at the median apex of the $\mathrm{W} ;(e)$ this mode of origin, and the much less complex gills of other bivalves, lead one to believe that there is on each side one gill, consisting of two gill plates formed from a series of united and reflected gill filaments. On the gills there are often parasitic mites (Atax).

\section{Excretory System.}

The paired kidney, which used to be called the "organ of Bojanus," lies beneath the floor of the pericardium. Each half is a nephridium bent upon itself, with the loop posterior, the two ends anterior. The lower part of this bent tube is the true kidney; it is dark in colour, spongy in texture, and excretes guanin and other nitrogenous waste from the blood which passes through it. It has an internal opening into the pericardium, which thus communicates indirectly with the exterior. The upper part of the bent tube, lying next the floor of the pericardium, is merely a 
ureter. It conveys waste products from the glandular part to the exterior, and opens anteriorly just under the place where the inner gill plate is attached to the visceral mass. As already mentioned, the "pericardial glands" probably aid in excretion, and possibly the same may be said of the mantle.

\section{The Reproductive Organs.}

These lie in the upper part of the foot, adjacent to the digestive gland. Ovaries and testes occur in different animals, and the two sexes are distinguishable, though not very distinctly, by the greater whiteness of the testes and by slight differences in the shells. The females are easily known when the larvæ begin to accumulate in crowds in the outer gill plates. The reproductive organs are branched and large; there are no accessory structures; the genital aperture lies on each side under that of the ureter.

Autumn and winter months seem to be the usual periods of reproduction. The ova are squeezed out of the foot, and appear to be moved to the exhalent region, whence, however, they do not escape, but are crowded backward till they pass into the cavity of the outer gill plate. At some stage they are fertilised by spermatozoa drawn in by the water currents, though it is difficult to believe that this is entirely a matter of chance. Development takes place in the gill cavity, which is often much distended with larvæ.

\section{Development and Life history.}

The development of Anodonta differs in certain details from that of most bivalves, perhaps in adaptation to fresh water conditions. Moreover, a temporary parasitism of the larva has complicated the later stages.

The egg cell is surrounded by a ritelline membrane, and attached to the wall of the ovary by a minute stalk, the insertion of which is marked on the liberated ovum by an aperture or micropyle, through which the spermatozoon enters.

Segmentation is total but unequal. A number of small clear yolkless cells are rapidly divided off from a large yolk-containing portion, which is slower in dividing. Eventually, a hollow ball of cells or blastosphere results (Fig. 120).

On the posterior dorsal region a number of large opaque cells form an internally convex plate, the beginning of the future shell sac. A pair of large cells are intruded into the central cavity, and begin the mesoderm.

On the under surface posteriorly there is a slight protrusion of ciliated cells forming a ciliated disc. In front of this, at an umusually late stage, 
an invagination establishes the archenteron and the embryo hecomes a gastrula (see Fig. I20).

The shell sac forms an embryonic shell, and many of the mesoderm cells combine in an adductor muscle. The mouth of the gastrula closes, and a definite mouth is subsequently formed by an ectodermic invagination. Gradually a larva peculiar to fresh water mussels, and known as a Glochidium, is built up.

The Glochidium has two triangular, delicate, and porous shell valves, each with a spiny incurved tooth on its free edge. The valves clap together by the action of the adductor muscle. The mantle lobes are very small, and their margins bear on each sicle three or four patches of sensory cells. The foot is not yet developed, but from the position which it will afterwards occuly there hang long attaching threads of

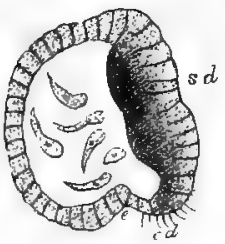

I

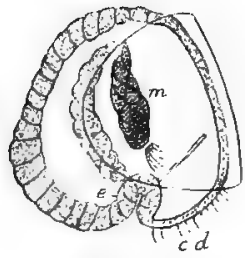

3

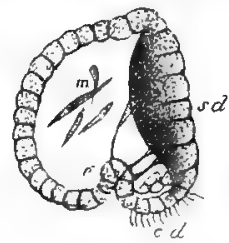

2

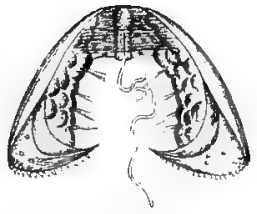

4

FIG. rzo.-Development of Anodonta. (After Griste.)

I. Section of blastosphere. $s d$,, shell gland; $c d$, ciliated disc; a., beginning of endodermic invagination. Note mesoderm cells in the cavity.

2. Later stage. $m$. mesoderm.

3. Embryonic shell has appeared.

4. Glochidium larva; note byssus threads, and teeth on shell valves.

"byssus," which moor the larva. If it manage to anchor itself on the tail, fins, or gills of a fish, the Glochidium shuts its valves and fixes itself more securely, and is soon surrounded by a pathological growth of its host's skin.

In this parasitic stage a remarkable metamorphosis occurs. The sensory or tactile patches not unnaturally disappear; the byssus and the embryonic byssus glands vanish, but a new byssus glanel (which remains 
quite rudimentary in Anodonta) appears; the single adductor atrophies and is replaced by two; the foot and the gills make their appearance; the embryonic mantle lobes increase greatly, or are replaced by fresh growths; and the permanent shell begins to be made.

After this metamorphosis, when the larva has virtually become a miniature adult, no longer so liable to be swept away, it drops from its temporary host to the bottom of the pond or river pool.

\section{Classificatron.}

Lamellibranchs are often classified as follows, the emphasis being laid on the adductor muscles :-

Order r. Isomya. Adductor muscles approximately equal.

Sub-order I. Integripallia. The mantle's line of attachment to the shell is not broken by a sinus into which inhalent and exhalent siphons may be retracted, but in most these siphons are present. Arca (Noah's Ark shell), Unio and Anodonta (fresh water), Lucina, Cyprina, Cardium (cockle), Cyclas (fresh water), Tridacna (the largest form).

Sub-order 2. Sinupallia. The mantle's line of attachment to the shell is inflected by a sinus into which the large siphons are retracted. Venzus, Mya, Saxicava (a boring bivalve), Solen (razor shell), Pholas (borer), Teredo (ship worm), Aspergillum (watering pot shell).

Order 2. Hetriomy. The anterior adductor is much smaller than the posterior, and siphons are rare. Mytilus (edible mussel), Modiola (horse mussel), Lithodomus (borer), Dreissena.

Order 3. Moxomya. One adductor, no siphon. Ostrea (oyster), Anomia, Lima, Pecten (scallop).

Pelseneer, however, lays emphasis on the nature of the gills, and classifies as follows:-

Order I. Protobranchia. The gill filaments, arranged in two divergent rows, are not reflected, e.g., Nucula, Solenomya.

Order 2. FILIBRANCHIA. The gill filaments lie parallel and are directed ventrally; they are refected, and united only by ciliated interfilamentar bridges, e.,., Anomia, Arca, Mytilus.

Order 3. Pseudolamilitifranchia. The gill filaments are loosely connected by connective or vascular bridges, e.g., Avicula, Ostrea, Pecten.

Order 4. Eulamellibranchia. The gill filaments are bound into plates, as in Anodon, Cardium, Mya, Venus.

Order 5. SEPTIBRANCHIA. The gills form a remarkable muscular septum extending round the foot from the anterior adductor to the separation of the two siphons. Poromya and Cuspidaria.

\section{General Notes on Lamellibranchs.}

The organs which most frequently vary in other bivalves, as compared with Anotonta, are the foot, the gills, the adductor muscles, and the mantle skirt. The foot varies much in size and shape; in Arca it has a flat sole-like surface which suggests the creeping foot of Gastero- 
pods ; the pedal gland of Gasteropods is often represented by a "byssus" gland, which secretes attaching threads, well seen in the edible mussel (Mytilus). In oysters the foot is absent. The gills show an interesting series of gradations, from a slight interlocking of separate gill filaments to the formation, by complicated processes of "concrescence," of platelike structures such as those of Anodonta. These processes are, however, much more closely related to the method of nutrition than of respiration, which, indeed, is probably largely performed by the mantle skirt. The mantle skirt is often united to a greater or less extent inferiorly, and is often prolonged and specialised posteriorly to form exhalent and inhalent "siphons." These siphons sometimes attain a considerable length ; they occur especially in forms, such as $M y a$, which live buried in sand or mud, or which burrow in wood or stone, e.g., Pholas. The variations of the adductor muscles afford one basis for classification.

We may associate with the sluggish habits and sedentary life of bivalves (I) the undeveloped state of the head region; (2) the largeness of the plate-like gills which waft food particles to the mouth; and $(3)$ the thick limy shells. We may reasonably associate these and other facts of structure (e.g., the absence of head eyes, biting or rasping organs) with the conditions of life, without being able to say very precisely what the relation is. It seems to some not improbable that sluggish habits have cumulative and manifold results in the course of generations; that structural changes produced by use and disuse of parts may have constitutional consequences which affect the germ cells, that is to say, the offspring. To others the adaptations seem to be most readily explainer as the result of the natural selection of indefinite germinal variations, which arise altogether apart from function or environment. In thinking about the sluggishness of most bivalves, we must not forget that the larval trochospheres and veligers are very active, perhaps almost too active, young creatures.

Habit. - Most bivalves, as every one knows, live in the sea, and their range extends from the sand of the shore to great depths. They occur in all parts of the world, though only a few forms like the edible mussel (Mytilus edulis) can be called cosmopolitan. Some, such as oysters, can be accustomed to brackish water. The fresh water forms may have found that habitat in two ways- $(a)$ a few may have crept slowly up from estuary to river, from river to lake; Dreissena polymorpha has been carried on the bottom of ships from the Black Sea to the rivers and canals of Northern Europe; and it is likely that aquatic birds have assisted in distributing little bivalves like Cyclas; $(b)$ on the other hand, it is more probable that the fresh water mussels (Unio, Anodonta, \&c.), are relics of a fauna which inhabited former inland seas, of which some lakes are the freshened residues.

Between the active Lima and Pecten, which swim by moving their shell valves and mantle flaps, and the entirely quiescent oyster, which has virtually no foot, there are many degrees of passivity, but most incline towards the oyster's habit. Of course, there is much internal activity, especially of ciliated cells, even in the most obviously sluggish. The cockle (Cardium) uses its bent foot to take small jumps on the sand; the razor fish (Solen) not only bores in the sand, but may swim backwards by squirting out water from within the mantle cavity; many 
(e.g., Teredo, Pholas, Lithodomus, Xylophaga), bore holes in stone or wood, but we do not certainly know how; in the great majority the foot is used for slow creeping motion.

The food consists of Diatoms and other Algæ, Infusorians and other Protozoa, minute Crustaceans and organic particles, which the ciliary action of the gills carries from the posterior end of the shell to the mouth. The bivalves are themselves eaten by worms, star fishes, gasteropods, fishes, birds, and even mammals.

Life History'- - The cggs are sometimes laid in the water, either freely or in attached capsules, or, more frequently, they are fertilised by spermatozoa drawn in with the inhalent water, and are subsequently sheltered within the body during part of the development. In the Unionidae the embryos are retained within the cavities of the outer gills; in Cyclas and Pisidizm there are special brood chambers at the base of the gills. In Cyclas the embryos are nourished by the maternal epithelial cells. Segmentation is always unequal; a gastrula may be formed by invagination or by overgrowth, the two cases being connected by a series of gradations. A trochosphere stage is more or less clearly indicated, being most obvious in cases where the eggs are laid in the water. The free swimming trochosphere becomes a veliger, and this is modified into the adult. The fresh water forms, with the exception of Dreissena polymorpha in which the habit is recently acquired, do not possess free swimming larvae; this must be regarded as an adaptation.

Past History of Bivalves. - Even in Cambrian rocks, which we may call the second olclest, a few bivalves have been discovered; in the Upper Silurian they becone abundant, and never fall off in numbers. About gooo extinct and 5000 living species were catalogued some years ago, so that we evidently have our full share now. Those with one closing muscle to the shell seem to have appeared after those which have two such muscles. Those which, from the shell markings, seem to have had an extension of the mantle into a protrusible tube or siphon, were also of later origin. The present fresh water forms were late of appearing. Of all the fossil forms the most remarkable are large twisted shells, called Hippurites (Rudistre), whose remains are often very abundant in deposits of the chalk period.

\section{Class V.-Cephalopoda. Cuttlefish. Examples:-Sepia, Octopus, Loligo, Nautilus.}

The Cephalopods are bilaterally symmetrical free suimming Molluscs. Part of the foot has come to surround the head, and is divided into numerous "arms" bearing tentacles or suckers. Another part forms a partial or complete tube-the "siphon" or "funnel" - through which water is forcibly expelled from the mantle cavity, driving the animal backwards. The muscular mantle flap which shelters the gills is posterior in position; the visceral hump shows no trace of spiral coiling, lut is elongated in a direction anatomically dorsal and posterior, 
though it may point forwards when the animal propels itself through the water. Except in the Pearly Nautilus, the shell of modern forms has been enclosed by the mantle, and is, in most cases, only hinted at. There is a very distinct head region, furnished zith eyes and other sensitive structures, and the mouth has strong beak-like jaws, as also a well developed radula. The nervous system shows considerable specialisation, and the chief ganglia are concentrated in the head. The true body cavity, pericardium of other Molluscs, is usually well developed, and frequently surrounds the chief organs. Except in Nautilus, it communicates with the exterior by the nephridia.

The vascular system is well developed, and, except in Nautilus, there are accessory branchial hearts. The sexes are separate. Development is direct. In habit, Cephalopods are predominantly active and predatory; in diet, carnivorous.

\section{TYPES OF CEPHALOPODA.}

First Type. The Common Cutrlefisrr (Sepia officinalis). Mode of Life.

This common cuttlefish is widely distributed, especially in warmer seas like the Mediterranean. Unlike the Octopus, which usually lurks passively, the Sepin is an active swimmer; it moves head foremost by working the fins which fringe the body, or it jerks itself energetically backwards by the outgush of water through the funnel. It likes the light, and is sometimes attracted by lanterns. The beautiful colours change according to external conditions and internal emotions; and a plentiful discharge of ink often covers its retreat from an enemy. Its food includes fish, other molluscs, and crabs. In spring the female attaches her encapsuled eggs to sea weeds and other objects, and often comes fatally near the shore in so doing. The "cuttles" are caught for food and bait. The "cuttle bone" and the pigment of the ink bag are sometimes utilised by man.

\section{External Appearance.}

A large Sepia measures about Io inches in length and 4 to 5 in breadth; the body, fringed by a fin, is shaped like a shield, the broad end of which bears a narrower head, 
with eight short and two long sucker-bearing arms. Besides the diffuse pigment cells, there are bands across the "back." The large eyes, the parrot-beak-like jaws protruding from the mouth, the spout-like funnel on the neck, and the mantle cavity are conspicuious.

The true orientation of the different regions in Sepia is not obvious. If the "arms" surrounding the mouth be divided portions of the anterior part of the "foot," the ventral surface is that on which the animal rests when we make it stand on its head. W!e can fancy how the "foot" of a snail might grow forward and surround the mouth, so as to bring that into the middle of the sole. Then the visceral mass has been elongated in an oblique dorso-posterior direction, so that the tip of the shield, directed forward when the cuttle jerks itself away from us, represents in anatomical strictness the dorsal surface tilted backwards. (As above noticed, the animal may also swim with foot and mouth in front.) The side of lighter colour, marked by the mantle cavity and the siphon or funnel, is posterior and slightly ventral ; the banded and more convex side on which the cerebral ganglia lie in the head region, and on which the shell lies concealed in the visceral region, is anterior and slightly dorsal.

\section{The Skin.}

The skin contains numerous actively changeful pigment cells or chromatophores lying in the connective tissue beneath the epidermis. Each cell is expanded by the contraction of fine muscle fibres which radiate from it, and contracts when the fibres relax. It is probable that these chromatophore cells have some protoplasmic spontaneity of their own, but the controlling fibres seem to be affected by nervous impulses from the central ganglia. As the cells dilate or contract the pigment is diffused or concentrated, and the colours change. The animal's beauty is further enhanced by numerous "iridocysts" or modified connective tissue cells, with fine markings which cause iridescence.

\section{Muscular System.}

The cuttlefish is very muscular, notably about the arms, the mantle flap, and the jaws. With great quickness it 
seizes its prey by throwing out its two long arms, which are often entirely retracted within pouches. With great force it jerks itself backwards by contracting the mantle cavity, and making the water gush out through the pedal funnel. This mode of locomotion is very quaint. At one time the mantle cavity is wide, and you can thrust your fingers into its gape; when about to contract, this gape is closed by a strange double hook and eye arrangement ; contraction occurs, and the water, no longer free to leave as it entered, gushes out by the funnel, the base of which is within the mantle cavity. Another muscular development is interesting, that of the suckers on the arms. 'They are muscular cups, borne on little stalks (unstalked in Octopus, $\xi_{0} 0$.), well innervated, and able to grip with a tenacity which in the giant cuttlefishes is dangerous even to men. The inner edge of the cup margin is supported by a chitinoid ring bearing small teeth. Each cup acts as a sucker, in a fashion which has many analogues, for a retractor muscle increases the size of the cavity after the margin has been applied to some object. The external pressure is then greater than that within the cup, and the little teeth keep the attachment from slipping.

It seems likely that the arms represent a propodium, and the siphon a mesopodium, and a valve within the siphon has been compared to a metapodium.

\section{Skeletal System.}

An internal skeleton is represented by supporting cartilaginous plates in various parts of the body, especially $(a)$ in the head, round about the brain, arching over the eyes, enclosing the "ears"; $(b)$ at the bases of the arms; $(c)$ as a crescent on the neck; $(d)$ at the hook and eye arrangement of the mantle flap; (e) along the fringing fins. Ramified "stellate" cells lie in the structureless transparent matrix of the cartilage.

On the shore one often finds the "cuttle bone" or sepiostaire, which is sometimes given to cage birds to peck at for lime, or used for polishing and other purposes. It lies on the dorsal side of the animal, covered over by the mantle sac. In outline it is somewhat ellipsoidal, thinned at the edges like a flint axe head, and with curved markings which indicate lines of growth. In the very young Sepia, it consists 
wholly of the organic basis conchiolin, but to this lime is added from the walls of the sac. Between the plates of lime there is gas, and though the structure may give the cuttle some stability, it is probably of more use as a float.

\section{Internal Appearance.}

When we cut open the mantle flap and fold the halves back, we at once see the two plume-like gills, and the lower end of the siphon. The dark outline of the ink bag followed along towards the head leads our eyes to the end of the food canal. Near this are the external apertures of the two kidneys and of the genital duct. On each side of the base of the funnel lies a very large and unmistakable "stellate" ganglion. Removing the skin as carefully as possible over the whole visceral region between the gills, and taking precautions not to burst the ink sac, we see the median heart, the saccular kidneys, contractile structures or branchial hearts at the base of each gill, and the essential reproductive organs near the apex of the visceral mass. Disturbing the arrangement of these organs, we can follow the food canal with its stomach, digestive gland, \&c.

\section{Nervous System.}

Three pairs of ganglia surround the gullet,-cerebral on the dorsal and anterior side, pedal and pleuro-visceral on the ventral and posterior side (Fig. I I 8).

The cerebral ganglia are three lobed, and are connected anteriorly by two commissures with a "supra-pharyngeal" ganglion, which gives off nerves to the mouth and lips, and is connected also with an "infrapharnygeal" ganglion. The cerebral ganglia are also connected by short clouble commissures, with the pedals and pleuro-viscerals on the ventral side of the gullet.

The following chief nerves are given off from the central system :-

(I) The very thick optic nerves are given off from the commissures between cerebrals and pleuro-viscerals, and lead to a large optic ganglion at the base of each eye.

(2) Ten nerves to the "arms" are given off by the pedal ganglion, and this is one of the reasons which have led most morphologists to regard these arms as portions of the "foot."

(3) Two large nerves from the more ventral portion of the pleurovisceral ganglia form a visceral loop, and give off many branches to the gills and other organs. From the pleural portion arise two mantle nerves, each of which ends in a large stellate ganglion. 
Sense Organs. - The eyes are large and efficient. They present a striking resemblance to those of Vertebrates, and, as they are not "brain eyes," they illustrate how superficially similar structures may be developed in different ways and in clivergent groups. In cuttlefishes, the eyes lie on the sides of the head, protected in part by the cartilage surrounding the brain, and in part by cartilages on their own walls.

The eye is a sensitive cup arising in great part from the skin. Its internal lining is a complex retina, on the posterior surface of which the nerves from the optic ganglion are disiributed. In the carity of the cup there is a clear vitreous humour.

The mouth of the cup is closed by a lens, supported by a "ciliary body." The lens seems to be formed in two parts-an outer and an inner plano-convex lens. The pupil or hole in front of the lens is fringed by a contractile iris.

The onter wall of the optic cup is ensheathed by a strong supporting layer-the sclerotic, which is in part strengthened by cartilage, covered by a silvery membrane, and continued into the iris.

In front of the eye there is a transparent cornea, and the skin also forms protecting lids.

Round about the optic ganglion there is a strange "white body," which seems to be a fatty cushion on which the eye rests.

The two ear sacs, containing a spherical otolith and a fluid, sometimes with calcareous particles, are enclosed in part of the head cartilage, close by the pedal ganglia. The nerves seem to come from the pedals, but it is said that their fibres can be traced up to the cerebrals.

A ciliated "olfactory sac" lies behind each eye, and is innervated from a special ganglion near the optic. There are no osphradia of the usual type.

Finally, there are tactile or otherwise sensitive cells on varions parts of the body, especially about the arms.

\section{Alimentary System.}

The cuttlefish eats food which requires tearing and chewing, and this is effected by the chitinous jaws worked by strong muscles, and by the toothed radula moving on a muscular cushion. The mouth lies in the midst of the arms, bordered by a circular lip. Through the ganglionic mass passes the narrow gullet, which leads into the globular stomach, lying near the dorsal end of the body. The stomach is followed by a cæcum or pyloric sac, and the intestine curves headwards again to end far forward in the mantle cavity. There do not seem to be any glands on the walls of the food canal, the stomach has a hard cuticle, the digestion which takes place there must therefore be due to the digestive juices of the glandular appendages. Of these the most important is usually called the liver; it is bilobed, and lies in front of the stomach attached to the osophagus. 
Its two ducts conduct the digestive juice to the region where stomach, "pyloric sac," and intestine meet, and these ducts are fringed by numerous vascular and glandular appendages which are called "pancreatic," though in reality formed as an outgrowth of the nephridia. Far forward, in front of the large digestive gland, lie two small white glands on each side of the gullet. Their ducts open into the mouth, and their secretion contains a diastatic ferment. At the other end of the food canal, the ink sac full of black pigment, probably of the nature of waste products, opens into the rectum close to the anus. This ink sac may be called a much enlarged anal gland, for while most of the bag is made of connective tissue and some muscle fibres, a distinct gland is constricted off at the closed end, and the neck is also glandular.

\section{Vascular System.}

The blood of Sepia is bluish, owing to the presence of hæmocyanin in the serum; the blood cells are colourless and amœboid. The median but somewhat oblique ventricle of the heart drives the

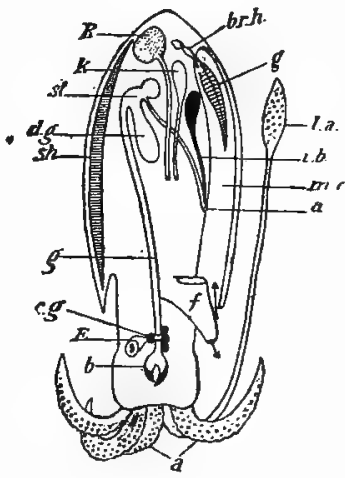

FIG. I21.-Diagram of the structure of Sepia. (Mainly after Pelseneler.)

a., Eight short arms around mouth ; l. $a$., one of the two long arms; $b_{.}$, beak of the mouth; c.g., cerebral ganglia with commissures to the others; $E$., eye; $g_{*}$, gullet ; $d_{. g} .$, digestive gland; st., stomach; $a$., anus ; sh., shell sac with sepiostaire; $k$, kidney; $R$., Reproductive organ; br.h., branchial heart; g., a gill ; $i . b$., ink bag; $w_{\text {.c. }}$, mantle cavity; $f$, funnel. blood forward and backward to all parts of the body. It reaches the tissues by capillaries, and apparently also by lacunar spaces. The venous blood of the head region is collected in an annular sinus round the basis of the arms, and passes towards the heart by a large vena cava which divides into two branchial veins, covered by spongy outgrowths of the nephridia. Joined by other vessels from the apical region of the viscera, each branchial vein enters a "branchial heart" at the base of each gill. The 
branchial heart is contractile, and drives the venous blood through the gills, whence purified it returns by two contractile auricles into the ventricle. There are valves preventing back flow from the ventricle to the auricles, or from the arteries to the ventricle. Beside each branchial heart lies an enigmatical glandular structure known as a "pericardial gland," possibly an excretory or incipiently excretory organ. The course of the blood differs from that in the mussel and snail in this, that none returns to the heart except from the respiratory organs. In the nephridial outgrowths around the branchial veins the interesting parasite Dicyema is found.

\section{Respiratory System.}

The blood is purified by being exposed on the two feather-like gills which are attached within the waterwashed mantle cavity.

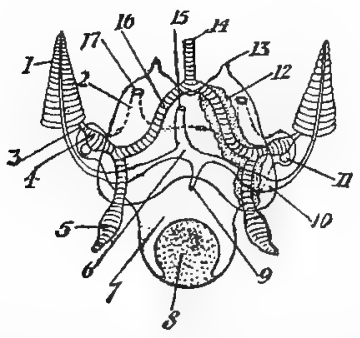

FIG. I22. - Dingram of circulatory and excretory systems in a Decapodlike Sepia. (After Pelseneer.)

I, Gill ; 2, renal sac ; 3, afferent branchial vessel; 4, branchial heart; 5 , abdominal vein; 6 , heart; 7 , pericardium ; 8 , genital organ ; 9 , posterior aorta ; ro, "auricle"; II, glandular appendage of branchial heart; 12, renal appendages of branchial vein ; 13 , external aperture of kidney; 14 , vena cava; 15 , anterior aorta: 16 , bifurcation of vena cava; 1 , reno-pericardial aperture.

The water penetrates them very thoroughly; the course of the blood is intricate. At the base of the gills there is some glandular tissue, which those impatient with enigmas have credited with blood making powers.

\section{Excretory System.}

The excretory system is difficult to dissect and to explain. On each side of the anus there is a little papilla through which uric acid and other waste products ooze out into the mantle cavity, and so into the water. A. bristle inserted into either of these two papillæ leads into a large sac-the nephridial sac. But the two sacs are united by two bridges, and they give off an unpaired dorsal elongation, which extends as far back 25 the reproductive organs.

The dorsal wall of each nephridial sac becomes intimately associated with the branchial veins, and follows their outlines faithfully. It is likely that waste material passes from the blood through the spongy appendages into the nephridial sacs. 
Into the terminal portion of each nephridial sac, a little below its aperture at the urinary papilla, there opens by a ciliated funnel another sac, which is virtually the body cavity. It surrounds the heart and other organs, and is often called the viscero-pericardial cavity. Through the kidneys or nephridial sacs it is in communication with the exterior.

\section{Reproductive System.}

The sexes are separate, but there is not much external difference between them, though the males are usually smaller, less rounded dorsally, and with slightly longer arms. When mature the male is easily known by a strange modification on his fifth left arm. The essential reproductive organs are unpaired, and lie in the body cavity towards the apex of the visceral mass.

The testis-an oval yellowish organ-lies freely in a peritoneal sac near the apex of the visceral mass. From this sac, the spermatozoa pass along a closely twisted duct-the vas cleferens. This expands into a twofold " seminal vesicle," and gives off two blind outgrowths, of which one is called the "prostate." The physiological interest of these parts is that within them the spermatozoa begin to be arranged in packets. In this form they are found within the next region-the spermatophore sac which opens to the exterior to the left of the anus. Each spermatophore is like a transparent worm of complex structure. Think of a little glass tube, closed at one end, drawn out and somewhat twisted at the other; see within the tube at the closed end a bag of dust attached to and kept in its place by a sort of spiral spring; this is prevented from expanding by the fact that its upper end is fixed by cement in the mouth of the tube. Suppose the cement be soluble in water, and that the tiny machine be thrown into a basin, the spring will expand violently as the cement is dissolved, and the bag of dust will be torn out and scattered. Somewhat similar but more complex is the spermatophore-with its clear case, its contained bag of spermatozoa, its spring-like arrangement, and its explosiveness in water. Even, indeed, on your scalpel, or on a dry slide, these strange but efficient bombs will explode. The liberated spermatozoa are of the usual sort.

The ovary-a large rounded white organ-lies freely in a peritoneal sac near the apex of the visceral mass. From this sac the eggs pass along a short direct oviduct, which opens into the mantle cavity to the left of the anus. Associated with the oviduct, and pouring viscid secretion into it, are two large " nidamental glands," of foliated structure. Close beside these are accessory glands, of a reddish or yellowish colour, with a median and two lateral lobes; while at the very end of the oviduct are two more glands. All seem to contribute to the external equipment of the egg.

The spermatophores pass from the genital duct of the male to the fifth left arm, which becomes covered with them and quaintly modified. This is usual among cuttlefish, indeed in some, e.g., Argonauta and Tremoctopus, the modified arm with its, load of spermatozoa is discharged 
bodily into the mantle cavity of the female. There its discoverers described it as a parasitic worm "Hectocotylus." The lost arm is afterwards regenerated. In Sepia, however, the modified arm is not discharged, but is simply thrust into the mantle cavity of the female. The spermatophores probably enter the oviduct and burst there.

The eggs when laid are enclosed within separate black capsules containing gelatinous stuff, but the stalks of the capsule are united so that a bunch of "sea grapes" results.

Second Type of Cephalopoda. The Pearly Nautilus (Nautilus pompilius).

The shells of the pearly nautilus are common on the shores of warm seas, but the animals are very rare. Naturalists do not seem to know how to get them, though the natives of Fiji and New Hebrides, who appreciate their flesh, trap them successfully in lobster pots baited with crustacean or sea urchin. The animal creeps or swims gently along the bottom at no great depth, and its appearance on the surface, "floating like a tortoiseshell cat," is probably the result of storms. It is called "pearly" on account of the appearance of the innermost layer of the shell. This is exposed after the soft organic stratum and the median layer which bears bands of colour have been worn away, or dissolved in a dolphin's stomach, or artificially treated with acid.

'The beautiful shell is a spiral in one plane, divided into a set of chambers, in the last of which the animal lives, while the others contain gas. The young creature inhabits a tiny shell curved like a horn; it grows too big for this, and proceeds to enlarge its dwelling, meanwhile drawing itself forward in the older part, and forming a door of lime behind it. This process is repeated again and again; as an addition is made in front, the animal draws itself forward a little, and shuts off a part of the chamber in which it has been living. The compartments seen on a divided shell are not exactly successive chambers, they are fractions of successive chambers abandoned and partitioned off as more space was gained in front. Moreover, all the compartments are in communication by a median tube of skin-the siphuncle-which is in part calcareous.

It has been suggested, that "each septum shutting off an air-containing chamber is formed during a period of quies- 
cence, probably after the reproductive act, when the visceral mass of the Nautilus may be slightly shrunk, and gas is secreted from the dorsal integument so as to fill up the space previously occupied by the animal."

The only other living Cephalopod which has a shell like that of the Nautilus is Spirula. In this form the shell is again chambered and spirally coiled in one plane. But it is without a siphuncle, and lies enveloped by folds of the mantle.

There can be no confusion between the beautiful shell of the cuttlefish called the paper Nautilus (Argonauta argo) and that of our type. For it is only the female Argonaut which bears a shell, it is not chambered, and is a shelter for the eggs-a cradle, not a house. It is usually stated to be formed by two of the arms, but it seems doubtful whether it is not in reality due to the activity of the mantle.

It is instructive also to compare the Nautilus shell with that of some Gasteropods, for there also chambers may be formed. But these arise from secondary alterations of an originally continuous spiral, and the resemblance is never very striking. The fresh water snail

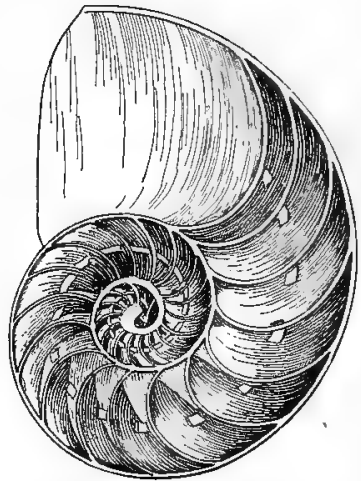

FItr. I23. - Section of shell of Nautilus. (After LENDENFELI).)

Planorbis has an unchambered shell spirally coiled in one plane, but in this and in similar Gasteropods, the foot is turned towards the internal curve of the coil, while that of Nautilus is directed externally.

There are only about half a dozen living species of Nautilus, but there are many hundred fossils of this and allied genera. This list is usually swelled by the addition of the extinct Ammonites, but there are some reasons for believing that these belong to the cuttlefish section of Cephalopods.

The following table states the chief points of distinction between Nautilus and the other series of Cephalopods. 


\section{Cephalopoda.}

\begin{tabular}{l} 
Tetrabranchiata (Nautilus). \\
\hline All extinct except one genus- \\
Nautilus; the extinct forms are \\
usually ranked as Nautiloid and \\
Ammonoid. \\
Shell external, chambered, straight \\
or bent or spirally coiled. That in \\
which Nautilus lives has been \\
described, with its siphuncle, gas- \\
containing compartments, \&c.
\end{tabular}

The part of the foot surrounding the mouth bears a large number of lobes, which carry tentacles in little sheaths, but no suckers.

The two mid lobes of the foot form a siphon, but they are not fused into a tube.

The eye is without a lens, and is bathed internally by sea water which enters by a small pinhole aperture. There are two "osphradia" or smelling patches at the bases of the gills.

Two pairs of gills; two pairs of nephridia; two genital ducts (the left rudimentary).

The cœlome sac opens directly to the exterior by two apertures.

The heart has two pairs of auricles, and there are no branchial hearts. No ink bag. No salivary glands.
Dibranchiata (Sepia, Octopus, Evc.).

Numerous living genera, ranked as Decapods or Octopods; along with the former the extinct Belemnites are included.

No living Dibranchiate lives in a shell. The shell is internal even in the extinct Belemnites, and in modern forms it occurs in various degrees of degeneration (cf. Spirula, Sepia, Loligo) or is quite absent (Octopoda).

The part of the foot surrounding the mouth is divided into ten or eight arms, which carry suckers, stalked in Decapods, sessile in Octopods.

The two mid lobes of the foot fuse to form a completely closed tubular siphon or funnel.

The covering of the eye may be perforated, but the mouth of the retinal cup is closed by a lens. There are no osphradia, though there may be "olfactory pits" behind the eyes.

One pair of gills; one pair of nephridial sacs ; two oviducts in Octopoda and Ommastrephes; two vasa deferentia in Electone moschata; in others an unpaired genital duct.

The colome opens in to the nephridia. by two pores, and thus to the exterior.

The heart has two auricles, and there are branchial hearts.

An ink bag and salivary glands.

\section{Classification of Cephalopoda.}

Order I. Tetrabranchiata (see table).

Family I. Nautilidae. Nautilus alone alive; but a great series of fossil forms, Orthoceras-Trochoceras.

Family II. Ammonitidæ. All extinct, but with shells well preserved, so that long series can be studied. They furnish striking evidence of progressive evolution in definite directions, e.g., Bactrites, Ceratites, Baculites, Turrilites, Heteroceras, and the whole series of genera formerly classed as Ammonites. 
Order II. Dibranchiata (see table).

Sub-Order Decapoda. Eight shorter and two longer arms. Suckers stalked and strengthened by a strong ring. Large eyes with a horizontal lid. Body elongated, with lateral fins. Mantle margin with a cartilaginous "hook and eye" arrangement. Some sort of internal "shell," enclosed by upgrowths of the mantle.

With calcareous internal "shell." Spimula; extinct Belemnites; Sepia.

With organic internal "shell."

(a) Eyes with closed cornea, e.g., Loligo.

(b) Eyes with open comea, e.g., Ommastrephes.

Sub-Order Octopoda. Eight arms only. Suckers sessile without horny ring. Small eyes with sphincter-like lid. Body short and rounded. No "hook and eye" arrangement. No "shell," except in the female Argonauta.

e.g., Octopus, Eledone, Aroonauta.

The classification given above is that usually adopted, but it may be noted that the Ammonites are included in the Tetrabranchiata on insufficient evidence.

The Cephalopods are the most specialised of the Molluscs, and present much variation of type. Nautizus appeared very early and has persisted, apparently unchanged, until the present, while the Ammonites and Belemnites, once so abundant, have entirely disappeared. Among recent forms we have Squid, Calamary, Octopus, Argonaut, and many others. All swim freely in the sea, or lurk and creep passively among the rocks. They are voracious eaters, and consume very diverse kinds of animals, their parrot-like jaws and powerful odontophore, as well as the numerous suckers, rendering them formidable adversaries.

A chambered external shell, serving as a house, is present in Nautilus alone among living Cephalopods. In Spirula, there is a spiral chambered shell, but it is very small, is enclosed by the folds of the mantle, and is quite useless for purposes of protection. Most of the extinct forms were furnished with large and efficient shells of very variable shape, some straight like Orthoceras, or coiled, with chambers separated by complex septa, as in the Ammonites, and so on. Most of the modern forms seem to be more active than their ancestors, and their shells have degenerated. While the fact of the degeneration is perfectly obvious, the line along which it has taken place is difficult and still 
debated. In Nautilus, although the animal lives within the shell, the mantle fold is for some distance reflected over it ; in the other series of Cephalopods, this process has gone further, and, where a shell is present, it is entirely enclosed within the mantle fold, and is at the same time much reduced in size. In the extinct Belemnites the internal shell was straight and chambered, but almost concealed by secondary deposits of lime, secreted by the walls of the shell sac. In Sepia, according to one view, the central laminated region of the "bone" represents the remains of the chambered shell ; the remainder corresponds to the secondary deposits of lime in the Belemnites. In Loligo, there is no deposit of lime, an organic chitinous pen only being left. In Octopus, there is no trace of shell at all. According to some, the shell sac, in which the shell or pen of Cephalopods is formed, is to be regarded as equivalent to the embryonic shell sac plus a mantle pocket. According to Ray Lankester, there is no relation between the secondary shell sac and the typical primitive Molluscan shell gland.

The sexes in Cephalopods are separate. The male elements are made up into packets or spermatophores, which usually pass out on to one of the "arms," more or less modified for copulatory purposes. The eggs are large, and often surrounded by capsules.

The eggs are always furnished with a large amount of yolk. In consequence, development is much modified as compared with other Molluscs, trochosphere and veliger stages not being recognisable.

\section{General Notes on Molluscs.}

The characters of the widely spread trochosphere larva, and many of the features of the simple Amphineura, suggest that Molluscs arose from some worm type, but beyond this all is hypothesis. Some of the Amphineura, both in the form of the body and in the arrangement of bristles on its surface, recall Annelids, but this can hardly be regarded as an evidence of affinity, for it is extremely improbable that such typically unsegmented animals can have arisen from segmented worms. It is perhaps more likely that Molluscs arose from a Turbellarian- 
like ancestor, an unsegmented form with a flat creeping ventral surface.

It is certain, however, that the great Mollusc branch must have divided at a very early stage into two. One branch bears those forms which live sluggishly, and have undeveloped heads and no odontophore-the bivalves or Lamellibranchs. The other branch bears more active forms, in which the head is well developed, and the characteristic radula is present in the mouth - the primitive Amphineura, the Gasteropods or snails, and the Cephalopods or cuttles.

Phylum Mollusca.

Branch Glossophora
(Syn. Odontophora).

Branch Lipocephata

(Syn. Acephala).

The position of the small class Scaphopoda is uncertain. Some place it between Gasteropods and Cephalopods.

Most Molluscs live in the sea from the shore to the great depths, but there are many fresh water Gasteropods and bivalves, and the terrestrial snails and slugs are legion.

The bivalves feed on microscopic animals and organic debris ; the Gasteropods are carnivorous or vegetarian; the Cephalopods are voracious flesh eaters.

The alimentary canal and its associated digestive gland often seem as if they were too big for the body; in bivalves, the gut tends to be displaced ventrally and coils about in the foot; in the others, it tends to be displaced dorsally, often protruding on the back as a visceral hump.

The vascular system in Molluscs always communicates freely with the lacunar spaces which constitute the apparent body cavity. These are never lined with epithelium, and are of secondary origin. A true epithelium, however, lines the pericardium and the cavity of the reproductive organs, which are both colomic in origin. 
The shell is a very characteristic molluscan structure, but in spite of all the years of conchology, we cannot answer the fundamental questions about shell making. Mollusc shells are very beautiful things alike in form and colour. They grow larger by month and year, and mark their progress by rings of growth and changing tints. That they afford their bearers efficient protection, is shown by the appreciation which some hermit crabs exhibit for stolen whelk or buckie shells. More precise observation shows us that the shell consists in great part of carbonate of lime; that it has a thin outer "horny" layer, a thick median "prismatic" stratum of lime, and an internal mother-ofpearl layer. This last exhibits iridescence, produced by the fine lines which mark successive deposits. The markings, fine as they are, were repeated, according to Brewster's famous experiments, on a piece of wax, which in consequence became iridescent. On the dorsal surface of almost every mollusc embryo, there is a little shell sac in which an embryonic shell is begun; the adult shell, however, begins on a separate area on the skin, and it is always lined and increased by the mantle. If the increase of the shell be carefully watched on young Molluscs, or if chemical analysis be made, it becomes plain that the shell is no mere deposition of carbonate of lime. Like other cuticular products, it has an organic basis (called conchiolin), along with which, in a manner that we do not clearly understand, the lime is associated.

Mr. Irvine's experiments at Granton Marine Station suggest that the lime salt originally absorbed is not the carbonate (of which there is a scant supply in sea water), but the sulphate (which is abundant), and that the internal transformation from sulphate to carbonate is perhaps associated with the diffuse decomposition of nitrogenous waste products. Thus, carbonate of ammonia, which seems to occur abundantly in the mantle of perfectly fresh mussels, would, with calcium sulphate, yield carbonate of lime and ammonium sulphate. I do not suppose that shell making is expressible in a chemical reaction of this simplicity, but it is time that we ceased to think that Molluscs simply absorb carbonate of lime from the sea water, and sweat it out on their skins. It is reasonable to inquire how far shell making 
may express a primitive mode of excretion to which a secondary significance has come to be attached, and in what way carbonate of lime shells are associated with preponderant sluggishness of habit. The thickness of the shell seems often to bear some relation to the external and internal activities of the mollusc, for it is thin in the active scallop (Pecten) and Lima, thick in the passive oyster and Tridacna, slight or absent in the pelagic Pteropods ("sea butterflies"), and in the more or less active cuttlefish, but heavy in most of the slowly creeping littoral forms. But that this is only one condition of shell development is evident in many ways, -for instance when we compare land snails with slugs; for the latter, though not more active than the former, are practically shell-less. In most cases, as Lang points out, the loss of the shell is justified by increased power of locomotion, by increased adaptation to peculiar habits of life, and so forth.

In their life history most Molluscs pass through two larval stages. The first of these is a pear shaped or barrel shaped form, with a curved gut, and with a ring of cilia in front of the mouth. It is a "trochosphere," such as that occurring in the development of many "worms." So far there is nothing characteristic.

Soon, however, the trochosphere grows into a yet more efficiently locomotor form-the veliger. Its head bears a ciliated area or "velum," often produced into retractile lobes ; its body already shows the beginning of "foot" and mantle; on the dorsal surface lies the little embryonic shell gland.

But although trochosphere and veliger occur in the development of most forms, they do not in any of the three types which we have particularly described,-not in Anodonta partly because it is a fresh water animal, with a peculiarly adhesive larva of its own, not in Helix partly because it is terrestrial, and not in Sepia partly because the eggs are rich in yolk.

The hard shells of extinct Molluscs are naturally well preserved in the rocks, and long series of fossil forms have been traced with much success. 


\section{CHAPTER XVII.}

\section{CLASS IEMICHORDA OR ENTEROPNEUSTA.}

\section{Type. Balanoglossus.}

A sPecies of Balanoglossus was described by Delle Chiaje at the end of the eighteenth century, but it is only within the last few years that the researches of Spengel, Bateson, and others have led to an appreciation of the importance of this type, and to a recognition of its peculiar features.

The class (Enteropneusta) which was erected for the reception of Balanoglossus is now known to include a few other forms, whose more or less distinct affinities with Vertebrates are suggested by the alternative title Hemichorda. Taken along with Tunicates and Amphioxus, they afford interesting illustrations of the gradualness with which Vertebrate characters have appeared in history.

General Characters. - The body is divisible into three regions-a pre-oral "proboscis," a "collar" around and behind the mouth, and a trunk, the anterior part of which bears gill siits. $A$ dorsal nerve cord arises from the epiblast along the middle line, and is connected, by a ring round the pharynx, with a ventral cord. In the skin, which is covered with ciliated ectoderm, there is also a nerve plexus. From the anterior region of the gut a diverticulum grows forward for a short distance, becomes a solid support for the proboscis, and is often called the "notochord." The gill slits open dorsally, are very numerous, and increase in number during life; in some details of development they recall those of Amphioxus. The mesoblast is formed by the outgrowth of pouches from the archenteron; i.e., the body cavity is enterocolic. An unpaired anterior pouch forms the pre-oral or proboscis cavity 
of the adult, and is compared to the anterior unpaired body cavity of Amphioxus.

Spengel, in his recent monograph, recognises I9 species and 4 genera - Balanoglossus, Ptychodera, Schizocardium, and Glandiceps. They are very widely, though locally, distributed, except, perhaps, on the Pacific coasts of America.

\section{Description of Balanoglossus.}

\section{Habit.}

The species which form this genus are worm-like marine animals, found in sand and mud in the English Channel, the Mediterranean, Chesapeake Bay, \&c. They vary in length from about an inch to over six inches, and are brightly coloured and of a peculiar odour. The sexes are distinct, and are marked externally by slight differences in colour.

Form.

The worm-like body consists of a prominent pre-oral region or "proboscis," a firm

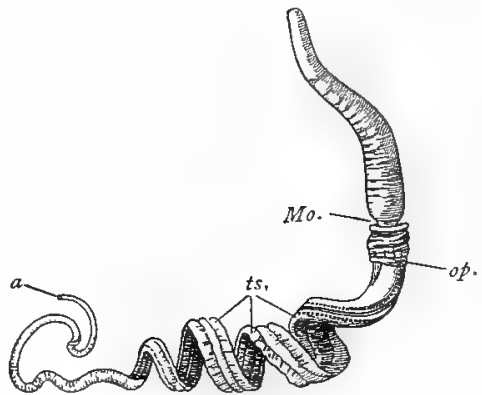

FIG. I24.-Male of Balanoglossus Kowalevskii. (After BATEson.)

Note anterior proboscis; Mo., mouth; op-, slight operculum behind the collar; then the region with gill slits; $t s$, , testes; a., anus.

"collar" behind the mouth, behind this a region with gill slits, and finally, a long, soft, slightly coiled portion.

Skin.

The epidermis is ciliated, and exudes abundant mucus from unicellular glands. In $B$. robinii the mucus sets firmly, and, with the addition of grains of sand, forms a tube round the body. Some species are phosphorescent.

\section{Muscular System.}

The muscular system is best developed about the proboscis and collar, which are used in leisurely locomotion 
through the soft sand. There are external circular and internal radial and longitudinal muscles. The fibres are unstriped.

\section{Nervous System.}

The dorsal nerve cord is most developed in the collar, but is continued along the whole length. It arises as a solid cord of epiblast, which is continued both forwards and backwards as a hollow tube. The cavity is said to be comparable to that of the spinal cord in Vertebrates. But the dorsal nerve cord in the collar is connected by a band round the pharynx with a ventral nerve. There is also a nervous plexus beneath the epidermis. There are no special sense organs, nor should we expect them in an animal which spends most of its life immersed in muddy sand. In the larvæ of some species there are two eye spots.

\section{Alimentary System.}

The mouth opens ventrally between the proboscis and the collar, and is adapted for swallowing the sand moved about by the wriggling proboscis and by the collar. The pharynx is constricted into a dorsal and ventral region, of which the former is respiratory (Fig. I $25, g^{1}$ ), and connected with the exterior by many gill slits, while the latter is nutritive (Fig. I 25, g), and conveys the food particles onwards. This ventral region may be compared with the "ventral groove" in Tunicates, with the "hypobranchial "groove in the lancelet, with a similar region in the lamprey, and even with part of the thyroid gland in higher Vertebrates. Behind the region with gill slits, the gut has a dorsal and a ventral ciliated groove, and bears, throughout the anterior part of its course, numerous glandular sacculations, which can be detected through the skin. The animal eats its way through the sand, and derives its food from the nutritive particles and small organisms therein contained.

\section{Skeletal System.}

The skeletal system is represented by the "notochord," which lies in the proboscis, and arises, like the notochord of indubitable Vertebrates, as a hypoblastic structure from the dorsal wall of the gut. Each gill-slit is furnished with a 
"chitinous" skeleton, which gives the slit a U shape on account of the growth downwards of a "tongue bar," the whole is suggestive of Amphioxus. Beneath the branchial skeleton there lies a "chitinous" rod, which divides into two in the collar.

\section{The Body Cavity.}

The body cavity is somewhat complex, consisting of five distinct parts, all of which are lined by mesoderm, and arise as pouches from the primitive gut or archenteron.

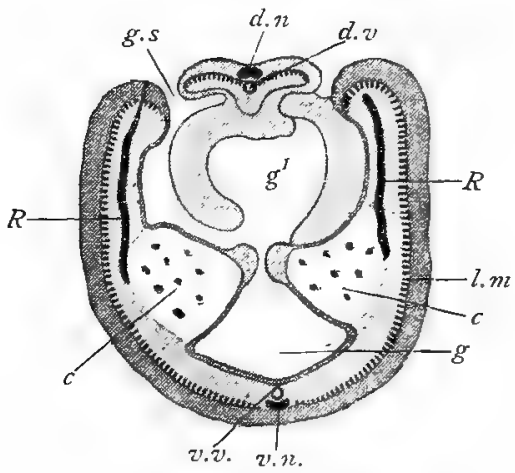

FIG. I25. - Transverse section through gill slit region of Ptychodera minuta. (After SPENGEL.)

The section, somewhat diagrammatic, shows a gill slit $(g . s$.$) to$ left, and a septum between two slits to the right; $d . n$., dorsal

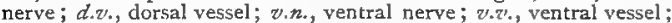
$g_{\text {, }}$ nutritive part of gut; $g^{\prime}$, , respiratory part of gut; $c_{v}$, lateral cœelomic spaces; l.m., longitudinal muscles; $R$., reproductive organs. As the gill slits are oblique, the whole of one could not be seen on a single cross section.

(a) There is first the unpaired cavity of the proboscis, which communicates with the exterior by a dorsal pore (or sometimes by two) at the base of the proboscis next the collar. It is possible that a glandular structure, which lies in front of the heart in the proboscis, may have excretory signifi- 
cance, but it seems to be quite enclosed. (b) In the collar region there are two small paired cœlomic cavities, from which two funnels open to the the exterior. Both these cavities and that of the proboscis tend to be obliterated by growth of connective tissue. (c) Two other cavities extend along the posterior region of the body, to some extent separated by the dorsal and ventral mesentery which moors the intestine. In these there is a body cavity fluid with cells.

\section{Respiratory System.}

The respiratory system consists of many pairs of ciliated gill slits. They open dorsally by small pores behind the collar. In development they begin as a pair, increase in number from in front backwards, and they go on increasing long after the adult structure has been attained. Water passes in by the mouth and out by the gill slits, where it washes branches of the dorsal blood vessels. There are no gill lamellæ associated with the slits.

\section{Vascular System.}

The vascular system includes a main dorsal blood vessel, which, at its anterior end, lies above the notochord; an anterior dilatation, which is sometimes called the "heart ;" a ventral vessel beneath the gut; and numerous smaller vessels. The blood flows forwards dorsally, backwards ventrally. This system should be contrasted with that of Amphioxus.

\section{Excretory System.}

The excretory system is slightly developed. No nephridia are known, but from the region of the collar two ciliated funnels open to the exterior, and we have already mentioned the enigmatical proboscis gland.

\section{Reproductive System.}

The sexes are separate. A number of simple paired genital organs lie dorsally in a series on each side of the body cavity in and behind the region with gill slits (Fig. 125, R). They open by minute dorsal pores in the skin, or in the American species by rupture. 


\section{Development.}

The eggs must be fertilised outside of the body. Segmentation is complete and approximately equal; a blastosphere or blastula results; this is invaginated in the normal fashion, and becomes a two-layered gastrula.

The American species ( $B$. kowalevskii) has a simpler development than the others, for it is without a remarkable larval form (Tornaria) which occurs in them. We shall take the simpler case first, though it is probably less primitive.

The blastopore or mouth of the gastrula narrows and closes; the external surface of the gastrula becomes ciliated;
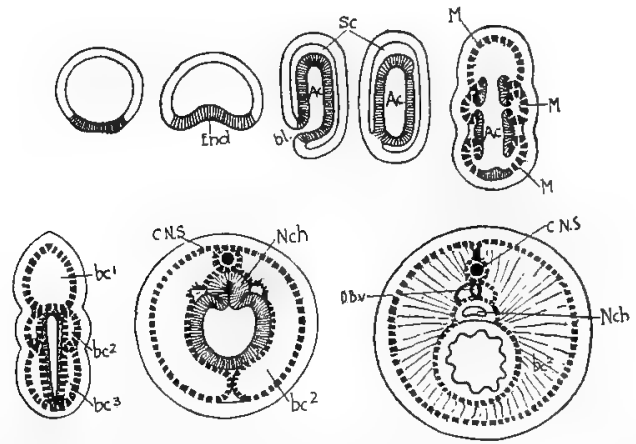

FIG. I26.-Development of Balanoglossus. (After BAtEson.)

The mesoderm is represented by the broken dark line.

In the upper row, from the left-

Section of blastula ; beginning of gastrulation, End, endoderm; section of gastrula, $b l$, blastopore; $A c$, Archenteron; $S c$, segmentation cavity; closure of blastopore, outgrowth of five coelome pouches $(M)$.

In the lower row, from the left-

Longitudinal section, showing the five parts of the body cavity $\left(b c c_{1} b c_{2}, b c_{3}\right.$ ) or coelome.

Cross section, $C N S$, central nervous system; $N c h$, notochord ; $b c 2$, body cavity in collar region.

Section at a later stage, D.b.v*, dorsal blood vessel.

the endoderm lies as an independent closed sac within the ectoderm. Meanwhile the embryo has become or is becoming free from the thin egg envelope, and begins to move about at the bottom in shallow water. It elongates and becomes more worm-like; there is an anterior tuft and a 
posterior ring of cilia; the primitive gut forms five colomic pouches; a mouth and an anus are formed, but there seem to be no fore gut nor hind gut invaginations. The regions of the body are defined at a very early stage.

The Tornaria larva found in other species is at first bell-shaped. A ventral mouth opens into the curved gut, which is furnished with a posterior terminal anus. There are external bands of cilia, something like those of an Echinoderm larva, and also an apical sensory plate (like that of many Annelid trochospheres), with two eye spots. The Tornaria is a pelagic form. During its period of free pelagic life it gradually

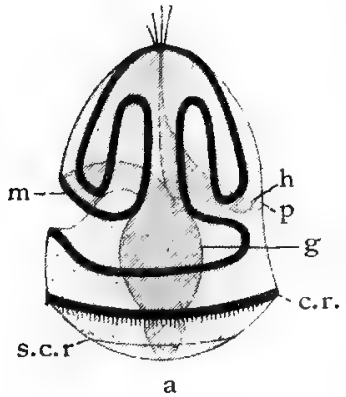

FIG. I27. - Tornaria larva, from the side. (After SPengel.)

$M$, mouth ; $g$, gut ; $a$, anus ; $h$, heart $; p$, pore entering proboscis cavity; c.r, chief ciliated ring; s.c.r, secondary ciliated ring. The dark wavy line indicates the margin of the lobes of the larval body.

loses its distinctive bands of cilia, becomes diffusely ciliated, acquires a proboscis and two gill slits, and thus approaches the form of the larva first described. The further development is the same in both cases. The Tornaria must be regarded as the more primitive larval form; the temporary absence of mouth and anus in the other type is probably an adaptation acquired after the pelagic habit was lost.

Johannes Miiller ranked the Tornaria larva, whose adult form was not then known, beside the larvæ of Echinoderms. The ciliated bands of the Tornaria resemble those of Echinoderm larvæ, but this is only a superficial characteristic. The anterior pouch, which forms the cavity of the proboscis and communicates with the exterior has also been compared with the beginning of the water vascular system in Echinoderms, and it is true that in both several independent colome pouches grow out from the primitive gut. But we might with as much force compare the Tornaria to an Annelid trochosphere, and it may be that it would be most profitable to compare certain features in the development of Balanoglossus with that of the lancelet.

Affinities with Vertebrates (especially emphasised by Mr. Bateson).

(I) "Notochord." -A dorsal outgrowth from the anterior region of the gut grows forward for a short distance into the proboscis and becomes a solid supporting rod (Fig. 126, Nch.). It may be compared with the notochord of Vertebrates, which also arises dorsally from the gut. But it lies below the main dorsal blood vessel, is of very limited extent, and may be merely an analogue of the notochord-a physiological necessity for the support of the elongated proboscis. 
(2)

"Gill slits."-Numerous gill slits (Fig. 124, gs.) open from the anterior region of the gut to the exterior, and are separated from one another by skeletal bars, which in some ways resemble the framework of the respiratory pharynx in Amphioxus. There are, however, many differences in detail, thus the slits open dorsally, not ventrally; the skeletal bars are differently disposed, the blood supply is different. Nor is it certain that the gullet of Balanoglossus is endodermic like that of Vertebrates. Still, the possession of these respiratory slits is one of the most satisfactory of the alleged Vertebrate-like characters of Balanoglossus.

(3) "Dorsal nerve cord."-A dorsal median insinking (Fig. I25, d.n) of ectoderm, especially strong in the region of the collar, may be compared with the medullary canal of Vertebrates. But it must be noticed that there is also a ventral nerve cord (Fig. 125, v.n) which cannot be ignored as subsidiary in character.

Mr. Bateson has also noted that the mesoblast arises, as in $A m p$ hioxus, $\& c$. , in the form of colome pouches, but this is true of many Invertebrates. He states that the history of the anterior colome pocket, which grows forward into the proboscis of Balanoglossus, is closely like that in Amphioxus, but this is denied by Spengel. He compares a slight fold, which grows backwards from in front of the gill slits, with the epipleural folds of Amphioxus (Fig. 124,op.), but the fold appears to be restricted to one species. It is still uncertain what weight should be attached to the fact that Balanoglossus is unsegmented.

Affinities with Annelids (after Prof. Spengel).

(I) The larva (Tornaria) (Fig. I27) may be regarded as a modified Trochosphere, but this points at most to a far-off common stock. Moreover, the nephridia, usually present in the Trochosphere, are unrepresented in the Tornaria.

(2) The body cavity is formed from segmentally arranged coelome pouches; but there is a pair of pre-oral pouches absent in Annelids, and segmental arrangement in the organs of the body in Balarzoglosszes, is, to say the least, very vague.

(3) The heart lies, as in some Annelids, dorsal to the gut, not ventral as in Vertebrates; the dorsal vessel carries blood forwards, the ventral backwards, as is usual in Annelids. But the double nervous system is essentially different from that of Annelids; and the gill slits are also, so far as we know, unrepresented. If there be a relationship between Enteropneusta and Annelids, it must be a very distant one, perhaps restricted to origin from some common stock.

Besides these affinities, others have been ingeniously detected. Those alleged to exist between Enteropneusta and Nemerteans, e.g., the external ciliation, the unsegmented musculature, the correspondence of the "notochord" and the Nemertean proboscis, are even more unsatisfac. 
tory than those above cited. Again, the resemblance between the Tornaria larva and that of Echinoderms is unsatisfactory, because of our relative ignorance of the development of the Tornaria.

Here, then, we have a lesson in uncertainties, for all that we can say is that the Enteropneusta seem to be synthetic, possibly transitional types, exhibiting affinities with various others, but differing markedly from all.

\section{Appendix (I) to Enteropneusta-Cephalodiscus.}

A single species (Cephalodiscus dodecalophus) was dredged by the "Challenger" in the Magellan Straits. It was at first described by $M$ 'Intosh as a divergent Polyzoon, but the researches of Harmer point to relationship with Balanoglosszes.

The minute stalked individuals occur associated together in a gelatinous investment, the colony may attain a size of 9 inches by 6 inches. The gut is curved, the anus being beside the mouth, beneath which are two rows of ciliated hollow tentacles. These two characters, formerly supposed to indicate Polyzoan affinities, may perhaps be adaptations to the sedentary life. With Balanoglosszes this type agrees in the possession of the following characters:- $(a)$ The body is divided into three regions which correspond to the proboscis, collar, and trunk of Balanoglosszes, this is especially obvious in the young bud; $(b)$ each of the three regions contains a colomic cavity, the most anterior being single, while the other two are divided by a median partition; $(c)$ the anterior pre-oral cavity opens to the exterior by two pores (cf. proboscis pores of Balanoglossus); (d) the collar region is also furnished with two collar pores, which open beneath a fold or operculum developed from the collar; (e) in the collar region the dorsal nervous system is also placed, and is continued to some extent into the proboscis; $(f)$ beneath the nervous system lies a diverticulum from the gut, which extends towards the proboscis region ("notochord"); $(g)$ the anterior region of the gut is perforated by a pair of lateral gill slits.

\section{Appendix (2) to Enteropneusta-Rhabdopleura.}

This genus is found at considerable depths in the North Sea. Like Cephalodiscus the individuals are minute and stalked, and occur in a colony; in this case, however, they remain attached to one another by a common stalk instead of being united only by an investment. In the head region there are two hollow lateral arms bearing numerous ciliated tentacles, which have a skeletal support. The gut, as in Cephalodiscus, has a U-shaped curvature, and an anterior diverticulum (" notochord "). There are five colomic cavities, of which the unpaired pre-oral part has two pores. There are no gill slits. The affinities between this type and Balanoglossus must still be held doubtful. 


\section{CHAPTER XVIII.}

\section{CLASS UROCHORDA OR TUNICATA.}

(Ascidians, Sea Seuirts, \&c.)

The Tunicates are remarkable animals, which seem to stumble on the border line between Invertebrates and Vertebrates. They were classified with Polyzoa and Brachiopoda as Molluscoidea, until, in 1866 , Kowalevsky described for the first time the development of a simple Ascidian, and correlated it, step by step, with that of Amphioxus. He showed that the larval Ascidian possesses a dorsal nerve cord, a notochord in the tail region, gill slits opening from the pharynx to the exterior, and an eye developing from the brain. It is true that in most cases the promise of youth is unfulfilled; the active larva settles down to a sedentary life, loses tail and notochord, nerve cord and eye, and becomes strangely deformed. Nevertheless we must now class Tunicates as degenerate Vertebrates. Of their possible relations to simpler forms nothing definite is known.

General Characters.-The Tunicates are marine Chordata, but the chordate characteristics-dorsal nervous system, notochord, gill slits, and brain eye-are in most cases discernible only in the free swimming larval stages. They usually degenerate in adolescence, and the adults, which are in most cases sedentary, tend to diverge very zeidely from the Vertebrate type. Thus the nervous system is generally reduced to a single ganglion. The body is invested by a thickened cuticular tunic, which contains cellulose. The pharynx is perforated by two (Larvacea), or in the majority by numerous ciliated gill slits, and is surrounded to a greater or less extent 
by a peribranchial chamber, which communicates with the exterior ly a special (atrial) opening. The heart is simple and tubular, and there is a periodic reversal in the direction of the blood current. Nephridia are absent, and the renal organs are always devoid of ducts. All are hermaphrodite. There is usually a metamorphosis in development. Colonies are frequently formed.

Though typically sedentary, the Tunicates show considerable variation in habit. Many grow fixed to stones or shells, or to the muddy bottom, and are common on or near the coasts of all seas. They live on minute organisms carried into the pharynx by the water of respiration. Throughout the group we see that antithesis between sessile vegetative forms and active pelagic forms, which is so vividly exhibited in the Coelentera. Some of the free swimming forms are indeed as typically pelagic in structure and habit as the medusie themselves. Of the sessile forms some are simple (e.g., Ascidia) ; others, in which the vegetative habit has more throughly permeated the organism, reproduce themselves freely by budding. The clusters so formed may consist of individuals united only by a common blood system, forming the so-called social Ascidians (e.g., Clavellina), or composite organisms may be formed as in Botrylluss. Again we have allied colonial forms, such as Pyrosoma, the phosphorescent fire flame, which are free swimming and pelagic, the whole colony, with its numerous individuals, moving as one creature. All these individuals are formed by budding from a rudimentary larva which arises from the fertilised egg.

All these types belong to the Ascidian series. Different from them, but connected by Pyrosoma, are the free swimming genera. Salpa and Doliolum, together with the strange abyssal, and probably sessile, "Challenger" genus Octacnemus, Both Salpa and Doliolum exhibit a complex alternation of generations, in the course of which both solitary and aggregated forms occur, the latter, like the floating colonies of Siphonophora, often showing considerable division of labour.

Finally, there are a few active free swimming forms, which retain many of the features of the larval Ascidian. Of these Appendicularia is the simplest type.

Type of Tunicata-a simple Ascidian (Ascidia mentula).

In form, an adult Ascidia is an irregular oval of three to four inches in length; one end is attached to stones or weed; the other is more tapering, and terminates in the mouth, close beside which, on the morphological dorsal surface, lies another opening, the exhalent or atrial aperture. During life, water is constantly being drawn in by the mouth, and passed out by the atrial opening. If irritated, the animal frequently drives a jet of water with considerable force from this aperture, whence the name "sea squirt." 


\section{Test and Skin.}

The whole body is clothed in a thick test or tunic, which can be readily peeled off from the underlying body wall. This tunic contains a carbohydrate allied to, if not identical with, the cellulose of plants, and also some proteid substance. The whole is at first a true cuticle, but cells soon migrate into it, while at one point blood vessels also enter it from the body, and ramify in all directions. The tunic is begun by secretory prolongations of ectoderm cells, and some ectoderm cells pass out into what is secreted. But the tunic also receives important contributions from mesenchyme cells which migrate into it. Some of them probably act as phagocytes in cases of injury or infection. The "Cellulose" or "tunicin" is common throughout the group, and it is interesting to find a characteristically vegetable product in the very passive cuticle of these passive animals. The ectoderm which secretes the tunic is a single layer of cells.

\section{The Muscular System.}

The muscular system forms beneath the epidermis a netted sheath of unstriped fibres, which are very numerous on the right side of the body, and almost absent on the left. Special sphincters surround the apertures.

\section{The Nervous System.}

The nervous system, which in the larva consists of a spinal cord with a slight anterior cerebral swelling, is represented in the degenerate adult only by a ganglionic mass, which lies between the two apertures, and gives off a few nerves.

\section{Sensory Structures.}

Sensory structures in the adult are few and unspecialised. In the larva there is a well developed eye and an auditory organ, both in close connection with the brain. These do not persist in the adult.

Beneath the ganglion in the adult there lies a small (sub-neural) gland from which a ciliated duct opens into the pharynx. According to some, this corresponds morphologically to the pituitary body (see page 436), and so partially to the pre-oral pit of $A$ mphioxzes (q. v.); its physiological 
significarce to the individual is uncertain; perhaps it secretes some of the mucus which entangles the food particles.

Further, it is probable that all the following structures have some sensory function,- the pigment spots between the lobes of the apertures, the tentacle-like processes beneatl the mouth, and other processes (languets) on the dorsal wall of the pharynx (absent in $A$. mentzla).

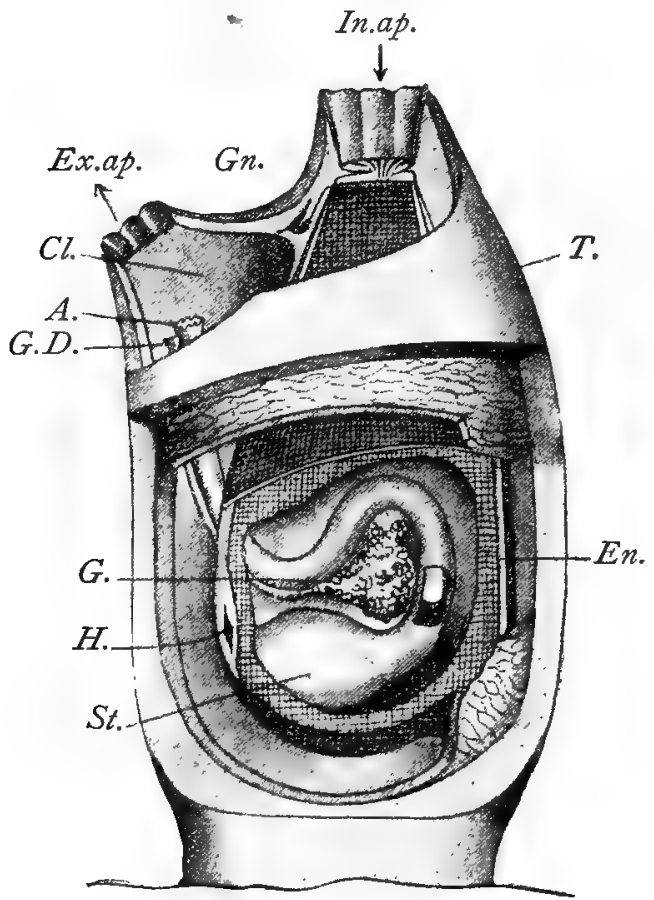

Fig. I28.-Dissection of Ascidian. (After Herdman.)

In.ap., Inhalent aperture; $T$, tunic, cut away below to show

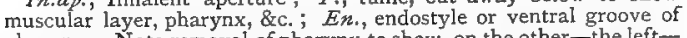
pharynx. Note removal of pharynx to show, on the other-the leftside, stomach (St.), intestine (with fold seen at incision), and reproductive organs (G.) ; $H$., heart; G.D., genital duct ; $A_{\text {., }}$ anus ; $C l$, cloacal chamber; $E_{x . a p .,}$ exhalent aperture; $G n_{.}$, lies above the ganglion which is seen between the two apertures. 


\section{Alimentary System.}

The mouth is surrounded by eight rounded lobes, between which are pigment spots. It leads into a large pharynx, or branchial sac, the walls of which are perforated by numerous gill slits. At the beginning of this pharynx there is a circle of tentacles, which point downwards from the posterior edge of the sphincter muscle. At its base in the dorsal middle line the pharynx opens into a short, curved, ciliated œsophagus, which leads to the large stomach, placed, like the rest of the gut, on the left side of the body. The intestine describes an S-shaped curve, and terminates in a cloacal chamber below the exhalent opening. Its wall is folded inwards as in the earthworm. With the stomach a mass of ramifying tubules is associated, which opens by means of a duct into the cavity of the stomach, and may be a digestive gland.

In feeding, water is drawn into the mouth by the action of the numerous small cilia which border the pharyngeal slits. This water contains small algæ and other organisms. These are glued together by a mucus secretion, and swept backwards to the cesophagus at the base of the pharynx, while the water passes through the slits into the peribranchial chamber. This lies between the body wall and the outer wall of the pharynx, and communicates with the exterior by the exhalent aperture.

On the internal ventral surface of the pharynx (the side on which the ganglion lies being morphologically dorsal) there is a longitudinal groove-the endostyle. This is furnished with very long cilia, and is in part a glandular region. It may secrete part of the mucus mentioned above. On the dorsal surface of the pharynx there is a projecting ciliated fold, called the dorsal lamina. In many Ascidians this is broken up into a series of processes, - the languets. Herdman considers that, as few of the endostyle cells are glandular, much of the mucus must be secreted elsewhere, and the food particles probably pass backwards, both on the ventral and dorsal surfaces.

The ventral endostyle is morphologically comparable to the rentral portion of the respiratory pharynx in Balanoglossus, and to the "hypobranchial groove" of Amphioxus. It may even be compared to the thyroid gland of Vertebrates, for that organ in the Ammoccete larva of 
the lamprey arises as an evagination of the floor of the pharynx, and for a long time, i.e., until the metamorphosis into the lamprey, has a glandular structure opening on the floor of the pharynx, remarkably like the endostyle of the Tunicate Oikopleura.

\section{Respiratory System.}

The water which enters by the mouth, after being deprived of some of its food particles, passes through the gill slits to the peribranchial chamber. On the walls of the pharynx

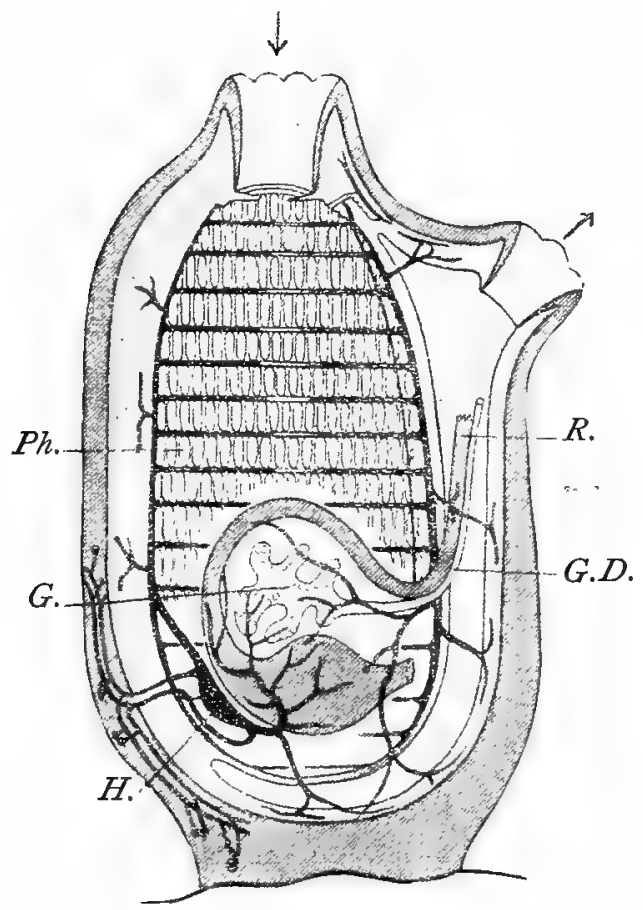

FIG. I29. -Diagram of Ascidian. (After HERDMAN.)

The arrows indicate the two openings, the dark border the tunic. $P h$, pharynx, with gill slits; $G$., reproductive organs; $H$., heart, with blood vessels; G.D., genital ducts; $R$., rectum ending in cloacal chamber. Surround ing the pharynx the peribranchial cavity is shown. 
the blood is spread out in vessels, and is thus aerated. The peribranchial chamber is lined by ectoderm, for it is formed in development by the union of two ectodermic invaginations, which grow towards each other. The first gill slits are formed by the fusion of small diverticula of the pharynx with two separate peribranchial invaginations. In the adult the slits are very numerous and of secondary origin; they are formed partly by the division of primary slits, partly by new perforations of the wall of the pharynx. With regard to the development of all these structures, however, there is as yet little certainty.

\section{Vascular System.}

The simple tubular heart (Fig. I29, H.) lies in a pericardial space at the side of the lower end of the pharynx. In development, two diverticula grow out from the pharynx; these meet and fuse, forming the pericardium. The heart arises as an evagination from its dorsal wall. According to some authorities, the cavities of the heart and of the blood vessels are blastocœlic in origin, i.e., they are said to be derived from the segmentation cavity of the embryo. A periodical reversal of the direction of the waves of contraction is discernible in the heart; for a certain number of beats the blood is driven upwards, and then the direction is reversed. This is said to be, at any rate partially, due to the differences in oxygenation of the blood at the two ends of the heart. This same reversal also occurs in Phoronis.

According to Professor Herdman, the ventro-dorsal contractions occasion the following circulation. The blood, which is spread out on the walls of the pharynx in vessels lying between the slits, collects into one large vessel, which, after receiving a vessel from the tunic, enters the ventral end of the heart. From the dorsal end it is poured into a great trunk, which sends one branch to the tunic and then breaks up among the viscera. From the visceral lacuna the blood is again collected to be distributed to the branchial sac. At the reversal of the contractions this circulation is also reversed. The blood is very colourless, but usually contains a few pigmented corpuscles.

\section{Excretory System.}

In the loop of the intestine there lies a mass of clear vesicles containing uric acid and other waste products. This, therefore, seems to be a renal organ, but there is no duct. Bacteria are usually found in the vesicles, and their 
activity may make diffusion easier. It is interesting to find such a plant-like method of storing up, instead of eliminating, waste products in these very passive animals. It has been suggested that the sub-neural gland may have some renal function.

\section{Reproductive System.}

Tunicates are hermaphrodite. The reproductive organs (Fig. $128, G$.) are very simple, and lie in the loop of the intestine. The ovary is the larger, and contains a cavity into which the ova are set free, and from which they pass outwards along an oviduct which opens into the cloacal chamber. The testis sur-

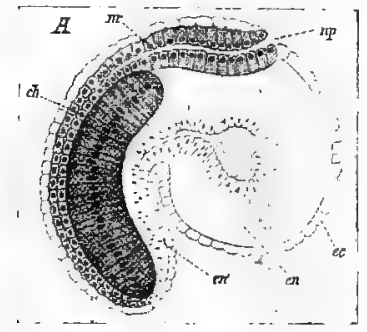

FIG. I30.-Young Embryo of Ascidian (Clavellina). (After VAN Beneden and Julin.)

np., Neuropore; nr., netural canal; ch., notochord; ec., ectoderm; en., endoderm. rounds the ovary, and is mature at a different time (dichogamy); its duct runs by the side of the oviduct. In some forms, where the gonads are near the cloaca, there are no ducts. The ova are surrounded by follicular cells, and are probably fertilised in the cloaca.

Development. - Most of the Ascidians exhibit the development with metamorphosis which is about to be described; a few in which the larve are retained for a long time within the body of the mother, show a much abbreviated life history.

The fertilised ovum divides completely and almost equally. The spherical blastosphere becomes slightly flattened, and ultimately forms a two-layered gastrula.

Along the dorsal median line of the gastrula, the ectoderm cells form the medullary groove, the sides of which arch together and form a canal-the medullary canal. This opens anteriorly to the exterior by the neuropore, and posteriorly commuricates with the archenteron by the neurenteric canal. In the posterior region of the gut, at the sides of the blastopore, a pair of diverticula, according to Van Beneden and Julin, grow ont. These form the mesoderm; the endoderm cells between them, roofing the gut, form the rudiment of the notochord. The mesoderm masses and the notochord grow forward together for a time, but later the mesoderm advances much further into the anterior region, the notochord being limited to the tail. The diverticula originally contain each a small cavity-the true colome, but this is soon 
obliterated. Two ectodermal invaginations form the originally double peribranchial chamber, and the primary gill slits break through the wall of the pharynx.

For some hours the tadpole-like larva enjoys a free swimming life, using its tail as an organ of locomotion. Then it fixes itself by a papilla on the head, and begins almost immediately to degenerate. The tail shrinks and disappears, the notochord being consumed by phagocytes. The nerve cord is lost, and with it the larval sense organs. The pharynx and peribranchial chamber assume their adult form, and the whole animal undergoes a metamorphosis, which one of the most signal instances of degeneration.

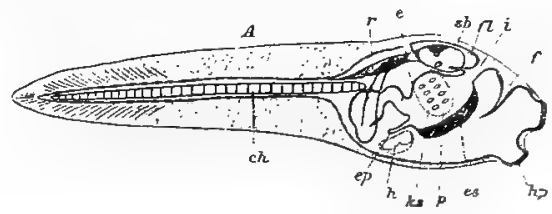

FIG. I3I.-Section of newly fixed larva of Clavellina. (After SEELIGER.)

$i$., Inhalent aperture; $f$., ciliated groove; $s b_{\text {. }}$, sensory vesicle; $\ell$., exhalent aperture; $r_{\text {., }}$, posterior part of medullary canal; $c h_{-}$, notochord; $h$., heart; $k s$. , gill slits; $\not$., peribranchial space; $e s$, endostyle; hp., attaching papilla.

\section{Classification.}

Order I. LaRvacea or Perennichordata. Appendicularia, Oikopleura, Fritillaria, Kowalevskia.

This order includes a few simple pelagic forms, which exhibit many of the characters of the larve of the Ascidians. All are minute, and furnished with a large locomotor tail which is bent forwards at an angle to the borly. Epidermic cells near the mouth secrete a slimy but consistent test, or "house," which is frequently abandoned and formed anew. The tail has a supporting notochord, and very large muscle cells. The nervous system consists of a lobed ganglionic mass above the mouth, from which a nerve cord is continued backwards and along the tail ; this is furnished with other ganglia in the tail region. It lies to the side of the notochord, and like the ganglia is said to be furnished with an axial canal. In connection with the cerebral ganglion there is a pigment spot, an otocyst (auditory?), and a tubular process communicating with the pharynx, and corresponding to the subneural gland and the ciliated duct of other Tunicates. There are two gill slits which communicate with two ectodernic atrial invaginations, as in the early larval stages of Ascidia. The mouth is almost at the anterior end, the anus at the root of the tail. The heart is very simple, and there are no distinct vessels. The hermaphrodite reproductive organs lie posteriorly and are cluctless. The eggs are difficult to obtain, and in consequence little is known of the development. 
The Larvacea form a group of great theoretical interest. As to their phylogenetic position two views have been maintained. According to one recently reasserted by Brooks, they are the slightly modified descendants of the primitive Tunicates, from which the Ascidians have diverged in the direction of degeneration; in other words, they are primitive forms. According to the other view, they have been derived from an Ascidian-like form, in which degeneration had already taken place, and may be compared to prematurely sexual larvæ.

\section{Order 2. Ascidiacea.}

(a) Ascidire simplices. Ascidia, Phallusia, Ciona.

(b) Ascidia Composite. Botryllus, Polyclinum.

(c) Pyrosomidze. Pyrosoma.

The characters of this order may be gathered from the description of Ascidia. Most are sedentary, with the notable exception of Pyrosoma. In several there is an alternation of sexual and asexual forms in the life history. In Pyrosoma the embryo develops within the body of the mother ; there is much yolk, and development is shortened and direct. The greater number of the Tunicates are included in this order, among them several well known British genera.

\section{Order 3. Thaliacea.}

(a) Salpa, Octacnemus.

(b) Doliolum, Anchinzio.

This order contains a few genera which show considerable modifica. tion from the Ascidian type. With the possible exception of the little known Octacnemus, all are free swimming, and, with the same exception, pelagic. The mouth is at one end of the body, the atrial aperture at the other; the animals swim by forcing the water out of the peribranchial chamber posteriorly. The cuticle is often very thin, as we should expect in animals so active; in some species it seems to be absent. In Doliolum, the muscles form hoops encircling the barrel shaped body; in Salpa the body is fusiform, and the muscle bands do not form complete rings. Both are devoid of reproductive ducts, and very simple in structure.

The life history shows an alternation of generations. In Salpa, a solitary asexual form, or " nurse," gives rise to a complex stolon, which segments to form a chain of embryos. This chain is set free, its members become sexual, and, either while still united, or after separation, produce ova, which give rise to the nurse form. In Doliolum, a small stolon forms a number of primitive buds, which creep over the parent and multiply. They form a lateral series of individuals, which furnish the parent form with food, and a median series which are set free as asexual "foster mothers." These carry with them some primitive buds, which divide into secondary buds, and these finally become the solitary sexual forms, producing ova which develop into "nurses." The "nurse" form during the budding process clegenerates greatly, until, like the swimming bells of the Siphonophora, it becomes a mere organ of locomotion, nourished by the lateral buds. There is thus considerable division of labour in the colonial form. 


\section{CHAPTER XIX.}

\section{CEPHALOCHORDA, AMPHIOXUS.}

(Synonyms, Acrania, Leptocardi, Pharyngobranchi.)

THere is but one well-defined genus in this class, - the lancelet (Amphioxus). Yet it deserves a class for itself, so distinct is it from other Chordata. It may be regarded as an offshoot from the primitive Vertebrate stock, lost, it is to be feared, for ever, as a far-off prophecy of a fish, or, perhaps, as a degenerate type, "a weed in the Vertebrate garden."

General Characters of Cephalochorda. - A class of simple Chordate animals, represented by one distinct typeAmphioxus. The nervous system has no well-defined brain region. The notochord is persistent and unsegmented; it is surrounded by a continuous sheath, and projects in a unique manner in front of the anterior end of the nerve cord. In the adult, the gill slits are very numerous. From Fishes, Amphioxus is zeidely renoved by the absence of limbs, skull, jares, definite brain, sympathetic nervous system, eye, ear, definite heart, spleen, and genital ducts. The species have a wide marine distribution near the coasts in rearm and temperate seas, are sluggish in habit, and feed on microscopic organisms or organic particles.

Description of Amphioxus lanceslatus, the best known species.

$$
\text { Mode of Life. }
$$

The lancelets are fond of lying in the sand in water about two fathoms deep, with only the fringed aperture of the 
mouth projecting. Into this diatoms and other small organisms are sucked. At times, and especially in the evening, the adults sometimes start up and swim about, but they are always less active than the larvæ. The early larva are indeed pelagic.

\section{Form.}

'The body, which rarely measures as much as two inches in length, is pointed at both ends, as the names suggest, and bears a dorsal and an anal cuticular fin, continuous around the tail. When alive the animal appears much plumper than the spirit specimens, and is translucent with a faint flesh colour. The muscles are arranged in sixty-two segments, or myotomes, readily visible externally. There are three unpaired apertures- $(a)$ the mouth is median and ventral, and overarched by a pre-oral hood, the edges of which are fringed with tentacle-like cirri; (b) the atriopore opens to the exterior in myotome thirty-six, and

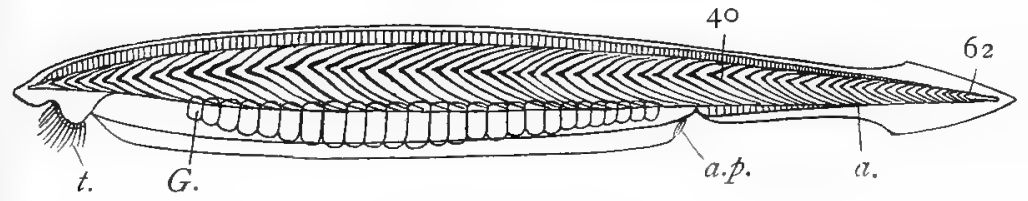

FIG. I32.-Lateral view of Amphioxus. (After RAY LANKESTER.)

Note the notochord running from tip to tip.

t., Tentacular cirri; G., reproductive organs; a.p., atriopore; $a$., position of anus; 40 and 62 , indicate number of myotomes.

gives exit to the water which enters by the mouth; (c) the anus is ventral and slightly to the left side, behind the atriopore, but some distance from the posterior end of the body. Along the back there is a median fin, which is continued around the tail, and along the ventral surface as far as the atriopore. In front of this region the ventral surface is flattened, the flattened area being fringed on either side by a slight fin-like "metapleural" fold. "These are continuations downwards of the walls of the atrial or branchial chamber, which extends from behind the mouth to the atriopore, and into which the gill slits of the pharynx open in the adult. 
Skin.

The epidermis is a single layer of columnar cells. Some of them project slightly from the surface, and are connected at the base with nerve fibres. These are sensory cells, and may be analogous with the cells of the lateral line in fishes and tadpoles. The epidermis lies upon a thin layer of clear

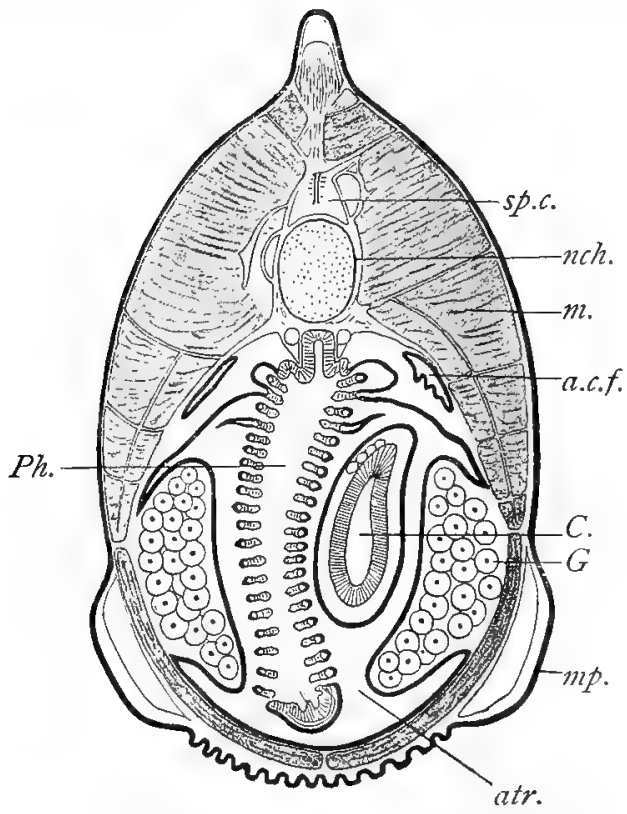

FIG. 133.-Transverse section through pharyngeal region of Amphioxus. (After RAY LAAKESTER.)

sp.c., Spinal cord; nch., notochord, beneath which the two dorsal aortæ; m., myotome; a.c.f.y atrio-coelomic funnel; $C_{\text {., }}$ cæcum; $G_{*}$ a genital sac with ova; mp. metapleural fold; atr. "iatrial cavity; $P h$., pharynx, with dorsal and ventral grooves, and bars between gill slits.

cutis. Beneath this there is a layer of fine tubes, which unite in a longitudinal canal running along each metapleural fold. These metapleural canals may be derived from the 
body cavity, or, according to another view, are spaces secondarily hollowed out in the tissues, and so pseudocalic.

\section{Skeleton.}

This is very slightly developed, for there is not only no bone, but the supporting material is not even definitely cartilaginous.

(a) The notochord runs from tip to tip. It consists of vacuolated cells, and probably owes its supporting power to their turgidity (cf. plants).

(b) The pharynx is supported by a system of chitinoid bars, which border the numerous gill slits. There is also a paired longitudinal plate along the mid ventral groove of the pharynx.

(c) The mouth is embraced by two curved bars, each segmented into about a dozen pieces, which bear filaments supporting the cirri.

(d) The sheath which envelops the notochord and is continued round the nerve cord, the septa of connective tissue which divide the muscle segments, and produce the $<$-shaped markings; the $25^{\circ}$ "fin rays" which support the dorsal and ventral fins, may also be included here.

\section{Muscular System.}

The swimming movements are caused by lateral wriggling of the body. This is effected by the segmented lateral muscles, in which the muscle fibres run longitudinally. On the ventral surface between the mouth and the atriopore there is a transverse set of fibres which help to drive out the water from the atrial cavity. Other muscles occur in the region of the mouth and elsewhere. Nearly all the fibres are striated.

\section{Nervous System.}

The dorsal nerve cord is shorter than the notochord, and has no anterior swelling. It gives origin to two sets of nerves, dorsal and ventral. The dorsal nerves correspond to the segments, except in the anterior region, where they are more numerous, and the first five pairs may be regarded as cerebral; the ventral nerves are minute and numerous. The two sets are compared to the single-rooted sensory 
dorsal nerves, and the many-rooted, motor, ventral nerves of higher Vertebrates. But the dorsal nerves of Amphioxus supply the muscles as well as the skin, so that they must be partly motor. Furthermore, there is no connection between the two sets, and the dorsal nerves have no ganglia. Nor are there any sympathetic ganglia.

The anterior region of the nerve cord is said to exhibit some histological, though no morphological distinctiveness. With it the following structures are associated :-

(a) Slightly to the left side there is a ciliated pit, often called olfactory. The development of this is interesting. The cavity of the medullary tube opens at first to the exterior by an anterior aperture, the neuropore. Later, an invagination of the ectoderm takes place at this point, and carries the neuropore in with it. This invagination forms the olfactory pit; it at first opens into the neural tube by the persistent neuropore; later this closes, and the pit becomes a mere blind sac. This invagination may perhaps correspond with the ciliated duct of the sub-neural gland of Tunicates, and so with part of the hypophysis of other Vertebrates.

(b) At the end of the nerve cord there is a pigment spot, sometimes called an eye spot. There are no true eyes.

(c) On the roof of the mouth there opens a small sac, the pre-oral pit, which may have a tasting or smelling function. It arises in development from the left of two pouches which grow out anteriorly from the gut of the embryo. The right of these potiches forms the head cavity of the adult, so that ontogenetically the pre-oral pit is the aborted head cavity of the left sicle. This is, however, only one of many explanations of the organ.

It is likely that the most important sensory structures of the adult are the sensitive cells of the epidermis.

We may connect the feeble development of sense organs with the almost sedentary habit.

\section{Alimentary System.}

The true mouth or velum lies well within the projecting pre-oral hood with its fringe of cirri. In the larva this hood is absent, and the mouth is flush with the surface.

The mouth opens into the pharynx, which, like it, is richly ciliated. The pharynx, like that of Tunicates, and indeed of Fishes also, is modified for respiration (Fig. I33, $P h$.). Its walls are perforated by numerous gill slits on each side, and between these lie supporting bars and arches, alternately split and unsplit.

Along the mid-dorsal and mid-ventral lines there are grooves respectively called hyper- and hypobranchial. The latter is comparable to the endostyle of Ascidians. 
The pharynx opens into the intestinal region of the gut which is straight and simple. Near its commencement a

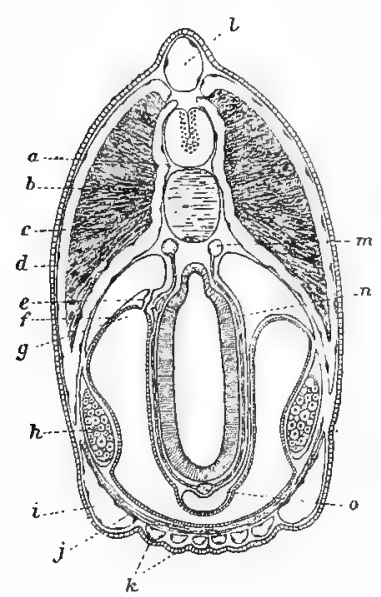

FIG. I34.-Cross section of Amphioxus through the gill slit region. (After Boveri and HATSCHEK.)

The spinal cord, notochord, pharynx, and atrial cavity are unlettered.

$a$, Sclera layer ; $b$, fascia ; $c$, muscle plate; $d$, cutis ; 2 , nephridial canal ; $f$, traverses the sub-chordal part of the colome on left side; $g$, glomerulus of kidney tube ; $h$, gonad ; $i$, metapleural cavity; $j$, transverse muscle; $k$, cavities in junction of metapleural folds; $l$, caviry in dorsal fin ; $m$, aorta; $n$, branchial vessel; $o$, branchial artery, the line traverses first the metapleural cavity, then the atrial cavity, and finally a minute part of the colome beneath the pharynx. is hollowed out at the expense of the true coelome. The coelomic spaces and canals contain coagulable fluid, and are in some regions continuous with the blood vessels. They represent the lymphatic system of higher forms. (Fig. I $33, C$.) arises, and extends forward on the right side of the pharynx. The anus is some distance from the end of the body (cf. Fishes); in the larva it is close to the caudal fin.

Body Cavity, - This can only be understood when its development is studied. From the archenteron of the embryo a pouch grows out on each side, and becomes almost at once segmented into a series of small sacs. These lie one behind the other, and soon lose all connection with the gut. Each ultimately divides into two portions, an upper, the true primitive segment, and a lower, corresponding to the lateral plate of other Vertebrates, The primitive segments form the body musculature, and retain their segmentation. Their cavity, the myocoele, persists to some extent in the adult, forming the system of lymph spaces and canals which lie below the cutis. In the region of the lateral plates the septa disappear, and the enclosed spaces, bounded by somatopleure and splanchnopleure, unite to form the "splanchnocoele" which surrounds the gut. Posteriorly, this space exists unconstricted in the adult; anteriorly, it is reduced to small spaces and ccelomic canals by the development of the atrial chamber. This pushes the somatopleure up before it as it develops, and so

pouch-like "liver" or cæcum 


\section{Respiratory System.}

The water which enters the mouth and passes down the pharynx leaves this by the numerous gill slits. In the embryo these open directly to the exterior; in the adult, into the atrial chamber, which opens by the single atriopore.

In development two folds appear laterally on the body wall, and form the hollow metapleural folds of the adult. On their inner apposed, but not united, surfaces, two ridges appear. These grow towards one another and unite, leaving only the atriopore open. Thus the floor of the atrial chamber (Fig. I33, atr.) is produced. The chamber, as first formed, is a tube with a very small lumen. Secondarily, it becomes enlarged, constricting the body cavity, as we noticed above, until it comes almost to surround the gut. At the same time, the metapleural folds increase in size until they assume the adult appearance (Fig. I33, mp.). The water currents are kept up by the action of cilia, and by the movements of the transverse muscles.

The gill slits gradually become more numerous as the animal grows older, and in the adult there are more than a hundred.

The original number of gill slits is doubled by the growth of a secondary bar down the centre of each slit, thus producing two gill slits where there was formerly only one. The primary bars are distinguished from the secondary in being split, and there are also histological differences between them.

\section{Circulatory System.}

The blood is colourless, with a few amoeboid cells. There is no definite heart, but the vessels are said to be contractile in several places.

Vessels from the body and from the cæcum unite to form a ventral vein, the cardiac aorta, which runs forward beneath the pharynx. From this vessel a series of smaller vessels arise, which pass up the primary branchial rods, and are termed aortic arches. The most anterior of the right side is larger than the rest, and sends branches to the head region. The aortic arches apparently open into two dorsal vessels, the right and left dorsal aortæe, which unite at the hinder end of the pharynx to form a single vessel running backward above the intestine. The blood vessels which are supposed to take blood from the intestine to the liver are termed portal veins ; those passing from the liver to the ventral vein are termed hepatic. The portal vein and the cardiac aorta are said to be specially contractile.

Although the names given above to the various vessels indicate the views generally held as to the course of the blood, our knowledge of this is only hypothetical.

Excretory System.-(a) Quite recently Prof. Boveri has discorered nephridial tubes in the adult. "These are found in the region of the 
pharynx, and are short tubes which place the sub-chordal coelome in communication with the atrial chamber." They open into the colome by three or four funnel shaped openings, and around them the vessels of the gill slits form a so-called glomerulus. They occur in relation to the gill slits, and open on the secondary branchial bars. Boveri regards them as equivalent to the pronephric tubules of other Vertebrates. Of their developmental history nothing is known.

(b) Professor Hatschek discovered in the anterior region of the larva a nephridial tube which is absent in the full grown adult; according to Van Wijhe, this is the residue of the communication between the left anterior diverticulum (or pre-oral pit) and the gut.

(c) Professor Ray Lankester discovered a pair of short pigmented funnel tubes (Fig. I33, a.c.f.), which lie in the twenty-seventh segment, and place the lymphatic spaces of the metapleural folds in communication with the atrial cavity. They may be compared with the pores which open from the collar region in Balanoglossus, and with the abdominal pores of higher Vertebrates. "It is doubtful whether they represent nephridia."

\section{Reproductive System.}

The sexes are separate and similar to one another. The organs are very simple, and are without ducts. They form twenty-six pairs of horse-shoe-shaped sacs, lying along the inner wall of the atrial cavity in segments ten to thirty-five on each side (Fig. I 33, G.). Each lies in a "genital chamber" formed in development by constriction from the cavity of the lower part of the primitive segment.

In the mature female the ovaries are large and conspicuous; the ova burst into the atrial cavity, whence they pass into the pharynx by the gill slits, and out by the mouth, or more directly by the atriopore.

The testes are like the ovaries; the spermatozoa burst into the atrial cavity, and pass out by the atriopore. The eggs are fertilised in the surrounding water.

\section{Development.}

The fertilised ovum is about $\frac{1}{\frac{1}{5}}$ inch in diameter. The segmentation is complete and almost equal. The first cleavage is vertical, and divides the ovum into two equal parts; the second is also vertical, along a meridional plane at right angles to the first, and the result is four equal cells. The third cleavage is equatorial, and gives rise to four larger cells (or macromeres) below or towards the vegetative pole, and to four smaller cells (or micromeres) above or towards 
the animal pole. The blastosphere, which is the final result of segmentation, invaginates to form a gastrula.

Along the mid-dorsal line of the gastrula the ectoderm cells sink in slightly so as to form a groove. This is the medullary groove, which here follows an unusual course of development. Instead of immediately closing to form a canal, the groove sinks inwards, and the lateral ectoderm grows over it before closing takes place. Later, the groove forms the medullary tube, which opens into the gut by the neurenteric canal; to the exterior by the anterior neuropore.

The cavity of the gastrula - the archenteron-becomes the gut of the adult. From it pouches grow out as was described above.

The notochord arises along the mid-dorsal line of the archenteron; its forward extension is secondary.

During the early part of larval life the ectodermal cells

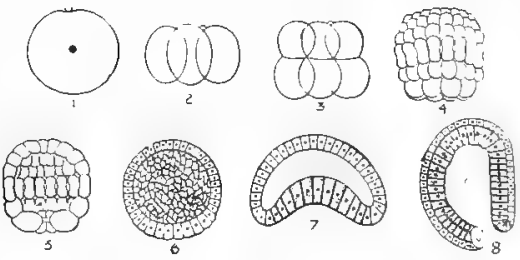

FIG. I35---Early stages in the development of Amphioxus. (After Hatschex.)

I. Ovum; 2. Four cell stage; 3. Eight cell stage ; 4. External appearance of blastula; 5,6 . Blastula in section (note the larger macromeres); 7. Beginning of gastrula stage; 8. Section of completed gastrula.

including those forming the medullary canal, are ciliated. At this stage the larva is much more active than the adult.

The later larvæ are more sedentary, lying much on the right side, and they are strongly asymmetrical. The mouth is placed at the left side; the gill slits of one side appear considerably before those of the other; the primitive segments of one side are not opposite those of the other, and so on. By the process known as the "symmetrisation " of the larva, the apparent symmetry of the adult is produced. 
The adult position of the anus and of the olfactory pit, both to the left side, and the position of the unpaired liver diverticulum, show how partial this process is.

Experimental Embryology.-As an illustration of what may be called experimental embryology, and of the developmental potentiality of the first few segmentation cells, reference may be made to the recent experiments of Prof. E. B. Wilson.

By shaking the water in which the the two-celled stages floated, Mr. Wilson separated the two cells, and the result was two quite separate and independent twins of half the normal size. Each of the isolated cells segments like a normal ovum, and gives origin, through blastula and gastrula stages, to a half-sized metameric larva.

If the shaking has separated the two first segmentation cells incompletely, double embryos-like Siamese twins-result, and also form short-lived (twenty-four hours) segmented larvæ.

Similar experiments with the four-celled stages succeeded, though development never continued long after the first appearance of metamerism. Complete isolation of the four cells resulted in four dwarf blastulæe, gastrulæ, and even larvæ. Separation into two pairs of cells

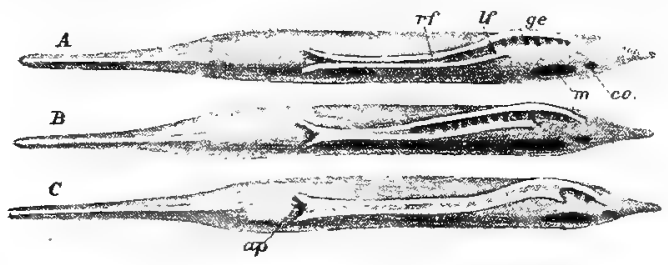

F1G. I36.-Three larval stages of Amphioxus. (After Ray LANKester and Willey.)

$A$, The metapleural folds still separate; $B$, united posteriorly ; $C$, united altogether; $\alpha p$, atriopore; $g c$, gill slits; $\mathscr{V}$, left metapleural fold; if, right metapleural fold; $m$, mouth ; $\mathrm{Co}_{1}$ ciliated pit.

resulted in two half-sized embryos. Incomplete separation resulted in one of three types- $(a)$ double embryos, $(b)$ triple embryos-one twice the size of the other two-and $(c)$ quadruple embryos, each a quarter size.

The eager observer proceeded to shake up the eight-celled stages, but in no case did he succeed in rearing a gastrula from an isolated unit of the eight-celled stages. Flat plates, curved plates, even one-eighth size blastula were formed, but none seemed capable of full development.

Thus, a unit from the four cell stage may form an embryo, but a unit from the eight cell stage does not. For various reasons it seems likely that this is due to qualitative limitations, not merely to the fact that the units of the eight cell stage are smaller. For although the separated cells of the eight cell stage have considerable vitality, and swim about 
actively, the difference between macromeres and micromeres has by this time been established; in fact the cells have begun to be specialised, and have no longer the primitive indifference, the absence of differentiation, which explains the flevelopmental potentiality of the separated units of the two-celled or four-celled stages.

Somewhat similar experiments have been made by other investigators on the developing ova of ascidians, sea urchins, \&c. Specialisation of segmentation cells appears to occur at different times in different animals, but it is illogical to infer the absence of specialisation from the fact that any of the first four blastomeres, let us say, can produce an entire embryo. For specialised cells may retain a power of regeneration. 


\section{CHAPTER XX.}

STRUCTURE AND DEVELOPMENT OF VERTEBRATA.

SincE the time of Aristotle-over two thousand years ago - the distinction between backboned and backboneless animals must have been more or less evident to all who, with any precision, thought of the forms of animal life.

Yet it was not till about a century ago that the line of separation was drawn with adequate firmness. This Lamarck did in 1797 .

But the doctrine of descent-the idea of organic evolution-with which Darwin impressed the thoughtful in 1859 , suggested inquiry into the apparently abrupt apartness of the group of Vertebrates.

The inquiry bore fruit in 1866 , when the Russian naturalist, Kowalevsky, worked out the development of the Vertebrate characteristics of Amphioxus, correlated this with the development of Ascidians, and discovered the pharyngeal gill slits of Balanoglossus.

From what has been said in regard to these three types, it will be plain that the apparent apartness of the Vertebrata was thus annulled.

General Characters. - Vertebrates are colomate Metazoa, with a segmental arrangement of parts. The central nervous system lies in the dorsal median line, and is tubular in its origin. A skeletal rod or notochord is formed as an outgrowth along the dorsal median line of the primitive gut, but though this is always present in the embryo at least, it tends to be replaced by a mesodermic axial skeleton-the backbone. Pharyngeal gill slits, which may or may not persist in adult life, are always developed, but gill-lamella do not occur above Amphibians. The heart is ventral. The eye begins to develop as an outgrowth from the brain. 


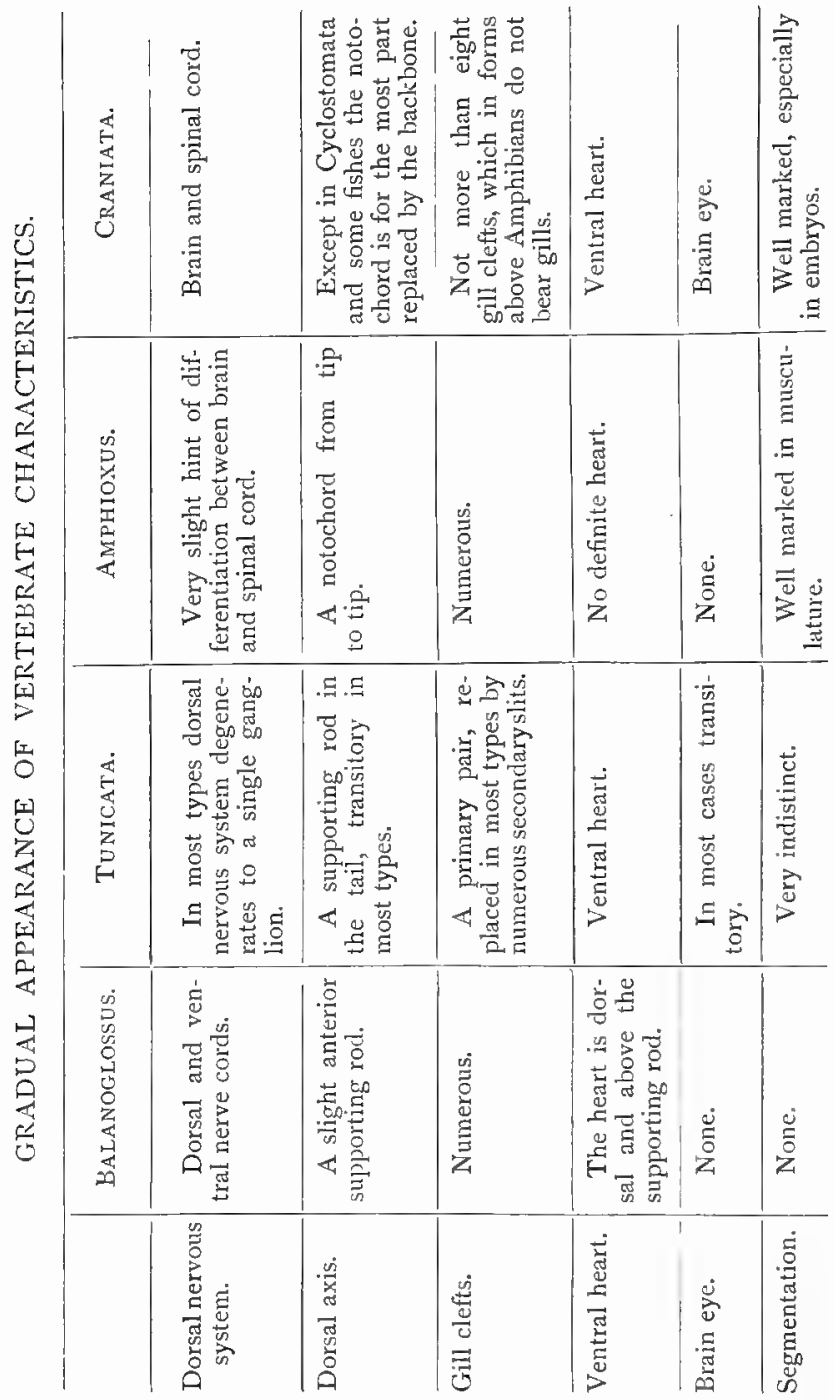




\section{General Classification.}

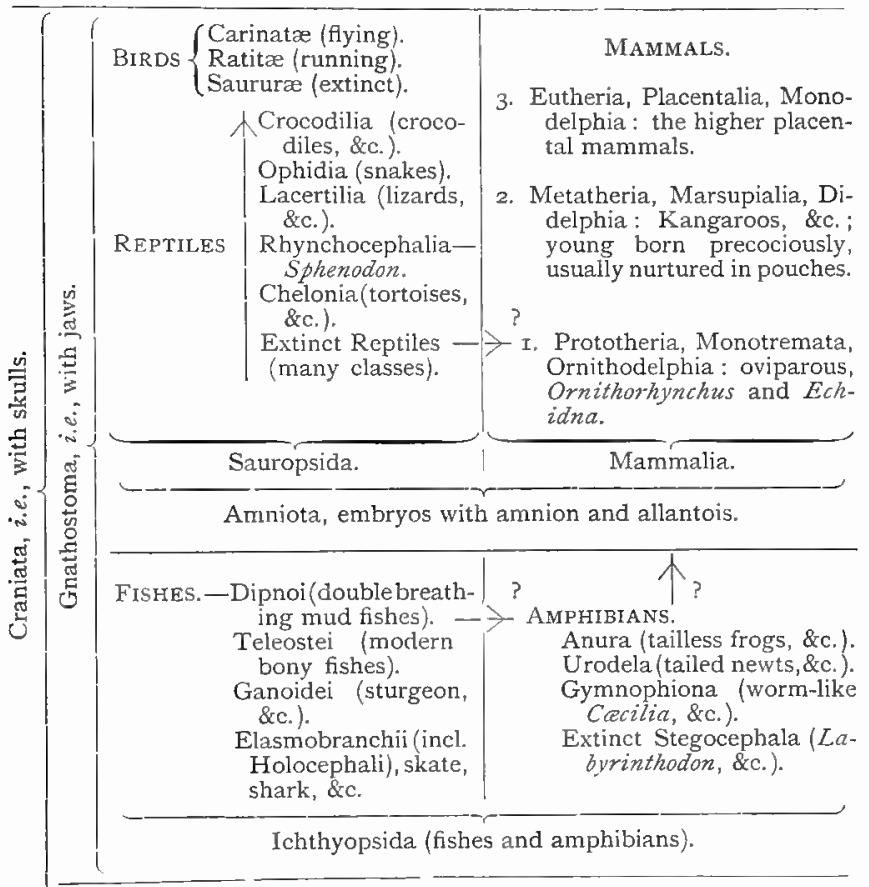

Cyclostomata (Round Mouths), without true jaws. Myxine, hag fish. Petromyzon, lamprey,

\begin{tabular}{|c|c|c|}
\hline $\begin{array}{c}\text { CEPHALOCHORDA. - Amphiuxus, or } \\
\text { Lancelet. }\end{array}$ & $\begin{array}{c}\text { UROCHORDA } \\
\text { or } \\
\text { TUNICATA. }\end{array}$ & $\left\{\begin{array}{l}\text { Salpa type. } \\
\text { Ascidian type } \\
\text { squirts). } \\
\text { Appendicularia } \\
\text { val type persisten }\end{array}\right.$ \\
\hline
\end{tabular}

Surviving offshoots of ancestral Vertebrates.

HEMICHORDA, or ENTEROPNEUSTA (offshoots of incipient Vertebrates?) Balanoglossus, \&c.; probably Cephalodiscus; possibly Rhabdopleura.

Nemertean affinities (?) Chætopod affinities (?) Arthropod affinities (?) 


\section{Ancestry of Vertebrates.}

It is not at present possible to trace the path along which Vertebrates have evolved, though our faith in the doctrine of evolution-as a modal theory of origins-leads us to believe that Vertebrates arose from forms which were not Vertebrates.

But, even when we recognise that $A m p h i o x u s$ is a Vertebrate very simple in its general features, and that the Tunicata, especially in their youth, are Vertebrates, we have to remember that degeneration seems to have been by no means uncommon in the history of animals, and that the types above mentioned may be less primitive than they seem.

The Enteropneusta carry us a little further back. For, while many of their alleged Vertebrate characteristics are debateable, one cannot gainsay, for instance, the possession of pharyngeal gill slits. But the affinities of the Enteropneusta with Invertebrate types are entirely obscure.

We have, in fact, to acknowledge frankly that the pedigree of Vertebrates remains unknown. At the same time, it is useful to enquire into certain convergences towards Vertebrate structure which are exhibited among various sets of Invertebrates.

In regard to these, speculation has been abundant. Alleged affinities have been discovered among Annelids, Nemerteans, Arachnids, Crustaceans, \&c. Indeed, there is almost no great class of Invertebrate Metazoa whose characters have not been ingeniously interpreted, or wrested, so as to reveal affinities with Vertebrates. It will be enough to select two illustrations.

Annelid Affuities.-Dohm, Semper, Beard, and others, maintain that Annelids have affinities with Vertebrates.

(1) Both Annelids and Vertebrates are segmented animals.

(2) The segmental nephridia of Annelids correspond to the primitive kidney tubes of a Vertebrate embryo.

(3) The ventral nerve cord of Annelids may be compared (in altered position) to the dorsal nerve cord of V'ertebrates. Both cords are bilateral, and it is likely enough that the tubular character of the spinal cord and brain is the necessary result of its mode of development, and without much morphological importance.

(4) Segmentally arranged ganglia about the appendages of some Chaetopod worms may correspond to the branchial and lateral sense organs of Ichthyopsida, and the ganglia associated with some of the nerves from the brain.

(5) The formation of the oral part of the pituitary body (see page 436 ) is suggestive of the way in which the mouth of Annelids is sometimes formed. Perhaps the pituitary body represents an old lost mouth and its ancient innervation.

To minor points, such as the red blood, well dereloped body carity, and slight internal skeleton of some Chretopods, little importance can be attached.

The absence of anything like gill slits in Annelids remains as a diffi- 
culty, even if we grant that no emphasis is to be laid on the tubular nerve cord of Vertebrates, and admit the possibility of an inversion bringing the ventral nerve cord to the dorsal surface.

Nemertean Affinities.-Hubrecht and others have emphasised the affinities between Nemerteans and Vertebrates.

In Nemerteans:-

(I) The lateral nerve cords sometimes approach one another ventrally, and in rare cases dorsally. An approximation dorsalwards, and union on that surface, would result in a double dorsal nerve cord.

(2) The firm dorsal sheath of the proboscis may correspond to a notochord.

(3) The proboscis itself may correspond to the hypophysis or pituitary process characteristic of Vertebrate brains.

(4) Two ciliated slits on the head may correspond to a pair of gill clefts.

(5) There is no segmentation, but the branches given off from the nerve cords are sometimes serially arranged.

It must be noted, that those who support these theories do not assert that any Nemertean or Annelid is in the direct line of Vertebrate ascent. They simply emphasise the demonstrable affinities. When these are thoroughly worked out, it will be possible to say what Invertebrate types are most nearly related to Vertebrates.

Structure and Development of Vertebrates.

Having separately discussed the Hemichorda, Urochorda, and Cephalochorda, we propose in this chapter to discuss the general structure of Craniata and the development of some of the important organs.

\section{The Skin.}

This forms a continuous covering over the surface of the body, serves as a protection to the underlying tissues, in some instances retains its primitive respiratory significance, and is frequently concerned in the excretion of waste and the regulation of the body temperature. As one or other of its many functions predominates, there are corresponding structural modifications. One function which we find oftenest emphasised at the expense of the others is that of protection, and yet the fossil Glyptodon, the sluggish Chelonia, the decadent Ganoids, seem to indicate that this, in itself or in its correlated variations, is not conducive to the continuance of the species. Indeed, the great develop- 
ment of exoskeleton may perhaps be regarded as the outcrop of a constitution which tends to extreme and unprofitable passivity.

The skin includes:-

(a) The epidermis, usually in several layers, the outer "horny" stratum corneum, the inner "mucous" stratum malpighii, or mucosum; both derived from the ectoderm or epiblast of the embryo.

(b) The dermis, cutis, corium, or under-skin, derived from the mesoderm or mesoblast of the embryo.

From the epidermis are derived feathers, hairs, and some kinds of scales. The dermis, as is natural when we consider its origin from the mesoblast (mesenchyme) or vascular layer, assists in nourishing these epidermic structures. From the dermis are derived the bony shields of armadillos and a few related mammals, the bony scutes of crocodiles and some other reptiles, and the scales of most bony (Teleostean) fishes. This again is readily explained by the fact that the mesenchyme is also the skeletal layer of the embryo. The ordinary teeth of Vertebrates, as well as the superficial or skin teeth of gristly fishes, are largely formed from the dermis, but are usually covered by a thin coating of ectodermic enamel. It should be noted, however, that Klaatsch has recently maintained the ectodermic origin of the skeleton forming cells (scleroblasts) which form the scales of Elasmobranchs and Teleosteans, and that there are hints in higher forms that the ectoderm has more to do with the skeleton than is usually allowed. There is, indeed, a growing tendency among morphologists to strip the mesoderm of its importance. It may be noted also that Klaatsch ventures to suggest that the beginning of skeleton in the ectoderm may have something to do with excretion.

Muscular System.-In all Vertebrates

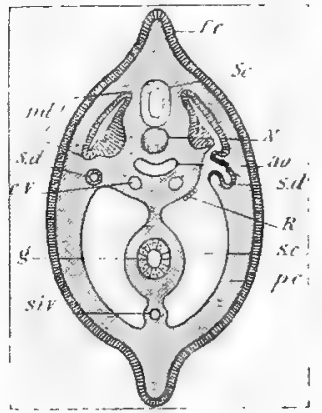

FIG. I37. - Transierse section through an Elasmobranch Embryo (diagrammatic). (After ZIEGLER.)

Ec., Ectoderm; S.c., spinal cord ; $N$., notochord ; ao., aorta ; s.d., segmental duct; $R$., reproductive cells; s.c., secondary coelome; p.c., primary coelome filled up with connective tissue; s.i.t', sub-intestinal vein; $g .$, gut; $c . v_{.}$, cardinal vein; s.d., segmental duct ; mt., myotome.

the muscles of the trunk arise from the primitive segments, or muscle plates, found in the embryo at the sides of the nerve cord. In $A m p h i$ oxus and Fishes the primitive segmented condition of the muscles is retained, as is seen in the myotomes visible externally in the lancelet. Above Fishes little trace of the segmented condition persists in the adult, except in the tail region. The muscles of the head arise from the primitive segments of that region. 
The muscles of the limbs arise in Elasmoluranchs as buds from the primitive segments; buds from several contiguous segments grow into each fin. In most other Vertebrates the formation of the limb muscles is more complicated ; they seem in some cases to arise independently of the primitive segments.

Most of the visceral muscles consist of unstriped fibres, but those of the trunk, head, and limbs, as well as of the heart, show the usual striped structure.

\section{Skeletal System.}

Apart from the exoskeleton of skin-teeth, scutes, shields, \&c., the skeleton consists of the following parts :-

(a) Axial
Skeleton

(The skull and its associated "arches." The backbone and associated ribs.

(The notochord is transitory except in the simplest Vertebrates).

(b) Appendicular (Fore limbs, and pectoral girdle.

Skeleton Hind limbs, and pelvic girdle.

\section{Skull.}

The notochord grows forward anteriorly as far as that region of the brain known as the optic thalami. Around notochord and brain the mesenchyme forms a continuous sheath which is the foundation of the skull.

As in the case of the notochordal sheath of the trunk region, so also here cartilage is formed in the primitive membranous cranium. The first cartilages to appear are the two parachordals, which lie on the lower surface of the head at the sides of the notochord, and the two trabeculæ lying in front. The parachordals grow round and above the notochord, producing the basilar plate, while the trabeculae unite in front to form the ethmoid plate. The continuance of the process of cartilage formation, together with the addition of cartilaginous nasal capsules in front and auditory capsules behind, completes the formation of the primitive cartilaginous brain box or chondrocranium of the lower Vertebrates.

Also connected with the head region, and of great importance are the visceral or gill arches which loop around the pharynx on either side, and separate the primitive gill clefts. At the time when cartilage begins to be formed in the mem- 
branous cranium, the arches also become chondrified, and at the same time divided into successive segments.

Of these arches, there are never more than eight. The most anterior is the mandibular arch which bounds the mouth, the second the hyoid; these two are of great importance in the development of the skull. The others, in Fishes and at least young Amphibians, bound open gill slits and support the pharynx; above Amphibians, they are less completely developed.

In the Elasmobranch fishes, the mandibular and hyoid arches do not form any direct part of the cartilaginous brain case, but in the Teleosteans and thence onwards, the cartilages, or bones, arising in connection with the mandibular and upper part of the hyoid arches, contribute directly to the formation of the skull. The hyoid proper, or lower part of the hyoid arch, forms the skeleton supporting the tongue. Cartilages arising in the lower part of the third risceral arch assist in the formation of the hyoid bones of the higher Vertebrates, and parts of two other arches appear to help in forming the laryngeal skeleton of Mammals.

The manclibular arch in Elasmobranchs and frogs divides into a lower portion-Meckel's cartilage-which forms the lower jaw or its basis, while from the upper portion a bud grows forward-the palato-pterygoquadrate cartilage, which forms the upper jaw in shark and skate, and has a closer union with the skull in the frog. In higher Vertebrates, the lower portion of the mandibular always forms the basis of the lower jaw, a quadrate element is segmented off from the upper part, but the palato-pterygoid part seems to arise more inclependently. The hyoid arch also divides into a lower portion, the hyoid proper, and an upper portion, the hyo-mandibular, which may connect the jaws with the skull, or from Amphibians onwards may be more remarkably displaced and modified as a columella or stapes connected with the ear. We adhere to the old interpretation, according to which the mandibular and hyoid form two arches; even if Dohrn's theory that they are equivalent to four be accepted, the general fact remains that certain arches aid in the development of the skull.

Returning now to the brain box itself, we must notice another complication, - the development of "membrane" bones. If we examine the skull of the skate, we find that the brain lies within a cartilaginous capsule, but this is not entirely closed, spaces (the fontanelles) being left in the roof, which during life are covered only by the tough skin with its numerous "dermal denticles." In the sturgeon, again, the small skin teeth are replaced by stout bony plates covering over the cartilaginous capsule. From such super- 
ficial bony plates it is supposed that the "membrane" bones or ossifications in membrane, which form so important an element in the skull of the higher Vertebrate, have originated.

In some bony fishes, notably the salmon, we find the brain enclosed in a double capsule. Inside there is a cartilaginous brain case in which what are called centres of ossification have appeared, and upon this a layer of membrane bones is placed, which can be readily removed without injury to the cartilage beneath. In general, however, we must recognise that, with the appearance of membrane bones, two changes tend to occur,--first, the cartilaginous cranium tends to be reduced and to exhibit consideralble openings; second, in the remaining cartilage centres of ossification appear, and we thus have "cartilage" bones formed. Further, in spite of the developmental differences, the membrane and cartilage bones become closely united to one another, or even fused, and there is thus formed "a firm, closed, bony receptacle of mixed origin," as exemplified by the skull of any of the higher Vertebrates.

We may thus say that in the evolution of the skull we have first a cartilaginous capsule, that this becomes invested to a greater or less extent by dermal ossifications, and that finally the dermal bones lose their superficial position, and, fusing with the ossified remainder of the cartilaginous cranium, form a complete bony capsule. In Cyclostomes and Elasmobranchs the brain box is wholly cartilaginous; above Elasmobranchs, the cartilage is more or less thoroughly replaced or covered by bones. In the individual development there is a parallel progress.

Although one is safe in saying that skeletal structures in Vertebrates are mostly mesodermic in origin, it should be noted (I) that the notochord is endodermic, and (2) that in the head certain ectodermic proliferations may give rise to skeletal rudiments of a connective tissue nature which subsequently become differentiated into cartilage (Goronowitsch, Platt). But there is still doubt as to this last point.

\section{Theory of the Skull.}

Near the beginning of this century, Oken and Goethe independently propounded what is known as the vertebral theory of the skull. Regarding the skull as an anterior portion of the vertebral column, composed of three or four vertebre, they compared the bones of the different regions to the parts of a vertebra. Thus in the hindmost region of the skull, the basi-occipital, the two ex-occipitals, and the supra-occipital 
were held to correspond to the centrum, the neural arches, and the neural spine of a vertebral body.

This undoubtedly suggestive theory, modified in various details, persisted for a long period, but ultimately gave way before the advances in comparative anatomy and embryology. Huxley gave it its death blow, and Gegenbaur replaced it by what may be called the segmental theory of the skull.

To realise this theory, we must go back in development to the period before the mesoblast has ensheathed the notochord. At this time the segmentation of the body is expressed, not in the skeleton (notochord), but in the primitive segments. These segments, though less obvious than in the trunk, are represented in the head region. Formerly nine were enumerated, but it appears that in Elasmobranchs they are more numerous. Subsequently, brain and spinal cord become alike enveloped in the mesoblastic sheath, which gives rise to the skeleton of both head and trunk.

The great development of the muscle segments of the trunk region induces a secondary segmentation of the mesoblastic skeleton (vertebral column), while the slight development of the muscles of the head region exercises no such influence upon its skeleton, this is therefore always quite devoid of segmentation. The segmentation of the head, in contradistinction to the skull, is expressed, although indistinctly, by the muscle segments and by the nerves supplying these, perhaps also by the lateral sense organs, the ganglia, and the arches. While it is quite certain that it is the head that is segmented and not the skull, the details of the segmentation are still much debated. 


\begin{tabular}{|c|c|}
\hline ELEMENTS. & ORIGIN. \\
\hline $\begin{array}{l}\text { I. Parachordals } \\
\text { and trabeculæ, } \\
\text { aided in some } \\
\text { cases by the end } \\
\text { of the notochord. }\end{array}$ & $\begin{array}{l}\text { Their precise } \\
\text { relations, e.g., to } \\
\text { the notochord are } \\
\text { unknown. }\end{array}$ \\
\hline $\begin{array}{l}\text { II. Sense-capsules. } \\
\text { (a) Nasal. } \\
\text { (b) Auditory. }\end{array}$ & $\begin{array}{l}\quad \text { From cartilage } \\
\text { surrounding the } \\
\text { ectodermic pits } \\
\text { which form the } \\
\text { foundation of } \\
\text { nose and ear. }\end{array}$ \\
\hline
\end{tabular}

III. Arches.

(a) Mandibular.

(b) Hyoid arch.

IV. Investing

membranebones.

(a) From the roof of the skull.

(b) On the floor of the skull, i.e., from the roof of the mouth.

(c) About the sides of the skull.

(d) About the upper jaw.

(e) About the lower jaw.
These arches, like those which follow them, are supports of the pharynx, lying between primitive or persistent gill slits. Perhaps they may be compared to ribs.

Originally of the nature of external bony plates, tooth structures, and the like.
RESULTS.

Occipital region, with four bones -basi-occipital, two ex-occipitals, and a supra-occipital (in part). The. basi-occipital is distinct only in Reptiles, Birds, and Mammals.

Sphenoidal and ethmoidal region, with basi-sphenoid and pre-sphenoid, paired alisphenoids and orbitosphenoids, the inter-orbital septum, the lateral or ectoethmoids, the internasal septum.

(a) Unite with ethmoidal region.

(b) May give origin to five bonespro-, sphen-, pter-, epi-, and opisthotics, or to the single periotic of Mansmals.

(a) Upper part = palato-pterygoquadrate cartilage of Elasmobranchs, palatine, pterygoid, and quadrate bones in the higher Vertebrates, but in Mammals the quadrate is believed by many to become the incus of the inner ear.

Lower part $=$ Meckel's cartilagethe basis of the lower jaw in all animals; the part next the quadrate becomes the articular bone, which in Mammals is believed by many to become the malleus of the inner ear,

(b) Upper part or hyo-mandibular = the "suspensorium" cartilage of Elasmobranchs, the hyo-mandibular and symplectic of Teleosteans, the columella auris of Amphibians, Reptiles, and Birds, the stapes of the Mammal's ear.

Lower part $=$ the hyoid proper (cartilage or bone).

(a) Parietals, frontals, nasals, \&c.

(b) Vomer, parasphenoid, \&c.

(c) Lachrymal, squamosal, orbitals, \&c.

(d) Premaxilla, maxilla, jugal, and quadrato-jugal (in part).

(e) Dentary, splenial, angular, supra-angular, coronoid. 


\section{The Vertebral Column.}

A dorsal skeletal axis is characteristic of Vertebrata, and its usefulness is evident. It gives coherent strength to the body; it is usually associated very closely with a skull, with limb girdles, and with ribs; it affords stable insertion to muscles; its dorsal parts usually form a protective arch around the spinal cord.

To understand this skeletal axis we must distinguish clearly between the notochord and the backbone.

The notochord is the first skeletal structure to appear in the embryo. It arises as an axial differentiation of endoderm along the dorsal wall of the embryonic gut or archenteron. The backbone, which in most Vertebrates replaces the notochord, has a mesoblastic origin; it develops as the substitute of the notochord, but not from it.

In Balanoglossus, what is sometimes dignified with the name of notochord, is restricted to the most anterior part of the body; in the Tunicata the notochord is confined to the tail, in Amphioxis it runs from tip to tip of the body, in Cyclostomata and Dipnoi it persists as an unsegmented gristly rod, in other Vertebrates it is more or less completely replaced by its better substitute-the backbone.

In Cyclostomata the notochord forms and is ensheathed by a cuticula chorde (or membrana limitans intema); outside this there is a meso. blastic or skeletogenous sheath; and outside this again lies a cuticula sceleti (or membrana limitans externa). It is likely that this represents a primitive condition. What happens in most Vertebrates is that the skeletogenous or mesoblastic sheath forms the backbone, and more or less completely obliterates the notochord. The formation of cartilage takes place at regular intervals in the notochordal sheath, and the vertebral bodies thus formed alternate regularly with the primitive muscle segments. This arrangement is necessary for the proper attachment of the muscles to the future vertebrie, and makes it probable, as we noticed above, that the segmentation of the backbone is secondary, and was only acquired, as a mechanical necessity, when the notochordal sheath became chondrified, and so rigicl. Thus we reach the conclusion that the primitive segmentation of the Vertebrates, alike in head and trunk, finds its expression in the arrangements of the primitive segments and the nerves supplying these; and not in the skeleton.

In the higher Vertebrates, soon after the formation of the bodies of the vertebra, the rudiments of the neural arches appear in the membrane surrounding the spinal cord. Finally, centres of ossification may occur, and so produce the segmented backlbone.

In Amphioxus, in Myxine, and in young lampreys (known as Ammo(cotes), the notochord persists, unsegmented and with a simple sheath. In the adult lamprey, there are rudimentary arches of cartilage forming a 
trough in which the spinal cord lies. In the cartilaginous Ganoid fishes, in the Chimera type, and in the Dipnoi, arches appear both above and below, but yet there are no vertebral bodies. These begin in the Elasmobranchs, in which the notochord is constricted by its encroaching sheath. In the bony Ganoids the vertebre are ossified, and so they are in all the higher Vertebrates. Moreover, the notochord is more and more completely obliterated as the backbone grows.

It will be remembered (see p. 34) that according to Kleinenberg the notochord supplies the necessary growthstimulus for the rise of its substitute, the backbone.

A vertebra generally consists of several more or less independent parts: the substantial centrum, the neural arches which form a tube for the spinal cord, and are crowned by a neural spine, the transverse processes which project laterally, and are, perhaps, homologous with the inferior hæmal processes in the posterior region of Fishes and some Amphibians.

The ribs which support the body wall and usually articulate with the transverse processes, or with the transverse processes and centra, perhaps bear the same relation to the vertebræ that the visceral arches do to the skull.

In Amphibians for the first time a breast bone or sternum is developed. It arises from two cartilaginous rods in a tendinous region on the ventral wall of the thorax, and seems to be different from that of higher animals. For the sternum which is present in some Reptiles, and in all Birds and Mammals, arises from a cartilaginous tract uniting the ventral ends of a number of ribs.

\section{Appendicular Skeleton.}

No secure conclusion has yet been reached as to the origin of the paired limbs. According to Gegenbaur, the pectoral and pelvic girdles are homologous with branchial arches, while the primitive limbs are made up of modified fin rays, originally like those of the unpaired fins. According to Dohrn, the limbs are residues of a longitudinal series of segmentally arranged outgrowths, perhaps comparable to the parapodia of an Annelid. According to Wiedersheim, the girdle portion is primarily due to the centripetal growth of the fin skeleton, which arose from a localisation of the supports of continuous lateral folds.

The pectoral or shoulder girdle consists of a dorsal 28 
scapular portion or shoulder blade, a ventral coracoid portion, with the articulation for the limb between them, and of a forward growing clavicle or collar bone.

The pelvic or hip girdle consists of a dorsal iliac portion, a ventral ischiac portion, with the articulation for the limb between them, and of a pubic region possibly homologous with the clavicle.

The fore limb-from Amphibians onwards-consists of a humerus articulating with the girdle, a lower arm composed of radius and ulna lying side by side, a wrist or carpus of several elements, a "hand" with metacarpal bones in the "palm," and with fingers composed of several phalanges.

'The hind limb-from Amphibians onwards-consists of a femur articulating with the girdle, a lower leg composed of a tibia and fibula lying side by side, an "ankle" region or tarsus of several elements, a foot with metatarsal bones in the "sole," and with toes composed of several phalanges.

The fin-like limbs peculiar to Fishes, are discussed along with the other characteristics of that class.

Distinct from the other bones are a few little sesamoids of occasional occurrence, e.g., the knee pan or patella. They develop in the tendons of muscles.

\section{Nervous System.}

This includes (a) the central nervous system, consisting of brain and spinal cord; (b) the peripheral, consisting of spinal and cranial nerves; and $(c)$ the sympathetic nervous system.

The central nervous system first appears as a superficial groove along the mid-dorsal line of the embryo. The sides of this ectodermic groove meet, and uniting, convert the medullary groove into the medullary canal. 'The greater part of this canal forms the spinal cord, the anterior portion of it is specialised as the brain. There is at first a posterior connection between the neural canal and the primitive gut of the embryo; when this is lost the cavity still persists as a little ciliated canal in the centre of the cord, and as the internal cavity of the brain. In Cyclostomes and Bony Fishes the central nervous system arises as a solid cord of cells, the cavities not appearing until a later stage; this condition does not seem to be primitive. 


\section{The Brain.}

At an early stage, even before the closing-in process is completed, certain portions of the anterior region of the medullary canal grow more rapidly than others, and form the three primary brain vesicles. By further processes of growth and constriction, these three form the five regions of the adult brain.

When first formed the brain vesicles lie in a straight line, but as a consequence, probably, of their rapid and unequal growth, this condition is soon lost, and a marked cranial flexure is produced. In the lower forms, e.g., Cyclostomata, the flexure is slight, and is corrected later, but in the higher types it is very distinct, and causes the marked overlapping of parts so obvious in the adult.

We must now follow the metamorphoses of the primary brain vesicles.

The first vesicle gives rise anteriorly to the cerebral hemi-

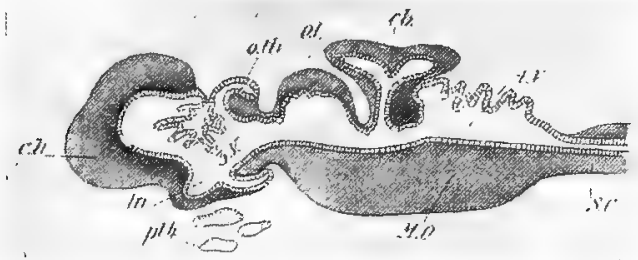

FIG. 138.-Longitudinal section of brain of young Dogfish (diagrammatic). (After GASKell.)

$C . h_{\text {. }}$ Cerebral hemispheres; o.th., optic thalami ; $. V .$, third ventricle; In., infundibulum; $\beta t . b$., pituitary body; 0.2. , optic lobes; ch., cerebellum; M.O., medulla oblongata; $4 . V$, fourth ventricle; S.C., spinal cord.

spheres, while the remainder forms the region of the optic thalami or thalamencephalon.

The cerebral hemispheres, also called prosencephalon, or fore brain, are exceedingly important. They predominate more and more as we ascend in the scale of Vertebrates, and become more and more the home of thought. Except in a few instances, the prosencephalon is divided into two parts-the cerebral hemispheres - which contain cavities known as the lateral ventricles. The two hemispheres are 
united by bridges or commissures, which have considerable classificatory importance. With the anterior region of the hemispheres olfactory lobes are associated.

In Cyclostomata, Ganoids, and Teleosteans, the fore brain has no nervous roof, but is covered by a epithelial pallium homologous with what is called the choroid plexus of the third ventricle in higher Vertebrates. This choroid plexus is a thin epithelium, with blood ressels in it. But in Elasmobranchs, Dipnoi, and Amphibians the basal parts of the fore brain have grown upwards to form a nervous roof, and this persists in higher Vertebrates.

The optic thalami (thalamencephalon, or tween-brain) form the second region of the adult brain. Hence arise the optic outgrowths, which form the optic nerves and some of the most essential parts of the eyes. The original cavity persists as the third ventricle of the brain; the thin roof gives off the dorsal pineal outgrowth or epiphysis, and uniting with the vascular pia mater, or brain membrane, forms a choroid plexus; the lateral walls become much thickened (optic thalami); the thin floor gives off a slight ventral evagination, or infundibulum, which bears the enigmatical pituitary body or hypophysis.

The Pituitary Body.-This is derived in part from the brain and in part from the mouth, and is extremely difficult to understand. It is apparently equivalent in part to the sub-neural gland of Tunicatcs, but this does not carry us much further. Dohrn connected it with two abortive gill slits, but the evidence seems insufficient. Beard has interpreted it as a
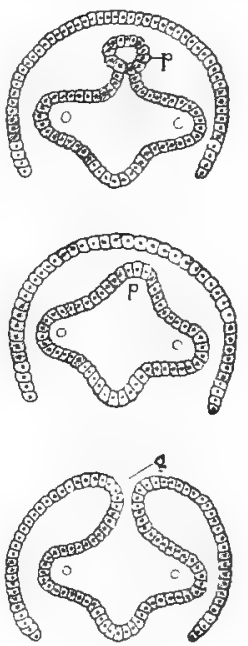

FIG. I39.-Origin of Pineal Body. (After BEARD.)

Lowest figure-a section through the first embryonic vesicle, while the medullary groove $(g)$ is still open; $o$, optic outgrowths. Middle figure shows beginning of pineal upgrowth $(p)$. Topmost figure show's a later stage.

residuum of the original mouth which Vertebrates are supposed to have possessed before the persistent one with which we are familiar was evolved, and of the innervation of that hypothetical structure, but again confirmation seems wanting. Of its physiological nature we 
know almost nothing, beyond that a pathological state of this organ is associated in man with certain diseases, e.g., acromegaly.

The Pineal Body. - The dorsal upgrowth (or epiphysis) from the roof of the thalamencephalon is represented, though to a varying extent, in all Vertebrates. It is terminally differentiated into a little body known as the pineal body. This was entirely an enigma until De Graaf discovered its eye-like structure in Anguis, and Baldwin Spencer securely confirmed this in the New Zealand "lizard" (Sphenodon) where the pineal body shows distinct traces of a retina.

In Elasmobranchs the pineal process is very long, and, perforating the skull, terminates below the skin in a closed vesicle. In the young frog it also comes to the surface above the skull, but degenerates in adolescence. In Splzenodon the stalk passes through the skull by the "parietal foramen," so that the "eye" itself lies close beneath the skin, the scales of which in this region are specialised and transparent.

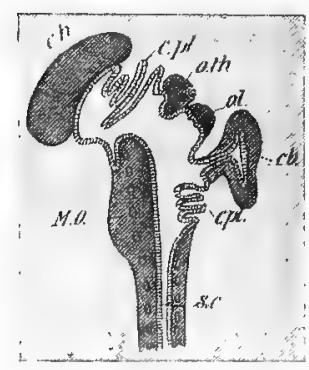

FIG. I40.-Diagram of the parts of the brain in Vertebrates. (After GASKLLL.)

c.t.., Cerebral hemispheres; c.ph., choroid plexus; o.th., optic thalami ; ol., optic lobes; $c b$., cerebellım; c.plo, choroid plexus; M.O., medulla oblongata; s.c., spinal cord.

pendent) degeneration. It has also been interpreted as an "organ for the perception of warmth." It may be, however, that the function discernible in some Reptiles is not primitive, but the result of a secondary modification of the structure in question. Thus, one of first interpretations (Dohrn's) connected the pineal and the pituitary outgrowths with a supposed passage of the original hypothetical mouth through the nerve cord.

The second primary vesicle of the brain undergoes little alteration, and forms the third region, that of the optic lobes (mesencephalon or mid brain) in the adult brain. The floor 
and lateral walls form the thickened crura cerebri, the roof becomes the two optic lobes, which are hollow in almost all Vertebrates. In Mammals a transverse furrow divides each optic lobe into two (corpora quadrigemina). The cavity of the vesicle becomes much contracted, and forms the narrow iter or aqueduct of Sylvius, a canal connecting the third ventricle with the fourth.

The third primary vesicle gives rise to the metencephalon, or hind brain, or region of the cerebellum, and to the myelencephalon, or after brain, or region of the medulla oblongata.

In the metencephalon the roof develops greatly, and gives rise to the cerebellum, which often has lateral lobes, and overlaps the next region. In the higher forms the floor forms a strong band of transverse fibres-the Pons Varolii.

From the region of the medulla oblongata most of the cranial nerves are given off. Here the roof, partly overlapped by the cerebellum, degenerates, becoming thin and epithelial, the cavity-called the fourth ventricle-is continuous with the canal of the spinal cord.

\section{Sumnary.}

(1) Cerebral hemispheres, prosencephalon, or fore brain. Note commissures, olfactory lobes and nerves, and first and second ventricles.

First Embryonic Vesicle.

Median Embryonic Vesicle.

Third Embryonic Vesicle.
Optic thalami, thalamencephalon, or tweenbrain. Note (a) optic, (b) pineal, (c) pituitary outgrowths, and the third ventricle.

(3) Optic lobes, mesencephalon, or mid brain. Note crura cerebri, and the aqueduct of Sylvius.

(4) Cerelvellum, metencephalon, or hind brain. Note Pons Varolii.

(5) Medulla oblongata, myelencephalon, or after brain. Note rudimentary roof, fourth ventricle, and origin of most of the cranial nerves.

Enswathing the brain, and following its irregularities is a delicate membrane-the pia mater-rich in blood vessels which supply the nervous system. Outside this, in higher Vertebrates, there is another nembrane-the arachnoid-which does not follow the minor irregulari- 
ties of the brain so carefully as does the pia mater. Thirdly, a firm membrane - the dura mater-lines the brain case, and is continued down the spinal canal. In lower Vertebrates the dura mater is clouble, in higher Vertebrates it is so in the region of the spinal cord, where the outer part lines the bony tunnel, while the inner ensheathes the cord itself. In Fishes the brain case is much larger than the brain, and a large lymph space lies between the dura and the pia mater.

An understanding of the relations of the different regions will be facilitated by a study of the following table which Dr. Gadow gives in his great work on Birds in Bronn's Thierreich :-

\begin{tabular}{|c|c|c|c|c|}
\hline Region. & FLOOR. & Sides. & Roof. & Cavity. \\
\hline Spinal cord. & $\begin{array}{l}\text { Anterior grey } \\
\text { and white com- } \\
\text { missure. }\end{array}$ & $\begin{array}{l}\text { White and grey } \\
\text { substance. }\end{array}$ & $\begin{array}{l}\text { Posterior commis- } \\
\text { sure. }\end{array}$ & Central canal. \\
\hline $\begin{array}{l}\text { Myelen- } \\
\text { cephalon. }\end{array}$ & \multicolumn{2}{|c|}{ Medulla oblongata. } & $\begin{array}{l}\text { Epithelium } \\
\text { choroid plexus. }\end{array}$ & $\begin{array}{l}\text { Posterior part of } \\
\text { fourth ventricle. }\end{array}$ \\
\hline $\begin{array}{l}\text { Meten- } \\
\text { cephalon. }\end{array}$ & $\begin{array}{l}\text { Commissural } \\
\text { part. }\end{array}$ & $\begin{array}{l}\text { Pedunculi or crura } \\
\text { cerebri. }\end{array}$ & Cerebellum. & $\begin{array}{l}\text { Anterior part of } \\
\text { fourth ventricle. }\end{array}$ \\
\hline Mesencephalon. & $\begin{array}{l}\text { Pedunculi } \\
\text { cerebri. }\end{array}$ & $\begin{array}{l}\text { Cortex of optic } \\
\text { lobes. }\end{array}$ & $\begin{array}{l}\text { Anterior commis- } \\
\text { sure, velum of Syl- } \\
\text { vius. }\end{array}$ & $\begin{array}{l}\text { Aqueduct of Syl- } \\
\text { vius and lateral } \\
\text { extensions. }\end{array}$ \\
\hline $\begin{array}{l}\text { Thalamen- } \\
\text { cephalon. }\end{array}$ & $\begin{array}{l}\text { Infundibulum, } \\
\text { hypophysis, } \\
\text { chiasma. }\end{array}$ & $\begin{array}{l}\text { Inner part of optic } \\
\text { lobes and optic thal- } \\
\text { ami. }\end{array}$ & $\begin{array}{l}\text { Epiphysis and } \\
\text { epithelium of cho- } \\
\text { roid plexus. } \\
\text { Corpus callosum. } \\
\text { Anterior commis- } \\
\text { sure. }\end{array}$ & Third ventricle. \\
\hline $\begin{array}{l}\text { Prosen- } \\
\text { cephalon. }\end{array}$ & $\begin{array}{l}\text { Corpus stria- } \\
\text { tum. } \\
\text { Lamina ter- } \\
\text { minalis. } \\
\text { Olfactory } \\
\text { lobes. }\end{array}$ & Cerebral he & emisphere. & $\begin{array}{l}\text { Lateral ven- } \\
\text { tricles. }\end{array}$ \\
\hline
\end{tabular}

\section{The Spinal Cord.}

After the formation of the brain vesicles, the remainder of the medullary canal forms the spinal cord.

The canal is for a time continuous posteriorly with the food canal beneath, so that a $\supset$-shaped tube results. The connection between them is called the neurenteric canal, and though it is only temporary its frequent occurrence is of much interest.

The wall of the medullary canal becomes very much 
thickened, the roof and floor grow less rapidly, and thus the cord is marked by ventral and dorsal longitudinal furrows. At the same time, the canal itself is constricted, and persists in the fully formed structure only as a minute canal lined by ciliated epithelium, and continuous with the cavity of the brain. It can hardly be said to have any function; it may be simply the result of a developmental necessity. But Sutton and Gaskell have independently suggested that the central canal of the nervous system represents a disused alimentary passage, which has been replaced by surrounding nervous material, and which ceased to be functional when the permanent gut became a tube open at each end. This suggestion, however, if indeed it was serious, has not been accepted by any morphologist.

In the cord it is usually easy to distinguish an external region of white matter, composed of medullated nerve fibres, and an internal region of grey matter, containing ganglionic cells, and non-medullated fibres.

The arrangement of the grey matter, together with the longitudinal fissures, give the cord a distinct bilateral symmetry, which is sometimes obvious at a very early stage.

The brain substance is also composed of grey and white matter, but there, at any rate in higher forms, the arrangement is very complicated.

Concerning the development of the peripheral nervous system there is far less certainty than with regard to the central.

The motor nerves, even the motor parts of the cranial nerves, appear to arise as fibrillar or as cellular outgrowths of the central nervous system. All the sensory nerves take their origin from peripheral ganglia, and the root fibres grow thence into the central system. According to some embryologists, certain sensory peripheral nerves, e.g., the lateralis of the tenth, arise in situ from the sensory epithelium. 
Cranial Nerves.

The origin and distribution of the cranial nerves may be summarised as follows :-

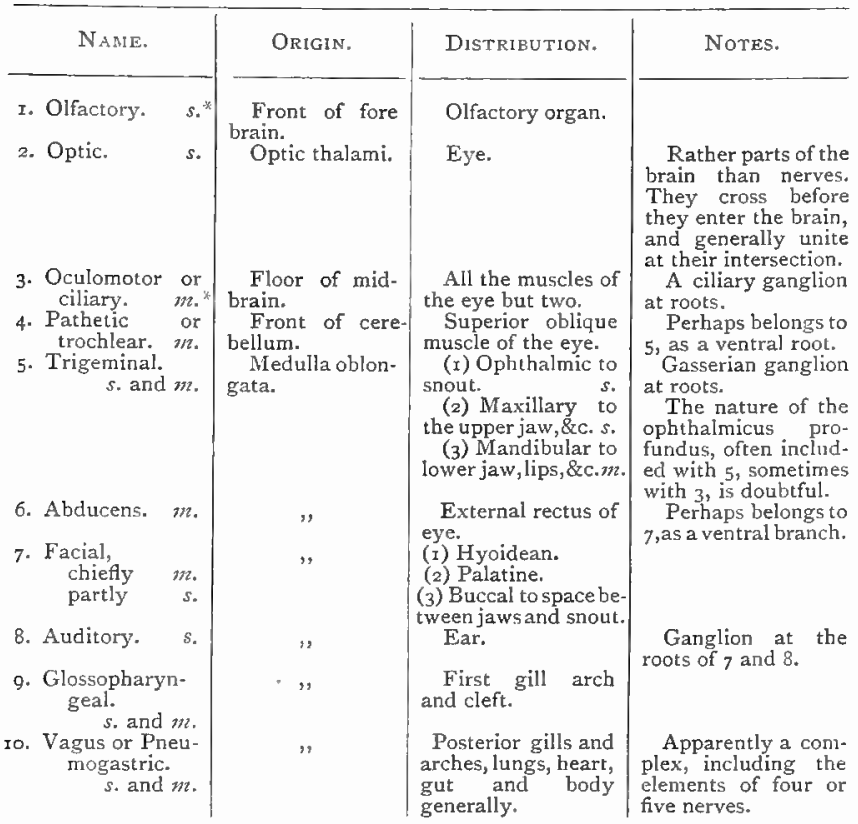

The fourth or pathetic nerve is peculiar anong motor nerves in that it arises from the extreme dorsal summit of the brain, between the mid and hind brain, from the region known as the "valve of Vieussens." In Fishes, the seventh nerve is mainly" a nerve of special sense ; in higher Vertebrates it has lost most of its sensory branches, and become chiefly motor.

* The letter $s$. is a contraction for sensory or afferent, i.e., transmitting impulses from a sensitive area to the centre; and $m$. is a contraction for motor or efferent, i.e., transmitting impulses from the centre to the body.

There is much uncertainty in regard to the morphological value of the various cranial nerves, but the following conclusions are important :-

(I) The nerves arise either as outgrowths of the central system, or as specialisations of peripheral cells. Each spinal nerve has two rootsa dorsal and a ventral, but in most cases at least a cranial nerve has primitively a single dorsal root developing from a neural ridge of the dorsal surface of the brain. In many cases this root divides 
into "dorsal," "ventral," and other branches. As these typically innervate a gill arch and slit, as may be well studied in 9, the branches may be called (as Beard proposes) supra-branchial (dorsal), postbranchial, pre-branchial, \&c. In the course of growth the nerve often shifts from the position whence its root originated.

(2) Some of the cranial nerves mark distinct segments of the head, while others are secondary derivatives. It is likely that $1,3,5,7,8,9$, and several parts of Io mark segments. It is possible that the oculomotor is a ventral root associated with the third or ciliary nerve, that the trochlear is a ventral root of the trigeminal, that the abducens is a ventral root of the facial.

(3) It is possible that each truly segmental nerve supplied a primitive gill slit, as 7 supplies the spiracle, 9 the first branchial, to the second, third, fourth, and fifth branchials.

(4) It is likely that each segmental yerve was associated with a branchial sense organ (Beard and Froriep). These organs arise above the gills, and grow thence into various parts of the head, and along the trunk as the "lateral line." It is possible a branchial sense organ

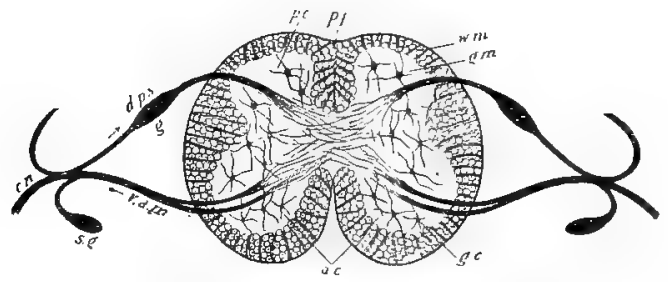

FIG. I4I.-Diagrammatic section of Spinal Cord.

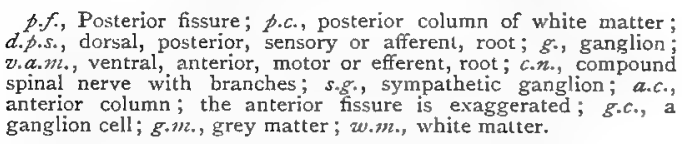

lay over each primitive gill cleft, and had an associated ganglion. The ganglia known as ciliary, gasserian, \&c., may be the ganglia of branchial sense organs, and it seems that parts of them arise in development independently of the brain. It may be that nose and ear were originally branchial sense organs.

\section{Spinal Nerves.}

Each spinal nerve has two roots-a dorsal, posterior, or sensory, and a ventral, anterior, or motor. These arise separately and independently, but combine in the vicinity of the cord to form a single nerve. The dorsal root exhibits at an early period a large ganglionic swelling-the spinal 
ganglion; the ventral root is apparently non-ganglionated. Moreover, the dorsal root has typically a single origin (as in the cranial nerves) while that of the ventral root is often multiple.

The dorsal roots are outgrowths of a continuous ridge or crest along the median dorsal line of the cord. As the cord grows the nerve roots of each side become separated. They shift sidewards and downwards to the sides of the cord. The ventral roots are later in arising; they spring as outgrowths from the latero-ventral angle of the cord.

Beard maintains that the spinal ganglia do not arise from the spinal cord, but have an independent origin from the deeper layers of the epiblast.

According to most authorities, the sympathetic ganglia are offshoots from the same rudiment as that from which the dorsal ganglia arise, and it is possible that they are the more or less vagrant ganglia of the ventral roots, with which they are connected by small fibres. On this view (Gaskell's) both roots may be said to be ganglionated. But the ganglion of the dorsal root is stationary in position, and the nerve fibres which pass through it come both from the visceral (splanchnic) and from the peripheral somatic parts, separating from one another within the cord. On the other hand, the supposed ganglion (sympathetic) of the ventral root is more or less vagrant, and off the main line of the root, from which it receives small fibres passing to splanchnic or visceral structures.

\section{Sense Organs.}

The central nervous system has doubtless arisen in the course of history from the insinking of external nerve cells; it does arise in development as an involution of ectoderm or epiblast. The same layer gives origin to the essential parts of the sense organs. The Vertebrate eye is formed in great part as an outgrowth from the brain, but as the brain is itself an involution of epiblast, the eye may be also referred to external nerve cells.

Branchial Sense Organs. - In many Fishes and Amphibians there are lateral sense organs which form the "lateral lines," while others lie in the head and were, in all likelihood, primitively connected with gill clefts. In Sauropsida and Mammals these branchial sense organs are no longer distinct as such.

The Nose.-It is possible that the sensory pits of skin which form the nasal sacs are two branchial sense organs. They are lined by epithelium in great part sensory, and are connected posteriorly with the olfactory nerves. In all Fishes, except Dipnoi, the nasal sacs remain blind; in 
Amphibians and in all the higher Vertebrates, they open posteriorly into the cavity of the mouth, and serve for the entrance of air. The peculiar nostril of hagfish and lamprey is referred to in the chapter on Cyclostomata.

The Ear in Invertebrates is formed by a simple invagination of the ectoderm forming a little sac, which may become entirely detached from the epidermis, or may retain its primitive connection: so in Vertebrates, at an early stage an insinking forms the auditory pit. This sinks further in, and the originally wide opening to the exterior becomes a long narrow tube. In Elasmobranchs, which exhibit many primitive features, this condition is retained in the adult, in other Vertebrates the tube loses its connection with the exterior, and becomes a blind prolongation of the inner ear-the aqueductus vestibuli, or ductus endolymphaticus.

The auditory vesicle, at first merely a simple sac, soon becomes very complicated. It divides into two chambers, the larger utriculus and the smaller sacculus. From the utriculus three semicircular canals are given off, except in the lamprey and hag, which have two and one respectively. From the sacculus an outgrowth called the cochlea or lagena originates; it is little more than a small hollow knob in Fishes and Amphibians, but becomes large and important in Sauropsida and Mammals.

As this differentiation of the parts of the internal ear takes place, the lining epithelium also becomes differentiated into flattened covering cells and sensory auditory cells. The auditory cells are arranged in patches to which branches of the auditory nerve are distributed. With these sensory patches calcareous concretions (otoliths) are associated, except in the cochlea of Mammals.

The fact that lime salts are often deposited in the skin, and that the ear sac arises as an insinking of epiblast, may perhaps shed some light on the origin of otoliths.

The parts which we have so far considered constitute together the membranous labyrinth of the ear. Round about them the mesoblast (mesenchyme) forms a two-layered envelope. Its inner layer disintegrates to produce a fluid, the perilymph, which bathes the whole outer surface of the membranous labyrinth. Its outer layer forms a firm case, the cartilaginous or bony labyrinth, surrounding the internal ear. The membranous labyrinth itself contains another fluid, the endolymph.

Certain facts of development suggest that the ear, like the olfactory organ, may be a branchial sense organ.

With regard to the function of the parts of the ear, the semicircular canals are believed by many to be concerned with the appreciation of a 
change in the direction or velocity of movement. How far the ears of lower Vertebrates (e.g., Crustacea and Molluscs), are adapted for any function except this is still doubtful, and we can hardly see that any other would be of much use to purely aquatic animals. It seems likely at any rate that the primitive function of the ear was the perception of vibrations, and that from this both the sense of hearing, and the sense of equilibration have been differentiated.

It is in accordance with the facts mentioned above, that we rarely find in Fishes any special path by which impressions of sound may travel from the external world to the ear. In Amphibians and higher Vertebrates, however, the ear has sunk further into the recesses of the sliull, and a special path for the sound is present. In Elasmobranchs the spiracle, or first gill cleft, is situated immediately behind the ear ; in higher forms, according to many authors, this first gill cleft is metamorphosed into the conducting apparatus of the ear. In development a depression beneath the closed gill cleft unites with an outgrowth from the pharynx, and thus forms the tympanic cavity, which communicates with the back of the mouth by the Eustachian tube. The tympanic cavity is closed externally by the drum or tympanum, which may be flush with the surface, as in the Frog, or may lie at the end of a narrow passage which in many Mammals is furnished externally with a projection or pinna. In Amphibia and Sauropsida the tympanic cavity is traversed by a bony rod-the columella, which extends from the drum to the fenestra ovalis, a little aperture in the wall of the bony labyrinth. In Mammals this is replaced by a chain of three ossicles, an outermost malleus, a median incus, an internal stapes.

The homologies of these ossicles are still uncertain. The following is Hertwig's interpretation :-

Malleus = Articular + angular elements of Meckel's cartilage.

Incus = Palato-quadrate of lower Vertebrates.

Stapes of Mammals has double origin, being formed from the upper part of hyoid arch tan ossification from the wall of the ear capsule $=($ wholly ? $)$ columella of Birds, Reptiles, and Amphibians.

The Eye.-There is no eye in Amphioxus, it is rarely more than larval in Tunicates, it is degenerate in Mixine, and in the young lamprey. In higher forms the eye is always present, though occasionally degenerate, e.g., in fishes from caves or from the deep sea. It is hidden under the skin in Proteus, an amphibian cave dweller, and in the subterranean amphibians like Cacitia, very small in a few snakes and lizards, and abortive as to its nerves in the mole.

The adult eye is more or less globular, and its walls consist of several distinct layers. The innermost layer bounding the posterior part of the globe is the sensitive retina, innervated by fine branches from the optic nerve. It may be compared to the nervous matter of the brain, from which, 
indeed, it arises. Outside of the retina is a pigmented epithelium, and outside of this a vascular membrane; together these are often called the choroid. The vascular part may be compared to the pia matter covering the brain, and like it is derived from mesoblast. Outside of the choroid is a protective layer or sclerotic, comparable to, and continuous with, the dura mater covering the brain, and also mesoblastic in origin. Occupying the front of the globe is the crystalline lens, a clear ball derived directly from the skin. It is fringed in front by a pigmented and muscular ring - the iris, which is for the most part a con-

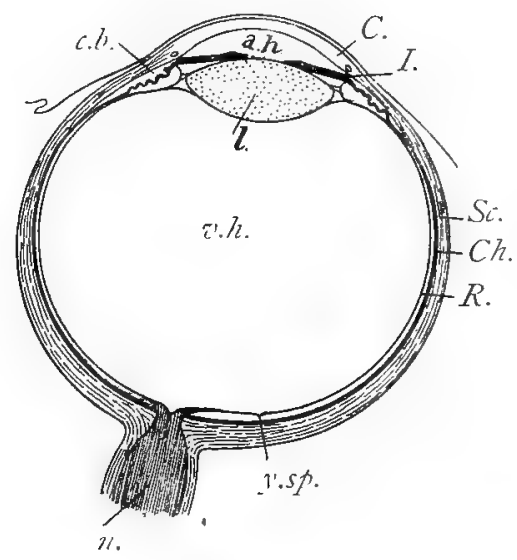

FIG. 142.-Diagram of the Eye. (Compiled).

C., Cornea ; $a . h$. , aqueous humour; $c . b .$, ciliary body; $l .$, lens; $I$, Iris; Sc., sclerotic; $C h$, choroid ; $R$., retina; $i . h .$, vitreons humour; $y . s p$., yellow spot ; $u_{\text {. }}$, optic nerve.

tinuation of the choroid. 'The space enclosed by the iris in front of the lens is called the pupil. Protecting and closing the front of the eye is the firm cornea continuous with the sclerotic, and covered externally by the conjunctiva -a delicate epithelium continuous with the epidermis. Between the cornea and the iris is a lymph space containing aqueous humour, while the inner chamber behind the lens contains a clear jelly - the vitreous humour. The lens is 
moored by "ciliary processes" of the choroid, and its shape is alterable by the action of accommodating muscles arranged in a circle at the junction of iris and sclerotic. In many Reptiles, and in Birds, a vascular fold called the pecten projects from the back of the eye into the vitreous humour. A similar fold in Fishes (processus falciformis) ends in a knot-like structure in the lens. The retina is a very complex structure with several layers of cells, partly supporting and partly nervous; the layer next the
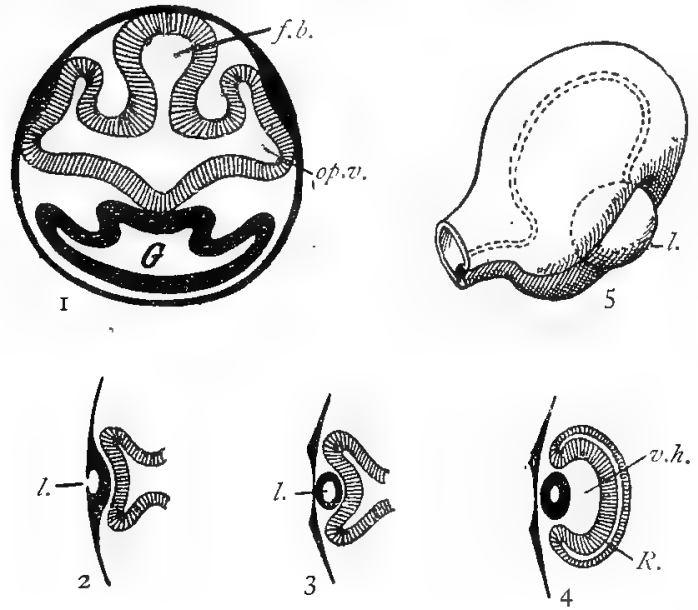

FIG. 143.-Developmental of the eye. (After BaLFour and HerTWig.)

I. Section through first embryonic vesicle, showing outgrowth of optic vesicles $\left(\not p, z\right.$.) to meet the skin; $f . b_{.}$, fore brain; $G$, the gut. $2-4$. The formation of the lens (l) from the skin, and the modification of the optic vesicle into an optic cup; $R$., retina; $v$.h., vitreous humour.

5. External aspect of embryonic eye; $l .$, lens.

vitreous humour consists of nerve fibres, while that furthest from the rays of light and next the pigment epithelium consists of sensitive rods and cones. The region where the optic nerve enters, and whence the fibres spread, is called the blind spot, and near 
this there lies the most sensitive region-the yellow spot, with its fovea centralis, where all the layers of the retina have thinned off except the cones.

Among the extrinsic structures, must be noted the six muscles which move the eyeball, the upper and lower eyelids which are often very slightly developed, and the third eyelid or nictitating membrane. Above Fishes there is a lachrymal gland associated with the upper lid, and a Harderian gland associated with the nictitating membrane. In Mammals there are also Meibomian glands. The secretions of all these glands keep the surface of the eye moist.

While the medullary groove is still open, the eyes arise from the first vesicle of the brain as hollow outgrowths or primary optic vesicles. Each grows till it reaches the skin, which forms a thickened involution in front of it. This afterwards becomes the compact lens. Meantime it sinks inwards, and the optic vesicle becomes invaginated to form a double walled optic cup. The two walls fuse, and the one next the cavity of the cup becomes the retina, while the outer forms the pigmented epithelium. Meanwhile surrounding mesoblast has insinuated itself past the lens into the cavity of the optic cup, there forming the vitreous humour, while externally the mesoblast also forms the vascular choroid, the firm often cartilaginous sclerotic, the inner layer of the cornea, \&c. Along the thinned stalk of the optic cup the optic nerve is developed. Its protective sheath is continuous with the sclerotic of the eye and the dura mater of the brain. As the nerves enter the optic thalami, they always cross one another in a chiasma, and their fibres usually interlace as they cross.

\section{The Alimentary System and Associated Structures.}

The alimentary tract exhibits much division of labour, for not only are there parts suited for the passage, digestion, and absorption of the food, but there are numerous outgrowths, e.g., lungs and allantois, which have nothing to do with the main function of the food canal.

By far the greater part of the food canal is lined by endoderm or hypoblast, and is derived from the original cavity of the gastrula-the primitive gut or archenteron. This is the mid gut or mesenteron. But the mouth cavity is lined by ectoderm, invaginated from in front to meet the 
mid gut. This region is the fore gut or stomatodæum. Finally, there is usually a slight posterior invagination of ectoderm, forming the anus. This is the hind gut or proctodæum.

Associated with the mouth cavity or stomatodæum are (a) teeth (ectodermic rudiments of enamel combined with a mesodermic papilia which forms dentine or ivory); (b) from Amphibians unwards special salivary glands; $(c)$ a tongue or muscular and sensitive outgrowth from the floor. The tongue develops as a fold of mucous membrane in front of the hyoid, and afterwards becomes increased by growth of connective tissue, \&c. In larval Amphibians muscle strands find their way into it, and it seems likely, as Gegenbaur has recently indicated, that their original function was to compress the glands. As they gained strength they became able for a new function, that of moving the tongue. In Myxine, Dipnoi, and higher animals, the nasal sac opens posteriorly into the mouth; in some Reptiles and Birds, and in all Mammals, the cavity of the mouth is divided by a palate into an upper nasal and lower buccal portion.

The origin of the oral aperture is not quite certain. In Tunicates it is formed by an ectodermic insinking which meets the archenteron; in Amphioxus it seems to be formed as a pore in an ectodermic disc; in other cases it is a simple ectodermic invagination, or it may owe its origin to the coalescence of an anterior pair of gill clefts innervated by the fifth nerve. If the last interpretation be true, its origin illustrates that change of function which has been a frequent occurrence in evolution. But if the mouth arose from a pair of gill clefts, and in some cases it actually has a paired origin, then there must have been an older mouth to start with. Thus Beard in his brilliant morphological studies, distinguishes between " the old mouth and the new." The new mouth is supposed to have resulted, as Dohrn suggested, from a pair of gill clefts; the old mouth was an antecedent stomatodxum, of which the so-called nose of Myxine and the oral hypophysis of higher forms may be vestiges. This theory harmonises with the observations of Kleinenberg, on the development of the mouth in some Annelids (Lopadorhynchus), in which the larval stomatodaum is replaced by a paired ectodermic invagination.

The mouth cavity leads into the pharynx, on whose walls there are the gill clefts. Of these the maximum number is eight, for the hundred slits in Amphioxus cannot be directly compared with the ordinary clefts. If we exclude the hypothetical clefts, such as those possibly represented in the mouth, the first pair form the spiracles-well seen in skates. In the position of the spiracles the Eustachian tubes of higher Vertebrates develop. In front of the spiracle there is sometimes a spiracular cartilage, which Dohrn dignifies as a distinct arch. The other gill clefts are associated with gills in Fishes and Amphibians while in 
Sauropsida and Mammals, in which there are no gills, four "visceral" clefts persist as practically functionless residual structures. In some cases their openings are very evanescent. The clefts are bordered by the branchial arches, and supplied by blood vessels and nerves.

With the anterior part of the alimentary canal, two strange structures are associated-the thyroid and the thymus.

The thyroid gland arises as a diverticulum from the ventral wall of the pharynx. It may be single (as in some Mammals), or bilobed (as in Birds), or double (as in some Mammals and Amphibians), or diffuse (as in bony fishes). Only in the larval lamprey does it retain its original connection with the pharynx, and is then a true gland.

As to its morphological nature, its mode of origin suggests comparison with the hypobranchial groove in Amphioxus and the endostyle of Ascidians. According to Dohrn it is a residue of the gill cleft between the hyo-mandibular and the hyoid.

Almost the only light which has been cast on the physiological nature of the thyroid is from the pathological side. Goitre and Derbyshire neck are associated with an enlargement and diseased state of this organ, and myxoedema with its degeneration or absence. As injection of extract of sheep's thyroid, or even eating this organ, alleviates myxcedema, it is concluded that the thyroid must naturally have some specific effect on the large quantity of blood which flows through it. It is probably safe to say that the thyroid aids in keeping the blood at a certain standard of health, perhaps through some specific ferment.

The thymus arises as a dorsal endodermic thickening where the outgrowths which form the gill clefts meet the ectoderm. It may be associated with a variable number of clefts-five in the skate, four in Teleosteans, three in the lizard, one in the chick ; in mammals it often seems to degenerate after youth. As it has from its first origin a distinct lymphoid nature, and apparently forms leucocytes, it has been interpreted (Beard) as a structure adapted for the phagocytic protection of the gills from Bacteria, parasites, and the effects of injury. If this be so, we can understand its diminishing importance in Sauropsida and Mammalia, where its place may be to some extent taken by the palatal and pharyngeal tonsils, which are believed by some (Stöhr, Killian, Gulland) to have a similar phagocytic function.

The pharynx leads into the gullet or œsophagus, which is a conducting tube, and this into the digestive stomach, which is followed by the digestive, absorptive, conducting intestine, ending in the rectum and anus.

From the csophagus, the air- or swim-bladder of most 
Fishes, and the lungs of higher Vertebrates, grow out. The air bladder usually lies dorsally and is almost always single ; the lungs lie ventrally and are double, though connected with the gullet by a single tube. It is not certain that these outgrowths are homologous, though the air bladder of Dipnoi acquires the functions of a lung.

'The beginning of the intestine gives origin to the liver which regulates the composition of the blood and secretes bile, and to the pancreas which secretes digestive juices.

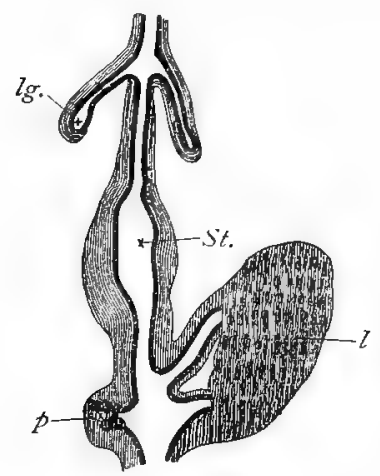

FIG. I44. - Origin of lungs, liver, and pancreas in the chick. (After GæTTE.)

The mesoderm is shaded; the endo. derm dark.

L., One of the lungs; st., stomach; $l$., liver; $p$., pancreas.

The pancreas has often a multiple rudiment.

From the hindmost region of the gut, the allantois grows out in all animals from Amphibians onwards. In Amphibians it is represented by an unimportant bladder; in the higher Vertebrates it is a vascular foetal membrane concerned with the respiration or nutrition of the embryo, or both.

Cilia are very common on the lining of the intestine in Invertebrates, but they are much rarer in Vertebrates. Yet as they occur in Amphioxus, lampreys, many fishes, Protopterus, some Amphibians, and in embryonic Mammals, it seems not unlikely that the alimentary tract was originally a ciliated tube.

Speculative.-The primitive gut was probably a smooth straight tube, but the rapid multiplication of well nourished cells would tend to its increase in diameter and in length. But on increase in both directions the slower growth of the general body would impose limitations, and in this we may find the immediate growth-condition determining the origin of folds, crypts, creca, and coils, which would be justified by the increase of absorptive and digestive surface. There are regular longitudinal folds in Myxine, cross folds traversing these would form crypts, which may be exaggerated into the pyloric creca of Teleosteans and Ganoids, while other modifications would give rise to "spiral valvess" and the like. In the same way it may be suggested that the numerous important 
outgrowths of the mid gut, such as lungs, liver, pancreas, and allantois, so thoroughly justified by their usefulness, may at first have been due to necessary conditions of growth-to the high nutrition, rapid growth, and rapid multiplication of the endoderm. It may be noted that in the development of the Amphibian Necturus, there are hints of more numerous endodermic diverticula (Platt). Even the notochord, which arises as a median dorsal fold, may be speculatively compared to a typhlosole-folded outwards instead of inwards. The future elaboration of the organs, which arise as outgrowths of the gut, would, however, depend on many factors, such as their correlation with other parts of the body, and would at each step be affected as usual by natural selection.

It is often said that, in some cases at least, as in lamprey, frog, and newt, the blastopore or opening of the primitive gastrula cavity persists as the anus of the adult, but it seems doubtful whether the anus is not always a new formation. In many cases, at least, an ectodermic invagination or proctodæum meets the closed archenteron, and at the junction the two epithelial layers give way, so that an open tube is formed.

The formation of the anus does not take place close to the posterior end of the primitive gut, but at a point some short distance in front of this. In consequence the so-called post-anal gut is formed. This is continuous with the neurenteric canal, and so communicates with the neural canal. The post-anal gut attains in Elasmobranchs a relatively considerable length. It has been very frequently found in Vertebrates, and is probably of universal occurrence. After a longer or shorter period it becomes completely atrophied, and with it the communication between neural and alimentary canals is completely destroyed.

\section{Alimentary System.-Summary.}

\begin{tabular}{l} 
REgIoN of THE GuT. \\
\hline Mouth cavity, \\
or Stomatodzeum, \\
or Fore gut, \\
originating as an ectodermic \\
invagination, or from a co- \\
alescence of two gill clefts.
\end{tabular}

Pharynx, gullet or cesophagus, stomach, small intestine, large intestine, and rectum; = the mesenteron or mid gut, originating from the cavity of the gastrula, the archenteron or primitive gut ; lined by endoderm.

Anal Region, or Proctodeum, or Hind gut.

Where the mouth of the gastrula persists, it forms the terminal aperture of the gut, in other cases there is an ectodermic invagination or proctodaeum.

\begin{tabular}{|c|c|}
\hline OUTGROWTHS. & Associated Structures. \\
\hline & $\begin{array}{l}\text { Teeth. } \\
\text { Salivary glands. } \\
\text { Tongue. } \\
\text { (Note relation between the } \\
\text { mouth and the oral part of } \\
\text { the hypopbysis.) }\end{array}$ \\
\hline \multirow[t]{2}{*}{$\begin{array}{l}\text { Thyroid yand the gill } \\
\text { Thymus clefts. } \\
\text { Air bladder; lungs. } \\
\text { Liver. } \\
\text { Pancreas. } \\
\text { Allantois. }\end{array}$} & $\begin{array}{l}\text { With the several out } \\
\text { growths the surrounding } \\
\text { mesodern becomes associated } \\
\text { often to a great extent. } \\
\text { (Note also the origin of the } \\
\text { notochord as an axial differ- } \\
\text { entiation of cells along the } \\
\text { mid dorsal line of the embry- } \\
\text { onic gut.) }\end{array}$ \\
\hline & $\begin{array}{l}\text { In some Fishes, all Amphi- } \\
\text { bians, all Sauropsida, and the } \\
\text { Prototherian Mammals, the } \\
\text { terminal part of the gut is a } \\
\text { cloaca or common chamber } \\
\text { into which the rectum, the } \\
\text { urinary and the genital ducts } \\
\text { open. }\end{array}$ \\
\hline
\end{tabular}




\section{Body Cavity.}

In Amphioxus a paired pouch grows out from the archenteron. Almost at once this becomes divided up on either side to form a series of small sacs, the cavities of which form ultimately the true body cavity or cœlome. According to Hertwig, this is in type, the method of formation of the cœlome throughout the Vertebrata. In the other Vertebrates, owing to modified processes of development, probably first arising from the presence of much yolk, we have solid cell masses growing out in place of hollow sacs, but the

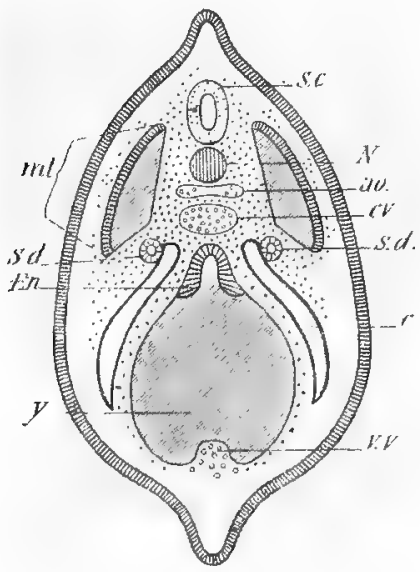

FIG. I45. - Transverse section through a Teleostean Embryo (diagrammatic). (After ZIEGLER.)

s.c., Spinal cord; $N$., notochord; ao., aorta; c.v., cardinal veins (united); s.d., segmental duct; $c .$, coelome or pleuro-peritoneal cavity ; v.v., position of median vitelline vein; $y$., yolk; En., Endoderm of gut ; s.d., segmental duct; $m t$., myotome. The dots represent mesenchyme cells, the little circles blood corpuscles. cavities which appear later, apparently by splitting of the cell mass, are in reality the retarded cavities of true gut pouches.

\section{Vascular System.}

From Cyclostomata onwards the blood fluid contains red corpuscles, i.e., cells coloured with hæmoglobin-a pigment which readily forms a loose union with oxygen, and bears this from the exterior (gills or lungs) to the tissues. These pigmented cells are usually oval and nucleated. In all Mammals except Camelidæ they are circular. Moreover, the full grown red corpuscles of Mammals have no visible nuclei. The blood fluid also contains uncoloured nucleated amœeboid cells, the white corpuscles or leucocytes, of much physiological importance, e.g., by bearing food particles from one part of the body to another, 
and by attacking and destroying micro-organisms within the body.

The heart receives blood from veins, and drives it forth through arteries. Its contractions in great part cause the inequality of pressure which makes the blood flow. It lies in a special part of the body cavity known as the pericardium, and develops from a single blood vessel in Cyclostomata, Fishes, and Amphibians, from a pair in Reptiles, Birds, and Mammals.

The receiving region of the heart is formed by an auricle or by two auricles, thence the blood passes into the muscular ventricle or ventricles, and is driven outwards. Except in adult Birds and Mammals the veins from the body enter the auricle (or the right auricle if there are two) by a porch known as the sinus venosus. In Fishes (except Teleosteans) and in Amphibians, the blood passes from the ventricle into a valved conus arteriosus which seems to be a continuation of the ventricle. In Teleosteans there is a superficially similar structure, but without valves and non-contractile, and apparently developed from the aorta, not from the ventricle; it is called the bulbus arteriosus, and may occur along with the conus arteriosus in other Fishes. In Vertebrates higher than Amphibians the conus is, to say the least, less distinct.

In Cyclostomata, and in all Fishes except Dipnoi, the heart has one auricle and one ventricle, and contains only impure blood, which it receives from the body and drives to the gills, whence purified it flows to the body.

In Dipnoi, the heart is incipiently three chambered.

In Amphibians, the heart has two auricles and a ventricle. The right auricle always receives venous or impure blood from the body, the left always receives arterial or pure blood from the lungs. The single ventricle of the Amphibian heart drives the blood to the body and to the lungs.

In all Reptiles, except Crocodilia, the heart has two auricles and an incompletely divided ventricle. By means of the partition in the ventricle much of the venous blood is sent to the lungs; indeed the heart, though possessing only three chambers, works almost as if it had four.

In Crocodilia, there are two auricles and two ventricles. But the dorsal aorta, which supplies the posterior parts of the body, is formed from the union of two aortic arches, one from each ventricle. Therefore it contains mixed blood.

In Birds and Mammals, the heart has two auricles and two rentricles, 
and one aortic arch supplies the body with wholly pure blood. This aortic arch always arises from the left ventricle, but in Bircls it curves over the right bronchus, i.en, is a right aortic arch, and in Mammals over the left, i.e, is a left aortic arch. Impure blood from the body enters the right auricle, passes into the right ventricle, is driven to the lungs, returns purified to the left auricle, enters the left ventricle, and is driven to the body.

The arterial system of a fish consists of a ventral aorta continued forwards from the heart, of a number of arching vessels diffusing the impure blood on the gills, and of efferent vessels collecting the purified blood into a dorsal aorta which runs along under the backbone and supplies the body.

So in the embryo of higher Vertebrates the same arrangement persists, though there are no gills beyond Amphibians. From a ventral arterial stem arches arise, which are connected so as to form the roots of the dorsal aorta. This aorta gives off vessels to the body, while in embryonic life it sends important vitelline arteries to the yolk, and (in Reptiles, Birds, and Mammals) equally important allantoic arteries to the allantois.

Returning to the arterial system of a fish, we must consider the arches more carefully, and compare them with those of Sauropsida and Mammals, where they are no longer connected with functional gill clefts, and also with those of Amphibians, where the complications due to lungs, \&c., begin.

\begin{tabular}{|c|c|c|}
\hline Fishes. & AMPHIBIANS. & Sauropsida and Maminals. \\
\hline $\begin{array}{l}\text { (a) Mandibular aortic arch } \\
\text { usually aborts; has } \\
\text { a persistent trace in } \\
\text { Elasmobranchs (spir- } \\
\text { acular artery). }\end{array}$ & $\begin{array}{l}\text { Aborts, or is not de- } \\
\text { veloped. }\end{array}$ & $\begin{array}{l}\text { At most merely em- } \\
\text { bryonic. }\end{array}$ \\
\hline $\begin{array}{l}\text { (b) Hyoid aortic arch } \\
\text { aborts, or is rudi- } \\
\text { mentary, persists in } \\
\text { Elasmobranchs and } \\
\text { some Ganoids. }\end{array}$ & Aborts. & $\begin{array}{l}\text { At most merely em. } \\
\text { bryonic. }\end{array}$ \\
\hline (c) Ist branchial. & Carotid. & Carotid. \\
\hline (d) and branchial. & $\begin{array}{l}\text { Systemic arches, } \\
\text { unite to form } \\
\text { dorsal aorta. }\end{array}$ & $\begin{array}{l}\text { Systemic. Only the right } \\
\text { persists in Birds; only } \\
\text { the left in Mammals. }\end{array}$ \\
\hline (e) 3rd branchial. & $\begin{array}{l}\text { Rudimentary or dis- } \\
\text { appears. }\end{array}$ & $\begin{array}{l}\text { Possibly the pulmonary } \\
\text { (unless that be } f \text {.). }\end{array}$ \\
\hline $\begin{array}{l}(f) 4^{\text {th }} \text { branchial (gives } \\
\text { off artery to " lung" } \\
\text { of Dipnoi). }\end{array}$ & Pulmonary. & $\begin{array}{l}\text { The pulmonary (unless } \\
\text { that be } e \text {.). }\end{array}$ \\
\hline
\end{tabular}


The important features in the development of the venous system are as follows:-

(a) In the embryo the vitelline veins bring back blood from the yolk sac, at first directly to the heart, and later to the liver. Into these veins, blond returned from the intestine is poured in increasing quantity by other veins. In the adult these persist to form the hepatic portal system by means of which blood from the

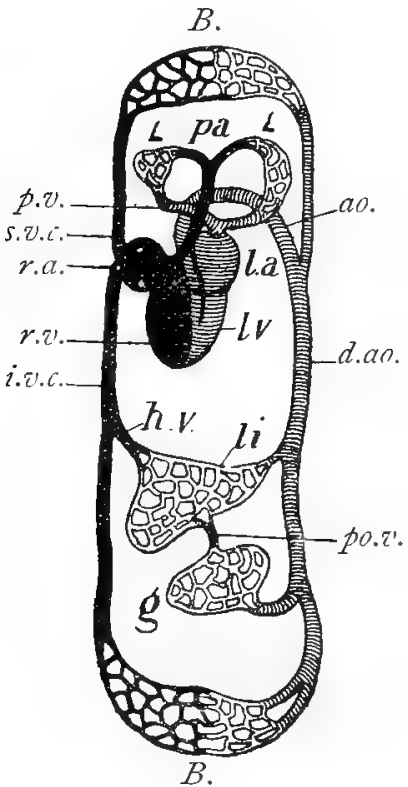

FIG. I46.-Diagram of Circulation. (After LEUNIS.)

r.a., Right auricle receiving superior vena cava (s.z.c.) and inferior vena cava $\left(i_{*} z_{1} c_{n}\right) ; r_{.} v_{n}$, right ventricle; $\not . a .$, pulmonary artery to

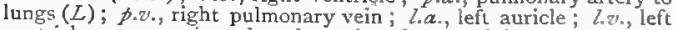
ventricle; ao., aorlic arch; $d$.ao., dorsal aorta giving off arteries to liver $\left(l i_{*}\right)$, to gut $\left(g_{*}\right)$, to body $(B$.$) ; po.z., portal veins; h_{.} v_{.}$, hepatic vein.

stomach and intestines is carried to the liver, and not directly to the heart.

(b) At an early stage in development the blood is brought back from the anterior region by the superior cardinal veins, from the posterior region by the inferior cardinals. The two cardinals on 
each side unite to form the short transverse ductus Cuvieri, the two ducts entering the sinus venosus of the heart. In Fishes the superior cardinals persist, the inferior cardinals bring back blood from the kidneys, and also to some extent, by means of their union with the caudal vein, from the posterior region of the body. In some cases this union with the caudal is only indirect, through the medium of the kidney (Elasmobranchs), in this way the renal portal system is constituted. In higher Vertebrates before development is completed, the superior cardinals are replaced by the superior vence cave (into which the superior cardinals open as external jugulars). The inferior cardinals at first return blood from the Wolffian bodies and the posterior region, later they atrophy, and are replaced by an unpaired inferior vena cava which brings back blood from the kidney (efferent renals), from the liver (hepatics), and from the hind limbs except when there is a renal portal system. In Mammals the azygos vein persists as a remnant of inferior cardinals.

(c) In Amphibia a vein known as the epigastric (anterior abdominal) carries blood from the hind limbs into the hepatic portal system. This vein also receives blood from the allantoic bladder, a fact which is of great theoretical importance. In all higher Vertebrates in embryonic life, the blood from the allantois passes through the liver, and to a greater or less extent into its capillaries, on its way to the heart. In Reptiles, the allantoic veins persist throughout life as the epigastric vein or veins. In Birds and Mammals, on the other hand, they atrophy completely at the close of foetal life. In Birds, however, a vein is developed which connects the veins coming from the posterior region with the allantoic veins, this persists when the remainder of the allantoic veins atrophy, and thus in Birds as in Amphibia there is a connection between the components of the inferior vena cava and the portal system. In Mammals no such connection occurs.

According to many authorities, the vascular system is developed in the mesoblast from the hollowing out of strands of cells, the outer cells forming the walls of the vessels, the inner forming the constituents of the blood. According to some, however, the endoderm plays an important part in the process. Thus, in Elasmobranch fishes, the aorta and the sinus venosus arise directly from the archenteron, and the cardinal veins arise from the fusion of segmental outgrowths of the aorta.

Associated with the vascular system is the spleen, which appears to be an area for the multiplication of blood corpuscles. It is usually believed to be of mesodermic origin, but there are some facts which point to its being endodermic.

Developed in mesoblastic spaces, and continuous with the body cavity on the one hand, and the blood vessels on the other, is the system of lymphatic spaces and vessels (see Chap. II.). 


\section{Respiratory System.}

In Balanoglossus, Tunicates, and Amphioxus, the walls of the pharynx bear slits, between which the blood is exposed in superficial blood vessels to the purifying and oxygenating influence of the water.

In Cyclostomata, Fishes, all young and some adult Amphibians, there are not only clefts on the walls of the pharynx, but gills associated with these. On the large surface of the feathery or plaited gills, the blood is exposed and purified.

In Reptiles, Birds, and Mammals, traces of gill clefts occur in the embryos, but without lamellæ or respiratory function. In the embryo the blood is purified, as will be explained afterwards, by aid of the foetal sac known as the allantois ; and after birth the animals breathe by lungs. All adult Amphibians also have lungs, to which the lung or swim-bladder of Dipnoi is physiologically equivalent.

The gill clefts arise as outgrowths of the endodermic gut which meet the ectoderm and open. The ventral paired lungs arise from an outgrowth of the gut, and such also is the swim-bladder of many Fishes, though that usually lies on the dorsal surface, has rarely more than a hydrostatic function, and has a blood supply different from that of the lungs. That lung and swim-bladder are homologous is by no means certain, but the comparison is plausible.

\section{Excretory System.}

The development of this is always complicated. In the embryos of Vertebrates at an early stage there are always traces of a pronephros or so-called head kidney. In its most developed condition this consists of $3-7$ pairs of segmentally arranged tubules, which communicate on the one hand with the body cavity, and on the other by a segmental duct with the exterior. The tubules are comparable to, and perhaps homologous with, the nephridia of Annelids. The pronephros persists, although apparently in a somewhat degenerate condition, in Myxine and Bdellostoma; in Bony Fishes and Amphibia it forms a large and functional organ in early life; in Elasmobranchs and onwards it is from the first rudimentary and functionless. 
The segmental duct has possibly an epiblastic origin, it grows gradually backwards from its place of origin, and it seems probable that the original excretory opening was far forwards. At a late period in those types in which the pronephros is a functional larval organ, but much earlier in the higher Vertebrates, another set of tubules are differentiated from the mesoblast, nearer the posterior end of the body, and acquiring a connection with the segmental or pronephric duct, constitute the mesonephros or mid kidney. Below the Amniota this forms the permanent excretory organ.

In higher forms another series of nephridial tubules

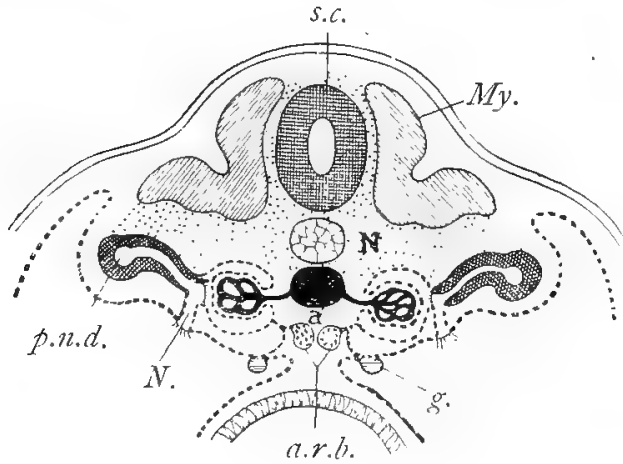

FIG. I47.-Transverse section through a Vertebrate Embryo. (After Semon.)

sc., Spinal cord ; My., Myotomes; N., notochord; $\alpha$., aorta, with vessels to two glomeruli; $g$, rudiment of genital organ; $p . n \cdot d$. , pronephric or segmental duct; $N$., nephrostome. The letters a.r.b. lie in the top part of the gut.

arises still further back in the body, and forms the metanephros, or permanent kidney.

In each case, when typically developed, the tubules consist $(a)$ of an internal ciliated funnel (nephrostoma) opening into the body cavity, but only rarely persistent; (b) of a dilatation (Malpighian body), into which blood vessels project; and $(c)$ of a coiled tube in part excretory, in part a conducting canal for the waste filtered from the blood. 


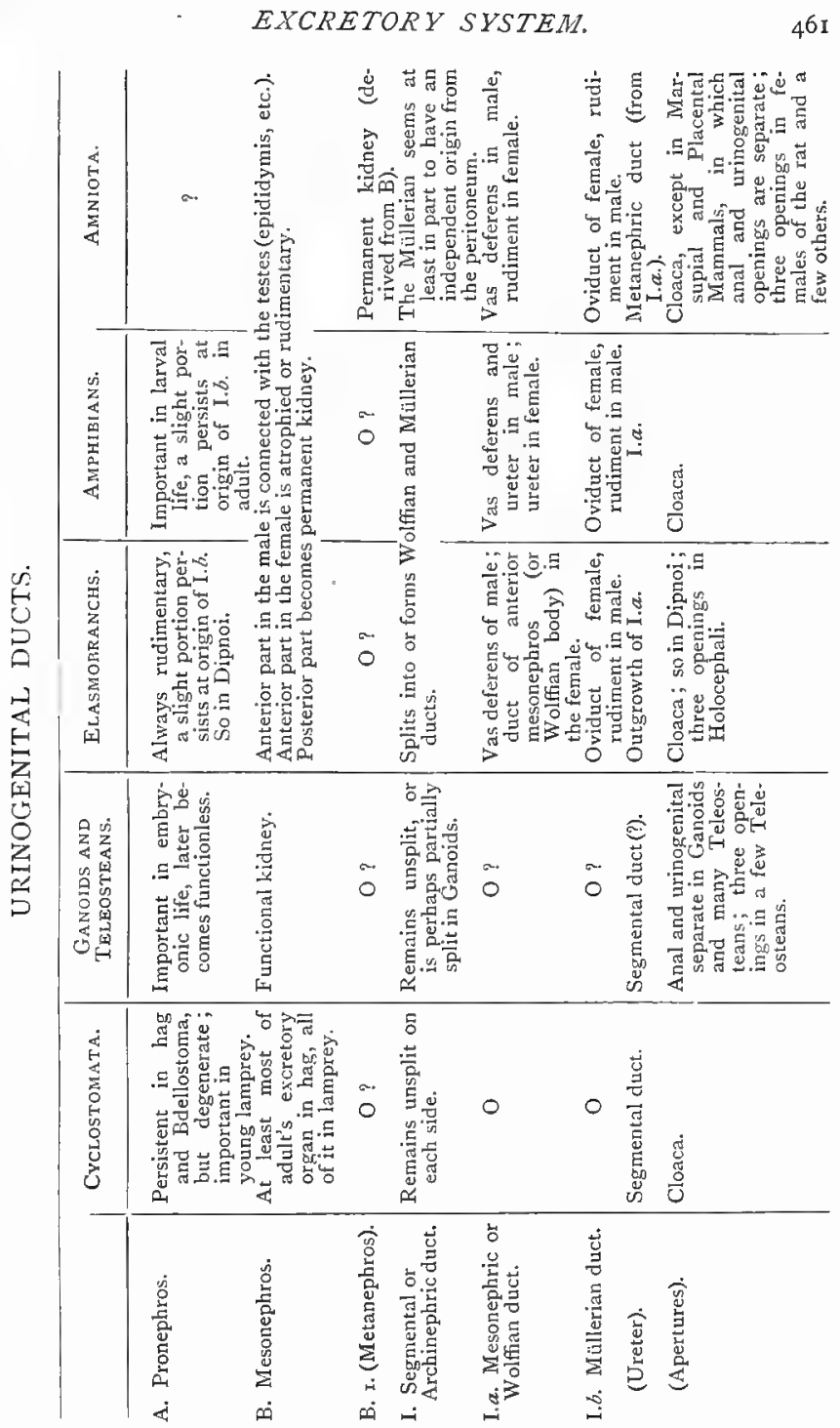


The segmental or pronephric duct on each side is, at any rate in some of the lower Vertebrates, divided into two ducts, the Muillerian duct and the mesonephric or Wolffian duct. In the Amniota the origin of the Müllerian duct is still somewhat obscure. It becomes the genital duct or oviduct of the female, while in the male the Wolffian duct becomes the genital duct or vas deferens.

The ureters or ducts from the persistent functional kidneys are either the original archinephric or segmental ducts (e.g., in Cyclostomata), or the Wolffian ducts (in Amphibians), or special posterior derivations of the latter.

Suprarenal Bodies. - These are found in most Vertebrates near the reproductive organs and kidneys. Structurally, each shows a distinction into a cortical and a medullary zone. It is usually asserted that these two areas have a different origin, the medullary region being derived from the sympathetic nervous system, the cortex from the pronephros. On the other hand, some investigators consider that the medulla is derived from metamorphosed cortical cells. Nor is the origin of the cortical part beyond dispute, for by some it is said to originate from a degeneration of the most anterior portion of the germinal epithelium, or from this in association with a part of the primitive kidney.

With regard to function, there is even more uncertainty. The suprarenal bodies are relatively very large in embryonic life, but fail to maintain their primitively rapid rate of growth. It has been suggested that they assist in breaking down or disposing of waste pigment.

\section{Reproductive System.}

The ovaries and testes are developed from a ridge formed by a part of the epithelium lining the abdominal cavity, this ridge constituting the so-called germinal epithelium.

In the male, the proliferating germinal epithelium is divided by embryonic connective tissue into numerous follicles. The cells of the follicles form seminal mother cells, which, by their ultimate divisions, give rise to spermatozoa. From the mesonephros, tubules grow out to the embryonic testes; these form the collecting tubes of the organ and open into the mesonephric duct, the vas deferens of the adult. This is the most typical Vertebrate condition, but is not universal. (See Table, page 46r.)

In the female, the ovary is similarly divided up into follicles. In this case, however, differentiation sets in among the originally equivalent cells of the follicle. One cell in each follicle is more successful than its neighbours, 
which are sacrificed to form an envelope of follicular cells around the single large ovum cell. The ova are usually shed into the body cavity, and pass thence to the exterior by the Müllerian ducts or oviducts.

In many cases between the follicular cells and the ovum there is a membrane, the zona radiata, which is traversed by fine pores, and, in consequence, has a striated appearance; other egg membranes, more or less transitory in nature, also occur. In the lower Vertebrates the layer of follicle cells is single, but in Mammals it is multiple, and a quantity of clear fluid accumulates between the cells and the ovum. The whole forms a "Graafian follicle," which bursts when the ovum is liberated.

Before fertilisation takes place the ovum undergoes a process of maturation, during which extrusion of polar bodies typically occurs; the technical difficulties in the way of the definite observation of this fact are, however, often very great. The ova are fertilised

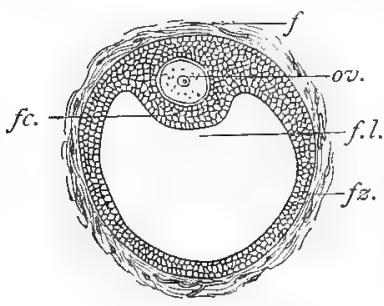

FIG. I49.-Mammalian Ovum. (After HFRTWIG.)

oo., Ovum; $f$, follicular capsule; fz., follicle cells; f.c., follicle cells forming discus proligerus; f.l., cavity occupied by liquor folliculi. outside the body in Cyclostomata, Ganoids, Teleosteans, Dipnoi, and tailless Amphibians; internally in the other Vertebrates.

Hermaphroditism occurs as a normal state in Tunicata, most of which are first functionally female and then male (protogynous); in Myxine (q. $\mathrm{v})$, which is first male and then female (protandrous) ; in some species of the Teleostean genera Chrysophrys and Serranus, of which the latter is regularly self-fertilising; and in a solitary Patrachian. It occurs casually in some Selachians, in the sturgeon, in about a score of Teleosteans, e.g., cod, in various Amphibians, and more rarely in Amniota. There are also embryological facts which suggest that the embryos of higher Vertebrates pass through a state of hermaphroditism before the unisexual condition is reached. On these grounds it has often been suggested that the original Vertebrate animals were hermaphrodite.

The quantity of yolk present in the egg varies very greatly in Vertebrates, and its presence or absence exercises a profound influence upon the processes of development. Following Hertwig we may notice that the presence of yolk has both a physiological and a morphological effect. Physiologically, the presence of a store of nutriment enables the developmental process to be carried on uninterruptedly, and the period of independent life to be postponed until more or less complexity of organisation has been attained. Again, morphologically, yolk acts as a check to the activity of the protoplasm, and by substituting an embryonic mode of nutrition for that for which the adult organism is 
fitted, tends to prevent a speedy establishment of the adult form. When much yolk is present it usually forms a hernia-like yolk sac, hanging down from the embryonic gut. As a further consequence, we may notice the tendency to the production of embryonic organs useful only during embryonic life. We must consider the formation of an organic connection between mother and unborn young as a further step in the same direction as the acquisition of yolk. This is hinted at in some Fishes and Reptiles, but culminates in the placental Mamma1s. It may be looked at in two different ways. On the one hand, the diversion of the nourishment from the ovary, during the period of gestation, tends to starve the remaining ovarian ova, and this check to fertility is further prolonged during lactation (Ryder); on the other hand, the chance of survival is much increased, and the maternal sacrifice finds its justification in the increased specialisation of the offspring.

In accordance with the effect of the presence of yolk as noted above, we find that segmentation is total (holoblastic) in the ova of the lamprey, the sturgeon, Ceratodus, Amphibians, and all Mammals except the Monotremes. In the ova of Elasmobranchs, Teleosteans, Reptiles, Birds, and Monotremes, the activity of the protoplasm is not sufficient to overcome the inertia of the yolk, and segmentation is partial (meroblastic).

Similarly we find that a gastrula is formed, in part at least, by distinct invagination in the development of the lamprey, the sturgeon, and Amphibians (recently the occurrence of invagination has been denied for the frog); it is more modified in Teleosteans and Elasmobranchs, whose ova have more yolk ; it is much disguised in Sauropsida and Mammals.

Most Vertebrates lay eggs in which the young are hatched, and these animals are usually called oviparous, though all animals do of course produce eggs. In some sharks, a few Teleosteans, some tailed Amphibians, a few lizards and snakes, the young are hatched before they leave the body of the mother animal. To such the awkward term ovo-viviparous is sometimes applied, but there is no real distinction between this mode of birth and that called oviparous, and both may occur in one animal (e.g., in the grass snake) in different conditions. In the placental Mammals, there is a close organic connection between the unborn young and the mother, and the parturition in this case is usually called viviparous. But this term is also objectionable, since all animals are in a sense viviparous. 


\section{CHAPTER XXI.}

\section{CLASS CYCLOSTOMATA.}

(Syn. Marsipobranchit.)

The hag (Myxine), the lamprey (Petromyzon), and a few others like them, are so different from Fishes that they must be ranked in a distinct class. 'They seem to represent an old-fashioned type, whose interest has been enhanced by the discovery of Palceospondylus in the Old Red Sandstone.

General Characters. - Unlike all higher Vertebrates (Gnathostomata), the Cyclostomata have round mouths without distinctly developed jazes. They are also without paired fins and without scales. Their respiratory system consists of 6-7 pairs of gill pouches, to which the term Marsipobranch refers. In the extant forms the skeleton is wholly cartilaginous, and the notochord persists unconstricted. The "nostril" is single, there is no sympathetic nervous system, no conus arteriosus, no pancreas, no spleen, and the segmental duct is unsplit.

First Type. Myxine-The Hag.

The glutinous hag (Myxine glutinosa) is not uncommon off the coast of Scotland, N. England, Scandinavia, \&c., living in the mud at depths of 40 to 300 fathoms. It often lies buried with only the nostril protruding from the mud, or it may swim about in search of prey. It eats the bait off the fisherman's long lines, and it also enters and devours the cod, \&c., which have been caught on the hooks. According to some reports, the hag also attacks free swimming fishes, boring its way into them, but the evidence is not satisfactory. Mr. J. T. Cunningham discovered that the young animals are hermaphrodite, containing immature ova 30 
and ripe spermatozoa, while older forms produce ova only, and Nansen has corroborated this. Of the development and early history nothing is known. They are said to spawn in late autumn.

\section{Form.}

The body is eel-like, measuring $15^{-24}$ inches in the adult. There is a slight median fin around the tail; beside the mouth and nostrils are four pairs of barbules. There are no paired fins.

\section{The Skin.}

The skin is scaleless, and rich in goblet cells, which secrete so much mucus that the ancients said the hag "could turn water into glue." Besides the diffuse goblet cells, there is a double row of glandular pits arranged segmentally on each side of the ventral surface along the entire length of the animal. Each opens by a distinct pore.

\section{Muscular System.}

The muscle segments or myomeres are to some extent traceable. Working the rasping teeth is a powerful muscular structure, sometimes called a "tongue."

\section{The Skeleton.}

The skeleton is wholly cartilaginous. The notochord persists unsegmented within a firm sheath, the skull is a simple unroofed trough, jaws are not distinctly developed, there is only a hint of the complicated basket work which supports the gill pouches of the lamprey, but the tongue, the barbules, \&c., are supported by cartilaginous rods. The end of the notochord in the tail is quite straight (protocercal or diphycercal).

\section{Nervous System.}

The brain has the usual parts, but is small and simple. It is much compressed, with practical obliteration of ventricles. the fore brain seems to agree with that of Ganoids and Teleosteans in having a non-nervous roof. The spinal cord is somewhat flattened. Throughout at least a portion of the cord there are two dorsal roots for each ventral root. The 
union of dorsal and ventral roots is only partial, and there is no sympathetic system.

The eye is degenerate (e.g., without a lens or iris), and is hidden beneath the skin; the ear has only one semicircular canal; the single nasal sac opens dorsally at the apex of the head, and communicates posteriorly with the pharynx by a naso-palatine duct. The absence of pigment and sensory structures in the skin, and the degeneracy of the eye may be associated with the hag's mode of life.

\section{Alimentary System.}

The mouth is suctorial. There is a median tooth above, and two rows of teeth are borne on each side of the muscular "tongue." These teeth are "horny," but sharp. Into the mouth, just in front of a fringed velum which

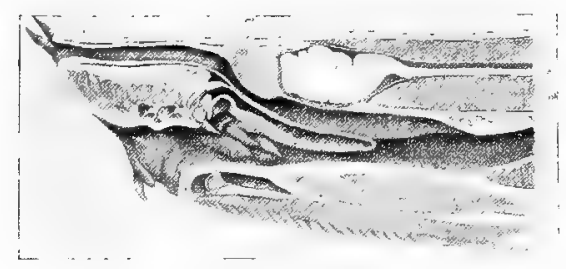

FIG. 150.-Median longitudinal section of anterior end of Myxine. (After Retzius.)

Showing barbules, nasal passage, mouth cavity, brain.

separates it from the pharynx, the nasal sac opens. Thus water passes from the nostril into the pharynx. It may be, as Beard suggests, that this passage is a persistent "old mouth." From the gullet open six respiratory pouches, each of which has an efferent tube, but the six efferent tubes of each side unite and have a common exhalent orifice. The gut is straight and uniform, with longitudinal ridges internally, with a two-lobed liver and a gall bladder, but without the usual pancreas.

\section{Respiratory System.}

Water enters by the nasal sac, passes into the pharynx, down the gullet, into the six pairs of respiratory pouches, 
out by their efferent tubes at a single aperture on each side. The respiratory pouches have much plaited internal walls, on which the blood vessels are spread out. On the left side, behind the sixth pouch, a tube opens from the cesophagus to the exhalent aperture.

\section{Vascular System.}

The blood contains nucleated red corpuscles. It is collected from the body in anterior and posterior cardinals, passes through a sinus venosus into the auricle of the heart, thence to the ventricle, thence along a ventral aorta which gives off arches to the respiratory pouches. From these the purified blood passes dorsalwards in efferent branchial vessels, which unite posteriorly to form the dorsal aorta, while from the most anterior a branch goes to the head.

\section{Excretory System.}

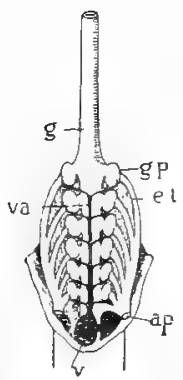

The segmental or archinephric ducts remain unsplit, and the kidney or nephridial system is represented by a series of small segmental tubules attached to the ducts. The pronephros or fore kidney persists, apart from the FIG. I51.-Respiratory System of Hag, from ventral surface.

g. Gullet; $g_{. p .}$. first gill pouch; e.t., exhalent tube of first gill pouch, uniting with those from the other five pouches ; $a . p$, exhalent aperture ; $v_{\text {., }}$ ventricle of heart; $y_{\text {.a. }}$, ventral aorta, carrying blood to gill pouches.

functional mesonephros, in a degenerate state on each side of the pericardium. The segmental ducts are said to end much in the same way as they do in the lamprey.

\section{Reproductive System.}

Myxine is a protandrous hermaphrodite, spermatozoa being formed at an early period, and ova afterwards. The reproductive organ is simple, unpaired, and moored by a median dorsal mesentery. Owing to the large size of the ova, the ovary is very conspicuous in full grown forms. 
When the ova are freed from the ovary they pass into the body cavity. Each has an oval horny case, with a circlet of knobbed processes at each end. By these they become entangled together. There are no genital ducts, and the expulsion of the products requires to be investigated. The development is still unknown.

Besides Myxine glutinosa, two other species are known, one from Japan, another from the Magellan Straits. The genus Bdellostoma, from Southern seas (off the Cape of Good Hope, \&c.), is nearly allied; it has six or more gill pouches which open apart from one another.

\section{Second Type of Cyclostomata.}

Petromyzon-The Lamprey.

There are three British species, the sea lamprey (Petromyzon marinus) over three feet in length, the river lampern ( $P$. fuviatilis) nearly two feet long, and the small lampern or "stone-grig" ( $P$. branchialis or planeri). They eat worms, small crustaceans, insect larvæ, dead animals, \&c., but they also fix themselves to living fishes and scrape holes in their skin. As their names suggest, they also fix their mouths to stones, and some draw these together into nests.

The spawning takes place in spring, usually far up rivers. Before laying the eggs, the lamprey seems to fast (cf. salmon, Protopterus, frog), and its muscles undergo a granular degeneration (cf. Protopterus, tadpole, \&c.). Soon after spawning the adults of both sexes die. For reproduction is often the beginning of death as well as of lifethough in higher animals the nemesis is often slow. The young are in many ways unlike the parents, and after two years or three years pass through a metamorphosis. To the larvæ before metamorphosis the old name Ammocates is often applied.

\section{Form.}

The body is eel-like, with two unpaired dorsal fins, and another round the tail. Two ridges, one on each side of the anus, Dohrn compares to rudimentary pelvic fins. Otherwise there is no trace of limbs. 


\section{The Skin.}

The skin is scaleless, slimy, and pigmented. Its structure, like that of Myxine, is complex. Sensory structures occur on the head and along the sides.

\section{Muscular System.}

The muscle segments are well marked. The suctorial mouth and the rasping "tongue" are very muscular.

\section{The Skeleton.}

The skeleton is wholly cartilaginous. The notochord persists unsegmented, but its firm sheath forms rudimentary neural arches. The skull is imperfectly roofed. There are no distinct jaws, but a cartilaginous ring supports the lips of the mouth. There is a complex basket work around the gill pouches, and it is likely that its elements correspond to visceral arches. Fin rays support the dorsal and caudal fins, and other skeletal parts occur about the "tongue." The caudal end of the notochord is quite straight.

\section{Nervous System.}

The brain has the usual parts, but is small and simple; the roof of the fore brain is composed of non-nervous epithelium; there is a distinct pineal body; the oral part of the hypophysis is developed from in front of the mouth, in close connection with the involution of epiblast which forms the nostril. The spinal cord is flattened; the anterior and posterior roots of the spinal nerves do not unite; there is no sympathetic system.

Though the larva sometimes receives the name of "nineeyes"-which expresses a popular estimate of the branchial apertures - it is blind, for the eyes are rudimentary and hidden. In the adult they rise to the surface, and are fairly well developed. The ear has only two semicircular canals instead of the usual three. The single nasal sac does not open posteriorly into the mouth as it does in Myxine; though prolonged backwards it ends blindly. Its external opening is at first ventral, but is shunted dorsally.

Alimentary System.

The oral funnel, at the base of which the mouth lies, has numerous horny teeth. It is applied to the lamprey's 
victim, and adheres like a vacuum sucker; the toothed "tongue" works like a piston; both flesh and blood are thus obtained. From the floor of the pharynx a groove is constricted off (cf. p. 450).

From the gullet of the young larva seven gill pouches open directly to the exterior; but in the adult this larval gullet becomes wholly a respiratory tube. It is closed posteriorly, and opens anteriorly into the gullet of the adult, which is a new structure. At the junction of the respiratory tube with the gullet of the adult, lie two flaps or vela.

The rest of the gut is straight and simple, with a singlelobed liver, but without a pancreas. There is a slight fold

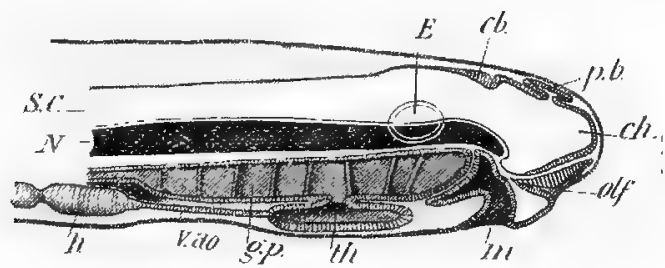

FIG. 152.- Longitudinal vertical section of anterior end of larval lamprey. (After BALFour).

m., Mouth ; th., thyroid ; g.p., one of the gill pouches; v.ao., ventral aorta; $h$., heart; $N$., notochord; S.C., spinal cord; $E$., auditory vesicle; $c b$. , cerebellum; $p . b .$, pineal body; $c h *$, cerebral hemispheres; olf, olfactory involution.

in the intestine, which may be compared with the spiral valve of Elasmobranchs.

\section{Respiratory System.}

Seven gill pouches with plaited walls open directly to the exterior on each side, and communicate indirectly with the gullet as already described.

When the lamprey is sucking a victim, and perhaps at other times, water passes in as well as out by the external openings of the gill pouches. In the larva there is an eighth most anterior pouch which does not open to the surface. It corresponds to the spiracle of Elasmobranchs. 


\title{
Vascular System.
}

The vascular system is essentially the same as in the hag. The red blood cells are biconcave, circular, nucleated discs.

\author{
Excretory System.
}

There are two elongated kidneys (mesonephros), each with a wide ureter. The ureters open terminally into a urinogenital sinus, the external aperture of which lies behind the anus and in the same depression.

\section{Reproductive System.}

The sexes are separate. The reproductive organ is elongated, unpaired, and moored by a median dorsal mesentery. There are no genital ducts. The ova and spermatozoa are liberated into the body cavity, and seem to pass by two pores into the urinogenital sinus, and thence to the exterior. In the male there is an ejaculatory structure, or so-called "penis." There are many more males than females.

Development of $P$. planeri.-In the ripe ovum, which has a considerable quantity of yolk, the nuclear substance of the germinal vesicle is expanded like a cup at the " animal pole," forming the so-called "pole plasma." Outside of this is a clear cupola, which several spermatozoa may enter, though only one really penetrates into the egg. After a spermatozoon has begun to make its way inwards, two polar bodies are formed. The elements of the sperm nucleus combine as usual in an intimate manner with those of the reduced nucleus of the ovum, and a segmentation nucleus is formed about three hours after fertilisation.

Segmentation is total, but slightly unequal owing to the yolk; a blastosphere results which is invaginated into a gastrula. The blastopore or mouth of the gastrula persists as the anus of the animal, and there is no neurenteric canal.

The formation of the central nervous system is peculiar, for the sides of the epiblastic infolding remain in contact instead of forming an open medullary canal.

In the head region, where the gut is not surrounded by yolk cells, the mesoblast is formed from hollow folds in "enterocolic "fashion; but in the trunk region the cushions of hypoblastic yolk cells change gradually into mesoblast, and acquire a coelome cavity in "schizocolic" fashion. Thus the two main ways in which a body cavity arises, $(a)$ from coelome pouches of the archenteron, $(b)$ from a splitting of solid mesoblast rudiments, are here combined.

The time between fertilisation and the hatching of the larva, or 1 mnocketes, varies with the temperature, being seventeen days in North Germany, but only eight at Naples. 
The larvæ live wallowing in the sand or mud of streams, and feed on minute animals. Those of $P$. planeri are so unlike the adults that they were once referred to a distinct genus Ammocates, and though a Strassburg fisherman, Baldner, is said to have discovered their true nature about 200 years ago, the fact was overlooked until August Muiller traced the metamorphosis in $185^{6}$. In the small lampern the change to the adult state is sometimes postponed until the autumn of the fourth or fifth year, when it completes itself rapidly. Less is known about the metamorphosis of the other species.

In the Ammorotes, or larva before metamorphosis, the head is small ; the dorsal fin is continuous, the upper lip is semicircular, the lower lip is small and separate; the mouth is toothless and not suctorial; the brain is long and narrow; the eyes are half made and hidden beneath the skin; the future gullet, as distinguished from the respiratory tube, is not yet developed.

Lampreys are distributed in the rivers and seas of north and south temperate regions. They are often used as food. Besides Petromyzon there are several related genera, e.g., Mordacia and Geotria, from the coasts of Chili and Australia, and Ichthyonzyzon, from the west coast of N. America. Certain structures called "conodonts" from very ancient (Silurian) strata have been interpreted as teeth of lampreys or hags.

\section{Cyclostomata.}

HAG (Myxine).

Exclusively marine.

The fin is confined to the tail.

Numerous large glands in the complex, slimy skin.

Mouth with barbules, no lips, few teeth.

Skull without any roof.

Skeletal system less developed han in the lamprey.

Eyes hidden and rudimentary.

Ear with one semicircular canal.

Nasal sac opens posteriorly into the pharynx.

Six pairs of gill pouches, opening directly into the gullet, less directly to the exterior.

Longitudinal ridges in the intestine.

Ova large and oval, with attaching threads.

(Development unknown).

\section{LAMPREX (Petromyzon),}

In rivers and seas.

Two unpaired dorsal fins.

Sensory structures in the complex, slimy, pigmented skin.

No barbules, but lips, and many teeth.

Skull very imperfectly roofed,

Hints of vertebral arches.

Cartilaginous basket work around gill-pouches.

Eyes hidden and retarded in the larva, exposed and complete in adult.

Ear with two semicircular canals.

Nasal sac ends blindly.

Seven pairs of gill pouches, opening directly to the exterior, less directly into the adult gullet.

A slight spiral fold in the intestine.

Ova small and spherical.

Development with metamorphosis. 


\section{Palaospondylus gunni.}

Under this title, Dr. Traquair has recently described a remarkable fossil form from the Old Red Sandstone of Caithness. He speaks of it as a "strange relic of early vertebrate life."

It is a dainty little creature, somewhat tadpole-like at first sight, usually under an inch in length. The following characters point strongly to its affinities with Cyclostomata:-

(I.) "The skull is apparently formed of calcified cartilage, and devoid of discrete ossifications." An anterior part is comparable to the trabecular and palatal region of a lamprey's skull; a posterior part is comparable to the parachordal region and auditory capsules,

(2.) "There is a median opening or ring, surrounded with cirri, and presumably nasal, in the front of the head" (n, Fig. I 53). limbs."

(3.) "There are neither jaws nor

(4.) "The rays which support the caudal fin expansion, apparently springing from the neural and hremal arches, are dichotomised (at least the neural ones), as are the corresponding rods in the lamprey."

Just behind the head lie two small oblong plates ( $x_{.}$, Fig. I 53 ), closely apposed to the commencement of the vertebral column, one on each side. The notochordal sheath is calcified in the form of ring-shaped or hollow vertebral centra with neural arches. Towards the tail,

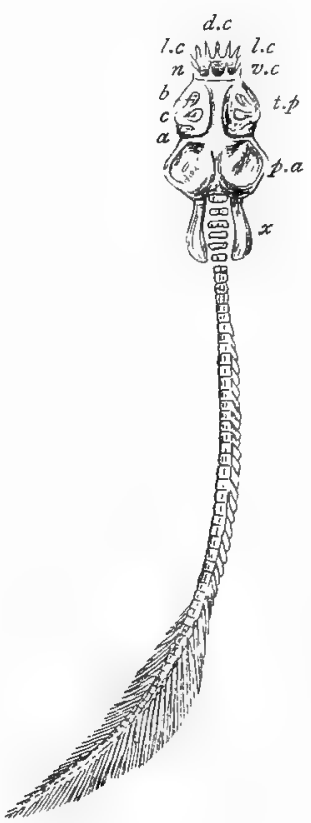

FIG. 153.-Restored skeleton of Palzeospondylus. - (After Traquair.)

d.c., cirri of dorsal margin; l.c., long lateral cirri ; v.c., cirri of ventral

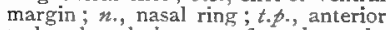
trabeculo-palatine part of cranium; $b$., anterior depression or fenestra; $c$., posterior depression or fenestra; $a_{\text {, }}$, lobe divided off from anterior part ; $p . x_{.,}$, posterior or parachordal part of

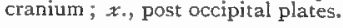

the arches are produced into slender neural spines, opposite which are shorter hæmal ones. 


\section{CHAPTER XXII.}

\section{CLASS PISCES-FISHES.}

Order I.-Elasmobranchii or Selachii, cartilaginous fishes, e.g., skates and sharks. With these may perhaps be ranked the Holocephali (Chimara and Callorhynchus.)

2.-Ganoidei, such as sturgeon (Acipenser) and bony pike (Lepidosteus); numerous extinct genera, only seven extant.

3.-Teleostei, bony fishes, such as cod, herring, salmon, flounder, eel.

4.-Dipnoi, mud fishes: Ceratodus, Protopterus, Lepidosiren.

The Dipnoi, or double breathers, are so distinct that some would remove them from among Fishes, and place them as an independent class between Fishes and Amphibians.

FISHES form the first markedly successful class of Vertebrates. For while the Tunicates are numerous, most of them are degenerate; the level attained by the lancelet is represented by, at most, two or three closely related genera; and the Cyclostomata are few in number and partially retrogressive.

In the possession of a Vertebrate axis and central nervous system, in the general integration of their structure, and in their great fecundity, Fishes have an easy pre-eminence over their Invertebrate inferiors. As successfully adapted forms -with typically wedge-like bodies, supple muscular tails, fin-like limbs, and the like-they may well compare with Birds in their mastery of the medium in which they live.

Their success may be read in the immense number of individuals, species, and genera, not only now but in the past; in the geological record which shows how the cartilaginous Elasmobranchs have persisted strongly from Silurian ages, or how the mysterious decadence of the Ganoid order has 
been followed by a yet richer predominance of the modern Bony Fishes; and, furthermore, in the plasticity with which many types appear to have assumed particular specialisations, such as that evolution of lungs which, in the double breathing Dipnoi, prophesies the epoch-making transition from water to terra firma.

General Characters. - Fishes are aquatic Vertebrates, breathing by gills attached to bony or gristly arches on the sides of the pharyngeal gill clefts. In Dipnoi, a single or double outgrowth from the gut-the air- or swim-bladderfunctions as a lung, air being inspired at the surface of the water. In Ganoids and in most Teleosteans the same structure is present, but though occasionally of some slight usefulness in respiration, usually serves as a hydrostatic organ.

Two pairs of limbs, in the form of fins, are usually present, and there are also unpaired median fins, supported by finrays. There are two great types of paired fin. In Dipnoi, and in some extinct forms, the fin has a central segmented axis, which (e.g., Ceratodus) bears on each side a series of radial pieces. In other fishes the radials diverge outwards from several basal pieces, and there is no median axis.

The skin usually bears numerous scales, in great part due to the dermis, but covered by a layer of epidermis, which may produce enamel. They vary greatly in form and texture, are suppressed in eels and electric fishes, and rudimentary in some other forms. Numerous glandular cells occur in the skin, but these are not compacted into multicellular glands, except in Dipnoi and a fere poisonous fishes. The skin also bears sensory structures, usually aggregated on the head, and arranged in one or more "lateral lines" along the trunk. There are no muscular elements in the dermis or cutis.

In many the gut ends in a cloaca, or a distinct anus may lie in front of the genital and urinary aperture, or apertures.

The heart is two-chambered, and contains only venous blood, except in the Dipnoi, where it showes hints of becoming three-chambered and receives pure blood from the hungs as well as impure blood from the body. Apart from the Dipnoi, the heart has a single auricle, receiving impure blood from the body, and a ventricle which drives this through a ventral aorta to the gills, whence the purified blood flow's to the 
head and by a dorsal aorta to the body. In addition to the two essential chambers of the heart, there is a sinus venosus, which serves as a porch to the auricle, and there is often a muscular conus arteriosus in front of the ventricle, or a bulbus arteriosus at the base of the ventral aorta. Except in Dipnoi, there is no vein which exactly corresponds to what is known in all higher Vertebrates as the inferior vena cava, i.e., a single vessel which receives hepatic veins from the liver, renal veins from the kidneys, and other veins from the posterior organs.

There is no distinct indication of an outgrowth from the hind end of the gut comparable to that which forms the bladder of Amphibians or the allantois of higher Vertebrates. Most fishes lay egos which are fertilised in the water.

First type of Frshes. The Skate (Raja)-of the order Elasmobranchii.

Various species of Raja-the grey skate ( $R$. batis), the thornback ( $R$. clavata), and the ray ( $R$. maculata)-are common off the coast of Britain. They are comparatively sluggish but very voracious fishes, and live along the bottom at considerable depths.

\section{Form.}

The body is flattened from above downwards or dorsoventrally, unlike that of the bony flat fishes, such as plaice and flounder, which are flattened from side to side. The skate rests on its ventral surface, the flounder on its side. The triangular snout, the broad pectoral fins, the long tail with small unpaired fins, are obvious features. On the dorsal surface the skin is pigmented and studded with placoid scales or skin teeth; on the top of the skull there are two unroofed areas or fontanelles; there are numerous jointed radials in the pectoral fins. Behind the lidless eyes are the spiracles - the first of the obvious gill slits, opening dorsally, containing a rudimentary gill, and communicating posteriorly with the mouth cavity. On the ventral surface, are seen the sensory mucous canals extending over the skin, the transverse mouth and the nostrils incompletely separated therefrom as if in double hare lip, the five pairs of gill apertures, the cloacal aperture and two abdominal pores 
beside it. We may feel the pectoral and pelvic girdles supporting the fore and hind fins. In the male, the latter are in part modified into copulatory "claspers."

\section{The Skin.}

On the dorsal pigmented surface, embedded in the dermis, there are many "skin teeth," or "dermal denticles," or "placoid scales." Each is based in bone, cored with dentine or ivory, tipped with enamel, the latter being due to the ectoderm (epidermis), the rest to the mesoderm (dermis or cutis) of the skin, the whole arising as a skin papilla. On the ventral unpigmented surface are numerous mucus canals or jelly tubes, ending in ampullæ. These are also present on the dorsal aspect, especially about the head. They have a sensory function. Most of the slime which exudes on the surface comes from glandular goblet cells in the epidermis.

\section{Muscular System.}

In the posterior part of the body and in the tail, the segmental arrangement of the muscles may be recognised. The large muscles which work the jaws are noteworthy. Professor Cossar Ewart has described a rudimentary electric organ in the tail region of Raja batis and $R$. clavata, apparently too incipient to be of any use.

Electric organs are best developed in two Teleostean fishes-a S. American eel (Gymnotzes) and an African Siluroid (Malapterztrzes), and in the Elasmobranch Torpedo. In Gymnotus they lie ventrally along the tail, in Malapterurus they extend as a sheath around the body, in Torpedo they lie on each side of the head, between the gills and the anterior part of the pectoral fin. In other cases where they are slightly developed, both in Elasmobranchs and Teleosteans, they lie in the tail. Separated from one another by connective tissue partitions, are numerous "electric plates," which consist of strangely modified muscle substance and numerous nerve endings. The electric discharge is very distinct in the three forms noted above, and is controlled in some measure at least by the animal.

\section{The Skeleton.}

The skeleton is for the most part cartilaginous, but here and there ossification has taken place, as a crust over many parts, but more deeply in the vertebral bodies, in the teeth, and in the tooth-like scales. 
The vertebral column consists of an anterior plate not divided into vertebræ, and of a posterior series of distinct vertebral bodies. Each of these has a biconcave or amphicœlous centrum. From each side of the centrum a transverse process projects backwards, and bears a minute hint of a rib. From the dorsal surface of each centrum rise two neural processes, which arch upwards on each side of the spinal cord; the arch is continued upwards in inter-neural plates which meet in a neural spine on the top. On the

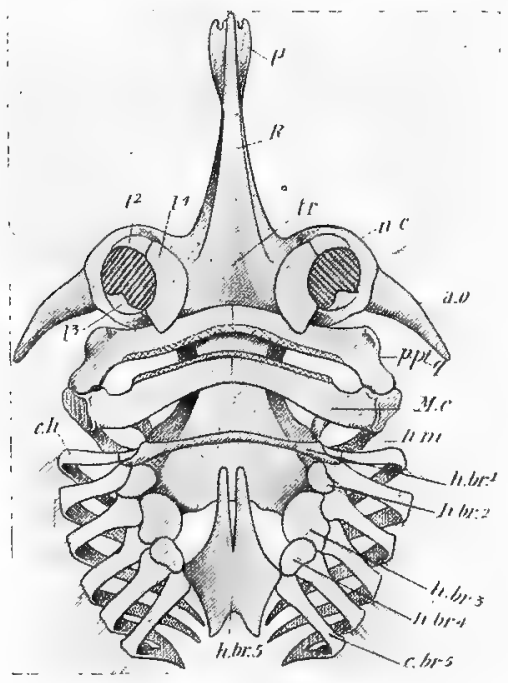

FIG. I54,-Under surface of skull and arches of skate. (After W. K. PARKER).

$l '$ First labial cartilage; $R$., rostrum; $t r_{\text {., }}$ trabecular region ; n.c., nasal capsule; a.o., ant-orbital cartilage; p.pt.q., palatopterygo-quadrate ; $M . c .$, Meckel's cartilage ; h.m., hyo-mandibular; h.br. I-5, hypo-branchials; c.br. 5, fifth cerato-branchial ; c.h., cerato-hyal; \& 2-4, labial cartilages.

caudal vertebræ, what seem to be the transverse processes are directed downwards, to form a hæmal arch enclosing an artery and a vein. In the lozenge-shaped spaces between the vertebræ lie gelatinous remains of the notochord. The 
vertebral column develops from the mesodermic sheath of the endodermic notochord.

'The skull is a cartilaginous case, with a spacious cavity for the brain, a large posterior aperture or foramen magnum through which the spinal cord passes, a large ear capsule on each side posteriorly, a similar nose capsule on each side anteriorly, a long snout or rostrum projecting in front, two incomplete regions or fontanelles on the roof. Compared with the skull of a cod or of a higher Vertebrate, that of a skate is simple; it is not ossified, nor divided into distinct regions, nor has it anything corresponding to the investing membrane bones, which in higher animals are added to the original foundations of the skull, nor do the visceral arches in the skate take part in forming the skull, which

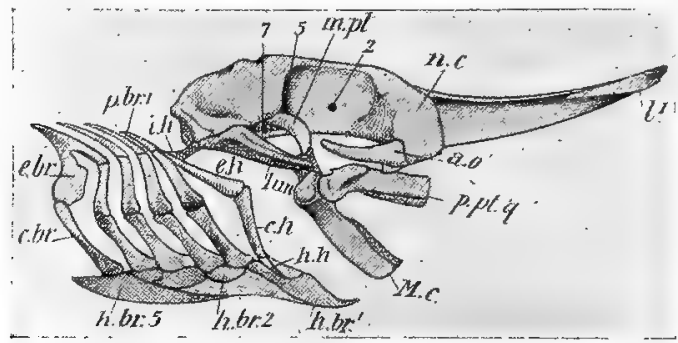

FIG. 155.-Side view of skate's skull. (After W. K. PARKER.)

l', First labial cartilage; n.c., nasal capsule ; a.o., ant-orbital ; $\not p . p t . q$. , palato-pterygo-quadrate; $M . c$. , Meckel's cartilage; $k_{. m} m$. ,

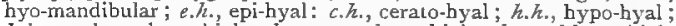
h.br. I-5, hypo-branchial; c.br, cerato-branchial ; e.br, , epi-branchial; $\not p . b r^{\prime}$., first pre-branchial; $i . h$. , inter-hyal ; $m . p t$., meta-pterygoid; $2,5,7$, foramina of these nerves.

arises, as usual (see p. 427), from parachordals, trabeculæ, sense capsules, \&c.

The visceral arches are primitively supports for the wall of the anterior part of the food canal, but at least two of them are much modified alike in position and function.

The upper jaw of the skate is a strong transverse bar, formed from the union of two palato-pterygo-quadrate cartilages. The lower jaw is a similar bar formed from the union of two Meckel's cartilages. 


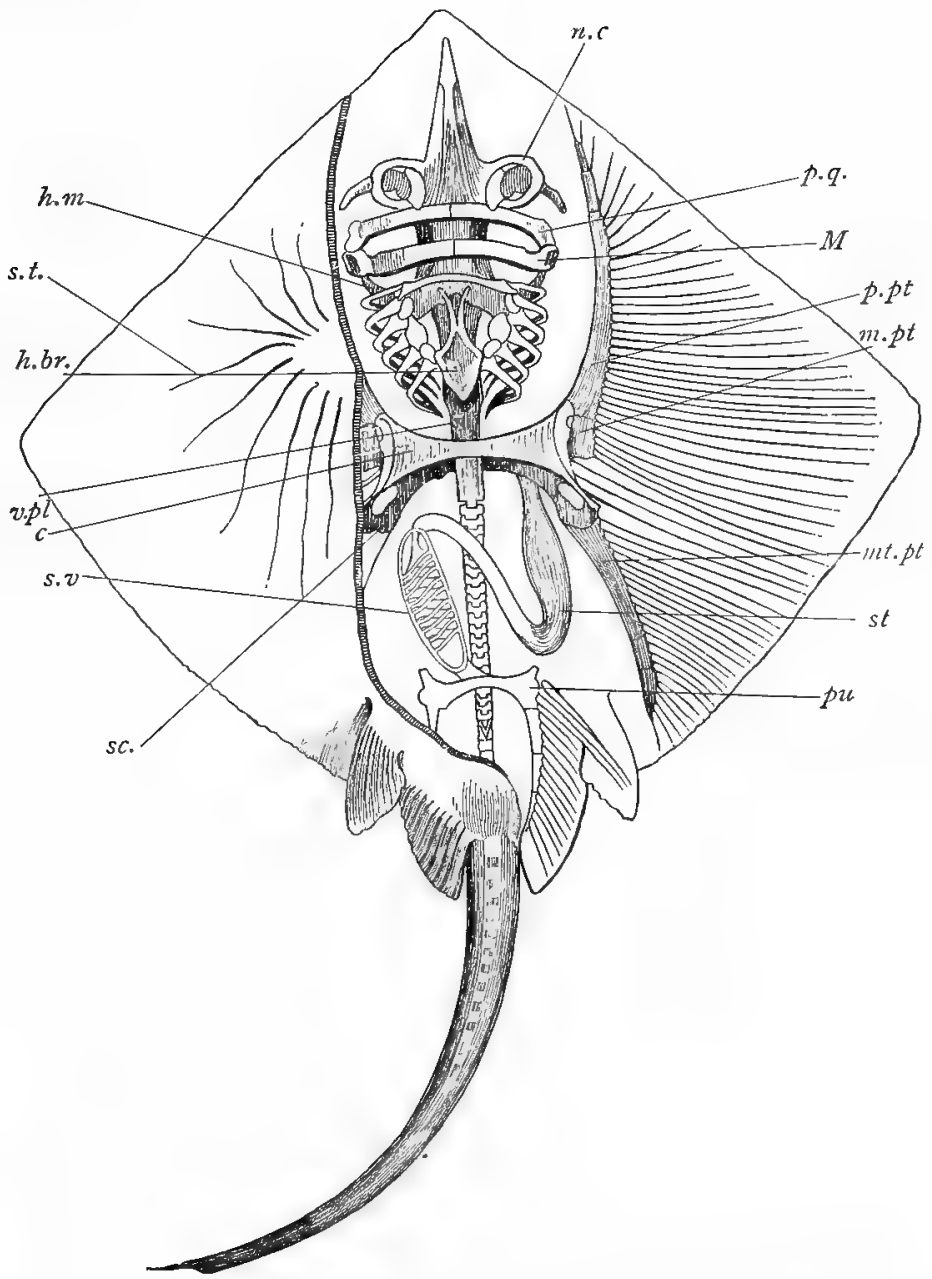

FIG. I 56.-Skeleton of Skate. (From a preparation.)

h.m., Hyo-mandibular; s.t., sensory tube; h.br., hypo-branchial, No. 5 ; $z^{\prime} . p l$, vertebral plate ; $c$., coracoid region; s. $v$., spiral valve ; s.c., scapular region; n.c., nasal capsule; $p . q$., palalo-quadratecartilage (upper jaw); M., Meckel's cartilage (lower jaw); p.pt. pro-pterygium ; m. pt., meso-pterygium ; ntt.pt., meta-pterygium : st., stomach ; pu., pubic bar. 
From the ear capsule to the articulation of upper and lower jaw there extends on each side a club shaped cartilage known as the hyo-mandibular. Attached to this is a slender three jointed rod-the hyoid.

Then follow five branchial arches, each primarily fourjointed, forming the framework of the gill-bearing region.

Of less importance are four labial cartilages about each nose capsule, an antorbital cartilage uniting the nose capsule with the end of the pectoral fin, and a spiracular cartilage supporting the rudimentary gill in the spiracle.

The dorsal or scapular region of the pectoral girdle is fixed on each side to the crest of the vertebral plate by means of a supra-scapula. The ventral region of the girdle is distinguished as the coracoid portion. The outer edge bears three facets, to which the three basal pieces of the pectoral fin are fixed.

Of these three basal pieces the anterior or propterygium and the posterior or metapterygium are large, the median or mesopterygium is small. All bear jointed radials. The true fin rays, comparable to the dermal rays in the fins of Bony Fishes, are represented by "horny" fibres.

The pelvic girdle is simpler than the pectoral, and is not fixed to the vertebral column. Its dorsal region is prolonged into an iliac process, while anteriorly a pre-pubic process projects from the ventral (pubic) bar. The girdle bears two articulating facets, to the posterior of which the the strong basal piece or metapterygium of the hind limb is attached. From this, and from the anterior facet of the girdle, the jointed radials proceed. The claspers of the males are closely connected with the hind limb, and have a very complex cartilaginous skeleton and an associated gland.

\section{The Brain.}

The brain (see p. 435) has the following parts :-

(I) The fused cerebral hemispheres or prosencephalon, with a nervous roof, and without ventricles.

(2) The thalamencephalon or region of the optic thalami, with a thread-like pineal body above, infundibulum and pituitary body below, thinly roofed third ventricle within.

(3) The mesencephalon or mid-brain with the optic lobes above, the crura cerebri below, the iter passing between.

(4) The cerebellum with an anterior and a posterior lobe, both marked by ridges and grooves. 
(5) The medulla oblongata, with thin vascular roof, with dorsolateral extensions called "restiform bodies."

The region beneath the thalamencephalon bears $(a)$ two ovoid inferior lobes, $(b)$ the infundibulum which carries the pituitary body, and $(c)$ a thin-walled three-lobed saccus vasculosus situated between the pituitary body and the inferior lobes.

\section{Cranial Nerves.}

Owing to the flat form of the skate and its frequently large size, the dissection of the cranial nerves is perhaps easier than in any other Vertebrate. Expecting practical verification, we shall describe their distribution in some detail, following in regard to certain points the investigations of Professor Cossar Ewart.

I. The olfactory, rising from the olfactory lobes of the cerebral hemispheres, extend to the nostrils, and there expand in olfactory bulbs, which give off nerves to the nostrils.

II. The optic, leaving the region of the optic thalami, cross in an optic chiasma, and extend to the retina of the eye.

III. The oculomotor or ciliary, arising from the crura cerebri, near the mid-ventral line, supply four of the six muscles of the eye. There is a ciliary ganglion in connection with III., and also with the ganglion of the ophthalmicus profundus.

IV. The pathetic or trochlear are small nerves arising dorsally from between the mid and hind brain, and supplying the superior oblique muscles of the eye. It is possible that they really belong to $\mathrm{V}$.

V. The trigeminal, or nerve of the "mouth-cleft," arising from the medulla oblongata (as do all that follow), has a (Gasserian) ganglion on its root, and three main branches - the sensory maxillary, which unites with the inner buccal of VII. ; the motor mandibular, which innervates the muscles of the jaws; and the sensory superficial ophthalmic (or orbitonasal), which runs over the eye to the snout, and comes into close relations with a

${ }^{1}$ I have to acknowledge indebtedness to Dr. Beard for his kindness in helping me to state the distribution of these nerves correctly, or as correctly as is at present possible. 
similar branch of VII. Internal to the mandibular branch lies the ganglionated ophthalmicus profundus, which sends branches to the eyeball, snout, \&c., and is referred by some to III., by others to V., and is regarded by others as an independent nerve.

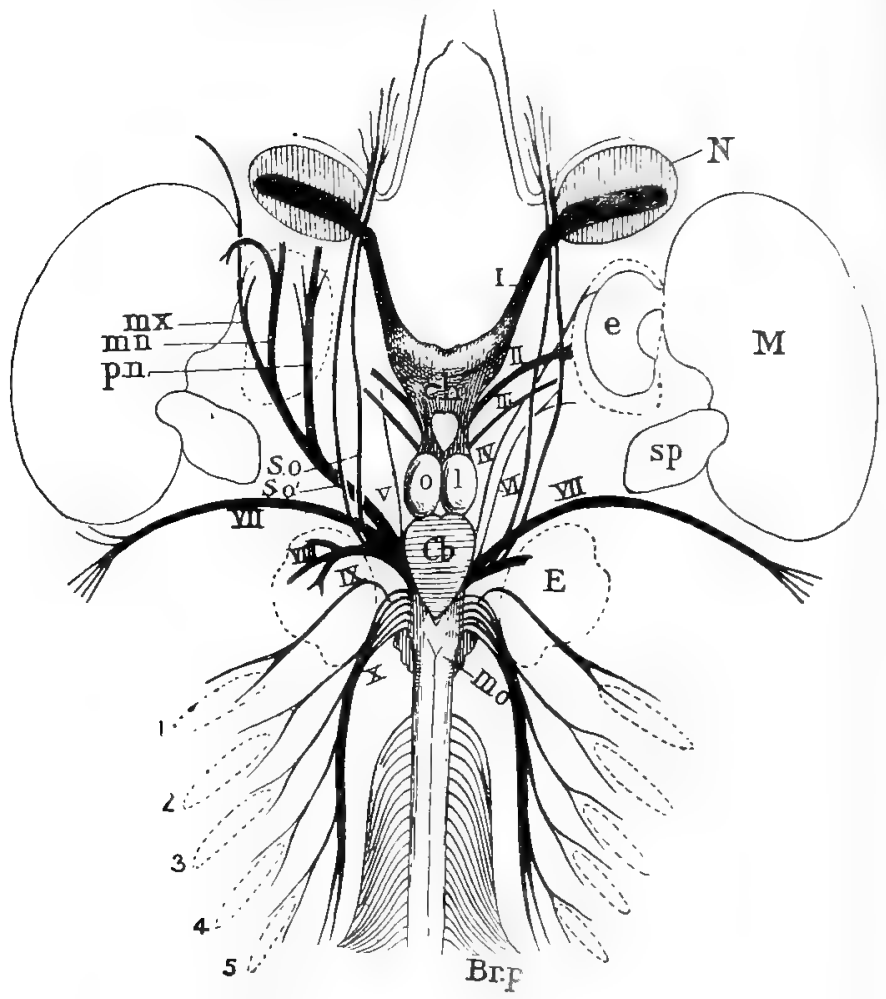

F1G. I 57.-Dissection of nerves of Skate.

$m x_{\text {, }}$ Maxillary of V.; mn, mandibular of V.; pm., ophthalmicus profundus; s.o., huperficial ophthalmic of V.; s.o'., superficial ophthalnic of VII.; V., VIl., VIII., IX., X., these nerves; I-5., gill clefts; o.l., optic lobes; $C b$., cerebellum; $N$., nostril; $c$, eye; $i{ }_{\text {. }}$, muscles of jaws; sp., spiracle; $E$., ear; m.o., medulla oblongata; $B$ r.p. brachial plexus ; I., II., III., IV., VI., VII., these nerves. 
VI. The abducens, a slender nerve, arising near the midventral line, adjacent to V. and VIII., and hidden by the former, supplies the external rectus muscle of the eye.

VII. The facial, morphologically the nerve of the spiracular cleft, supplies all the five groups of ampullæ on the head, and has seven main branches.

I. The ophthalmicus superficialis runs over and past the eye, unites with a similar branch of V., and supplies ampullae on the snout.

2. The inner buccal runs under the eye, through the nasal capsule, to inner buccal ampullæ. The outer buccal runs under the eye, over the antorbital cartilage, to outer buccal ampullae.

3. The hyomandibular runs directly outwards behind the spiracle to hyoid ampulle.

4. The external mandibular runs behind and under the spiracle along the jaw to mandibular ampulle, and is a branch of the hyo-mandibular.

5. The palatine runs over the spiracle to the roof of the mouth.

6. The "facial proper" supplies the muscles of the hyoid arch, and gives off-

7. The "chorda tympani," which runs under the spiracle to the inner side of the jaw.

With the loss of the sensory ampulize, the seventh nerve of higher Vertebrates becomes restricted to the last three branches.

A recurrent branch of the facial also runs under the auditory capsule to IX., and is equivalent to Jacolsson's anastomosis in higher forms.

VIII. The auditory, arising just behind VII., is the nerve of the ear.

IX. The glossopharyngeal, the most typical of all, is the nerve of the first functional gill cleft. Its root passes through the floor of the auditory capsule, and bears a ganglion above the cleft. Its branches, as named by Beard, are :-

(a) Post-branchial, to the muscles of the first branchial arch ;

(b) pre-branchial, arches over the cleft and runs along its front wall ;

(c) intestinal or visceral, to the pharynx ;

(d) supra-branchial or dorsal to a few sense organs on the mid dorsal line of the head. 
$\mathrm{X}$. The vagus, apparently made up of at least four cranial nerves, has five roots, and divides into six main ganglionated portions, which supply the four posterior clefts and arches, the posterior jelly-tubes, and the heart and stomach. It thus consists of :-

(a) A ganglionated root to the clefts and arches ( 2 to 5 inclusive), with post-branchial, præ-branchial, and pharyngeal branches, as in IX.

(b) A ganglionated root, arising in front of all the others, from which arises the lateral branch innervating all the posterior sensory tubes.

(c) From the fourth branchial branch arises the ganglionated intestinal which innervates the heart and the stomach.

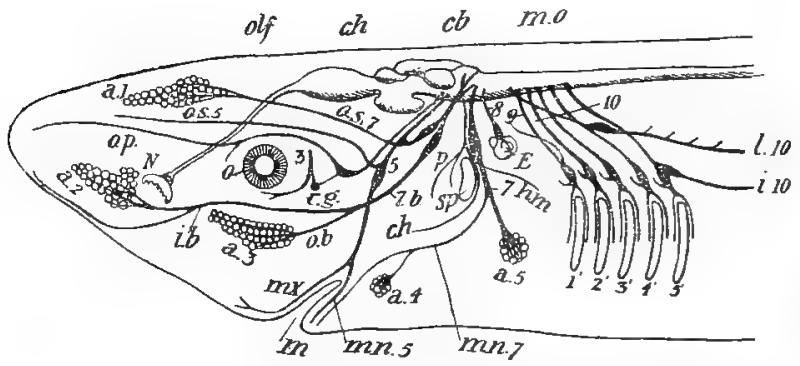

FIG. 158.- - Sidle view of chief cranial nerves of Elasmobranchs. (Slightly modified from CosSar EwarT.)

olf., Over olfactory nerve; ch., over cerebral hemispheres; ch. over cerebellum ; m.o., over medulla oblongata; m., mouth; mx., maxillary branch of 5 ; $m n .5$. , mandibular branch of $5 ; m n .7$. , mandibular branch of seventh nerve; $\boldsymbol{a}_{\text {. I-5., }}$ groups of ampullae; $0.5 .5 .$, superficial ophthalmic of $5 ; 0 . p$, ophthalmicus profundus; o.s.7., superficial ophthalmic of $7 ; N_{3}$, nostril ; 3 , oculomotor; c.g, ciliary ganglion; $5 .$, trigeminal ; $2 . b$., inner buccal ; $0 . b$., outer buccal ; $7 . b .$, buccal of $7 ; p$, palatal of $7 ; s p$, spiracle; $c h_{n}$, chorda tympani ; $7 . h m 2$, hyomandibular of $7 ; 8$, auditory; $E$, ear; 9 ., glossopharyn-

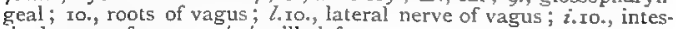
tinal nerve of vagus; $I^{\prime}-5^{\prime}$, gill clefts.

The spinal cord lies in the cartilaginous neural archway above the vertebral column, is divided by deep dorsal and ventral fissures, and gives off numerous spinal nerves, formed as usual from the union of dorsal (sensory) and 
ventral (motor) roots. The first sixteen or eighteen nerves form the brachial plexus, converging and uniting in a trunk which supplies the pectoral fin.

The sympathetic system consists of a longitudinal ganglionated cord along each side of the vertebral column. The ganglia of these cords are connected with the spinal nerves.

\section{Sense Organs.}

(a) The Eyes (see p. 445). The iris has a beautifully fringed upper margin.

(b) The Ears (see p. 444). The vestibule is connected with the surface by a delicate canal - the aqueductus vestibuli-a remnant of the original invagination. A small part of the wall of the auditory capsule is covered only by the skin forming a kind of tympanum. Within the vestibule are calcareous otolithic particles surrounded by a jelly.

(c) The Nasal sacs are cup-like cavities with plaited walls.

(d) The Sensory tubes are best seen on the ventral surface, where they lie just under the skin. They end in ampullæe, containing sensory cells.

\section{Alimentary System.}

The mouth is a transverse aperture; the teeth borne by the jaws are numerous, and those worn away in front are

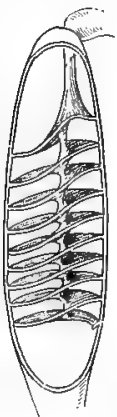

FIG. 159. - Spiral valve of Skate. (After T. J. PARKER.) replaced by fresh teeth from behind; naso-buccal grooves connect the nostrils with the corners of the mouth; the spiracles, which open dorsally behind the eyes, communicate with the buccal cavity; from the gullet five gill clefts open ventrally on each side; the stomach lying rather to the left is bent upon itself; the large brownish liver is trilobed, and has an associated gall bladder, from which the bile duct extends to the duodenum - the part of the gut immediately succeeding the stomach; the whitish pancreas lies in the duodenal loop between stomach and intestine, and its duct opens opposite the bile duct; the intestine contains an internal spiral fold-a membrane which increases the absorp- 
tive surface; a small rectal gland of unknown significance is attached to the terminal or rectal portion of the gut; the end of the gullet and the anterior portion of the stomach and the rectum are supported by folds of peritoneum,- the membrane which lines the body cavity, - the rest of the gut lies freely; into the terminal chamber or cloaca the rectum, the ureters, and the genital ducts all open; an abdominal pore opens on each side of the cloacal prorture. Excepting mouth-cavity and cloaca, the gut is li ondoderm.

Respiratory System.

The first apparent gill clefts-the spiracles - open dorsally behind the eyes. Each contains a rudimentary gill on the

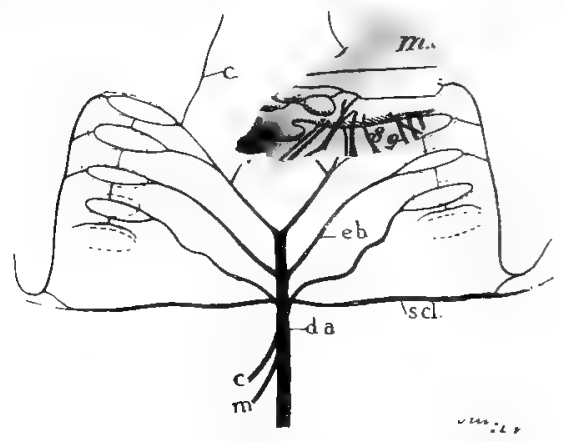

FIr. I60.-Upper part of the dorsal aorta in the skate. (After MONRO.)

d.c., Dorsal aorta; c., coeliac artery; m., superior mesenteric; s.cl., subclavian; $e . b$., efferent branchial vessels, three formed from the union of nine; $\%$, vertebral; c., carotid.

anterior wall, supported by a spiracular cartilage. Through the spiracles water may enter or leave the mouth.

There are five pairs of gill cavities, separated by partitions, and with ventral apertures. The first cavity is bounded anteriorly by the hyoid arch, posteriorly by the first branchial arch. The hyoid arch bears branchial filaments on its posterior surface; the first four branchial arches bear gill filaments on both surfaces; the fifth 
branchial arch bears none. Each of the first four branchial arches bears a half gill on each side; thus, including the gill filaments borne on the posterior side of the hyoid, there are four and a half gills. The absence of an operculum or gill cover is obvious. The gills are outgrowths from the wall of the gut, and therefore endodermic.

\section{Circulatory System.}

The impure blood from the body enters the heart by a bow-shaped sinus venosus, which leads into a large thinwalled auricle. Thence through a bivalved aperture the

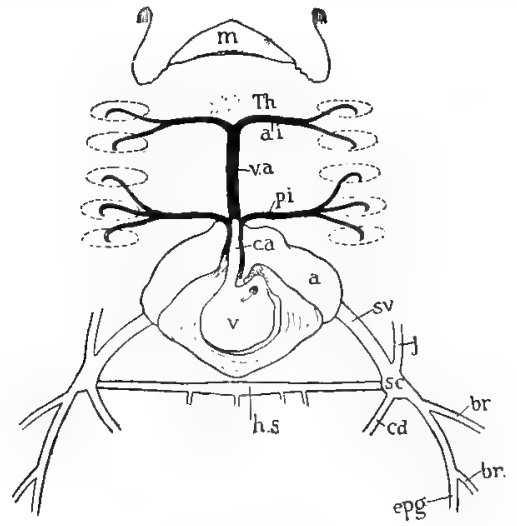

FIG. I6I.-Heart and adjacent vessels of Skate. (In part after MonRo.)

z., Ventricle; c.a., conus arteriosus; p.i., posterior innominate; y.a., ventral aorta; $a . i$, anterior innominate; $T h$., thyroid; $m$., nouth ; $\alpha_{\text {. }}$, auricle; s.v., sinus venosus; s.c., precaval sinus or sinus of Cuvier; h.s., hepatic sinus; $j$, jugular; br., brachials; $c d$. , cardinal ; epg., epigastric.

blood passes into the smaller muscular ventricle, and from this it is driven through a contractile conus arteriosus, with three longitudinal rows of five valves, into the ventral aorta.

The ventral aorta gives off a pair of posterior innominate arteries, which take blood to the three posterior gills, and a 
pair of anterior innominate arteries, which supply the anterior gill and the hyoid half gill on each side.

The purified blood passes from each half gill by an efferent branchial artery. To begin with, there are nine of these on each side, but by union they are reduced to three efferent trunks, which combine to form the dorsal aorta.

From the efferent branchial of the hyoid arch, a carotid arises, which divides into internal and external branches supplying the brain and head. From the first of the efferent trunks, a vertebral arises which supplies the brain and spinal cord.

The dorsal aorta gives off-(I) a subclavian to each pectoral fin; (2) a coeliac to the stomach, duodenum, and liver; (3) a superior mesenteric to the intestine, pancreas, and spleen; (4) spermatic arteries to the reproductive organs; (5) an inferior mesenteric to the rectum; (6) renal arteries to the kidneys; (7) arteries to the pelvic fins. It ends in the caudal artery.

At each end of the bow-shaped sinus venosus, there is a pre-caval sinus. This receives venous blood as follows :(a) from the head by a jugular vein; (b) from the liver by a hepatic sinus, which runs from one pre-caval sinus to the other like the string of the bow; (c) from a large posterior cardinal sinus (between the reproductive organs) by a cardinal vein on each side; $(d)$ from the hind-fin by an epigastric, with which brachials from the fore-limb unite anteriorly. The great cardinal sinus receives blood from the hind limbs, the kidneys, and other posterior parts.

Blood passes into the liver $(a)$ from the coeliac artery, and (b) by portal veins from the intestine (the hepatic portal system); blood leaves the liver by hepatic veins which enter the hepatic sinus.

Blood passes into the kidneys (a) from the renal arteries, and $(b)$ by renal portal veins from the caudal, pelvic, and lumbar regions (the renal portal system); blood leaves the kidneys by posterior cardinal veins, which enter the cardinal sinus.

Into the pre-caval sinus there also opens the lymphatic trunk, with nutritive fluid from the intestine.

The heart lies in a pericardial cavity, which is connected 
with the abdominal cavity by two fine canals, and is an anterior part of the colome. The blood contains, as usual, red blood corpuscles and leucocytes.

The dark red spleen lies in the curve of the stomach. The red thyroid gland lies just in front of the anterior end of the ventral aorta. The whitish thymus gland is a paired structure lying dorsally above the gills.

\section{Excretory System.}

Excretory System (see pp. 458-462).-The elongated, dark red kidneys lie posteriorly on each side of the vertebral

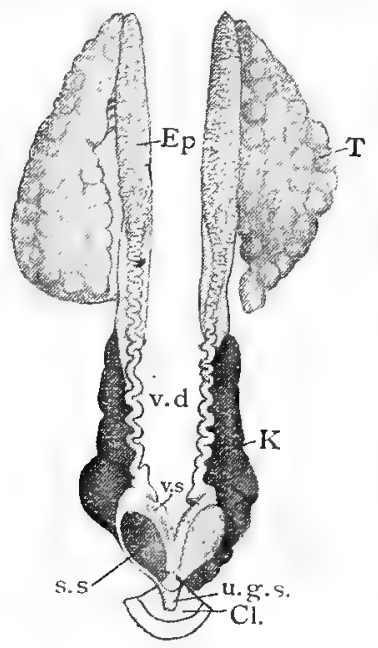

FIG. I62.-Urinogenital organs of male Skate. (From a specimen in Edinburgh Museum of Science and Art.)

$T$., Testis ; Ep., epididymis ; v.d., vas deferens : $K$., kidney ; v.s., seminal vesicle; s.s., sperm sac; H...s.s., urinogenital sinus; $C l$., cloaca.

column. They are developed from the hind part of the mesonephros. Several tubes from each kidney combine to form a ureter. The two ureters of the male open into the urinogenital sinus, whence the waste products pass out by 
the cloaca; in the female they open into little bladders-the dilated ends of the Wolffian ducts, and thence by a common aperture into the cloaca.

The archinephric or segmental duct of each side divides into a Wolffian and a Muillerian duct. The Wolffian duct becomes in the male the vas deferens, in the female an unimportant mesonephric duct; the Miillerian duct becomes in the female the oviduct, and in the male a mere rudiment.

The muscles and other organs of Elasmobranchs retain considerable quantities of nitrogenous waste products.

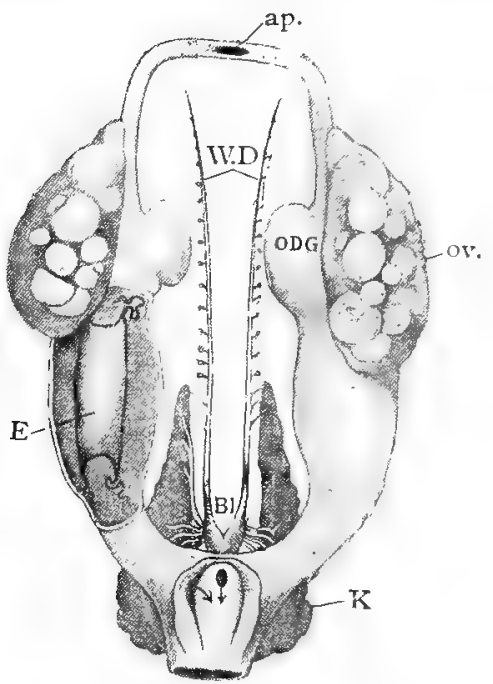

FIG. 163.-Urinogenital organs of female Skate. (In part after MONRO.)

$a \beta$, Aperture of united oviducts; $W . D .$, Wolffian duct; or'., ovary; O.D.G., oviducal gland; $E_{\text {. }}$, egg in mermaid's purse; $B L_{\text {, }}$ bladder at base of Wolffian ducts (arrow into cloaca); $K$, kidney (arrow from base of oviduct into clonca).

\section{Reproductive System.}

The male organs or testes lie on each side of the cardinal sinus, moored by a fold of peritoneum. Spermatozoa pass from the testis by vasa efferentia into a tube surrounded 
anteriorly by an epididymis. The tube of the epididymis is continued into the vas deferens, which is dilated posteriorly into a seminal vesicle and an adjacent sperm sac. Finally, the two vasa deferentia open into the urinogenital sinus, through which the spermatozoa pass into the cloaca. In copulation, the complex "claspers" of the male are inserted into the cloaca of the female.

'The female organs or ovaries lie on each side of the cardinal sinus, moored by a fold of peritoneum. In young skates they are like the young testes, but in the adults they are covered with large Graafian follicles, each containing an ovum. The ripe ova burst into the body cavity, and enter the single aperture of the oviducts, which are united anteriorly behind the heart. About the middle of each oviduct there is a large oviducal gland, which secretes the "purse;" the elastic lower portions open into the cloaca.

\section{Development.}

The ripe ovum which bursts from the ovary is a large sphere of yolk, with the formative protoplasm concentrated at one pole.

In Elasmobranchs formation of polar bodies (maturation) takes place at an early stage.

In the upper part of the oviduct the ovum is fertilised. It is said by some that numerous spermatozoa often enter the Elasmobranch ovum, although only one is actually concerned in fertilisation.

As the ovum descends further it is surrounded by albuminous material, and by the four-cornered "mermaid's purse" secreted by the walls of the oviducal gland. This purse is composed of keratin-a common skeletal substance which occurs for instance in hair and nails. Its corners are produced into long elastic tendrils, which may twine round sea weed, and thus moor the egg. Rocked by the waves, the embryo develops, and the young skate leaves the purse at one end.

The segmentation is meroblastic, being confined to the disc of formative protoplasm. From the edge of the blastoderm, or segmented area, some nuclei (so-called "merocytes") are formed in the outer part of the subjacent yolk (Fig. I64, n.). According to some, these yolk nuclei after- 
wards share in the making of the embryo. On the other hand, it has been said that they do nothing, even that they are the heads of numerous spermatozoa which have succeeded in entering the ovum.

At the close of segmentation the blastoderm is a lensshaped disc with two strata of cells. It is thicker at one end-where the embryo begins to be formed. Towards the other end, between the blastoderm and the yolk, lies a segmentation cavity (Fig. I64, sg.c.).

At the embryonic end, the outer layer or epiblast undergoes a slight invagination (Fig. $164, x$ ), beginning to form the roof of the future gut $(g \circ)$, in other words establishing the hypoblast. This inflected arc of the blastoderm corresponds to the blastopore or mouth of the gastrula, which is

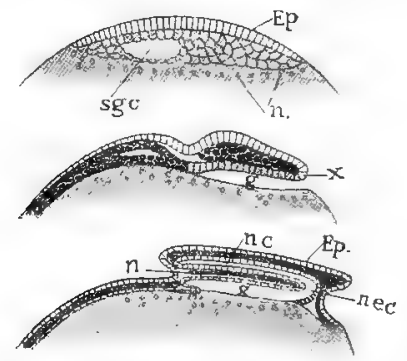

FIG. I64.-Elasmobranch development. (After BALfour.)

Uppermost figure shows blastoderm at an early stage. $E_{p}$, epiblast; so.c., segmentation cavity; $n_{\text {, }}$, yolk nuclei.

Middle figure shows the invagination which forms the gut. $x$, blastopore; $g$. archenteron. Mesoderm dark.

Lowest figure, a longitudinal section at a later stage $E p$, epiblast ; n.c., neural canal; ne.c., neurenteric canal ; $g$., gut ; $\eta_{\text {. }}$, notochord. Mesoderm dark.

much disguised by the presence of a large quantity of yolk. As the invagination proceeds, the segmentation cavity is obliterated. The floor of the gut is formed by infolding of the lateral walls.

Along the mid dorsal line of the epiblast a medullary groove appears - the beginning of the central nervous system. Its sides afterwards arch towards one another, and meet to form a medullary canal (Fig. $164, n . c$.). A posterior 
communication between this dorsal nervous tube above and the ventral alimentary tube persists for some time as the neurenteric canal (Fig. I6 6 , n.e.c.).

The mesoblast arises as two lateral plates, one on each side of the medullary groove. The plates seem to arise as a pair of solid outgrowths from the wall of the gut. They are afterwards divided into segments. Between the mesoblast plates, along the mid dorsal line of the gut, the notochord is established (Fig. I64, n.).

Besides the internal establishment and differentiation of layers, there are two important processes, (a) the growth of the blastoderm around the yolk, $(b)$ the folding off of the embryo from the yolk. The yolk is thus enclosed in a yolk sac, with which the embryo is finally connected only by a thin stalk-the umbilical cord. Through the canal of this cord nutriment is absorbed into the gut, and blood vessels also effect absorption.

What the different layers form, and how the organs arise, may be inferred from the general conclusions stated elsewhere.

Second type of Fishes. The Haddock (Gadus aglefinus) -A type of Teleosteans with closed swim bladder (Physoclisti).

\section{Form and External Features.}

The elongated wedge-like form is well adapted for rapid swimming. 'The terminal mouth bears a short barbule; this is long in the $\operatorname{cod}(G$. morrhua), and absent in the whiting ( $G$. merlangus). The nostrils, situated near the end of the snout, have double apertures. The eyes are lidless, but covered with transparent skin. Over the gill chamber and the four gills lies the operculum, supported by several bones. Distinct from one another, but closely adjacent, are the anal, genital, and urinary apertures,named in order from before backwards. Along the sides of the body runs the dark lateral line containing sensory cells. There are three dorsal and two anal fins, and an apparently symmetrical tail fin. 


\section{Skin.}

The small scales which cover the body are developed in the dermis, and are without any bone cells. Their free margin is even, a characteristic to which the term cycloid is applied, in contrast to ctenoid, which describes those scales which have a notched or comb-like free margin. Over the scales extends a delicate partially pigmented epidermis.

\section{Appendages.}

The pectoral fins are attached to the shoulder girdle just behind the branchial aperture. The pelvic or ventral fins, attached to what is at most a rudiment of the pelvic girdle, lie below and slightly in front of the pectorals-far from the normal position of hind limbs.

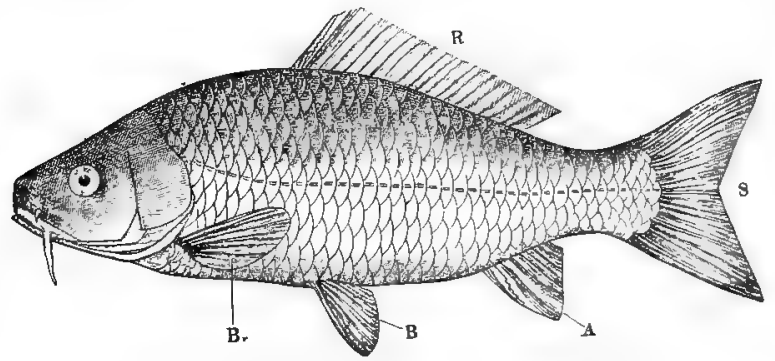

FiG. 165.-External characters of a Teleostean-a carp. (After Leunis.)

$R$., Dorsal unpaired fin ; $S$, homocercal caudal fin : $A$., anal fin; $\mathcal{B} . B$., Pectoral and pelvic paired fins. Note also the lateral line and barbule.

\section{Muscular System.}

The main muscles of the body are disposed in segments, -myotomes or myomeres, separated by partitions of connective tissue.

Skeleton.

The Vertebral column consists of biconcave or amphicœlous bony vertebræ. Each centrum in the trunk region bears superior neural processes, uniting in a neural arch crowned by a neural spine, and transverse processes pro- 
jecting from each side. Articulated to the distal ends of the transverse processes are the downward curving ribs, and also more delicate intermuscular bones which curve upwards. In the caudal vertebræ, the centra (c.) bear not only superior neural processes (n.a.), but also inferior hæmal processes (h.a.).

At the end of the vertebral column lies a fan-shaped hypural bone which helps to support the tail. The fin rays are jointed flexible rods, which in the dorsal and anal fins are attached to the ends of interspinous bones alternating with the neural and hæmal spines, and

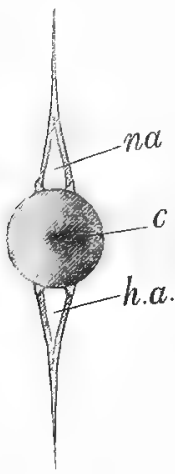

FIG. I66.-Caudal verte-

bra of haddock.

n.a., Neural arch ; c., centrum ;

$h, a$, hamal arch. attached to them by fibrous tissue.

The Skull includes the following bones, which may be grouped in the following regions (the membrane bones in italics) :-

(a) Around the foramen magnum; basi-occipital, two ex-occipitals, and a supra-occipital.

(b) Along the roof; supra-occipital, parietals, frontals, mesethmoid, nasals. Beneath the parietals lie the alisphenoids.

(c) Along the floor; basi-occipital, parasphenoid, vomers.

(d) Around the ear on each side; sphenotic, pterotic, and epiotic above, prootic and opisthotic (beneath).

(e) In front of and around the orbit; Parethmoid, lachrymal, orbitals.

The first or mandibular arch is believed by many to form Meckel's cartilage beneath, and the palato-pterygo-quadrate cartilage above. Meckel's cartilage becomes the foundation of the lower jaw, and bears a large tooth-bearing membrane bone-the dentary, a small corner bone-the angular, while the articular element is a cartilage bone. Of the bones associated with the upper part, the palatine lies in front, the quadrate articulates with the lower jaw; while between palatine and quadrate lie the pterygoid, the mesopterygoid, and the metapterygoid.

The second or hyoid arch is believed by many to form the hyomandibular and the symplectic above, and various 
hyoid bones beneath. The hyomandibular and its inferior segment the symplectic connect the quadrate with the side of the skull. Of the six hyal bones, the largest and most important is the ceratohyal, which bears seven long branchiostegal rays.

The toothed premaxilla forms the upper part of the gape,

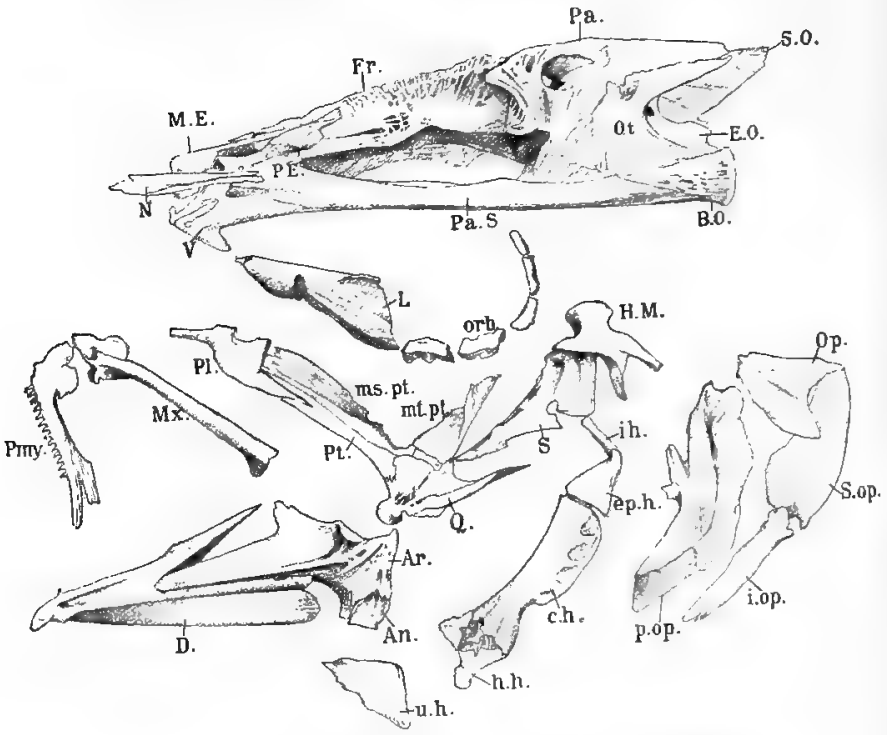

FIG, I67,--Disarticulated skull of Cod. (From Edinburgh Museum of Science and Art.)

S.O., Supra-occipital ; Pa., parietal ; Fr., frontal ; M.E., mesethmoid ; $N$., nasal ; $P . E$., parethmoid; Ot, otics; $E . O$. , ex-occipital ; $B . O$., basi-occipital ; $P a . S$, parasphenoid ; $V$, vomer ; $L$., lachrymal; orb., orbitals; H.M., hyomandibular; $S$. , symplectic; Q., quadrate ; Pt., pterygoid ; ntt.pt., metapterygoid; ms.pt., mesopterygoid ; $P l_{n}$, palatine; $D I x$, maxilla; $P m y$. premaxilla; Ar, articular; $A n_{\text {., }}$ angular; $D$., dentary; $24 . h_{n}$, urohyal; $h . h$., hypohyal ; $c . h .$, ceratohyal ; $e p h . h$. , epihyal ; $i h_{.}$inter-hyal; Op., opercular; S.op., sub-opercular; i.op., inter-opercular; p.op., praopercular.

while the maxilla which articulates dorsally with the vomer, and nearly reaches the quadrate posteriorly, does not enter into the gape. Both are membrane bones.

In the opercular fold are four membrane bones. 
The branchial arches are divided into various parts, of which the most interesting are the two superior pharyngeal bones which lie in the roof of the pharynx and bear teeth, and their counterpart, the inferior pharyngeal bone, which lies on the floor of the pharynx, and is likewise toothed.

The Limbs and Girdles.-The dermal rays of the pectoral fin are attached to four small brachial ossicles; these articulate with a dorsal scapula and a more ventral coracoid; both of these are attached to the inner face of a large clavicle, which almost meets its fellow of the other side

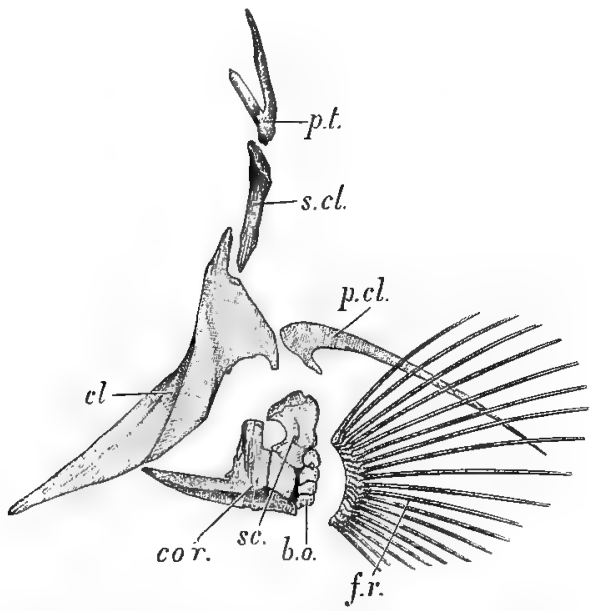

FIG. I68.-Pectoral girdle and fin of Cod. (From Edinburgh Museum of Science and Art.)

f.r., Fin rays; b.o,, brachial ossicles; cor., coracoid; sc., scapula ; $c l$., clavicle; $p . c l$. , post-clavicle; s.ch., supra-clavicle; p.t., posttemporal.

in the mid-ventral line of the throat. From the clavicle a slender post-clavicle extends backwards and downwards; while a stout supra-clavicle extends from the dorsal end of the clavicle upwards to articulate with a forked posttemporal, which articulates with the back of the skull. It must not be assumed that the elements of this girdle are directly comparable to those of a higher Vertebrate, although the nomenclature is the same. 
The fin rays of each pelvic fin are attached to a thin innominate bone, which may be a basal element of the fin, or the rudiment of a pelvic girdle.

\section{Nervous System.}

The relatively small cerebral hemispheres, the thalamencephalon with its inferior lobes and infundibulum, the large optic lobes, the tongue-shaped cerebellum which conceals most of the medulla oblongata, have their usual general relations. Each of the olfactory nerves is at first double; their bulb-like terminations lie far from the brain behind the nasal sacs. The large optic nerves cross one another without fusion at a slight distance from their origin, otherwise the nerves generally resemble those of the skate.

In the large eyes, the different parts will be readily identified; the small nasal sacs with plaited walls have double anterior apertures; the vestibule of the ear contains a large otolith, and another very small one in a posterior chamber. The dark lateral line, covered over by modified scales, lodges sensory tubes, and is innervated by a branch of the vagus.

\section{Alimentary System.}

Teeth are borne by the premaxillæ, the vomer, and the superior pharyngeal bones above, by the dentaries and the inferior pharyngeal bone beneath. There are no salivary glands, nor spiracles, nor posterior nares. A small tongue is supported by a ventral part of the hyoid arch. Five gill clefts open from the pharynx; their inner margins are fringed by horny gill rakers attached to the branchial arches and serving as strainers. The gullet leads into a curved stomach; at the junction of stomach and duodenum numerous tubular pyloric cæca are given off; into the duodenum opens the bile duct from the gall bladder and liver; the intestine passes gradually into the rectum, which has an aperture apart from those of the genital and urinary ducts. A pancreas is absent; perhaps the pyloric creca take its place. The peritoneal membrane which lines the abdominal cavity is darkly pigmented.

\section{Respiratory System.}

Water that passes in by the mouth may pass out by the gill clefts; the branchial chamber is also washed by water 
which passes both in and

out under the operculum. The gill filaments borne on

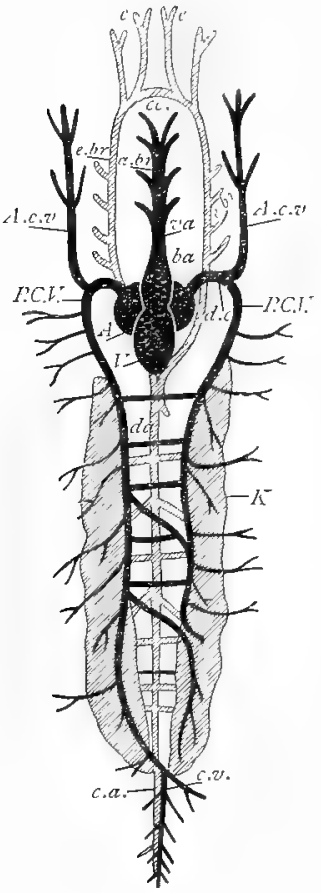

FIG. 169. - Diagram of Teleostean circulation. (After NuHN.)

The venous system is dark. $A$. , auricle; $V_{\text {., }}$ ventricle; b.a., bulbus arteriosus; $u_{\text {. a. }}$, ventral aorta; $a . b r$., afferent branchials; $2 . b r$., efferent branchials; c.c., cephalic circle; $c_{\text {. }}$, carotids; A.c.v, anterior cardinal veins; P.C.V., posterior cardinal veins; d.c., ductus Cuvierii ; d.a., dorsal aorta ; c. $z^{\prime}$, , caudal vein; c.a., caudal artery; $k$., kidney.

the heart by the sinus venosus, passes into the thin-walled the four anterior branchial arches are long triangular processes, whose free ends form a double row. As there are no partitions between the five gill clefts, the filaments project freely into the cavity covered by the operculum. Along each arch and filament there are blood vessels, bringing the impure blood, and removing it purified. On the internal surface of the operculum lies a red patch, the pseudobranch or rudimentary hyoidean gill.

The swim bladder lies along the dorsal wall of the abdomen; the duct which originally connected it with the gut has been closed. The dorsal wall of the bladder is so thin, that the kidneys and vertebræ are seen through it; the ventral wall is thick, and bears anteriorly a large vascular rete mirabile, which receives blood from the mesenteric artery and returns blood to the portal vein.

\section{Circulatory System.}

The heart lies within a pericardial chamber, separated by a partition from the abdominal cavity. The blood from the body and liver enters 
auricle, and thence to the muscular ventricle. From the ventricle it is driven up the ventral aorta, the base of which forms a white non-contractile bulbus arteriosus.

The ventral aorta gives off on each side four afferent branchial vessels to the gills. Thence the blood is collected by four efferent trunks, which unite on each side in an epibranchial artery. 'The two epibranchials are united posteriorly to form the dorsal aorta, while anteriorly they give off the carotids which are united by a transverse vessel closing the "cephalic circle."

Blood enters the sinus venosus by two vertical precaval veins, and by hepatics from the liver. Each precaval vein is composed dorsally of a jugular from the head and a cardinal from the body. The cardinals extend along the kidneys and are continuous posteriorly with the caudal vein, but the middle part of the left cardinal is obliterated.

\section{Excretory System.}

The kidneys are very long bodies, extending above the swim bladder under the vertebral column. The argest parts lie just in front of and just behind the swim bladder. From the posterior part an unpaired ureter extends to the urinary aperture, before reaching which it gives off a small bilobed bladder. The pronephros degenerates; the functional kidney is a mesonephros.

\section{Reproductive System.}

The testes are long lobed organs, conspicuous in mature males at the breeding season. The ovaries of the female are more compact sacs, more restrictedly posterior in position.

Two vasa deferentia combine in a single canal. The likewise single oviduct is continuous with the cavity of the ovaries. The genital aperture in either sex is in front of, but very close to, that of the ureter. According to some authorities the genital canals in Teleosteans are secondary structures, unconnected with the archinephric or segmental ducts, but the researches of Jungersen have made this very doubtful.

\section{Development.}

The ova of the haddock, like those of other Teleosteans, contain a considerable quantity of yolk, are fertilised after 
they have been laid, and undergo meroblastic segmentation. The eggs float, i.e., are pelagic; while those of the herring sink, i.e., are dimersal.

At one pole of a transparent sphere of yolk, lies a disc of formative protoplasm of a light terra cotta colour. The ovum is surrounded by a firm vitelline membrane. After fertilisation, the formative disc divides first into two, then into four, then into many cells which form the blastoderm. From the edge of the blastoderm certain yolk nuclei or periblast nuclei are formed which afterwards have some importance. At the end of segmentation, the blastoderm lies in the form of a doubly convex lens in a shallow concavity of the yolk.

The blastoderm extends for some distance laterally over the yolk; the central part raises itself, and thus forms a closed segmentation cavity; one radius of the blastoderm becomes thicker than the rest, and forms the first hint of the embryo; an inward growth from the edge of the blastoderm forms an invaginated layer-the dorsal hypoblast or roof of the gut; the periblast forms the floor of the gut, and afterwards aids the mesoblast which appears between epiblast and hypoblast; the medullary canal is formed as usual in the dorsal epiblast. It is likely that the edge of the blastoderm represents the blastopore or mouth of the gastrula, much disguised by the presence of yolk.

The newly hatched larva is still mouthless, and lives for a while on the residue of yolk, which, by its buoyancy, causes the young fish to be suspended in the water back downwards.

Third Type of Fishes. The Herring-Clupea harengus. A type of those Teleosteans which have the swim bladder communicating with the gut (Physostomi).

In habit the herring is pelagic and gregarious. It is found in the North Sea, the temperate and colder parts of the Atlantic, the Baltic, and the White Sea. A similar species lives in the N. Pacific.

\section{External Characters.}

The herring has the typical "fish" shape. Externally it differs from the haddock in the following features:- there is no barbule; the maxilla is divided into three parts; the nostrils have a single aperture on each side; there is no lateral line; the pelvic fins are abdominal, not jugular in position; there is one dorsal and one anal fin ; the body is more compressed; the ventral edge is covered by sharply keeled bony scales. 


\section{Nervous System.}

The brain has very small cerebral hemispheres and large optic lobes. The ear has a peculiar connection with the swim bladder. External to each of the well developed eyes are two immovable transparent folds of skin, with a vertical slit between.

\section{Alimentary System.}

The mouth has a narrowed gape. The upper jaw moves downwards and forwards when the mouth is opened. Small visible teeth are borne on the tongue and on the vomer, but those on both jaws are inconspicuous. The foodchiefly small crustaceans-is probably in part crushed by the gill rakers, which also prevent it passing out by the gill clefts. From the posterior end of the gullet a cæcum or crop is given off. A narrow communication leads from beneath this crop to a thick-walled, gizzard-like, muscular organ directed forwards. This in turn has an opening into the intestine, which runs straight to the anus. About twenty digestive cæca open into the beginning of the intestine. The swim bladder has a silvery exterior, and lies close under the back bone. The herring differs from most Physostomi as regards the connection between the swim bladder and gut, for the bladder does not communicate with the gullet but with the cæcum by means of a narrow, twisted canal. Anteriorly on each side the swim or air bladder gives off a thin duct which, passing through the walls of the skull, divides into two branches, each ending in a dilatation close to the ear. Posteriorly the swim bladder has a duct opening externally on the left side of the anus.

\section{Respiratory System, Bo.}

The gill filaments are fixed in a double row on the outer edge of each branchial arch. The specially wide opening behind the gill cover permits of a free current of water for respiration. The heart, kidneys, \&c, are much the same as in the haddock.

\section{Reproductive System.}

The testes or milt of the male and the ovaries or roe of the female, lie on each side of the abdominal cavity. In 
each sex there is a single external opening behind the anus. The number of mature eggs spawned at one time by the female has been variously estimated at from 10,000 to 30,000 .

In British waters there is a spring as well as an autumn spawning probably, however, by distinct shoals of herrings. When about to spawn, the herring come near the coasts into water of from ten to twenty fathoms depth. While the eggs are being shed by the females, the spermatic fluid is passed into the water by the males, and the eggs are thus fertilised before reaching the bottom, where they adhere to stones, zoophytes, and even crustaceans. The hatching of the eggs takes from 8 to 40 days, according to the temperature.

Development.--The young herring on emerging from the egg has the yolk sac attached; its skeleton is rudimentary; it has no scales; the ventral fins are undeveloped; one continuous fin passes along the back, round the tail to the anus. A month after hatching, the larva is about two-thirds of an inch long, and has absorbed all its yolk. About the third month the scales appear, and though only two inches in length, the form is then that of the adult. Growth continues at the rate of less than half an inch per month, and at the end of eighteen months the herring is sexually mature.

Closely allied to the herring are the sprat, the shad, and the pilchard. Thames "whitebait" are herring not six months old.

\section{THE ORDERS OF FISHES. (See Table, pp. 518-ig).}

Order I. Elasmobranchil-Cartilaginous Fishes.

Synonyms. Selachii. Plagiostomata (with transverse ventral mouth).

Sharks and skates represent the two distinct types included in this order. They are voracious carnivorous fishes. The scales are "skin teeth." There is no cover over the (5-7) gill apertures; anterior to these there is often a spiracle, the first gill cleft-with a rudimentary gill. The fins are large. The skeleton is mostly cartilaginous. The tail is asymmetrical or heterocercal. The mouth extends transversely on the under side of the head. The nostrils are also ventral. A spiral fold extends along the internal wall of the large intestine. Into the terminal chamber (or cloaca) of the gut, the genital and urinary ducts also open. The ventricle of the heart has an anterior auxiliary region-a contractile conus arteriosus. The males are provided with copulatory modifications of the hind limb, known as claspers. 
Fertilisation is internal. The ova are few and large. Large egg purses are common, but some Elasmobranchs are viviparous. The embryos have external gills.

Subdivisions.-The shark and the skate are types of two distinct suborders : - (I) the older Selachoidei, with approximately cylindrical bodies and lateral gill openings, as in shark and dog fish; (2) the more modified Batoidei, with flattened bodies and ventral gill openings, as in skates or rays.

Special Forms. - Mrustelues, Carcharias, Squalus, Torpedo, Acanthias, and others, are viviparous; Raja, Scyllium, Cestracion, and others, are oviparous. In two species of the genera first named, there is a placenta-like connection between the yolk sac of the embryo and the uterus of the mother. Zygana has a peculiar hammer-like head expansion; Pristis has the snout prolonged into a toothbearing saw; Torpedo has a powerful electric organ.

History. - The Elasmobranchs appear in the Upper Silurian, are very abundant from the Carboniferous onwards, but are now greatly out-numbered by the Bony Fishes. An increasing calcification of the axial skeleton is traceable through the ages, and in some of the ancient forms the exoskeleton was greatly developed, often including long spines or ichthyodorulites firmly fixed on the dorsal fins or on the neck. Among the most remarkiable

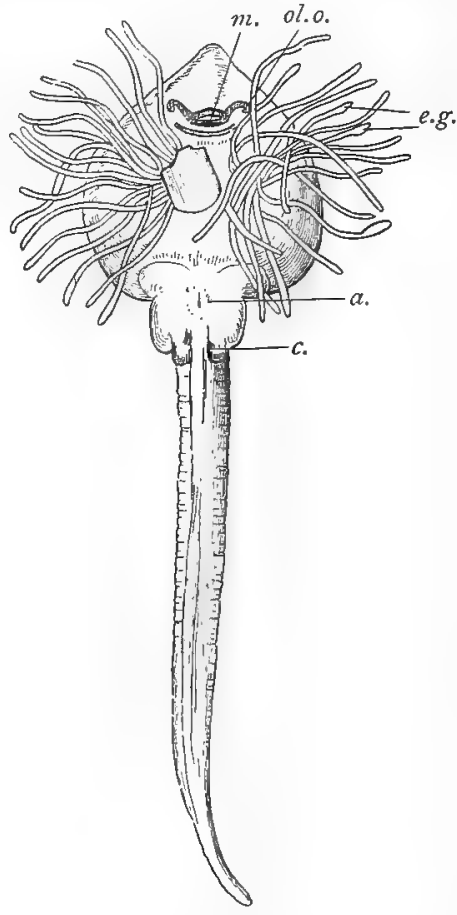

FIG. I70.-Young Skate. (From BEARD.)

(The yolk sac has been cut off, the yolk stalk

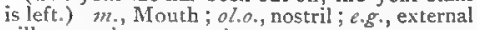
gills ; $a$., cloaca ; $c_{2,}$ claspers. extinct genera is Pleuracanthus, from Carboniferous to lower Permian. It had a terminal mouth, a naked body, a continuous dorsal fin, a symmetrical tail, and pectoral fins with an arrangement of rays resembling that in the biserial "archi-pterygium." 
The Holocephali are represented by the sea cat or Chimara from northern seas, and Callorhynchus from the south. There is a fold or operculum covering the gill clefts and leaving only one external opening on each side; the jaws are rigidly fixed to the cartilaginous skull; the skin is naked; the anus, the Miillerian and urinary ducts open separately. Otherwise the Holocephali resemble Elasmoluranchs, and may be regarded as a suborder. In some respects, however, e.g., in the structure of the skull, they suggest Dipnoi, and in this connection it is interesting to notice that there is an auricular septum in Chimara.

Teeth (of Ptyctodus, Rhynchodus, \&c.), which have been referred to Chimreroids occur in Devonian rocks, and some, at least of the detached spines of Carboniferous age, may have belonged to fishes of this order or sub-order. Undoubted Mesozoic Chimeroids are Squaloraja, Myriacanthus, Chimcropsis, Ischyodus, \&c., while others, including the recent genus Chimara, are found in strata of Tertiary age. The other recent genus, Callorhynchus, is also represented by a Cretaceous species, C. Hectori.

Another interesting but quite extinct group, whose position was for long a matter of dispute, but which is now usually referred to the

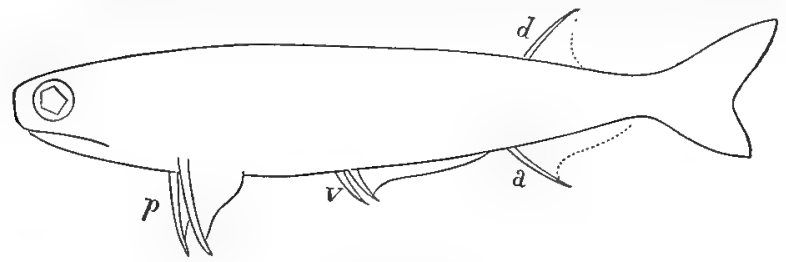

FIG. I71.-Outline of Acanthodes subcatus. (After TRAQUAIR.)

p., Pectoral fins ; v., ventrals ; a., anal ; d., dorsal.

Elasmobranchii, is that of the Acanthodei. These flourished principally in Devonian times, but lived on also through the Carboniferous to the Lower Permian. These are usually rather small fishes, with minute rhomboidal shagreen-like scales, and a strong spine in front of each fin, except the caudal. In some genera (Parexus, Climatizes) there are two rows of small intermediate spines between the proper pectorals and the ventrals.

\section{Order II. GanoIDEI.}

This ancient order of armoured fishes flourished in Devonian and Carboniferous ages, but is now represented by only seven genera, of which the Sturgeon (Acipenser) and the Bony Pike (Lepidosteus) are the most familiar.

The skin bears large scales, or bony scutes. The tail is either heterocercal or homocercal. Membrane bones invest 
the skull and shoulder girdle. The endoskeleton is in great part cartilaginous in Acipenser, Scaphirhynchus, and Spatularia, but is ossified in Lepidosteus, Polypterus, Calamoichthys, and Amia. In the first three the notochord is unconstricted; in the others there are distinct vertebral bodies, opisthocclous in Lepidosteus, amphicœlous in the other three genera. The fore-brain has a non-nervous roof. There is a spiral valve in the intestine, but it is very small in Lepidosteus. The food canal ends apart from and in front of the urinogenital aperture. There are also abdominal pores. An air bladder is present with a persistent open duct. The openings of the gill clefts are covered by an operculum supported by bones; in some of the genera there is a spiracle. A conus arteriosus is associated with the ventricle. The archinephric or segmental ducts do not divide; thus no Müllerian ducts are formed; the pronephros completely degenerates. The ova are small, and are fertilised in the water; they have comparatively little yolk, and so far as we know, their segmentation is holoblastic.

Genera.-The sturgeon (Acipenser) is one of the more cartilaginous Ganoids. The skin bears five rows of large bony scutes; the tail is asymmetrical or heterocercal; the notochord is unsegmented. A snout, bearing pendent barbules, extends in front of the ventral mouth, which is rounded and toothless. Sturgeons feed on other fishes, which they swallow whole. They are the largest fishes found in fresh water, for A. sturio may attain a length of is feet, and a weight of 600 pounds, while the $A$. huso of Southern Russia may measure 25 feet, and weigh nearly 3000 pounds! Most of the species are found both in the sea and in rivers or lakes. The flesh is edible, except in the case of the green sturgeon, $A$. medirostris, of the Pacific coasts, which is said to be poisonous; the roes or ovaries form caviare; the gelatinous internal layer of the swim bladder is used as isinglass. The genus Scaphirhynchus is represented in Asia and the United States; Polyodon or Spatularia spatula is the paddle fish or spoon bill of the Mississippi. In Pohptesus, from the Nile and other African rivers, the dorsal fin is divided into many parts, the nasal sac has a complex labyrinthine structure, the swim bladder arises from the ventral side of the gullet, the young are said to have external gills. In Old Calabar there is a related genus Calamoichthy's. The gar pike or bony pilie-Lefidosteus -is covered with rows of enamelled scales; the whole skeleton is well ossified, and the vertebral bodies arc opisthocnelous or concare behind; the swim bladder is like a lung in structure, and to a slight extent in function. The bow fin, Amia calzar, frequenting still waters in the United States, has a similar lung-like swim bladder. 
The fossil Ganoids appear in the Silurian about the same time as the Elasmobranchs, they are abundant from the Devonian to the Upper Cretaceous when the Teleosteans begin to become numerous. It is very doubtful whether the primitive armoured fishes (Tremataspis, Pterastis, Cephalaspis, Pterichthys, \&c, have any claim to be considered as Ganoids at all. They constitute the group of Ostracodermi, which, commencing in the Upper Silurian, seems to have become extinct at the conclusion of the Devonian era.

Fishes allied to the Ganoids of the present day appear in the Middle Devonian, and are found in abundance until the close of the Jurassic era, when they gave way to the more specialised Teleostei. In Devonian and Carboniferous rocks these Ganoids may be classed in two series :Crossopterygii (FIoloptychiidæ, Rhizodontidæ, Osteolepidæ, Colacanthidr), allied to the living Polypterus, and the Acipenseroidei (Palæoniscidae), allied to the Sturgeons. But already in the Permian era we begin to find representatives of that great semi-heterocercal series, which is represented at the present day by Lepidosteats and Amia, and which, in reality, passes gradually into the Physostomous Teleostei. These, represented by such forms as Lepidotus, Dapedius, Eugnathus,

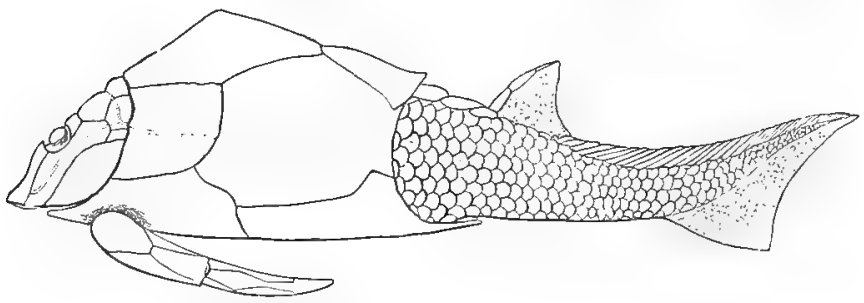

FIG. I72.-Pterichthys Milleri. (Lateral View. Restored by TRAQUAIR).

\&c., become very abundant in Jurassic rocks, while the Crossopterygii and Acipenseroiclei dwindle away. So cloes the Lepidosteid series in the Cretaceous era, and in Tertiary times the Ganoids were, as now, nearly a thing of the past.

\section{Order III. Tezeostei-the "Bony Fishes."}

This order includes most of the fishes now alive. Though comparatively modern fishes, they are older than was formerly supposed, as several Jurassic genera (Thrissops, Leptolepis, \&c.), which used to be classed as Ganoids, must be considered as actual Clupeoids, or Herring-like Teleostei. It is, however, not until the Upper Cretaceous and Tertiary 
epochs that they assume among fishes that overwhelming preponderance in numbers which they possess at the present day. The physostomous type of Teleostean is the most ancient, and probably stands in a continuous genetic line with the Lepidosteoid Ganoids.

The skeleton is well ossified, with numerous investing bones on the skull, others in the operculum, and on the shoulder girdle. The tail is sometimes quite symmetrical or diphycercal, but in most cases it is heterocercal at first, and acquires a secondary symmetry termed homocercal, for while the end of the notochord in the young forms is bent upwards as usual, the subsequent development of rays produces an apparent symmetry. The scales are in most cases relatively soft. As in Ganoids, the roof of the fore-brain is without nervous matter. The optic nerves are remarkable, because they cross one another without fusing (decussate). As in Ganoids, the partitions between the gill clefts disappear, so instead of the pouches seen in Elasmobranchs, there is, on each side, one branchial chamber, covered over by an opercular fold. Into this chamber the gill lamellæ borne by the branchial arches project freely. In most, a swim bladder is developed from the dorsal side of the gullet. There is no spiral valve in the intestine, and the food canal ends in front of and separate from the genital and urinary apertures or aperture. The base of the ventral aorta is swollen into a non-contractile bulbus arteriosus, but there is no conus, unless very exceptionally, as in Butirinus. According to some authorities, the archinephric duct is unsplit, and there is no Müllerian duct; according to Jungersen, the oviduct is a true Müllerian duct. The pronephros degenerates; the ova are numerous, and are fertilised in the water.

\section{Classification of Teleostei. (After Günther.)}

Dorsal, anal, and pelvic
fins in part spiny. $\left\{\begin{array}{lr}\text { Acanthopteri. Example-Perch. } \\ \text { Pharyngognathi. } & \text { Example - } \\ \text { Wrass. }\end{array}\right)$ Physoclisti, _- duct

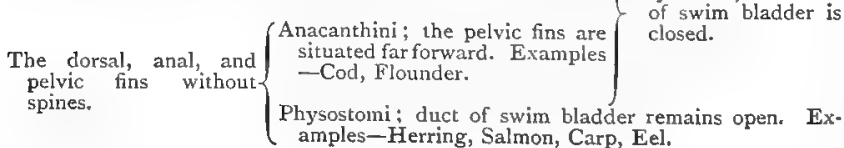


Besides these chief sub-orders, there are two sets of aberrant forms:-

(a) The sea horses, such as Hippocampus and Phyllopteryx, and the pipe fishes, such as Syngnathus, are distinguished as Lophobranchii. The gills, instead of being rows of filaments, are tufts of rounded lobes; the gill cover is a simple plate, leaving a small aperture; the skin is more or less protected by large derinal plates; the toothless mouth is at the end of a prolonged snout.

(b) The globe fishes, such as Tetrodon and Diodon, the trunk fishesOstracion, the sun fish-Orthagoriscus, and others, are distinguished as Plectognathi. The body is globular or compressed sideways; the skin bears bony scutes or spines, or is naked; the skeleton is incompletely ossified, and the vertebræ are few; the bones of the upper jaw are more or less fused; the pelvic fins are absent or reduced to spines; the gills are comb-like; the swim bladder has no duct.

It is likely that some of the loosely-built deep-sea fishes, such as the pelican fish Eurypharynx, are not referable to the orders usually recognised.

\section{Order IV. Dipnoi-"Mud Fishes."}

The Dipnoi, whose name means double breathers, are now represented by three genera-Ceratodus, from two rivers of Queensland; Protopterus, from certain African rivers, e.g., the Gambia; and Lepidosiren, from the Amazons. The wide distribution is noteworthy.

They are very ancient forms, for Ceratodus or a closely allied form has lived on from Mesozoic times, and there were also undoubted Dipnoi far back in Palæozoic times, such as Dipterus and Phaneropleuron of the Devonian,Ctenodus and Uronemus of the Carboniferous. According to some, the remarkable Devonian Coccosteidæ are also to be considered as an aberrant group of Dipnoi.

Prof. W. N. Parker regards them as "the isolated survivors of an exceedingly ancient group, which was probably nearly allied to the ancestors of existing Amphibians and Fishes, more particularly Elasmobranchs, though the Ganoid stock most likely arose not far off."

Were it not for the disadvantage of multiplying classes, one would be inclined to place them between Pisces, which they resemble in having cycloid scales, paired fins, a spiral valve, \&cc, and Amphibia, which they approach in having lungs, an incipiently three-chambered heart, a vena cava, a pulmonary vein, posterior nares, and multicellular skin glands. 
It must be noted, however, that it does not follow that the Dipnoi are the connecting links between Fishes and Amphibians because they possess certain characters of both these classes. We require further palæontological and embryological evidence. The Dipnoi are physiologically transitional between Fishes and Amphibians, having, for instance, acquired lungs while retaining gills, but it does not follow that they are morphologically transitional.

\section{(a) Ceratodus.}

The genus Ceratodus is abundantly represented by fossils in the Mesozoic beds of Europe, America, Asia, and Australia, but the living animal is now limited to the basins of two of the rivers of Queensland. C. forsteri, the best known and perhaps the only species, was first described by Krefft in 1870, and recently (1891) its habits have been studied by Professor Richard Semon of Jena. Like that other old-fashioned animal the duckmole, Ceratodus frequents the still deep places of the river's bed, the so-called "water-holes." At the bottom of these it lies sluggishly, occasionally rising to the surface to gulp in air. Its diet was formerly supposed to be exclusively vegetarian, but Semon holds that it crops the luxuriant vegetation of the river-banks only for the sake of the animal life-larvæ and eggs of insects, worms, molluscs, amphibians, and fishescontained among it. Certain it is, that natives and colonists catch it by means of animal bait. From this method of angling for it, and from its rosy-tinted flesh, considerable confusion has arisen between Ceratodus and a Teleostean fish, the true Barramunda or Dawson salmon, found in some of the Queensland rivers. Ceratodus is quite unable to live out of water, but its air-breathing powers enable it to exist in water which is laden with sand or rotten vegetable matter. According to Semon, its limited distribution is to be accounted for, first, by its sluggish nature, for it comes of a dying stock; and, secondly, by the fact that the eggs are very readily destroyed, and so incapable of distribution by any of the ordinary means. Nothing is known of the process of fertilisation, but the eggs, which are surrounded by a jelly-like envelope, are laid singly in the water. The development has not yet been fully worked out, but seg 
mentation is complete and unequal, and is followed by gastrulation. Segmentation of the embryo is obvious at a very early period; there is no trace of external gills. The

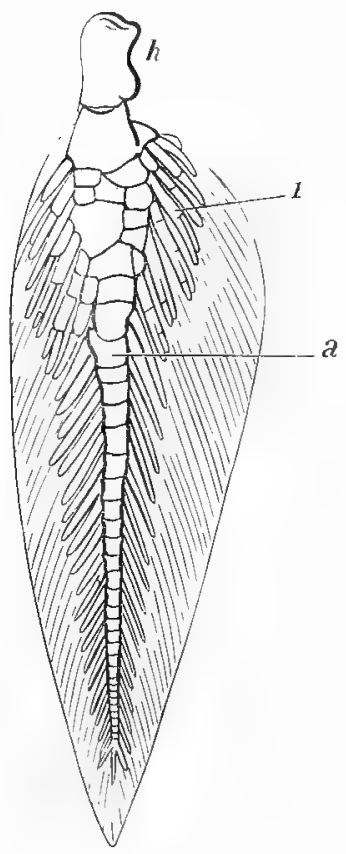

FIG. I73.-Skeleton of Cera. todus Fin. (From GeGerBAUR.)

a., Central axis; $r$, radials; $h$, basal piece. fourth epibranchial gives off the pulmonary artery. The pulmonary vein enters the left side of the auricle.

\section{(b) Protopterus.}

Protopterus lives in African rivers (Gambia, Quilimane, \&c.), is mainly but not exclusively carnivorous, and attains 33 
a length of 2 to 3 feet. It has extraordinary vitality, surviving severe wounds, long fasting, and desiccation. It appears to be most active at night, and to prefer shallow water, swimming rapidly with powerful tail-strokes, or "walking" slowly along the bottom with its filamentous fins moving alternately on each side, somewhat like the legs of a newt. At short intervals it comes to the surface to take mouthfuls of air, which passes out again through the opercular aperture.

As the dry season approaches, Protopterus burrows into the earth to a depth of about 18 inches, coils itself up,

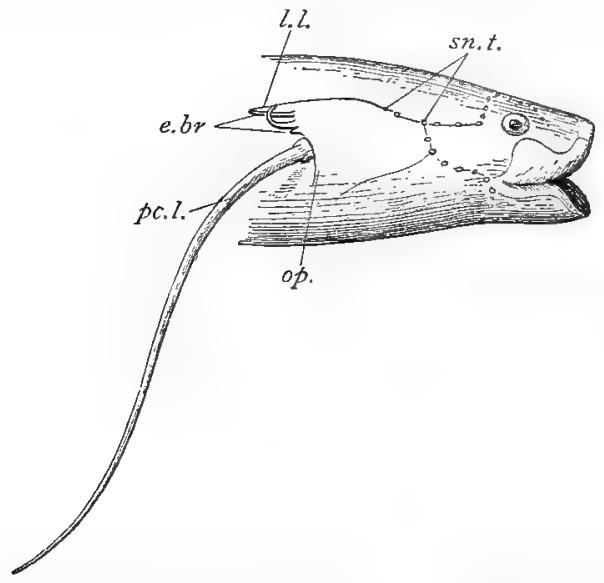

FIG. I74.-IIead region of Protopterus. (From W. N. PARkER.) sn.t., Sensory tubes; $l . l .$, lateral line; $c . b r .$, external gills; pc.l.,
pectoral fin ; op., operculum.

and secretes abundant mucus from its skin glands. This secretion forms a cocoon or capsule, with adherent earth externally, with moist slime internally, and with a lid, on which there is always a small aperture. Thus encapsuled, the animal may remain dormant for many months, e.g., from August to December. "The animal lies coiled up in such a manner that the head lies alongside the base of the tail, which from this point is again bent backwards over the 
head, so that it covers the head and body like a veil." These capsules, with the surrounding earth, have often been transported from Africa to northern Europe, without injury to the dormant life within. On emergence the animal makes peculiar sounds, probably due to the forcible expulsion of air from the lungs through the lips.

Two questions of much interest arise:-how does the encapsuled animal breathe, and how is it nourished?

Although the red vascular appearance of the tail led Wiedersheim to the opinion that caudal blood vessels might be the seat of a respiratory interchange of gases, it is almost certain that air passes directly from the mouth of the burrow, through the aperture of the capsule-lid (which is produced inwards in a short pipe) to the external nostrils, and thence to the lungs.

The nourishment appears to be derived from a store of fat deposited in the lymphoid tissue around the reproductive organs and kidneys, and among the lateral muscles of the tail (cf. fatty bodies in caterpillars, amphibians, \&c.). Moreover, some of the muscles are replaced by fat, and others undergo a pathological granular degeneration (cf. lamprey). To a certain extent, therefore, the dormant animal lives on its own tail. It is probable that leucocytes aid in the absorption and transportation of the degenerated muscles (cf. tadpoles).

A few of the anatomical characteristics of Protopterus may now be noted, following Prof. W. N. Parker.

The paired fins are filamentous, and seem degenerate when compared with those of Ceratodus, having only one series of short lateral horny rays on the cartilaginous segmented axis. The tail is symmetrical, and ends in a filament which, like the end of the fins, is often bitten off; often, however, there is a slight upward bending, which suggests a heterocercal condition. Both tail and fins may be regenerated after serious injuries.

In the skin are very numerous mucus-secreting goblet cells, and there are also (especially on the snout) multicellular glands, which are absent from most fishes, though common in Amphibians, Reptiles, and Mammals. There is a continuous lateral line, and apart from this there are other integumentary sense organs on the head and various parts of the body. There are taste buds on tongue and palate, olfactory organs with posterior as well as anterior nares-the latter concealed by the overhanging lips, relatively small, lidless eyes, and auditory organs. "The apparently anomalous position of the nostrils is probably to be explained as an adaptation to the habits of the animal in connection with its summer sleep."

There is a spiral valve in the large intestine; the cloaca has an associated "cæcum;" the pancreas surrounds the bile-duct, and though large, is almost bidden within the walls of the gut; the spleen is also large, but inconspicuous. Cilia are present throughout the stomach and intestine, and there are no differentiated gastric or intestinal glands. There is an unusually abundant investment of lymphoid tissue associated with the gut, "which, during the period when Protopterus is, as it 
were, parasitic upon itself, is probably of especial importance, not only in the formation of leucocytes and in the destruction of dying cells, but also in the processes of metabolism."

Behind the hyoid are five rudimentary branchial arches. There are five gill clefts, covered by an operculum, outside which are three external epidermic gills. Of the true internal gills the arrangement is as follows:- the hyoid has a small half row, the next two arches bear none, the third and fourth have the usual double rows of lamellæ, and the fifth has a single row.

The lungs are paired along almost their entire length, and extend under the notochord to the end of the body cavity. The glottis lies as usual on the median ventral floor of the pharynx, and by means of a vestibule ascending on the right side communicates with the unpaired anterior end of the lungs. Thus, although the lungs lie dorsally, they probably arise as a ventral diverticulum, as in higher animais.

The blood is remarkable for the large size of its elements and for the predominance of white over red corpuscles. In general structure the heart is like that of Ceratodus. There is but one auricle, but a dorsal fibrous ridge hints at its division. The conus arteriosus has a long spiral longitudinal valve and minute pocket-like valves. From the cone four branchial arteries arise on each side, and pass to the first four branchial arches, and the effect of the longitudinal valve is that the anterior pair contain blood already purified in the lungs; the posterior pair carry almost unmixed venous blood. The efferent branchials unite in a transverse trunk, and then form the dorsal aorta, and from the root of the aorta a paired pulmonary artery arises, the left supplying the ventral, and the right the dorsal aspect of the lungs. In regard to the veins, there is a single true postcaval, or inferior vena cava, along with a persistent left posterior cardinal. There is a single caudal vein giving rise to a right and left renal portal. Two pulmonary veins unite near the front of the lung in a single vessel, which enters the left side of the auricle.

The urinogenital organs are surrounded by lymphoid and fatty tissue ; the kidneys probably represent the mesonephros, and their duct the Wolffian duct; nephrostomes are absent. The vas deferens appears to be a special duct, probably formed in connection with the testes, quite independently of the excretory apparatus, and, therefore, to a certain extent comparable to that of Teleosteans; it opens into the base of the Miillerian duct, the rest of which gradually aborts in the male. The ovaries are strikingly like those of Amphibians; the oriduct seems to be the Muillerian duct. Ureters and genital ducts open beside one another into the cloaca.

(c) Lepidosiren. - Relatively little is known in regard to the third type-Lepidosiren-from the Amazons. It has an eel-shaped body with a continuous vertical fin. The limbs are reduced to cylindrical stems without any radials. There are no external gills. The air bladder or lung is double, and its relations to blood vessels are like those in Proto. pterzes.

There is an imperfect muscular septum dividing the auricle into two, and there is a similarly incomplete septum in the ventricle. The conus resembles that of Protoptemis. 


\section{General Notes on Fishes.}

\section{Form and Movenent.}

A fish may well compare with a bird in its mastery of the medium in which it lives. Thus a salmon is said to travel at the rate of about eight yards in a second, or over sixteen miles an hour. The motion depends mainly on the powerful muscles which produce the lateral strokes of the tail and posterior part of the body. It may be roughly compared to the motion of a boat propelled by an oar from the stern. So energetic are the strokes that a fish is often able to leap from the water to a considerable height. In some cases undulating movements of the unpaired fins, and even the rapid backward outrush of water from under the gill cover, seem to help in movement. The paired fins are chiefly used in ascending and descending, in steering and balancing, and some observers state that the pectoral fins of the flying fish are distinctly moved during the long skimming leaps. In a few cases, as in the climbing perch, and in the strange Periophthalmus, which clambers on the mangrove roots, the fore fins and tail are used in scrambling.

The characteristic form of the body, as seen in herring or trout, is an elongated laterally compressed spindle, thinning off behind like a wedge. In most cases the trunk passes quite gradually into head and tail. It is evident that this form is well adapted for rapid progression through the water. Flat fishes, whether flattened from above downwards, like the skate, or from side to side like the plaice and sole, usually live more or less on the bottom; eel-like forms often wallow in the mud, or creep in and out of crevices; globe fishes, like Diodon and Tetrodon, often float passively. There are many strange fishes, such as the sea horses (e.g., Hippocampus), which play among the sea-weeds in warm seas. Some of the deep-sea fishes have very quaint shapes.

\section{Colour.}

The colours of Fishes are often very bright. They depend partly on pigments in the cells of the skin, partly on the physical structure of the scales. The common silvery colour is often due to small crystals on the scales. In many cases the colours of the male are brighter than those of his 
mate, witness the gemmeous dragonet (Callionymus lyra) and the stickleback (Gasterosteus), and this is especially true at the breeding season. The colours of many fishes change with their surroundings. In the plaice and some others the change is rapid. Surrounding colour affects the eye, the influence passes from eye to brain, and from the brain down the sympathetic nervous system, thence by peripheral nerves to the skin, where the distribution of the pigment granules in the cells is aitered. In sballow and clear water this power of colour change may be of much protective value, but it seems likely that this has been exaggerated. An appreciation of the protective value of colouring demands careful attention to the habits and habitat of the animals, to the nature of the light in which they live, and to the enemies which are likely to attack them.

\section{Food.}

The food of Fishes is very diverse-from Protozoa to Cetaceans. Sharks and many others are voraciously carnivorous, many engulf worms, crustaceans, insects, molluscs, or other fishes; others browse on sea-weeds, or swallow mud for the sake of the living and dead organisms which it contains. Their appetite is often enormous, and cases are known (e.g., Chiasmodon niger), where a fish has swallowed another larger than its own normal size. Many fishes follow their food by sight; many by a diffuse sensitiveness, to which it is difficult to give a name; and others, it would seem, by a localised sense of smell.

Some Points of Structure-Fins. - Along the median line of the dorsal and ventral surfaces of some fishes, e.g., flounder, there is a continuous fin-a fold of skin with fin rays and underlying skeletal supports.

In the embryos of many fishes, the same continuous fringe is seen, while the adults have only isolated median fins. There is no doubt that these isolated median fins-of which there may be two dorsals, a caudal, and an anal or ventral - arise or have arisen from a modification of a once continuous fin, which is suppressed at one part and increased at another:

Now, the paired fins, which correspond to limbs, often resemble unpaired fins in their general structure, and in their mode of origin. In some Elasmobranch embryos, Balfour showed that the pectoral and pelvic fins were connected by transitory lateral ridges. It is therefore likely that the paired fins have arisen by a localisation of two once continuous lateral folds. Why there should be only two pairs we do not know. 
Two types of fish fin are distinguishable- $(a)$ that best illustrated among living fishes by Ceratodzes, in which a median jointed axis bears on each side a series of radial rays-a form often called an archipterygium; and $(b)$ the commoner type, in which the radials arise from a number of basal pieces (an ichthyopterygium). Experts do not seem to have yet come to a decision as to which of these types is the more ancient, or as to how they are related to one another.

Professor Huxley suggested that the fingered limb (cheiropterygium) of higher Vertebrates might arise from a limb of the Ceratodus type by an atrophy of its proximal fore-and-aft radials, and the hypertrophy of its distal radials. Thus the axis becomes the middle digit, while the other four digits are the terminations of the two distal radials on each side. But it seems just as easy or as difficult to trace the digitate limb to an ichthyopterygium.

Another interesting subject of inquiry is as to the origin of the girdles, whether as ingrowths from the bases of the limbs, or from modifications of branchial arches, or from both or neither.

Tail.-In Dipnoi and a few Teleosteans, e.g., the eels, the vertebral column runs straight to the tip of the tail, dividing it into two equal parts. This perfectly symmetrical condition is called diphycercal or protocercal, but it is not quite certain that its thorough symmetry is primitive.

In Elasmobranchs, Holocephali, cartilaginous and many extinct Ganoids, the vertebral column is bent dorsally at the end of the tail, and the ventral part of the caudal fin is smaller than, and at some little distance from, the upper part. This asymmetrical condition is called heterocercal.

In most Teleostei, and in extant bony Ganoids, the end of the vertebral column is also bent upwards, but the apex atrophies and, by the disproportionate development of rays on the ventral side, an apparent symmetry is produced. The vertebral column usually ends in a urostyle, -the undivided ossified sheath of the notochord. Most of the fin really lies to the ventral side of this. The condition is termed homocercal.

Scales,-In Elasmobranchs the scales (placoid) have the form of skin teeth (dermal denticles), tipped with enamel, cored with dentine, and based with bone sunk in the dermis. They arise from skin papillæ, the (ectodermic) epidermis forming the enamel, the (mesodermic) dermis forming the rest. It has been recently maintained, however, that the ectoderm forms most, if not all, of the scale (see p. 426). In other fishes the scales are almost wholly dermic, in marked contrast to those of Reptiles.

In most Teleosteans the scales are soft, and the epidermic covering is very thin. They are called cycloid or ctenoid, as their free margins projecting from sacs in the dermis are entire or notched. But bony scales also occur in many Teleosteans.

The sturgeon has five rows of bony dermic plates (scutes); the scales of the Bony Pike (Lepidostezs), Polypterzes, and many extinct Ganoids are covered with enamel.

The great interest of these exoskeletal structures is that those of Elasmobranchs are homologous with teeth, and that many bony scales often fuse into plates, suggesting the manner in which the membrane bones of 
the skull and pectoral girdle (e.g., the clavicle of Bony Fishes) are believed to have originated.

The simplest teeth of Elasmolranchs are precisely homologous with dermal denticles. But just as the skin teeth sometimes fuse in groups, so is it also with their homologues which form true teeth. Compound cuspiclate teeth in sharks arise from the fusion of adjacent simple cusps. But the fusion may go further; a complex crushing dental plate may be formed from the coalescence of several successional teeth. A further complication is brought about by the multiplication of cusps on individual teeth. These facts are, as Mr. A. Smith Woodward points out, of much interest, because it is by similar processes of fusion and of multiplication that the complex teeth of various Mammals arise.

Szoim bladder. - The swim bladder of fishes is one of the numerous outgrowths of the gut. It is absent in Elasmobranchs and some Teleosteans, such as most flat fish, and it forms the lung of Dipnoi. Unlike a lung, it opens dorsally into the gut, except in Dipnoi and the Ganoid Polypterzes, where the aperture is ventral. The original duct communicating with the gut may remain open, as in Physostomatous Teleosteans, or it may be closed as in Physoclystous Teleosteans. The bladder is usually single, but it is double in Protopterns, Lepidosiven, and Polypterus.

In regard to the use of the swim bladder, there is still considerable uncertainty. Where it is abundantly supplied with impure or partially purified blood, as in Dipnoi, Polypterns, and Amia, and where the yas within is periodically emptied and renewed, it is doubtless respiratory. But what of other cases, where its supply of blood is arterial, and what especially where it is entirely closed? In such cases it is usual to speak of its function as hydrostatic.

In greater detail, the function of the air bladder is (I) to render the fish, bulk for bulk, of the same weight as the medium in which it lives; moreover (2) the volume of the contained gas varies with increased secretion and absorption, and seems to adjust itself to different external pressures as the fish descends or ascends. (3) In many fishes the bladder may help indirectly in respiration by storing the superabundance of oxygen introduced into the blood by the gills. (4) There is in several Teleosteans, a remarkable connection between the swim bladder and the ear, sometimes by an anterior process of the bladder, as in the herring and perch-like fishes, sometimes by a chain of bones, as in Siluridie. This has suggested the riew that the connection serves to make the fish aware of the varying tensions of gas in the bladder, due to the varying hydrostatic pressure, and in the same connection it is interesting to notice the theory that the ear of fishes has to do through its semicircular canals with the equilibration and orientation of the animal's movements. It is also worthy of note that those fresh water fishes (Ostariophysia), which have the adjusting mechanism above referred to, have a marked ascendancy over all other fresh water species in which this mechanism is awanting (Bridge and Haddon).

$$
\text { lilat fishes. }
$$

In illustration of biological problems, let us briefly discuss some of the peculiarities of the flat fishes (Pleuronectidæ), 
such as flounder, plaice, sole, and turbot. These forms, we at once perceive, are flattened from side to side,-unlike the skates and rays, which are flattened from above downwards.

In adult life they swim and rest on one (the right or the left) side, and the hidden side is unpigmented. Moreover, the eye belonging to the downward side has come to lie beside its fellow on the upward side; the dorsal fin is extended anteriorly, separating the blind side of the head from that which bears the eyes; the inter-orbital parts of the frontal bones, which should be median, are bent to the upward side and compressed; and there may be further asymmetry in the skull, as in the greater development of jaws and teeth on the downward side. The skin of the downward side has an opaque reflecting layer (argenteum) and minute reflecting elements (iridocytes), but no pigment cells (chromatophores); all three contribute to the colour of the upturned surface.

In early life the larvæ swim for some time near the surface, and in the normal position, with the dorso-ventral plane vertical. Then they have an eye and chromatophores on each side. As they grow older they cease to swim vertically; one eye begins to move round the edge of the head (in Plagusia through an anterior extension of the dorsal fin); the body is held in a slanting position so that the line joining the eyes is kept horizontal; more or less rapidly the slant increases; the lower eye gets quite round to the upward side; the chromatophores on the shaded side disappear; and the fish rests and swims on one side at the bottom. In the turbot the right side is normally downward; in the flounder, the left side, but reversed specimens (especially of flounder) often occur. Occasionally these flat fishes are pigmented on both sides, and then it is sometimes noted that the migrating eye has not completed its movement.

Turbot and brill (species of Rhombus) have a well developed swim bladder during metamorphosis, and swim near the surface until the change is almost complete; flounder and other species of Pleuronectes have no swim bladder during metamorphosis, and begin to lie on the bottom almost as soon as the change commences. 
So far some of the more important facts, - what of their interpretation? That these asymmetrical forms have been derived from symmetrical ancestors is plainly suggested by their development. Of the original cause of the asymmetry we are quite ignorant. Did changes in the conditions of life induce the ancestral forms to leave the surface for the bottom? Or was the change due to certain peculiarities of structure-requiring, of course, previous explanation-such as the great depth of the body and the degeneration of the swim bladder? Or did both these causes operate at once?

But, supposing we had attained to some clearness in regard to the change of habitat and loss of vertical balance, we should then have to consider the twisting round of the downward turned eye and the absence of pigment cells on the downward side.

As to the change of the eye, it may be said (I) that this has gradually resulted from the efforts of the fish to continue to use the lower eye, a possible interpretation if acquired characters can be transmitted. (2) It may be said by those who do not believe in "use inheritance" that the twisting round of the lower eye is not a result of a transmitted growth tendency at all, but is wrought out by effort in each generation de novo. But young turbot and brill have nearly completed the twisting round of the lower eye long before they have abandoned their pelagic habit. (3) It may be said that the twisting round of the lower eye arose as a germinal variation, apart from any direct influence of function or environment, and that it has been retained and strengthened in the usual course of natural selection.

Again, as to the absence of chromatophores, it may be supposed that this also is a useful adaptive character persistent as the result of selection. But, apart perhaps from economy, it is not evident in what the advantage consists. It seems more likely that the under surface is unpigmented because it is shaded, and Mr. J. T. Cunningham, who has devoted special attention to the problem of flat fishes, has proved experimentally that artificial illumination of the lower sides by means of a mirror induces the development of pigment cells. It must be noted, however, that pigmentation of both sides occurs also as a natural variation. 


\section{Senses, \&c.}

Fishes do not seem to have much sense of taste or of smell, but diffuse sensitiveness to touch, chemical stimuli, $\& c$., is well developed, especially on the head and along the lateral line. Though there is no drum, and the ear is deeply buried, they certainly hear; thus there are well known cases of tame fishes coming to the sound of a bell or voice. Experiments have led some to believe that the semicircular canals of the fish's ear are indispensable in the direction or equilibration of movement, and it is obvious that this function is more important to a fish than the luxury of listening. But the results of experiment are still somewhat discordant. The sense of sight is, on the whole, well developed. As to the intellectual powers of their small brains we know little, but many show quickness in perceiving friends or foes, and many of their instincts are complex. At the breeding season there is sometimes an elaborate expression of excitement, well seen in the stickleback.

\section{Reproduction.}

The sexes are separate, except in Chrysophrys and Serranus, two hermaphrodite bony fishes, or when abnormal hermaphroditism occurs, as in herring, cod, mackerel. In many cases the males are smaller, brighter, and less numerous than the females. Courtship is illustrated by the sticklebacks (Gasterosteus, \&c.), the paradise fish (Macropodus), and others, and the bent lower jaw of the male salmon reminds us that some male fishes fight with their rivals.

Most Fishes lay eggs which are fertilised and develop outside of the body. They may be extruded on gravelly ground, or sown broadcast in the water. Sturgeon, salmon, and some others ascend rivers for spawning purposes, while the eels descend to the sea. In the case of trout, Barfurth has observed that the absence of suitable spawning ground may cause the fish to retain its ova. This results in ovarian disease, and in an inferior brood next season, a fact which should be compared with what Hertwig has observed in regard to Echinoderms, that ova which are retained beyond the normal period become over ripe and pathological. 
Except in Elasmobranchs the ova are relatively small, and large numbers are usually laid at once. In Elasmobranchs, the egg is large, and in the oviparous genera it is enclosed in a "mermaid's purse."

Most sharks and a few Teleosteans are viviparous, the eggs being hatched within the body of the mother, - in the lower part of the oviduct in sharks, in the ovary in Teleosteans. In two of the viviparous sharks (Mustelus lavis and Carcharias glaucus) there is an interesting union between the yolk sac and the wall of the oviduct, which should be compared with a similar occurrence in two lizards, and with the placenta of most Mammals.

As to fertilisation, the usual process is that the male deposits spermatozoa or "milt" upon the laid eggs or "spawn," but fertilisation is of course internal when the eggs are enveloped in a firm sheath, or when they are hatched within the mother.

Most Fishes have a great number of offspring, and parental care is proportionately little. Moreover, the conditions of their life are not suited for the development of that virtue. When it is exhibited, it is usually by the males, - witness the sea horse (Hippocampuss) and the pipe fish (Syngnathus), which hatch the eggs in external pouches, and "the male of some species of Arius, who carries the ova about with him in his capacious pharynx." The female of Aspredo carries the eggs on the under surface of the body until they are hatched, much in the same way as the Surinam toad bears her progeny on her back, while in Solenostoma a pouch for the eggs is formed by the ventral fins and skin. At least a dozen kinds of fishes make nests, of which the most familiar illustration is that of the male stickleback, who twines grass stems and water weeds together, glueing them by mucus threads exuded as semi-pathological products from the kidneys, which are compressed by the enlarged male organs.

Fishes have a less definite limit of growth than most other Vertebrates, and it is rare for a fish to exhibit any of the senile changes associated with old age in other Vertebrates. But surroundings and nutrition affect their size and colour very markedly. Some marine forms, such as flounders, may survive being shifted to fresh water, while others, such as salmon and sturgeon, pass from sea to rivers at spawning 
time. But many are sensitive to changes of medium. Many can endure prolonged fasting, and some may survive being frozen stiff. Lowered temperature may induce torpor, as seen in the winter sleep of the pike, while in the dry season of hot countries the mud fishes, the Siluroids, and others, encyst themselves in the mud, and remain for a long time in a state of "latent life."

\section{Inter-relations.}

Commensalism is illustrated by some small fishes which shelter inside large sea anemones, and by Fierasfer, which goes in and out of sea cucumbers and meduse. On the outside or about the gills of Fishes parasitic Crustaceans, fish lice, are often found; various Flukes are also common external parasites, and many Cestodes in bladderworm or tapeworm stage infest the viscera. The immature stages of Bothriocephalus latus occur in pike and burbot; a remarkable hydroid (Polypodium) is parasitic on the eggs of a sturgeon; the young of the fresh water mussel are temporarily parasitic on the stickleback; and the young of the Bitterling (Rhodeus amarus) live for a time within the gills of fresh water mussels.

Distribution in Space.-There are about 2300 species of fresh water fishes, three or four Dipnoi, about thirty Ganoids, and the rest Teleosteans, over a half being included in the two families of carps (Cyprinidæ) and cat fishes (Siluridæ).

Among marine fishes, about 3500 species frequent the coasts, rarely descending below 300 fathoms. A much smaller number, including many sharks, live and usually breed in the open sea, About 100 genera have been recorded from great depths.

In regard to the last, Dr. Guinther has shown that in forms living at depths from 80-200 fathoms, the eyes tend to be larger than usual, as if to make the most of the scanty light ; beyond the 200 fathom line small-eyed forms occur with highly developed organs of touch, and large-eyed forms which have no such organs, but seem to follow the gleams of "phosphorescent" organs; finally, in the greatest depths blind fishes occur with rudimentary organs. Many of these abyssal fishes are phosphorescent; the colouring is usually simple, mostly blackish or silvery; the skin exudes much mucus; the skeleton tends to be light and brittle; the forms are often very quaint; the diet is necessarily carnivorous. 


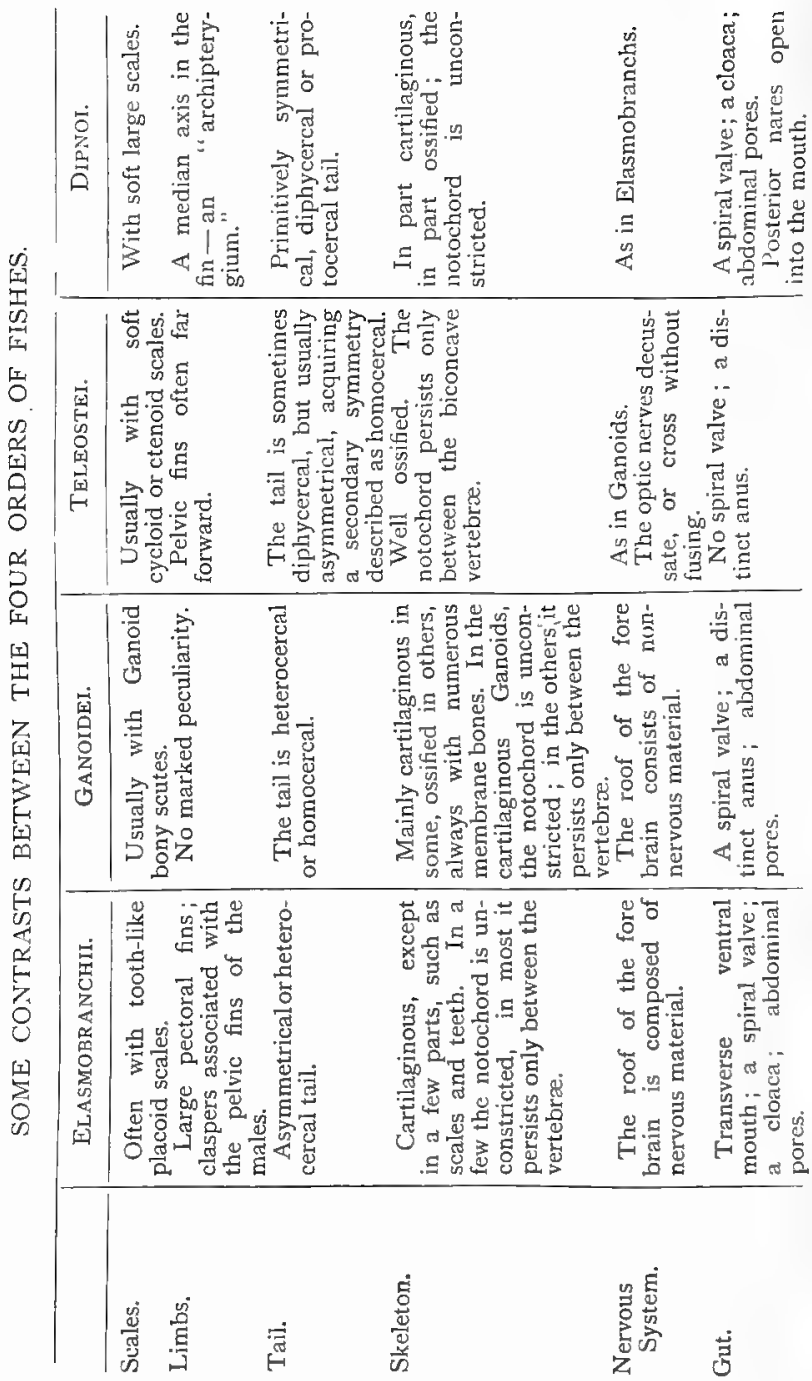




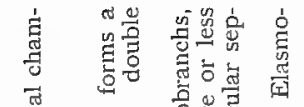

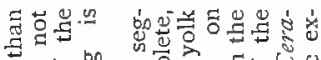

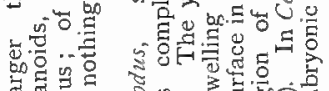

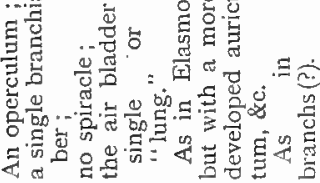

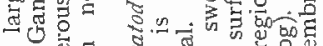

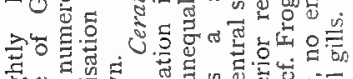

至焉

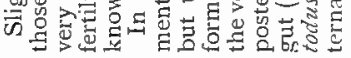

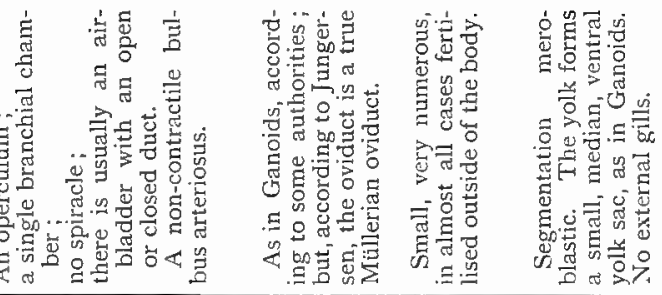

\begin{tabular}{|c|c|c|c|c|c|}
\hline 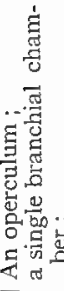 & \multicolumn{2}{|c|}{ 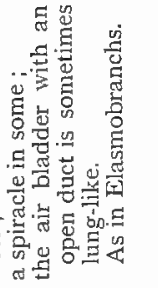 } & 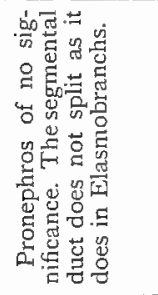 & 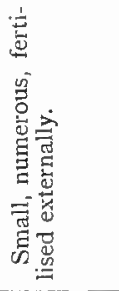 & 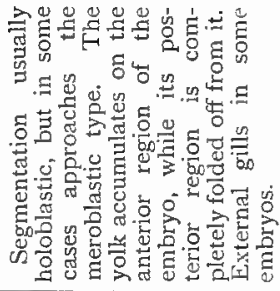 \\
\hline 。 & 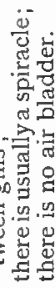 & 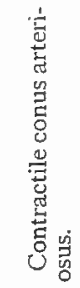 & 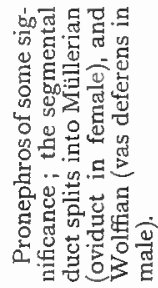 & 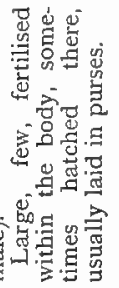 & 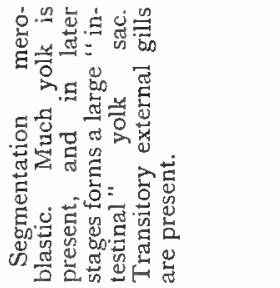 \\
\hline
\end{tabular}

起景

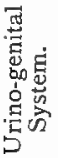

范 
The relationships of Fishes.

Balfour regarded the Elasmobranchs as nearest the ancestral stock; while from hypothetical Proto-Ganoids he derived on the one hand the Dipnoi, on the other hand the Ganoids, and thence the Teleosteans.

But it must be noted that the Dipnoi are markedly separated in many ways from living Ganoids. Moreover, the extinct Ganoids form a very large and diverse series, which cannot he fairly appreciated by a study of the few survivors. Nor does the palæontological evidence bear out the separateness of Teleosteans and Ganoids.

Günther distinguishes the sub-class Teleostei from the sub-class Palæichthyes, including under the latter the Chondropterygii (Elasmobranchs and Holocephali) and the Ganoidei (along with which Dipnoi are ranked). As two other sub-classes of Fishes, be recognises the Cyclostomata and the Leptocardii (Amphioxus).

Beard proposes the following classification of Ichthyopsida, insisting especially on the separateness of Dipnoi from Ganoids, and on their nearness to Amphibians :-

$$
\begin{aligned}
& \text { Ganodichthyidx, }\left\{\begin{array}{l}
\text { Marsipobranchii (Cyclostomata). } \\
\text { Ganoidei. } \\
\text { Teleostei. }
\end{array}\right. \\
& \text { Selachodichthyidxe. }\left\{\begin{array}{l}
\text { Selachil. } \\
\text { Pneumichthyidæ. }\left\{\begin{array}{l}
\text { Dipnoi. } \\
\text { Amphibia. }
\end{array}\right.
\end{array}\right.
\end{aligned}
$$




\section{CHAPTER XXIII.}

\section{CLASS AMPHIBIA.}

Order I. LABYRINTHODONTTA or STEGOCEPHALA (extinct).

II. GYMNOPHIONA or APODA (a small order).

III. URODElA ar CAUDATA, e.g., Newts and Salamanders.

IV. Anura or Ecaudata, e.g., Frogs and Toads.

Amphibians represent in the evolution of Vertebrates those forms which made the transition from aquatic to terrestrial life, but have lagged near the water. Certain acquisitions, such as lungs and a three-chambered heart, gained by the Dipnoi, are here firmly established. The race has dwindled in size of body since the early days of its beginning, but it seems to have been progressive, for Amphibians are not awanting in affinities with Reptiles or even with Mammals.

General Characters. - Amphibia are Vertebrates in which the visceral arches of the larva almost always bear gills, rehich may be retained throughout life, though the adults always possess functional lungs. When limbs are present they exhibit distinct digits, and conform to the same type as those of higher Vertebrates. Although unpaired fins are frequently present, both in larval and adult life, there are no fin rays. In existing forms there is rarely any exoskeleton, but some extinct forms were furnished with an armour of bony plates. The heart is three chambered, having two auricles and a ventricle. The gut ends in a cloacal chamber into which the urinogenital ducts open. A bladder, which grows out from the hind region of the gut, is probably homologous with the allanto is of the embryos of higher Vertebrates. The ova are small, numerous, uswally pigmented, and with yolk towards one pole. They are almost always laid in water; the segmentation is holoblastic, but unequal. There is often a marked metamorphosis in development. 
IIuxley was the first to recognise the affinities between Fishes and Amphibians, and to unite the two classes under the title Ichthyopsida.

Of the common characters of the two classes, we may emphasise the following :-gills are always present, but may be restricted to the larval stages; there is no amnion, and at most a homologue of the allantois ; there are lateral sensory structures, such as the "branchial senseorgans" and those of the "lateral line," but these may be diminished in the adults; unpaired fins are almost always represented, but may not persist in the adult life.

From the higher Vertebrates or Amniota the Ichthyopsida are clearly distinguished by the presence of gills (in youth at least) and by the absence of amnion and allantois. For though the bladder of Amphibians may be homologous with an allantoic outgrowth, it does not function as such, i.e., it does not aid in the respiration or the nutrition of the embryo.

It is more difficult to distinguish between Fishes and Amphibians, more especially if we include the Dipnoi in the former class. The most obvious clifferences are the absence of fin-rays and the development of fingers and toes. In the following table the two classes are contrasted :-

FisHes.

Gills persist throughout life.

The swim bladder functions as a lung in Dipnoi and less markedly in some Ganoids, but in most cases its respiratory significance is slight.

The heart is two-chambered (incipiently three-chambered in Dipnoi). There is no inferior vena cava, except in Dipnoi.

The limbs are fins.

The unpaired fins are supported by fin rays.

The skull has, in most cases, one occipital condyle.

There is usually an exoskeleton of scales or scutes.

Except in Dipnoi, the nasal sacs do not open posteriorly into the mouth.

There is no certain homologue of the allantois.
Amphibians.

Gills may disappear as the adult form is attained.

Lungs are always developed in the adults. It is doubtful whether they are directly comparable with the swim bladder.

The heart has three chambers. There is an inferior vena cava.

The limbs have digits.

There are no fin rays.

There are two occipital condyles.

There is no exoskeleton, except in a few exceptional cases, and in extinct forms.

There are posterior nares opening into the cavity of the mouth.

The bladder seems to be the homologue of the allantois.

\section{The Frog as a type of Amphibians.}

The common British frog (Rana temporaria) and the frequently imported Continental species ( $R$. esculenta) agree in essential features. A black patch on the side of the head behind the ear distinguishes our British species; the males of the edible frogs have special resonating sacs, and there are other trivial differences. 
'Though aquatic in youth, frogs often live in dry places, biding in great drought, reappearing when the rain returns. Everyone knows how they sit with humped back, how they leap, how they swim. They feed on insects and slugs. These are caught by the large viscid tongue, which, being fixed in front of the mouth and free behind, can be jerked out to some distance, and with even greater rapidity retracted. When we watch a frog, we see that the nostrils are alternately opened and closed, and that the under side of the throat is rhythmically expanded and compressed, the mouth remaining shut meanwhile; the movements are evidently connected with respiration. That the males trumpet in the early spring to their feebly responsive mates, that in our British species the pairing takes place soon after, that the young are tadpoles, that a notable metamorphosis takes place, are familiar facts of observation. In winter the frogs hibernate, buried in the mud of the pond, and breathing through their skin.

\section{Form and External Features.}

We notice the absence of neck and tail, the short forelimbs almost without thumbs, the longer hind limbs with five webbed nailless toes and with a long ankle region, the apparent hump back where the hip girdle is linked to the vertebral column. There is a very rudimentary thumb, and there is a horny knob at the base of the hallux or "great toe." At pairing time, the skin of the first finger is modified in the males into a rough cushion, darkly coloured in R. temporaria.

We see the wide mouth, the paired nostrils, the projecting eyes, the upper eyelid thick, pigmented, and almost immovable, the lower semi-transparent and moving very freely, the circular drum of the ear, the smooth skin, with patches of a deeper tint on its yellowish ground, and the slightly dorsal cloacal aperture.

\section{Skin.}

The smooth, moist skin is but loosely attached to some parts of the body; it consists of an external two-layered (ectodermic) epidermis, and an internal (mesodermic) dermis. The outer layer of the epidermis is shed periodically. The 
dermis differs markedly from that of a fish, for there is no exoskeleton, but this was present in the extinct Labyrinthodonts; there are multicellular glands, whose secretion makes the skin moist; and there is a stratum of unstriped muscle fibres. In the dermis there are also branched pigment cells, usually in two strata. Through a reflex nervous action they are slightly affected by the colour of the surroundings, the pigment-bearing internal cell substance contracting or expanding, and thus producing colour change. There are cutaneous blood vessels, by means of which the frog can, to a certain extent, breathe by its skin. The tadpole has sensory cells arranged in distinct lateral lines, but of these the adult retains no definite trace, though there are many nerveendings and "touch spots" in the skin.

\section{Skeleton.}

The vertebral column consists of nine vertebræ, and an unsegmented portion called the urostyle.

The first vertebra bears two facets for the two condyles of the skull and an odontoid process which lies between the condyles. Its arch is incompletely ossified. Each of the next six has an anteriorly concave or procœlous centrum, a neural arch surrounding the spinal cord, a trans-

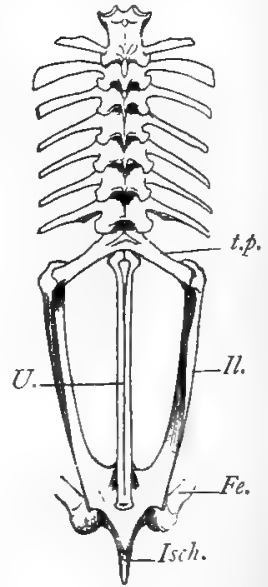

FIG. I75.- Vertebral column and pelvic girdle of Bull-frog.

t.f, Transverse processes of sacral vertebra; $I l$, ilium; $U$, urostyle; $F_{\mathcal{C}}$, femur; Isch, ischiac region. verse process from each side of the base of the arch, an anterior and a posterior pair of articular processes, and a short neural spine. The eighth vertebra has a biconcave or amphicclous centrum. The ninth is convex in front, with two convex tubercles behind, and bears large transverse processes with which the hip girdle articulates. The urostyle has anteriorly a dorsal arch enclosing a prolongation of the spinal cord, but both arch and nerve cord disappear posteriorly. The notochord, around which the 
vertebral column has developed, is finally represented only by vestiges in the centra of the vertebræ.

The skull consists $(a)$ of the persistent parts of the original cartilaginous brain box or chondrocranium, developed, as in the skate, from parachordals and trabeculæ,
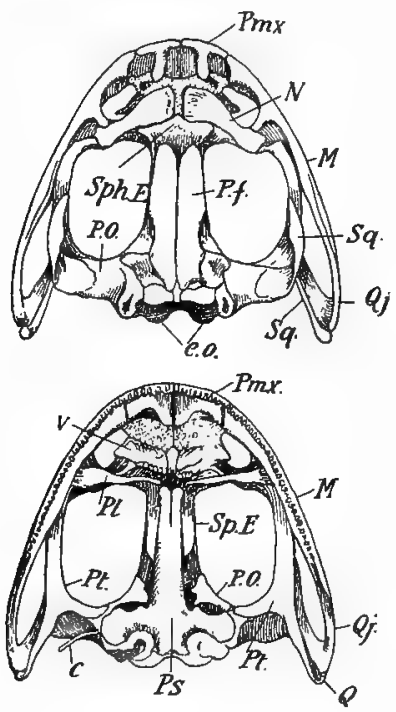

FIG. 176.-Skull of Frog-upper and lower surface. (After W. K. PARKER.)

Upper surface-

$P n x$, premaxilla ; $N$, nasal ; $M$, maxilla; $S q$, squamosal; $O j$, quadrato-jugal ; e.o., ex-occipitals ; $P . f$, parieto-frontals: Sph.E, sphenethmoid ; P.O., pro-otic.

Lower surface-

$P m x$, premaxilla; $M$, maxilla; $Q ;$, quadrato-jugal : $Q$, quadrate; $P t_{0}$ pterygoid; $P_{s}$, parasphenoid; $P . O$., pro-otic ; Sp.E, sphenethmoid ; $P l$, palatine; $V$, vomer; $c$, columella.

anterior end of the parasphenoid palatines.

Each half of the lower jaw, based on Meckel's cartilage, consists of capsules; (b) of ossifications of parts of the chondrocranium, cartilage bones; (c) of membrane or investing bones; and (d) of associated visceral arches.

Part of the chondrocranium persists as an encasement of the brain. Two exoccipitals bounding the foramen magnum and forming the condyles, two pro-otics or ossifications of the original auditory capsule, and an unpaired sphenethmoid forming the front of the braincase are cartilage bones. Two parieto-frontals and two nasals above, a paired vomer and an unpaired dagger shaped parasphenoid beneath, and two lateral hammer shaped squamosals are membrane bones. There is no basisphenoid ossification.

To these are added the small pre-maxillze in the very front of the skull, the long maxille on each side, the quadratojugals which continue the latter to the minute nodule which represents the quadrate bone.

On the roof of the mouth, extending from the quadrate forwards to near the vomers, are the triradiate pterygoids, while at right angles to the and behind the vomers are the

plus nasal and auditory 
three pieces, -the largest an articular angulo-splenial, outside this a thin dentary, and anteriorly uniting with its fellow a minute mentomeckelian.

A delicate rod-the columella auris-extends from the tympanum to the fenestra ovalis in the internal capsule of the ear. According to Parker, it represents the upper part of the hyoid arch, the lower portion of which forms the cartilaginous or partially ossified hyoid plate, which lies in the floor of the mouth and is produced into two anterior and two posterior cornua. According to Villy, however, the columella is morphologically connected with the ear capsule.

The teeth are borne by the premaxille, maxillæ, and vomers.

There is no parietal foramen, but in the Labyrinthodonts it is always distinct, and the pineal body is supposed to have been well developed. The foramen is also very distinct in some of the extinct Ganoid Fishes.

The cartilage which bears the quadrate at its lower end, and runs between pterygoid and squamosal, connecting the articulation of the lower jaw with the side of the skull at the auditory capsule, is called the suspensorium. In Elasmobranchs, the hyomandibular is the suspensorium ; in Teleosteans, the name is applied to the hyomandibular and symplectic; in Sauropsida, the quadrate occasionally gets the same confusing title.

When the lower jaw is connected with the skull wholly by elements of the hyoid arch, as in most Elasmobranchs and Ganoids, and all Teleosteans, the term hyostylic is used. When the connection is due to a quadrate element only, as in Amphibia and Sauropsida, it is called autostylic. When there is both a hyoid and a quadrate element, as in Lepidosteus among Ganoids, or a hyoid and a palatoquadrate, as in Cestracion among Elasmobranchs and perhaps also in Holocephali, the term amphistylic is used. Finally, it may be noted here that in Mammals the lower jaw articulates with the squamosal.

The first or mandibular arch gives origin inferiorly to Meckel's cartilage, which forms the basis and persistent core of the lower jaw, and superiorly to the palato-pterygo-quadrate cartilage which is represented in the adult by the minute quadrate bone, by the suspensorial cartilage, and by other cartilages which are invested by the pterygoid and palatine bones.

The second or hyoid arch gives origin inferiorly to the hyoid plate, superiorly, according to Parker, to the columella.

Of the four posterior branchial arches, there are in the adult some persistent remnants, e.g., in the larynx.

\section{The Limbs and Girdles.}

The shoulder girdle consists of a dorsal portion-the scapula and the partially cartilaginous supra-scapula, and of a ventral portion - the coracoid, and the clavicle, and the cartilaginous precoracoid on which the clavicle lies. There is some uncertainty, however, in regard to the relations of the last two; according to one view, the clavicle is unrepre- 
sented. The glenoid cavity with which the humerus articulates is formed as usual by the junction of scapula and coracoid.

Between the median ends of the coracoids lie two cartilaginous epicoracoids, behind which is a bony part of the sternum, prolonged posteriorly into a notched cartilaginous xiphisternum. Anteriorly lies a bony portion called the omosternum, which is prolonged forwards into an episternum cartilage. This sternum does not arise like that of higher Vertebrates from a fusion of the ventral ends of ribs. Indeed, there are no ribs in the frog, unless they be minute rudiments at the ends of the transverse processes.

The skeleton of the fore limb consists of an upper arm

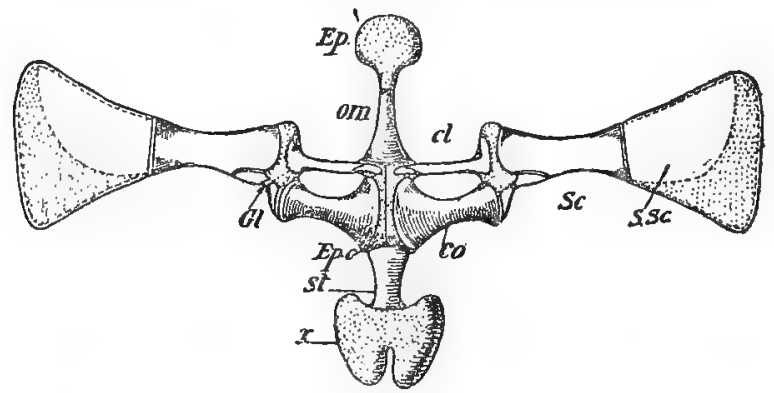

FIG. 177.-Pectoral girdle of Rana esculenta. (After Ecker.)

The cartilaginous parts are dotted. Ep, episternum; on, omosternum; Ep.c, epicoracoids; st, sternum; $x$, xiphisternum ; $c l$, clavicle with underlying precoracoid cartilage; $\mathrm{Co}$, coracoid; $\mathrm{Sc}$, scapula ; S.sc, supra-scapula ; $G l$, glenoid cavity for humerus.

or humerus, a fore arm in which the inner radius and the outer ulna are fused, a wrist or carpus including two proximal and three distal elements, and a central piece wedged in between them, five metacarpal bones, of which the first-corresponding to the absent thumb-is very small, and four fingers, of which the two innermost have two joints or phalanges, while the two others have three. 
The pelvic girdle has a long $V$-shape, the ends of which are cartilaginous and articulate with the expanded transverse processes of the ninth or sacral vertebra. Each limb of the $\mathrm{V}$ is an ilium; the united posterior part consists of a fused pair of ischia, and a ventral cartilaginous pubic portion. Ilia, ischium, and pubes unite in bounding the deep socket or acetabulum with which the femur articulates.

The skeleton of the hind limb consists of a thigh bone or femur, a lower leg formed from the united tibia and fibula, an ankle region or tarsus including two long proximal elements-the astragalus or tibiale and the calcaneum or fibulare-and two imperfectly ossified distal elements, five metatarsal bones, and five toes. The first toe or hallux has two phalanges, the second also two, the third three, the fourth four, the fifth three, and, finally, outside the

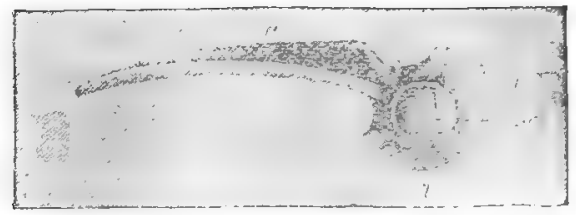

FIG. 178.-Side view of frog's Pelvis. (After Ecker.) $I l$, ilium ; $I s$, ischium; $P b$, pubis; $A c$, acetabulum.

hallux there is a "calcar," which looks like an extra toe and consists of three pieces. The astragalus is in line with the first toe.

\section{Muscular System.}

I shall not describe the musculature of any of the $V$ ertebrate types With the guides to practical work cited in the appendix the student will readily find out what he wishes to know.

The muscles are enswathed in connective tissue. They consist of bundles of muscle fibres, and at their ends or at one of them they are usually continued into strong tendons, which are more or less directly attached to parts of the skeleton. 
Nervous System.

'The brain, covered with a darkly pigmented pia mater, has the usual five parts.

The elongated cerebral hemispheres have "olfactory lobes" in front of them, and are connected by anterior and posterior commissures, and by a hint of a "corpus callosum" (?).

The thalamencephalon gives origin dorsally to a pineal outgrowth closely attached to the skull. On the ventral side will be seen the chiasma or interlaced crossing of the optic nerves, and a tongue-shaped mass (the tuber cinereum), to which the pituitary body is attached, and which is produced by the depression
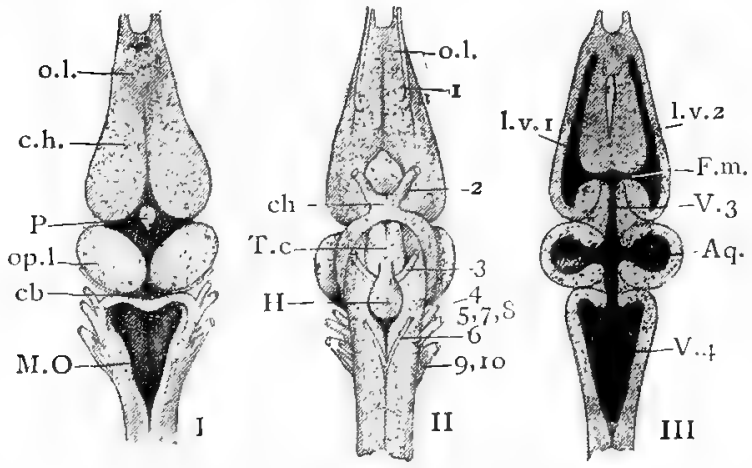

Fig. I79. - Brain of Frog. (After Wiedersheim.)

I. Dorsal Aspect, - o.l., olfactory lobes; $c_{.} h$., cerebral hemispheres $: P$., pineal body, rising from optic thalami; of.l., optic lobes; $c b$, rudimentary cerebellum; $M . O$, medulla oblongata.

II. VENTRAL ASPECT, - The numbers indicate the origins of the nerves ; ch. optic chiasma ; T.c., tuber cinereum ; $H$., hypophysis.

III. LoNGITUdinal SECTion.-l.v., I and 2 , lateral ventricles of cerebrum ; $F . m_{\text {, }}$ foramen of Monro; $V_{.}, 3$ and 4 , third and fourth ventricles; $A q$. cavities of optic lobes and aqueduct of Sylvius from third to fourth ventricle.

of the floor of the third ventricle to form the infundibulum.

The optic lobes, a pair of oval bodies between and below which is the iter.

The cerebellum, a very narrow transverse band. 
The medulla oblongata, on the roof of which the pia mater forms a very vascular "choroid plexus."

In the tadpole the pineal body lies outside the skull under the skin of the head; it atrophies at the metamorphosis, so that in the adult the stalk only is represented.

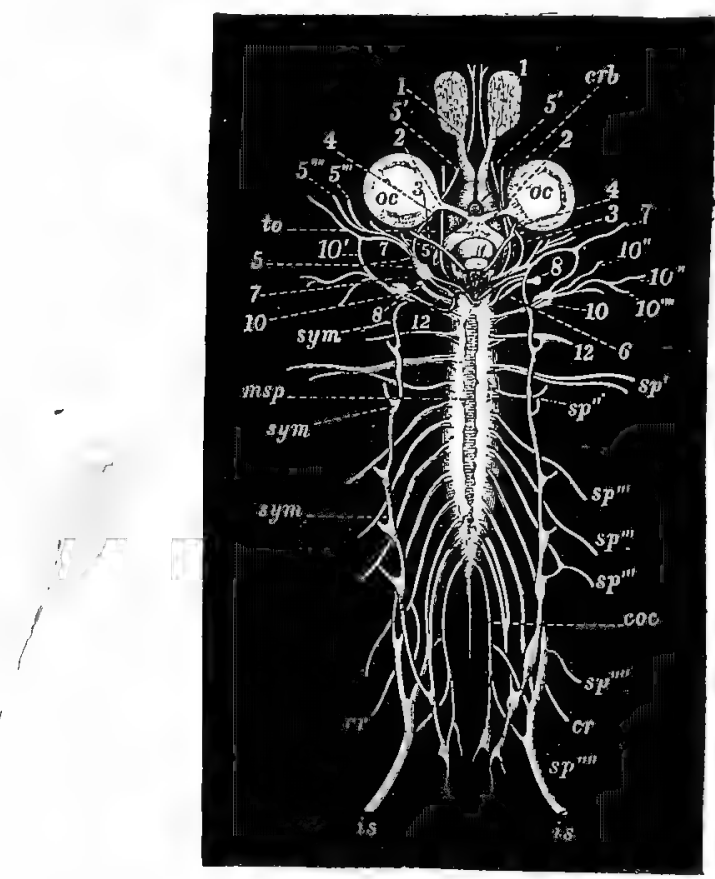

FIG. 180.-Nervous system of Frog. (After ECKer.)

I-Io. The cranial nerves; $o c$, eyes; $c r b$, in front of optic chiasma; to, optic tract; sym, sympathetic system: $m s p$, spinal cord; $s p$, spinal nerves.

The cranial nerves are, as usual, on each side the following :- -

(I) Olfactory, from the olfactory lobe to the nose ;

(2) Optic, crossing and interlacing with its fellow;

(3) Oculomotor, to four muscles of the eye; 
(4) Pathetic, to the superior oblique eye muscle;

(5) Trigeminal, with ophthalmic, maxillary, and mandilular branches ;

(6) Abducens, to the external rectus eye muscle ;

(7) Facial, arising along with the auditory, with a ganglion uniting with the Gasserian ganglion of the trigeminal, with a palatine branch to the roof of the mouth, and a hyoid branch to the lower jaw ;

(8) Auditory, to the ear ;

(9) Glossopharyngeal, to the tongue and some of its muscles; with a ganglion which unites with that of the tenth;

(ro) Vagus, with branches to lungs, heart, stomach, \&c.

The student should refer back to the description of the skate, and to the chapter on the structure of Vertebrates.

The spinal cord gives origin to ten pairs of spinal nerves, and is swollen at the origin of those which go to the limbs. Around the union of the anterior and posterior roots lie sacs with crystals of carbonate of lime.

The sympathetic system consists of about ten pairs of ganglia- $(a)$ united by branches to the spinal nerves; $(b)$ united to one another by longitudinal trunks which accompany the dorsal aorta and the systemic arches, and end anteriorly in the Gasserian ganglion; (c) giving off branches to the heart, the aorta, and the viscera in the pelvic region.

\section{Sense Organs.}

The eyes project on the top of the head and on the roof of the mouth. There are two lids, the upper thick and very slightly movable, the lower transparent and movable. The transparent cornea in front, the firm sclerotic surrounding the eyeball, and the sheath of the optic nerve, are as usual continuous. The next layer includes the vascular and pigmented choroid and the brilliant iris. Internally is the sensitive retina, while vitreous humour fills the cavity behind the lens.

The internal ears have the usual parts and lie within the auditory capsules, which are in great part bounded by the pro-otics. Connecting the fenestra ovalis of the ear with the tympanic membrane, which is flush with the skin, there is a delicate bony rod-the columella. This lies in the Eustachian tube, which opens into the mouth at the corner of the gape.

The nostrils open into small nasal cavities, with folded 
walls of sensitive membrane; the posterior nares open into the front of the mouth.

There are taste papillæ on the tongue, and touch spots on the skin.

\section{Alimentary System.}

The frog feeds in great part on insects, which it catches dexterously with its tongue. This is fixed in front and loose behind. There are teeth on the pre-maxillæ, maxillæ, and vomers. Into the cavity of the mouth the nasal sacs open anteriorly, and the Eustachian tubes posteriorly. The males of Rana esculenta have a pair of sacs which open into the mouth cavity at the angle of the jaw, and are dilated during croaking. The tongue bears numerous taste papilla. Behind the tongue on the floor of the mouth is the glottis, the opening of the short larynx which leads to the lungs. The larynx is supported by two arytenoid cartilages, and also by a ring; with the arytenoids the vocal cords are closely associated. The lungs lie so near the mouth that laryngeal, tracheal, and bronchial regions are hardly distinguishable. On the floor of the mouth is the hyoid cartilage, which serves for the insertion of muscles to tongue, \&c.

Of the (4) gill clefts which are borne on the walls of the pharynx in the tadpole, there are no distinct traces in the adult. The lungs develop as outgrowths from the gullet.

The gullet leads into a tubular stomach, which is not sharply separated from it. 'There is a pyloric constriction dividing the stomach from the duodenum, or first part of the small intestine. After several coils the small intestine opens into the wider large intestine or rectum, which enters the cloaca.

The liver has a right and a left lobe, the latter again subdivided. The gall bladder lies between the right and left lobes; bile flows into it from the liver by a number of hepatic ducts, which are continued onwards to the duodenum in a common bile duct. The pancreas lies in the mesentery between stomach and duodenum, and its secretion enters the distal portion of the bile duct. The bladder is a ventral outgrowth of the cloaca, has no connection with the ureters, and seems to be homologous with the allantois of Reptiles, Birds, and Mammals. 


\section{Vascular System.}

The heart, enclosed in a pericardium, is three-chambered, consisting of a muscular conical ventricle, which drives the blood to the body and the lungs, of a thin-walled right auricle receiving impure blood from the body, and of a thinwalled left auricle receiving purified blood from the lungs. From each of the auricles blood enters the ventricles. The two superior venæ cavæ which bring back blood from the anterior regions of the body, and the inferior vena cava which brings back blood from the posterior parts, unite on the dorsal surface of the heart in a thin-walled sinus venosus, which serves as a porch to the right auricle. From the ventricle the blood is driven up a truncus arteriosus, which soon divides into two branches, each of which divides into three aortic arches.

Thus we may distinguish five regions in the heart,--the ventricle, the right auricle, the left auricle, the sinus venosus, and the truncus arteriosus. The sinus venosus is the hindmost, the truncus arteriosus the most anterior part. The two auricles are often included in the term atrium, the undivided part of the truncus arteriosus next the ventricle is called the pylangium, the more anterior part from which the arches arise is known as the synangium. The truncus arteriosus corresponds, in greater part at least, to the conus arteriosus of many fishes.

As the heart continues to live after the frog is really dead, its contractions can be readily observed. The sinus venosus contracts first, then the two auricles simultaneously, and finally the ventricle. Although the ventricle receives both impure and pure blood, the structural arrangements are such that most of the impure blood is driven to the lungs, the purest blood to the head, and somewhat mixed blood to the body.

The blood contains in its fluid plasma- $(a)$ the oval "red" corpuscles with a definite rind, a distinct nucleus, and the pigment hæmoglobin ; $(b)$ white corpuscies or leucocytes, like small amcebæ in form and movements; $(c)$ very minute bodies, usually colourless and variable in shape. When the blood clots, the plasma becomes a colourless serum, traversed by coagulated fibrin filaments, the red corpuscles often arrange themselves in rows, and the white corpuscles are entangled in the coagulated shreds. When the web of a living frog is examined under the microscope, it will be seen that the flow of blood is most rapid in the arteries, more sluggish in the veins, most sluggish in the capillaries or fine branches which connect the arteries and 
the veins. The red corpuscles are swept along most rapidly, and are often deformed by pressure; the leucocytes tend to cling to the walls of the capillaries, and may indeed pass through them (diapedesis).

\section{The Arterial System.}

Each branch of the truncus arteriosus is triple, and divides into the following on each side :-

I. The carotid arch, the most anterior, corresponding

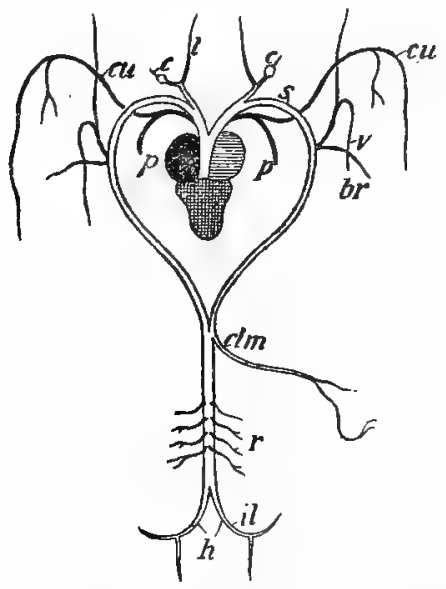

FIG. I8I.-Arterial system of Frog. (After Ecker.)

$l .$, Lingual; $\iota$, carotid ; $s$. , systemic ; $c u$, cutaneous ; $p$, pulmonary ; $v_{0}$, vertebral; $b r$, brachial ; clm., coliaco-mesenteric; $r_{.}$, renals ; il., common iliacs; $h_{\text {. }}$ hamorrhoidal.

to the first efferent branchial of the tadpole, gives off-

a lingual artery to the tongue,

a carotid artery, which bears near the origin of the lingual a spongy swelling (the "carotid gland"), and gives off an external carotid to the mouth and the orbit, and an internal carotid to the brain. 
II. The systemic arch, the median one of the three, corresponding to the second efferent branchial in the tadpole, gives off-

the laryngeal artery to the larynx, the osophageal to the osophagus,

the occipito-vertebral to the head and vertebral column,

the subclavian to the fore limb.

From the left aortic arch, just as it unites with its fellow of the other side to form the dorsal aorta, or from the beginning of the dorsal aorta, there is given off the coliaco-mesenteric to the stomach, intestine, liver, and spleen.

Further back the dorsal aorta gives off-

the renal arteries to the kidneys, and the genital arteries to the reproductive organs;

the inferior mesenteric to the large intestine;

Then it divides into two iliacs, each of which supplies the bladder (hypogastric), the ventral body wall (epigastric) and the leg (sciatic).

III. The pulmo-cutaneous arch, the most posterior, corresponding to the fourth efferent branchial in the tadpole, gives off-

the cutaneous artery to the skin,

the pulmonary artery to the lungs.

\section{The Venous System.}

I. Each superior vena cava is formed from the union of three veins, and each of these three is formed from two smaller vessels.

\begin{tabular}{|c|c|c|}
\hline \multirow{6}{*}{$\begin{array}{c}\text { Superior } \\
\text { vena cava. }\end{array}$} & External & $\begin{array}{l}\text { Lingual from the mouth and } \\
\text { tongue. }\end{array}$ \\
\hline & jugular. & Mandibular from the lower jaw. \\
\hline & Innominate. & of the skull. \\
\hline & timouminate. & $\begin{array}{l}\text { Subscapular from the back } \\
\text { the arm and the shoulder. }\end{array}$ \\
\hline & & Brachial from the arm. \\
\hline & Subclavian. & $\begin{array}{l}\text { Musculo-cutaneous from }{ }^{t} \\
\text { skin and sides of the body. }\end{array}$ \\
\hline
\end{tabular}


II. The inferior vena cava begins between the kidneys, and ends in the sinus venosus. Its components are as follows :-

Inferior $\quad\left\{\begin{array}{l}\text { Efferent renal veins from the kidneys. } \\ \text { Genital veins from the reproductive organs. } \\ \text { Efferent hepatic veins from the liver. }\end{array}\right.$

The renal portal system, by which venous blood from the posterior region filters through the kidneys on its way back to the heart, is as follows on each side :-

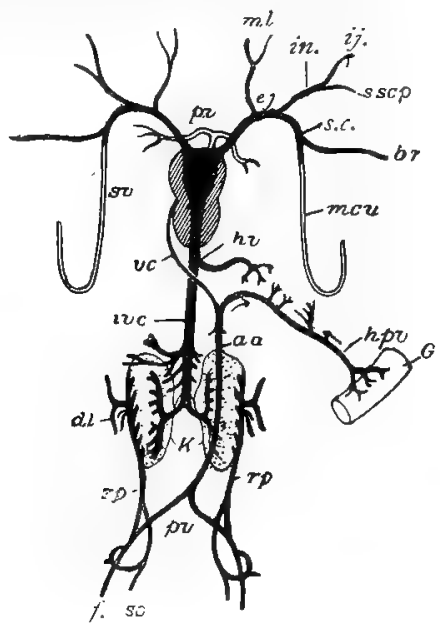

FIG. 182.-Venous system of Frog. (After EckER.)

$n l .$, Mandibular and lingual; ej, external jugular; $i j$, internal jugular; s.scp., subscapular; int., innominate; s.c., subclavian; br., brachial ; mcu., musculo-cutaneous; ho., hepatic vein; h.pro., hepatic portal ; $G_{0}$, gut ; $a_{\text {. }} a_{\text {. }}$, anterior abdominal ; rp., renal portal ; $\not z^{\prime}$, pelvic; $K$., kidneys; sc., sciatic ; $f_{.}$, femoral ; $d l$, dorsoIumbar ; ivc., inferior vena cava; vc., cardiac vein.

Renal portal system.
A posterior branch of the femoral vein from the hind limb forms the renal portal vein, which receives the sciatic from the back of the leg, and the dorso-lumbar veins from the dorsal wall of the body, and oviducal veins in the female.

The anterior branch of the femoral vein is called the pelvic, and unites with its fellow of the opposite side, and 
gives origin to a median vein which runs to the liver-the anterior abdominal. By means of an anastomosing branch, the anterior branch of the femoral is also connected to the sciatic.

The hepatic portal system, by which venous blood from the posterior region and from the gut passes through the liver on its way back to the heart is as follows :-

(Anterior abdominal vein, from the union of the two pelvics, receiving tributaries from

Hepatic portal the bladder, ventral body wall, and trunsystem. cus arteriosus.

Hepatic portal vein, from the union of veins from the stomach, intestine, and spleen.

III. The pulmonary veins which bring back purified blood from the lungs, unite just before they enter the left auricle.

\section{Lymphatic System.}

The lymph is a colourless fluid, like blood without red corpuscles. It is found in the spaces between the loose skin and the subjacent muscles, in the pleuro-peritoneal cavity in which heart, lungs, and other organs lie, in a sub-vertebral sinus extending along the backbone, and in special lymphatic vessels which pass fatty materials absorbed from the intestine into the venous system. There are two pairs of contractile "lymph hearts" at two regions where the lymphatic system communicates with the veins. A pair lie posteriorly near the end of the urostyle; the other two lie between the transverse processes of the third and fourth vertebræ.

\section{Mechanism of the Heart.}

We must now return to the heart to consider how it is that the blood is propelled from the ventricle along the proper channels. 'The right half of the ventricle being nearer the right auricle contains more impure blood, and it is from the right side of the ventricle that the truncus arteriosus arises. Therefore when the ventricle contracts, the blood which first fills the truncus is venous. It passes along the left side of a median longitudinal valve into the pulmonary arteries-along the path of least resistance. As the pulmonary arteries become distended, the next quantum of blood-that which 
has been mixed in the middle of the ventricle-is driven forwards, and passes on the right side of the longitudinal valve into the aortic arches. "And, as the truncus becomes more and more distended, the longitudinal valve, flapping over, tends more and more completely to shut off the openings of the pulmonary arteries, and to prevent any blood from flowing into them. Finally the last portion of blood from the ventricle, representing the completely arterialised blood of the left auricle, which is the last to arrive at the opening of the truncus, passes into the carotid trunks, and is distributed to the head." (The last two sentences are quoted from the "Text-book of Practical Biology," by Professors Huxley and Martin, Howes and Scott.)

Spleen, Thyroid, and Thymus.-The spleen, which is probably, as in some other animals, concerned with blood making, is a small red organ lying in the mesentery near the beginning of the large intestine. The thyroid, which is believed to have something to do with maintaining the health of the blood, is represented by two little bodies near the roots of the aortic arches. The thymus, perhaps originally associated with the gill-clefts, lies on each side just behind the angle of the lower jaw.

\section{Respiratory System.}

The larval frog breathes at first through its skin, then by external gills, and, finally, by internal gills. The adult frog breathes chiefly by its lungs, but some cutaneous respiration is still retained, for even without its lungs a frog may live for some time, and it does not use them when hibernating.

The lungs arise as outgrowths of the osophageal region of the gut, and are connected with the back of the mouth by a short laryngo-tracheal tube, whose slit-like aperture is the glottis. Each lung is a transparent oval sac, with muscle fibres in its walls. The cavity is lessened by the spongy nature of the internal walls, which form numerous little chambers bearing the fine branches of blood vessels.

In respiration, the mouth is kept shut, and air passes in and out through the nostrils. A frog will die of asphyxia if its mouth be artificially kept open for a considerable time. When the floor of the mouth is lowered, and the buccal cavity thus increased, air passes in. When the elastic lungs and the muscles of the sides of the body contract, air passes out. When the nostrils and also the opening of the gullet 
are shut, and the floor of the mouth at the same time raised, air is forced through the glottis into the lungs.

\section{Excretory System.}

The paired kidneys are elongated organs situated dorsally and posteriorly in the region of the urostyle. The waste products which they filter out of the blood pass backward by two ureters which open separately on the dorsal wall of the cloaca, without any communication with the bladder. The ureter or Wolffian duct is seen as a white line along the outer side of each kidney; in the male it functions also as the duct of a testis. On the surface of each kidney is a longitudinal yellowish streak, which is an adrenal gland of unknown significance, and little spots mark ciliated apertures or nephrostomes, which remain as communications between the abdominal cavity and the renal veins, though they are apparently in embryonic life connected with the urinary tubules.

\section{Reproductive System.}

The males are readily distinguished from the females by the swollen cushions on the first fingers, and there are some other slight external differences. The breeding season begins in spring, and then the males trumpet to their mates. The male clasps the female with his fore limbs, and retains his hold for several days, fertilising the ova as they pass out into the water.

The paired testes are oval yellowish bodies lying in front of the kidneys; the spermatozoa pass by vasa efferentia through the anterior part of the kidney into the Wolffian duct, which functions both as a ureter and as a vas deferens. In the male of $R$. esculenta, the vas deferens is dilated for some distance after leaving the kidney; in $R$. temporaria it bears on the outer side near the cloaca a dilated glandular mass or "seminal vesicle." In the males rudiments of the Müllerian ducts are sometimes seen.

The paired ovaries when mature are large plaited organs, bearing numerous follicles or sacs containing the pigmented ova. The ripe ova are liberated into the body cavity, and moved anteriorly towards the heart near which the oviducts open. These oviducts are long convoluted tubes, anteriorly 
thin-walled and straight, then glandular and coiled, terminally thin-walled and dilated. In the median part, the ova are surrounded with jelly; the terminal uterine parts open on the dorsal wall of the cloaca. In the females the Wolffian ducts act solely as ureters. There are occasional variations in the nature of the reproductive organs, and sometimes the hermaphrodite stage through which the tadpoles pass, is to some extent retained. Attached to the anterior end of the reproductive organs are yellow lobed

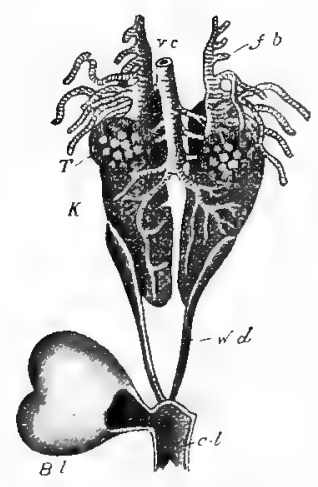

FIG. I83.-Urinogenital system of Male Frog. (After ECKER.)

$f . b_{2}$, Fatty bodies ; $\%, c, c$, vena cava; $T .$, testis ; $K$., kidney; zu.d., Wolffian duct; $c l$., cloaca; $B l$., bladder.

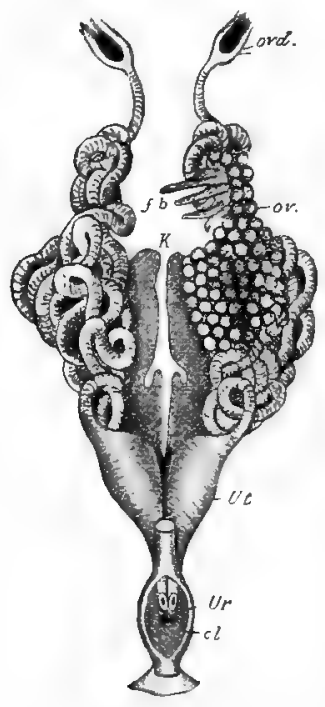

FIG. 184.-Urinogenital system of Female Frog. (After ECKER.)

oud., Opening of oviduct; ov., ovary; $f . b_{.}$, fatty body ; $K$., Kidney; $U t$. , uterus; $U_{r}$, opening of ureters into cloaca $(\mathrm{cl}$.)

"fatty bodies," largest in the males. It has been suggested that they contain stores of reserve material, which is absorbed at certain seasons. They seem to be fatty degenerations of the anterior part of the genital ridges. The head kidney or 
pronephros persists for some time in the embryo, but eventually degenerates. It does not seem to have anything to do with the fatty bodies.

\section{Development of the Frog.}

The ripe ovum exhibits "polar differentiation," its upper portion is deeply pigmented, the lower has no pigment and contains much yolk. This yolk-containing hemisphere is the heavier, and consequently is always the lower half of the egg, whatever position this may be originally placed in. Round the ovum there is a delicate vitelline membrane, and this is again surrounded by a gelatinous investment which swells up in water. The formation of polar bodies takes place before the liberation of the eggs.

The spheres of jelly preserve the eggs and embryos from friction, prevent their being eaten by most birds, appear to be distasteful to Gammarids, and often enclose in their interspaces groups of green Algæ, which help in æration. The spheres may also be of use in relation to the absorption and radiation of heat.

Fertilisation occurs immediately after the eggs are laid. The spermatozoa, which exhibit the usual features of male elements, work their way through the gelatinous envelopes, and one fertilises each ovum.

The first cleavage is vertical, and divides the ovum into a right and left half. If one of these two cells be punctured, the other will, according to Roux, form a one-sided half-embryo. This fact, disputed by Hertwig, suggests that the very first division of the frog's ovum is qualitative. At a certain stage, Roux's half-embryo regenerates the missing half, usually by re-vitalising the remains of the cell which was punctured. The second cleavage is also vertical, and at right angles to the first, dividing an anterior from a posterior half. The third cleavage is equatorial, at right angles to the first two, dividing the dorsal region from the ventral.

The segmentation is total but unequal, and results in the formation of a ball of cells, those of the upper hemisphere being smaller and more numerous than the yolk-laden cells below. Within there is a small segmentation cavity. Since 
the presence of yolk acts as a check on the activity of the protoplasm, we can understand why the smaller cells continue to divide much more rapidly than the large yolk-containing cells, and so how the smaller epiblastic cells gradually spread over the egg, covering in the larger ones. At one point, where upper and lower cells meet, a groove is formed. According to the older view, at this point the small cells are invaginated, and so form a cavity; according to recent research, the cavity is simply formed by the splitting of the large cells. However this may be, the cavity, which is the archenteron or embryonic gut, rapidly enlarges at the expense of the segmentation cavity, which soon disappears. The groove becomes a circular aperture in the epiblast, which has now
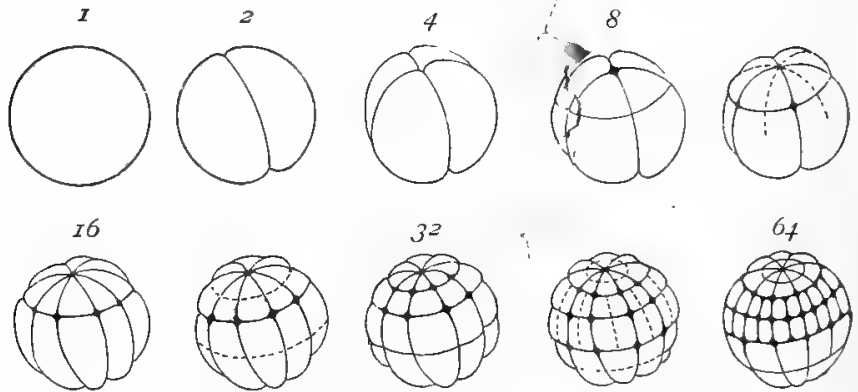

FIG. 185.-Division of Frog's Ovum. (After Eckier.)

The numbers indicate the number of cells or blastomeres.

spread over the whole egg except at this spot, the blastopore. The embryo elongates slightly, but the mass of yolk-laden cells which lie on the floor of the gut prevents the body acquiring at once the fish-like shape. The blastopore as usual marks the posterior region of the body.

The processes which follow are already in outline familiar to the student. Along the mid dorsal line an epiblastic neural plate is differentiated. The edges become raised into the neural folds, these approach one another and, fusing together, form the medullary or neural canal. At the posterior end this communicates with the archenteron for a time by the neurenteric canal. Internally, a differentiation 
of hypoblast forms the notochord along the mid dorsal line of the archenteron. At each side of this lie masses of mesoblast which have been split off from the hypoblast. Each of these divides into the primitive segments (protovertebræ) above, and the unsegmented lateral plates below. The lateral plates split into two layers, the splanchnic or inner investing the gut, the somatic or outer layer being applied to the epiblast; the space between the two layers is the body cavity. The body now becomes distinctly divided into regions, the eyes bud out from the brain, external gills grow out from the visceral arches, and the larva, still within its gelatinous case, exhibits peculiar lashing movements of the tail. Eventually, about a fortnight after the eggs are laid, it escapes from the surrounding jelly and swims freely in the water. At this time there is a cloacal opening, but

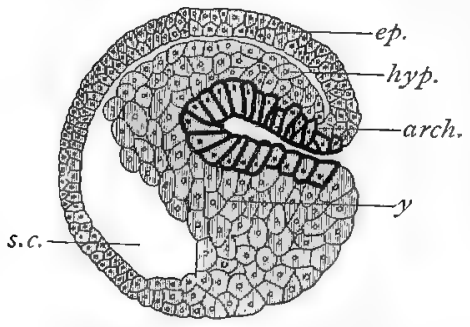

FIG. I 86.-Gastrula stage of Newt. (After IIerTwiG.)

ep., epiblast; hyp., hypoblast; arch., archenteron; $y$., yolk-cells; s.c., segmentation cavity.

the mouth has not yet appeared. There is a large horse-shoe-shaped sucker on the under surface of the head, by means of which the tadpole attaches itself to foreign objects.

In the next stage of development, which extends from the time of hatching until the commencement of the metamorphosis, many and important changes take place. The mouth, which has previously been merely a blind pit, opens into the gut, the gut itself lengthens rapidly, and becomes coiled like a watch spring; the tadpoles feed eagerly on vegetable matter and increase in size. The mouth is "bordered by a pair of horny jaws, and fringed with fleshy lips provided with horny papillæ," the sucker behind it becomes paired, and is gradually less used as the power of locomotion increases. About the time when the mouth is opened, four gill clefts open from the pharynx to the exterior. The external gills shrivel, and are replaced by an internal set; these are enclosed on either 
side by opercular folds, which form gill chambers. By the continued growth of these folds the gill chambers are closed, with the exception of a single exhalent aperture on the left side. Through this opening the water which is taken in by the mouth in respiration passes outwards, having washed the gills on its way.

Shortly afterwards the rudiments of the limbs appear. The fore limbs are concealed within the gill chambers, and so are not obvious until a much later stage; but the hind legs may be watched in the progress of development from small papillæ to the complete limb.

The lungs are developed as outgrowths from the osophagus, even before hatching, but increase in size very slowly. After the appearance of the hind legs, the larvæ come to the surface of the water to breathe, showing that the lungs are now to some extent functional. At this stage the tadpoles, now about two months old, are at the level of Dipnoi.

The changes in the relations of the blood vessels, which accompany the successive changes in the methods of respiration, and render these possible, are somewhat complicated.

When respiration is by the gills only, the circulation is essentially that of a fish. From the two-chambered heart 
the blood is driven by afferent branchials to the gills, from these it collects in efferent vessels which unite on each side to form the two aortæ. The aortæ send arteries to the head, and passing backwards unite to form the single dorsal aorta which supplies the body. For a time there are two dorsal aortæ. When the external gills are replaced by the internal, a new set of gill capillaries are developed, but otherwise the circulation remains the same. As in Ceratodus, a pulmonary artery arises from the fourth efferent branchial. At the time when the hind legs begin to be developed, a direct communication is established between afferent and efferent branchial vessels, so that blood can pass from the heart to the dorsal aorta without going through the gills. As the pulmonary circulation becomes increasingly important, the single auricle of the heart becomes divided into two by a septum, and the pulmonary veins are established. At the time of the metamorphosis an increasing quantity of blood avoids the gills in the manner indicated above, and these, being thrown out of connection with the rest of the body, soon atrophy, while the lungs become the important respiratory organs. The fate of the various branchial arteries may be gathered from the table which follows.

\begin{tabular}{|c|c|c|c|}
\hline ARCHES. & Clefis. & $\begin{array}{l}\text { AORTIC ARches } \\
\text { IN THE EMBRYo. }\end{array}$ & $\begin{array}{l}\text { Aortic Arches } \\
\text { IN THE Adult. }\end{array}$ \\
\hline Mandibular. & Eustachian & $\begin{array}{l}\text { Late in develop- } \\
\text { ment vessels } \\
\text { appear which } \\
\text { represent a } \\
\text { modification of } \\
\text { those of a } \\
\text { branchial arch. }\end{array}$ & Only a trace persists. \\
\hline Hyoid. & Eustacinan tuve. & $\begin{array}{l}\text { The arch is repre- } \\
\text { sented in a less } \\
\text { modified form. }\end{array}$ & Disappears entirely. \\
\hline First Branchial. & First cleft. & $\begin{array}{c}\text { First efferent } \\
\text { branchial. }\end{array}$ & Carotid arcb. \\
\hline Second Branchial. & second clett. & Second & Systemic arch. \\
\hline Third Branchial. & Fourth cleft. & Third " & Atrophies. \\
\hline Fourth Branchial. & & Fourth $\quad "$ & Pulmo-cutaneous. \\
\hline
\end{tabular}

Before, however, all these internal changes have taken place, the external form undergoes a striking metamorphosis. 
The tadpole has by this time grown large and strong while feeding eagerly on water weeds. Now it seems to fast, the tail shrinks, for from it wandering phagocytes carry nourishment to other parts of the body. The habit becomes less active, the structural adaptations to the aquatic life disappear. "The horny jaws are thrown off; the large frilled lips shrink up; the mouth loses its rounded suctorial form and becomes much wider; the tongue, previously small, increases considerably in size; the eyes, which as yet have been beneath the skin, become exposed; the forelimbs appear; the left one being pushed through the spout-
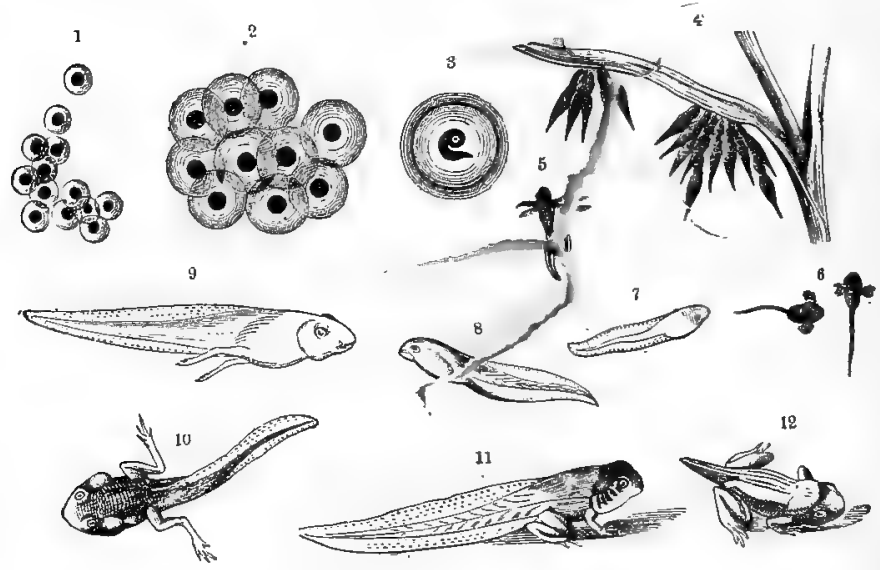

FIG. 188. - Life history of a Frog. (After BReHM.)

\begin{abstract}
I-3. Developing ova ; 4. newly hatched forms hanging to water weeds; $5-6$. stages with external gills; 7 -10. tadpoles during emergence of limbs; II. tadpoles with both pairs of limbs apparent; 12. metamorphosis to frog.
\end{abstract}

like opening of the branchial chamber, and the right one forcing its way through the opercular fold, in which it leaves a ragged hole." (Marshall.)

While these changes are in progress, and as the supply of food afforded by the tail begins to be exhausted, the tadpole recovers its appetite, but is now carnivorous, feeding on any available animal matter, or even on its fellows. The change 
is not, however, so great as it seems, for even at a very early stage, animal food is eagerly devoured.

With the change of diet, the abdomen shrinks, stomach and liver enlarge, the intestine becomes relatively narrower and shorter. The tail shortens more and more, and as it does so the disinclination for a purely aquatic life seems to increase. Eventually it is completely absorbed, the hind limbs lengthen, and the conversion into a frog is completed.

It seems that for a considerable time the tadpole is neither male nor female, but hermaphrodite. Differences in nutrition and other conditions cause one kind of sexual organ to predominate over the other, and the tadpole becomes unisexual. In nature there are usually about as many males as there are females, but Yung has shown that by increasing the quality of the food given to young tadpoles from fish-flesh to beef, and from beef to frog-flesh, he could raise the percentage of females to about ninety.

In many respects the development of the tadpole is very interesting, especially because it is a modified recapitulation of that transition from aquatic to aerial respiration, which must have marked one of the most momentous epochs in the evolution of Vertebrates.

\section{Cilassification of Amphibia.}

\section{Order ANURA or Ecaudata.}

The attainment of the adult form is associated with the loss of tail and gills. The bocly is broad. The long and very muscular hind-legs are powerful in leaping.

(a) The frog and its allies:-

The British frog (Kana temporaria), brown in colour, with a black patch on the side of the head:

the Erlible frog (R.esculenta), not indigenous in Britain, common on the Continent, greenish in colour, without the black patch :

the North American bull-frog ( $R$. catesbiana), sometimes eight inches in length, with a very sonorous croak:

some Asiatic and African "tree-frogs," such as Rhacothorus and Hyperolizes:

some toothless frogs, such as the American Dendrobates.

(b) Those allied to the toad, all toothless:-

the toads in the strict sense ( $B u f o$ ), with poisonous skin :

the crimson-bellied Bombinator igneus, the Fenerkrote of Germany : 
the obstetric toad-Alytes obstetrzcans, the male of which carries the eggs on his back and legs: Hylodes in tropical America, with rapid development without metamorphosis :

the South American Ceratophrys, of which some species have bony plates in the skin of the back: Pelobates, which like many others lives for the most part underground: the brightly coloured tree-toads, such as Hyla, with adhesive discs at the ends of the digits; Nototrema, with a dorsal egg-pouch in the females: Liopelna hochstetteri, the only Amphibian in New Zealand.

(c) The tongueless Surinam Toad (Pipa americana), in which the eggs develop in pouches on the back of the female; and the allied Ethiopian genus Xenopus, with a "tentacle" extending backwards on each side of the head.

\section{Order URODEla or CAudata.}

The tail persists in adult life; the body is elongated; the limbs are weak when compared with those of Anura.

(a) Forms like Proteus:-Two extant genera Proteus and Necturus both with persistent gills. Several species of Protens inhabit the water in the caves of Carinthia and Dalmatia in Austria. The gills persist, there are two pairs of limbs. The eyes are degenerate, the colours are pale, as we should expect in caveanimals. Two species of Necturus (or Menobranchus) occur in North America, in rivers and lakes, such as those of the Mississippi and Ohio basins. The pigment of the skin is welldeveloped.

(b) Forms like Siren:-Two extant genera, Siren and Pseudobranchus, both North American, both with persistent gills, with only the anterior limbs.

(c) Forms like the newts and salamanders:-The North American Amphiuma, with two pairs of rudimentary legs, with a slit persisting in adult life as a remnant of the gilled state: Meralobatrachus or Cryptobranchus maximus, the largest living Amphibian, found in Japan and Thibet, attains a length of three feet; Amblystoma and its gilled form the Axolotl; Desmognathus fusca, the common water salamancler of the United States, lays its eggs in a wreath which one of the sexes twines round its body; Salamandra maculosa and S. atra, both European, both viviparous; the newts - Triton or Molge - of which Triton alpestris becomes sexually mature while still larval.

\section{Order GyMNOPHIONA or APODA.}

Worm-like or snake-like forms, subterranean in habit; without limbs or girdles or tail ; with dermic scales concealed in the skin; in at least some forms, gills occur in early life; the eyes are rudimentary; peculiar "tentacles" are connected with the orbit, and are perhaps equivalent to the "balancers," which occur in larval Urodela in front of the first gill cleft. Cacilia in West Africa, Malabar, South America; Siphonops 
in Brazil and Mexico; Epicrizm in Ceylon; Rhinatrema in Cayenne; Cacilia compressicauda is viviparous.

\section{Order Labyinthodontia or Stegocephala.}

Extinct forms, occurring from Carboniferous to Triassic strata.

Dermal armour is present, the teeth are frequently folded in a complex manner. Mastodonsaurus, Dendrerpeton, Archegosazrus.

\section{Life of Amphibians.}

Most Amphibians live in or near fresh water ponds, swamps, ancl marshes. Even those adults which have lost all trace of gills are usually fond of water. The tree-toads, such as Hyla, are usually arboreal in habit, while the Gymnophiona and some toads are subterranean.

The black Salamander (Salanandra atra) of the Alps lives where pools of water are scarce, and instead of bringing forth gilled young, as its relative the spotted salamancler ( $S$. maculosa) does, bears them as lung-breathers, and only a pair at a time. But if the unborn young are

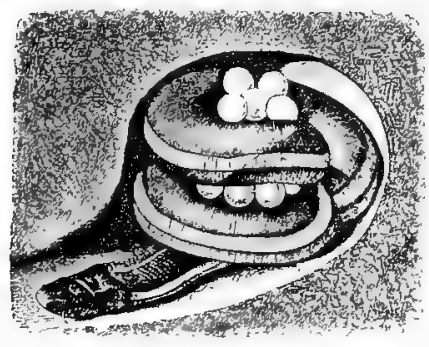

FIG. 189.-Crecilian (Icthyophis) with eggs. (After SARASIN.) removed from the body of the mother and placed in water, they form gills like other tadpoles. Within the mother, the respiration and nutrition of the young seems to be effected by crowds of red blood corpuscles which are discharged from the walls of the uterus.

Species of Hylodes, such as $H$. mantinicensis of the West Indian Islands, live in regions where there are few pools. In such cases the development is completed within the egg-case, and a lung-breathing tailed larra is hatched in about fourteen days. It is likely that the tail helps in respiration before hatching, but one observer reports the presence of small gills.

In some Mexican and $N$. American lakes, there is an interesting amphibian known as Amblystonia or Siredon. It has two forms, one losing its gills (Amblystoma), the other retaining them (Axolotl). Both these forms reproduce, and both may occur in the same lake. Formerly they were referred to different genera. But the fact that some Axolotls, kept in the Jardin des Plantes in Paris, lost their gills when their surroundings were allowed to become less moist than usual, led naturalists to recognise that the two forms were but different phases of one species. It has been shown repeatedly, that a gilled Axolotl may be transformed into a form without gills, and this metamorphosis seems to occur constantly in one of the Rocky Mountain lakes. The facts do not, however, justify the hasty conclusion that the change from the gilled to the gill-less form is cletermined only by differences in amount 
of moisture. The transformation may indeed take place in water, and both Axolotl and Amblystoma have been observed in the same lake. Further, the absence or presence of gills is not the only difference between the two forms.

Amphibians are very defenceless, but their colours often conceal them. Not a few have considerable power of colour-change.

Many Amphibians live alone, but they usually congregate at the breeding seasons when the amorous males often croak noisily. Alike in their love and their hunger they are most active in the twilight.

Their food usually consists of worms, insects, slugs, and other small animals, but some of the larval forms are for a time vegetarian in diet. They are able to survive prolonged fasting, and many hibernate in the mud. Though the familiar tales of "toads within stones" are for the most part inaccurate, there is no doubt that both; frogs and toads can survive prolonged imprisonment. Besides having great vital tenacity, Amphibians have considerable power of reparing injuries to the tail or limbs.

Although the life of Amphibians seems to have fatea; average a low potential, even the most sluggish wake up in conn th reproduction. The males often differ from their mates in Proteus : of their parental habits seem like strange experimof Prot

Thus in the Surinam toad (Pipa ameoncana), thelia in by the male on the back of the female and fertili becomes much changed-doubtless in response -and each fertilised ovum sinks into a little pocket, aich is closed by a gelatinous lid. In these pockets the embryos develop, perhaps absorb. ing some nutritive material from the skin. They are hatched as miniature adults. In Nototrena and Opisthodelphis, the female has a dorsal pouch of skin opening posteriorly, and within this tadpoles are hatched. In Rhinoderma darziniz, the male carries the ova in his capacious croaking-sacs. In the case of the obstetric toad (Alytes obstetricans), not uncommon in some parts of the Continent, the male carries the strings of ova on his back and about his hind legs, buries himself in damp earth until the development of the embryos is approaching completion, then plunges into a pool, where he is freed from his living burden. Thus among Amphibians, as among Fishes, the males sometimes take upon themselves the task of hatching the eggs.

In the Anura the ova are fertilised by the male as they leave the oviduct; in the newt the male deposits a spermatophore in the water close to the female; in Salamandra atra, S. maculosa, and Cicilia compressicauda the fertilisation must take place internally, for the young are hatched within the mother.

The eggs of the frog are laid in masses, each being surrounded by a globe of jelly; those of the toad are laid in long strings; those of newts are fixed singly to water plants; those of some tree-toads, such as Hylodes, are laid on or under leaves in moist places.

In Salamandra atra, Pipa americana, Hylodes, and Cacilia compressicauda, the young are hatched as miniature adults; and marked metamorphosis can hardly be said to occur in any Urodela.

There are about 900 living species of Amphibia, most of them tailless. All are averse to salt-water, hence their absence from almost all 
oceanic islands. The Anura are well-nigh cosmopolitan; the Urodela are limited to the temperate parts of the northern hemisphere.

\section{History.}

It is likely that Amphibians were derived from a stock from which the Dipnoi and perhaps also the Elasmobranchs sprang. The order Labyrinthodontia or Stegocephala does not seem very homogeneous; it perhaps includes two or more distinct orders. Of extant forms, the Gymnophiona are more old-fashioned than the others. The modern types gradually appear in Tertiary times. Some of the extinct forms were gigantic.

Huxley has emphasised the following affinities between Amphibians and Mammals :- the Amphibia, like Mammals, have two condyles on the skull; the pectoral girdle of Mammals is as much amphibian as it is sauropsidian; the mammalian carpus is directly reducible to that of Amphibians. In Amphibians only does the articular element of the mandibular arch remain cartilaginous; the quadrate ossification is small, and the squamosal extends down over it to the osseous elements of the mandible, thus affording easy transition to the mammalian condition of these parts.

There are many remarkable affinities between the Labyrinthodont Amphibians and a class of extinct Reptiles known as Anomodontia, and as the latter have also many affinities with Mammals, it is possible that both Mammals and Anomodonts diverged from an Amphibian stock. The strange extinct Eotetrapoda of Credner seem to unite the Stegocephala to the Rhychocephalia, a class of Reptiles now represented by the New Zealand "lizard" Sphenodon. 


\title{
CHAPTER XXIV.
}

\author{
REPTILES.
}

Classes-Chelonia. Rhynchocephalia. Lacertilia. Ophidia. Chelonia, and Extinct Classes.

The diverse animals-Tortoises, Lizards, Snakes, Crocodilians, \&c.- - which are classed together as Reptiles, are the modern representatives of those Vertebrates which first became independent of the water and began to possess the dry land. While almost all Amphibians spend at least their youth in the water, breathing by gills, this is not necessary for Reptiles, in which embryonic respiration is secured by a vascular fotal membrane known as the allantois. As in still higher Vertebrates, gill slits are present in the embryos, but they are not functional, and are without gills. Reptiles are essentially creatures of the earth, but many lizards, snakes, and turtles, and all the crocodilians, are aquatic. Partially marine forms are represented by the Galapagos lizard, which swims out among the seaweed, by some crocodilians which venture down the estuaries; some turtles live far out to sea and only seek the shores to lay their eggs; the Hydrophidæ, or sea-snakes, never leave the water.

Reptiles, Birds, and Mammals are often distinguished as Amniota from Amphibians and Fishes, which are called Anamnia, the terms referring to the presence or absence of a characteristic protective foetal membrane - the amnionwith which the allantois is always associated.

Of these three highest classes of Vertebrates, the Reptiles and Birds, so different in form and habit, are united by deep structural resemblances. These were first clearly recognised 


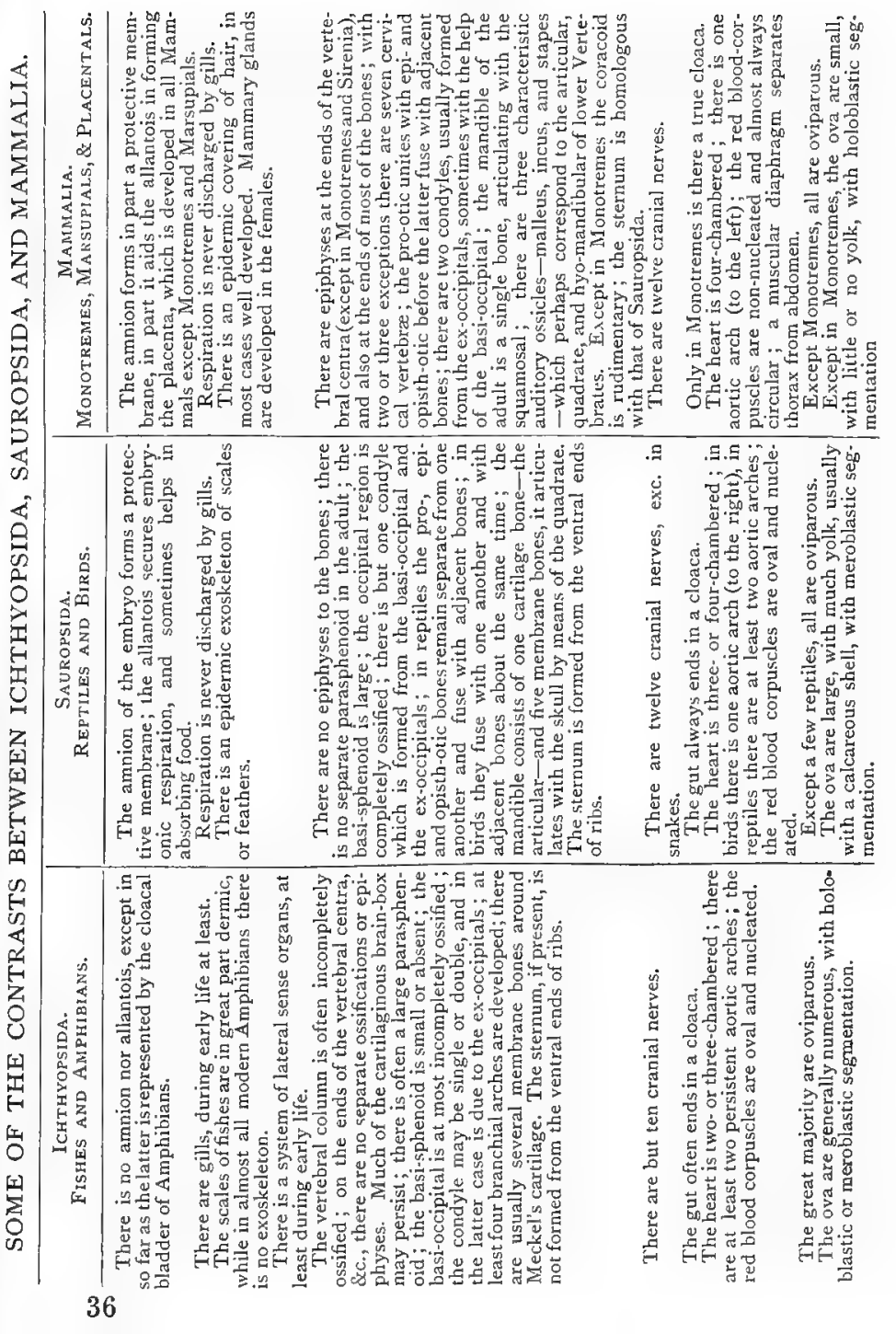


by Professor Huxley, when he united the two classes as Sauropsida, in contrast to Mammalia on the one hand, and Ichthyopsida (Amphibians and Fishes) on the other. Let us state some of the contrasts which he recognised, noting at the same time that Reptiles form among Vertebrates a great central assemblage, Iike "worms" among Invertebrates, rather a number of classes than a class, exhibiting affinities not only with Birds, but with Mammals and Amphibians as well.

Again we shall virtually quote from Huxley in noting some of the distinctions between Reptiles and Birds :-

REPTILES.

The exoskeleton consists of horny epidermal scales, or of bony dermal scutes, or of both.

The centra of the vertebrae are rarely like those of birds.

When there is a sacrum, its vertebra (usually two in number) have large expanded ribs, with the ends of which the ilia articulate.

The cartilaginous sternum may become bony, but is not replaced by membrane bones, unless perhaps in Pterodactyls.

When there is an interclavicle, it remains distinct from the clavicle and sternum.

The hand has more than three digits, and at least the three radials are clawed.

In living reptiles the ilia are prolonged further behind than in front of the acetabulum; the pubes slope downward and forward; there are pubic and ischiac symphyses.

There are often five toes; the tarsals and the metatarsals remain distinct.

Al least two aortic arches percist ; only the Crocodilia have a structurally fourchambered heart; more or less mixed blood always goes to the posterior body.

The body has approximately the temperature of the surrounding medium.

The optic lobes lie on the upper surface of the brain.
BURDS

There is an outer covering of feathers, and though there may be a few scales, there are never scutes.

The centra of the vertebra have usually a peculiar terminal curvature.

The two sacral vertebra bave no expanded ribs, they fuse with others to form a long composite sacrum.

The cartilaginous sternum is replaced by membrane "מonés from several centres.

When there is an interclavicle, it is confluent with the clavicles.

The hand has not more than three digits, and at most two radials are clawed. The fore-limbs are modified as wings; some carpals fuse with the metacarpals.

The ilia are greatly prolonged in front of the acetabulum, the inner wall of which is membranous. The pubes slope backwards, parallel with the ischia; only in Struthio is there a pubic symphysis, only in $R$ hica is there is an ischiac one.

There are not more tban four toes; the proximal tarsals unite with the tibia, forming a tibio-tarsus; the first metatarsal if present is free, but the three others are funed to one another and to the distal tarsals, forming a tarso-netatarsus.

There is but one aortic arch, to the right; the heart is four-chambered; the blood sent to the body is purely arterial.

The body temperature is very high.

The optic lobes lie on the side of the brain.

The lungs have associated air sacs.

The sutures between the bones of the skull are usually obliterated at an early stage.

i'he right ovary atrophies. 


\section{Class Chelonia. Tortoises and Turtles.}

General Characters. - The body is compact and broad in the region of the trunk. There is a dorsal and a ventral shield, within the shelter of which the head and neck, tail and limbs, can be more or less retracted.

The dorsal shield or carapace is formed from the neural spines of the vertebra, from the expanded ribs, and from a series of marginal plates around the outer edge.

The ventral shield or plastron consists of nine or so dermal plates. There is no sternum.

Overlapping, but in no way corresponding to the bony plates, are epidermic horny plates of "tortoise shell," which,

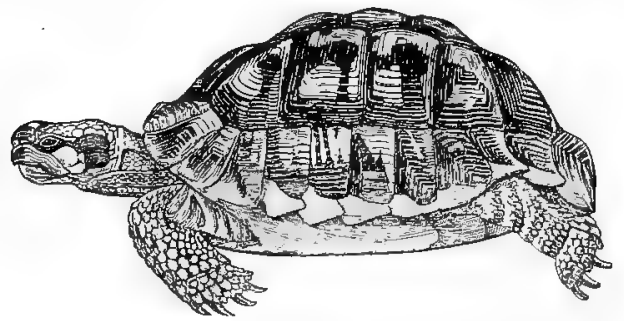

FIG. 190.-External appearance of Tortoise.

though very hard, are not without sensitiveness, numerous nerves ending upon them.

The quadrate is immovably fixed.

The jaws are covered by a horny sheath, and are without teeth, though rudiments of these har'e been seen in some embryos.

The average life of Chelonians is sluggish. Perhaps this is in part due to the way in which the ribs are lost in the carapace, for this must tend to make respiration less active.

All are oviparous. The eggs have firm usually calcareous shells.

Some Peculiarities in the Skeleton of Chelonia.

The dorsal vertebre seem to be without transverse processes, and along with the ribs are for the most part immovably fused in the carapace. The tail and the neck are the only flexible regions. 
Professor Berry Haycraft gives the following account of the development of the dorsal shield:-

If we compare a very early embryo turtle with that of a crocodile, we notice the following difference:-In the crocodile, each cartilaginous rib is completely invested by a tubular sheath of young connective tissue, and in the intercostal spaces are distinct muscle plates. In the turtle the cartilaginous ribs are simply embedded in young osteogenetic tissue, which foums the whole of the body wall, extending superficially up to the skin. As development proceeds in the crocodile, the tubular sheath of connective tissue (periosteum) investing each cartilaginous rib, grows in size, and forms bone (the rib) anteriorly, the cartilage being absorbed. Thus we get the adult cylindrical rib, separated from its neighbours by the intercostal muscles, developed from the muscle plates. In the green turtle bone begins to form upon the rib cartilage, the latter subsequently being absorbed, but as there is no investing periosteal sheath, this formation of bone spreads out on all sides, right up to the skin superficially, and as far as the neighbouring growths laterally, to form the solid bone of the carapace. In the mud turtles, the growth of bone which is extending laterally from each cartilaginous rib, does not meet its neigh. bour, for already the intercostal tissue has partly become differentiated into fibrous tissue, and a fibro-osseous carapace results. In the green turtle, the rib cartilage, at both its distal and proximal ends, is invested by true periosteum, which causes in these parts the formation of cylindrical bone.

What then is a costal plate?

It is more than a rib; it is a rib, which, in its development, has sprear into and involved the surrounding intercostal tissue.

Is it an intramembranous or intracartilaginous bone? We now know that all bones are developed through the agency of membranes, and that the humerus, for example, an intracartilaginous bone, is eventually formed entirely from its membranous periosteum.

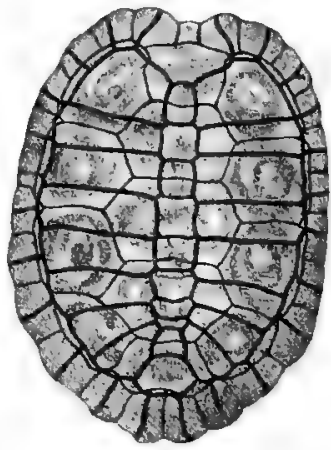

FIG. I9I.-Carapace of Tortoise. (From Edinburgh Museum of Science and Art.)

The dark contours are those of the bony pieces: the lighter contours are those of the scales which have been removed.

A membrane bone is therefore not a bone developed from a membrane, for every bone in the body is now known to be so formed, it is a bone whose place was never represented by cartilage.

If we accept this view of an intramembranous and intracartilaginous bone, a view forced upon us by modern inquiry, then the costal plate is an intracartilaginous bone, and comes out in its proper contrast from the marginal and plastron plates which are not preformed in cartilage. The neural plates may be looked upon as similar in their origin to the costal plates, bone encrusting the cartilaginous vertebre, and then ex- 
tending into the tissue between neighhouring spinous processes, and superficially up to the tissue which has differentiated into the thin layer of connective tissue below the scales.

The ventral shich or plastron consists of dermal bones; according to somo, three anteriog pieces represent clavicles and isterdavicle.

The cervical vertebre have at most little rudiments of ribs, are remarkably varied as resards their articular faces, and grive the neck many possibilities of motion. There are no lumbar vertebre.

The hones of the skull are immorably united: there are no ossified alisphenouds, hut dowm ard prolongations of the large parietals take their place: neither presphenod nur orbitosphenoids are ossifed : there we no distinct masal bones in mulern Chelonians: the prenasille are very small: there are no tweth: there is a complete bony palate formet

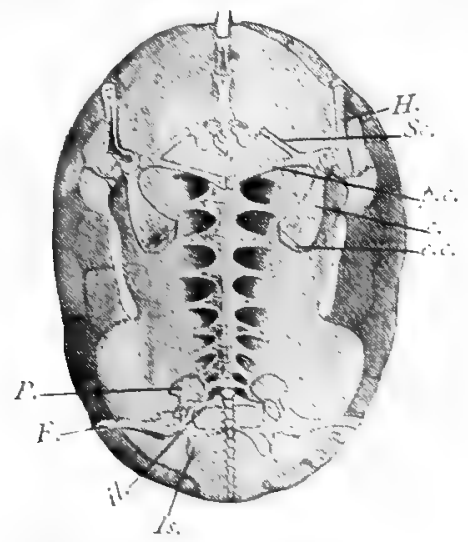

Fic, I92. - Internal vicw of skeleton of Turtle. (From Edinhurgh Museum of Science and Art.)

$H$. humerus: $S_{i}$, scapula, ruming dorsally: $\therefore$ wrokit : $\ldots$ epi-torawid: $A$ pre-coracuid: $f$, pules: it, ilium, running dursally: $L s$, ischium : F, femur.

in great purt from the junction of the perrygoids with the hasisphenoid and with one another.

There is no sternum.

The pectoral girale on each she consists of a thrsal stapula attached to the arapaces a ventral coracoid beang tominally a small epicora. coid, and anterior to the coracoid a "precorncoid."

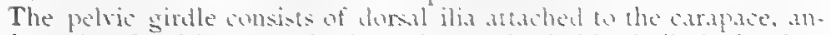
terior pules fused in a symphysis, and posterior ischiz similarly fuscd.

The sirdles orisinally lic in trone of or hehind the rils. hut ate we!archet by the carapace in the course of its development. 


\section{Some Peculiarities in the Organs of Chelonia.}

The brain of the adult shows a slight curvature. In Chelonians and in all higher animals except serpents, there are twelve cranial nerves, for in addition to the usual ten, a hypoglossal to the tongue, and a spinal accessory to cervical muscles are ranked as the eleventh and twelfth.

The gullet often bears internally pointed horny papillæe directed downwards. There are blind pockets or anal bursee connected with the cloaca.

The heart is three chambered, but an incomplete septum divides the ventricle into a right portion from which the pulmonary arteries and the left aortic arch arise, and a left portion from which the right aortic arch issues. From the right aortic arch, which contains more pure blood than the left, the carotid and subclavian arteries are given off. The left aortic arch gives off the coeliac artery before it unites with the right.

Unlike other Reptiles, the Chelonians are said to have no renal portal system.

The lungs are attached to the dorsal wall of the thorax, and have only a ventral investment of peritoneum;

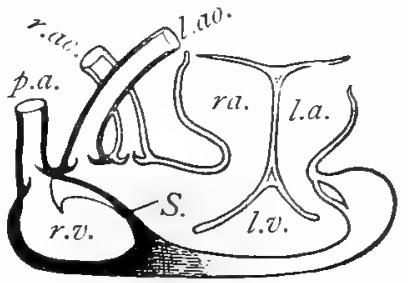

FIG. I93.-Dissection of Chelonian heart. (After Hoxley.)

r.r., Right half of ventricle; $s$, septum; $l . z^{\prime}$. , left half of ventricle; ra, right auricle; l.a., left auricle; l.ao, left aortic arch ; r.ao, right aortic arch ; p.a., pulmonary arch. each is divided into a series of compartments into which branches of the bronchus open. There is a slight muscular diaphragm.

In the males, the kidney, the epididymis, and the testes, lie adjacent to one another on each side. The males have a grooved penis attached to the anterior wall of the cloaca. There is a urinary bladder.

\section{Classification of Chelonia.}

I. ATHECÆ. Vertebrae and ribs free from bony shield. Skull without descending processes from parietals.

Sphargidx, leather turtles, with flexible carapace. Dermochelys coriacea, the only living species, the largest modern Chelonian, sometimes measuring six feet in length. It is widely, lut now sparsely, distributed in tropical and temperate seas, and is said to be herbivorous.

II. THECOPHORA. Dorsal vertehre and ribs fused in the carapace. Parietals prolonged downwards. Inclucting the following and other families.

Chelonid:c, marine turtles, with fin-like feet, and partially ossified carapace. They occur in intertropical seas, and bury their soft-shelled eggs on sandy shores. The green turtle (Chilone viritis) is much 
esteemed as food; the hawk's-bill turtle (Caretta imbricata) furnishes much of the commercial tortoise shell.

Testudinide, land tortoises, with convex perfectly ossified carapace and feet adapted for walking. They are found in the warmer regions of both the old and the new world, but not in Australia. In diet they are vegetarian. The common tortoise (Testudo greca), and the exterminated giant tortoises of the Mascarene and Galapagos Islands are good representatives.

Chelydidæe, fresh water tortoises, more or less aquatic, with perfectly ossified carapace, and feet with sharp claws. Examples-Chely's fmbriata, from Brazil and the Guianas, with warty growths of decep-

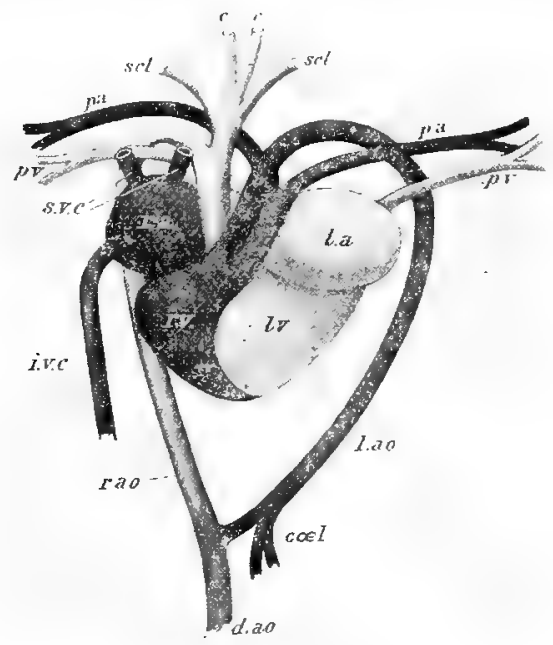

FIg. I94--Heart, and associated vessels, of Tortoise. (After NuHN.)

r.a, Right auricle; superior venæ cavæ $\left(s_{0} \varepsilon^{\prime}, c_{0}\right)$ and inferior vena cava (i.v.c.) enter it. r.v. Right half of ventricle; pulmonary arteries $(p . a)$ and left aortic arch (l.an) leave it; coel coliac; d.ao, dorsal aorta. l. $\alpha$, Left auricle; $p . z$, pulmonary veins enter it. $l . z$, Left half of ventricle; right aortic arch $(r, a o)$, giving off carotids $(c)$ and subclavians $(s . c l)$, leaves it.

tive appearance; Emy's orbicularis common in S. Europe; Chelydra and Macroclemmys, the aquatic terrapins of N. America.

Trionychide, fresh water turtles, with depressed carapace covered with soft skin, with webled digits. Each foot has sharp claws on the three inner digits. They are carnivorous in halbit. Examples-Triony, javanious, sangetious, niloticus, from Jara, the Ganges, and the Nile respectively. 


\section{Class Rhynchocephalia.}

The only living representative of this class is the New Zealand "Lizard"-Hatteria or Sphenodon punctatus, the Tuatara of the Maoris. Lizard-like in appearance, it measures from one to two feet in length, has a compressed crested tail, is dull olive-green spotted with yellow above and whitish below. It is now rare, but is being preserved in some small islands off the New Zealand coast. It lives in holes among the rocks or in small burrows, feeds on small animals, and is nocturnal in habit.

The skull, unlike that of any lizard, has an ossified quadrato-jugal, and therefore a complete infra-temporal arcade; the quadrate is firmly united to pterygoid, squamosal, and quadrato-jugal; the pterygoids meet the vomer and separate the palatines; there are teeth on the palatine

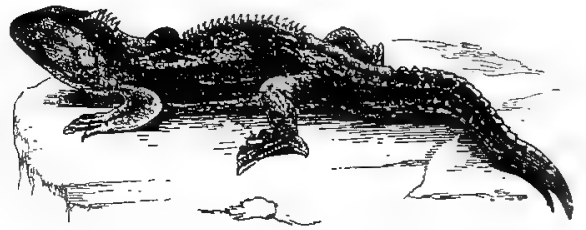

FIG. I95--Hatteria or Sphenodon. (After HAYEK.)

in a single longitudinal row, parallel with those on maxilla and mandible, and the three sets seem to wear one another away; there is also a single tooth on each side of a kind of beak formed by the premaxillæ; the nares are divided.

The vertebræ are biconcave, as in geckos among lizards and in many extinct Reptiles. Some of the ribs bear uncinate processes, as in Birds; as in crocodiles, there are numerous "abdominal ribs," ossifications in the sub-cutaneous fibrous tissue of the abdomen. The anterior end of the "plastron" thus formed overlaps the posterior end of the sternum. Clavicles and interclavicle present.

The pineal or parietal eye, which reaches the skin on the top of the head, is less degenerate than in other animals, retaining, for instance, distinct traces of a complex retina. 
Near the living Sphenodon, the Permian Palcohatteria, the Triassic Hyperodapedon, and some other important types may be ranked. Along with these may be included the remarkable Proterosauras from the Permian, though Seeley establishes for it a special order Proterosauria as distingushed from Rhynchocephalia. According to Baur, quoted by Nicholson and Lydekker, "the Rhynchocephalia, together with the Proterosauria, to which they are closely allied, are certainly the most generalised group of all Reptiles, and come nearest, in many respects, to that order of Reptiles from which all others took their origin." We have already noted how they are linked to the Amphibia.

\section{Class Lacertilia-Lizards.}

This class occupies a somewhat central position among Reptiles.

General Characters. - The body is usually zell covered with scales.

In most, both fore and hind limbs are developed and bear clawed digits, but either pair or both pairs may be absent. The shoulder and hip girdles are always present, in rudiment at least.

Unlike snakes, lizards have non-expansible mouths, and almost always movable eyelids and external ear openings.

The teeth are fused to the edge or to the ridge of the jazes, never planted in sockets.

The tongue, broad and short in some, e.g., Geckos and Iguanas, long and terminally clubbed in Chamceleons, is oftenest a narrow bifid organ of touch.

The opening of the cloaca is transverse.

There is a urinary bladder and a double penis.

Most are oviparous, but in a few the eggs are hatched within the body.

They are usually active agile animals, beautifully aud often protectively coloured.

The caudal region is often very brittle; lost tails and even legs may be regenerated.

The food generally consists of insects, worms, and other small animals, but some prey upon larger animals, and others are vegetarian.

Most are terrestrial, some arboreal, a few semi-aquatic, and there is one marine form.

Lizards are most abundant in the tropics, and are absent from very cold regions. 


\section{Some Peculiarities in the Skeleton of Lizards (mostly quoted from Huxley).}

The epidermic exoskeleton of scales is sometimes, as in Cyclodus, associated with scutes or ossifications in the dermis. In Geckos and Amphisbenas there is hardly any exoskeleton.

Except the Geckos, all living Lizards have procnelous vertebre. The sacral vertebre, two or rarely three in number, are not fused. Underneath the vertebra, in the anterior part of the tail, there are usually special "chevron" bones. In many cases there is an unossified septum across the middle of each cauclal vertebra, and it is across this that the tail so readily breaks.

In the skull, there is an interorbital septum except in Amphisbænas, there are no alisphenoids nor completely ossified presphenoid or orbitosphenoids, there is usually an unossified parietal foramen on the roof of the skull, in most an epipterygoid (or "columella ") runs from the parietal to the pterygoicl, in most there are prominent parotic processes formed from prolongations of the opisthotics, pro-otics and ex-occipitals, with the onter end of one of these processes the quadrate articulates, and is usually movable, the fronto-parietal region is often slightly movable on the occipito-sphenoidal part, the quadrato-jugal is usually represented by ligament only, from the union of the palatine and pterygoid a transverse bone extends to the maxilla, the two rami of the lower jaw are in most cases firmly connected.

Teeth occur on the premaxille, maxillx and dentaries, and sometimes also on palatines and pterygoids. They generally become fused to the bones which bear them. When they are attached by their bases to the ridge of the jaw, the dentition is described as acrodont; when they are attached by their sides to the side of the jaw, the dentition is described as pleurodont.

\section{DESCRIPTION OF A LIZARD.}

The following description applies especially to the longtailed green lizard (Lacerta viridis), found abundantly in Jersey, but, except in minor points, it will be found to apply equally to the small British grey lizard (Lacerta agilis) and to the viviparous lizard (Zootoca vivipara).

\section{Form and External Features.}

The depressed head is separated from the body by a distinct neck, but the posterior region of the body passes gradually into the long tail, which is often mutilated in captured specimens. Both fore and hind limbs are present, and both are furnished with five clawed digits. Of the apertures of the body, the large mouth is terminal, the external nares are close to the end of the snout, and the 
cloacal aperture is a considerable transverse opening placed at the root of the tail. There is no external ear, but the tympanic membrane at either side is slightly depressed below the level of the skin of the head. The eyes are furnished with both upper and lower eyelids, and also with a nictitating membrane.

Skin.

As contrasted with that of the frog, the skin is remarkable as possessing a distinct exoskeleton of epidermic scales. In the head region these exhibit a definite arrangement characteristic of the species. With the presence of an exoskeleton we must associate the absence of the numerous cutaneous glands of the frog; these are here represented only by a row of "femoral glands," which open by pores on the ventral surface of the thigh. Their secretion is most obvious in the male at pairing time. The histological composition of the skin is very similar to that of the frog's skin. Pigment is deposited here also in two layers, of which the outer is greenish, the inner black. It is of special interest to notice that over the parietal foramen (see Skull) the black pigment is absent, the green only feebly represented; in this region, therefore, the skin is almost transparent.

Many Lizards, such as the Chameleons, exhibit in a remarkable degree the power of rapidly changing the colour of their skin. This is due to the fact that the protoplasm of the pigment cells contracts or expands under nervous control. The change of colour is sometimes advantageously protective, but it seems often to be merely a reflex symptom of the nervous condition of the animals.

In a few cases, e.g., some of the skinks, there are minute dermal ossifications beneath the scales.

\section{Skeleton.}

The backbone consists of a variable number of vertebræ, and is divisible into cervical, dorsal, lumbar, sacral, and caudal regions. Except the atlas and the last caudal, all the vertebræ are procolous.

The atlas consists of three separate pieces, its centrum ossifies as usual as the odontoid process of the axis. There are two sacral vertebrie with large expanded sacral ribs. To the ventral surfaces of many of the caudal vertebre Y-shaped "chevron" bones are attached. Across the centre of the caudal vertebre there extends a merlian unossified zone, it is in this region that separation takes place when a startled lizard loses its tail. 
The ribs are numerous, but only five reach the sternum.

The skull is well ossified, but in the region of the nares, in the interorbital septum, \&c., the primitive cartilaginous brain-box persists. On the dorsal surface the bones exhibit numerous impressions made by the epidermic scales, which render it difficult to distinguish the true sutures of the bones.

Two fused parietals with the rounded median "parietal foramen," two frontals, and the two nasals, are the most important constituents of the roof of the skull. Anteriorly the premaxillæe appear between the nasals, while posteriorly the sickle-shaped squamosal is attached by a suture to the parietal, and is overlapped by one of the two small supra-temporal

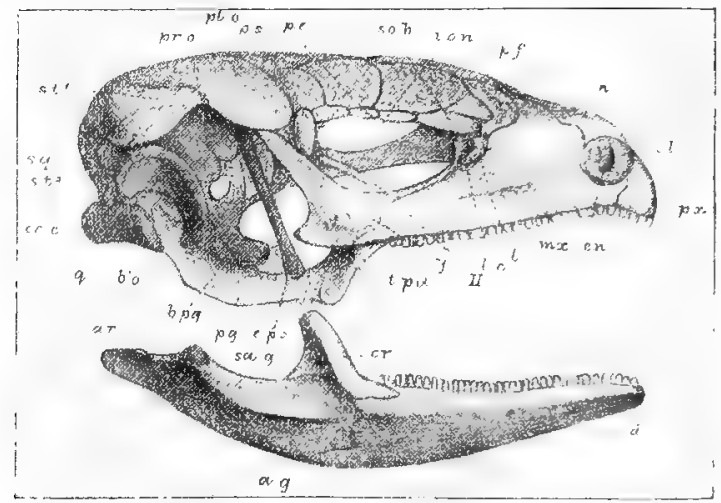

FIG. Ig6.-Side view of skull of Lacerta. (After W. K. PArker.)

$\not x_{\text {. }}$ Premaxilla; $e_{.} t_{\text {. }}$, external nostril ; $m x$., maxilla; $l$, lachrymal ; $j_{.}$, jugal; II., optic nerve; $t . p a$, trans-palatine; $e p \cdot g .$, , epipterygoid; $p g .$, pterygoid ; b.pg., basi-pterygoid ; $b_{0} 0_{.}$, basi-occipital ; $q .$, quadrate; $o c . c .$, occipital condyle; $s q .$, squamosal ; $\not r_{0} .0 .$, pro-otic;

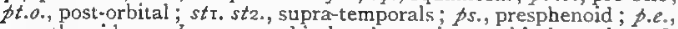
mesethmoid; s.o.b., supra-orbitals; i.o.k., inter-orbital notch; $\not f$. , prefrontal ; $n$, nasal ; 0.2 , olfactory sac ; $a r$, articular ; $a g .$, angular; sag., surangular; cr., coronary ; d., dentary.

bones. The orbit is roofed by a series of small bones, of which the anterior and posterior are respectively known as pre- and post-frontal.

On the floor of the adult skull there is a large basal bone, composed of fused occipital and sphenoidal elements, and continued forward as a slender bar (parasphenoid). This hone gives off two stout processes, the basipterygoid processes, which articulate with the pterygoids. Each pterygoid is connected posteriorly to the quadrate bone of the corresponding side, and anteriorly with the palatine. From the union of 
pterygoid and palatine, a stout os transversum or trans-palatine extends outwards to the maxilla. In front of the palatines lie the small vomers, which, in their turn, articulate with the premaxilla and maxilla, both of which are furnished with small pointed teeth. In the posterior region of the skull we have still to notice the large ex-occipitals with which the opisthotics are fused, and which are continued into the conspicuous parotic processes. The lateral walls of the brain case are largely formed by the paired pro-otics. Internally, an important bone, the epipterygoid or "columella" (not to be confounded with the columella or stapes of the ear), extends from the prootic to the pterygoid. The orbit is bounded posteriorly and inferiorly by the jugals. There is no ossified quadratojugal, and thus the lateral temporal fossa is open below in the dried skull (contrast Hatteria). The other fossæ of the dried skull are the supra-temporal on the upper surface, and the posterior-temporal on the posterior face.

Each half of the lower jaw is composed of six bones, which do not fuse in the adult.

\section{The Limbs and Girdles.}

In the shoulder-girdle, the flat coracoids, with an anterior precoracoidal region, articulate with the sternum, which is represented by a cartilaginous plate of rhomboidal shape. Over it projects the long limb of the $\mathbf{T}$-shaped interclavicle, which, at the sides, is continued backwards by the curved clavicles. 'The remaining elements are the scapulæ, which are continuous with the cartilaginous supra-scapulæ.

The fore limbs have the usual parts. In the carpus all the typical nine bones are represented, and there is in addition an accessory "pisiform" bone.

In the pelvic girdle, ilium, pubis, and ischium are represented as usual; there are both pubic and ischiac symphyses.

In the tarsus the fibulare and tibiale are united, and the distal row consists of only two bones.

\section{Nervous System.}

The brain consists of the usual parts. The cerebellum is small and only partially overlaps the fourth ventricle. In the region of the thalamus the epiphysis is distinct and conspicuous, but in the adult the pineal body is quite separated from it, and lies in its connective tissue capsule below the skin.

\section{Alimentary System.}

Small pointed teeth are present on the maxillæ, premaxillæ, palatines, and on the lower jaw; they are attached 
by their sides (pleurodont). Salivary glands occur on the floor of the mouth cavity. 'The narrow gullet passes gradually into the muscular stomach, which again passes into the coiled small intestine. Near the commencement of the large intestine there is a small cæcum. A voluminous liver, with a gall bladder embedded in it, and a pancreas are present as usual.

Embedded in the mesentery below the stomach lies the rounded spleen. A whitish thyroid gland lies on the ventral surface of the trachea a short distance in front of the heart.

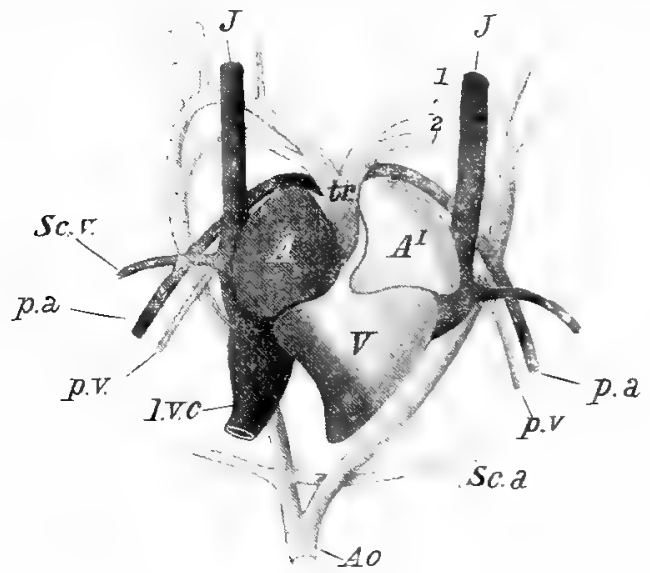

FIG. 197.-Heart, and associated ressels, or Lizard. (After NuHN.)

A., Right auricle ; jugulars ( $/$. ) subclavians $\left(S_{C . x^{\prime}}\right)$, and inferior vena cava (I.V.C.) enter it. $V_{\text {, }}$, ventricle; tr., truncus arteriosus; I, first aortic arch giving off carotids ; 2 , second aortic arch ; $\beta . \alpha$., pulmonary artery; $S c . a$, subclavian; fo, dorsal aorta. 41 , left auricle; pulmonary veins $\left(f, x^{\prime}\right.$ ) enter it. In the lizard described, the left jugular is not developed.

\section{Vascular Sy'stem.}

The heart is completely enveloped by the pericardium, and is three chambered, consisting of two thin-walled auricles and a muscular ventricle. From the ventral surface of the ventricle arises the conspicuous truncus arteriosus, 
which is formed by the bases of the aortic arches, and exhibits a division into two parts. From the more ventral part arises the left aortic arch, which curves round to the left side, first giving off a short connecting vessel (ductus Botallii) to the carotid arch. From the other division of the truncus arteriosus, a great arterial trunk arises, and this gives off the right aortic arch and the right and left carotid arches. The right aortic arch sends a ductus Botallii to the carotid arch of the right side, and then curves round the heart to join the left arch, when the two form the dorsal aorta. The carotid arches supply the head region with blood. From the base of the truncus arteriosus, the right and left pulmonary arteries also arise.

From the right aortic arch as it curves round, arise the right and left subclavian arteries, which carry blood to the fore limbs. A creliacomesenteric artery arises from the dorsal aorta and supplies the viscera. Smaller vessels are also given off to the genital organs, \&c., and then at the anterior end of the kichneys, the aorta divides into two femoral arteries which break up into a network of small vessels, supplying hind limbs and kidneys, and finally, at the posterior end of the kidneys, reunite to form the caudal artery, which runs down the tail.

The blood from the anterior region of the body is returned to the heart by the right and left precaval veins or superior venz cave. The right precaval is formed by the junction of external and internal jugulars with the subclavian vein ; on the left sicle the jugular is alsent. From the posterior region of the bodv, blood is brought back by the postcaval vein or inferior vena cava. The three great veins open into a thin-walled sints venosus, which opens into the right auricle.

The postcaval is formed by the union of two veins which run along the genital organs, and receive renal veins from the kidneys. In passing through the liver the postcaval receives important hepatic veins.

From the tail region the blood is brought back by a caudal which bifurcates in the region of the kidneys into two pelvics. The pelvic veins give off renal portals to the kidneys, and receive the femoral and sciatic veins from the hind limbs. They then unite to form the epigastric or anterior abdominal, which, carries blood to the liver. Except through the medium of the renal-portal system, there is no connection between the anterior abclominal and the postcaval. To the liver blood is carried as usual from the stomach, de., by the portal vein.

From the lungs blood is brought to the left auricle by the pulmonary veins.

A lymphatic system including a pair of lymph hearts is present.

\section{Respiratory System.}

The lungs are elongated oval structures which taper away posteriorly. The mouth does not, as in the frog, play any 
part in the respiratory movements. In some lizards (Chamæleon and Geckos) the lungs are prolonged in airsacs, suggesting those of Birds (Fig. 198).

\section{Excretory System.}

The paired kidneys lie in the extreme posterior region of the abdominal cavity, and extend a little further back than the level of the cloaca. Each is furnished with a very short ureter. In the male the ureters unite with the vasa deferentia; in the female they open separately into the cloaca. Into the cloaca opens also a large thin-walled "urinary bladder"; this is a remnant of the fotal allantois, and has no functional connection with excretion. The urine is semi-solid, and consists largely of uric acid.

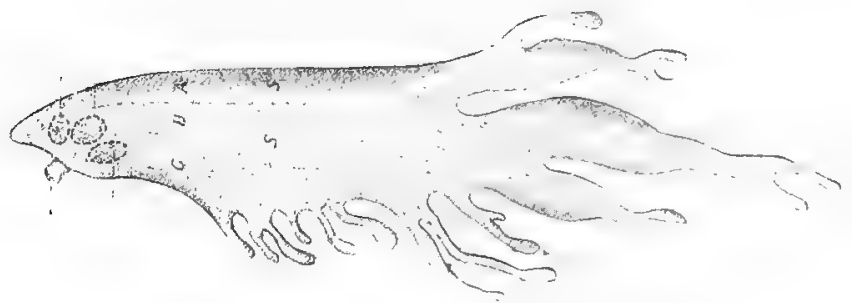

FIG. 198.-Lung of Chamaleo vulgaris, showing air sacs. (After WiEIERSHEIM.)

Reproductive System.

In the male the testes are two white oval bodies suspended in a dorsal fold of mesentery. Along the inner surface of each runs the epididymis, which receives the vasa efferentia, and is continuous posteriorly with the vas deferens. The two vasa deferentia, after receiving the ureters, open by small papillæ into the cloaca. In connection with the cloaca there is a pair of eversible copulatory organs.

In the female the ovaries occupy a similar position to that of the testes in the male. The oviducts open far forward by wide ciliated funnels; as they pass backward they show a gradual increase in cross section, but there is no line of demarcation between an oviducal and a uterine portion. Posteriorly, the oviducts open into the cloaca. 
The right reproductive organ tends to be larger and in front of the left. In many of the males, the Wolffian body is well developed. Viviparous, or what is clumsily called ovo-viviparous, parturition is well illustrated by Zootoca ziziparus, Angwis fragilis, Seps, \&c., but most lay eggs with more or less calcareous shells. In 7rathydosaurus and Cyilodus the embryo seems to absorb food from the wall of the uterus. It is likely that Lacertilians existed in Permian ages, but their remains are not numerous before the Tertiary strata.

Many instructive illustrations of evolutionary change are afforded by lizards. Thus there are numerous gradations in the reduction of the limbs, from a decrease in the toes to entire absence of limbs. The diverse forms of tongue and the varied positions of the teeth are also connected by gradations. From the variations of the wall lizard (Lacerto muralis), Eimer elaborated most of his theory of evolution.

\section{Some Families of Lacertilia.}

The class includes great variety of form.

In the Geckos (Geckonidie) the vertebre are biconcave or amphicolous, the tongue is short and fleshy, the eyelids are rudimentary, the teeth are pleurodont, the toes bear numerous plaits, by means of which they adhere to smooth surfaces. The Geckos have been observed to eat their own young and even their own tails. The name Gecko indicates their call. Examples:-Platydaily/us maurianizus (\$. Europe), $H_{c}$ 'midactylus in most warm countries, Ptychosnon, with lateral webs of skin which serve as parachutes.

The Agamas (Agamidx) are acrodont lizards common in the eastern hemisphere. Examples:-Agama; Draco, with the skin extended on long prolongations of five or six posterior ribs; Chlamydostzurus, an Australian lizard, with a large scaled frill around the neck; Moloch, another Australian form bristling with sharp spikes.

The Iguanas (Igumidic) are pleworlont lizards, represented in the warmer parts of the New World. Examples:-Iguana, an arboreal lizard, with a large distensible dewlap: Amblyhymihus or Orociphalus wistatus, a marine lizard confined to the Galapagos Islands; Basiliscus, in S. Mexico, with none of the marvellous qualities of the mythological basilisk; Anolis, the American chamieleon, with powers of rapid colour change; Phrymosoma, the American "horned tond," with numerous horny scales, and a collar of sharp spines suggesting in miniature that of some of the extinct Reptiles.

The slow worms (Anguidi), are limbless lizards, with serpentine body, long tail, rudimentary girdles and sternum. The British species, -tugrais fragilis, is neither blind nor poisonous; the tail breaks very readily; the young are hatched within the mother. The American "glass snake" Opheosaurus a'entratis-is in many ways ljke our slow worm.

The poisonous Mexican lizard (Hildirma suspectum) measures over a foot in length, and is covered with bead-like scales. Its bite is poisonous, and rapiclly fatal to small Mammals. It is interesting to find poisonous powers like those of many serpents exhibited by this exceptional lizard.

The water lizards (Varanide) are large semi-aquatic forms of carni37 
vorous habit, most at home in Africa, but represented also in Asia and Australia. The Monitor of the Nile, Varanus nzloticus, may attain a length of five or six feet, and is noteworthy because of its fondness for the eggs and young of Crocodiles.

The family Teiidae inciudes many New World pleurodont lizards, mostly terrestrial in habit, for example, Teizs teguexim, the variegated lizard of tropical Brazil, sometimes measuring five feet in length; Ameiva dorsalis, the common ground lizard of Jamaica.

The Amphisbenidæe are degenerate subterranean lizards, without limbs, with rudimentary girdles, with no sternum, with small covered eyes, with hardly any scales. The sooty Amphisbaena ( $A$. fuliginosa), at home in the warmer parts of S. America, is the commonest species.

The Lacertidx are Old World acrodont lizards, such as Pseudopzes (Europe and S. Asia), Lacerta viridis, the green lizard of Jersey and S. Europe, L. agilis, the British grey lizard, L. muralis, abundant about ruins in S. Europe, L. or Zootoca vivipara, the British scaly lizard.

The Scincidxe are common in tropical countries, e.g., Scincus, Cyclodus, Seps, Acontias (without limbs), Oligosoma (abundant in the Southern States of America), Eumeces (common in America and elsewhere).

The Chamzeleons (Chamæleontidæ) are very divergent lizards, mostly African. There is one genus Chancleo. The head and the body are compressed; the scales are minute; the eyes are very large and movable, with circular eyelids pierced by a hole; the tympanum is hidden ; the tongue is club shaped and viscid; the digits are divided into two sets, and well adapted for prehension; the tail is prehensile; the power of colour change is remarkably developed.

The Chamaleons exhibit numerous anatomical peculiarities. As in the Amphisbenas, there is no epipterygoid nor interorbital septum. The pterygoid does not directly articulate with the quadrate which is ankylosed to the adjacent bones of the skull.

\section{Class Ophidia. Serpents or Snakes.}

The elongated limbless form of snakes seems at first sight almost enough to define this order from other Reptiles, but it must be carefully noticed that there are limbless lizards, limbless amphibians, and limbless fishes, which resemble serpents in shape though very different in internal structure. For the external shape seems in great part an adaptation to the mode of life, to the habit of creeping through crevices or among obstacles. Even in the thin-bodied weasels is there not some suggestion of the serpent? Yet the limblessness of serpents is not a merely superficial abortion, for there is no pectoral girdle nor sternum, and never more than a hint of a pelvis.

The skin is covered with scales, which being simply folds 
of the epidermis have much coherence, and are periodically shed in a continuous slough. The scales on the head form large plates, and those on the ventral surface are transverse shields. There are no separate eyelids, but the thin transparent epidermis extends over the staring eyes. The nostrils lie near the tip of the head; there are no external ear openings. In many cases there are odoriferous glands near the cloacal aperture.

The muscular system is very highly developed, and the limbless serpent, Owen says, "can outclimb the monkey, outswim the fish, outleap the zebra, outwrestle the athlete, and crush the tiger."

There are many remarkable peculiarities in the skeleton.

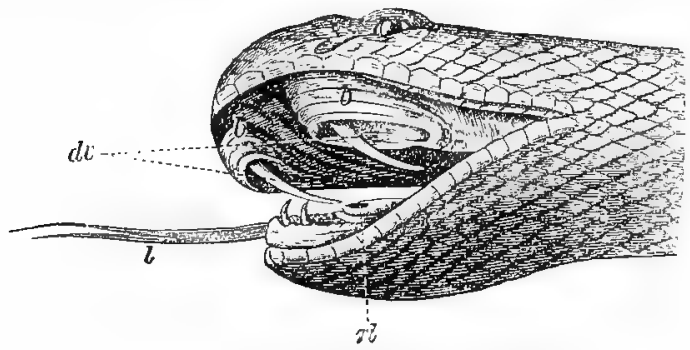

FIG. I99.-Snake's head. (After NuHN.)

$d \psi_{*}$, Poison fangs; $b$, , sheath of fang; $l$. tongue ; $r$, muscles of tongue.

The vertebræ are very numerous, some pythons having four hundred; they are procoelous, and are distinguishable only into a pre-caudal and caudal series.

All the pre-caudal vertebræ except the first-the atlashave associated ribs, which are movably articulated and used as limbs in locomotion. In the caudal region, the transverse processes, which are elsewhere very small, take the place of ribs.

The serpent "literally rows on the earth, with every scale for an oar; it bites the dust with the ridges of its body." On a perfectly smooth surface it can make no headway, but in normal conditions the edges of the anterior ventral scales are fixed against the roughnesses 
of the ground, the ribs are drawn together first on one side then on another, the body is thus wriggled forward to the place of attachment, the front part shoots out as the hind part fixes itself, an anterior attachment is again effected, and thus the serpent flows onward. But this account of the mechanism of movement does not suggest the swiftness or the beauty of what Ruskin calls " one soundless, causeless march of sequent rings, and spectral procession of spotted dust, with dissolution in its fangs, dislocation in its coils." "Startle it; the winding stream will become a twisted arrow;--the wave of poisoned life will lash through the grass like a cast lance."

One of the most distinctive characteristics of the skull, is the mobility of some of the bones. Many of the Ophidians swallow animals which are larger than the normal size of the mouth and throat. The mobility of the skull bones is an adaptation to this habit. Thus, the rami of the mandible are united by an elastic ligament; the quadrates and the squamosals are also movable, forming "a kind of jointed lever, the straightening of which permits of the separation of the mandibles from the base of the skull." The nasal region may also be movable. On the other hand, the bones of the brain case proper are firmly united. The premaxillae are very small and rarely bear teeth; the palatines are usually connected with the maxillæe by transverse bones, and through the pterygoids with the movable quadrates.

Teeth, fused to the bones which bear them, occur on the dentaries beneath, and above on the maxilla, palatines, and pterygoids, and very rarely on the premaxillz. The fanglike teeth of venomous serpents are borne by the maxillæ, and are few in number. Each fang has a groove or canal down which the poison flows. When the functional fangs are broken, they are replaced by reserve fangs which lie behind them. In the egg eating African Rachiodon the teeth are rudimentary, but the inferior spines of some of the anterior vertebra project on the dorsal wall of the gullet, and serve to break the egg shells.

When a venomous snake strikes, the mandible is lowered, the distal end of the quadrate is thrust forward, this 


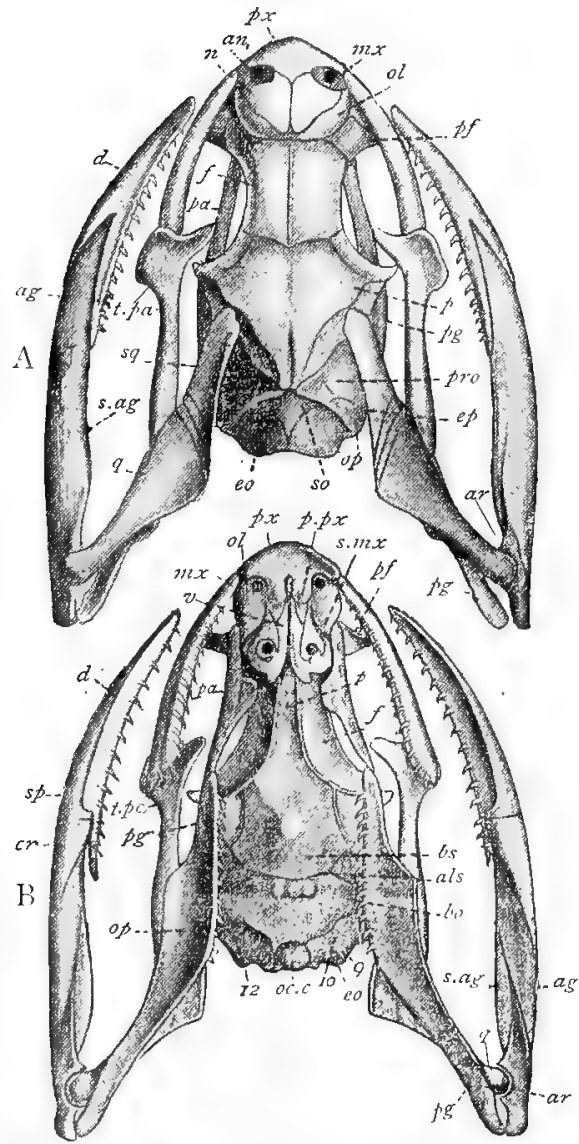

Fig. 200.-Skull of Grass Snake. (From W. K. PARker).

A. Dorsal surface-pax, premaxilla; $m x$, maxilla ; an., external nostril ; $n$. , nasal; ol., nasal cartilages; $\not f$. , prefronto-lachrymal ; $p_{0}$, parietal ; $f$., frontal ; pa., palatine; t.pa., trans-palatine; $p g$., pterygoid; pro., pro-otic; $e p$., epiotic: op., opisthotic; so., supra-occipital ; eo., ex-occipital ; $a \%$., articular ; s.ag., sur-angular; $a g_{.}$, angular; $d_{\text {. }}$, dentary ; $q$. , quadrate; $s q .$, squamosal ; B. Ventral surface- $p_{x}$, , premaxilla; ol., nasal cartilage; mx., maxilla; $v$, vomer; pa., palatine;

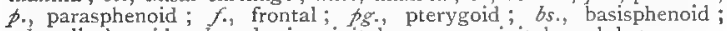
als., alisphenoid ; b.o., basi-occipital ; oc.c., occipital condyle; co., exoccipital ; q., quadrate ; ar., articular ; ag., angular ; s.ag., sur-angular ; $c r$. , coronary ; $s p$., splenial ; $d_{\text {, }}$, dentary ; op., opisthotic region. 
pushes forward the pterygoid, the pterygo-palatine joint is bent, the maxilla is rotated on its lachrymal joint, the fangs borne by the maxilla are erected into a vertical position, the poison gland is compressed by a muscle, and the venom is forced through the fang.

While there are no hints of anterior appendages, pythons, boas, and some other snakes, have rudiments of a pelvis and even small clawed structures which represent hind legs.

Some of the peculiarities in the internal organs of Ophidia may be connected with the elongated and narrow shape of the body. Thus one lung, usually the left, is always smaller than its neighbour, or only one is developed; the liver is much elongated; the kidneys are not opposite one another.

The brain presents no remarkable peculiarities: there are only ten cranial nerves; the sense of hearing is often slightly developed, and there is no tympanic carity: the eyelids are fused and transparent; the bifid, mobile, retractile tongue is a specialised organ of touch.

The poison gland is a specialised salivary gland; the venom is useful in defence, and in killing the prey, which is always swallowed whole.

The heart is three chambered, the ventricular septum being incomplete, as in all other Reptiles except Crocodilians.

There is a transverse cloacal aperture. In the males, a double saccular and spiny copulatory organ is eversible from the cloaca.

The British adder (Pelias berus) is viviparous, and so are a few others. The great majority are oviparous, but confinement and abnormal conditions may make oriparous forms, like the Boa constrictor and the British grass snake (Tropidonotus natrix), viviparous. The female python incubates the eggs.

Many Ophidians become lethargic during extremes of temperature, or after a heavy meal.

Though most abundant in the Tropics, snakes occur in most parts of the world. They are absent from many islands; thus there are none in New Zealand, and we all know that there are no snakes in Iceland. Most are ter- 
restrial, but not a few readily take to the water, and there are many habitual sea serpents.

The serpent still bites the heel of progressive man, the number of deaths from snake bite in India alone amounting to many thousands yearly, though there can be little doubt that the snakes are often innocent scape goats.

True Ophidians first occur in Tertiary strata.

\section{Classification of Ophidia.}

Suld-order I. Typhlopidre. The lowest and most divergent Ophidians, occurring in most of the warmer parts of the earth, generally smaller than earthworms, usually subterranean burrowers, with eyes hidden under scales, with a non-distensible mouth, with teeth restricted either to the upper or to the lower jaw. "The palatine bones meet, or nearly meet, in the base of the skull, and their long axes are transverse; there is no transverse bone; the pterygoids are not connected with the quadrates" (Huxley).

Example:-Typhlops, British India.

In all other Ophidians, the palatines are widely separated, and their long axes are longitudinal ; there are transverse bones connecting palatines and maxillre; the pterygoids are connected with the quadrates.

Sub-order 2. Colubriformes (innocuous Snakes). The poison gland is not developed as such; the maxillary teeth are not grooved.

Examples:-The British smooth snake (Coronella lavis), the British grass snake (Tropidonotzes natrix), the Pythons, the Boas. The Anaconda (Boa murina), which may attain a length of almost thirty feet, is the largest living Ophidian.

Sub-order 3. Colubriformes Venenosi.

Examples:-Cobras, Naja tripudians (Indian), Naja haje (African); the Hamadryad (Ophiophagus elaps), eating other snakes; Coral snakes (Elaps, \&c.); Sea snakes (Hydrophis, \&c.), with paddle-shaped tails.

Sub-order 4. Viperiformes.

Examples:-The British adder (Pelias or Viperaberus); the Rattlesnake (Crotalzcs), with a rattle formed chiefly from epidermic remnants of successive sloughings; the African Puff arlder (Clotho arietans).

Crocodilia. Crocodiles, Alligators, Gavials.

General Characters. - The Crocodilians are carnivorous fresh water reptiles of large size, now represented by three genera-Crocodilus, Alligator, and Gavialis.

The skin bears epidermic scales, underneath some of which there are dermic bones or sutes. 
The tail is laterally compressed and assists in swimming.

Teeth occur in distinct sockets in the premaxilla, maxille, and dentaries.

In modern Crocodilians, almost all the vertebre are procalons.

The skull has many characteristic features, such as the union of maxilla, palatines, and pterygoids in the middle line on the roof of the mouth, and the consequent shunting of the posterior nares to the very back of the mouth.

Some of the ribs have double articulating heads, and bear small uncinate processes somewhat like those of birds; transverse ossifications associated with the subcutaneous fibrous tissue of the abdomen from socalled abdominal ribs.

The heart is four chambered; a muscular diaphragm partially separates the thoracic from the abdominal cavity.

The cloaca has a longitudinal opening. The males have a grooved penis.

The Crocodilians are oviparous. The eggs have firm calcareous shells, and are laid in holes in the ground.

Some of the Characteristic Features in the Skeletal Sy'stem of Crocodilians.

(These notes on the skeleton are in great part taken from Huxley's Manual.)

Numerous transverse rows of sculptured bony plates or scutes, ossified in the dermis, form a dorsal shield. On the ventral surface the scutes are absent, except in some alligators, in which they are partially ossified. But besides and above the scutes, there are horny epidermic scales like those in other Reptiles. The hide is often used as leather.

The vertebral column consists of distinct cervical, dorsal, lumbar, 
sacral, and caudal vertebræ, all procœlous except the first two cervicals, the two sacrals, and the first caudal. In most of the pre-cretaceous Crocodilians, however, the vertebre were amphicolous. The centra of the vertebræ are united by fibro-cartilages, and the sutures between the neural arch and the centrum persist at least for a long time. Chevron bones are formed beneath the centra of many of the caudal vertebræ.

Many of the ribs have two heads-capitulum and tubercle-by which they articulate with the vertebre. From seven to nine of the anterior

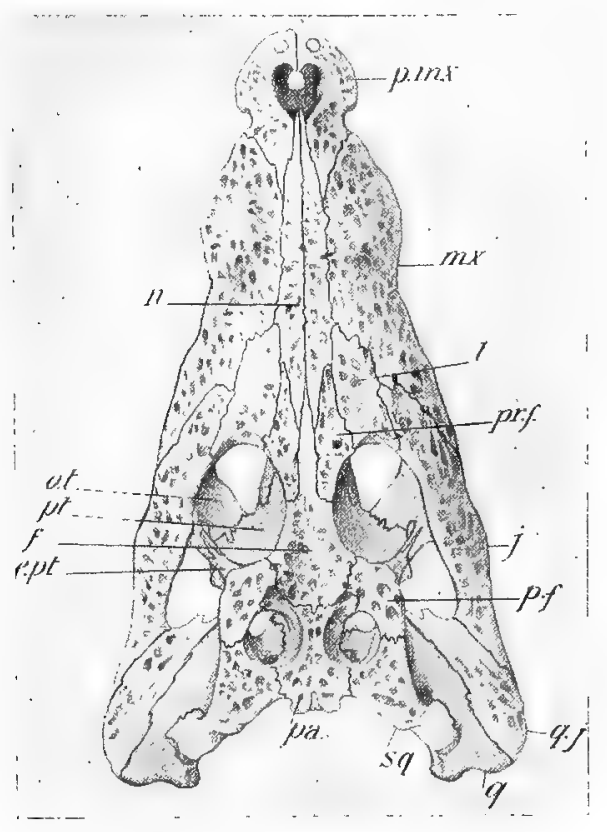

FIG. 202,-Crocodile's skull from dorsal surface.

p.mx., Pre-maxilla ; mx., maxilla ; l., lachrymal ; pr.f., pre-frontal ; $j$. , jugal ; $p . f$. , post-frontal ; $q \cdot j$. , quadrato-jugal ; $q$. , quadrate ; $s q$. , squamosal ; $p a$. , parietal ; $c \cdot p t$., epi-pterygoid ; $f_{\text {. }}$, frontal ; $p t .$, pterygoid (on lower surface); o.t., os transversum (on lower surface); $n$., nasal.

dorsal ribs are connected with the sternum by sternal ribs, and from several of these anterior ribs cartilaginous or partially ossified uncinate processes project backwards. The so-cal led abdominal ribs have nothing to do with ribs, but are ossifications in the fibrous tissue which lies 
under the skin and above the muscles. They form seven transverse series, each composed of several ossicles.

As to the skull, there is an interorbital septum with large alisphenoids ; the presphenoid and orlbitosphenoids are at best incompletely ossified ; all the bones are firmly united by persistent sutures; both upper and lower temporal arcades are completely ossified; the maxillex, the palatines, and the pterygoid, meet in the middle line of the roof of the mouth, covering the vomers, and determining the position of the posterior naresat the very back of the mouth; an os transversum extends between the maxilla and the junction of palatine and pterygoid; an epi-pterygoid runs down from post-frontal to os transversum ; the quadrate is large and immovable; there are large parotic processes; the tympanic cavity is completely bounded by bone; the teeth, which are borne by premaxillæe, maxillæ, and den-

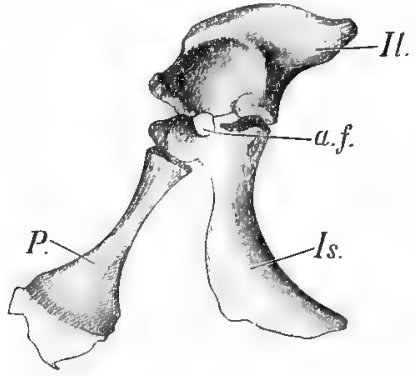

Frg. 203.-IIalf of the pelvic girdle of a young Crocodile.

Il., Ilium ; a.f, acetabulum ; Is, ischium; $P$, pubis.

taries, are lodged in distinct cavities; beside and eventually beneath the teeth lie reserve "germs" of others.

Each ramus of the mandible consists, as in most Reptiles, of a cartilagebone-the articular - working on the quadrate, and five membrane bones - dentary, splenial, coronoid, angular, and surangular.

The hyoid region is very simple.

In the pectoral arch there are no clavicles nor epicoracoids, but there is a so-called interclavicle or episternum; the fore limb is well though not strongly developed; there are five digits, webbed and clawed.

In the pelvic arch, large ilia are united to the strong ribs of the two sacral vertebre; the puhes slope forward and inward and have a cartilaginous symphysis; the ischia slope backwarl and have a symphysis; ilia and ischia form almost the whole of the acetabulum. The hindlimbs bear four digits, webbed and clawed.

\section{Some of the Characteristics of the varions organs of Crocodilians.}

The Crocodilians are seen to best advantage in the water, swimming by powerful tail strokes. The limbs are too weak for very effective locomotion on land, the borly drags on the ground, and the animals are stiff necked. Although many, especially in their youth, feed on fishes and small aninals, the larger forms lurk by the edge of the water, lying in wait for mammals of considerable size. These they grasp in their extremely powerful jaws, and drown by holding them under water. If the dead booty cannot be readily torn, it is often buried and left until it 
begins to rot. In connection with their way of feeding, we should notice several peculiarities of structure; as the nostrils are at the upper end of the snout, and the cyes and ears also near the upper surface, the Crocodilians can breathe, see, and hear, while the body is altogether immersed except the upper surface of the head; as the nostrils can be closed by valves, and the eyes by transparent third eyelids, and the ears by movable flaps, the head can be comfortably immersed; a flat tongue is fixed to the floor of the mouth, and the cavity of the mouth is bounded behind by two soft transverse membranes which, meeting when the reptile is drowning its prey, prevent water rushing down the gullet; the posterior opening of the nostrils is situated at the very back of the mouth, and when the booty is being drowned, the Crocodilian keeps the tip of its snont above water, the glottis is pushed forwarl to meet the posterior nares, a complete channel for the passage of air is thus established, and respiration can go on unimpeded. For their shore work the Crocodilians prefer the darkness, but they often float basking in the sun, with only the tip of the snout and the ridge of the back exposed.

Glands with a secretion which smells like musk are usually developed on the margin of the lower jaw, at the side of the cloacal aperture, and on the posterior margins of the dorsal scutes. The musky odour is very strong during the pairing season, and when the animals are attacked.

In connection with the muscular system, the presence of what is often called an incipient diaphragm between the thoracic and the alsdominal cavity is of interest.

The brain seems very small in relation to the size of the skull.

The eyes are provided with a third eyelid, as in most Reptiles, Birds, and Mammals; there are large lachrymal glands, but there is no special deceitfulness about "crocodile's tears."

The ears open by horizontal slits, over which lies a flap of skin; three Eustachian tubes-one median and one on each side-open into the mouth behind the posterior nares.

The nostrils also can be closed, and, as we have already noticed, their internal openings lie at the back of the mouth.

The stomach suggests a bird's gizzard, for it has strong muscular walls, and its pyloric end is twisted upward so as to lie near the cardiac part.

The heart is four chambered, the septum between the ventricles being complete as in Birds and Mammals. But as the dorsal aorta is formed from the union of a left aortic arch containing venous blood, and a right aortic containing arterial blood, the blood which is driven to many parts of the body is "mixed blood," i.e., blood partly venous, partly arterial, with some of its red blood corpuscles carrying hæmoglobin and others oxy-hemoglobin. At the roots of the two aortic arches there is a minute communication between them-the foramen Panizzæ.

Into the right auricle venous blood is brought by the two superior vene cave and by the inferior vena cava. The llood passes through a valved aperture into the right ventricle, and is clriven thence $(a)$ by the pulmonary artery to either lung, or (b) by the left aortic arch to the body. From this left aortic arch, before it unites with its fellow on the right to form the dlorsal aorta, is given off the great coliac artery. The anterior viscera thus receive wholly venous blood from the heart. 
The blood driven to the lungs is purified there, and returns by pulmonary veins to the left auricle. Thence it passes through a valved aperture in to the left ventricle. Thence it is driven into the right aortic arch. From this the carotids to the head and the subclavians to the fore limbs are given off. These parts of the body thus receive wholly arterial blood from the heart.

The renous blood returning from the posterior regions may pass through the kidneys in a renal portal system, and thence into the inferior vena cara; or it may pass through the liver in a hepatic portal system, and thence by hepatic veins into the inferior vena cava; or some of it may pass directly into the inferior vena cara. The renal portal reins arise from a transverse vessel uniting the two branches of the caudal, but the latter are also continued forward as lateral epigastrics which enter the liver.

The temperature of the blood is not above that of the surrounding medium.

In regard to the respiratory system, we should notice that the lungs are invested by pleural sacs as is the case in Mammals.

The ureters of the kidneys, the vasa deferentia from the testes in the male, the uriducts from the ovaries in the female open into the cloaca, which has a longitudinal opening.

The eggs, which in size are like those of geese, have a thin calcareous shell, are buried in excavated hollows, and, warmed by the sun, hatch without incubation.

Of one species of crocodile it is known that the mother opens up the nest when the young, ready to be hatched, are heard to cry from within the eggs. The mothers take some care of the young, which require to be defended even from the appetite of the males.

Crocodiles are relatively sluggish, and fond of basking passirely, sometimes hiding in the mud during the hot season. They are remarkable for the long continuance of growth, which does not seem to have so definite a limit as in most other animals.

\section{Classification of Crocodilia.}

(a) The true crocodiles, of the genus Crocodilus, occur in Africa, Southern Asia, tropical Australia, Central America, and the IVest Indies.

The Indian Crocodile (C. forosus) may measure about eighteen feet in length, and even larger forms have been recorded. The sacred African crocodile (C. a'mlgaris) is still formidably common in some of the fresh waters of tropical Africa.

The eggs and the young are often eaten by a mammal called the Ichneumon, and by a species of lizard. The adults have few enemies except man. They seem to live in friendly partnership with little birds (Plurianus equpticus). which remove parasites from the body, and in their familiarity almost justify the account which Herodotus gives of their cleaning the reptile's teeth.

(b) The Alligators, of the genus Allisator, are, with the exception of one Chinese species, confined to North and South America. In North America A. mississifficusis, in South America A. silerofs, are common. 
(c) The gavials or gharials, of the genus Gavialis, are distinguished by their long narrow snout. In the Ganges and its tributaries, G. gangeticus, said to attain a length of twenty feet, is common. They feed chiefly on fishes. "Old males have a large cartilaginous hump on the extremity of the snout, containing a small cavity for the retention of air, by which means these individuals are enabled to remain under water for a longer time than females or young."

\section{DIFFERENCES BETWEEN CROCODILES, ALLIGATORS, AND GAVIALS.}

Alligators.
The head is short and
broad.
First and fourth lower
teeth bite into pits in the
upper jaw.
The union of the two rami
of the lower jaw does not ex-
tend beyond the fifth tooth.
The nasal bones form part
of the nasal aperture.
The teeth are very un-
equal.
The scutes on the neck are
distinct from those on the
back. All American, except one
All Ame
Chinese species.

\begin{tabular}{l|l} 
Crocodiles. & Gavials. \\
\hline Longer. & The snout is very long.
\end{tabular}

The first bites into a pit; the fourth into a groove.

Not beyond the eighth.

As in the alligator.

Unequal.

Sometimes distinct, sometimes continuous.

Living in Africa, Living in India, Borneo, India, Australia, jN. Australia.
First and fourth lower teeth bite into grooves in the upper jaw.

The union extends at least to the fourteenth.

The nasal bones do not form part of the nasal aperture.

Almost equal.

Continuous.

History of Crocodilians. - These giant reptiles form a dlecadent order. Fossil forms are found in Triassic strata (e.g., Belodon, Parasuchuzs, and Stagonolepis); their remains are abundant in Jurassic rocks. In Cretaceous strata, crocodilians with procoelous vertebræe first occur, the pre-Cretaceous forms having centra of the amphicolous type. Huxley has worked out an "almost unbroken" series from the ancient Triassic crocodilians down to those of to-day.

\section{Development of Reptiles.}

As the development of Birds will be discussed in the next chapter, a few notes on that of Reptiles, which is in many respects sinilar, will be sufficient.

The ovum contains much yolk, at one pole of which there is a small quantity of formative protoplasm surrounding the germinal vesicle. Formation of polar globules has not been observed. The segmentation is necessarily meroblastic and discoidal, as in Birds.

The segmented area or blastoderm, originally at one pole, gradually grows round the yolk. The central region of the dorsal blastoderm is separated from the yolk by a shallow space filled with fluid, and is clearer than the rest of the blastoderm. In this central region or area pellucida, the germinal layers and subsequently the parts of the embryo are established, while the rest of the blastoderm-the area opaca- 
simply forms a sac round the yolk. One of the first signs of development is the appearance of a thickened band of cells extending forward in the micklle line from the posterior margin of the area pellucida. This band is called the primitive streak, and seems to represent a fusion of the two edges of the blastoderm behind the future embryonic region. The embryo develops in front of the primitive streak, and one of the first signs of its development is the formation of a primitive or medullary groove in a line with the primitive streak. As development proceeds, folds appear around the embryo, constricting it off from the subjacent yolk or yolk sac.

Fotal Membranes.-It is with Reptiles that the series of higher Vertebrates or Amniota begins. It is here that the fotal membranes known as amnion and allantois are first formed. Let us consider their development.

(a) The Amnion.-At an early stage in development, the head end of the embryo seems to sink into the subjacent yolk. A semilunar fold of the blastoderm, including epiblast and mesoblast, rises up in front. Similar folds appear laterally. All the folds increase in size, arch upwards, and unite above, forming a dome over the embryo. Each of these folds is double; the inner limbs unite to form "the true amnion;" the outer limbs unite to form "the false amnion," "serous membrane," or subzonal membrane. The cavity bounded by the true amnion contains an amniotic fluid bathing the outer surface of the embryo; the cavity between the true and the false amnion is lined by mesoblast, and is continuous with the pleuro-

FIG. 204.-Origin of Amnion and Allantois. (After Balfour.)

x. Rise of annniotic folds $\left(a, f_{0}\right)$; around embryo $(e) ; \not p_{0}$, , pleuro-peritoneal cavity; $y_{*}$, yolk.

2. Further growth of amniotic folds $\left(a_{2} f\right)$ over embryo and around yolk.

3. Fusion of amniotic folds above embryo $a \cdot \dot{p}$, , amnion proper; $s . z . m$. , sub-zonal membrane; $y . s$. , yolk sac.

4: Outgrowth of allantois (al.); amniotic cavity (a.c.); $h_{2}$, head end ; $t$., tail end.

5. Complete enclosure and reduction of yolk sac $\left(y . s_{0}\right)$; s.z.m, sub-zonal menibrane; a.p.., amnion proper; al., allantois: $g_{r}$, gut of embryo.
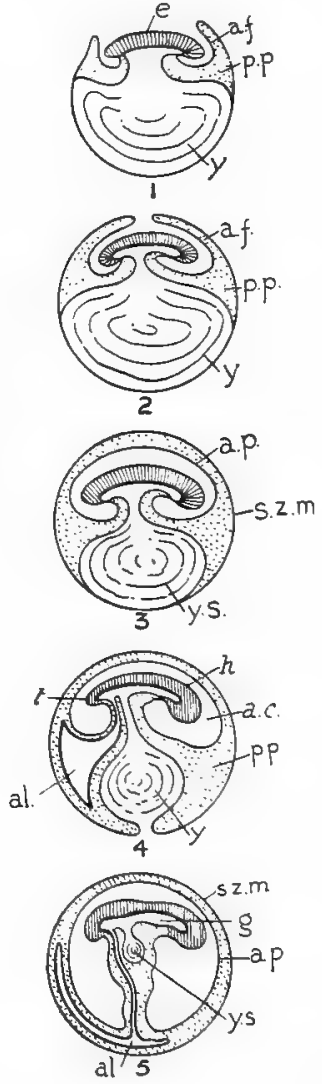
peritoneal or body cavity of the embryo. The amniotic folds extend not only over the embryo, but ventrally around the yolk sac which they completely invest.

(b) The Allantois.-- While the amnion is being formed, a sac grows out from the hind end of the embryonic gut. This is the allantois, lined internally by hypoblast, externally by mesoblast. It rapidly insinuates itself between the two limbs of the amnion, eventually surrounding both embryo and yolk sac.

The amnion is a protective membrane, forming a kind of water bag around the embryo. It may be due in part to the embryo sinking into the yolk sac by its own weight.

The allantoic sac is vascular, and has respiratory and perhaps also some yolk absorbing functions. It seems to be homologous with the outgrowth which forms the cloacal bladder of Amphibians; it has been called "a precociously developed urinary bladder."

Before the amnion is developed, the heavy head end of the embryo has already sunk into a depression (in Lizards, Chelonians, Birds (?) and Mammals), and is surrounded by a modification of the head fold termed the pro-amnion. This does not include any mesoblast, and is afterwards replaced by the amnion.

Sone Peculiarities in Chelonians. - Mitsukuri has recently investigated the development of the foetal membranes in Chelonians (Clemmys and Triony $x$ ), and has demonstrated some interesting peculiarities.

The amnion has at first the nature of a pro-amnion, consisting in the region of the sunken head of epiblast and hypoblast, and in the dorsal region of epiblast alone, being as yet non-mesoblastic. The coelomic cavities of the amniotic folds are not united with each other dorsally in the usual fashion; a connection between the "true amnion" and the "serous membrane" separates the cavities to the very end of the development. The anterior and lateral amniotic folds are continued backward beyond the posterior end of the embryo, as a long tube connecting the amniotic sac with the exterior. This tube perhaps conveys nutritive matter from the albumen into the amniotic cavity. In Clemmys, a process from the fotal membranes projects into a small persistent mass of albumen, and seems to absorb nutritive particles.

Hints of a Placenta before Manmals.-As will be explained afterwards, the placenta, which characterises most Mammals, is an organic connection between mother and unborn young. Its embryonic part is chieffy formed from a union of the serous or sub-zonal membrane and the allantois, but in some cases the yolk sac and the sub-zonal membrane form a provisional placenta. The placenta establishes a vital union between the embryo and the mother.

Now it is interesting to notice, that there are some hints of placenta connection in animals which are much lower than Mammals. In some species of Mustelus and Carcharids, there is a connection between the yolk sac and the wall of the uterus; in the Teleostean Anableps, the yolk sac has small absorbing outgrowths or villi; in Trachydosaumes and Cyclodus among Lizards, the vascular yolk sac is separated from the wall of the uterus "only by the porous and friable rudiment of the egg shell;" in Clemmys among Chelonians, there is, as above described, an absorbing prourusion of the fotal membranes. 
In Birds also small villi of the yolk sac absorb yolk, and others on the allantois absorb albumen. (See A. C. Haddon's Embryology.)

\section{Extinct Reptiles.}

The first known occurrence of fossil Reptiles is in Permian strata; in the Trias most of the orders or classes are represented; while the "golden age" of the group was undoubtedly during Jurassic and Cretaceous times.

Some of the modern Reptiles are linked by a series of fine gradations to very ancient progenitors, the Crocodiles of to-day lead back to those of the Trias, the New Zealand Hatteria to the Triassic Rhynchocephalia, but we have no example of a Reptilian genus which has persisted from age to age as Ceratodus has done among fishes. It follows naturally from this linking of the present with the past, that among the fossil forms we find "generalised" types, types which exhibit affinities with groups, which in our classification of recent forms may be very widely separated. It is indeed, as has been said, only because of our ignorance of their past history that we are able to classify living genera into separate orders at all.

The following types of extinct reptiles seem to have entirely disappeared:-

Anomodontiz. - Lizard-like animals with limbs adapted for walking, found in the Permian and Trias. The most generalised forms approach the Labyrinthodont Amphibians very closely, especially in the characters of the skull and pelvis. They, however, also exhibit affinities with the Monotreme Mammals. In the more specialised types the nature of the dentition is the most interesting feature. In Galesaum, for example, which is a carnivorous form, the teeth are arranged in three series, the anterior series (incisors) are separated by a tusk-like tooth (canine) from a lateral series of cheek teeth (molars). It is hardly necessary to insist upon the close affinity between such a dentition and that of carnivorous Marsupials, and we cannot doubt that the Anomodontia are in some way related to Mammals.

Examples:-Pariasaurus, Galesaurus, Dicynoion.

Sauropterysia. - Reptiles represented from the Trias to the Chalk, without exoskeleton, usually with a long neck and short tail. The limbs vary; in the earlier, more generalised, forms they are adapted for walking on land, but in the more specialised types they are modified into powerful paddles, like those of Chelonia. The nearest affinities are with the Chelonia. Notoszurus hard limbs adlapted for progression on land; Ptesiosaums and Pliosazmus were carnivorous forms adapted to an aquatic life. Plcsiosaurus had a very long neck, and sometimes attained a length of 40 feet. In Fliosiam the neck was much shorter, 
while the head was very large. In both, the limbs form powerful elongated paddles, with apparently no trace of nails.

Ichthyopterysia.-Large marine carnivorous Reptiles, represented from the Trias to the Chalk, with tapering body like that of a shark, large dorsal and caudal fins, and two pairs of paddle-like linus. There is no exoskeleton. The length of the body is sometimes 30 to 40 feet. In the paddle the number of digits is often more than five, and the phalanges of each are often very numerous. The skull has a large parietal foramen, and shows other affinities with that of Sphenodon. Some species were apparently viviparous.

Examples:- Tchthyosaurus, Ophthalmosaurus.

Mosasauria. - These strange Cretaceous Reptiles should probably be placed between the Lacertilia and the Rhynchocephalia. They are specially characterised by the enormous elongation of the body, which sometimes reached a length of 75 to 80 feet. The skull is like that of the Monitor among the lizards, but according to Cope it also presents affinities with snakes. The body is snake-like, but there are two well-

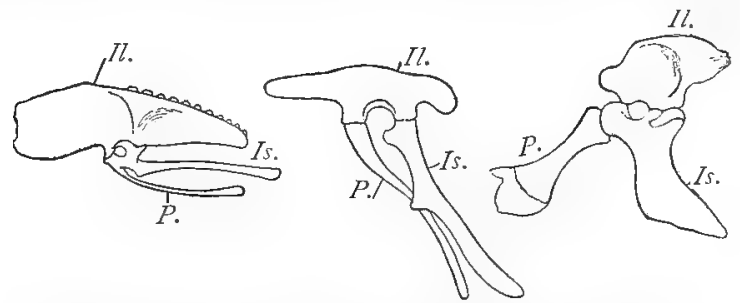

FIG. 205.-Comparison of pelvic girdles of Cassowary (to left); Iguanodon, an extinct Reptile (in centre); Crocodile (to right).

$$
I l ., \text { Ilium; Is., ischium; } P_{\text {., pubis. }}
$$

developed pairs of limbs, forming swimming paddles. All were carnivorous and marine; the distribution was cosmopolitan.

Mlosasaurus, Clidastes, Liodon.

Dinosauria.-Terrestrial Reptiles, ranging from the Trias to the Chalk, often very large, and, like Marsupials, specialised in various directions. They exhibit many points of resemblance to Crocodiles on the one side and to Birds on the other. Brontosaurus, a gigantic, herbivorous form, nearly sixty feet in length was probably amphibious. Atlantosaumas was even larger, the femur measuring over six feet in length. Compsognathus, Igzanodon, and Camptosaurus are examples of the "bird-footed" herbivorous Dinosaurs. In all these the form of the pelvis and of the hind limbs presents very strong affinities with the conditions seen in Birds. Compsognathus only reached a length of two feet, and hopped on its hind legs like a bird. Ionanodon habitually walked on its hind limbs, and, like several others, had hollow bones; it reached a height of fifteen feet. Of the carnivorous Dinosaurs, 
Megalosaurus is a good type. The pelvis has a Crocodilian aspect, for the pubes slope forwards instead of backwards as in Birds and Iguanodon, \&c. The limbs were furnished with powerful claws, and the teeth show much specialisation. Stegosaurus was furnished with heavy armour of plates and spines. Triceratops had three horns on its enormous head. The point of greatest interest about the Dinosaurs is the resemblance to Birds. This was first insisted on by Huxley, and since then it has been generally held that Birds have diverged from a Dinosaur stock. It is, however, fair to notice that by some these resemblances have been declared to be unimportant, while the points of resemblance between Birds and the next order of Reptiles are much dwelt upon.

Omithosanria.-Flying Reptiles, represented from the lower Jurassic to the Upper Chalk, exhibiting many points of resemblance to Carinate Birds, but still distinctly Reptilian in type. An expansion of the skin seems to have been stretched on the much elongated outermost finger, and to have extended backwards to the hind legs and the tail. The long bones contained air-sacs as in many Birds. The sternum is keeled, and teeth are often present on both jaws. Some are said to have had an expanse of wing of nearly twenty-five feet, but others were no larger than sparrows. It is a question how far the resemblances of these forms to Birds are a consequence of similar habits, and how far they can be regarded as indicating true affinities.

Examples :-Pterodactylus, Rhamphorhynihus, Pteranodon.

\section{Relationships.}

While it is still rash to venture on general conclusions, this much seems clear that the Reptiles, in their widest sense, form a central assemblage among Vertebrates. As we have noted above, some of the extinct forms exhibit affinities with Amphibians, others with Birds, others again with Mammals. Though we cannot with certainty point to any of the extinct types as directly ancestral to either Birds or Mammals, it seems likely that the ancestors of both were derived from the plastic Saurian stock. 


\section{CHAPTER XXV}

\section{CLASS AVES. BIRDS.}

I. Sub-class. Archæornithes (or Saurure) extinct Archeopteryy.

II. Sub-class. NeORNITHES.

r. Division, Ratitæ. "Running Birds." Ostrich, \&c.

2. Division, Odontolcæ. Extinct toothed birds, Hesperornis, \&c.

3. Division, Carinatæ, "Flying birds" with keeled sternum.

BiRus share with Mammals the rank of the highest Vertebrates. Their muscles and skeleton, heart and lungsindeed, most of their structural arrangements-are not less differentiated than those of Mammals. Nor are they inferior in integration. The body temperature, exceeding that of all other animals, is a physiological index to their rapid metabolism, to their intense activity. Most Mammals show a higher degree of brain development, and a closer organic connection between mother and offspring, but the truth is that the two classes represent markedly divergent lines of progress.

Life having begun in the waters, in all likelihood not far from the sea-shore, slowly gained possession of the dry land and then of the air. Insects among the lower animals, and Birds among the higher, are pre-eminently the creatures of the air ; intensely vivacious, typically beautiful in form and colour, lovely and delightful in their ways.

In the Birds we observe a marked increase of emotional life, so that their affection for their mates, their care of their young, the joyousness of their mood, often bursting forth in song, have become proverbial among us. With their power of flight they are emblems of freedom. 
General Characters. - The fore-limbs are generally modified as wings capable of fight; the neck is long, and the tail is short exiept in the extinct Saurura.

The epidermic exoskeleton is represented by feathers, with sometimes a few scales; there are no scutes.

- Almost the only skin gland is an oil or preen gland at the root of the tail.

The pectoral muscles used in flight are generally large; in many there is a muscular gizzard; the diaphragm is only hinted at.

In the brain, the predominance of the basalparts of cerebrum and cerebellum has resulted in displacing the optic lobes to the sides.

The nostrits are often overhung by a sensitive cere; there is no external ear; the connection between tympanum and inner ear is by means of a columella; the eveball is strengthened by sclerotic ossicles, there is a well-developed third eyelid and a large nutritive pecten.

There are no epiphyses in connection with the bones, many of which contain prolongations of the air sacs connected with the lungs, and are in the adult without marrow. The curvature of the vertebral centra, viezed from in front, is concave from side to side, and convex from aboz'e downwards. The cervical vertebra have small ribs. A large number of vertebra (one to three dorsals, all the lumbars, and some caudals) fuse with the two or three true sacrals. The terminal vertebra fuse in a ploughshare bone.

Most of the bones of the skull fuse, the sutures being obliterated. Only the lower jaw, the quadrate, the columella, and hyoid are alu'ay's movable, but the ptergoids usually articulate freely with the basi-sphenoid, the lachrymals may remain free, and there may be a joint in the beak at the end of the premaxilla. There is but one condyle. A membrane bone called the basi-temporal covers the basi-sphenoid. There is an interorbital septum formed from presphenoid and mesethmoid. The otic bones fuse with adjacent bones and with one another about the same time. In modern birds there are no teeth, but the jaws are cocered by horny sheaths. The premaxilla are large, and form most of the beak. The lower jaw consists on each side of five membrane bones and a cartilage bone-the articular-which works on the quadrate. Many of the skull 
bones have a spongy texture, due to cavities fllled with air from the nasal and Eustachian tubes.

There is a well-developed sternum, generally with a keel, to which the pectoral muscles are in part attached. The strong coracoids reach and articulate with the sternum. In flying birds, the clavicles are well developed, and are usually connected by an interclavicle, which is often fused to the top of the breast bone. The fore-limb has not more than three digits, the three metacarpals are fused (except in Archæopteryx),

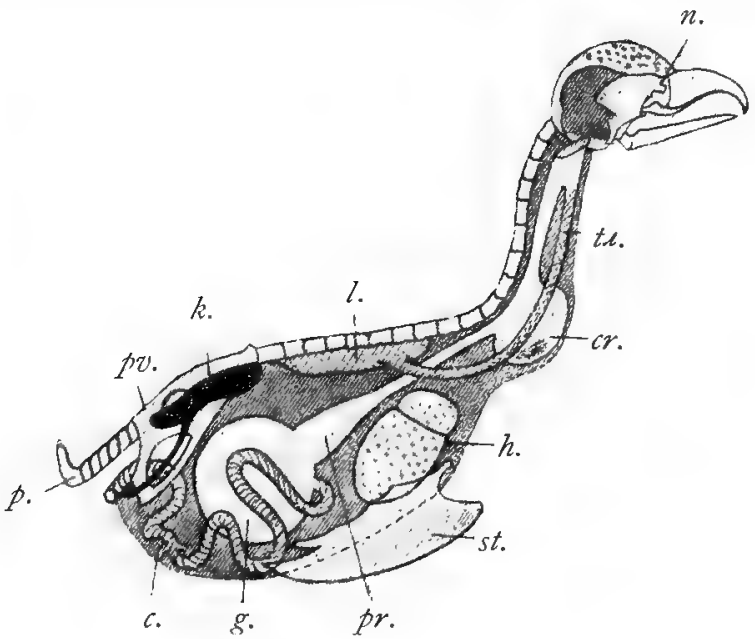

FIG. 206. - Position of Organs in a Bircl. (After SELENKA.)

n., Nostrils; tr., trachea ; cr., crop ; h., heart ; st., sternum ; pr.,

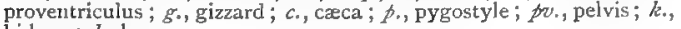
kidney; l., lung.

and there are only two separate carpals, the others fusing with the metacarpals, and thus forming a carpo-metacarpus. The metacarpals and digits bear the primary feathers or quills.

The ilia of the pelvis are firmly fused to the complex sacrum; the acetabulum is incompletely ossified; the pubes (or post-pubic 
processes) are directed backwards parallel to the ischia. There is no pubic symphysis except in the African ostrich (Struthio), and no ischiac symphysis except in the American ostrich (Rhea). In the hind-limb, the fibula is incomplete and united to the tibia; there are no free tarsal bones, half of them being united to the distal end of the tibia (which is therefore called a tibiotarsus), the others being united to the proximal end of three united metatarsals (which thus form a tarso-metatarsus). In other words, the ankle joint is intertarsal. The maximum number of toes is four, of which the first is the hallux, and if there be four, the netatarsal of the hallux is free from the other three.

In regard to the alimentary system, the absence of teeth, the frequent occurrence of a crop and a gizzard, the usual shortness of the large intestine, the presence of a cloaca, may be noted.

The heart is four-chambered; the single aortic arch curves to the right side: only the pulmonary artery rises from the right ventricle; the two valves between the right auricle and the right ventricle are in part muscular; the red blood corpuscles are oval and nucleated; the temperature of the body is from $2^{\circ}-14^{\circ}$ F. higher than that of Mammals.

The lungs are fixed to the dorsal wall of the thorax; the bronchial tubes expand in irregular branches in the lungs; the ends of some of these branches are continued into surrounding air

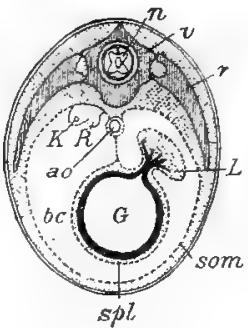

FIG, 207.-Diagrammatic section of young Bird. (After GADOW.)

n., Spinal cord; z., vertebra; r., rib; $L$., liver; $G$., gut; somt. (dotted), somatic layer of mesoblast; spl. (dotted), splanchnic layer of mesoblast; ao., aorta; $R_{\text {., }}$ reproductive organ; $K$., kidney. sacs; and these are continued into air spaces in the bones. The trachea is supported by bony rings, and has a voiceless larynx at its upper end, and a syrinw or song-box (zeith vocal cords) at the origin of the bronchi.

The kidneys are threctoled, and lie cmbadd'd in the poleis; the areters open into the clalca: there is nobladder: the wrine is semi-solid, and consists chiefly of urates.

The testes lie beside the kidneys; the rasa deferentia run 
beside the ureters, and open into the middle region of the cloaca. The right ovary atrophies, the right oviduct is rudimentary.

The eggs have much yolk and hard calcareous shells. The segmentation is meroblastic and discoidal. The allantois is chiefly respiratory, though it may also help in absorbing the nutritive substance of the egg.

The Pigeon (Columba) considered as a type of Birds.

The varieties of domesticated pigeon with which we are familiar, are all descended from the rock-dove, Columba livia, and afford vivid illustrations of variation, and of the results of artificial selection. Certain variations, e.g., in beak or tail, crop up, we know not how; and similar forms are bred together until a new breed is established. The power of rapid flight, the diet of seeds, the wooing of mates, the feeding of the young by both parents, are well known.

\section{Form and External Characters.}

The body, well suited for rapid flight, ceases to be graceful when stripped of its feathers. The cere above the nostrils, the third eyelid hidden in the anterior upper corner of the eyeball, the external opening of the ear concealed by the feathers, the preen gland on the dorsal surface at the root of the tail, the cloacal aperture, are external features easily recognised.

\section{Feathers.}

'The feathers most important in flight are the remiges of the wing, divided into primaries borne by the metacarpals and phalanges of the two fingers, and secondaries by the ulna. The feathers of the tail help to guide the flight, and are called rectrices. A distinct tuft of feathers borne by the thumb is called the bastard wing. Covering the bases of the large feathers are the coverts, - wing-coverts and tailcoverts,-- while the contour feathers give shape to the whole body. In the pigeon there are no true down feathers or plumules, but among the ordinary contour feathers or penna, 
there are little hair-like feathers (filoplumes) with only a few terminal barbs.

Any one of the large feathers consists of an axis or scapus divided into a lower hollow portion-the calamus or quill, and an upper solid portion-the rachis, which forms the axis of the vane. This vane consists of parallel rows of lateral barbs, linked to one another by barbules, which may be joined to one another by microscopic hooklets. The quill is fixed in a pit or follicle of the skin, with which muscle fibres are connected. At the base of the quill there is a little hole-the inferior umbilicus - through which a nutritive papilla of dermis is continued into the growing feather. At the base of the vane there is a little chink -the superior umbilicus-but this has no importance, except that parasites sometimes enter by it. Close to this region, however, in many birds, a tuft or branch arises, which is called the aftershaft. In the Emu and Cassowary, the aftershaft is so long that each feather seems double.

A feather grows from a papilla of skin, but the whole of the feather is really formed from the cornification of the inner layer of the epidermis. The papille rarely occur diffusely on the skin, but are usually disposed along definite feather-tracts. Each papilla consists externally of epidermis and internally of dermis, and lsecomes surrounded by a depression or moat, which deepens to furm the feather-follicle or the sac in which the base of the quill is sunk. The epidermis has two layers-(a) an outer stratum corneum, which in the developing feather forms merely a protective external sheath, and $(b)$ an inner stratum Malpighii, which becomes cornified and forms the whole feather. The process by which this cylinder of cells becomes horny is remarkable; in the upper part ridges are formed, which separate from one another as a set of barbs, the lower part remains intact as the quill. When we pull the horny sheath off a young feather, we disclose a set of barbs lying almost parallel with one another, yet slightly divergent. The central one predominates as the rachis, and its neighbours gradually become the lateral barbs. The external sheath falls off; the core of dermis is wholly nutritive, and disappears as the feather ceases to grow.

On the toes and on the base of the legs small epidermic scales occur. The toes are clawed, and in some birds the same is true of the thumb and first finger. Only in the embryos of the hoatzin (Opisthocomus) and of the ostriches (Struthio and Rhea) is the second finger clawed. The beak is covered by a horny sheath, which is annually moulted in the puffin. The dermis is very thin and vascular, and is rich in tactile nerve endings or Paccinian corpuscles, which are especially abundant in the cere. The only skin gland - the preen gland -secretes an oily fluid, with which the bird anoints its feathers. It is absent in the ostrich, emu, cassowary, and kiwi, and in a few Carinate birds. 


\section{Aluscular Sistem.}

The largest pectornl muscle (pectoralis major) arises from the sternum and its keel, and from the clavicle; is inserted on the humerus; and depresses the wing. The smaller but longer muscle (pectoralis minor), exposed when the large one is reflected, elevates the wing. It arises from the keel and sides of the sternum, and is continued over the shoulder to its insertion on the dorsal surface of the humerus. Arising chiefly from the curacoid, but in part from the stemum, and inserted on the humerus is a small coraco-brachialis which helps a little in raising the wing. 'There are several yet smaller muscles.

Interesting also is the mechanism of perching. When the bircl sits on its perch, the toes clasp this tightly. The flexor tentons of the toes are continued upwats in flexor muscles orer the metatarsal joint to the tibia, and are flexed automatically when the leg is bent during perching. Furthermore, an ambiens muscle, inserted on the front of the pulvis, is continued down the anterior side of the femur, and its tendon bending round the knee to the opposite side of the tibia, is inferiorly connected with the flexors of two digits. When the leg is bent in silling, the ambiens tendon is stretched, and the digits clasp the brancl. Thus the bird, when asleep, does not fall off its perch.

In connection with the muscular sistem, we may also notice that the walls of the gizzard consist of thick muscles radiating around tendinous dises. Two small sternotracheal muscles ascend from sternum to trachea. Complex muscles are associated with the song-box.

\section{Skilitem.}

In Birds there is a marked tendency to fusion of bones, as seen in the skull, rertebal column, pelvis, and limbs. In the pigeon most of the bones, except those of the tail, fore-arm, hand, and hind-limb, contain air spaces.

The rertebral column is divided into five regions-cervical, thoracic, lumbar, sacral, and caudal. In the pigeon the mobile neck consists of fourteen cervical rertebre with cervical ribs, short except in the last two, which have them well-dereloped. of the thoracic rertebre, namely those whose ribs reach the sternum, the anterior four are fused to one another, while the tifth is fused to the sacral 
region. The complex sacral region consists of the last thoracic (with ribs), two or three lumbars, three or four sacrals, and six caudals all fused. Lastly, there are about six free caudals, ending in a pygostyle or ploughshare bone, which represents a fusion of several vertebræ.

When we examine one of the cervical vertebræ, we notice that the anterior surface of the centrum has a complex and distinctive curvature, often described as saddle-shaped. It is concave from side to side, convex from above downwards. Posteriorly the curvatures are, of course, the reverse. The vertebra also bears expanded transverse processes, perforated on each side by an aperture for the vertebral artery, anterior articular processes or zygapophyses, posterior articular pro-

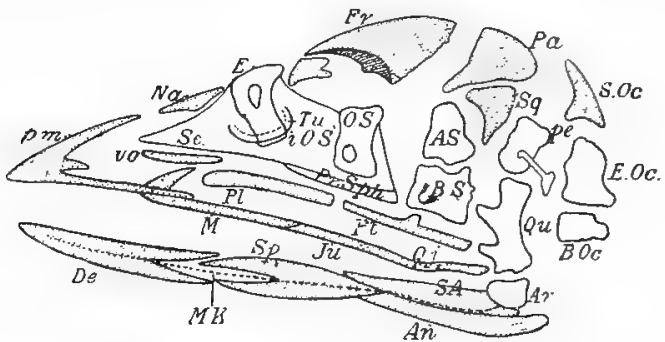

FIG. 208.-Disarticulation of Bird's Skull. (After GADow.) Membrane bones shaded.

B. Oc., basi-occipital ; $E$. Oc., ex-occipital; $S$. Oc., supraoccipital ; $P a$, parietal ; Fr, frontal ; $N$ a., nasal ; $p m$. , premaxilla; $M .$, maxilla ; $J u$., jugal ; $Q i$., quadrato-jugal ; $Q u$, quadrate ; pe., periotic ; $S q$. , squamosal; $B S$., basi-spheroid ; $O S$., orbito-sphenoid; Pr.Sph., pre-sphenoid ; vo., vomer ; ios., interorbital septum ; $E_{\text {., }}$ ethmoid; Se., nasal septum; $D e$. , dentary; $S_{p}$, splenial; $A n$, angular; Sa., surangular; Ar., arlicular; $M K$., Meckel's cartilage.

cesses, and a large neural arch culminating in a neural spine.

The ribs, borne by five vertebræ, have two heads-a capitulum articulating with a centrum, a tubercle articulating with a transverse process. The ventral part of the rib which reaches the sternum is called the sternal rib, and is joined at an angle to the dorsal part, which articulates with a vertebra. On the posterior surface of each of the first 
four ribs there is an uncinate process, absent only in the horned screamers (Palamedeæ).

The skull has a rounded cranial cavity and a narrow beak, which is mostly composed of the premaxillæ. All the bones are fixed except the quadrate, lower jaw, columella,

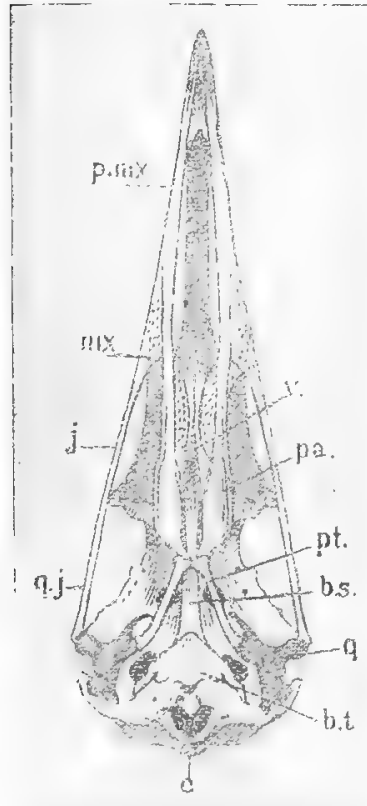

FIG. 209. - Under surface of Gull's Skull. (From Edinburgh Museum of Science and Art.)

c., Condyle ; b.t., basi-temporal ; b.s., basi-sphenoidal rostrum ; pt., pterygoid; sa., palatine; $z$, vomer; $p . m x$, premaxilla ; m.x., maxilla; $j$, jugal; $q-j .$, quadrato-jugal : $q .$, quadrate. and hyoid. The surface is polished, the sutures are obliterated very early in life.

The back part of the skull is formed by the basi-occipital, the two ex-occipitals, and the supra-occipital. These bound the foramen magnum through which the spinal cord passes. The basi-occipital forms most of the single condyle on which the skull rotates.

The top of the skull is formed from the paired parietals, frontals, and nasals, the last being small and in part superseded by the upward extension of the premaxillæ.

The line of the upper jaw consists of premaxilla, small maxilla, jugal, and quadratojugal, the last abutting on the movable quadrate.

Of the membrane bones on the side of the skull, the lachrymal in front of the orbit, and the squamosal above the quadrate, are the most important.

On the roof of the mouth the basisphenoid, which lies just in front of the basi-occipital, is covered over by a membrane bone-the basi-temporal. In front of this is a sharp "basisphenoidal rostrum," or parasphenoid, also a membrane bone. Articulating with the quadrate and with 
the rostrum are the pterygoids, in front of these lie the palatines, between which a part of the vomer may be seen. The bony front of the palate is formed from inward exten-

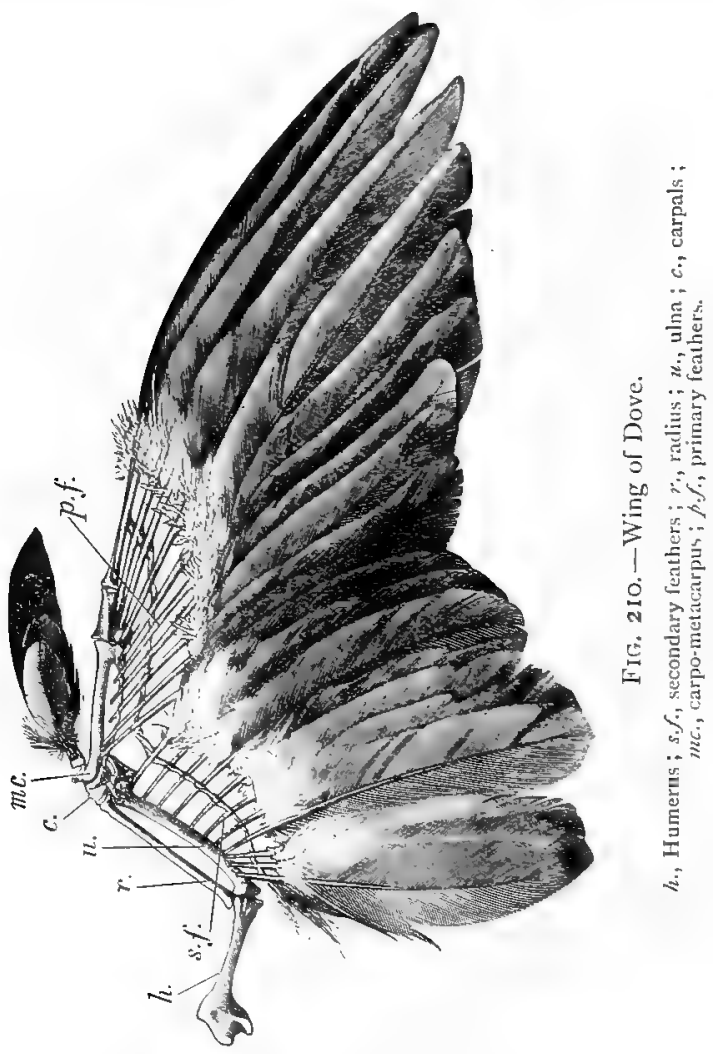

sions of the premaxillie and maxiller. The inter-orbital septum is formed chiefly from the mesethmoid but also from the presphenoid. From the tympanum to the inner ear 
extends the rod-like columella. The lower jaw originally consists of four membrane bones-dentary, splenial, angular, and surangular; and one cartilage bone-the articular. The hyoid consists of a flat "body," with anterior and posterior "horns."

The pectoral girdle consists of sabre-like scapulæ extending dorsally over the ribs, of stout coracoids sloping ventrally and articulating with the sternum, of the clavicles which are united by the interclavicle to form the merrythought.

The sternum bears a conspicuous keel, is produced laterally and posteriorly into two xiphoid processes, and bears articular surfaces for the coracoids anteriorly, for the ribs laterally.

The skeleton of the wing includes the stout humerus, the

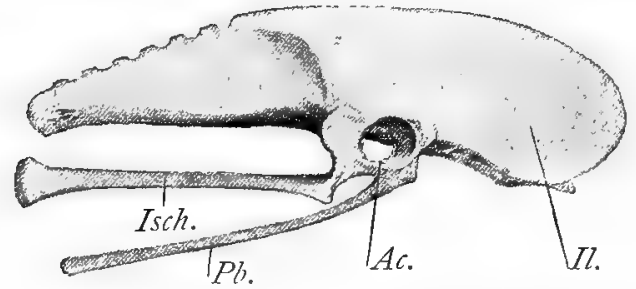

FIG. 2II.-Side view of pelvis of Cassowary.

Il., ilium; Isch., ischium ; $P b$., pubis ; $A C$., acetabulum.

separate radius and ulna (the latter the larger), two free carpals, a carpo-metacarpus of three metacarpals fused to one another and to some carpal elements, and three digits - the thumb with one joint, the first finger with two joints, the second with one.

The pelvic girdle consists of dorsal ilia fused to the complex sacral region, of ischia sloping backwards, and of pubes or post-pubic processes running parallel to the ischia. The incomplete ossification of the socket or acetabulum, and the absence of ventral symphyses, are also noteworthy.

The hind limb consists of a short stout femur, a tibia to which the proximal tarsals are fused (forming a tibio-tarsus), 
an incomplete fibula joined to the tibia, three metatarsals fused to one another and to the distal tarsals (forming the tarso-metatarsus), and, finally, three toes, of which the innermost has two phalanges, the next three, and the outermost four.

\section{Nervous System.}

In contrast to the brain of crocodiles and other Reptiles, the brain of the pigeon and other Birds fills the cranial cavity. 'The cerebral hemispheres are large and smooth. Their roof is thin, their main mass consists of the large corpora striata which bulge into the ventricles. They meet the cerebellum and throw the solid optic lobes to the sides. The olfactory lobes are very small. Between the cerebral hemispheres and the cerebellum, the pineal body rises to the surface, and a slight posterior separation of the hemispheres will disclose the region of the optic thalami. The cerebellum is ridged transversely and divided into a median and two lateral regions. The curvature of the brain is now well marked in the adult, thus the medulla is

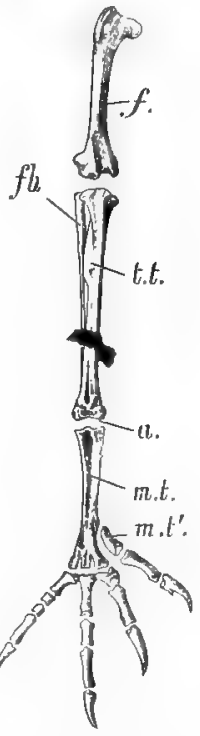

FIG, 212.-Bones of hind leg of Eagle.

$f .$, Femur : $t h$., tibio-tarsus : $f h .$, fibula; $a_{\text {. }}$, ankle joint ; $m t$. tarso-metatarsus; $m t^{1}$, ist metatarsal (free). quite hidden by, and descends almost vertically from, the cerebellum.

There are as usual twelve cranial nerves.

In connection with the spinal cord, the brachial plexus of nerves to the fore-arm, and the sacral plexus to the leg, should be noticed. In the lumbar region the halves of the corts diverge for a short distance, forming a wide space-the rhomboilal sinus-roofed only by the pia mater. The cervical part of the sympathetic nervous system is clouble on each side. 
Sense Orgens.

'The sense of smell does not seem to be keenly developed in many birds. 'The nostrils are longitudinal slits overhung by the swollen, more or less sensitive, cere.

The sense of hearing is acute. Extemally the ear is marked by an open tube-the external auditory meatus; the aperture of which is surrounded by a regular circlet of feathers. Within the tube beneath the surface lies the drum or tympanum; connecting this with the fenestra ovalis of the inner ear is the well-developed columella ; the tympanic

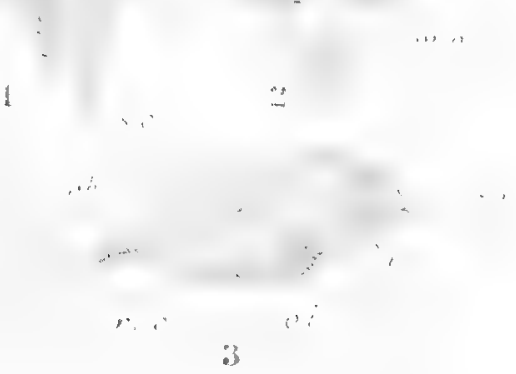

Ftg. 2I3.-Brain of Pigeon. (After Bronn.)

1, Dorsal view; 2 , ventral vicw; 3 , side view; of , olfactory lobes; $c_{\text {. }}$ cerebral hemispheres; $b . l$, optic lubes; $c h$, cerebellum; m.o., medulla ohlongata: s.c., spinal cord.

chamber is continued past the ear as the Eustachian tube which unites with that of the opposite side, and opens into the mouth cavity in front of the basi-sphenoid bone. The cochlea, or curved protuberance of the sacculus, which is incipient in Amphibians, and larger in Reptiles, is yet more marked in Birds.

As to the eye, its protection by an upper, a lower, and a 
third eyelid or nictitating membrane, is obvious. The front of the sclerotic protrudes in a rounded cone, and is strengthened by a ring of little bones. Into the vitreous humour, the vascular, nutritive pecten projects. Birds have remarkable powers of optic accommodation.

\section{The Alimentary System.}

The jaws are ensheathed in horn. There are no hints of teeth except that a "dental ridge" (see Mammals) has been detected in some embryos. A narrow tongue lies in the floor of the mouth; it is unimportant in the pigeon, but is thick in parrots, and long in woodpeckers and hummingbirds. Associated with the tongue there are numerous glands. Into the mouth there open the posterior nares, and the united Eustachian tubes.

The gullet expands into a thinwalled, slightly bilobed, nonglandular crop, in which are stored the hurriedly swallowed seeds. While it remains within the crop, the food is softened a little. Especially at the breeding season the cells lining the crop undergo a strange degeneration, forming "pigeon's milk," which both males and females give to the young birds.

From the crop the food canal is continued into the glandular

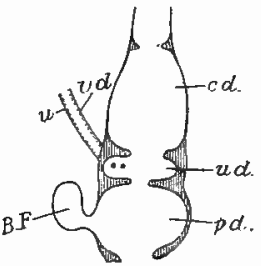

FIG. 214.-Diagrammatic section of cloaca of Male Bird. (After Gadow.)

$c d$, Upper region of cloaca into which rectum opens; ud, median region into which urelers $(u)$ and vas deferens $(v d)$ open; $p d$, posterior region into which Bursa Fabricii $(B . F)$ opens. part of the stomach (the proventriculus) where gastric juice is secreted.

Beneath the proventriculus is the gizzard, in which the food is ground. The walls are very muscular, the fibres radiating from two tendinous discs; the internal surface is lined by a hard cuticle, and within the cavity are small stones which the bird has swallowed. The pyloric opening from the gizzard into the duodenum, is very near the opening from the proventriculus into the gizzard.

In the fold of the long duodenum lies the pancreas with three ducts, and into the same region open two bile ducts 
from the liver, which is without a gall bladder, though this is present in most birds.

The small intestine is long; the large intestine is very short; in fact, it is not more than a rectum two inches in length. At the junction of the small and the large intestine,

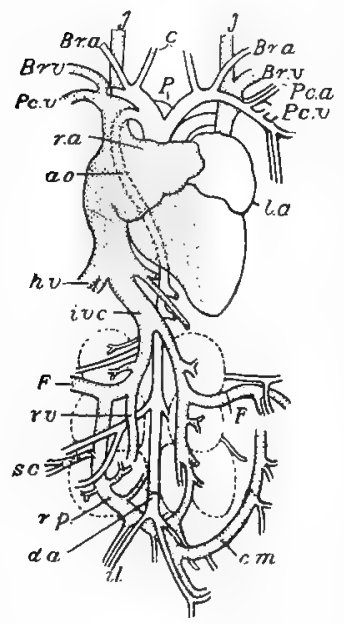

FIG. 215.-Circulation of Pigeon. (From PARKER, see Preface.)

$r a$, Right auricle; $l a$, left auricle ; $P$, pulmonary artery ; $C$, carotid artery ; Br.a, brachial artery; $P C$. a, pectoral artery; $j$, jugular vein ; $B r . \nu$, brachial vein; $P C . v$, pectoral vein; $\alpha_{0}$, aorta; $h v$, hepatic veins; iv $c$, inferior vena cava; $d a$, dorsal aorta; $i l$, iliac artery and vein; $r p$, renal portal or hypogastric; $s c$, sciatic artery and vein; $r v$, renal vein; $F$, femoral vein; $c m$, coccygeo-mesenteric to liver.

there are two short cæca. In some birds, e.g., the fowl, these are of considerable length.

The cloaca has three divisions, an upper part into which the rectum opens, a median part into which the ureters and the genital ducts open, and a posterior region opening into which from the dorsal surface is a vascular and glandular sac called the bursa Fabricii, which usually disappears during adolescence. Its function is obscure.

\section{Vascular System.}

The four-chambered heart, the single aortic arch bending over to the right side, the hot blood, are the most important characteristics.

The impure blood having returned by the venæ cavæ to the right auricle, passes through the auriculo-ventricular valve, which has two muscular flaps, into the right ventricle, and is thence driven to the lungs. From the lungs, the purified blood returns to the left auricle, and passes through two membranous valves into the left ventricle. Thence it is driven through the arterial trunk into the carotids, the subclavians, and the dorsal aorta. The bases of the aortic and pulmonary trunks are guarded by three semilunar valves. From the capillaries, the impure blood is collected 
anteriorly in two superior venæ cavæ, and posteriorly in an inferior vena cava, composed of veins from hind legs and kidneys, and receiving as it approaches the heart the hepatic veins from the liver.

The right auricle of the heart is larger than the left; the right ventricle has thin walls, and partly surrounds the more muscular left ventricle.

The arterial system consists of the following vessels :-

(a) The arterial trunk, as it rises from the heart, gives off on each side an innominate artery. Each innominate gives off a carotid and a subclavian, and the subclavian immediately divides into a brachial to the arm and a pectoral to the breast muscles.

(b) The dorsal aorta, formed by a continuation of the arterial trunk bending round on the right side, gives off cœliac, mesenteric, renal, femoral, sciatic, iliac, and other arteries.

(c) The pulmonary arteries (with impure blood) from right ventricle to lungs.

The venozes system consists of the following vessels :-

(a) Two superior vene cava, each formed from the union of jugulars from the head, a brachial from the arm, and a pectoral from the breast.

(b) The inferior vena cava is formed from the junction of two iliac veins just in front of the kidneys. Each of these iliacs results from the union of a femoral from the leg, an efferent renal from the kidney, and a renal portal, or hypogastric, which passes upwards through the kidney. To understand this renal portal, it is convenient to begin at the tail. A short caudal vein divides anteriorly into right and left branches, each of these receives an internal iliac from the sides of the pelvic region; thus the hypogastric is formed, which passing upwards through the kidney receives the sciatic, and finally joins with the femoral and with the renal.

(c) The pulmonary veins, with pure blood, from lungs to left auricle.

The hepatic portal system is as usual:-mesenteric veins from the intestine combine in portal veins; the blood filters through the liver; and is collected in hepatic veins, which unite with the anterior end of the inferior vena cava.

A functional renal portal system is represented by branches which the femoral and sciatic give off to the kidney.

From the transverse vein formed between the two hypogastrics or by the division of the caudal vein, a coccygeo-mesenteric arises, which receives vessels from the cloaca and large intestine, and is continued along the mesentery to join the hepatic portal system.

As there are rarely any valves in the renal portal veins, the blood from the viscera and hind limbs can pass freely either through the iliac veins and thence to the inferior vena cava, or through the coccygeomesenteric vein to the hepatic portal system.

The epigastric vein of the bird takes blood from the fat-laden sheet 
or great omentum which covers the abdominal viscera. It leads not into the liver, but into one of the hepatic veins.

Associated with the blood vascular system, there is a lymphatic system with a few lymphatic glands. The spleen lies on the right side of the proventriculus, the paired thyroid lies beside the origin of the carotid arteries, and a paired thymus is found in young birds in the neck region.

\section{Respiratory System.}

The important facts are, that there is as yet no diaphragm, that some of the bronchial branches in the lungs are continued into adjacent air sacs, that expiration is a more active process than inspiration.

The nostrils lie at the base of the beak overlapped by the cere. Only in the kiwi are they at the tip of the beak.

The trachea is strengthened by bony rings, and is moved by two sterno-tracheal muscles from the sternum. It has a larynx at its anterior end, and a syrinx, with vocal cords, at its lower end, where the bronchi diverge. The bronchial tubes branch in a kind of tree-like fashion in the lungs. These lie attached to the dorsal wall of the thorax, indented by six of the ribs, and covered with pleural membrane on their ventral surface only.

Around the lungs, and connected with the ends of some of the bronchial tubes, are nine air sacs, four lateral and one median. In order from behind forwards, lie posterior or abdominal sacs, the posterior thoracics, the anterior thoracics, the cervicals, and the interclavicular in the middle line in front. The anterior and posterior air sacs are continuous with air spaces in the bones.

\section{Excretory System.}

The kidneys are three lobed, and lie embedded in the ilia. They receive blood from the dorsal aorta by renal arteries, and the filtered blood leaves them by renal veins which unite with femorals and renal portals to form the iliacs, or, we may almost say, the inferior vena cava. But the kidney also receives venous blood from the sciatic and other posterior veins. Thus to some extent there is a renal portal system, which does not occur in Mammals.

The waste products, consisting for the most part of urates, pass in semi-solid form down the ureters into the median compartment of the 
cloaca. The predominance of urates in the active birds, with high oxidation and blood very rich in red corpuscles should be borne in mind when the physiological relations of uric acid to metabolism are studied.

In front of each kidney, at the base of the iliac vein, there lies a suprarenal body.

\section{Reproductive System.}

The testes lie in front of the kidneys. They increase in size before the breeding season. The sexual period in birds is often narrowly limited.

The spermatozoa pass from the testis into a vas deferens, which lies to the outside of the corresponding ureter. The

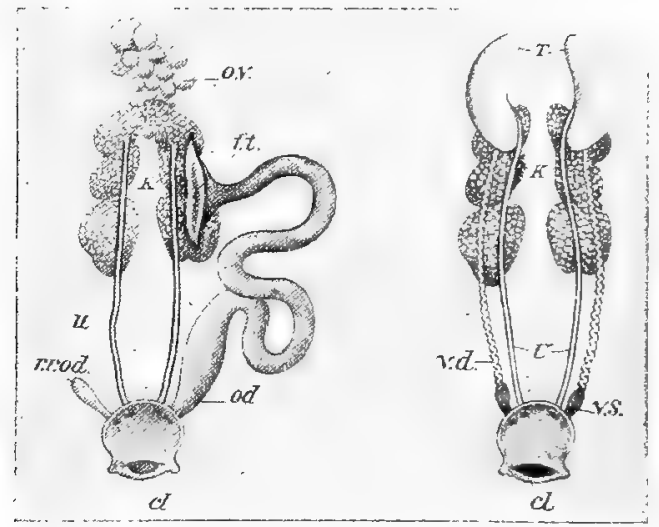

FIG. 2I6.-Urinogenital organs of Pigeon.

$K$., kidney; $U_{\text {., }}$ ureter; $c l$., cloaca; $T$., Testis; $y . d$., vas deferens; $v . s$. , seminal vesicle ; ov, ovary; od., oviduct; r.r.od., rudimentary right oviduct; $f . t$. , funnel at end of oviduct.

vasa deferentia, slightly convoluted when full of sperms, open separately into the median compartment of the cloaca.

In the adult pigeon, and in most birds, there is only one ovary; that of the right side atrophies very early in life. The right oviduct is represented by a small rudiment close to the cloaca.

The ovary is covered with follicles containing ova at various stages of ripeness. As these ova become dilated with yolk and otherwise mature they burst from the ovary, 
and are caught by the dilated end of the oviduct. The first part of the duct is narrow, and there the ova may be fertilised; the second part is wide and glandular, secreting the white of egg ; in the third region, which is muscular and glandular, the shell of the egg is made.

How the shell is made we do not precisely know, but it seems certain that it is not by the mechanical apposition of the secretions of the oviduct. A rudiment is present from the first, and this rudiment uses materials provided by the oviduct, and, although not cellular, grows by "intussusception."

In sexual union the cloaca of the male is closely apposed to that of the female; only in a few cases (in ducks and geese, and in the Ratitæ), is there a copulatory organ.

\section{Development of the Chick.}

The ovarian ovum of the hen is a large spherical body, consisting largely of yolk, but exhibiting at one region a disc of formative proto-

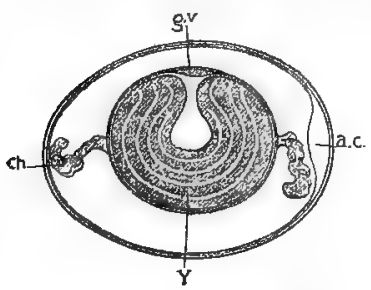

FIG. 217.-Diagrammatic section of Egg. (After AlleN Thomson.)

$g . v$, , Position of germinal vesicle; a.c., air chamber ; $Y$., yolk (alternate layers of "yellow" and "white"); ch. chalaza. result of these processes, we find that when laid the egg is surrounded first by a firm porous shell of carbonate of lime, formed in the lowest part of the oviduct; beneath this there is a double shell membrane, the two layers of which are separated at the broad end of the shell to form. an air chamber. This chamber grows larger as development proceeds, and is of some importance, in connection with respiration, as an intermediate region between the embryo and the external medium. Beneath the shell membranes lies the albumen, or "white of egg," which is secreted by the thin-walled region of the oviduct; in it lie two spirally twisted cords or chalazze, produced by the rotation of the egg in the 
oviduct. Within the enveloping albumen lies the ovum proper, with its enormous mass of yolk. The yolk is not homogeneous, but consists of two substances, known respectively as white and yellow yolk. The white yolk forms a central flask-shaped mass, and occurs also as thin concentric layers in the yellow yolk.

On the upper surface of the yolk, in whatever position the egg be held, lies the segmented blastoderm, whose exact origin we must consider more precisely.

In accordance with the principles of development, with which the student is already familiar, yolk is to be regarded as an inert and passive substance. In the hen's egg we have an increased specialisation along the line indicated by the egg of the frog. Here we have a small patch of formative protoplasm at one pole, and a large aggregate of yolk composing the remainder of the egg. In consequence, the activity of the protoplasm is unable to overcome the inertia of the yolk, and segmentation is meroblastic and discoidal.

In the protoplasm of the egg horizontal and vertical furrows appear in rapid succession. The result, as exhibited by vertical sections, is to produce an upper epithelial layer of cells, separated by a small space from larger, more irregular cells, which are still in connection with the yolk on which they lie. At the circular border of the germinal disc the two sets of cells are continuous. According to some authorities, this stage represents the blastula, the upper layer of cells corresponding to the cells of the animal pole in the frog, the lower with the enormous mass of yolk on which they lie to the cells of the vegetative pole, the space to the segmentation cavity.

At the next stage there appears at the future posterior end a crescentshaped groove. In this region there is an ingrowth of cells, which probably represents a modified process of gastrulation, and results in the obliteration of the segmentation cavity, and the formation of a "subgerminal" cavity or archenteron. The floor of the sub-germinal cavity is formed by the yolk, in which, by a process of supplementary cleavage, yolk nuclei appear.

This condition is that attained when the egg is laid. On surface view we see a central ill-defined "pellucid area." This, which becomes much more distinct during the early hours of inculation, is the area of the blastoderm which overlies the sub-germinal cavity, and is contrasted with the surrounding "opaque area," which lies directly on the yolk. At the posterior region of the opaque area, as already noted, there is the crescentic groove, where the outer and inner layers are continuous.

After the commencement of incubation, the blastoderm spreads rapidly over the yolk, chiefly by the extension of the area opaca; the area pelucida meanwhile elongates and becomes oval.

Another important change which also occurs in the early hours of incubation is the conversion of the transverse crescentic groove into the longiturlinal primitive streak. The precise meaning of this change is difficult and uncertain, but there seens no doult that the primitive streak represents the anterior lip of the blastopore of the frog. It runs down the centre of the area pellucida, and is narked by a central furrow, the primitive groove. At its sicles two wings of cells are obvious; these soon spread out laterally and anteriorly, and constitute the mesoblast. 

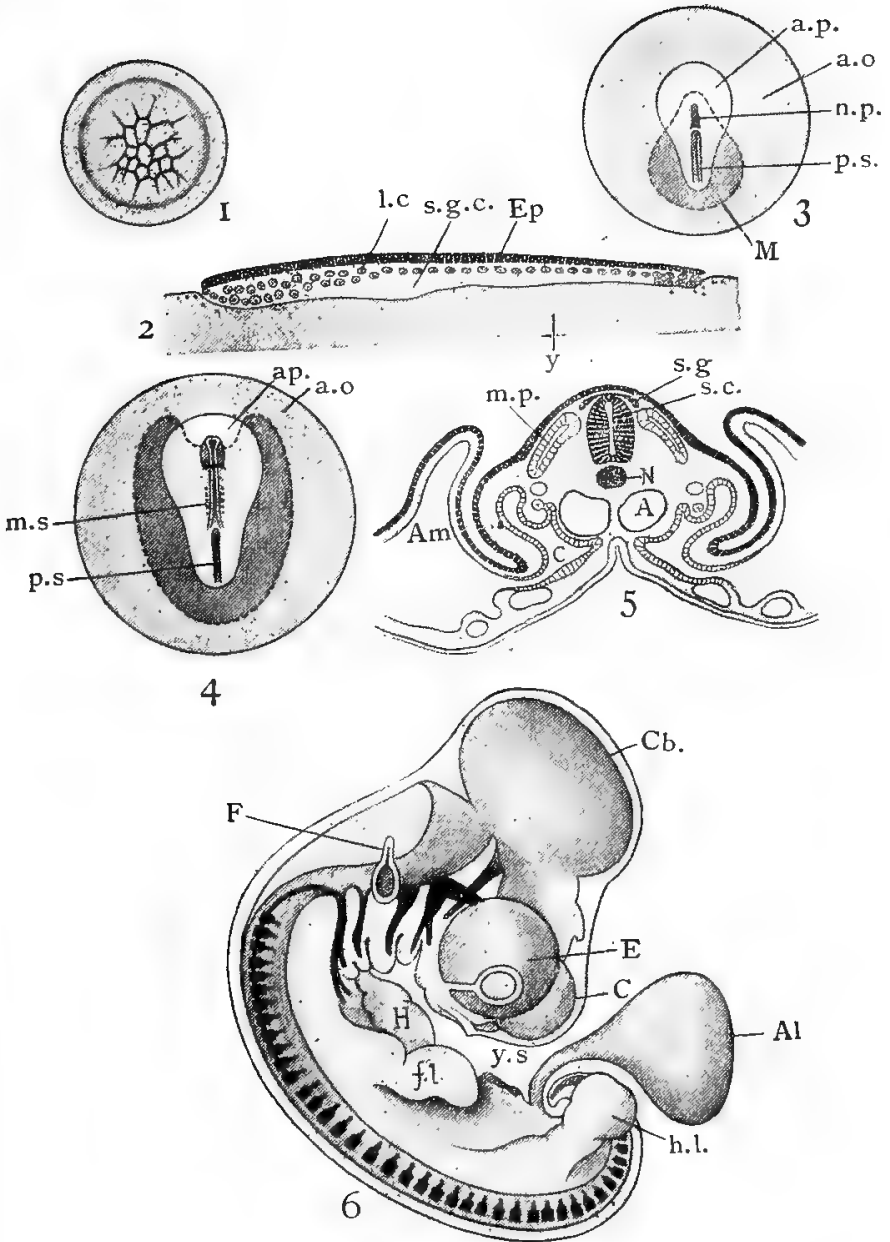

Fig. 218.-Stages in development of Chick. (After Marshall.)

I. Segmentation, superficial view of blastoderm.

2. Vertical section of blastoderm; Ep., epiblast ; l.c., lower layer of cells; s.g.c., segmentation cavity ; $y$, yolk.

3. Diagrammatic surface view; a.p., area pellucida; $a .0$. , area opaca; n.p., neural groove; p.s., primitive streak.

4. Diagrammatic surface view at later stage; $a \beta_{1}$, area pellucida; $a .0_{.1}$, area opaca; m.s., mesoblast segments ; p.s., primitive streak.

5- Cross section; s.c., spinal cord; s.g., rudiment of spinal ganglia ; $N$., notochord ;

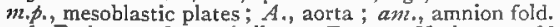

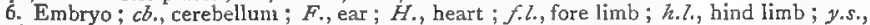
stalk of cut-off yolk sac; $A Z_{\text {. }}$, allantois ; $E$., eye ; $c$., cerebrum. 
The precise origin of the constituents of this middle layer is uncertain, but it is important to notice that all three layers of the embryo are connected at the sides of the primitive streak, as at the margin of the blastopore in the frog.

In the region in front of the primitive streak, a row of hypoblast cells becomes differentiated to form the notochord. At its sides the sheets of mesoblastic celis split into an inner or splanchnic layer, and an outer or somatic layer. A little later the mesoblast divides into the segmentally arranged mesoblastic somites, lying at the sides of the notochord, and the unsegmented lateral plate, whose outer and inner walls form the corresponding boundaries of the colome.

At the time when the notochord has appeared internally, the external epiblast becomes differentiated to form the medullary groove, which gives rise in the usual way to the medullary canal. The folds at first diverge posteriorly on either side of the primitive streak, but as the union travels backwards, this is included in the medullary canal, and so disappears.

During the course of the second day, the embryo seems to sink further into the yolk, while both anteriorly and posteriorly double folds known respectively as the head and the tail folds, rise up. In the course of their development the embryo becomes completely "folded off" from the yolk. At a slightly later stage, side folds also appear; all the folds now consist of a double layer of somatopleure covered externally by epiblast. The folds meet above the back of the embryo and coalesce. The inner layer forms the true amnion, the outer the false amnion or subzonal membrane. Into the space between the amniotic folds, an outgrowth from the posterior region of the gut, the allantois, grows out.

Before the end of the first day, blood vessels begin to be developed in the extra-embryonic region of the blastoderm. These form the beginning of the vitelline vessels, which are of great importance in the early

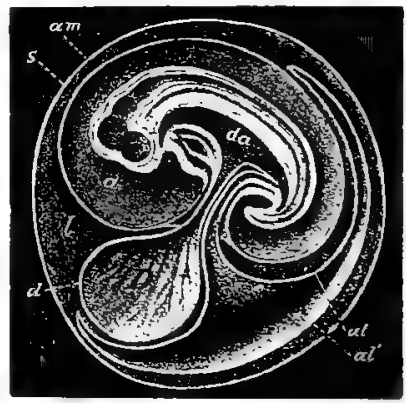

FIG. 219.-Diagrammatic section of embryo within Egg. (After KENNEL.)

$D$., Yolk sac ; $d^{t} a$, gut of embryo; $a l$, $a t^{\prime}$., inner and outer wall of the allantois; am., amnion proper; $a$., within amniotic cavity ; s., sub-zonal membrane. stages of development, and have probably at first some respiratory importance. As development proceeds, the allantois increases greatly, and, fusing with the subzonal membrane, approaches close to the egs shell. It has a large blood supply, and functions as an organ of respiration, in addition it absorbs the white of egg, thus serving as an organ of nutrition, and also receives deposits of urates, thus functioning as an organ of excretion in the narrow sense of the term.

We have spoken of the "folding off" of the embryo; it is important 
to realise that, as a result of this, the still small embryo is attached by a relatively narrow stalk to the large yolk sac, over which the blastoderm is now slowly spreading. In the young tadpole, the yolk lies heaped up on the floor of the gut, and causes a certain amount of distortion. In the chick the amount of yolk is so great that it forms a hernia-like protrusion of the gut, and only at a very late stage is the greatly reduced sac withdrawn into the gut, after which the dermal and intestinal umbilical openings are closed.

With regard to the development of the various organs of the body, the conditions are much the same as for the frog. The chick embryo never exhibits any trace of gills, but yet gill clefts perforate the pharynx. The embryonic organ of respiration is the allantois, but yet that arrangement of aortic arches by means of which in the tadpole blood is carried to the gills, is repeated here.

About the twentieth day, the beak perforates the membranes of the air chamber, and, the air rushing in, expands the hitherto functionless lungs. At the same time important changes occur in the circulatory system, " the umbilicus becomes completely closed, the allantois shrivels up, and the chick, piercing the broad end of the shell with repeated blows of its beak, steps out into the world."

\section{CLASSIFICATION OF BIRDS.}

I. - Sub-Class. Archeornithes or Saururf. Ancient extinct birds, with reptilian affinities more marked than in any living forms.

The oldest known bird is Archaopteryx, remains of which have been found in the Solenhofen slates in the Upper Oolite (Jurassic) of Bavaria. "The stone is so fine grained that, besicles the bones of the wings, the furculum or merrythought, the pelvis, the legs, and the tail, we have actually casts or impressions on the stone (made when it was as yet only soft mud) of all the feathers of the wings, and of the tail." - (Nicholson and Lydekker).

This link between Birds and Reptiles seems to have been a land bird about the size of a crow. It had feathers, and the upper jaw bore conical teeth. In the fossil specimen the feathers are confined to the wings, legs, and tail, those on the head, neck, and trunk, having perhaps fallen off. Each joint of the long tail bears a pair of lateral feathers - a unique arrangement. The vertebre have flat ends; the ribs are very slender, without uncinate processes; the sternum is not known; there seem to have been abdominal ribs. There are separate metacarpals; the first finger has two phalanges, the second three, the third three or four, and all are clawed. Dr. Hurst has recently maintained that the metacarpals of digits 4 and 5 are also present. The tail was long like that of a lizard, with about a score of vertebra, and no ploughshare bone.

\section{II.-Sub-Class. NeOrivithes.}

The metacarpals are fused. The second finger is the longest, ancl the third is reduced. Caudal vertebre are apparently not more than thirteen in number. 
The extant Neornithes are conveniently distinguished as Ratitæ and Carinatæ, but the distinctions do not seem very well grounded, and to most of them there are exceptions.

\section{Sone Contrasts between modern Ratita and modern Carinata.}

RATITÆ.
Running Birds, with wings more or
less degenerate and unused in
fight, with a keelless raft-like
breast bone.

'The skull is dromæognathous, i.e., the vomer is interposed between the palatines, the pterygoids, and the basi-sphenoidal rostrum.

The sutures in the skull remain for a long time distinct.

The long axes of the adjacent portions of the scapula and coracoid lie almost in the same line, or form a very obtuse angle, and the two bones are fused.

The clavicles are small or absent.

The ilium and ischium are not united behind, except in adult Rhea and Dromaus. There is no ploughshare bone.

The feathers of the adult have free barbs. There is no oil gland.
CARINATAE.

Flying Birds, with wings almost always well exercised in flight, with a keeled breast bone.

(The keel is rudimentary in the New Zealand parrot Stringops, in the exterminated Dodo (Didus), and in the extinct Aptornis-one of the rails. The penguins do not fly at all, the Tinamou, the Hoatzin, and some other birds, fly very little.

Except in the Tinamous, the skull is never dromæognathous, $i_{.} e$, the vomer is not fused with the neighbouring bones of the palate, and the palatines articulate with the basi-sphenoidal rostrum.

The sutures in the skull almost always disappear very early.

The scapula and coracoid meet at a sharp angle, and are separate from one another.

The clavicles are in most cases very well developed.

The ilium and jschium unite, enclosing a sciatic foramen. The terminal caudal vertebræ fuse to form a ploughshare bone or pygostyle.

The barbs of the feathers are generally united.

\section{Division Ratice. Running Birds with raft-like unkeeled breastbone.}

The African Ostrich (Struthio) is represented by two or three species, at home in the plains and deserts of Africa, and notable for their size, swiftness of foot, and beauty. There are but two toes, the third and the fourth, with stunted nails. There are no clavicles. The pubes form a ventral symphysis. The enormous size of rectum and creca is a unique character. The ostrich is monogamous, and at the breeding season the hen lays its eggs, at intervals of two days, in a hollow dug out in the sand by the male. The eggs are incubated loy the parents alternately, the inale sitting during the night, but in the hottest regions 
they are sometimes left during part of the day simply covered by the sand.

The American Ostrich (Rhea) is represented by three species in the S. American Pampas. In the Rhea there are three toes, all clawed, and the ischia form a ventral symphysis. There are no clavicles. Only here among Ratite is there a well-developed syrinx. The caca are large. The male excavates a shallow nest in the ground, and there, surrounded by a few leaves and grasses, the numerous eggs are usually laid. It seems that the male bird alone hatches the eggs. Single eggs are often laid here and there on the plains, but these are not incubated.

The Emu (Dromaus) is represented by two species in Australian deserts and plains. The fore limb is greatly reduced, the feathers have long aftershafts. Nearly related are the Cassowaries (Casuarius) living in the Austral-Malayan region, eight species in the Papuan Islands, one in N. E. Australia, and one in Ceram. They live in the forests and scrub. The fore limb is very small, with the shafts of the wing feathers reduced to spines; the ordinary feathers having long aftershafts. On the top of the skull there is a horny helmet, covering a core of light spongy bone; this protects the bent head as the bird rushes through the scrub. There are three toes, the inner one with a long sharp claw-a formidable weapon. In both these genera the clavicles are rudimentary and the cæca small.

The Kiwi (Aptery'x) forms a very distinct genus of Ratitze, represented by four species, restricted to New Zealand. It is not larger than a hen, and has simple hair-like or bristle-like feathers, a long bill and terminal nostrils, a very rudimentary wing and no clavicles, and no distinct tail feathers. There are four clawed toes. The caeca are large. It is a nocturnal bird, swift and noiseless in its movements, feeding in great part on earthworms. The egg is very large for the size of the bircl.

Among the extinct forms are the gigantic Moas (Dinornis), which seem to have been exterminated in New Zealand in comparatively recent times. The fore limbs were almost completely reduced, the hind legs were very large, and some forms attained a height of ten feet or even more.

Another recently lost order of giant birds is represented by remains of Epyornis found in Madagascar. Some of these indicate birds as large as ostriches, but eggs have been found holding six times as much as that of an ostrich.

Besides these there are other extinct Ratite (Stereornithes) such as Brontornis, Dasomis, and Gastomis.

We may think of the Ratite, according to W. K. Parker, as "overgrown, degenerate birds that were once on the right road for becoming flying fowl, but through greediness and idleness never reached the 'goal,' went back, indeed, and lost their sternal keel, and almost lost their unexercised wings,"

2. Division Odontol.ce. Extinct toothed birds from N. American Cretaceous strata, e.g., Hesperornis, somewhat like a swimming ostrich, with sharp teeth sunk in a groove, with vertebre like those of modern birds. 
3. Division Carinate. Flying Birds with a keeled breast bone.

The detailed classification of Birds is difficult. There are so many that ornithologists have not yet been able to decipher all their relationships. It is only of recent years that anatomists like Huxley, and embryologists like Parker, have placed the classification on a secure foundation.

For though the old classification of birds into snatchers (Raptores), perchers (Insessores), climbers (Scansores), scratchers (Rasores), stiltwalkers (Grallatores), and swimmers (Natatores) was interesting and suggestive, yet it is easy to understand that an arrangement of this sort may be misleading, since birds of entirely different structure may have similar habits.

Huxley classified birds accorcling to the structure of their skulls, and though this might seem a one-sided method of classification, its naturalness depends, as Parker notes, on the striking fact that "the structure of the skull and face govern the whole body, as it were; every other part of the organism corresponds to what is observable there."

Huxley's classification, slightly altered by Parker, is as follows :-

A. The vomer broad behind, and interposing between the pterygoids, the palatines, and the basi-sphenoidal rostrum:-DromæoGNATH龙.

The Tinamous.

B. The vomer narrow behind; the pterygoids and palatines articulating largely with the basisphenoidal rostrum.

a. The maxillo-palatines free.

1. The vomer pointed in front:-SchizOGNATHA.

The plovers, gulls, penguins, cranes, fowls, sand grouse, pigeons, the hoatzin, the goat suckers, the humming birds.

2. The vomer truncated in front:- EGITHOGNATHE. The passerines, swifts, and the hemipods.

3. The vomerine halves permanently distinct, and the maxillo-palatines arrested:-SAUROGNATH.E.

The woodpeckers.

b. The maxillo-palatines united:-Desmognath Æ.

The birds of prey, parrots, cuckoos and kingfishers, ducks and geese, flamingoes, storks, and cormorants.

We give an outline of the arrangement of Carinatie proposed by Professor Gartow :-

BRIGADE I.

I. Legion Colymbomomphr.

I. Ichthyomithes; the extinct Icthyomis, with teeth and biconcave vertebree.

2. Colymbiformes, e.g., grebe and dabchict.

3. Sphenisciformes, e.g., penguins.

4. Procellariformes, e.g., petrels.

2. Legion Pelargomorphie.

5. Ciconiiformes, e.g:, storks, herons, solan goose, cormorant.

6. Anseriformes, $e_{. g}$., clucks, geese, and horned screamers.

7. Falconiformes, e.g., falcons, vultures, hawks, eagles. 
BRIGADE II.

3. Legion Alectomorphre.

8. Tinamiformes, tinamou.

9. Galliformes, e.g., fowl, pheasants, Opisthocomuss.

Io. Gruiformes, e.g., cranes, rails, bustards,

I I. Charadrifformes, e.g., plovers, gulls, sand grouse, pigeons.

4. Legion Coracomorphæ.

12. Cuculiformes, e.g., cuckoos, parrots.

13. Coracioformes, e.g., rollers, owls, woodpeckers.

14. Passeriformes, e.g., crows, birds of paradise, finches, thrushes, swallows, \&c. \&c., in fact more than half the known birds.

\section{GENERAL LIFE.}

FLIGHT.-As birds are characteristically flying animals, many of their peculiarities may be interpreted in adaptation to this mode of motion.

(a) Shape and General Structure of the Body.-The resistance offered by the air to the passage of a body through it depends in part on the shape of the body, and the boatlike shape of the bird is such that it offers relatively little resistance. The attachment of the wings high up on the thorax, the high position of such light organs as lungs and air sacs, the low position of the heavy muscles and digestive organs, the consequently low centre of gravity, are also structural facts of some importance. It must be remembered, however, that the frictional resistance of the air is slight.

(b) The Muscles of Flight.-The most important is that which covers the whole of the breast bone, the pectoralis major. It brings the wing downward, forward, and backward, keeping the bird up and carrying it forward. As it has most work to do, it is by far the largest. Internal to it lies a second muscle, the pectoralis minor, which raises the wing for the next stroke. Besides these two great muscles, there are others of minor importance, the deltoides externus and three coraco-brachials, all of which help to raise the wing. On an average these muscles of flight weigh about one-sixth of the whole bird, but the proportion is often much greater, amounting to nearly one-half in some pigeons. Buffon noted that eagles disappeared from sight in about three minutes, and a common rate of flight is about fifty feet per second. 
(c) The Skeleton-The rigidity of the greater part of the backbone, due to fusion of vertebræ, is of advantage in affording a firm fulcrum for the wing strokes, while the arched clavicles (meeting in an interclavicle and often fused in front to the sternum) and the strong coracoids (which articulate with the sternum) are adapted to resist the inward pressure of the down stroke. As the keel of the breast bone serves in part for the insertion of the two chief muscles, its size bears some proportion to the strength of flight. It is absent in the running birds, such as the ostriches, and has degenerated in the New Zealand parrot

(Stringops), which has ceased to fly and taken to burrowing.

(d) Air Sacs and Air Spaces. - The lungs of birds open into a number of air sacs, which have a larger cubic content than the lungs, and in many cases these air sacs are continued in development into air spaces within the bones and even under the skin. From a broken bone it is possible to inflate the air sacs, and through a broken bone a

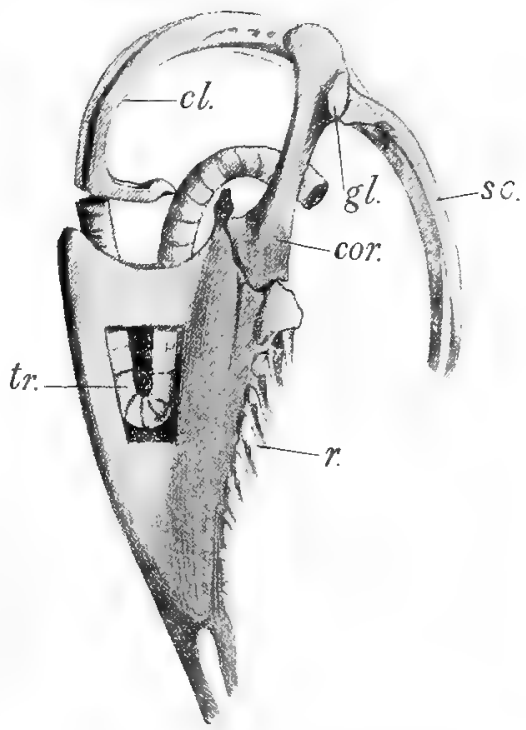

FIG. 220.-Pectoral girdle and sternum of Swan.

A part of carina removed shows peculiar loop of trachea $(t r.) ; c l$, clavicle; cor., coracoid; sc., scapula ; $g$ ?., glenoid cavity for head of bumerus; $\%$., sternal parts of ribs.

bird with choked windpipe may for a time breathe. The whole system of air-containing cavities is continuous, except in the case of the skull bones, whose spaces receive air from the nasal and Eustachian tubes. In view of these facts, it used to be supposed that a bird with heated air in the sacs and spaces 
was comparable to a balloon. But this is fallacious. The air must indeed lessen the specific gravity of the bird, but a few mouthfuls of food are sufficient to counteract the lightening. Moreover, in many small birds which are good flyers all the large bones, or all except the humerus, contain marrow, and are therefore not "pneumatic," and the hornbill, which is but a poor flyer, is one of the most pneumatic of birds. It is likely that the chief importance of the air sacs and air spaces is in connection with respiration and with the regulation of the body temperature. It is certain that in ordinary flight the lightest of birds has to keep

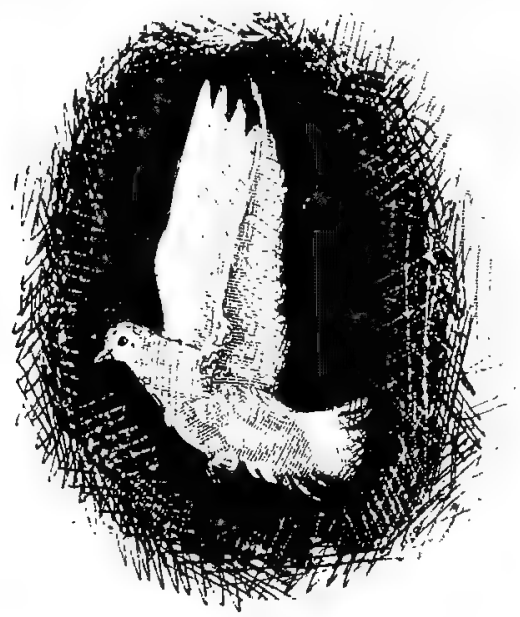

FIG. 221.-Position of wings in Pigeon at maximum elevation. (From MAREY.)

itself from falling by constant effort. The bird is not comparable to a balloon, but to a flying machine ; "it has to be not a buoyant cork, but a buoyant bullet."

There is no motion more marvellous or more beautiful than the flight of a bird. It is harmonious with the bird's true nature. For there is more than poetic insight in Ruskin's description:- "The bird is little more than a drift of the air brought into form by plumes; the air is in all its quills, it breathes through its whole frame and flesh, and glows with air in its flying; like a blown flame it rests upon the air, subdues 
it, surpasses it, outraces it ; is the air, conscious of itself, conquering itself, ruling itself."

Ruskin has compared the flight of a bird to the sailing of a boat. "In a boat, the air strikes the sail ; in a bird, the sail strikes the air; in a

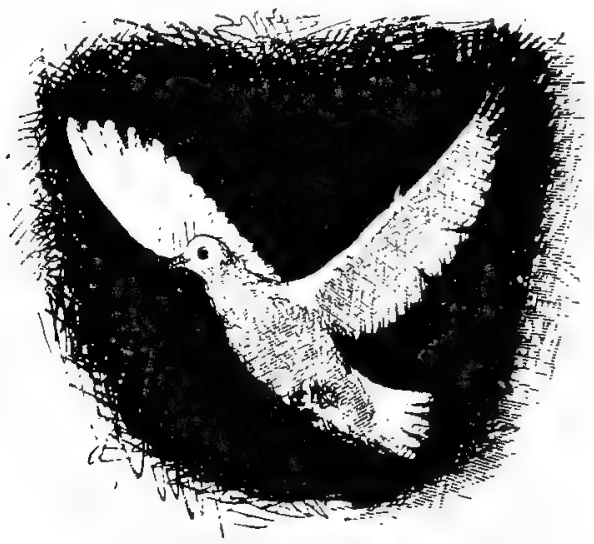

FIG. 222. -Wings coming down. (From MAREy.)

boat, the force is lateral, and in a bird downwards; and it has its sail on both sides." But, as he says, the sail of a boat serves only to carry it onwards, while wings have not only to waft the bird onwards, but to

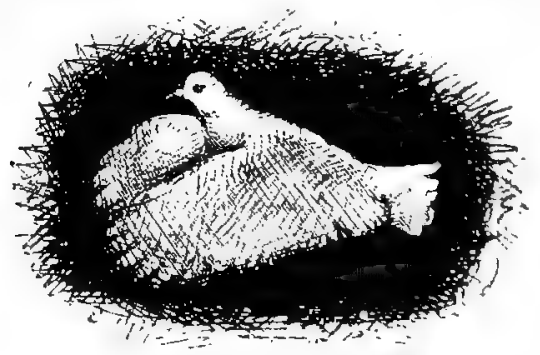

FIG. 223.-Wings completely depressed. (From MAREY.)

keep it up. To carry the weight of the bird the wings strike vertically, to carry the bird onwards they strike obliquely; sometimes the direction of the stroke is more vertical, and then the bird mounts upwards; 
sometimes it is more oblique, and then the bird speeds onwards ; usually both directions are combined. The raising of the wing after each stroke requires relatively little effort, the resistance to be overcome being very slight. In steering, the feathers of the tail often bear to the wings a relation comparable to that between rudder and sail.

Modes of fight. - There are three chief modes of flight :-

(I) By glicling or skimming, during which the bird has its wings spread, but does not flap them, depending for its movement on the velocity acquired by previous strokes, by descending from a higher to a lower level, or by the wind. This may be readily ouserved in gull and heron, in a pigeon gliding from its loft to the ground, or in a falcon swooping upon its quarry.

(2) By active strokes of the wings, in which the wings move downward and forward, backward and upward, in a complex curve. This is of course the commonest mode of flight. It has been carefully studied and photographed by Marey in the gull and pigeon.

(3) By sailing or soaring with motionless spread wings, in which the bird does not necessarily lose in velocity or in vertical position as is the case in gliding. It is illustrated by such bircls as crow, falcon, stork, and albatros, and has been observed only when there was wind. It is still imperfectly understood, but there seems most to be said in favour of the theory that it depends on the varying velocity of the wind at different heights.

The Song of Birds._-Singing is a natural expression of emotional intensity. The song rises in the bird, Richard Jefferies said, as naturally as the sap in the bough. It is richest at the climax of emotion in the breeding season, and is always best and often solely developed in the males. But song in any excellence is the gift of comparatively few birds, though nearly all have a voice of some sort, often so characteristic that the species may be recognised by its call alone. The twittering of swallows, the cawing of rooks, the melancholy voice of the sea-mew, the lapwing's prayerful cry, the weird call of the curlew, are familiar to most of us. A few birds, notably the parrot and the jackdaw, can be taught to pronounce articulate words: but the power of imitation is widespread among birds, the case of the canary learning the song of the nightingale being a well known instance. This power of imitation has some importance in relation to the general theory of instinct, for the song of all birds is probably in great part imitative, though to a certain extent the musical talent is really inherited. Young birds taken away from their nests when very young, so that they have hardly heard the voices of their kind, will sing the characteristic song of the species, but do so imperfectly. 
Many birds, apart from those who have been educated, have "words," expressing pleasure, pain, sense of danger, presence of food, and the like. But there is a difference between this power of utterance and the possession of language, which implies the expression of a judgment, e.g., food is good.

The vocal organ of Birds is not situated in the larynx as it is in Mammals, but in the syrinx - a song box at the base of the windpipe. In this syrinx there are vocal membranes or folds of skin; their vibration as the air passes over them causes sound; the note varies with the muscular tension of the folds, with the muscular state of the complex associated parts, and with the column of air in the windpipe.

Courtship.-Birds usually pair in the springtime, but there are many exceptions. Some, such as eagles, live alone except at the pairing time; others, notably the doves, always live together in pairs; many, such as rooks, parrots, and cranes, are sociable gregarious birds. A few, like the fowls, are polygamous; the cuckoo is polyandrous.

In most cases, however, birds pair, and the mates are true to one another for a season. The pairing is often preceded by a courtship in which the more decorative, more vocal males win their desired mates, being, according to Darwin, chosen by them. Darwin attriluted the captivating characteristics of the males, well seen in peacocks and birds of paraclise, or as regards musical powers in most of our own British songsters, to the sexual selection exercised by the females; for if the more decorative or the more melodious males always got the preference in courtship, the qualities which contributed to their success would tend to predominate in the race. He believed, moreover, that characteristics of male parents were entailed on male offspring. Wallace regarded the differences between males and females in another way, arguing that in the course of natural selection the more conspicuous females had been eliminated, brightmess being disadvantageous during incubation. It seems likely enough that both conclusions are to some extent true, while there is much to be said in favour of a deeper explanation, to which Wallace inclines, that the secondary differences between the sexes are natural and necessary expressions of the fundamental constitutional differences involved in maleness and femaleness.

Nests.-After pairing, the work of nest-building is begun. Almost all bircls build nests; the well-known habit is a characteristic expression of their parental care. Other creatures, indeed, such as stichlebacks among Fishes, and squirrels among Mammals, besides numerous Insects, build nests, but the habit is most perfectly developed among Birds. As is well known, each species has its own peculiar style of nest, and builds it of special materials. Generally the nest is solitary,
hidden in sume private nook. The perfection of art which is reached by some bircls in the making of their nests is marvellous; they use their bills and their feet, and smooth the inside by twisting round and round. 
Usually the hen does most of the work, but her mate sometimes helps, both in building the nest and in hatching the young.

The nest is a cradle rather than a house, for its chief use is to secure an approximately constant warmth for the young which are being formed within the eggs, and to afford protection for the helpless fledglings. At the same time, the nest secures the comfort of the parent bird during the days and nights of brooding.

The variety of nests may be illustrated by mentioning the burrowed nests of sand-martins and kingfishers, the ground-nests of game-birds and gulls, the mud-nests of house-swallow and flamingo, the holes which the woodpecker fashions in the tree stem, the platforms built by doves and eagles, storks and cranes, the basket-nests of most singingbirds, the structures delicately woven by the goldfinch, bullfinch, and humming-birds, the sewed nest of the tailor-bird, the mossy nests of the wrens, the edible nest of the Collocalia, which is chiefly composed of mucin secreted by the salivary glands.

The Eggs of Birds. - When the nest is finished, the eggs are ready to be laid. After they are laid, the patience of brooding begins. With the great care that Birds take of their young we may associate the comparatively small number of the eggs; but it is more accurate to recognise that, as animals become more highly evolved, the number of offspring decreases. Yet it must be remembered that inductions of this kind are only generally true, for subsidiary conditions often bring about the apparent contradiction of a general truth. Thus we are not justified in saying that the Apteryx, which lays one egg, is a more highly differentiated bird than the ostrich, which lays many.

The size of the egg usually bears some relation to the size of the bird. Of European lirds, the swans have the largest eggs, the golden-crested wren the smallest. It is said that the egg of the extinct Noo sometimes measured nine inches in breadth and twelve inches in length; while that of the extinct Aipyornis held over two gallons, some six times as much as an an ostrich's egg, or a hundred and fifty times as niuch as a fowl's. Yet the size of the egg is only generally proportional to that of the bird ; for while the cuckoo is much larger than the lark, the eggs of the two are about the same size; and while the guillemot and the raven are almost of equal size, the eggs of the former are in volume about ten times larger than those of the latter. The eggs of birds, whose young are rapidly hatched and soon leave the nest are large. Professor Newton remarks that "the number of eggs to be covered at one time seems also to have some relation to their size," while from what one notices in the poultry yard, and from a comparison of the habits of different birds, it seems probable that a highly nutritive, sluggish bird, will have larger eggs than a bird of more active habit and sparser diet.

The shell of the egg is often very beautifully coloured; there is a predominant tint upon which are spots, streaks, and blotches of varied colour and disposition, so that the egg is almost always characteristic of the species. The colouring matter consists of pigments related to those of the blood and the bile, and is deposited while the shell is being formed in the lower part of the oviduct. As the eggs may move before the pigments are fixed, blotchings and markings naturally result. But the most interesting fact in regard to the colouring of the egg shells, is 
that the tints are often protectively harmonious with those of the surroundings. Thus, eggs laid almost on the ground are often brownish like the soil, those laid in rocky places by the sea often look very like stones, while conspicuous eggs are usually found in covered nests.

The state of the newly hatched young is very various. Some are born naked, blind, and helpless, and have to be carefully fed by their parents until they are fully fledged. This is true of the thrush and of many other song-birds. Others are born covered with down but still helpless; while a few, like the chicks, are able to run about and feed themselves a few minutes after they leave the egg. Those which require to be fed and brooded over are sometimes called Altrices or Insessores, while those which are at once active and able to feed themselves are called Præcoces or Autophagæ.

Moulting.-Every year birds lose their old feathers. This moulting generally takes place after the fatigue of the breeding season, but in the case of the swallows and the diurnal birds of prey and some others the moult is in mid-winter. The process is comparable to the casting of scales in Reptiles, and to the shedding of hair in Mammals. Feathers are so easily injured that the advantage of the annual renewal is evident, especially when it takes place just before the time at which it may be necessary to set forth on a long migratory flight.

In moulting, the feathers fall out and are replaced gradually, but sometimes they are shed so rapidly that the bird is left very bare, thus moulting ducks are unable to fly. There are many birds that moult, more or less completely, more than once a year; thus the garden warbler sheds its feathers twice. The males of many bright birds assume special decorations after a partial moult which occurs before the time of pairing. Most remarkable is the case of the ptarmigan, which changes its dress three times in the year; after the breeding season is over the plumage becomes grey; as the winter sets in it grows white, and suited to the surrounding snow; in the spring, the season of courtship, the wedding robes are put on.

Diet. - The food of birds varies greatly, not only in different kinds, l)ut also at different seasons. Many are herbivorous, feeding on the soft green parts of plants, and in these birds the intestine is long. Some confine themselves to grain, and these have large crops and strong grinding gizzards, while those which combine cereals and insects bave in most cases no crop. A few sip honey, and may even help in the cross-fertilisation of flowers ; those that feed on fruits play an important part in the dissemination of seeds; those that devour insects are of great service to man. In fruit-eating and insectirorous birds the crop is usually small, and the gizzard only slightly muscular. But many birds feed on worms, molluscs, fishes, and small mammals; in these the glandular part of the stomach is more developed than the muscular part. It has been shown that the nature of the stomach in the Shetland gull changes twice a year, as the bird changes a summer diet of grain and seeds for a winter diet of fish, and vice versa. In the case of canaries, bullfinches, parrots, etc., it has been noted that the food influences the colouring of the plumage.

Migration of Birds. - Migration remains in no small degree a zoological inystery. On certain points we need more facts, and even 
where facts are abundant we but imperfectly understand them. Let us first state some of the outstanding facts.

(I) Most birds seem to be more or less migratory, but the range differs greatly. It is said that the clotterel may sup on the North African steppe and breakfast next morning on the Arctic tundra, and although the alleged rate may not be demonstrable, there is no doubt that a distance of about 2000 miles is traversed by this bird and by many others. Indeed, flights of 7-10,000 miles are said to occur. In the Tropics, on the other hand, the migration may simply be from valley to hillside.

(2) Observers in temperate countries long ago noticed that the birds they saw might be grouped in reference to their migrations. Thus (a) some arrive in spring from the South, remain to breed, and leave for the South in autumn, e.g., swallow and cuckoo in Britain; (b) some arrive in autumn chiefly from the North, stay throughout the winter, and fly northwards again in spring, e.g., the fieldfare and the redwing in Britain; (c) some-the "Birds of Passage"-are seen only for a short time twice a year on their way to colder or warmer countries in spring or autumn, e.g., sandpipers; and $(d)$ some seem to deserve the name of "residents," but really exhibit a partial migration, such as the songthrush and redbreast in Britain. In spring the tide is on the whole northwards, in autumn southwards, but the paths are great curves, and easterly and westerly waves pass from one country to another. The migrants always breed in the colder countries included in their range.

(3) There is striking regularity in the advent and departure of many of the migrants. In spite of the immense distances which many of our immigrants travel, and in spite of unpropitious weather, they are often punctual within a day or two to their average time of arrival for many years. Similarly some birds, such as the swifts, are hardly less precise in leaving our shores.

(4) It is beyond all doubt that many individual birds find their way back to the same district, even to the same spot, where they had made their nest in previous years. Not less marvellous is the security with which the flight from country to country is continued in darkness, at great heights, and over the trackless sea. At the same time it must be noticed that the mortality during migration is very great.

Having stated a few of the outstanding facts, let us note some of the interpretations and suggestions which help us to understand them.

The impulse to migrate is instinctive; it is exhibited by well-fed caged birds; migrating is an inherited habit. But it is likely that there are always immediate causes which prompt the habit, such as scarcity of food, and to a less degree, increasing cold in the case of many birds which leave us in autumn. It is more difficult to recognise the immediate causes prompting their return.

It seems likely that the origin of the migrating habit is wrapped up with the history of climates, and we can understand how the setting in of glacial conditions from the north would gradually force birds, century by century, to a longer flight southwards. And if the climatic conditions limit the area of safe and comfortable breeding to one country (the more northerly), and the possibility of food cluring winter to another country (the more southerly), we can understand, with Wallace, "that 
those birds which do not leave the breeding area at the proper season will suffer, and ultimately become extinct; which will also be the fate of those which do not leave the feeding area at the proper time." In short, given environmental changes of climate on the one hand, and a measure of plasticity and initiative on the part of the organism, the habit of migrating would be perfected in the course of natural elimination.

But while this view is so far satisfactory, it leaves us face to face with the problem how birds migrate as safely and surely as they do on their pathless way. To say that they do so by instinct only shelves the difficulty, even if it were true; and to point out that the merciless elimination which continually goes on keeps up the standard of racial fitness, leaves us still wondering how any became fit at all.

One welcomes therefore any suggestion as to the manner in which birds learn or have learned to find their way. The power has been compared to the "homing" faculty of some pigeons, but most believe that pigeons are guided solely by noticing landmarks, which could hardly be done over 10,000 miles of land, and obviously not over 1000 miles of sea, or during the night. Some have urged that birds follow river valleys, the lines of old "land bridges" connecting continents, the roll of the waves, and so forth, but the difficulty remains of flight by night and at very great heights. Attractive is the suggestion that birds are guided by what may be called a "tradition" based on experience ; those guide well one year who have followed well in previous years. But some young birds fly apart from their parents, and some birds do not fly in flocks at all. Moreover, it is difficult to understand how the experience could be gained except by sight, which in many cases is excluded by the darkness. In face of these difficulties, some authorities, such as Professor Newton, have been led to believe that birds have, in an unusual degree, "a sense of direction."

\section{Pedigree of Birds.}

Birds have many structural affinities with Reptiles, some of the ancient Dinosaurs present approximations to Birds, the extinct flying Pterodactyls show that it was possible for flight to be developed among Reptiles, the oldest bird-Archcoptery $x$ - is in many ways a connecting link between the two classes, and the development of some Birds reveals many remarkable resemblances with that of Reptiles,-therefore, with the strength of the general argument for evolution to corroborate us, we conclude that Birds evolved from a Reptile stock.

Speaking of his work on the development of the fowl, W. K. Parker wrote in 1868:- "Whilst at work I seemed to myself to have been endeavouring to decipher a palimpsest, and one not erased and written upon again just once, but five or six times over. Having erased, as it were, 
the characters of the culminating type-those of the gaudy Indian bird-I seemed to be amongst the sombre Grouse; and then, towards incubation, the characters of the Sandgrouse and Hemipod stood out before me. Rubbing these away, in my downward work the form of the Tinamou looked me in the face; then the aberrant Ostrich seemed to be described in large archaic characters; a little while, and these faded into what could just be read off as pertaining to the Sea Turtle; whilst underlying the whole, the Fish, in its simplest Myxinoid form, could be traced in morphological hieroglyphics."

More than twenty years later, the same accomplished embryologist described the development of the "Reptilian Bird"-Opisthocomus cristatus. In this form the unhatched chick has a paw-like hand, three clawed fingers and a rudiment of a fourth, a wrist of numerous carpal elements, and many other features suggestive of reptilian descent. It is not surprising, then, that to Parker, a bird seemed as "a transformed and, one might even say, a glorified Reptile."

It is likely that Birds arose from the Dinosaurian stock, but by what steps and under what impulses we do not know. To one it is enough to say that the evolution was accomplished gradually in the course of natural selection by the fostering of fit variations and the elimination of the disadvantageous; to another it seems that the incipient birds were "fevered representatives of reptiles, progressing in the direction of greater and greater constitutional activity;" but both these suggestions leave much in the dark, leave us still to "wonder how the slow, cold-blooded, scaly beast ever became transformed into the quick, hot-blooded, feathered bird, the joy of creation." 


\section{CHAPTER XXVI}

\section{CLASS MAMMALIA. ${ }^{1}$}

As Birds and Mammals have evolved along very different lines, Birds possessing the air and Mammals the earth, it is difficult to say that either class is the higher. But apart from the fact, which prejudices us, that man himself is zoologically included among Mammals, this class is superior to Birds in two ways-in brain development and in the relation between mother and offspring. In most Mammals there is a prolonged organic connection between the mother and the unborn young, and perhaps Robert Chambers was right in suggesting that this prolonged gestation was one of the conditions of progress, connected, it may be, with the development of large brains. Moreover, it is characteristic of Mammals that the young are nourished after birth by their mother's milk, and it has been suggested that the prolonged infancy of young Mammals was one of the factors in the evolution of gentleness. It is certain at least that the carefulness and sacrifice of the mothers has been a condition of the survival and success of Mammals, and of Birds also. We may find in the term Mammalia, which Linnæus first applied to the class, a hint of the idea that in the evolution of these forms of life, the mothers led the way.

$$
\text { General Survey of Mammals. }
$$

There are three grades of Mammalian development:(A.) The duckmole (Ornithorhy'nchus) and the spiny ant-

I In the systematic part of this chapter I have been especially indebted to the "Introduction to the Study of Mlammals," by Sir W. H. Flower and Mr. Lydekker.-(Lond., I891.) 
eaters (Echidna and Proechidna) differ markedly from other Mammals. The young are hatched outside of the body; in other words, the mothers are oviparous. The brain is poorly developed when compared with that of other Mammals. Some of the characteristics of the skeleton, \&c., suggest Reptilian affinities. To this small sub-class, the titles Monotremata, Ornithodelphia, and Prototheria are applied.

(B.) The kangaroos and bandicoots, phalangers and opossums, and the like, form the second sub-class. In these the young are born prematurely after a short gestation, during which the organic connection between the mother and the young is comparatively slight. Most female Marsupials have an external pouch or marsupium, to which the tender young are transferred, and within which they are nourished and protected for some time. Moreover, the brains even of the most intelligent Marsupials are not so well developed as those of higher Mammals. To this heterogeneous sub-class, the titles Marsupialia, Didelphia, and Metatheria are applied.

(C.) In all the other Mammals there is a placenta uniting the unborn young to the mother. It is among these placental Mammals that the brains begin to be much convoluted,- -as it were, wrinkled with thought. To this subclass, including sloths and ant-eaters (Edentata), sea-cows (Sirenia), hoofed-animals (Ungulata), Cetaceans, Rodents, Carnivores, Insectivores, Bats, Lemurs, and Monkeys, the titles Placentalia, Monodelphia, and Eutheria are applied.

Among these orders of placental Mammals, it seems likely that the Edentata and Sirenia should be placed lowest, for many of their characteristics are old-fashioned. The rest may be provisionally grouped in three sets, perhaps representing three main lines of evolution.

On one side we place the great series of hoofed animals or Ungulata, including (a) those with an even number of toes (Artiodactyla), such as pigs, hippopotamus, camels, cattle, and deer; $(b)$ those with an odd number of toes (Perissodactyla), such as tapir, rhinoceros, and horse; $(c)$ the elephants (Proboscidea); (d) the Hyraxes (Hyracoidea). And near the Ungulata it seems legitimate to rank $(a)$ the whales and dolphins (Cetacea), and $(b)$ the rabbits and hares, rats and mice, \&c. (Rodentia). 
On the other side we place the great series of Carnivora, such as cats, dogs, bears, and seals. Beside these may be ranked the Insectivora, such as hedgehog, mole, and shrew, and the bats or Chiroptera, which seem to be specialised Insectivores.

In the middle we place the series which, beginning with the Lemurs, Jeads through various grades of monkeys to a climax in man. Among the monkeys are the small and simple marmosets, the flat-nosed American monkeys, the dog-like apes of the Old World, and the anthropoid apes, which most nearly approach ourselves.

But it must be carefully noted that these orders are often linked by extinct types. Thus, to take one instance only, it is believed by some that the extinct Phenacodus has affinities with Ungulates, Carnivores, and Lemurs.

We may summarise our general classification thus :-

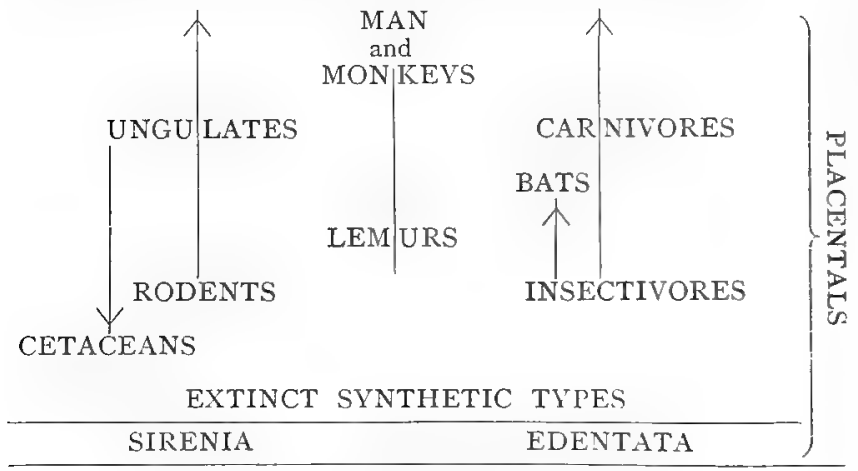

MARSUPIALS

MONOTREMES

General Characters.-All Mammals are quadrupeds, except the Cetaceans and Sirenians, in which the hind limbs have disappeared, leaving at most internal vestiges. There is generally a distinct neck betzreen the head and the trunk, and the vertebral column is, in most cases, prolonged into a tail.

Hairs are never entirely absent. In most they form a thick covering, but they are scanty in Sirenians and in the hippo- 
potamus, and almost absent in Cetaceans, in which they are sometimes restricted to early stages in life. The skin has abundant sebaceous and sudorific glands. In the female, milk-giving or mammary glands develop, as specialisations of sebaceous glands, except in Monotremes, where they are nearer the sudorific type.

A complete muscular partition or diaphragm separates the chest cavity containing the heart and lungs from the abdominal cavity, and is of great importance in respiration.

All the important bones have distinct terminal ossifications or epiphyses, absent, however, in the vertebra of Monotremes and Sirenia. The centra of the vertebre have generally flat faces, and there are seven cervical vertebra, except in the manatee and the two-toed sloth (Choloepus hoffmanni), which have six; the three-toed sloth (Bradypus tridactylus), which has nine; and the pangolin (Manis), which has sometimes eight, -variations which, it will be observed, are limited to the two most old-fashioned orders of placental Mammals.

The bones of the skull are firmly united by sutures, which generally persist. Only the lower jaw, the ear ossicles, and the hyoid are movable. There are two occipital condyles, as in Amphibians. It may be noted, however, that for various reasons, e.g., that some Birds and Reptiles are not very clearly single-condyled, morphologists no longer attach so much importance to this character as they once did. The lower jaw on each side consists, in adult life, of a single bone which works on the squamosal; the quadrate which intervenes in Sauropsida has disappeared, or has been shunted to become one of the ear ossicles. For it is one theory of the three ossicles-malleus, incus, and stapes-zehich connect the drum with the inner ear, that they correspond respectively to the articular, quadrate, and columella or hyo-mandibular of other Vertebrates. The otic bones fuse to form a compact periotic. A bony palate, formed from premaxilla, maxilla, and palatines, separates the buccal cavity from the nasal passages. In most cases there are teeth, borne in sockets by the premaxilla, maxille, and mandible.

Except in Monotremes, the coracoid is represented by a small process from the scapula, forming part of the glenoid cavity in which the head of the humerus works, but not reaching the sternum. The latter includes (a) a 
presternum with which in Monotremes an interclavicle is fused, and with which the clavicles (if well-developed) articulate; (b) a mesosternum divided into segments, with which the sternal parts of the ribs articulate; and (c) a xiphisternum, often cartilaginous. There are generally two sacral vertebra, but to these several caudals, and more rarely a lumbar, may be fused. The ilia slope downwards and backwards, the ischia have no symphysis, but the pubes are almost always united ventrally.

The cerebral hemispheres have usually a convoluted surface, and always cover the optic thalami and the optic lobes (now four-fold corpora quadrigemina), and in higher forms the cerebellum as well. The commissural system is well developed, being especially represented by a large corpus callosum, except in Monotremes and Marsupials, in which the anterior commissure is large and the corpus callosum small (according to some), or absent (according to others). There is also an important set of longitudinal flores called the fornix.

Except in Monotremes, in which there is a cloaca, the food canal ends separately from the urinogenital aperture.

The heart is four-chambered, and the temperature of the blood is high, though less than that of Birds. There is but one aortic trunk, which curves over the left bronchus. The red blood corpuscles are, when fully formed, non-nucleated, and are circular in outline, except in the Camelida where they are oval.

The lungs are invested by pleural sacs, and lie freely in the chest iavity. Within the lungs the bronchial tubes fork repeatedly into finer and finer branches. At the top of the trachea there is a complex larynx with the vocal cords.

The kidneys are generally compact and rounded bodies; the ureters open into the bladder, except in Monotremes in which they enter a urinogenital sinus. Except in Monotremes, the outlet or urethra of the bladder unites in the male with the genital duct, to form a urinogenital canal; in the female, except in Monotremes and a fere other cases, the urethra and the genital duct open into a common vestibule.

In the more primitive mammals the testes lie in the abdomen; in the majority they descend permanently (in a fere cases temporarity) into a single or paired scrotal sac, bying, except in Marsupials, behind the penis. 
The ovaries are small. Except in Monotremes, the genital ducts of the fenale are differentiated into (a) Fallopian tubes, which catch the ova as they burst from the ovaries; (b) a uterine portion in which the young develop; and (c) a vaginal portion ending in the urinogenital aperture. In Monotremes the two ducts are simple, and open separately into the cloaca; in Marsupials there are two uteri and two vagine; in Placental Manmals the uterine regions are more or less united, and the vaginal regions are always fused.

In Monotremes the eggs are large and rich in yolk; in all others they are small and almost yolkless. In the ovary each ovum lies embedded in a nest of cells, within a swelling or Graafian follicle which eventually bursts and liberates the egg cell. In Monotremes the segmentation is necessarily meroblastic, in other cases it is holoblastic. As in Sauropsida there are two fotal membranes- the amnion and the allantois, both of which share in forming the placenta of the Placental Mammals.

The Monotremes are oviparous; the Marsupials bring forth their young prematurely after a short gestation; the Placental Mammals have a longer gestation, during which the young are vitally connected to the wall of the uterus by means of the placenta.

\section{General Life of Mammals.}

Most Mammals live on dry land. The bats, however, have the power of flight, and not a few forms, belonging to diverse orders, are able to take long swooping leaps from tree to tree. Thus, there are "flying phalangers," such as Petaurus, among Marsupials; "flying squirrels," such as Pteromys, among Rodents; "flying lemurs" (Galeopithecus), allied to Insectivores. Not a few are aquatic, - all the Cetaceans, the two Sirenians, and the Pinniped Carnivores, such as seals and walruses; while water voles, beavers, otters, polar bear, and many others are also at home in the water. Burrowers are well represented by moles and rabbits ; aboreal forms by squirrels and monkeys.

As to the diet, man, most monkeys, pigs, and many others, may be called omnivorous; kangaroos, hoofed animals, and most rodents are herbivorous; the Echidna, the ant eaters, hedgehogs and shrews, and most bats are insectivorous; 
most of the Carnivora are carnivorous; dolphins and seals feed chiefly on fishes; but in most cases the diet varies not a little with the available food supply.

The struggle for existence among Mammals is sometimes keen among fellows of the same kind; thus the brown rat (Mus decumanus) tends to drive away the black rat ( $M$. rattus), but stress, due to over-population, is sometimes mitigated by migration, as in the case of the lemmings. The struggle seems to be keener between foes of different kinds, between carnivores and herbivores, between birds of prey and small mammals; but combination for mutual defence often mitigates the intensity of the conflict. Teeth and claws, hoofs and horns are the chief weapons, while the scales of pangolins, the bony shields of armadillos, the spines of hedgehogs and porcupines, and the thick hide of the rhinoceros may be regarded as protective armature. In keeping their foothold some mammals are helped by the harmony between their colouring and that of their surroundings; thus, the white Arctic fox and hare are inconspicuous on the snow, the striped tiger is hidden in the jungle, and many tawny animals harmonise with the sandy background of the desert.

The majority of Mammals are gregarious, witness the herds of herbivores, the cities of the prairie dogs, the packs of wolves, the schools of porpoises, the bands of monkeys. Combinations for attack and for defence are common; sentinels are posted and social conventions are respected; such migrations as those of the lemming and reindeer are characteristically social. In the beaver village and among monkeys there is combination in work, and their communal life seems prophetic of that sociality which is distinctively human.

Among Birds, mates are won by beauty of song and plumage; Mammals not less characteristically woo by force. Rival males fight with one another, and are usually larger and stronger than their mates. The antlers of male deer, the tusk of the male narwhal, the large canine teeth of boars illustrate secondary sexual characters useful as weapons. But manes and beards, bright colours and odoriferous glands are often more developed in the males than in the females, and may be of advantage in the rough mammalian court- 
ship. At the breeding season, a remarkable organic reaction often affects the animal, the timid hare becomes a fierce combatant, and love is often stronger than hunger. The courtship of Mammals is usually like a storm-violent but passing; for, after pairing, the males return to their ordinary life, and the females become maternal. Some monkeys are faithfully monogamous; and exceptional pairs, such as beavers and some antelopes, remain constant year after year; but this is not the way of the majority.

The duckmole lays eggs and brings up her young in the shelter of the burrow; the Echidna has a temporary pouch. In Marsupials the time of gestation is very short, and there is no truly placental union between the unborn young and the mother. The new-born Marsupials are very helpless, and are in most cases transferred to an external pouch or marsupium, within which they are nurtured. In Placental Mammals the gestation usually lasts much longer than in Marsupials,-its duration varying to some extent with the rank in the mammalian series, but there are great differences in the condition of the young at birth. "In those forms," Professor Flower says, "which habitually live in holes, like many Rodents, the young are always very helpless at birth; and the same is also true of many of the Carnivora, which are well able to defend their young from attack. In the great order of Ungulates or Hoofed Mammals, where in the majority of cases defence from foes depends upon fleetness of foot, or upon huge corporeal bulk, the young are born in a very highly developed condition, and are able almost at once to run by the side of the parent. This state of relative maturity at birth reaches its highest development in the Cetacea, where it is evidently associated with the peculiar conditions under which these animals pass their existence." The importance of prolonged infancy, as illustrated among monkeys, should be recognised in connection with the evolution of sympathy.

The maternal sacrifice involved in the placental union between the mother and her "foetal parasite," in the prolonged gestation, in the nourishment of the young on milk, and in the frequently brave defence of the young against attack, has been rewarded in the success of the mammalian race, and has been justified in the course of 
natural selection. But it is important to recognise that the maternal sacrifice--whatever its origin may have beenexpresses a subordination of self-preserving to species-maintaining. Thus, other-regarding as well as self-regarding activities have been factors in evolution.

History of Mammals. - As to the origin of Mammals we can only speculate. There are some remarkable resemblances between Monotremes and certain extinct Reptilian types, known as Anomodontia or Theromorpha, and these again exhibit affinities with the extinct Labyrinththodont Amphibians. Amphibians and Mammals agree in having two occipital condyles, small quadrates, large squamosals, and in certain characteristics of pectoral and pelvic girdles. Possibly the ancestral Mammals and the Anomodont Reptiles diverged from a common Amphibian stock.

The oldest known remains of Mammals are some fossils from Triassic rocks, and similar types have been found in Cretaceous and Jurassic beds; most of these Mesozoic fossils are but small pieces of small animals, and secure conclusions as to their nature are not readily reached. The earliest suggest affinities with Marsupials and Insectivora. Many of the Mesozoic mammals belong to a group which has received the name of Multituberculata, on account of the longitudinal rows of tubercles on the back teeth. It is possible that these forms, $e_{0} g$., Plagiaulax, Tritylodon, Polymastodon, should be ranked beside the Monotremes, but they are usually placed nearer Marsupials.

Other Mesozoic forms, such as Dromatherium, Triconolon, Amphitherium, Spalacotherizm, are often referred to the Marsupial series beside opossum, dasyure, and bandicoot.

The first certain remains of Placental Mammals are found in Eocene strata, and give evidence of the existence of generalised types connecting rather than referable to the modern orders. Many are characterised by the presence of three tubercles on the back teeth, and of five digits on the limbs, and by having brains relatively smaller than those of their modern successors.

Among extinct Tertiary types, we may especially notice the ground sloths (e.g:, Mesathcrium) and Glyptodonts allied to the modern Edentata, the Zeuglodonts, sometimes included among Cetaceans, numerous ancestral Ungulates, and the Creodonts allied to modern Carnivores.

\section{More detailed account of some of the structures of Mammals.}

Skin.

The skin consists of a superficial epidermis derived from the outer or ectodermic layer of the embryo, and of a subjacent mesodermic dermis or cutis.

The most characteristic modification of the mammalian epidermis is the hair. Each hair arises from the cornifica- 
tion of an ingrowing epidermic papilla, surrounded at its base by a moat-like follicle, and nourished during growth by a vascular projection of the dermis.

Each hair consists of a spongy central part and a denser cortex, but there are many diversities of form and structure, such as short fur and long tresses, the soft wool of sheep and the bristles of pigs, the spines of hedgehog, porcupine, and Echidna, the cilia of the eyelids and the tactile vibrisse of the lips and cheeks.

It is generally believed that the hairs of Mammals are homologous with the feathers of Birds and the scales of Reptiles, but Maurer maintains that the facts of development upset the homology and point rather to a resemblance between hairs and the sensory papillie of Amphibians. But this is still under discussion.

The hair keeps the animal dry and warm; in the practically hairless Cetacea the layer of fat or blubber underneath the skin also serves to sustain the temperature of the body. Like feathers, hairs die away and are cast off, being replaced by fresh growths. A few mammals, such as the Arctic fox, the mountain hare, and the ermine, become white in winter, harmonising with the snow. In the case of Ross's lemming, we know that this change is due to the influence of the cold, and depends in great part on the appearance of gas bubbles inside the hairs.

That the colouring is sometimes of protective advantage we have already noticed; but in many cases no utilitarian interpretation can be read into the stripes and markings. Those of related species often form regular series, and are superficial outcrops of constitutional changes hardly to be analysed. Sometimes there is considerable change during the lifetime of the animal, thus most young deer have spots, but only the Fallow and Axis deer retain these when adult. To an excess of pigment is due the variation known as melanism or blackness, e.g., in black wolves and rabbits; to a dearth of pigment albinism is due, as in white mice and white elephants. In tropical countries the skin is sometimes very darkly coloured, as in Indian cattle, and many monkeysespecially males-are notable for the bright colours of the bare parts of the body.

Among other tegumentary structures are the scales which occur along with hairs on the pangolins (Manis); the scales on the tails of rats and beavers and some other forms; the thickened skin-pads or callosities on the ischia of apes, the breast of camels, the legs of horses ; the nails, claws, or hoofs which ensheath the ends of the digits in all mammals except Cetaceans. Unique is the armature of the armadillos, for it consists of bony plates developed in the dermis, overlaid by epidermic scales. The median solid horns of the rhinoceros are epidermic outgrowths, comparable to exaggerated warts ; the paired horns of the Ruminants con41 
sist of epidermic sheaths covering outgrowths of the frontal bones, but extending far beyond these; the antlers of stags are outgrowths of the frontal bones, and, except in the reindeer, are cast and regrown each year, and possessed by the males only.

The skin of Mammals, unlike that of Birds, is rich in glands. Sebaceous glands are always associated with the hair-follicles, and sudorific or sweat glands are scattered over the skin.

Specialised glands are also very common, especially those which secrete some strongly odoriferous stuff, scenting which the animals recognise their fellows, their foes, or their prey. Often they are most developed in the males, and their activity increases at the pairing season.

Among the numerous special glands may be noted those which are connected with a perforated spur on the hind-legs of male Monotremes, the sub-orbital glands of antelopes and deer, the anal glands of carnivores, the perineal glands of the civet, the preputial glands of the musk-deer and beaver, the inter-digital glands of the sheep.

Most characteristic, however, are the mammary glands, functional in female Mammals after parturition. They seem to be specialisations of sebaceous glands, except in Monotremes, in which they are nearer the sudorific type. They consist of branching tubes opening by one or several apertures on the skin. From the white blood corpuscles of the abundant vascular supply, and from a degeneration of the cells lining the glandular tubes, the milk is produced. It begins to be produced when the young are born, when, in Placental Mammals, the demand upon the mother through the placenta has ceased.

In Monotremes, the simple glands, compressed by muscles, open by several pores on a bare patch of skin. This is depressed into a slight cup from which the young lick the milk. In Marsupials, the glands open by teats or mammæ, generally hidden within a marsupium; and again the action of surrounding muscles forces the milk into the mouths of the young, which do not seem to be able to suck. An anterior prolongation of the larynx to meet the posterior nares, establishes a complete air passage, and enables the young to continue breathing while they are being fed. "In the Cetacea, where the prolonged action of sucking would be incompatible with their subaqueous life, the 
ducts of the glands are dilated into large reservoirs, from which the contents are injected into the mouth of the young animal by the action of a compressor muscle." In all other Mammals the young suck the milk from the mammæ.

\section{Dentition.}

The teeth of Mammals are developed in the gum or soft tissue which covers the borders of the premaxillæ, maxillæ, and mandibles. As in other animals, they are in part of epidermic, in part of dermic origin. In the course of their development their bases are enclosed in sockets formed in the subjacent bones.

In most teeth there are three or four different kinds of tissue. The greater part consists of dentine or ivory ; outside of this there is a layer of very hard glistening enamel ; in the interior there is a cavity which in growing teeth contains a gelatinous tissue or pulp, supplied by blood vessels and by branches of the fifth nerve, and contributing to the increase of the dentine; lastly, around the narrowed bases or roots of the tooth, or between the folds of the enamel if these have been developed, there is a bone-like tissue called the crusta petrosa or cement.

The development of teeth begins with the formation of a dental ridge, an invagination of the ectodermic epithelium. From this ridge a number of "enamel germs" are next differentiated. Beneath each germ a papilla of the vascular mesodermic dermis is defined off as the "dentine germ." The crown of this papilla becomes hard, and the ossification proceeds downwards and inwards, while above the dentine crown the enamel begins to form a hard cap. Meantime the tissue around the base of the toothpapilla becomes differentiated into an enclosing follicle or sac, from the inner layer of which the cement is developed. The pulp is but the uncalcified core of the papilla.

The base of a tooth may remain unconstricted, and the core of pulp may persist. Such a tooth goes on growing, its growth usually keeping pace with the rate at which the apex is worn away with use, and it is described as "rootless" and "with persistent pulp." The incisors of Rodents and of Elephants illustrate this condition.

In the development of most teeth, however, the base is narrowed and prolonged into a root or several roots which become firmly fixed in the socket. Through a minute aperture at the end of the root, 
blood vessels and nerves still enter the pulp cavity and keep the tooth alive, but as the limit of growth is reached the residue of soft pulp tends to disappear. Of these "rooted" teeth there are many kinds, differing in size and shape, in the number of roots, and in the period at which these are definitely established. Mammals also differ not a little in regard to the period at which the teeth-usually concealed at the time of birth-appear on the surface or cut the gum.

Whereas Fishes and Reptiles have a practically unlimited succession of teeth, the succession in Mammals is practically limited to two sets, though traces of at least a third set have been seen. It was until recently the custom to distinguish between monophyodont Mammals, with only one set of teeth, and diphyodont Mammals with two sets. But more careful investigation has shown that there are no strictly monophyodont Mammals. Even the baleen whales, which have no functional teeth at all, have the rudiments of two sets. In most cases we have to distinguish a more important replacing set which is functional through the greater part of life, and a less important transient first set the members of which, often being developed during the period of sucking, are called milk teeth. The milk teeth may dwindle, as in seals, before or shortly after birth; or they may remain, as in Ungulates, for a long time, being gradually replaced by the permanent set; or they may remain as the permanent dentition, in Marsupials and Cetaceans.

Some recent investigators distinguish four generations of teeth, viz. :-

Ist, or pre-milk dentition, non-functional vestiges, e.g., Myrmecobius. 2nd, or milk dentition, generally functional for a time, permanent in Marsupials and toothed Cetaceans, usually in great part temporary. In most Mammals, except Hyrax and a few others, the first premolar is a persisting milk tooth.

3 rd, or replacing dentition, usually the permanent dentition, rudimentary in Marsupials and Cetaceans;

$4^{\text {th }}$ dentition, doubtful, in Phoca (?), in Desmodus (?), sometimes in man (?).

By a set or generation of teeth we mean those which differentiate contemporaneously, or almost contemporaneously, from the dental ridge. It used to be supposed that the replacing teeth develop from the milk set, but both are derived, as sister dentitions, from the dental ridge.

M. F. Woodward, in careful account of recent work, says that consideration of the facts "leads us to the belief (I) that the living Mammalia show traces of from three to four distinct generations of teeth, and consequently (2) that they are potentially polyphyodont; (3) that the first set is vestigial and not functional in any living mainmal; (4) that the second, which is so important in the lower mammals, is more or less replaced by the third in the higher forms; and (5) that this third dentition remains dormant in the Marsupials and Cetaceans."

Mr. Woodward points out that the evolution of the specialised heterodont dentition of the Mammalia from the simple homodont and polyphyodont dentition of the lower Reptilia "would necessarily cause a reduction in the number of successional sets of teeth, due to an 
enlargement of one set and a consequent abstraction of growth-energy and material from the underlying sets. The specialisation would not appear in the first generation of teeth, which must necessarily be of small size from its early development and consequent adaptation to the small jaw of the young animal, and which would, moreover, be required for temporary use, while the larger and more complicated dentition was developing. The increased size of the second set of teeth might well abstract the growth-energy from the succeeding sets and retard them." The diet of milk would, however, do away with the function of the first teeth, while the third gradually gained in prominence.

The following series, after Leche, is of interest-

(I) In toothed Cetaceans, the persisting dentition is wholly of the milk set (Odontoceti).

(2) In Marsupials, the persisting dentition is of the first set, except the third (or fourth) premolar.

(3) In the hedgehog, the persisting dentition is mixed, thus the incisors, canines, and premolars are partly of the milk set and partly replacing teeth.

(4) In the great majority of Mammals the persisting dentition consists of replacing teeth, excepting in most the first premolar, and also excepting (according to many) the molars.

In a few Mammals, for instance in the dolphins, the teeth are very uniform, almost all alike from beginning to end. Such a dentition is called homodont, in contrast to the common heterodont dentition, in which the teeth are more or less markedly different in form and function. It is necessary now to consider these differences.

In the typical dentition of Mammals there are forty-four permanent teeth, eleven on each side above and below. The eleven on each of the upper jaws may be divided into four sets. Most anteriorly, associated with the premaxilla, are three simple, single-rooted teeth, usually adapted for cutting or seizing. These are called incisors. Posteriorly, there are crushing or grinding teeth, whose crowns bear cusps or cones, or are variously ridged, and which have two or more roots associated with the maxilla. But of these grinders, the last three occur as one set, having no successors. They are therefore distinguished as true molars, from the four more anterior, and often simpler premolars, which occur in two sets, the milk set being replaced by a permanent set, except in most cases the first. Finally, the tooth just behind the incisors, that is to say, immediately posterior to the suture between premaxilla and maxilla, is distinguished as the canine, and is often long and sharp. 
There is still much uncertainty in regard to the nature of the molar teeth. Thus some regard them as resulting from a fusion of several dentitions, others regard them as milk teeth which are not replaced, others as belonging to the replacing set. There seems no doubt that traces of both preceding and succeeding dental structures have been seen associated with molar teeth; the question is, which set has been suppressed?

This classification of teeth is in great part one of convenience ; thus, the distinction between incisors and grinding teeth is anatomical, that between molars and premolars refers to the history of these teeth ; the connection between the teeth and the subjacent bones is a secondary matter; there is often little to differentiate canine from premolar. Moreover, the teeth of the lower jaw, which is a single bone on each side, cannot be so certainly classified as those of the upper jaw.

No part of a Vertebrate is more distinctive than the skull, and no mammalian characteristic is more useful in diagnosis than the dentition. It is convenient, therefore, to have some notation expressing the nature of the dentition. Thus we use "dental formulæ," in which the incisors, canines, premolars, and molars are enumerated in order, and in which the teeth of the upper jaw are ranked above the analogous teeth of the lower jaw. The typical mammalian dentition already referred to may be expressed as follows :-

$$
\text { Incisors } \frac{3-3}{3-3} \text {, canines } \frac{x-I}{I-x} \text {, premolars } \frac{4-4}{4-4} \text {, molars } \frac{3-3}{3-3}=\frac{I I-x I}{I I-I I}=\text { total } 44 \text {. }
$$

or using initial letters :-

$$
\text { i. } \frac{3-3}{3-3}, \text { c. } \frac{x-x}{x-1}, \operatorname{pm} \cdot \frac{4-4}{4-4}, \mathrm{~m} \cdot \frac{3-3}{3-3}=44 \text {. }
$$

or, recognising that the right and left side are almost invariably identical. and omitting the initial letters : $-\frac{3143}{3^{1} 43}$.

We may cite the formulae for the adult dentition of some representative mammals :-

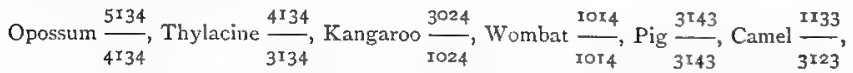

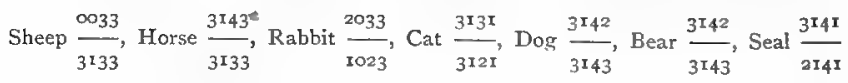

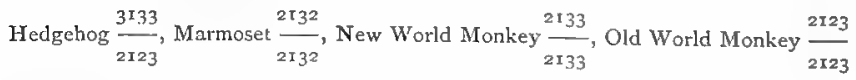
$\operatorname{Man} \frac{2123}{2123}$

It is more interesting, however, to try to associate different kinds of dentition with different kinds of diet. Thus, dolphins, which feed on 
fish and swallow them whole, have numerous almost uniform, sharp, recurved, conical teeth, well suited to take a firm grasp of the slippery and struggling booty. To a slight extent the same piscivorous dentition may be seen in seals. In the more strictly carnivorous mammals, the incisors are small; the canines are long and sharp, piercing the prey with a deathful grip, while the back teeth have more or less knife-like edges which sever flesh and bone. In typical insectivorous mammals the upper and lower incisors meet precisely, "so as readily to secure small active prey, quick to elude capture but powerless to resist when once seized," while the crowns of the molars bear many sharp points. Herbivorous mammals have front teeth suited for cropping the herbage or gnawing parts of plants, the canines are small or absent, the molars have broad grinding crowns with transverse ridges. In omnivorous mammals, the incisors are suited for cutting, the canines are often formidable weapons in the male sex, the molars have crowns raised into rounded tubercles.

It is likely that the most primitive type of mammalian tooth was a simple cone, such as may be seen in toothed whales. In some of the extinct mammals, e.g., Triconodon, the tooth is a main cone with two lateral cusps, and this type leads to what is called the tritubercular tooth, in which the crown bears three cusps disposed in a triangle. From this tritubercular type most of the more complex forms of teeth may be derived. But it remains doubtful whether the tritubercular type is the result of the fusion of three cones, or the budding of one.

\section{Development and Placentation.}

The ova of placental mammals are small; even those of the Whales are "no larger than fern seed." They are formed from germinal epithelium, the cells of which grow inwards in clustered masses into the connective tissue or stroma of the ovary. In each cluster one cell predominates over its neighbours; it becomes an ovum; the others invest and nourish it, and are called follicle cells.

In the middle of each clump or Graafian follicle, a cavity is formed containing fluid, and into this cavity the follicle cells immediately surrounding the ovum project as what is called the discus proligerus.

When mature the ovum protrudes on the surface of the ovary, and is liberated by the bursting of the Graafian follicle. Some blood, which fills up the empty follicle, degenerates into what is called the corpus luteum.

The spermatozoa are formed from germinal epithelium in the testes. The primitive male cells or spermatogonia give rise by division to daughter cells or spermatocytes, which with or without further division form spermatozoa.

The homologue of the ovum is the spermatogonium or 
mother-sperm-cell, but the physiological equivalent of the ovum is the spermatozoon.

No one has succeeded in satisfactorily observing an extrusion of polar bodies in the maturation of the mammalian ovum, but analogous processes occur at an early stage.

'The ovum having burst from the ovary is immediately caught by the fimbriated mouth of the Fallopian tube and begins to pass down the oviduct. There it is met by ascending spermatozoa, received by the female as the result of sexual union, and is fertilised. One of the spermatozoa enters the ovum, and sperm nucleus unites with ovum nucleus in an intimate and orderly manner. It is interesting to remember that it was only in 1843 that the union of spermatozoon and ovum was for the first time detected by Martin Barry, and in the case of the rabbit.

The Connection between Embryo and Mother.--(a) The lowest Mammals, the Duckmole (Ornithorhynchus) and the Porcupine Ant Eater (Echidna) resemble Birds and most Reptiles in bringing forth their young as eggs, i.e., in
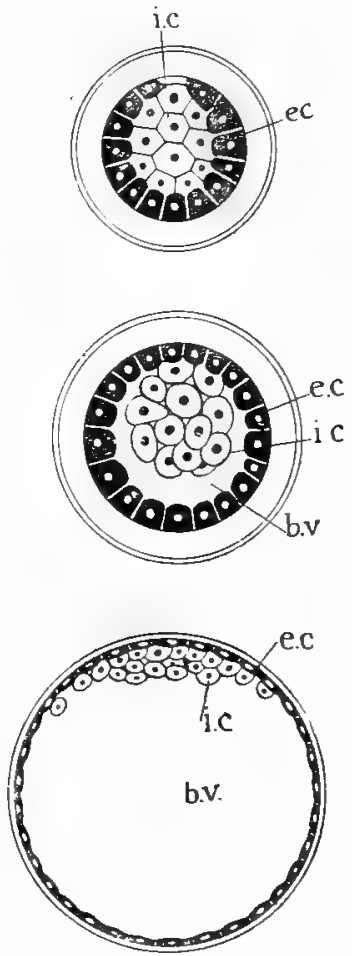

being oviparous. The eggs are large, with a considerable quantity of yolk, and after fertilisation divide partially, i.e., 
exhibit meroblastic segmentation like the eggs of Birds and Reptiles. The tunic formed round about them in the Graafian follicles of the ovary consists as in Birds and Reptiles of a single layer

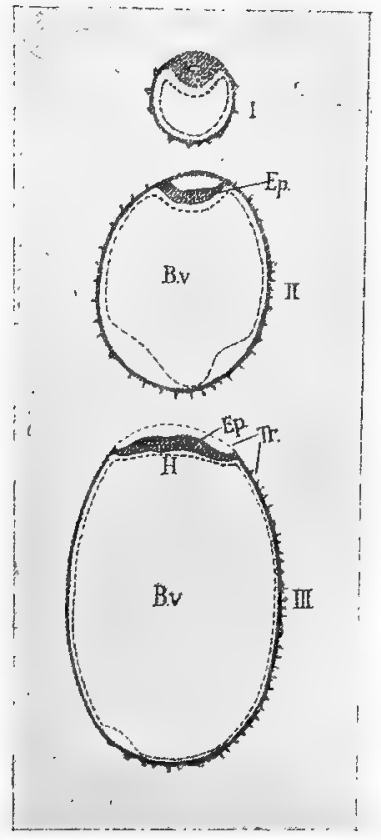

FIG. 225. - Development of Hedgehog. Three early stages. (After HuBRECHT.)

I. Shows internal vesicle of hypoblast, the disc and external sheath of epiblast. II. Shows villi arising from trophoblast; the disc of formative epiblast ( $\left.E p_{0}\right)$; the blastodermic vesicle $(B, v$.) III. A more advanced stage, $T r_{\text {. }}$, trophoblast ; $E p_{\text {. }}$, disc of formative epiblast; $B . v_{-}$, blastodernic vesicle; H., hypoblast. of cells. As they develop they are unattached to the walls of the oviducts. They are laid in a nest by the Duckmole; in the Echidna they are hatched in a slight, periodically developed, external pouch.

(b) In the Marsupials, the connection between mother and offspring has become closer. The embryo is born alive, though prematurely, after a short uterine life, during which, however, it is either not vitally attached at all to the uterus, or only to a slight degree by villi from the yolk sac. In the opossum, it lies surrounded by a quantity of nutritive albuminoid material. Here it may be recalled that in two Elasmobranch fishes and in two lizards, there is a connection between the yolk sac of the embryo and the wall of the oviduct. As we shall see, there is a preliminary yolk sac placenta in three orders of Placentals.

(c) In all the other Mainmals, the maternal sacrifice prior to birth is greater, for a close connection is established between the embryo and the wall of the uterus, by means 
of a special adaptation - the placenta. This, in rough physiological language, is a double vascular sponge, partly embryonic, partly maternal, by means of which the blood of the mother nourishes and purifies that of the embryo.

As many of the most fundamental structural and functional problems in connection with placentation are still being investigated, it is impossible to discuss even the leading questions with definiteness and certainty. The authority here followed is Hubrecht, in his study of the placentation of the hedgehog, which is at once a simple and a central type.

First, then, let us seek to define the embryonic and maternal structures which are associated with placentation. (I) At a very early stage, the divided ovum of the hedgehog consists of a sac of cells, an outer layer, epiblastic or ectodermic, enclosing another aggregate - the future inner layer, endoderm or hypoblast (Fig. 226, I.). (2) The epiblast divides into an embryonic disc which will form the epidermis, nervous system, \&c., of the embryo, and an external layer, the wall of the embryonic sac or blastocyst, with which the disc retains a slight connection until the protective amnion is formed.

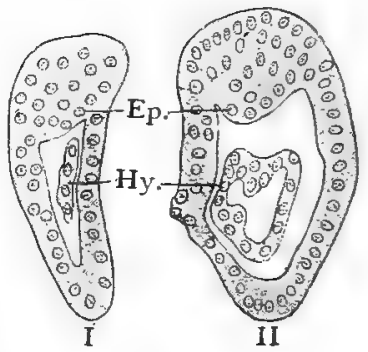

FIG. 226. - Two stages in segmented ovum of Hedgehog. (After HUBRECHT.)

Ep., Epiblast; Hy., Hypoblast.

In the outer epiblastic wall lacunæ develop, which are bathed by the maternal blood, and the pillars of tissue between the lacunæ grow out into villi, which aid in this earliest connection between mother and offspring. Long before any vascular area or foetal placenta is devel. oped, the outer epiblastic wall has the above nutritive function, and deserves its name of trophoblast (Fig. 225, Tr.). (3) The hypoblast or inner mass, which is at first a solid aggregate of cells (Fig. 224, i.c.), becomes a sac, as a morula may become a blastosphere. The upper part of this sac 
forms the lining of the incipient gut, while the lower portion, following the contour of the blastocyst wall, becomes the yolkless yolk sac or umbilical vesicle. Its connection with the upper part is narrowed into a canal-the vitelline duct, which is part of the "umbilical cord," entering the embryo at the future navel. (4) Between the epiblast and the hypoblast of the embryo, the mesoblast develops, splitting into an outer, parietal, or somatic, and an inner, visceral, or splanchnic layer. The cavity between these is the incipient body cavity. A double fold of somatic mesoblast, carrying with it a single sheet of epiblast, rises up round about the embryo, arching over it to form the amnion. Over the embryo the folds of amnion meet in a cupola, and the inner layers of the double fold unite to form the "amnion proper," while the outer layers also unite to form a layer lying internally to the epiblastic blastocyst wall,-and termed by Sir William Turner the subzonal membrane. The folds of amnion are continued, as the diagram shows, ventrally as well as dorsally, so that the subzonal membrane surrounds the embryo beneath the blastocyst wall, while a splanchnic layer of mesoblast grows round about the hypoblastic yolk sac. The space between the two layers of mesoblast, which are shortly termed somatopleure and splanchnopleure, is obviously continuous with the body cavity of the embryo. The epiblastic outer wall or trophoblast, and the mesoblastic subzonal membrane, are included in Hubrecht's termdiplotrophoblast. (5) From the hind wall of the gut there grows out a hypoblastic sac, the allantois, insinuating itself and spreading out in the space between the two layers of mesoblast. As an outgrowth of the gut, homologous with the bladder of the frog, the allantois is of course lined by hypoblast or endoderm, but it is covered externally by a layer of mesoblast, which it bears with it as it grows. In all placental mammals the allantois, which becomes richly vascular, unites with the subzonal membrane, and therefore with the external epiblast as well, to form the foetal part of the placenta, with outgrowing vascular processes or villi which fit into corresponding depressions or crypts on the wall of the uterus. [To the mesoblastic wall of the allantois, plus the subzonal membrane, the term "chorion" is sometimes applied, but as the word has been used in many different 
senses, its abandonment, except perhaps in human embryology, is almost imperative.] The complex union of allantois with diplotrophoblast, Hubrecht calls the allantoidean trophoblast. (6) But in the hedgehog, rabbit, and some other types, there is a mode of embryonic nutrition between that attained by the epiblastic trophoblast and that effected by the final placenta. The wall of the yolk sac,

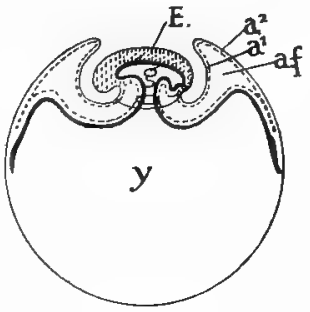
hypoblastic internally, mesoblastic externally, unites with the subzonal membrane, and becomes the seat of villous processes, which through the external epiblast are connected with the uterine wall. Thus is formed what Hubrecht calls an omphaloidean trophoblast. Neither omphaloidean nor allantoic villi ever directly interlock with maternal tissue, but always through the agency of the external epiblastic trophoblast.

(7) It is now time to turn for a little to the maternal tissue.

FIG. 227.-Development of Fœtal Membranes. (After HerTwig.)

Uppermost figure shows up-growth and down-growth of amnion folds, $E$., embryo; $a_{*} f_{\text {. }}$, amnion fold; $a_{I_{\text {* }} \text {, aminion }}$ proper ; $a_{2 .}$, subzonal membrane; $\delta$, the

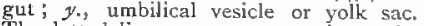
The dotted line represents mesoderm, the dark, hypoblast. The second figure shows origin of allantois, and the amnion folds have met. The third figure shows increase of allantois (al.); the dwindling yolk sac (y.s.); a.c., ammiotic cavity; s.a.m., subzonal membrane. The fourth figure shows the embryo apart from its nembranes; m., mouth; $a_{\text {., anus. Note }}$
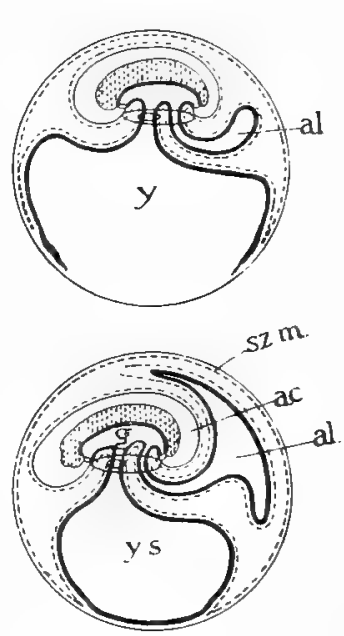
umbilical connection with yolk sac. 
The embryo lay at first in a groove of the uterine wall, moored by the preliminary blastocyst villi, which are as it were pathfinders for those subsequently developed from yolk sac and allantoic regions. At the point of attachment, the mucous lining of the uterus ceases to be glandular, and becomes much more vascular. As the embryo becomes fixed, the blastocyst almost eating its way in, the outer epithelium degenerates and disappears; below this the outer layer of the mucous membrane becomes spongy and exhibits unique blood spaces, forming what Hubrecht calls the trophospongia; below this there is the vascular and vitally active remainder of the mucosa, less modified than the

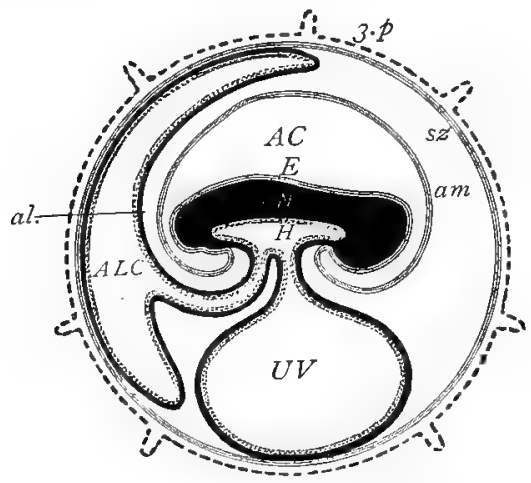

FIG. 228.-Diagram of Foetal Membranes. (After TURner.)

$E$, Embryo ; $H$, gut lined by hypoblast dotted ; the dark is mesoblast ; $U V$, umbilical vesicle or yolk sac; $A C$, amniotic cavity; am, amnion proper ; sz., sub-zonal membrane; $A L C$, allantoic cavity; al., allantois; $3 . p$., may be here taken to represent the early epiblastic trophoblast.

above mentioned sponge; below this again, there are the muscular and other elements of the uterine wall, with which we are not now concerned. The most important fact to emphasise is, that the maternal blood in the spaces of the spongy outer layer of the mucous membrane directly bathes the fotal tissue represented by the trophoblast. By the activity of the trophoblast cells, the nutritive and respiratory advantages of the maternal blood are secured for the villi of 
the allantois and yolk sac. It ought also to be mentioned that mainly by a folding of the uterine wall, the hedgehog embryo is virtually enclosed in a maternal sheath, homologous with a fold called the decidua reflexa in human embryology, and analogous with a similar capsule in the rabbit.

To sum up :-

(I) At an early stage, a wall of epiblast encloses an aggregate of hypoblast (Figs. 224, 225, I., 226).

(2) The epiblast divides into an embryonic disc and an outer blastocyst wall, with fixing and nutritive functions, - the trophoblast (Figs. 225, I. and II.).

(3) The hypoblast becomes a sac, of which the upper portion lines the gut, while the lower part forms the yolk sac (Fig. 225, III.).

(4) The mesoblast divides into somatic and splanchnic layers; a double fold of the somatic layer (along with a slight sheet of epiblast) forms the amnion, of which the outer limbs unite as the subzonal membrane, and form along with the external epiblast-the diplotrophoblast. The splanchnic layer of the mesoblast is continued round the yolk sac (Fig. 227).

(5) The allantois grows out from the hind region of the gut, being lined internally by hypoblast, externally by splanchnic mesoblast. The allantois plus the diplotrophoblast always forms the true placenta (Fig. 228).

(6) Part of the yolk-sac wall, uniting with the diplotrophoblast, also forms an efficient but temporary placenta.

(7) At the area of fixing the uterine epithelium degenerates, the glands disappear, vascularity increases. The outer part of the modified mucous membrane (or decidua) becomes a spongy tissue, with spaces filled with maternal blood. This maternal blood bathes the trophoblast, which is intermediate between it and the placental villi.

The three modes of embryonic nutrition are as follows:(a) At first the maternal blood bathes the lacunæ in the epiblastic outer wall-the trophoblast with its preliminary path-finding villi. 
(b) An efficient yolk sac placenta functions for a time, but decreases and shrivels as the final allantoidean placenta develops. The maternal blood in the spaces of the outer layer of the mucous layer of the uterus bathes the trophoblast. Thus it comes into indirect connection with the vascular villi from the region where the yolk-sac wall unites with the diplotrophoblast. This yolk sac placenta is found in Insectivora, Chiroptera, and Rodents.

(c) The final placenta is allantoidean, it replaces the yolk-sac placenta, if there be one. In Insectivora Chiroptera, and Rodentia, and probably in other cases, the trophoblast is always intermediate between the maternal blood and the villi, and is the only intervening tissue. The customary Classification of Placentation
is as follows :-

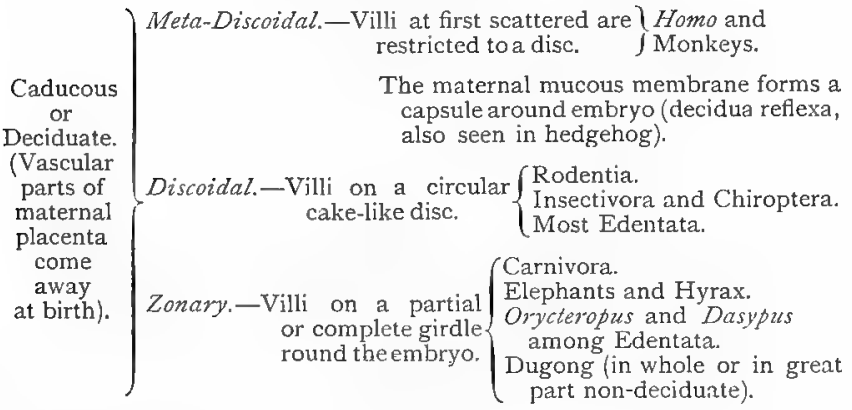

Non-Caducous Cotyledonary.-Villi in patches. Ruminants. or Indeciduate.

(Maternal part of placenta does not come away at birth). \} Diffuse.-Scattered Villi. $\left\{\begin{array}{l}\text { Lemurs. } \\ \text { Most Ungulates except } \\ \text { Ruminants. } \\ \text { Cetacea. } \\ \text { Manis among Edentata. }\end{array}\right.$ 


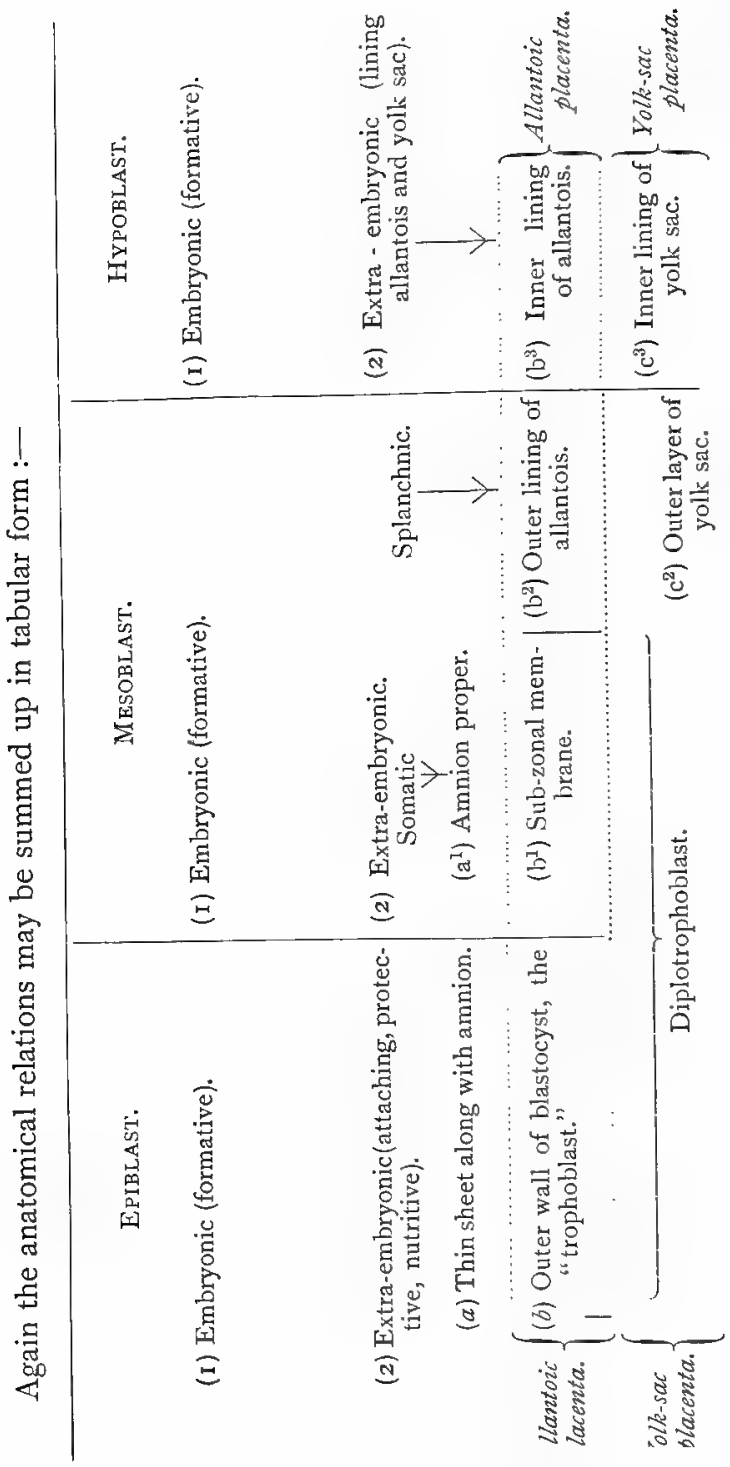


While Sir William Turner, "the grand master of placental research," in his arrangement of placentas, allots the lowest place to such diffuse forms as that of the pig, passes thence to the cotyledonary of Ruminants, thence to the zonary of Carnivores, and finally to the discoidal of monkeys and man, others maintain that the discoidal, as illustrated in the Insectivora, is the most primitive type.

To avoid confusion it may be better, as Hubrecht suggests, to revert to the old terms caducous and non-caducous, instead of deciduate and indeciduate, for all placental mammals have a "decidua," i.e., a specially active region of the

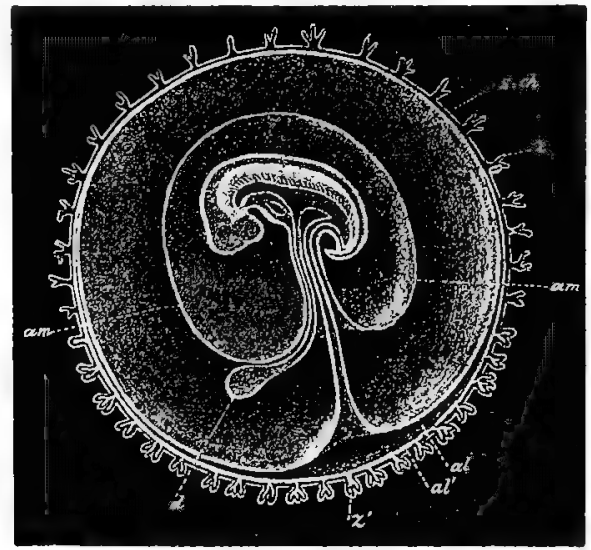

FIG. 229.-View of Embryo with its foetal membranes. (After KenNet..)

am., Amnion; $d$. , dwindled yolk sac ; al. allantois; al.1, subzonal membrane; $z$., villi.

mucous membrane of the uterus to which the embryo is attached. Moreover, the distinction between deciduate and indeciduate is one of degree, for no sharp line can be drawn between the two types.

The RABbit as a type of Mammals.

The rabbit (Lepus cuniculus) is a familiar representative of the Rodent order, to which rats and mice, voles and beavers, 42 
lemmings and marmots also belong. Like the hare (Lepus timidus) and other species of the same genus, and like the Picas or tailless hares (Lagomvs), the rabbit has two pairs of incisors in the upper jaw, while other Rodents have a single pair. Therefore the genera Eepus and Lagomys are ranked in the sub-order Duplicidentata, in contrast to all other Rodents which form the sub-order Simplicidentata.

With the rabbit's mode of life all are familiar. It is herbivorous, and often leaves softer food for the succulent bark of young trees ; it is gregarious and a burrower; it is very prolific, often breeding four to eight times in a year. It is said to live, in normal conditions, seven or eight years. The rabbit seems to have had its original home in the western Mediterranean region, but it has spread widely throughout Europe, and is now abundant in countries, such as Scotland and Ireland, in which not many years ago it was quite rare. Introduced into Australia and New Zealand it has multiplied exceedingly, and has become a scourge. There are many varieties of rabbit, some in isolated regions perhaps illustrating the effect of segregation in fostering divergent types. According to Darwin, the rabbits introduced early in the fifteenth century into Porto Santo, an island near Madeira, are now represented by a dwarf race of about half the normal size, and these are said to be incapable of breeding with the ordinary forms. But the varieties with which we are familiar in the breeds of tame rabbits, illustrate variation under domestication and the efficacy of artificial selection.

\section{External Appearance.}

The head bears long external ears which are freely movable. The black patch at the tip of the ears in the hare is either absent or very small in the wild rabbit. This external ear is characteristic of most Mammals, and collects the sound like an ear trumpet. In the rabbit it is longitudinally folded, thin and soft towards its tip, firm and cartilaginous at its base. The large eyes have eyelids with few eyelashes, and a third eyelid or nictitating membrane-a white fold of skin-lies in the anterior corner. This third eyelid, which also occurs in Reptiles and Birds, is present in most Mammals, and is of use in cleaning the cornea. It is absent in Cetaceans, where the front of the 
eye is bathed by the water, and it is rudimentary in man and monkeys where its absence is compensated for by the habitual winking of the upper eyelid. The nostrils are two slits at the end of the snout, and are connected with the mouth by a "hare lip" cleft in the middle of the upper lip. In front of the mouth are seen the chisel-edged incisors, a pair on the mandibles, and two pairs on the premaxillæ, the smaller pair hidden behind the larger pair. The first milk incisors above and below never cut the gum, but are absorbed before birth; the second milk incisors above (there are none below) are functional, but are shed about the third week of extra-uterine life; the same is true of the milk premolars. Into the toothless gap or diastema between the front and back teeth, the hairy skin of the lips projects into the mouth. On the sides of the snout, and about the eyes, there are tactile hairs or vibrissæ.

The plump trunk is separated from the head by a short neck. The tail is very short, but in the scampering wild rabbit it is conspicuous as a white tuft, which some naturalists interpret as a directive signal. Beneath the base of the tail the food canal ends, and beside the anus are the openings of the perineal glands, whose secretion has a characteristic odour. In front of the anus is the urinogenital aperture, -in the male at the end of an ensheathed penis, in the female a slit or vulva, with an anterior process or clitoris-the homologue of the penis. Beside the penis in the male lie the scrotal sacs, into which the testes descend when the rabbit becomes sexually mature. Along the ventral surface of the thorax and abdomen in the female there are four or five pairs of small teats or mammæ.

The limbs have clawed digits, five on the fore feet, four on the hind feet; they are very hairy.

\section{Skin and Muscles.}

The skin is thickly covered with hair, and has the usual sebaceous and sudorific glands, besides special glands, such as the perineal glands beside the anus, the glands of the eyelids, the lachrymal glands, and the mammary glands developed in the females. Between the skin and the subjacent muscles there is a layer of fatty tissue, known as the panniculus adiposus; it is present in all Mammals 
except the common hare, and forms the blubber of whales and seals. Beneath the skin is a thin sheet of muscle (the panniculus carnosus), and when this is removed with the skin, many of the muscles of head and neck, limbs and trunk are disclosed. [The student who wishes to study these, and to compare them with their homologues in man, will find practical directions in Parker's Zootomy.]

\section{The Skeleton.}

The bones, like those of other Vertebrates, are developed either as replacements of pre-existent cartilages, or independent of any such preformations, but in all cases through the agency of active periosteal membranes. By themselves, however, must be ranked little sesamoid bones, which are developed within tendons and near joints, notably, for instance, the patella or knee pan. There is no bony exoskeleton in any mammals except the armadillos, unless we rank the teeth, which develop in connection with the skin of the jaws, as in a sense exoskeletal. The vertebral centra of Mammals, except in Monotremes and Sirenians, have distinct terminal epiphyses, and the same distinctness of ossification is seen in many of the larger bones.

\section{Vertebral Column.}

The vertebræ may be grouped as cervical (seven in number), thoracic (with ribs), lumbar (without ribs), sacral (fused to support the pelvis), and caudal. The faces of the centra are more or less flat, and between adjacent vertebræ there are inter-vertebral discs of fibro-cartilage.

The first vertebra or atlas is ring-like, its neural canal being very large, its centrum unrepresented except by the odontoid process which fuses to the second vertebra. The ring is divided transversely by a ligament, through the upper part the spinal cord passes, into the lower the odontoid process projects. The transverse processes are very broad; the articular surfaces for the two condyles of the skull are large and deep.

The second vertebra or axis has a broad flat centrum produced in front in the odontoid process. 'The neural spine forms a prominent crest, the transverse processes are small, the anterior articular surfaces are large. 
A typical lumbar vertebra will show the centrum and its epiphyses, the neural arch and neural spine, the transverse processes, the anterior and posterior articular processes or zygapophyses, the median ventral hypapophysis, the small anapophyses from the neural arch below the posterior zygapophyses, below the anapophyses the posterior intervertebral notches-passages through which the spinal nerves pass out, and anteriorly a similar pair of notches. There are twelve or thirteen pairs of ribs which support the wall of the thorax, and aid in the mechanism of respiration. The first seven pairs articulate with the breast bone, the eighth and ninth are connected to the ribs in front, the others are free. Any one of the first seven or more typical ribs consists of two parts, a vertebral portion articulating with a vertebra, an imperfectly ossified sternal portion connecting the end of the vertebral portion with the sternum. Each of the first nine ribs has a double head - the capitulum articulating with the centrum of the corresponding vertebra, and partly with that of the one in front, the tubercle articulating with the transverse process of the corresponding vertebra. The posterior ribs have no tubercles, and the capitular articulations are restricted to the corresponding vertebre.

The sternum is a narrow jointed plate, with a large keeled præsternum or manubrium, then five segments composing the mesosternum, then a posterior xiphisternum ending in cartilage.

\section{The Skull.}

The skull consists, as in all the higher Vertebrates, of two sets of bones, - cartilage-bones preformed in the cartilage of the original gristly brain-box and its associated arches, membrane bones developing in the investing membrane and not preformed in cartilage. (The names of the membrane bones are printed in italics.)

We have already noticed the chief characteristics of the mammalian skull, such as the usual persistence of sutures, the two condyles, the bony palate, the fusion of the periotic bones, the articulation of the mandible with the squamosal, the fusion of the parts of each ramus of the mandible into a single bone in the adult, and the three ossicles of the ear. 
In studying the skull it is convenient to consider the bones in groups.

On the posterior surface of the skull, the foramen magnum, through which the spinal cord issues from the cranial cavity, is bounded by the basi-occipital beneath, the ex-occipital on the sides, the supra-occipital above. The ex-occipitals form most of the occipital condyles, but the basi-occipital contributes a small part. In many mammals the exoccipitals alone form the condyles. From each ex-occipital a par-occipital process descends and is applied to the tympanic bulla- a dilatation at the base of the tympanic bone which protects the external auditory tube.

Along the roof of the skull from behind forwards lie the supra-occipital, the parietals, the frontals, and the nasals. Between the supraoccipital and the parietals there is a small interparietal.

On the very front of the skull are the prentaxille bearing the incisor teeth. Behind each premaxilla is a maxilla, bearing the premolars and molars, behind this, along the zygomatic or temporal arch projecting

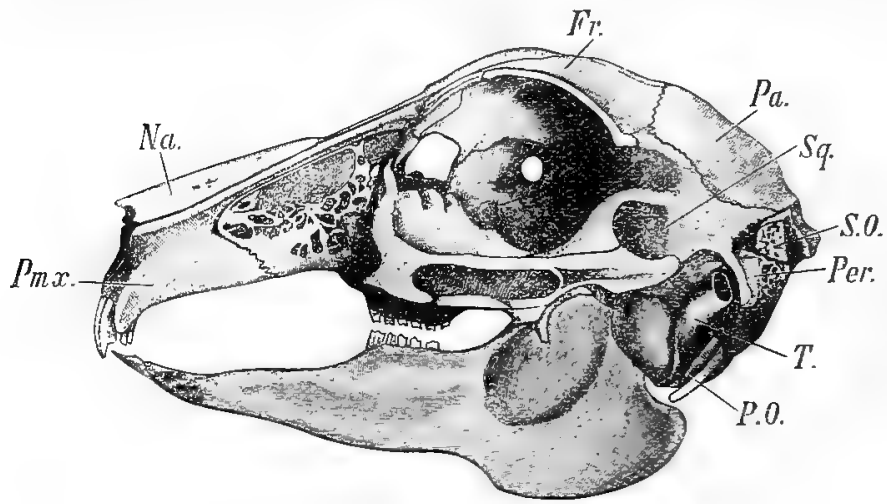

FIG. 230.-Side view of Rabbit's skull.

$P m x$., Premaxilla; $N a$., nasal; Fr., frontal; $P$ a., parietal ; $S_{q .}$, squamosal; S.O., supra-occipital ; $P_{\mathcal{E}} \%$, periotic; $T$., tympanic; $P . O$., par-occipital process.

beneath the orbit is the jugal or malar which unites posteriorly with the squamosal. This zygomatic arch bridges over the deep temporal fossa behind the orbit, and serves for the insertion of muscles, and its "squamoso-maxillary" structure occurs outside of Mammalia in the Anomodont reptiles only. The sifuamosats form a great part of the posterior side walls of the sliull, and articulate with the parietals, frontals, orbilosphenoids, and alisphenoids. At the posterior end of the zygomatic arch is the longitudinally elongated glenoid cavity in which the mandible moves backwards anel forwards.

In connection with the floor of the skull and the roof of the month, there lie from behind forwards the following components :-the median 
basi-occipital ; the median basisphenoid which lodges the pituitary body in a dorsal depression called the sella turcica; the paired alisphenoids fused to the sides of the basisphenoid; the median presphenoid which forms the lower margin of the optic foramen between the two orbits; the paired orbitosphenoids, fused to the presphenoid, sutured to the alisphenoids and squamosals, and surrounding the optic foramen; the vertical pterygoids attached at the junction of basisphenoid and alisphenoids; the partly vertical palatines, united above to the presphenoid and behind to the pterygoids and alisphenoids, separating the posterior nasal passages from the orbits, and uniting in front to form the posterior part of the bony palate; the median vertical mesethmoid cartilage extending in front of the presphenoid, separating the two nasal cavities, posteriorly

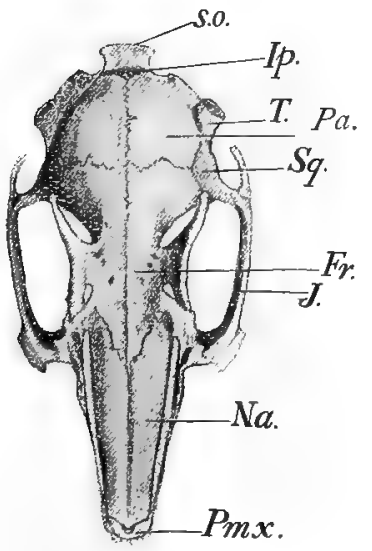

Fig. 231. - Dorsal view of Rabbit's skull.

S.O., Top of supra-occipital ; Ip.. interparietal; $T$., tympanic; $P \alpha$., parietal; $S q$., squamosal ; Fr., frontal; J. jugal ; Na., nasal; Pmux., premaxilla.

pharynx. Stretching from the tympanum to the fenestra ovalis of the inner ear is the chain of minute ear ossicles, the three links of which -malleus, incus, and stapes-possibly correspond respectively to the articular, the quadrate, and hyo-manclibular or columella of most other Vertebrates.

The orbits are bounded anteriorly by the lachrymals and the maxilla, and above by the frontals. The interorbital septum is formed above and behind by the orbitosphenoids, below by the presphenoid.

Associated with the olfactory chambers, are the nasals above, the 
vomers beneath, the mesethmoid in the median line, while internally there are several thin scroll-like turbinal bones.

The lower jaw or mandible consists in adult life of a single bone or ramus on each side, but this is formed around Meckel's cartilage from several centres of ossification. Its condyle works on the squamosal.

The hyoid lies between the rami of the mandible, in the back of the mouth, and consists of a median "body," and two pairs of horns or cornua extencling backwards.

The Appendicular Skeleton consists of the bones of the limbs and the girdles.

The pectoral girdle, which supports the fore limbs and is itself attached by muscles and ligaments to the vertebral column, virtually consists of one bone - the scapula - on each side. For in all Mammals except Monotremes, the coracoid, though a distinct ossification, forms only a small (epicoracoid) process on the anterior margin of the glenoid cavity in which the head of the humerus works. The last of the metacoracoid is seen in Monotremes. The clavicle is also much reduced in the rabbit, being only about an inch in length and very slender. It is a membrane bone, and lies in the ligament between the scapula and the sternum. The triangular

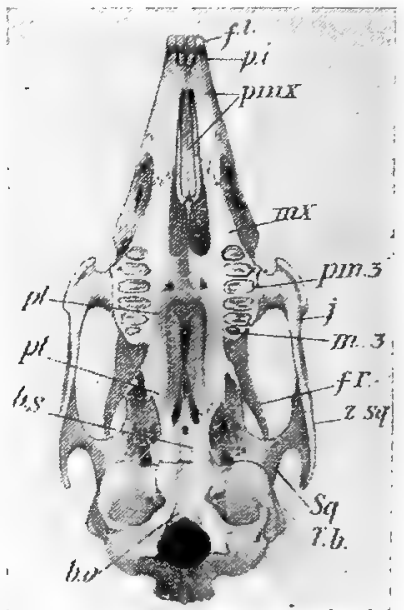

FIG, 232. - Under surface of Rabbit's skull.

$f_{i}$, , Front incisors ; $\not i_{i}$, small posterior incisors; pmx., premaxilla; $m x$, maxilla ; pm. 3 , third premolar; $m$. 3 , third molar; $j$. , jugal ; $f_{r}$, supra-orbital ridge of frontal (on dorsal surface); $z . s g .$, zygomatic process of squamosal; $S_{q}$., squamosal; $T_{.} b_{\text {. }}$, tympanic bulla ; b.o., basi-occipital; b.s., basisphenoid; $p t$. , pterygoid ; pl., palatine.

scapula has a prominent external ridge or spine, continued ventrally into an acromion with a long metacromion process. The scapula is usually strong, and the clavicle is usually present in mammals which grasp or climb or burrow. 
The fore limb consists of an upper arm or humerus, a fore arm of two bones-the radius and the ulna, a wrist or

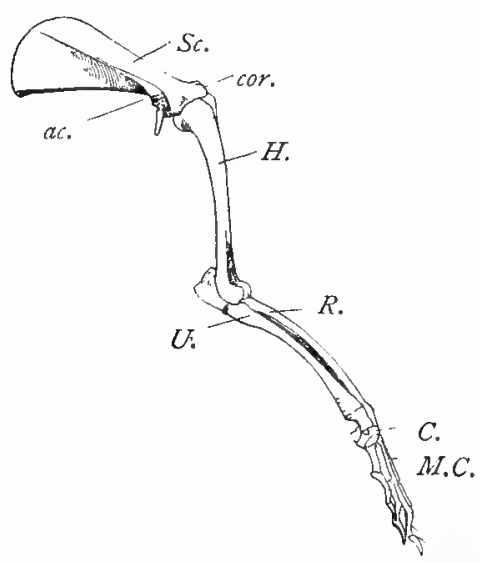
carpus, five palm bones or metacarpals, and five digits with joints or phalanges.

The head of the humerus works in the glenoid cavity formed by the scapula and the coracoid process.

When the arm of a mam. mal is directed outwards at right angles to the body, with

FIG. 233.-Rabbit's fore leg.

Sc., Scapula ; cor, coracoid process; ac., acromion; $H_{\text {. }}$, humerus ; $R$., radius ; $U_{\text {., ulna; }}$ $C$., carpal region; $M$.C., metacarpal region.

the palm vertical and the thumb uppermost, the thumb and the radius are in a preaxial position, the little finger and the ulna are in a postaxial position. But in the normal position of the limb in most mammals, the radius and the ulna cross one another in the fore arm, so that the preaxial radius is external at the upper end, internal at the lower end.

The typical mammalian wrist or carpus consists of two

rows or bones, with a central bone between the two rows. In the rabbit all the bones - nine in number - are present, viz :-

Ulnare or Cuneiform. Intermedium or Lunar. Rarliale or Scaphoid. Centrale.

Carpale 5 and 4 Unciform.
Carpale 3 Carpale 2 or or Magnum. Trapezoid.
Carpale I or Trapezium. 
In Mammals the fourth and fifth carpals are always fused; the centrale is often absent. In the tendons of the flexor muscles there are often two sesamoid bones, of which the ulnar is called the pisiform.

In the rabbit there are five metacarpal bones and five digits, each with three phalanges except the thumb or pollex which has but two.

The pelvic girdle is articulated to the backbone, and bears externally a cup-like socket or acetabulum in which the head of the thigh bone works. Each half of the girdleforming what is called the innominate bone-really consists of three bones which meet in the acetabulum. The dorsal bone or ilium, which corresponds to the scapula, articulates with the sacral vertebræ; the pubis - the anterior of the two lower bones--unites with its fellow on the opposite side in the pubic symphysis; the two ischia, which correspond to the coracoids, extend backwards, separated from the pubes by the large obturator foramen, and expand into posterior tuberosities. The ischia of mammals may touch one another ventrally, but do not fuse in a symphysis; the pubic symphysis is almost invariably present. Only in Cetacea and Sirenia is the pelvis markedly rudimentary.

The hind leg consists of a thigh or femur, a lower leg with two bones - the tibia and the fibula, an ankle or tarsus, the sole bones or metatarsals, the toes with several joints or phalanges.

The head of the femur works in the acetabulum of the pelvis. Near the head are several processes or trochanters, serving for the insertion of muscles; in the rabbit there are three--the great trochanter, the lesser trochanter, and the third trochanter.

In front of the knee there is a sesamoid bone-the knee-pan or patella -and posteriorly there are smaller fabellee.

In the lower leg, the tibia, which corresponds to the radius, is preaxial, and in the normal position interior; the fibula, which corresponds to the ulna, is postaxial, and in the normal position exterior. In the rabbit the fibula is slender, and is fused distally with the tibia.

In the mammalian tarsus there are two rows of bones, and a central bone interposed between the two rows on the inner or tibial side.
Calcanerum
or Fibulare.
Astragalus
( = Intermedium and Tibiale).
Centrale
or Navicular.
Tarsale 5 and 4
Tarsale 3 or
Tarsale 2
External
or
Middle
Cuneiform. Cuneiform.
Tarsale I or Internal Cuneiform.

In the rabbit the first tarsal and the corresponding toe or hallux are 
wanting. There are thus only four metatarsals and digits. Each digit has four phalanges.

\section{Nervous System.}

The brain has the usual five parts-cerebral hemispheres, optic thalami, optic lobes, cerebellum, and medulla oblongata, but the cerebral hemispheres cover the next two parts, and the cerebellum conceals the medulla. Of the brain membranes, the dura mater lines the cranial cavity, projecting longitudinally between the cerebral hemispheres, and transversely between the latter and the cerebellum, while the

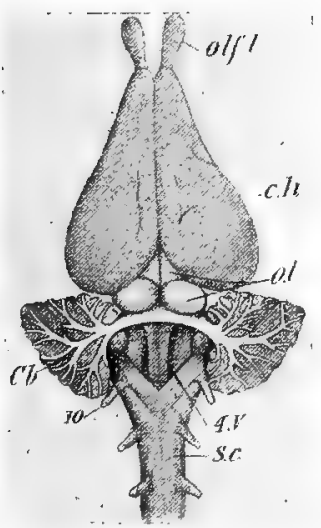

FIG. 235.-Dorsal view of Rabbit's brain, with most of cerebellum cut away. (After KRAUSE.)

olf.l., Olfactory lobes; c.h., cerebral hemispheres; o.l., optic lobes; 4.\%., fourth ventricle (exposed); s.c., spinal cord; ro, root of vagus; $C b$., lobe of cerebellum.

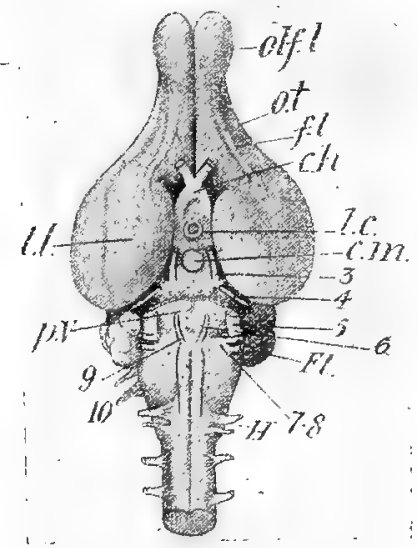

Fig. 236. - Under surface of Rabbit's brain. (After KRAUSE.)

olf.l., Olfactory lobes; $0 . t$, olfactory tract ; f.l., frontal lobe; c/e, optic chiasma; t.c., infundibulum; $c . m$. corpus mammillare; 3 , root of oculomotor ; 4, root of pathetic; 5, root of trigeninal; 6, root of abducens ; 7-8, roots of facial and auditory ; $F l$, flocculi of cerebellum ; $H$., izth or hypoglossal nerve; xo, roots of vagus; 9 , the line runs in front of the root of the glossopharyngeal to the root of $6 ; p_{1}, v_{4}$, pons Varolii ; t.l., temporal lobe.

vascular pia mater invests the brain closely. There are the usual twelve pairs of cranial nerves. The spinal cord gives off the usual spinal nerves, and there is a sympathetic system as in most other Vertebrates. 
The cerebral hemispheres of the rabbit are very slightly convoluted, and they leave the cerebellum quite uncovered. They are connected transversely by a broad bridge-the corpus callosum-and beneath this there is a longitudinal band of fibres - the fornix. The corpus callosum is readily disclosed by gently separating the hemispheres. The outer wall and floor of the anterior part of the cavity or ventricle of each hemisphere is formed by a thick mass, called the corpus striatum, and the internal cavity is lessened by a prominent convex ridge, called the hippocampus major. The ventricles of the cerebrum communicate with the third ventricle, between the optic thalami, by a small aperture, called the foramen of Munro. In front of the hemispheres two club-shaped olfactory lobes project. The thin cortical layer of the cerebrum consists of grey (ganglionic) matter, and so does the thick corpus striatum, while the central part consists of white matter (nerve fibres).

The thalamencephalon is entirely hidden, but gives origin as usual to the dorsal epiphysis, ending in a pineal body, which lies on the surface between the cerebrum and cerebellum, and to the ventral infundibulum, at the end of which the pituitary body lies, lodged in a fossa of the basisphenoid. Immediately in front of the infundibulum the optic nerves cross in a chiasma, from which optic tracts can be traced to the optic lobes. Immediately behind the infundibulum lies a rounded elevation, called the mamillary body. Anteriorly on the ventral surface of each side of the thalamencephalon there is a rounded swelling, called the corpus geniculatum. The roof of the third ventricle is formed by a thin membrane or velum, with a plexus of blood vessels. In the anterior wall of the third ventricle lies the small anterior commissure, across the third ventricle the large middle commissure runs, in the roof of the hind part of the ventricle lies a small posterior commissure.

The optic lobes are fourfold-corpora quadrigemina. They are almost quite covered by the cerebrum. Between them runs the iter connecting the third ventricle and the fourth. The floor of this passage is formed by the thick crura cerebri, which connect the medulla with the cerebrum.

The cerebellum is divided into a median and two lateral lobes, and is marked by numerous folds, mostly transverse. The two sides are connected ventrally by the pons Varolii, lying across the anterior ventral surface of the medulla.

The medulla oblongata lies beneath and behind the cerebellum, and is continued into the spinal cord. The cavity of the fourth ventricle is roofed by a thin membrane or velum, above which lies the cerebellum. On the ventral surface the medulla is marked by a deep fissure, bordered by two narrow bands or ventral pyramids.

The spinal cord presents its usual appearance, with its dorsal sensory nerve roots with ganglia, its ventral motor nerve roots apparently without ganglia, and the spinal nerves formed from the union of these. The ganglia of the adjacent sympathetic system perhaps belong to the ventral roots of the spinal nerves.

A large number of nerves pass down the neck. Of these the following are most important :--

(i) The eleventh cranial nerve or spinal accessory, leaving the skull with the ninth and tenth, and distributed to the muscles of the neck. 
(2) The twelfth cranial nerve or hypoglossal, lying at first close to the ninth, tenth, and eleventh, turning, however, to the muscles of the tongue.

(3) The tenth cranial nerve, the pneumogastric or vagus, lies outside the carotid artery, and gives off a superior laryngeal to the larynx with a depressor branch to the heart, an inferior or recurrent laryngeal which loops round the subclavian artery and runs forward to the larynx, and other nerves to the heart, lungs, and gullet.

(4) The cervical part of the sympathetic, lying alongside of the trachea, with two ganglia.

(5) The great auricular, a branch of the third spinal nerve, running to the outer ear.

(6) The phrenic nerve, a branch of the fourth cervical nerve, with a branch from the fifth and sometimes from the sixth, runs along the backbone to the diaphragm.

For details as to these nerves, the student should consult the practical manuals of Marshall and Hurst and of Parker.

As to the sense organs little need be said, for their general structure is like that of other Vertebrates, while the detailed peculiarities are beyond our present scope.

The third eyelid, present in all mammals except the Cetaceans and the Primates, is well developed. The lachrymal gland (absent in Cetacea) lies under the upper lid, and the lids are kept moist by the secretion of Harderian and Meibomian glands. The external ear or pinna is conspicuously large. The cochlea of the inner ear is large and spirally twisted. The nostrils are externally connected with the mouth by a characteristic cleft lip. The tongue bears numerous papillæ with taste bulbs. The long hairs or vibrissæe on the snout are tactile.

\section{Alimentary System.}

In connection with the cavity of the mouth we notice the characteristic dentition, the hairy pad of skin intruded in the gap between incisors and premolars, the long and natrow, in part bony, palate separating the nasal from the buccal cavity, the muscular tongue with its taste papillæ, the glottis which leads into the windpipe, and the bilobed flap or epiglottis which guards the opening, the paired apertures of the Eustachian tubes opening into the posterior nasal passage, the end of this passage above the glottis, and the beginning of the pharynx. Luess obvious are the organs of Jacobson, paired tubular bodies lying enclosed in cartilage in the front of the nasal chamber, and communicating on the one hand with the nostrils, and on the other hand with the mouth by two naso-palatine canals which open a little way behind the posterior incisors. Opening into the 
mouth and bearing the salivary juice, whose ferment alters the starchy parts of the food, are the ducts of four pairs of salivary glands. The parotid, which is largest, lies between the external ear chamber and the angle of the mandible; the infra-orbital lies below and in front of the eye; the submaxillary lies between the angles of the mandible; the sub-lingual lies along the inner side of each ramus of the mandible.

The pharynx passes into the gullet, and that leads through the diaphragm to the expanded stomach, which is dilated at its upper or cardiac end, and narrows to the curved pyloric end. Partly covering the stomach is the large liver. The first portion of the intestine, which is called the duodenum, receives the bile duct, and has the pancreas in its folds. Then follows the much coiled small intestine measuring many feet in length. The lower end of the small intestine is expanded into a sacculus rotundus. Here the large cacum - a blind diverticulum - - is given off; it ends in a fingerlike vermiform appendix. Its proximal end is continuous with the colon or first part of the large intestine, the beginning of which is much sacculated. The large intestine narrows into the long rectum in which lie little freal pellets.

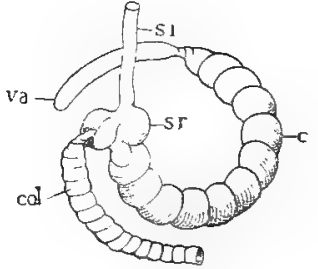

FIG. 237.-Diigram of cæcum in Rabbit.

s.z., Small intestine; s.r., sacculus rotundus; col., sacculated colon; $c_{0,}$ crecum; $z, a$, vermiform appendix.

On the last two inches of the rectum there are paired yellowish glands. Beside the anus are two perineal sacs of skin, into which open the ducts of the perineal glands, whose secretion has a characteristic and strong odour.

The liver is attached to the diaphragm by a fold of peritoneum-the glistening membrane which lines the abdominal cavity. In the liver there are five lobes. From these lobes the bile is collected by hepatic ducts into a common bile duct, which is also connected to the gall bladder by the cystic duct.

The very diffuse pancreas lies in the mesentery of the 
duodenal loop. Its secretion is gathered by several tubes into the pancreatic duct which opens into the duodenum.

The mesentery which supports the alimentary canal, is a double layer of peritoneum reflected from the dorsal abdominal wall.

The dark-red spleen (of importance in connection with the blood), lies behind the stomach. In the mesentery, not far from the top of the right kidney, lie a pair of coliac ganglia,

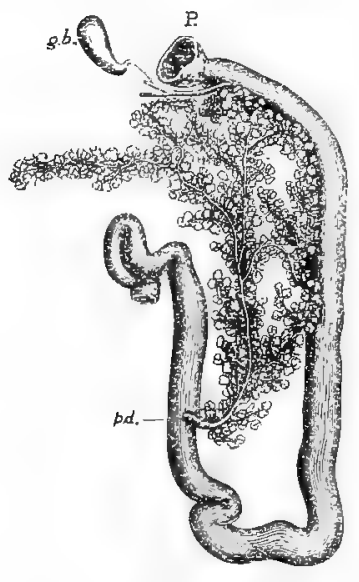

Fig. 238.-Duodenum of Rabbit. (From KRAUSE, in part after Claude Bernard.)

$P .$, Pyloric end of stomach ; g.b., gall bladder with bile duct and hepatic ducts; $p . d$. pancreatic duct. which receive nerves from the thoracic sympathetic system, and give off branches to the gut.

Vascular System.

The four-chambered heart lies in the thoracic cavity between the lungs. It is surrounded by a thin pericardium, and immediately in front of it there lies the soft thymus, which is larger in the young than in the adult animal.

By two superior venæ cavæ, and by the inferior vena cava, the venous blood collected from the body enters the right auricle. Thence the blood passes into the right ventricle through a crescentic opening, bordered by a threefold (tricuspid) membranous valve (worked by chordæ tendineæ attached to papillary muscles projecting from the wall of the ventricle).

The right ventricle is not so muscular as the left, which it partly surrounds. By its contraction the blood is driven into the pulmonary trunk, whose orifice is guarded by three semilunar valves. During contraction, the tricuspid valves are pressed together, so that no regurgitation into the right auricle can take place. 
The pulmonary trunk divides into two pulmonary arteries, which branch into capillaries on the walls of the lungs. There the red blood corpuscles gain oxygen, and the blood is freed from much of the carbonic acid gas which it has borne away from the tissues. The purified blood returns to the heart by two pulmonary veins, which unite as they enter the left auricle.

FIG. 239.-Circulatory system of the Rabbit. (In part after Professors PARKeR and KRAUSE.)

(a) Letters to right-

e.c. External carotid.

i.c. Internal carotid.

e.j. External jugular.

scl.a. Subclavian artery.

scl.v. Subclavian vein.

p.a. Pulmonary artery (cut short).

p.z. Pulmonary vein.

L.A. Left auricle.

L.V. Left ventricle.

a.ao. Dorsal aorta.

h.v. Hepatic veins.

c. Cœliac artery.

a.m. Anterior mesenteric.

s.r.b. Supra-renal body.

l.r.a. Left renal artery.

l.r. $\%$. Left renal vein.

$K$. Kidney.

p.m. Posterior mesenteric artery.

$s p m$. Spermatic artery and vein.

c.il.a. Common iliac artery.

(b) Letters to left-

$\not p . f$. and a.f. Posterior and anterior facial.

e.j. External jugular vein.

$i, j$. Internal jugular.

$R . S c l$. Right subclavian artery.

$S . V . C$. Superior vena cava.

$R \cdot A$. Right auricle.

R.V. Right Ventricle.

I.V.C. Inferior vena cava.

$r, r . a$. Right renal artery.

r.r.v. Right renal vein.

s.r.b. Supra-renal body.

$s p m$. Spermatic artery and vein.

i.l. llio-lumbar vein.

f. $z$. Femoral vein.

i.il.v. Internal iliac veins.

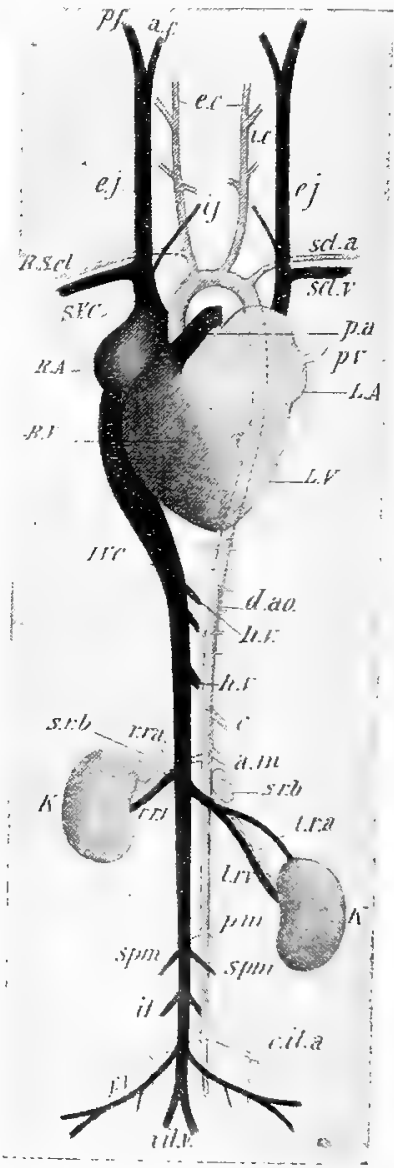

From the left auricle, the pure blood passes into the left ventricle through a funnel-like opening, bordered by a 
(mitral) valve with two membranous flaps, with chordæ tendineæ and musculi papillares as on the right side, but the muscles here are larger.

The left ventricle receives the pure blood and drives it to the body. During contraction, the mitral valve is closed, so that no blood can flow back into the auricle. The blood leaves the left ventricle by an aortic trunk, whose base is guarded by three semilunar valves, just above which coronary arteries arise from the aortic trunk and supply the heart itself.

The aortic trunk bends over to the left, and passes backward under the backbone, dividing near the pelvis into two common iliac arteries, which supply the hind legs and posterior parts. The arteries given off near the heart and in the abdominal region may be grouped as follows:-

The aortic trunk

gives off the innominate artery, which divides into $(a)$ the right subclavian, continued as the brachial to the fore limb, but giving off the vertebral to the spinal cord and brain, and the internal mam. mary to the ventral wall of the thorax :

(b) the right carotid, running along the trachea, dividing into the right internal carotid to the brain, and the right external carotid to the head and face :

(c) the left carotid, with a similar course:

thereafter the aorta gives off

the left subclavian artery, with branches like the right,

the cœeliac artery to the liver, stomach, and spleen,

the anterior mesenteric to the pancreas and intestine,

the renal arteries to the kidneys,

the spermatic or ovarian arteries to the reproductive organs, the posterior mesenteric to the rectum, the lumbar arteries to the posterior body walls.

The aorta is continued terminally in the median sacral artery to the tail, and laterally in the common iliacs which form the femorals of the hind legs, and give off in the abdomen several branches to the abdominal walls, the pelvic cavity, the bladder, and the uterus.

The Venous System.-The two superior venæe cavae bring blood from the head, neck, thorax, and fore limbs. Each is formed from the union of

a subclavian from the shoulder and fore limb, an external jugular from the face and ear, an internal jugular from the brain, 
an anterior intercostal from the spaces between the anterior ribs,

an internal mammary from the ventral wall of the thorax; and the right superior vena cava also receives an azygos cardinal vein, which runs along the mid-dorsal line and collects blood from the posterior intercostal spaces.

The inferior vena cava is a large median vein lying beside the aorta beneath the backbone. Anteriorly it is embedded in the liver, and receives the hepatic veins. Thence it passes through the diaphragm into the right auricle. Posteriorly the inferior vena cava has the following components :-

internal iliacs from the back of the thighs, forming by their union the beginning of the inferior vena cava;

femoral veins from the inner borders of the thighs, continued into external iliacs which open into the inferior vena cava;

paired ilio-lumbars from the posterior abdominal walls;

spermatic or ovarian veins from the reproductive organs;

renal veins from the kidneys.

There is no renal portal system.

The food which has been digested-rendered soluble and diffusiblepasses from the food canal into the vascular system by two paths:-

(a) All except the fatty material is absorbed by veins from the stomach and intestine. These unite in a main trunk the portal vein. The components of the portal vein are-the lieno-gastric from the stomach (and also from the spleen), the duodenal from the duodenum (and also from the pancreas), the anterior mesenteric from the intestine, the posterior mesenteric from the rectum. The portal vein breaks up into branches in the liver, whence the modified blood passes by hepatic veins into the inferior vena cava.

(b) The fat passes through the intestinal villi into the lymphatic vessels, which combine to form a thoracic duct which runs forward, and opens into the left subclavian vein at its junction with the left external jugular. Here and there lie lymphatic glands.

\section{Respiratory System.}

The lungs are pink, spongy bodies, lying in the thorax, connected to the exterior by the bronchial tubes and the trachea, and to the heart by blood vessels. The pleural membrane which invests the surface of the lungs is reflected from the sides of the thoracic cavity. When the lungs expand, the pleural cavity - between the two folds of pleural membrane-is almost obliterated. The thoracic cavity is separated from the abdominal cavity by a partly muscular diaphragm, which is supplied by two phrenic nerves, arising from the fourth cervical spinal nerves. By its contraction the diaphragm alters the size of the thoracic cavity, and 
thus shares in the mechanism of respiration. At the top of the trachea lies the complex larynx, the seat of the voice in mammals.

Anteriorly the larynx is supported on its sides and beneath by the thyroid cartilage, behind this lies the ring-like cricoid, dorsally to the cricoid are two small triangular arytenoids.

Within the larynx there are stretched membranous bands-the vocal cords. Beside the larynx is the paired thyroid gland.

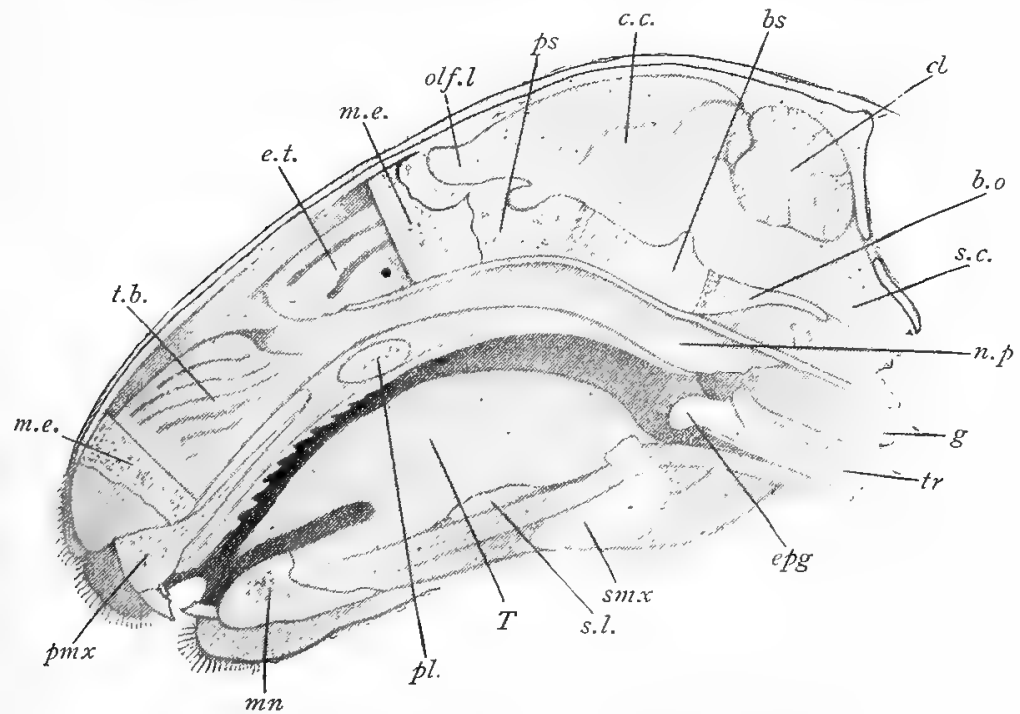

FIG. 240.-Vertical section through Rabbit's head. (From a section, with help from PARkeR's Zootomy and KRAUSE.)

pmx., Premaxilla with incisors; m.e., part of mesethnoid partition; t.b., maxillary turbinals; e.t., ethmoidal turbinal; m.e., part of mesethmoid ; olf.l., olfactory lobe of cerebrum; $p s .$, presphenoid ; c.c., position of corpus callosum ; bs., basisphenoid with depression for pituitary body; $c b$., cerebellum; $b .0$. , basi-occipital ; s.c., spinal

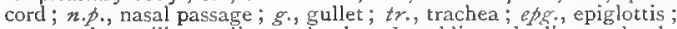
smex, sub-maxillary salivary gland; s.l., sublingual salivary gland; $\tau$., tongue; $p l$., transverse portion of palatine; $m n$., anterior end of mandible.

\section{Excretory System.}

The excretory system includes the blood filtering kidneys, their ducts the ureters, and a reservoir or bladder, into 
which these open. The kidneys and their ducts are formed from the metanephros and metanephric ducts of the embryo. The bladder arises as a diverticulum from the hind end of the gut, being in fact a remnant of the intra-embryonic part of the allantois. It loses its connection with the gut, and the ureters which originally opened into the rectum follow the bladder and open into it.

The kidneys are dark-red ovoid bodies lying on the dorsal wall of the abdomen; the one on the left is further down than that on the right, because of the position of the stomach on the left side. When a kidney is dissected, a marked difference is seen between the superficial cortical part and the deeper medullary substance. On papillæ or pyramids in the very centre, the coiled excretory tubules open, and empty the water and waste products into the "pelvis" or mouth of the ureter.

The ureters run backward along the dorsal wall of the abdomen, and open into the bladder, a thin-walled sac lying in front of the pelvic girdle.

In front of each kidney lies a yellow suprarenal body of doubtful physiological significance.

\section{Reproductive Organs.}

(a) Male.-The testes arise on the dorsal abdominal wall near the kidney, but as the rabbit becomes sexually mature, they are loosened from their original attachment, and pass out on the ventral surface, as if by a normal rupture, into the scrotal sacs. A spermatic cord, consisting of an artery, a vein, and a little connective tissue, runs from the abdomen to the testis.

The testis is attached to the base of the scrotal sac, and is bordered by a mass of convoluted tubes--the epididymis -consisting of the caput epididymis anteriorly, the larger cauda epididymis posteriorly, and a narrow band between them. The cauda epididymis is connected to the scrotal sac by a short cord or gubernaculum.

Through the tubes of the epididymis (the modified mesonephros) the spermatozoa developed in the testis are collected into the vas deferens (the modified Volffian duct), which arises from the cauda epididymis, ascends to the abdomen, extends round to the dorsal surface of the neck of 
the bladder, and there opens beside its fellow into a median sac called the uterus masculinus. In many Mammals paired diverticula, known as seminal vesicles, are connected with

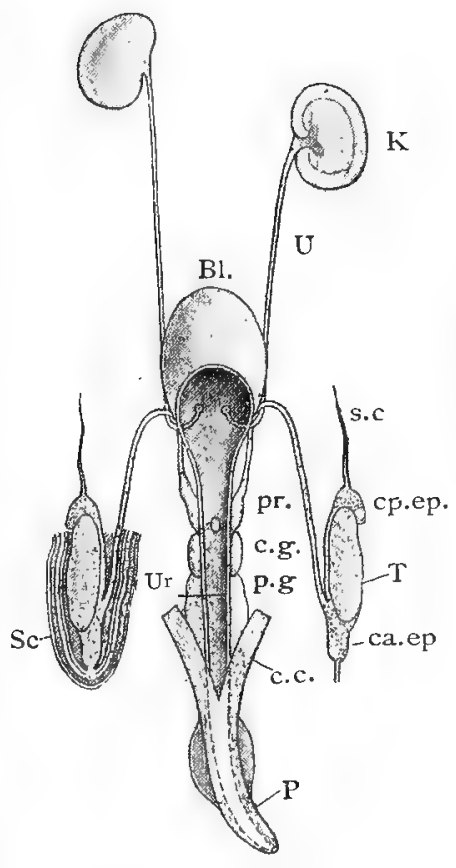

FIG. 24I,-Urinogenital organs of Male Rabbit.

$K$., Kidney ; $U .$, ureter ; $B l$., bladder: $T$., testis; $S_{.} C_{\text {. }}$, spermatic cord ; cp.ep., caput epididymis ; ca.ep., cauda epididymis; Sc., scrotal sac; pr., prostates; c.g., Cowper's glands; $p . g$. , perineal glands; Ur., urethra; c.c., corpus cavernosum; $P$., penis.

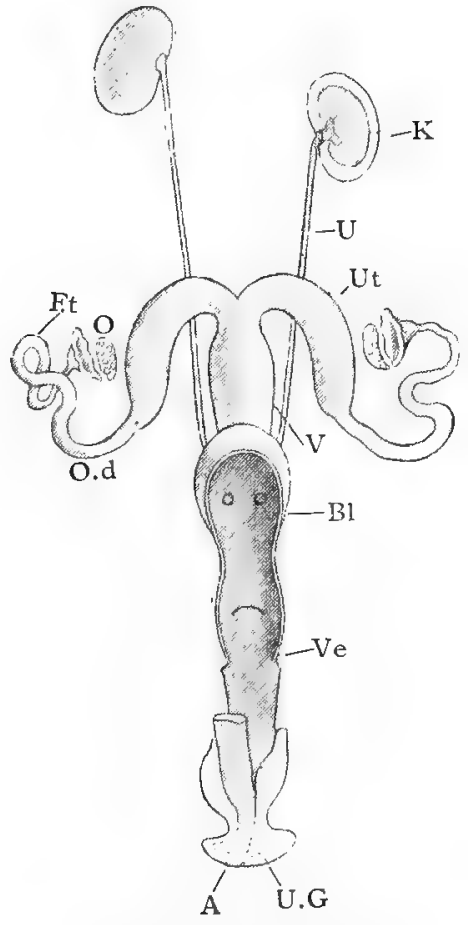

FIG. 242.-Urinogenital organs of Female Rabbit.

$K .$, Kidney; $U$., ureter ; $O$, ovary ; $F . t .$, Fallopian tube; $O . d$. , oviduct: $U t$., uterus ; $V$., vagina; $B l$., bladder; $V E_{\text {., }}$ vestibule; $U . G_{\text {, }}$, trinogenilal aperture; $A .$, anus. Bladder and vestibule are cut open.

the ends of the vasa deferentia, but they are not developed in the rabbit. 
The uterus masculinus is the homologue of the vagina in the female, and seems to arise from the Müllerian ducts. It opens into the urethra, which runs backwards from the bladder, and the urinogenital canal thus formed is continued through the penis.

Beside the uterus masculinus and the vasa deferentia, there are lobed prostate glands opening by several ducts into the urinogenital canal. Behind the prostate, on the dorsal wall of the urinogenital canal, lie two Cowper's glands.

'The penis projects in front of the anus behind the pubic symphysis, has vascular dorsal walls (corpus spongiosum), stiff ventral walls (corpora cavernosa), and is invested by a loose sheath of skin-the prepuce. At the side of the penis lie two perineal glands.

(b) Female.-The ovaries are small oval bodies about three quarters of an inch in length, attached behind the kidneys to the dorsal abdominal wall, exhibiting on their surface several clear projections or Graafian follicles, each of which encloses an ovum.

The ova, when mature, burst from the ovaries, and are caught by the adjacent anterior openings of the oviducts. The oviducts are modified Müllerian ducts, differentiated into three regions. The anterior portion or Fallopian tube is narrow, slightly convoluted, with a funnel-shaped, fimbriated mouth lying close to the ovary. The median portion or uterus is the region in which the fertilised ova become attached and develop. In the rabbit, the uterine regions of the two oviducts are distinct, forming what is called a double uterus. In most cases the uterine regions of the two oviducts coalesce, forming a bicornuate or a single uterus, according to the completeness of the fusion. In all mammals above Marsupials, the posterior parts of the two oviducts unite in a median tube-the vagina.

The vagina unites with the neck of the bladder, and forms the wide but short urinogenital canal or restibule, which opens at the vulva ventral to the anus. On the ventral wall of the vestibule lies the clitoris, a small rod-like body-the homologue of the penis. On the dorsal wall lie two small Cowper's glands, and there are also perineal glands as in the male. 
The development of the fertilised ovum is in most respects like that of the hedgehog (pp. 648-56). In the guinea-pig and some other Rodents, but not in the rabbit, there is a remarkable inversion of the germinal layers.

There is in the rabbit, as in all Rodents, a provisional yolk sac placenta. The allantoic placenta is discoidal and deciduate.

Systematic Survey of the Orders of Mammalia.

I. Sub-Class-Prototheria or Ornithodelphia-Order Monotremata.

II. $"$-Metatheria or Didelphia-Order Marsupialia.

III.,$\quad$ Eutheria or Monodelphia or PlacenTALIA.

Orders of Eutheria.

I. Edentata.

2. Sirenia.

3. Ungulata.

Prtiodactyla $\}$ Ungulata Vera.

Hyracoidea.

Proboscidea.

Extinct sub-orders.

4. Cetacea.

Mystacoceti-baleen cetaceans.

Archroceti-(extinct types).

Odontoceti-toothed cetaceans.

5. Rodentia.

Simplicidentata.

Dupliciclentata.

6. Carnivora.

Carnivora Vera.

Pinnipedia.

Creodonta (extinct).

7. Insectivora.

Insectivora Vera.

Dermoptera.

8. Chiroptera.

Megachiroptera.

Microchiroptera.

$\left.\begin{array}{l}\text { 9. Lemuroidea. } \\ \text { r. Anthropoidea. }\end{array}\right\}=$ Primates. 


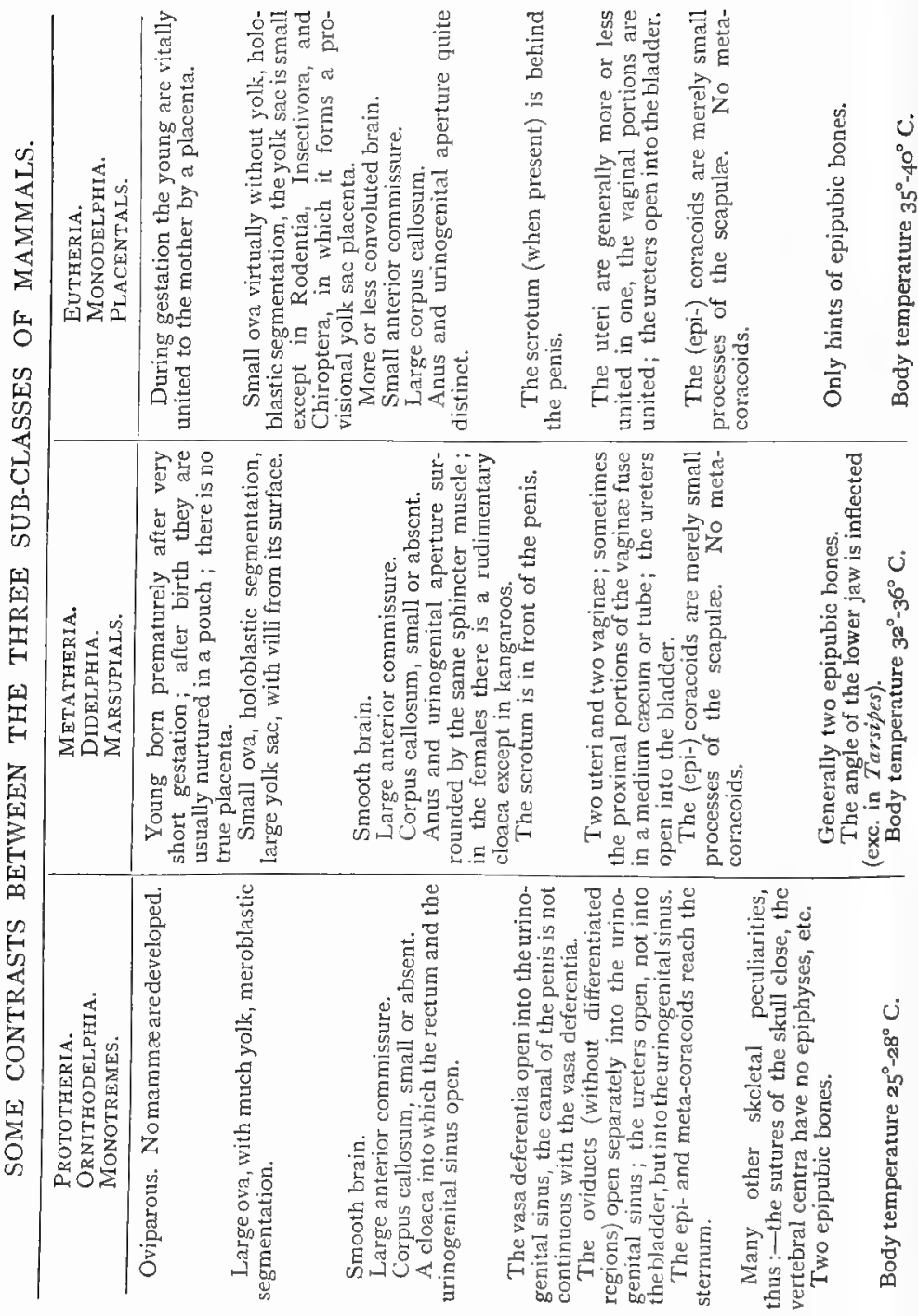




\section{Sub-class Prototheria (Syn. Ornithodelphia),}

Order Monotremata.

This sub-class includes the duckmole (Ornithorhynchus anatinus), the spiny ant eater (Eckidna aculeata), and a third form resembling Echidna, but often referred to a distinct genus as Proechidna. These are the lowest Mammals, and exhibit affinities with Sauropsida, and perhaps even with Amphibia. It need hardly be said that they have no special affinities with Birds.

\section{General Characters of Prototheria.}

The duckmole is found in the rivers of Australia and Tasmania; Echidna, in Australia, Tasmania, and New

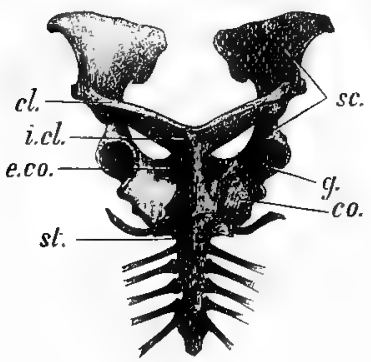

FIG. 243.-Pectoral girdle of Echidna. (From Edinburgh Museum of Science and Art.)

Sc., Scapula; cl., clavicle; i.cl., interclavicle ; co., metacoracoid ; e.co., epicoracoid ; st., sternum.

Guinea; Proechidna in New Guinea.

In Ornithorhynchus, the skin is covered with soft fur; in Echidna and Proechidna, there are spines among the hairs. The mammary glands in the female Ornithorhynchus open on a flat patch; in Echidna, in a depressed area around which a temporary pouch seems to be developed. There are no distinct mammæ.

The vertebral centra bear no epiphyses. The skull is smooth and polished as in Birds, for the sutures disappear. The rami of the lower jaw do not unite in front, and have no ascending process. In Ornithorhynchus, there are true mammalian teeth, but only in the young; in Echidna, none are present. Cervical ribs remain distinct for a time at least; the odontoid process of the second vertebra is long and not fused to the centrum. The (meta-) coracoids reach the præsternum, there are also large epicoracoids and a $T$-shaped interclavicle, the whole girdle resembling that of Lizards. An interclavicle is, however, recognisable in he 
embryos of some Placentals also. In Ornithorhynchus, the ischia form a long ventral symphysis; in Echidna, the acetabulum socket for the femur is incompletely ossified as in Birds; the pubes bear epipubic bones, as in Marsupials. On the side of the tarsus, in the duckmole, there is a spur perforated by the duct of a gland. This spur persists in the males, is rudimentary in the females. The male Echidna has a similar but smaller spur.

The brain is smooth, the cerebellum is not covered by the cerebrum, there is a large anterior commissure and the corpus callosum is rudimentary, or, according to Symington, absent.

The food canal ends in a cloaca.

The right auriculo-ventricular valve in Ornithorhynchus is partly muscular as in Birds, while in other Mammals it is membranous and is worked by papillary muscles attached to it by tendon-like cords (chordæ tendineæ). The temperature of the body is said to be about $25-28^{\circ} \mathrm{C}$.

The ureters open, not into the bladder, but into the urinogenital canal.

The testes remain in the abdomen. The left ovary is larger than the right, as in Birds. The vasa deferentia open separately into the urinogenital canal. So in

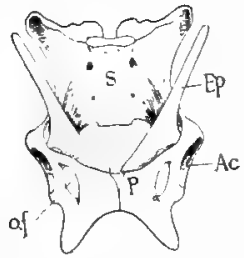

FIg. 244,-Pelvis of Echidna. (From Edinburgh Museum of Science and Art.)

$S$, sacrum; $E p$, epipubic bones; 1c, acetabulum : o. $f_{\text {, }}$ obturator foramen berween ischium and pubis (p.). the female do the oviducts, and these hare no fringed fimbriated apertures nor distinct uterine region. The penis is attached to the ventral wall of the cloaca, and its canal is not continuous with the urinogenital canal.

The ova are large, with abundant yolk, and undergo meroblastic segmentation. The Prototheria are oviparous.

The duckmole, duck-lilled platypus, or water mole, lives beside lakes and rivers. It swims by means of its fore limbs, which are webbed as well as clawed; it grubs for aguatic insects, crustaceans, and worms in the mud at the lrottom of the water. It collects small animals in its cheek pouches, and chews them at leisure with its eight horny jaw 
plates. It makes long burrows in the banks, often with two openings, one above, one under the water. The animal is shy, and dives swiftly when alarmed. When about to sleep, it rolls itself into a luall. In the recesses of the burrows the eggs are laid, two at a time. The egg measures about three quarters of an inch in length, and is enclosed in a "strong, flexible, white shell," through which the young animal has to break its way.

The full grown duckmole measures from eighteen to twenty inches in length; the male slightly exceeds his mate. The fur is short and soft, dark brown above, lighter beneath. The jaws are flattened like the bill of a duck, and covered with naked skin, which forms a soft, sensitive, collar around the region where the bill joins the skull. The eyes are very small ; there is no external ear flap or pinna; the nostrils lie near the end of the upper part of the bill. The tail is short and flat.

Horny plates, two on each jaw above and below, serve as teeth in the adult. True teeth, three on each jaw above and below, are calcified, but

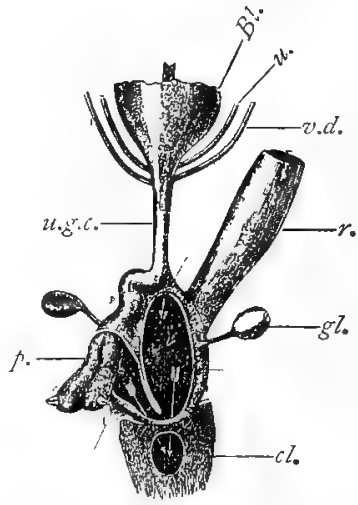

FIG. 245. -Urinogenital organs of Male Duckmole. (After OWEN.)

$B l .$, Bladder; $u$, ureter; $u$.d., vas deferens; $r_{\text {., }}$ rectum ; $g l$. , gland ; $c l$, cloaca; p., penis; u.g.c., urinogenital canal.

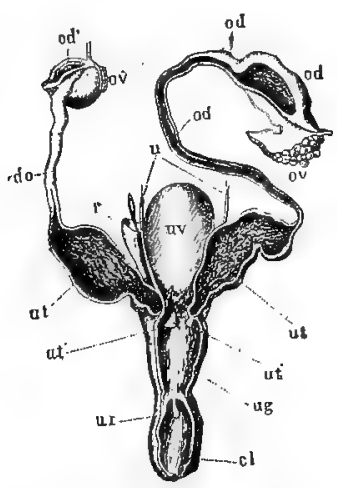

FIG. 246.-Urinogenital organs of Female Duckmole. (After OWEN.)

Ov, ovary; od, oviduct; $u t$, "uterine" region; $w$, ureter; $r$, rectum; $u\langle 2$, bladder; $u g$, urinogenital sinus; $c l$, cloaca.

soon worn away and shed. The digits are clawed and connected by a web which is best developed on the fore limbs. The spur borne on the heel seems to be sometimes used as a weapon, and as it persists only in the males, is perhaps useful in contests between rivals.

Echidna and Proechidna live in rocky regions, are mainly nocturnal in habit, and burrow rapidly, legs foremost. They feed on ants, which are caught on the rapidly mobile, slender, viscid tongue. No traces of teeth have as yet been seen. 
Strong spines occur thickly in Eilzidna, more sparsely in Proechidna among the hairs. The snout is prolonged into a slender tube. The limbs bear five toes, two of which in Proechidna are often without claws and somewhat rudimentary. In Echidna, the eggs seem to be hatched in a temporarily developed pouch.

Sub-class Metatheria or Didelphia,-Order Marsupialia.

With the exception of the $\mathrm{N}$. American opossums, all the Marsupials now alive are natives of Australasia. But fossil remains found in Europe and America show that they once had a wide range. As there are no higher mammals indisputably indigenous to Australasia, it seems as if the insulation of that region had occurred after the Marsupials had gained possession, but before higher mammalian competitors had arrived. Thus saved and insulated, the

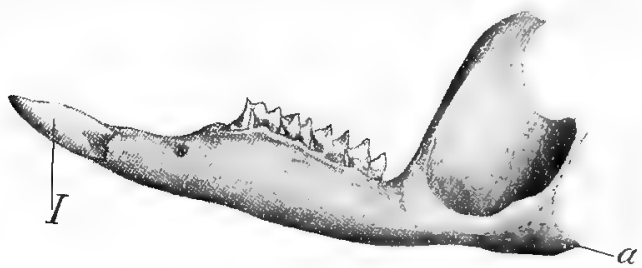

FIG. 247.-Lower jaw of Kangaroo.

$a .$, Inflected angle; $I$, single incisor.

Marsupials have developed in many different directions, the families included in this order being very diverse.

\section{General Characters of Marsupials.}

The brain is less developed than in placental Mammals, for the convolutions are simple or absent, the anterior commissure is large, the corpus callosum is small or even absent. In the skeleton there are several peculiarities; thus the angle of the lower jaw is more or less inflected, except in the genus Tarsipes; there are generally two epipubic or "marsupial" bones in front of the pubic symphysis; there are more incisors above than below (except in the wombat), and the number of incisors sometimes exceeds three on each side. 
The functional teeth are those of the milk set, with the probable exception of the third (or rather fourth) premolar. It may be, however, that this successional premolar is a retarded milk tooth, intermediate in position between pm. 2 and pm. 3. In living Marsupials, there seems to be a suppression of what, in typical placentals, would be called the second premolar.

A common sphincter muscle surrounds the anus and the urinogenital aperture, and in the females (except kangaroos) the anus lies so much within the urinogenital sinus that the arrangement may be described as cloacal. 'The scrotal sac containing the testes lies in front of the penis. The genital ducts of the females are often separate throughout, so that there are two uteri and two vaginæ. But the bent proximal parts of the vaginæ sometimes fuse and form a cæcum, which, according to the degree of fusion, may be a single tube or divided by a partition. Moreover, in Bennett's kangaroo, the cæcum opens independently into the cloaca between the apertures of the distal portions of the vaginæ.

The allantois may be small, and may fail to reach the subzonal membrane. In no case is there an allantoic placenta. The yolk-sac is large and adheres to a portion of the subzonal membrane. From this region in some cases, e.g., opossums, non-vascular villi are given off, which enter into close connection with the glands of the uterine wall. The embryo is also attached to the uterus by amœboid processes from the subzonal membrane.

The gestation is short, only lasting a fortnight in the opossum, about five weeks in the kangaroo, whereas, that of the mare, for instance, is about eleven months. Except in some opossums, there is a marsupial pouch, usually with a forward directed aperture. Within this pouch are the teats, and here the delicate young are nurtured after birth. As they are unable to suck, the milk is forced down their throat, the mammary gland being compressed by the cremaster muscle which covers it. Vague vestiges of a marsupium are said to be visible in some Placentals.

Families of Marsupials.

A. Polyprotodontia. "Incisors numerous, small, subequal Canines larger than the incisors. Molars with sharp cusps." 
Family, Didelphyida:-American opossums, distributed from the United States to Patagonia, arboreal in habit, usually carnivorous or insectivorous in diet. The limbs have five digits with claws, the hallux is opposable. The tail is generally long and often prehensile. The stomach is simple, the cæcum small. The pouch is generally absent, but the young are often carried on the back of the mother, their tails coiled round hers. Dentition, 5134

4.34

Examples:-The Virginian or crab-eating opossum (Didelphys marsupialis), with a pouch; the woolly opossum ( $D$. lanigera); the aquatic Yapock (Chironectes) which feeds on fish and smaller water animals.

Family Dasyuridxe:-Carnivorous or insectivorous Marsupials. The limbs have clawed digits, five in front, four or five behind. The canines are generally large. The stomach is simple, there is no cacum.

Examples:--The Tasmanian wolf (Thylacimzis), of dog-like form, dentition, $\frac{4134}{3^{1} 34}$, and the Dasyure (Dasyurus), civet-like, dentition, $\frac{4124}{3124}$, are specialised as carnivores. The members of the genus Phascogale are small and insectivorous. The banded ant eater (Myrnecobius) of W. and S. Australia, a somewhat squirrel-like animal, has a long thread-like protrusible tongue and more teeth than any other Marsupial $\frac{4{ }^{1} 35 \text { or } 6}{3 \pi 35 \text { or } 6}$.

Family Peramelidx:-The burrowing bandicoots, all small in size, insectivorous or omnivorous in diet. In the fore feet, two or three of the middle toes are well developed and clawed, the others being rudimentary; in the hind feet, the hallux is small or absent, the second and third toes are very slender and united in the same fold of skin, the fourth toe is very large, the fifth smaller, - the whole foot suggesting that of the kangaroo. The stomach is simple, the crecum not large. Clavicles are absent. Dentition, $\frac{4 \text { or } 5 \text { I } 34}{3 \text { I } 34}$.

Examples:- The true bandicoot (Perameles); the native rabbit (Peragale lagotis); the rat-like Charopus.

B. Diprotodontia. "Incisors usually $\frac{3}{1}$; the first large and cutting; the upper canines generally, the lower canines always small or absent ; the molars with bluntly tuberculated or transversely ridged crowns."

Family Phascolomyida:-The Wombats, terrestrial, vegetarian, nocturnal Marsupials, somewhat bear-like in appearance. The dentition is rodent-like, $\frac{\text { ror } 4}{\text { Ior }}$, the teeth have persistent pulps, the incisors are chisel-cdged, there heing no enamel except in front. 
In the embryo, however, there are four upper incisors, of which the first persists, and five lower incisors, of which the third persists. The fore feet have five distinct toes with strong nails : the hind feet have a small nailless hallux, the second, third, and fourth toes partly united by skin, the fifth distinct. The tail is very short. The stomach is simple, the crecum very short.

There is but one genus-Phascolonns, with three species.

Family Phalangeride :--Small woolly arboreal nocturnal Marsupials, with vegetarian or mixed diet. The fore feet have five distinct toes; the hind feet have a large, nailless, opposable hallux, the second and third toes are narrow and bound together by skin, the fourth and fifth free. The tail is generally long and prehensile. The stomach is simple, the cæcum usually large. Average dental formula, $\frac{3, \mathrm{x}, 2-3,3-4}{\mathrm{I}, \mathrm{O}, \mathrm{0-2}, 3-4}$.

Examples :-The grey Cuscus (Phalanger orientalis) ; Tarsipes, a small mouse-like animal which feeds on honey, and is remarkable in having no inflection of the angle of the mandible and no crecum; the flying phalangers (Petaumzs), with a parachute of skin extending from the little finger to the ankle; the Koala or "native bear" (Phascolarctos cinerezes), a relatively large form about two feet in length. An extinct form, Thylacoleo, of the late Tertiary period of Australia, is interesting in its extraordinary dentition, the functional teeth being reduced to large front incisors and third premolars, both adapted for sharp cutting.

Family Macropodidæe:-Kangaroos, herbivorous terrestrial Marsupials. Dentition, $\frac{3,0-1,2,4}{\mathrm{x}, 0,2,4}$. The incisors are sharp and suited for cropping herbage. The hind legs are usually larger than the fore legs, and the animals move by leaps.

Examples:-The true Kangaroos, e.g., Macropus; the rat-kangaroos or potoroos (Potorous); the genus Hypsiprymzodon, with a foot approaching that of the Phalangers.

The true Kangaroos, belonging to the genus Macropus, include the largest living Marsupials, but within the genus there is much difference in size.

The grey Kangaroo ( $M$. gigantezes) lives on the grassy plains of Eastern Australia and Tasmania, and is as tall as a man; the Wallabies, at home in the bush, are smaller, and some are no bigger than rabbits.

The hind limbs seem disproportionately long, and are well suited for rapid bounding. The long tail, carried horizontally, helps to balance the stooping body as the animal leaps, and it gives additional stability to the erect pose. The fore limbs sometimes come to the ground when the animal is feeding, and in the largest species they are strong enough to throttle a man.

The fore limbs bear five clawed digits, the hind feet have only four. The hallux is absent; the fourth toe is very long; the fifth is about half as large ; the third and second are too slender to be useful for more than scratching, and are bound together by the skin (syndactylous). The 
length of the hind limb is due to the tibia and fibula, and to the foot. The clavicles and fore arm are well developed. The epipubic or marsupial bones are large.

The Kangaroos feed on herbage, and are often hunted down on account of the damage which they do to pastures and crops. The sharp incisors are suited for cropping the grass and herbs, which the ridged and tuberculated molars crush.

As the Kangaroos are exclusively herbivorous, it is not surprising to find that the stomach is large and complex, with numerous saccules on its walls. The whole gut is long, and there is a well-developed cacum.

Among extinct Marsupials there were some gigantic forms, notably Diprotodon australis, as large as a Rhinoceros. It is likely that many of the early Mesozoic mammals were Marsupials. The discovery of extinct Thylacine-like marsupials in the lower Tertiaries of Patagonia suggests an ancient land connection between Australia and the southern extremity of America. No other fossil Marsupials of Australian type are known in other parts of the world.

A remarkable new type of Marsupial (Notoryctes typhlops) has been recently discovered by Dr. E. C. Stirling. Four or five cervical vertebræe are fused, there is a keeled sternum, and a bird-like pelvis. The eyes are rudimentary and beneath the skin. The marsupial bones are small nodules. There is a functional pouch. The animal is a rapid burrower. In

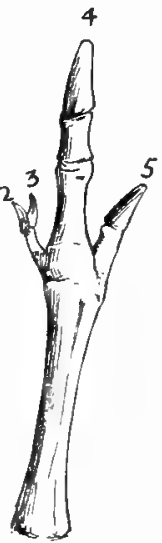

FIG. 248.-Foot of young Kangaroo.

2-3, Small syndactylous toes, 4 , large fourth toe; 5 , fifth toe. its mole-like characters it is a good illustration of "convergence," i.e., the appearance of similar structures in forms not nearly related, appar. ently in response to similar conditions of life.

Most palæontologists recognise, besides Marsupials and Monotremes, another order of non-placental Mammals, - the Multi-tuberculata, wholly extinct.

\section{Sub-class Eutheria-Order I. Edentata.}

This order includes five very distinct families with living representatives-the sloths, the ant eaters, the armadillos, the pangolins, and the aard-varks. The first three families are found in the New World, the last two in the Old World.

Functional teeth are alssent in several, but the ant eaters (Myrmecophagidæe) are the only forms which still appear strictly edentulous. When present the teeth are uniform, usually simple, without roots, and with persistent pulp. They are never developed on the fore 
part of the mouth, and they have not more than hints of enamel. Till recently the dentition was described as monophyodont, but we now know that there is evidence of two sets in Tatusia, Orycteropus, Dasypus, and others. It is the milk set which disappears.

The modern Edentata are specialised survivors of a waning order, whose extinct representatives seem to have been larger and more primitive. The modern forms usually have protective peculiarities of structure and habit which secure their persistence. Thus some are arboreal, others are burrowers, and many are covered with strong armature of bone or of horn. It is interesting to observe how very varied the nature of the placenta is:-

a dome-shaped disc (deciduate) in the sloths, dome-shaped or discoidal (deciduate) in the ant eaters,

discoidal (deciduate) in the armadillos, diffuse (non-deciduate) in the pangolins, zonary (deciduate) in the aard-varks.

\section{Families of Edentata.}

I. Bradypodidre-Sloths. - The three-toed sloths (Bradypus) and the two-toed sloths (Cholocpus) are restricted to the forests of South and Central America. They are the most arboreal of mammals, passing their whole life among the branches, to which they hang, and along which they move back downwards. They are solitary, nocturnal, vegetarian animals, sluggish, as their name suggests, and with a very firm grip of life. Their shaggy hides harmonise with the mosses and lichens on the branches, and the protective resemblance is increased by the presence of a green alga on the hair. Their food consists of leaves and shoots and fruits.

The body is covered with coarse shaggy hair ; the head is rounded, and bears very small external ears; the fore limbs are longer than the hind limbs, and the two or three digits are bound together by skin, and have long claws; the tail is rudimentary.

Concerning the skeleton we may note the $\frac{5}{4}$ rootless, unenamelled teeth, the incomplete zygomatic arch with a descending process from the jugal, the presence of clavicles, the rod-like appearance of the embryonic stapes, the occurrence of nine cervical vertebræ in Bradypus, of six in Cholopus. The adult Bradypus has a distinct coracoid or epicoracoid.

As in most herbivorous animals, the stomach is complex, but there is no cacum. In the limbs the main blood vessels break up into numerous parallel branches. The uterus is simple, the vagina seems to be origin. 
ally divided by a median partition, the placenta is a deciduate domeshaped disc. One young one is born at a time.

2. Megatheriide or Ground Sloths_extinct forms of large size, intermediate between the sloths and the ant eaters. Their remains are found in Pleistocene deposits in $\mathrm{N}$. and S. America. Megatherium exceeded the Rhinoceros in size.

3. Myrmecophagidx-the Ant Eaters, hairy animals, without even traces of teeth, with long threatl-like protrusible tongues, viscid with the secretion of greatly enlarged submaxillary glands. One form, Myrmecophaga jubata, is terrestrial, the others, belonging to the genera Tamandua and Cycloturus, are arboreal. All feed on insects. All are Neotropical. The skull is long; the third finger is greatly developed, the others are small; the pes has four or five almost equal clawed toes; the clavicles are rudimentary ; the tail is long and sometimes prehensile. The brain is well convoluted. The uterus is simple. The placenta is dome-like or discoidal.

4. Dasypodidee-the Armadillos, all S. American except Tatusia novencincta, which extends as far north as Texas. They are nocturnal, omnivorous animals, able to run and burrow rapidly. They are unique among living mammals in having a dermal armature of bony scutes united into shields and rings, and covered by horny epidermis. The teeth are numerous, simple, and of persistent growth. Clavicles are well-developed. The digits have strong claws or nails. The brain has large olfactory lobes; the cerebral hemispheres have few convolutions. The tongue is long and protrusible, and the submaxillary glands are large. The stomach is simple. The uterus is simple. The placenta is discoidal and deciduate.

Examples:-Dasypus, Chlanydophorus, Tatusia.

5. Glyptodontidx-extinct Pleistocene types, mostly S. American, but represented in Mexico and Texas. The body was often huge, and was covered by a solid carapace of great strength, "Why such a form as the Glyptodon should have failed to keep his ground is a great mystery; nature seems to have built him, as Rome was built, for eternity." (W. K. Parker.)

6. Manidae-the Ethiopian and Oriental Pangolins, covered dorsally with overlapping horny scales. They are terrestrial, burrowing animals, but sometimes climb trees. They usually feed on termites. Teeth are rudimentary, the tongue is long and protrusible. The uterus is bicornuate, the placenta diffuse and non-deciduate. There is one extant genus Manis.

7. Orycteropidie-the Ethiopian Aard-rarks, represented by two species of Oryctiropus, ranging from S. Africa to Egypt. They are shy, nocturnal animals, living in burrows, feeding on termites. There are numerous complex teeth. The skin bears scanty bristles. The mouth is tubular, and the tongue is narrow and protrusible. The cligits bear nails suited for digging. The uterus is bicornuate, the placenta broadly
zonary. 


\section{Order 2. Sirenia--Sea Cows.}

A small moribund order of sluggish, aquatic, vegetarian Mammals, in no direct way connected with Cetaceans, possibly related to Ungulates, but certainly primitive. There are two living genera, Halicore (I)ugong), and Manatus (Manatee), and one recently exterminated (Rhytina).

The Sirenia are sluggish animals, with massive heavy bones, a plump body, some oil, and sparse hair. They are aquatic, with fish-like form, no trace of hind limbs, flipper-like fore limbs, no external ear, valved nostrils, networks in the arteries (useful in prolonged immersion). They are herbivorous, and like others of similar habit have a chambered stomach, a long intestine, and a cæcum.

They are primitive, and with this fact may be associated the abdominal testes, the absence of distinct epiphyses on the vertebræ (as in Prototheria), and the small, rather smooth brain.

The body is fish-like, the head rounded, the skin is thick and tough, with sparse bristles, mostly about the mouth.

The paddle-shaped fore limbs have, at most, rudimentary nails; there are no hind limbs. The skull is not like that of Cetaceans. The nasals are, at most, rudimentary. There are no canine teeth. There are chevron bones below the tail. There are no clavicles. The pelvis is rudimentary, and there is no sacrum.

The brain is small and has few convolutions. The nostrils are valved, and lie at the end of the snout. The small eyes have imperfect eyelids, but have a nictitating membrane. There are no external ears. In the mouth there are horny crushing plates. The stomach is chambered, and there is a cæcum. The ventricles are separated by a cleft. There are retia mirabilia on the arteries. The testes are abdominal in position. The uterus is bicornuate. Two teats lie behind the arm pits. The placenta of the dugong is zonary, wholly or in great part non-deciduate. The placenta of the manatee has not yet been investigated. The food consists of Algæ and estuarine plants. 
Neck vertebrxe reduced to six. Abortive incisors ( $\left.\frac{2}{2}\right)$ in both sexes.

Molars (11) six or so at a time, uniform, with square enamelled crowns, and tuberculated transverse grinding ridges.

Premaxillæ almost straight.

Tail rounded.

Rudimentary nails on fingers.

Caecun divided.

M. australis and $M$. senegalensis live in the mouths of great rivers which flow into the tropical Atlantic.
The usual seven neck vertebra.

Two tusk-like incisors persist in the the male.

Molars (品 or $\frac{n}{6}, 2$ or 3 at a time), primitive, with persistent pulps and no enamel.

Premaxillæ crooked downwards.

Deeply notched tail.

Nailless digits.

Thick and single cæcum.

H. tabernaculi, E. African coast and Red Sea; $H$.dugong, Indian and Pacific Oceans, eastward from the home of the last species tothe Philippines; H.australis, E. and N. Australia.

The genus Rhytina was toothless, with a slightly crooked snout, small head and arms, and thick naked skin. Steller's Sea Cow (R. stelleri), the only known species, from the North Pacific, seems to have been exterminated in the last century.

The order was once much larger. Fossil forms occur in Tertiary strata. The most important is Halitherium, a less specialised Sirenian than those still extant, e.g., with traces of hind limbs.

\section{Order 3. Ungulata.}

Hoofed Animals-Artiodactyla, Perissodactyla, Hyracoidea, Proboscidea, and extinct sub-orders.

This large and somewhat heterogeneous order includes pigs, hippopotamus, camels, cattle, deer, tapirs, rhinoceros, horses, hyrax, elephants, and some other distinct types.

They are terrestrial, and for the most part herbivorous animals. Their digits generally end in hoofs or at least in broad nails. In the adults of the modern types there are no clavicles. The teeth are diverse, the milk set in part persistent until the animal attains maturity.

\section{Ungulata Vera:-Artiodactyla and Perissodactyla.}

In these typical Ungulates, the feet are never plantigrade. In modern types there are never more than four functional toes. The os magnum of the carpus articulates freely with the scaphoid. The brain is well convoluted. The testes descend into a scrotum. The uterus is bicornuate. The placenta is non-deciduate, and either diffuse or cotyledonary. 
Artiodactrla-Pigs, Camels, Chevrotains, and Ruminants.

The third and fourth digits of each foot are equally developed, and the line halving the foot runs between them.

The premolars and molars are usually different.

There are nineteen dorso-lumbar vertebra.

The femur has no third trochanter.

The astragalus has always equal articular facets for the navicular and for the cuboid. The calcaneum has an articular facet for the fibula.

The stomach tends to be complex, and the crecum is small.

The mamma are few and inguinal, or numerous and abdominal.

The placenta is diffuse or cotyledonary.

\section{Perissodactyla-Tapiks,}

Rhinoceros, Horses.

The third digit occupies the middle of the foot, is largest, and is symmetrical on itself, so that the line halving the foot bisects the third digit.

The premolars resemble the molars.

There are almost always twenty-three dorso-lumbar vertebræ.

The femur has a third trochanter.

The astragalus has a large facet for the navicular, a small facet for the cuboid. The calcaneum does not articulate with the lower end of the fibula.

The stomach is always simple, and the crecum is large.

The mamma are always inguinal.

The placenta is always diffuse.

\section{Sub-Order Artiodactyla-Even-toed Ungulates.}

Pigs and Hippopotamus (Suina), Camels (Tylopoda), Chevrotains (Tragulina), and Ruminants (Pecora) like Cattle and Deer.

The general characters of this sub-order have been stated above in contrast to those of Perissodactyla. The equal development of the third and fourth digits, the fact that the premolars have a single lobe while the molars have two, the nature of the tarsal bones, the tendency that the stomach has to be complex (as in Camels and Ruminants) are important characteristics. There are others of less obvious importance, such as the absence of the alisphenoid canal, which in Perissodactyla encloses the external carotid artery as it passes along the alisphenoid.

There are primitive extinct Artiodactyla which connect the four modern groups-Suina, Tylopoda, Tragulina, and Pecora. Thus they unite the bunodont types, such as pigs, with cone-like tubercles on the crowns of the molars, and the selenodont types, such as cattle, with the tubercles expanded from before backwards and curved in crescents.

Group I. Suina-Hippopotanus, Pigs, and Peccaries. The molars are bunodont; the third and fourth metacarpals and metatarsals are not completely fused as "cannon bones."

Hippopotamicle: - Huge African mammals, incluter in the single genus Hippopotamus. They spend the day in the rivers and lakes, swimming and diving well, but usually remaining con- 
cealed. At night they come on land and browse on grass and herbage. The skin is extremely thick, with a few hairs restricted to the snout, head, neck, and tail. There are four toes on each foot, all reaching the ground. The rootless incisors continue growing; so do the large curved canines; the dental formula is $\frac{2-3,143}{1-3,143}$ The stomach has three chambers; there is no cæecum.

Suidae:-The Old World boars and pigs, characterised by the mobile snout and terminal nostrils. There are four well-developed digits on the narrow feet, but the second and fifth do not reach the

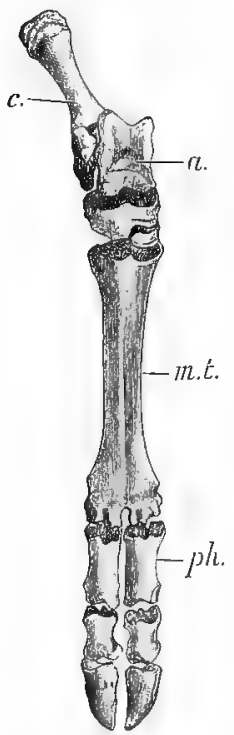

FiG. 249. - Foot of Ox.

a., Astragalus; c., os calcis; n.t., cannon bone (fused third and fourth metatarsals); $\not h$., phalanges.

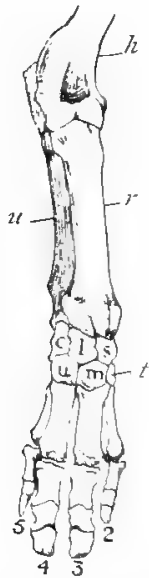

FIG. 250.-Fore leg of Pig.

$h$., Humerus; $r .$, radius; $t u$., ulna ; s., scaphoid ; $l$., lunar ; $c$., cuneiform ; t., trapezoid; $1 t_{n}$, os magnum; z.., unciform; $2-5$, digits.

ground in walking. The incisors are rooted, the upper canine curves outwards or upwards. The stomach is almost simple, but has more or less of a carliac pouch; there is a cacum.

Examples:-Sus, $\frac{3^{1}+3}{3^{\mathrm{I}} 43}$; Babinsa $\frac{2123}{3623}$, the male with remarkable canines, the upper pair growing upwards from their base 
through the skin, arching backwarls as far as the forehead, and sometimes forwards and downwards again, the lower pair with a more or less parallel course; Phacocharus, the wart hog.

Dicotylidx:-The New World Peccaries (Dicotyles), with a snout like that of pigs, with four toes on the fore feet, and three behind. The incisors are rooted, the upper canines are directed downwards, the dental formula is $\frac{2133}{3+33}$. The stomach is complex, and there is a cæcum.

Group 2.-Tylopoda, comprising the family Camelida-the Camels of the Old World and the Llamas of S. America. The limbs are long, with only the third and fourth digits developed; the two metacarpals and metatarsals are united for the greater part of their length, but there is a deep distal cleft ; the tips of the digits

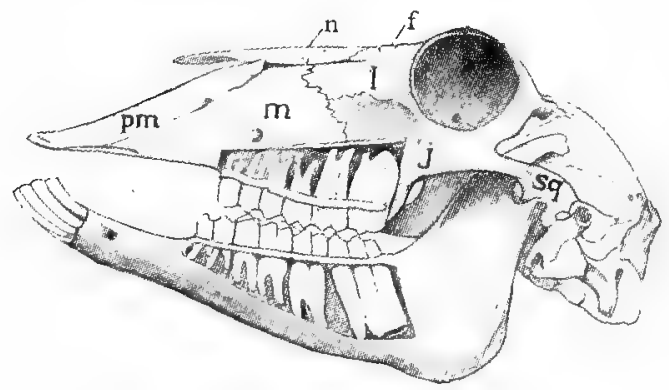

FiG. 25I.-Side view of Sheep's skull, with roots of back teeth exposed. (From Edinburgh Museum of Science and Art.)

$f$, Frontal ; $n$, nasal ; $p m$, premaxilla ; $m$, maxilla ; $j$, jugal ; $s q$, squamosal; $l$, lachrymal.

have very incomplete hoofs, and the animals walk on a broad pad of skin surrounding the middle phalanges. The femur is long and vertical, and the knee is low down. Of the three upper incisors only one persists in adult life, as an isolated sharp tooth, those of the lower jaw are long and slope forwards. There are canines both above and below. The molars are selenodont. The animals are ruminant in habit, and the stomach is divided into three chambers, of which the first two have on their walls remarkable pouches, which can be filled with fluid, and closed by sphincter muscles. The Camelidxe are unique among Mammals in having oval instead of circular red blood corpuscles. The placenta is diffuse. 
Examples:-Camelus, $\frac{\mathrm{Ir}_{33}}{3 \mathrm{r} 23}$, the Arabian camel (C. dromedarius) has a dorsal hump of fat, the Bactrian camel (C. bactrianus) has two humps. The genus Auchenia $\frac{1123}{3 \times 23}$, includes the llama, alpaca, huanaco, and vicugna of S. America, smaller forms than the camels, and without humps.

Group 3.- Tragulina, comprising the family Tragulidx or Chevrotains. These are small animals, "intermediate in their structure between the Deer, the Camels, and the Pigs." There are four complete toes on each foot, but the second and fifth are slender; the third and fourth metacarpals and metatarsals are fused in Tragulus, free in the other genus Dorcatherium; the fibula is complete. There are no upper incisors, the upper canines are long and pointed especially in the males, the lower canines are like incisors, the dental formula is $\frac{{ }^{2} 33}{3 \mathrm{~T} 33}$. The Chevrotains ruminate, and the stomach is divided into three chambers. The placenta

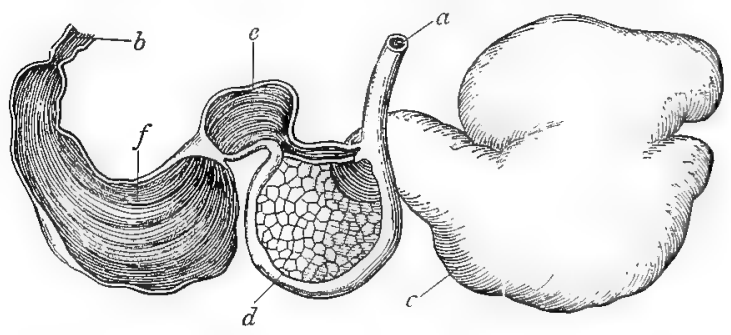

FIG. 252.-Stomach of Sheep. (From Leunis.)

$a$, Esophagus ; $c$, rumen or paunch ; $d$, reticulum or honeycomb bag; $\varepsilon$, psalterium or many-plies ; $f$, abomasum or reed ; $b$, beginning of duodenum.

is diffuse. The Chevrotains are often confusedly associated with the musk deer (Moschus) with which they have no special affinities. Species of Tragulus (smallest among living Ungulates) occur in IndoMalaya, India, and Ceylon; one species of Dorcatherium, of aquatic piglike habits, is found on the west coast of Africa.

Group 4.-Pecora or Cotylophora - the truc Ruminants, including deer, giraffes, cattle, and sheep. Only the third and fourth digits are complete, the fused third and fourth metacarpals and metatarsals form "cannon bones." In the embryos of ox and sheep, the second and fifth metacarpals and metatarsals are also represented; the second metacarpal and fifth metatarsal are unstable and soon disappear; small traces of the fifth metacarpal and second metatarsal persist. Paired outgrowths of the frontal bones are common, capped with horny sheaths in the Bovidre, deciduous 
and restricted to the males in almost all Cervidæ. There are no upper incisors, and rarely upper canines; there are three pairs of lower incisors which bite against the hardened gum above, and the lower canine resembles and is in the same series as the incisors; the typical dentition is $\frac{0^{0} 33}{3 \mathbf{1} 33}$. The stomach has four distinct compartments, a psalterium or many-plies in addition to the three which are present in Camels and Chevrotains. The placenta is cotyledonary, the villi occurring on a number of distinct patches.

The process of rumination or chewing the cud cannot be understood without considering the complex stomach. It is divided into four chambers, the paunch or rumen, the honeycomb bag or reticulum, the many-plies or psalterium, the reed or abo-

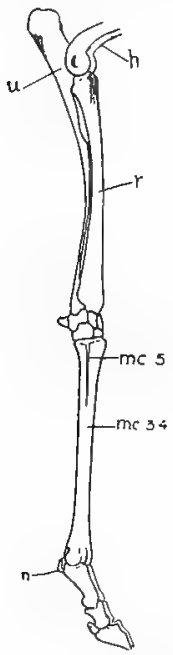

FIG. 253. - Side view of Calf's fore leg.

$h .$, Distal end of humerus; u., olecranon process of ulna ;

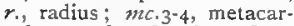
pals 3 and 4 fused to form cannon bone; $m c .5$, fifth metcarpal; n., nodule. masum. The swallowed food passes into the capacious paunch, the walls of which are beset with close set villi resembling velvet pile. After the food has been softened in the paunch, it is regurgitated into the mouth where it is chewed over again and mixed with more saliva. Swallowed a second time the food passes not into the paunch, but along a muscular groove on the upper wall of the globular honeycomb bag into the third chamber or many-plies. The honeycomb bag owes its name to the hexagonal pattern formed by the mucous memlurane on its walls. The many-plies or psalterium is a filter, its lining membrane being raised into numerous leaf-like folds covered with papillæ. Along these the food passes to the reed, which secretes the gastric juice.

Cervidæ-the widely distributed deer, absent only from the Ethiopian and Australian regions. The second and fifth digits are usually represented, often along with the distal parts of the corresponding metacarpals and metatarsals. The upper canines are usually present in both sexes. The horns, if present, are antlers, confined to the males and deciduous, except in the reindeer, where they are possessed by both sexes and are permanent. They are outgrowths of the frontal bones, are covered during growth by vascular skin-the velvet-and attain each year to a certain limit of growth. After the breeding season the blood supply ceases, the velvet dies off, and an 
annular absorption occurs near the base. Then the antlers are shed, leaving a stump, from which a fresh but larger growth takes place in the next year. The earliest (Lower Miocene) deer had no antlers, thus resembling young stags of the first year; the Middle Miocene deer had simple antlers with not more than two branches, thus resembling two-year-old stags. Thus there is a parallelism between the history of the race and the individual development.

Examples:-Cervus, most Old World deer; Rangifer, the reindeer; Alces, the elk or moose; Capreohus, the roe deer; Hydropotes, the water deer, without antlers; Moschus, the musk deer, without antlers, with long sharp upper canines in the males, with large musk glands.

Giraffidæ, represented solely by the giraffe (Giraffa camelopardalis), a tall Ethiopian animal, notable for its enormously elongated cervical vertebre, and for its long limbs. It is gregarious in its habits, and feeds on the leaves of trees. The lateral digits are entirely absent. The dental formula is $\frac{0033}{3^{\mathrm{T}} 33^{\circ}}$. On both sexes there are on the forehead short erect prominences, over the union of parietals and frontals, which arise from two distinct centres of ossification, but afterwards fuse with the skull. In front of these there is median protuberance.

Antilocapridæe, represented solely by the prongbuck (Antilocapra americana), a North American animal, with most of the characteristics of Buvidae, but with deciduous and branched horns.

Bovidæ, the hollow-horned Ruminants, widely distributed throughout the world, but without indigenous representatives in Australia, South or Central America. The second and fifth digits may be completely absent, but are often represented by minute hoofs and supporting nodules of bone. The frontal appendages, if present, consist of a solid bony core growing from the frontal, and a much longer sheath of horn, which grows at the base as it is worn away at the tip. They are not deciduous, and are usually present in both sexes, though larger in the males.

Examples:-Antilope, Gazella, Capra, Ovis, Bos.

\section{Sub-Order Perissodactyla.}

Horses, Tapirs, Rhinoceros, and their extinct Allies.

The middle or third digit of fore and hind feet is larger than the others, and symmetrical on itself. It may be the only complete digit, as in the horse, or it may be accompanied by a second and a fourth, and in the fore foot of Tapirs and some extinct forms, by a fifth digit. No modern forms have any trace of a first digit. The astragalus has a pulley-like surface above for articulation with the tibia; its distal surface is flattened and unites to a much 
greater extent with the navicular than with the cuboid. The last-named bone is of less importance than in the

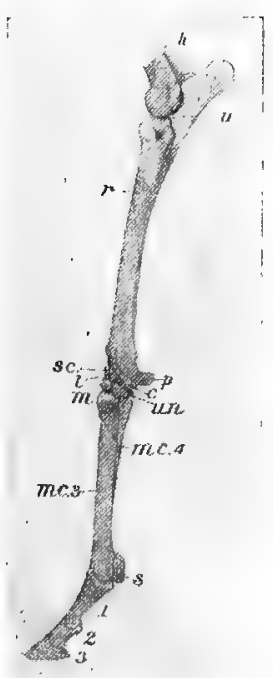

FIG, 254,-Side view of lower part of Pony's fore leg. (From Edinburgh Museum of Science and Art.)

h. Distal end of humerus; $u$, olecranon process of ulna; $r$, radius; sc, scaphoid; $\ell$, lunar; $c$, cuneiform; th, os magnum; $u n$, unciform ; $p$, pisiform; mc. 4, splint of fourth metacarpal ; mcc. 3 , third metacarpal; $s$, sesamoid; I, $z, 3$, phalanges of third digit.

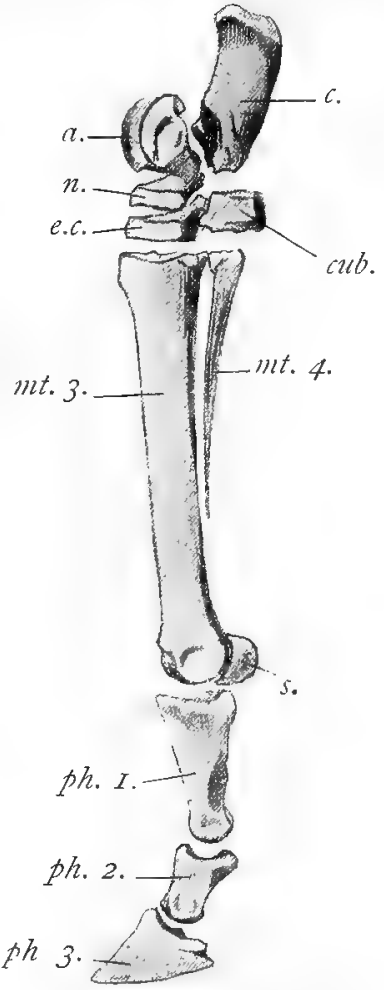

FIG. 255.-Side view of ankle and foot of Horse. (From Edinburgh Museum of Science and Art.)

a., Astragalus ; $c_{\text {., }}$ calcaneum ; $n_{\text {., }}$ navicular; e.c., external cuneiform; cztb., cuboid; mt. 3 , third metatarsal ; met. 4 , splint of fourth metatarsal; s., sesamoid ; phe. $\mathbf{r}-3$, phalanges of third digit.

Artiodactyla. The calcaneum does not articulate with the lower or distal extremity of the fibula. The femur has a 
third trochanter or process for the insertion of muscles. There are usually twenty-three dorso-lumbar vertebræ.

As to the dentition, the premolars and molars form a continuous series, with broad transversely ridged crowns, the last premolars often very like the molars.

The stomach is simple, the cæcum is large, there is no gall bladder.

The mammæ are inguinal; the placenta is diffuse and non-deciduate.

\section{Families of Perissodactyla.}

Family Tapiridie. In the Tapirs (Tapirus), there are four digits in the manus, but the third finger is still practically median, as the

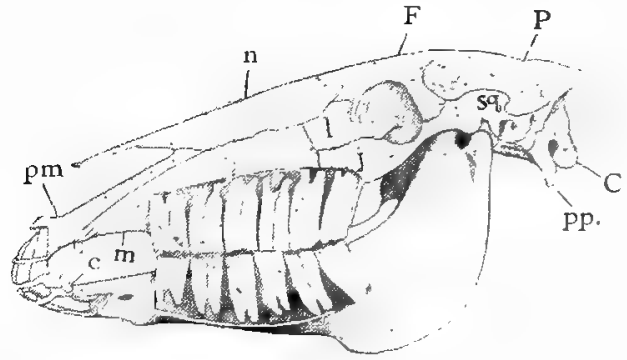

FIG. 256,-Side view of Horse's skull, roots of back teeth exposed. (From Edinburgh Museum of Science and Art.)

$P$, Parietal ; $F$, frontal ; $n$, nasal ; $p m$, premaxilla ; $n$, maxilla; $j$, jugal ; $l$, lachrymal ; $s q$, squamosal ; $p p$, paroccipital process; $c_{2}$ canine ; $C$, condyle.

fifth digit scarcely reaches the ground. The hind foot has three digits. The dentition of the genus is $\frac{3^{\mathrm{T}} 43}{3^{\mathrm{I}} 33}$. The orbit and temporal fossa are continuous. The nose and upper lip form a short proboscis. The thick skin has but scanty hair. In hahit, the Tapirs are shy and nocturnal, fond of forests and water, feeding on tender shoots and leaves. The distribution is somewhat remarkable, for four species live in Central and South America, while a fifth is Malayan. The genus was once widespread, it has survived in these two widely separated regions.

Jamily Equida. In the modem horses (Equrs), there is on each foot one functional digit-the third, with splints representing the metacarpals and metatarsals of the second and fourth. Professor 
Cossar Ewart has recently found in the embryo of the horse the rudiments of the three phalanges of the second and fourth digits. The vestigial phalanges of these digits subsequently fuse with one another and with the respective metacarpals or metatarsals, forming "buttons" at the end of the splints. The ulna and fibula are incomplete. The dentition is $\frac{3^{\mathrm{I}} 43}{4}$, but the first premolar is rudimentary. The orbit is completely surrounded by bone.

The modern horses are connected by a very complete series of forms with ancestral Eocene types. The progress shows an increase of size, a diminution in the number of digits, an increased folding of the back teeth, and other differentiations. The Eocene Phenacodus is regarded
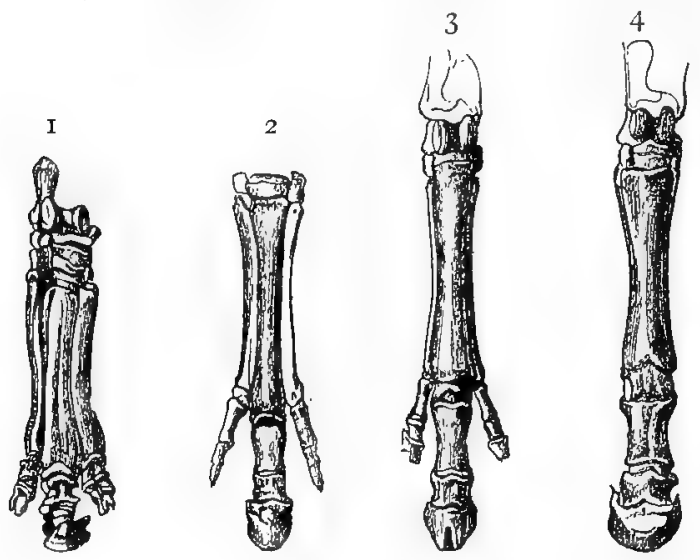

FIG. 257.-Feet of Horse and its progenitors.

(From Neumayr.)

x. Palcotherium; 2. Anchitherium; 3. Hippotherium; 4. Equus.

by some as near the origin of the stock, it had five complete digits on each foot; Hyracotherizm and Systemodon had only four functional digits in the manus; Anchitherium from the Miocene, an animal about the size of a sheep, had three digits, or three and a rudiment: Hippotherium and Protohippus from the Pliocene, were as large as donkeys, and show a marked diminution of the second and fourth digits; finally, in the Pleistocene, the modern forms appeared.

The living species are the horses (Equus caballus), apparently originating in Asia, domesticated in prehistoric times, artificially selected into many breeds, sometimes reverting to wildness as in the case of those imported into America and Australia by European settlers; the wild horse of Central Asia ( $E$. prezevalskii); the donkey ( $E$. asinus) of 
African origin, the wild asses of Africa and Asia, the striped African species-the zeloras and the (exterminated) quagga.

Family Rhinocerotidae. There is now but one genus Rhinocsros, species of which occur in Africa and in some parts of India and Indo-Malaya. They are large heavy Ungulates, shy and nocturnal, fond of wallowing in water or mud, feeding on herbage, shoots, and leaves. The skin is very thick, with scanty hair. One or two median horns grow as huge warts from the snout and forehead. The dentition is very variable, but the back teeth $\frac{4,3}{4,3}$ are almost uniform, there are no upper canines, but sometimes a large lower pair, there are a few incisors, but these are often small and deciduous.

There are several entirely extinct families of Perissodactyla, such as Lophiodontidæ (Eocene), e.g., Lophiodon, Hyracotherium, Systemodon,-a family perhaps ancestral to most of the modern Perissodactyla.

Palæotheriidæe (Eocene to Miocene), e.g., Palcotherium and Anchitherium.

Other remarkable types-

Lambdotherium, Chalicotherium, Titanotherium, of elephantine size, and the specialised Macrauchenia-are referred to distinct families.

\section{Sub-Order HyRACOIDEA.}

Small Rodent-like Ungulates, represented by one genus - Hyrax or Procavia, living in rocky regions and on trees in Africa and Syria. The species (I4) are adept climbers.

The upper incisors have persistent pulps and are curved as in Rodents, but they are sharply pointed, not chisel-edged. The outer lower incisors are straight and have trilobed crowns. There are no canines in the second set, but the upper milk canine sometimes persists; and there is a wide space between incisors and premolars. The back teeth are very uniform and like those of Perissodactyla. The milk dentition is $\frac{3^{124}}{214}$, the permanent is $\frac{1043}{2043}$. Hyrax is one of the few Mammals in which the first premolar is a replacing tooth.

In the fore feet, the thumb is rudimentary, the little finger is smaller than the median three, which are almost equal. In the hind feet, which are like miniatures of those of the rhinoceros, the hallux is absent, and the fifth toe is rudimentary. There are no clavicles. The tail is very short.

The brain is like that of Ungulates. The stomach is 
divided into two parts by a constriction. In addition to the short but broad cæcum, there are two supplemental cæca lower down on the intestine. The testes are abdominal. Of the mammæ, four are on the groin and two are axillary. The placenta is zonary as in the Proboscidea and Carnivora. No extinct forms are known.

\section{Sub-Order Proboscidea.}

This sub-order is now represented by two species of elephant (Elephas). They occupy a somewhat isolated position, though distinctly Ungulates. As regards skull, proboscis, and teeth they are highly specialised, but their limbs are of a generalised type.

The elephants are confined to the Ethiopian and Oriental regions. They feed on leaves, young branches, and herbage. By means of the mobile proboscis they gather their food, and they drink by filling the proboscis and then ejecting the water into the mouth.

The proboscis is a muscular extension of the nose, and bears the nostrils at its tip.

The skin is strong and the hair somewhat scanty.

In the limbs, radius and ulna, tibia and fibula, are quite distinct; the radius and ulna are fixed in a crossed position; owing to the length of the humerus, and yet more of the femur, elbow and knee are lower than usual ; the carpal and tarsal bones have flat surfaces; the feet are broad and bear five hoofed toes embedded in a common integument. There are no clavicles.

The skull is very large, being adapted to support the proboscis and tusks, and to afford a broad insertion for the large muscles. In most of the bones there is during growth an extraordinary development of air spaces, which communicate with the nasal passages. The nasal bones are very short; the zygomatic arch (formed anteriorly by the maxilla, medianly by the small jugal) is slender and straight. The neck is very short.

The dentition is unique. The two upper incisors or tusks are mainly composed of solid ivory; the enamel is restricted to the apex and soon wears off. As the tusks grow, their roots sink through the premaxillæ into the maxillæ. There are no canines nor premolars. The molars are very large, 
and the enamel is very much plaited, forming a series of transverse ridges enclosing the dentine, and united to one another by cement. 'Thus on the worn tooth there are numerous successive layers of enamel, dentine, and cement. Extinct forms show transitions between this complex type and the horse's tooth. In a lifetime there may be six molar teeth on each side of each jaw, but of these only one, or portions of two, can find space at a time. The series gradually moves forward as the front parts are worn away and cast out.

The brain is highly developed.

The stomach is simple, and there is a large cæcum.

There are two superior venæ cavæ entering the right auricle.

The testes remain abdominal in position.

There are two pectoral mammæ; the uterus is bicornuate; the placenta is non-deciduate and zonary.

Elephas, $\frac{106}{\infty 06}$, now represented by the Indian Elephant ( $E$. indicus), with parallel folds of enamel on the molars and ears of moderate size, and the African Elephant (E. africanus), with lozenge-shaped folds of enamel and very large ears.

The mammoth ( $E$. primigenius) belonged to the Pleistocene period, and had a wide geographical range, occurring for instance in Britain.

The genus Mastodon is represented by fossil remains in Miocene, Pliocene, and even in Pleistocene strata, in Europe, India, and America. The molar teeth show transitions between those of elephants and those of other Ungulates.

In Dinotherium, found in Miocene and Pliocene strata in Europe and Asia, the lower jaw bore an enormous pair of tusks projecting vertically downwards, and all the back teeth seem to have been in use at the same time.

\section{Several Extinct Sub-Orders.}

Although we cannot describe the following remarkable types, it is important to notice their existence, for they serve to impress us with the original connectedness of what are now separate orders.

The huge Amblypoda, found in Eocene formations in W. America, had three pairs of remarkable protuberances on the top of the skull, no upper incisors, large upper canines, especially in the males, and six back teeth. Example-Uintatherizm; the genus Coryphodon may also be related.

Cope includes a number of generalised Eocene Ungulates under the title Condylarthra. Some seem ancestral to the Perissodactyla and Artiodactyla; some suggest a union of ancestral Ungulates and ancestral Carnivores. The genus Periptychus may he regarded as an ancestral 
Bunodont, and Phenacodus as near the origin of the horse stock. But Phenacodus is so generalised that Cope has suggested affinities between it and not only Ungulates, but also Carnivores and Lemurs.

The tertiary strata of S. America have yielded a number of strange types, e.g., Toxodon, Nesodon, and Typotherium, ranked in the suborders Toxodontia and Typotheria.

From the Eocene of $\mathrm{N}$. America, Marsh has disentombed a group of animals which he calls Tillodontia, e.g., Tillotherium, which seem to combine the characters of the Ungulata, Rodentia, and Carnivora.

\section{Order 4.-Cetacea.}

The Cetaceans, including whales and dolphins and their numerous relatives, are aquatic mammals of fish-like form.

The spindle-shaped body has

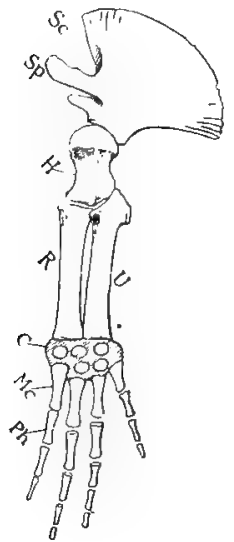

FIG. 258.-Left fore-limb of Balænoptera.

Sc., Scapula with spine $(s p)$; $H$., humerus; $R$., radius; $U$., ulna; $C$., carpals embedded in matrix ; Mc., metacarpals ; $P h$., phalanges.

eye-cleansing nictitating membrane, the dorsal position and valvular aperture of the single or double nostril, the sponginess of the bones, the retia mirabilia in different parts of the body, may be associated with the aquatic life of these mammals. relatively large head and the trunk, and tapers to a notched tail, the horizontal expansions of which form the flukes. 'The fore limbs are paddle-like, and there are no external hints of hind limbs. Most forms have a median dorsal fin. Hairs are generally absent, though a few bristles may persist near the mouth. The thick layer of fat or biubber beneath the skin serves to retain the warmth of the body, and thus compensates for the absence of hair. In one of the dolphins dermal ossicles occur, a fact which has suggested the idea that the toothed whales may have had mailed ancestors. Traces of dermal armour have also been found in the extinct Zeuglodonts.

The general shape, the absence of external ears, the absence of an no distinct neck between the 
The cervical vertebræ are thin and more or less fused. There is no union of vertebræ to form a sacrum, for the hind-limbs are at most very rudimentary. Under the caudal vertebræ there are wedge-shaped chevron bones.

The brain-case is almost spherical; the supra-occipital meets the frontals and shuts out the parietals from the roof of the skull; the frontals arch over the orbit; the snout or rostrum of the skull is composed of premaxillæ, maxilla, and vomer, and of the mesethmoid cartilage.

There are at least rudiments of two sets of teeth, as in other Mammals, but in baleen whales only the teeth of the milk set are calcified, and they come withal to nothing, being to some extent replaced by the horny baleen plates developed on the palate. In toothed whales the two sets are said by Kükenthal to fuse, but the usual interpretation is that the functional teeth belong to the milk set. It is possible that the simple, homodont, conical teeth of Odontoceti have resulted from a splitting of more complex cusped teeth. No clavicles are developed. Excepting the humerus, the bones of the fore-limb are stiffly jointed and flattened. The carpals are fixed in a fibrous matrix, tend to be rudimentary, and are often unossified. There are four or five digits, of which the second and third have more than the usual number of phalanges, a peculiarity possibly due to a duplication and separation of epiphyses. The pelvis may exhibit two rudimentary ischia, with small vestiges of femur and tibia.

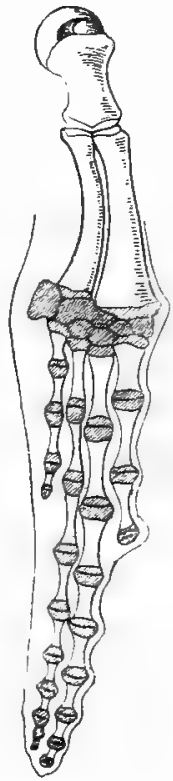

FII. 259.-Forelimb of thale (IIE) suatera longimana). (After Struthers.)

The rounded brain is relatively large, with well-convoluted cerebral hemispheres.

As to the alimentary system, salivary glands are rudimentary or absent, the stomach is chambered, the intestine 
has rarely a cæcum, the liver is but slightly lobed, there is no gall bladder.

The heart is often cleft between the ventricles. Both arteries and veins tend to form retia mirabilia.

The larynx is elongated so that it meets the posterior nares, and forms a continuous canal down which air passes from nostrils to lungs. Cetaceans must, of course, rise to the surface to inspire, but the expiration occurs at longer intervals than in terrestrial mammals. The water vapour expelled along with the air from the lungs, condenses into

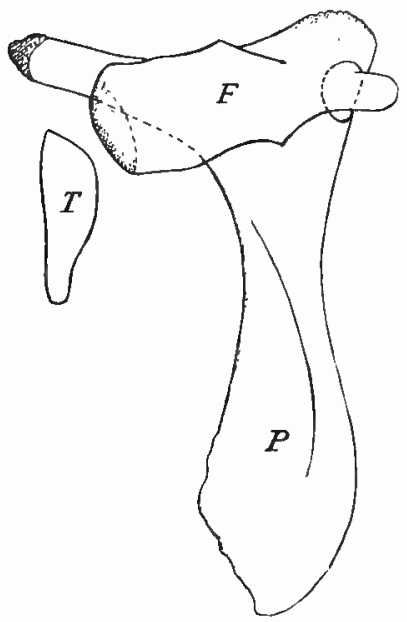

FIG, 260.-Pelvis and hind-limb of Greenland Whale (Balæna).

(After Struthers.)

$P$., Pelvis ; $F$, femur ; $T$., tibia.

a cloud, which is sometimes increased by an accidental puff of spray.

The kidneys are lobulated. The testes are abdominal. There are no seminal vesicles. The uterus is bicornuate. The placenta is nondeciduate and diffuse. The two mammæ lie in depressions beside the genital aperture, and the milk is squeezed from special reservoirs into the mouth of the young. Usually a single young one is born at a time, and there are never more than two.

All are carnivorous, but while many feed on small pelagic animals, others swallow cuttles and fish, and Orca attacks other

Cetaceans and seals. Most are gregarious and live in schools or herds.

The living Cetaceans are ranked in two sub-orders--the Mystacoceti or Balænoidea without functional teeth but with whalebone or baleen plates on the palate, and the Odontoceti or Delphinoidea, with functional teeth and without baleen. 
Some Eocene fossils, known as Zeuglodonts, are regarded by some (Lydekker, Dames) as primitive CetaceansArchæoceti-less specialised than modern forms, but Prof. D'Arcy Thompson has advanced strong arguments in favour of their affinities with Pinniped Carnivores.

In regard to the possible affinities of the Cetacea, Sir Wm. Flower maintains (I) that the hypothesis of their descent from Ichthyopterygian reptiles is untenable, (2) that they are separated from an alliance with Carnivora by

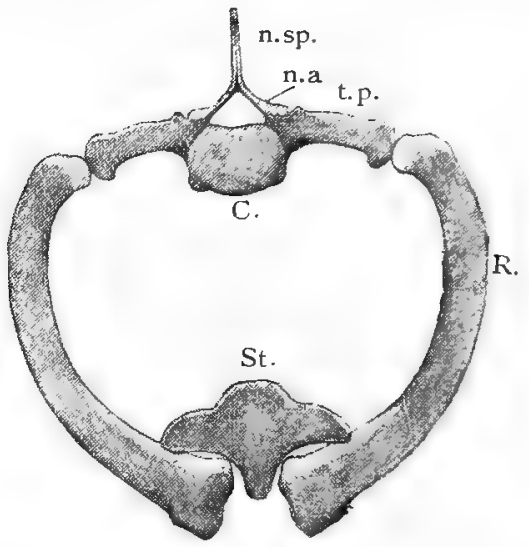

FIG. 26I.-Vertebra, rib, and sternum of Balenoptera. (From specimen in Anatomical Museum, Edinburgh.)

C., Centrum; $\boldsymbol{n} . a_{.}$, neural arch; $n . s p .$, neural spine; t.p., transverse process ; $R+$, rib; $S t$, sternum.

many essential characters, (3) that they exhibit several, though by no means close, affinities with Ungulata.

The same authority refers to several facts which suggest that, in their transition from terrestrial to marine life, the Cetaceans may have passed through a stage in which they lived in fresh water. 
The two Sub-Orders of living Cetaceans may be contrasted as follows (after Flower) :--

\section{Mystacoceti or Balenoidea, baleen Cetaceans.}

The teeth are absorbed before birth.

Whalebone or baleen plates develop as processes from the palate.

The skull is symmetrical.

The nasals roof the anterior nasal passages, which are directed upwards and forwards.

The maxilla does not overlap the orbital process of the frontal.

The lachrymal is small, and distinct from the jugal.

The tympanic is ankylosed to the periotic.

The rami of the mandible are arched outwards, and have no true symphysis.

All the ribs articulate only with the transverse processes of the vertebra.

The sternum is a single piece, and articulates with a single pair of ribs.

The external nostrils are separate.

The olfactory organ is distinctly developed.

There is a short cæcum.

Examples:-

The right-whale (Balcena), the hump-back (Mepaptera), the rorqual (Balcenoptera).

\section{OdONTOCETI OR DeLPHINOIDEA.} toothed Cetaceans.

The teeth persist after birth, and are generally numerous and functional.

There is no baleen.

The skull on its upper surface is more or less asymmetrical.

The nasals, often small, do not roof the anterior nasal passages, which are directed upwards and backwards.

The maxilla covers most of the orbital process of the frontal.

The lachrymal is fused to the jugal, or is large, and helps to roof the orbit.

The tympanic is not ankylosed to the periotic.

The rami of the mandible are straight, and form a symphysis.

Several anterior ribs articulate by capitula with the centra of vertebra.

The sternum has usually several segments with which several sternal ribs articulate.

The nostrils unite in a single blow-hole on the top of the head.

The olfactory organ is rudimentary or absent.

There is no cæcum, except in Platanista.

Examples:-

The Sperm whale (Physeter), the dolphin (Delphinus), the porpoise (Phocena), the "Grampus" (Orca), the Ca'ing whale (Globicephalus), Grampus, the Narwhal (Monodon), with a horn-like tusk in the male only, the Beluga (Delphinapterns), the blind Platanista of the Ganges. 


\section{Order 5. RoDentia.}

Rodents are represented in all parts of the world, and by more species than any other order of mammals. Most of them are small, and most are terrestrial, but there are some arboreal and aquatic forms. All are herbivorous, and gnaw their food in a characteristic way.

The dentition is characteristic. The incisors have chisellike edges, for as the enamel is either entirely restricted to the front, or is at most thin posteriorly, the back part wears away more rapidly. The incisors are always rootless, growing from persistent pulps as they are worn away, and the same is sometimes true also of the back teeth. On the lower jaw there is never more than a pair of incisors, and in most cases the upper jaw also has only a pair. There are no canines, and the skin projects as a hairy pad into the mouth through the large gap between incisors and premolars.

The feet are plantigrade or semi-plantigrade, generally with five clawed or slightly hoofed digits. Clavicles, though often rudimentary, are generally present. The scapula has usually a long acromion process.

The condyle of the mandible is elongated from before backwards, and in gnawing the jaw moves backward and forward (unimpeded by any postglenoid process of the squamosal). The mandible has an abruptly narrowed and rounded symphysis, and a very large angular portion. The orbits are confluent with the temporal fosse. The zygomatic arch is complete. There is generally a distinct interparietal bone. The tympanic bullæ are always developed, and are often large.

The cerebral hemispheres are smooth, and leave the cerebellum uncovered.

The skin is generally thin, and the panniculus carnosus but slightly developed.

The intestine has a large cæcum, except in Myoxidæ. Special anal or perineal or other glands secreting odoriferous substances are usually developed.

The testes are inguinal or abdominal in position; only in the hares and rabbits do they completely descend into scrotal sacs. 
The mammæ are on the abdomen, or on the abdomen and thorax.

The uterus is double or very markedly bicornuate. There is a provisional yolk sac placenta; the allantoic placenta is discoidal and deciduate.

Sub-order Simplicinentata.-Rodents with only one pair of upper incisors, with the enamel restricted to the front.

Squirrel-like (Sciuromorpha), including the following and some other families :-

Anomaluridæ-the Ethiopian arboreal genus Anomalumes, with a lateral parachute of skin.

Sciuridæ-the squirrels (Scizorus), the flying squirrelsPteromys and Sciuropterus - with a parachute of skin connecting the fore and hind limbs, the marmots (Arctomys), the prairie dogs (Cynomys), the pouched marmots or sousliks (Spermophilus).

Castoridæ-the beaver (Castor).

Mouse-like (Myomorpha), including the following and some other families :-

Myoxidæ-the dormice (Myoxus, E尺c.)

Muridæ-e.g., the brown rat (Mus decumanus), the black rat (M. rattus), the house mouse (M. mutsculzus), the woodmouse (M. sylvaticus), the harvest mouse ( $M$. minutus), the water-voles (Arvicola), the American musk rat (Fiber zibethicus), the lemming (Myodes), the hamsters (Cricetzis).

Geomyidx-e.g., the American pouched rat (Geomys bursarius).

Dipodida-e.g., the Jerboas (Dipus, \& $\sigma_{0}$ )

Porcupine-like (Hystricomorpha), including the following and some other families :-

Octodontidæ-e.g., the aquatic Coypu (Myopotamus coypu).

Hystricidx-e.g., the porcupine (Hystrix, $\mathcal{E}^{*} c_{\text {. }}$ )

Chinchillidæ-e.g., the squirrel-like Chinchilla.

Dasyproctidx-e.g., the Agoutis (Dasyprocta), and the paca (Calogenzys.)

Caviidæ-e.g., the guinea pig (Cavia), and the S. American Capybara (Hydrocho:rzss), the largest living Rodent, measuring about four feet in length.

Sub-order Duplicidentata. - Rodents with two pairs of incisors in the upper jaw, the second pair small and behind the first pair; the enamel extends to the posterior surfaces, but is thinner there.

Lagomyidx-The Picas or tailless hares (Lagomys), guineapig-like animals found on the mountains of N. Asia, in S.-E. Europe, and on the Rocky Mountains.

Leporidæ-e.g., the common hare (Lepus timidus), the mountain hare ( $L$. variabilis), the rabbit ( $L$. cuniculuss). 


\section{Order 6. CaRnivora.}

This order includes $(a)$ the true Carnivores, such as lions and tigers, foxes and dogs, bears and otters ; $(b)$ the aquatic Pinnipedia, such as seals and walruses; and $(c)$ the extinct Creodonta with several generalised types.

Most of the Carnivora feed on animal food, and the most

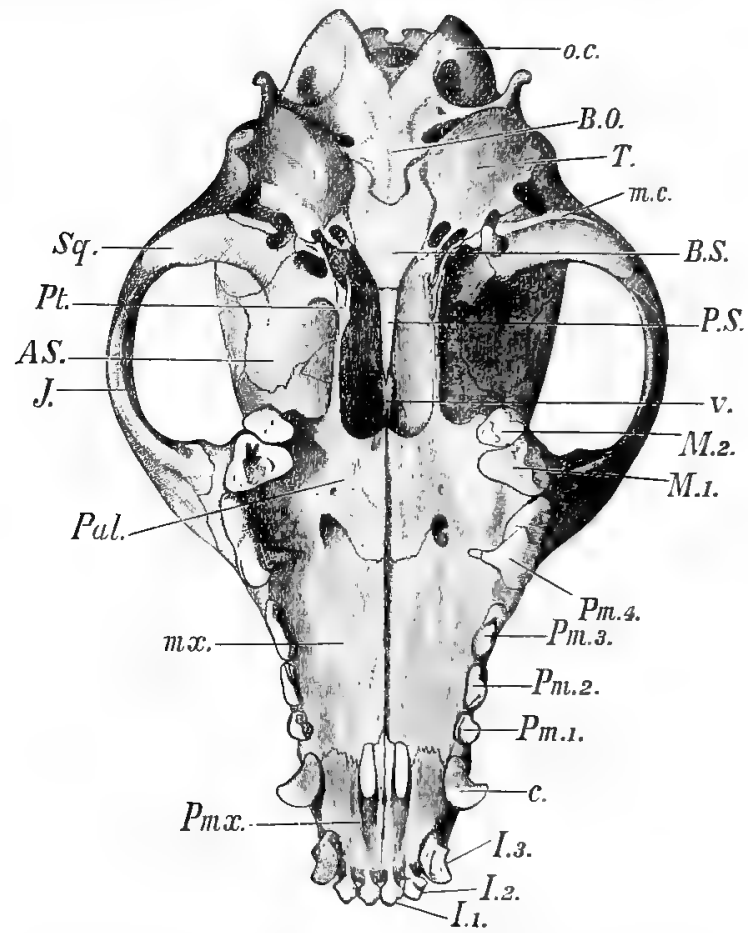

FIG. 262.-Lower surface of Dog's skull.

o.c., Occipital condyle; B.O., basi-occipital; T., tympanic bulla; m.c., post-glenoid process behind fossa for condyle of mandible; B.S., basi-sphenoid: P.S., base of presphenoid ; I", vomer; $M .2$, second molar ; $M$. I, first molar ; $P_{n, I-4}$, premolars ; $c_{c}$, canine I. I-3, incisors: Pmx., premaxilla; mx., maxilla ; Fal, palatine; $J_{.,}$jugal ; $A S$. , alisphenoid ; $P t_{\text {. }}$ pterygoid; $S q$., squamosal. 
typical forms prey upon other animals and devour their warm flesh. Most are bold and fierce animals, with keen senses and quick intelligence.

Almost all have well-developed claws; there are never fewer than four toes. The teeth are always rooted except in the case of the tusks of the walrus; the canines are strong and sharp ; some of the back teeth are generally sharp and specially adapted for cutting.

"The condyle of the lower jaw is a transversely placed half-cylinder, working in a deep glenoid fossa of corresponding form." Thus the jaw moves only up and down. The zygomatic arch within which lie the powerful jaw muscles is generally prominent, and the widening of this has, as it were, broken the bridge behind the orbit, so that the orbit is confluent with the temporal fossa. There are generally strong occipital and sagittal crests for the insertion of muscles. The tympanic bullæ are in most cases large.

The clavicles are incomplete or absent; the radius and ulna are always distinct; the fibula is slender but distinct.

The cerebrum is well convoluted, and the cerebellum is more or less covered by the cerebrum.

The stomach is always simple; the cæcum is absent, or short, or simple; the colon is not sacculated.

There are no vesiculæ seminales. The uterus is bicornuate. The mammæ are abdominal. The placenta is deciduate and zonary.

Representatives of Carnivora are found in all parts of the world.

Sub-Order Carnivora Vera or Fissipedia.

The true Carnivores are for the most part terrestrial. The incisors are almost always ${ }^{3}$, the canines are usually large, one of the back teeth is modified as a trenchant carnassial or sectorial. The digits generally have sharp claws, which may be retractile. Within the sub-order there are three sectionsEluroidea, Cynoidea, and Arctoidea-represented respectively by cat, dog, and bear, but these types are connected by extinct forms. 


\begin{tabular}{|c|c|}
\hline $\begin{array}{c}\text { ELUROIDEA. } \\
\text { c.g., cat, civet, hyæna. }\end{array}$ & $\begin{array}{c}\text { CYNOIDEA. } \\
\text { e.g., dog, fox, wolf, jackal. }\end{array}$ \\
\hline Digitigrade. & Digitigrade. \\
\hline Typical dentition, $\frac{313 I}{3^{I 2 I}}$ & Typical dentition, $\frac{3 \pi 42}{3 \sqrt{13}}$ \\
\hline $\begin{array}{l}\text { The tympanic bulla is much } \\
\text { dilated, rounded and thin- } \\
\text { walled, and is divided in- } \\
\text { to two chambers by an } \\
\text { internal septum (except } \\
\text { in Hyænidæ). }\end{array}$ & $\begin{array}{l}\text { The tympanic bulla is dila- } \\
\text { ted, but the internal sep- } \\
\text { tum is rudimentary. }\end{array}$ \\
\hline $\begin{array}{l}\text { The paroccipital process of } \\
\text { the ex-occipital is applied } \\
\text { to the hinder part of the } \\
\text { tympanic bulla. }\end{array}$ & $\begin{array}{l}\text { The paroccipital process is } \\
\text { in contact with the bulla, } \\
\text { but it is prominent. }\end{array}$ \\
\hline $\begin{array}{l}\text { The crecum is small, rarely } \\
\text { absent. }\end{array}$ & $\begin{array}{l}\text { The cacum is sometimes } \\
\text { short and simple, some- } \\
\text { times long and peculiarly } \\
\text { folded. }\end{array}$ \\
\hline
\end{tabular}

ARCTOIDEA.

e.g., bear, otter.

Plantigrade or sub-plantigrade.

Typical dentition, $\frac{3142}{3143}$.

The tympanic bulla is often depressed, and there is no hint of an internal septum.

The paroccipital process is quite apart from the bulla.

The cæcum is absent.

In retractile claws, the last phalanx of the digit with its attached claw is drawn back into a sheath on the outer side of the middle phalanx in the fore-foot, on the upper side in the hind-foot. When the animal is at rest or is walking, the claw is retained in this bent position by an elastic ligament, and is in this way protected. When the animal straightens the phalanges, the claws are protruded.

Digitigrade animals walk on their toes only, plantigrade forms plant the whole sole of the foot on the ground, but between these conditions there are all possible gradations. Most Carnivores are sub-plantigrade, often when at rest applying the whole of the sole to the ground, but keeping the heel raised to a greater or less extent when walking.

\section{ALuroidea-Cat-like Carnivores.}

Family Felide, including the most specialised forms. The canines are large, the molars are reduced to $\frac{I}{\mathrm{I}}$, the carnassials are the last premolars above (with a three-lobed blade), and the molars beneath (with a two-lobed blade). The skull is generally rounded, the zygomatic arches are wide and strong, the tympanic bulle are large and smooth. The limlss are digitigrade, the claws retractile. There is no alisphenoid canal. The dentition of the typical genus Felis is $\frac{3 \mathrm{I} 3 \mathrm{I}}{312 \mathrm{I}}$.

Examples:-The lion (Felis leo) in Africa, Mesopotamia, Persia, N.-W. India; the tiger $(F$. Tigris), widely distributed in Asia; the leopard ( $F$. Pardus) in Africa, India, Ceylon, Sumatra, Borneo, \&c. ; the wild cat $(F$. catus); the Caffre 
cat ( $F$. caffra) of Africa and S. Asia, venerated and mummified by the Egyptians, perhaps ancestral to the domestic cat; the puma or couguar ( $F$. concolar) from Canada to Patagonia ; the jaguar $(F$. onca), also American.

A high degree of specialisation for carnivorous habit is well illustrated by the sabre-toothed tigers (Macharodus) of Tertiary ages, whose serrated upper canines were sometimes seven inches long.

Family Viverridæ-Old World forms, such as civets (Viverra), of Africa and India, genets (Genetta), of S. Europe, Africa, and S. W. Asia, ichneumons or mongooses (Herpestes), from Spain, Africa, Inclia, Indo-Malaya.

Family Proteleida-represented by Proteles cristatus, the hyæena-like Aard-wolf of Cape Colony.

Family Hyænidre-represented by the genus Hycna, found in Africa and S. Asia. The tympanic bulla is not divided by a septum.

\section{CynoInea-Dog-like Carnivores.}

Family Canidre-including forms intermediate between the cats and the bears. The dentition is more generalised than in the Felidæ, its usual formula is $\frac{3^{\tau+42}}{3^{1} 43}$. Within the tympanic bulla there is only a rudimentary septum. The paroccipital process in contact with the bulla is prominent. The cæecum is either short and simple, or long and peculiarly folded upon itself.

Examples:-The genus Canis has representatives in all parts of the world, the wolves ( $C$. lupus, \&c.), the jackals (C. aureus), mesomelas, \&c.), the domestic dogs (C. familiaris), the foxes (C. vulpes, \&c.), the Cape hunting dog (Lycaon), the bush$\operatorname{dog}$ (Icticyon) of Guiana and Brazil, and the primitive Otocyon megalotis from $\mathrm{S}$. Africa, with the maximum number of back teeth $\frac{3,1,4,3-4}{3, x, 4,4}$. In the dog the dental formula is $\frac{3^{1}+2}{3 x_{43}}$; the upper carnassial or fourth premolar has a stout bilobed blade, the lower carnassial or first molar has a compressed bilobed blade. The skull is more elongated than in the cats; the orbits are very widely open posteriorly; the clavicles are very small; the limbs are digitigrade; there are five toes on the fore-feet, but the short thumb does not reach the ground; there are only four toes on the hind-feet, but in domestic dogs the rudiment of the hallux is sometimes enlarged as the "dewclaw;" the claws are non-retractile and blunt.

\section{Arctoidea-Bear-like Carnivores.}

The tympanic bulla shows no trace of an internal septum; the paroccipital process of the ex-occipital is quite apart from the bulla, and widely separated from the mastoid process of the periotic. The limbs are plantigrade or sub-plantigrade, and always bear five toes. There is no cæecum. 
Family Ursida - Bears. The molars have broad tuberculated crowns. The three anterior premolars are usually rudimentary, The auditory bulla is depressed. Ursus, $\frac{3142}{3^{1} 43}$, absent from Ethiopian and Australian regions, represented in the Neotropical region by only one species, elsewhere widespread.

Family Procyonidx-The Himalayan Panda (Elurus fulgens), the American raccoon (Procyon).

Family Mustelidre-The otter (Lutra), the sea-otter (Latax luiris), the skunk (Mephitis), the badger (Meles), the ratel (Mellivora), the marten, sable, polecat, stoat, weasel (Mustela).

\section{Sub-Order Prnnipedia. Seals, Eared Seals, and Walruses.}

These are marine Camivores, unable to move readily on land, but coming ashore for breeding purposes. They feed for the most part on fish, molluscs, and crustaceans. Absent from the Tropics, they are represented on most of the coasts in Temperate and Arctic zones. Many are markedly gregarious.

The upper parts of the limbs are included within the skin and general contour of the body. There are five well-developed digits connected by a web of skin. In the hind-foot the first and fifth toes are generally stouter and longer than the rest. There are no clavicles. The tail is very short.

The small milk-teeth are absorbed before or immediately after birth. The incisors are always fewer $\operatorname{than} \frac{3}{3}$; there are no carnassials ; the back teeth have pointed cusps often sloping slightly backwards.

The brain is large and well-convoluted. The eyes are large and prominent, with a flat cornea. The external ear is small or absent.

The cæcum is very short. The kidneys are divided into lobules. The mamma are two or four in number, and lie on the abdomen.

Family Otariida-Eared or fur-seals, connecting the Pinnipeds with the Fissipeds. The hind-feet can be turned forward and used on land in the usual fashion. The palms and soles are naked. There is a small external ear. The testes lie in an external scrotum.

The sea-lion Otaria, $\frac{3, r, 4, x-2}{2, x, 4, x}$, supplies the seal skin of commerce.

Family Trichechidre-Walruses, intermediate between the Otariidxe and the seals. The hind feet can be turned forwards and used on land. The upper canines form large tusks; the other teeth are small, single rooted, and apt to fall out; those generally in use are $\frac{113^{\circ}}{0_{130}}$, but the dentition of the fotus is $\frac{3^{132}}{3131}$.

The jaw seems relatively short, an adaptation perhaps to musselcrushing instead of fish-catching.

There are no external ears.

The walrus or morse, Trichechus.

Family Phocida-Seals, the most specialised Pinnipeds. The hind- 
limbs are stretched out behind, and the strange jumping movements on land are effected by the trunk, sometimes helped by the fore-limbs. The palms and soles are hairy. There are welldeveloped canines, the upper incisors have pointed crowns, there are $\frac{5}{5}$ back teeth. There is no external ear. The testes are abdóminal.

The common seal (Phoca), $\frac{3^{\mathrm{T}} 4^{\mathrm{I}}}{214 \mathrm{I}}$; the grey seal (Halicharus), the monk seal (Monachus), the large elephant seal (Macrorhinus leoninus).

Sub-Order Crgononta (extinct).

In Eocene and early Miocene strata, in Europe and America, there are remains of what seem to be generalised Carnivora, ancestral to the modern types, and apparently related to Insectivora as well. Those included in the sub-order Creodonta have strong canines but no single carnassials, while the molars are often like those of Marsupials. The brain seems to have been small.

Examples:-Hyonodon, Pterodon, Proviverra, Arctocyon.

\section{Order 7. InSECTIVORA.}

This order includes hedgehog, mole, shrews, and related mammals. There is much diversity of type, so that a statement of general characters is very difficult.

Most Insectivores run about on the earth; the mole (Talpa), and others like it, are burrowers; Potamogale, Myogale, and others are aquatic: Tupaia and its relatives live like squirrels among the branches; and the aberrant "flying Lemur"-Galeopithecus takes swoops from tree to tree.

Most feed on Insects, but Galeopithecus and some other arboreal forms eat leaves as well, the moles eat worms, Potamogale is said to feed on fish.

The body is usually covered with soft fur, but the hedgehog (Erinaceus) is spiny, and so to a less extent is Centetes, the groundhog of Madagascar. The digits, usually five in number, are clawed, and the animals walk in plantigrade or semi-plantigrade fashion. In most, the mammæ are thoracic or abdominal ; in Galeopithecus, there are two pairs in the axillary region.

The cranial cavity is small; the skull is never high; the facial region is long; the zygomatic arch is slender or absent. Except in Potamogale, there are clavicles. 
There are more than two incisors in the mandible. The enamelled molars have tuberculated crowns and well-developed roots. In many cases it is not easy to distinguish the usual division of the teeth into incisors, canines, premolars, and molars, but in many the dentition is typical$3, \mathrm{I}, 4,3=44$.

In the hedgehog, according to Leche, i. 3, pm. 2, m. I-3, of the upper jaw, and i. $3, \mathrm{c}, \mathrm{pm} .3, \mathrm{~m}$. I-3, of the lower jaw, are persistent milk teeth, - a mixed and primitive condition.

The cerebral hemispheres are smooth and leave the cerebellum uncovered; the olfactory lobes are large; the corpus callosum is short and thin. Thus, as regards the brain, the Insectivora represent a low grade of organisation.

Except in Galeopithecus, the stomach is a simple sac; the intestine is long and simple, but the vegetarian forms have a cæcum. In most, there are odoriferous glands, axillary in shrews, but usually near the anus.

The testes are inguinal or in the groin, or near the kidneys, not in a scrotum. The penis may be pendent from the wall of the abdomen, but is usually retractile. There is a bicornuate or two-horned uterus. Except in Guleopithecus, several offspring and usually many are born at once.

The allantoic placenta is discoidal and deciduate. There is a provisional yolk sac placenta.

Insectivora are represented in the temperate and tropical zones of both hemispheres, but not in S. America nor Australia.

Sub-Order Insectivora Vera :-Insectivores with free limbs suited for movement on land, climbing, burrowing, or swimming. "The upper and lower incisors are conical, unicuspidate or with basal cusps only, the lower not pectinated."

Examples:-the hedgehogs (Erinaceus), throughout Europe, Africa, and most of Asia, dentition $\frac{3 \text { I } 33}{2 \text { T23 }}$; the shrews (Sorex), in Europe, Asia, and N. America, dentition $\frac{4123}{2013}$; the moles (Talpa), throughout the Palaarctic region; the tailless tenrec (Centetes) of Madagascar; the S. African golden moles (Chrysochloris); the African jumping shrews (Macroscelides); the Oriental tree shrews (Tupaia).

Sub-Order Dermoptera :-represented by the very divergent Galeopithecus, which almost requires an order for itself. The fore and hind 
limbs are connected by a parachute, and the animals can glide from tree to tree, "sometimes traversing a space of seventy yards with a descent of only about one in five." The upper and lower incisors are compressed, multicuspidate, the lower deeply pectinated. Two species of this genus live in the forests of the Malayan region. They are nocturnal, and feed on leaves and fruit. The dentition is $\frac{2123}{3123}$. There are numerous skeletal peculiarities.

\section{Order 8. Chiroptera-Bats.}

Bats are specialised Mammals related to Insectivores. They have the power of flight, the fore limbs being modified as wings. The wing is mainly due to an extension of the skin stretched between the very long fingers. The fold of skin usually begins from the shoulder, extends along the upper margin of the arm to the base of the thumb, thence between the fingers, and along the sides of the body to the hind legs or even to the tail. Contrasted with the wing of a bird, that of a bat has a rudimentary ulna beside a long curved radius, a wrist with six bones, five free digits with long metacarpals on the four fingers. The shoulder girdle is very strong, there is a long curved clavicle, a large triangular scapula, a long coracoid process; the presternum bears a slight keel on which are inserted some of the muscles used in flight. The thumb is always clawed; the other digits are unclawed, except in most frugivorous bats, where the second digit bears a claw.

The hind limb is relatively short and weak, the pelvic girdle is also weak, and in most cases the pubic symphysis is loose in the males, unformed in the females. The knee is turned backwards like the elbow, the ankle has a cartilaginous prolongation or calcar which supports the fold of skin between limb and tail, the five toes are clawed.

The vertebral column is short, there is little mobility between the vertebræ, neural spines are absent behind the third cervical except in Pteropidæ, the caudal vertebræ are very simple. The ribs are usually flat. The maximum dentition is $\frac{2 \pi 33}{3 \pi 33}$; the milk teeth are very different from the permanent set. All the bones are slender, and have relatively large medullary canals.

The cerebral hemispheres are smooth and leave the cerebellum uncovered. The spinal cord is at first very 
broad, but narrows rapidly behind the neck. The sense of touch is remarkably developed in the hot skin of the wing, the large mobile external ears, the whisker hairs of the snout, and in the strange plaited "nose leaves" around the nostrils. Even when deprived of sight, hearing, and smell,

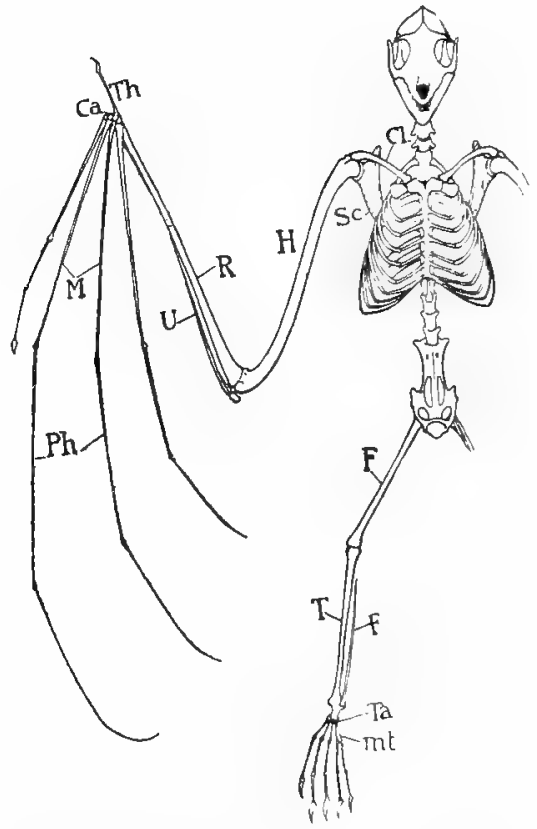

Fig. 263.-Skeleton of Fox Bat,-Pteropus.

$C l$, Clavicle; $H$, humerus ; $R$, radius ; $U$, incomplete ulna ; $T / \ell$, thumb; $C a$, carpals; $M T$, netacarpals 3 and $4 ; P / h$, phalanges; $F$, femur; $T$, tibia; $F$, fibula; $r \alpha$, tarsals; $n t t$, metatarsals.

bats will fly about in a room without striking numerous wires stretched across it.

The temperature of the body is high. The testes are abdominal or inguinal; the penis is pendent. The uterus is simple, with cornua generally short. There is usually but 
one offspring at a time. The mammæ are thoracic, generally post-axillary. As in Insectivora and Rodents, the yolk sac forms a provisional placenta, and the allantoic placenta is discoidal and deciduate.

Fossil Chiroptera occur in Upper Eocene strata, but are quite like the modern forms.

The two sub-orders of bats may be contrasted as follows:-

Megachiroptera.

Frugivorous bats, usually large.

The molars have smooth crowns, with a longitudinal groove.

The thumb is clawed, and generally also the second digit.

The tail, if present, is below, not bound up with the interfenoral membrane.

The pyloric part of the stomach is in most cases much elongated.

Found in warm and tropical parts of the Eastern hemisphere.

\section{Examples :-}

The "flying-foxes " or fox-bats (Pteropus), large, tailless bats, distributed from Madagascar to India, Ceylon, Malaya, S. Japan, Australia, Polynesia. The largest species ( $P$. ectulis) measures five feet across its spread wings. Dentition, $2 \frac{1}{13} \frac{2}{3}$.

In India, Cynopterus narginatus is very common. Xantharpyia agyptiaca inhabits the Pyramids.
Microchiroptera.

Usually insectivorous bats, small in size.

The molars have cusped crowns, with transverse grooves.

In the hand the thumb only is clawed.

The tail, if present, is bound up with the interfemoral membrane, or lies along its upper surface.

Except in one family the stomach is simple.

Found in the tropical and temperate regions of both hemispheres.

Examples:-

The horse-shoe bats ( $R$ hinolophus), the common pipistrelle (Vesperugo pipistrel. (zis), the genus Vespertilio with four British species, Vampyrus spectrum, a large Brazilian form, which seens to have been erroneously credited with blood. sucking habits, the common vampire (Desmodus rufus) an American bat-a formidable blood-sucker.

\section{Order 9. Lemuroidea. Lemurs.}

Opinions differ as to whether the monkey-like animals known as Lemurs should be ranked with monkeys as a suborder of Primates or referred to a separate order. They differ from monkeys and men (Anthropoidea) in the following points :-The orbit opens freely into the temporal fossa (except in Tarsius); the lachrymal foramen lies outside the orbit; the first pair of upper incisors is separated in the middle line (except in Chiromys); the cerebral hemispheres are but slightly convoluted and do not completely overlap 
the cerebellum; "the middle or transverse portion of the colon is almost always folded or convoluted on itself ;" there may be abdominal mammre; the uterus is bicornuate; the placenta is diffuse. The dentition of Lemurs varies greatly; in some it is $\frac{2 \pi 33}{2 \pi 33^{*}}$.

'The Lemurs are small, furry, monkey-like quadrupeds. Many are nocturnal, all arboreal. They feed on fruits and leaves, on eggs and small animals. Seven genera live in Madagascar, three genera occur in the African continent, and other three genera are represented here and there in Oriental forests as far east as the Philippines and Celebes.

As remains of extinct Lemurs are found in Europe and N. America, the distribution of the order is now greatly restricted, and no less than thirty out of the total of fifty species are confined to Madagascar. Wallace conclucles from the distribution of Lemurs that there must have been "a large tract of land in what is now the Indian Ocean. connecting Madagascar on the one hand with Ceylon, and with the Malay countries on the other. About the same time (but perhaps not contemporaneously) Madagascar must have been connected with some portion of Southern Africa; and the whole of the country would possess no other Primates but Lemuroidea." Whether this be altogether true or not, it is certain that the Lemurs are absent from regions where once they lived, that nost of the modern forms are found (like the Marsupials) on an island, that this insulated race has evolved in several specialised directions, that outside of Madagascar the Lemurs maintain their existence on a few other islands, or by hiding in the forests.

There are three chief types:-

(a) That of the Lemurida, e.g., in Madagascar Lemur, and the large Indris (2 feet long), in Africa Galago, in Malay Nycticebus, in India and Ceylon Loris.

(b) Tarsius, a specialised Indo-Malayan type with many peculiarities, e.g., the calcaneum and navicular are elongated like the calcaneum and astragalus in the frog.

(c) Chiromy's, the Aye-Aye, a specialised Madagascar type, with many peculiarities, $e_{.}$. ., with incisors like those of Rodents, and with a very much attenuated middle finger.

\section{Order Io. AnTHROPOIDEA.}

This order includes five families.

Family 5. Hominidæ. Man.

4. Simiidæe. Anthropoid Apes. ) Old World

3. Cercopithecidæ. Baboons, $\}$ Catarrhini.

2. Cebida. American Monkeys.) New World

I. Hapalidae. Marmosets. Platyrrhini. 
The following characteristics are generally true.

The body is hairy, least so in man; the dentition is

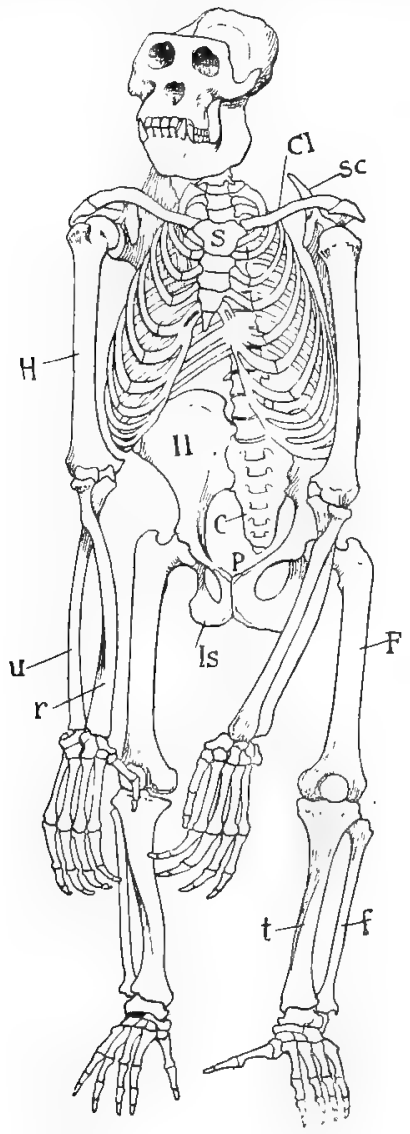

FIG. 264.-Skeleton of Male Gorilla. (From Edinburgh Museum of Science and Art.)

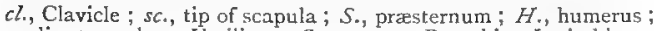
$r_{\text {., }}$ radius; $u$., ulna; $I l$., ilium ; $C$., coccyx; $P_{\text {, }}$ pubis ; $I_{s}$, ischium ; $F$., femur; $t$., tibia ; $f$, fibula. 
diphyodont and heterodont; the incisors do not exceed ${ }_{2}^{2}$; the molars are ${ }^{3}$ except in the marmosets where they are ${ }_{2}^{2}$; the axis of the orbit is directed forward, and the orbit is closed off from the temporal fossa; the clavicles are well developed; the radius and ulna are never united; the scaphoid, the lunar, and usually the os centrale remain distinct from one another; there are usually five fingers and five toes, but the thumb may be absent or rudimentary; the big toe is opposable except in man, and has a flat nail except in the orang; the thumb is usually more or less opposable; the cerebral hemispheres have numerous convolutions and overlap the cerebellum; the stomach is simple except in Semnopithecus and its relatives, in which it is sacculated, and there is a cæcum which is often large; there are two mamma on the breast; the uterus is simple; the testes lie in a scrotum; the placenta is meta-discoidal, being developed by the concentration of the villi from a diffuse area into a well-defined disc.

Some of the characteristics in which the Anthropoidea differ from Lemuroidea may be re-emphasised:-the orbit is separated from the temporal fossa by a bony partition; the lachrymal foramen is situated within the margin of the orbit; the median upper incisors are in contact; the cerebral hemispheres are richly convoluted and hide or almost cover the cerebellum; "the transverse portion of the colon extends uninterruptedly across the abdomen ;" the mammæ are never abdominal; the uterus is not bicornuate but simple; the placentation is meta-discoidal.

Family I. Hapalide (= Arctopithecini). Marmosets.

The marmosets are the smallest monkeys, being no larger than squirrels. They live in companies in the Neotropical forests, especially in Brazil, and feed on insects and fruit.

Their dentition ${ }_{2132}^{2132}$ is distinctive, for other Anthropoidea have ${ }_{3}^{3}$ molars. There is a broad septum between the nostrils, as in the other New World monkeys; the external auditory meatus is not bony. The tail is long, hairy, and 
non-prehensile. The arms are not longer than the legs; there are no cheek pouches nor ischial callosities. The thumb or pollex is long but not opposable; all the digits have a pointed claw except the great toe or hallux which is very small. The marmosets often bear three young ones at a birth, whereas the other monkeys usually bear but one. There are two genera, Hapale and Midas.

Family 2. Ceride (= Platyrrhini). American Monkeys.

In the American monkeys the nose is flat, with a broad internarial septum. They occur throughout tropical America, but are most at home in Brazil. All are arboreal, and many have prehensile tails. The digits have nails, not claws; the thumb, though not opposable, is divergent from the fingers, except in the spider monkey-Ateles - in which it is rudimentary. The skull is rounded, and the frontals form a V-shaped suture with the parietals. The dentition is characteristic, for there are six back teeth; the formula being 2 I 33 .

Examples:-The howling monkeys (Atycetes), with large vocal organs protected by the expanded mandibles, and with an inflated hyoid bone forming a resonating chamber; the sakis (Pithecia) with very long tail ; Nyctipithecus; Chrysothrix; the spider monkeys (Ateles) with exceedingly prehensile tail; the capuchins (Cebus), often imported into Europe.

Family 3. Cercopithecide (= Cynomorph Catarrhini). Old World dog-like Apes.

The OId World apes of this family are still quadrupeds, and the snout or muzzle often justifies the term Cynomorph or dog-like. There is a narrow internarial septum, to which the term Catarrhini refers. The dentition is like that of the anthropoid apes and man, 2123. The external auditory meatus is bony. The thumb is opposable, except when it is rudimentary as in Colobus. The tail is not prehensile. Over the rough surfaces of the cverted ischia the skin forms callosities often brightly coloured. The breast bone is narrow. The cæcum has no vermiform appendix.

In the sub-family Cercopithecinæe, there are cheek pouches, the stomach is simple, the fore and hind limbs are almost equal.

Examples :- the African baboons (Cynocephaless) e.g., the mandrill (C. maimon) notable for the bright colours of the face and hips 
in the adult males, the macaques (Macacus) all Asiatic except the Barbary ape ( $M$. inutus) of N. Africa and Gibraltar; the African Cercopithecus.

In the sub-family Semnopithecine, there are no cheek pouches, the stomach is sacculated in a complex fashion, the hind linıbs are longer than the fore limbs.

Examples:-the sacred Indian apes (Semnopithecus), the African Colobus, and the proboscis monkey (Nasalis) of Borneo.

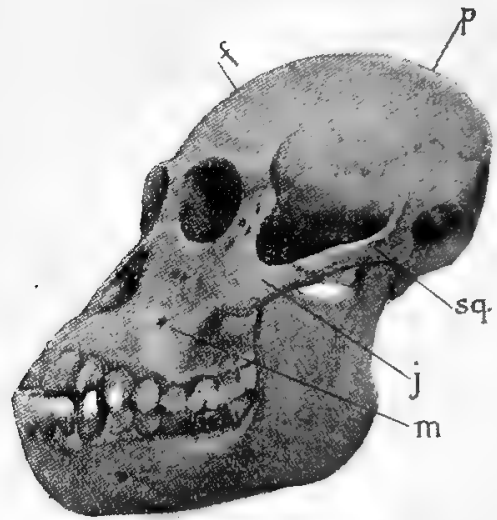

Fig. 265.-Skull of Orang-Utan. (From Edinburgh Museum of Science and Art.)

$p_{*}$, Parietal ; $f_{n}$, frontal ; sq., squamosal ; $j$. jugal ; $m$. , maxilla.

Family 4. Simrdes (= Anthropomorph Catarrhini). Anthropoid Apes.

The Old World apes of this family are the Gibbons (Hylobates), the Orangs (Simia), the Chimpanzees (Troglodytes or Anthropopithecus), and the Gorillas (Gorilla). As 
they are the highest apes and likest man, they are called Anthropoid.

These apes are less like quadrupeds than the others; they have no distinct tail nor cheek pouches. Only in the Gibbon are there ischial callosities, and these are small. 'The arms are much longer than the legs. The sternum is broad. The cæcum has a vermiform appendix. As in the lower Old World apes, the dentition is like that of man-2 I 23 .

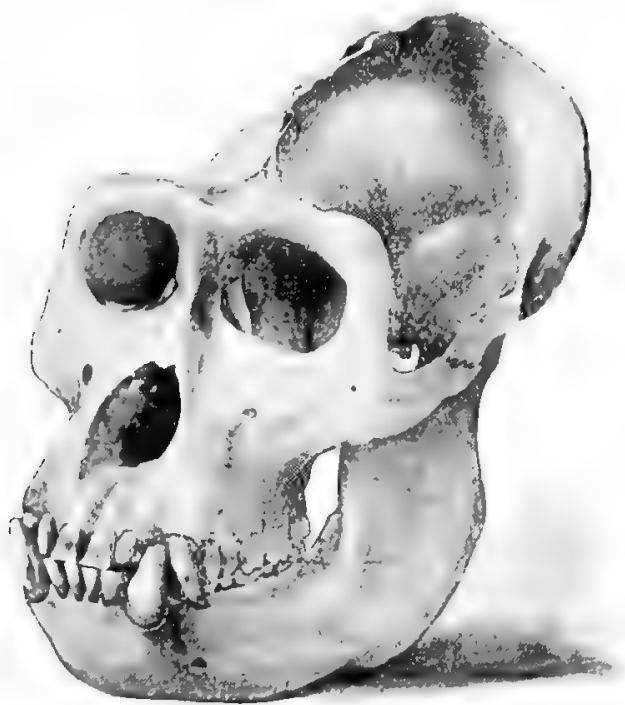

FIG. 266.-Skull of Gorilla. (From Edinburgh Museum of Science and Art.)

The Gilubons (Hylobalis) live in S.-E. Asia, especially in the Malayan region. The largest attains a height of three feet. They walk erect with the hands reaching the ground. The skull is not prolonged into a vertical crest. There is an os centrale in the carpus. The hallux is well-developed. They are the highest apes with hints of ischial callusities. They are mainly arloreal in their halits. They fecel on fruits, leaves, shoots, eggs, young lirels, spidery, and innects. Their voice is powerful. As regards teeth, the gibbons are most like man.

The Orangs (Simia) live in swatnpy forests in Simbatra and Borneo. The males measure over four feet. They walk on their knuckles and 
on the outer edges of the feet. The skull is prolonged into a vertical crest. There are but slight supra-orbital ridges. The canines are very large. There are twelve ribs as in man, and sixteen dorso-lumbar vertebræ. The larynx is connected with two large sacs which unite ventrally. There are no ischial callosities. They are arboreal in their habits, and make nests in the branches. They are exclusively vegetarian. As regards the structure of the brain, the orangs are most like man.

The Gorillas (Gorilla) live in Western Equatorial Africa. They are larger than all other apes, and larger than man, though not over $5 \frac{1}{2}$ feet in height. The arms reach to the middle of the lower leg, and the animals walk with the backs of their closed hands and the flat soles of their feet on the ground. The skull is not prolonged into a vertical crest. There are prominent supra-orbital ridges. The canines of the males are very large. The cervical vertebræ bear very high neural spines, on which are inserted the muscles which support the heavy skull. There are thirteen ribs, and seventeen dorso-lumbar vertebre. There is no os centrale in the carpus. There are no ischial callosities. They live in families in the forest, and feed on fruits. As regards size, the gorillas are most like man. The males are much larger than the females.

The Chimpanzees (Anthropopithecus) live in Western and Central Equatorial Africa. They do not exceed a height of 5 feet. The arms reach a little below the knee. They walk on the backs of their closed hands and on their soles or closed toes. The skull has no high crests. The supra-orbital ridges are distinct. The canines are smaller than in Gorilla or Orang. There is no centrale in the carpus. There are no ischial callosities. The chimpanzees live in families in the forest, and are chiefly arboreal, making nests in trees. They seem to feed on fruits. In the sigmoid curvature of the vertebral column the chimpanzees are most like man.

\section{Family 5. Hominid e. Genus Homo.}

The distinctiveness of man from his nearest allies depends on his power of building up ideas and of guiding his conduct by ideals. But there are some structural peculiarities of interest.

Man alone, after his infancy is past, walks thoroughly erect. Though his head is weighted by a heavy brain, it does not droop forwards. With his upright attitude, the increased command of vocal mechanism is perhaps in part connected.

Man plants the soles of his feet flat on the ground; the great toes are often longer, never shorter than the others, and lie in a line with them; he has a better heel than monkeys have. No emphasis can be laid on the old distinction which separated two-handed men (Bimana) from 
the "four-handed" monkeys (Quadrumana), nor on the fact that men are peculiarly naked. But "the arms are shorter than the legs, and, after birth, the latter grow faster than the rest of the body."

Compared with the anthropoid apes, man has a bigger forehead, a less protrusive face, smaller cheek bones and supra-orbital ridges, a true chin, and more uniform teeth $(2,1,2,3)$, forming an uninterrupted horse-shoe-shaped series without conspicuous canines.

More important, however, is the fact that the weight of the gorilla's brain bears to that of the smallest brain of an adult man the ratio of $2: 3$, and to the largest human brain the ratio of $x: 3$; in other words, a man may have a brain three times as heavy as that of a gorilla. The brain of a healthy human adult never weighs less than $3 \mathrm{I}$ or 32 ounces; the average human brain weighs 48 or 49 ounces; the heaviest gorilla brain does not exceed 20 ounces. "The cranial capacity is never less than 55 cubic inches in any normal human subject, while in the Orang and Chimpanzee, it is but 26 and $27 \frac{1}{2}$ cubic inches respectively."

But, as Owen allowed long since, there is an "all-pervading similitude of structure " between man and the anthropoid apes. As far as structure is concerned, there is much less difference between man and the gorilla than there is between the gorilla and the marmoset.

The arguments by which Darwin and others have sought to show that man arose from an ancestral type common to him and to the higher apes, are the same as those used to substantiate the general doctrine of descent. The "Descent of Man" is the expansion of a chapter in the "Origin of Species." "The arguments may be briefly summarised.

(I) Physiological. The bodily life of man is like that of monkeys; men and monkeys are subject to similar diseases ; various human traits of gesture, expression, \&c., are paralleled among the "brutes;" reversions and monsters corroborate the alliance sadly enough.

(2) Morphological. The structure of man is like that of the anthropoid apes; none of his distinctions, except that of a heavy brain, are momentous; there are about eighty vestigial structures in his muscular, skeletal, and other systems. 
(3) Historical. Certainties in regard to remains of primitive man are few, but his individual development reads like a recapitulation of ancestral history.

To many, man seems too marvellous to have been naturally evolved, to others the evidence seems insufficient, but if the doctrine of descent is true for other organisms, it is surely true for man also.

As to the antiquity of the human race, it is certain that men lived in Europe in the later stages of the Ice Age, and there are indications of human life in Pliocene times. But as it is certain that man could not have arisen from any of the known anthropoid apes, and likely that he arose from an ancestral stock common to them and to him, it seems justifiable to date the antiquity of the race not later than the time when the anthropoid apes are known to have existed as a distinct race. This takes us back to Miocene ages.

If man was naturally evolved, the factors in the process require elucidation, but in regard to these we can only speculate. From what we know of men and monkeys, it seems likely that in the struggles of primitive man wits were of more use than strength. When the habits of using sticks and stone, of building shelters, of living in families began--and they have begun among monkeys-it is likely that wits would grow rapidly. The prolonged infancy, characteristic of human offspring, would help to evolve gentleness. But even more important is the fact that among monkeys there are distinct societies. Families combine for protection, the combination favours the development of emotional and intellectual strength. "Man did not make society; society made man."

Finally, it is plain that all repugnance to the doctrine of descent as applied to man should disappear when we clearly realise the great axiom of evolution, that "there is nothing in the end which was not also in the beginning." 


\section{CHAPTER XXVII.}

\section{COMPARATIVE PHYSIOLOGY.}

THE comparative study of the Physiology of the Invertebrates has not as yet been carried very far, though there are several careful investigations of particular problems. This chapter is an attempt to gather up some of the most important facts, in order especially to show what is sometimes forgotten, that physiology has much to say upon the general problem of the origin and maintenance of particular characters. A short note on abnormal physiological conditions and their bearing upon evolution has also been added.

The Physiology of the Nervous System has been very fully investigated in several cases among the Invertebrates, and we will therefore begin our survey with it.

We may say, in the most general way, that the function of the nervous system is to bring the organism into relation with the external world. The mechanism by which this is effected consists typically of three parts:-(1) the peripheral nerve endings, which receive the stimuli ; (2) the nerves, or paths by which the stimuli are conveyed to or from-(3) the central nerve cells. The peripheral end-organs with which we are most familiar are those of eye, ear, and the other special senses; but we must not forget that the termination of nerve in muscle-the so-called end-plate-is equally a peripheral nerve ending. All nerves are in $\mathrm{com}^{-}$ munication on the one hand with a peripheral organ, and on the other with central cells.

It is obvious, from the above definition, that neither Protozoa nor Sponges possess a nervous system. For in a Protozoon the receptive and perceptive mechanism is contained in the single cell, - any part of the protoplasm will 
respond to external stimuli. In Sponges, the transmission of stimuli is effected by the general protoplasm of the cellslittle division of labour being apparent-though here and there so-called nerve cells have been described.

Among the Coelentera, we find in Hydra special nerve cells, but, as proved by the familiar regeneration experiments, these are all similar and equivalent. On the other hand, among the "jellyfish," we find nerve centres and nerves quite distinctly differentiated. As we should expect, the nerve physiology differs in the Craspedota and the Acraspeda.

In the Craspedote forms the nervous system consists of a ring round the margin of the bell, giving off nerves which form a plexus among the muscles, and furnished with slight thickenings - the marginal bodies - at the bases of the tentacles. The ring controls the movements of the swimming bell; if it is totally destroyed the movement ceases, but the retention of a very small part is sufficient to maintain the movement. The parts of the ring are apparently equivalent to each other, any part being capable of transmitting motor impulses to the whole of the muscles effecting movement. The thickened areas of the ring seem to have a slightly more powerful effect than the undifferentiated parts, but the difference is not very marked; the marginal bodies are, however, distinctly sensitive to light. If a strong beam of light be thrown upon a swimming bell, it responds by more active contractions, and as the organisms are more active in light than in darkness, we may conclude that light (along with heat) acts as a constant stimulus. If the nerve ring is totally destroyed, the animal becomes motionless, and does not recover itself; if stimulated electrically or mechanically, it responds by a single contraction, or occasionally, in very vigorous specimens, by several.

In the Acraspeda the eight separate nerve centres preside over the swimming movements; if these are all destroyed, the movements cease. If the specimen is vigorous, however, it not infrequently, after a period of rest, resumes its movements, sometimes only fecbly, sometimes with a speed quite comparable to that of an uninjured specimen. If stimulated during the latent period, the Medusa usually responds with more than one contraction, thus being again 
contrasted with the Craspedote forms. Sensitiveness to light is exhibited in the same way as in the latter. The central nervous system is connected by a nerve plexus with the muscles which effect movement. Although little is known histologically of the way in which the nerves end in the muscles, yet physiologically, in its relation to poisons, the peripheral termination shows a remarkable resemblance to the "end plate," which characteristically occurs in the muscles of Vertebrates. We find here, therefore, even at this low stage, that the three distinct parts of a nervous system are quite clearly defined. It seems unlikely that division of labour has gone so far as to definitely differentiate sensory and motor nerves, but it is important to note that muscular contraction does follow the application of a stimulus. The difference as to the effect of the removal of the nerve centre in the two types is extremely interesting, but as yet unexplained.

In Sea Anemones the nervous system has been less fully investigated than in the Medusæ. There are no specialised nerve centres; nearly all parts of the body when separated seem to be able to respond to stimuli, so that the nerve cells must be scattered. The relation of the muscles to the nervous tissue has the same physiological complexity as in the Medusæ. An interesting point is the absence of the spontaneous movement which is so characteristic of the Medusæ. We have the same contrast often presented even in the life history of the individual, - compare the sessile hydroid and the active swimming bell, the fixed hydra-tuba and the pelagic jellyfish. Recently in the Great Barrier reef of Australia an Alcyonarian has been found, in which the polypes, though sessile, exhibit a constant rhythmic contraction and expansion of their tentacles, so that the tendency to exhibit continuous rhythm is widely spread in the Coelentera. The nervous system is apparently tolerably uniform in type throughout the group; what determines the physiological peculiarities has yet to be investigated. There are two rival explanations of rhythmic movements, such as those of the umbrella of the Medusæ. According to the first, it is caused by rhythmic stimuli, passing out from the nerve centres to the muscles concerned, and thereby causing the contractions. 'The other 
view is that the regular contractions are due to the activity of the muscles themselves. On this hypothesis, building-up processes go on in the muscles until extremely unstable substances are produced; these explode and break down into simpler compounds, the process being accompanied by an evolution of energy manifested by the contraction of the muscle. The process, repeated at regular intervals, causes the regular contractions. But this view seems to minimise unduly the function of nerve cells.

In Beroe, representing the Ctenophora, we can only notice that the sense organ, which is placed at the aboral pole, has to do with the movements. In contra-distinction to the conditions found in the Medusæ, we find that special parts of the central nervous system preside over special areas of the organism. This is a distinct advance in the direction of division of labour, and recalls the state of affairs in higher forms, where clusters of brain cells form what are called centres, which preside over particular organs.

Little is known of the nerve physiology of the members of the very heterogeneous group of "Worms." It is said that a decapitated earthworm can regenerate the anterior end with its cerebral ganglia. This would seem to indicate that there is little centralisation of the nervous system, and that the ganglia are all of nearly equal physiological importance. It seems more likely, however, that in, at any rate, most Annelids, the so-called "brain" does perform to some extent the function of a central nervous system, although the centralisation is only partial. In Lumbricus, sensory and motor nerve fibres are differentiated.

The nerve physiology of the Echinoderms has been very fully worked out, except in the case of the Holothurians. In the starfish, the nervous system consists of a ring round the mouth, from which nerves pass out to the rays, giving off branches to the tube feet. The whole surface of the body is sensitive to stimuli. The ring round the mouth co-ordinates the action of the different rays; if it is severed, the rays lose their power of acting in concert.

In Echimus also the ring round the mouth has a coordinating function; only when it is intact do the segments of the body act in unison. The ambulacral nerves branch freely to form the inner nerve plexus; from this, nerves pass 
out through the shell to the outer nerve plexus. If any spot on the outside of the shell be lightly stimulated, all the spines, pedicellariæ, and tube-feet in the neighbourhood bend towards the spot; if it be more strongly irritated, the spines and tube feet of the other segments come into play, and by their co-ordinated activity move the animal in a straight line away from the point of injury. The spines and tube feet thus exhibit two different forms of activity-one a mere local response to stimuli, the other a more complicated and co-ordinated action. The first is presided over by the external plexus, but for its complete accomplishment the internal plexus must be intact; a connection with the gullet ring is unnecessary, as the action is quite as efficiently performed when the ambulacral nerves are severed. Over the co-ordinated action of the spines and tube feet the internal nerve plexus presides, but connection with the gullet ring is absolutely necessary. The gullet ring is thus of great importance, but the co-ordinating action is not entirely limited to it. Each ambulacral nerve can coordinate the action of the tube feet of its own segment, when quite detached from the ring and the other ambulacral nerves. This nervous system is a considerable advance on that of the jellyfish, but the centralisation is still small.

In the Arthropods, as in the Annelids, the question of the value of the supra-cesophageal ganglia has been much debated. In Insects, according to Krukenberg, they are not of great importance as a co-ordinating centre, many complex movements being performed without the head. But this argument is hardly conclusive, for a decapitated tortoise may continue to walk along for several yards. The respiratory movements appear to be presided over by the ganglia of the abdomen; they, are still performed by separated segments, though their depth or frequency is often disturbed by the separation from the brain. In spite, however, of the independence of the ganglia of the ventral chain, the brain here, as in higher animals, directs the movements. In the Crayfish, while voluntary movements and the maintenance of equilibrium depend on the supra-œsophageal ganglia, the infra-œesophageal contain the centres for the co-ordination of the movements of eating, being reflex centres, as are all the remaining ganglia. In the Crab there 
is both morphologically and physiologically a much greater amount of concentration.

Among the Mollusca we find that in the Lamellibranchs the three sets of ganglia are of nearly equal importance. There is no defined central nervous system, a fact which we may correlate with the sedentary habit. The motor nerves to the great retractor muscles pass out from the adjacent ganglia; that is, the cerebral ganglia innervate the anterior retractor, the visceral the posterior. The closing of the shell is active, and is caused by the passage of impulses to the muscles along the motor nerves. The opening is more passive, as the elastic ligament causes the valves to gape when the muscles relax. This relaxation is caused by inhibitory nerves which inhibit the action of the motor nerves, and the muscles in consequence return to their former condition. The inhibitory nerves to both muscles pass out from the cerebral ganglia, but there is no evidence to justify the assumption that these have any "brain" function. The motor cells of the cerebral and visceral ganglia can be stimulated through many peripheral sensory nerves. The heart is innervated from the visceral ganglia, but some physiologists who minimise the importanee of the innervation maintain that the heart's activity is largely protoplasmic, and that the nerves have chiefly or wholly an inhibitory or trophic function.

Among the Gasteropods there is not much of special interest in regard to the nervous system.

In the Cephalopoda the supra-oesophageal mass is undoubtedly a true "brain." When it is destroyed the ordinary vital functions, such as respiration, circulation, \&c., are unaltered; the animal continues to respond to external stimuli, but the power of "volition" is gone; if left to itself, it remains in one position until death ensues. From this fact we see that the centres, or presiding nerve cells, for all the automatic functions are placed elsewhere than in the brain, but that this originates all the "voluntary" muscular movements. Of the various centres, the respiratory is located in the pleural ganglia; from it nerves pass out which end in the stellate ganglia, and are both motor and sensory for the mantle. This centre is not self-acting, that is, not automatic, as are the corresponding centres in higher 
Vertebrates, but is only reflexly stimulated into activity by impulses borne by afferent nerves from some part of the body. It seems most reasonable to suppose that this condition is primitive, and that the automatic form of activity is derived. The centre for the movement of the chromatophores is in the sub-œsophageal mass. The activity of the heart is said by some to be purely "protoplasmic," but co-ordination of the parts of the heart, the branchial hearts, \&c., is effected by means of the ganglia placed in the course of the visceral nerves and their branches. The arms are very well innervated, containing a central nervous axis ; even a severed arm is said to exhibit powerful reflex movements. This property is probably of some use in the free hectocotylised arm of the male.

\section{The Physiology of Nutrition.}

We have seen that by means of the nervous system the animal is brought into relations with the external world. It is in consequence constantly evolving energy in the form of movement, heat, electrical energy (Gymmotzs, \&rc.), or light (phosphorescent animals). We now proceed to consider the manner by which this loss of energy is made good, that is the Nutrition of the Tissues. Inasmuch, however, as the food of animals typically consists of very complex organic substances, the process of Digestion must first be considered. Digestion is the process by which the organic substances of the food are broken down into simpler substances, which are soluble and diffusible, and capable of being assimilated and built up into the substance of the tissues.

\section{Digestion.}

In the familiar case of the Amoba, solid food particles are ingested, they are surrounded by fluid, and eventually the fuid is absorbed with the products of digestion, while the useless and indigestible residue is rejected. Primarily, this process differs from that found in Vertebrates in that it is intra-cellular, instead of being the result of the action of extra-cellular ferments. There is some doubt as to whether the Protozoan type of digestion is also due to ferments, or whether the living protoplasm has the power of directly 
inducing changes in substances brought into contact with it. Krukenberg succeeded in extracting a peptic ferment from the plasmodium of "Flowers of Tan," but did not believe that it could have a digestive function on account of the alkalinity of normal protoplasm. Metchnikoff, however, has demonstrated in some cases that the fluid of "food vacuoles" is acid, and seems to hold that all digestion is due to ferment action. Miss Greenwood has also demonstrated an acid in the vacuoles of several Protozoa, and described the process of digestion. In any case we must note that the formation of ferments appears to be a characteristic of protoplasm; but that as we ascend in the scale of being these ferments are more and more utilised in the digestive processes, and tend to be limited to the walls and outgrowths of the alimentary canal. We may note here (as is more fully explained in the section on Comparative Pathology) that in most animals certain cells retain the primitive Protozoan capacity for taking up and digesting solid particles, while the general body cells have lost it.

It is a fact of common observation that in parasites the alimentary canal tends to be absent or degenerate; nutrition is usually affected by simple absorption of the juices of the host. The exact physiological reason for the disappearance of the gut is not obvious. Further, the method by which such parasites are protected from the action of the ferments of their hosts is not clear. The reason is perhaps in part the thickness of the cuticle, which is composed of substances not amenable to ferment action. Again, Frenzel claims to have found an anti-enzyme in Gregarines, which neutralises the action of the host's intestinal juices. The problem is analogous to that suggested by the fact that the cells of the gut escape during life the action of its juices, by which they are often attacked after death. Frenzel, indeed, compares a Gregarine to an absorbing intestinal cell.

\section{Digestion in the Invertebrata.}

In the Cœlentera, ferments have been extracted from the bodies of jellyfish and sea anemones. In some cases a tryptic ferment was extracted from the reproductive organs, a peptic from the tentacles and mesenteries. The secretion 
of ferment is thus not confined to the digestive region, and according to Krukenberg the ferments are not employed for the digestion of food outside the formative cells. In his experiments he found that solution and absorption of food particles only took place when the particles were in actual contact with the digestive region. In Sponges, digestion is purely intra-cellular; in Hydra, both intra- and extracellular digestion seem to occur.

Among the higher worms, Hirudo is distinguished by the absence of an enzyme containing secretion. The blood contained in its pouched gut is simply absorbed by the walls. The similarity of this method of nutrition to the purely parasitic one found in Cestodes and Trematodes, has been advanced as an additional reason for associating the leeches with flat worms rather than with the Chætopoda. The habit of feeding on the blood of other animals may, however, have led to some of the leech's peculiarities.

In most of the other Annelida-Aphrodite, Arenicola, Lumbricus, \&c.-a ferment capable of acting upon proteids has been found. It is closely allied to the tryptic ferment of Vertebrates, but is not identical in all its chemical reactions. It has been termed iso-trypsin, and like trypsin it is only active in neutral or alkaline solution. It appears to be confined to the Annelida. The intestinal "cæca" found in Aphrodite and others are not absorptive areas, but merely reservoirs of secretion. They are rendered necessary by the fact that the gland cells are constantly active, and not merely, as in Vertebrates, stimulated to action by the presence of food in the intestine. The process is therefore closely analogous to the secretion of bile by the vertebrate liver, where the liver cells are constantly active, and the gall bladder, like the cæca of worms, serves as a store chamber. But as the bile is probably not to any extent a digestive fluid, and as the true digestive glands of Vertebrates are not constantly active, the conclusion is suggested that the constant activity of the cells in the worm is a primitive condition. In most Annelida a diastatic ferment also occurs, which possesses as usual the power of converting starch into sugar.

Turning to the Echinoderms, we find that in star fishes tryptic, peptic, and diastatic ferments are all found. The 
voluminous cæca are not areas where digestion goes on, but, as in Aphrodite, merely reservoirs for the secretion. In the Holothurians no digestive glands have been, as yet, found in connection with the gut, nor can any ferment be extracted from its walls. The contents of the gut are, however, mixed with a peptic ferment; this can also be extracted from extra-intestinal parts of the body, so that ferment secreting glands must exist. A similar diffuseness in the occurrence of ferments is very common among the Echinoderma. It is therefore asserted that digestion must go on in various parts of the body, and that it is not limited to the alimentary I tract. Diastatic ferments are very frequently present.

In Arthropods, peptic, tryptic, and diastatic ferments are common. The peptic ferment is uniform throughout the group, and has been termed "homaropepsin," to indicate that it differs considerably from the pepsin of Vertebrates. On the other hand, the tryptic ferment is not distinguishable from that of Vertebrates. Both peptic and tryptic ferments are often secreted by the same gland. The reason for this and its physiological consequences are unknown.

In the Mollusca, œsophageal glands, usually called "salivary," are very common, and often large. In some cases, as in Dolium and others, these glands secrete only mineral acids (sulphuric in Dolium). According to Bunge, these acids, like the hydrochloric of the Vertebrate stomach, have chiefly an antiseptic action, destroying Bacteria introduced with the food. If this be correct, the advantage of the cesophageal position is very obvious. The true digestive gland of Molluscs is the "liver," which is usually very large, and often secretes diastatic, peptic, and tryptic ferments. Its secretion, like the perivisceral fluid, is always neutral or slightly alkaline. Peptic digestion may be rendered possible (r) by the presence of acid derived from the cesophageal glands, or (2) by the acid nature of the food; but nothing is known with certainty. In the Eolide, the gut gives off prolongations which pass upwards into the dermal papillæ. Into these the contents of the alimentary canal pass, and here both digestion and absorption take place. They thus become filled with chyle, which is directly absorbed by the tissues.

In Ascidians, Krukenberg was in many cases quite unable 
to find clear indications of the presence of ferments in the gut. He inclined to the opinion that in many members of the class, the digestive processes are as simple as those of the Coelentera.

\section{The Nutrition of the Tissues.}

After the complex food substances have been broken down into simpler ones, they must be carried to the tissues, there to be employed in repairing waste, or in growth. In a simple Protozoon there is no difficulty; like a primitive community, the single cell supplies its own wants, and the question of transport is never raised. In a Metazoon, on the other hand, as in a civilised state, there is much division of labour, and the question of the transport of manufactured material becomes very important.

In a Vertebrate the blood is the great transporting agent; into it the products of digestion are ultimately poured; from it waste products are filtered. It is itself, however, confined to closed vessels, and does not come into close connecton with the tissues; these are, strictly speaking, nourished by the lymph, which bathes the tissues throughout, and also communicates freely with the blood stream. Thus the lymph is the "middleman" between blood and tissues. In Vertebrates the lymph has not the respiratory significance which the blood has in virtue of its red corpuscles.

In most of the lower aquatic forms of life, the fluid within the body differs little from that which surrounds it. Thus, as we should expect, the fluid which bathes the cavity of a sea anemone or a jellyfish, filling the hollow tentacles of the one and the canal system of the other, is little more than sea water. It contains no formed elements, no dissolved albumens, no organic substances capable of forming a loose combination with oxygen-that is, no respiratory pigment. It is thus certainly not a nutritive fluid; the tissues must be nourished by the products of digestion passing from cell to cell. It is, however, of use in respiration. Like other sea water, it contains dissolved oxygen ; and we must suppose that the endoderm cells take up the oxygen they require directly from it, as the ectoderm cells do from the surrounding water. The fluid has also an excretory signi- 
ficance; it carries away waste products, both solid and gaseous, and removes these from the body.

The fluids of Ascidians, Lamellibranchs, and of a few Gasteropods, are all classed by Krukenberg as hydrolymph. They consist largely of water, but contain in addition formed elements, or dissolved proteids. In Ascidians, the body fluid contains a small amount of dissolved proteids, and some pigmented corpuscles. Its real function has not been fully investigated; the presence of dissolved proteids seems to suggest digestion by ferments in spite of Krukenberg's negative results.

In Echinoderms we find that both a perivisceral fluid and blood enclosed in special blood vessels are present. Of the blood little or nothing is known, the technical difficulties in the way of isolation being very great. The perivisceral fluid contains numerous formed elements, and a small amount of dissolved proteids. It probably performs the functions of the lymph of Vertebrates, but is said to have a respiratory function in addition.

In Insects the blood is of the nature of Vertebrate lymph. It is very rich in dissolved proteids, and undoubtedly serves for the nutrition of the tissues. It has no respiratory function, in spite of the frequent occurrence of various pigments in it-a point of some theoretical interest. The tracheal tubes carry air, and so oxygen, to every part of the body; an oxygen carrying fluid formed by the organism itself thus becomes quite unnecessary. We may, physiologically, compare the tracheal system of the Insect with the canal system of the Medusa. In both cases the external medium is carried by special channels to the tissues themselves; in both cases the body fluids have in consequence no respiratory significance.

In "Worms," Crustaceans, most Gasteropods, and Cephalopods, the blood is both respiratory and nutritive. It is "hæmolymph," combining the functions of the blood and the lymph in Vertebrates.

In Annelid worms the blood contains small formed elements, and a number of respiratory pigments, some of which will be discussed later.

In Cephalopods the blood contains formed elements similar to leucocytes, while in the plasma a respiratory 
pigment known as hæmocyanin is dissolved. This consists of a proteid substance united to copper, and is the only albuminoid present in the plasma. It is very widely spread among Gasteropods, Crustaceans, \&c., but is not universal. Its absence in some Crabs, which have apparently no compensating metal containing pigment, perhaps indicates that too much stress should not be laid upon its respiratory significance. Lipochrome pigments are very frequently present in the blood of Crustaceans and Cephalopods; their use is unknown.

If we compare the condition seen in Cephalopods with that found in Vertebrates, we find that in the latter it is the red blood corpuscles which are the oxygen carriers, while in the former the plasma alone subserves respiration. Even in Vertebrates, however, the waste carbonic acid is carried in the plasma in combination with its soda, so that the plasma is not entirely unconcerned with respiration. In both Vertebrates and Cephalopods the plasma has a nutritive function.

\section{Products of Metabolism.}

In the course of those processes of breaking down and building up of protoplasm which constitute what is called the metabolism of the animal, we constantly find that certain by-products are formed. These may be simply waste matters, capable of subserving no useful purpose in the animal economy, or they may have important functions. As we ascend in the scale we find that these by-products are more and more utilized for different purposes. Thus many pigments which are widely distributed seem to be practically functionless, but in particular cases they come to be of importance in producing protective coloration, and so on. Among the products of metabolism, we will discuss here only two groups, the skeletal tissues and the colouring matters.

\section{The Skeletal Tissues of Animals.}

Even in the very simplest forms of life we find that the soft protoplasm is frequently provided with protective structures. In many cases the organism merely takes up inorganic particles from the surrounding medium, and with these fashions a shell for itself, as we find in some of the Foraminifera. In most of the Foraminifera, however, a true 
shell of lime is "secreted" by the protoplasm. This taking up of inorganic particles is not the only way in which the tendency to form a protective covering is manifested in the Protozoa. The Corticata are encased in a firm sheath which shows many of the characters of true skeletal substances; while familiar organic compounds such as cellulose, gelatine, and horny substances, are not unknown. Even in the Protozoa, therefore, we see in germ the power, so characteristic of higher animals, of producing by modifications of their protoplasm, specific substances capable of affording both support and protection.

Skeletal tissues are usually characterised by the physical property of being firm and often hard to the touch, while generally retaining some elasticity, and the chemical one of offering great resistance to ordinary chemical agencies. They are naturally passive and inert, and, so far as the internal skeleton is concerned, are formed in the connective tissues, and not in relation to important organs, except in pathological conditions. Lime salts are frequently associated with some of the common skeletal substances, but this is by no means universal even for the same substance. Thus the collagen of the bones of Vertebrates is associated with abundant lime salts, while that of the cartilages contains an inconsiderable quantity. Again, chitin in the Crustacea is strongly impregnated with lime, while in Insecta lime salts are practically absent. Within the limits of the Cephalopoda, the conchiolin of the "shell" may be associated with lime in one genus and quite devoid of it in another. Within the Mollusca, indeed, we find every stage in shell development represented ; from the papery "shell" of Aplysia to the enormous edifices seen in some of the tropical forms. It seems difficult in these cases to avoid the conclusion that the disproportionate bulk is due to necessities of growth, and has no relation to the needs of the animal.

The following is a brief account of some of the more important skeletal substances :-

\section{Tunicin.}

Tunicin, or animal cellulose, is a carbohydrate very similar to, if not identical with, the cellulose of plants. It 
occurs in the test of Tunicates as a cuticular product of the epidermal cells, and is said to have been also found in some cases in the body of the animal. Dr. Ambronn asserts that he has found a body giving similar chemical reactions in connection with the chitin of Arthropods, and also in some Molluscs.

\section{Chitin and Conchiolin.}

Chitin and Conchiolin may serve as examples of skeletal substances containing nitrogen, but giving only one of the proteid reactions. Several other well-known substances are included in this group, such as spongin, byssus-substance, \&c. All are characterised by their great resistance to chemical agents.

Chitin is characteristic of Arthropods, but also occurs in the shell of Lingula and in "cuttle-bone." It yields on decomposition reducing substances of the nature of sugar, and is a derivative of a carbohydrate. It is a product of ectodermal cells, and is the only organic skeletal substance in Arthropods. In the Crustacea it is usually associated with lime salts and with various pigments.

Conchiolin is found in Bivalves, Gasteropods, and some Cephalopods. It strongly resists the action of mineral acids, and, like Chitin, is unaffected by ferments. It varies greatly in composition, even within the limits of a species, and is probably a mixture of nearly related substances. The substance which forms the horny axis in Gorgonida and Antipatharia is closely allied to conchiolin.

\section{Collagen and Keratin.}

Collagen and Keratin are well-known examples of skeletal substances which contain sulphur as well as nitrogen, and give some, though not all, of the chemical reactions of proteids. Collagen is found in the bones and cartilages of Vertebrates; it is characterised by yielding gelatin when boiled with water. Unlike the substances previously mentioned, it is readily digested by pepsin, but is not affected by tryptic ferments. In Vertebrates it is found as an intracellular matrix, secreted by little patches of formative cells. In Cephalopods in the head region there is a modified form of collagen which is readily acted on by trypsin. Collagen 
is said to have been found in Sipunculus, in Holothurians, and in Brachiopods.

The dead epidermal cells of many Vertebrates form a cuticle of keratin over the living cells below. The process is said to be one of dehydration; but it is not a simple drying up as it occurs quite as markedly in aquatic animals. In the hairs and nails of mammals, the feathers of birds, the scales of fishes, keratin forms a protective covering; in some mammals it further furnishes powerful offensive "horns." Keratin is also found in the egg shells of Birds, Reptiles, and Selachians; in the first group it is associated with lime salts. It also occurs in the sheath of nerve fibres, which is explicable enough when we remember that in development the nerves arise from the ectoderm. Keratin has also been found among worms. It is extremely resistant to the action of ferments.

\section{The Colouring Matters of Animals.}

Colour in animais is either due directly to pigments, or, as in the case of structural colours, is simply a light effect. To the latter division belong the often brilliant colours of some Annelids, and the gorgeous metallic tints of the plumage of some birds. In this section we confine ourselves to the pigments.

Physiologically, we may classify pigments in various ways: there are the respiratory pigments, of which Hæmoglobin is perhaps the best example; the waste products, such as the pigments of some butterflies' wings (which are allied to uric acid), and probably the pigments of bile; finally, there are numerous pigments of whose primary physiological meaning we can say nothing, but which may be secondarily of use in producing protective, warning, or sexual colouring. Such are the pigments of the skin in Crustacea, caterpillars, Amphibians, and so on.

The most important respiratory pigments are Hæmoglobin, Hæmocyanin, and Hremerythrin; some others have been named by different authors, but their respiratory significance seems uncertain.

Homoglobin occurs in all the Craniate Vertebrates, and also not infrequently among the different Invertebrate 
classes, usually in isolated members of groups. It consists of a pigment, Hæmatin, united to a proteid; the pigment contains iron in its molecule. In the higher Vertebrates, Hæmoglobin is during life continually undergoing decomposition. The jron is mostly retained within the body, and is probably re-utilised in metabolism; the proteid is probably also utilised, while the iron-free Hæmatin undergoes chemical changes, and is excreted as the pigments of bile and urine. In pathological conditions Hæmatin may be deposited in the tissues in different forms. This deposition of pigments derived from Hæmatin, which only occurs in disease in Vertebrates, seems to occur normaily in certain Invertebrates, in the shells of some Gasteropods, the skin of star fishes, \&c., apparently in some cases in forms in which Hæmatin itself does not occur. With regard to the distribution of Hæmoglobin, we must note that the occurrence of the same pigment in widely separated forms indicates similar physiological processes, but not necessarily a similar function. Thus, Hæmoglobin is said to occur in considerable quantity in the perivisceral fluid of Holothurians, where we can hardly suppose that its respiratory importance is very well marked. In fact, the wide and irregular distribution of Hæmoglobin among Invertebrata forbids the supposition that it can there possess the supreme importance which it has in higher Vertebrates.

The efficiency of Hæmoglobin is of course due to its power of forming a loose combination with oxygen; it is, however, also capable of uniting with other gases, as $\mathrm{CO}$ and $\mathrm{CO}_{2}$.

Hemocyanin is found in many Crustacea, and in Molluscs. In the oxidised state it is a colourless substance, but turns blue when reduced. It is absent in the few Crustaceans (Daphnia, \&c.), which contain Hæmoglobin, and is a true respiratory pigment. It consists of a proteid united to copper, but in a few cases it is said that the copper is replaced by iron. There is said to be more difficulty in reducing Hæmocyanin than there is with Hæmoglobin.

Hcemerythrin occurs in the blood of Gephyreans; it undergoes a colour change dependent on processes of oxidation and reduction.

The number of pigments which we can definitely classify 
as respiratory, or as waste products resulting from the decomposition of such, is very small; in the great majority of cases we can say nothing as to function. In some cases, however, we can point to the physical or chemical conditions which favour the development of pigments. Thus in some animals the pigments indicate the normal reaction of the tissues. For example, those sea anemones which contain peptic ferments are red, those which contain tryptic, yellow or brown. Again, light and absence of oxygen are necessary for the development of certain of the black pigments; the black pigment in a frog's skin disappears in an atmosphere of pure oxygen. It is a fact of common observation that portions of animal's bodies which are shaded from the light tend to be pale in colour. Another interesting point about colouring matters is that they are not always produced by the animal in which they occur. Thus green oysters owe their colour to an insoluble pigment taken from the diatoms of the food, and deposited in the tissues; the colour of "red mullet" is perhaps due to the crustaceans of the food. It has been suggested that the uniformity of tint observed in many animals living in the same environment, as, for example; in the Sargasso Sea, may be due to a similar cause.

Most of the pigments fall into chemical groups; of these the best defined and perhaps most widely spread is the Lipochrome group. The Lipochromes are characterised (I) by their colour, which varies from yellow through orange to red; (2) by giving in the dry state a blue coloration with strong $\mathrm{H}_{2} \mathrm{SO}_{4}$; (3) by their ready decomposition when exposed to light, when they lose their colour and yield cholesterin; (4) by the fact that they consist only of carbon, hydrogen, and oxygen. They occur in both plants and animals, especially in the "liver" in Arthropods and Molluscs, in the skin and body fluids of Crustacea, in the skin of many Vertebrates, \&c. According to Krukenberg, the lipochromes probably often take origin from fatty bodies; if this is correct, their association with the "liver" in Invertebrates is interesting.

The above is a very brief account of some of the chief known facts of animal coloration. It may serve to show that much must be discovered as to the primary physiological 
import of pigments, before the vexed subject of their secondary import can be definitely dealt with.

\section{Comparative Pathology.}

Within recent years pathologists have begun to study diseased conditions comparatively-an obviously rational method which promises to lead to very important results, both practical and theoretical. For man has no monopoly of disease, and some of the processes by which unhealthy conditions are dealt with by the organism are more readily studied in lower animals than in him. Of this we shall give one illustration. In 1862 , Hreckel observed that grains of indigo injected into the mollusc Thetys were surrounded by the amœboid blood corpuscles. Other observers followed the hint which this suggestive fact supplied, and Metchnikoff, above all others, has shown the important rôle which these amoboid cells fill in waging war against intruding germs and parasites, in surrounding irritant particles, in repairing injuries, and the like. In fact, Metchnikoff has worked out the evolution of the phagocyte, as he terms the amcoboid cell whose function it is to discharge the rôle above indicated. It is this evolution, as stated in Metchnikoff $s$ lectures on the comparative pathology of inflammation (Trans., London, I893), which we shall take in illustration of comparative pathology.

The simplest conditions are of course illustrated by the Protozoa. These enjoy comparative immunity from the injurious effects of wounds and from infectious disease. For injuries are very rapidly repaired; a fragment, if nucleated, can usually regrow the whole; infecting organisms are in most cases digested, and irritant particles are got rid off. This is particularly true of the amceboid Protozoa, the Rhizopods. Sometimes, moreover, the Bacteria or other micro-organisms which produce disease are actually avoided, for some of the Protozoa exhibit that sensitiveness (or chemiotaxis) which distinguishes the wandering amoeboid cells or phagocytes of higher animals. Thus, a Myxomycete will creep towards a decoction of dead leaves and away from a salt solution, and will "prefer" a nutritive fluid which is not swarming with Bacteria to one that is. 
In Sponges, infection is often avoided and parasites are excluded by the closure of the inhalent pores. But if entrance be effected, the microbe or irritant is dealt with by the amoeboid cells of the middle stratum, which have also to do with ordinary digestion. Thus disease in Sponges is very rare. In Hydra, where there is virtually no mesogloea, the flagellate or amœboid cells lining the gut act as so many "stationary phagocytes." Thus, in these two cases, the functions of intra-cellular digestion and of "phagocytosis" are combined.

In other Coelentera, as in Hydra, the ordinary digestive functions are restricted to the endoderm cells lining the gut, but most of them have, what Hydra has not, wandering amceboid cells in the mesoglœa, and these deal with microbes, parasites, and irritants. The same is true of simple worms, such as Turbellarians.

In higher worms and in Echinoderms the phagocytic cells are usually situated on the peritoneal epithelium, or float in the perivisceral fluid. They may have many functions, respiratory and excretory, for instance, but the phagocytic function is of great importance, all the more so that the gut has now lost its power of intra-cellular digestion.

Crustaceans, insects, molluscs have a more or less welldeveloped blood vascular system, and there are often amoboid cells in the blood like the white blood corpuscles of most Vertebrates. But the phagocytic function still depends, largely at least, on wandering phagocytes in the body cavity or in the mesodermic tissues. But as the vascular system in these forms is usually lacunar, no rigid distinction can be drawn between phagocytes in the blood and phagocytes in the body cavity. No case is known, however, in which the leucocytes or white blood corpuscles of an Invertebrate exhibit the power of migrating through the walls of the blood vessels to the seat of irritation or injury, as is common among Vertebrates.

Among Vertebrates, as the circulatory system becomes gradually more highly developed from Tunicates onwards, the number of extra-vascular phagocytes is reduced, and more and more depends on those of the blood. In the fin of a young newt an injury or an infection may be dealt with solely by the migratory phagocytes of the connective tissue; 
in the most frequently observed case- the tail of a tadpole in which the blood vessels are formed - the extra-vascular phagocytes are greatly aided by leucocytes, which work their way through the walls of the vessels or are liberated by a lesion; in other cases all may depend on these leucocytes. It is important also to notice that the endothelial cells of the blood vessels seem by their contractility to assist the passage (or diapedesis) of the leucocytes; sometimes, moreover, they may themselves leave the wall of the vessel to deal with Bacteria introduced into the blood.

We are not here concerned with Metchnikoff's thesis that "inflammation generally must be regarded as a phagocytic reaction on the part of the organism against irritants - a reaction carried out by the mobile phagocytes sometimes alone, sometimes with the aid of the vascular phagocytes or of the nervous system." We are immediately interested only in noticing how these mobile cells, retaining many of the qualities of the ancestral Amobæ, perform in the animal body numerous functions, struggling with invading Bacteria, surrounding and engulfing irritant particles, and repairing wounds. And from the most general point of view it is evident that one of the numerous factors determining the fate of an organism in the struggle for existence is its power of resisting Bacteria. If phagocytes be not present, there must be some other means of defence; thus the Nematodes have found this in their firm resistent cuticles.

The processes of disease in higher animals have been very carefully investigated from the evolutionist's point of view by Sutton. He points out that some of the causes which pathologists recognise as operating to produce disease, (viz., hypertrophy or atrophy of organs or structures, and coalescence of parts originally distinct), are also "factors in evolution," which biologists recognise in their theories of the progress of life. Thus, descending to particular cases, we find that the long claws of the sloth and bat, the great curved teeth of the Babirussa, are paralleled in pathological conditions by the elongated nails and hoofs of Birds and Ungulates kept in unnatural conditions, by the curved incisors of Rodents which have lost the corresponding teeth of the other jaw. It is unnecessary here to multiply examples of greatly hypertrophied organs, normally present in certain 
animals, but occurring in disease in others; many will occur on reflection. In considering many of these cases, we must recognise the law of correlation, and realise that the structures of a particular animal are not commonly the best conceivable, but the best that can be attained under the given conditions.

Pathological new formations may arise in response to mechanical stimulation, as in the case of corns and warts, or may be due to aberrant physiological processes. Thus, cancer is regarded by many as in origin an aberrant glandformation, and only occurs in regions of the body where glands are normally found. It . is a senile modification of an ordinary developmental process. Pathological bony growths seem to have their origin in patches of cartilage remaining from the primitive cartilage of limb or brain case, and so are continuations of the ordinary process by which cartilage is replaced by bone.

Rudimentary or imperfectly formed organs are in general specially liable to disease. Such rudimentary organs are usually relics of past history. We must thus recognise that, just as in the history of civilisation some of the most cruel wrongs are only good institutions belated, so in the history of disease the most dire pathological conditions may be historically only the result of belated physiological processes. In some cases we may perhaps say more hopefully that pathological processes may be the starting point for new physiological evolutions.

Brief as the above comparative survey of Physiology and Pathology is, it may serve to give the student some impression of the intricacy of life, and act as a relief from mechanical theories of Variation, Selection, and Heredity. It is an attempt to look from the inner side upon the great problem which is constantly being worked out before us:-given the potentialities of protoplasm and certain chemical and physical conditions, to find the best adaptation to a given environment. 


\section{CHAPTER XXVIII.}

\section{GEOGRAPHICAL DISTRIBUTION OF ANIMALS.}

As similar animals tend to occur where the conditions of life are similar, we are warranted in speaking of a pelagic fauna, an abyssal fauna, a littoral fauna, and so on. Let us briefly consider this grouping of animals according to their haunts.

\section{The Pelagic Fauna.}

The pelagic fauna includes all the animals of the open sea, both drifters (Plankton) and swimmers (Nekton). The physical conditions in which they live are very favourable:there is room for all, sunshine without risk of drought, and an evener life throughout the day and throughout the year than is to be found elsewhere except in the abysses of the deep sea. Moreover, the minute pelagic Algæ afford an inexhaustible food supply to the animals. It is not surprising, therefore, to find that the open sea has been peopled from the earliest times of which the rocks give us any life record.

The fauna is representative, exhibiting great variety of types, from the minute Noctiluca which sets the waves aflame in the short summer darkness, to the giants of modern times -the whales. It includes a few genera of Foraminifera, rich in species, all the Radiolarians, many Infusorians, Medusæ and Medusoids, Siphonophora and Ctenophora, many "worm" types and a Holothurian, a legion of Crustaceans and a few Insects (Halobatidæ), such Molluscs as Pteropods, Heteropods, and many of the Cephalopods, such Tunicates as Salpa and Pyrosoma, many fishes, a few turtles and snakes, besides some well-known birds and mammals. 
The fauna of the open sea is representative, but there are few of the types which we can suppose to have lived there always. It may be that forms like the minute water-fleas have been there almost from the first, $k$ ut most bear the impress of lessons which the open sea fould never have taught them.

Pelagic animals tend to be delica in and translucent; many are phosphorescent. The nulis of species, differing from one another whin a relatively narrow range, is often enormous, thus ahuut 5000 species of Radiolarians are known. The hưge number of individuals, which frequently occur in great swarms, is equally characteristic. Perhaps both facts indicate that the conditions of life are relatively easy, as is also implied in the limitless food supply afforded by the unicellular Algæ.

\section{The Abyssal Fauna.}

Through the researches of the Challenger and similar expeditions, we know that there is practically no depth-limit to the distribution of animal life, though the population is denser at moderate depths than in the deepest abysses, and though there is probably a thinly peopled intermediate zone between the light-limit and the greatest depths. We know, too, that there are representatives of most types from Protozoa to Fishes, though Sponges and Echinoderms preponderate, and that the distribution tends to be cosmopolitan, in correspondence with the uniformity of the physical conditions.

The abyssal fauna includes many flinty sponges, some corals and sea-anemones, possibly a few medusæ, annelids and other "worms" on the so-called red clay, representatives of the five extant orders of Echinoderms, abundant Crustaceans, representatives of most of the Mollusc types, and peculiarly modified fishes, many more than half-blind, others catching with darkness-eyes the fitful gleams of phosphorescence.

As to the physical conditions, the deep-sea world is in darkness, for a photographic plate is not influenced below 250-500 fathoms; it is extremely cold, about $34^{\circ} \mathrm{F}$., for the sun's heat is virtually lost at about 150 fathoms; the 
pressure is enormous, thus at 2500 fathoms it is about $2 \frac{1}{2}$ tons per square inch; the cold water in sinking brings down much oxygen; it is quite calm, for even the greatest storms are relatively shallow in their influence; there are no plants (except perhaps the resting phases of some Algæ), for typical vegetable life depends upon light, and not even Bacteria, otherwise almost omnipresent, are known to flourish in the great depths. A strange, silent, cold, dark, plantless world! The animals feed upon one another and upon the debris which sinks from above, including the rain of pelagic Protozoa, whose continual dying seems rather to contradict Weismann's doctrine of their immortality.

We do not clearly know when the colonising of the depths began, but there is much to be said for the view that an abyssal fauna was, at most, scanty before Cretaceous ages. One of the arguments is as follows :-In ancient days, when warmth-loving plants flourished in the far north, when there was no ice-bound polar sea, the abyssal water cannot have been so cold as it is now, it would therefore contain less abundant oxygen, and this scantiness would make life more difficult. But whenever the peopling of the abysses occurred, it must have been gradual. It is likely that most of the pioneers migrated outwards and downwards from the shore region (in a wide sense), following the drift of food; it is possible that others, e.g., some Crustaceans, sank from the surface of the open sea. The boreal character of many deep-sea animals has been often remarked, and it is plausible to suppose that there was a particularly abundant colonisation in the Polar regions, and a gradual spreading towards the Equator as the Poles became colder. Perhaps the richness of the fauna at the Equator may be thought of as in part due to the meeting of two great waves of life from the Poles.

The abyssal conditions of life tend to uniformity over vast areas, just as in the open sea. But, on the whole, life must always have been harder in the depths than on the surface. The absence of plants, for instance, involves a keener struggle for existence among animals. Thus, although many abyssal forms, e.g., sea anemones, live a passive sedentary life, waiting for food to drop into their mouths, the majority are less easy-going. The deep-sea has been a sterner school of life than the surface.

\section{The Littoral Fauna.}

At a very early date the shores were peopled, and the fauna is very rich and representative. From the strictly 
Littoral zone, exposed at low tide, with its acorn shells and periwinkles, limpets and cockles, to the Laminarian zone (to I 5 fathoms) with its sea slugs and oysters, where the great seaweeds wave listlessly amid an extraordinary keen battle, to the Coralline zone (I 5-40 fathoms), with its carnivorous buckies, what variety and abundance, what crowding and struggle!

There are Infusorians and Foraminifera, sponges horny, flinty, and limy, zoophytes and sea-anemones, a mob of worms, star-fishes and sea-urchins, crabs and shrimps, acorn shells on the rocks and sandhoppers among the jetsam, a few insects about high-tide mark, sea-spiders clambering on the seaweeds, abundant bivalves and gasteropods, sea-squirts in their degeneracy, besides fishes, a few reptiles, numerous shore birds, and an occasional mammal. The shore fauna is thus very representative, rivalling in its range that of the open sea, far exceeding that of the abysses.

The conditions of life on the shore are in some ways the most stimulating in the world. It is the meeting place of air, water, and land. Vicissitudes are not exceptional, but normal. Ebb and flow of tides, fresh-water floods and desiccation under a hot sun, the alternation of day and night felt much more markedly than on the open sea, the endless variations between gently lapping waves and blasting breakers, the slow changes of subsidence or elevation,these are some of the vicissitudes to which shore animals are exposed. The shore is rich in illustrations of keen struggle for existence and of life-saving shifts or adaptations, such as masking, protective coloration, surrender of parts, and "death feigning." We may think of it as a great school where many of the great lessons of life, such as moving head foremost, were learned.

\section{The Fresh Water Fauna.}

Perhaps the most striking fact in regard to the animals which live in fresh water is their uniformity. The number of individuals in a lake is often immense, but the number of species is relatively small, the number of types still smaller. In widely separated basins and in different countries the same forms occur. 
We may distinguish a littoral, a surface, and a deepwater lacustrine fauna. The deep-water forms are chiefly Rhizopods, Turbellarians, Nematodes, Leeches, Chætopods, Amphipods, Isopods, Entomostraca, a few Arachnids, some insect larvæ, and molluscs, and the general opinion is that these are derivable from the shore-fauna of the lake, which includes similar forms, along with a few others, such as the fresh-water sponge and Hydra. On the other hand, the surface lacustrine fauna, consisting of water-fleas, Rotifers, Infusorians, \&c., widely and uniformly distributed, is said not to be derivable from the shore forms. In transparency, in gregariousness, in nocturnal habit, and in other ways they present a marked analogy with the marine Plankton. How are we to account for their origin and wide distribution?

(I.) To explain the uniformity Darwin referred to the birds which carry organisms from watershed to watershed, to the carrying power of the wind, and to changes of land level which bring different river beds into communication. But this is not enough.

(2.) It seems very likely that some of the fresh water forms have migrated from the sea and seashore through brackish water to rivers and lakes. As the possibility of making the transition depends on the constitution of the animal, it is intelligible that similar forms should succeed in different areas.

(3.) There seems much force in what Credner and Sollas emphasise that many lakes are dwindling relict-seas of ancient origin. Granted a fairly uniform pelagic fauna, e.g., before Cretaceous times, we can understand that the conversion of land-locked seas into lakes would imply a decimating elimination, and, as the conditions of elimination would be much the same everywhere, the result would be uniformity in the survivors.

\section{Minor Faunas.}

(a.) Of Brackish Water. - We are warranted in speaking of a brackish-water fauna, because of its uniformity in widely separated regions. It does not seem to be a mere physiological assemblage, varying in each locality, but rather a transition fauna of ancient date, a relic of a littoral fauna once more uniform. The fact is that the power to live in brackish water is not very common; it runs in families.

(b.) Cave fauna. - In America, thanks very largely to the labours of Packard, about Ioo cave animals are known; in Europe the number is about 300 , the increase being largely due to the occurrence of about Ioo species of two genera of beetles in European caves. In the famous Mammoth Cave of Kentucky, which has over 100 miles of passages, with streams, pools, and dry ground, there are over 40 different species 
of animals. The temperature is very equable, varying little more than a degree throughout the year; it is, of course, dark; and there are no plants other than a few Fungi. Thus the conditions present some analogy with those of the deep sea. The fauna is of much interest to the evolutionists, for we wonder how far the peculiarities of the caveanimals, $e_{. g}$, absence of coloration and frequent blindness, are due to the cumulative effect of the environment and disuse, or how far they represent the survival of fortuitous variations, and the result of the cessation of natural selection along certain lines. Have the seeing animals found their way out, leaving only the blind sports, which crop up even in daylight? or is the loss of eyes the result of disuse and absence of stimulus? Or again, if it be granted that pigment is an organic constitutional necessity, e.g., a waste-product, while coloration is explicable as an adaptation wrought out in the course of natural elimination, then the question arises whether the cessation of natural selection-a condition awkwardly called "panmixia" - which might account for the disappearance of the coloration when there is no premium set upon it, can also account for the loss of pigment, that is of a character which was not acquired in the course of natural selection? (See Beddard's Animal Coloration). Our only answer at present is that there is need for experiment.

(c.) Parasitic fauna. - It seems legitimate to rank together those animals whose habitat is in or on other organisms, from which they derive subsistence, without in most cases killing them quickly, if at all, nor on the other hand, rendering them any service. Among ectoparasites, there are such forms as fish lice and many other Crustaceans, numerous insects such as lice and fleas, and Arachnids such as mites. Among endoparasites, there are Gregarines, some Mesozoa, many Nematodes, most Trematodes, all the Cestodes, many Crustaceans, insect larvæ, and Arachnids.

The parasitic habit is a common one, illustrated by many different types. It is associated with degeneration, varying according to the degree of dependence, with great nutritive security and prolific reproduction, but with enormous hazards in the fulfilment of the life history.

Parasitic animals must be distinguished $(a)$ from epiphytic or epizoic animals which live attached to plants or animals, but are in no way dependent upon them, e.g., barnacle on Norway lobster ; (b) from commensals (p. 160), who live in some degree of partnership, but without in any way preying upon one another, e.g., craband sea anemone; and $(c)$ from symbions, who live in close partnership, or symbiosis (p. II4), e.g., Radiolarians and Algre. But between these habits there are many gradations, and from close association there is always an easy transition to parasitism.

\section{Terrestrial Fauna.}

The colonising of dry land has doubtless been a gradual process, as different types wandered inland from the shore, or became able to survive the drying up of fresh water basins. The fauna includes some Protozoa, e.g., Amoba terricola, 
which lives in moist earth, some of the Planarians, Nematodes, Leeches, Chætopods, and other "worms," a few Crustaceans like the wood lice (Oniscus), many insects and Arachnids, a legion of slugs and snails, most adult Amphibians, most Reptiles, many Birds, and most Mammals. Among Vertebrates certain fishes are of interest in having learned to gulp mouthfuls of air at the surface of the water, to clamber on the roots of the mangrove trees, or to lie dormant through seasons of drought. But among Vertebrates, Amphibians were the first successfully to make the transition from water to dry land.

It is important to bear in mind that many a stock may, in the course of its evolution, have passed through a variety of environments. Thus the thoroughly aquatic Cetaceans were probably derived from a land stock common to them and to the Ungulates, and may have passed through a fresh water stage. Without going further back, we have here an illustration of the zigzag course of evolution.

We cannot believe in any alrupt transition from the shore to terra firma. It has been a slow ascent, slow as the origin of dry land itself. Thus, mud-inhabiting worms, dwellers in damp humus, bankfrequenting animals, those which find a safe retreat in rottenness or within bolder forms, dot the path from the shore inland. Many have lingered by the way, many have diverged into cul-de-sacs, many have been content to keep within hearing of the sea's lullaby, which soothed them in their cradles.

Simroth, in his work on the origin of land animals, seeks to show that hard skins, cross-striped muscle, brains worthy of the name, red blood, and so on, were acquired as the transition to terrestrial life was effected. Let us take the last point by way of illustration. Iron in some form seems essential to the making of hremoglobin, but iron compounds are relatively scarce and not readily available in the sea, they are more abundant in fresh water, and yet more so as the land is reached. Therefore it is suggested that it was as littoral animals forsook the shore for the land, via fresh water paths, that iron, in some form, entered into their composition, became part and parcel of them, helped to form hæmoglobin or some analogous pigment, and thus opened the way to a higher and more vigorous life.

\section{The Aerial Fauna.}

The last region to be conquered was the air. Insects were the first to possess it, but it was long before they were followed. The flying fishes flapped their fore-fins above the foam as they leapt; the web-footed tree frogs, Draco volans, with skin spread out on elongated ribs, and various 
lizards began to swoop from branch to branch; some of the ancient Saurians flopped their leathery skin-wings; a few arboreal mammals essayed what the bats perfected; and the feverish birds flew aloft gladly.

Perhaps a keen struggle among insects, or such events as floods, storms, and lava-flows would prompt to flight, perhaps it was the eager males who led the way, perhaps the additional respiratory efficiency, produced by the outgrowth of wings, gave these a new use. Perhaps the high temperature of bircls-an index to the intensity of their metabolism-may have had to do with the development of those most elaborate epidermic growths which we call feathers. But we must still be resigned to a more or less ingenious "perhaps."

\section{The Relation of the different Faunas to one another.}

As we have already hinted, the problem of the evolution of faunas is still beyond solution, and as this is not the place for the marshalling of arguments, I shall content myself with stating various possibilities.

(a.) According to Moseley, "The fauna of the coast has not only given origin to the terrestrial and fresh-water faunas, it has throughout all time, since life originated, given additions to the pelagic fauna in return for having received from it its starting point. It has also received some of these pelagic forms back again to assume a fresh littoral existence. The terrestrial fauna has returned some forms to the shores, such as certain shore-birds, seals, and the polar bear; and some of these, such as the whales and a small oceanic insect, Halobates, have returned thence to pelagic life."

"The deep-sea has probably been formed almost entirely from the littoral, not in the most remote antiquity, but only after food, derived from the debris of the littoral and terrestrial faunas and floras, became abundant in deep water."

"It was in the littoral region that all the primary branches of the zoological family tree were formed; all terrestrial and deep-sea forms have passed through a littoral phase, and amongst the representatives of the littoral fauna the recapitulative history, in the form of series of larval conditions, is most completely retained."

(b.) According to Agassiz, Simroth, and others, if one may venture to compress their views into a sentence, a littoral fauna was the original one, whence have been derived, on the one hand, the pelagic and abyssal faunas; on the other hand, the fresh-water and terrestrial faunas.

(c.) According to Broolis, a pelagic fauna was primitive, whence have been derived the tenants of the shore and the inhabitants of the deep sea. To the latter, however, a possibility of ascending again is not denied. 
(d.) Personally, I regard the most probable ancestral home of animals as some region not far from the shore, and I picture the relations as follows :-

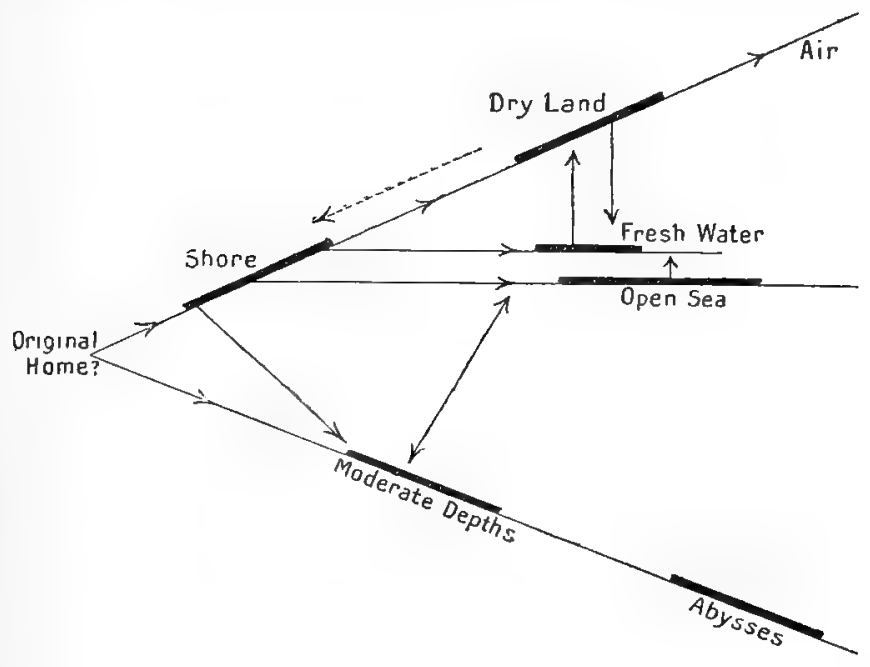

The more detailed Problems of Geographical Distribution.

Leaving the general, and at present very obscure, problem of the evolution of faunas, let us briefly notice some of the more detailed questions of distribution. We shall content ourselves with stating (I) a few of the outstanding facts, (2) the factors determining why some animals are here and others there, and (3) the usually recognised zoo-geographical regions.

Some of the Outstnnding Facts of Geographical Distribution.

(a.) Widely separated countries may have an essentially similar fauna. Thus, there is much in common between Britain and Northern Japan, and there is so much agreement between the North European (Palæarctic) and the North American (Nearctic) fauna that many unite the two regions in one (Holarctic). . 
(b.) Closely adjacent countries may have quite different faunas. Thus, the Bahamas and Florida, Australia and New Zealand are peopled by very different animals. But the best illustration is that of two little islands, Bali and Lombok, in the Malay Archipelago, which are separated by "Wallace's Line," a strait only fifteen miles wide at its narrowest part. They differ from each other in their birds and quadrupeds far more widely than Britain and Japan.

(c.) Regions with very different faunas are in many cases connected by transition areas. Thus a journey from the North of Canada to Brazil would show a fairly gradual transition from an Arctic to a tropical fauna.

(d.) At the same time there are regions whose fauna is exceedingly distinctive and sharply defined. Thus the Mammalian fauna of Australia is distinctively Marsupial, and nowadays there is only one family of Marsupials-the American opossums-found beyond the Australasian limits.

(e.) Another striking fact is the "discontinuous distribution" of certain types, by which we mean that examples of a type may occur in widely separated regions without there being any representatives in the intermediate area. The general explanation is that the type in question once enjoyed a wide distribution, as the rock record shows, and that the conditions favourable to survival have been found in widely separated places. Thus, of the genus Tapir, there are some four species in South and Central America, while the only other species occurs in Malacca and Borneo. Similarly the Camelidæ are represented by one genus in the Old World and another in South America, and the insectivorous Centetidæ are represented by five genera in Madagascar, and one in Cuba and Hayti.

\section{The Factors determining Distribution.}

There are six factors which comline to determine the particular distribution of an animal. These may be conveniently considered in pairs.

(a) Distribution is in part determined by the constitution of the animal and the physical conditions of the region. Thus snakes diminish rapidly in numbers towards the poles, their constitution being in most cases ill-adapted to withstand cold ; thus crayfishes are absent from districts where the fresh water does not contain sufficient lime salts for their needs.

(b) Distribution is in part determined by the position of the animal's original home (which is often an unknown fact), and by the available 
means of dispersal. Thus, so far as we know, the Old World has been the exclusive home of the anthropoid apes, and there they have remained; thus bats, being able to fly, have a more cosmopolitan distribution than most other mammals; thus amphibians, being unable to withstand salt water, are absent from almost all oceanic islands.

(c) Distribution is in part determined by the actual changes (geological, climatic, \&c.) which have affected different regions, and by "bionomic" factors, i.e., the relations between the animal in question and other organisms, whether animals, plants, or man. Thus it is plain that we cannot understand the fauna of Australia without knowing the geological fact that part of this island was once connected with the Oriental continent by a bridge of land across the Java Sea. The Australasian mammalian fauna consists of survivals and descendants of a Mesozoic mammalian fauna which has been exterminated everywhere else, except in the case of the American opossums. The original Australian mammals were saved, not by any virtue of their own, but by the earth-change which insulated them. Similarly, it is the geologist who helps us to understand the faunal diversity on the two sides of "Wallace's line," or the absence of amphibians, reptiles, and mammals from the Canaries. That much will also depend on the animal's power of surviving the struggle for existence in different regions is too obvious to require exposition. We need only think of the way in which man has in a few years altered the distribution of many birds and mammals, sometimes indeed reducing it to $n i l$, or increasing it to desperation.

To sum up, the chief factors determining geographical distribution are- $(x)$ the constitution of the animal, $(2)$ the physical conditions of the region, (3) the position of the original home, (4) the means of dispersal, (5) the historical changes of the earth and its climate, and (6) the bionomic relations.

\section{Zoo-Geographical Regions.}

I shall simply quote a paragraph from Professor Heilprin's work-The Geographical and Geological Distribution of Animals (Internat. Sci. Series. London, 1887), a very valuable book for the student, especially as it considers distribution in space and time together.

"By most naturalists (Wallace, Sclater, and others) the terrestrial portion of the earth's surface is recognised as consisting of six primary zoological regions, which correspond in considerable part with the continental masses of geographers. These six regions are:-

"I. The Palcarctic, which comprises Europe, temperate Asia (with Japan), and Africa north of the Atlas Mountains; also Iceland, and the numerous oceanic islands of the North Atlantic: 
"2. The Ethiopian, embracing all of Africa south of the Atlas Mountains, the southern portion of the Arabian Peninsula, Madagascar, and the Mascarene Islands, and which, consequently, nearly coincides with the Africa of geographers :

"3. The Oriental or Indian, which embraces India south of the Himalayas, Farther India, Southern China, Sumatra, Java, Bali, Borneo, and the Philippines:

"4. The Australian, comprising the continent of Australia, with Papua or New Guinea, Celebes, Lombok, and the numerous islands of the Pacific:

" 5. The Nearctic, which embraces Greenland, and the greater portion of the continent of North America (excluding Mexico):

"6. The Neotropical, corresponding to the continent of South America, with Central America, the West Indies, and the greater portion of Mexico."

Professor Heilprin makes several modifications on this scheme of distribution : (a) uniting Palæarctic and Nearctic in one Holarctic realm; (b) establishing a special Polynesian realm for the scattered island groups of the Pacific; and (c) defining three transition regions, (I) around the Mediterranean, intermediate between Palæarctic, Ethiopian, and Oriental, (2) Lower California between IVestern Holarctic and Neotropical, and (3) the Austro-Malaysian islands lying to the east of Bali and Borneo, inclusive of the Solomon islands, a region intermediate between Oriental, Australian, and Polynesian. It seems also convenient to recognise two polar regions,-Arctic and Antarctic. Of the last, we have had as yet only glimpses.

It may be useful to map out the divisions as follows :-

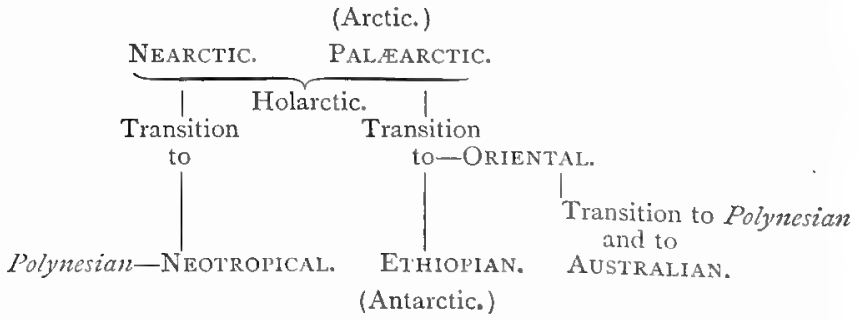




\section{CHAPTER XXIX.}

\section{THEORY OF EVOLUTION.}

IN Chapter VI. we indicated the nature of the evidence which has led naturalists all but unanimously to accept the doctrine of descent as a modal interpretation of organic nature. The data of physiology and morphology, combined with what is known of the history of the race and the development of the individual, have led us to believe that the forms of life now around us are descended from simpler ancestors (except in cases of degeneration), and these from still simpler, and so on, back to the mist of life's beginnings. In other words, we believe that the present is the child of the past and the parent of the future. This is the general idea of evolution.

But while this general idea, which is a very grand one, is usually recognised as the simplest interpretation of the facts, we remain in doubt as to the factors of the process by which the world of life has come to be what it is. This uncertainty is in part due to the complexity of the problem, in part to the relative novelty of the inquiry - for precise etiology is not yet fifty years old, in part also to the fact that while there has been much theorising, there has been comparatively little experimenting or connected observation as to the modes and causes of evolution.

With the exception of Alfred Russel Wallace and a few others who believe that it is necessary to postulate spiritual influxes to account for certain obscure beginnings, e.g., of the higher human qualities, evolutionists are agreed in seeking to explain the evolution of plants and animals as a continuous "natural" process, the end of which was 
implicit in the beginning. In so doing, they follow the method of analysis, endeavouring to explain the facts in their lowest terms. But as the biologist's lowest term is living matter, and as one aspect of this is, in favourable conditions, known as thought, there is no reason to call the evolutionist's analysis "materialistic"-if anything opprobrious be meant by that adjective. The common denominator of the biologist is as inexpressibly marvellous as the philosopher's greatest common measure, if indeed, they are not practically the same.

\section{The Tzeo Great Problems.}

Our uncertainty in regard to the factors of evolution is so great, that I cannot venture here to do more than indicate (a.) what the great problems are, and (b.) the general drift of the most important suggestions which have been made towards their solution.

The two great problems before the evolutionist are :-

(a.) What is the nature and origin of variations, i.e., of those organic changes which make an organism appreciably different from its parents or its species?

(b.) What are the directive factors which may operate upon given variations, determining their elimination or their persistence, and helping towards the familiar but puzzling result-the existence of distinct and relatively well-adapted species?

Secure answers to these two questions must be found in reference to the present; as our data accumulate it will be more possible to argue back to the past.

It may be convenient to speak of the factors which cause variation as primary or originative, and of the factors which operate upon or direct the course of variation as secondary or directive. As far as practical results are concerned, the two sets of factors are of equal importance.

\section{The Nature of Variations.}

We mean by variations those changes in organisms which make them appreciably different from their parents or from their species. 
The term of course includes not only material differences, but also those whose only demonstrable expression is psychical. Thus, an increase in maternal affection is as important and real a variation as the sharpening of a canine tooth.

It may also be useful to distinguish variations in size, symmetry, number of appendages, and so on, from more qualitative variations in chemical composition, such as the appearance of a new pigment, but this distinction is only a matter of convenience, as it is only a matter of degree.

Again, variations occur which may be called continuous, being merely minute increments or diminutions of certain parental or specific characters. These are related to one another much in the same way as are the successive stages in the continuous growth of an individual.

But other variations occur which deserve to be called discontinuous. For, without the appearance of transitional stages, marked variations crop up, reaching with apparent suddenness to what must be called new and may withal exhibit a measure of perfectness.

That both kinds of variations occur is a fact of life; the possibility of both is probably a primary quality of organisms; but we are only beginning to know the relative frequency of the two kinds and their respective limits, and we know almost nothing as to their causes (see Bateson's "Materials for the Study of Variation, I894").

\section{Primary or Originative Factors.}

What causes variation? This is the fundamental question, but it is the least answerable.

It is, indeed, an axiom or a truism, that changes in any animate system are evoked by changes in the larger system of which the organism forms a part. In other words, the stimulus to organic change must always be ultimately traceable to the environment, but this is implied in our conception of living matter, and does not help us to understand the immediate conditions which lead to change.

In the absence of sufficiently precise data, we can do little more than point out various possibilities :- 


\section{(a) Variations due to Change in the Environment.}

There is abundant proof that changes in surrounding pressure, in the chemical composition of the medium, in food supply, in heat, light, \&c., may be followed by changes in the organism upon which these influences play. Changes in the body of the organism follow changes in the environment. But (I) it is difficult to discriminate between variations which may be spoken of as the direct results of environmental influence, and those to which the organism was already definitely predisposed, and to which the environmental change supplied only the stimulus. (2) We have not at present sufficient data to enable us to state that variations arising in or acquired by the body of an individual organism as the result of surrounding change, do as such in any degree specifically affect the reproductive cells. In other words, we cannot at present say that "environmental variations" are transmissible. And if they are not, their importance in evolution is only indirect.

(b) Variations due to Change in Function.

It is an undoubted fact that the bodily structure of an animal may be changed by the increased use of certain parts, or the disuse of others, in short, by some change of function. This change of or in function may be directly prompted by some change in the external conditions of life, or it may be the expression of a deeper variation in the animal's material constitution or mental character. But important as these functional variations and their results are to the individual, we are uncertain as to their importance for the race, for we do not know to what extent (if any) the results are transmissible.

\section{(c) Variations due to Changes in the Germ Cells.}

In many cases of variation, particularly those which appear in early life, it is not possible to suggest any environmental or functional condition which may be regarded as the stimulus or the cause. We are led in such cases to believe that the variation in bodily structure or habit is the expression of some novelty in the protoplasmic constitution of the germ-cells. Then, hiding our ignorance, we say that the variation is germinal, constitutional, congenital, or blastogenic. 
But why should there be changes in the germ cells? Perhaps, because living matter is very complex and unstable, and because it is of its very nature to differentiate and integrate; perhaps because the immediate environment of the germ cells (blood, body cavity fluid, sea water, \&c.) is complex and variable. But it may be more important to recognise that every multicellular organism, reproduced in the usual way, arises from an egg cell fertilised by a spermatozoon, and that the changes involved in and preparatory to this fertilisation, or "amphimixis," make new permutations and combinations of living substances or vital qualities not only possible but necessary.

\section{Secondary or Directive Factors.}

(I.) Natural Selection. - The distinctive contribution which Charles Darwin and Alfred Russel Wallace made to etiology was their theory of Natural Selection.

By Natural Selection is meant that process whereby, in the ordinary course of nature, certain organisms, e.g., certain members of a species, are more or less rapidly eliminated, while others are allowed to survive longer.

That some forms, e.g., in one family, should succeed less well than others, depends obviously on the fact that all are not born alike, depends, in other words, on the fact of variation.

That there should be elimination is necessary $(a)$ because a pair of animals usually produce many more than a pair, and the population tends to outrun the means of subsistence, and (b) because organisms are at the best only relatively well-adapted to their conditions of life, which are variable. These two primary facts and their subsequent consequences, e.g, that some animals feed upon others, that there may be more males than females, \&c., render some struggle for existence necessary, though this phrase must be used, as Darwin said, "in a wide and metaphorical sense," including all endeavours for the well-being, not only of the individual, but of its offspring.

'The facts then are-that variations constantly occur, that some members of a species or family are necessarily less fitly adapted than others, and that the course of nature is such that these relatively less fit forms will tend to be 
eliminated, while the relatively more fit will tend to survive. As many variations re-appear generation after generation, and may become gradually increased in amount, the continuance of the selective or eliminating process will work towards the origin of new adaptations and new species.

The importance of Natural Selection as a secondary factor in evolution will vary according to stringency of the eliminating process, and it must be noted that the "struggle for existence" varies in intensity within wide limits, that it requires to be investigated for each case, and cannot be postulated as a force of nature.

The importance of the factor will also depend on the number, nature, and limits of the variations which occur. Thus a new species might arise, either by the occurrence of a discontinuous variation of considerable magnitude, or by the eliminating process acting for many generations on a series of minute continuous variations.

Darwin also believed in the importance of sexual selection, in which the females choose the more attractive males, which, succeeding in reproduction better than their neighbours, tend to transmit their qualities to their numerous male heirs. But this and other forms of reproductive selection may be regarded as special cases of natural selection, and require no particular emphasis. Nor is the importance of sexual selection or preferential mating admitted by so great an authority as Wallace.

(2.) "Ysolation."-Under this title Romanes, Gulick, and others include the various ways in which free intercrossing is prevented between members of a species, e.g., by geographical separation, or by a reproductive variation causing mutual sterility between two sections of a species living on a common area. Without some "isolation" tending to limit the range of mutual fertility within a species, or bringing similar variations to breed together, a new variation is liable, they say, to be "swamped" by intercrossing. But definite facts as to this "swamping," and in many cases as to the alleged "isolation," are hard to find, nor can we say that a strong variation will not persist unless it be "isolated." Romanes's view, however, was that "without isolation, or the prevention of free intercrossing, organic evolution is in no case possible. Isolation has been the universal condition 
of modification. Heredity and variability being given, the whole theory of organic evolution becomes a theory of the causes and conditions which lead to isolation."

SUMMARY OF EVOLUTION THEORIES.

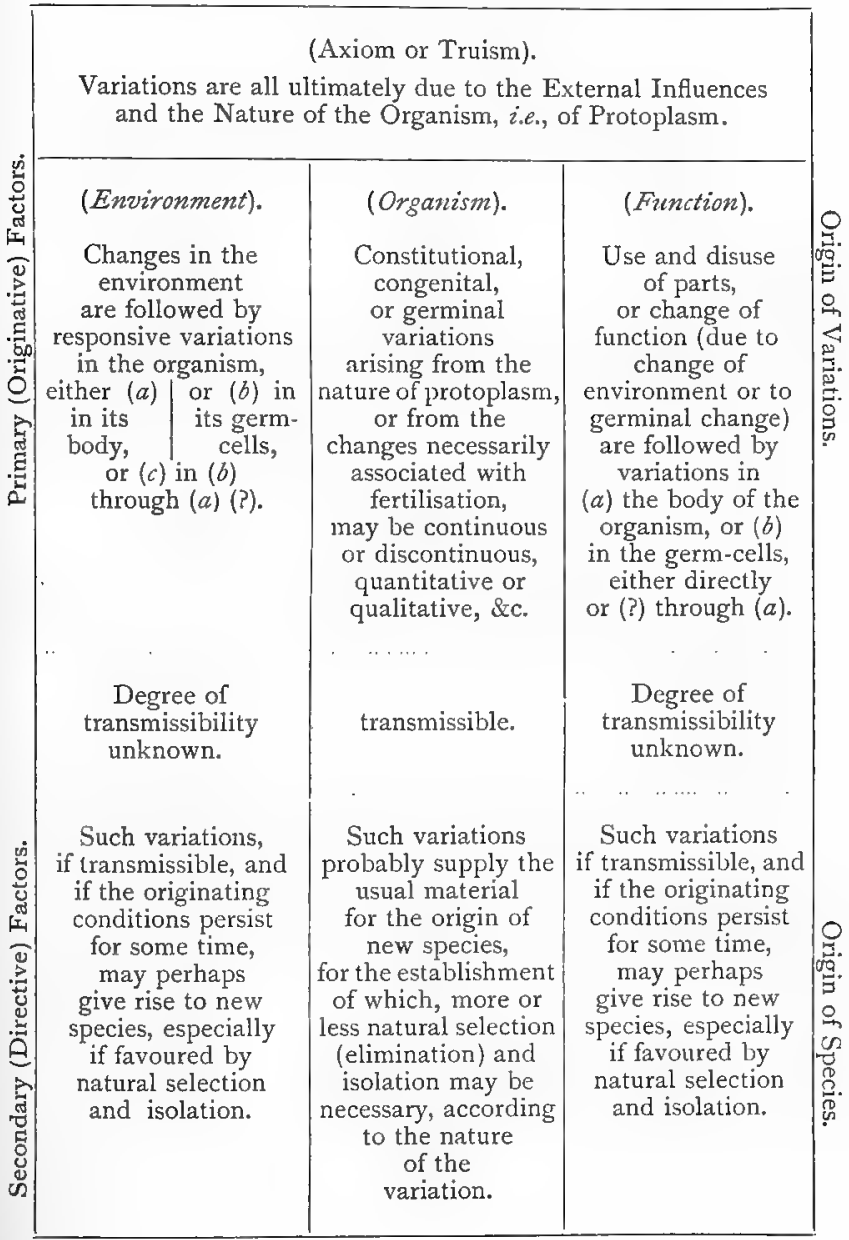





\section{APPENDIX.}

SOME ZOOLOGICAL BOOKS.

\section{INTRODUCTORY :-}

F. Jeffrey Bell, Comparative Anatomy and Physiology (Lond. I 887 ).

C. Lloyd Morgan, Animeal Biology (Lond. I889).

T. H. Huxley and II. N. Martin, A Course of Elementary Instruction in Practical Biology (Lond. I888), revised edition by G. B. Howes and D. H. Scott.

A. Milnes Marshall and C. H. Hurst, A Course of Practical Zoology (3rd Ed., Lond. I892).

T. Jeffrey Parker, Elementary Biology (2nd Ed., Lond. I893).

J. Arthur Thomson, The Study of Animal Life (Lond. 1892).

\section{TEXT-BOOKS OF ZOOLOGY :-}

T. H. Huxley, Anatomy of Invertebrates (I877), and Anatony of Vertebrated Animals (1871).

C. Claus, Grundzïge der Zoologie (4th Ed., 1880-82), and his smaller Text-Book of Zoology (translated by Sedgwick, I884-5.)

Hatchett Jackson's edition of Rolleston's Forms of Animal Life (Oxford, I888).

Text-Books (mostly with similar titles) by Boas, R. Hertwig, Kennel, I1. A. Nicholson, A. S. Packard, R. Perrier, Shipley, and many others.

J. Ritzema Bos, Agricullural Zoology (translated by Davis, Lond. I894.)

Books as Guides to Practical Work:-

C. Vogt and E. Yung, Traité d'Anatomie Comparée pratique (Paris, I 885-95); also in German.

T. J. Parker, Zootomy (Lond. 1884).

Huxley and Martin, op. cit.

A. Milnes Marshall and C. H. Hurst, op. cit.

W. K. Brooks, Handbook of Invertebrate Zoology for Laboratories and Sea-side Work (Boston, 1882).

P. Girod, Manipulations de Zoologie (1879-8I). 
Books as Guides to Practical Work-continued.

A. Bolles Lee, Microtomist's Vade-Mecum (3rd Ed., 1893).

An Atlas will help the student greatly, if he does not use it too much, e.g.:-

G. B. Howes, Atlas of Practical Elementary Biology (Lond. I885). W. R. Smith and J. S. Norwell, Illustrations of Zoology (Edin. 1889). A. de Vayssièro, Atlas d'Anatomie Comparée des Invertébrés (Paris, I889).

C. B. Briuhl, Zootomie aller Thierklassen (Wien).

General Morphology:-

Ernst Hæckel, Generelle Morphologie (Berlin, I866).

Herbert Spencer, Principles of Biology (Lond. 1864-66).

W. His, Unsere Körperform (1875).

G. Jaeger, Allgemeine Zoologie (1878).

P. Geddes, Art. "Morphology" (Encyclop. Brit.)

Classification, see:-

E. Ray Lankester, Art. "Zoology" (Encyclop. Brit.)

W. A. Herdman, Phylogenetic Classification of Animals.

Works on Comparative ANaTOMy:-

Besides the classic works of Cuvier, Meckel, Milne Edwards, \&c.Richard Owen, Comparative Anatomy of Vertebrate Animals (4th Ed, I87I).

T. H. Huxley, op. cit.

C. Gegenbaur, Elements of Comparative Anatomy (trans. by F. Jeffrey Bell, Lond. 1878).

A Lang, Text-Book of Comparative Anatomy (trans. by H. M. and M. Bernard, Lond, in progress).

R. Wiedersheim, Comparative Anatomy of Vertebrata (trans. by W. N. Parker, Lond. I886). And a larger work untranslater.

B. Hatschek, Lehrbuch der Zoologie (1888).

F. Leydig, Lehrbuch der Histologie (Comparative) (I857).

Works on Comparative Physiology:-

Claude Bernard, Phinomines de la Vie Commune aux Animanx et aux Végétaux (1878).

Paul Bert, Lécons sum la Physiologie comparie de la Respiration (1870).

C. F. W. Krukenherg, Vergleichend-Physiologische Studien and Vorträge ( 188 I-89).

F. Jeffrey Bell, Comparative Anatomy and Physiology (Lond. I887).

A. B. Griffiths, Comparative Physiology (1891).

Halliburton, Physiological Chemistry (1891).

Bunge, Physiological and Pathological Chemistry (trans. I8go). 
Embryology :-

F. M. Balfour, Comparative Embryology (2 vols., Lond. I880-8I).

M. Foster and F. M. Balfour, revised by A. Sedgwick and W. Heape, Elements of Embryology (Lond. I883).

A. C. Haddon, Introduction to the Study of Embryology (Lond. I887).

O. Hertwig, Lehrbuch der Entwichlungsgeschichte des Menschen und der Wirbelthiere (trans. by E. L. Mark, 3rd Ed., I893).

E. Korschelt and K. Heider, Lehrbuch der Vergleichenden Entwicklungsgeschichte der Wirbellosen Thiere (Jena, 1890-93).

L. Roule, Embryologie Générale (Paris, I892).

A. Milnes Marshall, Vertebrate Embryology (1893).

C. S. Minot, Human Embryology (I892).

PALAONTOLOGY :-

H. A, Nicholson and R. Lydekker, Mamal of Palaontology (2 vols., Lond. and Edin. I889).

K. A. von Zittel, Handbuch der Palcontologie (completed I893).

M. Neumayr, Die Stämme des Thierreichs (vol. I., Wien and Prag, I 889).

Gaudry, Les Enchainements du Monde Animal (1889-90).

Carus Sterne (Ernst Krause), Werden und Vergehen (3rd Ed., Berlin, I886).

Also text-books by Bernard (1893), Koken (1893).

Geographical Distribution :-

A. R. Wallace, Geographical Distribution (2 vols, Lond. 1876).

A. Heilprin, The Geographical and Geological Distribution of Animals (Lond, 1887).

Trouessart, La Geographie Zoologique (Paris, 1890).

W. Marshall, in Berghaus' Physikal Atlas (Leipzig, I887).

Boots of Naturalist Travellers, e.g.:-

Charles Darwin, Voyage of the Beagle (Lond. I844, New Ed., I890).

H. W. Bates, Naturalist on the Amasons (New Ed., Lond. 1892).

T. Belt, Naturalist in Nicaragua (2nd Ed., I888).

A. R. Wallace, Malay Arihipelago (1869), Tropical Nature (1878), Island Life (I880).

Wyville Thomson, The Depths of the Sea (1873), Voyage of the Challenger (1885).

H. N. Moseley, Naturalist on the Challenger (1879, New Ed., I892).

W. H. Hudson, Naturalist in La Plata.

A. E. Brehm, Fron North Pole to Equator (translation Ed. by J. Arthur Thomson, with bibliography, I 895). 
Comparative Psychology:-

G. J. Romanes, Animal Intelligence and Mental Evolution of Animals.

C. Lloyd Morgan, Aninual Life and Intelligence and Introduction to Comparative Psychology (Lond. 1894).

General Natural History:-

Brehm's Thierleben (3rd Ed., by Pechuel-Losche, Io vols., Leipzig and Wien, I890-1893).

Cassell's Natural History (Ed. by P. Martin Duncan, 6 vols., I882).

Standard or Riverside Natural History (Ed. by J. S. Kingsley, 6 vols., I 888).

Royal Natural History (Ed. by R. Lydekker, in progress).

Books on EvoluTion :-

Charles Darwin, Origin of Species (1859, \&c.).

Alfred Russel Wallace, Darwinism (1889).

Herbert Spencer, Principles of Biology (I866, \&c.).

Ernst Hæecke], Generelle Morphologie (I866, \&c.).

For more recent books, see my "Study of Animal Life." To the list there given must be added two books in particular, Weismann's Germ-Plasm (1893) and Bateson's Materials for the Study of Variation (I894).

General Works of Reference:-

W. Hatchett Jackson's edition of Rolleston's Forms of Animal Life (Oxford, I888). A very valuable work, with special bibliographies.

Leunis, Synopsis des Thierreiths, re-edited by Ludwig (I Hanover, I886).

Bronn, Klassen und Ordmungen des Thierreichs (I859-I895).

E. Ray Lankester and others, Zoological Articles reprinted from Encyclop. Brit. (Lond. I89I).

Monograpinic Series, e.g.-

Reports of the Voyage of H.M.S. "Challenger."

Fauna und Flora des Golfes von Neapel.

Catalogues of the British Museum-Natural History.

RECORDS OF RESEARCH :-

Joumal of Royal Dicroscopial Soizety, edited by F. Jeffrey Bell (6 parts in the year).

Zoological Record, edited by D. Sharp (yearly).

Zoologisches Jahresbericht (Naples) (yearly).

Anatonische Ergebnisse (Merkel \& Bonnet) (yearly).

Science Progress, edited by $\mathbf{F}$. Bretland Farmer (monthly).

Natural Science (monthly). 


\section{HISTORY OF ZOOLOGY :-}

W. Whewell, History of Inductive Sciences (1840).

J. V. Carus, Geschichte der Zoologie (I872).

E. Perrier, La Philosophie Zoologique avant Darwin ( 1884 ).

E. Hæckel, Natural History of Creation (trans. Lond. 1870).

E. Ray Lankester, Article "Zoology" (Encyclop. Brit.).

H. A. Nicholson, Natural Fistory: its Rise and Progress in Britain (1888).

E. Krause, Die Allgemeine Weltanschaumngen (1889).

H. F. Osborn, From the Greeks to Darwin (1894).

\section{BIBLIOGRAPHY :-}

Hatchett Jackson and Rolleston, op. cit.

L. von Graff, Bibliotheh des Professors der Zoologie atnd veroleichenden Anatomie (Leiprig, I89I).

Bibliotheca Historico-naturalis, Engelmann, (I700-I846).

Bibliotheca Zoologica, Carus and Engelmann, (1846-6I).

Bibliotheca Zoologica, continued by Taschenberg, (I86I-I880).

Bibliotheca Zoologice et Geologiar, I. Agassiz, edited by Strickland and Jardine (Lond. I848-1854).

Royal Society's Catalogue of Scientific Papers (fr. I800).

Annual Bibliographies in Zoological Record, \&c., cited above.

C. Davies Sherborn, Books of Reference int the Natural Sciences, Natural Science, V. (August I894). 
- 
I N D E X. 



\section{N D E X.}

PAGE

Aard-vark, . . . . 690

Aard-wolf, . . . . 7 I 5

Abdominal pores, . . 472,477

" ribs, . . 568, 584

Abducens nerve, . . . $44 \mathrm{I}$

Abomasum, . . . . . 697

Absorption, . . . . 23

Abyssal Fauna, . . . 754

Acanthias, . . . . 506

Acanthin, . . . . $\mathrm{IO2}$

Acanthocephala, . . . I85

Acanthodrilini, . . . 209

Acanthometra, . . . 103

Acanthopteri, . . . 510

Acarina, . . . . 336

Acephala, . . . . 362

Acetabulum, . . . 605,666

Achromatin, . . . . 43

Acineta, . . . . 105

Acinetaria, . . . . I05

Acipenser, . . . . 508

Acoela, . . . . 162

Acontia, . . . . I 5 I

Acontias, . . . , 578

Acornshell, . . . . 274

Acquired Characters, . 7 7,768

Acrania = Cephalochorda, . 410

Acraspeda, . . I42, I48, I 54

Acrodont teeth, . . 570

Acromion, . . . . 664

Actinia . . . . I 56

Actiniaria, . . . I53, I 56

Actinomma, . . . . 103

Actinophrys, . . . . IOO

Actinospharium, . . . IoO
Actinotrocha (larva), . $\quad 225$

Actinozoa, . . . 129, I 56

Adambulacrals, . . . 230

Adamsia, . . . . I60

Adder, . . . . 583

Aclhesive cells, . . . I57

Adrenal body of Pigeon, . 612

, , Rabbit, . 676

Eginopsis, . . . . 156

Egithognathre, . . . 620

Aluroidea, . . . . 714

Alurus, . . . . 716

Apyornis, . . . . 619

Aerial Fauna, . . . 759

Athalium, . . . . 99

Atiology, . . . . 765

Agama, . . . . 577

Agamidae, . . . . 577

Agouti, . . . . 7 I I

Air bladder of Fishes, . . 476

Air sacs of Bircls, . . . 622

", of Lizards, . . 576

Albumen gland of Snail, , 354

, of bird's egg, . . 613

", of frog's egg, . 549

Alces, . . . . 698

Alciope, . . . . 209

Alcippe, . . . . 275

Alcyonaria, . . . . I57

," and Zoantharia, . I53

Alcyonidium, . . $\quad 225$

Alcyonium, . . . I53, I56

Alecithal, . . . 65

Alimentary System of-
Amphibians, , 540 


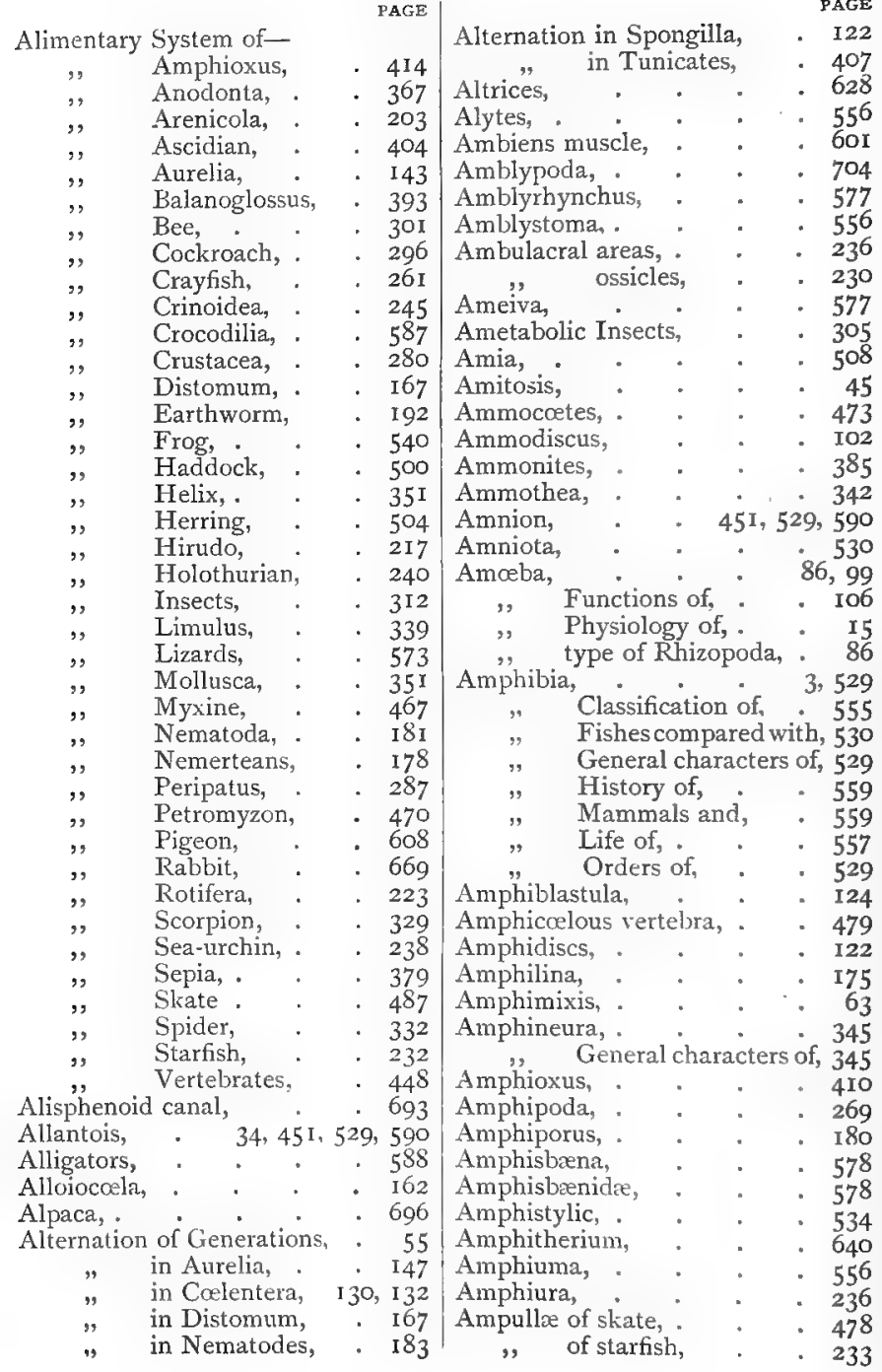


PAGE

Anableps, "Placenta" of, . 59I Anabolism,

Anthropoid Apes, .

PAGE

Anacanthini, : : 510

Anaconda,

Anal cerci of Cockroach, . 295

Analogous organs,

Anamniota,

33

Anapophyses,

530

Anchinia,

$66 \mathrm{I}$

Anchitherium, . . . 7OI

Ancylus, . . . 358

Androctonus, . . . 33I

Anemonia,

Angiostomum,

156

Anguillulidæ,

182

Anguis, .

185

Angular bone.

577

Animalculists,

$43 r, 586,605$

Animal Kingdom - General

$$
\text { survey of, . . . I-I2 }
$$

Animals and plants contrasted, I7-I 9

Anisopleura, . . . . 388

Anisopoda, . . . . 276

Annelids.

I86

" Development of, . 196

," and Vertebrates, . 424

Anoclonta, . . $\quad 363,372$

722

Anthropoidea, . . . 722

Anthropomorph, . . . 726

Anthropopithecus, . . . 726

Antilocapra, . . . . 698

Antilope, . . . 698

Antipatharia, . . I53, I57

Antiquity of man, . $\quad .730$

Antlers, . . . . 698

Ant-lion, . . . 304

Ants, . . . . 304

Anura, . . . . 555

Anus of Vertelorates, . . $45^{2}$

Apes, . . . . . 726

Aphaniptera, . . 304

Aphides, . . . 305

Aphrodite, . . . . 209

Apis, . . . 298

Aplacophora,. . . . 347

Aplysia, . . . . 357

Apneustic Insects, . . . 3I4

Apoda (Echinoderma) . . 243

Apoda = Gymnophiona . . 556

Apodemata (Crayfish) . . 257

Appendages of Arachnoidea, . 329

,$\quad$ Arenicola, . 202

, External appearance of, 363

"Internal , 364

" Life history of," . 370

". Mode of life of, : 363

"Shell of, . : 364

, Structure of, . . 363

Anolis, . . , 577

Anomalurus, . . . . 7 I I

Anomia, . . . 372

Anomodontia, . . . 592

Anomura, . . . . 279

Anoplodium, . . . I62

Ant-eaters, . . . . 68I

Antedon, . . . . 243

Structure of, . . 244

Antenna of Cockroach, . 295

" of Crayfish, . . 256

," of Myriopods, . . 292

" of Peripatus, . . 287

of Trilobites, . . 34I

Antennules of Crayfish, . . 256

Anthomedusæ, . . . I55

Anthozoa,

- 156

Appendicularia, . 40I, 408

Cockroach, . 295

", Crayfish, 255, 257

", Crustacea, . 255

, Eurypterina, . 340

, Haddock, - 496

, Insects, . . 307

"Limulus, . 339

, Myriopods, . 292

", Peripatus, . 287

,Polychreta, . 209

"Spider, . . 332

, Trilobita, $34 \mathrm{I}$

Appendicular skeleton of

Vertebrates,

\begin{tabular}{ll} 
Appendix vermiformis, . & .433 \\
\hline & 670
\end{tabular}

Apseudes, . . . . 277

Apterygota, . . . 304

Apteryx, . . . 6I9

Aptornis, . . . 618

Apus, . . . . . 269

Aquatic Mammals, . . 638

Aqueduct of Sylvius, . . 438

Aqueductus Vestibuli, . . 444 
PAGE

Aqueous humour of Eye, $\quad 446$

Arachnoidea,

Arachnoid membrane, .

Araneidæ, fluid, : * : 438

Arca, . . . . . 372

Arcella,

Archaeoceti, .

Archæopteryx,

Archegosaurus,

Archenteron,

Archerina,

Archi-Annelida,

Archi-cerebrum,

Archi-Chretopoda,

Archicoele,

Archigetes,

Archinephric duct $=$ Segmental duct,

Archipterygium of Fishes,

Archoplasm,

Arctocyon,

Arctoidea,

Arctomys,

Arctopithecini,

Arenicola,

$$
\text { Structure of, }
$$

Argonauta

Argulus,

Argyroneta,

Arion,

Aristotle's lantern,

Arius,

Armadillo (Crustacean),

$$
\text { , (Mammal), Exo- }
$$

skeleton of,

Armadillos, .

99

708

617

557

67,448

98

2 II

$27 \mathrm{I}$

211

I 76

I 7 I

$46 \mathrm{I}$

521

45

7 I 7

715

711

724

201

202-6

386

269

336

358

237

526

277

690

690

Arrow-worms,

222

Artemia,

Arterial arches of Vertebrates, system of embryo of higher Vertebrates,

35 ," of Fish,

,

of Frog,

455

9

$$
\text { is }
$$

of Pigeon,

of Rabbit,

Arthrobranch" of Crayfish,

Arthropoda, Classes of, .

, General characters of, Arthrostraca, .
542

610

673 264

7,252

253
Articular, . . 43I, 586, 605

Articulata, . . . 246

Artiodactyla, . . . . 693 and Perisso"dactyla contrasted, . 693

Arvicola, . . . . 7II

Asaphus, . . . . 34I

Ascaridx, . . . . I84

Ascaris, . . . . I83

Ascetta, . . . II7

Ascidia, . . 40I, 409

Ascidiacea, . . . 409

Ascon type (of Sponge), . I 8

Asellus, . . . . 277

Asexual reproduction, . . $5 \mathbf{I}$

Aspergillum, . . . . 372

Aspredo, . . . . . 526

Astacus, . . 253-69

Asteracanthion, . . . 235

Asterias, . . . 229, 235

Asteroidea, . . . . 229

Astrea, . . . I54, I57

Astropecten, . . . . 235

Astrophyton, . . . 236

Astrorhizidx, . . . 106

Atavisms or Reversions, . 83

Atax, . . . . . 336

Ateles, . . . . 725

Atheca, . . . . 566

Atlanta, . . . . 357

Atlantosaurus, . . . 593

Atlas vertebra, . . 660

Atrial cavity, . . 40I, 416

Atriopore of Amphioxus, $\quad 416$

Attida, . . . . 336

Atypus, . . . . 336

Auchenia, . . . . 696

Auditory capsules of skull, : 427

" nerve, . . 44I

") organs of Insects, - 3 I I

Aulastoma, . . . . 214

Aulosphera, . . . 103

Aurelia, . I $33,142,156$

, Life history of, I46, I47

, Relatives of, . I47

Auricles of Vertebrate heart, . 454

Auricularia (of Holothuria), : 243

Australian region, . . . 764

Autostylic, . . . 534

Aves, see Birds, . . . 2, 595 
PAGE

Avicula, $\cdot \quad \cdot \quad \cdot 372$

Axial Skeleton, see Backbone, 432 Axis vertebra, . . . 660 Axolotl, . . . . 556 Aye-Aye, . . . . 722 Azygobranchs, . . . 357

Babirusa, . . . . 694 Baboons, . . . . 725 Backhone, . . . . 432 Bactrites, . . . . 385

Baculites, . . . $\quad 385$

Badger, . . . . 716

Balæena, . . . . 709

Balæenoidea, . . . . 709

Balænoptera, . . . 709

Balanoglossus-

". Description of, . . 39I

", General characters of, 39I

"Habits of, . . . 392

,, Invertebrate characters of, 398

"Species of, . . . 392

," Vertebrate characters of, 397

Balantidium, . . . I05

Balanus, . . . . 269

Baleen, . . . . 706

Bandicoot, . . . . 686

Barbary ape, . . . . 726

Barbule, . . . . 495

Barnacle, . . . . 272

Basiliscus, . . . 577

Basitemporal, . . . 596

Basipodite of Crayfish, . . 255

Batoidei, . . . 506

Bats, . . . . 719

Bdellostoma, . . . . 469

Bears, . . . . 716

Beaver, . . . . . 7II

Bees, . . . . . 298

Beetles, . . . . 304

Belemnites, - . . . 386

Belinurus, . . . 340

Belodon, . . . . 589

Beluga, . . . . 709

Bernhardus, . . . . I60

Beroë, . . . . . I 157

Bilateral symmetry, . 3I, I6I

Bile,

Bilharzia, . . . . I70

Bimana,
Bionomics-

Birds, . . . . 62 I

Colentera, . . . I60

Crustacea, . . . $\quad 283$

Fishes, . . . $55^{17}$

Insects, . . . . 324

Mollusca, . . . . $36 \mathrm{I}$

Spiders, . . . 334

Sponges, . $\quad . \quad$ - 125

Bipalium, . . . . 163

Bipinnaria, . . . 234, 249

Bird lice, . . . . 305

Birds, . . . . 595

", and Reptiles, . . 562

, Classes of, . . . 3

"Classification of, . . 617

" Courtship of, . . . 626

", Diet of, . . . 626

". Eggs of, . . . 627

"Feathers of, . . . 699

", Flight of, . . . 62I

," General characters of, - 596

" ", survey of, . 596

, Intelligence of, . 626

". Life of, . . 62I

", Migration of, : . 628

", Moulting of, . . 628

"Nests of, . . 626

, Pedigree of, . . . 630

," Song of, . . . 625

Birgus, . . . . 279

Bitterling, . . . . 527

Bivalves, . . . 362

Classification of, . 372

General characters of, 363

Habits of, notes on, 372
. 373

Life history of, - 374

Past history of, * 374

Bivium of Sea urchin, . . 237

". of Starfish, . . 230

Bladder of Frog, . . . 540

,, of Mammals, . . 676

,, -worms, . . . I73

Blastocyst, . . . . $65^{\circ}$

Blastoderm, . . . 65

Blastoidea, . . . . 246

Blastomere, . . . . 420

Blastopore, . . . . 67

Blastosphere,. . . . 65 
Blastula,

PAGE

Blatta,

Blind spot of Eye, . . 447

Blood, of Vertebrates,

Boa

Body cavity-

Characters of a true, . 315

of Amphioxus, . . 4I5

,, Arenicola, . . 203

"Balanoglossus, . . 394

", Crayfish, . . . 262

, Earthworm, . . 190

"Hirudo, . . . 216

", Insects, . . . 3I5

", Lamprey, . . . 472

,, Peripatus, . . 315

"Scorpion, . . 330

,, Spider, . . . 332

"Starfish, . . . 232

"Teleostean, . . 453

"Vertebrates, . . 453

" $" \quad$ Primary, . 426

", Secondary, 426

Bombinator, . . 555

Bone,

Bonellia,

38,373

Books, Notes on,

Book scorpions, . . . 33I

Bopyridæ,

Bos,

Bothriocephalus, . . . I7 I

Botryllus,

Bougainvillea,

Bovida, .

Brachiolaria, .

Brachionus,

Brachiopoda, .

Brachyura,

Bradypodida,

Bradypus,

Brain of Skate,

" of Vertebrates, . . 435

", Summary of parts of, . 438

Branchellion, .

$2 \mathrm{I} 4$

Branchixe $=$ Gills.

Branchial arches, . . 428, $43 \mathrm{I}$

" sense organs, .

Branchiobdella,

443

Branchiopoda, 269
Branchiostegal rays,

PAGE

. $\quad \cdot 498$

Branchipus, • • . $\quad 269$

Branchiura, . . . 209

Breast bone, . . . . 433

Brisinga, . . . . 235

Brittle-stars, . . . 235

Brontornis. . . 6I9

Brontosaurus, . . . 593

Bryozoa, . . . 224

Buccinum, . • • 357

Buckie $=$ Buccinum.

Budding,

52

Bufo, . . . . 555

Bugs, . . . . 305

Bulbus arteriosus, . . . 454

Bulla, . . . . . 357

Bunodont, . . . . 693

Bursa Fabricii, . . . 609

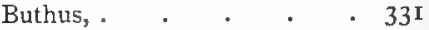

Butirinus, . . . . 5 IO

Butterflies, . . . 304

Byssus, . . . . 37 I

Caddis flies, . . . 304

Cxca, . . . . 739

Cæcilia, . . . . 556

Cæcum of rabbic, . . . 670

Ca'ing whale, . . . 709

Calamoichthys, . . . 508

Calcarea, . . . II6

Calciferous glands, . . 192

Calcispongiæ, . . . I16

Caligus, . . . . 272

Callionymus, . . . 520

Callorhynchus, . . . 507

Calymene, . . . . 34I

Calyptoblastic, . . . I55

Camelida, . . . . 696

Camelus, . . . . 696

Campanularia, . . . I55

Campodea, . . . . 305

Campodeiform larva, . . 323

Camptosaurus, . . 593

Cancer, . . . . 279

Canida, . . . . 715

Canis, . . . . 715

Cannon-bone, . . . 6196

Capitellidie, . . . 209

Capitulum of rib, . . . 66I

Capra,

- 698 
Caprella, $\because 277$

Capreolus, . . . . 698

Capuchins, . . . . 725

Capybara, . . 7II

Carapace of Chelonia, . . 254

of Crayfish, . . 564

Carcharias, . . . 506

Carchesium, . . . . 93

Carcinus, . . . . 279

Cardium, . . . . 372

Cardo. . . . . 295

Caretta, . . . . 567

Carina of Cirripedia, . . 274

Carinaria,

597,605

Carinatæ,

. 357

, and Ratita con-

Carinella, trasted, . 6I8

Carinoma, : : $:$ I80

Carmarina, . . . 156

Carnassial teeth, . . . 7I3

Carnivora, . . . . 7I2

Carp, . . . . 5IO

Carpo-metacarpus, . . . 597

Carpus, . . . . 665

Cartilage, . . . . 38 -bones, . $\quad 428,660$

Caryophyllæus, . . . I7 I

Cassiopeia, . . . . 148

Cassowary, . . . . 619

Castor, . . . . 7II

Casuarius, . . . . 619

Cat, . . . . 7it

Catallacta, . . . $\quad 99$

Catarrhini, . . . . 722

Caterpillar, . . . . 322

Caudata, . . . . 556

Cave Fauna, . . . . 757

Cavia, . . . . 7II

Caviidx, . . . . 7II

Cebidx,. . . . . 725

Cebus, . . . . 725

Cell cycle among Protozoa, . 97

"division. . . 45-48

" , Rationale of, . 46

"Nucleus of, . . . 43

"Substance of, . . . 42

"Theory,. 37,4r, 50, 69

.Wall of, . . 44-45

Cells, • • . 40

"Forms of, . . . 42

, History, , , 4I

"Structure of, . , 42

Cellepora. . . . 225

Cellulose in Tunicates, . . 402

Centetes, . . . 7I7

Centipedes, . . 42, 285,29I

" and Millipedes, 286, 292

Central corpuscles, . . 44

Centrolecithal, . . . 65

Centrosomes, . . . 44

Cephalaspis, . . . 509

Cephalochorda, . . 410

Cephalodiscus, $\quad 399$

Cephalopoda, . . . 374

" Classification of, 385

Cephalothorax of Crayfish, . 254

Eurypterina, 340

" Limulus, - 338

" Scorpion, : 329

" Spider, - 332

" Trilobita, - 34I

Cephalothrix, . . . 180

Ceratiocaris, . . . 276

Ceratites, . . . . 385

Ceratium, . . . . 105

Ceratodus, . . . . 5 II

Ceratophrys, . . . . 556

Ceratospongice, . . . II8

Cercariæe, . . . . I69

Cerci of cockroach, . . 295

Cercopithecina, . . . 725

Cercopithecus, . . . 725

Cere of pigeon, . . . 607

Cerebellum, . . . . 438

Cerebral hemispheres, - . 435

Cerebratulus, . . . I80

Cerianthus, . . . . I57

Cervidx, . . . . 697

Cervus, . . . . 697

Cestoda, . . . I7I

Cestracion, . . . . 506

Cestum Veneris, . . . I57

Cetacea, . . . 705

Cetochilus, . . . . 272

Chretoderma,. . . . 347

Chretognatha, . . , 222

Chæetopoda, . . I87,2I2 
PAGE

Chætopoda, General survey of, 209 Chætopterus, . . . . 2II

Chalicotherium, . . . 702 Chalina, . . . 125

Chamæleo, . . . 578

Chamaleon, the American, - 577

Chamæleonidæ, . . . 578

Charybdea, . . . 156

Cheliceræ of Limulus, . $\quad 338$

" Scorpion, . · 329

Chelifer, Spider, · 332

Chelone, • • 331

Chelonia, . . 560, 563

"Classification of, 566

" General characters of, 563

Chelydidæ,

Organs of, . . 566

$\cdot .567$

Chelydra, . . . . 567

Chelys, . . . . 567

Chemotaxis, . . . . I07

Chernes, . . . . $33 \mathrm{I}$

Chevron bones, . . . 706

Chevrotain, . . . . 696

Chiasma of optic nerves, $\quad \cdot 484$

Chilognatha, . . . 292

Chilopoda, . . . . 292

Chimæra, . . . . 507

Chimpanzee, . . . . 726

Chinchilla, . . . . 7II

Chiromys, . . . . 722

Chiroptera, . . . 719

Chitin, . . . 254,745

Chiton,. . . . 345

Chlamydomyxa, . . . 100

Chlamydophorus, . . . 690

Chlamydosaurus, . . . 577

Chlorophyll, .

Choeropus,

Choløepus,

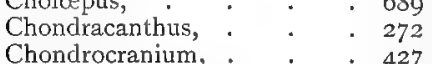

Chondrosia, . . . 125

Chordata and Non-chordata, .

Chordx tendinex,

Chordotonal organs,

Chorion,

Choroid of Eye,

I9

686

689

125

673

3 II

651

446

Chromatin, .
Chromosomes,

Chrysalis,

Chrysaora,

Chrysochloris,

Chrysophrys, .

Chrysothrix,

Chyle,

Chyme, .

Cicada, .

Cidaris,

Cilia,

Ciliary nerve,

, processes of the choroid, 447

Ciliata,. . . . I05

Cilium, . . . . 106

Ciona, . . . . 409

Circulatory system, see Vascular system.

Cirri of Chætopods, . . 209

"Crinoids, . . . 244

$\begin{array}{ll}\text { Lancelet, * } \quad * \quad & 411 \\ \text { Cirripedia, : } & \text {. } 269\end{array}$

Cirrus sac, . . . . 167

Civet, . . . . 715

Cladocera, . . . . 271

Claspers of Skate, . . $\quad .478$

Classification of Animals, . I3

I3

" The basis of of organs, . 36

Clathrulina grades of, . . 12

Clavellina, . . . 401

Clavicle, . . . 434, 664

Clemmys, "Placenta" of, 59I

Clepsine, . . . 208, 214

Clidastes, . . . . 593

Clio, . . . . . 358

Clione, . . . . I25

Clitellum of Earthworm, . I89

Clitoris, . . . . 678

Cloaca of Vertebrates, . . $46 \mathrm{I}$

Clotho, . . . . $5^{83}$

Clupea, . . . . 503

Clypeaster, . . . . 240

Cnidoblasts, . . . . I36

Cnidocil, . . . I 36

Cobra, . . . . $\quad 583$

Coccidium, . . . . 104

Coccus insects, . . . 305

43 Cochlea, . . . . 444 
PAGE

Cockle, . . . 372

Cockroach, . . 293-98, 305

Cocoon,

Cod,

. 510

Colentera, · . . . 129

" Classification of, I29, I 54-5

., General characters of, . 129

" , life of, . . 134

" $"$ scheme of, - I59

" History of, . . . I57

$"$ Pedigree of, . . . 158

Coeliac ganglia, . . . $67 \mathrm{I}$

Coelogenys, . . . 7 II

Colomata and Coelentera, 8, I30

", Worms the first, . I6r

Colome, see Body cavity, - 453

Coloplana, . . . . I64

Coenurus, . . . . 173

Coleoptera, . . . . 304

Collagen, . . . . 745

Collembola, . . . . 305

Collozoum, . . . . I03

Colobus, . . . . 726

Colon, . . . . 670

Coloration, . . . . 758

Colour Sense, Theory of, . 28

Colouring matters of Animals, 746

Protective, among Insects, 317

Colubriformes,

- 583

" venenosi, - 583

Columba, . . . . 599

" livia, . . 83, 599

Columella of Vertebrate ear, - 445

,$\quad$ or epi-pterygoid, 573,586

, in cotals, . I 54

Comatula, snail, * 349

Commensalism among Crustacea, . . 283

Commissures of Mammalian brain, . . 636,668

Complemental males among Cirripedia, .
Myzostomata,

280

Compsognathus,

Conchifera, see Bivalves, - 362

Conchiolin, .

Condylarthra,
Cone shells,

PAGE

Conjugation, 357

Multiple, . Ir

of Paramœeium, 92

of Protozoa, . III

of Vorticella, . 94

Conjunctiva, . . . 446

Connective tissue, . . $\quad 21,38$

Contractile Vacuoles, . . 86

Contraction of muscle, . . 39

Conus, - . . . 357

Conus arteriosus, . . . 454

Convergence, . . . 688

Convoluta, . . . . 162

Copelata = Larvacea, . $\quad .400$

Copepoda, . . . . 269

Coracoid, . . . 434, 664

Corallium, . . . I53, I57

Corals, . I I30, I33, I57

Cordylophora, . . I55, I60

Corium,. . . . 426

Cornea, . . . . 446

Coronary, . - 431, 586, 605

Coronella, . . $\quad 5^{83}$

Corpora adiposa, , . 548

Corpus callosum, . . . 668

,, geniculatum, . . 668

," striatum, . . . 668

Corrodentia, . . . 305

Corticata, . . . . 97

Coryphodon,. . . . 704

Costal plates, . . . 564

Cotylophora, . . . 696

Couguar, . . . 7I5

Courtship of Spiders, . $\quad 334$

Cowper's glands, . . . 678

Coxa, . . . 295

Coxal glands of Limulus, . 339

" " Peripatus, . 285

" " $"$ Scorpion, "330

" "Spider, . 332

Coxopodite of Crayfish, . . 255

Coypu, . . . . 7II

Crab, . . . 279

Crangon, . . . . 279

Crania, . . . . 226

Cranial nerves, . . 44I, 483

Craniata, . . 425

Craspedon of Swimming bell, I40

Craspedota, . . . I40 
Craspedota and Acraspeda, I48

Crayfish, The Fresh water, 253-269 "The Sea, .

Creodonta,

Cribrella,

Cribriform plate, . . . 663

Cricetus,

Cricket,

Crinoidea,

Cristalella,

$$
\text { Classification of, : } 246
$$

Crocodiles, Alligators, and

$$
\text { Gavials, . . } 585,5^{89}
$$

Crocodilia,

\section{"Classification of, . 588}

" General characters of, 583 Crocodilus, History of, - 589
588

Crop of Earthworm, . . 192 " of Leech, . . . 2I8

", of Pigeon, . . . 608

" of Snail, . . . 352

Crossopterygii. . . . 509

Crotalus,

Crumb of Bread Spunge, Crura cerebri,

Crustacea-

" General characters of, . 253

" Habits and Habitats of, 283

" History of, . . . 279

"Life History of, . . 28 $\mathrm{r}$

"Systematic survey of, 269-79

Cryptobranchus,

556

Cryptoniscidæ,

Cryptophialus,

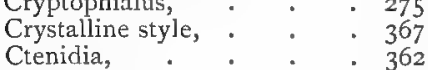

Cteniza, .

Ctenodus,

Ctenoid scale,

Ctenophora,

Ctenoplana,

Cubomedusæ,

Cucullanus,

Cucumaria,

Cucumerina, .

Cuma,

Cumacea,

Cunina, .

Cuscus, .
253 305

243

Cuspidaria, . . . $\quad 372$

Cuticle, . . . . 254

Cutis, . . . . 426

Cuttlebone, . . . . 377

Cuttlefish, . . . . 375

" Characteristics of, . 375

Cuvierian organs, . . . 24I

Cyamidæ, . . . 277

Cyanea,. . . . 148, 156

Cyanosoma, . . . 240

Cyclas, . . . . 372

Cyclodus, . $\quad . \quad$. 578

" "Placenta" of, . 59I

Cycloid scale, . . . 521

Cycloporus, . . . . 163

Cyclops, . . . 269

Cyclostomata, . . 465

General characters of, 465

Cycloturus, . . . 690

Cydippe, . . . . 157

Cymbulia, . . . . 358

Cymothoa, . . . . 277

Cymothoidæ, . . . . 277

Cynocephalus, . . . 725

Cynoidea, . . . 715

Cynomorph monkeys, . . 725

Cynomys, . • • . 7II

Cynopterus, . . . $72 \mathrm{I}$

Cyprea, . . . . 372

Cypridina, . . . . 269

Cyprina, . . . . 372

Cypris, . . . . 269

Cysticercus, . . . . 175

Cystoidea, . . . . 246

Cysts of Protozoa, . . . I Io

Cytoplasm, . . . . 42

Daphnia, . . . 269

Dart sac of Snail, - . $\quad$. 356

Dasornis, . . . . 619

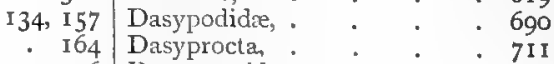

Dasyproctidre, . . . 7II

Dasypus, . . . . 690

Dasyurida, . . . . 686

Dasyurus, . . . 686

Dead man's fingers, . . I 57

Decapoda (Crustacea), : $\quad 276$

I 56 (Cephalopods), $\quad 386$

687 
Decidua reflexa

Deer,

• * 697

Delamination,

Delphinapterus,

Delphinoidea,

Delphinus,

Demodex,

Demospongix,

Dendrerpetum,

Dendrobates, .

Dendrocœlida,

Dendroccelum,

Dendrosoma, .

Dentalium,

Dentary,

Dentition of Mammals,

Dermal denticles, .

Dermaptera,

Dermatobranchia,

Dermis of Vertebrates,

Dermochelys,

Dermoptera, .

Descent, Doctrine of, of man,

Desmodus,

Desmognathæ,

Desmognathus,

Desmosticha, .

Desor, Larva of

Deutoplasm, .

Development of-

Amphioxus,$$
79
$$$$
\text { , }
$$$$
9
$$$$
\text { , }
$$$$
\text { , }
$$
505

"Mammalian teeth, - 643

"Mammals, . . . 647

"Nemerteans, . . . 183

" Peripatus, . . . 289

"Petromyzon,. . . 472

"Placenta, . . . 647

"Polychæta, . . . 206

"Reptilia, . . . 589

"Scorpion, . . . 330

"Sea anemone, . . 152

"Skate, . . . . 493

"Skull, . . . . 429

"Sponges, . . . I22

"Tunicata, . . . 407

" Vertebrata, . . . $42 \mathrm{I}$

Diadema, . . . . 240

Diapedesis, . . . . $54 \mathrm{I}$

Diaphragm, . . . . 674

Dibranchiata, . 385,386

Dichogamy in Cymothoidæ, 277

"Tunicata, . 407

Dicotyles, . . . . . 695

Dicyema, . . . . I27

Dicyemidæ, . . . . I27

Dicynodon, . . . 592

Didelphia, . . . . 684

Didelphyidæ, . . . 686

Didelphys, . . . . . 686

Differentiation, . . $\quad 32$

Difflugia, · • • 99

Digenetic Trematodes, . . I70

Digestion, . .22-23, 737

"Intra-cellular in Protozoa, 86

,$\quad$, $\quad$ in Sponges, $12 \mathrm{I}$

Digitigrade, . . . 714

Dimorphism of sexes, . . 50

Dinofiagellata, . . 105

Dinophilus, . . . . 223

Dinornis, . . . . 619

Dinosauria, . . . 593

Dinotherium, . . . 704

Diodon, . . . . 5I I

Diphycercal, . . . . 518

Diphyes, . . 156

Diphyodont dentition, . . 644

Diploblastic, .

I 6 
PAGE

Diplopoda $=$ Millipedes, $\quad$. 292

Diplotrophoblast, . . . 65I

Diplozoon,

Dipneumones,

Dipnoi, .

Dipodidæe,

Diprotodon,

Diptera,

Dipterus,

Dipus,

Direct division,

Discoidal segmentation,

Discomedusæ,

Discophora, ".

Distomum, : : : : 164

Distribution, Geographical, . 753

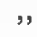

,

of Lemurs,

,

of Marsupials, of Tapirs, .

Geological,

Division of Labour,

Dochmius,

Doctrine of Descent,

Dodo,

Dog,

Dogfish,

Dogwhelk,

Dolabella,

Doliolum,

Dolphin,

Donkey,

Dorcatherium

Doris,

Dormouse,

Dorsal lamina of Tunicate,

Draco,

Dracunculus, .

Dragonet,

Dragon-flies,

Dreissena,

Drepanidium,

Drepanophorus,

Drepanophors, . . " 176

Dromagnathr, : . 620

Dromæus, . . . . 619

Dromatherium,

Dromia,

Duckmole,

Ductus endolymphaticus,

Dugong,

Duodenum,
Duplicidentata, . . . 7 I I

Dura mater, . . . 439

Ear of Arenicola, . . . 203

Crayfish, . . . 260

Myxine, . . . 467

Vertebrates, . . 444

Earthworm, . . . 187

Development of, I96-20I

" Structure of, I87-196

Earwigs, . . . 305

Ecardines, . . . . 226

Ecaudata, . . . 555

Ecdysis or moulting of Arthropods,

Echidna,

Echinochrome, : : $: 238$

Echinococcus, . . . I73

Echinoderma, - . 7,227

Contrast between the classes of, . $25 \mathrm{I}$

Development of, . 247

General characters of, $\mathbf{2 2 7}$

Larvæ of, . . 250

Relationships of, $\quad 250$

Echinoidea, . . . $\quad 236$

357 , Classification of, . 240

Echinorhynchus, . . . 185

Echinus, . . . . 236

Echiuridæ, . . . - 2 II

Ectoderm, - . 36, 68

Ectoplasm, . . . . Iro

Ectoprocta, . . . . 225

Edentata, : : : 688

404 , Families of, . . 689

Edriophthalmata, . . . 276

Eel, . . . 5 I0

Egg case of Skate, . . 493

Egg cases of Buccinum, Ian-

thina, Purpura, . . 356

Eggs, see Ova, : . $\quad 56$

," of Birds, . . . 627

Eimeria, . . . " 104

Elaps, . : : . 583

Elasipoda, . . . . 243

279 Elasmobranchii, : 477,505

$68 \mathrm{I}, 682$ Electric organ of Skate, $\begin{array}{r}477,505 \\ \quad 478\end{array}$

$444, \quad$ organs of Teleostei, . 478

Eledone, . . . 386

Elephant's tooth shells, - : 362 
Elpidia,

698

Elytra, C : 243

Embolé,

Emu, . . . .

56

619

Emys, . . . . . 567

Enchytræida, . . . 209

Encystation, . . . 87,88

Endoderm, - . . 36,68

Endolymph, . . . . 444

Endoplasm, . . . I IO

Endopodite (crayfish), . . 255

Endoprocta, . . . 200

Endostyle of Tunicates, . . 404

Enoplidæ, . . . . 183

Entalium, • . . 362

Enterochlorophyll, . 232, 352

Enteroccle, . . $\quad 472$

Enteron=gut, . . . 448

Enteropneusta, . . . 39 I

Entoconcha, . . . 360

Entomostraca, . . . 269

Entoprocta, . . . 225

Entosternite of Limulus, · 339

, Scorpion; . 329

. "Spider, . . 332

Environment, influence of, $\quad 768$

Eolis, . . . . 357

Epeira, . . . . 334, 336

Ephemerida, . . . 305

Ephyra, . . . . I47

Epiblast, . . . 36, 67

Epibolé,

67

Epicrium, * . . 557

Epidermis of Insects, . . 310

Vertebrates, . 426

Epididymis of Skate, - . 493

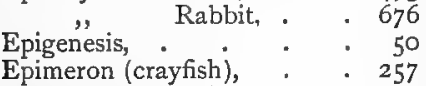

Epiphragm of snail, : : 349

Epiphyses, . . . 660

Epiphysis = Pineal Body, - 437

Epipodite of Crayfish, : 264

Epipubic bones, . 680, 682, 684

Epistoma (crayfish), . . 257
Epithelial Tissue, . . . 37

Equidæ, . . . . 700

Equus, . . . . 700

Erinaceus, . . . 718

Eruciform larva, . . . $\quad .323$

Estheria, . . . . 270

Ethiopian region, . . . 764

Eucyrtidium . . . . IO3

Eudrilini, . . . . 209

Euglena, . . . . 105

Eulamellibranchia, . $\quad 372$

Eulima. . . . 360

Eumeces, . . . . 578

Eunice, . . . . 210

Eupagurus, . . . . 279

Euphausia, . . . . 278

Euplectella, . . . . I25

Eupomatus, . . . . 206

Euryalida, . . . . 236

Eurypharynx, . . . 5I I

Eurypterina, . . . . 340

Eurypterus, . . . . 340

Euscorpius, . . . . 33I

Euspongia, . . , II8

Eustachian tube, . . . 449

Eustrongylus, . . . 185

Eutheria, . . . . 688

Euthyneura, . . . . 357

"Evolution," . . . 49

Evolution of Animals, - . 8 $\mathrm{r}$

, Evidences of, . , 82

". Factors in,. . 765

Summary of theories of, 771

of Man, . . . 729

of Sex, . . 52-53

Excretion in Animals, . $\quad 25-26$

Excretory system of-

Amphioxus, . . 416

Anodonta, . $\quad 369$

Arenicola, . . 206

Balanoglossus, . 395

Bee, . . 306

Cockroach, - . 297

Crayfish, . . 264

Crocodilia, . 588

Crustacea, . . 280

Earthworm, . . 194

Frog, . . . 547

Haddock, . . 502

Helix, • . 353 
Excretory System of -

PAGE

Hirudo,

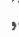

in

Insects, .

Lizards,

Mammalia,

Myxine,

Nematoda,

Nemerteans,

Peripatus,

Petromyzon, .

Pigeon,

Rabbit, .

Scorpion,

Sea urchin,

Sepia,

Skate,

Starfish,

Tunicates,

Vertebrates,

Exopodite (crayfish),

Exoskeleton of Vertebrates,

Extinction of Animals, .

Eyes of Cæcilia,

$$
\text { Crayfish, . }
$$

Cuttlefish, .

Helix,

Insects,

Lamprey,

Myxine,

Proteus,

Tunicata, .

Vertebrata,

ment of,

Develop-

",

$$
\text { ment of, }
$$

Facial nerve,

Freces,

Fallopian tube,

Fangs of Ophidia,

Fasciola,

Fat body of Insects, Frog,

Feathers of Pigeon,

Development of

Feather stars,

Felidee, .

Felis,

Femur,

Fenestra ovalis

Ferments,
317

576

675

468

I82

I79

287

472

6II

675

330

- 239

$38 \mathrm{I}$

490

234

406

458

255

426

77

556

260

376

350

3 II

470

467

445

445

445

447

44 I

23

678

580

164

313

548

599

600

243

714

714

- 434

- 445

22,28
Fertilisation, . . . 54, $6 \mathbf{r}$

Fiber, . . . .7II

Fibula, . . . . 434

Fierasfer. . . . . 527

Filaria, . . . . I84

Filibranchia, . . . . 372

Fins, . . 476, 518, 520

Fishes, Abyssal, . . . 527

, and Amphibians com-

pared, . . 530

Colour of, . . 5 I7

Commensalism in, . 527

Contrasts between, - 518

Fins of, . . 517,520

Flat, . . . 522

Food of, . . 520

General characters of, 476

Hermaphrodite, . 525

Orders of, . $\quad 4,475,505$

Parental care among, . 526

Relationships of, $\quad 527$

Reproduction of, . 525

Senses of, . . $\quad 525$

Viviparous, . . 526

Fission, . . . 52

Fissipedia, . . . . 7I3

Flagella, . . . 106

Flagellata, . . . . 104

Flagellum of snail, . $\quad 354$

Fleas, . . . . 304

Flies, . . . 304

Flight of Birds, . . . 625

Floridine in Sponges, . . I2I

Floscularia, . . . 223

Flounder, . . . 510

Flustra, . . . . 225

Flying Foxes, . . . $72 \mathrm{I}$

"Lemur, . . . 637

" Mammals, . . . 637

, Phalangers, . . 687

, Squirrels, . . 71 I

Fotal membranes of Birds, . 616

, " Mammals, 653

". Reptiles, 590

Follicie cells, . . . 463

Fontanelles in Skull of the

Skate, .

477

Food vacuoles, . . . 86

Foot of Anodonta, : : 363

Cephalopoda, : : 376 
Foot of Dibranchiata PAGE

$$
\begin{array}{ll}
\text { " } & \text { Masteropoda, : } \\
\text { " Scaphopoda, : } & \text { Tetrabranchiata, }
\end{array}
$$

Foramen of Munro, , . 668 Foraminifera, : : 587

Fore brain $=$ Prosencephalon, Roof of, in Vertebrates, .

Fore gut $=$ Stomatodæum,

Fornix,

Fossils, .

Fovea centralis,

Fox,

Fresh-water Fauna,

Fritillaria, Mussel,

Frog,

Fuligo, .

Functions, Change of, of Animals,

Fungia, . Secondary, of organs, 34

Gad fly,

Gadus, .

Galago, .

Galea, .

Galeodes,

Galeopithecus,

Galesaurus,

Galithea,

Gall bladder, .

Gall fly,

Galls on Plants, : : 336

Gamasus, . . . . 336

Gammarus, . . . . 278

Ganglion,

Ganodichthyidæ,

Ganoid scales,

Ganoidei,

Garden Spider,

Gasteropoda, .

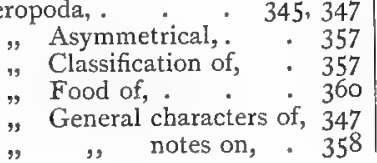

528

518,521
Gasteropoda, Life history of, . 360 Mode of life of,. 359 Parasitic, . 360 Past history of, $36 \mathrm{I}$ Symmetrical, - 345 Torsion of, - 359

Gasterosteus, . . . 520

Gastornis, . . . 619

Gastræa, . . . 3I, 67

" Theory, . . . 69

Gastric Juice, . . . 22 "Mill of Crayfish, , 26I "Mill of Crustacea, . 280 Gastroliths of Crayfish, , . 26 I Gastro-vascular canals, . , 144 Gastrula, . . 3I, 67 , of Vertebrates, : 464

Gavials. . . . 589

Gazella, . . . . 698

Gecarcinus, . . . . 279

Geckos, . . . . 577

Gelasimus, . . . 279

Gemmation $=$ Budding,.$\quad 5^{2}$

Gemmules in pangenesis, . 7 I

,, of Spongilla, . . I2I

Genealogical tree, . . . II

Genetta, . . . 7I5

Geodesmus, . . . . 163

Geodia, . . . . 125

Geographical areas, . . 762

,$\quad$ Distribution, . 753

Geological Record, . . 74

Geomys, . . . 7 II

Geophilus, . • . . 291

Geoscolecini,. . . . 209

Geotria, . . . 473

Gephyrea, • • . . 224

Germ cells, . . . $5 \mathrm{I}$

,, plasm, . . 72, I32, I 56

Germinal variations, . . 768

Geryonia,

I $32, \quad 156$

Gharials= Gavials, . . 589

"Giant fibres," . . . I9I

Gibbon, . . . . 726

Gill clefts, . . . . 449

slits of Balanoglossus, - 395

Gills of Amphibians, . . 557

Anodonta, . . 369

Crayfish, . . . 263 
280

Fishes, . . 476

Gasteropoda, . $\quad 357$

Polychieta,

Sepia,

Giraffe, .

Gizzard of Cockroach,

Crayfish,

Earthworm,

Pigeon,

Glandiceps,

3

Croc

,

Dipnoi, .

Insects

Mammals

Myxine,

Glass rope sponge, .

, snake,

Globe fishes, .

Globicephalus,

Globigerina, .

Glochidium of Anodonta,

Glossopharyngeal nerve,

Glossophora, .

Glycogen,

Glyptodon,

Gnathia,

Gnathobdellidx,

Gnathostomata

Gnats,

Gonads $=$ Reproductive organs,

36, I7 I

Gonapophyses of Cockroach,. 295

Goniaster,

229,235

Gonophores, .

Gordiidæ,

I 42 , I 55

Gordius,

- I85

Gorgonia,

I 85

Gorgonocephalus,

Gorilla, .

Graafian follicle,

Graafilla,

Grampus,

Grantia,

Graptolites,

Green gland of Crayfish,

Gregarina,

Grey matter of brain,

Gromia, .

Guinea pig,

515

642

466

I25

577

5 I I

709

IOI

$37 \mathrm{I}$

$44 \mathrm{I}$

388

24,255

690

277

221

465

304

I 57

463

162

709

I 25

I 58

264

85

439

IOI
Gunda,

PAGE

Gymnoblastic,

Gymnomyxa,

Gymnophiona,

Gymnosomata,

Gymnotus,

Gynæcophorus,

Gyrodactylus,

Haddock,

495

Hæmadipsa

Hæmatin,

213

Hæmerythrin,

Hæmocœle, .

Hæmocyanin,

747

Hæmoglobin of blood, 25, 26, 747

Hrmopsis, . . . 214

Hagfish, . . . . 465

Haimea, . . . . I53

Hair, . . . . . 641

") worms, . . . I80

Halichœrus, . . . . 717

Halichondria, . . . I25

Halicore, . . . . 69I

Halicryptus, . . . . 224

Haliotis, - . . . 357

Haliphysema, . . . 102

Halisarca, . . . . 125

Halitherium, . . . . 692

Halobates, . . . 325, 753

Hamadryad, . . . $\quad 583$

HTamster, . . . . 7II

Hapale, . . . . 725

Hapalidæ, . . . . 724

Harderian gland, . . . 448

Hare, . . . . 7II

Harelip, . . . 477,659

Harvest bus

- $33 \mathrm{I}$

, men,

mites,

$33 I$

Hastigerina, .

336

Hatteria,

IOI

. 568

Heart of Vertebrates, . . 454

Hectocotylus of Cuttlefish, - 383

Hedgehog, Dentition of, . 718

Development of, . 650

Heliopora, Placenta of, . 7I4,7 18

Heliozoa, . . . I00

Helix, . . . 348-57 
PAGE

Helix, External appearance of,

" Mode of life of, .

"Shell of, Structure of,

\section{$\mathrm{H}$} " Str

Hemiaspis, . . . . 340

Hemiaster, . . . . 240

Hemichorda, . . . 39 I

Henidactylus, . . . 577

Hemimetabolic Insects, . . 322

Hemiptera, . . . 305

Hepatopancreas, . . . 262

Heredity, . . . 7 I

Hermaphrodite duct of Snail, 354

Hermaphroditism, . . 54

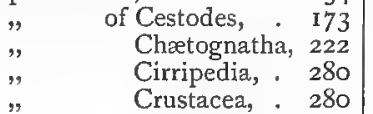

" Cymothoidæ, 277

, Earthworm, I94

" $\quad$ Frog tadpole, 555

" Leech, . 219

" Myxine, . 460

" Myzostomata, 212

" Tardigrada, 337

" Trematoda, I70

" Tunicata, . 407

"Turbellaria, I62

Hermione,

Herpestes, . . . . 715

Herring, . * . 503, 510

Hesperornis, . . . 619

Heteroceras, . . . 385

Heterocercal, . . . 518

Heterocœla, . . . , 125

Heterodera, . . . . 185

Heterodont dentition, . . 645

Heteromya, . . . . 372

Heterophrys, . . . . 100

Heteropods, . . . . 357

Heterotricha, . . . 105

Hexacoralla, . . . . 157

Hexactinellida, . . . 116

Hexapoda $=$ Insects, . . 313

Hind gut=Proctodæum, . 449

Hippocampus, . . . 511

", of brain, . 668
PAGE

Hippopotamus, . . . 693

Hippotherium, . . . 701

Hippurites, . . . . 374

Hirudinea, . . . . 213

" Classification of, . $22 \mathrm{I}$

Hirudo, . . 2 I4

H" Structure of, " 2 I4 2 II

Histriodrilus, : : $: 212$

Hoatzin=Opisthocomus, $\quad 618$

Holoblastic segmentation, . 65

Holocephali, . . . . 507

Holophytic, . . . . 95

Holopneustic, . . . 314

Holopus, . . . . 244

Holothuria, . . . . 243

Holothuroidea, . . . 240

" Classification of, 243

Holotricha, . . . 90

Homarus, . . . 253,278

$\mathrm{HomO}_{4}$. . . . 728-30

Homocercal, . . . 518

Homocœla, . . . . 125

Homodont dentition, . . 645

Homology of organs, . . 33

Homoplastic, . . . 33

Honeycomb bag, . . . 697

Hoplonemertea, . . . 180

Horns, . . . . 64I

Horse, . . . . 700

Horse-shoe Crab, . . . 337

House fly, . . . 304

Howling monkeys, . . 725

Huanaco, . . . . 696

Humerus, . . • . 434

Hyana, . . . . 715

Hyænodon, . . . . 717

Hyalea, . . . . $35^{8}$

Hyalonema, . . . . I25

Hydatina, . . . 223

Hydra, . 32, 37, I3I, I34

" Budding of, . . 5I

" Development of, . I38

, General life of, . . I34

" , structure of, . I35

" Minute ", I36

". Muscle system of, . 38

"Physiology of, . . I5

". Reproduction of, . 49, I 38

Hydrachna, . . . 336 
PAGE

Hydractinia, - . I3I, I55

Hydra-tuba of Aurelia, . - I47

Hydrochœrus, . . . 7 II

Hydrocorallinæ, . . . I56

Hydroid colonies, . . . I30

Hydromedusæ, . I29, I40, I55

Hydrophis, . . . . 583

Hydrophora, . . . I55

Hydropotes, . . . 698

Hydrozoa, . , I33, I55

Types of, . I40, I 55

Hydrozoon and Scyphozoon contrasted, . . I48

Hyla, . . . • . 556

Hylobates, . . • . 726

Hylodes, . . . . 556

Hymenaster, . . . . 235

Hymenocaris, . . . 276

Hymenoptera, . . 298,304

Hyoid arch of 'Vertebrates, - 43I

Hyo-mandibular arch, . • 43I

Hyostylic, . . . . 534

Hypapophysis, . . . 66r

Hyperia, . . . . 278

Hyperolins, • * . $\quad 555$

Hypoblast, . . . 36,67

Hypodermis = Epidermis, $\quad$. 254

Hypophysis, . . . 436

Hypostome, . . . . I35

Hypotricha, , . , , 105

Hypural bone, . . . 497

Hyracoidea, . . . . 702

Hyracotherium, . . . 70I

Hyrax, • • • 702

Hystricomorpha, . . . 7II

Hystrix, . . . . 7II

Ianthina,

357

Ichneumon, . * * . 7 I5

Ichthyomyzon,
Ichthyopsida, Sauropsida and Mammalia contrasted,

Ichthyopterygia, 473

Ichthyopterygium of Fishes,

Ich thyosaurus,

Idant,

Idotea, · • • • • 277

Iguana, . • • . 577

Iguanodon, - . • . 593

Ilium,

593

60
Incus of Ear, . . . . . 445

Indirect development, - . 228

division, . . . 45

Indris, . . . . . 722

Infundibulum, . . . 436

Infusoria, . . . . 85

Ciliary movement in, 106

Ink bag of Cephalopods, - 378

Innominate Bone, . . 666

Insecta, . . . 285, 292

"Classification of, 304-5

,, General characters, . 293

, , life of, . - 324

Insectivora, . . . . 717

Insects and flowers, $\quad \cdot 325$

" History of, . . 326

" Injurious, . . . 325

" Pedigree of, - . 326

Instinct, . . . . 629

Integration of individual, . $\quad 32$

Integripallia, . . . . 372

Intestine, - . - . 448

Intracellular digestion. 22, 86, 121

Invagination, . . . . 67

Invertebrata. Classes of, .6-10

Iris, . . . . . 446

Ischium, . $\quad . \quad$. $\quad$. 434

Isis, . . . . 153

Isolation, . . . . 770

Isomya, . . - . 372

Isopleura, . . . 345

Isopoda, . $\quad$ - $\quad$. 269

Isotrypsin, . . . . 739

Iter, . . . . . 438

Ixodes, . . . . . 336

Jackal, . . . . 715

Jacobson's organ, . . . 669

Jaguar, . . . . 715

Jellyfish, . . . 130, 142

Jerboa, . . . 7 II

593 Jugal, · . . . 429,662

520 Julus, . . . . . 292

Kangaroo,

687

Karyokinesis, : : : 45

Katabolism, - : : : 28

Keber s organ, . . . 368

Keratin, 
Kidney, Functions of,

PAGE of Vertebrates,

King crab,

Kiwi,

Kolga,

Kowalevskia,

Labial Cartilages of Skull, "Palp of Cockroach,

Labium of Insects,

. 295

Labrum of Insects,

Labyrinth of Ear, .

Labyrinthodontia, .

Labyrinthula,

Labyrinthulidea,

Lacerta,

Lacertidx,

Lacertilia,

Lace-winged flies, .

Lachrymal gland, .

Lacinia, .

Lacteals,

of Cockrroach,

Lactic acid,

Lagena, .

Lagomys,

$=$ Cochlea,

Lambdotherium,

Lamellibranchiata,

Lamprey,

Lamp shells, .

Lancelet,

Langia, .

Language,

Lanice, .

Larva of Amphibians,

Anodonta,

Antedon,

Ascidian,

Aurelia,

Chretopods,

Crustaceans,

Holothuria,

Insects, .

Lamprey,

Molluscs,

Nemerteans,

Ophiuroids,

Polygordius,
25

458

337

619

243

408

482

295

308

308

295

444

557

100

99

570

578

560,569

304

448

308

295

23

$2 I$

IOI

444

7 I I

702

362

469

225

410

176

626

2 II

$55 \mathrm{I}$

37 I

246

408

I 46

208

28I-2

243

322

473

345

I 80

236

208
Larva of Sea urchin,

PAGE

" Star fish, .

Larvacea,

Larynx, . . 428,534,675

Iatax, . . . 716

Lateral line system, . 495, 525

Laurer-Stieda canal, . . I67

Layers of Coelomata and

Hydra contrasted, . I38

Leech, . . . . 213

Lemming, . . . . 7II

Lemur, . . . . 721

Lemuridæ, . . . . 721

Lemuroidea, . . . . 721

Lens of Eye, . . . . . 446

Leopard, . . . . 714

Lepas, . . . . 269

Lepidoptera, . . . . 304

Lepidosiren, . . 5II,5I6

Lepidosteus, . . . 508

Lepisma, . . . . 305

Leporidx, . . . 7II

Leptocardii=Cephalochorda, ' 410

Leptodera, . . . . 183

Leptodiscus, . . . . 105

Leptodora, . . . . 27I

Leptomedusæ, . . . I55

Leptoplana, . . . . 163

Leptostraca, . . . . 269

Lepus, . . . 657,7II

Lerniea, . . . 272

Lencocytes of blood. - 453,75I

Leucon type of sponge, . . I 8

Leucones, . . . . 125

Leucosolenia, : , , 125

Lice, • . . 305

Life History of Amcba, . 87

Anodonta, . 370

Aurelia, . 146

Cestodes, I7I, I75

Crustaceans, . 282

Distomum, . 167

Frog. . . 549

Gregarina, . 88

Hydra, . . I38

Insects, . $32 \pi$

Monocystis, . 90

Nematodes . 183

Paramœcium, 92 


$\begin{array}{cc}\text { Life History of Spongilla, } \\ \text { " } & \text { Tænia, } \\ \text { " } & \text { Trichina, } \\ " & \text { Tunicates, } \\ \text { " } & \text { Vorticella, }\end{array}$

Lima,

Limax, .

Limbs and Girdles of-

Chelonia,

Crocodilia,

Frog.

Haddock,

Lizards, .

Pigeon,

Rabbit,

Skate,

Theories as to origin of,

Limnadia,

Limnæus,

Limnocodium,

\section{Movement of,}

Limnoria, .

Limpet, .

Limulus,

Lineus, . . . . 176

Linguatulida, . . . . 336

Lingula,

Liodon, .

Lion,

Liopelma,

Lipocephala,

Lipochrome pigment,

Lithobius,

Lithodes,

Lithodomus,

Littoral life,

Littorina,

Liver, Functions of, of Vertebrates,

Liver Fluke. .

Lizards,

"Classification of, General characters of,

Llama,

Lobosa,

Lobster,

Lobworm,

Locust, .
PAGE

I 22

I) I

184

407

94

95

I 7 I, 295

- 372

- 358

565

- 586

- 534

- 499

573

605

664

482

- $52 \mathrm{I}$

- 270

I69, 358

- 359

- 160

- 277

357

337

226

593

- 7 I4

- 556

- 362

I2I, 748

- 292

- 279

- 372

- 755

- 357

23,24

$45 \mathrm{I}$

I6 4

569

577

569 696 86, 99

305
Loligo, .

Lophiodontidæ,

Lophobdella, .

Lophobranchii,

Lophogaster, .

Lophophore, .

Lophopus,

Loxosoma,

Lucernaria,

Lucifer, .

PAGE

Lucina, .

Luidia,

Lumbricini

Lumbricus,

Lung-books of Scorpion,

Lungs, .

and Air-bladder,

Function of,

Lutra,

Lycaon,

Lycosa, .

Lymnæus = Limnæus, . $\quad .358$

Lymph, . . . . 23,74I

", hearts, . . 545

Lymphatic system of Frog, - 545

$$
\text { " } \quad \text { " Rabbit, . } 674
$$

Macacus,

726

Machairodus,

715

Macrauchenia, . . . 702

Macrobdella, . . . 213

Macroclemmys, . . . 567

Macromere, . . . 417

Macronucleus, . . . II2

Macropodidæ, . . . 687

Macropus; . . . . 687

Macrorhinus, . . . 717

Macroscelides, . . . 718

Madrepora, . . . . 157

Madreporaria, - . I53, I57

Madreporic plate of Brittle star, 235

,",$\quad$ Sea urchin, 238

". " Starfish, 232

Magosphera, • • . I57

.$\quad \cdot 99$

$\cdot \quad \cdot 279$

Malacobdella, . . . 180

Malacostraca, . . . 269

Malapterurus, Electric organ of, 478 

tubule,

Mandibular arch of Vertebrates, 428

Mandril, . . . 725

Manidæ, . . . . 690

Manis, . . . . 690

"Vertebre of, . . 635

Mantle of Molluscs, . . 343

Manubrium of Swimming bell, i 40 $\because$ Sternum, . 66I

Many-plies, . . . . 697

Margelis, . . . . 155

Marmosets, . . . . 724

Marmot, . . . 710

Marsipobranchii, see Cyclos-

$\begin{array}{cr}\text { tomata, } \\ \text { Marsupial bones, . } \quad ~ & 682,685\end{array}$ Marsupials, . . . 684 Families of, * . 685 General characters of, 684

Marsupites, . . . 246

Marten, . . . . 7I6

Mastigamceba, . . . 105

Mastodon, . . . . 704

Mastodonsaurus, . . $\quad 557$

Mastoid process, . . . 663

Maxilla of Vertebrate Skull, . 43I

Maxillæe of Cockroach, . . 295

Maxillæ of Insects, . . 308

,' Myriopods, . 29I

Maxillipedes of Crayfish, . . 256

May-flies, . . 305

Meckel's cartilage, , . $43 \mathrm{r}$

Medulla, . . . 438

Medullary canal, . . . 434

" groove, . . 434

Meduse,, . . . . I47

Medusoids, . . . I30, 140

Medusomes, . . . . 156

Megachiroptera, . . . 721

Megalobatrachus, . . . 556

Megalopa, . . . 282

Megalosaurus, . . . 594

Megaptera, . . . . 709

Megascolides, . . . 209

Megatheriidæ, . . . 690

Megatherium, . . 640,690

Meibomian gland, . . . 448

Meles, . . . . 716

Melicerta, . . . . 223

Mellivora, . . . . 716

Membrane bones, . . . 662

Membranipora, . . . 225

Menobranchus, . . . 556

Menognatha, . . 304, 305

Menorhyncha, . . . 305

Mento-meckelian, . . 534

Mentum of Cockroach, . . 295

Insects, . . 308

Mephitis, . . . . 716

Mermaid's gloves, . . I25

$"$ purse, . . . 493

Mermis, . . . 183

Meroblastic segmentation, . 65

Merostomata, . . 340

Mesencephalon, . . . 438

Mesenchyme cells, . 38,67

Mesenteries of sea anemone, . I $5^{\circ}$

Mesenteric filaments, . . I5I

Mesenteron, . . . 448

Mesentery, . . 4 487,670

Mesoderm (or Mesoblast), 36, 67

Mesoglcea of Coelentera, : 430
I30

" Sponges, . . II6

Mesonephros, . . 459 "' in the different "Vertebrate groups, . 46I 


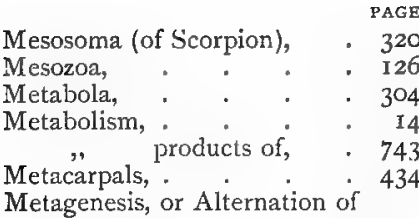
Generations,

Metagnatha, .

Metakinesis, . . . 46

Metamere, . . . . 419

Metamorphosis of-

Anodonta, . . . $37 \mathrm{I}$

Crustacea, . . . 28I

Echinoderma, . . 250

Frog, . . . . 552

Insects, . . 32I-24

Lamprey, . . . 473

Tunicata, . . . 40I

Metanephros, . . . 459

" in the different

Vertebrate groups, . 46I

Metapleural fold, . . . 4II

Metasoma (Scorpion), . $\quad 329$

Metatarsals, . . . 434

Metatheria, . . . . 684

Metazoa,

Me" and Protozoa,

$$
\text { and Protozoa, : I I5 }
$$

Metencephalon, . . . $43^{8}$

Microchiroptera, . . . $72 I$

Microgromia, . . IOI

Microhydra, . . . 140, 160

Micromere, . . . . 4I7

Micronucleus, . . . II2

Micropyle, . . . . 298

Microstoma, . . . . I62

Microzooids, . . . . 94

Midas,

Midge,

Mid gut = Mesenteron, . . 448

Miliolina.

Milk, . , . . . 642

Millepora, . . . . 156

Millipedes, . " . 285,29I

Milt,

Mites, - . . . . 336

Mitosis,

Moa,

Modiola,

Moina, .
Molars, . . . . . 646

Mole, . . . . 718

Mole Cricket, . . . 305

Mollusca, . . . . 343

Classification of, $\quad 388$

General characters of, 343

Notes on, . 387

Life history of, .

Shell of,

Molluscoidea, . • . 224

Moloch, . . . . 577

Monachus, . . . . 717

Monaxonida, . . . . II6

Monera, . . . . I09

Moniligastres, . . . 209

Monitor, . . . . 578

Monkeys, . . . . 725

Monobia, . . . 98

Monocystis, . . . 88, 104

Monocyttaria, . . . I03

Monodon, . . . 709

Monogenetic Trematodes, . I70

Monomeniscous eyes, . . 3II

Monomya, . . . . 372

Monostomum, . . . I70

Monotremata, . . . 68I

Monoxenia, . . . I53, 157

Moose, . . . 698

Morphology, . . . $\quad 30+3 \mathrm{I}$

Morse, . . . .716

Morula, . . . . 65

Mosasauria, . . . . 593

Moschus, . . . 698

Mother of pearl, . . . 389

Moths, . . . 304

Moulting of cuticle of crayfish, 255

Mouse, . . . 7II

Mouth, Origin of Vertebrate, 449 of Lopadorhynchus, . 449

Mucous canals of Skate, . 487 , glands of Myxine, . 466

" Snail, . 349, 356

Mud Fishes, . . . 5 II

Muillerian duct, : : : 462

in different

Vertebrate groups, *46I

Multiple conjugation, . : II2

Multituberculata, . . : 688

Mungoose, . . . . 592

Murex, . . . . . 357 
Muridæ,

PAGE

Muscle, Contraction of, : Fibres, markings, Smooth, Striped,

Muscular system of -

Amphioxus,

Anodonta,

Arenicola,

Ascidian,

Aurelia,

Balanoglossus,

Birds,

Cockroach,

Crayfish,

Crocodilia

Earthworm,

Frog,

Haddock,

Helix,

Hirudo,

Insects,

Mammals,

Myxine,

Peripatus,

Petromyzon,

Pigeon,

Rabbit,

Sepia,

Skate,

Snakes,

Starfish,

Vertebrates,

Muscular tissue,

Musk deer,

$$
\text { activity in animals, }
$$

", glands,

"rat,

Mussel, Edible,

Mustela Horse,

Mustelidx,

Mustelus,

Mya,

Mycetes,

Mycetozoa,

Myelencephalon,

- $7 \mathrm{Ir}$

- 39

2I, 39

39

21, 39

$2 \mathrm{I}, 39$

4I 3

365

202

402

I 43

392

600

294

257

587

189

536

496

350

216

310

659

466

287

470

600

659

376

478

579

230

426

38

2 I

698

698

7 II

372

372

716

716

506

506 372

725

98

439
Mygale,

PAGE

Myodes,

333

Myogale,

7 II

Myomeres or Myotomes,

7 I7

Myomorpha,

413

Myopotamus,

Myotomes,

7 II

Myoxid:

Myriopoda,

Myrmecobius,

Myrmecophagidæ,

Myrmecophagus,

Mysis,

Mystacoceti, .

Mytilus,

Myxidium,

Myxine,

" and Petromyzon contrasted,

Myxospongiæ,

125

Myzostomata,

212

Nais,

209

Naja,

Narcomedusæ,

583

Narwhal,

Nasal Capsule,

Nasalis,

Naso-buccal groove, . . 487

Naso-palatine canal, . . 669

Natural Selection, . . . 769

Nauplius,

269

Nautilus,

Nearctic region,

383,385

Nebalia, 764

Nebendarm, "

Nebendarm, " 209

Necturus, . . . . 556

Nemathelminthes, . . . 186

Nematocysts, . . . $\mathbf{1 3 6}$

Nematoda, . . . 180

Nemertea or Nemertines, - 175 ,$\quad$ Affinities of, to

Vertebrates, I80, 425

Nemertes,

Classification of, . 180

Neo-crinoidea. $\cdot$. 246

Neomenia, . . . . 347

Neornithes, . . . . 617

Neotropical region, . . 764

Nephelis, 
PAGE

Nephridioblast, • . . 199

Nephridia of Crayfish, . . 264 Earthworm, . 194 ", Gasteropoda,

,

Leech, 345 ,

354,357

3

,

Mussel,

Peripatus,

Vertebrates,

Nephrops,

Nephrostoma of kidney, .

Nephthys,

Nereis,

Nerve Cells, .

, Fatigue,

" Fibres,

, Tissue,

Nerves, Cranial, Morphology of,

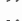
Origin of, Structure of,

Nervous Activities in Animals,

Nervous System of-

Amphioxus,

Anodonta,

Arenicola,

Ascidian,

Aurelia, .

Balanoglossus,

Bee,

Birds,

Cephalopoda, .

Chretognatha. .

Cockroach,

Crayfish,

Crinoid,

Distomum,

Earthworm,

Frog,

Haddock,

Helix ?

Herring. .

Hirudo,

Holothurian,

Hydra,

Insects,

Limulus,

Lizards, .

Mammals,

259

- 244

167

- 190

536

500

- $35^{\circ}$

504

- $2 \mathrm{I} 6$

241

136

3 IO

339

573
Nervous System of -

Myxine, . . . . 466

Nematoda, . . . I8I

Nemerteans, . . . 176

Peripatus, . . 287

Petromyzon, . . 470

Pigeon, . . . 606

Rabbit, . . . 667

Rotifers, . . . 223

Scorpion, . . . 329

Sea-urchin, . . . 237

Sepia, . . . 378

Skate, . . . 483

Spider. . . . . 332

Starfish, . . . 23I

Vertebrates, . . . 434

Nervures, . . . 308

Nesodon, . . . 705

Neurapophysis $=$ Neural

spine, . .

$433,66 I$

Neurilemma, . . . . 40

Neuroblast, . . . . 199

Neurochord, . . . . I9I

Neuro-muscular cells, . . 39

Neuro-nephroblast, . . 220

366 Neuropodium, . . . 209

Neuroptera, . . . . 304

Newts, . . . 556

Nictitating membrane of Eye, 658

Noah's ark-shell, . . . 372

Noctiluca, . . .. . I05

Nose, The, . . . . 443

," of Myxine, . . . 466

Nothosanus, . . . 592

Notochord of Balanoglossus, . 432

of Fishes, . * 432

- 667
Origin of, - . 432

Sheath of, : . 432

Notommata, . . . 223

Notopodium, . . . 209

Notopteris, . . . . 72I

Notoryctes, . . . . 688

Nototrema, . . . . 556

Nucleus,

N" $\cdot 43$

43

Nudibranchs, . $\quad .357$

Nummulites, . . . . IO2

Nycticebus, . . . . 722

Nyctipithecus, : : : 725 
Nymph, . . . . . 322

Nymphon, . . . . 342

Obelia, . . . . I55

Ocellatæ, . . . . I4I

Ocelli, . . . . 3II

Octacnemus, . . . . 409

Octocoralla, . . . . I57

Octodontid $x, \quad . \quad$. 7 I I

Octopoda, . . . . 386.

Octopus, . . . . 386

Oculomotor nerve,. . . 44I

Odonata, . . . . 305

Odontoceti, . . . . 709

Odontoid process, . . . 660

Odontolcx, . . . . 619

Odontophora, see Glossophora, . . . 388

Odontophore of Cephalopoda, 375

", "Snail, . 35I

Csophagus of Vertebrates, . 450

Oikapleura, . . . . 408

Olfactory lobes, . . . . 436

$$
\text { "nerves, . . . 44I }
$$

". .

Oligosoma, : . $\quad \cdot \quad 578$

Ollulanus, . . . . 183

Omentum, . . . . 6I I

Ommastrephes, . . . 386

Oniscus, . . . $\quad 277$

Ontogeny, . . . . 70

Ooze, Atlantic, . . . IOI

Opalina, . . . . 105

Opalinopsis, . . . . 109

Operculum of Gasteropods, . 357 $\begin{array}{lll}" & \text { Limulus, . } & 340 \\ " & \text { Scorpion, } & \text {. } \\ " & \text { Teleostei, } & 510\end{array}$

Opheosaurus, . . 577

Ophidia, . . 560,578

Ophiocoma, 583

Ophiophagus,

Ophiopholis, .

Ophiothrix, .

Ophiuroidea, . . . . 235

Ophryodendron, . . . . 105

Ophryotrocha, . . . . 212

Ophthalmosaurus, . . 593
Opisthobranchs, . . . 357

Opisthocœlous, . . . 508

Opisthocomus, . . . 63I

Opossums, . . . . 686

Optic chiasma, . . . 484

, foramen, . . . 663

, lobes, . . . 436

,, nerves,. . . . 44I

" thalami, . . . 44I

,,,$\quad$ Structures con-

nected with, 436

• $\cdot 726$

Orca, . . . . . 709

Orchestia, . . . 278

Oreocephalus, . . . 577

Organs, . . . . 32

" Analogous. . . 33

" Classification of, - 36

" Correlation of, . - 33

" Homologous, . . 33

" Origin of, . . . 68

" Rudimentary, . . 35

" Substitution of, - 34

Organ Pipe Coral, . . I57

Oriental region, . . . 764

Ornithodelphia, . . . 68I

Ornithorhynchus, . . . 68r

Ornithosauria, . . . 594

Orthagoriscus, . . . 511

Orthoceras. . . . $3^{85}$

Orthonectidx, . . . I27

Orthoptera, . . . 305

Orycteropus,. . . 690

Oscarella, . . . . II9

Osculum, . . . . II7

Osphradium of Molluscs, . 367

Ossicles of Ear, . . . 445

Osteoblasts, . . . 38

Ostracion, . . . . 5I I

Ostracoda, . . . . 269

Ostrea, . . . . . 372

Ostriches, . . . . 618

Otaria, . . . . 716

Otocyon, . . . 7I5

Otocysts, see Otoliths, - $35 \mathrm{I}, 367$

Otoliths of Anodonta, . $\quad 367$

Aurelia, . . I43

Crayfish, . 260

Haddock, . . 500 
Otoliths of Sepia, . . . $\quad 382$

, Skate, . . . 487

, Vertebrates,. . 444

Otter, . . . 7I6

Ova of-

Amphibia, . . . 549

Anodonta, . . . 370

Birds, . . . 6I3

Cockroach, . . . 298

Crayfish, . . . 265

Earthworm, . . . 195

Echinoderms, . . . 247

Fishes, . . . 519

Fluke, . . . . 167

Hydra, . . . . I38

Monotremes, . . . 682

Myxine . . . . 469

Peripatus, . . . 288

Placental Mammals, . 678

Reptilia, . . . 589

Vertebrates, . . . 463

Oviducal gland of Skate, - 493

Oviduct of Vertebrates,. . . 462

Oviparous Vertebrates, . . 464

Ovis, . . . . 698

Ovists, The, . . . 49

Ovo-testis of Snail, . . 354

Ovo-viviparous Vertebrates, . 464

Ovum, The, . . . . 56

" Maturation of the, 59-60

" Membranes around the Vertebrate, . 466

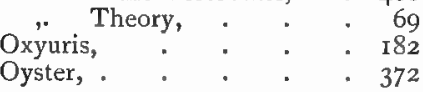

Paca, . . . . . 7II

Pachymatisma, . . . I20

Pagurus, . • . . 279

Palæarctic region, . . . 763

Palæichthyes, . . . 528

Palemon, . . . . 279

Palæo-Crinoidea, . . . 246

," Echinoidea, . . 240

Paloenemertea, . . . I80

Palreontolugical series, . . 76

Palæontology, . . . 74

Palæospondylus, . . 5, 574

Palicostraca, . . . 327,337

Palæotherium, . . 702
PAGE

Palate of Mammals, . . 669

Palato-pterygo-quadrate car-

$$
\text { tilage, }
$$

Palinurus,

Palisade Worm,

- $43 \mathrm{I}$

Pallial line, . . . . 364

Pallium, . . . . 343

Palmipes, . . . . 229

Palp, . . . . 308

Paludicella, . . . . . 225

Paludina, . . . . 76

Pancreatic juice, . . . 23

Panda, . . . . 716

Pandalus, . $\quad . \quad$. $\quad 279$

Pangenesis, . . . . $7 \mathbf{I}$

Pangolin, . . . 690

Panmixia, . . . 758

Panniculus adiposus, . . 659

" carnosus, - 660

Pantopoda, : : : : : $\quad \cdot 342$

Parachordals of Skull, . - 431

Paraglossæ, . . . 308

Parn" " 295

90,105

Paranucleus, . . . I05

Parapodia, . . . 187,210

Parasphenoid, . . . 533

Parasuchus, . . . 589

Parasitic Fauna, . . . $75^{8}$

Parasitism of-

Acarina,

Cestoda,

Cirripedia, : : : 275

Copepoda, . . . 271

Crustacea, . . $\quad 279$

Cyamidæe, - . $\quad 277$

Cymothoidæ, . . . 277

Gasteropods, . . . 360

Insects, . . . 325

Nematoda, . . . I8I

Nemertea, . . . I84

Pentastomum, . . 336

Trematoda, . . . 164

Parenchymula, . . . 124

Parental care in-

Amphibians, - . 558

Asteroids, . . 235

Birds, . . . . .626-7

Clepsine, . . 220 
Parental care in-

Penella,

Crayfish,

Crocodiles,

Echinoids,

588

Fishes,

Mammals,

526

Spiders, .

639

Pariasaurus,

Paroccipital process,

592

Parovarium $=$ Wolffian body in

663 female,

Parthenogenesis,

$46 \mathrm{I}$

49,55

$\begin{array}{ll}" & \text { in Apus, } \\ " & \text { Artemia, } \\ " & \text { Crustacea, } \\ " & \text { Insects, } \\ " & \text { Limnadia, } \\ & \text { Rotifers, }\end{array}$

Patella,".

Pathetic nerve,

Pathology, Comparative,

Paunch,

Pauropoda,

Pauropus,

Peccaries,

Pecora,

Pecten,

$$
\text { of Eye of Birds, \&c., . }
$$

Pectines of Scorpion,

Pectoral girdle,

270

270

280 318

270

223

357

$44 \mathrm{I}$

749

697

292

292

695

696

372

447

329

Pedal ganglion,

434

Pedalion,

366

Pedata,

Pedicellariæ of sea-urchin,

Pedicellina, starfish, .

Pedipalpi,

Pedipalps of Scorpion,

237

- 230

225

331

329

Pelagia, Spider,

332

Pelagic Life, .

I $33,148,156$

Pelagonemertes,

Pelagothuria,

Pelecypoda, see Bivalves,

Pelias,

Yelican fish,

Pelobates,

Pelomyxa,

Pelvic girdle,

Penæus,
Penis of Mammals,

272

Pennatula, .

678

Pentacrinus,

I $53, \mathrm{I} 57$

Pentastomum,

244

Pepsin,

336

Peptic digestion,

22

Peragale,

740

Peramelidæ, . . . 686

Perch, . . . . 510

Perching of Birds, . . . 601

Perennichordata, see Larvacea, 408

Peribranchial cavity, . . 406, 4I6

Pericardium, . . . 454

Perícolpa, . . . . I56

Peridinium, . . . . I05

Perigenesis, . . . . $7 \mathbf{I}$

Perilymph, . • . 444

Perineal glands, . $642,670,678$

Peripatus, . . . . 285

, Detailed account of, 286

", and Annelids, . 285, $29 \mathrm{I}$

Peripheral segmentation, . 65

Periplaneta, . . . 293

Periptychus, . . . 704

Perissodactyla, , . . 698

Peristaltic action, . . . 23

Peritoneum, . . . 670

Peritricha, . . . 93

Periwinkle, . . . . 357

Perla, . . . . 305

Peromedusæ, . . . 156

Petalosticha, . . . 240

Petaurus, . . . 687

Petromyzon, . • . . 469

Phacochorus, . . 695

Phacops, : . . 341

Phagocytes, . . . . 749

Phagocytosis, . . . 749

Phalanger, . . . . 687

Phalangeridx, . . '. 687

Phalanges, . . . . 434

Phalangidæe, . . . 33r

Phalangium, . . . . 33I

Phallusia, . . . . 409

Pharyngobranchii, . . 4ro

Pharyngognathi, . . . 5IO

Pharynx, . . . . 449

Phascogale, . . . 686 
PAGE

Phascolarctos, . . . 687

Phascolomyide, . . . 686

Phascolomys, $_{4} \quad$. $\quad$. 687

Phascolosoma, . . . 224

Phenacodus, . . . 70I

Phoca, . . . . 7I7

Phocæna, . . . . 709

Phocidx, . . . . 716

Pholas, . . , . 372

Phoronidea, . . . . 224

Phoronis, . . . . 225

Phronima, . . . . 278

Phrynosoma, . . . . 577

Phrynus, . . . . 33I

Phyllopoda, . . . . 269

Phyllopteryx, * . * 5I I

Phyllosoma, . . . . 278

Phylloxera, . . . . 305

Phylogeny, . . . . 70

Physalia, . . . . I56

Physeter, . . . . 709

Physiology, . . 14, 73I

," History of, . I6-I7

Physoclisti, . . . . 495

Physostomi, . . . . 503

Phytoptus, . . . . 336

Phytozoa, . . . . I29

Pia mater, . . . . 438

Pica, . . . . 7II

Pigs, . . . . . 694

Pigeon, . . . . 599

Pigeon's milk, . . . 608

Pigments, . . . . 746

Pilema, . . . . I48

Pilidium larva, . . . I76

Pineal body, . . . . 437

,$\quad$ in Hatteria, , 437

$\begin{array}{ll} & \text { in Iguana, : : } 437 \\ \text { Pinnipedia, . . } 716\end{array}$

Pinnotheres, . . . . 279

Pipa, . . . . 556

Pipe fishes, . . . . 5II

Pipistrelle, . . . . 721

Pisces, see Fishes, d 4, 475

Pisiformis, . . . . I75

Pithecia, . . . 725

Pituitary body, . . . 436

," Hypotheses regarding, . 436

Placenta, Hints of a, before Mammalia,

Placenta of Mammals, . . 648

Placentation, Classification of, 655

Placoid scales, • . • 478

Plagiaulax, . . . . 640

Plagiostomata, . . . 505

Plakina, . . . 125

Planaria, . . . 162,163

Planorbis, . . . $76,35^{8}$

Plantigrade, . . . 714

Plants and Animals, . I7-I9

Planula larva, . . . I4I

Plasmodium, . . . 98, I12

Plasticity of Organisms, $\quad . \quad 83$

Plastidules, . . . 71

Plastron, . . . 563, 565

Platanista, . . . 709

Plathelminthes, . . . 162

Platydactylus, . . . 577

Platyrrhini, . . . . 725

Plecoptera, . . . . 305

Plectognathi,. . . . 5II

Plesiosaurus, . . . . 592

Pleura of Crayfish, . . 257

Pleural membrane, . . 674

Pleuracanthus, . . . 506

Pleurobranchs of Crayfish, . 264

Pleurodont teeth, . . . 570

Pleuronectidæe, . . . 522

Pliosaurus, . . . . 592

Ploughshare bone, . . .60I

Plumularia, . . . . 155

Pluteus larva, . . 236, 249

Pluvianus, . . . . 588

Pneumatic bones, . . . 623

Pneumoderma, . . . 358

Pneumodichthyda, . . 528

Pneumogastric nerve, . - 44I

Podical plate of Cockroach, , 295

Podobranchs of Crayfish, . 264

Podophthalmata, . . . 278

Podura, . . . . 305

Poison gland of Snakes, . 582

Polar globules, . . . 60

: in Earthworm, 197

Pole $"$ in Vertebrates, 463

Polia, : : : 180

Polian Vesicle (Holothuria), : 242

Polychreta, . 187,201, 209

"Development of, . 206 
PAGE

Polycladida,

PAGE

Polyclinum,

Polycyttaria,

Polygordius,

Polymastodon,

Polymeniscous eye

Polynoë,

I'olyodon,

Polyphemus, .

Polyplacophora,

Polypodium, .

Polyprotodontia,

Polypterus,

Polysperny,

Polystomum, .

Polyzoa,

Pondsnail,

Pons Varolii,

Pontobdella,

Porcellana,

Porcellenaster,

Porcellio,

Porcupine,

Porifera,

Poromya,

Porpites,

Porpoise,

Portal veins of Mammals,

Portuguese Man-of-war,.

Portunus,

Post-anal gut,

Post-pubic processes,

Potamogale,

Præcoces,

Prairie dog,

Prawn, .

Preen gland of Pigeon, .

Preformation theory,

Priapulus,

Primary vesicles of brain,

Primates,

Primitive streak,

Pristis,

Proboscidea, .

Procavia,

Procolous,

Proctodreum, .

Procyon,

Procyonidxe,

Proechidna,

409

IO3

$208,2 \mathrm{I} 2$

640

- 3 II

- 210

- 508

269

- 345

I40, 160

685

508

63

170

224,225

357

668

214

279

235

277

7 II

I I6

372

I 56

709

674

I $32, I 56$

- 279

- $45^{2}$

- 605

- 717

- 628

- 7II

- 279

- 599

49

224

435

679

614

506

703

702

532

449

. 716

716

. $68 \mathrm{I}$
Proglottis,

PAGE

Proneomenia,

I7 I

Pronephros,

347

Pronephros in the different

Vertebrate groups, . 46I

Prongbuck, . . . . 698

Proscolex, . . . . I7I

Prosencephalon, . . . 439

Prosopygii, . . . . 224

Prostate glands, . . 219,678

Protandrous, . . 463,468

Protective colouring of Insects, 324

Proteleid $x_{\text {, . . . 715 }}$

Proteles, . . . . 715

Proteolepas, . . . . 275

Proteomyxa, . . . . 98

Proterosaurus, . . . 569

Proterospongia, . . . 105

Proteus, . . . 556

Proteus animalcule, see Amoba.

Protobathybius,

98

Protobranchia, . . . 372

Protocercal, . . . . 521

Protodrilus, . . . . 2 I2

Protoelastin, . . . . 87

Protogenes, . . . . 98

Protogynous, . . . 96, 963

Protohippus, . . . 701

Protohydra, . . . . I40

Protomyxa, , . . . 98

Protoplasm, . . . 26, 42, 47

Protopodite of Crayfish, . 255

Protopterus, . . 5 II, $5^{\mathrm{I}} 3$

Protospongia, . . I25

Prototheria . . 68I

Protovertebræ $=$ Mesoblastic

segments, . . 426, 430

Protozoa, . . 85-115

"Classification of, $97-98$

, Functions in the, . 106

" General interest of, I I 4

,2, Notes on the, 106

, History of the, I05-6

, Immortality of, . II5

, and Metazoa, . II5

"Reproduction in, . IIO

, Structure of, . , IO9

," Survey of the, - 98

Protracheata,. • . . 285

Proviverra, . . . . 717 
Psalterium, . , . . 697

Pseudobranchus, . . . 556

Pseudogastrula, . . . 123

Pseudolamellibranchia, . $\quad 372$

Pseudonavicellæ, . . . 90

Pseudopodia, . . . 86

Pseudopus, . . . . 578

Pseudoscorpionidæe, . . 331

Psolus, . . . . 243

Pteranodon, . . . 594

Pteraster, . . . . 235

Pterichthys, . . . . 509

Pterodactyl, . . . . 594

Pterodon, . . . . 7I7

Pteromys, . . . . 7II

Pteropods, . . . . $35^{8}$

Pteropus, . . . . 721

Pterotrachea, . . . 357

Pterygota, . . . 304

Ptyalin, . . . . 22

Ptychodera, . . . . 392

Ptychozoon, . . . . 577

Puff Adder, . . . . . 583

Pulmonary sacs of Arachnids, 327

" "Scorpion, - 330

Pulmonata, ". Spider, • • 3337

Puma, . . . . 7I5

Pupa, . . . . . 322

Pupil of Eye, . . . 446

Purpura, . . . . 357

Pycnogonidæe, . . . 342

Pycnogonum, . . . 342

Pycnopodia, . . . . 235

Pygostyle, . . . 60I

Pylangium, . . . . 54 I

Pyloric cæeca of Insects, \&c., . 3I3

Pyrosoma, . . . 40I, 409

Python, . . . . 583

Quadrate,

Quadrumana, . . . 729

Quagga, . . . 702

Rabbit, . . . . . 657

Raccoon, . . . . 7166

Rachiodon, . . . . 500

Radial symmetry, . . . 3I

Radials of a fin, . . . 52 I

Radiolaria, . . . 102
Radius,

Radius, • • 434

Radula of Cuttlefish. • • 375

Gasteropods, . $35 \mathrm{I}$

Snail, . . 35I

Raja, . . . . 477, 506

Rana, see Frog, . * . 530

Rangifer, . . . 698

Raphidiophrys, . . . I00

Rat, . . . . 7 II

Ratel, . . . . . 716

Ratitæ, . . . . 618

Rattlesnake, . . . $\quad 583$

Ray, . . . . . 477

Razor-shell, . • . . 372

Recapitulation of Ancestral

History, . . . 69

Rectal gland, . . . 487

" respiration, . . 280

Red Coral, . . . . 157

Redia . . . . 169

Reducing division,. . . 60

Reed, . . . . . 697

Reindeer, . . . . 698

Relative age of Animals, $\quad 79,80$

Reproduction, . . 15,49

\begin{tabular}{|c|c|c|c|}
\hline & & & \\
\hline & 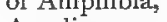 & & 5 \\
\hline & Aurelia, & . & 145 \\
\hline & Crustacea, & - & $28 c$ \\
\hline & $\begin{array}{l}\text { Earthworm. } \\
\text { Echinoderms, }\end{array}$ & - & 19 \\
\hline & Fishes. & & \\
\hline & Hydra, & " & \\
\hline & Insects, . & $3 I 7$ & $3 I$ \\
\hline & Mammals, & 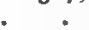 & \\
\hline & Modes of, & . & \\
\hline & Rotifers, & - & \\
\hline & Sponges, & . & 2 \\
\hline & Trenia, . & - & \\
\hline
\end{tabular}

Reproductive system of-

Amphioxus, . . . 4I7

Anodonta, . . . 370

Arenicola, . . . 206

Ascidian, . . . 407

Aurelia. : . . 145

Balanoglossus, . . 395

Bee, . . . 306

Chretognatha, . . 222

Cockroach, . . . 297

Crayfish, . . . 264

Crinoid, . . . . 246 
Reproductive System of-

Crocodilia,

Distomum,

Earthworm, - . . 194

Frog, . . . . 549

Haddock, . . . 502

Helix, . . . 354

Herring, . . . 504

Hirudo, . , . 2 I9

Holothurian, . . . 243

Insects, . . . 317

Limulus, . . 340

Lizards, . . . . 576

Mammals, . . . 676

Myxine, . . . . 468

Nematoda, . . . 182

Nemerteans, . . . 179

Ophiuroids, . . . 235

Peripatus. . . . 288

Petromyzon, . . . 472

Pigeon, . . . 612

Rabbit, . . . 676

Scorpion, . . 330

Sea urchin, . . . 240

Sepia, . . . . 382

Skate, . . . . 492

Spider, . . . . 330

Starfish, . . . 234

Tænia, . . . I72

Vertebrates, . . . 462

Reptilia,

3, 360

$"$ and Birds, . . 562

" Development of, . 589

"Extinct, . . . 592

" Pedigree of, . . 594

Respiration in Animals,

Respiratory System of-

Acarina, * · . 336

Amphioxus, • $\cdot 4 \mathrm{I} 6$

Anodonta, . . . 369

Arenicola, . . . 205

Ascidian, . . . 405

Balanoglossus, . . 395

Bee, . . . 306

Cephalopoda, . . . 38I

Cockroach, . . . 297

Crayfish, . . . 263

Crinoid,

Crocodilia,

Crustacea,
Respiratory System of-

PAGE

Frog, : .

Helix, . . . . 353

Herring, . . . 504

Holothurian, . . . 24I

Insects, . . . 3I4

Limulus, . . . 340

Lizards, . . . . 575

Mammals, . . . 674

Myxine, . . . 467

Peripatus, . . . 287

Petromyzon, . . . $47 \mathrm{I}$

Pigeon, . . . 6II

Rabbit, . . . . 674

Scorpion, . . . 330

Sea urchin, . . . 239

Sepia, . . . $38 \mathbf{I}$

Skate, . . . . 488

Spider, * • • . 333

Starfish, . . . . 234

Tracheata, . . . 314

Vertebrates, . . . 458

Retia mirabilia of Cetacea, - 705

Sirenia, - 69I

Sloths, . 689

Reticulum, . . . . 445

Retina, . . . . 697

Reversion, . . . . 83

Rhabdites, . . . . 162

Rhabdocolida, . . . I62

Rhabdonema, . . . 183

Rhabdopleura, . . $\quad 399$

Rhacophorus, . . . 555

Rhamphorhynchus, . . 594

Rhea, . . . 619

Rhinatrema, . . . 557

Rhinoceros, . * . . 702

Rhinoderma, . . . $55^{8}$

Rhinolophus, . . . $72 \mathrm{I}$

Rhizocrinus, . . . 246

Rhizopoda, . . 85

Rhizostoma, . . 148,156

Rhodeus, . . . . 527

Rhomboidal sinus,. . . 606

Rhopalia, . . . . I43

Rhopalodina, . . . 243

Rhopalura, . . . . I27

Rhynchobdellidx, . . . $22 \mathrm{I}$

Rhynchocephalia, . . . 568 
PAGE

Rhynchoflagellata, · • 105

Rhynchota, . . . 305

Rhytina,

691

Ribs of Vertebrates, · . 433

Rock-dove, . . . . 599

Rodentia, . . . 710

Roe deer, . . . . 698

Rorqual, . . . 709

Rostrum of Crayfish, . . 257

Rotatoria $=$ Rotifers, . . 223

Rudimentary organs, . . 35

Rudistæ,

374

Rugosa, . . . . I57

Rumen, . . . . 697

Ruminants, . . . . 696

Rumination, . . . . 697

Sabella, . . . . 209

Sable, . . . . . 716

Saccocirrus, . . . . $2 \mathrm{I} 2$

Sacculina, . . . . 275

Sacculus of ear, . • . 444

, rotundus, . . 670

Sacrum, . . . 601,660

Sagitta, . . . . 222

Sakis, . . . . . 725

Salamander, . . . . 556

Salinella, · • • . 127

Saliva, . . . . 22

Salivary glands of Ant-eaters, . 690

$\begin{array}{llll}, & \text { " } & \text { Cockroach, } & 296 \\ " & \text { ". } & \text { Collocalia, } & 627 \\ \prime \prime & \text { Helix, } & 352\end{array}$

" $\quad$ " Helix, - 352

" " $"$ Insects, . 3I3

" . Mammals, . 670

Salmon, ". Rabbit, . 670

Salpa, . . 5 . 510

Sapphirina, . . . . 272

Sarcocystis, . . . . IO4

Sarcode, . . . . 41

Sarcolemma, . . . . 2 I

Sarcoptes, . . . . 336

Sarsia, . . . . I55

Saurognathre, . . 620

Sauropsirla, Ichthyopsida, and Mammalia, . . 56I

Sauropterygia, . . 592

Saururæ,

Saw flies, . . . 304

Saxicava, . . . . 372

Scales of Birds, . . . 600

"Fishes, • • 52 I

" Mammals, . 64I

" Reptiles, 562, 563, 579, 583

Scallop,. . . . 372

Scalpellum, . . . . 274

Scaphirhynchus, . . 508

Scaphognathite of Crayfish, . 263

Scaphopoda, . • . 362

Scapula, . . . . 434

Schizocardium, . . . 392

Schizocoele, . . . $I 76,472$

Schizogenes, . . . . 98

Schizognathæ, . . . 620

Schizonemertea, . . . I80

Schizopoda, . . . . 276

Scincida, . . . . 578

Scincus, . . . . 578

Sciuromorpha, . . . 711

Sciuropterus, . . . 7II

Sciurus, . . . . 7 II

Sclerobasic, . • • . I54

Sclerodermic, . . . 154

Sclerotic, . . . 446

, ossicles, . . . 608

Scolex, . . . . 173

Scolopendra, . . . 292

Scolopendrella, . . . 29 I

Scorpion, . . . . 327

, flies, . . 304

Scrotum, . . . . 677

Scuta, . . . . . 272

Scutes, . . . . 426

Scyllium, . . . . 506

Scyphistoma, . . . . I47

Scyphomedusæ, . . 142,156

Scyphozoa, . . . I42, I56

Sea anemone, . . $\quad 149$

" butterfies, . . . 358

" cows, . . . . 691

" cucumbers, . . . 240

" horse, . . . . 5II

"lion, . . 716

. mat, . . . 225

" mouse, . . . 2 IO

" otter, . . . 716

"pen, . . . . I57

" snakes, : : : : 583 
Sea squirts, vide Tunicata, $\quad \begin{array}{r}\text { PAGE } \\ 401\end{array}$ ", urchin, . . . 236-40 Seals, . . . . 716 Sebaceous glands, . . . 642 Sedentaria, . . . 2 Io Segmental duct of Vertebrates, 462 ," Organs, . . I94 "Papillæ, . . 255 Segmentation of Ovum, . . 63 " in Amphioxus, . . 4I7 " Anodonta, . . 370 " Ascidian, . . 407 " Balanoglossus, . 396 " Birds, . . . 613 " Chætognatha, . 222 " Crustacea, . . 28I " Dipnoi,. . . 513 " Earthworm, . . 194 " Echinoderms, . 247 " Fowl, . . . 613 " Frog, . . . 549 "Ganoids, . . 508 " Gasteropoda, . . 356 " Haddock, . . 503 " Hydra, . . . I39 $" \quad$ Insects, . . 319

" Lamprey, . . 472

" Monotremata, 649

" Monotremata, : 649

" Placental Mammals, 648

" Reptilia, . . 56 I

"Scorpion, . . 330

" Skate, . . . 493

" Spider, . . 333

" Teleosteans, . . 503 " Vertebrata, * - 463

Seison, .

Selachii = Elasmobranchs, . 505 Selachodichthyidæ, . . 528 Selachoidei, . . . . 506 Selection, . . . . 769 Selenodont, . . . . 693 Self-fertilisation in Hydra, . 138

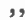

7

,9 in Serranus, . 463 in Tapeworms, I7I in Trematoda, 164

Sella turcica, .

Semicircular canals of Ear, . 444 Seminal vesicle, . . 219,677 Semnopithecina; . . $\quad 726$ Semnopithecus, . • $\quad 726$
Sense organs of -

Amphioxus, . . . 4I4

Anodonta, . . . 367

Ascidian, . . . 402

Aurelia, . . . 143

Crayfish, . . . 259

Crocodilia, . . . 587

Crustacea, . . . 280

Frog, . . . . 539

Haddock, . . . 500

Hagfish, . . . . 467

Helix, . . . 350

Hirudo, . . . 216

Holothurian, . . . $24 \mathrm{I}$

Insects, . . . 3I I

Lamprey, . . . 470

Limulus, . . . 295

Pigeon, . . . . 607

Rabbit, . . . . 669

Scorpion, . . . 329

Sea-urchin, . . . 237

Sepia, . . . 379

Skate, . . . 486-7

Spider, . . . . 332

Starfish, . . . . 231

Vertebrates, . . . 443

Sepia, . . . 375, 386

Sepiostaire, . . . . 375

Seps, . . . . 578

Septibranchia, . . . 372

Sergestes, . . . . 278

Serpents, . • . . 578

Serpula, . . . . 2 II

Serranus, . . . . 525

Sertularians, . . . . I55

Sesamoid bones, . . . 660

Setæe, . . . . 189

Sex, . . . 52-53

Sexual selection, . . . 770

" selection among Birds, 626

" " among Spiders, 334

" reproduction, . 50

" Divergent modes of

Sharks, sexual reproduction, $54-56$

Shell of-

Anodonta, . . . 364

Argonauta, . . . 386

Cephalopoda, . . . 375

Chiton, . . . 346 
Shell of-

Helix,

Molluscs

Nautilus,

Planorbis,

Scaphopoda, . $\quad .362$

Spirula, .

Shell gland or sac, . $36 \mathrm{I}, 37 \mathrm{I}, 387$

Shepheardella,

Shrews,

IOI

Shrimp,

Sida,

Silicispongiæ,

Simia, . . . . 726

Simiidæe, . . . . 726

Simplicidentata, . . . 710

Sinews, . . . . 2 I

Sinupallia, . . . $\quad 372$

Sinus venosus, . . $\quad$. 454

Siphon of Cephalopods, . $\quad 374$

, Gasteropods, . 357

Siphonaptera,

Lamellibranchs, - 373

Siphonophoræ, . . . I56

Siphonops, . . . 556

Siphuncle of Nautilus, . 384

Sipunculoidea, . $\quad$. 224

Sipunculus, . . . . 224

Siredon, . . . $\quad 557$

Siren, . . . . . 556

Sirenia, , . . . 69I

Skate, . . • • . 477

" Development of, - 493

" Structure of, . 477, 493

Skeletal tissues of animals, . 743 Skeleton of-

Amphioxus, . . . 4I2

Balanoglossus, • . 393

Birds, . . . . 622

Chelonia, . . $\quad 563$

Crayfish, . . . 257

Crinoids, . . . 244

Crocodilia, . . . $5^{84}$

Frog, . . . . 532

Haddock, . . . 496

Hatteria, . . . 568

Holothurian, . . . 24I

Limulus, . . . 338

Lizards, .

- $57 \mathrm{I}$

Skeleton of -

Mammals, . . . 635

Myxine, . . . 466

Petromyzon, . . . 470

Pigeon, . . . 60I

Rabbit, . . . 660

Sea urchin, . . . 236

Sepia, * • • • 377

Skate, . . . . 478

Snakes, . . . . 579

Spiders, . . . 332

Starfish, . . . 230

Vertebrates, . . . 427

Skin of-

Amphioxus, . . . 412

Anodonta, . . . 365

Balanoglossus, . . 392

Birds, . . . . 596

Cockroach, . . . 293

Crayfish, . . . 254

Crocodilia, . . 583,584

Earthworm, . . 189

Fishes, . . . 476

Frog, . . . 53I

Haddock, . . . 496

Helix, . . . 350

Insects, . . . 3IO

Leech, . . . 215

Lizards, . . . 57 I

Mammals, . . . 640

Myxine, . . . . 466

Peripatus, . . . 287

Petromyzon. . . . 470

Rabbit, . . . . 659

Sepia, . . . . 376

Skate, . . . . 478

Snakes, . . . 578

Tunicate, . . . 402

Skull,

Vertebrates, . . . 425

• 427, 429,43I

Crocodilia, . . 584,586

Frog, . . . 533

Haddock, . . . 497

Hatteria, . . . 568

Lizards, . . . 572

Mammals, . . . 635

Pigeon, . . . 602

Rabbit, . . . . $66 \mathrm{I}$

Skate, . . . . 480 
PAGE

Skull of Snakes, . . . 580

Skunk, . . . . 716

Sloth animalcules, .

Slotbs,

" Vertebra of,

Slow worm,

Slug,

Smynthurus,

Snails,

Snail, see Helix,

Snakes,

Solaster,

Solen,

Solenomya,

Solenostoma,

Solpuga,

Solpugidæ or Solifingæ,

Somatic cells,

Somatopleure,

Somites $=$ segments,

Sorex,

Sousliks,

Sparlella,

Spalacotherium

Spatangus,

Spatularia,

Species,

Spermathecr,

Spermatophores,

Spermatozoa,

Spermophilus,

Sphæridia,

Sphærophrya,

Sphærozoum,

Sphzerularia, .

Sphargidæ,

Sphargis,

Sphenethmoid, 566

Sphenethmoid, • • 533

Sphenodon, . . . 568

Sphenotic, . . . . 497

Sphincter muscles, - . I49

Spicules (of Sponge), . . I20

Spiders,

" Classification of

Spider monkeys,

Spinning glands of Insects,

Spinal cord," Spiders,

331

- 336

- 725

- 318

- 333 ganglia

s)

nerves,
- 439

440,443

440,442
Spiracles of Skate,

PAGF

Spiracular cartilage,

477

Spiral valve, .

482

Spirula, .

Splanchnopleure, .

Spleen,

of Frog,

of Pigeon, .

of Rabbit, .

of Skate,

Splenial,

386

$4 \mathrm{I} 5,6 \mathrm{I} 6$

- 457

- 546

- 6II

671

. 490

586,605

Sponge, Development of a, . 122 Structure of $a$, .

I $17 \cdot 120$

Spongelia,

124

Sponges,

I16-1 28

Spongicola, . . . 148

Spongilla, . . . . I22

Sporocyst, . . . . 169

Sporozoa, . . . . 103

Spring-tails, . . . 305

Squalus, . . . . 506

Squamosal, - $\quad 43 \mathrm{I}, 533,662$

Squilla, :

269

Squirrels, . . • . 7 7 I

Stagonolepis, . . . . 589

Stapes of ear, . . . 445

Starfish,

229,235

Statoblasts, . . . . I2I

Stegocephala, . . $\quad 557$

Stegosaurus, . . . . 594

Steller's Sea Cow, . . . 692

Stentor, . . . . IO5

Stephanoceros, . . . 223

Sterno-tracheal muscles, . 60I

Sternum of Amphibia, . . 535

Crustacean segment, 257

Lizards, . . 573

Monotremes, . 68I

Pigeon, . . 605

Rabbit, . . 664

Stickleback, . . . . 520

Stigmata, . . . 295

Sting of scorpion, . . . 328

Stinging Animals, . I29-I60

," cells, . . I30, I36

Stipes, . . . . . 295

Stoat, . . . . 716

Stomatodæum, . . . . 449

Stomato-gastric nerves, . . 259

Stomatopoda, . . . 276 


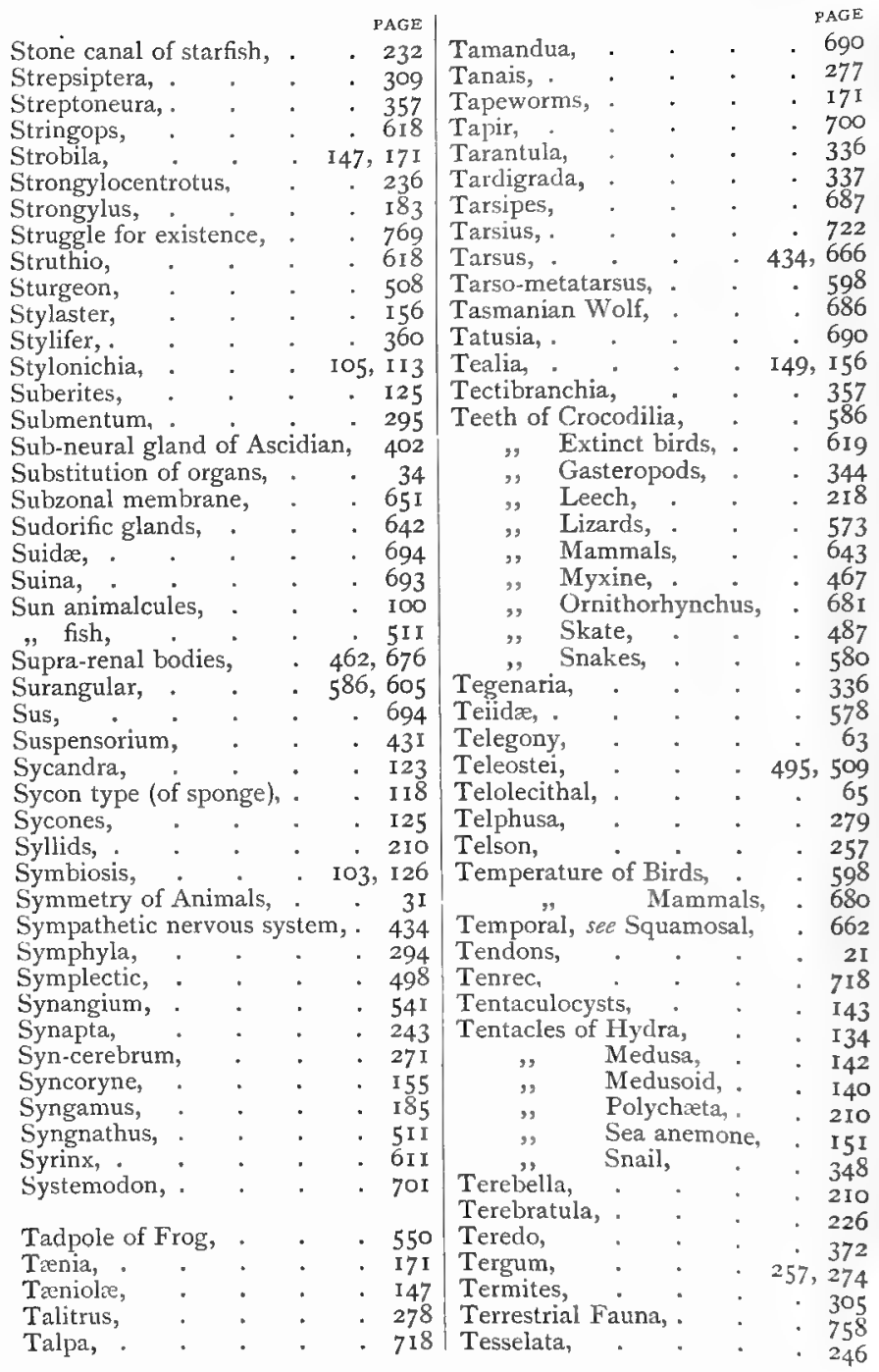


Test of ascidian,

Testicardidian, . . 402

Testurdines, . . . 226

Testudo, . . 567

Tetrab, . $\quad 567$

Tetrabranchiata, . . . 385

Tetracoralla = Rugosa, . . $\quad$ I 57

Tetractinellida, : : . 116

Tetrapneumones, : . $\quad .336$

Tetrarhyncus, . . . 336

Tetrodon, . . . 5 II

Tetronerythrin, . . . 263

Thalamencephalon, . . 439

Thalassema, . . . 2 II

Thalassicola, . . . 103

Thaliacea, . . . . 409

Theca,

Thecophora, .

I 54

Thecosomata,

566

Thelyphonus,

Thoracic duct, . . . . 674

Thoracostraca, . . . 269

Thornback, . . . 477

Thread cells = stinging cells, . I36

Threadworms,

Thrips,

Thylacinus,

180

305

Thylacoleo,

Thymus,

Thyroid,

," of Frog, .

686

687

$45^{\circ}$

546

$67 \mathrm{I}$

490

450

" of Frog,

$" \quad$ of Rabbit,

$"$ of Skate,

Thysanoptera,

Thysanozoon,

Thysanura,

Tibia,

Tibio-tarsus, .

Ticks, .

Tiedemann's bodies,

546

675

490

305

I63

305

434

598

336

233

Tiger,

Tillotherium,

Tinamou,

Tissues,

Titanotherium,

Nutrition of,

714

705

620

$37-40$

741

702

Toads, .

555

Tornaria,

396

Torpedo,

PAGE

, Electric organ of, . 478

Tortoises,

563

Toxodon, . . . 705

Trabeculæ of Skull, . . 43 I

Trachea, . . . 3I4

, of Arachnoidea, . 327

,

Mites, .

Peripatus, . . 285

" Spider, . . 333

Tracheal gills, . . . 314

Tracheata, . . . 285

Trachomedusæ, . . . 156

Trachydosaurus, "Placenta" of, 59I

Trachymeduse, . . . I56

Tragulina, . . . . 696

Tragulus, . . . . 696

Trematoda, . . . . 165

Treptoplax, . . . . 127

Triceratops, . . . . 594

Trichechidæe, . . . 716

Trichechus, . . . 716

Trichina, . . . I84

Trichocephalus, . . . I84

Trichocysts, . . . 91

Trichodes, . . . . 183

Trichodina, . . . . 105

Trichoplax, . . . . 127

Trichoptera, . . . . 304

Tricladida, . . . . 163

Triconodon, . . . 640

Tridacna, . . . . 372

Trigeminal, . . . . 44I

Trilobites, . . . . 34I

Trionychide, . . . 567

Trionyx, . . . 567

Tristomum, . . . 170

Triton, . . . . 556

Tritylodon, . . . . 640

Trivium, . . . 230

Trochanter, . . 308, 666

Trochoceras, . . 385

Trochlear nerve, . . . 441

Trochosphere, . . 208

Troglodytes, . . . . 726

Trombidium, . . . 336

Trophoblast, . . . 65 1

Trophospongia, . . . 653

Tropidonotus, . . . 583 
Truncus arteriosus, . . 54I

Trunk fishes, . . . 5II

Trypsin, . . . . 22

Tuber cinereum, . . . 537

Tubercle of rib, . . . 66 I

Tube feet of Brittle star, - 235

Sea urchin, . 239

Starfish, . 229

Tubifex, . . . I74, 209

Tubipora, . . . 153, I57

Tubularia, . . . I40, I55

Tunicata, . . . . 400

Tunicin, Classification of, - 408

Tupaia, * * 744

Turbellaria, , , I62

$\begin{array}{lll}\text { Turrilites, Classification of, } & \text { I62 } \\ \cdot & \cdot & 385\end{array}$

Turtles, . . . . 563

Tylenchus, . . . . 183

Tylopoda, . . . . 695

Tympanic bulla, . . . 663

Tympanum, . . . . 445

Typhlopidæ, . . . . 583

Typhlops, . . . $\quad 5^{83}$

Typhlosole, . . . . I92

Typotherium, . . . 705

Tyroglyphus, . . . 336

Uintatherium, . . . 704

Ulna, • . . . . 434

Umbilical Cord, . . 495, 65 I

"Vesicle, . . 651

Umbo, . . . . 364

Umbrella, . . . . 357

Uncinate processes, . . 602

Ungulata, . . . . 693

Unio, . . . . 372

Ureters, . . . . 46 I

Urethra, . . . . 678

Urinogenital ducts, . . 46I

Urnatella, . . . . 225

Urochorda, . . . . 400

Urodela, . . . . 556

Urostyle, . . . . 532

Ursidæ, . . . . 7 7 6

Ursus, . . . . 716

Uterus, . . . . . 678

, masculinus, . '. 677

Utricles,

TAGE

Utriculus of Ear, . . . 444

Vacuoles, . . . 92, 108

" Contractile, . 86, IoI

" Food, . . 86, I 10

Vagina, . . . . 678

Vagus nerve, . . . 44I

Valves of Mammalian heart, . $67 \mathrm{I}$

Vampire bat, . . . 721

Vampyrella, . . . . 98

Vampyrus, . . . . 721

Varanidæ, . . . . 577

Variation, . . . . 766

Vas deferens, . . . 462

Vascular System of-

Amphioxus, . . . $4 \mathrm{I} 6$

Anodonta, . . . 367

Arenicola, . . . 204

Ascidian, . . . 406

Balanoglossus, . $\quad 395$

Bee, . . . 306

Cockroach, . . . 297

Crayfish, . . . 263

Crinoid, . . . 245

Crocodilia, . $\quad 584,587$

Dipnoi, . . . 516

Earthworm, . . . 192

Frog, . . . 54I

Haddock, . . . 500

Helix, . . . . 353

Hirudo, . . . 218

Insects, . . . $3 I 5$

Limulus, . . . 339

Lizards, . . . 574

Mammalia, . . . 671

Myxine, . . . 468

Nemerteans, . . . 179

Peripatus, . . . 287

Petromyzon, . . . 472

Pigeon, . . . 609

Rabbit, . . . 67I

Scorpion, . . . 330

Sea urchin. . . . 239

Sepia, . . . 380

Skate, . . . . 488

Spider, . . . 332

Starfish, . . . 234

Vertebrates, . . . 453

Velarium, . . . 142 
P'AGE

Velella,

Veliger,

Velum,

Ventricles of brain,

heart,

Venus",

Venus' flower basket, girdle,

Vermiform appendix,

Vertebra, Parts of a,

Vertebral column,

Vertebral column of Crocodile, 584

\begin{tabular}{|c|c|c|c|}
\hline$"$ & , & Frog, & \\
\hline$"$ & , & Haddock & \\
\hline " & $"$ & Lizard, & \\
\hline$"$ & "' & Pigeon, & \\
\hline ", & " & Rabbit, & \\
\hline$"$ & "heory of & Skate, & \\
\hline
\end{tabular}

Vertebrata, • . 4 42I

$42 \mathrm{I}$

"Affinities of Annelids with, 424

$" \quad, \quad$ of Nemerteans with, .

425

Ancestry of, . . . 424

"Development of, . 425-464

" General characters of, . 421

" $" \quad$ classification of, 423

"Gill clefts of, . . . 449

"Heart of, . . . 454

", and Invertebrata, . 5

"Nervous system of, . 434

", Notochord of, . . 432

" Origin of, . . . 42I

"Segmental symmetry of, $42 \mathrm{I}$

." Structure of, . . 425, 464

Vesiculæ seminales of Earthworm,

I I4

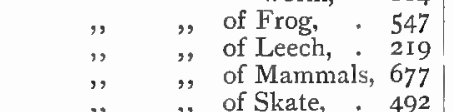

Vesiculatxe, ", of Skate, . I4I

Vespertilio, . . . . 721

Vesperugo, . • . . 721

Vestigial structures, . . 36

Vibrissæ,

669

Vicugna,

696

Villi.

23

Vipera, .

Viperiformes, .
Visceral arches, , $\quad 427$

clefts, . . . 449

nerves, . . . I9I

membrane of ovum, 57,613

Vitreous humour, . . . 446

Viverra, . . . . 7I5

Viviparous Fishes, . . . 526

Insects, . . 3I8

Lizards, . . 577

Vertebrates, . 464

Voice of Birds, . . . 625

Vole, . . . . 7II

Volvox, . . . . 69,94

Vortex, . . . . I62

Vorticella, . . . 93, 105

Waldheimia, , , , 226

Walking-stick insect, . . 305

Wallaby, leaf, : $\quad \cdot 305$
687

Walrus, . . . . 716

Wasps, . . . 304

Water bears, . . . . 337

,, Scorpion, . . . 305

"Vascular System, of-

$\because \quad$ Crinoid, : . 245

"Ophiuroid, . . 235

,$\quad$ Sea urchin, . . 239

", Starfish. . . . 232

Weasel, . . . . 7 I6

Web of Spiders, . . . 334

Whales, . . . . 709

Wheel animalcule,. . . 223

Whelk, . . . . 357

Whip scorpions, . . . 33I

White matter of Brain, . . 439

" Spinal Cord, 440

White of eyes, . . 613

Wing of bat, . . . 719

birds, . . . 624

insect, . . 308

pterodactyl, . . 594

Wolf, . . . 7 75

Wolffian duct, . . . 46I

Wombat, . . . 686

Worms, . . $7,8,16 \mathrm{I}-226$

Wrass, . . 510

. 583 Xantharpyia, . . $72 \mathrm{I}$ 


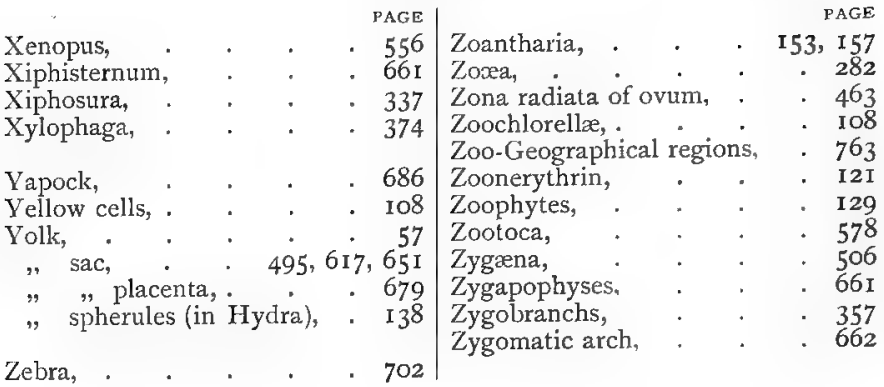



,

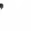




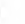


